\title{
State Energy Price and Expenditure Report 1989
}

\section{September 1991}

Energy Information Administration

Office of Energy Markets and End Use

U.S. Department of Energy

Washington, DC 20585

DISTRIBUTION OF THIS OOCUMENT IS UNLMTED $\mathrm{C}^{i^{2}}$

This report was prepared by the Energy Information Administration, the independent statistical and analytical agency within the Department of Energy. The information contained herein should not be construed as advocating or reflecting any policy position of the Department of Energy or any other organization. 


\section{Contacts}

The State Energy Price and Expenditure Report is prepared in the Energy Information Administration (EIA). General information may be obtained from W. Calvin Kilgore, Director, Office of Energy Markets and End Use, 202-586-1617; Arthur T. Andersen, Director, Economics and Statistics Division, 202-586-1441; Katherine E. Seiferlein, Chief, Statistics Branch, 202-586-5692.

Questions concerning the contents of the State Energy Price and Expenditure Report may be referred to Julia F. Hutchins 202-586-5138, Roy M. Stanley 202-586-5839, or Raymond Boyer 202-586-1680 in the Statistics Branch. 


\section{Contents

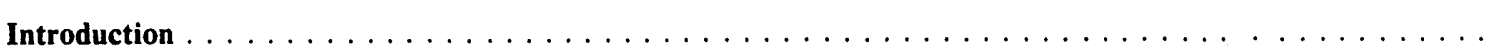

Page

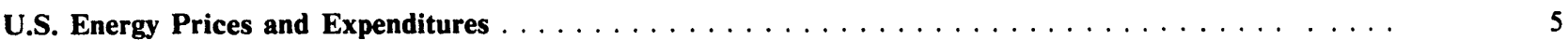

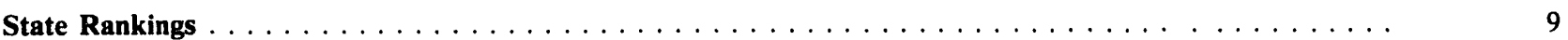

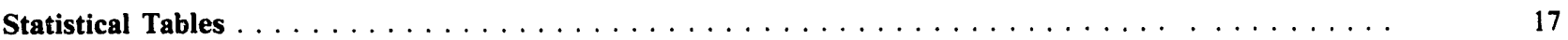

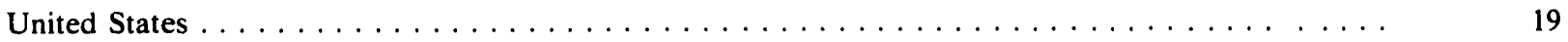

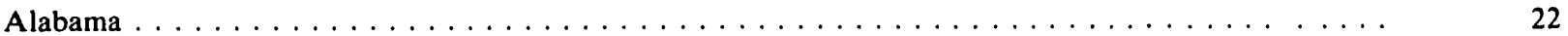

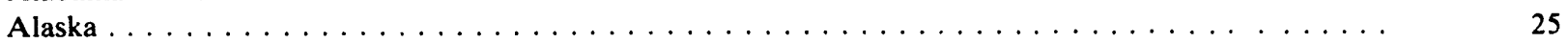

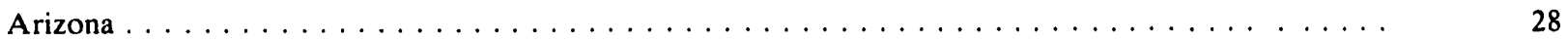

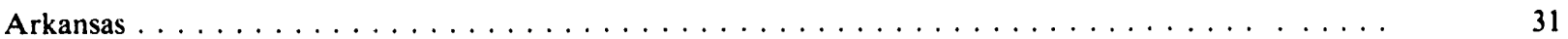

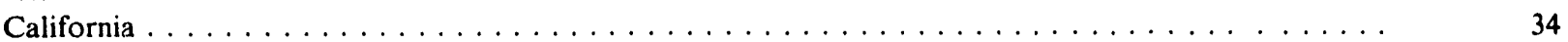

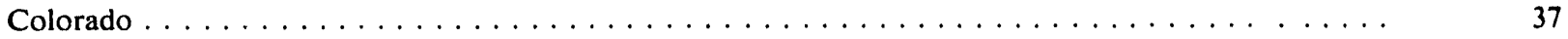

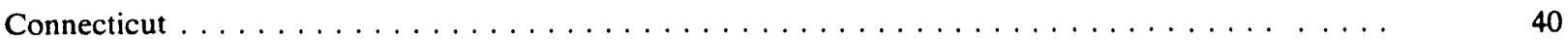

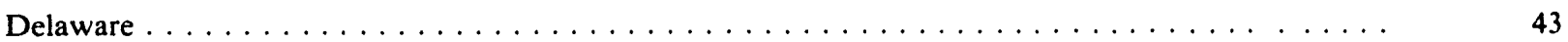

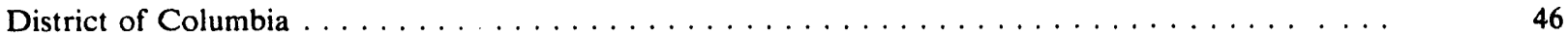

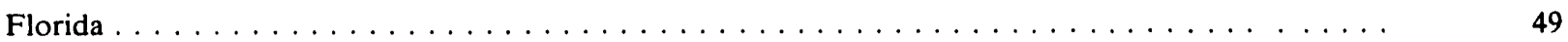

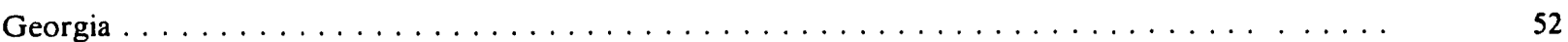

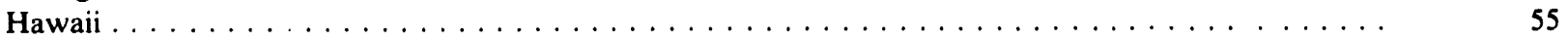

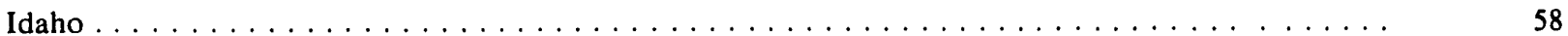

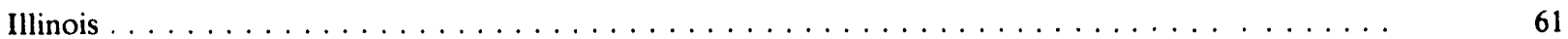

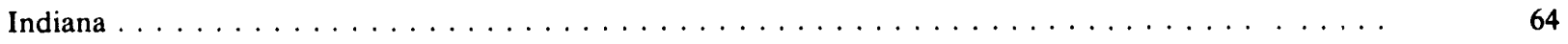

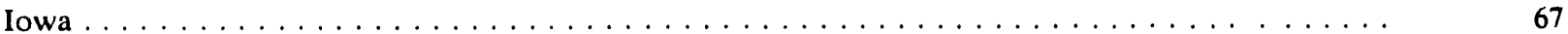

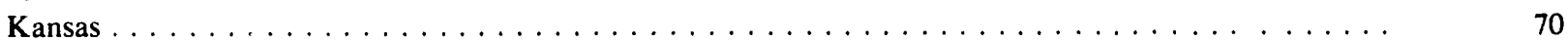

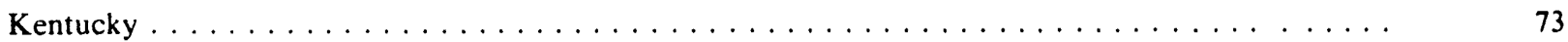

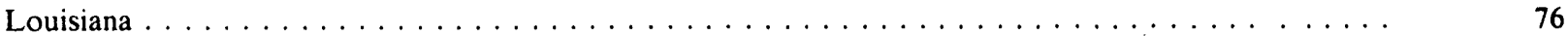

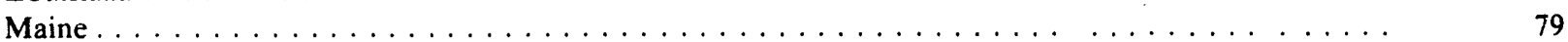

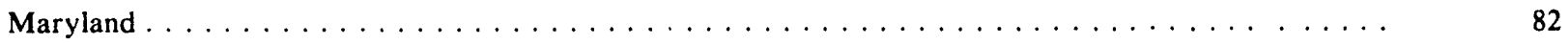

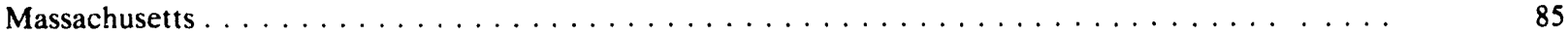

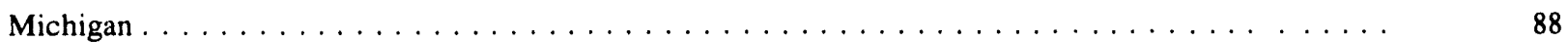

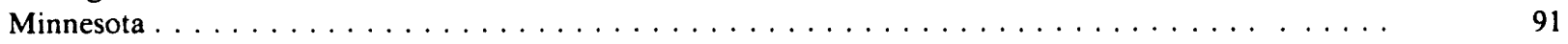

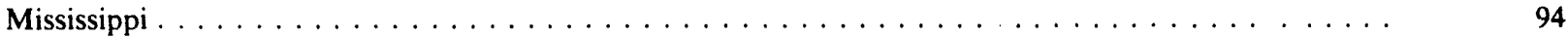

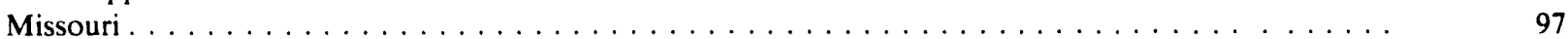

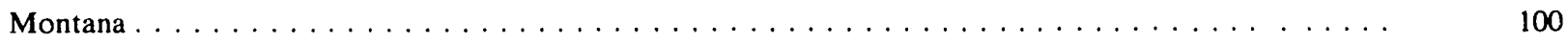

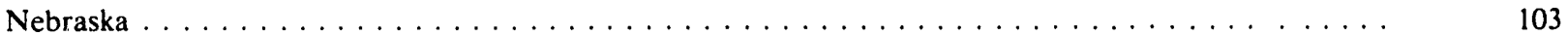

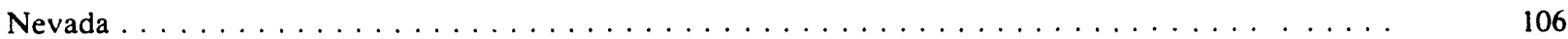

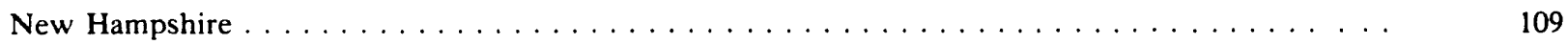

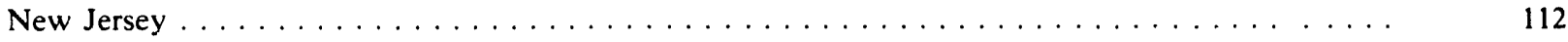

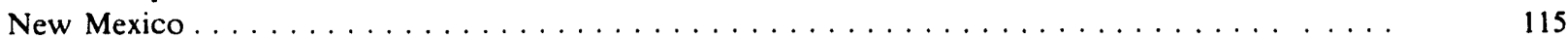

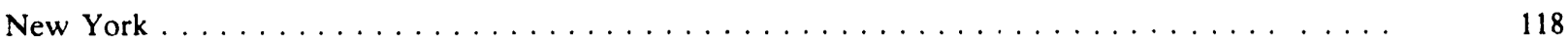

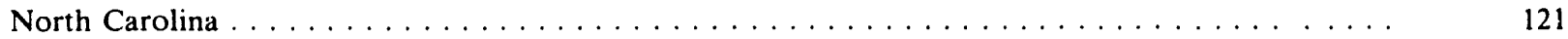

North Dakota . . . . . . . . . . . . . . . . . . . . . . . . . . . . . . . . . . . . . . . . 124

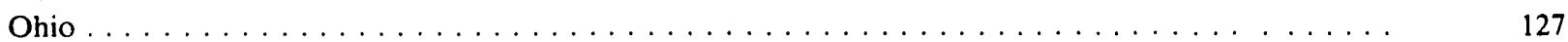

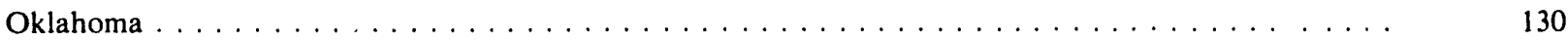

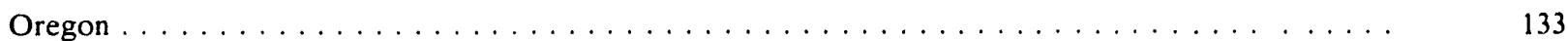

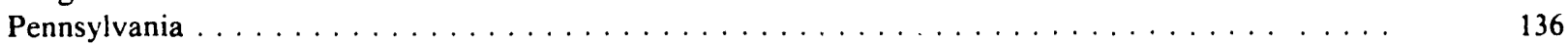

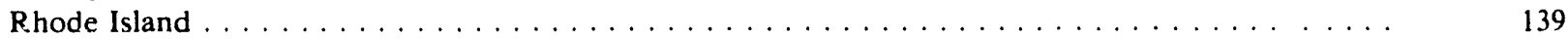

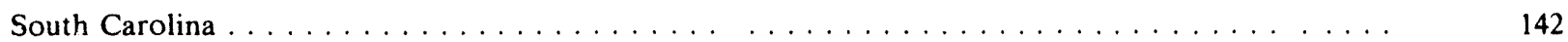

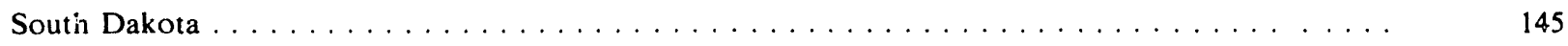

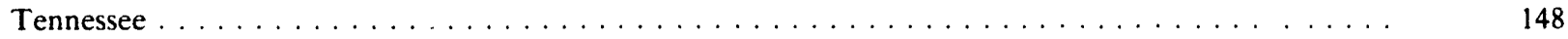

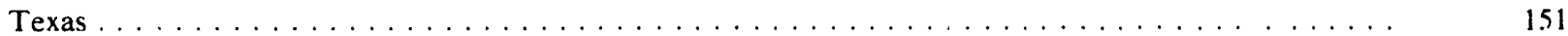

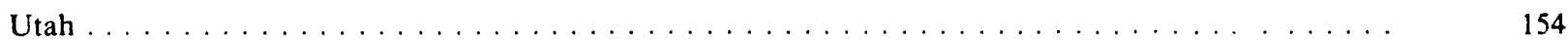




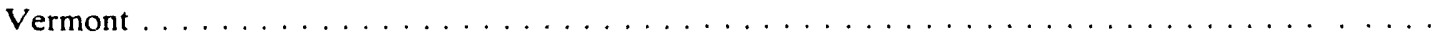

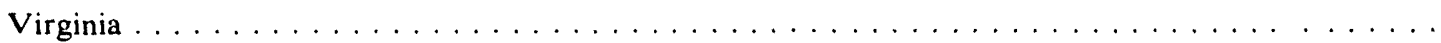

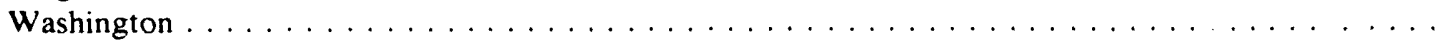

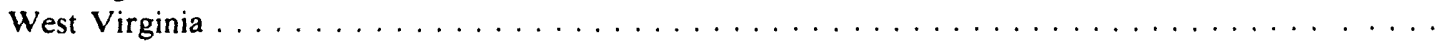

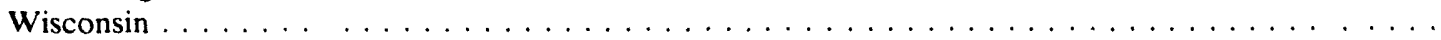

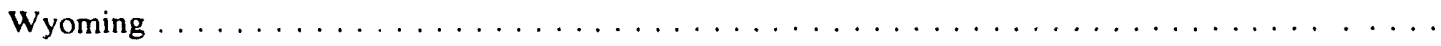

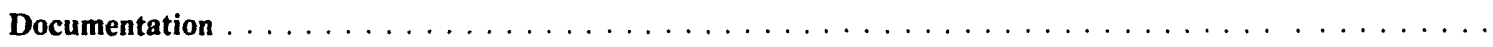

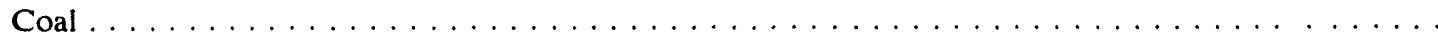

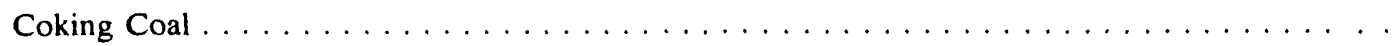

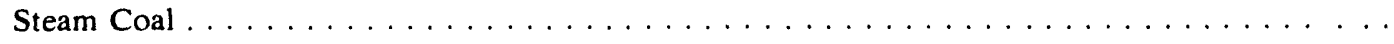

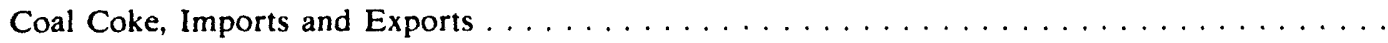

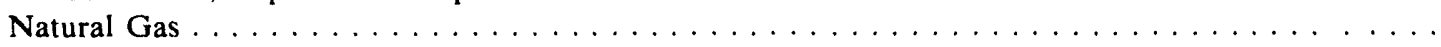

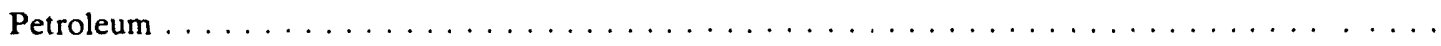

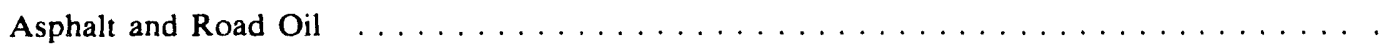

Aviation Gasoline . . . . . . . . . . . . . . . . . . . . . .

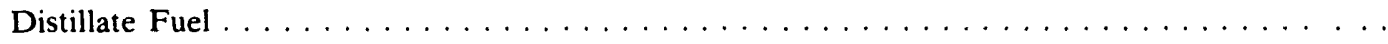

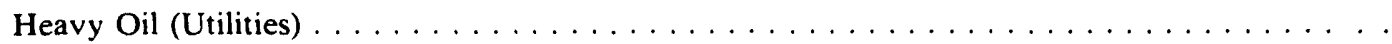

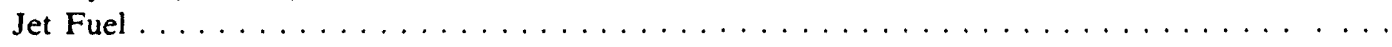

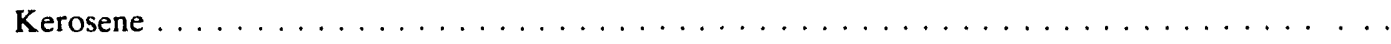

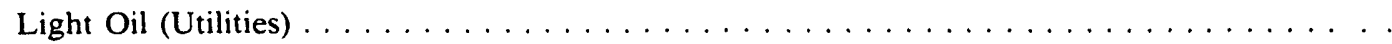

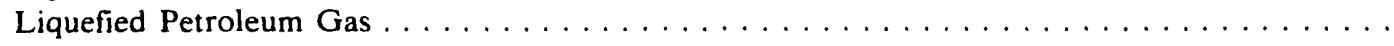

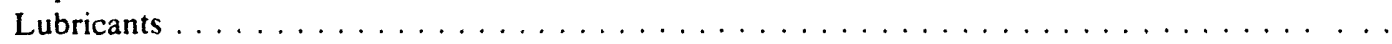

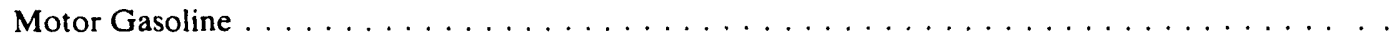

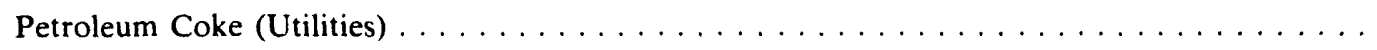

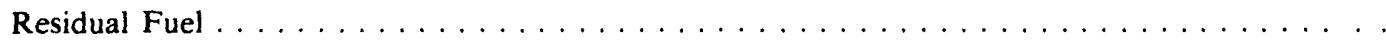

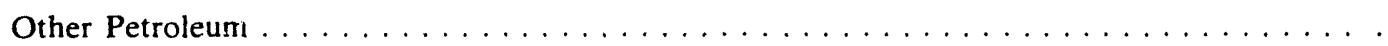

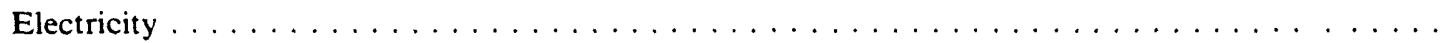

Electricity Consumed by End-Use Sectors $\ldots \ldots \ldots \ldots \ldots \ldots \ldots \ldots \ldots \ldots \ldots$

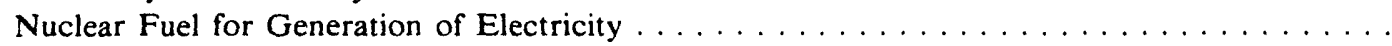

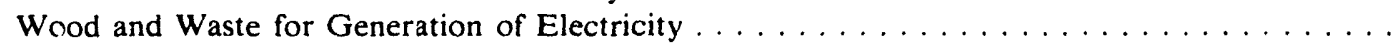

Consumption Adjustments for Process Fuel and Intermediate Products . . . . . . . . . . . .

Summary of Changes Since the State Energy Price and Expenditure Report $1988 \ldots \ldots \ldots \ldots \ldots \ldots$

Additional Measures of Energy Consumption, Expenditures, and Price $\ldots \ldots \ldots \ldots \ldots$ 


\section{Introduction}

The State Energy Price and Expenditure Report (SEPER) presents energy price and expenditure estimates for the 50 States, the District of Columbia, and the United States. The estimates are provided by energy source and economic sector. This report is an update of the State Energy Price and Expenditure Report 1988 published in September 1990. Changes from the last report are summarized in a section of the documentation.

Energy price and expenditure estimates are published for the years 1970,1975, 1980, and 1985 through 1989. Documentation follows the tables and describes how the price estimates are developed, including sources of data, methods of estimation, and conversion factors applied. Data for all years, 1970 through 1989, are available on personal computer diskettes. Consumption estimates used to calculate expenditures, and the documentation for those estimates, are from the State Energy Data Report, Consumption Estimates, 1960-1989 (SEDR), published in May 1991. Expenditures are calculated by multiplying the price estimates by the consumption estimates, adjusted to remove process fuel and intermediate product consumption. All expenditures are consumer expenditures, that is, they represent estimates of money directly spent by consumers to purchase energy, generally including taxes.

Although all prices and expenditures in this report are expressed in nominal dollars, an appendix entitled "Additional Measures of Energy Consumption, Expenditures, and Prices" describes the methodology used to develop energy expenditure estimates in constant 1982 dollars, an implicit energy price deflator, and a fixedweight energy price index. A national-level analysis illustrating the use of these estimates is also presented in the appendix.

Reliable State $^{1}$ data rarely exist, especially as series that are consistent over a long period. Estimates and assumptions are applied to fill data gaps and to maintain consistent definitions in the data series over time. $S E P E R$ incorporates the most consistent series and procedures possible. Users of this report (and of the personal computer diskettes) should recognize the limitations imposed on the system due to changing and inadequate data sources. Estimates often are based on a variety of surrogate measures that are selected on the basis of availability, applicability as indicators, continuity over time, and consistency among the various energy commodities. Original source documents for data used in SEPER (cited in the documentation) include descriptions of collection methodologies, universes, imputation or adjustment techniques (if any), and errors associated with the individual processes. Due to the numerous collection forms and procedures associated with these reports, it is not possible to develop a meaningful numerical estimate of the overall errors of the material published in SEPER.

The five economic sectors used in SEPER correspond to those used in $S E D R$ as follows:

- Residential Sector--The residential sector is considered to consist of all private residences, whether occupied or vacant, owned or rented, including single-family homes, multifamily housing units, and mobile homes. Secondary homes, such as summer homes, are also included. Institutional housing, such as school dormitories, hospitals, and military barracks generally are not included in the residential sector; they are included in the commercial sector. The SIC code used to classify an establishment as residential is 88 (Household).

- Commercial Sector--The commercial sectcr, as defined economically, consists of business establishments that are not engaged in transportation or in manufacturing or other types of industrial activity (agriculture, mining, or construction). Commercial establishments include hotels, motels, restaurants, wholesale businesses, retail stores, laundries, and other service enterprises; religious and nonprofit organizations; health, social, and educational institutions; and Federal, State, and local governments. Street lights, pumps, bridges, and public services are also included if the establishment operating them is considered commercial. SIC codes used to classify an establishment as commercial are 50 through 87, 89, and 91 through 97.

- Industrial Sector--The industrial sector comprises manufacturing industries, which make up the largest part of the sector, along with mining, construction, agriculture, fisheries, and forestry. Establishments in the sector range from steel mills, to small

${ }^{1}$ Throughout this report, the term "State" includes the District of Columbia. 
farms, to companies assembling electronic components. The SIC codes used to classify establishments as industrial are 1 through 39.

- Transportation Sector--Private and public vehicles that move people and commodities. Included are automobiles, trucks, buses, motorcycles, railroads and railways (including streetcars), aircraft, ships, barges, and natural gas pipelines. The SIC codes used to classify establishments as belonging to the transportation sector are 40 through 49 .

- Electric Utility Sector--Privately and publicly owned establishments that generate electricity primarily for use by the public.
Although end-use allocations of energy consumption and expenditures follow those guidelines as closely as possible, some data are collected using different classifications. For example, agricultural use of natural gas should be included in the industrial sector. However, since it is not separately identified on the data collection form, there is no basis for allocating it to the industrial sector, and it remains in the commercial sector. Another example is master-metered condominiums, apartments, and establishments with a combination of residential and commercial units. In many cases, residential use is counted in the commercial sector because it is metered and billed as commercial. In those cases, there is no basis for separating residential from commercial use. Readers are advised to consult the

\section{Taxes in SEPER}

While the objective is to provide price estimates that include all taxes, the data sources used in SEPER do not treat taxes uniformly. In general, where taxes are included in the source data, they are included in SEPER. Where taxes are not included but can be separately estimated, they are generally added. In many cases, particularly in the early 1970's, it is not clear whether taxes are included in the source data and no adjustments are made. EIA is continuing to analyze these cases to see if the presence of taxes can be determined. The status of tax data in this year's report is summarized below and described more fully in the documentation for each energy source and sector.

\section{End-Use Sectors}

Coal. Residential steam coal and industrial coking coal prices include taxes in all years. Commercial, industrial, and transportation sector steam coal prices include taxes from 1980 forward; it is not clear from the data sources whether taxes are included in the prices for earlier years. Appropriately, coal imports and exports in the industrial sector do not include taxes.

Natural Gas. Natural gas prices are intended to include all federal, State, and local taxes, surcharges, and adjustments billed to consumers. However, sales and other taxes itemized directly on customers' bills are typically not reported as revenues and therefore are not included in calculating the prices.

Petroleum. Motor gasoline and diesel fuel prices include excise and other per-gallon taxes but do not include general sales taxes due to wide variation at the local level. Residual fuel oil and asphalt and road oil prices include all taxes. Distillate fuel oil and kerosene prices include taxes, generally from 1983 forward; it is not clear from the data sources whether taxes are included in the prices for earlier years. Residential liquefied petroleum gas prices include taxes in all years, but commercial, industrial, and transportation sector prices do not include taxes from 1985 forward. Jet fuel, aviation gasoline, lubricants and other petroleum products do not include taxes. Other petroleum products are miscellaneous products, petrochemical feedstocks (naphtha, other oils, and still gas), industrial petroleum coke, special naphthas, and waxes.

Electricity. Taxes paid directly by electric utilities (rather than end users) are considered operating costs and are passed on to end users as part of the price. Depending on jurisdiction, taxes collected from end users and turned over directly to a governmental authority are not included in reported revenues and therefore are not included in calculating the prices.

\section{Electric Utility Sector}

Coal, natural gas, petroleum, petroleum coke, nuclear, and wood and waste prices include all taxes, transportation, and handling costs. There are no direct fuel costs (or taxes) for hydroelectric, geothermal, centralized solar, or wind energy. Capital costs and related taxes associated with these energy sources are included indirectly because electricity prices reflect their presence in the rate base. 
$S E D R$ documentation for specific assumptions regarding the consumption estimates.

Where prices for an energy source and sector are not available, comparable prices are substituted. For example, the transportation sector motor gasoline prices are also applied to the commercial and industrial sectors. In some cases, the average of adjacent States' prices is assigned to a missing State price. The documentation elaborates on these price assumptions. There are no direct fuel costs for hydroelectric, geothermal, centralized solar, or wind energy. Capital costs associated with these energy sources frequently are extensive and are included indirectly because the prices charged for electricity reflect their presence in the rate base.

Except where specified, it is not generally possible to describe the prices in this report as "wholesale" or "retail." The prices paid in each consuming sector are actually a combination of both sets of prices depending on a number of closely interrelated factors, and the data reflect the combination of prices actually paid by each sector. Almost all residential sector prices are close to retail, reflecting the relatively small quantities of individual purchases and the increased costs of ex- tensive, diffuse, and multilayered distribution systems. Similarly, in the transportation sector almost everyone pays the same retail-like price for motor gasoline, regardless of volume purchased or location of purchase. Conversely, residual fuel oil prices in the transportation sector are certainly more wholesale-like as a result of large deliveries to bulk facilities in major ports. In the same manner, most large industrial and many large commercial expenditures can be thought of as near wholesale, frequently involving direct access to a producer or bulk distribution facility for very large quantities. Many smaller industrial and commercial facilities pay something much closer to retail prices as a result of the small quantities involved and their institutional distance from primary suppliers. Notable exceptions to these relationships include natural gas and electric utilities, which typically establish fixed rates for each of several classes of service depending on representative quantities, service factors, and distribution expenses.

Under contract to the Energy Information Administration, the Battelle Pacific Northwest Laboratories developed many of the price series and estimating procedures incorporated into this report. Descriptions of those data series and procedures are included in the documentation section of this report. 


\section{U.S. Energy Prices and Expenditures}

The U.S. energy bill totaled $\$ 437$ billion in $1989 .^{2}$ While the total amount of energy consumed in the United States grew by 1 percent in 1989 reaching 81 quadrillion Btu, the average price of energy increased by 6 percent to $\$ 7.72$ per million Btu, causing energy expenditures to increase by 7 percent. In 1989, consumers used almost the same amount of energy per person as they had in 1970; however, including inflation, they spent 4 times as much money per person for that same amount of energy (Table 1).

Expenditures by Energy Source. Americans purchased $\$ 209$ billion worth of petroleum products in 1989, 48 percent of their total expenditures on energy (Figure 1). Motor gasoline purchases alone cost $\$ 113$ billion, 26 percent of total expenditures. While these shares represent significant portions of the total, they are reduced from the 1970 shares when petroleum took 58 percent of all expenditures and motor gasoline accounted for 38 percent. The Nation spent $\$ 65$ billion in natural gas purchases in 1989,15 percent of the total

Table 1. Energy Consumption and Expenditures per Capita, 1970-1989

\begin{tabular}{lcccc}
\hline & \multicolumn{2}{c}{$\begin{array}{c}\text { Consumption } \\
\text { per Capita }\end{array}$} & \multicolumn{2}{c}{$\begin{array}{c}\text { Expenditures } \\
\text { per Capita }\end{array}$} \\
\hline & $\begin{array}{c}\text { Million } \\
\text { Btu }\end{array}$ & $\begin{array}{c}\text { Index } \\
\mathbf{1 9 7 0} \mathbf{1 . 0 0}\end{array}$ & Dollars & $\begin{array}{c}\text { Index } \\
\mathbf{1 9 7 0}=\mathbf{1 . 0 0}\end{array}$ \\
\hline 1970 & 326 & 1.00 & 406 & 1.00 \\
1971 & 328 & 1.00 & 434 & 1.07 \\
1972 & 341 & 1.04 & 467 & 1.15 \\
1973 & 352 & 1.08 & 528 & 1.30 \\
1974 & 340 & 1.04 & 718 & 1.77 \\
1975 & 328 & 1.00 & 797 & 1.96 \\
1976 & 342 & 1.05 & 890 & 2.19 \\
1977 & 347 & 1.06 & 1,002 & 2.47 \\
1978 & 352 & 1.08 & 1,076 & 2.65 \\
1979 & 351 & 1.08 & 1,323 & 3.26 \\
1980 & 335 & 1.03 & 1,650 & 4.06 \\
1981 & 322 & 0.99 & 1,857 & 4.57 \\
1982 & 305 & 0.94 & 1,831 & 4.51 \\
1983 & 301 & 0.92 & 1,775 & 4.37 \\
1984 & 313 & 0.96 & 1,833 & 4.51 \\
1985 & 310 & 0.95 & 1,825 & 4.49 \\
1986 & 308 & 0.94 & 1,582 & 3.89 \\
1987 & 315 & 0.97 & 1,617 & 3.98 \\
1988 & 326 & 1.00 & 1,658 & 4.08 \\
1989 & 328 & 1.00 & 1,759 & 4.33 \\
\hline
\end{tabular}

Note: Indices are based on unrounded data

Sources: State Energy Data System 1989 and State Energy Price and Expenditure Data System 1989. amount spent on energy. The natural gas portion of the U.S. total energy bill ranged from a low of 11 percent in 1974 to a high of 18 percent in 1984. Coal purchases in 1989 totaled $\$ 28$ billion, 6 percent of the national total energy expenditures. During the 1970-89 period, coal's portion of the national energy expenditures remained steady at 5 percent to 8 percent of the total.

In 1989, purchases of primary energy sources by the four major end-use sectors, which exclude electric utilites, cost $\$ 267$ billion while electricity purchases totaled $\$ 169$ billion, 61 percent and 39 percent of total expenditures, respectively. In comparison, primary energy purchases in 1970 accounted for 72 percent of total expenses while electricity's share was only 28 percent.

Expenditures by End-Use Sector. The largest portion of the 1989 U.S. energy expenditure was spent in the transportation sector, $\$ 157$ billion. The residential

Figure 1. U.S. Energy Expenditures by Source,

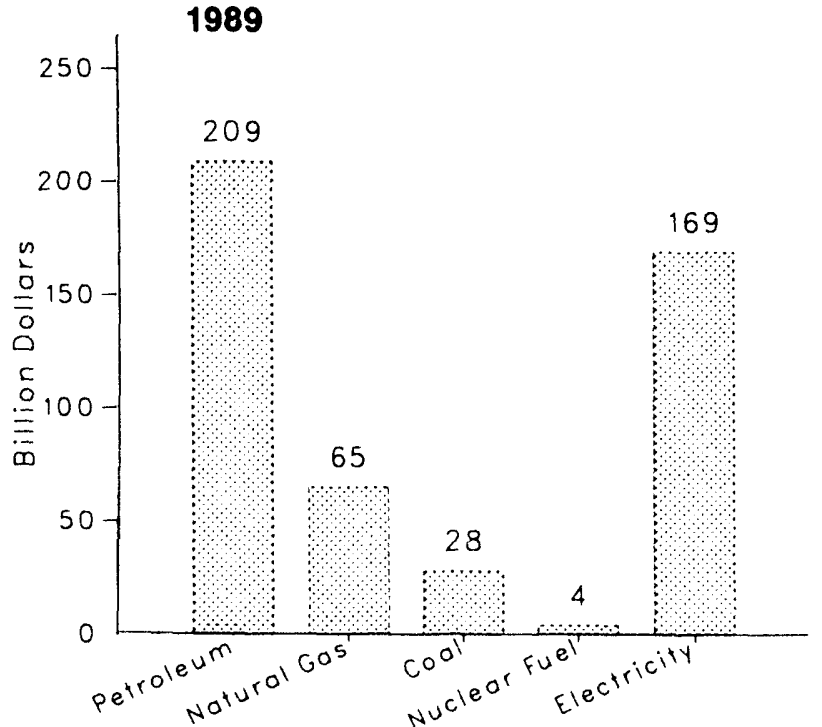

Note: Doto are not odditive due to expenditure components not shown and the double-counting of primary fuels in electricity. See U.S. expenditures table on poge 19 1989 . Source: Stote Energy Price and Expenditure Doto System

${ }^{2} \mathrm{All}$ prices and expenditures are expressed in nominal dollars. 
U sector was next, at $\$ 109$ billion, followed by the indusN trial and commercial sectors, spending $\$ 95$ billion and N $\$ 76$ billion, respectively. While the transportation secI tor exceeded 0 :her sectors in expenditures throughout T the $1970-89$ period, its relative share of the total genE erally decreased (Figure 2). The commercial sector's D share of expenditures expanded the most beginning in the early 1980 's, reflecting the consumption growth in that sector. The industrial and residential sectors each 5 spent close to one quarter of the Nation's total energy $T$ expenses during the period studied, with the industrial sector's portion larger in 1974-85 and the residential sector spending more at the beginning and ending of the period. In 1970, the sectors' shares of total expenditures were transportation, 43 percent; residential, 24 percent; industrial, 20 percent; and commercial, 13 percent. By 1989, the transportation sector's share was 7 percentage points lower at 36 percent; the residential and industrial sectors' portions were about the same--residential, 25 percent, and industrial, 22 percent; and the commercial sector's share increased to 17 percent.

In 1989, electricity purchases accounted for the largest portion of the residential, commercial, and industrial sectors' energy bills (Figure 3). The transportation sector purchased petroleum products for energy almost exclusively. The residential and commercial sectors' spending for energy were parallel; natural gas, as the second-largest expense, accounted for over twice as much as the petroleum purchases did. Although electricity accounted for the largest energy expenditure in the industrial sector in 1989, industries relied more heavily on petroleum products than did the residential
Figure 2. End-Use Sector Shares of U.S. Total Energy Expenditures, 1970-1989

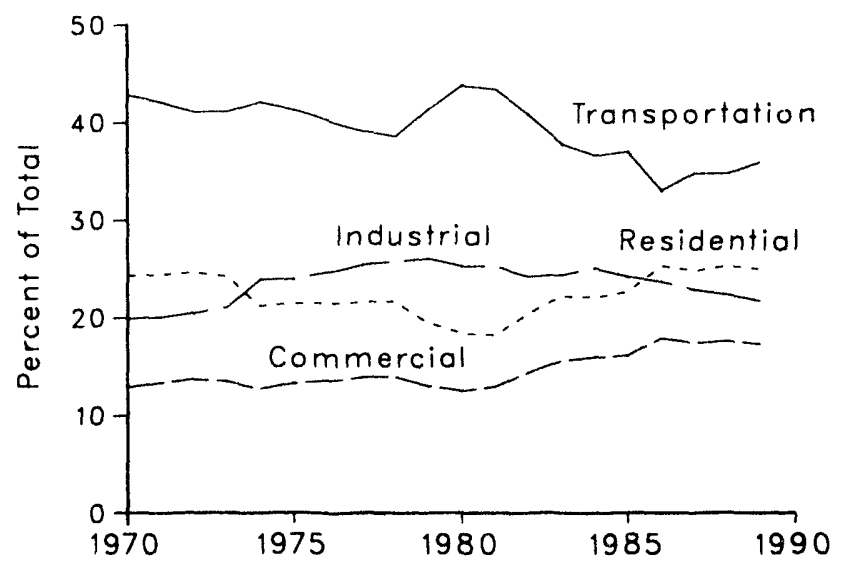

Source: State Energy Price ond Expenditure Data System 989

and commercial sectors. Natural gas and coal expenses were lower than petroleum in the industrial sector, and their combined costs were less than the amount spent on petroleum products.

Prices by Energy Source. Of the major energy sources in 1989, electricity registered the highest price per million Btu (\$18.98), followed by petroleum (\$6.48), natural gas (\$3.85), coal (\$1.48), and nuclear fuel (\$0.70) (Figure 4). The prices of these energy sources have remained in the same relative order throughout the

Figure 3. U.S. Energy Expenditures by End-Use Sector and Source, 1989

\section{(Billion Dollars)}

Resldentlal

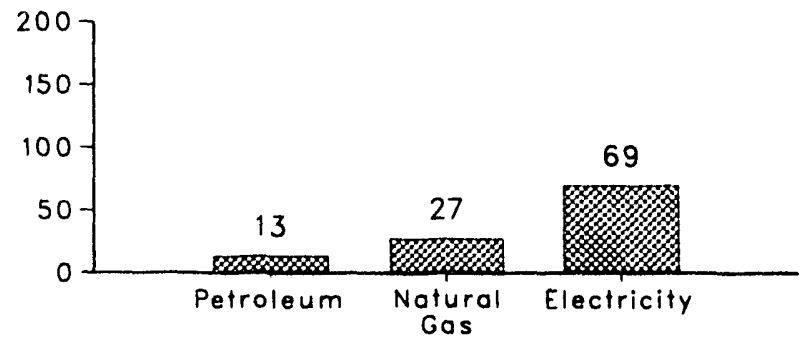

Induetrlal

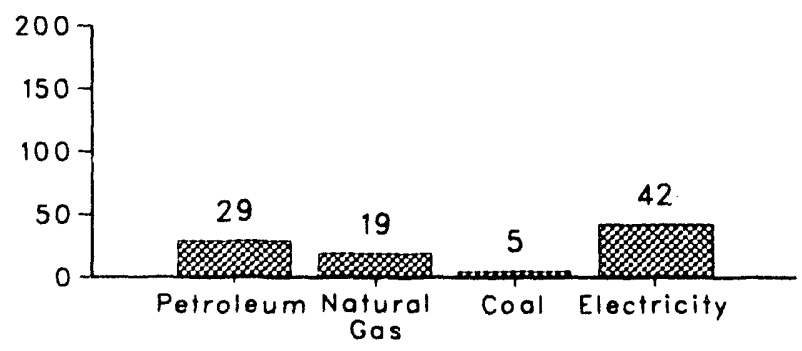

$(s)=$ Less than 0.5 billion dollors.

Source: State Energy Price and Expenditure Doto System 1989.

\section{Commerclal}

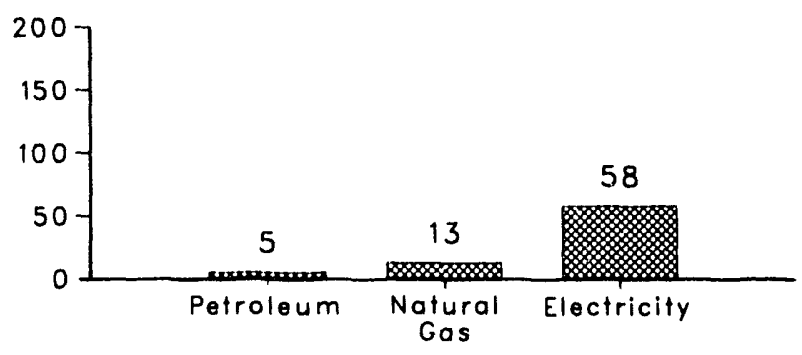

Traneportation

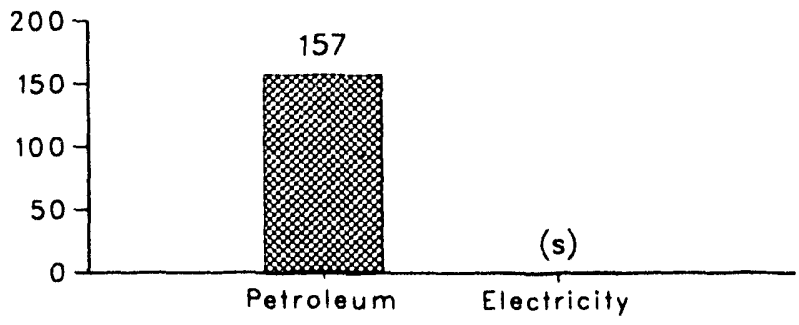


Figure 4. U.S. Energy Prices by Source, 1989

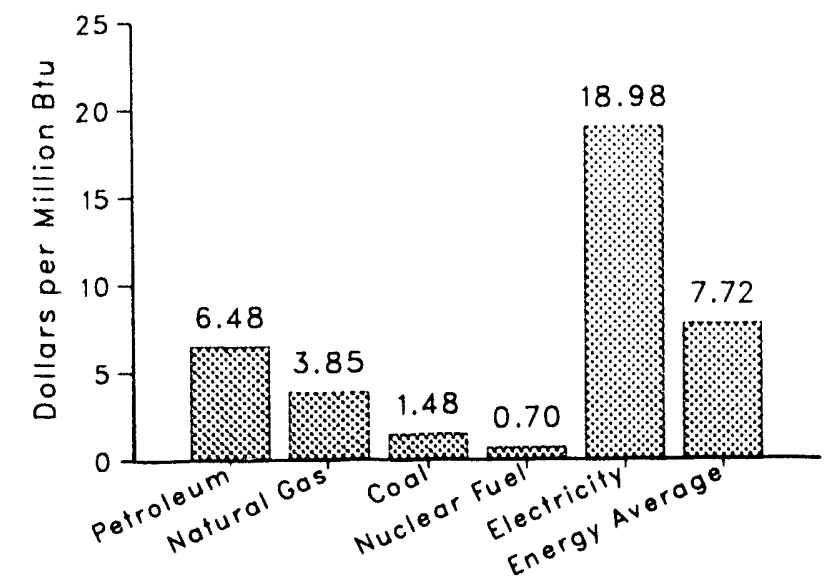
1989

1970-89 period. The price of electricity is considerably higher because of significant costs for converting energy from various forms (e.g., fossil fuels, nuclear fuel, hydroelectric energy, and geothermal energy) into electricity as well as somewhat smaller costs for transmitting and distributing electricity to end users. In addition, electricity is a premium form of energy because of its flexibility and clean nature at energy consumers' sites.
Figure 5. U.S. Energy Prices by End-Use Sector, 1989

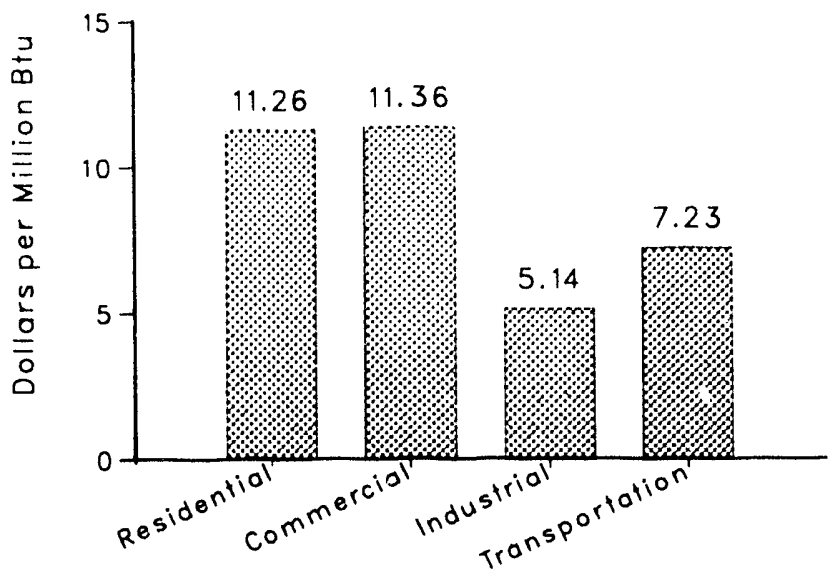
Source: State Energy Price and Expenditure Dato System 1989 .

Prices by End-Use Sector. Because of the differing mixes of the energy sources consumed, the average prices paid by major end-use sectors in the U.S. economy varied considerably (Figure 5). The commercial and residential sectors' average prices were the highest in $1989, \$ 11.36$ per million Btu in the commercial sector and \$11.26 per million Btu in the residential sector. The transportation sector's average price of $\$ 7.23$ per million Btu reflects that sector's reliance on petroleum products. Although the industrial sector consumed

Figure 6. U.S. Energy Prices by End-Use Sector and Source, 1989 (Dollars per Million Btu)
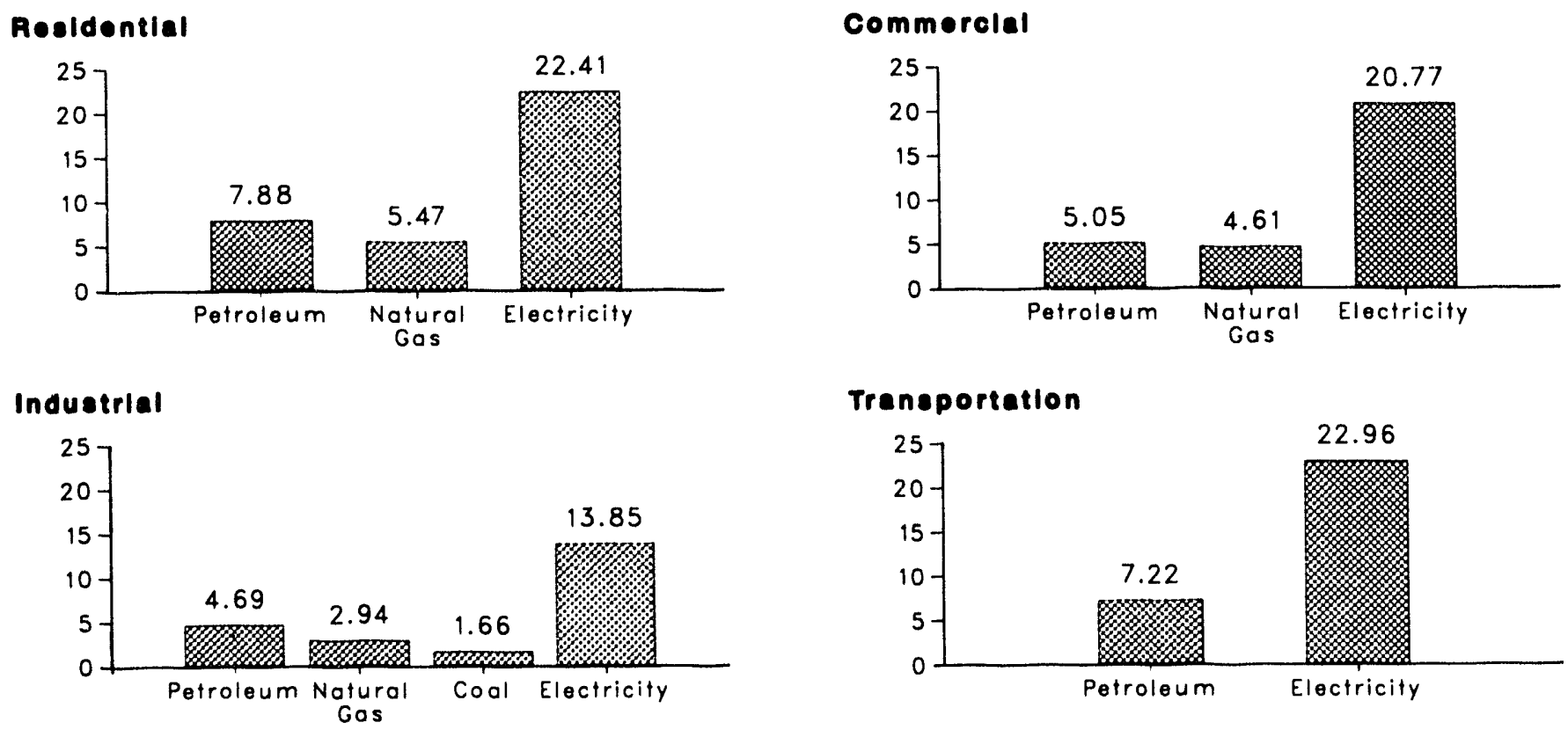

Source: State Energy Price and Expenditure Data System 1989. 
U more energy than the other sectors, its lower average $N$ price of $\$ 5.14$ per million Btu reflects the use of large contracted purchases, which are less costly, and the use of lower-priced fuels such as coal and residual fuel (Figure 6). The higher average prices paid by commercial and residential consumers are a result, in part, of those sectors' greater reliance on higher-priced electricity. Throughout the 1970 's, the residential, commercial, and transportation sectors' average prices for energy were very close, with industrial sector prices about half of the residential prices but following the same year-to-year pattern in price fluctuations. For instance, in 1970, the average price for energy in the residential sector was $\$ 2.12$ per million Btu, in the commercial sector was $\$ 1.97$ per million Btu, in the transportation sector was $\$ 2.31$ per million Btu, and in the industrial sector was $\$ .83$ per million Btu. Throughout the 1980 's, the transportation sector average prices dropped significantly below the average residential and commercial prices, following the trend in motor gasoline prices. Industrial prices continued to follow the same fluctuations as the residential and commercial sectors and remained about half of those prices.

Prices at Electric Utilities. The average price of fuel consumed at electric utilities in 1989 was $\$ 1.48$ per million Btu, compared with the peak price of $\$ 2.01$ per million Btu paid in 1982, when petroleum and natural gas prices were near their highest. Petroleum was consistently the highest-priced fuel used by electric utilities during the 1970-89 period. In 1970-1975, coal prices were slightly higher than natural gas prices. After 1975 , natural gas prices rose rapidly until 1985 , while coal prices grew more slowly. The prices of both fuels declined in the second half of the 1980's. In 1989, electric utilities paid an average price of $\$ 1.45$ per million Btu for coal and $\$ 0.70$ per million Btu for nuclear fuel versus $\$ 2.95$ per million Btu for petroleum and $\$ 2.36$ per million Btu for natural gas (Figure 7).

Expenditures at Electric Utilities. Of the $\$ 39$ billion spent by electric utilities to purchase fuel in 1989, 60 percent ( $\$ 23$ billion) was used to purchase coal (Figure 8). Far less money was spent purchasing other fuels-- $\$ 7$ billion for natural gas, $\$ 5$ billion for petroleum products, and $\$ 4$ billion for nuclear fuel. Coal consumption by electric utilities doubled during the 1970-89 period, as its portion of electric utilities' annual energy expenditures ranged between 40 percent to 65 percent of the total. Petroleum took increasing shares of the electric utility sector fuel budget in the early 1970's and peaked in 1974 at 44 percent. After that, electric utilities spent proportionally less each year on petroleum and by 1986, petroleum purchases were only 10 percent of electric utilities' fuel expenditures. Purchases of natural
Figure 7. U.S. Energy Prices at

Electric Utilities, 1989

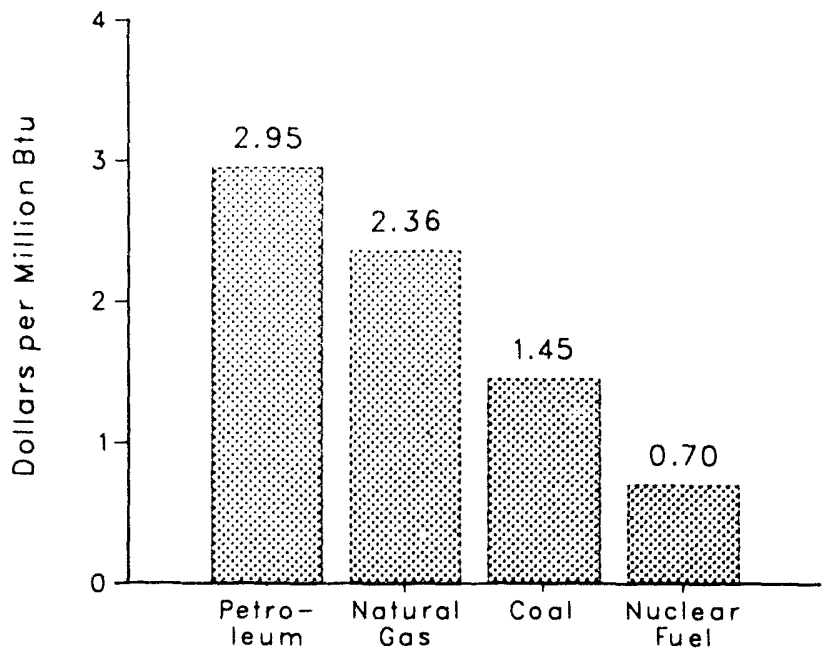

Source: State Energy Price and Expenditure Dota System 1989.

gas were a more constant portion of electric utilities' expenditures, accounting for 20 percent to 25 percent of the electric utility budget in 1970-73 and 1979-85 and slipping to 16 percent to 18 percent in the late 1980 's. The trend over the 20-year period was for electric utilities to spend proportionally less each year on petroleum products and more on nuclear fuel. During 1989 , however, there was a slight shift back with increased consumption and higher prices for petroleum and natural gas along with decreased prices for coal and nuclear fuel.

\section{Figure 8. U.S. Energy Expenditures at} Electric Utilities, 1989

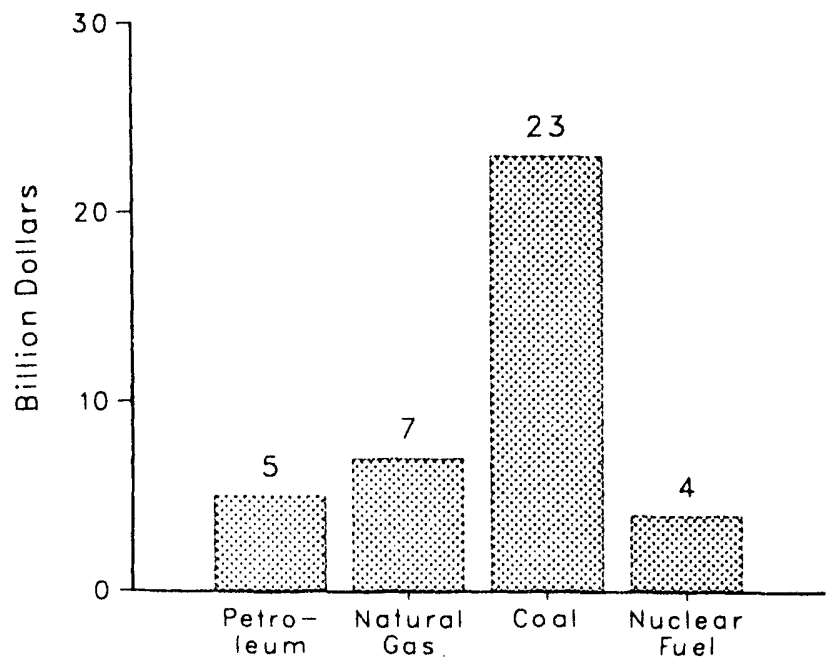
Source: State Energy Price ond Expenditure Dota System
1989 . 
State Rankings

Energy Prices. It is important to note that, even within a State, ${ }^{3}$ a single average price may have limited meaning in that it represents a consumption-weighted average over a whole State. For example, urban and rural electricity prices can vary significantly from a State's weighted average, and prices in one region of a State may differ from another because of access to less expensive hydroelectricity. Differences within a State may be greater than differences among adjacent States. Thus, the principal value of the estimates in this report lies in general comparisons among the States, interstate comparisons for a given year, and the analysis of trends over several years.

The average price paid for energy in 1989 ranged from $\$ 5.18$ per million Btu in Louisiana to $\$ 10.68$ per million Btu in Connecticut (Table 2). The State rankings of average energy prices in 1989 varied from their rankings in 1970. The District of Columbia, with the secondhighest price in 1989 ( $\$ 10.58$ per million Btu), ranked 28 th among the States in 1970. Conversely, North Dakota, which ranked 49th in energy prices in 1989, ranked 7th in 1970.

Energy Expenditures. State energy expenditures vary widely due to differences in population, climate, and size and type of industry, among other factors. In 1989, energy expenditures ranged from $\$ 43.6$ billion in California to $\$ 1.0$ billion in Vermont (Table 3). Nine of the top 10 States in energy expenditures in 1989 were among the 10 largest spending States in 1970 in slightly different order--California, New York, Texas, Illinois, Pennsylvania, Ohio, Michigan, New Jersey, and Florida. The top 10 States accounted for over half of the Nation's total energy expenditures in both years, 53 percent in 1989 and 55 percent in 1970. California and Texas, the two States with the largest energy purchases in 1989, accounted for 19 percent of the total U.S. energy expenditures that year.

Energy Expenditures per Capita. Energy expenditures per capita in 1989 ranged from the lowest rate in New York $(\$ 1,404)$ to the highest in Alaska $(\$ 3,362)$ (Table 4). State-level energy expenditures per capita demonstrate some interesting facts of energy usage: for example, California spent the highest sum for euer $y$ in 1989 but ranked 48th in spending per person; New York's total expenditures ranked 3rd, but its per capita expenditures ranked last; and Florida was 7th in expenditures in 1989 but 49th in per capita expenditures.

Petroleum Prices and Expenditures. Vermont paid the highest average price for petroleum products in 1989, $\$ 8.03$ per million Btu, while Texas paid the lowest, $\$ 5.15$ per million Btu (Table 5). The States that paid the most for petroleum products in 1970 varied from those in 1989. Only Ohio, Iowa, South Dakota, and Minnesota were among the 10 States with the highest petroleum prices in both years. Mississippi paid the $2 \mathrm{~d}$ highest price for petroleum products in 1970 but ranked 48th in petroleum prices in 1989. The District of Columbia, which had one of the lowest prices for petroleum in 1970, paid the eighth highest price in 1989. Even with its high price, the District of Columbia spent the smallest amount on petroleum products of all the States in 1989 (Table 6). Similarly, Vermont paid the highest price for petroleum in 1989 but had the second smallest petroleum expenditures that year. The 10 States that spent the most for all energy in 1989 were also the leading petrcleum-purchasing States, in slightly different order. The top 10 petroleum purchasers in 1970 were still the top 10 in 1989 with the exception of Louisiana, which replaced Massachusetts in 1989. The top 10 States accounted for 53 percent of all expenditures for petroleum in 1970 and 51 percent in 1989.

Motor Gasoline Prices and Expenditures. The State rankings for motor gasoline prices changed significantly when 1970 and 1989 are compared (Table 7). Although Hawaii had the highest price in both years, 6 States in the top 10 changed. In 1970, Alaska, Nevada, Illinois, Nebraska, New Jersey, and Indiana were among the States paying the most for motor gasoline. The States with the highest motor gasoline prices in 1989 were eastern States with the exception of Hawaii, Minnesota, and North Dakota. The most substantial increase in relative prices occurred in North Dakota, which ranked 33d in 1970 and 6th in 1989. The nine States with the largest petroleum and motor gasoline purchases were the same in 1989 (Table 8). With the exception of slight ranking differences, the nine States with the largest motor gasoline expenditures were the same in 1970 and 1989. The three States with the lowest motor gasoline expenditures in 1970 and 1989 were the District of Columbia, Alaska, and Vermont.

${ }^{3}$ Throughout this report, the term "Siate" includes the District of Columbia. 
Natural Gas Prices and Expenditures. When 1970 and 1989 are compared, the State rankings in natural gas prices and expenditures shifted very little, with the exception of Hawaii's disparate high price for liquefied natural gas in 1989. The highest prices paid for natural gas in both years were in the the Eastern United States (Table 9). Mississippi, Oklahoma, Louisiana, and Texas paid among the lowest prices in 1970 and 1989. The States with the largest expenditures on natural gas in 1989 were Texas, $\$ 7.8$ billion dollars, and California, $\$ 7.4$ billion dollars (Table 10). Their portion of the U.S. total natural gas expenditures in 1989 was 23 percent, 6 percentage points less than their share in 1970 . The 10 largest purchasers of natural gas in 1989 were responsible for 64 percent of the U.S. total, up from 62 percent in 1970. The States that spent the least on natural gas in 1970 and 1989 were Vermont, Maine, and Hawaii.

Coal Prices and Expenditures. Vermont paid the highest price for coal in $1989, \$ 3.12$ dollars per million Btu (Table 11) and had the smallest expenditure, $\$ 1$ million (Table 12). The 10 States that paid the highest prices for coal in 1989 were well below the median in expenditures, with the exceptions of Alabama and Florida, which were 8th and 10th, respectively, in both coal prices and expenditures. Comparing data for 1970 and 1989, State rankings in coal prices and expenditures show more change than in other energy sources. In 1970 , there were 5 States that consumed no coal, and the 10 States that spent the most on coal purchases accounted for 74 percent of the national total. In 1989 , all States had some expenditures for coal, and the 10 largest purchasers accounted for only 56 percent of the total. The District of Columbia, whose price for coal ranked 40th in 1970, paid the 5th highest price in 1989. Texas showed the most dramatic change in coal expenditures, ranking 30th in 1970 and 4th in 1989.

Electricity Prices and Expenditures. While Alaska paid the highest price for electricity in both 1977 and 1989 (Table 13), it's iotal expenditures for electricity ranked 51st in 1970 and 50th in 1989. Washington, which relies extensively on hydroelectricity, paid the lowest electricity price in 1970 and 1989. California experienced the largest increase in its electricity price ranking, moving from 34th in 1970 to 5 th in 1989 . Several States experienced considerable declines in their price rankings comparing 1970 and 1989--Wisconsin, Minnescia, Iowa, North Dakota, and South Dakota. States' rankings in electricity expenditures in 1970 and 1989 were remarkably similar with 9 States remaining among the top 10 States in slightly different order (Table 14). The 10 States that spent the most for electricity accounted for 55 percent of the national total in 1970 and 54 percent in 1989. In 1989, the three States (California, Texas, and New York) that had the highest expenditures on total energy and petroleum products and natural gas, also spent the most on electricity accounting for 24 percent of the U.S. total electricity expenditures.
Table 2. Energy Prices

by State, 1989

(Dollars per Million Btu)

\begin{tabular}{|c|c|c|}
\hline Ranking & State & Price \\
\hline 1 & Connecticut & 10.68 \\
\hline 2 & District of Columbia & 10.58 \\
\hline 3 & New Hampshire & 10.39 \\
\hline 4 & Arizona & 10.38 \\
\hline 5 & Vermont & 10.34 \\
\hline 0 & New York & 9.94 \\
\hline 7 & Florida & 9.83 \\
\hline 8 & Massachusetts & 9.66 \\
\hline 9 & Rhode Island & 9.59 \\
\hline 10 & North Carolina & 9.31 \\
\hline 11 & Maine & 9.13 \\
\hline 12 & New Jersey & 8.68 \\
\hline 13 & Maryland & 8.60 \\
\hline 14 & Delaware & 8.57 \\
\hline 15 & Sovih Carolina & 8.52 \\
\hline 16 & California & 8.42 \\
\hline 17 & Virginia & 8.39 \\
\hline 18 & Hawaii & 8.25 \\
\hline 19 & Nevada & 8.22 \\
\hline 20 & Georgia & 8.15 \\
\hline 21 & New Mexico & 8.13 \\
\hline 22 & Tennessee & 8.03 \\
\hline 23 & South Dakota & 8.03 \\
\hline 24 & Missouri & 8.01 \\
\hline 25 & Illinois & 7.99 \\
\hline 26 & Oregon & 7.92 \\
\hline 27 & Pennsylvania & 7.88 \\
\hline 28 & Wisconsin & 7.80 \\
\hline \multirow[t]{2}{*}{29} & Michigan & 7.78 \\
\hline & United States & 7.72 \\
\hline 30 & Minnesota & 7.68 \\
\hline 31 & Ohio & 7.68 \\
\hline 32 & Nebraska & 7.61 \\
\hline 33 & Colorada & 7.60 \\
\hline 34 & Arkansas & 7.56 \\
\hline 35 & Kentucky & 7.47 \\
\hline 36 & Idaho & 7.36 \\
\hline 37 & Kansas & 7.25 \\
\hline 38 & Ir a & 7.16 \\
\hline 39 & Montana & 7.13 \\
\hline 40 & Mississippi & 7.13 \\
\hline 41 & Alabama & 7.04 \\
\hline 42 & Washington & 6.98 \\
\hline 43 & Oklahoma & 6.73 \\
\hline 44 & Utah & 6.69 \\
\hline 45 & Wyoming & 6.45 \\
\hline 46 & Indiana & 6.29 \\
\hline 47 & Alaska & 6.25 \\
\hline 48 & West Virginia & 6.13 \\
\hline 49 & North Dakota & 6.05 \\
\hline 50 & Texas & 5.70 \\
\hline 51 & Louisiana & 5.18 \\
\hline
\end{tabular}

Note: Rankings are based on unrounded data.

Source: State Energy Price and Expenditure Data System 1989 
Table 3. Energy Expenditures

by State, 1969

(Million Dollars)

\begin{tabular}{|c|c|c|}
\hline Ranking & State & Expenditure \\
\hline 1 & California & 43,619 \\
\hline 2 & Texas & 38,375 \\
\hline 3 & New York & 25,210 \\
\hline 4 & Ohio. & 20,599 \\
\hline 5 & Illinois & 20,456 \\
\hline 6 & Pennsylvania & 20,365 \\
\hline 7 & Florida & 18,864 \\
\hline 8 & Michigan & 16,096 \\
\hline 9 & New Jersey & 15,343 \\
\hline 10 & Louisiana & 11,983 \\
\hline 11 & Indiana & 11,685 \\
\hline 12 & Georgia & 11,560 \\
\hline 13 & North Carolina & 11,530 \\
\hline 14 & Virginia & 10,513 \\
\hline 15 & Massachusetts & 9,838 \\
\hline 16 & Tennessee & 9,196 \\
\hline 17 & Missouri & 8,854 \\
\hline 18 & Alabama & 7,992 \\
\hline 19 & Wisconsin & 7,983 \\
\hline 20 & Washington & 7,756 \\
\hline 21 & Maryland & 7,587 \\
\hline 22 & Minnesota & 7,217 \\
\hline 23 & Kentucky & 7,195 \\
\hline 24 & South Carolina & 6,348 \\
\hline 25 & Arizona & 6,014 \\
\hline 26 & Connecticut & 5,948 \\
\hline 27 & Oklahoma & 5,570 \\
\hline 28 & Kansas & 5,062 \\
\hline 29 & Iowa & 4,971 \\
\hline 30 & Colorado & 4,869 \\
\hline 31 & Mississippi & 4,778 \\
\hline 32 & Oregon & 4,628 \\
\hline 33 & Arkansas & 4,376 \\
\hline 34 & West Virginia & 3,488 \\
\hline 35 & Nebraska & 2,942 \\
\hline 36 & New Mexico & 2,763 \\
\hline 37 & Utah & 2,595 \\
\hline 38 & Maine & 2,227 \\
\hline 39 & Nevada & 2,173 \\
\hline 40 & New Hampshire & 1,861 \\
\hline 41 & Hawaii & 1,856 \\
\hline 42 & Alaska & 1,772 \\
\hline 43 & Idaho & 1,696 \\
\hline 44 & Montana & 1,598 \\
\hline 45 & North Dakota & 1,525 \\
\hline 46 & Rhode Island & 1,505 \\
\hline 47 & Wyoming & 1,453 \\
\hline 48 & South Dakota & 1,308 \\
\hline 49 & Delaware & 1,308 \\
\hline 50 & District of Columbia & 1,067 \\
\hline \multirow[t]{2}{*}{51} & Vermont & 983 \\
\hline & United States & 436,643 \\
\hline
\end{tabular}

Note: Rankings are based on unrounded data.

Source: State Energy Price and Expenditure Data System 1989
Table 4. Energy Expenditures

per Capita by State, 1989

([ollars per Capita)

\begin{tabular}{|c|c|c|}
\hline Ranking & State & Expenditure \\
\hline 1 & Alaska & 3,362 \\
\hline 2 & Wyoming & 3,060 \\
\hline 3 & Louisiana & 2,735 \\
\hline 4 & North Dakota & 2,311 \\
\hline 5 & Texas & 2,259 \\
\hline 6 & Indiana & 2,089 \\
\hline 7 & Kansas & 2,014 \\
\hline 8 & New Jersey & 1,983 \\
\hline 9 & Montana & 1,982 \\
\hline 10 & Nevada & 1,956 \\
\hline 11 & Delaware & 1,943 \\
\hline 12 & Alabama & 1,941 \\
\hline
\end{tabular}

Kentucky $\quad 1,93$

Ohio $\quad 1,889$

West Virginia $\quad 1,878$

Tennessee $\quad 1,862$

Connecticut $\quad 1,836$

South Dakota $\quad 1,830$

Nebraska $\quad 1,826$

Mississippi $\quad 1,823$

Maine $\quad 1,822$

Arkansas $\quad 1,819$

New Mexico $\quad 1,808$

South Carolina $\quad 1,808$

Georgia $\quad 1,796$

District of Columbia $\quad 1,766$

United States $\quad 1,759$

North Carolina $\quad 1,755$

Illinois $\quad 1,755$

Iowa $\quad 1,750$

Michigan $\quad 1,736$

Vermont $\quad 1,734$

Oklahoma $\quad 1,728$

Virginia $\quad 1,724$

Missouri $\quad 1,716$

Pennsylvania $\quad 1,691$

Arizona $\quad 1,691$

New Hampshire $\quad 1,681$

Idaho $\quad 1,673$

Hawaii $\quad 1,669$

Massachusetts $\quad 1,664$

Minnesota $\quad 1,658$

Oregon $\quad 1,641$

Wisconsin $\quad 1,640$

Washington $\quad 1,629$

Maryland $\quad 1,616$

Utah $\quad 1,520$

Rhode Island $\quad 1,508$

California $\quad 1,501$

Florida $\quad 1,489$

Colorado $\quad 1,468$

New York

1,404

Note: Rankings are based on unrounded data.

Source: State Energy Price and Expenditure Data System 1989. 
Table 5. Petroleum Prices

by State, 1989

(Dollars per Million Btu)

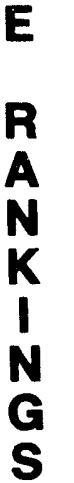

\begin{tabular}{|c|c|c|}
\hline Ranking & State & Price \\
\hline 1 & Vermont & 8.03 \\
\hline 2 & Wisconsin & 7.72 \\
\hline 3 & Minnesota & 7.59 \\
\hline 4 & Iowa & 7.50 \\
\hline 5 & Ohio & 7.44 \\
\hline 6 & Michigan & 7.43 \\
\hline 7 & South Dakota & 7.36 \\
\hline 8 & District of Columbia & 7.36 \\
\hline 9 & Rhode Island & 7.32 \\
\hline 10 & Illinois & 7.31 \\
\hline 11 & Arizona & 7.30 \\
\hline 12 & Nebraska & 7.27 \\
\hline 13 & North Carolina & 7.27 \\
\hline 14 & Tennessee & 7.27 \\
\hline 15 & Idaho & 7.24 \\
\hline 16 & Colorado & 7.15 \\
\hline 17 & Kentucky & 7.07 \\
\hline 18 & South Carolina & 7.05 \\
\hline 19 & Oregon & 7.02 \\
\hline 20 & Nevada & 6.98 \\
\hline 21 & Virginia & 6.97 \\
\hline 22 & West Virginia & 6.97 \\
\hline 23 & Maryland & 6.94 \\
\hline 24 & North Dakota & 6.93 \\
\hline 25 & Kansas & 6.90 \\
\hline 26 & Wyoming & 6.85 \\
\hline 27 & Pennsylvania & 6.79 \\
\hline 28 & Oklahoma & 6.77 \\
\hline 29 & Utah & 6.75 \\
\hline 30 & Arkansas & 6.74 \\
\hline 31 & Missouri & 6.74 \\
\hline 32 & New Mexico & 6.73 \\
\hline 33 & Montana & 6.72 \\
\hline 34 & Connecticut & 6.71 \\
\hline 35 & New Hampshire & 6.57 \\
\hline 36 & Indiana & 6.56 \\
\hline \multirow[t]{2}{*}{37} & Maine & 6.52 \\
\hline & United States & 6.48 \\
\hline 38 & New Jersey & 6.44 \\
\hline 39 & Alabama & 6.43 \\
\hline 40 & Georgia & 6.41 \\
\hline 41 & California & 6.40 \\
\hline 42 & Massachusetts & 6.26 \\
\hline 43 & Alaska & 6.25 \\
\hline 44 & Washington & 6.23 \\
\hline 45 & Delaware & 6.23 \\
\hline 46 & New York & 6.15 \\
\hline 47 & Florida & 6.04 \\
\hline 48 & Mississippi & 5.84 \\
\hline 49 & Hawaii & 5.27 \\
\hline 50 & Louisiana & 5.26 \\
\hline 51 & Texas & 5.15 \\
\hline
\end{tabular}

Note: Raıkings are based on unrounded data.

Source: State Energy Price and Expenditure Data System 1089.
Table 6. Petroleum Expenditures by State, 1989

(Million Dollars)

\begin{tabular}{|c|c|c|}
\hline Ranking & State & Expenditure \\
\hline 1 & California & 20,868 \\
\hline 2 & Texas & 20,062 \\
\hline 3 & New York & 10,784 \\
\hline 4 & Florida & 9,383 \\
\hline 5 & Ohio & 8,333 \\
\hline 6 & Pennsylvania & 8,310 \\
\hline 7 & Illinois & 7.744 \\
\hline 8 & New Jersey & 7,739 \\
\hline 9 & Michigan & 6,855 \\
\hline 10 & Louisiana & 6,375 \\
\hline 11 & Massachusetts & 5,312 \\
\hline 12 & Virginia & 5,293 \\
\hline 13 & North Carolina & 5,234 \\
\hline 14 & Georgia & 5,111 \\
\hline is & Indiana & 5,028 \\
\hline 16 & Missouri & 4,257 \\
\hline 17 & Tennessee & 4,227 \\
\hline 18 & Washington & 4,174 \\
\hline 19 & Maryland & 3,809 \\
\hline 20 & Wisconsin & 3,801 \\
\hline 21 & Alabama & 3,770 \\
\hline 22 & Minnesota & 3,666 \\
\hline 23 & Kentucky & 3,547 \\
\hline 24 & Connecticut & 3,281 \\
\hline 25 & South Carolina & 2,722 \\
\hline 26 & Oklahoma & 2,722 \\
\hline 27 & Kansas & 2,623 \\
\hline 28 & Arizona & 2,572 \\
\hline 29 & Mississippi & 2,563 \\
\hline 30 & Oregon & 2,465 \\
\hline 31 & Iowa & 2,428 \\
\hline 32 & Colorado & 2,257 \\
\hline 33 & Arizona & 2,051 \\
\hline 34 & West Virginia & 1,758 \\
\hline 35 & Nebraska & 1,533 \\
\hline 36 & New Mexico & 1,511 \\
\hline 37 & Maine & 1,475 \\
\hline 38 & Hawaii & 1,475 \\
\hline 39 & Alaska & 1,273 \\
\hline 40 & Utah & $i, 251$ \\
\hline 41 & Nevada & 1,217 \\
\hline 42 & New Hampshire & 1,088 \\
\hline 43 & Montana & 907 \\
\hline 44 & Idaho & 854 \\
\hline 45 & South Dakota & 821 \\
\hline 46 & Wyoming & 805 \\
\hline 47 & Rhode Island & 779 \\
\hline 48 & North Dakota & 772 \\
\hline 49 & Delaware & 745 \\
\hline 50 & Vermont & 590 \\
\hline \multirow[t]{2}{*}{51} & District of Columbia & 310 \\
\hline & United States & 208,536 \\
\hline
\end{tabular}

Note: Rankings are based on unrounded data.

Source: State Energy Price and Expenditure Data System 1989. 
Table 7. Motor Gasoline Prices

by State, 1989

(Dollars per Million Btu)

\begin{tabular}{|c|c|c|}
\hline Ranking & State & Price \\
\hline 1 & Hawaii & 10.42 \\
\hline 2 & District of Columbia & 9.50 \\
\hline 3 & Connecticut & 9.30 \\
\hline 4 & Rhode Island & 9.11 \\
\hline 5 & Delaware & 8.88 \\
\hline 6 & North Dakota & 8.88 \\
\hline 7 & Vermont & 8.84 \\
\hline 8 & Maryland & 8.84 \\
\hline 9 & Minnesota & 8.72 \\
\hline 10 & Maine & 8.69 \\
\hline 11 & Alaska & 8.65 \\
\hline 12 & New Hampshire & 8.60 \\
\hline 13 & Montana & 8.59 \\
\hline 14 & Virginia & 8.55 \\
\hline 15 & West Virginia & 8.52 \\
\hline 16 & South Dakota & 8.51 \\
\hline 17 & Massachusetts & 8.50 \\
\hline 18 & Oregon & 8.50 \\
\hline 19 & New Jersey & 8.48 \\
\hline 20 & Nebraska & 8.42 \\
\hline 21 & Inwa & 8.40 \\
\hline 22 & Wisconsin & 8.29 \\
\hline 23 & Tennessee & 8.25 \\
\hline 24 & Utah & 8.24 \\
\hline 25 & New Mexico & 8.24 \\
\hline 26 & Idaho & 8.21 \\
\hline 27 & Arizona & 8.19 \\
\hline 28 & Nevada & 8.19 \\
\hline 29 & Ohio & 8.18 \\
\hline 30 & Colorado & 8.13 \\
\hline 31 & North Carolina & 8.10 \\
\hline 32 & Kentucky & 8.08 \\
\hline 33 & Mississippi & 8.07 \\
\hline \multirow[t]{2}{*}{34} & Washington & 8.03 \\
\hline & United Stgtes & 8.01 \\
\hline 35 & Illinois & 8.00 \\
\hline 36 & Alabama & 7.96 \\
\hline 37 & Texas & 7.92 \\
\hline 38 & Louisiana & 7.90 \\
\hline 39 & South Carolina & 7.84 \\
\hline 40 & New York & 7.82 \\
\hline 41 & Oklahoma & 7.79 \\
\hline 42 & Pennsylvania & 7.79 \\
\hline 43 & Wyoming & 7.79 \\
\hline 44 & Michigan & 7.77 \\
\hline 45 & Arkansas & 7.69 \\
\hline 46 & Florida & 7.68 \\
\hline 47 & California & 7.66 \\
\hline 48 & Kansas & 7.66 \\
\hline 49 & Indiana & 7.60 \\
\hline 50 & Missouri & 7.45 \\
\hline 51 & Georgia & 7.12 \\
\hline
\end{tabular}

Note: Rankings are based on unrounded data.

Source: State Energy Price and Expenditure Data System 1989.
Table 8. Motor Gasoline Expenditures by State, 1989

(Million Dollars)

\begin{tabular}{clcc}
\cline { 1 - 3 } Ranking & \multicolumn{1}{c}{ Saate } & Expenditure & E \\
\cline { 1 - 3 } 1 & California & 12,504 & \\
2 & Texas & 8,458 & R \\
3 & Florida & 5,732 & A \\
4 & New York & 5,480 & N \\
5 & Ohio & 4,994 & \\
6 & Illinois & 4,853 & K \\
7 & Pennsylvania & 4,453 & I \\
8 & Michigan & 4,127 & N \\
9 & New Jersey & 3,626 & G \\
10 & North Carolina & 3,306 & G \\
11 & Virginia & 3,183 & S \\
12 & Georgia & 3,122 &
\end{tabular}

Massachusetts

Tennessee

Missouri 2.493

Indiana 2,4i

Maryland 2,2.9

Washington 2,268

Minnesota

Wisconsin 2,139

Alabama $\quad 2,067$

Louisiana $\quad 1,945$

Kentucky 1,841

South Carolina $\quad 1,755$

Arizona $\quad 1,747$

Oklahoma $\quad 1,590$

Connecticut $\quad 1,576$

Colorado $\quad 1,513$

Iowa $\quad 1,436$

Oregon $\quad 1,423$

Mississippi $\quad 1,229$

Kansas $\quad 1,201$

Arizona $\quad 1,188$

West Virginia $\quad 872$

New Mexico 817

Nebraska $\quad 814$

Utah $\quad 749$

Maine $\quad 647$

Nevada $\quad 626$

New Hampshire 555

Idaho 487

Hawaii $\quad 479$

Montana 465

Rhode Island 424

South Dakota $\quad 408$

North Dakota 392

Delaware 380

Wyoming 309

Vermont 304

Alaska 231

District of Columbia 209

United States $\quad \mathbf{1 1 2 , 5 8 5}$

Note: Rankings are based on unrounded data

Source: State Energy Price and Expenditure Data System 1989 


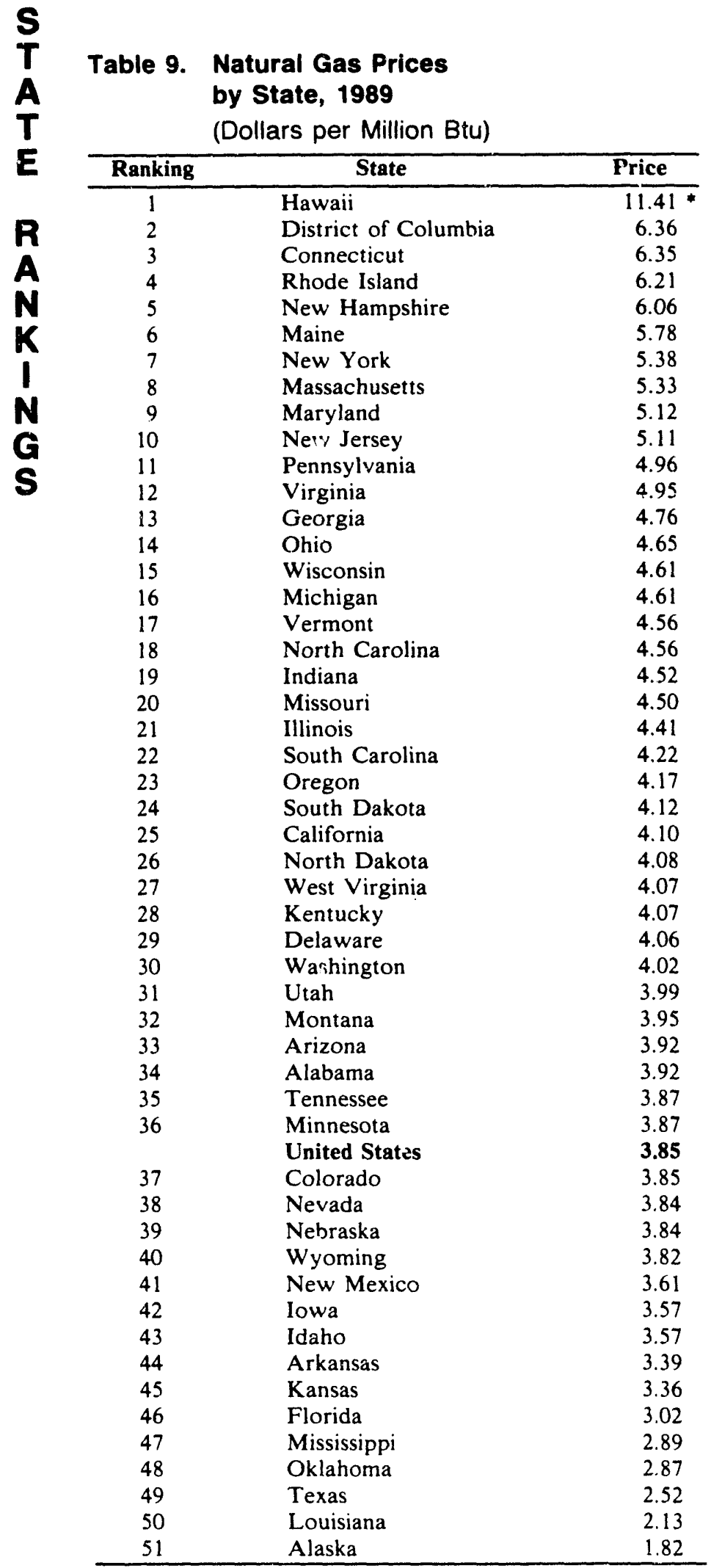

* Based on small quantities of liquefied natural gas. Note: Rankings are based on unrounded data.

Source: State Energy Price and Expenditure Data System 1989.
Table 10. Natural Gas Expenditures by State, 1989

(Million Dollars)

\begin{tabular}{|c|c|c|}
\hline Ranking & State & Expenditure \\
\hline 1 & Texas & 7,821 \\
\hline 2 & California & 7,426 \\
\hline 3 & New York & 4,627 \\
\hline 4 & Illinois & 4,359 \\
\hline 5 & Ohio & 3,826 \\
\hline 6 & Michigan & 3,517 \\
\hline 7 & Pennsylvania & 3,295 \\
\hline 8 & Louisiana & 2,431 \\
\hline 9 & New Jersey & 2,360 \\
\hline 10 & Indiana & 2,045 \\
\hline 11 & Wisconsin & 1,491 \\
\hline 12 & Georgia & 1,486 \\
\hline 13 & Massachusetts & 1,367 \\
\hline 14 & Oklahoma & 1,353 \\
\hline 15 & Missouri & 1,161 \\
\hline 16 & Minnesota & 1,109 \\
\hline 17 & Maryland & 990 \\
\hline 18 & Florida & 977 \\
\hline 19 & Virginia & 851 \\
\hline 20 & Alabama & 838 \\
\hline 21 & Colorado & 837 \\
\hline 22 & Kansas & 829 \\
\hline 23 & Tennessee & 797 \\
\hline 24 & Iowa & 778 \\
\hline 25 & Arizona & 725 \\
\hline 26 & North Carolina & 719 \\
\hline 27 & Kentucky & 690 \\
\hline 28 & Washington & 628 \\
\hline 29 & Connecticut & 620 \\
\hline 30 & Mississippi & 525 \\
\hline 31 & Arizona & 511 \\
\hline 32 & South Carolina & 495 \\
\hline 33 & West Virginia & 475 \\
\hline 34 & Nebraska & 437 \\
\hline 35 & Oregon & 430 \\
\hline 36 & Utah & 406 \\
\hline 37 & New Mexico & 355 \\
\hline 38 & Nevada & 246 \\
\hline 39 & Rhode Island & 216 \\
\hline 40 & District of Columbia & 213 \\
\hline 41 & Alaska & 187 \\
\hline 42 & Montana & 165 \\
\hline 43 & Idaho & 149 \\
\hline 44 & Delaware & 143 \\
\hline 45 & Wyoming & 135 \\
\hline 46 & South Dakota & 106 \\
\hline 47 & North Dakota & 105 \\
\hline 48 & New Hampshire & 86 \\
\hline 49 & Hawaii & 33 \\
\hline 50 & Vermont & 28 \\
\hline \multirow[t]{2}{*}{51} & Maine & 21 \\
\hline & United States & 65,419 \\
\hline
\end{tabular}

Note: Rankings are based on unrounded data.

Source: State Energy Price and Expenditure Data System 1989. 
Table 11. Coal Prices

by State, 1989

(Dollars per Million Btu)

\begin{tabular}{|c|c|c|}
\hline Ranking & State & Price \\
\hline 1 & Vermont & 3.12 \\
\hline 2 & Rhode Island & 2.70 \\
\hline 3 & Maine & 2.66 \\
\hline 4 & Connecticut & 2.16 \\
\hline 5 & District of Columbia & 1.97 \\
\hline 6 & Alaska & 1.95 \\
\hline 7 & California & 1.93 \\
\hline 8 & Alabama & 1.83 \\
\hline 9 & Idaho & 1.82 \\
\hline 10 & Florida & 1.79 \\
\hline 11 & Illinois & 1.77 \\
\hline 12 & North Carolina & 1.77 \\
\hline 13 & Hawaii & 1.76 \\
\hline 14 & Delaware & 1.75 \\
\hline 15 & New Hampshire & 1.75 \\
\hline 16 & Georgia & 1.75 \\
\hline 17 & Michigan & 1.72 \\
\hline 18 & South Carolina & 1.71 \\
\hline 19 & Mississippi & 1.67 \\
\hline 20 & Massachusetts & 1.64 \\
\hline 21 & Arkansas & 1.64 \\
\hline 22 & New York & 1.63 \\
\hline 23 & New Jersey & 1.63 \\
\hline 24 & Washington & 1.62 \\
\hline 25 & Louisiana & 1.62 \\
\hline 26 & Maryland & 1.60 \\
\hline 27 & Virginia & 1.57 \\
\hline 28 & Ohio & 1.51 \\
\hline 29 & Pennsylvania & 1.50 \\
\hline 30 & Wisconsin & 1.50 \\
\hline 31 & Indiana & 1.49 \\
\hline \multirow[t]{2}{*}{32} & Nevada & 1.49 \\
\hline & United States & 1.48 \\
\hline 33 & Texas & 1.44 \\
\hline 34 & West Virginia & 1.43 \\
\hline 35 & Arizona & 1.38 \\
\hline 36 & Tennessee & 1.36 \\
\hline 37 & Missouri & 1.35 \\
\hline 38 & Utah & 1.31 \\
\hline 39 & Oklahoma & 1.29 \\
\hline 40 & Minnesota & 1.26 \\
\hline 41 & Iowa & 1.25 \\
\hline 42 & Oregon & 1.25 \\
\hline 43 & Kansas & 1.24 \\
\hline 44 & New Mexico & 1.23 \\
\hline 45 & Kentucky & 1.22 \\
\hline 46 & North Dakota & 1.21 \\
\hline 47 & Colorado & 1.08 \\
\hline 48 & South Dakota & 1.07 \\
\hline 49 & Wyoming & 0.89 \\
\hline 50 & Nebraska & 0.85 \\
\hline 51 & Montana & 0.61 \\
\hline
\end{tabular}

Note: Rankings are based on unrounded data.

Source: State Energy Price and Expenditure Data System 1989
Table 12. Coal Expenditures

by State, 1989

(Million Dollars)

\begin{tabular}{|c|c|c|}
\hline Ranking & State & Expenditure \\
\hline 1 & Ohio & 2,208 \\
\hline 2 & Pennsylvania & 2,200 \\
\hline 3 & Indiana & 1,953 \\
\hline 4 & Texas & 1,910 \\
\hline 5 & Michigan & 1,377 \\
\hline 6 & West Virginia & 1,326 \\
\hline 7 & Illinois & 1,261 \\
\hline 8 & Alabama & 1,234 \\
\hline 9 & Georgia & 1,182 \\
\hline 10 & Florida & 1,128 \\
\hline 11 & North Carolina & 982 \\
\hline 12 & Kentucky & 934 \\
\hline
\end{tabular}

Tennessee

Missouri 740

New York 590

Wisconsin 590

Virginia 569

South Carolina $\quad 514$

Arizona $\quad 494$

Maryland 473

Utah 452

North Dakota 437

Minnesota 406

Iowa 398

Wyoming 375

Colorado 349

Oklahoma $\quad 346$

New Mexico 345

Louisiana 336

Arizona 333

Kansas $\quad 330$

Nevada 253

Massachusetts $\quad 198$

Mississippi 161

Washington $\quad 157$

New Jersey 153

Nebraska 113

California $\quad 112$

Montana 108

Delaware 106

New Hampshire 55

Connecticut 51

South Dakota 35

Maine 18

Idaho 18

Alaska 9

Oregon 9

District of Columbia 3

Rhode Island 2

Hawaii

Vermont

United States

28,101

Note: Rankings are based on unrounded data.

Source: State Energy Price and Expenditure Data System 1989. 


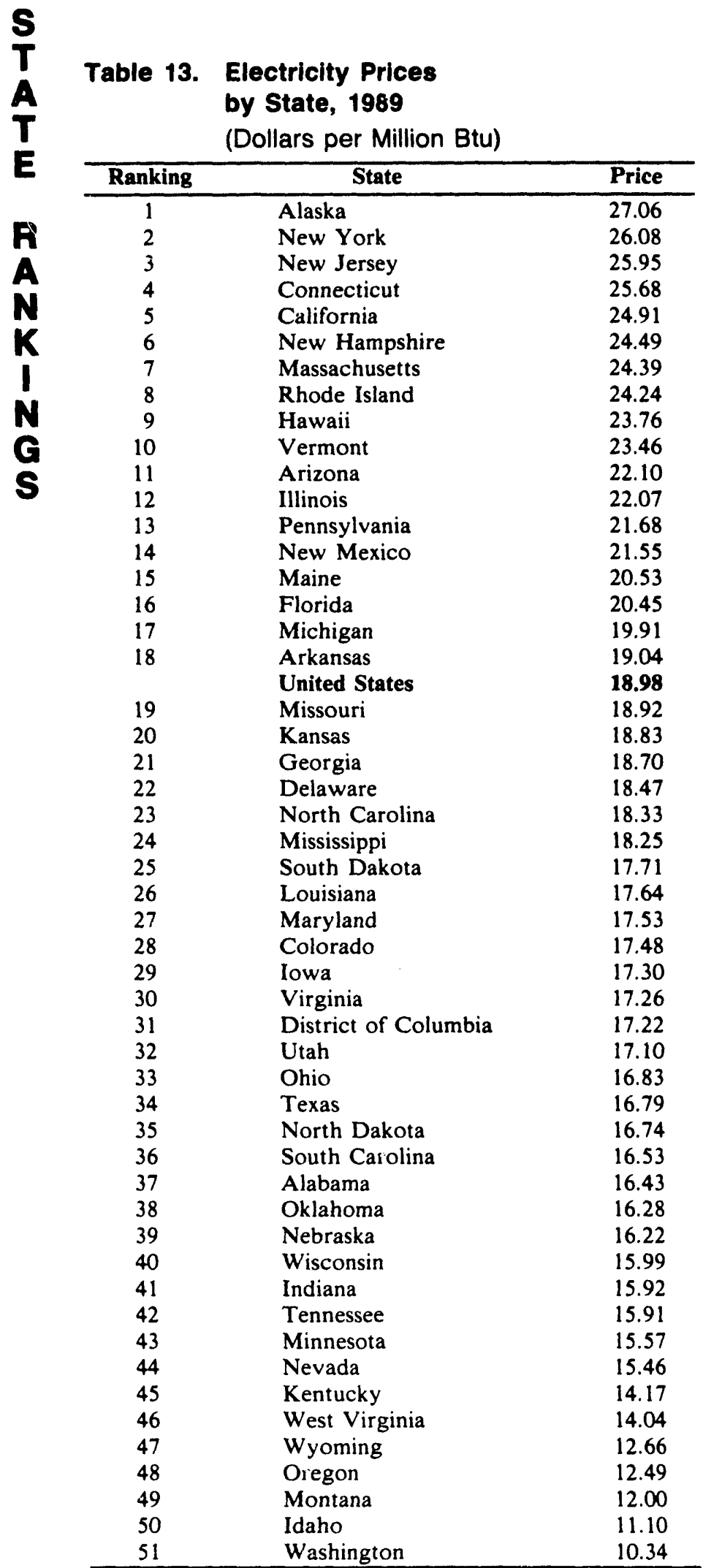

Note: Rankings are based on unrounded data.

Source: State Energy Price and Expenditure Data System 1989.

Table 14. Electricity Expenditures by State, 1989

(Million Dollars)

\begin{tabular}{|c|c|c|}
\hline Ranking & State & Expenditure \\
\hline 1 & California & 17,085 \\
\hline 2 & Texas & 12,782 \\
\hline 3 & New York & 11,353 \\
\hline 4 & Florida & 9,661 \\
\hline 5 & Pennsylvania & 8,361 \\
\hline 6 & Illinois & 8,158 \\
\hline 7 & Ohio & 8,033 \\
\hline 8 & New Jersey & 5,583 \\
\hline 9 & Michigan & 5,572 \\
\hline 10 & North Carolina & 5,479 \\
\hline 11 & Georgia & 4,885 \\
\hline 12 & Virginia & 4,308 \\
\hline 13 & Tennessee & 4,031 \\
\hline 14 & Indiana & 3,913 \\
\hline 15 & Maine & 3,801 \\
\hline 16 & Louisiana & 3,604 \\
\hline 17 & Missouri & 3,396 \\
\hline 18 & Alabama & 3,140 \\
\hline 19 & South Carolina & 3,038 \\
\hline 20 & Arizona & 3,030 \\
\hline 21 & Washington & 2,984 \\
\hline 22 & Maryland & 2,946 \\
\hline 23 & Kentucky & 2,792 \\
\hline 24 & Wisconsin & 2,614 \\
\hline 25 & Minnesota & 2,410 \\
\hline 26 & Connecticut & 2,398 \\
\hline 27 & Oklahoma & 2,040 \\
\hline 28 & Mississippi & 1,787 \\
\hline 29 & Colorado & 1,775 \\
\hline 30 & Oregon & 1,774 \\
\hline 31 & Iowa & 1,695 \\
\hline 32 & Kansas & 1,647 \\
\hline 33 & Arizona & 1,645 \\
\hline 34 & West Virginia & 1,085 \\
\hline 35 & Nebraska & 972 \\
\hline 36 & New Mexico & 958 \\
\hline 37 & Utah & 854 \\
\hline 38 & Maine & 801 \\
\hline 39 & Nevada & 773 \\
\hline 40 & New Hampshire & 759 \\
\hline 41 & Idaho & 675 \\
\hline 42 & Hawaii & 630 \\
\hline 43 & District of Columbia & 567 \\
\hline 44 & Rhode Island & 526 \\
\hline 45 & Montana & 519 \\
\hline 46 & Delaware & 504 \\
\hline 47 & Wyoming & 467 \\
\hline 48 & North Dakota & 401 \\
\hline 49 & South Dakota & 383 \\
\hline 50 & Alaska & 380 \\
\hline \multirow[t]{2}{*}{51} & Vermont & 366 \\
\hline & United States & 169,340 \\
\hline
\end{tabular}

Note: Rankings are based on unrounded data

Source: State Energy Price and Expenditure Data System 1989 . 


\section{Statistical Tables}




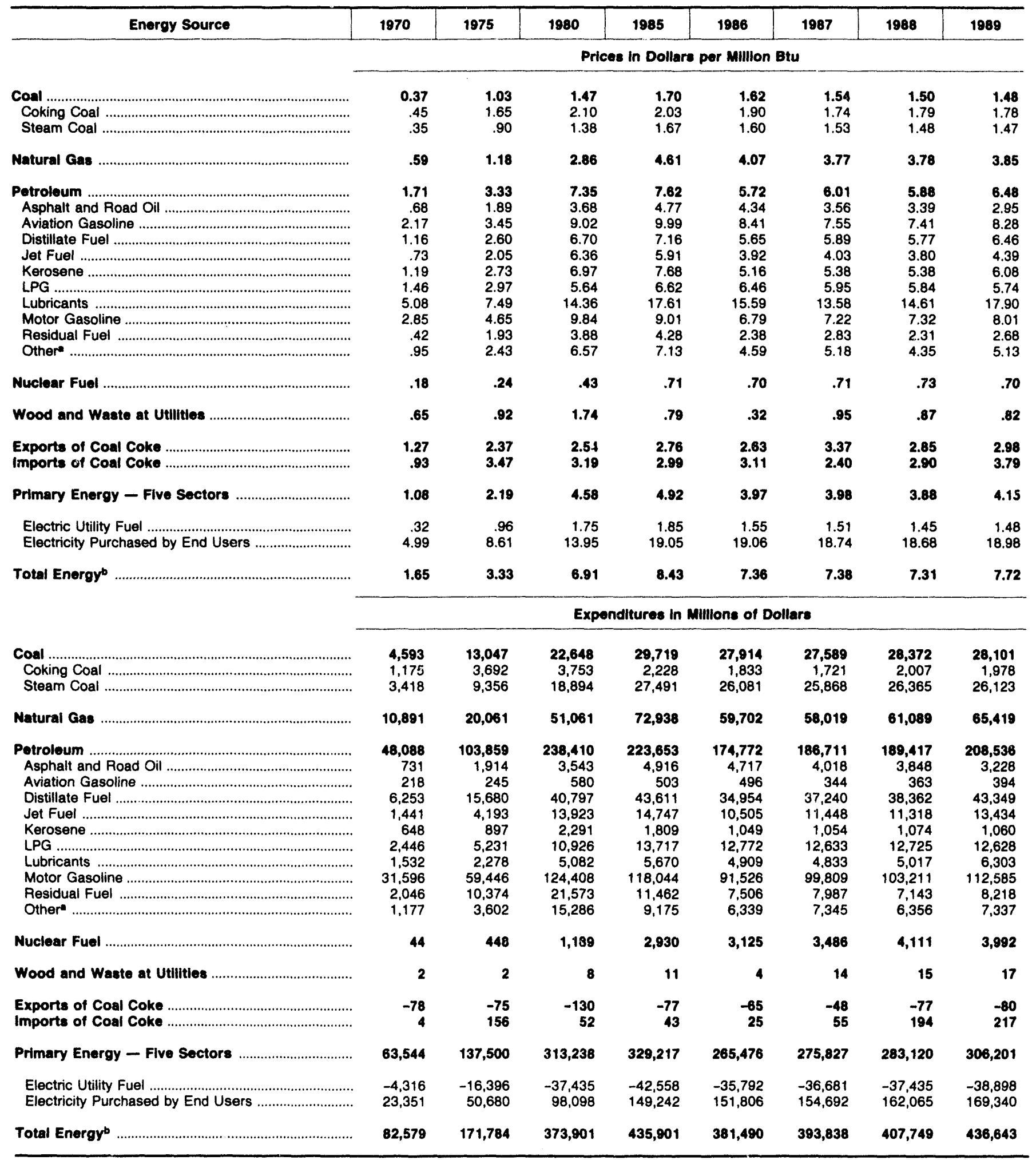

Includes petroleum coke used at electric utilities.

bThere are no direct fuel costs for hydroelectric, geothermal, centralized solar, or wind energy. Wood and other biomass fuels are not included, except those consumed at electric utilities.

Note: Expenditure totals may not equal sum of components due to independent rounding

Sources: Data sources, estimation procedures, and assumptions are described in the "Documentation" section of this report. 
U Energy Price Estimates by Sector, United States

N $1970,1975,1980$, and 1985-1989

I (Dollars per Million Btu)

\begin{tabular}{|c|c|c|c|c|c|c|c|c|}
\hline Sector and Energy Source & 1970 & 1975 & 1980 & 1985 & 1986 & 1987 & 1988 & 1989 \\
\hline $\begin{array}{l}\text { Residential Sector } \\
\text { Primary Energy } \\
\text { Coal } \\
\text { Natural Gas } \\
\text { Petroleum } \\
\text { Distillate Fuel } \\
\text { Kerosene } \\
\text { LPG } \\
\text { Electricity }\end{array}$ & $\begin{array}{l}2.12 \\
1.24 \\
1.13 \\
1.06 \\
1.56 \\
1.39 \\
1.54 \\
2.12 \\
6.51\end{array}$ & $\begin{array}{r}3.83 \\
2.13 \\
2.47 \\
1.67 \\
3.04 \\
2.74 \\
3.14 \\
4.02 \\
10.29\end{array}$ & $\begin{array}{r}7.55 \\
4.56 \\
2.90 \\
3.60 \\
7.26 \\
7.02 \\
8.32 \\
7.92 \\
15.71\end{array}$ & $\begin{array}{r}11.12 \\
6.49 \\
3.25 \\
5.94 \\
8.26 \\
7.92 \\
8.68 \\
8.94 \\
21.66\end{array}$ & $\begin{array}{r}10.97 \\
5.96 \\
3.11 \\
5.67 \\
6.94 \\
6.35 \\
6.88 \\
8.39 \\
21.75\end{array}$ & $\begin{array}{r}10.93 \\
5.71 \\
2.76 \\
5.39 \\
6.74 \\
6.05 \\
6.48 \\
8.46 \\
21.82\end{array}$ & $\begin{array}{r}10.87 \\
5.65 \\
2.64 \\
5.32 \\
6.73 \\
6.11 \\
6.50 \\
8.22 \\
21.92\end{array}$ & $\begin{array}{r}11.26 \\
6.04 \\
2.67 \\
5.47 \\
7.88 \\
6.76 \\
7.24 \\
10.29 \\
22.41\end{array}$ \\
\hline 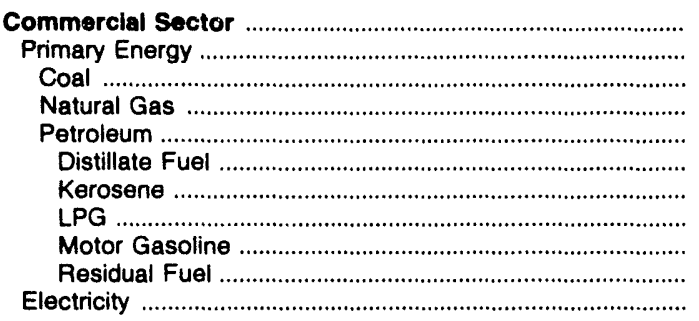 & $\begin{array}{r}1.97 \\
.79 \\
.45 \\
.75 \\
.90 \\
1.10 \\
.77 \\
1.24 \\
2.86 \\
.45 \\
6.10\end{array}$ & $\begin{array}{r}4.09 \\
1.67 \\
1.31 \\
1.32 \\
2.39 \\
2.42 \\
2.32 \\
2.60 \\
4.66 \\
1.91 \\
10.11\end{array}$ & $\begin{array}{r}7.88 \\
4.02 \\
1.54 \\
3.32 \\
5.64 \\
6.45 \\
6.46 \\
5.15 \\
9.77 \\
4.12 \\
16.06\end{array}$ & $\begin{array}{r}11.72 \\
5.54 \\
1.80 \\
5.34 \\
6.41 \\
6.35 \\
6.82 \\
8.47 \\
9.01 \\
4.40 \\
21.31\end{array}$ & $\begin{array}{r}11.40 \\
4.73 \\
1.66 \\
4.94 \\
4.58 \\
4.59 \\
4.49 \\
8.19 \\
6.77 \\
2.58 \\
21.10\end{array}$ & $\begin{array}{r}11.05 \\
4.56 \\
1.54 \\
4.64 \\
4.64 \\
4.52 \\
4.86 \\
7.61 \\
7.22 \\
2.96 \\
20.45\end{array}$ & $\begin{array}{r}10.88 \\
4.40 \\
1.54 \\
4.51 \\
4.41 \\
4.33 \\
4.34 \\
7.69 \\
7.33 \\
2.37 \\
20.34\end{array}$ & $\begin{array}{r}11.36 \\
4.65 \\
1.54 \\
4.61 \\
5.05 \\
5.10 \\
5.08 \\
7.22 \\
8.03 \\
2.67 \\
20.77\end{array}$ \\
\hline $\begin{array}{l}\text { Industrial Sector } \\
\text { Primary Energy } \\
\text { Coal } \\
\text { Coking Coal } \\
\text { Steam Coal } \\
\text { Natural Gas } \\
\text { Petroleum } \\
\text { Asphalt and Road Oil } \\
\text { Distillate Fuel }\end{array}$ & $\begin{array}{r}.83 \\
.60 \\
.45 \\
.45 \\
.44 \\
.38 \\
.96 \\
.68 \\
.72 \\
.77 \\
1.10 \\
5.08 \\
2.86 \\
.46 \\
.96 \\
1.27 \\
.93 \\
2.99\end{array}$ & $\begin{array}{l}2.20 \\
1.66 \\
1.50 \\
1.65 \\
1.28 \\
.95 \\
2.41 \\
1.89 \\
2.23 \\
2.34 \\
2.51 \\
7.49 \\
4.65 \\
1.91 \\
2.43 \\
2.37 \\
3.47 \\
6.07\end{array}$ & $\begin{array}{r}4.71 \\
3.77 \\
1.87 \\
2.10 \\
1.56 \\
2.52 \\
5.59 \\
3.68 \\
5.54 \\
6.30 \\
5.18 \\
14.36 \\
9.82 \\
3.69 \\
6.58 \\
2.54 \\
3.19 \\
10.81\end{array}$ & $\begin{array}{r}6.11 \\
4.50 \\
1.89 \\
2.03 \\
1.81 \\
3.87 \\
6.25 \\
4.77 \\
6.10 \\
6.60 \\
5.86 \\
17.61 \\
9.07 \\
4.17 \\
7.16 \\
2.76 \\
2.99 \\
14.57\end{array}$ & $\begin{array}{r}5.39 \\
3.64 \\
1.80 \\
1.90 \\
1.75 \\
3.20 \\
4.76 \\
4.34 \\
3.76 \\
3.55 \\
5.80 \\
15.59 \\
6.80 \\
2.38 \\
4.61 \\
2.63 \\
3.11 \\
14.45\end{array}$ & $\begin{array}{r}5.18 \\
3.50 \\
1.68 \\
1.74 \\
1.64 \\
2.88 \\
4.78 \\
3.56 \\
4.16 \\
4.22 \\
5.19 \\
13.58 \\
7.25 \\
2.78 \\
5.22 \\
3.37 \\
2.40 \\
13.98\end{array}$ & $\begin{array}{r}5.01 \\
3.32 \\
1.68 \\
1.79 \\
1.61 \\
2.90 \\
4.44 \\
3.39 \\
3.83 \\
4.04 \\
5.06 \\
14.61 \\
7.32 \\
2.17 \\
4.38 \\
2.85 \\
2.90 \\
13.78\end{array}$ & $\begin{array}{r}5.14 \\
3.41 \\
1.66 \\
1.78 \\
1.58 \\
2.94 \\
4.69 \\
2.95 \\
4.78 \\
4.95 \\
4.19 \\
17.90 \\
8.05 \\
2.50 \\
5.18 \\
2.98 \\
3.79 \\
13.85\end{array}$ \\
\hline 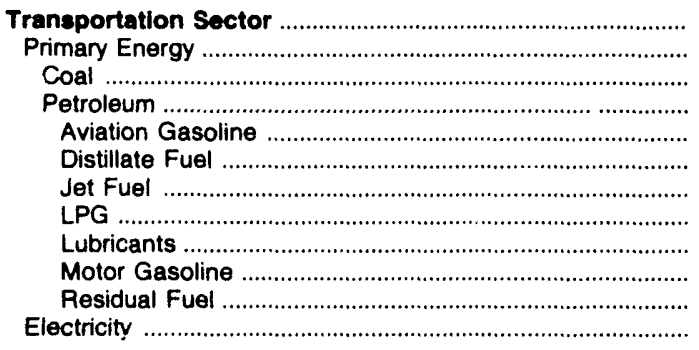 & $\begin{array}{r}2.31 \\
2.31 \\
.41 \\
2.31 \\
2.17 \\
1.31 \\
.73 \\
1.11 \\
5.08 \\
2.85 \\
.88 \\
4.65\end{array}$ & $\begin{array}{r}4.02 \\
4.02 \\
1.26 \\
4.02 \\
3.45 \\
2.80 \\
2.05 \\
2.51 \\
7.49 \\
4.64 \\
1.72 \\
11.72\end{array}$ & $\begin{array}{r}8.61 \\
8.60 \\
- \\
8.60 \\
9.02 \\
7.19 \\
6.36 \\
5.20 \\
14.36 \\
9.84 \\
3.31 \\
14.71\end{array}$ & $\begin{array}{r}8.25 \\
8.25 \\
- \\
8.25 \\
9.99 \\
7.50 \\
5.91 \\
8.52 \\
17.61 \\
9.01 \\
4.41 \\
19.74\end{array}$ & $\begin{array}{r}6.23 \\
6.23 \\
- \\
6.23 \\
8.41 \\
6.36 \\
3.92 \\
8.05 \\
15.59 \\
6.79 \\
2.25 \\
19.63\end{array}$ & $\begin{array}{r}6.59 \\
6.58 \\
- \\
6.58 \\
7.55 \\
6.75 \\
4.03 \\
7.60 \\
13.58 \\
7.22 \\
2.62 \\
23.03\end{array}$ & $\begin{array}{r}6.58 \\
6.57 \\
- \\
6.57 \\
7.41 \\
6.59 \\
80 \\
7.65 \\
14.61 \\
7.32 \\
2.23 \\
22.05\end{array}$ & $\begin{array}{r}7.23 \\
7.22 \\
- \\
7.22 \\
8.28 \\
7.16 \\
4.39 \\
7.22 \\
17.90 \\
8.01 \\
2.47 \\
22.96\end{array}$ \\
\hline 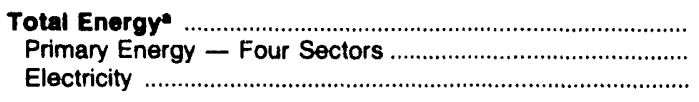 & $\begin{array}{l}1.65 \\
1.30 \\
4.99\end{array}$ & $\begin{array}{l}3.33 \\
2.65 \\
8.61\end{array}$ & $\begin{array}{r}6.91 \\
5.85 \\
13.95\end{array}$ & $\begin{array}{r}8.43 \\
6.54 \\
19.05\end{array}$ & $\begin{array}{r}7.36 \\
5.24 \\
19.06\end{array}$ & $\begin{array}{r}7.38 \\
5.30 \\
18.74\end{array}$ & $\begin{array}{r}7.31 \\
5.21 \\
18.68\end{array}$ & $\begin{array}{r}7.72 \\
5.62 \\
18.98\end{array}$ \\
\hline 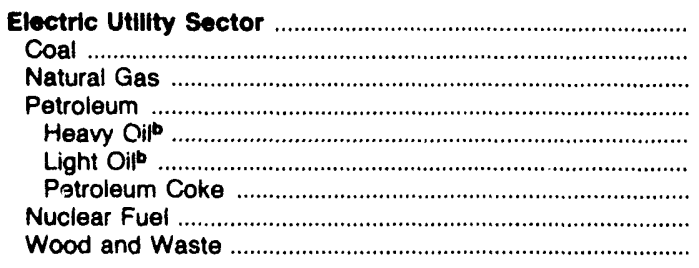 & $\begin{array}{l}.32 \\
.31 \\
.28 \\
.42 \\
.41 \\
.57 \\
.29 \\
.18 \\
.65\end{array}$ & $\begin{array}{r}.96 \\
.82 \\
.75 \\
2.00 \\
1.99 \\
2.22 \\
.53 \\
.24 \\
.92\end{array}$ & $\begin{array}{r}1.75 \\
1.35 \\
2.20 \\
4.34 \\
4.25 \\
5.75 \\
2.61 \\
.43 \\
1.74\end{array}$ & $\begin{array}{r}1.85 \\
1.65 \\
3.43 \\
4.35 \\
4.24 \\
5.89 \\
1.27 \\
.71 \\
.79\end{array}$ & $\begin{array}{l}1.55 \\
1.58 \\
2.35 \\
2.48 \\
2.42 \\
3.67 \\
1.04 \\
.70 \\
.32\end{array}$ & $\begin{array}{l}1.51 \\
1.51 \\
2.24 \\
3.03 \\
2.97 \\
1.05 \\
1.04 \\
.71 \\
.95\end{array}$ & $\begin{array}{r}1.45 \\
1.47 \\
2.26 \\
2.49 \\
2.41 \\
3.81 \\
.99 \\
.73 \\
.87\end{array}$ & $\begin{array}{r}1.48 \\
1.45 \\
2.36 \\
2.95 \\
2.85 \\
4.26 \\
.86 \\
.70 \\
.82\end{array}$ \\
\hline Primary Energy - Five Sectors & 1.08 & 2.19 & 4.58 & 4.92 & 3.97 & 3.98 & 3.88 & 4.15 \\
\hline
\end{tabular}

-There are no direct fuel costs for hydroelectric, geothermal, centralized solar, or wind energy. Wood and other biomass fuels are not included, except those consumed at electric utilities.

b Heavy oil includes Grade Nos. 4, 5, and 6, and residual fuel oils. Light oil includes Grade No. 2 heating oil, kerosene, and jet fuel.

-No consumption, including cases where adjustments were made. See "Consumption Adjustments for Process Fuel and Intermediate Products."

Sources: Data sources, estimation procedures, and assumptions are described in the "Documentation" section of this report. 


\begin{tabular}{|c|c|c|c|c|c|c|c|c|}
\hline Sector and Energy Source & 1970 & 1975 & 1980 & 1985 & 1986 & 1987 & 1988 & 1989 \\
\hline $\begin{array}{l}\text { Resldential Sector } \\
\text { Primary Energy } \\
\text { Coal } \\
\text { Natural Gas } \\
\text { Petroleum } \\
\text { Distillate Fuel } \\
\text { Kerosene } \\
\text { LPG }\end{array}$ & $\begin{array}{r}20,083 \\
9,731 \\
173 \\
5,272 \\
4,286 \\
2,603 \\
459 \\
1,225 \\
10,352\end{array}$ & $\begin{array}{r}36,844 \\
16,201 \\
209 \\
8,410 \\
7,582 \\
4,954 \\
504 \\
2,124 \\
20,644\end{array}$ & $\begin{array}{r}68,825 \\
30,367 \\
175 \\
17,497 \\
12,695 \\
9,234 \\
887 \\
2,575 \\
38,458 \\
\end{array}$ & $\begin{array}{r}98,729 \\
40,057 \\
225 \\
27,136 \\
12,696 \\
7,910 \\
1,011 \\
3,775 \\
58,672\end{array}$ & $\begin{array}{r}96,628 \\
35,851 \\
215 \\
25,147 \\
10,489 \\
6,428 \\
596 \\
3,465 \\
60,776\end{array}$ & $\begin{array}{r}97,814 \\
34,496 \\
181 \\
23,926 \\
10,389 \\
6,236 \\
588 \\
3,566 \\
63,318\end{array}$ & $\begin{array}{r}103,209 \\
36,415 \\
174 \\
25,332 \\
10,909 \\
6,497 \\
675 \\
3,737 \\
66,793\end{array}$ & $\begin{array}{r}109,171 \\
39,927 \\
156 \\
26,934 \\
12,837 \\
7,038 \\
604 \\
5,196 \\
69,243\end{array}$ \\
\hline $\begin{array}{l}\text { Commerclal Sector } \\
\text { Primary Energy } \\
\text { Coal } \\
\text { Natural Gas } \\
\text { Petroleum } \\
\text { Distillate Fuel } \\
\text { Kerosene } \\
\text { LPG }\end{array}$ & $\begin{array}{r}10,668 \\
3,333 \\
98 \\
1,844 \\
1,391 \\
646 \\
47 \\
127 \\
247 \\
323 \\
7,335\end{array}$ & $\begin{array}{r}22,835 \\
6,680 \\
162 \\
3,385 \\
3,133 \\
1,423 \\
114 \\
242 \\
415 \\
939 \\
16,155\end{array}$ & $\begin{array}{r}46,881 \\
16,260 \\
135 \\
8,858 \\
7,267 \\
3,337 \\
262 \\
296 \\
1,046 \\
2,325 \\
30,621\end{array}$ & $\begin{array}{r}70,325 \\
20,201 \\
193 \\
13,368 \\
6,640 \\
3,965 \\
338 \\
632 \\
866 \\
840 \\
50,124\end{array}$ & $\begin{array}{r}68,320 \\
16,822 \\
178 \\
11,770 \\
4,874 \\
2,731 \\
186 \\
597 \\
720 \\
639 \\
51,498\end{array}$ & $\begin{array}{r}68,717 \\
16,746 \\
154 \\
11,601 \\
4,992 \\
2,680 \\
175 \\
566 \\
795 \\
775 \\
51,970\end{array}$ & $\begin{array}{r}71,725 \\
17,256 \\
158 \\
12,377 \\
4,721 \\
2,487 \\
180 \\
617 \\
810 \\
628 \\
54,469\end{array}$ & $\begin{array}{r}75,605 \\
18,076 \\
135 \\
12,915 \\
5,026 \\
2,731 \\
240 \\
643 \\
818 \\
594 \\
57,529\end{array}$ \\
\hline 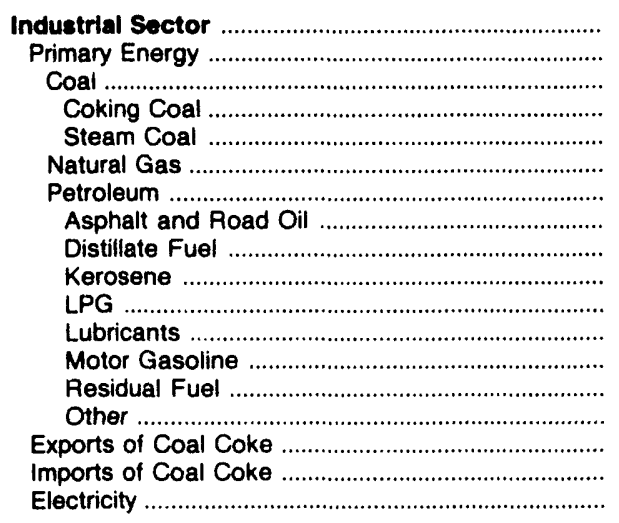 & $\begin{array}{r}16,458 \\
10,834 \\
2,082 \\
1,175 \\
907 \\
2,625 \\
6,202 \\
731 \\
866 \\
142 \\
1,046 \\
787 \\
824 \\
635 \\
1,171 \\
-78 \\
4 \\
5,624\end{array}$ & $\begin{array}{r}41,170 \\
27,409 \\
5,498 \\
3,692 \\
1,806 \\
5,844 \\
15,986 \\
1,914 \\
2,907 \\
278 \\
2,760 \\
1,119 \\
1,039 \\
2,367 \\
3,601 \\
-75 \\
156 \\
13,760\end{array}$ & $\begin{array}{r}94,521 \\
65,658 \\
5,888 \\
3,753 \\
2,135 \\
16,350 \\
43,498 \\
3,543 \\
7,232 \\
1,143 \\
7,967 \\
2,614 \\
1,553 \\
4,175 \\
15,271 \\
-130 \\
52 \\
28,863\end{array}$ & $\begin{array}{r}105,529 \\
65,339 \\
5,244 \\
2,228 \\
3,616 \\
21,615 \\
38,513 \\
4,916 \\
7,208 \\
460 \\
9,074 \\
2,916 \\
1,977 \\
2,797 \\
9,166 \\
-77 \\
43 \\
40,190\end{array}$ & $\begin{array}{r}90,436 \\
51,164 \\
4,766 \\
1,833 \\
2,934 \\
16,479 \\
29,959 \\
4,717 \\
4,478 \\
267 \\
8,502 \\
2,524 \\
1,402 \\
1,741 \\
6,329 \\
-65 \\
25 \\
39,271 \\
\end{array}$ & $\begin{array}{r}90,186 \\
51,077 \\
4,469 \\
1,721 \\
2,748 \\
15,909 \\
30,691 \\
4,018 \\
5,082 \\
291 \\
8,345 \\
2,486 \\
1,487 \\
1,649 \\
7,335 \\
-48 \\
55 \\
39,109\end{array}$ & $\begin{array}{r}91,143 \\
50,641 \\
4,750 \\
2,007 \\
2,743 \\
17,257 \\
28,517 \\
3,848 \\
4,652 \\
219 \\
8,201 \\
2,580 \\
1,414 \\
1,260 \\
6,344 \\
-77 \\
194 \\
40,503\end{array}$ & $\begin{array}{r}94,691 \\
52,636 \\
4,645 \\
1,978 \\
2,667 \\
18,820 \\
29,034 \\
3,228 \\
5,473 \\
216 \\
6,632 \\
3,241 \\
1,599 \\
1,321 \\
7,324 \\
-80 \\
217 \\
42,255\end{array}$ \\
\hline $\begin{array}{l}\text { Transportation Sector } \\
\text { Primary Energy . } \\
\text { Coal .. } \\
\text { Petroleum } \\
\text { Aviation Gasoline } \\
\text { Distillate Fuel } \\
\text { Jet Fuel } \\
\text { LPG } \\
\text { Lubricants } \\
\text { Motor Gasoline } \\
\text { Residual Fuel } \\
\text { Electricity }\end{array}$ & $\begin{array}{r}35,370 \\
35,330 \\
3 \\
35,327 \\
218 \\
2,058 \\
1,441 \\
49 \\
746 \\
30,525 \\
291 \\
40\end{array}$ & $\begin{array}{r}70,935 \\
70,814 \\
1 \\
70,814 \\
245 \\
5,938 \\
4,150 \\
105 \\
1,159 \\
57,992 \\
1,226 \\
120\end{array}$ & $\begin{array}{r}163,674 \\
163,518 \\
- \\
163,518 \\
580 \\
20,090 \\
13,856 \\
88 \\
2,469 \\
121,809 \\
4,626 \\
156\end{array}$ & $\begin{array}{r}161,318 \\
161,062 \\
- \\
161,062 \\
503 \\
24,027 \\
14,747 \\
236 \\
2,754 \\
115,201 \\
3,594 \\
256\end{array}$ & $\begin{array}{r}126,107 \\
125,847 \\
- \\
125,847 \\
496 \\
21,012 \\
10,505 \\
208 \\
2,384 \\
89,404 \\
1,838 \\
260\end{array}$ & $\begin{array}{r}137,121 \\
136,826 \\
- \\
136,826 \\
344 \\
22,879 \\
11,448 \\
156 \\
2,348 \\
97,527 \\
2,124 \\
295\end{array}$ & $\begin{array}{r}141,673 \\
141,373 \\
- \\
141,373 \\
363 \\
24,310 \\
11,318 \\
170 \\
2,437 \\
100,988 \\
1,787 \\
300\end{array}$ & $\begin{array}{r}156,977 \\
156,664 \\
- \\
156,664 \\
394 \\
27,475 \\
13,434 \\
157 \\
3,061 \\
110,168 \\
1,974 \\
313\end{array}$ \\
\hline $\begin{array}{l}\text { Total Energy" } \\
\text { Primary Energy - Four Sectors } \\
\text { Electricity }\end{array}$ & $\begin{array}{l}82,579 \\
59,228 \\
23,351\end{array}$ & $\begin{array}{r}171,784 \\
121,104 \\
50,680\end{array}$ & $\begin{array}{r}373,901 \\
275,803 \\
98,098\end{array}$ & $\begin{array}{l}435,901 \\
286,659 \\
149,242\end{array}$ & $\begin{array}{l}381,490 \\
229,684 \\
151,806\end{array}$ & $\begin{array}{l}393,838 \\
239,146 \\
154,692\end{array}$ & $\begin{array}{l}407,749 \\
245,685 \\
162,065\end{array}$ & $\begin{array}{l}436,643 \\
267,303 \\
169,340\end{array}$ \\
\hline $\begin{array}{l}\text { Eloctric Utllity Sector } \\
\text { Coal } \\
\text { Natural Gas } \\
\text { Petroleum } \\
\text { Heavy Oib } \\
\text { Light Oib } \\
\text { Petroleum Coke } \\
\text { Nuclear Fuel } \\
\text { Wood and Waste }\end{array}$ & $\begin{array}{r}4,316 \\
2,237 \\
1,151 \\
882 \\
797 \\
80 \\
6 \\
44 \\
2\end{array}$ & $\begin{array}{r}16,396 \\
7,178 \\
2,422 \\
6,345 \\
5,842 \\
502 \\
1 \\
448 \\
2\end{array}$ & $\begin{array}{r}37,435 \\
16,450 \\
8,357 \\
11,432 \\
10,446 \\
972 \\
14 \\
1,189 \\
8\end{array}$ & $\begin{array}{r}42,558 \\
24,056 \\
10,819 \\
4,742 \\
4,232 \\
502 \\
9 \\
2,930 \\
11\end{array}$ & $\begin{array}{r}35,792 \\
22,755 \\
6,305 \\
3,603 \\
3,288 \\
306 \\
10 \\
3,125 \\
4\end{array}$ & $\begin{array}{r}36,681 \\
22,785 \\
6,583 \\
3,812 \\
3,439 \\
362 \\
11 \\
3,486 \\
14\end{array}$ & $\begin{array}{r}37,435 \\
23,290 \\
6,123 \\
3,896 \\
3,468 \\
416 \\
12 \\
4,111 \\
15\end{array}$ & $\begin{array}{r}38,898 \\
23,165 \\
6,750 \\
4,974 \\
4,328 \\
632 \\
13 \\
3,992 \\
17\end{array}$ \\
\hline Primary Energy - Five Sectors ............................... & 63,544 & 137,500 & 313,238 & 329,217 & 265,476 & 275,827 & 283,120 & 306,201 \\
\hline
\end{tabular}

-There are no direct fuel costs for hydroelectric, geothermal, centralized solar, or wind energy. Wood and other biomass fuels are not included, except those consumed at electric utilities.

bHeavy oil includes Grade Nos. 4, 5, and 6, and residual fuel oils. Light oil includes Grade No. 2 heating oil, kerosene, and jet fuel.

- No consumption, including cases where adjustments were made. See "Consumption Adjustments for Process Fuel and intermediate Products."

Note: Totals may not equal sum of components due to independent rounding.

Sources: Data sources, estimation procedures, and assumptions are described in the "Documentation" section of this report. 

A Energy Price and Expenditure Estimates by Source, Alabama
L 1970, 1975, 1980, and 1985-1989

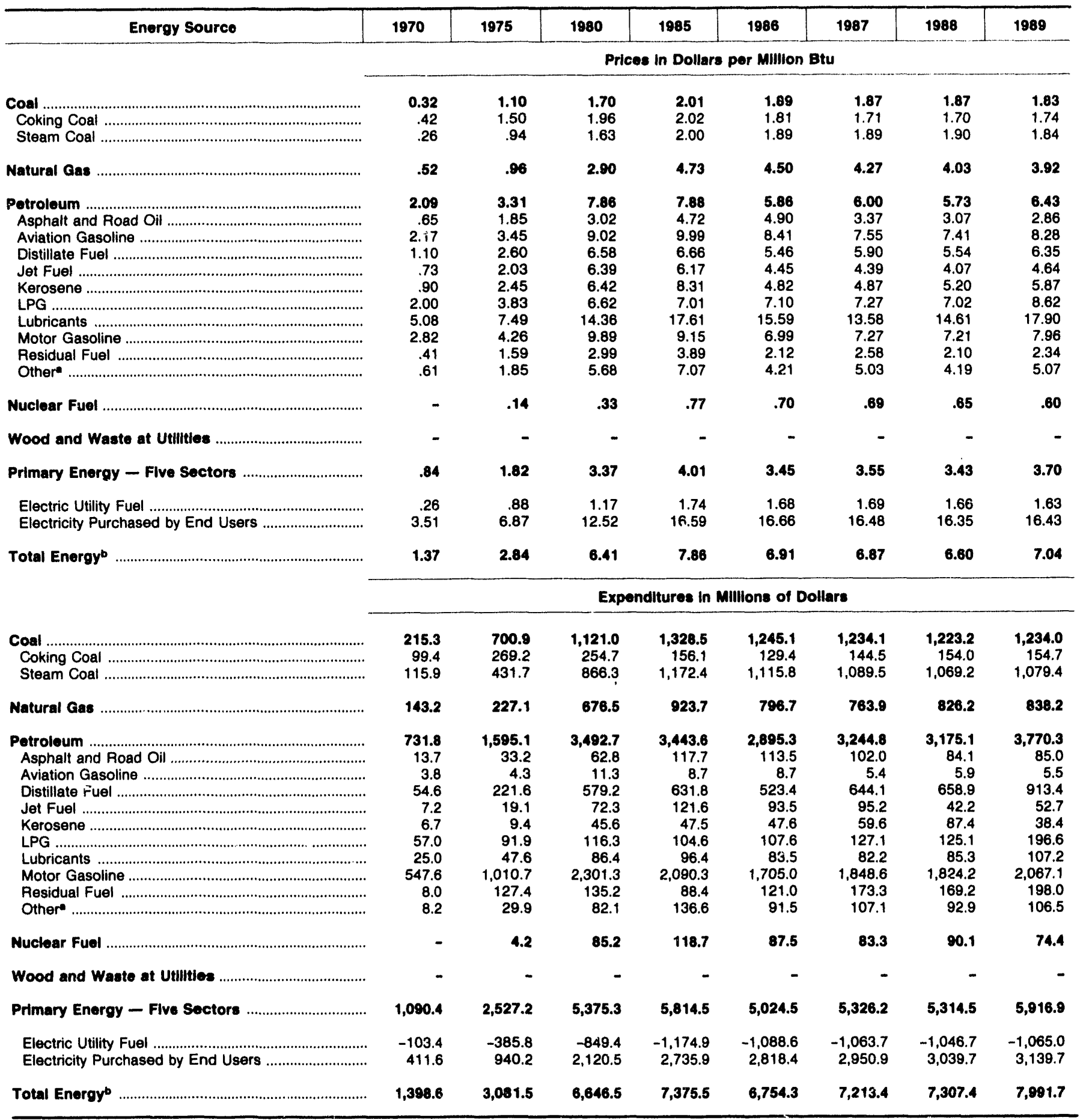

-Includes petroleum coke used at electric utilities.

There are no direct fuel costs for hydroelectric, geothermal, centralized solar, or wind energy. Wood and other biomass fuels are not included, except those consumed at electric utilities.

- No consumption, including cases where adjustments were made. See "Consumption Adjustments for Process Fuel and Intermediate Products."

Note: Expenditure totals may not equal sum of components due to independent rounding.

Sources: Data sources, estimation procedures, and assumptions are described in the "Documentation" section of this report. 


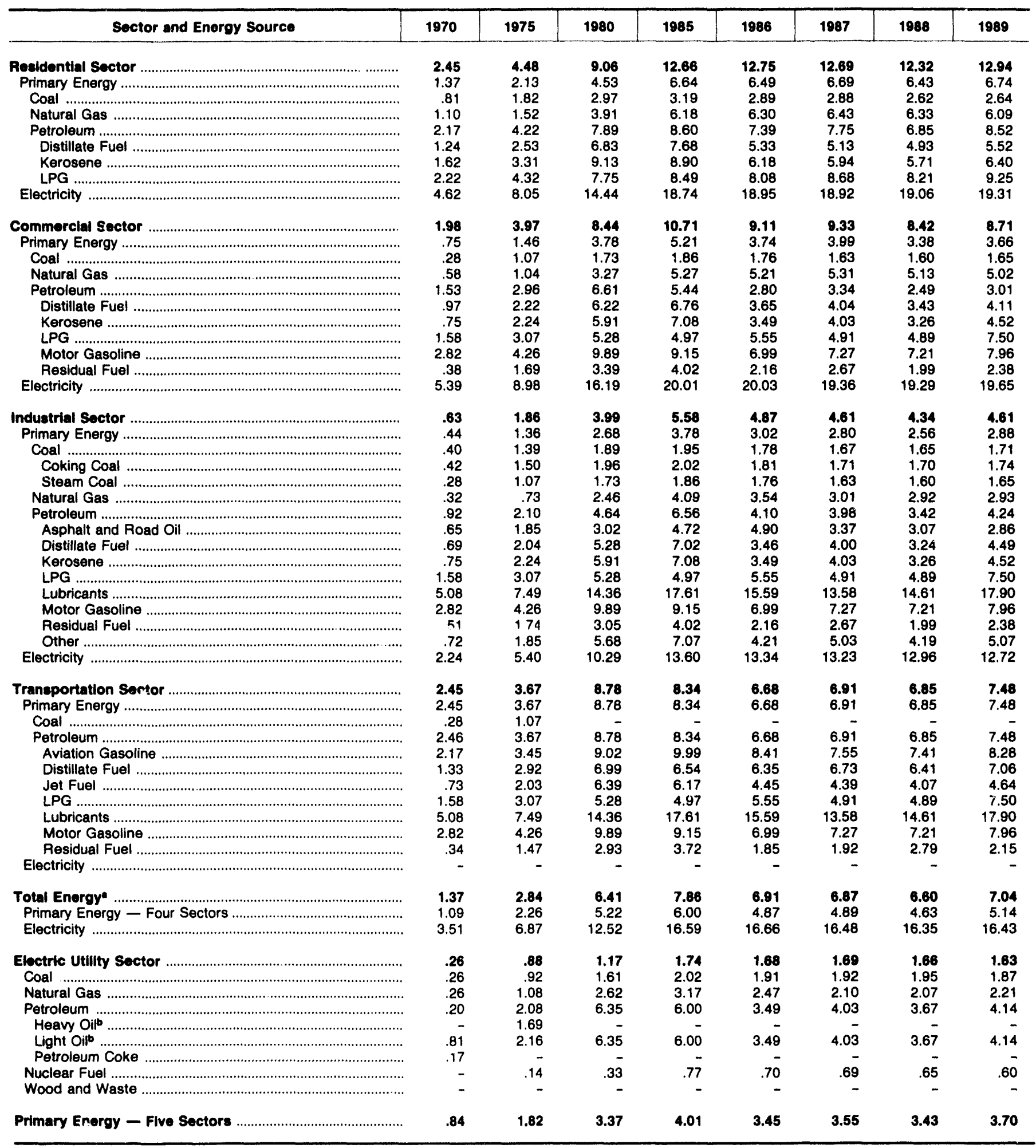

-There are no direct fuel costs for hydroelectric, geothermal, centralized solar, or wind energy. Wood and other biomass fuels are not included, except those consumed at electric utilities.

bHeavy oil includes Grade Nos. 4, 5, and 6, and residual fuel oils. Light oil includes Grade No. 2 heating oil, kerosene, and jet fuel.

-No consumption, including cases where adjustments were made. See "Consumption Adjustments for Process Fuel and Intermediate Products."

Sources: Data sources, estimation procedures, and assumptions are described in the "Documentation" section of this report. 


\section{A Energy Expenditure Estimates by Sector, Alabama \\ L $1970,1975,1980$, and $1985-1989$ \\ A (Million Dollars)}

\begin{tabular}{|c|c|c|c|c|c|c|c|c|}
\hline Sector and Energy Source & 1970 & 1975 & 1980 & 1985 & 1986 & 1987 & 1988 & 1989 \\
\hline Realdentlal Sector & 289.4 & 517.3 & $1,113.2$ & $1,491.2$ & $1,581.2$ & $1,702.0$ & $1,770.1$ & $1,775.3$ \\
\hline 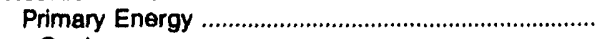 & 107.7 & 148.8 & 301.9 & 392.8 & 400.8 & 456.8 & 493.1 & 468.3 \\
\hline Coal & .9 & .3 & 5.7 & 3.4 & 3.2 & 3.4 & 3.5 & 1.6 \\
\hline 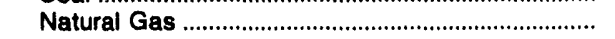 & 63.0 & 82.5 & 211.7 & 280.1 & 291.3 & 325.9 & 318.4 & 300.5 \\
\hline 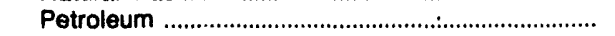 & 43.8 & 66.4 & 84.5 & 109.4 & 106.3 & 127.6 & 171.2 & 166.1 \\
\hline Distillate Fuel ...………………………………….... & .3 & 1.1 & .5 & 1.5 & 1.2 & .9 & 1.2 & 1.1 \\
\hline 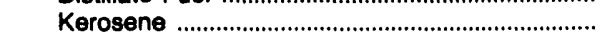 & 2.2 & 2.5 & 10.2 & 34.4 & 30.2 & 31.8 & 76.1 & 30.1 \\
\hline LPG & 41.4 & 62.8 & 73.7 & 73.5 & 74.9 & 94.9 & 93.9 & 134.9 \\
\hline 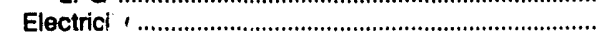 & 181.7 & 368.5 & 811.2 & $1,098.4$ & $1,180.4$ & $1,245.2$ & $1,277.0$ & $1,307.0$ \\
\hline Commerclal Sector & 131.2 & 263.4 & 551.3 & 866.2 & 876.3 & 911.0 & 929.3 & $1,045.3$ \\
\hline Primary Energy …………………………………....... & 36.6 & 64.4 & 154.2 & 265.0 & 241.1 & 254.9 & 255.3 & 300.4 \\
\hline Coal ...1. & .5 & .3 & 6.2 & 3.6 & 3.6 & 3.5 & 3.9 & 1.8 \\
\hline Natural Gas ………………………………………. & 21.8 & 35.9 & 96.5 & 141.3 & 135.7 & 122.7 & 135.0 & 136.7 \\
\hline Potroleum …………………………………………... & 14.2 & 28.2 & 51.5 & 120.1 & 101.8 & 128.8 & 116.4 & 161.8 \\
\hline 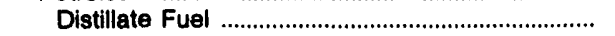 & 1.5 & 7.1 & 23.2 & 50.8 & 20.6 & 27.1 & 22.5 & 29.4 \\
\hline Kerosene ……………………………………… & 1.8 & 3.1 & 5.9 & 5.9 & 7.6 & 17.7 & 4.3 & 3.8 \\
\hline 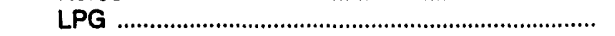 & 5.2 & 7.9 & 8.9 & 7.6 & 9.1 & 9.5 & 9.9 & 19.3 \\
\hline 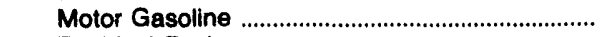 & 5.8 & 10.1 & 13.4 & 12.1 & 9.3 & 9.9 & 9.2 & 9.3 \\
\hline 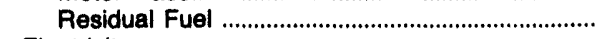 & & & .1 & 43.7 & 55.2 & 64.7 & 70.5 & 100.0 \\
\hline 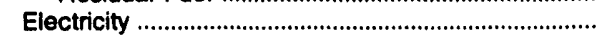 & 94.6 & 199.0 & 397.2 & 601.1 & 635.2 & 656.1 & 674.0 & 745.0 \\
\hline & 369.6 & $1,032.8$ & $2,058.2$ & $2,321.0$ & $2,041.1$ & $2,108.9$ & $2,136.4$ & 2,247.2 \\
\hline 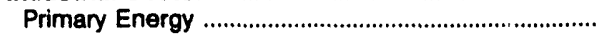 & 234.3 & 660.1 & $1,146.0$ & $1,284.6$ & $1,038.2$ & $1,059.3$ & $1,047.7$ & $1,159.5$ \\
\hline 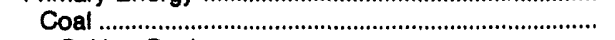 & 115.2 & 332.8 & 353.9 & 272.2 & 241.4 & 251.5 & 268.3 & 249.2 \\
\hline 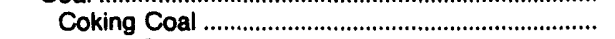 & 99.4 & 269.2 & 254.7 & 156.1 & 129.4 & 144.5 & 154.0 & 154.7 \\
\hline 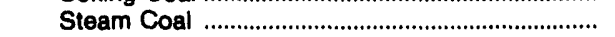 & 15.8 & 63.6 & 99.2 & 116.1 & 112.0 & 106.9 & 114.3 & 94.5 \\
\hline 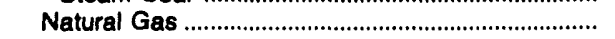 & 54.2 & 102.4 & 364.1 & 498.5 & 366.8 & 312.1 & 367.2 & 397.0 \\
\hline Petroleum & 64.9 & 224.9 & 428.0 & 514.0 & 430.0 & 495.7 & 412.2 & 513.4 \\
\hline Asphalt and Road Oil .............................................. & 13.7 & 33.2 & 62.8 & 117.7 & 113.5 & 102.0 & 84.1 & 85.0 \\
\hline 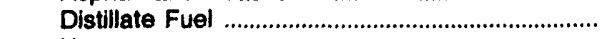 & 11.4 & 52.4 & 100.8 & 150.0 & 82.3 & 103.8 & 82.7 & 135.1 \\
\hline 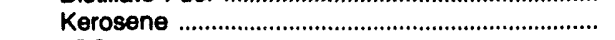 & 2.7 & 3.8 & 29.5 & 7.3 & 9.8 & 10.1 & 7.1 & 4.5 \\
\hline 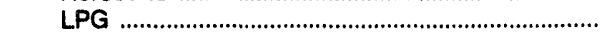 & 9.9 & 20.1 & 32.8 & 20.8 & 21.1 & 21.0 & 19.7 & 39.8 \\
\hline 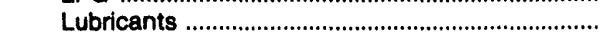 & 12.1 & 20.0 & 44.1 & 49.2 & 42.6 & 42.0 & 43.5 & 54.7 \\
\hline 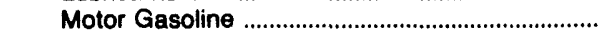 & 3.0 & 4.4 & 5.4 & 24.4 & 15.8 & 16.7 & 14.6 & 20.7 \\
\hline Residual Fuel .......................................................... & 4.4 & 61.1 & 70.5 & 7.9 & 53.5 & 92.9 & 67.6 & 67.1 \\
\hline Other & 7.7 & 29.9 & 82.1 & 136.6 & 91.5 & 107.1 & 92.9 & 106.5 \\
\hline 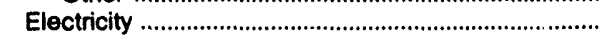 & 135.3 & 372.7 & 912.1 & $1,036.4$ & $1,002.8$ & $1,049.6$ & $1,088.7$ & $1,087.7$ \\
\hline $\begin{array}{l}\text { Transportation Sector } \\
\text { Primary Energy }\end{array}$ & $\begin{array}{l}608.4 \\
608.4\end{array}$ & $\begin{array}{l}1,268.0 \\
1,268.0\end{array}$ & $\begin{array}{l}2,923.8 \\
2,923.8\end{array}$ & $\begin{array}{l}2,697.1 \\
2,697.1\end{array}$ & $\begin{array}{l}2,255.8 \\
2,255.8\end{array}$ & $\begin{array}{l}2,491.4 \\
2,491.4\end{array}$ & $\begin{array}{l}2,471.6 \\
2,471.6\end{array}$ & $\begin{array}{l}2,923.8 \\
2,923.8\end{array}$ \\
\hline Coal ....... & .1 & & - & - & & - & - & $2,0<0.0$ \\
\hline 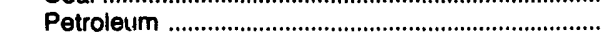 & 608.3 & $1,268.0$ & $2,923.8$ & $2,697.1$ & $2,255.8$ & $2,491.4$ & $2,471.6$ & $2,923.8$ \\
\hline 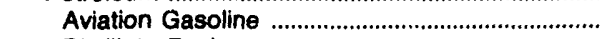 & 3.8 & 4.3 & 11.3 & 8.7 & 8.7 & 5.4 & 5.9 & 5.5 \\
\hline 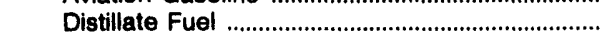 & 41.3 & 154.6 & 449.8 & 426.4 & 418.0 & 510.9 & 548.8 & 742.6 \\
\hline 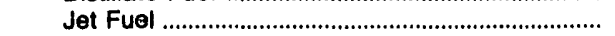 & 7.2 & 19.1 & 72.3 & 121.6 & 93.5 & 95.2 & 42.2 & 52.7 \\
\hline LPG & .6 & 1.0 & .9 & 2.7 & 2.6 & 1.8 & 1.6 & 2.6 \\
\hline 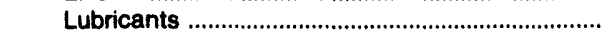 & 13.0 & 27.7 & 42.3 & 47.2 & 40.9 & 40.2 & 41.8 & 52.5 \\
\hline Motor Gasoline ................................................... & 538.8 & 996.1 & $2,282.5$ & $2,053.8$ & $1,679.9$ & $1,822.0$ & $1,800.4$ & $2,037.0$ \\
\hline 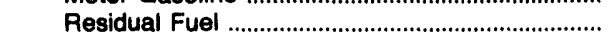 & 3.5 & 65.2 & 64.6 & 36.8 & 12.3 & 15.8 & 31.0 & 30.9 \\
\hline 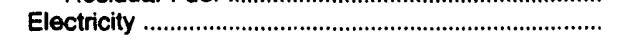 & - & - & - & - & - & - & - & \\
\hline 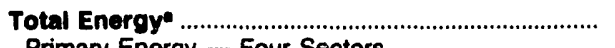 & $1,398.6$ & $3,081.5$ & $6,646.5$ & $7,375.5$ & $6,754.3$ & $\begin{array}{r}7,213.4 \\
4,2625\end{array}$ & $\begin{array}{l}7,307.4 \\
4,267\end{array}$ & $\begin{array}{l}7,991.7 \\
4,8519\end{array}$ \\
\hline $\begin{array}{l}\text { Primary Energy - Four Sectors } \\
\text { Electricity }\end{array}$ & $\begin{array}{l}987.0 \\
4 i 1.6\end{array}$ & $\begin{array}{r}2,141.4 \\
940.2\end{array}$ & $\begin{array}{l}4,525.9 \\
2,120.5\end{array}$ & $\begin{array}{l}4,639.6 \\
2,735.9\end{array}$ & $\begin{array}{l}3,935.9 \\
2,818.4\end{array}$ & $\begin{array}{l}4,262.5 \\
2,950.9\end{array}$ & $\begin{array}{l}4,267.7 \\
3,039.7\end{array}$ & $\begin{array}{l}4,851.9 \\
3,138.7\end{array}$ \\
\hline Eloctric Utility Soctor & 103.4 & 385.8 & 849.4 & $1,174.9$ & $1,088.6$ & $1,063.7$ & $1,046.7$ & $1,065.0$ \\
\hline 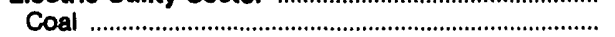 & 98.6 & 367.5 & 755.2 & $1,049.4$ & 996.9 & 975.7 & 947.4 & 981.4 \\
\hline Natural Gas & 4.2 & 6.7 & 4.1 & 3.8 & 2.8 & 3.3 & 5.5 & 4.0 \\
\hline Petroleum & .6 & 7.5 & 4.8 & 3.1 & 1.4 & 1.4 & 3.7 & 5.2 \\
\hline 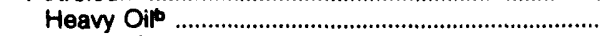 & - & 1.0 & - & - & - & - & - & - \\
\hline 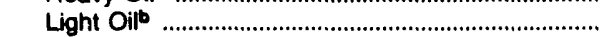 & .1 & 6.5 & 4.8 & 3.1 & 1.4 & 1.4 & 3.7 & 5.2 \\
\hline Petroleum Coke & .4 & - & - & & - & & - & - \\
\hline Nuclear Fuel ....................................................... & - & 4.2 & 85.2 & 118.7 & 87.5 & 83.3 & 90.1 & 74.4 \\
\hline Wood and Waste & - & - & - & - & - & - & - & - \\
\hline Primary Energy - Five Sectors ………………….......... & $1,090.4$ & $2,527.2$ & $5,375.3$ & $5,814.5$ & $5,024.5$ & $5,326.2$ & $5,314.5$ & $5,916.9$ \\
\hline
\end{tabular}

-There are no direct fuel costs for hydroelectric, geothermal, centralized solar, or wind energy. Wood and other biomass fuels are not included, except those consumed at electric utlitities.

byeavy oil includes Grade Nos. 4, 5, and 6, and residual fuel oils. Light oil includes Grade No. 2 heating oil, kerosene, and jet fuel.

- No consumption, including cases where adjustments were made. See "Consumption Adjustments for Process Fuel and Intermediate Products."

- Value less than 0.05 million dollars.

Note: Totals may not equal sum of components due to independent rounding

Sources: Data sources, estimation procedures, and assumptions are described in the "Documentation" section of this report. 


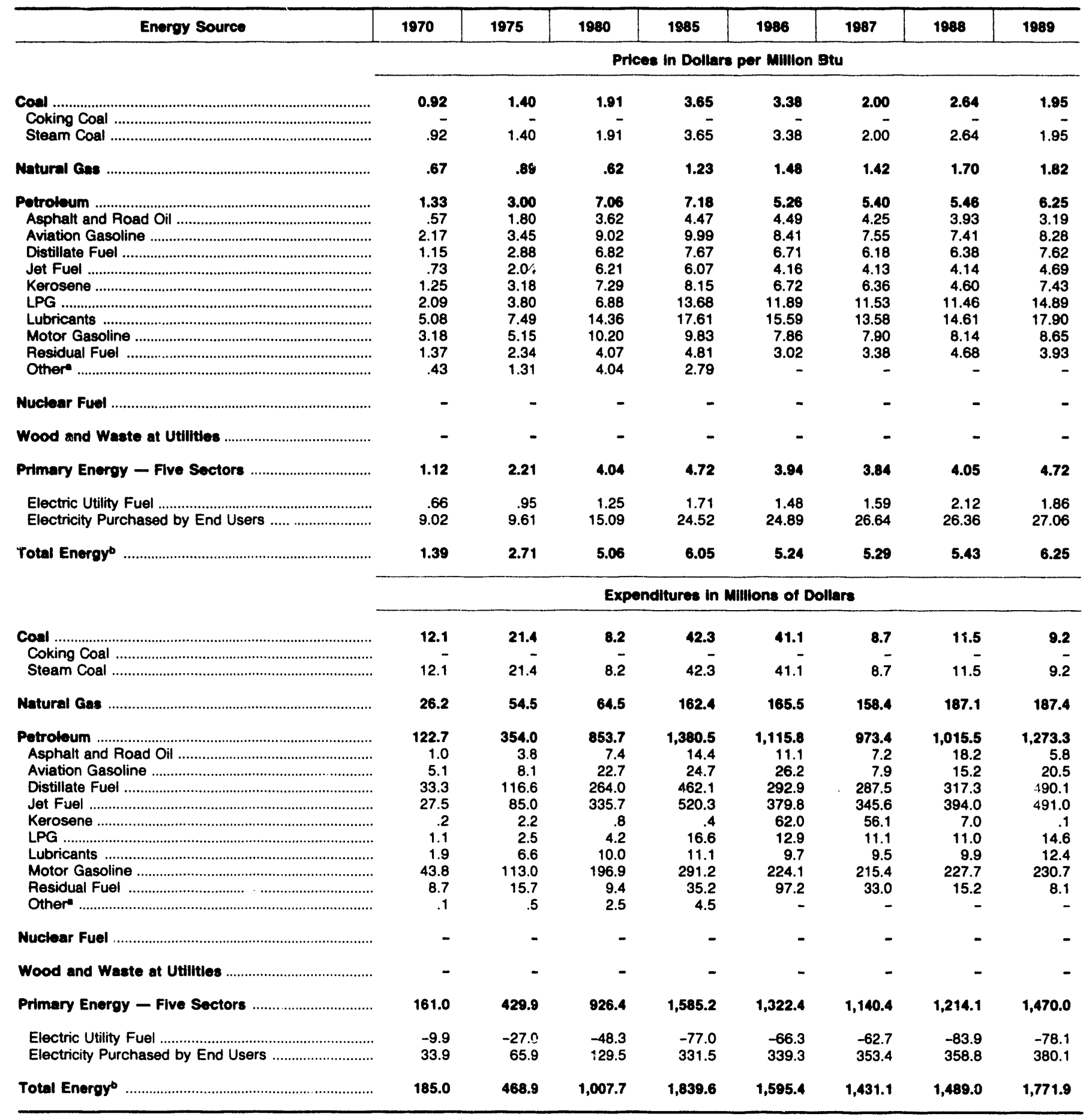

Includes petroleum coke used at electric utilities.

bThere are no direct fuel costs for hydroelectric, geothermal, centralized solar, or wind energy. Wood and uther biomass fuels are not included, except those consumed at electric utilities.

-No consumption, including cases where adjustments were made. See "Consumption Adjustments for Process Fuel and Intermediate Products."

Note: Expenditure totals may not equal sum of components due to independent rounding.

Sources: Data sources, estimation procedures, and assumptions are described ir the "Documentation" section of this report. 


\section{A Energy Price Estimates by Sector, Alaska \\ L $1970,1975,1980$, and $1985-1989$ \\ A (Dollars per Million Btu)}

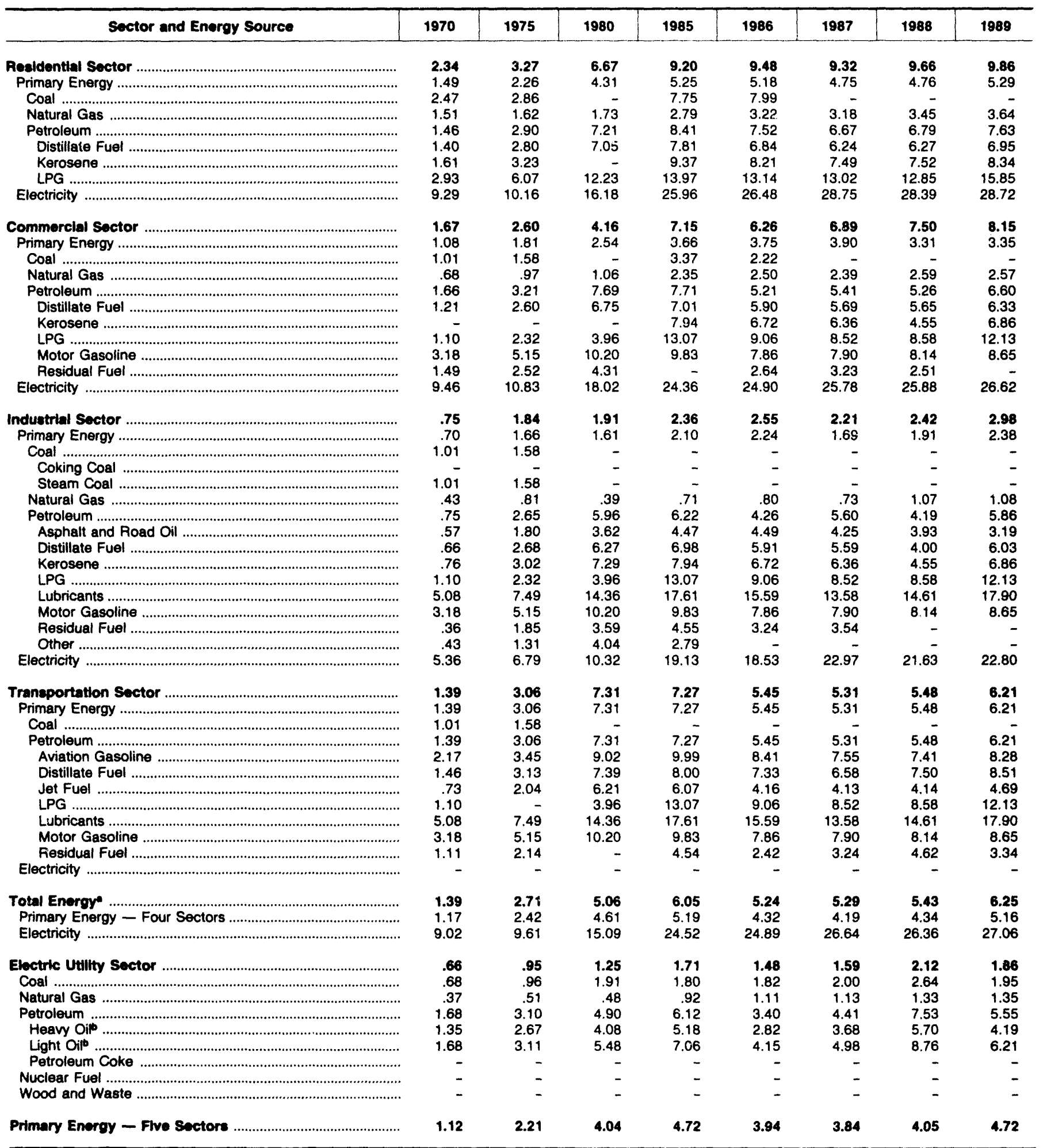

- There are no direct fuel costs for hydroetectric, geothermal, centralized solar, or wind energy. Wood and other biomass fuels are not included, except those consumed at electric utilities.

Heavy oil includes Grade Nos. 4, 5, and 6, and residual fuel oils. Light oil includes Grade No. 2 heating oil, kerosene, and jet fuel.

-No consumption, including cases where adjustments were made. See "Consumption Adjustments for Process Fuel and Intermediate Products."

Sources: Data sources, estimation procedures, and assumptions are described in the "Documentation" section of this report. 


\begin{tabular}{|c|c|c|c|c|c|c|c|c|}
\hline Sector and Energy Source & 1970 & 1975 & 1980 & 1985 & 1986 & 1987 & 1988 & 1989 \\
\hline $\begin{array}{l}\text { Reeldential Sector } \\
\text { Primary Energy }\end{array}$ & 38.6 & 77.9 & 124.8 & 275.5 & 260.4 & 258.3 & 252.8 & 283.4 \\
\hline Primary Energy & 21.9 & 46.8 & 64.5 & 127.2 & 113.6 & 106.4 & 98.8 & 122.3 \\
\hline 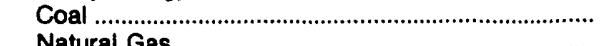 & .4 & .3 & - & 18.7 & 22.0 & - & - & - \\
\hline 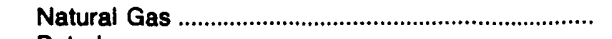 & 9.4 & 16.9 & 13.8 & 37.3 & 39.3 & 39.3 & 43.4 & 49.3 \\
\hline 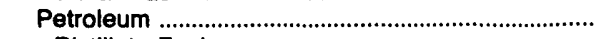 & 12.1 & 29.6 & 50.8 & 71.2 & 52.3 & 67.0 & 55.5 & 73.0 \\
\hline 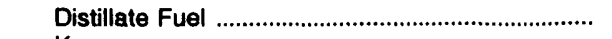 & 11.1 & 26.4 & 48.2 & 59.6 & 42.4 & 58.6 & 46.9 & 61.5 \\
\hline 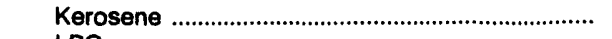 & .2 & 1.7 & - & .1 & $\dot{0}$ & 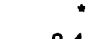 & .2 & .1 \\
\hline 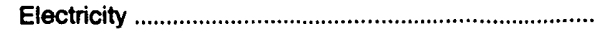 & 16.7 & 31.1 & 60.3 & 148.3 & 146.8 & 151.9 & 154.0 & 161.0 \\
\hline 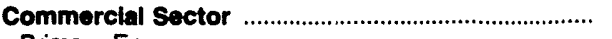 & 39.0 & 66.4 & 99.0 & 274.6 & 351.6 & 325.9 & 263.7 & 276.2 \\
\hline 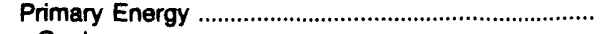 & 23.5 & 42.1 & 54.2 & 116.9 & 185.3 & 159.2 & 94.8 & 90.2 \\
\hline Coal & .3 & .3 & - & 15.1 & 11.3 & - & - & - \\
\hline 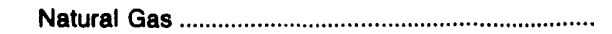 & 8.6 & 14.0 & 17.5 & 48.1 & 52.7 & 48.7 & 54.2 & 55.9 \\
\hline Petroleum & 14.7 & 27.8 & 36.8 & 53.7 & 121.2 & 110.5 & 40.6 & 34.4 \\
\hline 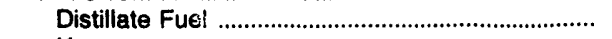 & 3.0 & 7.6 & 22.7 & 37.8 & 28.8 & 34.9 & 28.8 & 30.4 \\
\hline Kerosene & - & - & - & .1 & 61.9 & 56.1 & 6.7 & .1 \\
\hline Residual Fuel & 7.5 & 8.9 & .1 & - & 21.1 & 16.4 & 1.9 & - \\
\hline 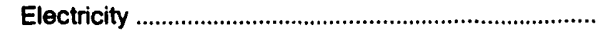 & 15.4 & 24.3 & 44.8 & 157.7 & 166.3 & 166.6 & 168.9 & 186.0 \\
\hline Industrial Sector & 25.7 & 81.2 & 128.0 & 205.5 & 193.7 & 136.4 & 154.4 & 147.1 \\
\hline Primary Energy & 23.9 & 70.7 & 103.5 & 180.0 & 167.5 & 101.5 & 118.5 & 114.0 \\
\hline Coal & 8.6 & 16.5 & - & - & - & - & - & - \\
\hline Coking Coal & - & - & - & - & - & - & - & - \\
\hline Steam Coal & 8.6 & 16.5 & - & - & - & - & - & - \\
\hline 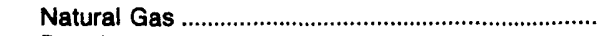 & 5.1 & 13.5 & 19.5 & 45.2 & 34.9 & 35.5 & 48.3 & 37.7 \\
\hline 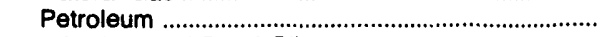 & 10.2 & 40.7 & 84.0 & 134.9 & 132.5 & 66.0 & 70.3 & 76.3 \\
\hline 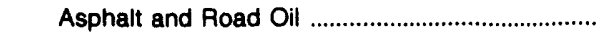 & 1.0 & 3.8 & 7.4 & 14.4 & 11.1 & 7.2 & 18.2 & 5.8 \\
\hline 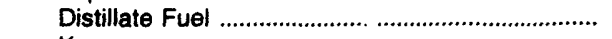 & 6.9 & 30.8 & 64.0 & 71.2 & 37.7 & 31.9 & 46.0 & 64.1 \\
\hline Kerosene & .1 & .5 & .8 & .2 & * & $\bullet$ & $\bullet$ & * \\
\hline LPG & .2 & .8 & 1.3 & 2.6 & 1.5 & 1.5 & 1.5 & 1.2 \\
\hline 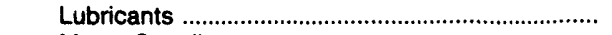 & • & 1.1 & 1.8 & 2.0 & 1.7 & 1.7 & 1.8 & 2.2 \\
\hline 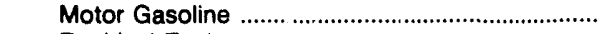 & 1.8 & 2.9 & 5.9 & 21.0 & 15.9 & 16.6 & 2.7 & 2.9 \\
\hline 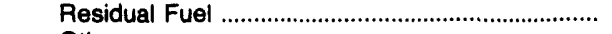 & .1 & .3 & .3 & 19.1 & 64.6 & 6.9 & - & - \\
\hline Other & .1 & .5 & 2.5 & 4.5 & - & - & - & - \\
\hline 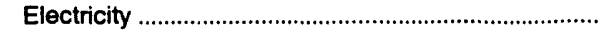 & 1.7 & 10.6 & 24.5 & 25.5 & 26.2 & 34.9 & 35.8 & 33.0 \\
\hline 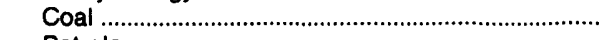 & & & - & - & - & $\bar{z}$ & - & 10 \\
\hline 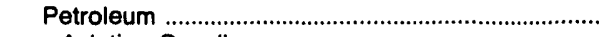 & 81.7 & 243.3 & 655.9 & $1,084.0$ & 789.8 & 710.5 & 818.1 & 1.065 .3 \\
\hline 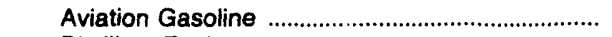 & 5.1 & 8.1 & 22.7 & 24.7 & 26.2 & 7.9 & 15.2 & 20.5 \\
\hline 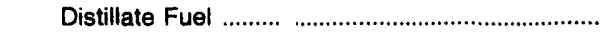 & 8.5 & 39.3 & 112.1 & 272.2 & 173.5 & 149.8 & 173.9 & 315.8 \\
\hline 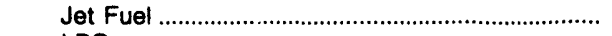 & 27.5 & 85.0 & 335.7 & 520.3 & 379.8 & 345.6 & 394.0 & 491.0 \\
\hline LPG & & - & .1 & .6 & .3 & .2 & .2 & .3 \\
\hline 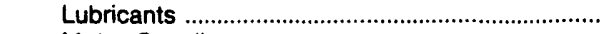 & 1.8 & 5.5 & 8.2 & 9.1 & 7.9 & 7.8 & 8.1 & 10.2 \\
\hline Motor Gasoline & 37.9 & 98.9 & 177.1 & 256.4 & 199.9 & 196.6 & 222.8 & 225.4 \\
\hline 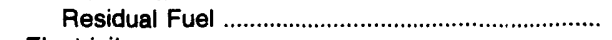 & .9 & 6.5 & - & .6 & 2.2 & 2.6 & 3.9 & 2.1 \\
\hline 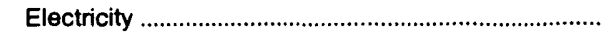 & - & - & - & - & - & - & - & - \\
\hline Total Energy" & 185.0 & 468.9 & $1,007.7$ & $1,839.6$ & $1,595.4$ & $1,431.1$ & $1,489.0$ & $1,771.9$ \\
\hline 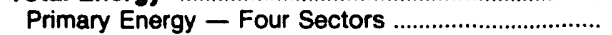 & 151.2 & 403.0 & 878.2 & $1,508.2$ & $1,256.1$ & $1,077.7$ & $1,130.2$ & $1,391.9$ \\
\hline 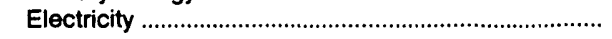 & 33.9 & 65.9 & 129.5 & 331.5 & 339.3 & 353.4 & 358.8 & 380.1 \\
\hline Electric Utility Sector & 9.9 & 27.0 & 48.3 & 77.0 & 66.3 & 62.7 & 83.9 & 78.1 \\
\hline Coal & 2.9 & 4.3 & 8.2 & 8.4 & 7.8 & 8.7 & 11.5 & 9.2 \\
\hline 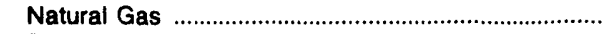 & 3.1 & 10.1 & 13.8 & 31.8 & 38.5 & 34.8 & 41.3 & 44.5 \\
\hline Petroleum & 3.9 & 12.6 & 26.3 & 36.8 & 19.9 & 19.3 & 31.1 & 24.4 \\
\hline 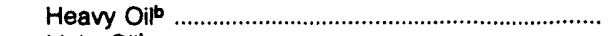 & & 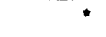 & 9.1 & 15.5 & 9.4 & 7.1 & 9.4 & 6.1 \\
\hline 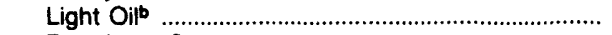 & 3.9 & 12.6 & 17.2 & 21.3 & 10.6 & 12.2 & 21.6 & 18.3 \\
\hline 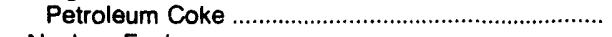 & - & - & - & - & - & - & - & - \\
\hline 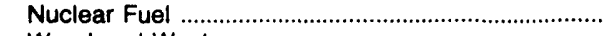 & - & - & - & - & - & - & - & - \\
\hline 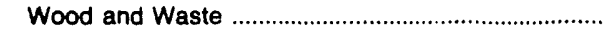 & - & - & - & - & - & - & - & - \\
\hline Primary Energy - Five Sectors ................................ & 161.0 & 429.9 & 926.4 & $1,585.2$ & $1,322.4$ & $1,140.4$ & $1,214.1$ & $1,470.0$ \\
\hline
\end{tabular}

-There are no direct fuel costs for hydroelectric, geothermal, centralized solar, or wind energy. Wood and other biomass fuels are not included, except those consumed at electric utilities.

beavy oil includes Grade Nos. 4, 5, and 6, and residual fuel oils. Light oil includes Grade No. 2 heating oil, kerosene, and jet fuel.

-No consumption, including cases where adjustments were made. See "Consumption Adjustments for Process Fuel and Intermediate Products."

- Value less than 0.05 million dollars.

Note: Totals may not equal sum of components due to independent rounding.

Sources: Data sources, estimation procedures, and assumptions are described in the "Documentation" section of this report. 


\section{A Energy Price and Expenditure Estimates by Source, Arizona \\ R $1970,1975,1980$, and 1985-1989}

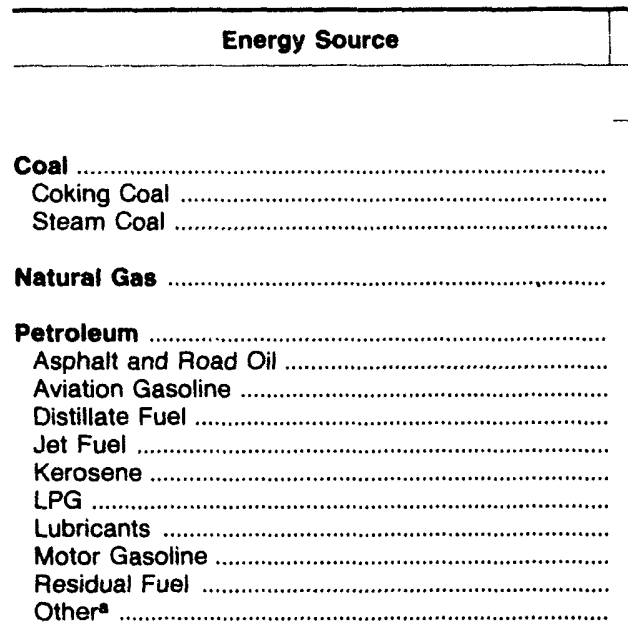

.

Nuclear Fuel

Wood and Waste at Utilities

Primary Energy - Five Sectors

Electric Utility Fuel

Electricity Purchased by End Users

\begin{tabular}{c|c|c|c|c|c}
\hline 1970 & 1975 & 1980 & 1985 & 1986 & 1987 \\
Prices in Dollars per Million Btu
\end{tabular}

Total Energy ${ }^{b}$

0.21
.21
.54
1.96
.60
2.17
1.10
.76
1.63
2.08
5.08
2.80
.48
-

0.23
.23

$\begin{array}{rr}1.01 & 1.36 \\ 1.01 & 1 \\ 2.86 & 4.92 \\ 8.14 & 8 \\ 3.63 & 4.76 \\ 9.02 & 9 \\ 6.57 & 6 \\ 6.59 & 6 \\ 5.45 & 5 \\ 6.85 & 9 \\ 14.36 & 17 \\ 9.68 & 9 \\ 3.92 & 3.01 \\ 4.04 & \\ & \end{array}$

1.36
-
1.36
4.92
8.08
4.76
9.99
6.45
6.20
5.79
9.98
17.61
9.06
3.74
-

$\begin{array}{rr}1.49 & 1.33 \\ - & -\end{array}$

$1.3 \overline{3}$

$1.42 \quad 1.38$

.54

.60
2.17

1.10

.76
1.63

2.08

5.08

.48

1.01

\subsection{5}

1.87

3.45

2.49
2.12

3.19

4.06

7.49

4.62
2.08

1.31

1.49

1.42

1.38

$\begin{array}{lll}-4.031 & 4.04\end{array}$

Coal
Coking Coal
Sieam Coal

Natural Gas

Petroleum

Asphalt and Road Oil

Aviation Gasoline

Oil

Distillate Fuel

Jet Fuel

Kerosene

LPG .............

.

Gasoline

Residual Fue

Other"

Nuclear Fuel

Wood and Waste at Utilities

Primary Energy - Five Sectors

$516.4 \quad 1,226.3$

$3,129.1$

$3,622.2$

$4.12 \quad 4.10$

4.45

3.92

Electric Utility Fuel

Electricity Purchased by End Users

Total Energyb

$\begin{array}{lll}-23.5 & -129.6 & -398.7\end{array}$

$250.1 \quad 697.1 \quad 1,431.6$

$\mathbf{7 4 3 . 0}$

$4,161.9$

$\begin{array}{ll}6.47 & 6.87 \\ 4.33 & 3.21\end{array}$

$8.41 \quad 7.55$

$\begin{array}{ll}8.41 & 7.55 \\ 5.32 & 6.02\end{array}$

$4.25 \quad 4.36$

$3.60 \quad 3.55$

$9.58 \quad 9.89$

$15.59 \quad 13.58$

$\begin{array}{ll}7.24 & 7.77\end{array}$

$2.38 \quad 2.28$

$\begin{array}{ll}6.55 & 7.30\end{array}$

$\begin{array}{ll}3.28 & 2.84 \\ 7.41 & 8.28\end{array}$

$\begin{array}{ll}7.41 & 8.28 \\ 5.97 & 6.33\end{array}$

$\begin{array}{ll}4.15 & 4.80\end{array}$

$4.82 \quad 4.40$

$9.06 \quad 11.51$

$14.61 \quad 17.90$

$\begin{array}{rr}7.31 & 8.19 \\ 2.41 & 2.76 \\ - & -\end{array}$

$\begin{array}{lll}.65 & .82 \quad .77 \quad .85\end{array}$

Includes petroleum coke used at electric utilities.

Dhere are no direct fuel costs for hydroelectric, geothermal, centralized solar, or wind energy. Wood and other biomass fuels are not included, except those consumed at electric utilities.

-No consumption, including cases where adjustments were made. See "Consumption Ad;jstments for Process Fuel and Intermediate Products."

- Value less than 0.05 million dollars.

Note: Expenditure totals may not equal sum of components due to independent rounding.

Sources: Data sources, estimation procedures, and assumptions are described in the "Documentation" section of this report. 


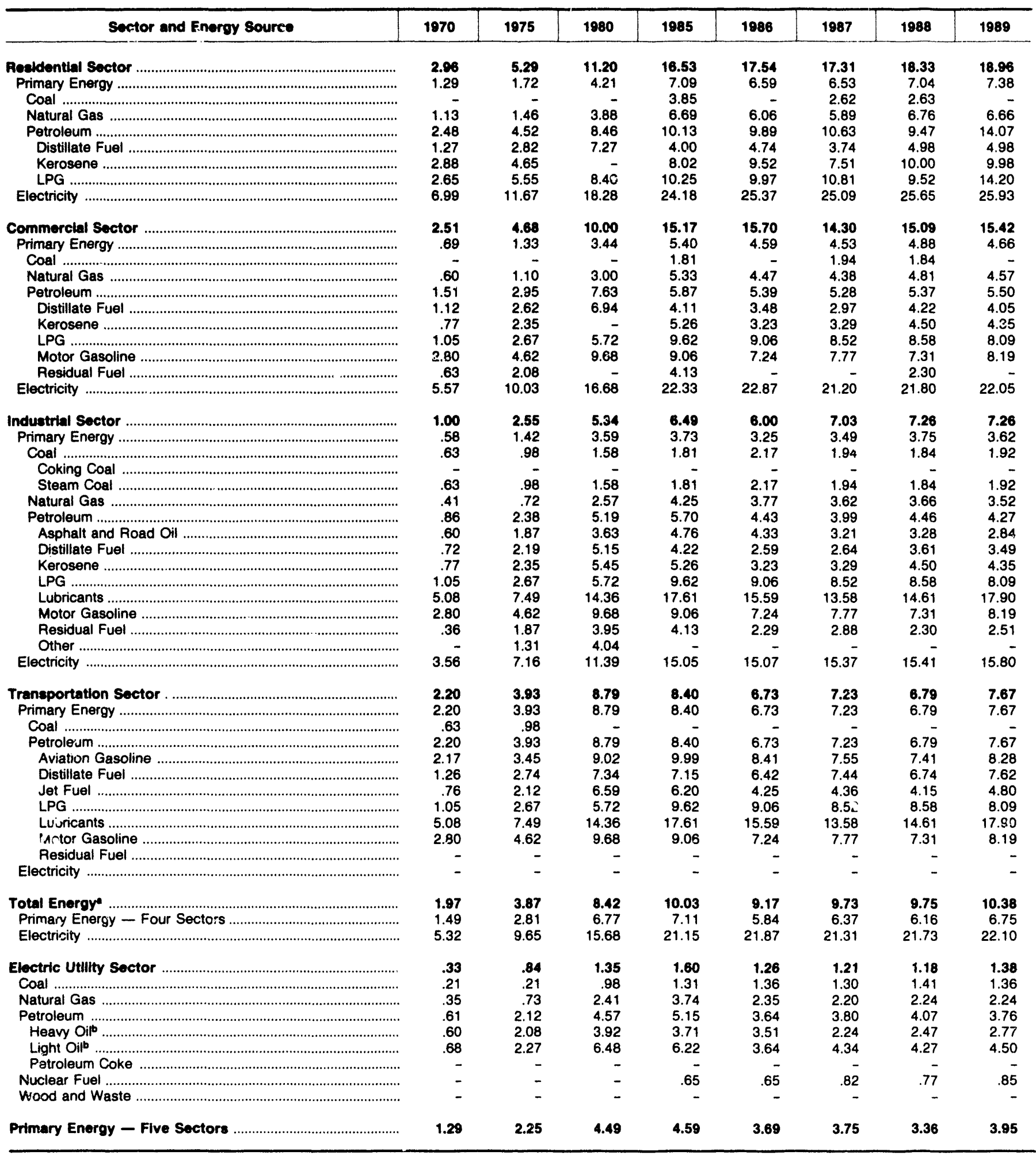

-There are no direct fuel costs for hydroelectric, geothermal, centralized solar, or wind energy. Wood and other biomass fuels are not included, except those consumed at electric utilities.

b Heavy oil includes Grade Nos. 4,5, and 6, and residual fuel oils. Light oil includns Grade No. 2 heating oil, kerosene, and jet fuel.

- No consumption, including cases where adjustments were made. See "Consumption Adjustments for Process Fuel and Intermediate Products."

Sources: Data sources, estimation procedures, and assumptions are described in the "Documentation" section of this report. 


\section{A Energy Expenditure Estimates by Sector, Arizona \\ R $1970,1975,1980$, and $1985-1989$ (Million Dollars)}

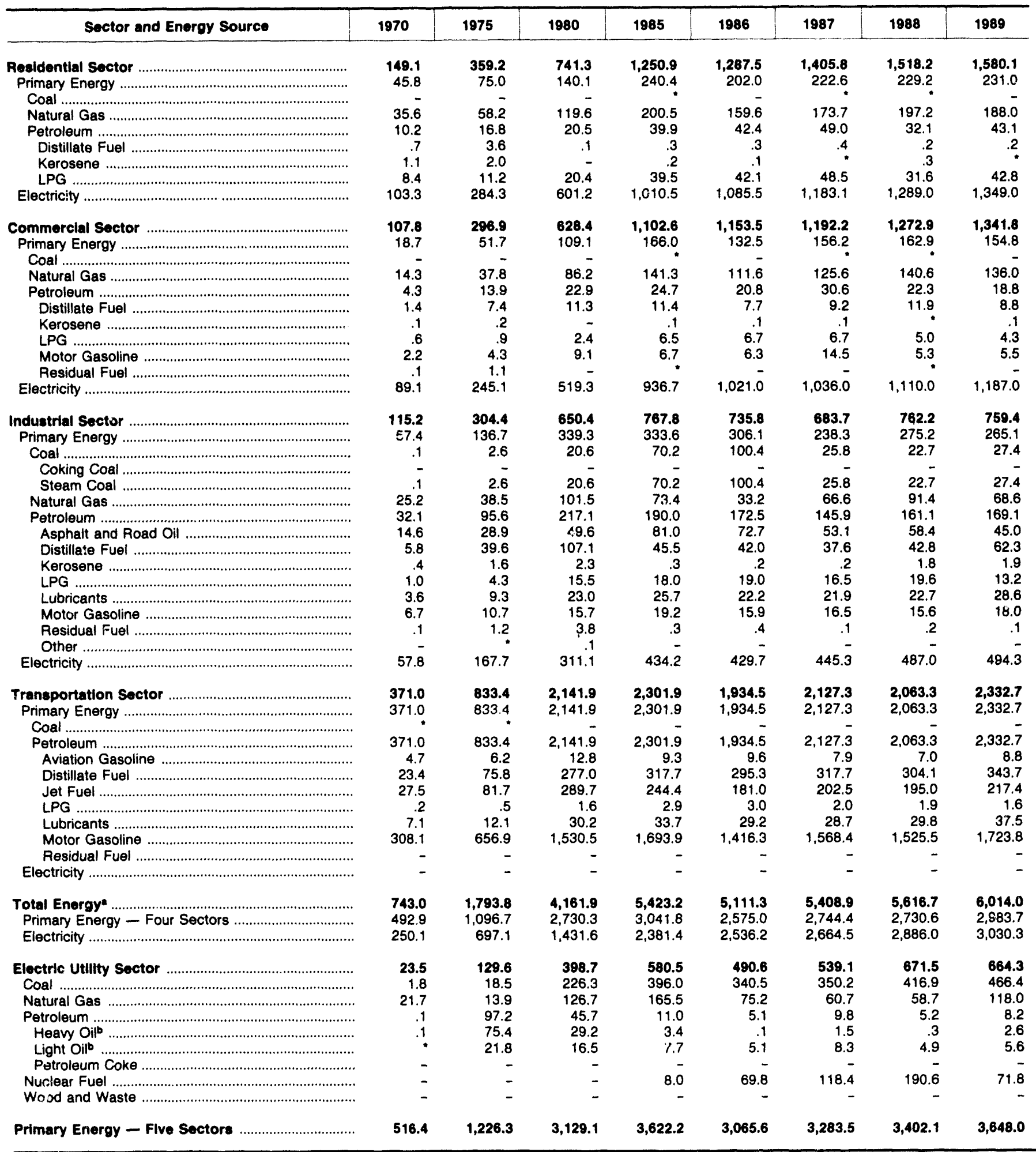

-There are no direct fuel costs for hydroelectric, geothermal, centralized solar, or wind energy. Wood and cther biomass fuels are not included, except those consumed at electric utilities.

bHeavy oil includes Grade Nos. 4, 5, and 6, and residual fuel oils. Light oil includes Grade No. 2 heating oil, kerosene, and jet fuel.

- No consumption, including cases where adjustments were made. See "Consumption Adjustments for Process Fuel and Intermediate Products." - Value less than 0.05 million dollars.

Note: Totals may not equal sum of components due to independent rounding.

Sources: Data sources, estimation procedures, and assumptions are described in the "Documentation" section of this report. 


\begin{tabular}{|c|c|c|c|c|c|c|c|c|}
\hline Energy Source & 1970 & 1975 & 1980 & 1985 & 1986 & 1987 & 1988 & 1989 \\
\hline & \multicolumn{8}{|c|}{ Prices in Dollars per Million Btu } \\
\hline Conal & - & 1.22 & 1.44 & 1.60 & 1.58 & 1.57 & 1.60 & 1.64 \\
\hline 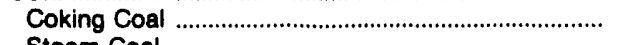 & - & - & - & - & - & - & - & - \\
\hline 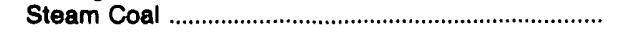 & - & 1.22 & 1.44 & 1.60 & 1.58 & 1.57 & 1.60 & 1.64 \\
\hline 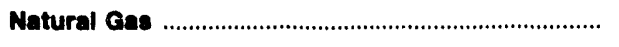 & 0.38 & .79 & 2.27 & 3.83 & 3.53 & 3.34 & 3.43 & 3.39 \\
\hline Potroloum & 1.96 & 3.30 & 7.56 & 8.17 & 5.70 & 6.43 & 6.22 & 6.74 \\
\hline 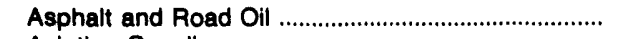 & .68 & 1.81 & 3.58 & 4.21 & 4.62 & 3.33 & 3.27 & 2.90 \\
\hline 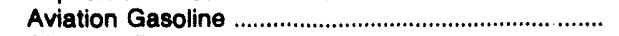 & 2.17 & 3.45 & 9.02 & 9.99 & 8.41 & 7.55 & 7.41 & 8.28 \\
\hline 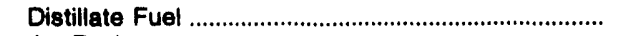 & .98 & 2.39 & 6.04 & 7.92 & 4.76 & 5.68 & 5.13 & 5.92 \\
\hline Jet Fuel & .72 & 2.01 & 6.34 & 5.96 & 3.93 & 4.07 & 3.66 & 4.27 \\
\hline 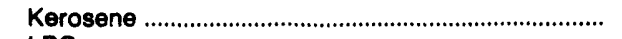 & .88 & 2.41 & 5.51 & 10.29 & 3.85 & 5.04 & 4.29 & 4.92 \\
\hline LPG & 1.63 & 3.12 & 6.97 & 8.54 & 7.54 & 7.29 & 7.71 & 7.16 \\
\hline 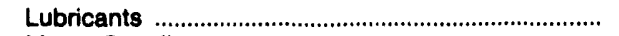 & 5.08 & 7.49 & 14.36 & 17.61 & 15.59 & 13.58 & 14.61 & 17.80 \\
\hline 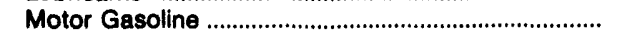 & 2.74 & 4.60 & 9.93 & 8.80 & 6.84 & 7.28 & 7.13 & 7.69 \\
\hline 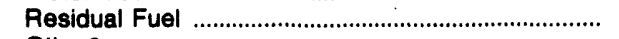 & .43 & 1.72 & 3.23 & 4.01 & 2.41 & 2.74 & 2.58 & 2.71 \\
\hline 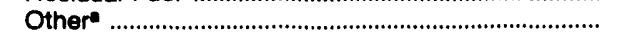 & .52 & 2.11 & 5.99 & 7.20 & 6.65 & 7.34 & 6.49 & 6.64 \\
\hline 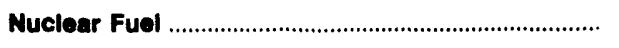 & - & .24 & .54 & .77 & .71 & .75 & .74 & .75 \\
\hline Wood and Waste at Utilties & - & - & - & - & - & - & - & - \\
\hline 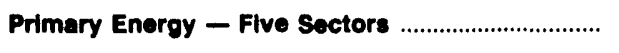 & 1.02 & 2.10 & 4.37 & 4.43 & 3.48 & 3.60 & 3.65 & 3.90 \\
\hline $\begin{array}{l}\text { Electric Utility Fuel ... } \\
\text { Electricity Purchased by End Users }\end{array}$ & $\begin{array}{r}.26 \\
4.78\end{array}$ & $\begin{array}{r}.72 \\
7.80\end{array}$ & $\begin{array}{r}1.46 \\
12.77\end{array}$ & $\begin{array}{r}1.36 \\
18.24\end{array}$ & $\begin{array}{r}1.32 \\
19.61\end{array}$ & $\begin{array}{r}1.28 \\
19.00\end{array}$ & $\begin{array}{r}1.34 \\
18.76\end{array}$ & $\begin{array}{r}1.39 \\
19.04\end{array}$ \\
\hline \multirow[t]{2}{*}{ Total Enorgy' } & 1.51 & 2.88 & 6.68 & 8.34 & 7.16 & 7.85 & 7.35 & 7.56 \\
\hline & \multicolumn{8}{|c|}{ Expenditures in Millions of Dollars } \\
\hline Coal & - & 1.1 & 52.6 & 351.1 & 354.7 & 330.7 & 349.4 & 332.8 \\
\hline 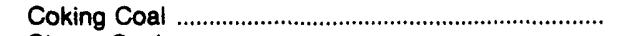 & - & - & - & & & & & \\
\hline 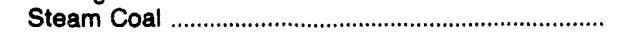 & - & 1.1 & 52.6 & 351.1 & 354.7 & 330.7 & 349.4 & 332.8 \\
\hline 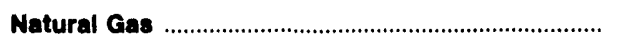 & 133.8 & 185.8 & 581.7 & 636.9 & 613.0 & 486.6 & 632.7 & 724.6 \\
\hline Potroloum n. & 465.7 & $1,125.6$ & $2,313.0$ & 2,426.4 & $1,752.8$ & $1,857.4$ & $1,858.5$ & $2,051.3$ \\
\hline 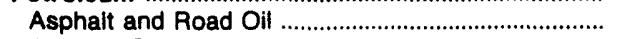 & 9.4 & 27.4 & 65.9 & 35.3 & 30.1 & 22.5 & 29.8 & 15.0 \\
\hline 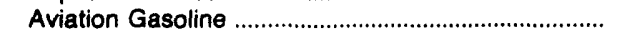 & 3.2 & 4.4 & 12.5 & 4.4 & 4.7 & 3.5 & 3.7 & 4.3 \\
\hline 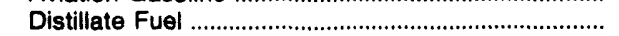 & 31.1 & 133.2 & 376.2 & 687.9 & 367.7 & 444.5 & 423.4 & 500.9 \\
\hline 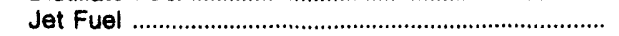 & 8.5 & 21.7 & 70.0 & 65.7 & 41.1 & 45.8 & 44.5 & 45.2 \\
\hline 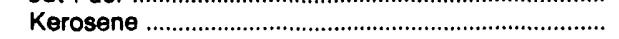 & 4.1 & 9.4 & 17.8 & 85.4 & 15.1 & 19.6 & 22.5 & 13.3 \\
\hline IPG & 62.4 & 100.4 & 123.7 & 129.1 & 109.3 & 96.3 & 109.4 & 127.9 \\
\hline 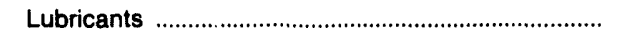 & 16.4 & 28.0 & 61.0 & 68.0 & 58.9 & 58.0 & 60.2 & 75.6 \\
\hline 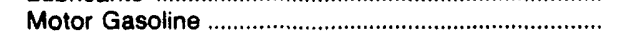 & 323.7 & 666.5 & $1,381.9$ & $1,235.5$ & $1,001.9$ & $1,089.6$ & $1,108.1$ & $1,187.7$ \\
\hline 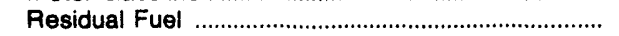 & 2.4 & 97.6 & 100.3 & 60.3 & 95.1 & 44.7 & 29.8 & 54.8 \\
\hline Other" & 4.4 & 28.0 & 103.6 & 54.8 & 28.9 & 32.7 & 26.9 & 26.5 \\
\hline 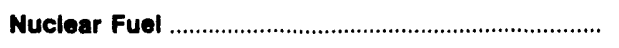 & - & 12.7 & 46.0 & 82.8 & 68.0 & 91.6 & 70.4 & 71.4 \\
\hline 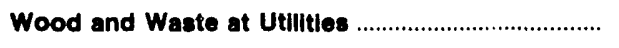 & - & - & - & - & - & - & - & - \\
\hline Primary Energy - Five Sectors ............................... & 599.4 & 1,325.1 & $2,993.3$ & $3,497.2$ & $2,788.5$ & $2,766.2$ & $2,911.0$ & $3,180.0$ \\
\hline $\begin{array}{l}\text { Electric Utility Fuel } \\
\text { Electricity Purchased by End Users }\end{array}$ & $\begin{array}{l}-29.3 \\
217.4\end{array}$ & $\begin{array}{l}-82.2 \\
480.4\end{array}$ & $\begin{array}{r}-286.3 \\
1.149 .8\end{array}$ & $\begin{array}{r}-451.4 \\
1.440 .1\end{array}$ & $\begin{array}{r}-454.4 \\
1,490.5\end{array}$ & $\begin{array}{r}-459.1 \\
1,521.8\end{array}$ & $\begin{array}{r}-445.9 \\
1,566.2\end{array}$ & $\begin{array}{r}-448.2 \\
1,644.7\end{array}$ \\
\hline Total Energyb & 787.6 & $1,723.3$ & $3,856.8$ & 4,485.8 & $3,824.6$ & $3,828.8$ & $4,031.3$ & $4,376.5$ \\
\hline
\end{tabular}

-Includes petroleum coke used at electric utilities.

-There are no direct fuel costs for hydroelectric, geothermal, centralized solar, or wind energy. Wood and other biomass fuels are not included, except those consumed at electric utilities.

- No consumption, including cases where adjustments were made. See "Consumption Adjustments for Process Fuel and Intermediate Products."

Note: Expenditure totals may not equal sum of components due to independent rounding.

Sources: Data sources, estimation procedures, and assumptions are described in the "Documentation" section of this report. 


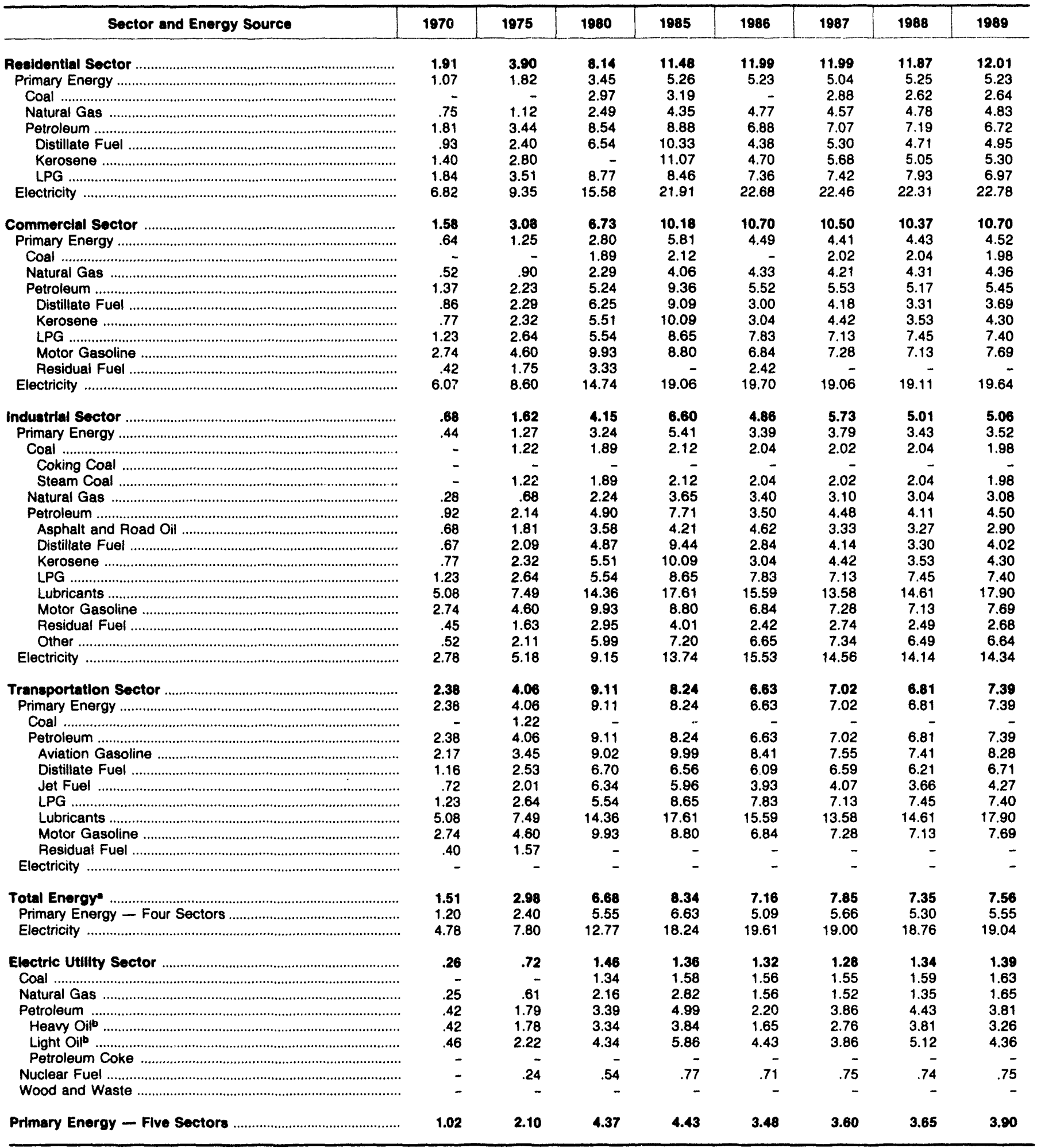

-There are no direct fuel costs for hydroelectric, geothermal, centralized solar, or wind energy. Wood and other biomass fuels are not included, except those consumed at electric utilities.

b Heavy oil includes Grade Nos. 4, 5, and 6, and residual fuel oils. Light oil includes Grade No. 2 heating oil, kerosene, and jet fuel.

- No consumption, including cases where adjustments were made. See "Consumption Adjustments for Process Fuel and Intermediate Products." Sources: Data sources, estimation procedures, and assumptions are described in the "Documentation" section of this report. 


\begin{tabular}{|c|c|c|c|c|c|c|c|c|}
\hline Sector and Energy Source & 1970 & 1975 & 1980 & 1985 & 1986 & 1987 & 1988 & 1989 \\
\hline $\begin{array}{l}\text { Pealdential Sector } \\
\text { Primary Energy }\end{array}$ & $\begin{array}{r}192.8 \\
92.3\end{array}$ & $\begin{array}{l}373.2 \\
125.8\end{array}$ & $\begin{array}{l}734.6 \\
190.8\end{array}$ & $\begin{array}{l}937.2 \\
269.3\end{array}$ & $\begin{array}{l}977.1 \\
260.9\end{array}$ & $\begin{array}{l}995.1 \\
251.2\end{array}$ & $\begin{array}{r}1,037.8 \\
280.8\end{array}$ & $\begin{array}{r}1,056.5 \\
282.5\end{array}$ \\
\hline Coal & - & - & .1 & 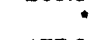 & - & 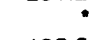 & : & .1 \\
\hline 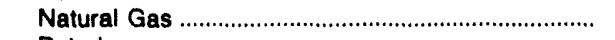 & 45.1 & 54.2 & 115.9 & 177.9 & 186.2 & 185.5 & 206.2 & 205.2 \\
\hline 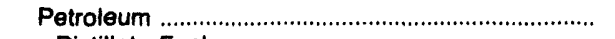 & 47.2 & 71.7 & 74.8 & 91.3 & 74.7 & 65.8 & 74.6 & 77.2 \\
\hline 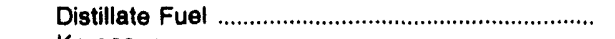 & .4 & 2.2 & 5.8 & 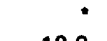 & 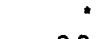 & * & $\cdot$ & * \\
\hline Kerosene & $\begin{array}{r}1.2 \\
45.8\end{array}$ & $\begin{array}{r}2.0 \\
67.4\end{array}$ & $69 . \overline{0}$ & $\begin{array}{l}18.2 \\
73.1\end{array}$ & $\begin{array}{r}9.0 \\
65.6\end{array}$ & $\begin{array}{r}10.8 \\
54.9\end{array}$ & $\begin{array}{r}13.2 \\
61.4\end{array}$ & $\begin{array}{r}8.9 \\
68.3\end{array}$ \\
\hline 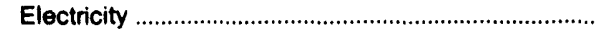 & 100.5 & 247.4 & 543.7 & 667.9 & 716.2 & 743.8 & 757.0 & 774.0 \\
\hline 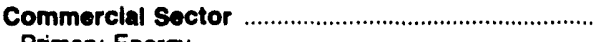 & 87.1 & 184.7 & 371.3 & 616.7 & 528.7 & 528.5 & 559.4 & 586.7 \\
\hline 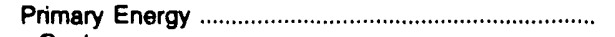 & 29.3 & 58.2 & 103.5 & 236.3 & 131.2 & 129.8 & 142.4 & 146.7 \\
\hline Coal & - & - & .2 & • & - & $\cdot$ & • & .1 \\
\hline 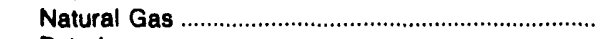 & 20.6 & 29.7 & 69.9 & 110.5 & 109.5 & 104.8 & 119.2 & 119.5 \\
\hline 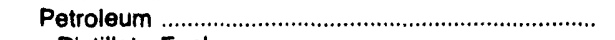 & 8.7 & 26.5 & 33.5 & 125.8 & 21.7 & 24.9 & 23.2 & 27.1 \\
\hline 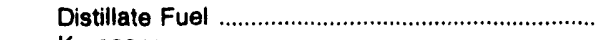 & .2 & 1.2 & 4.1 & 62.0 & 3.3 & 8.8 & 4.9 & 9.4 \\
\hline 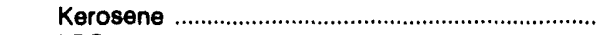 & .4 & 1.0 & 4.1 & 45.1 & 1.5 & 1.9 & 3.5 & .5 \\
\hline LPG & 5.4 & 8.9 & 7.7 & 13.2 & 12.3 & 9.3 & 10.2 & 12.8 \\
\hline 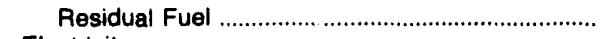 & .1 & 11.9 & 9.2 & - & 4 & - & - & - \\
\hline 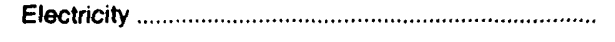 & 57.8 & 128.6 & 267.8 & 380.4 & 397.5 & 398.8 & 417.0 & 440.0 \\
\hline 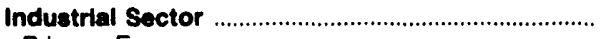 & 143.8 & 365.2 & 994.5 & $1,321.2$ & 976.9 & 828.1 & 941.0 & $1,063.0$ \\
\hline 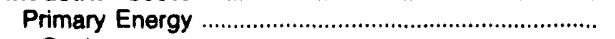 & 84.8 & 260.7 & 656.2 & 929.4 & 600.1 & 449.0 & 548.8 & 632.4 \\
\hline 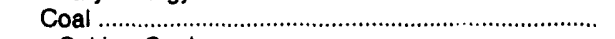 & - & 1.1 & 12.0 & 17.0 & 15.8 & 13.6 & 11.7 & 11.8 \\
\hline 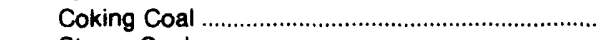 & - & - & - & - & - & - & - & - \\
\hline Steam Coal . & - & 1.1 & 12.0 & 17.0 & 15.8 & 13.6 & 11.7 & 11.8 \\
\hline 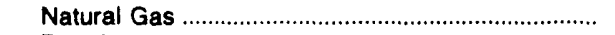 & 40.7 & 82.3 & 265.8 & 314.5 & 270.6 & 146.0 & 276.7 & 350.2 \\
\hline 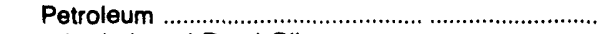 & 44.1 & 177.4 & 378.4 & 597.9 & 313.7 & 289.3 & 260.4 & 270.3 \\
\hline 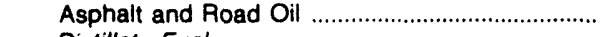 & 9.4 & 27.4 & 65.9 & 35.3 & 30.1 & 22.5 & 29.8 & 15.0 \\
\hline 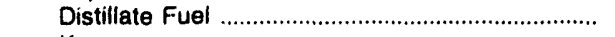 & 7.7 & 34.5 & 100.5 & 331.9 & 87.0 & 112.1 & 95.5 & 84.7 \\
\hline 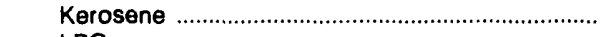 & 2.6 & 6.3 & 13.7 & 22.1 & 4.6 & 7.0 & 5.8 & 3.9 \\
\hline LPG & 8.2 & 26.4 & 42.8 & 38.5 & 29.1 & 30.2 & 35.8 & 44.7 \\
\hline 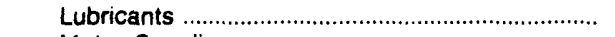 & 7.1 & 14.0 & 23.3 & 26.0 & 22.5 & 22.2 & 23.0 & 29.0 \\
\hline Motor Gasoline & 4.2 & 4.1 & 2.7 & 29.1 & 17.3 & 17.9 & 16.9 & 14.5 \\
\hline 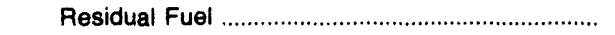 & .5 & 36.7 & 25.9 & 60.1 & 94.2 & 44.7 & 26.6 & 52.1 \\
\hline Other & 4.4 & 28.0 & 103.6 & 54.8 & 28.9 & 32.7 & 26.9 & 26.5 \\
\hline 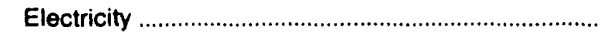 & 59.1 & 104.4 & 338.3 & 391.8 & 376.8 & 379.1 & 392.2 & 430.7 \\
\hline 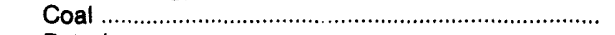 & - & 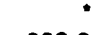 & - & - & - & - & - & - \\
\hline 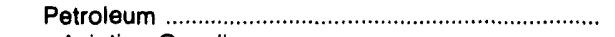 & 363.9 & 800.2 & $1,756.4$ & $1,610.8$ & $1,341.9$ & $1,477.1$ & $1,493.1$ & $1,670.3$ \\
\hline Aviation Gasoline ................................................. & 3.2 & 4.4 & 12.5 & 4.4 & 4.7 & 3.5 & 3.7 & 4.3 \\
\hline 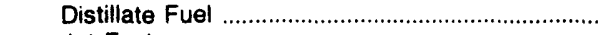 & 22.8 & 94.4 & 261.3 & 293.5 & 277.1 & 323.4 & 319.1 & 403.2 \\
\hline 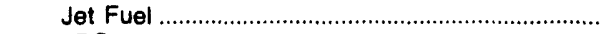 & 8.5 & 21.7 & 70.0 & 65.7 & 41.1 & 45.8 & 44.5 & 45.2 \\
\hline LPG & 3.2 & 6.7 & 4.2 & 4.3 & 2.3 & 1.9 & 2.1 & 2.0 \\
\hline Lubricants & 9.2 & 14.0 & 37.6 & 42.0 & 36.3 & 35.8 & 37.1 & 46.7 \\
\hline 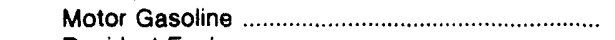 & 316.9 & 658.9 & $1,370.7$ & $1,200.9$ & 980.4 & $1,066.7$ & $1,086.5$ & $1,168.9$ \\
\hline 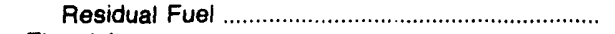 & $\bullet$ & .1 & - & - & - & - & - & - \\
\hline 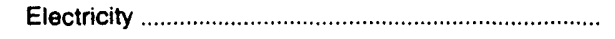 & - & - & - & - & - & - & - & - \\
\hline 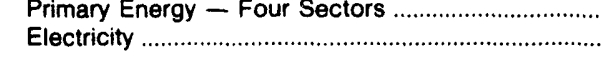 & 217.4 & 480.4 & $1,149.8$ & $1,440.1$ & $1,490.5$ & $1,521.8$ & $1,566.2$ & $1,644.7$ \\
\hline Electric Utillty Sector & 29.3 & 82.2 & 286.3 & 451.4 & 454.4 & 459.1 & 445.9 & 448.2 \\
\hline 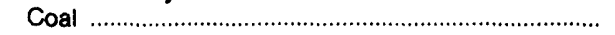 & - & - & 40.3 & 334.0 & 338.9 & 317.0 & 337.7 & 320.8 \\
\hline Natural Gas & 27.4 & 19.7 & 130.1 & 34.0 & 46.7 & 50.3 & 30.7 & 49.7 \\
\hline 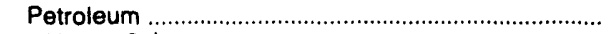 & 1.9 & 49.8 & 69.8 & .6 & .8 & .2 & 7.1 & 6.4 \\
\hline 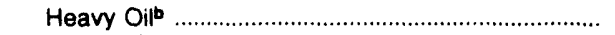 & 1.8 & 49.0 & 65.3 & .2 & .5 & 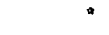 & 3.2 & 2.8 \\
\hline 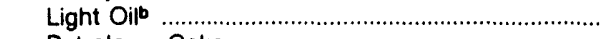 & $\bullet$ & .8 & 4.5 & .4 & .3 & .2 & 3.9 & 3.6 \\
\hline Petroleum Coke & - & - & - & - & - & - & - & - \\
\hline 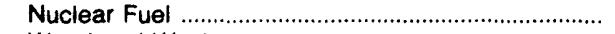 & - & 12.7 & 46.0 & 82.8 & 68.0 & 91.6 & 70.4 & 71.4 \\
\hline Wood and Waste & - & - & - & - & - & - & - & - \\
\hline Primary Energy - Five Sectors & 599.4 & 1,325.1 & $2,993.3$ & $3,497.2$ & $2,788.5$ & $2,766.2$ & $2,911.0$ & $3,180.0$ \\
\hline
\end{tabular}

-There are no direct fuel costs for hydroelectric, geothermal, centralized solar, or wind energy. Wood and other biomass fuels are not included, except those consumed at electric utilities.

b Heavy oil includes Grade Nos. 4, 5, and 6, and residual fuel oils. Light oil includes Grade No. 2 heating oil, kerosene, and jet fuel.

-No consumption, including cases where adjustments were made. See "Consumption Adjustments for Process Fuel and Intermediate Products."

- Value less than 0.05 million dollars.

Note: Totals may not equal sum of components due to independent rounding.

Sources: Data sources, estimation procedures, and assumptions are described in the "Documentation" section of this report. 
Energy Price and Expenditure Estimates by Source, California $1970,1975,1980$, and $1985-1989$

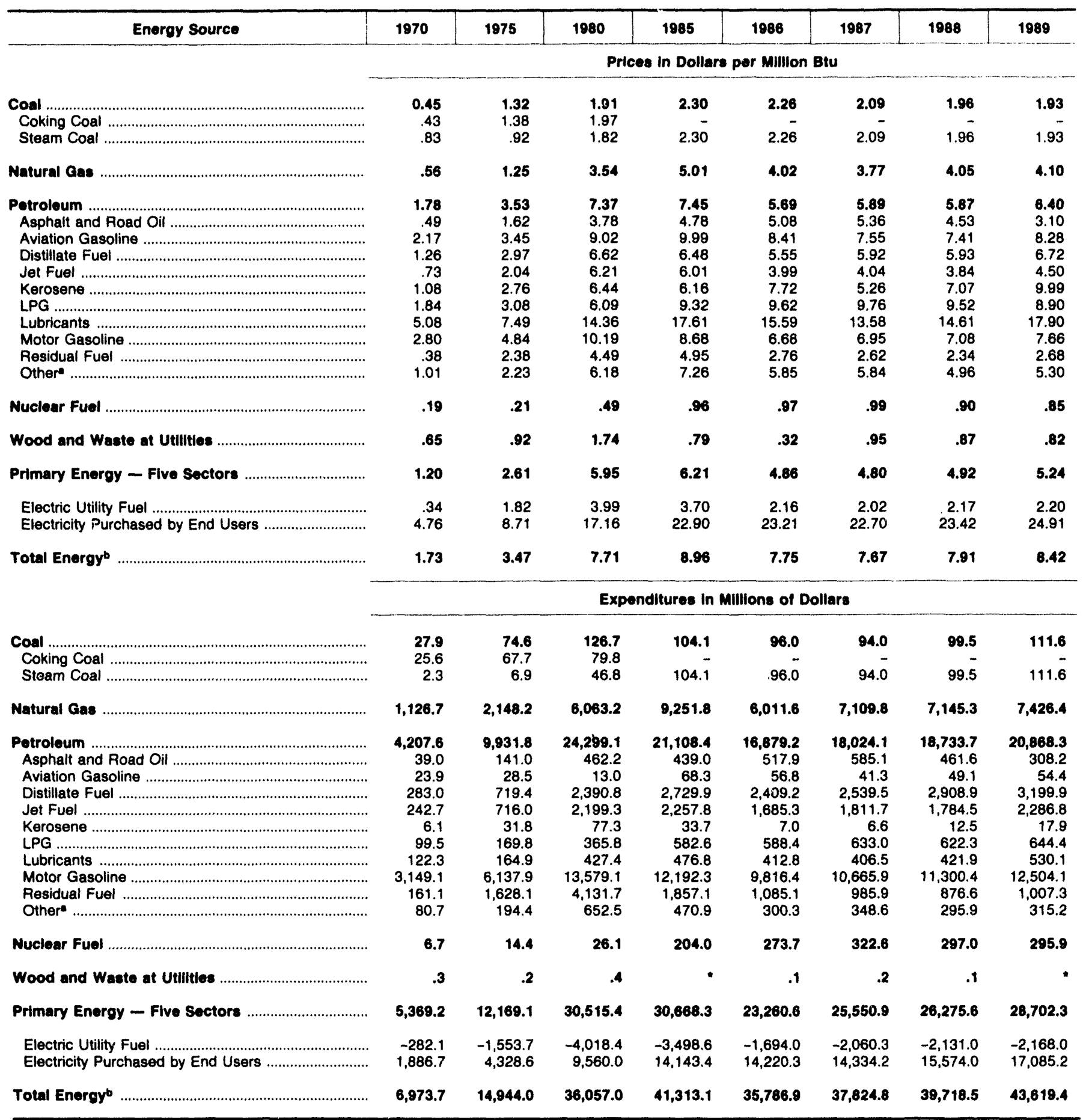

- Includes petroleum coke used at electric utilities.

bThere are no direct fuel costs for hydroelectric, geothermal, centralized solar, or wind energy. Wood and other biomass fuels are not included, except those consumed at electric utilities.

- No consumption, including cases where adjustments were made. See "Consumption Adjustments for Process Fuel and Intermediate Products."

- Value less than 0.05 million dollars.

Note: Expenditure totals may not equal sum of components due to independent roundinc

Sources: Data sources, estimation procedures, and assumptions are described in the "Documentation" section of this report. 


\begin{tabular}{|c|c|c|c|c|c|c|c|c|}
\hline Sector and Energy Source & 1970 & 1975 & 1980 & 1985 & 1986 & 1987 & 1988 & 1989 \\
\hline 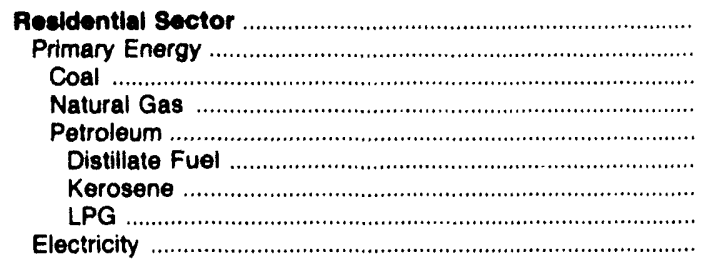 & $\begin{array}{r}1.92 \\
.99 \\
1.31 \\
.93 \\
2.49 \\
1.27 \\
2.57 \\
2.67 \\
6.53\end{array}$ & $\begin{array}{r}3.20 \\
1.55 \\
1.49 \\
4.17 \\
2.80 \\
5.08 \\
4.45 \\
10.68\end{array}$ & $\begin{array}{r}6.76 \\
3.53 \\
5.13 \\
3.37 \\
8.14 \\
6.92 \\
13.04 \\
8.15 \\
17.18\end{array}$ & $\begin{array}{r}10.02 \\
5.63 \\
4.54 \\
5.51 \\
8.54 \\
5.25 \\
9.18 \\
8.66 \\
22.80\end{array}$ & $\begin{array}{r}10.27 \\
5.19 \\
- \\
4.95 \\
10.76 \\
7.58 \\
13.26 \\
10.95 \\
23.26\end{array}$ & $\begin{array}{r}10.43 \\
5.42 \\
3.76 \\
5.13 \\
11.91 \\
6.05 \\
10.57 \\
12.37 \\
23.55\end{array}$ & $\begin{array}{r}11.36 \\
5.74 \\
3.37 \\
5.48 \\
11.05 \\
5.84 \\
10.22 \\
11.39 \\
25.02\end{array}$ & $\begin{array}{r}11.89 \\
5.67 \\
3.66 \\
5.40 \\
11.49 \\
7.63 \\
13.34 \\
11.65 \\
27.69\end{array}$ \\
\hline $\begin{array}{l}\text { Commercial Sector } \\
\text { Primary Energy } \\
\text { Coal .. } \\
\text { Natural Gas } \\
\text { Petroleum } \\
\text { Distillate Fuel } \\
\text { Kerosene } \\
\text { LPG } \\
\text { Motor Gesoline } \\
\text { Residual Fuel } \\
\text { Electricity }\end{array}$ & $\begin{array}{r}2.09 \\
.71 \\
.63 \\
.69 \\
.76 \\
1.12 \\
.78 \\
1.37 \\
2.80 \\
.40 \\
5.02\end{array}$ & $\begin{array}{r}4.36 \\
1.48 \\
- \\
1.22 \\
2.93 \\
2.60 \\
2.50 \\
2.77 \\
4.84 \\
2.45 \\
8.73\end{array}$ & $\begin{array}{r}9.58 \\
4.30 \\
1.82 \\
3.82 \\
6.02 \\
6.60 \\
6.38 \\
5.21 \\
1019 \\
4.90 \\
17.99\end{array}$ & $\begin{array}{r}15.05 \\
6.42 \\
2.28 \\
6.39 \\
6.72 \\
5.40 \\
5.90 \\
9.65 \\
8.68 \\
3.93 \\
23.61\end{array}$ & $\begin{array}{r}15.21 \\
5.65 \\
- \\
5.65 \\
5.67 \\
5.56 \\
4.41 \\
9.02 \\
6.68 \\
2.16 \\
24.19\end{array}$ & $\begin{array}{r}14.18 \\
5.31 \\
2.09 \\
5.28 \\
5.42 \\
4.81 \\
4.54 \\
8.47 \\
6.95 \\
2.60 \\
23.47\end{array}$ & $\begin{array}{r}13.88 \\
4.71 \\
1.96 \\
4.55 \\
5.48 \\
4.94 \\
4.51 \\
8.53 \\
7.08 \\
2.09 \\
24.15\end{array}$ & $\begin{array}{r}14.64 \\
4.92 \\
1.92 \\
4.71 \\
6.15 \\
6.22 \\
5.70 \\
7.63 \\
7.66 \\
2.30 \\
25.33\end{array}$ \\
\hline $\begin{array}{l}\text { Industrial Sector } \\
\text { Primary Energy } \\
\text { Coal .. } \\
\text { Coking Coal } \\
\text { Steam Coal } \\
\text { Natural Gas } \\
\text { Petroleum } \\
\text { Aspinalt and Road Oil } \\
\text { Distillate Fuel } \\
\text { Kerosene } \\
\text { LPG } \\
\text { Lubricants } \\
\text { Motor Gasoline } \\
\text { Residual Fuel } \\
\text { Other } \\
\text { Electricity }\end{array}$ & $\begin{array}{r}.87 \\
.57 \\
.43 \\
.43 \\
.63 \\
.38 \\
.91 \\
.49 \\
.68 \\
.78 \\
1.37 \\
5.08 \\
2.80 \\
.35 \\
1.01 \\
2.90\end{array}$ & $\begin{array}{r}2.24 \\
1.51 \\
1.32 \\
1.38 \\
.92 \\
1.05 \\
2.25 \\
1.62 \\
2.21 \\
2.50 \\
2.77 \\
7.49 \\
4.84 \\
1.66 \\
2.23 \\
6.70\end{array}$ & $\begin{array}{r}6.19 \\
4.32 \\
1.91 \\
1.97 \\
1.82 \\
3.64 \\
5.20 \\
3.78 \\
5.49 \\
6.38 \\
5.21 \\
14.36 \\
10.19 \\
3.16 \\
6.18 \\
16.04\end{array}$ & $\begin{array}{r}8.24 \\
5.28 \\
2.28 \\
-2 \\
2.28 \\
4.54 \\
6.44 \\
4.78 \\
5.54 \\
5.90 \\
9.65 \\
17.61 \\
8.68 \\
3.93 \\
7.26 \\
22.00\end{array}$ & $\begin{array}{r}7.25 \\
4.24 \\
2.26 \\
- \\
2.26 \\
3.46 \\
5.25 \\
5.08 \\
4.15 \\
4.41 \\
9.02 \\
15.59 \\
6.68 \\
2.16 \\
5.85 \\
21.63\end{array}$ & $\begin{array}{r}6.66 \\
4.23 \\
2.09 \\
- \\
2.09 \\
3.39 \\
.59 \\
5.36 \\
4.27 \\
4.54 \\
8.47 \\
13.58 \\
6.95 \\
2.60 \\
5.84 \\
20.38\end{array}$ & $\begin{array}{r}6.96 \\
4.24 \\
1.96 \\
- \\
1.96 \\
3.65 \\
5.33 \\
4.53 \\
4.24 \\
4.51 \\
8.53 \\
14.61 \\
7.08 \\
2.09 \\
4.96 \\
20.15\end{array}$ & $\begin{array}{r}7.05 \\
4.21 \\
1.92 \\
- \\
1.92 \\
3.62 \\
5.41 \\
3.10 \\
5.35 \\
5.70 \\
7.63 \\
17.90 \\
7.66 \\
2.30 \\
5.30 \\
20.89\end{array}$ \\
\hline 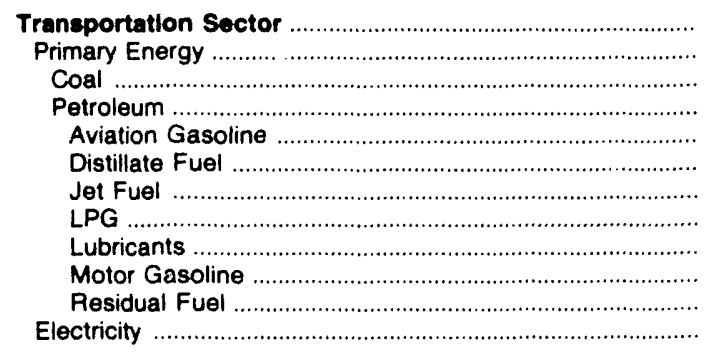 & $\begin{array}{r}2.07 \\
2.07 \\
.63 \\
2.07 \\
2.17 \\
1.42 \\
.73 \\
1.37 \\
5.08 \\
2.80 \\
.36 \\
2.88\end{array}$ & $\begin{array}{r}4.02 \\
4.02 \\
.92 \\
4.02 \\
3.45 \\
3.22 \\
2.04 \\
2.77 \\
7.49 \\
4.84 \\
2.12 \\
4.34\end{array}$ & $\begin{array}{r}8.22 \\
8.22 \\
- \\
8.22 \\
9.02 \\
7.07 \\
6.21 \\
5.21 \\
14.36 \\
10.19 \\
4.14 \\
11.39\end{array}$ & $\begin{array}{r}7.63 \\
7.62 \\
- \\
7.62 \\
9.99 \\
6.90 \\
6.01 \\
9.65 \\
17.61 \\
8.68 \\
5.02 \\
18.29\end{array}$ & $\begin{array}{r}5.75 \\
5.75 \\
- \\
5.75 \\
8.41 \\
5.93 \\
3.99 \\
9.02 \\
15.59 \\
6.68 \\
2.86 \\
18.21\end{array}$ & $\begin{array}{r}5.92 \\
5.92 \\
- \\
5.92 \\
7.55 \\
6.70 \\
4.04 \\
8.47 \\
13.58 \\
6.95 \\
2.60 \\
13.47\end{array}$ & $\begin{array}{r}6.00 \\
5.99 \\
- \\
5.99 \\
7.41 \\
6.51 \\
3.84 \\
8.53 \\
14.61 \\
7.08 \\
2.26 \\
14.99\end{array}$ & $\begin{array}{r}6.60 \\
6.60 \\
- \\
6.60 \\
8.28 \\
7.14 \\
4.50 \\
7.63 \\
17.90 \\
7.66 \\
2.57 \\
13.15\end{array}$ \\
\hline $\begin{array}{l}\text { Total Energye } \\
\text { Primary Energy - Four Sectors } \\
\text { Electricity }\end{array}$ & $\begin{array}{l}1.73 \\
1.40 \\
4.76\end{array}$ & $\begin{array}{l}3.47 \\
2.78 \\
8.71\end{array}$ & $\begin{array}{r}7.71 \\
6.43 \\
17.16\end{array}$ & $\begin{array}{r}8.96 \\
6.80 \\
22.90\end{array}$ & $\begin{array}{r}7.75 \\
5.38 \\
23.21\end{array}$ & $\begin{array}{r}7.67 \\
5.46 \\
22.70\end{array}$ & $\begin{array}{r}7.91 \\
5.54 \\
23.42\end{array}$ & $\begin{array}{r}8.42 \\
5.90 \\
24.91\end{array}$ \\
\hline $\begin{array}{l}\text { Eloctric Utility Sector } \\
\text { Coal } \\
\text { Natural Gas } \\
\text { Petroleum } \\
\text { Heavy Oib } \\
\text { Light Oib }\end{array}$ & $\begin{array}{l}.34 \\
.33 \\
.40 \\
.40 \\
.36\end{array}$ & $\begin{array}{l}1.82 \\
1.0 \overline{5} \\
2.50 \\
2.50 \\
2.43\end{array}$ & $\begin{array}{l}3.99 \\
- \\
3.53 \\
5.06 \\
5.03 \\
5.84\end{array}$ & $\begin{array}{r}3.70 \\
- \\
4.47 \\
5.33 \\
5.31 \\
5.69\end{array}$ & $\begin{array}{r}2.16 \\
- \\
2.81 \\
3.26 \\
3.16 \\
4.79\end{array}$ & $\begin{array}{l}2.02 \\
2.50 \\
2.93 \\
2.82 \\
4.10\end{array}$ & $\begin{array}{r}2.17 \\
- \\
2.83 \\
2.67 \\
2.66 \\
4.03\end{array}$ & $\begin{array}{r}2.20 \\
- \\
2.92 \\
3.09 \\
3.08 \\
3.64\end{array}$ \\
\hline $\begin{array}{l}\text { Petroleum Coke } \\
\text { Nuclear Fuel } \\
\text { Wood and Waste }\end{array}$ & $\begin{array}{r}. \\
.19 \\
.65\end{array}$ & $\begin{array}{r}- \\
.21 \\
.92\end{array}$ & $\begin{array}{r}.49 \\
1.74\end{array}$ & $\begin{array}{r}. \\
.96 \\
.79\end{array}$ & $\begin{array}{r}- \\
.97 \\
.32\end{array}$ & $\begin{array}{r}.99 \\
.95\end{array}$ & $\begin{array}{r}. \\
.80 \\
.87\end{array}$ & $\begin{array}{r}- \\
.85 \\
.82\end{array}$ \\
\hline 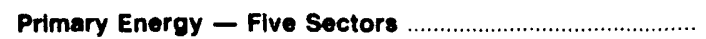 & 1.20 & 2.61 & 5.95 & 6.21 & 4.86 & 4.80 & 4.92 & 5.24 \\
\hline
\end{tabular}

-There are no direct fuel costs for hydroelectric, geothermal, centralized solar, or wind energy. Wood and other biomass fuels are not included, except those consumed at electric utilities.

b Heavy oil includes Grade Nos. 4, 5, and 6, and residual fuel oils. Light oil includes Grade No. 2 heating oil, kerosene, and jet fuel.

- No consumption, including cases where adjustments were made. See "Consumption Adjustments for Process Fuel and Intermediate Products."

Sources: Data sources, estimation procedures, and assumptions are described in the "Documentation" section of this report. 
C Energy Expenditure Estimates by Sector, California

A $1970,1975,1980$, and $1985-1989$ (Willion Dollars)

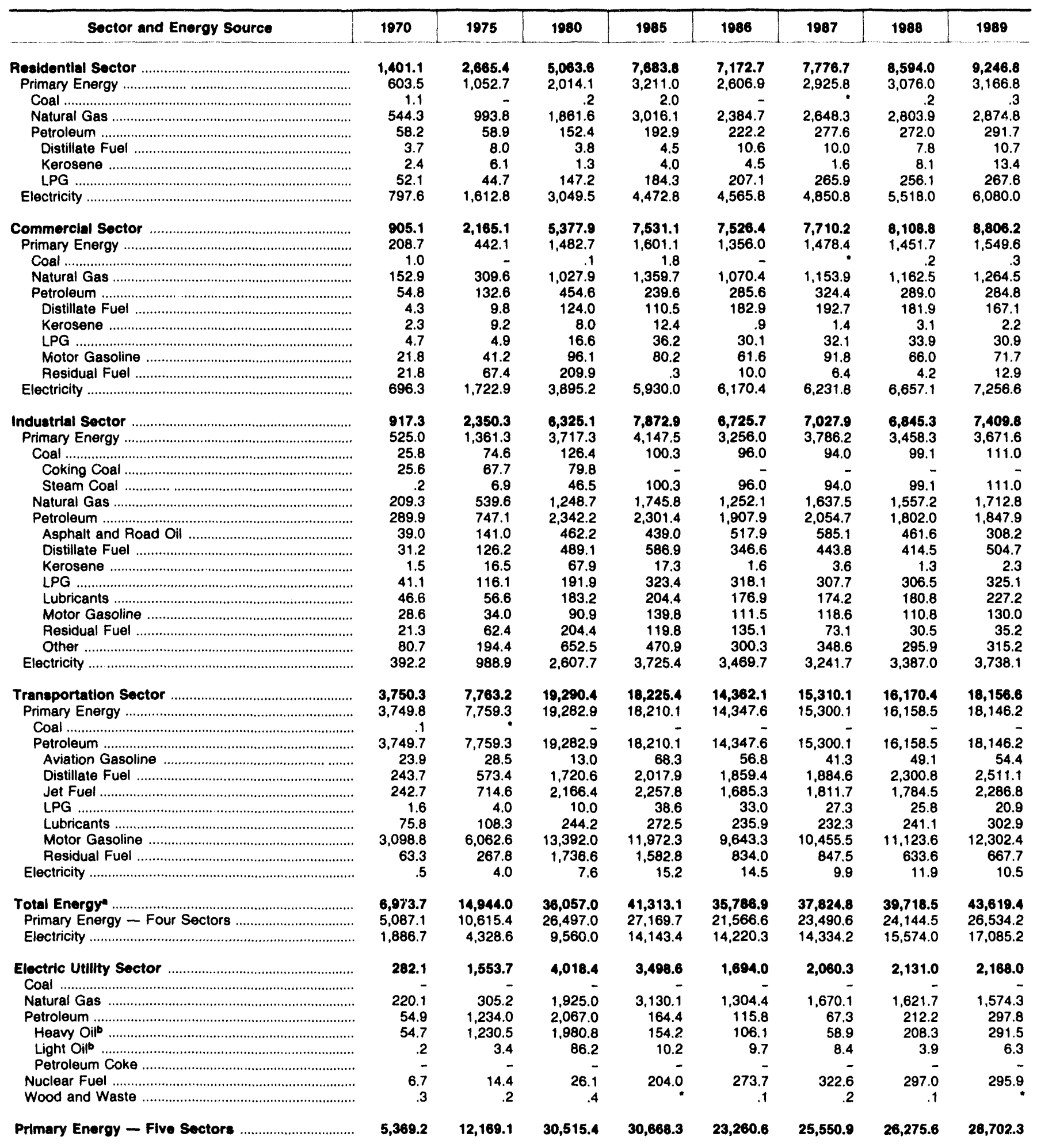

-There are no direct fuel costs for hydroelectric, geothermal, centralized solar, or wind energy. Wood and other biomass fuels are not included, except those consumed at electric utilities.

bHeavy oil includes Grade Nos. 4, 5, and 6, and residual fuel oils. Light oil includes Grade No. 2 heating oil, kerosene, and jet fuel.

- No consumption, including cases where adjustments were made. See "Consumption Adjustments for Process Fuel and Intermediate Products."

- Value less than 0.05 million dollars.

Note: Totals may not equal sum of components due to independent rounding.

Sources: Data sources, estimation procedures, and assumptions are described in the "Documentation" section of this report. 


\begin{tabular}{|c|c|c|c|c|c|c|c|c|}
\hline Energy Source & 1970 & 1976 & 1800 & 1885 & 1986 & 1087 & 1988 & 1989 \\
\hline & \multicolumn{8}{|c|}{ Prices in Dollare per Million Btu } \\
\hline Cool & 0.33 & 0.68 & 1.00 & 1.17 & 1.18 & 1.14 & 1.08 & 1.08 \\
\hline 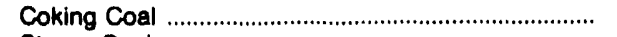 &, 43 & 1.38 & 1.97 & - & - & - & - & - \\
\hline Steam Coal & .30 & .53 & .89 & 1.17 & 1.18 & 1.14 & 1.08 & 1.08 \\
\hline 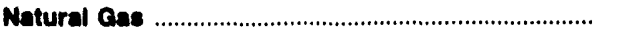 & .48 & .9 & 2.98 & 4.71 & 4.54 & 4.26 & 3.95 & 3.85 \\
\hline Potroleum & 1.88 & 3.54 & 7.93 & 8.00 & 6.02 & 6.47 & 6.24 & 7.15 \\
\hline 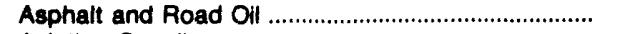 & .58 & 2.01 & 3.40 & 4.73 & 4.30 & 3.03 & 3.19 & 2.73 \\
\hline Aviation Gasoline & 2.17 & 3.45 & 9.02 & 9.99 & 8.41 & 7.55 & 7.41 & 8.28 \\
\hline 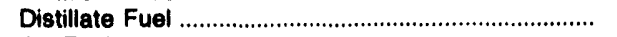 & 1.04 & 2.30 & 6.45 & 6.26 & 5.52 & 5.86 & 5.46 & 6.66 \\
\hline 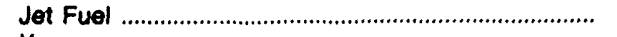 & .76 & 2.12 & 6.59 & 5.94 & 3.92 & 4.05 & 3.78 & 4.30 \\
\hline 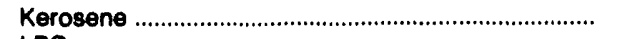 & .98 & 2.22 & 5.78 & 7.55 & 4.56 & 4.09 & 3.98 & 5.18 \\
\hline LPG & 1.60 & 3.02 & 5.88 & 6.39 & 6.23 & 4.80 & 3.28 & 5.97 \\
\hline 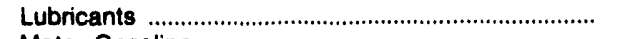 & 5.08 & 7.48 & 14.36 & 17.61 & 15.59 & 13.58 & 14.61 & 17.90 \\
\hline 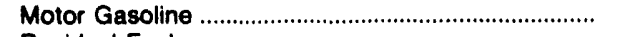 & 2.72 & 4.67 & 9.36 & 9.28 & 6.76 & 7.59 & 7.34 & 8.13 \\
\hline Residual Fuel & .44 & 1.59 & 3.88 & 4.05 & 2.34 & 2.68 & 2.44 & 1.81 \\
\hline & & & & & & & 4.00 & \\
\hline Nuclear Fuel & - & - & .21 & - & .27 & .28 & .29 & .28 \\
\hline 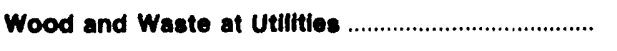 & - & - & - & .79 & .32 & .95 & .87 & .82 \\
\hline Primary Energy - Flve Sectors …............................... & 1.03 & 2.02 & 4.33 & 4.73 & 3.93 & 4.01 & 3.77 & 3.99 \\
\hline 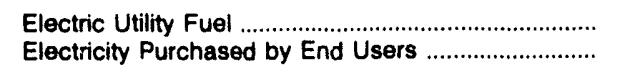 & $\begin{array}{r}.25 \\
6.09\end{array}$ & $\begin{array}{r}.60 \\
7.95\end{array}$ & $\begin{array}{r}1.12 \\
12.84\end{array}$ & $\begin{array}{r}1.21 \\
17.88\end{array}$ & $\begin{array}{r}1.21 \\
17.69\end{array}$ & $\begin{array}{r}1.15 \\
17.12\end{array}$ & $\begin{array}{r}1.09 \\
17.32\end{array}$ & $\begin{array}{r}1.08 \\
17.48\end{array}$ \\
\hline \multirow[t]{2}{*}{ Total Energy } & 1.51 & 2.02 & 6.49 & 8.20 & 7.20 & 7.35 & 7.14 & 7.60 \\
\hline & \multicolumn{8}{|c|}{ Expenditures in Millions of Dollars } \\
\hline Coal & 38.3 & 108.4 & 248.2 & 340.0 & 349.5 & 336.8 & 337.3 & 349.0 \\
\hline 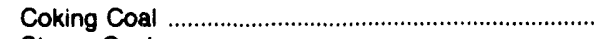 & 12.0 & 39.5 & 50.2 & - & - & - & $\overline{0}$ & - \\
\hline 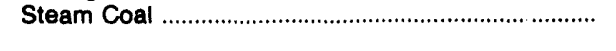 & 26.3 & 68.0 & 197.9 & 349.9 & 349.5 & 336.8 & 337.3 & 349.0 \\
\hline 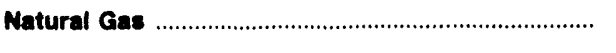 & 128.2 & 262.9 & 706.8 & 931.2 & 810.9 & 793.7 & 817.3 & $\mathbf{8 3 7 . 0}$ \\
\hline Potroloum & 503.1 & $1,130.9$ & $2,577.0$ & $2,604.8$ & $2,019.1$ & $2,140.1$ & $2,074.1$ & $2,257.3$ \\
\hline 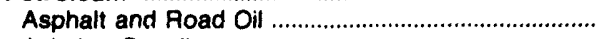 & 12.3 & 29.8 & 51.6 & 97.5 & 88.2 & 62.6 & 75.2 & 53.1 \\
\hline Aviation Gasoline & 3.7 & 4.6 & 12.1 & 7.1 & 7.5 & 5.8 & 6.2 & 7.6 \\
\hline 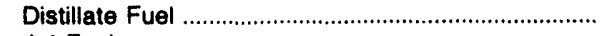 & 30.9 & 118.1 & 422.1 & 348.2 & 325.5 & 336.7 & 355.7 & 395.9 \\
\hline Jet Fuel & 32.0 & $\mathbf{8 5 . 7}$ & 175.9 & 264.1 & 178.8 & 192.0 & 138.0 & 129.8 \\
\hline 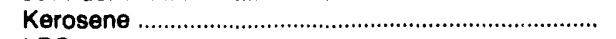 & 4.5 & 3.5 & 13.5 & 4.5 & 1.4 & 1.5 & 1.6 & 3.2 \\
\hline LPG & 27.5 & 55.7 & 83.3 & 56.6 & 48.3 & 38.5 & 29.6 & 76.7 \\
\hline 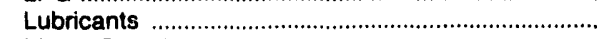 & 13.1 & 20.8 & 55.8 & 62.3 & 53.9 & 53.1 & 55.1 & 69.2 \\
\hline 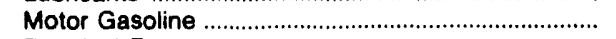 & 372.5 & 782.3 & $1,685.6$ & $1,742.4$ & $1,297.1$ & $1,439.3$ & $1,404.9$ & $1,512.7$ \\
\hline 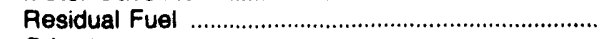 & 3.9 & 32.7 & 43.6 & 4.0 & 10.9 & 2.2 & .6 & 1.4 \\
\hline 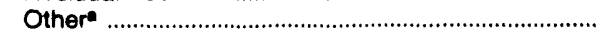 & 2.7 & 6.7 & 33.5 & 18.0 & 7.6 & 8.4 & 7.1 & 7.7 \\
\hline 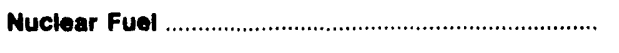 & - & - & 1.6 & - & .2 & .5 & 2.1 & 1.6 \\
\hline 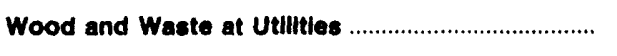 & - & - & - & $\cdot$ & $\cdot$ & $\bullet$ & - & * \\
\hline Primary Energy - Five Sectors ................................ & 689.6 & $1,511.2$ & $\mathbf{3 , 6 3 3 . 4}$ & $3,895.9$ & $3,179.7$ & $3,271.2$ & $3,230.7$ & $3,444.9$ \\
\hline $\begin{array}{l}\text { Electric Utility Fuel } \\
\text { Electricity Purchased by End Users }\end{array}$ & $\begin{array}{l}-30.6 \\
222.3\end{array}$ & $\begin{array}{r}-105.4 \\
426.0\end{array}$ & $\begin{array}{r}-272.5 \\
918.2\end{array}$ & $\begin{array}{r}-342.6 \\
1,608.3\end{array}$ & $\begin{array}{r}-340.8 \\
1,621.9\end{array}$ & $\begin{array}{r}-332.3 \\
1,622.8\end{array}$ & $\begin{array}{r}-337.3 \\
1.716 .9\end{array}$ & $\begin{array}{r}-350.7 \\
1,775.2\end{array}$ \\
\hline 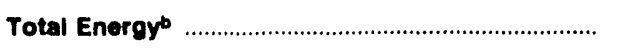 & 861.3 & $1,831.8$ & $4,170.1$ & $5,151.5$ & $4,460.8$ & $4,561.7$ & $4,610.3$ & $4,869.4$ \\
\hline
\end{tabular}

-Includes petroieum coke used at electric utilities.

b There are no direct fuel costs for hydroelectric, geothermal, centralized solar, or wind energy. Wood and other biomass fuels are not included, except those consumed at electric utilities.

- No consumption, inciuding cases where adjustments were made. See "Consumption Adjustments for Process Fuel and Intermediate Products."

- Value less than 0.05 million doliars.

Note: Expenditure totals may not equal sum of components due to independent rounding.

Sources: Data sources, estimation procedures, and assumptions are described in the "Documentation" section of this report. 


\section{Energy Price Estimates by Sector, Colorado \\ O 1970, 1975, 1980, and 1985-1989 \\ L (Dollars per Million Btu)}

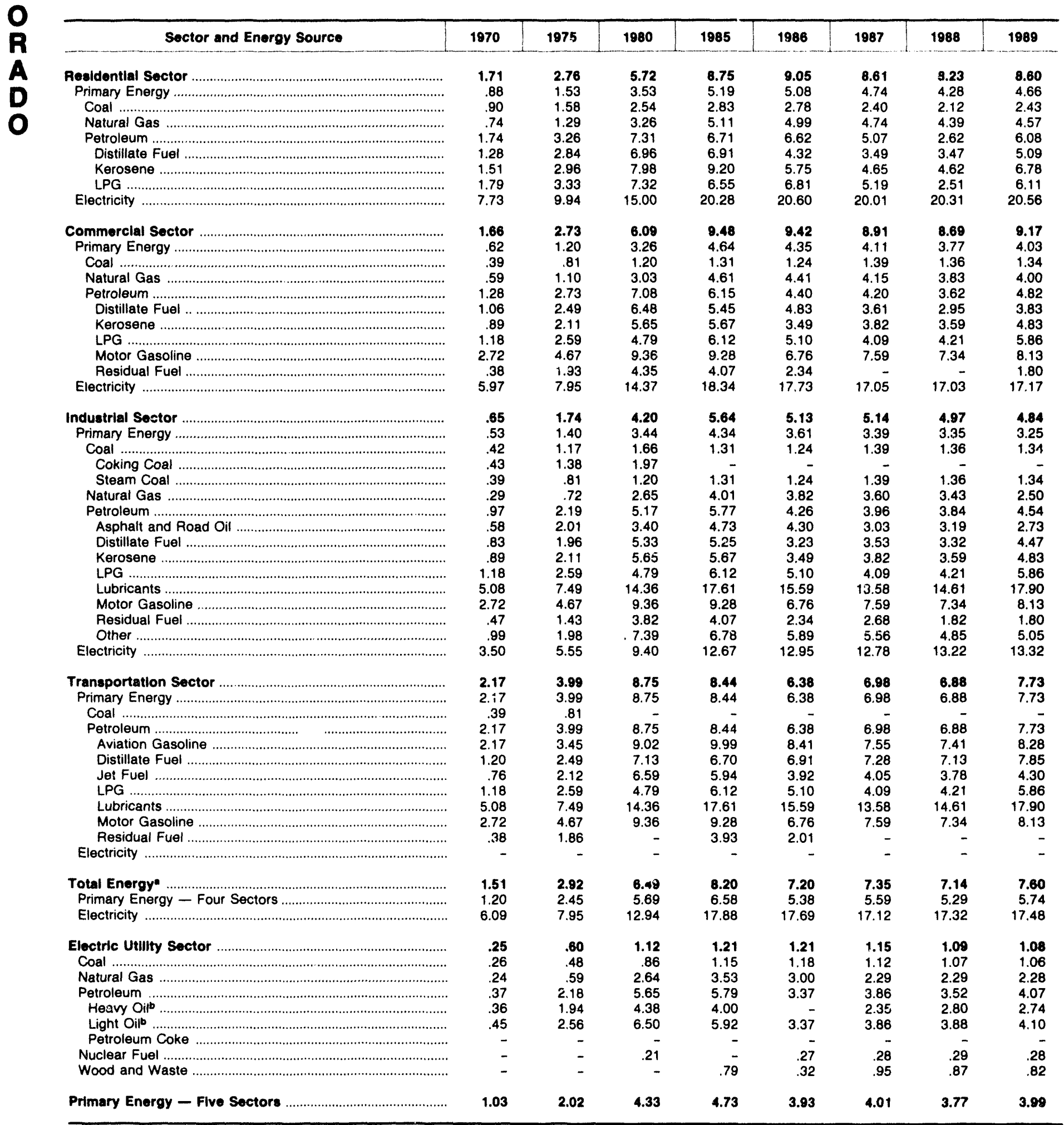

- There are no direct fuel costs for hydroelectric, geothermal, centralized solar, or wind energy. Wood and other biomass fuels are not included, except those consumed at electric utilities.

beavy oil includes Grade Nos. 4, 5, and 6, and residual fuel oils. Light oil includes Grade No. 2 heating oil, kerosene, and jet fuel.

- No consumption, including cases where adjustments were made. See "Consumption Adjustments for Process Fuel and Intermediate Products."

Sources: Data sources, estimation procedures, and assumptions are described in the "Documentation" section of this report. 


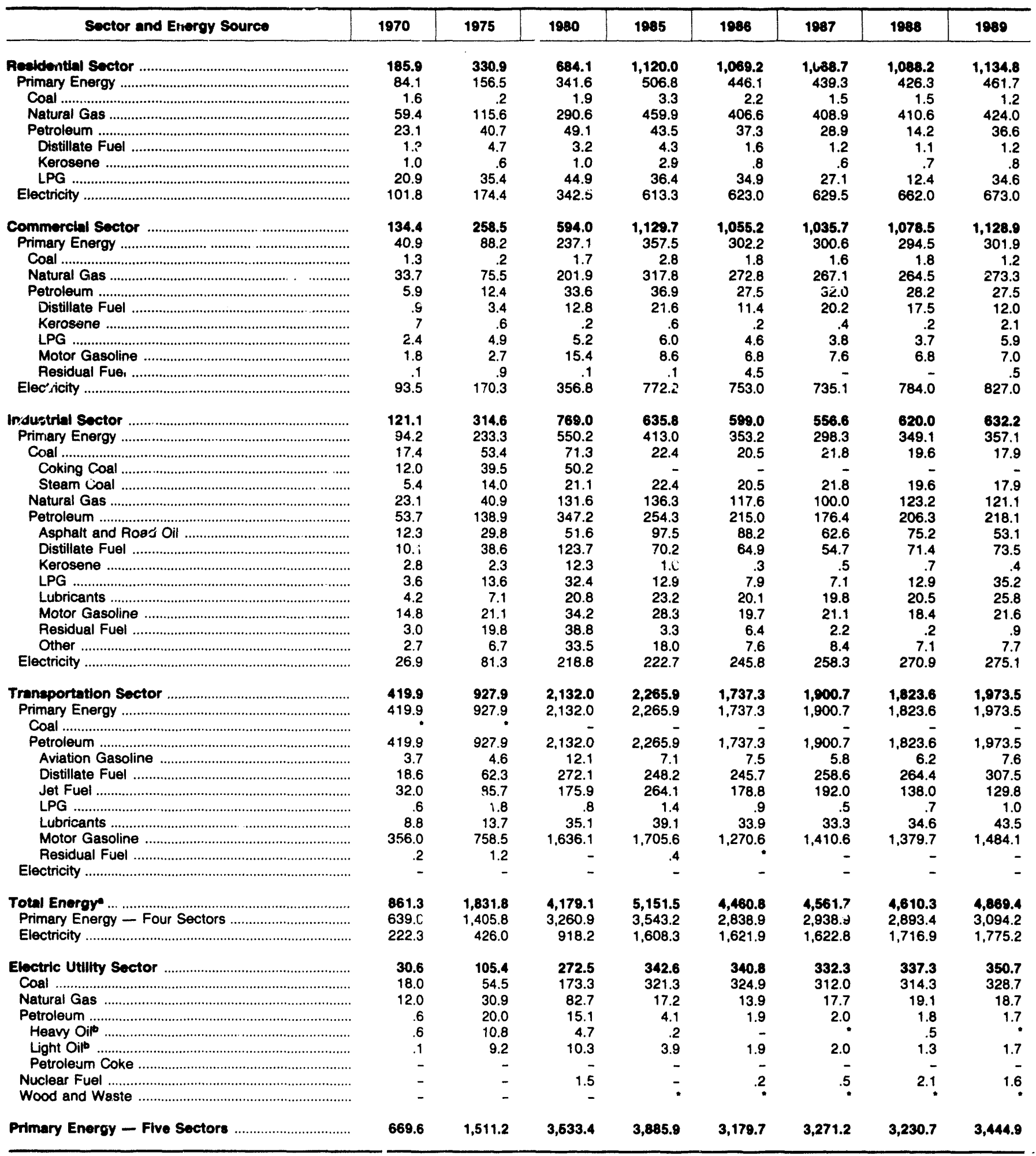

-There are no direct fuel costs for hydroelectric, geo'nermal, centralized solar, or wind energy. Wood and other biomass fuels are not included, except those consumed at electric utilities.

bHeary oil includes Grade Nos. 4, 5, and 6, and res Jua! fuel oils. Light oil includes Grade No. 2 heating oil, kerosene, and jet fuel.

- No consumption, including cases where adjustr ents were made. See "Consumption A.jjustments for Process Fuel and Intermediate Products."

- Value less than 0.05 million dollars.

Note: Totals may not equal sum of components dut to independent rounding.

Sources: Data sources, estimation procedures, and assumptions are described in the "Documentation" section of this report. 
Energy Price and Expenditure Estimates by Source, Connecticut $1970,1975,1980$, and $1985-1989$

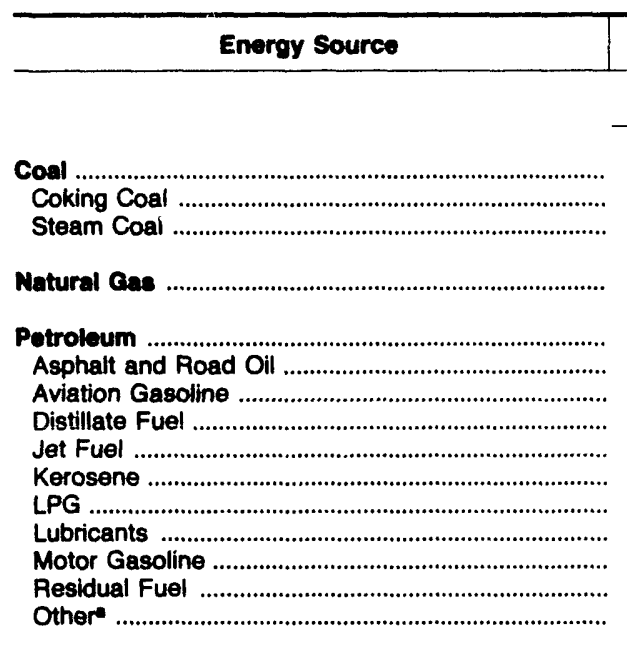

Nuclear Fuel

Wood and Waste at Utilties

Primary Energy - Five Sectors ................................

Electric Utility Fuel

Electricity Purchased by End Users

Total Energyb

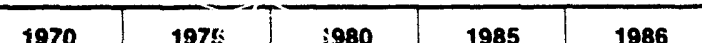

1987

1988

1989

Prices in Dollars per Million Btu

\begin{tabular}{|c|c|c|c|c|c|c|c|}
\hline 0.48 & 2.09 & 2.68 & 2.40 & 2.32 & 2.45 & 2.31 & 2.16 \\
\hline - & - & - & - & - & - & - & - \\
\hline .48 & 2.09 & 2.68 & 2.40 & 2.32 & 2.45 & 2.31 & 2.16 \\
\hline 1.57 & 2.86 & 4.97 & 7.20 & 6.86 & 5.94 & 6.04 & 6.35 \\
\hline 1.37 & 3.07 & 7.09 & 7.49 & 5.72 & 6.02 & 6.18 & 6.71 \\
\hline .68 & 1.91 & 3.67 & 5.20 & 4.86 & 3.58 & 3.39 & 3.20 \\
\hline 2.17 & 3.45 & 9.02 & 9.99 & 8.41 & 7.55 & 7.41 & 8.28 \\
\hline 1.29 & 2.73 & 6.82 & 8.25 & 6.61 & 6.28 & 6.49 & 7.19 \\
\hline .75 & 2.11 & 6.50 & 6.29 & 4.50 & 4.34 & 4.00 & 4.67 \\
\hline 1.40 & 2.91 & 7.17 & 8.70 & 6.77 & 6.42 & 6.15 & 6.70 \\
\hline 1.98 & 3.63 & 6.85 & 10.91 & 10.65 & 9.98 & 10.80 & 11.17 \\
\hline 5.08 & 7.49 & 14.36 & 17.61 & 15.59 & 13.58 & 14.61 & 17.90 \\
\hline 2.96 & 4.61 & 10.10 & 9.37 & 7.45 & 7.93 & 8.91 & 9.30 \\
\hline .40 & 2.04 & 4.66 & 4.30 & 2.54 & 2.96 & 2.42 & 2.87 \\
\hline .91 & 3.08 & 7.76 & 7.80 & 5.06 & 5.93 & 4.97 & 5.73 \\
\hline .13 & .29 & .38 & .91 & .91 & .92 & .86 & .89 \\
\hline - & - & - & - & - & - & .87 & .82 \\
\hline 1.26 & 2.68 & 5.65 & 5.95 & 4.47 & 4.47 & 4.52 & 5.03 \\
\hline $\begin{array}{r}.35 \\
6.27\end{array}$ & $\begin{array}{r}1.35 \\
13.15\end{array}$ & $\begin{array}{r}2.60 \\
19.10\end{array}$ & $\begin{array}{r}2.38 \\
26.62\end{array}$ & $\begin{array}{r}1.54 \\
24.65\end{array}$ & $\begin{array}{r}1.61 \\
24.27\end{array}$ & $\begin{array}{r}1.42 \\
24.55\end{array}$ & $\begin{array}{r}1.64 \\
25.68\end{array}$ \\
\hline 2.09 & 4.53 & 9.07 & 11.30 & 9.79 & 9.70 & 10.06 & 10.68 \\
\hline
\end{tabular}

Coal .

Coking Coal

Steam Coal

Natural Cae

Petroleum

Asphalt and Road Oil

Aviation Gasoline

Distillate Fuel .

Jet Fuel

Kerosene

LPG

Lubricants

Motor Gasoline

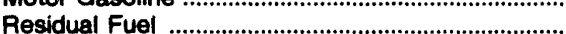

Othere

Nuclear Fuel

Wood and Wante at Uthlies

Primary Energy - Five Sectors

Electric Utility Fuel

Electricity Purchased by End Users

Total Energy

Includes petroleum coke used at electric utilities.

-There are no direct fuel costs for hydroelectric, geothermal, centralized solar, or wind energy. Wood and other biomass fuels are not included, except those consumed at electric utilities.

- No consumption, including cases where adjustments were made. See "Cionsumption Adjustments for Process Fuel and Intermediate Products." Note: Expenditure totals may not equal sum of components due to independent rounding.

Sources: Data sources, estimation procedures, and assumptions are described in the "Documentation" section of this report. 


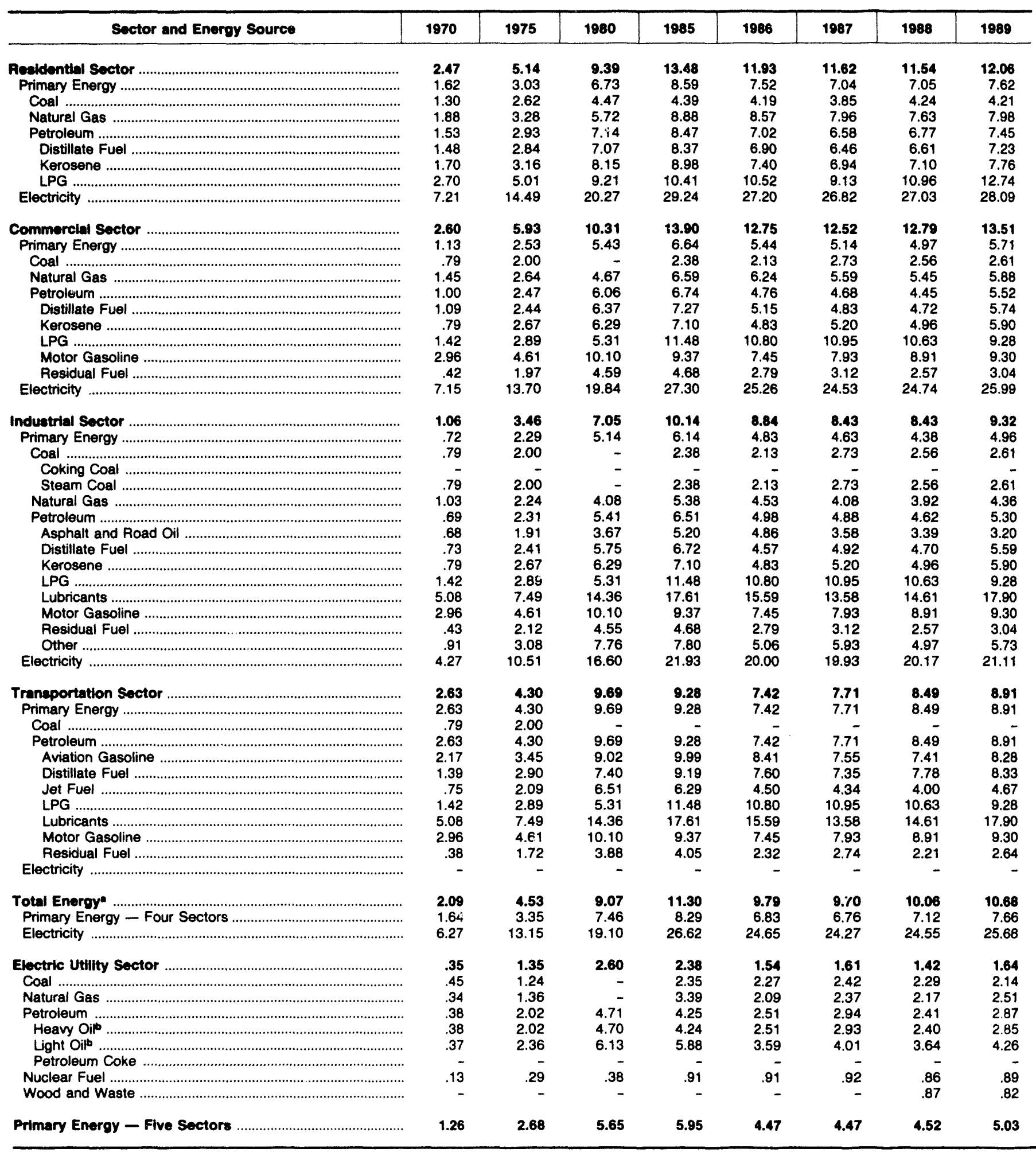

-There are no direct fuel costs for hydroelectric, geothermal, centralized solar, or wind energy. Wood and other biomass fuels are not included, except those consumed at electric utilities.

bHeavy oil includes Grade Nos. 4, 5, and 6, and residual fuel oils. Light oil includes Grade No. 2 heating oil, kerosene, and jet fuel.

- No consumption, including cases where adjustments were made. See "Consumption Adjustments for Process Fuel and Intermediate Products."

Sources: Data sources, estimation procedures, and assumptions are described in the "Documentation" section of this report. 


\section{Energy Expenditure Estimates by Sector, Connecticut \\ O 1970, 1975, 1980, and 1985-1989 \\ N (Million Dollars)}

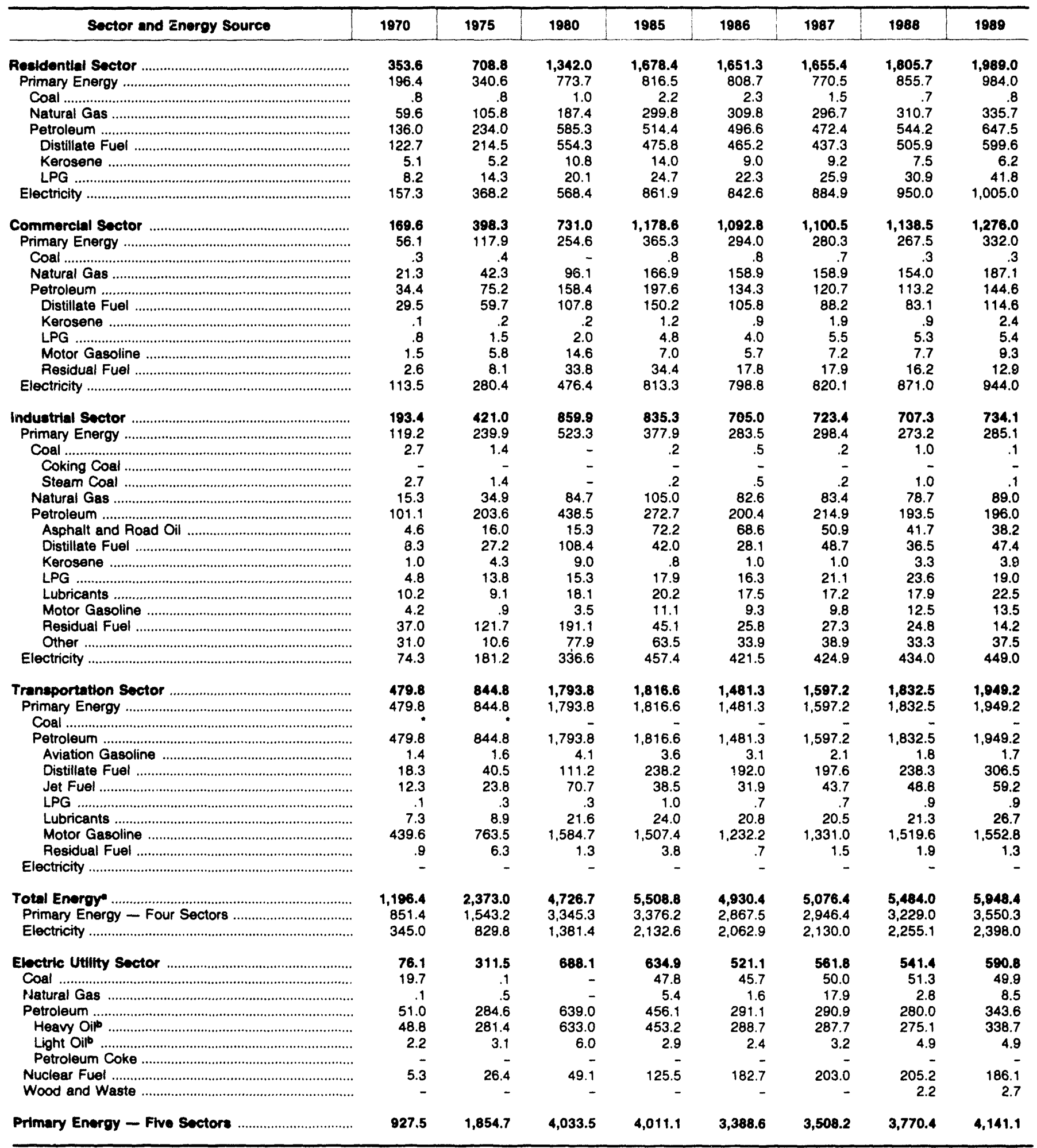

-There are no direct fuel costs for hydroelectric, geothermal, centralized solar, or wind energy. Wood and other biomass fuels are not included, except those consumed at electric utilities.

- Heary oil includes Grade Nos. 4, 5, and 6, and residual fuel oils. Light oil includes Grade No. 2 heating oil, kerosene, and jet fuel.

- No consumption, including cases where adjustments were made. See "Consumption Adjustments for Process Fuel and intermediate Products."

Value less than 0.05 million dollars.

Note: Totals may not equal sum of components due to independent rounding.

Sources: Data sources, estimation procedures, and assumptions are described in the "Documentation" section of this report. 


\begin{tabular}{|c|c|c|c|c|c|c|c|c|}
\hline Energy Source & 1970 & 1975 & 1980 & 1885 & 1986 & 1987 & 1988 & 1989 \\
\hline & \multicolumn{8}{|c|}{ Prices In Dollars per Million Btu } \\
\hline Coal & 0.39 & 1.16 & 1.57 & 1.87 & 1.87 & 1.78 & 1.77 & 1.75 \\
\hline 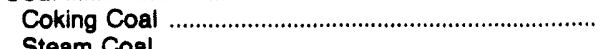 & - & - & $\overline{-}$ & $-\overline{-}$ & $\overline{7}$ & - & - & $\overline{1}$ \\
\hline 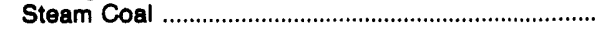 & .39 & 1.16 & 1.57 & 1.87 & 1.87 & 1.78 & 1.77 & 1.75 \\
\hline 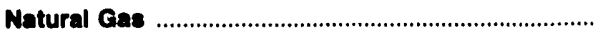 & .91 & 1.80 & 3.37 & 4.87 & 4.43 & 3.93 & 4.04 & 4.06 \\
\hline Potroleum & 1.27 & 2.75 & 6.00 & 7.41 & 5.28 & 5.64 & 5.43 & 6.23 \\
\hline 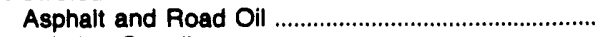 & .66 & 1.76 & 3.58 & 4.97 & 4.16 & 3.17 & 3.13 & 2.90 \\
\hline 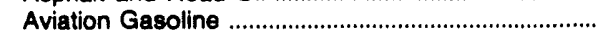 & 2.17 & 3.45 & 9.02 & 9.99 & 8.41 & 7.55 & 7.41 & 8.28 \\
\hline 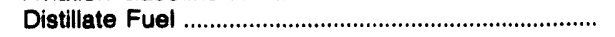 & 1.16 & 2.53 & 6.77 & 7.58 & 5.94 & 5.70 & 5.68 & 6.33 \\
\hline Jet Fuel & .73 & 2.03 & 6.46 & 6.63 & 4.87 & 4.03 & 4.16 & 4.80 \\
\hline Kerosene & 1.26 & 3.14 & 8.36 & 8.66 & 7.03 & 6.51 & 0.48 & 7.30 \\
\hline LPG & 1.25 & 3.60 & 5.20 & 10.64 & 10.77 & 10.37 & 10.14 & 11.47 \\
\hline 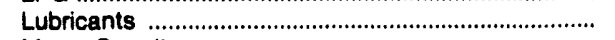 & 5.08 & 7.49 & 14.36 & 17.61 & 15.59 & 13.58 & 14.61 & 17.90 \\
\hline 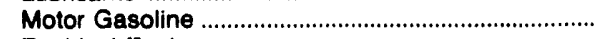 & 2.86 & 4.54 & 9.60 & 9.39 & 6.97 & 7.61 & 8.07 & 8.88 \\
\hline 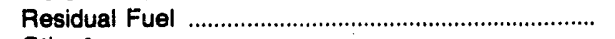 & .45 & 1.92 & 4.23 & 4.15 & 2.31 & 2.74 & 2.25 & 2.67 \\
\hline Other & .35 & 1.68 & 5.84 & 4.94 & 2.88 & 3.30 & 2.92 & 3.47 \\
\hline 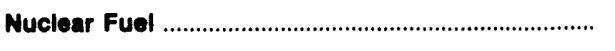 & - & - & - & - & - & - & - & - \\
\hline 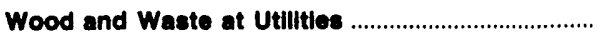 & - & - & - & - & - & - & - & - \\
\hline 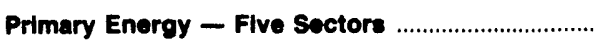 & 1.06 & 2.48 & 5.13 & 5.14 & 4.07 & 4.11 & 4.11 & 4.61 \\
\hline 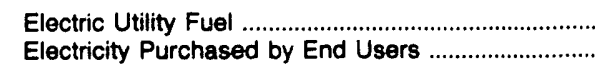 & $\begin{array}{r}.39 \\
4.94\end{array}$ & $\begin{array}{r}1.63 \\
11.69\end{array}$ & $\begin{array}{r}3.35 \\
18.84\end{array}$ & $\begin{array}{r}2.48 \\
21.42\end{array}$ & $\begin{array}{r}2.01 \\
19.09\end{array}$ & $\begin{array}{r}2.05 \\
18.79\end{array}$ & $\begin{array}{r}1.96 \\
19.54\end{array}$ & $\begin{array}{r}2.12 \\
18.47\end{array}$ \\
\hline \multirow[t]{2}{*}{ Total Energy } & 1.69 & 3.89 & 7.28 & 9.22 & 7.75 & 7.64 & 8.03 & 8.57 \\
\hline & \multicolumn{8}{|c|}{ Expenditures in Millions of Dollars } \\
\hline Coal tan & 14.5 & 26.6 & 44.1 & 133.3 & 124.1 & 125.1 & 121.8 & 106.4 \\
\hline 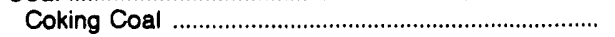 & - & - & - & - & - & - & - & - \\
\hline 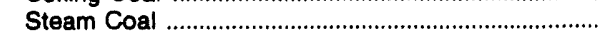 & 14.5 & 26.6 & 44.1 & 133.3 & 124.1 & 125.1 & 121.8 & 106.4 \\
\hline 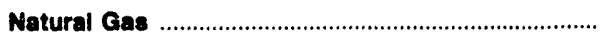 & 24.4 & 34.0 & 102.9 & 188.6 & 145.4 & 144.1 & 118.9 & 143.4 \\
\hline Petroleum n. & 173.0 & 435.9 & $1,059.8$ & 793.3 & 588.1 & 627.1 & 669.8 & 745.4 \\
\hline 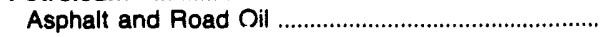 & 2.3 & 7.6 & 8.3 & 27.3 & 16.8 & 12.1 & 8.5 & 10.0 \\
\hline Aviation Gasoline & .2 & .3 & .5 & .8 & .8 & .6 & .7 & .7 \\
\hline 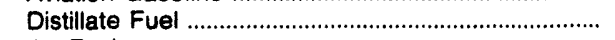 & 29.1 & 62.2 & 146.5 & 150.8 & 114.3 & 126.9 & 127.3 & 155.3 \\
\hline Jet Fuel & 8.1 & 18.0 & 54.6 & 56.0 & 35.2 & 27.9 & 30.5 & 32.4 \\
\hline Kerosene & 3.1 & 4.9 & 14.3 & 15.7 & 6.8 & 7.8 & 5.8 & 5.8 \\
\hline LPG & 10.6 & 34.9 & 56.7 & 38.5 & 34.4 & 36.3 & 38.0 & 42.0 \\
\hline 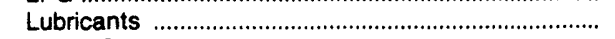 & 3.3 & $3 . ?$ & 12.1 & 13.5 & 11.7 & 11.5 & 11.9 & 15.0 \\
\hline 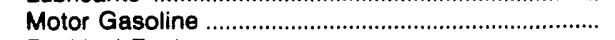 & 93.8 & 168.4 & 333.5 & 372.5 & 282.7 & 314.6 & 347.3 & 380.3 \\
\hline 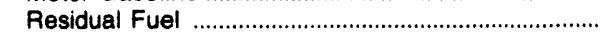 & 18.6 & 123.3 & 335.5 & 86.2 & 65.1 & 65.4 & 78.7 & 79.1 \\
\hline Other & 3.8 & 12.5 & 97.8 & 32.1 & 20.2 & 24.0 & 20.9 & 24.8 \\
\hline 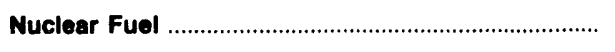 & - & - & - & - & - & - & - & - \\
\hline 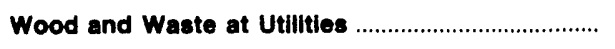 & - & - & - & - & - & - & - & - \\
\hline 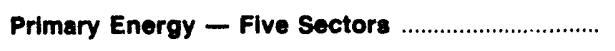 & 211.8 & 496.5 & $1,206.8$ & $1,115.2$ & 857.7 & 896.2 & 910.5 & 995.2 \\
\hline 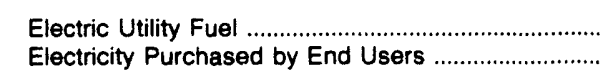 & $\begin{array}{r}-23.1 \\
75.7\end{array}$ & $\begin{array}{r}-106.3 \\
202.1\end{array}$ & $\begin{array}{r}-239.3 \\
368.7\end{array}$ & $\begin{array}{r}-229.9 \\
457.9\end{array}$ & $\begin{array}{r}-179.4 \\
440.0\end{array}$ & $\begin{array}{r}-186.2 \\
446.3\end{array}$ & $\begin{array}{r}-185.6 \\
499.4\end{array}$ & $\begin{array}{r}-191.6 \\
504.3\end{array}$ \\
\hline Total Energy' & 264.4 & 592.2 & $1,336.2$ & $1,343.2$ & $1,118.3$ & $1,156.3$ & $1,224.3$ & $1,307.9$ \\
\hline
\end{tabular}

Includes petroleum coke used at electric utitities.

-There are no direct fuel costs for hydroelectric, geothermal, centralized solar, or wind energy. Wood and other biomass fuels are not included, except those consumed at electric utilities.

-No consumption, including cases where adjustments were made. See "Consumption Adjustments for Process Fuel and Intermediate Products."

Note: Expenditure totals may not equal sum of components due to independent rounding.

Sources: Data sources, estimation procedures, and assumptions are described in the "Documentation" section of this report 


Sector and Enorgy Source
Pealdential Sector
Primary Energy
Noal
Petroleul Gas
Distillate Fuel
Kerosene
LPG

\begin{tabular}{|l|l|}
\hline 1970 & 1975 \\
\hline
\end{tabular}

$2.39 \quad 5.15$

2.39

1.52

1.13
1.55

1.51

1.51
1.42
1.34
2.40

2.40

7.53

1.55
.76
.28

.76
.28
1.22

1.22

1.12

1.12
.85

.85
.99
.89

2.86

2.86
.46
0.56

6.56

1.02

.66

.28

.28
.57

.69

.86
.78

.78
.85

.89
5.08
2.86

2.86
.46

.46
.50

3.10

Transportation Sector

Primary Energy .

Coa

Petroleum ......

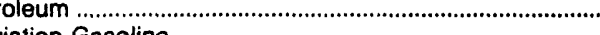

Gasoline

Distillate Fuel

Jet Fue

Lubricants

Motor Gasoline

Residual Fuel

Electricity

Total Energy".

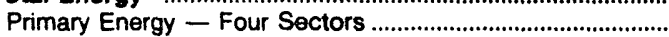

Electricity

Electric Utility Sector

Coal

Natural Gas ...

Petroleum

Heavy Oip

Light Oilb

Petroleum Coke

Nuclear Fuel

Wood and Waste

Primary Energy - Five Sectors

2.78

2.73

2.39

2.99
2.71

2.71
3.37

4.73

4.54

4.54
2.11

1.20

1.87

2.17

2.38

2.36

3.54
1.85

1.95

12.76

1.20

1.20

1.37

2.31

1.76

2.19
2.36

3.40

7.48

4.54
1.87

1.87
1.96

1.86
0.25

$2.13 \quad 3.74$

2.13

.28
2.13

2.13
2.17

2.17
1.24

1.24
.73

.73
.99
5.08
2.86

2.86
.42

3.45
2.81

3.40
7.48

7.48
4.54
1.72

1.89
1.34
4.94

2.18

1.06

$1975]$

$\begin{array}{ll}5.16 & 10.19\end{array}$

\subsection{4}

2.14

\subsection{74}

1.20
3.74

3.74
3.45

2.03
3.40

1.72

\section{$1.89 \quad 3.89$}

2.89

2.89
11.69

.39
.39
.37
.40
.46
.47
.28

\subsection{3}

1.63
1.15

1.02

1.92
1.97

1.97
2.18

2.48

5.13

5.14

4.07

$\begin{array}{rrr}8.41 & 8.79 & 6.50 \\ 8.41 & 8.79 & 6.50 \\ - & - & - \\ 8.41 & 8.79 & 6.50 \\ 9.02 & 9.99 & 8.41 \\ 7.72 & 8.52 & 6.56 \\ 6.46 & 6.63 & 4.87 \\ 4.72 & 11.06 & 10.37 \\ 14.36 & 17.61 & 15.59 \\ 9.60 & 9.39 & 6.97 \\ 3.93 & 3.98 & 2.13 \\ - & - & -\end{array}$

1087

11.85

$\begin{array}{rrr}11.85 & 12.08 & 12.62\end{array}$

6.05
3.38

4.16

7.34

6.88
8.55

8.53
21.76

6.60

4.47

1.20
3.92

4.54

6.30

6.36
4.72

9.60

4.24

20.78

5.69

4.36

1.20

1.20

5.23

3.58

6.36

4.72
14.36

14.36
8.60

4.19

6.16

15.28

\subsection{8}

5.90

18.84

9.22

7.12
21.42

\subsection{5}

5.59

19.09

$\begin{array}{rr}3.35 & 2 . \\ 1.64 & 1.81 \\ 3.47 & 3.88 \\ 4.33 & 3.86 \\ 4.27 & 4 . \\ 6.21 & 5.51 \\ 4.32 & 1 . \\ - & \\ - & \end{array}$

-There are no direct fuel costs for hydroelectric, geothermal, centralized solar, or wind energy. Wood and other biomass fuels are not included, except those consumed at electric utilities.

beavy oil includes Grade Nos. 4, 5, and 6, and residual fuel oils. Light oil includes Grade No. 2 heating oil, kerosene, and jet fuel.

-No consumption, including cases where adjustments were made. See "Consumption Adjustments for Process Fuel and Intermediate Products."

Sources: Data sources, estimation procedures, and assumptions are described in the "Documentation" section of this report. 


\begin{tabular}{|c|c|c|c|c|c|c|c|c|}
\hline Sector and Energy Source & 1970 & 1975 & 1980 & 1985 & 1986 & 1987 & 1988 & 1989 \\
\hline 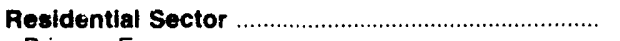 & 65.9 & 135.5 & 246.2 & 319.2 & 290.8 & 309.4 & 332.1 & 345.8 \\
\hline 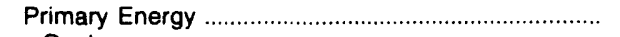 & 35.9 & 57.5 & 107.7 & 140.0 & 110.7 & 117.0 & 119.1 & 131.8 \\
\hline Coal & .1 & .2 & .2 & .2 & .4 & 1.3 & .6 & .6 \\
\hline 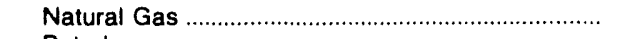 & 12.4 & 16.9 & 29.7 & 43.9 & 48.6 & 44.7 & 45.5 & 48.8 \\
\hline 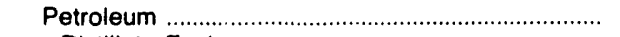 & 23.4 & 40.4 & 77.8 & 95.9 & 61.7 & 71.0 & 73.0 & 82.4 \\
\hline 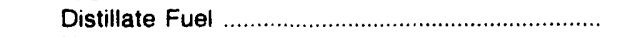 & 16.8 & 29.4 & 52.7 & 58.5 & 37.7 & 44.7 & 46.9 & 48.9 \\
\hline 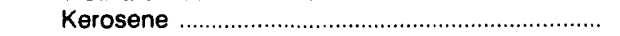 & 2.8 & 4.1 & 13.3 & 14.7 & 6.6 & 7.3 & 5.4 & 5.5 \\
\hline LPG & 3.8 & 6.9 & 11.7 & 22.8 & 17.4 & 19.1 & 20.8 & 28.0 \\
\hline 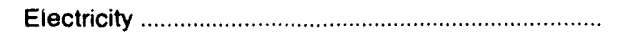 & 30.0 & 77.9 & 138.6 & 179.1 & 180.1 & 192.4 & 213.0 & 214.0 \\
\hline Commercial Sector & 34.4 & 90.5 & 261.3 & 176.4 & 165.4 & 170.6 & 189.6 & 193.1 \\
\hline 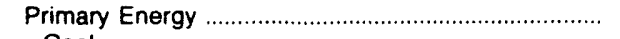 & 14.5 & 32.5 & 154.0 & 43.3 & 35.7 & 36.6 & 30.6 & 39.1 \\
\hline 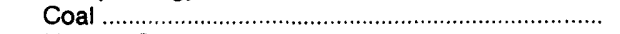 & $\bullet$ & $\bullet$ & .1 & .1 & .2 & .8 & .4 & .4 \\
\hline 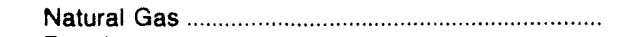 & 3.5 & 5.6 & 13.1 & 22.0 & 22.4 & 19.3 & 19.9 & 22.6 \\
\hline 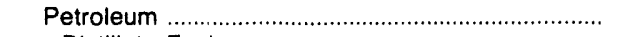 & 11.0 & 26.8 & 140.8 & 21.2 & 13.2 & 16.5 & 18.3 & 16.2 \\
\hline 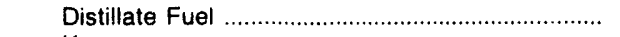 & 5.1 & 10.0 & 23.3 & 12.8 & 7.0 & 9.4 & 10.6 & 9.0 \\
\hline 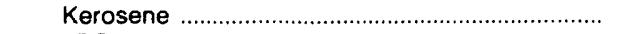 & .2 & .4 & .3 & .9 & .2 & .3 & .3 & .1 \\
\hline LPG & .3 & .9 & 1.1 & 4.3 & 2.9 & 3.1 & 3.7 & 3.5 \\
\hline 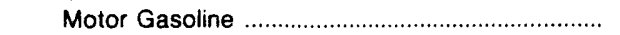 & .4 & .8 & 2.3 & 1.9 & 1.4 & 1.7 & 1.7 & 1.8 \\
\hline 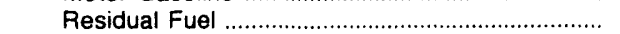 & 5.0 & 14.7 & 113.8 & 1.3 & 1.7 & 2.1 & 1.9 & 1.8 \\
\hline 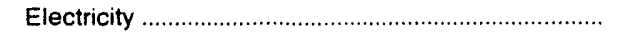 & 19.9 & 58.0 & 107.3 & 133.0 & 129.6 & 133.9 & 151.0 & 154.0 \\
\hline Industrial Sector & 57.1 & 160.3 & 375.0 & 349.8 & 278.3 & 260.9 & 258.7 & 264.3 \\
\hline Primary Energy & 31.3 & 94.2 & 252.1 & 204.1 & 148.9 & 140.9 & 123.4 & 128.0 \\
\hline 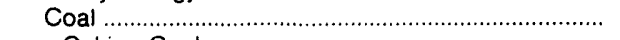 & .2 & .8 & 5.4 & 7.0 & 6.7 & 7.1 & 8.1 & 6.6 \\
\hline 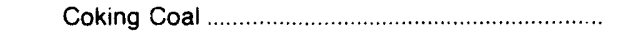 & - & - & - & - & - & - & - & - \\
\hline 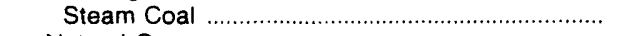 & .2 & .8 & 5.4 & 7.0 & 6.7 & 7.1 & 8.1 & 6.6 \\
\hline 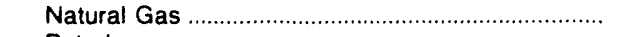 & 7.0 & 9.5 & 34.8 & 93.5 & 69.7 & 57.7 & 46.2 & 50.2 \\
\hline 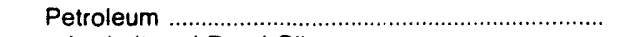 & 24.1 & 83.9 & 212.0 & 103.7 & 71.6 & 76.2 & 69.1 & 71.2 \\
\hline 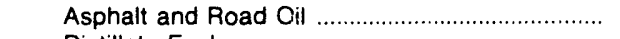 & 2.3 & 7.6 & 8.3 & 27.3 & 16.8 & 12.1 & 8.5 & 10.0 \\
\hline 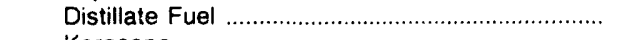 & 3.6 & 12.7 & 20.5 & 15.0 & 10.8 & 10.2 & 10.1 & 12.0 \\
\hline 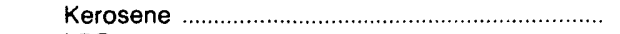 & .1 & .4 & .6 & .1 & * & .2 & .1 & .1 \\
\hline LPG & 6.5 & 26.7 & 43.6 & 11.2 & 12.8 & 14.1 & 13.4 & 10.4 \\
\hline 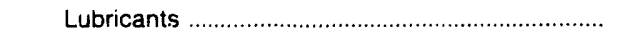 & 1.3 & 1.4 & 6.6 & 7.3 & 6.3 & 6.2 & 6.5 & 8.1 \\
\hline 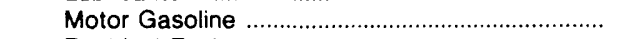 & 1.4 & 1.5 & 1.8 & 2.7 & 2.2 & 2.3 & 2.4 & 3.0 \\
\hline Residual Fuel . & 7.3 & 21.7 & 45.1 & 10.7 & 5.2 & 9.3 & 9.2 & 4.6 \\
\hline 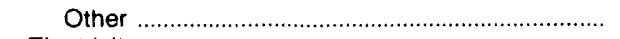 & 1.6 & 11.8 & 85.6 & 29.4 & 17.4 & 21.6 & 18.9 & 23.0 \\
\hline 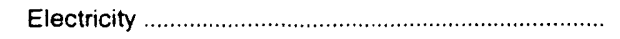 & 25.7 & 66.1 & 122.9 & 145.7 & 130.3 & 119.9 & 135.4 & 136.3 \\
\hline Transportation Sector & 107.0 & 206.0 & 453.7 & 497.8 & 383.8 & 415.4 & 443.9 & 504.6 \\
\hline Primary Energy & 107.0 & 206.0 & 453.7 & 497.8 & 383.8 & 415.4 & 443.9 & 504.6 \\
\hline Coal & $\bullet$ & $\bullet$ & - & - & - & - & - & - \\
\hline 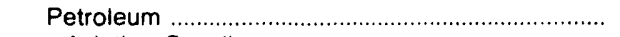 & 107.0 & 206.0 & 453.7 & 497.8 & 383.8 & 415.4 & 443.9 & 504.6 \\
\hline 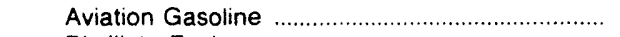 & .2 & .3 & .5 & .8 & .8 & .6 & .7 & .7 \\
\hline 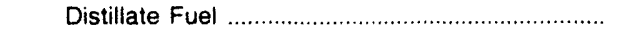 & 2.8 & 8.4 & 43.3 & 61.3 & 56.5 & 59.6 & 56.3 & 79.1 \\
\hline 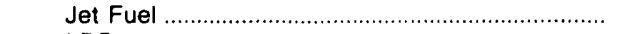 & 8.1 & 18.0 & 54.6 & 56.0 & 35.2 & 27.9 & 30.5 & 32.4 \\
\hline LPG & .1 & .5 & .2 & .1 & 1.3 & .1 & .2 & .2 \\
\hline 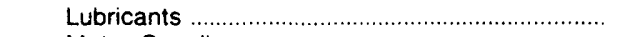 & 2.1 & 2.3 & 5.5 & 6.2 & 5.4 & 5.3 & 5.5 & 6.9 \\
\hline 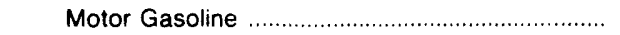 & 92.1 & 166.2 & 329.4 & 367.9 & 279.0 & 310.5 & 343.2 & 375.5 \\
\hline Residual Fuel & 1.8 & 10.4 & 20.1 & 5.4 & 5.6 & 11.4 & 7.6 & 9.8 \\
\hline 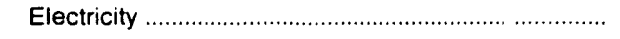 & - & - & - & - & - & - & - & - \\
\hline Total Energy a & 264.4 & 592.2 & $1,336.2$ & $1,343.2$ & $1,118.3$ & $1,156.3$ & $1,224.3$ & $1,307.9$ \\
\hline 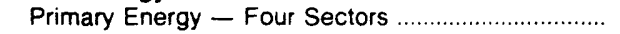 & 188.8 & 390.2 & 967.5 & 885.3 & 678.3 & 710.0 & 724.9 & 803.6 \\
\hline 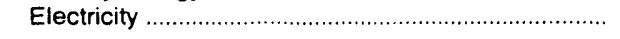 & 75.7 & 202.1 & 368.7 & 457.9 & 440.0 & 446.3 & 499.4 & 504.3 \\
\hline Electric Utility Sector & 23.1 & 106.3 & 239.3 & 229.9 & 179.4 & 186.2 & 185.6 & 191.6 \\
\hline Coal fon. & 14.2 & 25.6 & 38.5 & 125.9 & 116.9 & 115.9 & 112.8 & 98.8 \\
\hline Natural Gas & 1.4 & 1.9 & 25.3 & 29.3 & 4.8 & 22.3 & 7.3 & 21.9 \\
\hline 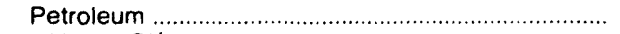 & 7.5 & 78.8 & 175.6 & 74.7 & 57.7 & 48.0 & 65.5 & 70.9 \\
\hline 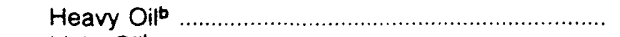 & 4.5 & 76.4 & 156.5 & 68.8 & 52.6 & 42.6 & 60.0 & 62.8 \\
\hline 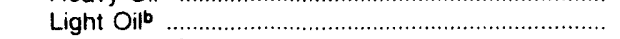 & .8 & 1.7 & 6.8 & 3.2 & 2.3 & 3.1 & 3.5 & 6.3 \\
\hline 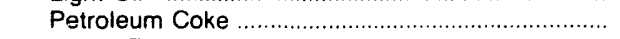 & 2.2 & .7 & 12.2 & 2.7 & 2.8 & 2.4 & 2.0 & 1.8 \\
\hline 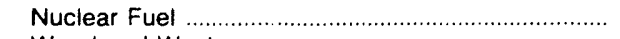 & - & - & - & - & - & - & - & - \\
\hline Wood and Waste & - & - & - & - & - & - & - & - \\
\hline 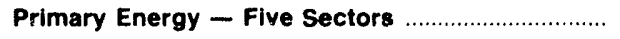 & 211.8 & 496.5 & $1,206.8$ & $1,115.2$ & 857.7 & 896.2 & 910.5 & 995.2 \\
\hline
\end{tabular}

-There are no direct fuel costs for hydroelectric, geothermal, centralized solar, or wind energy. Wood and other biomass fuels are not included, except those consumed at electric utilities.

- Heavy oil includes Grade Nos. 4, 5, and 6, and residual fuel oils. Light oil includes Grade No. 2 heating oil, kerosene, and jet fuel

-No consumption, including cases where adjustments were made. See "Consumption Adjustments for Process Fuel and Intermediate Products."

- Value less than 0.05 million dollars.

Note: Totals may not equal sum of components due to independent rounding

Sources: Data sources, estimation procedures, and assumptions are described in the "Documentation" section of this report. 


\section{Energy Price and Expenditure Estimates by Source, District of Columbia I 1970, 1975, 1980, and 1985-1989}

\begin{tabular}{|c|c|c|c|c|c|c|c|c|}
\hline Energy Source & 1970 & 1975 & 1980 & 1985 & 1986 & 1987 & 1988 & 1989 \\
\hline & \multicolumn{8}{|c|}{ Prices in Dollars per Million Btu } \\
\hline 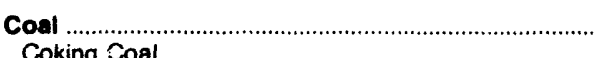 & 0.29 & 1.32 & 1.76 & 2.00 & 1.99 & 1.96 & 1.92 & 1.97 \\
\hline Coking Coal & .29 & 1.32 & $1.7 \overline{6}$ & 2.00 & $1.9 \overline{9}$ & 1.96 & 1.92 & 1.97 \\
\hline 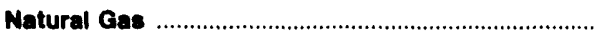 & 1.27 & 2.13 & 4.36 & 7.30 & 6.77 & 6.06 & 6.00 & 6.36 \\
\hline Petroleum & 1.19 & 3.30 & 7.86 & 8.73 & 6.43 & 6.93 & 7.01 & 7.36 \\
\hline 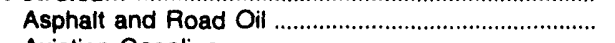 & .67 & 1.80 & 3.58 & 4.95 & 4.18 & 3.22 & 3.16 & 2.88 \\
\hline & - & - & - & - & - & - & - & - \\
\hline $\begin{array}{l}\text { Distillate Fuel } \\
\text { Jet Fuel }\end{array}$ & $\begin{array}{r}1.09 \\
73\end{array}$ & 2.61 & $\begin{array}{l}7.18 \\
6.46\end{array}$ & $\begin{array}{l}7.95 \\
5.80\end{array}$ & $\begin{array}{l}6.22 \\
4.01\end{array}$ & & $\begin{array}{r}6.04 \\
375\end{array}$ & 6.42 \\
\hline Jet Fuel & $\begin{array}{r}.73 \\
1.41\end{array}$ & 2.74 & 8.50 & 7.08 & 6.84 & $\begin{array}{l}4.01 \\
6.74\end{array}$ & $\begin{array}{l}3.75 \\
6.09\end{array}$ & 5.82 \\
\hline LPG & 1.49 & 3.37 & 6.00 & 11.55 & 11.19 & 10.88 & 11.04 & 10.29 \\
\hline 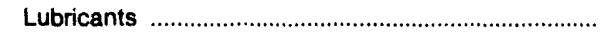 & 5.08 & 7.49 & 14.36 & 17.61 & 15.59 & 13.58 & 14.61 & 17.90 \\
\hline 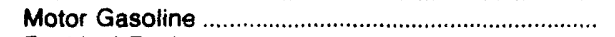 & 2.86 & 4.85 & 9.97 & 10.19 & 8.11 & 8.36 & 8.80 & 9.50 \\
\hline 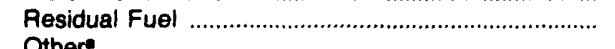 & .50 & 1.97 & 4.46 & 4.19 & 2.78 & 3.23 & 2.37 & 2.74 \\
\hline 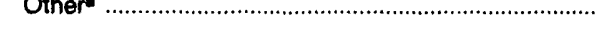 & - & - & - & - & - & - & - & - \\
\hline 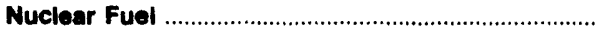 & - & - & - & - & - & - & - & - \\
\hline 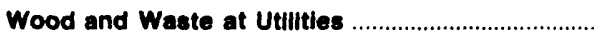 & - & - & - & - & - & - & - & - \\
\hline Primary Energy - Five Sectors ..................................... & 1.06 & 2.85 & 6.36 & 7.81 & 6.48 & 6.45 & 6.52 & 6.82 \\
\hline $\begin{array}{l}\text { Electric Utiiity Fuel ... } \\
\text { Electricity Purchased by End Users . }\end{array}$ & $\begin{array}{r}.43 \\
5.39\end{array}$ & $\begin{array}{r}1.92 \\
10.74\end{array}$ & $\begin{array}{r}4.59 \\
14.92\end{array}$ & $\begin{array}{r}4.24 \\
20.89\end{array}$ & $\begin{array}{r}2.28 \\
20.14\end{array}$ & $\begin{array}{r}3.04 \\
18.84\end{array}$ & $\begin{array}{r}2.40 \\
18.00\end{array}$ & $\begin{array}{r}2.89 \\
17.22\end{array}$ \\
\hline \multirow[t]{2}{*}{ Total Energyb } & 1.72 & 4.33 & 8.75 & 11.67 & 10.51 & 10.35 & 10.46 & 10.58 \\
\hline & \multicolumn{8}{|c|}{ Expenditures in Millions of Dollars } \\
\hline 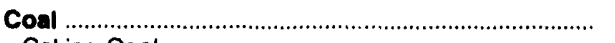 & 8.4 & 13.4 & 5.8 & 7.0 & 2.7 & 3.4 & 1.5 & 2.9 \\
\hline 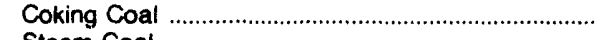 & - & - & - & - & - & - & - & - \\
\hline 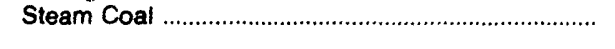 & 8.4 & 13.4 & 5.8 & 7.0 & 2.7 & 3.4 & 1.5 & 2.9 \\
\hline Natural Gas & 33.5 & 55.7 & 121.8 & 211.5 & 200.6 & 188.7 & 197.1 & 213.1 \\
\hline Petroleum & 154.1 & 250.8 & 374.9 & 334.6 & 290.4 & 285.2 & 290.5 & 310.3 \\
\hline 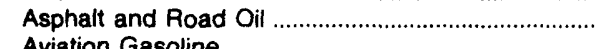 & .1 & .2 & .4 & .9 & .9 & .7 & .7 & .5 \\
\hline 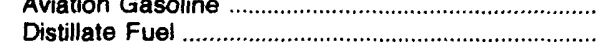 & $31 . \overline{4}$ & $48 . \overline{1}$ & $95 . \overline{6}$ & $103 . \overline{2}$ & $86 . \overline{8}$ & 70.6 & 65.7 & 68.8 \\
\hline 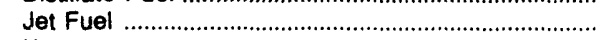 & & - & 12.1 & .2 & 11.4 & . & .1 & - \\
\hline 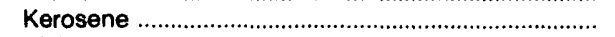 & .4 & 1.7 & 12.9 & 1.2 & .3 & 3 & .2 & .9 \\
\hline LPG & • & .1 & .1 & .2 & .1 & .1 & .2 & .2 \\
\hline 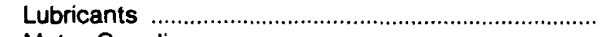 & 1.7 & 2.7 & 5.3 & 5.9 & 5.1 & 5.0 & 5.2 & 6.6 \\
\hline 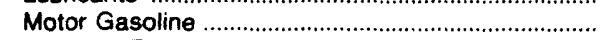 & 85.4 & 146.4 & 203.3 & 203.4 & 165.2 & 185.9 & 201.8 & 209.4 \\
\hline 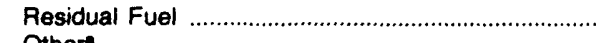 & 35.1 & 51.6 & 45.2 & 19.5 & 20.6 & 22.5 & 16.5 & 23.8 \\
\hline Othere & - & - & - & - & - & - & - & - \\
\hline 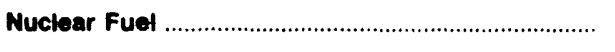 & - & - & - & - & - & - & - & - \\
\hline 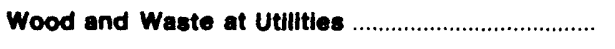 & - & - & - & - & - & - & - & - \\
\hline Primary Energy - Five Sectors …………….............. & 195.9 & 319.9 & 502.4 & 553.1 & 493.7 & 477.3 & 489.1 & 526.3 \\
\hline 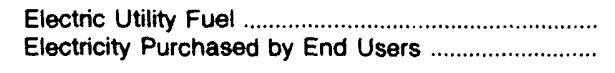 & $\begin{array}{r}-18.0 \\
99.2\end{array}$ & $\begin{array}{l}-31.7 \\
212.3\end{array}$ & $\begin{array}{l}-45.1 \\
356.5\end{array}$ & $\begin{array}{r}-8.3 \\
585.3\end{array}$ & $\begin{array}{r}-6.7 \\
590.1\end{array}$ & $\begin{array}{l}-11.7 \\
578.9\end{array}$ & $\begin{array}{l}-16.4 \\
576.0\end{array}$ & $\begin{array}{l}-26.8 \\
567.0\end{array}$ \\
\hline Total Energy' & 277.2 & 500.5 & 813.8 & $1,130.1$ & $1,077.1$ & $1,044.6$ & $1,048.7$ & $1,066.5$ \\
\hline
\end{tabular}

Includes petroleum coke used at electric utilities.

bThere are no direct fuel costs for hydroelectric, geothermal, centralized solar, or wind energy. Wood and other biomass fuels are not included, except those consumed at electric utilities.

-No consumption, including cases where adjustments were made. See "Consumption Adjustments for Process Fuel and Intermediate Products."

Value less than 0.05 million dollars.

Note: Expenditure totals may not equal sum of components due to independent rounding.

Sources: Data sources, estimation procedures, and assumptions are described in the "Documentation" sertion of this report. 


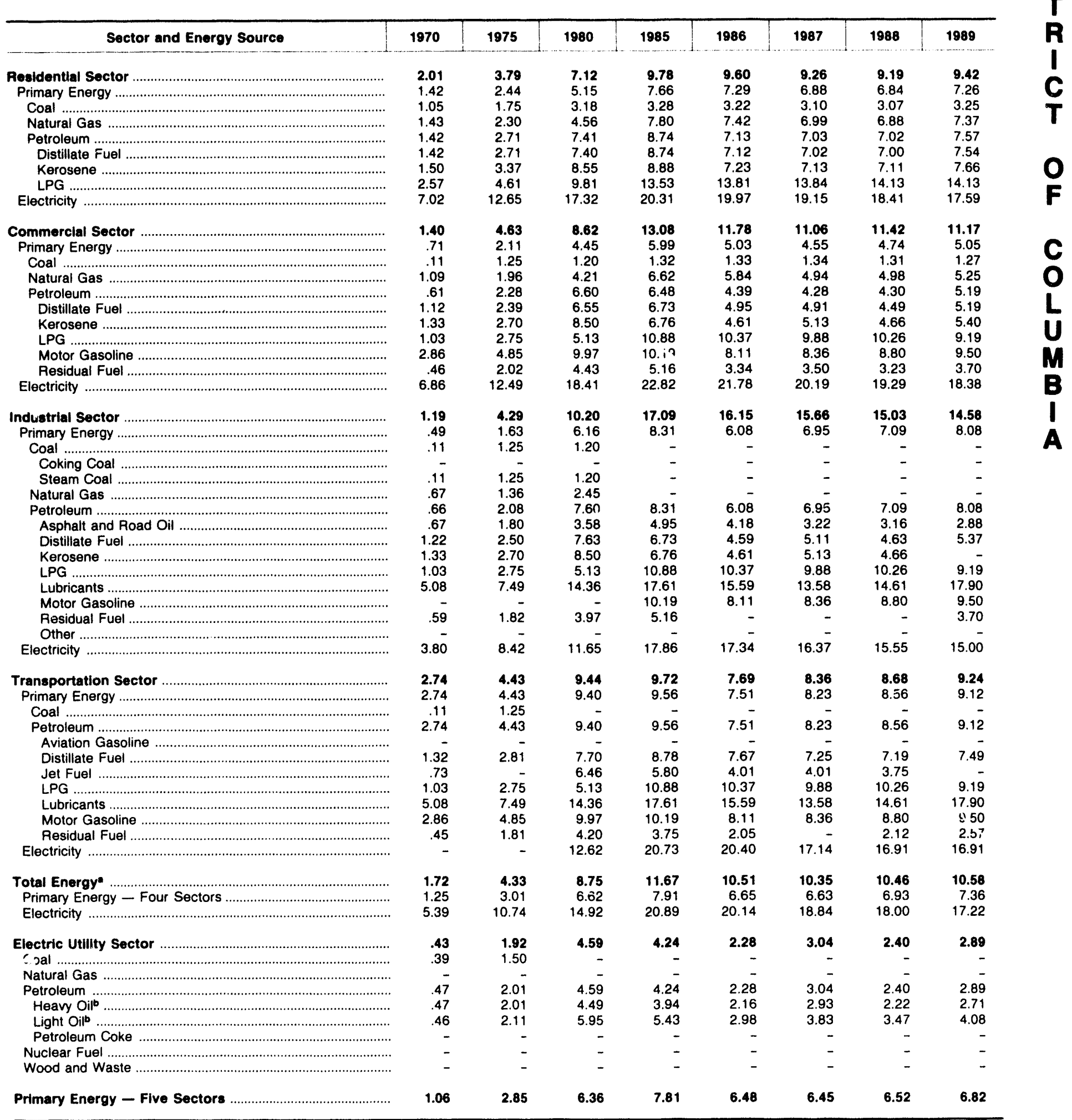

- There are no direct fuel costs for hydroelectric, geothermal, centralized solar, or wind energy. Wood and other biomass fuels are not included, except those consumed at electric utilities.

bHeavy oil includes Grade Nos. 4, 5, and 6, and residual fuel oils. Light oil includes Grade No. 2 heating oil, kerosene, and jet fuel.

-No consumption, including cases where adjustments were made. See "Consumption Adjustments for Process Fuel and Intermediate Products."

Sources: Data sources, estimation procedures, and assumptions are described in the "Documentation" section of this report. 


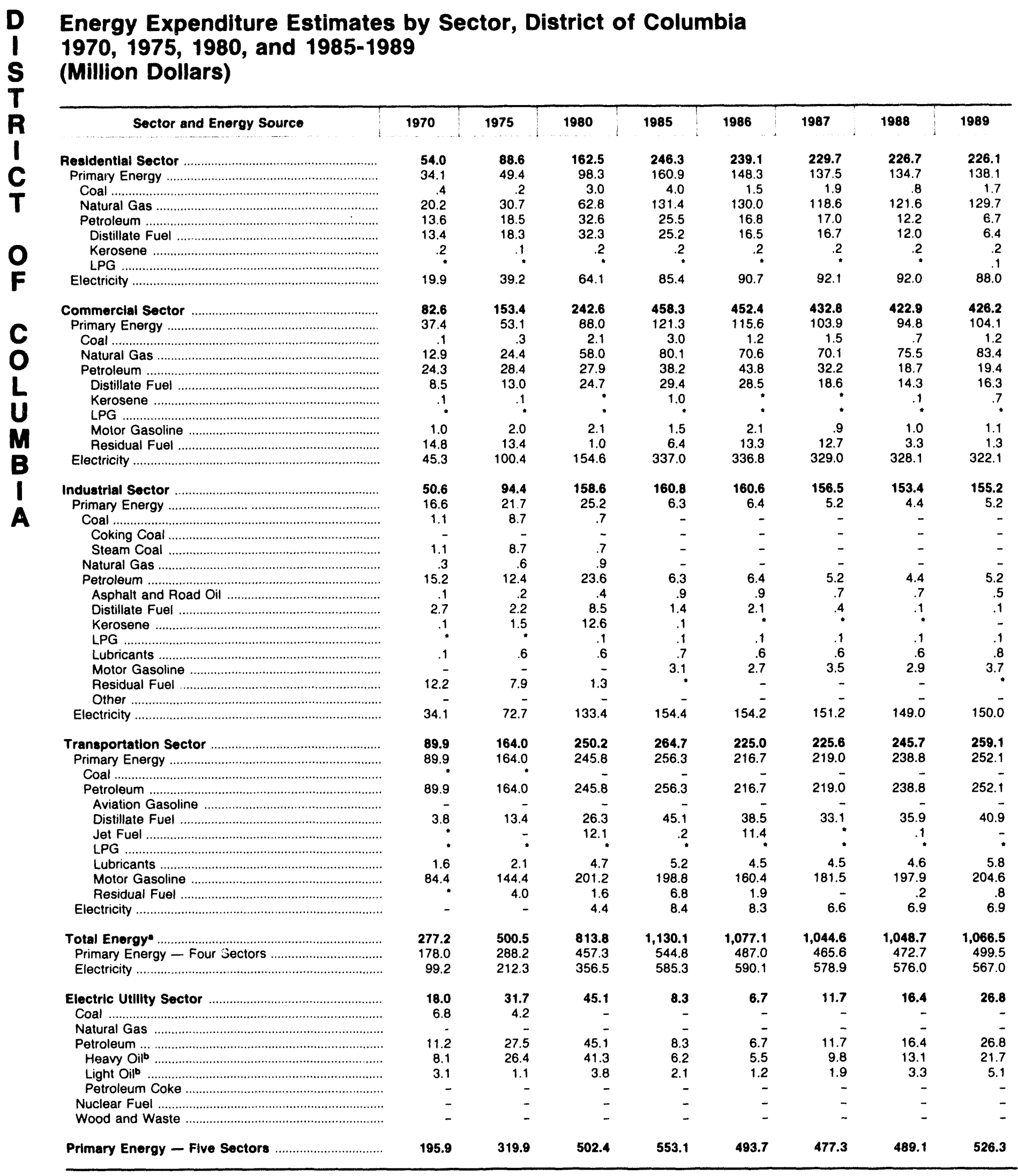

- There are no direct fuel costs for hydroelectric, geothermal, centralized solar, or wind energy. Wood and other biomass fuels are not included, except those consumed at electric utilities.

bHeavy oil includes Grade Nos. 4, 5, and 6, and residual fuel oils. Light oil includes Grade No. 2 heating oil, kerosene, and jet fuel.

-No consumption, including cases where adjustments were made. See "Consumption Adjustments for Process Fuel and Intermediate Products." - Value less than 0.05 million dollars.

Note: Totals may not equal sum of components due to independent rounding

Sources: Data sources, estimation procedures, and assumptions are described in the "Documentation" section of this report. 


\begin{tabular}{|c|c|c|c|c|c|c|c|c|}
\hline Energy Source & 1870 & 1975 & 1980 & 1985 & 1986 & 1987 & 1988 & 1989 \\
\hline & \multicolumn{8}{|c|}{ Prices in Dollars per Million Btu } \\
\hline Coa! & 0.31 & 1.01 & 1.80 & 2.12 & 1.83 & 1.82 & 1.78 & 1.79 \\
\hline Coking Coal & - & - & - & - & - & - & - & - \\
\hline 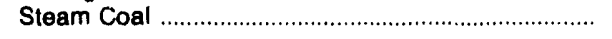 & .31 & 1.01 & 1.80 & 2.12 & 1.93 & 1.82 & 1.78 & 1.79 \\
\hline (1) & .49 & 1.00 & 2.19 & 3.73 & 2.76 & 3.21 & 2.80 & 3.02 \\
\hline Petroleum & 1.53 & 3.02 & 6.66 & 7.51 & 5.38 & 5.88 & 5.54 & 6.04 \\
\hline 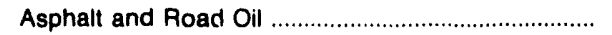 & .67 & 1.78 & 3.57 & 4.99 & 4.16 & 3.20 & 3.13 & 2.87 \\
\hline Aviation Gasoline & 2.17 & 3.45 & 9.02 & 9.99 & 8.41 & 7.55 & 7.41 & 8.28 \\
\hline 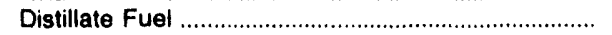 & 1.08 & 2.53 & 6.91 & 6.78 & 5.90 & 6.12 & 5.83 & 6.16 \\
\hline 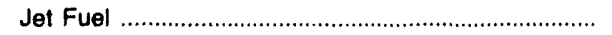 & .73 & 2.03 & 6.46 & 5.90 & 4.19 & 4.07 & 3.82 & 4.43 \\
\hline 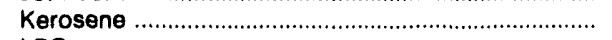 & 1.28 & 3.12 & 8.45 & 7.56 & 7.87 & 7.76 & 7.99 & 7.35 \\
\hline LPG & 2.61 & 5.14 & 7.48 & 10.90 & 10.28 & 11.00 & 11.21 & 10.17 \\
\hline Lubricants & 5.08 & 7.49 & 14.36 & 17.61 & 15.59 & 13.58 & 14.61 & 17.90 \\
\hline Motor Gasoline & 2.81 & 4.39 & 9.80 & 9.03 & 6.70 & 7.11 & 7.12 & 7.68 \\
\hline 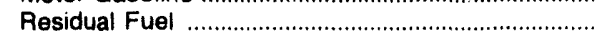 & .33 & 1.84 & 3.61 & 3.88 & 2.15 & 2.90 & 2.18 & 2.65 \\
\hline Other" & 1.21 & 3.02 & 7.89 & 8.29 & 5.31 & 6.17 & 5.20 & 5.84 \\
\hline Nuclear Fuel & - & .17 & .35 & .65 & .73 & .69 & .94 & .66 \\
\hline Wood and Waste at Utilities & - & - & - & - & - & - & - & - \\
\hline Primary Energy - Five Sectors & 1.20 & 2.44 & 5.12 & 5.18 & 3.98 & 4.20 & 3.94 & 4.26 \\
\hline 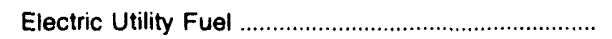 & .33 & 1.35 & 2.40 & 2.21 & 1.76 & 1.96 & 1.72 & 1.90 \\
\hline 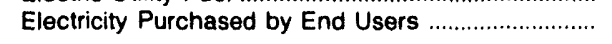 & 5.67 & 10.46 & 16.24 & 22.59 & 21.37 & 20.55 & 20.70 & 20.45 \\
\hline \multirow[t]{2}{*}{ Total Energyb } & 2.35 & 4.62 & 8.84 & 10.93 & 9.43 & 9.46 & 9.32 & 9.83 \\
\hline & \multicolumn{8}{|c|}{ Expenditures in Millions of Dollars } \\
\hline Coal tan. & 35.8 & 135.0 & 405.4 & 999.9 & 887.6 & $1,066.6$ & $1,088.2$ & $1,128.3$ \\
\hline Coking Coal & - & - & - & - & - & - & - & - \\
\hline 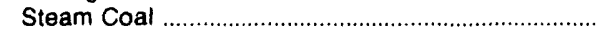 & 35.8 & 135.0 & 405.4 & 999.9 & 887.6 & $1,066.6$ & $1,088.2$ & $1,128.3$ \\
\hline (1) & 170.1 & 283.6 & 693.8 & $1,081.0$ & 787.7 & 964.7 & 825.1 & 976.6 \\
\hline 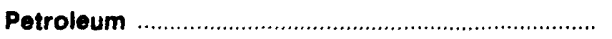 & $1,630.7$ & $4,166.9$ & $11,048.0$ & $9,699.0$ & $7,902.4$ & $8,434.0$ & $8,608.1$ & $9,383.4$ \\
\hline Asphalt and Road Oil & 18.0 & 43.3 & 106.4 & 220.8 & 227.3 & 160.8 & 164.6 & 123.5 \\
\hline Aviation Gasoline & 34.4 & 33.4 & 61.0 & 42.4 & 43.4 & 29.6 & 33.0 & 40.8 \\
\hline Distillate Fuel & 98.0 & 343.6 & $1,183.7$ & $1,198.8$ & $1,089.5$ & $1,172.6$ & $1,169.0$ & $1,269.2$ \\
\hline 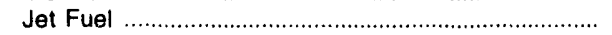 & 96.6 & 275.6 & $1,302.3$ & 762.5 & 587.3 & 604.7 & 685.3 & 835.0 \\
\hline Kerosene & 26.6 & 15.5 & 45.6 & 49.2 & 32.6 & 28.7 & 24.1 & 17.3 \\
\hline LPG & 77.1 & 142.5 & 294.6 & 390.4 & 401.1 & 340.5 & 336.6 & 313.6 \\
\hline 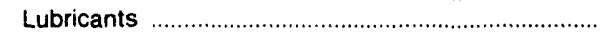 & 33.6 & 54.0 & 122.7 & 136.9 & 118.5 & 116.7 & 121.2 & 152.2 \\
\hline Motor Gasoline & $1,125.2$ & $2,319.6$ & $5,627.4$ & $5,947.5$ & $4,614.0$ & $5,132.4$ & $5,310.7$ & $5,731.5$ \\
\hline 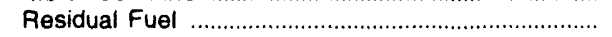 & 112.8 & 915.2 & $2,193.5$ & 835.2 & 705.2 & 752.3 & 682.4 & 811.4 \\
\hline 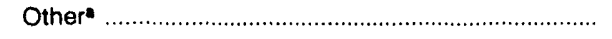 & 8.4 & 24.1 & 110.8 & 115.2 & 83.4 & 95.6 & 81.1 & 88.9 \\
\hline (1) & - & 15.8 & 63.8 & 165.1 & 172.9 & 138.6 & 265.0 & 148.5 \\
\hline Wood and Waste at Utilities & - & - & - & - & - & - & - & - \\
\hline Primary Energy - Five Sectors & $1,836.6$ & $4,601.2$ & $12,211.0$ & $11,945.0$ & $9,750.6$ & $10,603.9$ & $10,786.5$ & $11,636.8$ \\
\hline 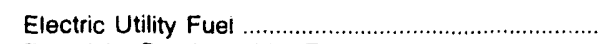 & -196.0 & $-1,114.2$ & $-2,439.2$ & $-2,244.7$ & $-1,980.3$ & $-2,256.9$ & $-2,200.2$ & $-2,433.9$ \\
\hline 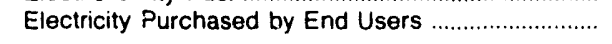 & 971.7 & $2,532.9$ & $5,029.8$ & $8,548.0$ & $8,480.2$ & $8,586.9$ & $9,197.0$ & $9,661.0$ \\
\hline Total Energyb & $2,612.4$ & $6,019.9$ & $14,801.6$ & $18,248.3$ & $16,250.4$ & $16,933.8$ & $17,783.3$ & $18,863.8$ \\
\hline
\end{tabular}

-Includes petroleum coke used at electric utilities.

bThere are no direct fuel costs for hydroelectric, geothermal, centralized solar, or wind energy. Wood and other biomass fuels are not included, except those consumed at electric utilities.

-No consumption, including cases where adjustments were made. See "Consumption Adjustments for Process Fuel and Intermediate Products."

Note: Expenditure totals may not equal sum of components due to independent rounding.

Sources: Data sources, estimation procedures, and assumptions are described in the "Documentation" section of this report. 


\begin{tabular}{|c|c|c|c|c|c|c|c|c|}
\hline Sector and Energy Source & 1970 & 1975 & 1980 & 1985 & 1986 & 1987 & 1998 & 1989 \\
\hline Aealdential Soctor & 4.60 & 9.04 & 14.66 & 21.68 & 20.69 & 20.44 & 20.86 & 20.85 \\
\hline 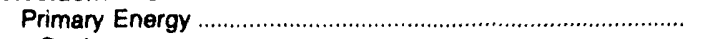 & 2.37 & 4.19 & 7.48 & 8.80 & 8.83 & 9.30 & 9.47 & 9.19 \\
\hline Coal & - & & 3.12 & 3.31 & 3.07 & 2.96 & 2.95 & 3.05 \\
\hline 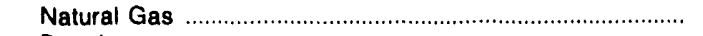 & 2.42 & 2.54 & 4.49 & 6.72 & 6.70 & 6.83 & 6.94 & 7.42 \\
\hline Petroleum & 2.35 & 5.10 & 9.24 & 10.12 & 10.11 & 10.94 & 11.22 & 10.32 \\
\hline Distillate Fuel & 1.25 & 2.62 & 6.92 & 6.73 & 7.67 & 6.75 & 7.17 & 6.56 \\
\hline 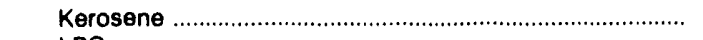 & 1.63 & 3.27 & 8.92 & 9.34 & 10.63 & 9.36 & 9.94 & 9.10 \\
\hline 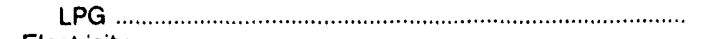 & 3.11 & 6.32 & 10.34 & 10.70 & 10.35 & 12.01 & 12.01 & 10.81 \\
\hline 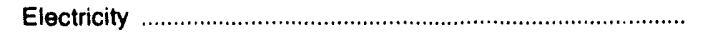 & 6.10 & 10.92 & 16.74 & 24.73 & 23.36 & 22.73 & 22.91 & 22.67 \\
\hline Commerclal Sector & 3.53 & 7.13 & 12.27 & 16.09 & 14.72 & 14.38 & 14.84 & 15.35 \\
\hline 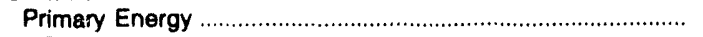 & 1.07 & 2.03 & 4.68 & 5.67 & 4.63 & 4.74 & 4.53 & 4.90 \\
\hline Coal & - & - & 1.77 & 2.04 & 1.98 & 1.87 & 1.84 & 1.86 \\
\hline Natural Gas & .89 & 1.58 & 3.21 & 4.80 & 4.11 & 4.28 & 4.20 & 4.47 \\
\hline Petroleum & 1.23 & 2.52 & 6.24 & 6.48 & 5.16 & 5.22 & 4.86 & 5.41 \\
\hline 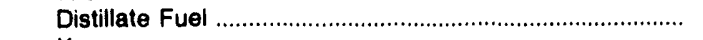 & .98 & 2.26 & 6.30 & 5.79 & 5.01 & 4.85 & 4.46 & 4.60 \\
\hline 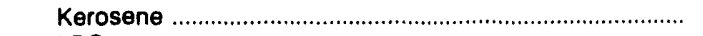 & .61 & 2.38 & 6.41 & 6.64 & 6.12 & 5.77 & 5.45 & 5.93 \\
\hline LPG & 1.27 & 2.51 & 5.46 & 11.24 & 10.15 & 9.27 & 9.94 & 9.15 \\
\hline 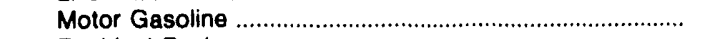 & 2.81 & 4.39 & 9.80 & 9.03 & 6.70 & 7.11 & 7.12 & 7.68 \\
\hline 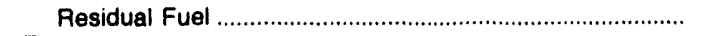 & .33 & 1.85 & 3.71 & 4.08 & 2.27 & 2.98 & 2.29 & 2.75 \\
\hline Electricity & 6.24 & 11.44 & 17.38 & 22.03 & 20.45 & 19.57 & 19.75 & 19.40 \\
\hline Induatrial Sector & .93 & 2.71 & 5.48 & 7.24 & 6.56 & 6.15 & 5.71 & 6.14 \\
\hline 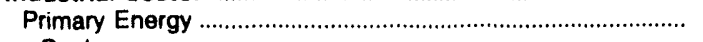 & .55 & 1.63 & 3.87 & 5.02 & 4.24 & 3.94 & 3.53 & 3.79 \\
\hline Coal & - & .53 & 1.77 & 2.04 & 1.98 & 1.87 & 1.84 & 1.86 \\
\hline 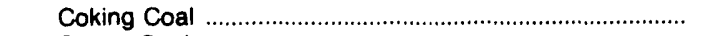 & - & - & - & - & - & - & - & - \\
\hline Steam Coal & - & .53 & 1.77 & 2.04 & 1.98 & 1.87 & 1.84 & 1.86 \\
\hline Natural Gas & .37 & .95 & 2.61 & 3.71 & 2.85 & 3.01 & 2.63 & 2.88 \\
\hline Petroleum & .68 & 2.17 & 4.70 & 6.29 & 5.25 & 4.89 & 4.46 & 5.02 \\
\hline Asphalt and Road Oil & .67 & 1.78 & 3.57 & 4.99 & 4.16 & 3.20 & 3.13 & 2.87 \\
\hline 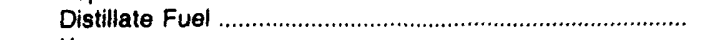 & .56 & 2.20 & 5.75 & 5.81 & 5.36 & 5.05 & 4.77 & 5.19 \\
\hline 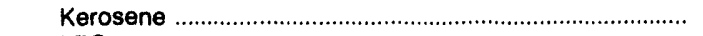 & .61 & 2.38 & 6.41 & 6.64 & 6.12 & 5.77 & 5.45 & 5.93 \\
\hline 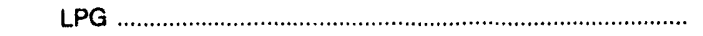 & 1.27 & 2.51 & 5.46 & 11.24 & 10.15 & 9.27 & 9.94 & 9.15 \\
\hline 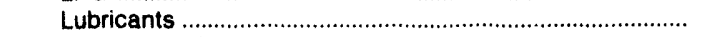 & 5.08 & 7.49 & 14.36 & 17.61 & 15.59 & 13.58 & 14.61 & 17.90 \\
\hline 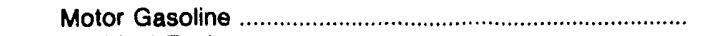 & 2.81 & 4.39 & 9.80 & 9.03 & 6.70 & 7.11 & 7.12 & 7.68 \\
\hline 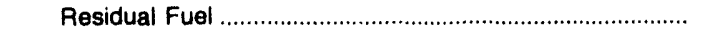 & .37 & 1.75 & 3.44 & 4.08 & 2.27 & 2.98 & 2.29 & 2.75 \\
\hline Other & 1.21 & 3.02 & 7.89 & 8.29 & 5.31 & 6.17 & 5.20 & 5.84 \\
\hline 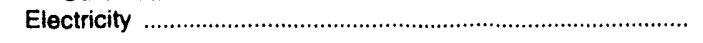 & 3.56 & 7.57 & 13.38 & 16.63 & 16.29 & 14.99 & 14.93 & 14.84 \\
\hline Transportation Sector & 2.19 & 3.79 & 8.39 & 8.20 & 6.18 & 6.50 & 6.34 & 6.86 \\
\hline 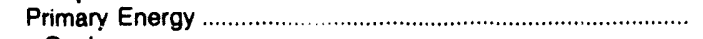 & 2.19 & 3.79 & 8.39 & 8.20 & 6.18 & 6.50 & 6.34 & 6.86 \\
\hline Coal & - & .53 & - & - & - & & & - \\
\hline Petroleum & 2.19 & 3.79 & 8.39 & 8.20 & 6.18 & 6.50 & 6.34 & 6.86 \\
\hline Aviation Gasoline & 2.17 & 3.45 & 9.02 & 9.99 & 8.41 & 7.55 & 7.41 & 8.28 \\
\hline 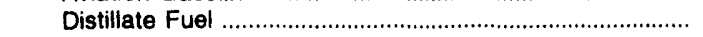 & 1.44 & 2.89 & 7.72 & 7.24 & 6.24 & 6.66 & 6.25 & 6.64 \\
\hline Jet Fuel & .73 & 2.03 & 6.46 & 5.90 & 4.19 & 4.07 & 3.82 & 4.43 \\
\hline LPG & 1.27 & 2.51 & 5.46 & 11.24 & 10.15 & 9.27 & 9.94 & 9.15 \\
\hline Lubricants & 5.08 & 7.49 & 14.36 & 17.61 & 15.59 & 13.58 & 14.61 & 17.90 \\
\hline 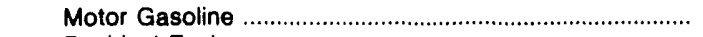 & 2.81 & 4.39 & 9.80 & 9.03 & 6.70 & 7.11 & 7.12 & 7.68 \\
\hline 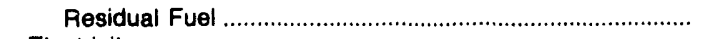 & .29 & 1.60 & 3.14 & 3.75 & 2.01 & 2.83 & 1.94 & 2.33 \\
\hline 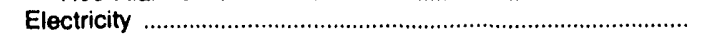 & & & - & 22.04 & 19.81 & 19.74 & 19.83 & 19.67 \\
\hline Total Energy" & 2.35 & 4.62 & 8.84 & 10.93 & 9.43 & 9.46 & 9.32 & 9.83 \\
\hline Primary Energy - Four Sectors ................................................ & 1.74 & 3.28 & 7.16 & 7.51 & 5.86 & 6.08 & 5.87 & 6.36 \\
\hline Electricity . & 5.67 & 10.46 & 16.24 & 22.59 & 21.37 & 20.55 & 20.70 & 20.45 \\
\hline Eloctric Utility Sector & .33 & 1.35 & 2.40 & 2.21 & 1.76 & 1.96 & 1.72 & 1.90 \\
\hline Coal & .31 & 1.01 & 1.80 & 2.12 & 1.93 & 1.82 & 1.78 & 1.79 \\
\hline 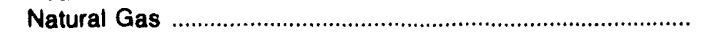 & .35 & .72 & 1.53 & 3.25 & 2.08 & 2.71 & 2.10 & 2.46 \\
\hline Petroleum & .33 & 1.88 & 3.80 & 3.96 & 2.18 & 2.94 & 2.23 & 2.77 \\
\hline 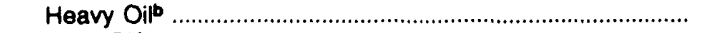 & .33 & 1.85 & 3.72 & 3.87 & 2.15 & 2.91 & 2.20 & 2.69 \\
\hline 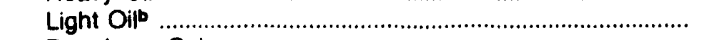 & .36 & 2.21 & 5.76 & 5.71 & 3.41 & 3.93 & 3.54 & 4.27 \\
\hline Petroleum Coke & - & - & - & & - & - & - & - \\
\hline 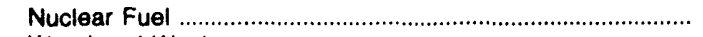 & - & 17 & .35 & .65 & .73 & 69 & .94 & .66 \\
\hline Wood and Waste & - & - & - & - & - & - & - & - \\
\hline 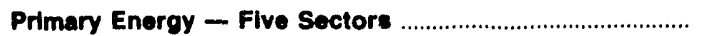 & 1.20 & 2.44 & 5.12 & 5.18 & 3.98 & 4.20 & 3.94 & 4.26 \\
\hline
\end{tabular}

-There are no direct fuel costs for hydroelectric, geothermal, centralized solar, or wind energy. Wood and other biomass fuels are not included, except those consumed at electric utilities.

bHeavy oil includes Grade Nos. 4, 5, and 6, and residual fuel oils. Light oil includes Grade No. 2 heating oil, kerosene, and jet fuel.

-No consumption, including cases where adjustments were made. See "Consumption Adjustments for Process Fuel and Intermediate Products."

Sources: Data sources, estimation procedures, and assumptions are described in the "Documentation" section of this report. 
Energy Expenditure Estimates by Sector, Florida

$1970,1975,1980$, and $1985-1989$

(Million Dollars)

\begin{tabular}{|c|c|c|c|c|c|c|c|c|}
\hline Sector and Energy Source & 1970 & 1975 & 1980 & 1985 & 1986 & 1987 & 1988 & 1989 \\
\hline $\begin{array}{l}\text { Residential Sector } \\
\text { Primary Energy }\end{array}$ & 645.6 & $1,488.1$ & $2,884.5$ & $4,951.9$ & $4,987.5$ & $\begin{array}{r}5,076.8 \\
3929\end{array}$ & $5,373.2$ & $5,606.4$ \\
\hline $\begin{array}{l}\text { Primary Energy } \\
\text { Coal }\end{array}$ & $\begin{array}{r}133.6 \\
-\end{array}$ & $\begin{array}{r}192.8 \\
-\end{array}$ & $\begin{array}{r}329.5 \\
.3\end{array}$ & $\begin{array}{r}385.1 \\
3.2\end{array}$ & $\begin{array}{r}390.9 \\
2.6\end{array}$ & $\begin{array}{r}392.9 \\
1.5\end{array}$ & $\begin{array}{r}372.2 \\
.1\end{array}$ & 333.4 \\
\hline 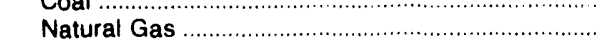 & $37 . \overline{0}$ & 41.7 & 72.7 & 100.9 & 99.9 & 109.0 & 111.5 & 105.5 \\
\hline 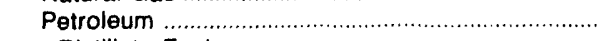 & 96.6 & 151.1 & 256.5 & 281.0 & 288.4 & 282.5 & 260.6 & 227.9 \\
\hline 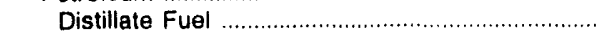 & 7.4 & 16.7 & 49.0 & 22.3 & 20.7 & 28.3 & 21.8 & 13.9 \\
\hline 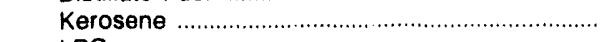 & 22.3 & 13.4 & 39.1 & 20.7 & 17.0 & 19.2 & 17.0 & 9.6 \\
\hline LPG & 66.9 & 121.0 & 168.4 & 238.0 & 250.8 & 235.0 & 221.8 & 204.5 \\
\hline 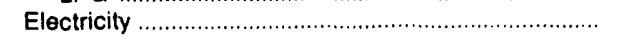 & 512.1 & $1,295.3$ & $2,555.0$ & $4,566.8$ & $4,596.6$ & $4,683.9$ & $5,001.0$ & $5,273.0$ \\
\hline Commercial Sector & 411.3 & $1,028.4$ & $1,921.0$ & $3,559.3$ & $3,464.6$ & $3, \mathbf{5 1 5 . 3}$ & $3,728.0$ & $3,868.1$ \\
\hline 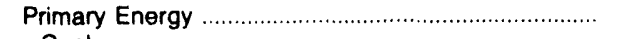 & 65.3 & 134.2 & 294.8 & 455.9 & 395.1 & 404.9 & 367.2 & 345.3 \\
\hline 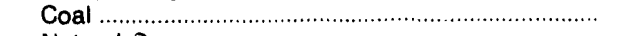 & - & - & .3 & 3.6 & 3.1 & 1.6 & .1 & $\bullet$ \\
\hline 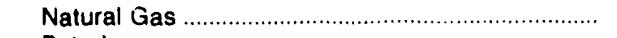 & 24.9 & 53.9 & 103.6 & 163.4 & 158.6 & 175.8 & 171.8 & 170.3 \\
\hline 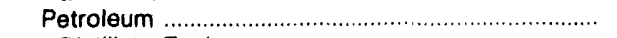 & 40.4 & 80.3 & 190.9 & 288.9 & 233.5 & 227.5 & 195.3 & 175.0 \\
\hline 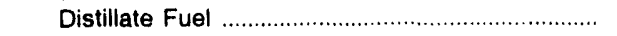 & 11.7 & 29.3 & 70.7 & 123.3 & 99.7 & 109.1 & $\mathbf{8 6 . 0}$ & 74.4 \\
\hline 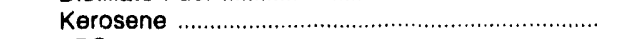 & .5 & .5 & 1.0 & 17.9 & 14.9 & 8.8 & 6.0 & 5.9 \\
\hline LPG & 4.8 & 8.5 & 15.7 & 44.1 & 43.4 & 32.0 & 32.4 & 30.5 \\
\hline 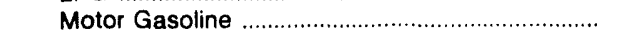 & 20.4 & 23.9 & 69.0 & 64.9 & 50.2 & 51.0 & 48.8 & 49.1 \\
\hline 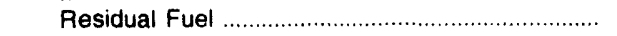 & 3.1 & 18.0 & 34.4 & 38.7 & 25.2 & 26.6 & 22.2 & 15.0 \\
\hline 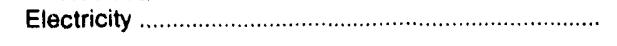 & 345.9 & 894.1 & $1,626.2$ & $3,103,3$ & $3,069.5$ & $3,110.4$ & $3,360.8$ & $3,522.8$ \\
\hline Industrial Sector & 233.9 & 678.0 & $2,056.5$ & $1,995.9$ & $1,697.4$ & $1,620.1$ & $1,665.4$ & $1,679.9$ \\
\hline 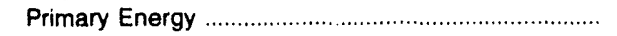 & 120.2 & 334.6 & $1,20 \% .9$ & $1,119.2$ & 885.8 & 830.2 & 832.4 & 816.9 \\
\hline 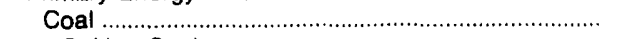 & - & .3 & 30.2 & 45.4 & 37.9 & 46.4 & 49.2 & 53.2 \\
\hline 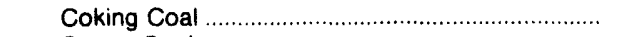 & - & - & - & - & - & - & - & - \\
\hline Steam Coal & - & .3 & 30.2 & 45.4 & 37.9 & 46.4 & 49.2 & 53.2 \\
\hline 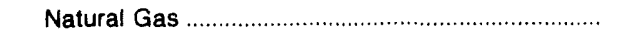 & 35.8 & 85.1 & 259.6 & 272.5 & 174.5 & 199.3 & 214.5 & 236.3 \\
\hline 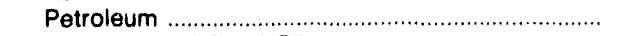 & 84.4 & 249.2 & 918.0 & 801.4 & 673.4 & 584.5 & 568.8 & 527.5 \\
\hline 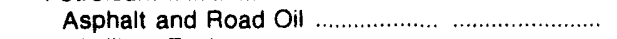 & 18.0 & 43.3 & 106.4 & 220.8 & 227.3 & 160.8 & 164.6 & 123.5 \\
\hline 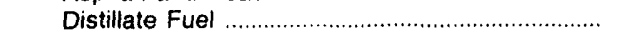 & 14.7 & 60.0 & 236.8 & 154.0 & 156.6 & 148.7 & 116.4 & 111.9 \\
\hline 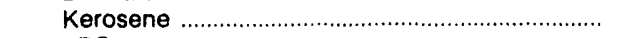 & 3.9 & 1.6 & 5.5 & 10.6 & .6 & .8 & 1.2 & 1.9 \\
\hline LPG & 4.4 & 11.5 & 107.2 & 96.1 & 94.3 & 67.8 & 76.2 & 72.6 \\
\hline 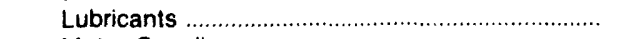 & 12.9 & 25.8 & 52.6 & 58.7 & 50.8 & 50.0 & 51.9 & 65.3 \\
\hline 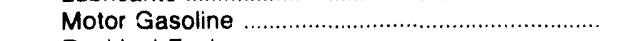 & 3.0 & 2.1 & 4.5 & 48.5 & 36.1 & 37.2 & 35.2 & 41.6 \\
\hline 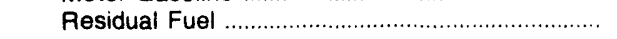 & 19.1 & 81.0 & 294.2 & 97.5 & 24.3 & 23.5 & 42.1 & 21.8 \\
\hline Other & 8.4 & 24.1 & 110.8 & 115.2 & 83.4 & 95.6 & 81.1 & 88.9 \\
\hline 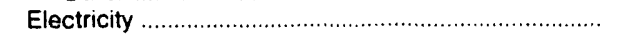 & 113.7 & 343.4 & 848.6 & 876.6 & 811.6 & 789.9 & 833.0 & 863.0 \\
\hline Transportation Sector & $1,321.5$ & $2,825.4$ & $7,939.7$ & $7,741.3$ & $6,100.9$ & $6,721.6$ & $7,016.7$ & $7,709.4$ \\
\hline 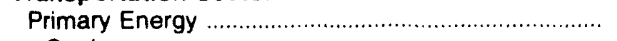 & $1,321.5$ & $2,825.4$ & $7,939.7$ & $7,740.0$ & $6,098.4$ & $6,718.9$ & $7,014.5$ & $7,707.2$ \\
\hline Coal & - & & - & - & - & - & - & - \\
\hline 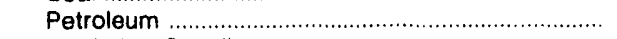 & $1,321.5$ & $2,825.4$ & $7,939.7$ & $7,740.0$ & $6,098.4$ & $6,718.9$ & $7,014.5$ & $7,707.2$ \\
\hline 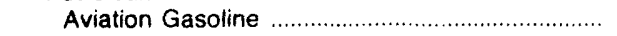 & 34.4 & 33.4 & 61.0 & 42.4 & 43.4 & 29.6 & 33.0 & 40.8 \\
\hline 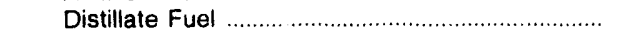 & 63.0 & 171.1 & 719.9 & 857.8 & 792.2 & 862.6 & 923.5 & $1,008.4$ \\
\hline 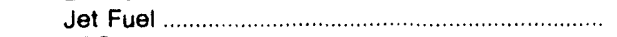 & 96.6 & 275.3 & $1,302.3$ & 762.5 & 587.3 & 604.7 & 685.3 & 835.0 \\
\hline LPG & .9 & 1.6 & 3.2 & 12.2 & 12.6 & 5.6 & 6.2 & 6.0 \\
\hline 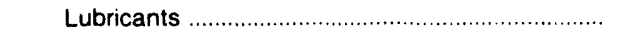 & 20.6 & 28.3 & 70.1 & 78.2 & 67.7 & 66.7 & 69.2 & 87.0 \\
\hline 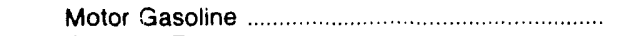 & $1,101.8$ & $2,293.5$ & $5,553.9$ & $5,834.1$ & $4,527.7$ & $5,044.1$ & $5,226.7$ & $5,640.8$ \\
\hline 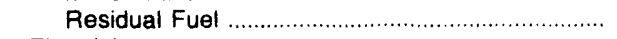 & 4.2 & 22.3 & 229.2 & 152.8 & 67.5 & 105.5 & 70.6 & 89.3 \\
\hline 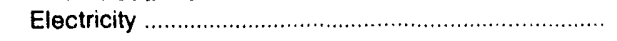 & - & - & - & 1.3 & 2.5 & 2.7 & 2.2 & 2.2 \\
\hline Total Energy" & $2,612.4$ & $6,019.9$ & $14,801.6$ & $18,248.3$ & $16,250.4$ & $16,933.8$ & $17,783.3$ & $18,863.8$ \\
\hline 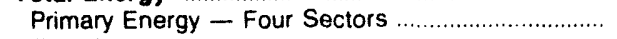 & $1,640.7$ & $3,487.0$ & $9,771.8$ & $9,700.3$ & $7,770.2$ & $8,346.9$ & $8,586.3$ & $9,202.9$ \\
\hline 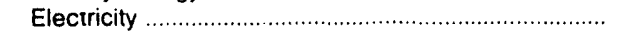 & 971.7 & $2,532.9$ & $5,029.8$ & $8,548.0$ & $8,480.2$ & $8,586.9$ & 9.197 .0 & 9.661 .0 \\
\hline Electric Utility Sector. & 196.0 & $1,114.2$ & $2,439.2$ & $2,244.7$ & $1,980.3$ & $2,256.9$ & $2,200.2$ & $2,433.8$ \\
\hline 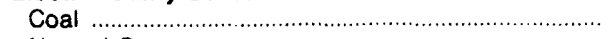 & 35.8 & 134.7 & 374.6 & 947.7 & 844.1 & $1,017.1$ & $1,038.9$ & $1,075.1$ \\
\hline 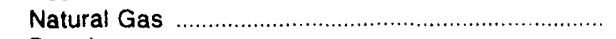 & 72.4 & 102.9 & 257.9 & 544.2 & 354.8 & 480.6 & 327.3 & 464.5 \\
\hline 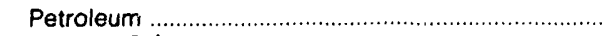 & 87.8 & 860.8 & $1,743.0$ & 587.7 & 608.6 & 620.6 & 568.9 & 745.8 \\
\hline 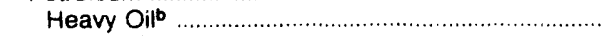 & 86.5 & 794.0 & $1,635.7$ & $54-2$ & 588.3 & 596.7 & 547.5 & 685.2 \\
\hline 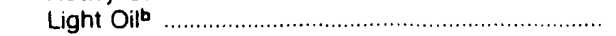 & 1.3 & 66.8 & 107.3 & 41.5 & 20.3 & 23.9 & 21.4 & 60.6 \\
\hline 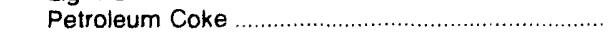 & - & - & - & - & - & - & - & - \\
\hline 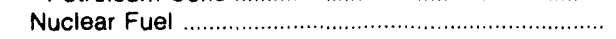 & - & 15.8 & 63.8 & 165.1 & 172.9 & 138.6 & 265.0 & 148.5 \\
\hline Wood and Waste & - & - & - & - & - & - & - & - \\
\hline 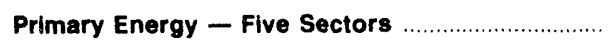 & $1,836.6$ & $4,601.2$ & $12,211.0$ & $11,945.0$ & $9,750.6$ & $10,603.9$ & $10,786.5$ & $11,636.8$ \\
\hline
\end{tabular}

"There are no direct fuel costs for hydroelectric, geothermal, centralized solar, or wind energy. Wood and other biomass fuels are not included, excep those consumed at electric utilities.

bHeavy oil includes Grade Nos. 4, 5, and 6, and residual fuel oils. Light oil includes Grade No. 2 heating oil, kerosene, and jet fuel

-No consumption, including cases where adjustments were made. See "Consumption Adjustments for Process Fuel and Intermediate Products."

* Value less than 0.05 million dollars

Note: Totals may not equal sum of components due to independent rounding.

Sources: Data sources, estimation procedures, and assumptions are described in the "Documentation" section of this report 


\begin{tabular}{|c|c|c|c|c|c|c|c|c|}
\hline Energy Source & 1970 & 1975 & 1980 & 1985 & 1986 & 1987 & 1988 & 1989 \\
\hline & \multicolumn{8}{|c|}{ Prices in Dollars per Million Btu } \\
\hline 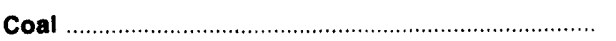 & 0.39 & 0.95 & 1.50 & 1.87 & 1.81 & 1.79 & 1.75 & 1.75 \\
\hline 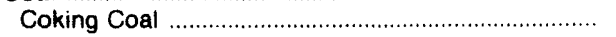 & - & - & - & - & - & - & - & - \\
\hline 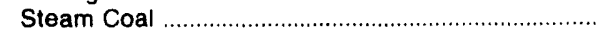 & .39 & .95 & 1.50 & 1.87 & 1.81 & 1.79 & 1.75 & 1.75 \\
\hline 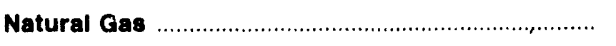 & .58 & 1.02 & 3.06 & 5.25 & 4.95 & 4.78 & 4.72 & 4.76 \\
\hline Petroleum . & 1.93 & 3.65 & 8.01 & 7.65 & 5.73 & 5.96 & 5.75 & 6.41 \\
\hline 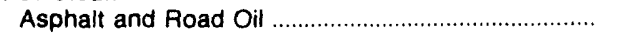 & .76 & 1.74 & 3.72 & 4.97 & 4.19 & 3.21 & 3.15 & 2.85 \\
\hline Aviation Gasoline & 2.17 & 3.45 & 9.02 & 9.99 & 8.41 & 7.55 & 7.41 & 8.28 \\
\hline 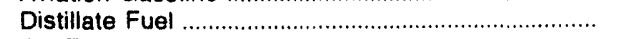 & 1.06 & 2.71 & 7.00 & 6.68 & 5.43 & 5.71 & 5.37 & 5.79 \\
\hline 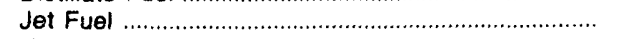 & .73 & 2.03 & 6.46 & 5.66 & 3.51 & 3.77 & 3.54 & 4.12 \\
\hline 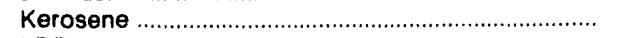 & .85 & 2.38 & 6.51 & 9.43 & 6.21 & 6.33 & 6.28 & 6.25 \\
\hline LPG & 2.01 & 3.58 & 6.40 & 9.48 & 8.83 & 8.77 & 8.37 & 9.11 \\
\hline 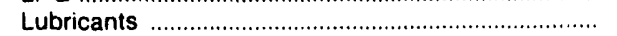 & 5.08 & 7.49 & 14.36 & 17.61 & 15.59 & 13.58 & 14.61 & 17.90 \\
\hline Motor Gasoline & 2.80 & 4.73 & 9.91 & 8.76 & 6.29 & 6.66 & 6.51 & 7.12 \\
\hline 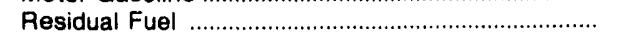 & .38 & 1.70 & 3.27 & 4.10 & 2.19 & 2.91 & 2.29 & 2.55 \\
\hline Other" & 1.99 & 3.05 & 8.42 & 8.88 & 5.79 & 6.61 & 5.62 & ع 36 \\
\hline Nuclear Fuel & - & .13 & .45 & .72 & .84 & .94 & 1.01 & 1.00 \\
\hline 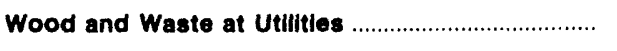 & - & - & - & - & - & - & - & - \\
\hline 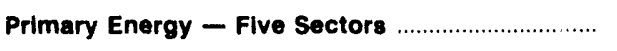 & 1.24 & 2.27 & 4.55 & 4.62 & 3.89 & 3.85 & 3.81 & 3.92 \\
\hline 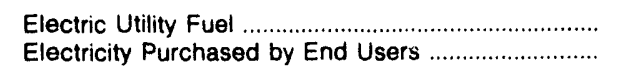 & $\begin{array}{r}.35 \\
4.58\end{array}$ & $\begin{array}{r}.91 \\
8.93\end{array}$ & $\begin{array}{r}1.38 \\
12.75\end{array}$ & $\begin{array}{r}1.73 \\
17.09\end{array}$ & $\begin{array}{r}1.71 \\
16.91\end{array}$ & $\begin{array}{r}1.63 \\
17.44\end{array}$ & $\begin{array}{r}1.61 \\
18.29\end{array}$ & $\begin{array}{r}1.53 \\
18.70\end{array}$ \\
\hline \multirow[t]{2}{*}{ Total Energyb } & 1.85 & 3.68 & 7.34 & 8.55 & 7.46 & 7.58 & 7.58 & 8.15 \\
\hline & \multicolumn{8}{|c|}{ Expenditures in Millions of Dollars } \\
\hline 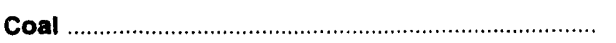 & 75.7 & 295.9 & 784.3 & $1,359.4$ & $1,249.4$ & $1,267.9$ & $1,220.7$ & $1,182.3$ \\
\hline Coking Coal & - & - & - & - & - & - & - & - \\
\hline Steam Coal & 75.7 & 295.9 & 784.3 & $1,359.4$ & $1,249.4$ & $1,267.9$ & $1,220.7$ & $1,182.3$ \\
\hline Natural Gas & 195.4 & 336.1 & 970.9 & $1,467.5$ & $1,370.2$ & $1,437.2$ & $1,508.1$ & $1,486.2$ \\
\hline 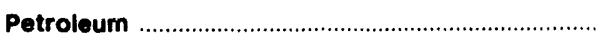 & $1,065.5$ & $2,389.1$ & $5,626.4$ & $5,683.2$ & $4,300.5$ & $4,686.0$ & $4,730.9$ & $5,111.2$ \\
\hline Asphalt and Road Oil & 19.7 & 48.4 & 118.2 & 151.2 & 156.7 & 127.4 & 126.4 & 93.9 \\
\hline Aviation Gasoline & 6.6 & 6.9 & 17.6 & 10.7 & 10.8 & 8.3 & 8.5 & 8.8 \\
\hline Distillate Fuel & 79.1 & $\therefore 4.0$ & 792.6 & 924.0 & 776.2 & 865.8 & 872.4 & 948.5 \\
\hline Jet Fuel & 42.8 & 147.4 & 598.1 & 518.0 & 351.4 & 419.2 & 405.8 & 405.7 \\
\hline Kerosene & 2.2 & 3.3 & 20.4 & 8.9 & 6.0 & 4.5 & 5.5 & 5.4 \\
\hline LPG & 56.5 & 108.2 & 175.0 & 234.9 & 208.9 & 197.1 & 209.0 & 255.2 \\
\hline 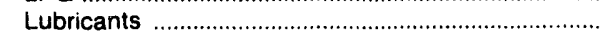 & 31.5 & 51.1 & 108.8 & 121.4 & 105.1 & 103.5 & 107.4 & 135.0 \\
\hline 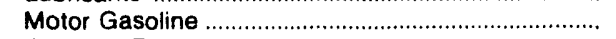 & 795.3 & $1,628.9$ & $3,409.4$ & $3,356.1$ & $2,543.8$ & 2.796 .6 & $=859.1$ & $3,122.4$ \\
\hline 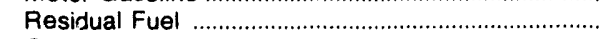 & 24.5 & 115.5 & 185.0 & 195.9 & 29.9 & 36.9 & 29.5 & 17.8 \\
\hline Other" & 7.3 & 25.3 & 201.3 & 162.0 & 111.7 & 126.8 & 107.3 & 118.5 \\
\hline 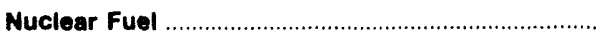 & - & 4.3 & 41.7 & 79.4 & 66.0 & 154.6 & 164.6 & 267.5 \\
\hline 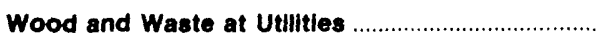 & - & - & - & - & - & - & - & - \\
\hline 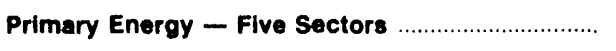 & $1,336.5$ & $3,025.4$ & $7,423.2$ & $8,589.4$ & $6,986.0$ & $7,545.7$ & $7,624.2$ & $8,047.2$ \\
\hline 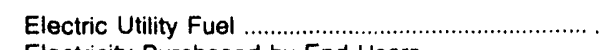 & -88.1 & -372.6 & -837.7 & $-1,379.9$ & $-1,256.5$ & $-1,347.1$ & $-1,305.3$ & $-1,372.2$ \\
\hline 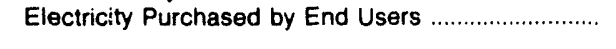 & 491.7 & $1,265.9$ & $2,227.3$ & $3,690.2$ & $3,916.7$ & $4,236.8$ & $4,608.9$ & $4,885.1$ \\
\hline (1) & $1,740.2$ & $3,918.7$ & $8,812.8$ & $10,809.7$ & $9,646.2$ & $10,435.4$ & $10,927.9$ & $11,560.1$ \\
\hline
\end{tabular}

Includes petroleum coke used at electric utilities.

bThere are no direct fuel costs for hydroelectric, geothermal, centralized solar, or wind energy. Wood and other biomass fuels are not included, except those consumed at electric utilities.

- No consumption, including cases where adjustments were made. See "Consumption Adjustments for Process Fuel and Intermediate Products."

Note: Expenditure totals may not equal sum of components due to indepondent rounding.

Sources: Data sources, estimation procedures, and assumptions are described in the "Documentation" section of this report. 


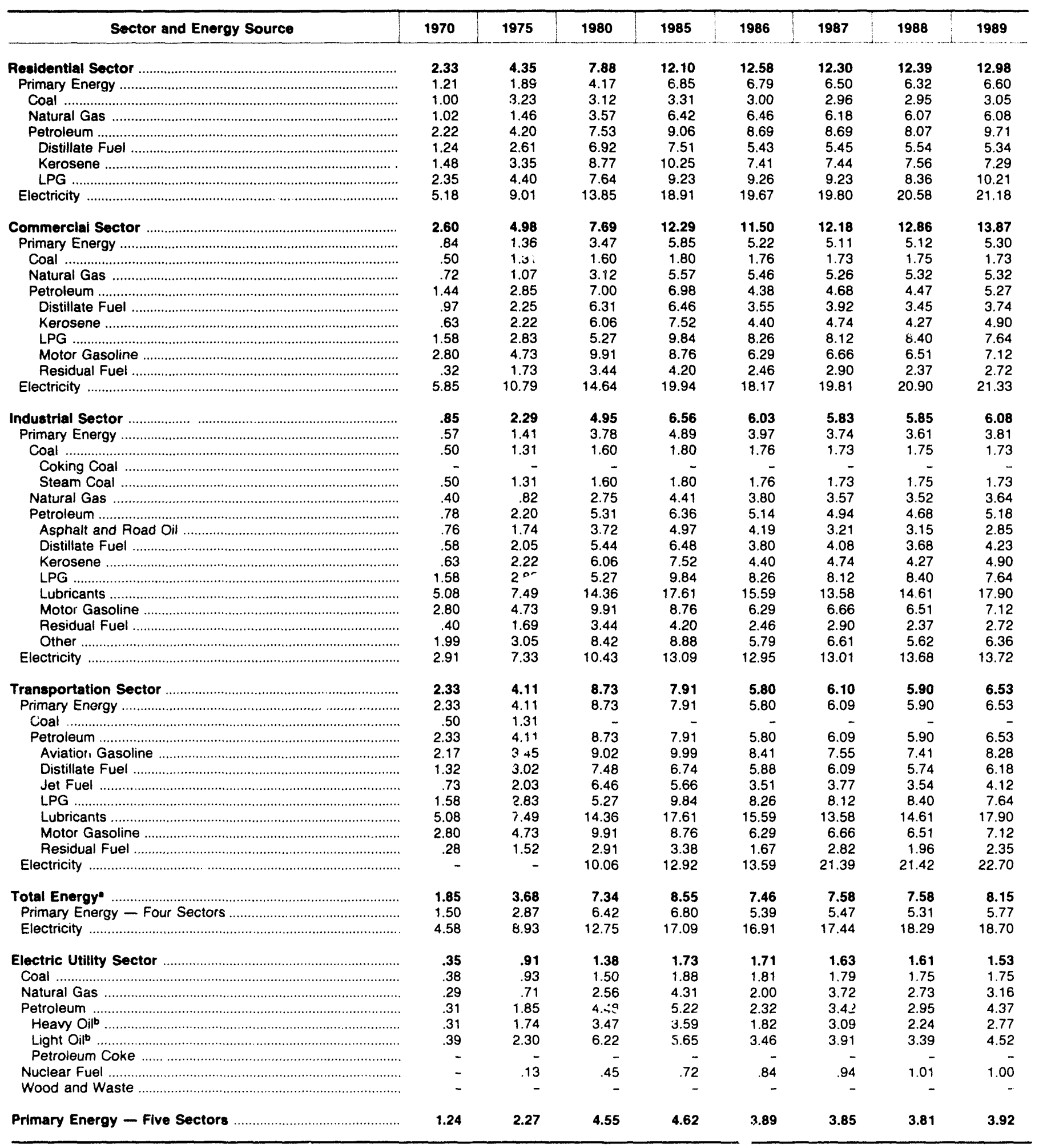

-There are no direct fuel costs for hydroelectric, geothermal, centralized solar, or wind energy. Wood and other biomass fuels are not included, except those consumed at electrir utilities.

Heaw oil includes Grade Nos. 4, 5, and 6, and residual fuel oils. Light oil includes Grade No. 2 heating oil, kerosene, and jet fuel.

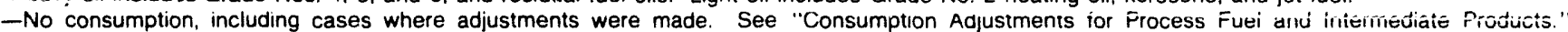

Sources: Data cources, estimation procedures, and assumptions are described in the "Documentation" section of this report. 
G Energy Expenditure Estimates by Sector, Georgia

E $1970,1975,1980$, and $1985-1989$

O (Million Dollars)

\begin{tabular}{|c|c|c|c|c|c|c|c|c|}
\hline Sector and Energy Source & 1970 & 1975 & 1980 & 1985 & 1986 & 1987 & 1988 & 1989 \\
\hline Residential Sector & 353.0 & 706.7 & $1,406.8$ & $2,230.0$ & $2,463.7$ & $2,598.1$ & $2,742.6$ & $2,871.3$ \\
\hline 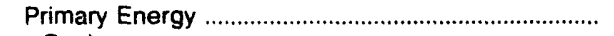 & 132.3 & 200.7 & 460.2 & 713.7 & 731.4 & 774.9 & 803.6 & 822.3 \\
\hline Coal & 1.1 & 1.4 & .6 & 1.1 & .2 & 1.0 & .7 & .3 \\
\hline 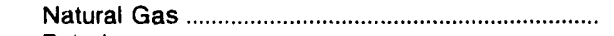 & 91.6 & 130.5 & 332.0 & 555.0 & 591.3 & 638.0 & 672.5 & 646.8 \\
\hline 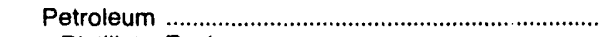 & 39.7 & 68.9 & 127.6 & 157.5 & 139.9 & 135.9 & 130.4 & 175.1 \\
\hline 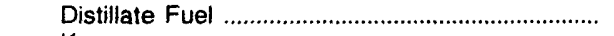 & 1.8 & 4.5 & 23.3 & 15.5 & 11.5 & 10.9 & 8.3 & 8.3 \\
\hline 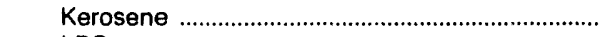 & 1.0 & .7 & 4.5 & 6.8 & 4.3 & 3.1 & 4.1 & 3.6 \\
\hline 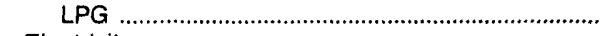 & 36.9 & 63.7 & 99.7 & 135.3 & 124.1 & 121.9 & 118.0 & 163.2 \\
\hline 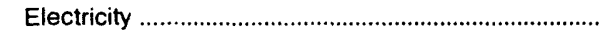 & 220.7 & 505.9 & 946.6 & $1,516.4$ & $1,732.3$ & $1,823.2$ & $1,939.0$ & $2,049.0$ \\
\hline Commerclal Sector & 206.7 & 497.0 & 830.4 & $1,561.0$ & $1,477.8$ & $1,677.3$ & $1,850.7$ & $1,971.6$ \\
\hline 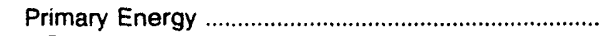 & 43.5 & 83.8 & 232.9 & 403.6 & 345.9 & 365.2 & 375.0 & 351.2 \\
\hline Coal & 1.0 & 1.0 & .6 & 1.1 & .2 & 1.0 & .8 & .4 \\
\hline 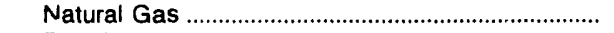 & 28.6 & 54.2 & 189.1 & 295.1 & 282.6 & 294.8 & 305.0 & 290.0 \\
\hline 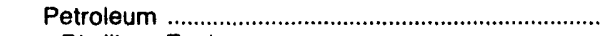 & 13.9 & 28.6 & 43.2 & 107.3 & 63.0 & 69.4 & 69.2 & 60.8 \\
\hline 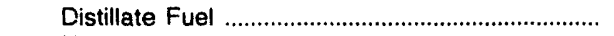 & 4.0 & 11.2 & 11.6 & 58.2 & 20.5 & 22.9 & 24.1 & 21.3 \\
\hline 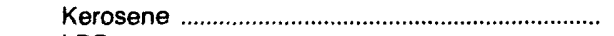 & .1 & .1 & .4 & .9 & .9 & .5 & .2 & 1.0 \\
\hline LPG & 4.4 & 7.2 & 12.1 & 25.5 & 19.5 & 18.9 & 20.9 & 21.6 \\
\hline 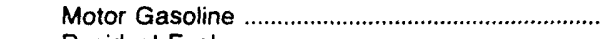 & 5.1 & 9.2 & 18.9 & 14.2 & 11.9 & 14.3 & 15.5 & 15.1 \\
\hline 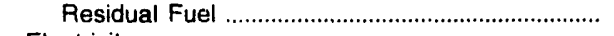 & .2 & .9 & .2 & 8.6 & 10.2 & 12.7 & 8.4 & 1.9 \\
\hline Electricity & 163.1 & 413.2 & 597.5 & $1,157.5$ & $1,131.9$ & $1,312.1$ & $1,475.7$ & $1,620.4$ \\
\hline Industrial Sector & 265.4 & 732.2 & $1,844.0$ & $2,499.9$ & $2,130.6$ & $2,185.6$ & $2,290.4$ & $2,349.0$ \\
\hline 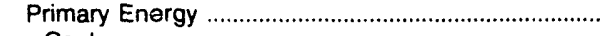 & 157.5 & 385.4 & $1,161.3$ & $1,486.0$ & $1,080.8$ & $1,087.8$ & $1,100.6$ & $1,137.9$ \\
\hline Coal & 6.0 & 13.3 & 26.5 & 69.5 & 77.8 & 84.1 & 91.6 & 88.0 \\
\hline 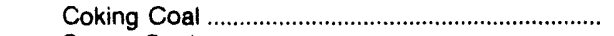 & - & - & - & - & - & - & & \\
\hline 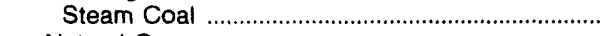 & 6.0 & 13.3 & 26.5 & 69.5 & 77.8 & 84.1 & 91.6 & 88.0 \\
\hline 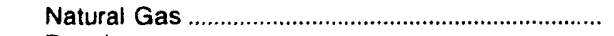 & 58.0 & 122.1 & 440.0 & 613.4 & 484.3 & 501.3 & 526.1 & 547.1 \\
\hline 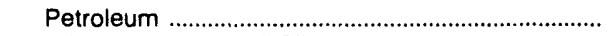 & 93.5 & 250.0 & 694.8 & 803.1 & 518.7 & 502.5 & 482.8 & 502.8 \\
\hline 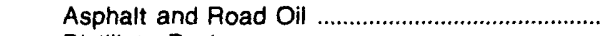 & 19.7 & 48.4 & 118.2 & 151.2 & 156.7 & 127.4 & 126.4 & 93.9 \\
\hline Distillate Fuel & 13.5 & 42.2 & 126.4 & 135.3 & 86.4 & 84.8 & 72.8 & 97.2 \\
\hline 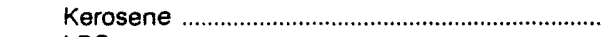 & 1.1 & 2.5 & 15.4 & 1.2 & .8 & .9 & 1.2 & .9 \\
\hline LPG & 14.5 & 36.2 & 61.7 & 68.4 & 60.9 & 53.0 & 66.4 & 67.7 \\
\hline Lubricants & 14.6 & 27.7 & 55.0 & 61.4 & 53.2 & 52.4 & 54.3 & 68.3 \\
\hline 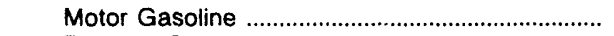 & 1.8 & 1.5 & 1.4 & 57.5 & 38.3 & 42.6 & 39.5 & 48.5 \\
\hline 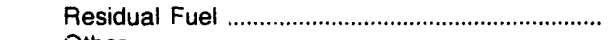 & 21.0 & 66.2 & 115.4 & 166.2 & 10.7 & 14.8 & 14.5 & 7.8 \\
\hline Other & 7.3 & 25.3 & 201.3 & 162.0 & 111.7 & 126.8 & 107.3 & 118.5 \\
\hline 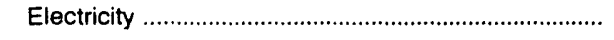 & 107.9 & 346.8 & 682.6 & $1,013.9$ & $1,049.8$ & $1,097.7$ & $1,189.8$ & $1,211.1$ \\
\hline 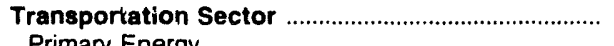 & 915.2 & $1,982.9$ & $4,731.6$ & $\begin{array}{l}4,608.7 \\
4,606.3\end{array}$ & $3,574.1$ & $3,974.5$ & $\begin{array}{l}4,044.1 \\
4039.8\end{array}$ & $4,368.2$ \\
\hline 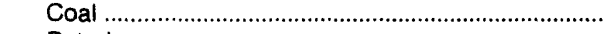 & & & & - & - & (20)- & & \\
\hline 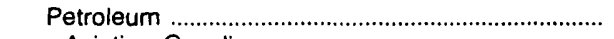 & 915.2 & $1,982.9$ & $4,731.1$ & $4,606.3$ & $3,571.4$ & $3,970.7$ & $4,039.8$ & $4,363.6$ \\
\hline 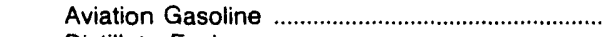 & 6.6 & 6.9 & 17.6 & 10.7 & 10.8 & 8.3 & 8.5 & 8.8 \\
\hline 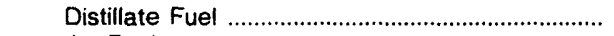 & 59.6 & 181.7 & 616.2 & 707.4 & 654.4 & 743.7 & 761.0 & 813.3 \\
\hline Jet Fuel & 42.8 & 147.4 & 598.1 & 518.0 & 351.4 & 419.2 & 405.8 & 405.7 \\
\hline LPG & .6 & 1.1 & 1.5 & 5.8 & 4.4 & 3.3 & 3.3 & 2.8 \\
\hline 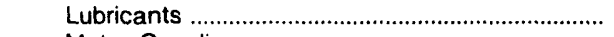 & 16.9 & 23.4 & 53.8 & 60.0 & 52.0 & 51.2 & 53.1 & 66.7 \\
\hline 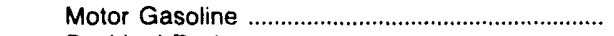 & 788.3 & $1,618.2$ & $3,389.1$ & $3,284.4$ & $2,493.6$ & $2,739.7$ & $2,804.1$ & $3,058.8$ \\
\hline 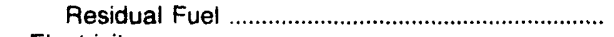 & .3 & 4.1 & 54.8 & 19.9 & 4.9 & 5.4 & 4.0 & 7.6 \\
\hline 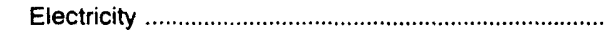 & - & - & .5 & 2.4 & 2.7 & 3.7 & 4.3 & 4.6̂ \\
\hline 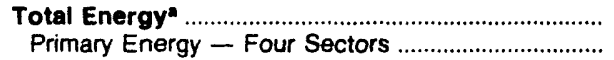 & $\begin{array}{l}1,740.2 \\
1,248.5\end{array}$ & $\begin{array}{l}3,918.7 \\
2,652.9\end{array}$ & $\begin{array}{l}8,812.8 \\
6,585.5\end{array}$ & $\begin{array}{r}10,899.7 \\
7,209.5\end{array}$ & $\begin{array}{l}9,646.2 \\
5,729.5\end{array}$ & $\begin{array}{r}10,435.4 \\
6,198.6\end{array}$ & $\begin{array}{r}10,927.9 \\
6,319.0\end{array}$ & $\begin{array}{r}11,560.1 \\
6,675.0\end{array}$ \\
\hline Electricity ... & 491.7 & $1,265.9$ & $2,227.3$ & $3,690.2$ & $3,916.7$ & $\begin{array}{l}0,1986.6 \\
4.236 .8\end{array}$ & $4,608.9$ & $4,885.1$ \\
\hline 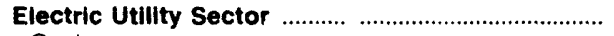 & 88.1 & 372.6 & 837.7 & $1,379.9$ & $1,256.5$ & $1,347.1$ & $1,305.3$ & $1,372.2$ \\
\hline Coal & 67.7 & 280.1 & 756.7 & $1,287.7$ & $1,171.2$ & $1,181.8$ & $1,127.6$ & $1,093.6$ \\
\hline Natural Gas & 17.3 & 29.3 & 9.7 & 3.9 & 11.9 & 3.1 & 4.4 & 2.2 \\
\hline Petroleum & 3.1 & 58.7 & 29.7 & 9.0 & 7.5 & 7.5 & 8.7 & 8.9 \\
\hline 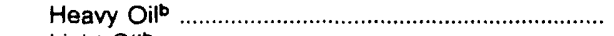 & 3.0 & 44.3 & 14.6 & 1.3 & 4.1 & 4.0 & 2.5 & .5 \\
\hline 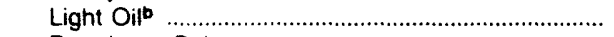 & .1 & 14.4 & 15.1 & 7.7 & 3.4 & 3.5 & 6.2 & 8.4 \\
\hline 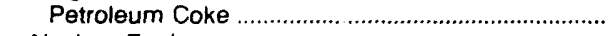 & - & - & 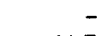 & - & - & - & - & \\
\hline 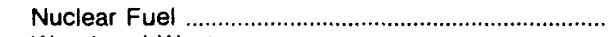 & - & 4.3 & 41.7 & 79.4 & 66.0 & 154.6 & 164.6 & 267.5 \\
\hline Wood and Waste & - & - & - & - & - & - & - & \\
\hline Primary Energy - Five Sector …………………...... & $1,336.5$ & $3,025.4$ & $7,423.2$ & $8,589.4$ & $6,986.0$ & $\mathbf{7 , 5 4 5 . 7}$ & $7,624.2$ & $8,047.2$ \\
\hline
\end{tabular}

-There are no direct fuel costs for hydroelectric, geothermal, centralized solar, or wind energy. Wood and other biomass fuels are not included, except those consumed at electric utilities.

bHeavy oil includes Grade Nos, 4, 5, and 6, and residual fuel oils. Light oil includes Grade No. 2 heating oil, kerosene, and jet fuel.

-No consumption, including cases where adjustments were made. See "Consumption Adjustments for Process Fuel and Intermediate Products."

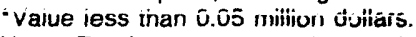

Note: Totals may not equal sum of components due to independent rounding.

Sources: Data sources, estimation procedures, and assumptions are described in the "Documentation" section of this report. 


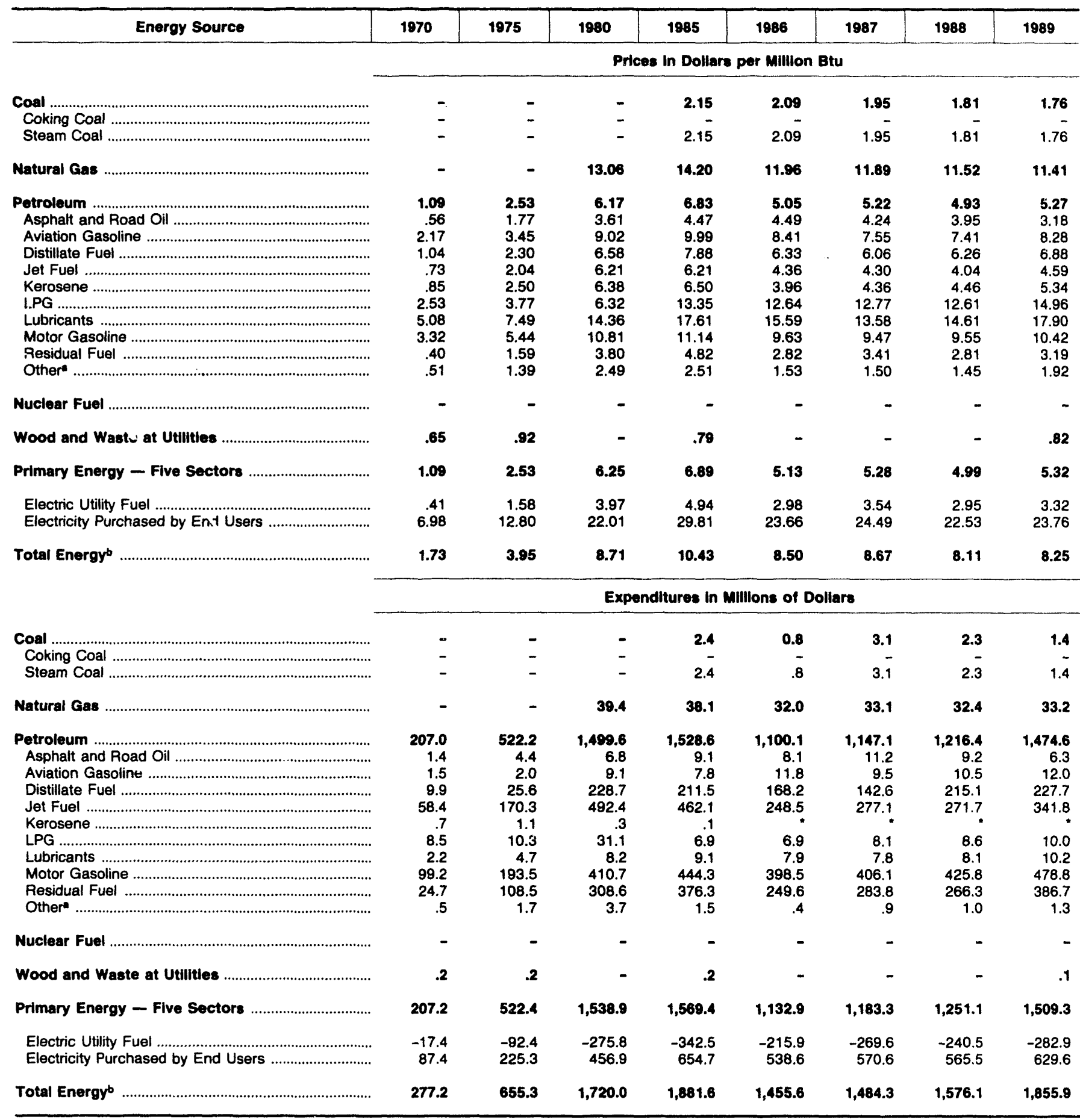

Includes petroleum coke used at electric utilities.

bThere are no direct fuel costs for hydroelectric, geothermal, centralized solar, or wind energy. Wood and other biomass fuels are not included, except those consumed at electric utilities.

- No consumption, including cases where adjustments were made. See "Consumption Adjustments for Process Fuel and Intermediate Products." "Value less than 0.05 million dollars.

Note: Expenditure totals may not equal sum of components due to independent rounding.

Sources: Data sources, estimation procedures, and assumptions are described in the "Documentation" section of this report. 
H Energy Price Estimates by Sector, Hawaii

A $1970,1975,1980$, and 1985-1989

W (Dollars per Million Btu)

\begin{tabular}{|c|c|c|c|c|c|c|c|c|}
\hline Sector and Energy Source & 1970 & 1975 & 1980 & 1985 & 1986 & 1987 & 1988 & 1989 \\
\hline Residential Sector & 7.07 & 13.13 & 20.08 & 30.92 & 25.50 & 25.82 & 24.31 & 25.71 \\
\hline Primary Energy & 4.11 & 6.19 & 12.48 & 16.14 & 14.53 & 14.47 & 14.15 & 15.65 \\
\hline 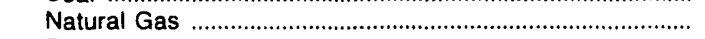 & - & - & 13.50 & 16.74 & 14.82 & 14.89 & 14.55 & 14.50 \\
\hline 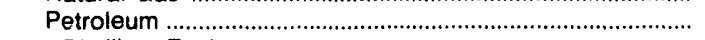 & 4.11 & 6.19 & 11.61 & 15.01 & 14.07 & 13.94 & 13.68 & 17.02 \\
\hline 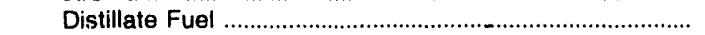 & 1.27 & 2.80 & 6.92 & 7.57 & 5.86 & 5.83 & 5.77 & 6.49 \\
\hline Kerosene & - & - & - & - & - & - & - & - \\
\hline LPG & 4.12 & 6.20 & 11.63 & 15.04 & 14.16 & 14.02 & 13.84 & 17.07 \\
\hline 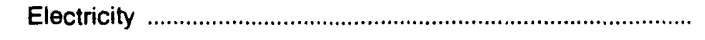 & 8.22 & 14.59 & 23.64 & 33.29 & 27.17 & 27.63 & 25.89 & 27.19 \\
\hline Commercial Sector & 5.65 & 12.66 & 17.85 & 25.97 & 20.26 & 15.30 & 11.67 & 8.58 \\
\hline Primary Energy & 1.55 & 3.37 & 8.75 & 11.28 & 8.50 & 5.90 & 4.67 & 4.02 \\
\hline Coal & - & - & - & - & - & - & - & - \\
\hline 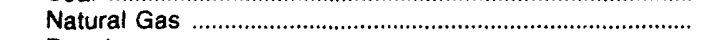 & - & - & 12.70 & 13.34 & 11.08 & 11.01 & 10.69 & 10.59 \\
\hline Petroleum & 1.55 & 3.37 & 6.60 & 7.67 & 5.37 & 4.19 & 3.56 & 3.46 \\
\hline 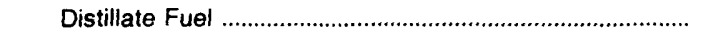 & 1.12 & 2.60 & 6.60 & 6.61 & 4.95 & 4.64 & 4.87 & 5.76 \\
\hline 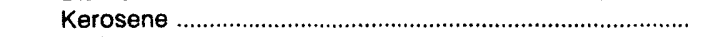 & .85 & 2.50 & - & 6.50 & 3.96 & 4.36 & 4.46 & 5.34 \\
\hline LPG & .91 & 1.91 & 3.81 & 9.62 & 9.06 & 8.52 & 8.58 & 8.09 \\
\hline 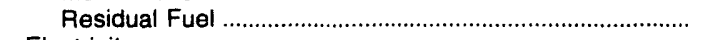 & .42 & 1.59 & 3.86 & 4.60 & 2.81 & 3.33 & 2.71 & 3.09 \\
\hline 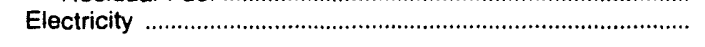 & 9.92 & 16.50 & 26.40 & 34.41 & 27.29 & 27.52 & 25.60 & 26.97 \\
\hline Industrial Sector & 1.55 & 4.80 & 8.67 & 15.45 & 9.81 & 10.93 & 10.46 & 10.48 \\
\hline 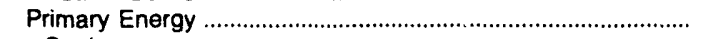 & .60 & 2.05 & 4.44 & 5.15 & 3.55 & 3.91 & 3.75 & 3.80 \\
\hline Coal & - & - & - & 2.15 & 2.09 & 1.95 & 1.81 & 1.76 \\
\hline 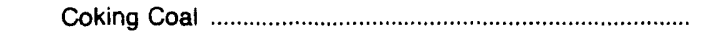 & - & - & - & - & - & - & - & - \\
\hline 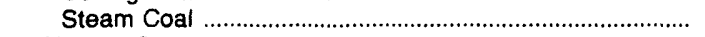 & - & - & - & $2 .: 5$ & 2.09 & 1.95 & 1.81 & 1.76 \\
\hline Natural Gas & - & - & - & - & - & - & - & - \\
\hline Petroleum & 60 & 2.05 & 4.44 & 5.56 & 3.59 & 4.19 & 3.97 & 3.91 \\
\hline 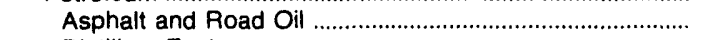 & .56 & 1.77 & 3.61 & 4.47 & 4.49 & 4.24 & 3.95 & 3.18 \\
\hline Distillate Fuel & .74 & 2.22 & 5.49 & 6.07 & 3.70 & 4.07 & 4.16 & 4.99 \\
\hline 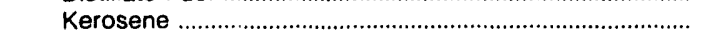 & .85 & 2.50 & 6.38 & 6.50 & 3.96 & 4.36 & 4.46 & 5.34 \\
\hline LPG & .91 & 1.91 & 3.81 & 9.62 & 9.06 & 8.52 & 8.58 & 8.09 \\
\hline 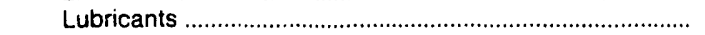 & 5.08 & 7.49 & 14.36 & 17.61 & 15.59 & 13.58 & 14.61 & 17.90 \\
\hline Motor Gasoline & 3.32 & 5.44 & 10.81 & 11.14 & 9.63 & 9.47 & 9.55 & 10.42 \\
\hline 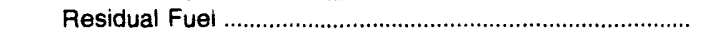 & .42 & 1.92 & 3.82 & 4.60 & 2.81 & 3.33 & 2.71 & 3.09 \\
\hline Other & .51 & 1.39 & 2.49 & 2.51 & 1.53 & 1.50 & 1.45 & 1.92 \\
\hline 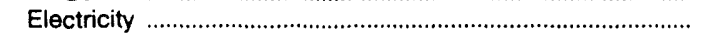 & 4.59 & 9.84 & 18.63 & 25.08 & 18.96 & 20.08 & 18.20 & 19.42 \\
\hline Coal . & - & - & - & - & - & - & - & - \\
\hline 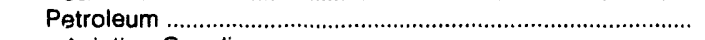 & 1.34 & 2.96 & 7.40 & 7.78 & 6.33 & 6.35 & 6.23 & 6.77 \\
\hline 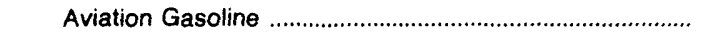 & 2.17 & 3.45 & 9.02 & 9.99 & 8.41 & 7.55 & 7.41 & 8.28 \\
\hline 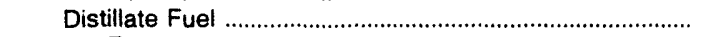 & 1.37 & 2.63 & 7.39 & 8.53 & 7.31 & 8.21 & 7.75 & 8.73 \\
\hline Jet Fuel & .73 & 2.04 & 6.21 & 6.21 & 4.36 & 4.30 & 4.04 & 4.59 \\
\hline LPG & .91 & 1.91 & 3.81 & 9.62 & 9.06 & 8.52 & 8.58 & 8.09 \\
\hline 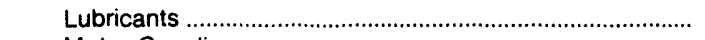 & 5.08 & 7.49 & 14.36 & 17.61 & 15.59 & 13.58 & 14.61 & 17.90 \\
\hline Motor Gasoline & 3.32 & 5.44 & 10.81 & 11.14 & 9.63 & 9.47 & 9.55 & 10.42 \\
\hline Residual Fuel & .37 & 1.37 & 3.27 & 4.64 & 2.62 & 3.18 & 2.50 & 2.81 \\
\hline 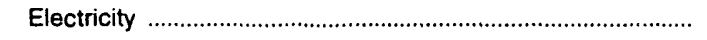 & - & - & - & - & - & - & - & - \\
\hline Total Energy & 1.73 & 3.95 & 8.71 & 10.43 & 8.50 & 8.67 & 8.11 & 8.25 \\
\hline 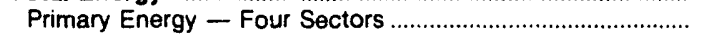 & 1.29 & 2.90 & 7.15 & 7.74 & 6.17 & 6.18 & 5.97 & 6.18 \\
\hline 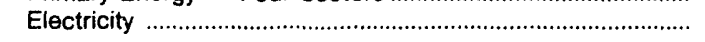 & 6.98 & 12.80 & 22.01 & 29.81 & 23.66 & 24.49 & 22.53 & 23.76 \\
\hline Electric Utility Sector & .41 & 1.58 & 3.97 & 4.94 & 2.98 & 3.54 & 2.95 & 3.32 \\
\hline Coal & - & - & - & - & - & - & - & - \\
\hline 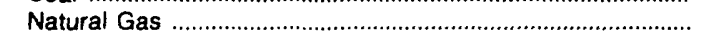 & - & - & - & - & - & - & - & - \\
\hline Petroleum & .40 & 1.58 & 3.97 & 4.95 & 2.98 & 3.54 & 2.95 & 3.32 \\
\hline 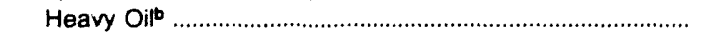 & .40 & 1.57 & 3.87 & 4.86 & 2.86 & 3.44 & 2.87 & 3.29 \\
\hline 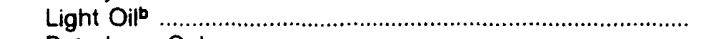 & .43 & 1.71 & 5.19 & 6.40 & 4.69 & 4.63 & 3.82 & 3.61 \\
\hline Petroleum Coke & - & - & - & - & - & - & - & - \\
\hline 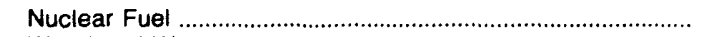 & - & - & - & - & - & - & - & - \\
\hline Wood and Waste & .65 & .92 & - & .79 & - & - & - & .82 \\
\hline 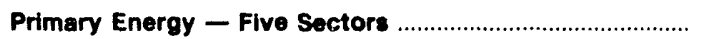 & 1.09 & 2.53 & 6.25 & 6.89 & 5.13 & 5.28 & 4.99 & 5.32 \\
\hline
\end{tabular}

- There are no direct fuel costs for hydroelectric, geothermal, centralized solar, or wind energy. Wood and other biomass fuels are not included, except those consumed at electric utilities.

bHeavy oil includes Grade Nos. 4, 5, and 6, and residual fuel oils. Light oil includes Grade No. 2 heating oil, kerosene, and jet fuel

-No consumption, including cases where adjustments were made. See "Consumption Adjustments for Process Fuel and Intermediate Products."

Sources: Data sources, estimation procedures, and assumptions are described in the "Documentation" section of this report 


\begin{tabular}{|c|c|c|c|c|c|c|c|c|}
\hline Sector and Energy Source & 1970 & 1975 & 1980 & 1985 & 1986 & 1987 & 1988 & 1989 \\
\hline Residential Sector & 43.0 & 90.1 & 185.3 & 230.1 & 196.7 & 211.7 & 206.1 & 225.6 \\
\hline 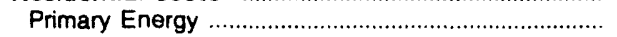 & 7.0 & 7.4 & 36.8 & 16.7 & 14.8 & 16.3 & 16.1 & 17.6 \\
\hline Coal & - & - & - & - & - & - & - & \\
\hline 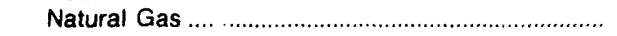 & - & - & 18.4 & 11.3 & 9.3 & 9.4 & 8.8 & 8.8 \\
\hline 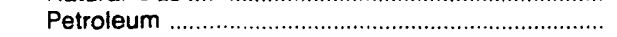 & 7.0 & 7.4 & 18.4 & 5.3 & 5.5 & 6.9 & 7.3 & 8.7 \\
\hline 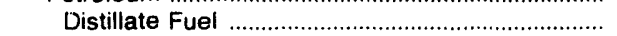 & • & $\because$ & ? & . & . & " & .1 & \\
\hline 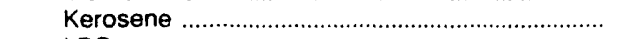 & - & - & - & - & - & - & - & - \\
\hline 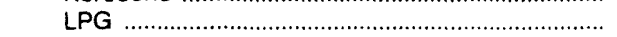 & 7.0 & 7.4 & 18.4 & 5.3 & 5.5 & 6.9 & 7.2 & 8.7 \\
\hline 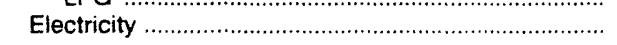 & 36.0 & 82.8 & 148.5 & 213.4 & 181.9 & 195.4 & 190.0 & 208.0 \\
\hline Commercial Sector & 30.3 & 67.7 & 172.7 & 225.0 & 202.2 & 233.1 & 246.8 & 316.9 \\
\hline 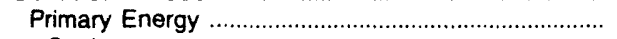 & 4.2 & 5.3 & 41.0 & 35.7 & 31.7 & 50.8 & 65.8 & 118.9 \\
\hline Coal & - & - & - & - & - & & - & \\
\hline 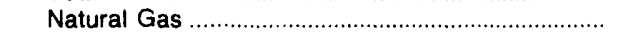 & - & - & 21.0 & 26.8 & 22.7 & 23.7 & 23.6 & 24.4 \\
\hline 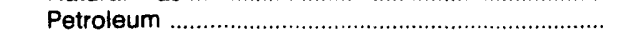 & 4.2 & 5.3 & 20.0 & 8.8 & 9.1 & 27.0 & 42.2 & 94.5 \\
\hline 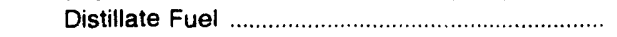 & 1.1 & 1.3 & 15.3 & 5.2 & 5.2 & 13.1 & 17.1 & 16.6 \\
\hline 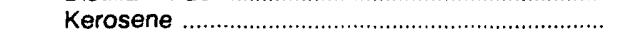 & .4 & .6 & - & .1 & . & * & ? & \\
\hline 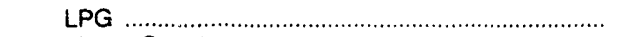 & .3 & .4 & 1.1 & .6 & .6 & .7 & .8 & .7 \\
\hline 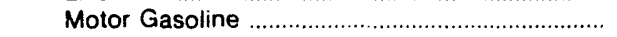 & 2.3 & 2.8 & 3.1 & 2.8 & 2.3 & 2.2 & 2.7 & 2.8 \\
\hline 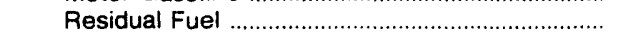 & .1 & .2 & .6 & .2 & .9 & 11.0 & 21.6 & 74.3 \\
\hline 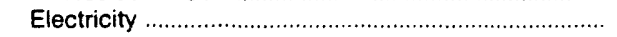 & 26.1 & 62.5 & 131.7 & 189.3 & 170.5 & 182.4 & 181.0 & 198.0 \\
\hline Industrial Sector & 36.0 & 110.9 & 275.7 & 300.3 & 237.2 & 241.8 & 240.7 & 282.0 \\
\hline 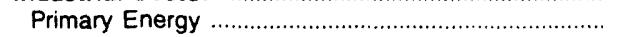 & 10.7 & 30.8 & 99.1 & 48.4 & 50.9 & 48.9 & 46.3 & 58.5 \\
\hline 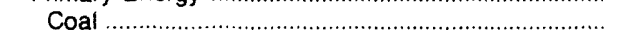 & - & - & - & 2.4 & .8 & 3.1 & 2.3 & 1.4 \\
\hline 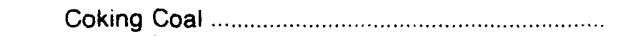 & - & - & $\sim$ & - & - & - & - & - \\
\hline 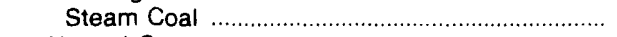 & - & - & - & 2.4 & .8 & 3.1 & 2.3 & 1.4 \\
\hline 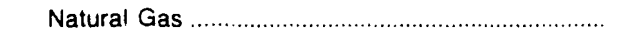 & - & - & - & - & - & - & - & \\
\hline 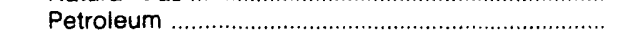 & 10.7 & 30.8 & 99.1 & 45.9 & 50.1 & 45.9 & 44.0 & 57.1 \\
\hline Asphalt and Road Oil ............................................ & 1.4 & 4.4 & 6.8 & 9.1 & 8.1 & 11.2 & 9.2 & 6.3 \\
\hline 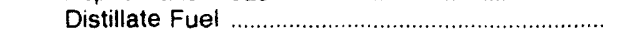 & 2.8 & 7.3 & 43.0 & 16.5 & 11,1 & 18.0 & 18.2 & 14.9 \\
\hline 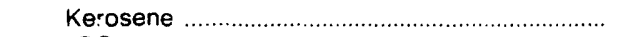 & .3 & .4 & .3 & * & • & & & \\
\hline 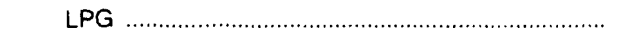 & 1.2 & 2.4 & 11.3 & .8 & .7 & .3 & .3 & .3 \\
\hline 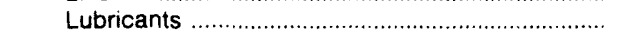 & .1 & 1.3 & 1.7 & 1.9 & 1.7 & 1.6 & 1.7 & 2.1 \\
\hline 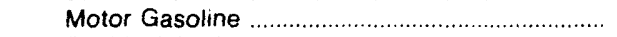 & .9 & 1.5 & 2.8 & 6.1 & 5.1 & 5.3 & 5.5 & 7.0 \\
\hline Residual Fuel ................................................ & 3.5 & 11.7 & 29.4 & 10.1 & 22.9 & 8.5 & 8.0 & 25.2 \\
\hline 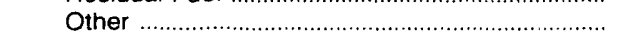 & .5 & 1.7 & 3.7 & 1.5 & .4 & .9 & 1.0 & 1.3 \\
\hline 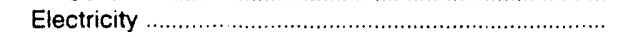 & 25.3 & 80.1 & 176.7 & 252.0 & 186.3 & 192.9 & 194.5 & 223.6 \\
\hline Transportation Sector & 167.9 & 386.6 & $1,086.3$ & $1,126.3$ & 819.6 & 797.7 & 882.4 & $1,031.4$ \\
\hline 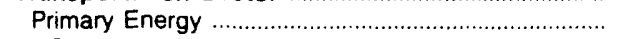 & 167.9 & 386.6 & $1,086.3$ & $1,126.3$ & 819.6 & 797.7 & 882.4 & $1,031.4$ \\
\hline 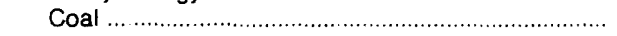 & & & & & & & & \\
\hline 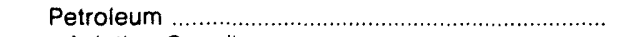 & 167.9 & 386.6 & $1,086.3$ & $1,126.3$ & 819.6 & 797.7 & 882.4 & $1,031.4$ \\
\hline Aviation Gasoline …………………………….... & 1.5 & 2.0 & 9.1 & 7.8 & 11.8 & 9.5 & 10.5 & 12.0 \\
\hline 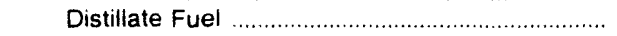 & 5.7 & 12.7 & 143.5 & 161.7 & 129.4 & 82.7 & 151.3 & 166.8 \\
\hline Jet Fuel & 58.4 & 170.3 & 492.4 & 462.1 & 248.5 & 277.1 & 271.7 & 341.8 \\
\hline 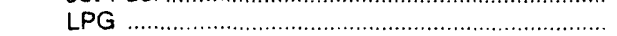 & 1 & .2 & .4 & .2 & .2 & .2 & .2 & 2 \\
\hline Lubricants ……… & 2.1 & 3.4 & 6.5 & 7.2 & 6.3 & 6.2 & 6.4 & 8.0 \\
\hline 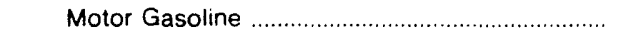 & 96.0 & 189.2 & 404.9 & 435.4 & 391.0 & 398.5 & 417.6 & 468.9 \\
\hline 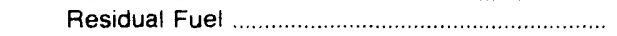 & 4.1 & 8.7 & 29.7 & 51.9 & 32.4 & 23.5 & 24.6 & 33.7 \\
\hline 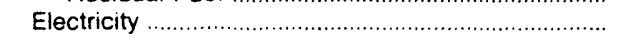 & - & - & - & - & - & - & - & - \\
\hline Total Energy" & 277.2 & 655.3 & $1,720.0$ & $1,881.6$ & $1,455.6$ & $1,484.3$ & $1,576.1$ & $1,855.9$ \\
\hline Primary Energy — Four Sectors .............................. & 189.8 & $\Delta 30.0$ & $1,263.2$ & $1,226.9$ & 917.0 & 913.7 & $1,010.6$ & $1,226.3$ \\
\hline 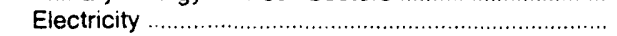 & 87.4 & 225.3 & 456.9 & 654.7 & 538.6 & 570.6 & 565.5 & 629.6 \\
\hline Electric Utility Sector & 17.4 & 92.4 & 275.8 & 342.5 & 215.9 & 269.6 & 240.5 & 282.9 \\
\hline Coal & & & - & & - & - & - & \\
\hline 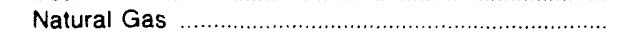 & - & - & - & - & - & - & - & - \\
\hline Petroleum & 17.2 & 92.2 & 275.8 & 342.3 & 215.9 & 269.6 & 240.5 & 282.8 \\
\hline 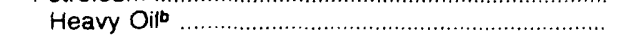 & 17.0 & 87.9 & 248.9 & 314.2 & 193.4 & 240.7 & 212.2 & 253.5 \\
\hline Light Oilb & .2 & 4.3 & 26.8 & 28.0 & 22.5 & 28.9 & 28.3 & 29.3 \\
\hline Petroleum Cuke ……………………………… & - & - & - & - & - & - & - & - \\
\hline 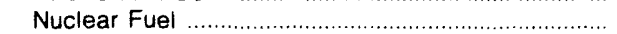 & - & - & - & - & - & - & - & - \\
\hline 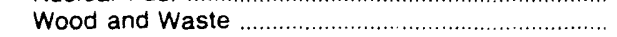 & .2 & .2 & - & .2 & - & - & - & .1 \\
\hline Primary Energy - Five Sectors & 207.2 & 522.4 & $1,538.9$ & $1,569.4$ & $1,132.9$ & $1,183.3$ & $1,251.1$ & $1,509.3$ \\
\hline
\end{tabular}

-There are no direct fuel costs for hydroelectric, geothermal, centralized solar, or wind energy. Wood and other biomass fuels are not included, except those consumed at electric utilities.

DHeavy oil includes Grade Nos. 4, 5, and 6, and residual fuel oils. Light oil includes Grade No. 2 heating oil, kerosene, and jet fuel.

-No consumption, including cases where adjustments were made. See "Consumption Adjustments for Process Fuel and Intermediate Products." - Value less than 0.05 million dollars.

Note: Totals may not equal sum of components due to independent rounding

Sources: Data sources, estimation procedures, and assumptions are described in the "Documentation" section of this report. 
I Energy Price and Expenditure Estimates by Source, Idaho
D 1970, 1975, 1980, and 1985-1989

A
$H$
$O$

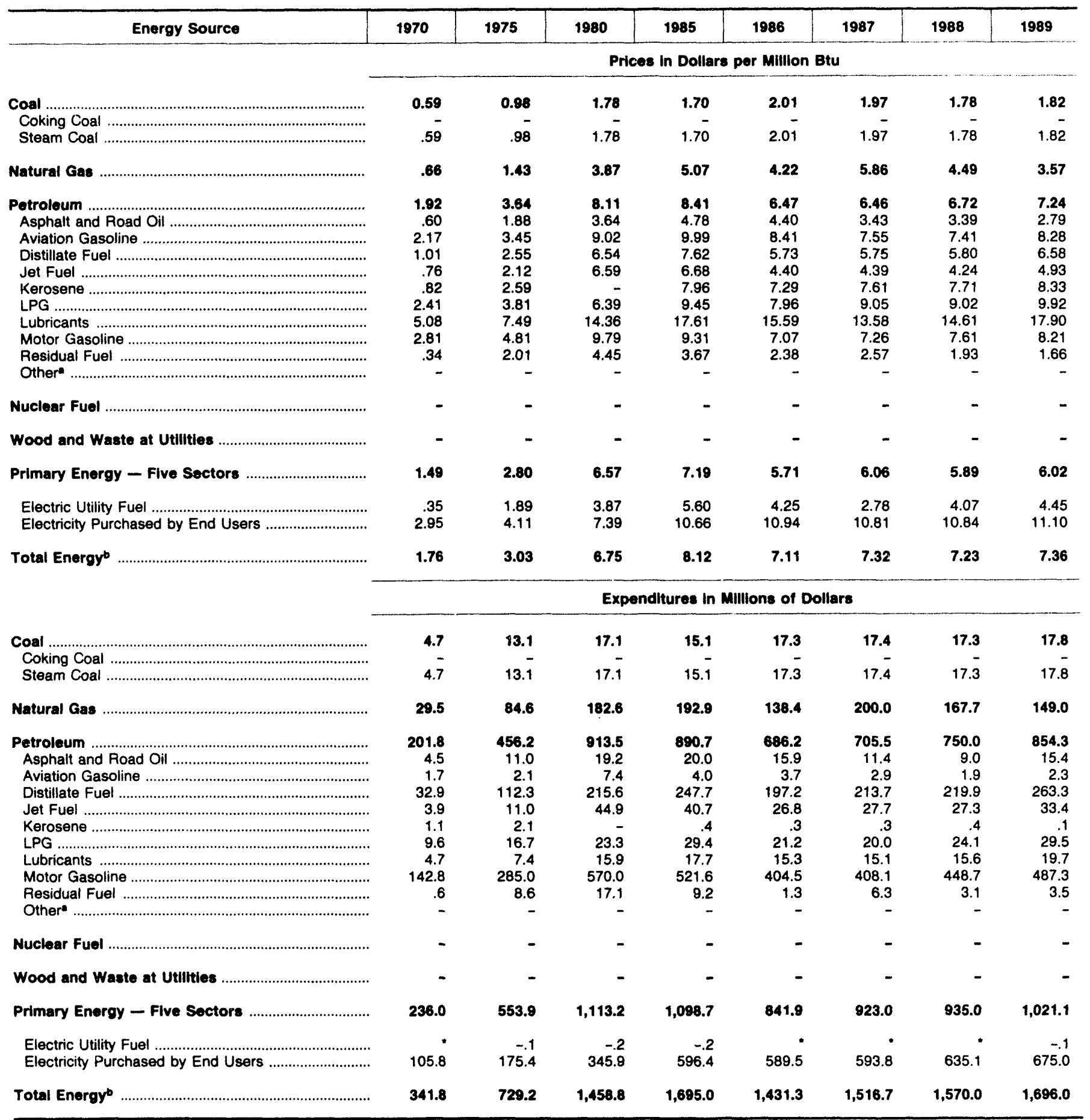

Includes petroleum coke used at electric utilities.

bThere are no direct fuel costs for hydroelectric, geothermal, centralized solar, or wind energy. Wood and other biomass fuels are not included, except those consumed at electric utilities.

-No consumption, including cases where adjustments were made. See "Consumption Adjustments for Process Fuel and Intermediate Products."

"Value less than 0.05 million dollars.

Note: Expenditure totals may not equal sum of components due to independent rounding.

Sources: Data sources, estimation procedures, and assumptions are described in the "Documentation" section of this report. 


\begin{tabular}{|c|c|c|c|c|c|c|c|c|}
\hline Sector and Energy Source & 1970 & 1975 & 1980 & 1985 & 1986 & 1987 & 1988 & 1989 \\
\hline 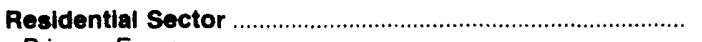 & 2.58 & 3.43 & 7.14 & 10.29 & 10.28 & 10.39 & 10.44 & 10.57 \\
\hline Primary Energy & 1.54 & 2.45 & 5.28 & 6.93 & 6.05 & 5.70 & 5.59 & 5.75 \\
\hline 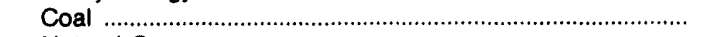 & .99 & 1.78 & 2.56 & 1.97 & 1.91 & 1.54 & 1.26 & 1.56 \\
\hline 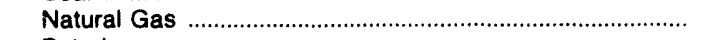 & 1.31 & 2.07 & 4.73 & 6.57 & 5.89 & 5.41 & 5.38 & 4.92 \\
\hline 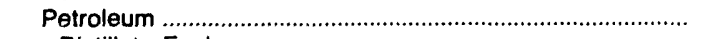 & 1.91 & 3.25 & 6.97 & 7.89 & 6.54 & 6.37 & 6.48 & 7.90 \\
\hline 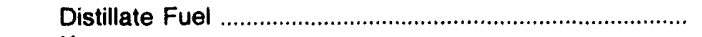 & 1.40 & 2.82 & 6.60 & 7.29 & 5.59 & 5.21 & 5.21 & 5.89 \\
\hline 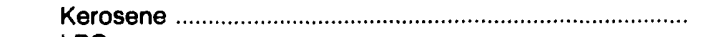 & - & - & - & 12.00 & 9.20 & 8.58 & 8.58 & 9.70 \\
\hline LPG & 2.83 & 4.17 & 7.85 & 9.55 & 9.70 & 10.64 & 10.38 & 12.49 \\
\hline Electricity & 4.81 & 5.27 & 8.54 & 12.60 & 13.14 & 13.45 & 13.88 & 14.11 \\
\hline 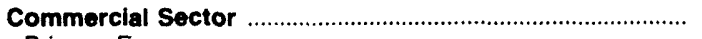 & 2.12 & 2.91 & 6.49 & 9.05 & 9.18 & 8.97 & 8.65 & 8.66 \\
\hline 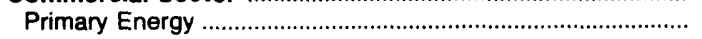 & .96 & 1.66 & 4.53 & 5.62 & 4.87 & 4.60 & 4.68 & 4.44 \\
\hline 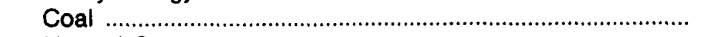 & .50 & .87 & 1.70 & 1.69 & 2.01 & 1.98 & 1.82 & 1.84 \\
\hline 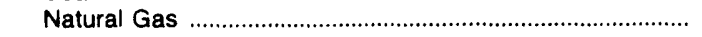 & .96 & 1.47 & 4.36 & 5.42 & 4.68 & 4.44 & 4.43 & 4.09 \\
\hline 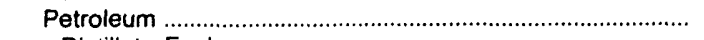 & 1.35 & 3.01 & 5.65 & 6.83 & 6.06 & 5.15 & 5.76 & 5.59 \\
\hline 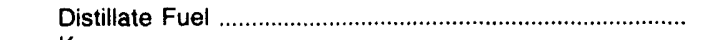 & 1.21 & 2.62 & 6.41 & 6.82 & 5.74 & 4.72 & 4.41 & 5.27 \\
\hline 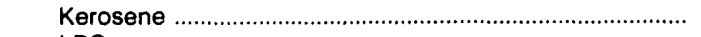 & .82 & 2.59 & - & 6.91 & 4.20 & 4.96 & 5.00 & 5.70 \\
\hline LPG & 1.55 & 3.25 & 5.72 & 9.38 & 6.76 & 7.98 & 7.99 & 7.58 \\
\hline Motor Gasoline & 2.81 & 4.81 & 9.79 & 9.31 & 7.07 & 7.26 & 7.61 & 8.21 \\
\hline Residual Fuel & - & - & 4.63 & 3.67 & 2.38 & 2.57 & 1.93 & 1.66 \\
\hline Electricity & 4.10 & 4.88 & 8.33 & 12.10 & 12.58 & 12.25 & 12.06 & 12.58 \\
\hline Industrial Sector & .96 & 1.97 & 4.53 & $\mathbf{5 . 6 5}$ & 4.99 & 5.73 & 5.15 & 4.86 \\
\hline 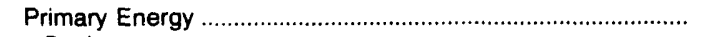 & .69 & 1.81 & 4.30 & 4.78 & 3.77 & 4.95 & 4.07 & 3.67 \\
\hline Coal & .50 & .87 & 1.70 & 1.69 & 2.01 & 1.98 & 1.82 & 1.84 \\
\hline 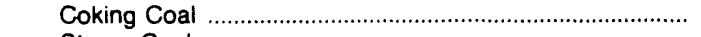 & - & - & - & - & - & - & - & - \\
\hline 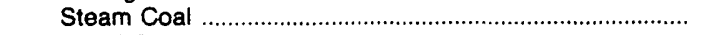 & .50 & .87 & 1.70 & 1.69 & 2.01 & 1.98 & 1.82 & 1.84 \\
\hline 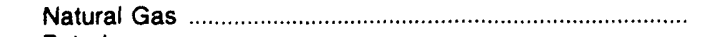 & .42 & 1.11 & 3.58 & 4.32 & 3.23 & 6.59 & 4.18 & 2.84 \\
\hline 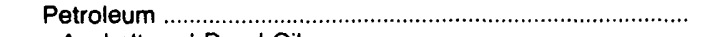 & .95 & 2.60 & 6.02 & 6.42 & 4.87 & 4.66 & 4.78 & 4.99 \\
\hline 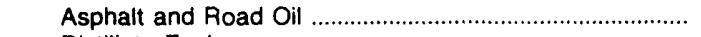 & .60 & 1.88 & 3.64 & 4.78 & 4.40 & 3.43 & 3.39 & 2.79 \\
\hline 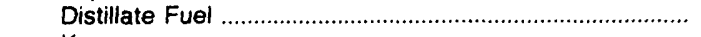 & .77 & 2.40 & 6.02 & 6.15 & 4.26 & 4.41 & 4.44 & 5.07 \\
\hline 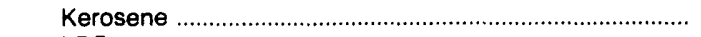 & .82 & 2.59 & - & 6.91 & 4.20 & 4.96 & 5.00 & 5.70 \\
\hline LPG & 1.55 & 3.25 & 5.72 & 9.38 & 6.76 & 7.98 & 7.99 & 7.58 \\
\hline 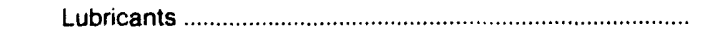 & 5.08 & 7.49 & 14.36 & 17.61 & 15.59 & 13.58 & 14.61 & 17.90 \\
\hline 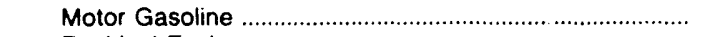 & 2.81 & 4.81 & 9.79 & 9.31 & 7.07 & 7.26 & 7.61 & 8.21 \\
\hline 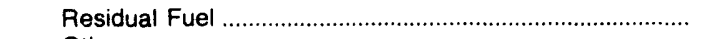 & .34 & 2.01 & 3.76 & 3.67 & 2.38 & 2.57 & 1.93 & 1.66 \\
\hline 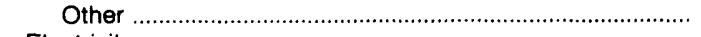 & - & - & - & - & - & - & - & - \\
\hline 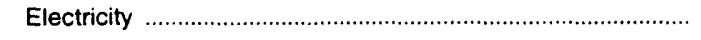 & 1.84 & 2.70 & 5.44 & 7.69 & 7.70 & 7.56 & 7.53 & 7.67 \\
\hline 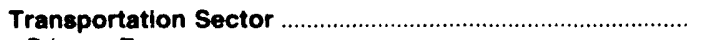 & 2.47 & 4.25 & 8.98 & 9.05 & 6.90 & 7.05 & 7.33 & 8.01 \\
\hline 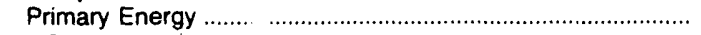 & 2.47 & 4.25 & 8.98 & 9.05 & 6.90 & 7.05 & 7.33 & 8.01 \\
\hline 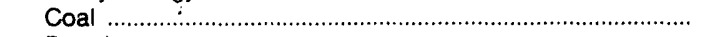 & .50 & .87 & - & - & - & - & - & - \\
\hline 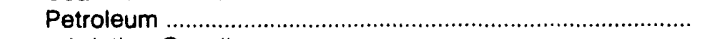 & 2.47 & 4.25 & 8.98 & 9.05 & 6.90 & 7.05 & 7.33 & 8.01 \\
\hline Aviation Gasoline & 2.17 & 3.45 & 9.02 & 9.99 & 8.41 & 7.55 & 7.41 & 8.28 \\
\hline 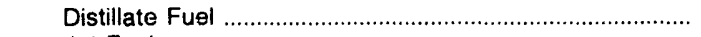 & 1.31 & 2.68 & 6.95 & 8.70 & 6.86 & 7.06 & 7.23 & 8.08 \\
\hline Jet Fuel . . & .76 & 2.12 & 6.59 & 6.68 & 4.40 & 4.39 & 4.24 & 4.93 \\
\hline LPG & 1.55 & 3.25 & 5.72 & 9.38 & 6.76 & 7.98 & 7.99 & 7.58 \\
\hline Lubricants & 5.08 & 7.49 & 14.36 & 17.61 & 15.59 & 13.58 & 14.61 & 17.90 \\
\hline Motor Gasoline & 2.81 & 4.81 & 9.79 & 9.31 & 7.07 & 7.26 & 7.61 & 8.21 \\
\hline 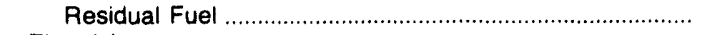 & .39 & - & - & - & - & - & - & - \\
\hline 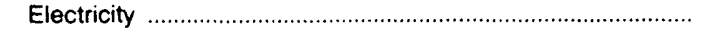 & - & - & - & - & - & - & - & - \\
\hline 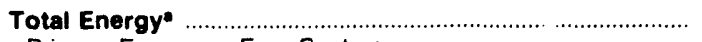 & 1.76 & 3.03 & 6.75 & 8.12 & 7.11 & 7.32 & 7.23 & 7.36 \\
\hline 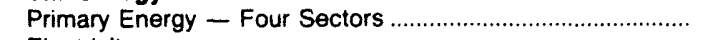 & 1.49 & 2.80 & 6.57 & 7.19 & 5.71 & 6.06 & 5.89 & 6.02 \\
\hline Electricity & 2.95 & 4.11 & 7.39 & 10.66 & 10.94 & 10.81 & 10.84 & 11.10 \\
\hline Electric Utility Sector & .35 & 1.89 & 3.87 & $\mathbf{5 . 6 0}$ & 4.25 & 2.78 & 4.07 & 4.45 \\
\hline 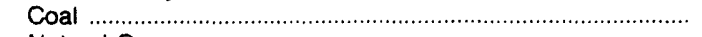 & - & - & - & - & - & - & - & - \\
\hline 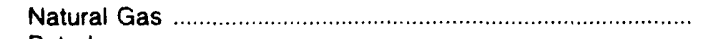 & - & 1.38 & 3.76 & 5.44 & 4.70 & 2.31 & $\overline{-}$ & - \\
\hline 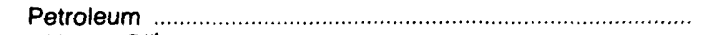 & .35 & 2.20 & 6.39 & 6.07 & 3.74 & 4.25 & 4.07 & 4.45 \\
\hline 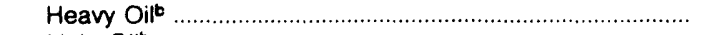 & - & - & - & - & - & - & - & - \\
\hline 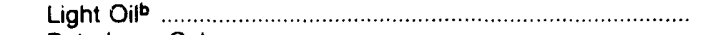 & .35 & 2.20 & 6.39 & 6.07 & 3.74 & 4.25 & 4.07 & 4.45 \\
\hline 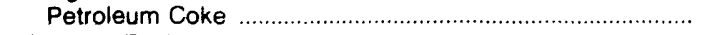 & - & - & - & - & - & - & - & - \\
\hline 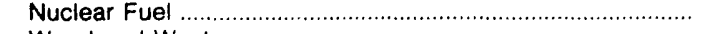 & - & - & - & - & - & - & - & - \\
\hline Wood and Waste & - & - & - & - & - & - & - & - \\
\hline 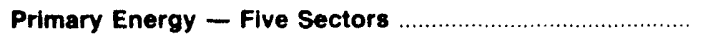 & 1.49 & 2.80 & 6.57 & 7.19 & 5.71 & 6.06 & 5.89 & 6.02 \\
\hline
\end{tabular}

- There are no direct fuel costs for hydroelectric, geothermal, centralized solar, or wind energy. Wood and other biomass fuels are not included, except those consumed at electric utilities.

-Heavy oil includes Grade Nos. 4, 5, and 6, and residual fuel oils. Light oil includes Grade No. 2 heating oil, kerosene, and jet fuel.

- No consumption, including cases where adjustments were made. See "Consumption Adjustments for Process Fuel and Intermediate Products.

Sources: Data sources, estimation procedures, and assumptions are described in the "Documentation" section of this report. 


\section{Energy Expenditure Estimates by Sector, Idaho \\ D $1970,1975,1980$, and $1985-1989$ \\ A (Million Dollars)}

H

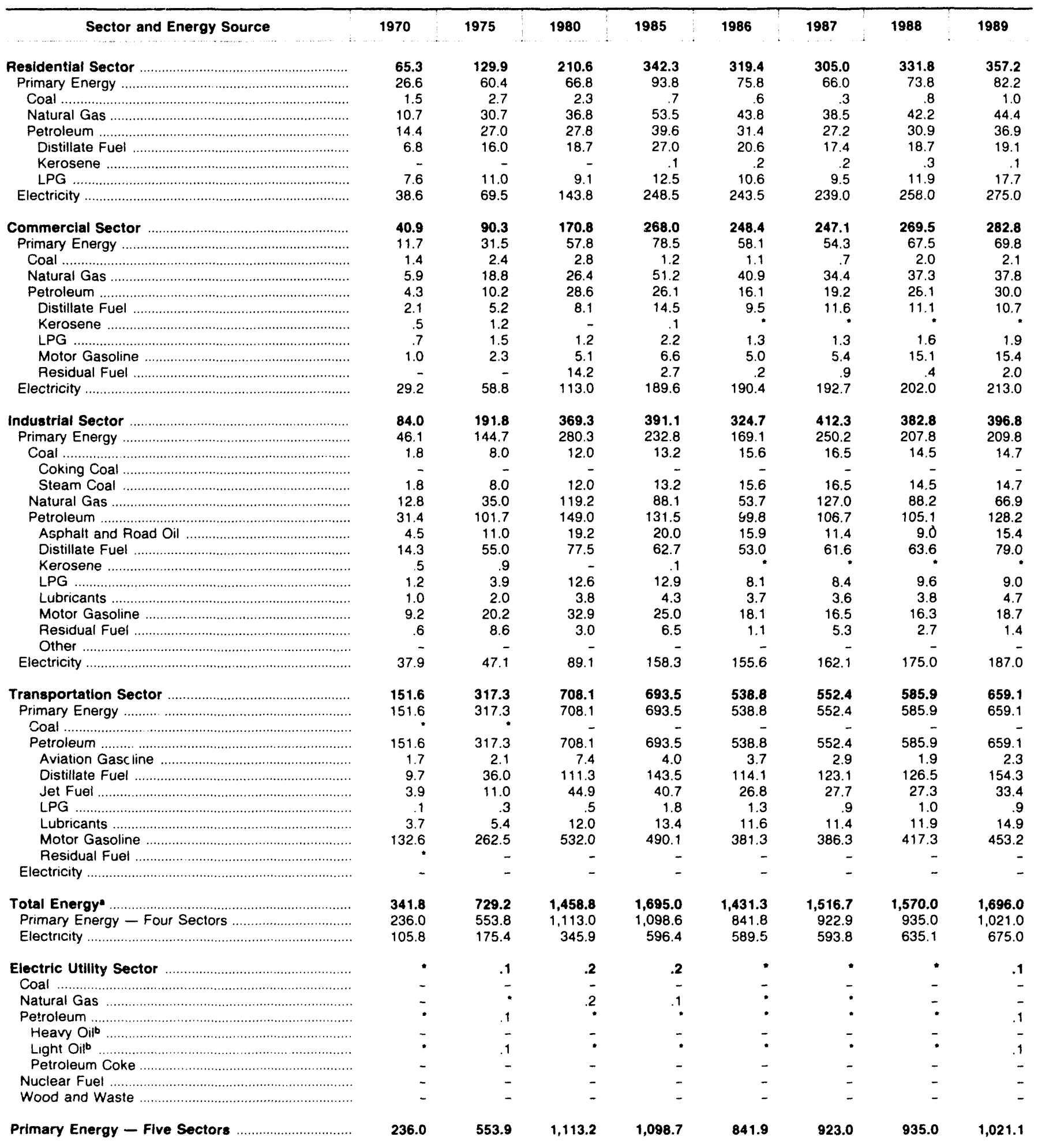

-There are no direct fuel costs for hydroelectric, geothermal, centralized solar, or wind energy. Wood and other biomass fuels are not included, except those consumed at electric utilities.

bHeavy oil includes Grade Nos. 4, 5, and 6, and residual fuel oils. Light oil includes Grade No. 2 heating oil, kerosene, and jet fuel.

- No consumption, including cases where adjustments were made. See "Consumption Adjustments for Process Fuel and Intermediate Products."

- Value less than 0.05 million dollars.

Note: Totals may not equal sum of components due to independent rounding

Sources: Data sources, estimation procedures, and assumptions are described in the "Documentation" section of this report 


\begin{tabular}{|c|c|c|c|c|c|c|c|c|}
\hline Energy Source & 1970 & 1975 & 1980 & 1985 & 1986 & 1987 & 1988 & 1989 \\
\hline & \multicolumn{8}{|c|}{ Prices in Dollars per Miliion Btu } \\
\hline $\begin{array}{l}\text { Coal } \\
\text { Coking Coal } \\
\text { Steam Coal }\end{array}$ & $\begin{array}{r}0.36 \\
.42 \\
.35\end{array}$ & $\begin{array}{r}0.89 \\
1.49 \\
.82\end{array}$ & $\begin{array}{l}1.64 \\
1.93 \\
1.63\end{array}$ & $\begin{array}{l}2.12 \\
2.08 \\
2.12\end{array}$ & $\begin{array}{l}2.06 \\
2.05 \\
2.06\end{array}$ & $\begin{array}{l}1.93 \\
1.92 \\
1.93\end{array}$ & $\begin{array}{l}1.83 \\
2.02 \\
1.82\end{array}$ & $\begin{array}{l}1.77 \\
1.92 \\
1.75\end{array}$ \\
\hline 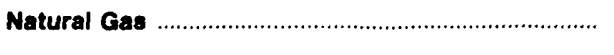 & .72 & 1.38 & 3.33 & 5.00 & 4.59 & 4.36 & 4.10 & 4.41 \\
\hline $\begin{array}{l}\text { Petroleum } \\
\text { Asphalt and Road Oil } \\
\text { Aviation Gasoline } \\
\text { Distillate Fuel } \\
\text { Jet Fuel } \\
\text { Kerosene } \\
\text { LPG } \\
\text { Mubricants } \\
\text { Retor Gasoline } \\
\text { Other }\end{array}$ & $\begin{array}{r}1.87 \\
.64 \\
2.17 \\
1.11 \\
.74 \\
1.13 \\
1.39 \\
5.08 \\
3.05 \\
.60 \\
1.33\end{array}$ & $\begin{array}{l}3.39 \\
1.99 \\
3.45 \\
2.58 \\
2.09 \\
2.82 \\
2.68 \\
7.49 \\
4.73 \\
1.68 \\
2.36\end{array}$ & $\begin{array}{r}7.67 \\
3.83 \\
9.02 \\
6.88 \\
6.38 \\
6.67 \\
5.16 \\
14.36 \\
9.81 \\
4.92 \\
6.75\end{array}$ & $\begin{array}{r}8.47 \\
4.91 \\
9.99 \\
7.62 \\
6.00 \\
8.42 \\
8.71 \\
17.61 \\
9.03 \\
5.07 \\
7.67\end{array}$ & $\begin{array}{r}6.49 \\
4.57 \\
8.41 \\
5.75 \\
4.22 \\
5.66 \\
7.24 \\
15.59 \\
6.78 \\
3.92 \\
5.65\end{array}$ & $\begin{array}{r}6.69 \\
3.34 \\
7.55 \\
6.00 \\
4.21 \\
6.07 \\
6.86 \\
13.58 \\
7.23 \\
3.26 \\
6.10\end{array}$ & $\begin{array}{r}6.68 \\
3.51 \\
7.41 \\
5.97 \\
3.84 \\
6.14 \\
6.94 \\
14.61 \\
7.27 \\
2.62 \\
5.13\end{array}$ & $\begin{array}{r}7.31 \\
2.92 \\
8.28 \\
6.67 \\
4.32 \\
6.80 \\
7.44 \\
17.90 \\
8.00 \\
2.79 \\
5.95\end{array}$ \\
\hline 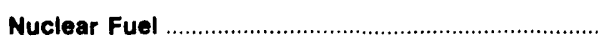 & .15 & .18 & .33 & .64 & .64 & .64 & .66 & .60 \\
\hline Wood and Waste at Utilities & .65 & - & - & - & - & - & - & - \\
\hline Primary Energy - Five Sectors & 1.08 & 2.00 & 4.32 & 4.86 & 4.06 & 3.97 & 3.76 & 3.89 \\
\hline $\begin{array}{l}\text { Electric Utility Fuel ... } \\
\text { Electricity Purchased by End Users }\end{array}$ & $\begin{array}{r}.32 \\
5.98\end{array}$ & $\begin{array}{r}.69 \\
9.35\end{array}$ & $\begin{array}{r}1.60 \\
15.33\end{array}$ & $\begin{array}{r}1.67 \\
21.07\end{array}$ & $\begin{array}{r}1.61 \\
22.37\end{array}$ & $\begin{array}{r}1.42 \\
22.86\end{array}$ & $\begin{array}{r}1.23 \\
21.49\end{array}$ & $\begin{array}{r}1.13 \\
22.07\end{array}$ \\
\hline \multirow[t]{2}{*}{ 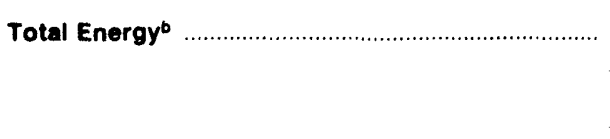 } & 1.69 & 3.15 & 6.69 & 8.44 & 7.75 & 7.95 & 7.57 & 7.99 \\
\hline & \multicolumn{8}{|c|}{ Expenditures in Millions of Dollars } \\
\hline 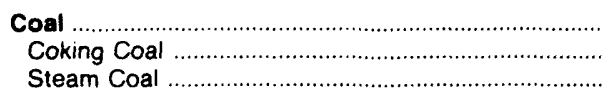 & $\begin{array}{r}329.4 \\
41.6 \\
287.8\end{array}$ & $\begin{array}{l}750.5 \\
120.7 \\
629.8\end{array}$ & $\begin{array}{r}1,388.1 \\
93.7 \\
1,294.5\end{array}$ & $\begin{array}{r}1,720.0 \\
131.6 \\
1,588.3\end{array}$ & $\begin{array}{r}1,619.4 \\
88.3 \\
1,531.1\end{array}$ & $\begin{array}{r}1,459.3 \\
80.5 \\
1,378.8\end{array}$ & $\begin{array}{r}1,319.7 \\
91.0 \\
1,228.7\end{array}$ & $\begin{array}{r}1,261.3 \\
131.2 \\
1,130.1\end{array}$ \\
\hline Natural Gas & 831.7 & $1,512.9$ & $3,601.8$ & $4,873.0$ & $4,234.0$ & $3,771.4$ & $3,933.5$ & $4,358.8$ \\
\hline $\begin{array}{l}\text { Petroleum } \\
\text { Asphalt and Road Oil ... } \\
\text { Aviation Gasoline } \\
\text { Distillate Fuel } \\
\text { Jet Fuel } \\
\text { Kerosene } \\
\text { LPG } \\
\text { Mobricants } \\
\text { Residual Fuel } \\
\text { Other }\end{array}$ & $\begin{array}{r}2,596.3 \\
53.8 \\
2.9 \\
287.9 \\
95.2 \\
22.9 \\
148.4 \\
100.4 \\
1.715 .3 \\
89.2 \\
80.3\end{array}$ & $\begin{array}{r}5,050.5 \\
135.0 \\
1.4 \\
770.9 \\
292.9 \\
41.9 \\
329.8 \\
141.7 \\
2,945.6 \\
223.0 \\
168.3\end{array}$ & $\begin{array}{r}10,365.9 \\
205.7 \\
6.0 \\
1,464.6 \\
710.2 \\
22.9 \\
702.6 \\
302.5 \\
5,622.7 \\
764.2 \\
564.5\end{array}$ & $\begin{array}{r}9,071.0 \\
244.5 \\
10.7 \\
1.427 .6 \\
92.2 \\
54.8 \\
1,042.7 \\
337.5 \\
5,272.2 \\
176.5 \\
412.2\end{array}$ & $\begin{array}{r}7,092.3 \\
187.4 \\
8.9 \\
1,174.5 \\
48.4 \\
13.1 \\
946.7 \\
292.1 \\
3,869.4 \\
194.6 \\
357.3\end{array}$ & $\begin{array}{r}7,444.1 \\
140.1 \\
6.1 \\
1,191.9 \\
46.8 \\
9.2 \\
1,039.2 \\
287.7 \\
4,186.1 \\
129.4 \\
407.6\end{array}$ & $\begin{array}{r}7,729.7 \\
130.4 \\
7.0 \\
1,169.7 \\
85.3 \\
11.0 \\
1,155.9 \\
298.6 \\
4,436.3 \\
86.6 \\
349.0\end{array}$ & $\begin{array}{r}7,744.2 \\
156.0 \\
8.0 \\
1,342.8 \\
109.3 \\
15.3 \\
424.6 \\
375.1 \\
4,852.9 \\
70.9 \\
389.2\end{array}$ \\
\hline 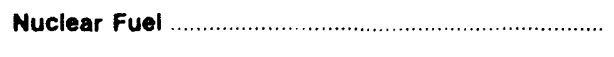 & 4.1 & 45.2 & 99.4 & 270.5 & 294.6 & 347.5 & 488.7 & 484.5 \\
\hline Wood and Waste at Utilities & - & - & - & - & - & - & - & - \\
\hline Primary Energy - Five Sectors & $3,761.6$ & $7,359.1$ & $15,455.2$ & $15,934.4$ & $13,240.3$ & $13,022.4$ & $13,471.7$ & $13,848.8$ \\
\hline $\begin{array}{l}\text { Electric Utility Fuel } \\
\text { Electricity Purchased by End Users }\end{array}$ & $\begin{array}{r}-254.5 \\
1,417.5\end{array}$ & $\begin{array}{r}-689.6 \\
2,644.9\end{array}$ & $\begin{array}{r}-1,794.2 \\
4,948.6\end{array}$ & $\begin{array}{r}-1,856.1 \\
7,063.0\end{array}$ & $\begin{array}{r}-1,849.5 \\
7,689.4\end{array}$ & $\begin{array}{r}-1,678.7 \\
8,095.9\end{array}$ & $\begin{array}{r}-1,638.8 \\
7,995.8\end{array}$ & $\begin{array}{r}-1,550.4 \\
8,157.9\end{array}$ \\
\hline 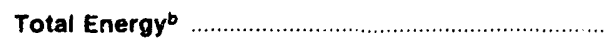 & $4,924.5$ & $9,314.3$ & $18,609.5$ & $21,141.3$ & $19,080.2$ & $19,439.7$ & $19,828.7$ & $20,456.2$ \\
\hline
\end{tabular}

- includes petroleum coke used at electric utilities.

bThere are no direct fuel costs for hydroelectric, geothermal, centralized solar, or wind energy. Wood and other biomass fuels are not included, except those consumed at electric utilities.

-No consumption, including cases where adjustments were made. See "Consumption Adjustments for Process Fuel and intermediate Products." - Value less than 0.05 million dollars.

Note: Expenditure totals may not equal sum of components due to independent rounding.

Sources: Data sources, estimation procedures, and assumptions are described in the "Documentation" section of this report. 


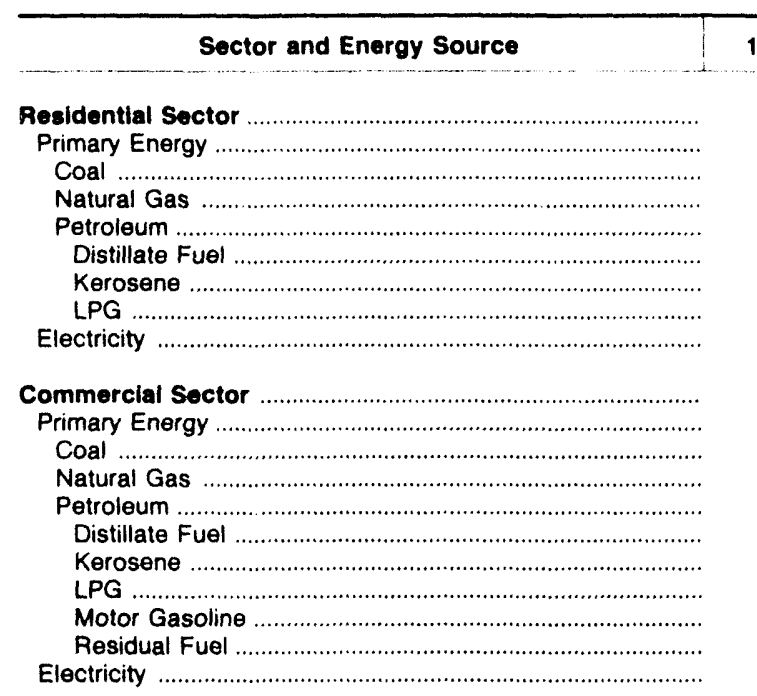
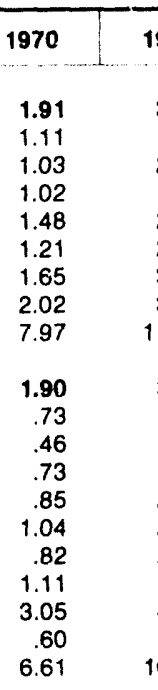

Industrial Sector

Primary Energy

Coal

Coking Coal

Steam Coal

Natural Gas

Petroleum

Asphalt and Road Oil

Distillate Fuel

Kerosene

LPG

Lubricants

Motor Gasoline

Residual Fuel

Other

Electricity .

Transportation Sector

Primary Energy ...

Coal

Petroleum

Aviation Gasoline

Distillate Fue

Jet Fue

Lubricants

Motor Gasoline

Residual Fuel

Electricity

Total Energy*

Primary Energy - Four Sectors

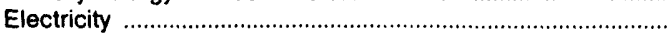

Electric Utility Sector

Coal

Natural Gas

Natural

Petroleum

Heavy Oilb

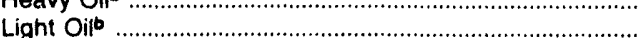

Petroleum Coke

Nuclear Fuel

Wood and Waste

Primary Energy - Five Sectors
3.06

1.91

1.11

.03

48

21

.65

.02
7.97

1.90

1.90
.73

43

.46

.73

1.04

1.11

3.05

.60
6.61

$$
1.83
$$$$
2.11
$$$$
\begin{aligned}
& 1.57 \\
& 2.95
\end{aligned}
$$$$
\begin{aligned}
& 2.95 \\
& 2.57
\end{aligned}
$$$$
2.57
$$$$
3.18
$$

3.72

11.41

\subsection{2}

1.43

1.19

1.28
2.01

2.39

2.51

2.29

4.73

1.36
10.38

05

.95
.74

2.23
1.78

1.78
1.33

1.49

1.19

1.19
2.52

2.52
1.99

2.33

2.51

2.29

7.49

4.73
2.14

2.14
2.36

6.43

\subsection{7}

4.06

1.19
4.06

4.06
3.45

3.45
2.84

2.08

2.29

7.49
4.73
1.61
6.11

1.61
6.11

\subsection{5}

3.15
2.49

2.49
9.35

5.56

5.56
15.33

$\begin{array}{ll}.69 & \mathbf{1 . 6 0} \\ 75 & 1.62\end{array}$

1.13

1.63

1.35

2.21

.18

$\begin{array}{ll}2.00 & 4.32\end{array}$

3.19

5.64
5.60

6.38

.33

1.08

4.32

$\begin{array}{ll}1.62 & 2.18 \\ 3.19 & 5.18\end{array}$

4.86

1985

1986

1987

1988

1989

9.04
5.52

2.34

5.34

7.83
7.38

9.07

7.82

26.42

10.22

5.01
1.71

1.71

4.84
6.53

6.11

6.45

8.89

9.03

4.14
22.36

\subsection{3}

5.03

2.05
4.96

6.12

5.47
6.72

6.72

6.55
27.74

10.69

4.41
1.60

1.60

4.47

4.34
4.03

4.59

7.35

6.78

2.38

23.72

6.70

5.20

1.88

2.08

1.71

4.57

7.60

4.91
6.05

6.05
6.45

8.89

17.61

9.03

4.14
7.67

7.67
15.35

6.29

4.50

1.75

2.05
1.60

4.60

4.06
6.08

4.57

4.31
4.59

7.35

15.59

6.78

2.38

5.65

16.46

9.00

8.98

6.84
6.81

$6.8 \overline{1}$

8.41

6.60

4.22
7.35

7.35

15.59

6.78

4.87
20.87

9.77

$\begin{array}{ll}9.10 & 9.07\end{array}$

2.27

4.74

6.61
6.04

6.04
7.43

6.94

29.82

10.97

4.30

1.44

4.33

4.64

4.22
4.76

6.85

7.23

2.93

2.93
22.95

6.13

4.36

1.59

1.92
1.44

3.47

5.94

3.34

4.46
4.76

6.85

13.58
7.23

7.23

2.93

6.10
16.54

7.22

7.22
7.20

7.20

7.55

6.89

4.21

6.85
13.58

13.58

7.23
3.32

3.32
20.31

7.75

7.95

7.95
5.43

5.43
22.86

22.37

1.42

2.00

3.66

3.63

3.56

4.22

.54

3.97

3.76

$2.08 \quad 2.20$

$4.52 \quad 4.81$

$\begin{array}{ll}6.43 & 7.49 \\ 5.87 & 6.12\end{array}$

$\begin{array}{ll}7.22 & 7.53\end{array}$

$6.77 \quad 8.16$

$\begin{array}{rr}28.54 & 29.21\end{array}$

$10.07 \quad 11.04$

$4.11 \quad 4.46$

$1.35 \quad 1.37$

$\begin{array}{ll}4.12 & 4.45 \\ 4.57 & 5.47\end{array}$

$\begin{array}{ll}4.00 & 4.72\end{array}$

$4.38 \quad 5.85$

$6.96 \quad 7.08$

$\begin{array}{ll}7.27 & 8.00 \\ 2.39 & 2.43\end{array}$

$\begin{array}{ll}21.38 & 22.24\end{array}$

$4.12 \quad 3.91$

$1.56 \quad 1.61$

$\begin{array}{ll}1.56 & 1.92 \\ 1.35 & 1.37\end{array}$ 


\begin{tabular}{|c|c|c|c|c|c|c|c|c|}
\hline Sector and Energy Source & 1970 & 1975 & 1980 & 1985 & 1986 & 1987 & 1988 & 1989 \\
\hline 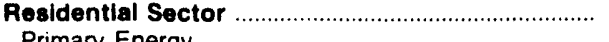 & $1,252.7$ & $2,144.7$ & $3,801.3$ & $5,493.0$ & $5,342.4$ & $5,412.0$ & $5,640.5$ & $5,895.2$ \\
\hline 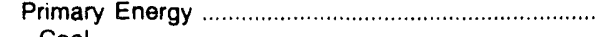 & 639.8 & $1,118.4$ & 1.985 .7 & $2,790.8$ & $2,411.4$ & $2,157.1$ & $2,331.5$ & $2,668.2$ \\
\hline 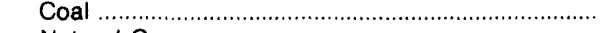 & 18.2 & 12.7 & 3.1 & 4.9 & 4.3 & 5.2 & 4.4 & 5.4 \\
\hline 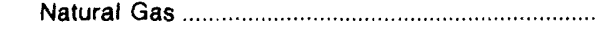 & 459.4 & 772.0 & $1,728.1$ & $2,480.4$ & $2,214.8$ & $1,961.9$ & $2,126.8$ & $2,442.7$ \\
\hline 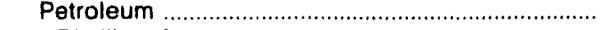 & 162.2 & 333.7 & 254.4 & 305.5 & 192.3 & 190.1 & 200.3 & 220.1 \\
\hline 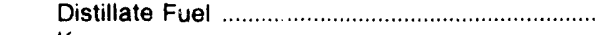 & 84.1 & 185.3 & 141.3 & 97.1 & 70.0 & 67.1 & 72.6 & 56.4 \\
\hline 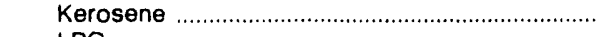 & 12.5 & 22.1 & 7.9 & 44.4 & 7.8 & 5.5 & 8.0 & 9.6 \\
\hline 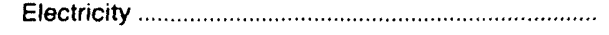 & 612.9 & $1,026.4$ & $1,815.6$ & $2,702.2$ & $2,930.9$ & $3,254.9$ & $3,309.0$ & $3,227.0$ \\
\hline 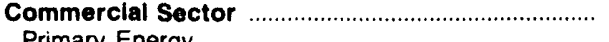 & 733.8 & $1,419.3$ & $2,802.2$ & $3,785.3$ & $3,772.9$ & $3,754.5$ & $3,761.0$ & $3,877.1$ \\
\hline 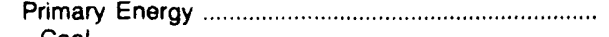 & 226.9 & 424.6 & $1,002.3$ & $1,297.0$ & $1,049.4$ & 945.3 & $1,006.0$ & 987.4 \\
\hline 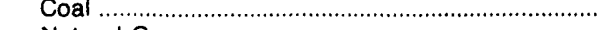 & 15.2 & 13.4 & 4.5 & 6.7 & 6.2 & 6.1 & 5.3 & 6.2 \\
\hline 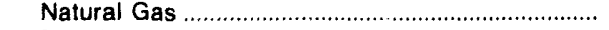 & 144.9 & 283.2 & 761.8 & $1,073.9$ & 934.7 & 838.7 & 901.9 & 892.4 \\
\hline 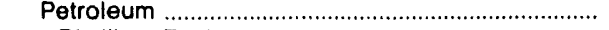 & 66.8 & 128.0 & 236.0 & 216.5 & 108.4 & 100.5 & 98.8 & 88.8 \\
\hline 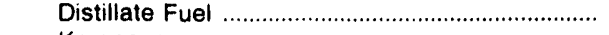 & 22.9 & 54.4 & 79.4 & 141.5 & 46.6 & 40.5 & 45.6 & 38.8 \\
\hline 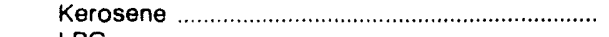 & .2 & .7 & .5 & 5.3 & 2.6 & 1.0 & 1.3 & 2.3 \\
\hline LPG & 6.4 & 13.7 & 12.9 & 32.9 & 22.7 & 20.5 & 21.7 & 23.6 \\
\hline 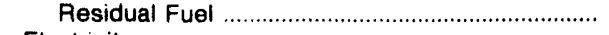 & 28.8 & 42.4 & 91.1 & 10.7 & 16.1 & 17.5 & 9.4 & 4.4 \\
\hline 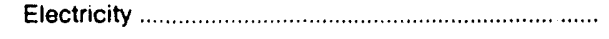 & 506.9 & 994.7 & $1,799.9$ & $2,488.3$ & $2,723.6$ & $2,809.3$ & $2,755.0$ & $2,889.7$ \\
\hline Industrial Sector & $1,060.9$ & $2,224.8$ & $4,771.9$ & $5,453.2$ & $5,130.5$ & $5,130.3$ & $4,954.6$ & $4,580.1$ \\
\hline 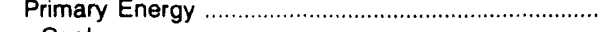 & 766.6 & $1,606.5$ & $3,449.8$ & $3,603.4$ & $3,120.6$ & $3,121.9$ & $3,045.8$ & $2,563.3$ \\
\hline Coal & 115.5 & 230.2 & 228.7 & 266.9 & 229.5 & 211.6 & 228.3 & 251.0 \\
\hline 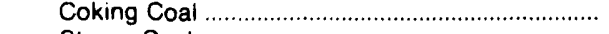 & 41.6 & 120.7 & 93.7 & 131.6 & 88.3 & 80.5 & 91.0 & 131.2 \\
\hline 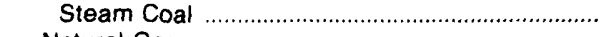 & 73.9 & 109.5 & 135.1 & 135.2 & 141.2 & 131.1 & 127.2 & 119.8 \\
\hline 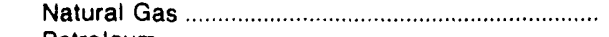 & 179.9 & 418.0 & $1,049.4$ & $1,287.4$ & $1,055.7$ & 959.0 & 885.8 & $1,000.5$ \\
\hline Petroleum & 471.2 & 958.4 & $2,171.7$ & $2,049.2$ & $1,835.4$ & $1,951.3$ & $1,931.7$ & $1,311.7$ \\
\hline 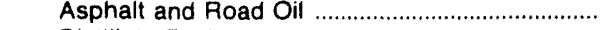 & 53.8 & 135.0 & 205.7 & 244.5 & 187.4 & 140.1 & 130.4 & 156.0 \\
\hline 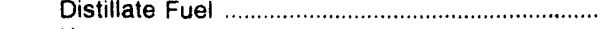 & 47.4 & 150.9 & 240.0 & 224.0 & 231.3 & 248.8 & 186.8 & 220.6 \\
\hline 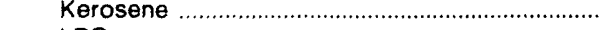 & 10.2 & 19.2 & 14.4 & 5.1 & 2.8 & 2.7 & 1.7 & 3.5 \\
\hline LPG & 74.2 & 185.6 & 581.2 & 828.3 & 797.3 & 893.0 & $1,004.2$ & 238.4 \\
\hline Lubricants & 62.1 & 75.8 & 170.6 & 190.3 & 164.8 & 162.2 & 168.4 & 211.6 \\
\hline Motor Gasoline & 96.4 & 106.5 & 180.7 & 82.5 & 56.0 & 59.5 & 57.2 & 59.6 \\
\hline 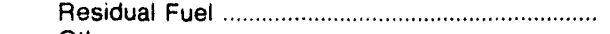 & 46.8 & 117.0 & 214.4 & 62.4 & 38.6 & 37.4 & 34.0 & 32.9 \\
\hline 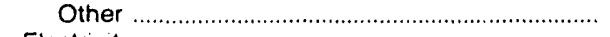 & 80.3 & 168.3 & 564.5 & 412.2 & 357.3 & 407.6 & 349.0 & 389.2 \\
\hline Electricity & 294.3 & 618.3 & $1,322.1$ & $1,849.8$ & $2,009.9$ & $2,008.4$ & $1,908.8$ & $2,016.8$ \\
\hline 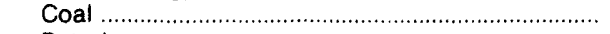 & .2 & $*$ & - & - & - & - & - & - \\
\hline 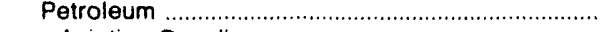 & $1,873.5$ & $3,519.9$ & $7,223.2$ & $6,387.1$ & $4,809.4$ & $5,119.5$ & $5,449.6$ & $6,079.5$ \\
\hline 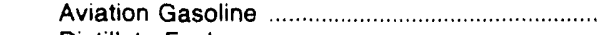 & 2.9 & 1.4 & 6.0 & 10.7 & 8.9 & 6.1 & 7.0 & 8.0 \\
\hline Distillate Fuel & 123.2 & 338.4 & 978.7 & 949.7 & 816.9 & 825.1 & 852.6 & $1,015.4$ \\
\hline 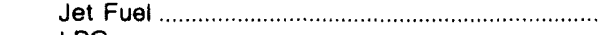 & 95.2 & 285.7 & 704.0 & 92.2 & 48.4 & 46.8 & 85.3 & 109.3 \\
\hline LPG & 2.2 & 4.1 & 3.2 & 17.6 & 12.1 & 8.3 & 10.3 & 8.5 \\
\hline 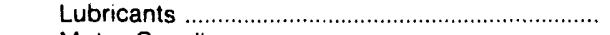 & 38.2 & 65.9 & 131.9 & 147.1 & 127.4 & 125.4 & 130.2 & 163.6 \\
\hline Motor Gasoline & $1,610.4$ & $2,822.2$ & $5,390.1$ & $5,163.7$ & $3,792.9$ & $4,105.6$ & $4,358.2$ & $4,773,6$ \\
\hline 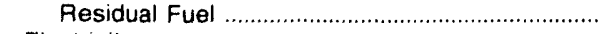 & 1.5 & 2.2 & 9.4 & 6.1 & 2.8 & 2.2 & 6.0 & 1.1 \\
\hline 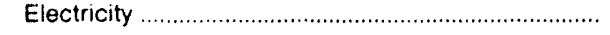 & 3.3 & 5.5 & 10.9 & 22.7 & 24.9 & 23.3 & 22.9 & 24.3 \\
\hline Total Energy & $4,924.5$ & $9,314.3$ & $18,609.5$ & $21,141.3$ & $19,080.2$ & $19,439.7$ & $19,826.7$ & $20,456.2$ \\
\hline 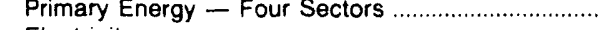 & $3,507.0$ & $6,669.5$ & $13,661.0$ & $14,078.3$ & $11,390.8$ & $11,343.7$ & $11,832.9$ & $12,298.3$ \\
\hline 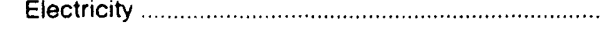 & $1,417.5$ & $2,644.9$ & $4,948.6$ & $7,063.0$ & $7,689.4$ & $8,095.9$ & $7,995.8$ & $8,157.9$ \\
\hline Electric Utility Sector & 254.5 & 689.6 & $1,794.2$ & $1,856.1$ & $1,849.5$ & $1,678.7$ & $1,638.8$ & $1,550.4$ \\
\hline 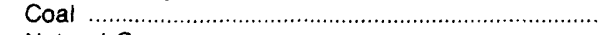 & 180.2 & 494.2 & $1,151.8$ & $1,441.6$ & $1,379.4$ & $1,236.5$ & $1,081.7$ & 998.7 \\
\hline (1) & 47.7 & 39.8 & 62.5 & 31.3 & 28.8 & 11.9 & 19.0 & 23.1 \\
\hline (2) & 22.5 & 110.5 & 480.6 & 112.7 & 146.8 & 82.8 & 49.3 & 44.1 \\
\hline (.). & 12.2 & 61.4 & 449.3 & 97.4 & 137.1 & 72.3 & 37.2 & 32.5 \\
\hline 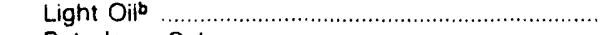 & 10.3 & 49.1 & 31.3 & 15.4 & 9.7 & 10.4 & 12.1 & 11.6 \\
\hline Petroleum Coke & - & - & - & - & - & - & - & - \\
\hline 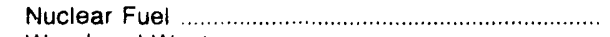 & 4.1 & 45.2 & 99.4 & 270.5 & 294.6 & 347.5 & 488.7 & 484.5 \\
\hline Wood and Waste & • & - & - & - & - & - & - & - \\
\hline 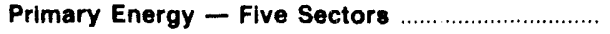 & $3,761.6$ & $7,359.1$ & $15,455.2$ & $15,934.4$ & $13,240.3$ & $13,022.4$ & $13,471.7$ & $13,848.8$ \\
\hline
\end{tabular}

- There are no direct fuel costs for hydroelectric, geothermal, centralized solar, or wind energy. Wood and other biomass fuels are not included, except those consumed at electric utilities.

b heavy oil includes Grade Nos. 4, 5, and 6, and residual fuel oils. Light oil includes Grade No. 2 heating oil, kerosene, and jet fuel.

- No consumption, includirig cases where adjustments were made. See "Consumption Adjustments for Process Fuel and intermediate Products."

"Value less than 0.05 million dollars.

Note: Totals may not equal sum of components due to independent rounding.

Sources: Data sources, estimation procedures, and assumptions are described in the "Documentation" section of this report. 


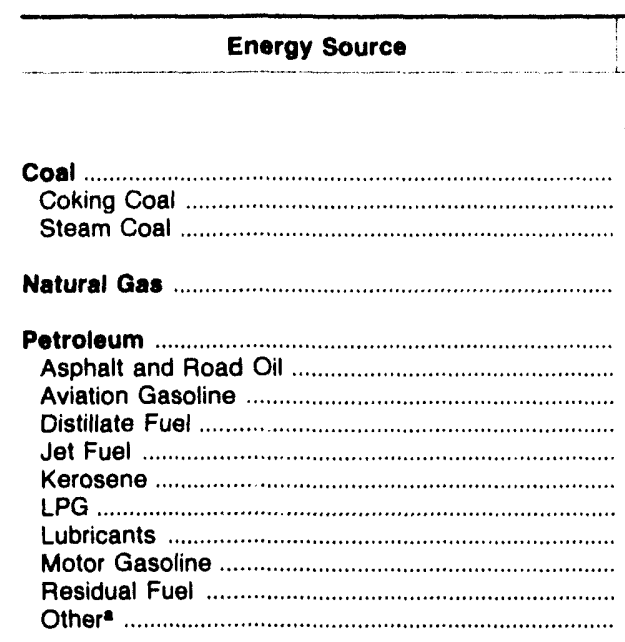

1970 1975

1980

1985

1986

1987

1988

1989

Prices in Dollars per Mililon Btu

0.36
.44
.32
.68
2.01
.76
2.17
1.03
.74
1.48
1.86
5.08
2.98
.57
.76

Nuclear Fuel

Wood and Waste at Utllitles

Primary Energy - Five Sectors

Electric Utility Fuel

Electricity Purchased by End Users

Total Energyb

$1.09 \quad 1.53$

$\begin{array}{ll}1.53 & 1.77\end{array}$

2.13
1.31

$1.77 \quad 1.67$

2.24
1.64

2.05
1.58

1.56
1.92

$\begin{array}{ll}.32 & .73\end{array}$

2.88

4.71

\subsection{9}

1.46

1.92

$68 \quad 1.16$

7.78
3.72

3.51
2.05

3.45

7.86

4.81

5.77

9.02

6.85

2.49
2.08

9.99
7.66

7.66
5.81

5.81
7.90

8.71
17.61

3.34

7.49

7.94

6.08

14.36

10.00

1.81

8.71
17.61

8.85

4.40
6.23

3.94

8.41
5.77

5.77

3.80
5.41

5.41
7.79

7.79
15.59

6.48

2.62

4.33

4.08

4.31

4.52

4.65

5.99

3.30

7.55

6.11

3.96

6.04

7.45

13.58
6.90

6.90
2.63

2.63
4.42

5.94

$\begin{array}{ll}5.94 & 6.56 \\ 3.33 & 3.03\end{array}$

$\begin{array}{ll}7.41 & 8.28\end{array}$

$\begin{array}{ll}5.77 & 6.41\end{array}$

6.04

7.45

14.61

6.94

2.10
3.65

6.41
4.33

6.91

8.88

17.90

7.60

2.33

4.41

-

- -

- $\quad-$
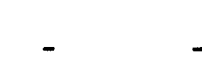

$-$

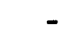

$\begin{array}{rrr}.93 & 1.90 & 3.67 \\ .26 & .62 & 1.30\end{array}$

$\begin{array}{lll}.26 & .62 & 1.30\end{array}$

$1.43 \quad 2.82$

4.14

3.50

3.43

3.38

3.57

1.66

1.59

1.47

1.44

16.53

1.37

15.92

6.94

6.23

6.04

6.01

6.29

Expenditures in Millions of Dollars

Coal

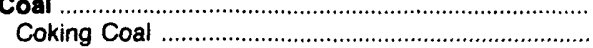

Steam Coal

Natural Gas

Petroleum

Asphalt and Road Oil

Aviation Gasoline

Distillate Fuel ....

Jet Fuel.

Kerosene

LPG ..

Lubricants

Motor Gasoline

.

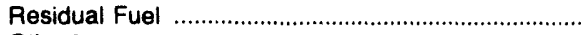

Other"

.........

Nuclear Fuel

Wood and Waste at Utilities

Primary Energy - Five Sectors

Electric Utility Fuel

Electricity Purchased by End Users

Total Energy ${ }^{b}$

$\begin{array}{lr}364.5 & 1,155.2 \\ 151.8 & 651.7\end{array}$

151.8

212.7

359.0

503.5

\section{$1,775.9$}

$1,775.9$
684.0

$1,091.9$

\section{$2,108.1$}

$2,108.1$
560.1

$1,548.0$

$1,921.0$

468.5
$1,452.5$

$1,861.3$

$1,861.3$
503.6

$1,357.7$

$2,040.2$
612.4

612.4
$1,427.8$

$1,776.1$

$1,639.6$

$1,920.7$

$4,618.5$

$1,313.5$
30.7

30.7
4.0

176.3

10.6

17.9

62.9

48.8

921.2

14.2

26.9

$2,643.5$

843.5
82.5
3.8

$5,384.3$

$5,384.3$
127.5
11.8

$1,995$.

$4,275.8$

$5,482.6$
170.2

$4,275.8$
158.5

19.8
179.4

$1,227.3$

473.9

$\begin{array}{r}1,227.5 \\ \hline\end{array}$

76.5
29.7
174.3

$1,373.4$

$1,373.4$
507.4

14.3
150.9

72.9
150.9

1.614 .2

120.0

155.8

$3,162.9$

$3,162.9$
261.7

156.8

49.8
246.7

246.7
173.8

$2,694.3$

78.8

$\begin{array}{r}168.4 \\ \hline\end{array}$

18.4
$1,067.7$

$1,067.7$
400.2

400.2
22.6

249.6

$\begin{array}{r}150.4 \\ \hline\end{array}$

$2,043.5$

59.3

59.3
105.6

166.4
14.4

14.4
$1,126.8$

$1,126.8$
428.4

18.1

254.5

148.1

$2,289.7$

46.3

125.9

$4,412.7$

$\begin{array}{r}453.5 \\ \hline\end{array}$

16.2

965.7

350.1

21.9

266.4

2340

33.0

33.0
112.2

$1,952.9$

544.0

$1,409.0$

$2,044.9$

$5,028.3$

128.4

12.0
$1,235.7$

429.8

27.6

361.7

193.2

$2,460.7$

48.0

131.2

-Includes petroleum coke used at electric utilities.

bThere are no direct fuel costs for hydroelectric, geothermal, centralized solar, or wind energy. Wood and other biomass fuels are not included, except those consumed at electric utilities.

-No consumption, including cases where adjustments were made. See "Consumption Adjustments for Process Fuel and Intermediate Products."

Note: Expenditure totals may not equal sum of components due to independent rounding.

Sources: Data sources, estimation procedures, and assumptions are described in the "Documentation" section of this report. 


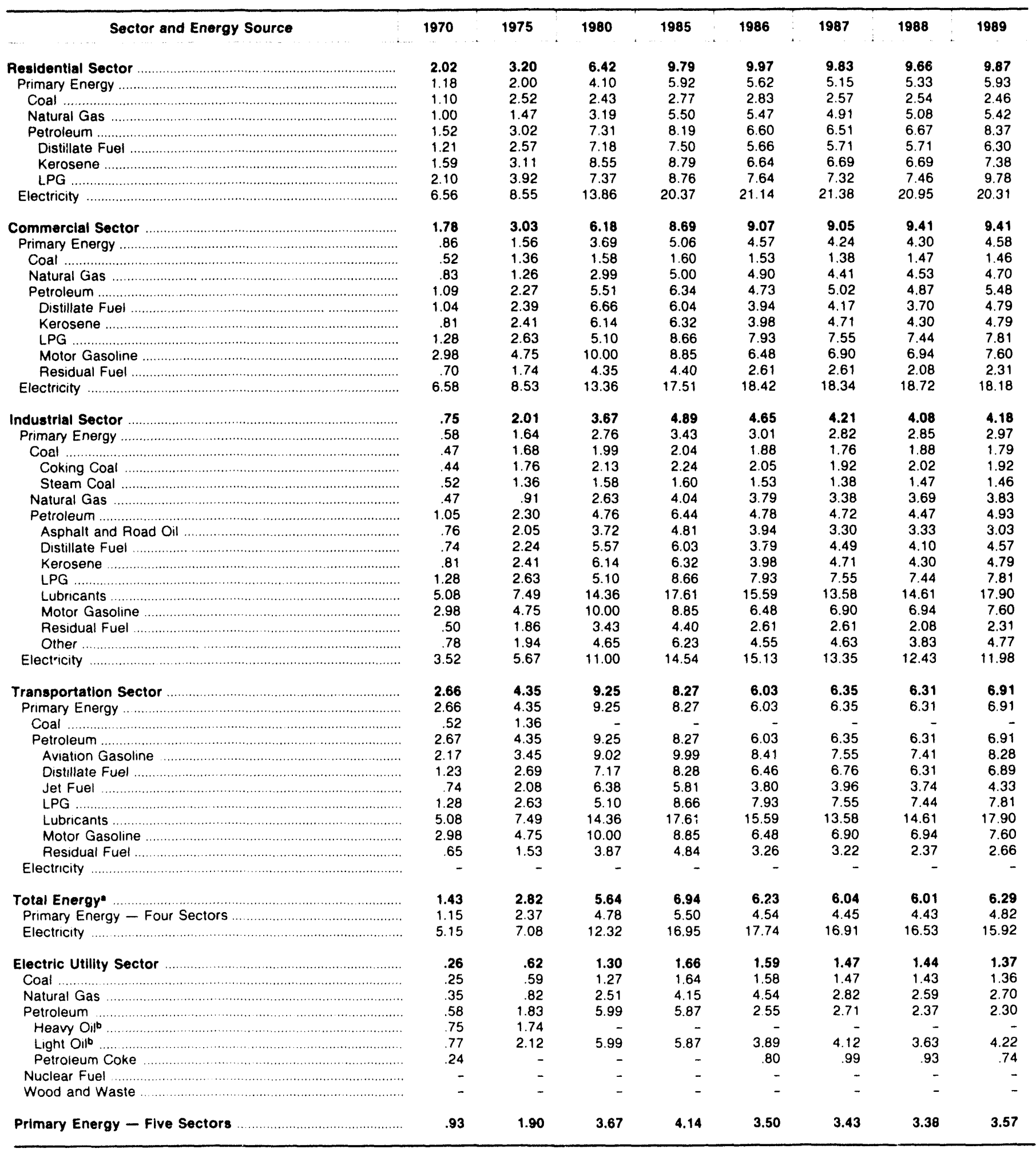

- There are no direct fuel costs for hydroelectric, geothermal, centralized solar, or wind energy. Wood and other biomass fuels are not included, except those consumed at electric utilities.

b Heavy oil includes Grade Nos. 4, 5, and 6, and residual fuel oils. Light oil includes Grade No. 2 heating oil, kerosene, and jet fuel.

- No consumption, including cases where adjustments were made. See "Consumption Adjustments for Process Fuel and Intermediate Products."

Sources: Data sources, estimation procedures, and assumptions are described in the "Documentation" section of this report. 


\begin{tabular}{|c|c|c|c|c|c|c|c|c|}
\hline Sector and Energy Source & 1970 & 1975 & 1980 & 1985 & 1986 & 1987 & 1988 & 1989 \\
\hline Residential Sector & 591.5 & 971.1 & $1,771.6$ & $2,467.3$ & $2,489.4$ & $2,461.4$ & $2,669.5$ & $2,737.1$ \\
\hline 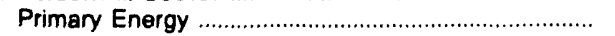 & 289.6 & 493.7 & 860.9 & 1.091 .0 & 1.010 .0 & 917.0 & 1.062 .4 & $1,193.1$ \\
\hline 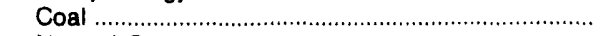 & 6.3 & 17.6 & 4.1 & 11.3 & 12.2 & 11.1 & 12.7 & 10.0 \\
\hline 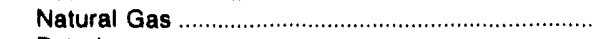 & 160.3 & 237.0 & 516.3 & 810.4 & 772.9 & 688.3 & 792.6 & 858.9 \\
\hline 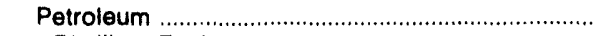 & 123.1 & 239.1 & 340.4 & 269.3 & 224.9 & 217.6 & 257.1 & 324.2 \\
\hline 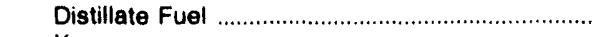 & 56.3 & 129.4 & 225.8 & 111.8 & 95.1 & 91.8 & 92.9 & 84.9 \\
\hline 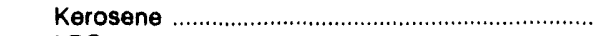 & 16.6 & 12.6 & 23.8 & 35.3 & 14.9 & 13.5 & 17.7 & 24.1 \\
\hline LPG & 50.2 & 97.1 & 90.8 & 122.2 & 114.9 & 112.3 & 146.5 & 215.2 \\
\hline 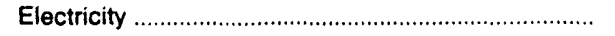 & 301.8 & 477.5 & 910.8 & $1,376.4$ & $1,479.4$ & $1,544.4$ & $1,607.0$ & 1.544 .0 \\
\hline 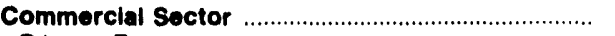 & 247.2 & 444.7 & 854.4 & $1,246.0$ & $1,233.2$ & $1,218.7$ & $1,423.8$ & $1, \mathbf{4 3 4 . 2}$ \\
\hline 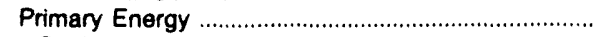 & 100.8 & 180.7 & 379.3 & 513.7 & 420.1 & 376.9 & 419.8 & 450.2 \\
\hline 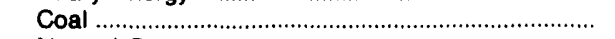 & 5.5 & 17.6 & 5.0 & 12.1 & 12.1 & 11.0 & 13.7 & 11.0 \\
\hline 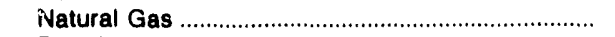 & 64.5 & 87.7 & 206.9 & 350.9 & 320.3 & 288.8 & 329.9 & 351.9 \\
\hline Petroleum & 30.7 & 75.3 & 167.4 & 150.7 & 87.7 & 77.1 & 76.3 & 87.4 \\
\hline 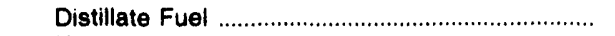 & 16.9 & 41.9 & 77.0 & 92.8 & 42.2 & 33.9 & 28.8 & 32.2 \\
\hline Kerosene & .8 & 1.0 & 1.1 & 7.3 & 3.1 & 1.2 & 1.7 & 1.2 \\
\hline 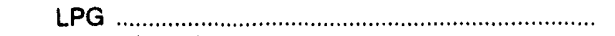 & 5.4 & 11.5 & 11.1 & 21.3 & 21.0 & 20.4 & 25.8 & 30.3 \\
\hline 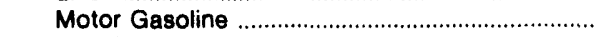 & 3.9 & 3.0 & 11.7 & 16.3 & 16.6 & 16.8 & 16.5 & 17.1 \\
\hline 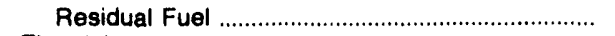 & 3.7 & 18.0 & 66.5 & 12.9 & 4.8 & 4.8 & 3.4 & 6.5 \\
\hline 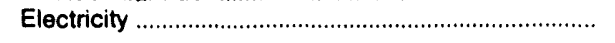 & 146.4 & 264.0 & 475.1 & 732.3 & 813.0 & 841.8 & $1,004.0$ & 984.0 \\
\hline Industrial Sector & 743.2 & $1,906.2$ & $3,446,9$ & $3,926.9$ & $3,539.5$ & $3,500.8$ & $3,550.8$ & $3,592.9$ \\
\hline Primary Energy …………………………………. & 534.2 & $1,455.4$ & $2,308.3$ & $2,387.8$ & $1,985.3$ & $2,038.1$ & $2,167.9$ & $2,208.1$ \\
\hline 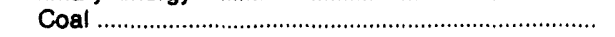 & 228.6 & 776.8 & 845.6 & 744.0 & 639.3 & 658.7 & 770.8 & 599.7 \\
\hline 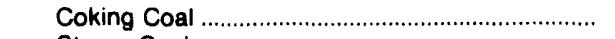 & 151.8 & 651.7 & 684.0 & 560.1 & 468.5 & 503.6 & 612.4 & 544.0 \\
\hline 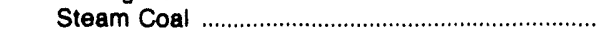 & 76.9 & 125.1 & 161.6 & 183.9 & 170.7 & 155.0 & 158.4 & 155.7 \\
\hline 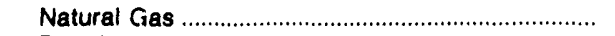 & 123.9 & 198.3 & 615.0 & 829.5 & 677.9 & 658.8 & 789.2 & 823.1 \\
\hline 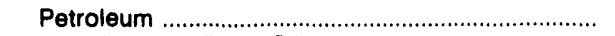 & 181.6 & 480.3 & 847.7 & 814.3 & 668.1 & 720.7 & 607.9 & 685.3 \\
\hline Asphalt and Road Oil & 30.7 & 82.5 & 127.5 & 170.2 & 158.5 & 166.4 & 153.5 & 128.4 \\
\hline 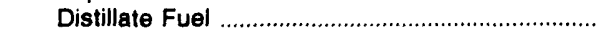 & 43.8 & 121.5 & 162.6 & 157.8 & 118.2 & 148.4 & 101.6 & 128.2 \\
\hline 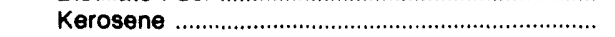 & .5 & .8 & 4.7 & 7.2 & 4.6 & 3.5 & 2.5 & 2.3 \\
\hline LPG …… & 6.9 & 41.0 & 70.8 & 97.2 & 108.6 & 117.9 & 89.2 & 110.9 \\
\hline 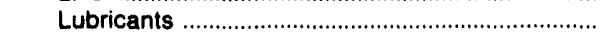 & 30.0 & 38.2 & 95.5 & 106.5 & 92.2 & 90.8 & 94.3 & 118.4 \\
\hline 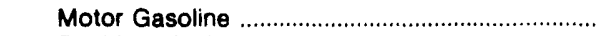 & 35.0 & 31.5 & 39.5 & 41.9 & 28.3 & 30.3 & 29.3 & 30.2 \\
\hline 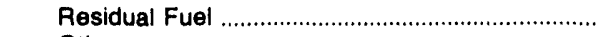 & 8.2 & 84.2 & 190.3 & 65.1 & 53.4 & 39.2 & 27.2 & 37.7 \\
\hline Other & 26.5 & 80.6 & 156.8 & 168.4 & 104.4 & 124.2 & 110.4 & 129.2 \\
\hline 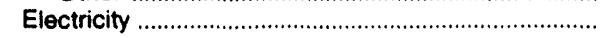 & 209.0 & 510.8 & $1,138.6$ & $1,539.1$ & $1,554.2$ & $1,462.6$ & $1,382.9$ & $1,384.8$ \\
\hline $\begin{array}{l}\text { Traneportation Sector } \\
\text { Primary Energy }\end{array}$ & $\begin{array}{l}975.9 \\
975.9\end{array}$ & $\begin{array}{l}1,828.2 \\
1,828.2\end{array}$ & $\begin{array}{l}4,003.3 \\
4,003.3\end{array}$ & $\begin{array}{l}4,234.2 \\
4,234.2\end{array}$ & $\begin{array}{l}3,286.5 \\
3,286.5\end{array}$ & $\begin{array}{l}3,593.1 \\
3,593.1\end{array}$ & $\begin{array}{l}3,461.6 \\
3,461.6\end{array}$ & $\begin{array}{l}3,920.4 \\
3,920.4\end{array}$ \\
\hline Coal & .4 & .1 & - & - & - & - & - & - \\
\hline 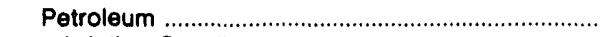 & 975.5 & $1,828.2$ & $4,003.3$ & $4,234.2$ & $3,286.5$ & $3,593.1$ & $3,461.6$ & $3,920.4$ \\
\hline 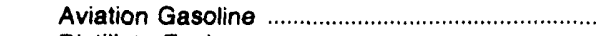 & 4.0 & 3.8 & 11.8 & 19.8 & 18.4 & 14.4 & 16.2 & 12.0 \\
\hline 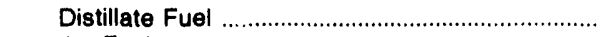 & 58.1 & 175.3 & 736.5 & 996.8 & 805.0 & 844.2 & 734.3 & 981.3 \\
\hline 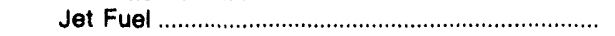 & 10.6 & 30.4 & 76.5 & 507.4 & 400.2 & 428.4 & 350.1 & 429.8 \\
\hline LPG & .5 & 1.2 & 1.6 & 6.0 & 5.1 & 3.9 & 4.9 & 5.3 \\
\hline Lubricants ………………………………………......... & 18.8 & 34.6 & 60.3 & 67.2 & 58.2 & 57.3 & 59.5 & 74.8 \\
\hline Motor Gasoline ................................................. & 882.2 & $1,579.7$ & $3,111.7$ & $2,636.0$ & $1,998.6$ & $2,242.6$ & $2,294.2$ & $2,413.4$ \\
\hline 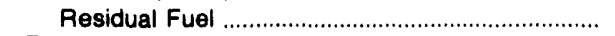 & 1.3 & 3.2 & 4.9 & .8 & 1.1 & 2.4 & 2.4 & 3.8 \\
\hline 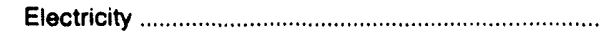 & - & - & - & - & - & - & - & - \\
\hline Total Energye & $2,557.8$ & & $10,076.3$ & $11,874.4$ & $10,548.6$ & $10,773.9$ & $11,105.7$ & $11,684.6$ \\
\hline Primary Energy - Four Sectors .................................. & $1,900.5$ & $3,958.0$ & $7,551.8$ & $8,226.6$ & $6,702.0$ & 6.925 .0 & $7,111.7$ & $7,771.8$ \\
\hline 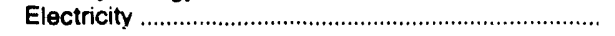 & 657.3 & $1,252.3$ & $2,524.5$ & $3,647.8$ & $3,846.6$ & $3,848.9$ & $3,993.9$ & $3,912.8$ \\
\hline Eloctric Utility Sector & 136.5 & 372.6 & 951.4 & $1,359.6$ & $1,270.9$ & $1,194,4$ & $1,261.8$ & $1,254.3$ \\
\hline 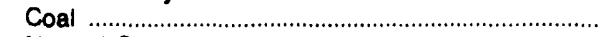 & 123.7 & 343.1 & 921.2 & $1,340.7$ & $1,257.4$ & $1,180.5$ & $1,243.0$ & $1,232.3$ \\
\hline 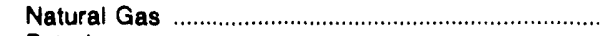 & 10.3 & 9.0 & 4.8 & 4.7 & 5.0 & 3.7 & 9.0 & 11.0 \\
\hline Petroleum & 2.5 & 20.6 & 25.4 & 14.2 & 8.5 & 10.1 & 9.8 & 11.1 \\
\hline 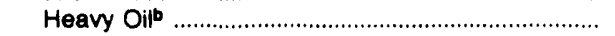 & 1.0 & 14.7 & - & - & - & - & - & - \\
\hline 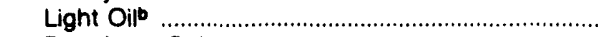 & 1.2 & 5.9 & 25.4 & 14.2 & 7.3 & 8.5 & 8.0 & 9.1 \\
\hline 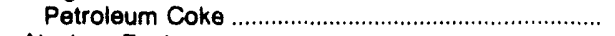 & .4 & - & - & - & 1.2 & 1.7 & 1.8 & 2.0 \\
\hline 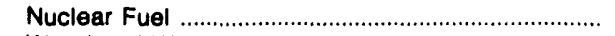 & - & - & - & - & - & - & - & - \\
\hline Wood and Waste & - & - & - & - & - & - & - & - \\
\hline Primary Energy - Five Sectors ………………......... & $2,037.0$ & $4,330.6$ & $8,503.3$ & $9,586.2$ & $7,972.9$ & $8,119.4$ & 8,373.5 & $9,026.1$ \\
\hline
\end{tabular}

-There are no direct fuel costs for hydroelectric, geothermal, centralized solar, or wind energy. Wood and other biomass fuels are not included, except those consumed at electric utilities.

beavy oil includes Grade Nos. 4, 5, and 6, and residual fuel oils. Light oil includes Grade No. 2 heating oil, kerosene, and jet fuel.

-No consumption, including casas where adjustments were made. See "Consumption Adjustments for Process Fuel and intermediate Prodjcts."

Note: Totals may not equal sum of components due to independent rounding.

Sources: Data sources, estimation procedures, and assuinptions are described in the "Documentation" section of this report. 


\begin{tabular}{|c|c|c|c|c|c|c|c|c|}
\hline Energy Source & 1970 & 1975 & 1980 & 1985 & 1986 & 1987 & 1988 & 1989 \\
\hline & \multicolumn{8}{|c|}{ Prices in Dollars per Million Btu } \\
\hline Coal & 0.36 & 0.95 & 1.42 & 1.52 & 1.41 & 1.31 & 1.28 & 1.25 \\
\hline Coking Coal & - & - & - & - & - & - & - & - \\
\hline 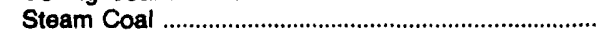 & .36 & .95 & 1.42 & 1.52 & 1.41 & 1.31 & 1.28 & 1.25 \\
\hline 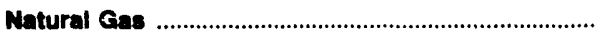 & .57 & 1.00 & 2.79 & 4.60 & 4.22 & 3.63 & 3.80 & 3.57 \\
\hline Potrolouvn & 2.11 & 3.74 & 8.19 & 8.10 & 6.59 & 6.70 & 6.51 & 7.50 \\
\hline Asphalt and Road Oil & .72 & 2.06 & 3.77 & 5.03 & 3.89 & 3.55 & 3.46 & 3.00 \\
\hline Av ion Gasoline & 2.17 & 3.45 & 9.02 & 9.99 & 8.41 & 7.55 & 7.41 & 8.28 \\
\hline Distillate Fuel & 1.01 & 2.45 & 6.41 & 6.16 & 5.47 & 5.95 & 5.48 & 6.27 \\
\hline Jet Fuel & .75 & 2.09 & 6.47 & 6.28 & 4.36 & 4.27 & 4.12 & 4.57 \\
\hline Kerosene & 1.31 & 2.74 & 6.23 & 6.93 & 5.77 & 5.80 & 5.41 & 5.36 \\
\hline LPG & 1.59 & 3.00 & 5.57 & 7.33 & 7.23 & 5.87 & 5.79 & 6.88 \\
\hline Lubricants & 5.08 & 7.49 & 14.36 & 17.61 & 15.59 & 13.58 & 14.61 & 17.90 \\
\hline Motor Gasoline & 2.83 & 4.59 & 9.97 & 9.47 & 7.13 & 7.36 & 7.37 & 8.40 \\
\hline Residual Fuel & .61 & 1.88 & 3.19 & 4.07 & 2.86 & 2.48 & 2.38 & 2.25 \\
\hline Other" & 2.19 & 3.20 & 7.69 & 8.60 & 7.14 & 7.78 & 6.70 & 8.21 \\
\hline 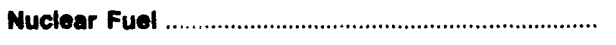 & - & .25 & .39 & .94 & .75 & .71 & .68 & .67 \\
\hline 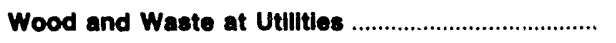 & .65 & .92 & 1.74 & .79 & .32 & .95 & .87 & .82 \\
\hline 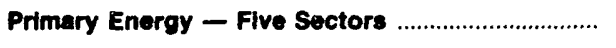 & 1.19 & 2.16 & 4.60 & 4.93 & 4.17 & 3.91 & 3.82 & 4.05 \\
\hline Electric Utility Fuel & .30 & .75 & 1.32 & 1.46 & 1.31 & 1.21 & 1.19 & 1.17 \\
\hline 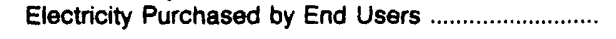 & 6.39 & 9.11 & 13.97 & 19.02 & 19.25 & 18.30 & 17.59 & 17.30 \\
\hline \multirow[t]{2}{*}{ Total Energy ${ }^{6}$} & 1.80 & 3.12 & 6.73 & 8.02 & 7.28 & 7.05 & 6.85 & 7.16 \\
\hline & \multicolumn{8}{|c|}{ Expenditures in Millons of Dollars } \\
\hline Coal & 47.5 & 125.4 & 333.4 & 407.9 & 370.6 & 375.2 & 390.6 & 397.8 \\
\hline 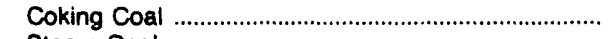 & - & - & - & - & - & - & $\overline{0}$ & - \\
\hline 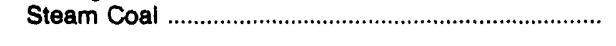 & 47.5 & 125.4 & 333.4 & 407.9 & 370.6 & 375.2 & 390.6 & 397.8 \\
\hline 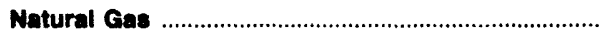 & 110.2 & 332.4 & 719.9 & $1,003.4$ & 851.8 & 713.4 & 874.1 & 777.6 \\
\hline Petroloum & 730.8 & $1,403.3$ & $3,032.4$ & $2,746.5$ & $2,241.4$ & $2,135.4$ & $2,162.7$ & $2,428.0$ \\
\hline 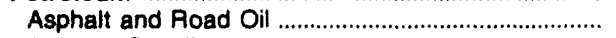 & 13.9 & 31.4 & 42.5 & 67.6 & 52.6 & 42.1 & 50.8 & 34.1 \\
\hline Aviation Gasoline & 2.8 & 3.3 & 8.4 & 4.2 & 6.4 & 4.2 & 5.4 & 4.6 \\
\hline 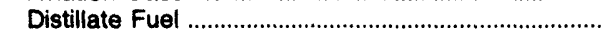 & 80.7 & 207.6 & 594.5 & 555.9 & 508.6 & 546.5 & 508.9 & 546.1 \\
\hline 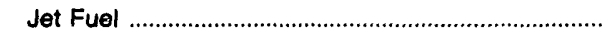 & 3.0 & 9.8 & 29.6 & 20.9 & 14.5 & 18.7 & 16.5 & 19.3 \\
\hline 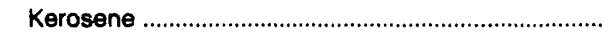 & 3.6 & 3.3 & 6.0 & 9.3 & 3.8 & 3.2 & 2.9 & 2.3 \\
\hline LPG & 66.2 & 152.2 & 228.5 & 372.1 & 369.0 & 192.0 & 213.7 & 259.6 \\
\hline 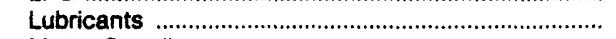 & 21.6 & 29.8 & 62.2 & 69.3 & 60.0 & 59.1 & 61.3 & 77.1 \\
\hline 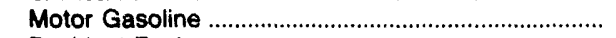 & 530.1 & 942.1 & $1,853.2$ & $1,565.7$ & $1,174.7$ & $1,222.9$ & $1,260.5$ & $1,435.8$ \\
\hline 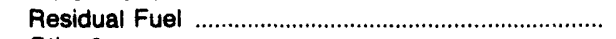 & 1.5 & 7.2 & 6.3 & 5.6 & 11.0 & 1.9 & 4.2 & 3.3 \\
\hline Other & 7.4 & 16.7 & 199.1 & 75.9 & 40.7 & 44.7 & 38.2 & 45.7 \\
\hline 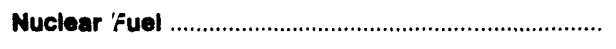 & - & 6.3 & 10.9 & 19.6 & 24.4 & 19.3 & 23.0 & 22.6 \\
\hline 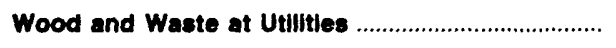 & .3 & .4 & .5 & .5 & .2 & .7 & .5 & .2 \\
\hline 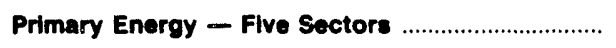 & 968.7 & $1,867.8$ & $4,097.0$ & $4,177.2$ & $3,488.4$ & $3,244.0$ & $3,450.9$ & $3,626.2$ \\
\hline 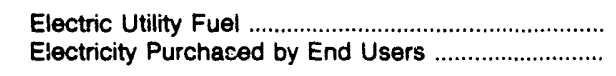 & $\begin{array}{r}-50.4 \\
337.5\end{array}$ & $\begin{array}{r}-132.5 \\
624.4\end{array}$ & $\begin{array}{r}-313.1 \\
1.184 .5\end{array}$ & $\begin{array}{r}-3567 \\
1,5666\end{array}$ & $\begin{array}{r}-335.2 \\
1,731.1\end{array}$ & $\begin{array}{r}-326.2 \\
1.687 .4\end{array}$ & $\begin{array}{r}-354.1 \\
1.731 .0\end{array}$ & $\begin{array}{l}-250.1 \\
1,69 \pm . ?\end{array}$ \\
\hline Total Energyb & $1,255.8$ & $2,359.7$ & $4,968.4$ & $5,477.7$ & $4,884.3$ & $4,605.2$ & $4,827.8$ & $4,971.2$ \\
\hline
\end{tabular}

Includes petroleum coke uzed at electric utilities.

-There are no direct fuol costs for hydroelectric, geothermal, centralized solar, or wind energy. Wood and other biomass fuels are not included, except those consumed at electric utilities.

-No consumption, including cases where adjustments were made. See "Consumption Adjustments for Process Fuel and Intermediate Products."

Note: Expenditure totals may not equal sum of components due to independent rounding.

Sources: Data sources, estimation procedures, and assumptions are described in the "Documentation" section of this report. 
I Energy Price Estimates by Sector, lowa

O 1970, 1975, 1980, and 1985-1989

W (Dollars per Million Btu)

A

\begin{tabular}{|c|c|c|c|c|c|c|c|c|}
\hline Sector and Energy Source & 1970 & 1975 & 1980 & 1985 & 1986 & 1987 & 1988 & 1989 \\
\hline Residential Sector & 2.06 & 3.44 & 6.87 & 9.44 & 9.44 & 9.82 & 9.31 & 9.61 \\
\hline 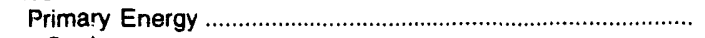 & 1.16 & 1.94 & 4.09 & 5.40 & 5.14 & 4.76 & 4.80 & 5.34 \\
\hline Coal & 1.27 & 3.69 & 3.31 & 3.41 & 3.12 & 2.89 & 2.51 & 2.60 \\
\hline 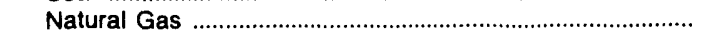 & .96 & 1.42 & 3.18 & 5.33 & 5.07 & 4.71 & 4.76 & 4.65 \\
\hline Petroleum & 1.63 & 3.25 & 6.84 & 5.78 & 5.47 & 5.13 & 5.19 & 7.69 \\
\hline 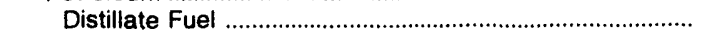 & 1.22 & 2.56 & 6.79 & 5.94 & 5.32 & 5.33 & 4.80 & 4.86 \\
\hline Kerosene & 1.57 & 2.99 & 8.10 & 7.33 & 6.57 & 6.57 & 5.92 & 5.99 \\
\hline LPG & 1.85 & 3.55 & 6.86 & 5.62 & 5.51 & 5.00 & 5.31 & 8.68 \\
\hline Electricity & 7.75 & 10.46 & 16.13 & 22.53 & 22.56 & 22.91 & 22.23 & 22.19 \\
\hline Commercial Sector & 1.80 & 2.94 & 6.33 & 9.23 & 9.38 & 8.85 & 8.35 & 8.39 \\
\hline 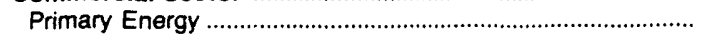 & .77 & 1.29 & 3.38 & 4.91 & 4.48 & 3.88 & 3.96 & 3.91 \\
\hline 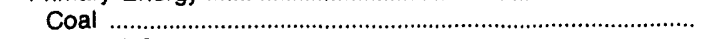 & .41 & 1.24 & 1.59 & 1.66 & 1.57 & 1.52 & 1.42 & 1.35 \\
\hline 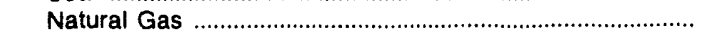 & .68 & 1.05 & 2.84 & 4.80 & 4.41 & 3.84 & 4.00 & 3.87 \\
\hline Petroleum & 1.30 & 2.71 & 6.57 & 6.50 & 5.95 & 5.33 & 5.15 & 5.03 \\
\hline 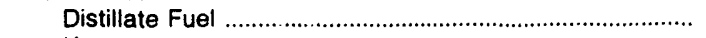 & 1.05 & 2.40 & 6.44 & 5.10 & 3.81 & 4.18 & 3.41 & 3.65 \\
\hline 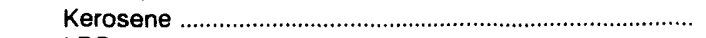 & .81 & 2.30 & 5.52 & 5.78 & 4.32 & 4.95 & 4.02 & 4.48 \\
\hline LPG & 1.16 & 2.46 & 4.88 & 8.25 & 8.25 & 6.45 & 6.23 & 5.21 \\
\hline Motor Gasoline & 2.83 & 4.59 & 9.97 & 9.47 & 7.13 & 7.36 & 7.37 & 8.40 \\
\hline 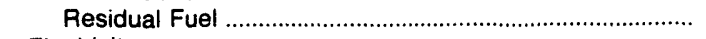 & .66 & 1.69 & 3.80 & 4.07 & 2.86 & 2.49 & 2.38 & 2.25 \\
\hline Electricity & 7.68 & 10.55 & 15.93 & 21.88 & 21.81 & 19.95 & 19.06 & 18.63 \\
\hline Industrial Sector & 1.05 & 2.01 & 4.70 & 6.00 & 5.51 & 4.85 & 4.67 & 4.38 \\
\hline 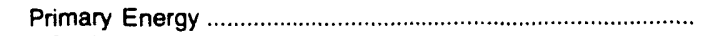 & .83 & 1.61 & 3.97 & 4.86 & 4.14 & 3.51 & 3.40 & 3.06 \\
\hline Coal & .41 & 1.24 & 1.59 & 1.66 & 1.57 & 1.52 & 1.42 & 1.35 \\
\hline 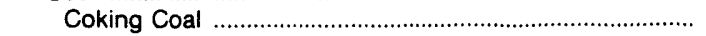 & - & - & - & - & - & - & - & - \\
\hline 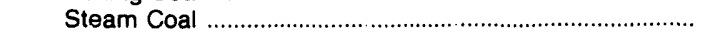 & .41 & 1.24 & 1.59 & 1.66 & 1.57 & 1.52 & 1.42 & 1.35 \\
\hline 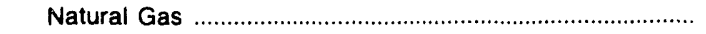 & .36 & .77 & 2.51 & 3.87 & 3.36 & 2.80 & 3.09 & 2.51 \\
\hline 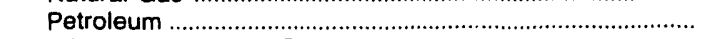 & 1.48 & 2.85 & 6.29 & 7.07 & 5.78 & 5.52 & 4.89 & 5.19 \\
\hline 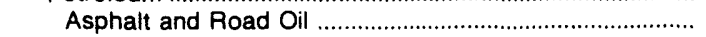 & .72 & 2.06 & 3.77 & 5.03 & 3.89 & 3.55 & 3.46 & 3.00 \\
\hline Distillate Fuel & .75 & 2.15 & 5.28 & 5.32 & 3.97 & 4.56 & 3.70 & 4.12 \\
\hline 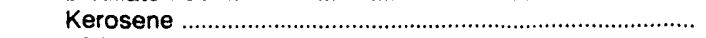 & .81 & 2.30 & 5.52 & 5.78 & 4.32 & 4.95 & 4.02 & 4.48 \\
\hline LPG & 1.16 & 2.46 & 4.88 & 8.25 & 8.25 & 6.45 & 6.23 & 5.21 \\
\hline 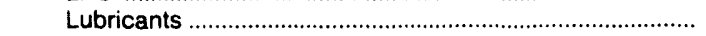 & 5.08 & 7.49 & 14.36 & 17.61 & 15.59 & 13.58 & 14.61 & 17.90 \\
\hline Motor Gasoline & 2.83 & 4.59 & 9.97 & 9.47 & 7.13 & 7.36 & 7.37 & 8.40 \\
\hline 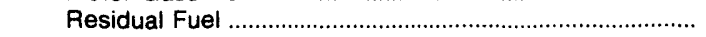 & .57 & 1.92 & 2.88 & 4.07 & 2.86 & 2.49 & 2.38 & 2.25 \\
\hline Other & 2.19 & 3.20 & 7.69 & 8.60 & 7.14 & 7.78 & 6.70 & 8.21 \\
\hline 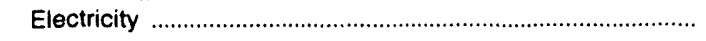 & 3.87 & 6.31 & 10.47 & 13.50 & 14.16 & 12.71 & 12.15 & 11.81 \\
\hline 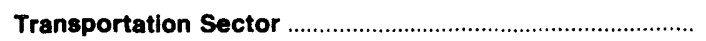 & 2.60 & 4.24 & 9.34 & 8.95 & 7.13 & 7.31 & 7.27 & 8.25 \\
\hline Primary Energy & 2.60 & 4.24 & 9.34 & 8.95 & 7.13 & 7.31 & 7.27 & 8.25 \\
\hline 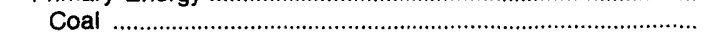 & .41 & 1.24 & - & - & - & - & - & - \\
\hline Petroleum & 2.60 & 4.24 & 9.34 & 8.95 & 7.13 & 7.31 & 7.27 & 8.25 \\
\hline 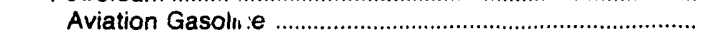 & 2.17 & 3.45 & 9.02 & 9.99 & 8.41 & 7.55 & 7.41 & 8.28 \\
\hline 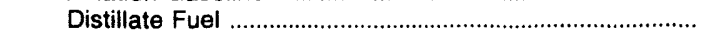 & 1.27 & 2.65 & 6.97 & 6.85 & 6.76 & 7.01 & 6.77 & 7.55 \\
\hline 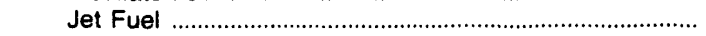 & .75 & 2.09 & 6.47 & 6.28 & 4.36 & 4.27 & 4.12 & 4.57 \\
\hline LPG & 1.16 & 2.46 & 4.88 & 8.25 & 8.25 & 6.45 & 6.23 & 5.21 \\
\hline 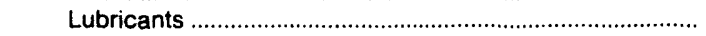 & 5.08 & 7.49 & 14.36 & 17.61 & 15.59 & 13.58 & 14.61 & 17.90 \\
\hline Motor Gasoline . & 2.83 & 4.59 & 9.97 & 9.47 & 7.13 & 7.36 & 7.37 & 8.40 \\
\hline 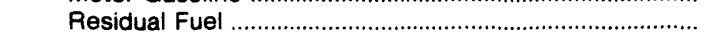 & .66 & - & - & - & - & 2.33 & - & 2.08 \\
\hline 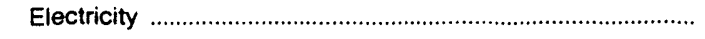 & - & - & - & - & - & - & - & - \\
\hline Yotal Energy" & 1.80 & 3.12 & 6.73 & 8.02 & 7.28 & 7.05 & 6.85 & 7.16 \\
\hline 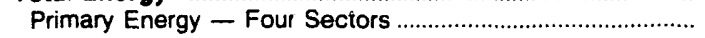 & 1.42 & 2.52 & 5.79 & 6.40 & 5.43 & 5.20 & 5.11 & 5.49 \\
\hline 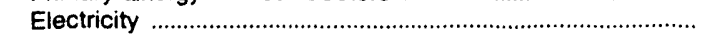 & 6.39 & 9.11 & 13.97 & 19.02 & 19.25 & 18.30 & 17.59 & 17.30 \\
\hline Electric Utility Sector & .30 & .75 & 1.32 & 1.48 & 1.31 & 1.21 & 1.19 & 1.17 \\
\hline 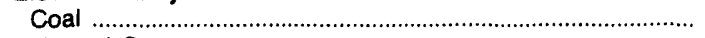 & .32 & .85 & 1.39 & 1.48 & 1.37 & 1.25 & 1.24 & 1.22 \\
\hline 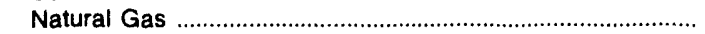 & .27 & .68 & 2.41 & 3.61 & 3.42 & 2.15 & 2.03 & 2.67 \\
\hline Petroleum & .74 & 2.05 & 5.41 & 5.88 & 3.63 & 4.06 & 3.72 & 4.45 \\
\hline 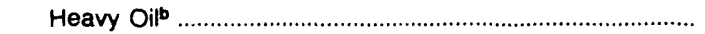 & .70 & 1.93 & 3.78 & 3.99 & - & - & - & - \\
\hline 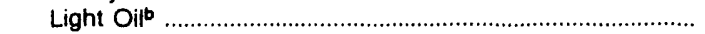 & .75 & 2.11 & 6.06 & 5.93 & 3.63 & 4.06 & 3.72 & 4.45 \\
\hline 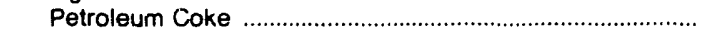 & - & - & - & - & - & - & - & - \\
\hline Nuclear Fuel & - & .25 & .39 & .94 & .75 & .71 & .63 & .67 \\
\hline Wood and Waste & .65 & .92 & 1.74 & .79 & .32 & .95 & .87 & .82 \\
\hline 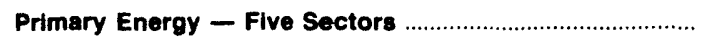 & 1.19 & 2.16 & 4.60 & 4.93 & 4.17 & 3.91 & 3.82 & 4.05 \\
\hline
\end{tabular}

-There are no direct fuel costs for hydroelectric, geothermal, centralized solar, or wind energy. Wood and other biomass fuels are not included, except those consumed at electric utilities.

beavy oil includes Grade Nos. 4, 5, and 6, and residual fuel oils. Light oil includes Grade No. 2 heating oil, kerosene, and jet fuel.

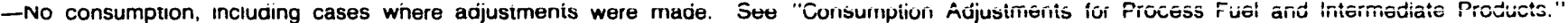

Sources: Data sources, estimation procedures, and assumptions are described in the "Documentation" section of this report. 


\begin{tabular}{|c|c|c|c|c|c|c|c|c|}
\hline Sector and Energy Source & 1970 & 1975 & 1980 & 1985 & 1986 & 1987 & 1888 & 1989 \\
\hline Residential Sector & 332.2 & 554.3 & $1,020.5$ & $1,345.8$ & $1,306.0$ & $1,206.8$ & $1,310.1$ & $1,343.9$ \\
\hline Primary Energy & 160.9 & 256.7 & 467.9 & 588.4 & 535.6 & 421.7 & 500.1 & 556.9 \\
\hline Coal & 1.6 & 3.3 & 2.1 & 7.1 & 5.9 & 7.1 & 7.3 & 3.7 \\
\hline 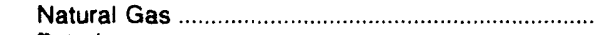 & 92.9 & 134.7 & 271.2 & 424.1 & 379.8 & 309.9 & 364.6 & 363.8 \\
\hline 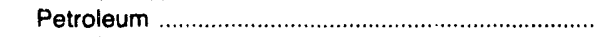 & 66.4 & 118.8 & 194.6 & 157.2 & 149.8 & 104.7 & 128.2 & 189.3 \\
\hline 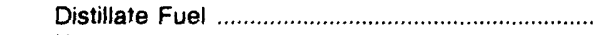 & 15.8 & 26.9 & 94.5 & 49.7 & 43.0 & 37.8 & 31.2 & 30.2 \\
\hline 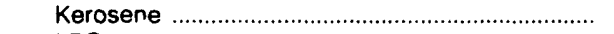 & 2.9 & 2.3 & 2.2 & 7.3 & 2.8 & 1.9 & 2.4 & 1.5 \\
\hline LPG & 47.6 & 89.5 & 98.0 & 100.2 & 104.0 & 65.0 & 94.7 & 157.7 \\
\hline 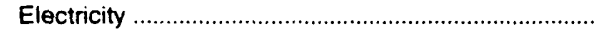 & 171.3 & 297.5 & 552.6 & 757.4 & 770.4 & 785.1 & 810.0 & 787.0 \\
\hline Commercial Sector & 151.3 & 287.6 & 505.7 & 780.1 & 741.3 & 655.4 & 699.2 & 686.0 \\
\hline Primary Energy & 55.5 & 103.3 & 206.7 & 309.3 & 253.9 & 198.2 & 235.2 & 222.0 \\
\hline Coal & 1.0 & 2.0 & 1.9 & 6.4 & 5.5 & 6.9 & 7.2 & 3.5 \\
\hline 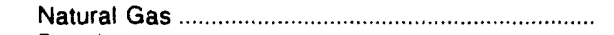 & 39.4 & 71.1 & 144.0 & 231.3 & 194.7 & 147.3 & 181.2 & 180.4 \\
\hline 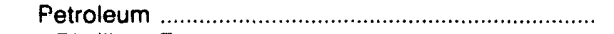 & 15.1 & 30.2 & 60.8 & 71.6 & 53.7 & 44.0 & 46.8 & 38.2 \\
\hline 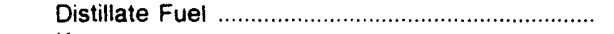 & 5.5 & 10.1 & 28.2 & 33.4 & 15.1 & 18.5 & 13.6 & 10.4 \\
\hline 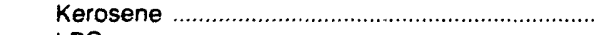 & .1 & .1 & .2 & .4 & $\dot{0}$ & .1 & .1 & .2 \\
\hline LPG & 5.3 & 11.0 & 12.3 & 26.0 & 27.5 & 14.8 & 19.6 & 16.7 \\
\hline Motor Gasoline ................................................... & 4.0 & 7.8 & 18.3 & 11.8 & 10.2 & 10.3 & 13.1 & 10.3 \\
\hline 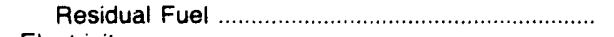 & .3 & 1.2 & 1.9 & $\cdot$ & .8 & .3 & .3 & .6 \\
\hline 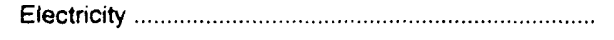 & 95.8 & 184.3 & 299.0 & 470.8 & 487.4 & 457.3 & 464.0 & 464.0 \\
\hline 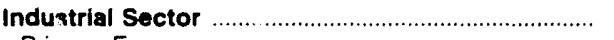 & 273.1 & 533.0 & $1,338.4$ & $1,482.6$ & $1,346.2$ & $1,164.5$ & $1,207.1$ & $1,087.8$ \\
\hline Primary Energy & 202.6 & 390.4 & $1,005.5$ & $1,044.3$ & 873.0 & 719.4 & 750.1 & 643.8 \\
\hline Coal & 17.8 & 35.1 & 51.6 & 59.1 & 55.6 & 64.8 & 59.4 & 72.7 \\
\hline 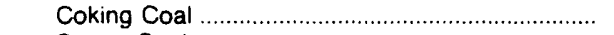 & - & - & - & - & - & - & - & - \\
\hline 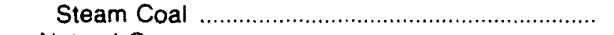 & 17.8 & 35.1 & 51.6 & 59.1 & 55.6 & 64.8 & 59.4 & 72.7 \\
\hline Natural Gas & 36.3 & 94.6 & 288.2 & 340.2 & 272.6 & 249.1 & 317.3 & 226.9 \\
\hline 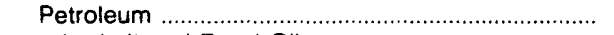 & 148.5 & 260.6 & 665.7 & 645.0 & 544.8 & 405.5 & 373.5 & 344.1 \\
\hline Asphalt and Road Oil ............................................ & 13.9 & 31.4 & 42.5 & 67.6 & 52.6 & 42.1 & 50.8 & 34.1 \\
\hline 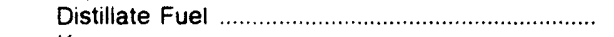 & 25.8 & 58.6 & 144.4 & 148.4 & 135.4 & 131.6 & 110.8 & 98.7 \\
\hline 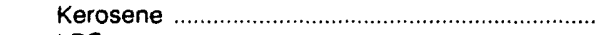 & .7 & .9 & 3.7 & 1.6 & 1.0 & 1.2 & .5 & .7 \\
\hline (.). & 13.0 & 51.2 & 117.6 & 242.4 & 232.4 & 111.0 & 98.2 & 84.0 \\
\hline 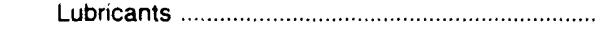 & 6.8 & 7.0 & 16.7 & 18.6 & 16.1 & 15.8 & 16.5 & 20.7 \\
\hline Motor Gasoline & 80.0 & 91.5 & 136.7 & 84.8 & 56.5 & 57.5 & 54.6 & 57.5 \\
\hline 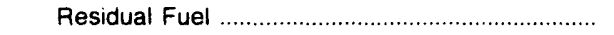 & .9 & 3.4 & 5.0 & 5.5 & 10.2 & 1.5 & 3.8 & 2.7 \\
\hline Other & 7.4 & 16.7 & 199.1 & 75.9 & 40.7 & 44.7 & 38.2 & 45.7 \\
\hline 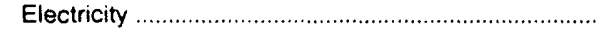 & 70.5 & 142.6 & 332.9 & 438.4 & 473.2 & 445.1 & 457.0 & 444.0 \\
\hline Transportation Sector & 499.2 & 984.9 & $2,103.8$ & $\lceil, 869.2$ & $1,490.8$ & $1,578.4$ & $1,611.5$ & $1,653.5$ \\
\hline 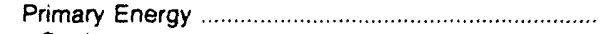 & 499.2 & 984.9 & $2,103.8$ & $1,869.2$ & $1,490.8$ & $1,578.4$ & $1,611.5$ & $1,853.5$ \\
\hline 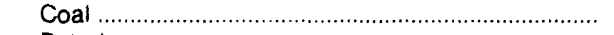 & 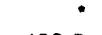 & $\cdot$ & - & - & - & - & - & - \\
\hline Petroleum & 499.2 & 984.9 & $2,103.8$ & $1,869.2$ & $1,490.8$ & $1,578.4$ & $1,611.5$ & $1,853.5$ \\
\hline 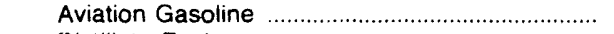 & 2.8 & 3.3 & 8.4 & 4.2 & 6.4 & 4.2 & 5.4 & 4.6 \\
\hline 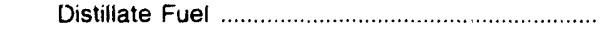 & 32.2 & 105.7 & 321.6 & 320.9 & 312.8 & 355.8 & 350.6 & 403.9 \\
\hline Jet Fuel & 3.0 & 9.8 & 29.6 & 20.9 & 14.5 & 18.7 & 16.5 & 19.3 \\
\hline LPG & .3 & .5 & .6 & 3.5 & 5.1 & 1.2 & 1.3 & 1.2 \\
\hline 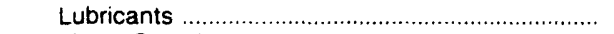 & 14.8 & 22.7 & 45.4 & 50.7 & 43.9 & 43.2 & 44.9 & 56.4 \\
\hline 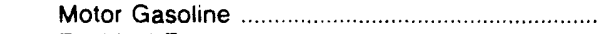 & 446.0 & 842.8 & $1,698.2$ & $1,469.1$ & $1,108.0$ & $1,155.2$ & $1,192.8$ & $1,368.0$ \\
\hline 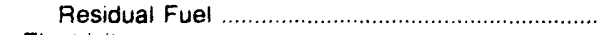 & .1 & - & - & - & - & .2 & - & • \\
\hline 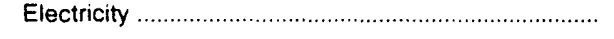 & - & - & - & - & - & - & - & - \\
\hline Total Energya & $1,255.8$ & $2,359.7$ & $4,968.4$ & $5,477.7$ & $4,884.3$ & $4,605.2$ & $4,827.8$ & $4,971.2$ \\
\hline 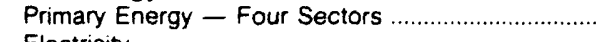 & 918.3 & $1,735.3$ & $3,783.9$ & $3,811.2$ & $3,153.2$ & $2,917.8$ & $3,096.8$ & $3,276.1$ \\
\hline Electricity & 337.5 & 624.4 & $1,184.5$ & $1,666.6$ & $1,731.1$ & $1,687.4$ & $1,731.0$ & $1,695.0$ \\
\hline Electric Utility Sector & 50.4 & 132.5 & 313.1 & 366.7 & 335.2 & 326.2 & 354.1 & 350.1 \\
\hline 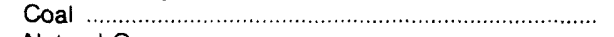 & 27.0 & 85.0 & 277.7 & 335.3 & 303.6 & 296.4 & 316.7 & 317.9 \\
\hline Natural Gas & 21.5 & 32.0 & 16.6 & 7.7 & 4.7 & 7.1 & 11.1 & 6.5 \\
\hline Petroleum & 1.6 & 8.8 & 7.4 & 3.6 & 2.2 & 2.7 & 2.7 & 2.9 \\
\hline (2) & .2 & 2.6 & 1.5 & .1 & - & - & - & - \\
\hline Light Oib & 1.4 & 6.2 & 5.9 & 3.5 & 2.2 & 2.7 & 2.7 & 2.9 \\
\hline 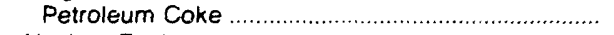 & - & - & - & - & - & - & - & - \\
\hline 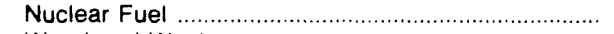 & - & 6.3 & 10.9 & 19.6 & 24.4 & 19.3 & 23.0 & 22.6 \\
\hline Wood and Waste & .3 & .4 & .5 & .5 & .2 & .7 & .5 & .2 \\
\hline 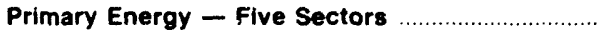 & 968.7 & $1,867.8$ & $4,097.0$ & $4,177.9$ & $3,488,4$ & $3,244.0$ & $3,450.8$ & $3,626.2$ \\
\hline
\end{tabular}

-There are no direct fuel costs for hydroelectric, geothermal, centralized solar, or wind energy. Wood and other biomass fuels are not included, except those consumed at electric utilities.

b Heavy oil includes Grade Nos. 4, 5, and 6, and residual fuel oils. Light oil includes Grade No. 2 heating oil, kerosene, and jet fuel.

-No consumption, including cases where adjustments were made. See "Consumption Adjustments for Process Fuel and intermediate Products."

- Value less tnan úú miliion doiliars.

Note: Totals may not equal sum of components due to independent rounding.

Sources: Data sources, estimation procedures, and assumptions are described in the "Documentation" section of this report. 


\section{K Energy Price and Expenditure Estimates by Source, Kansas \\ A $1970,1975,1980$, and $1985-1989$}

\begin{tabular}{|c|c|c|c|c|c|c|c|c|}
\hline Energy Source & 1970 & 1975 & 1980 & 1985 & 1986 & 1887 & 1988 & 1989 \\
\hline & \multicolumn{8}{|c|}{ Prices in Dollars per Mililon Btu } \\
\hline 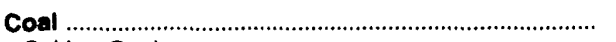 & 0.34 & 0.68 & 1.08 & 1.41 & 1.33 & 1.27 & 1.24 & 1.24 \\
\hline 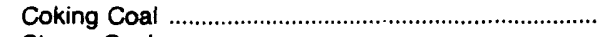 & - & - & - & - & - & - & - & - \\
\hline Steam Coal & .34 & .68 & 1.08 & 1.41 & 1.33 & 1.27 & 1.24 & 1.24 \\
\hline 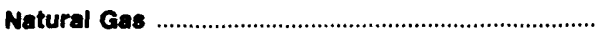 & .39 & .67 & 2.14 & 3.58 & 3.30 & 3.15 & 3.03 & 3.36 \\
\hline Petroleum & 1.86 & 3.41 & 7.38 & 7.20 & 6.31 & 6.45 & 6.11 & 6.90 \\
\hline 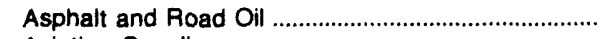 & .74 & 2.10 & 4.02 & 5.22 & 3.88 & 3.70 & 3.49 & 3.00 \\
\hline Aviation Gasoline & 2.17 & 3.45 & 9.02 & 9.99 & 8.41 & 7.55 & 7.41 & 8.28 \\
\hline 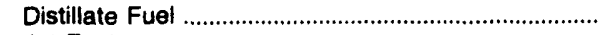 & .89 & 2.43 & 6.52 & 6.42 & 5.54 & 5.66 & 5.16 & 6.30 \\
\hline Jet Fuel & .75 & 2.09 & 6.47 & 5.94 & 3.73 & 4.01 & 3.81 & 4.27 \\
\hline Kerosene & .96 & 2.61 & 5.25 & 6.76 & 4.72 & 3.44 & 3.49 & 5.82 \\
\hline LPG & 1.27 & 2.65 & 4.41 & 4.39 & 7.93 & 7.43 & 7.38 & 7.29 \\
\hline 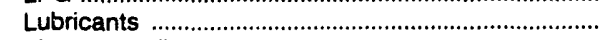 & 5.08 & 7.49 & 14.36 & 17.61 & 15.59 & 13.58 & 14.61 & 17.90 \\
\hline Motor Gasoline & 2.64 & 4.50 & 9.27 & 9.28 & 6.72 & 7.05 & 6.78 & 7.66 \\
\hline Residual Fuel & .47 & 1.60 & 3.24 & 3.90 & 2.28 & 2.36 & 1.87 & 2.05 \\
\hline 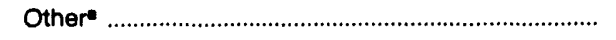 & .68 & 1.65 & 3.72 & 5.81 & 6.35 & 6.22 & 5.28 & 6.11 \\
\hline 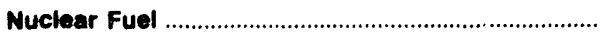 & - & - & $\sim$ & .84 & .63 & .51 & .45 & .32 \\
\hline 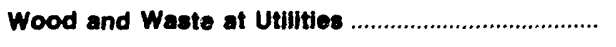 & - & - & - & - & - & - & - & - \\
\hline 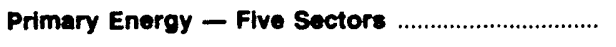 & .98 & 1.88 & 3.87 & 4.29 & 3.76 & 3.71 & 3.61 & 3.83 \\
\hline 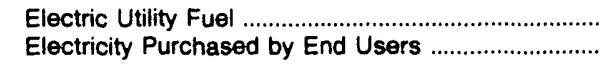 & $\begin{array}{r}.30 \\
5.74\end{array}$ & $\begin{array}{r}.72 \\
7.72\end{array}$ & $\begin{array}{r}1.38 \\
13.75\end{array}$ & $\begin{array}{r}1.44 \\
19.07\end{array}$ & $\begin{array}{r}1.22 \\
20.53\end{array}$ & $\begin{array}{r}1.16 \\
20.06\end{array}$ & $\begin{array}{r}1.13 \\
19.22\end{array}$ & $\begin{array}{r}1.04 \\
18.83\end{array}$ \\
\hline \multirow[t]{2}{*}{ Total Enorgy } & 1.54 & 2.86 & 5.93 & 7.23 & 7.01 & 6.81 & 6.68 & 7.25 \\
\hline & \multicolumn{8}{|c|}{ Expenditures in Millions of Dollars } \\
\hline 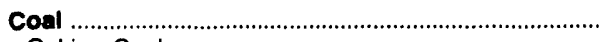 & 3.7 & 42.5 & 207.0 & 365.8 & 334.3 & 340.5 & 334.5 & 330.4 \\
\hline 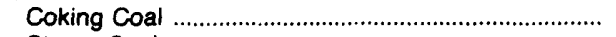 & - & - & - & - & - & - & - & - \\
\hline 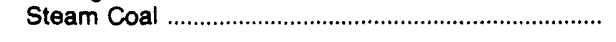 & 3.7 & 42.5 & 207.0 & 365.8 & 334.3 & 340.5 & 334.5 & 330.4 \\
\hline 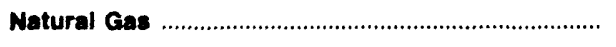 & 175.6 & 248.1 & 808.1 & 960.1 & 768.5 & 809.7 & 772.4 & 828.8 \\
\hline Petroleum & 5.32 .8 & $1,160.4$ & $2,501.4$ & $2,676.8$ & $2,353.4$ & $2,403.7$ & $2,465.4$ & $2,623.0$ \\
\hline 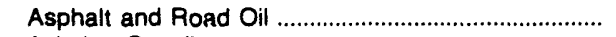 & 10.7 & 30.1 & 80.5 & 58.9 & 68.4 & 64.2 & 101.4 & 61.8 \\
\hline Aviation Gasoline & 3.6 & 3.1 & 10.1 & 6.9 & 6.9 & 4.6 & 5.5 & 6.5 \\
\hline 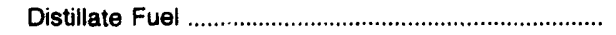 & 43.3 & 159.8 & 560.3 & 561.8 & 461.6 & 550.4 & 498.4 & 578.8 \\
\hline 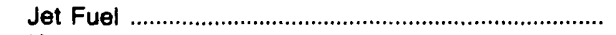 & 6.4 & 15.0 & 89.3 & 147.6 & 148.2 & 96.7 & 89.2 & 91.5 \\
\hline 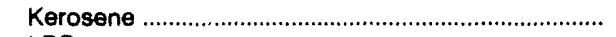 & 1.7 & 1.5 & 14.6 & 3.3 & 2.0 & 1.2 & .7 & 2.0 \\
\hline LPG & 37.1 & 83.9 & 131.0 & 358.7 & 506.6 & 448.9 & 514.6 & 500.4 \\
\hline 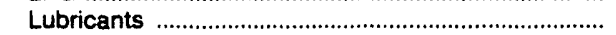 & 20.2 & 35.1 & 88.1 & 98.3 & 85.1 & 83.8 & 86.9 & 108.2 \\
\hline 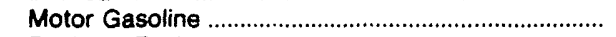 & 399.6 & 756.2 & $1,440.7$ & $1,375.2$ & $1,005.0$ & $1,077.4$ & $1,099.1$ & $1,200.6$ \\
\hline 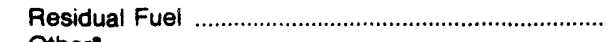 & 1.5 & 49.8 & 17.9 & 1.6 & 5.8 & 4.0 & 7.6 & 4.7 \\
\hline Othere & 8.8 & 25.8 & 69.1 & 64.4 & 63.9 & 72.6 & 62.0 & 67.4 \\
\hline 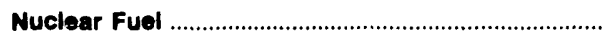 & - & - & - & 34.9 & 47.7 & 35.7 & 32.4 & 33.3 \\
\hline 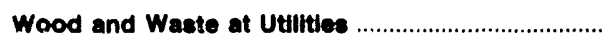 & - & - & - & - & - & - & - & - \\
\hline Primary Energy - Five Sectors .................................. & 712.2 & $1,451.0$ & $3,516.5$ & $4,037.6$ & $3,504,0$ & $3,589.7$ & $3,604.7$ & $3,815.5$ \\
\hline 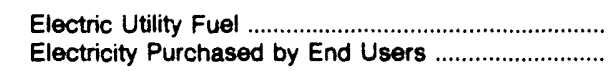 & $\begin{array}{l}-53.9 \\
259.0\end{array}$ & $\begin{array}{r}-159.5 \\
444.0\end{array}$ & $\begin{array}{r}-394.3 \\
986.7\end{array}$ & $\begin{array}{r}-453.4 \\
1,520.6\end{array}$ & $\begin{array}{r}-412.3 \\
1,656.3\end{array}$ & $\begin{array}{r}-405.4 \\
1,658.4\end{array}$ & $\begin{array}{r}-404.4 \\
1,680.9\end{array}$ & $\begin{array}{r}-400.5 \\
1,646.7\end{array}$ \\
\hline Total Energy & 917.3 & $1,735.4$ & $4,108.8$ & $5,104.8$ & $4,748.0$ & $4,842.6$ & $4,881.3$ & $5,061.6$ \\
\hline
\end{tabular}

- Includes petroleum coke used at electric utilities.

-There are no direct fuel costs for hydroelectric, geothermal, centralized solar, or wind energy. Wood and other biomass fuels are not included, except those consumed at electric utilities.

-No consumption, including cases where adjustments were made. See "Consumption Adjustments for Process Fuel and Intermediate Products."

Note: Expenditure totals may not equal sum of components due to independent rounding.

Sources: Data sources, estimation procedures, and assumptions are described in the "Documentation" section of this report. 


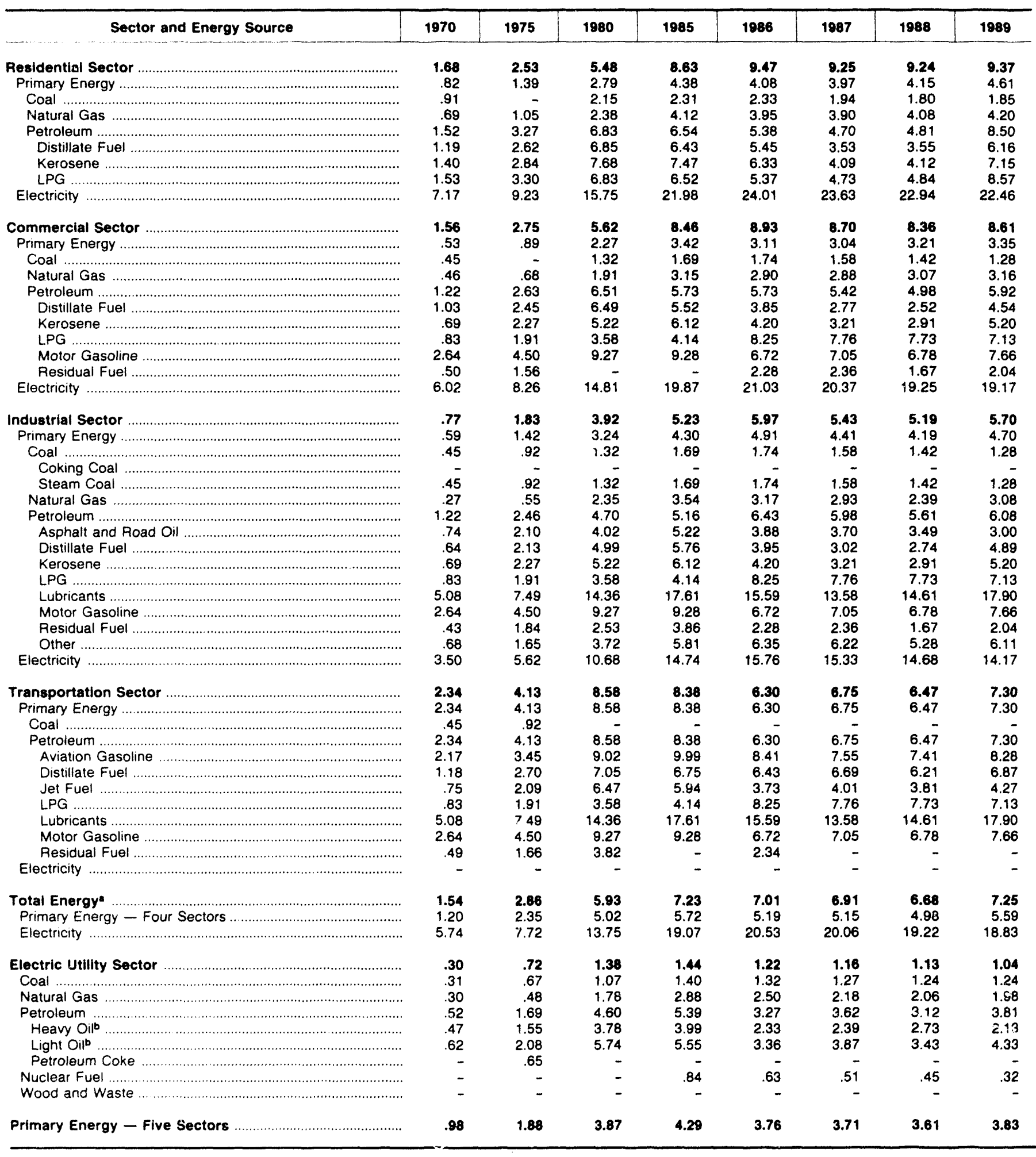

- There are no direct fuel costs for hydroelectric, jeothermal, centralized solar, or wind energy. Wood and other biomass fuels are not included, except those consumed at electric utlities.

bHeavy oil includes Grade Nos. 4, 5, and 6, and residual fuel oils. Light oil includes Grade No. 2 heating oil, kerosene, and jet fuel.

- No consumption. including cases where adjustments were made. See "Consumption Adjustments for Process Fuel and Intermediate Products."

Sources: Data sources, estimation procedures, and assumptions are described in the "Documentation" section of this report. 
K Energy Expenditure Estimates by Sector, Kansas

A $1970,1975,1980$, and $1985-1989$

N (Million Dollars)

$\mathbf{S}$

\begin{tabular}{|c|c|c|c|c|c|c|c|c|}
\hline Sector and Energy Source & 1970 & 1975 & 1980 & 1985 & 1986 & 1987 & 1988 & 1989 \\
\hline $\begin{array}{l}\text { Residential Sector } \\
\text { Primary Energy }\end{array}$ & $\begin{array}{r}226.7 \\
95.9\end{array}$ & $\begin{array}{l}338.9 \\
159.5\end{array}$ & $\begin{array}{l}646.6 \\
260.4\end{array}$ & $\begin{array}{l}998.6 \\
384.0\end{array}$ & $\begin{array}{l}997.3 \\
313.7\end{array}$ & $\begin{array}{r}1,011.7 \\
317.1\end{array}$ & $\begin{array}{r}1,062.6 \\
348.6\end{array}$ & $\begin{array}{r}1,068.1 \\
386.2\end{array}$ \\
\hline Coal & .1 & & .1 & & & & & .1 \\
\hline 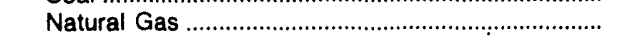 & 66.7 & 101.2 & 201.9 & 322.7 & 274.8 & 284.9 & 307.2 & 317.1 \\
\hline Petroleum ........... & 29.2 & 58.3 & 58.4 & 61.3 & 38.9 & 32.2 & 41.3 & 69.0 \\
\hline 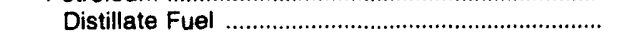 & .4 & 1.5 & 6.0 & 2.5 & .8 & .4 & 6 & 1.1 \\
\hline 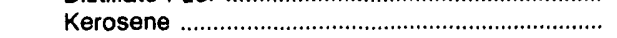 & .9 & 1.0 & .2 & 1.7 & .7 & .4 & .4 & .8 \\
\hline LPG & 27.9 & 55.9 & 52.2 & 57.1 & 37.5 & 31.4 & 40.3 & 67.1 \\
\hline 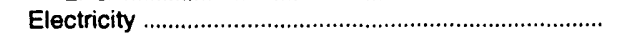 & 130.8 & 179.4 & 386.2 & 614.6 & 683.6 & 694.6 & 714.0 & 682.0 \\
\hline Commercial Sector & 112.0 & 208.4 & 488.0 & 770.3 & 783.8 & 776.6 & 799.3 & 806.5 \\
\hline 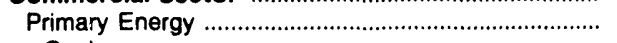 & 30.6 & 50.3 & 144.1 & 216.1 & 184.0 & 182.6 & 208.3 & 209.5 \\
\hline Coal & .1 & & .1 & & & & & .1 \\
\hline 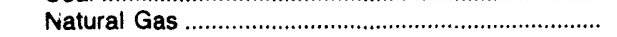 & 23.9 & 34.7 & 111.7 & 178.0 & 159.6 & 161.9 & 185.2 & 183.9 \\
\hline 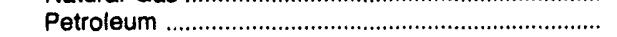 & 6.6 & 15.6 & 32.3 & 38.0 & 24.4 & 20.7 & 23.1 & 25.5 \\
\hline 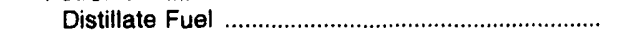 & .7 & 3.0 & 13.6 & 22.5 & 7.7 & 4.4 & 5.7 & 8.8 \\
\hline 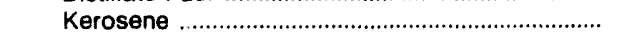 & .1 & .2 & .3 & .5 & .2 & .2 & .1 & .5 \\
\hline 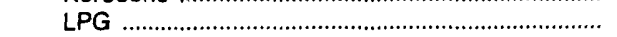 & 2.7 & 5.7 & 4.8 & 6.4 & 10.2 & 9.1 & 11.3 & 9.8 \\
\hline 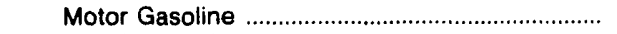 & 3.0 & 6.3 & 13.6 & 8.6 & 6.2 & 7.0 & 5.9 & 6.2 \\
\hline Residual Fuel ......................................................... & .1 & .4 & - & - & .1 & & & .2 \\
\hline Electricity & 81.4 & 158.1 & 343.9 & 554.2 & 599.8 & 593.9 & 591.0 & 597.0 \\
\hline Industrial Sector ........ & 163.3 & 357.7 & $1,025.1$ & $1,406.5$ & $1,446.5$ & $1,398.1$ & $1,387.0$ & $1,404.8$ \\
\hline 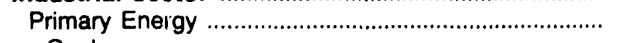 & 116.5 & 251.2 & 768.6 & $1,054.7$ & $1,073.6$ & $1,028.4$ & $1,011.1$ & $1,037.1$ \\
\hline Coal & 1.0 & 2.5 & 9.4 & 13.1 & 9.7 & 8.7 & 6.5 & 5.2 \\
\hline 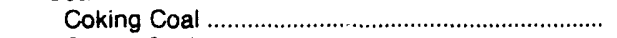 & - & - & - & & & - & - & - \\
\hline 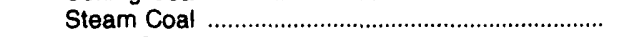 & 1.0 & 2.5 & 9.4 & 13.1 & 9.7 & 8.7 & 6.5 & 5.2 \\
\hline 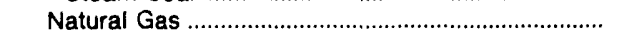 & 35.5 & 51.5 & 322.0 & 400.3 & 297.7 & 328.4 & 241.3 & 291.1 \\
\hline 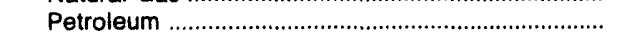 & 80.0 & 197.1 & 437.1 & 641.3 & 766.1 & 691.3 & 763.3 & 740.8 \\
\hline 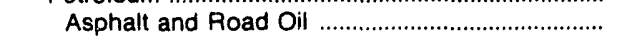 & 10.7 & 30.1 & 80.5 & 58.9 & 68.4 & 64.2 & 101.4 & 61.9 \\
\hline 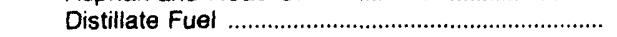 & 9.4 & 43.8 & 101.0 & 130.8 & 104.8 & 75.4 & 70.9 & 111.7 \\
\hline 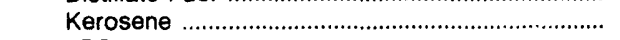 & .6 & .3 & 14.1 & 1.1 & 1.1 & .6 & .2 & .7 \\
\hline LPG & 5.4 & 19.7 & 72.5 & 293.4 & 455.3 & 405.0 & 458.1 & 417.5 \\
\hline 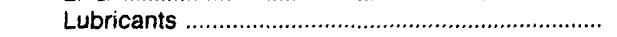 & 6.4 & 11.5 & 35.5 & 39.6 & 34.3 & 33.8 & 35.1 & 44.1 \\
\hline 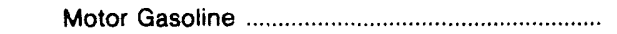 & 38.5 & 56.8 & 58.3 & 51.9 & 32.8 & 36.1 & 30.2 & 33.7 \\
\hline 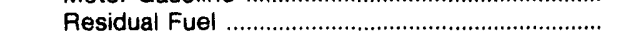 & .2 & 9.0 & 6.1 & 1.1 & 5.4 & 3.6 & 5.5 & 3.8 \\
\hline Other & 8.8 & 25.8 & 69.1 & 64.4 & 63.9 & 72.6 & 62.0 & 67.4 \\
\hline 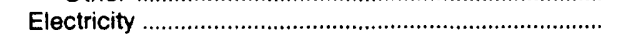 & 46.8 & 106.5 & 256.6 & 351.8 & 372.9 & 369.8 & 375.9 & 367.7 \\
\hline 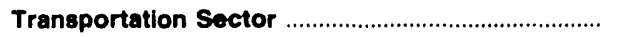 & 415.3 & 830.4 & $1,949.1$ & $1,929.5$ & $1,520.4$ & $1,656.2$ & $1,632.4$ & $1,782.2$ \\
\hline 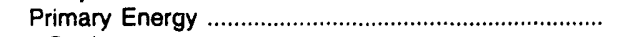 & 415.3 & 830.4 & $1,949.1$ & $1,929.5$ & $1,520,4$ & $1,656.2$ & $1,632.4$ & $1,782.2$ \\
\hline 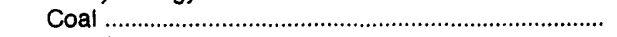 & & & - & & & & & \\
\hline 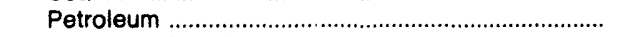 & 415.3 & 830.4 & $1,949.1$ & $1,929.5$ & $1,520.4$ & $1,656.2$ & $1,632.4$ & $1,782.2$ \\
\hline 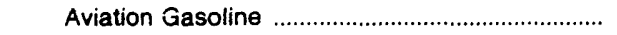 & 3.6 & 3.1 & 10.1 & 6.9 & 6.9 & 4.6 & 5.5 & 6.5 \\
\hline 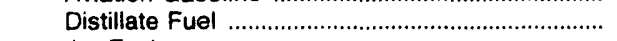 & 32.2 & 92.9 & 426.9 & 399.8 & 344.9 & 467.2 & 418.1 & 452.3 \\
\hline Jet Fuel ................ & 6.4 & 15.0 & 89.3 & 147.6 & 148.2 & 96.7 & 89.2 & 91.5 \\
\hline 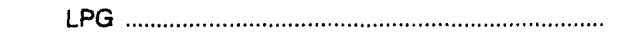 & 1.1 & 2.6 & 1.5 & 1.8 & 3.6 & 3.4 & 4.8 & 5.9 \\
\hline Lubricants .......................................................... & 13.8 & 23.6 & 52.5 & 58.6 & 50.8 & 50.0 & 51.9 & 65.2 \\
\hline 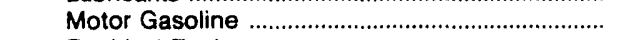 & 358.2 & 693.1 & $1,368.8$ & $1,314.7$ & 966.1 & 1.034 .3 & $1,063.0$ & $1,160.7$ \\
\hline 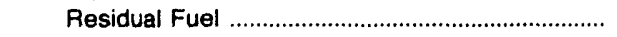 & • & .2 & " & - & • & - & - & - \\
\hline 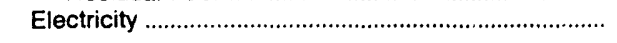 & - & - & - & - & - & - & - & - \\
\hline 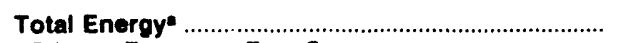 & 917.3 & $1,735.4$ & $4,108.8$ & $5,104.9$ & $4,748.0$ & $4,842.6$ & $4,881.3$ & $5,061.6$ \\
\hline Primary Energy - Four Sectors ................................... & 658.3 & $1,291.5$ & $3,122.2$ & $3,584.2$ & $3,091.7$ & $3,184.2$ & $3,200.4$ & $3,415.0$ \\
\hline 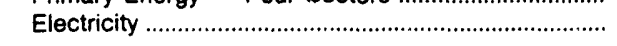 & 259.0 & 444.0 & 986.7 & $1,520.6$ & 1.656 .3 & $1,658.4$ & $1,680.9$ & $1,646.7$ \\
\hline Electric Utility Sector & 53.9 & 159.5 & 394.3 & 453.4 & 412.3 & 405.4 & 404.4 & 400.5 \\
\hline 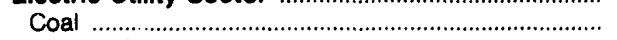 & 2.6 & 39.9 & 197.4 & 352.6 & 324.6 & 331.9 & 328.0 & 324.9 \\
\hline 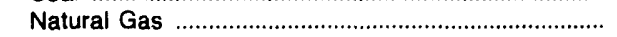 & 49.5 & 60.6 & 172.4 & 59.1 & 36.4 & 34.5 & 38.6 & 36.7 \\
\hline 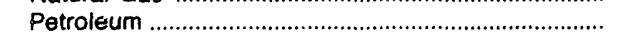 & 1.8 & 58.9 & 24.5 & 6.8 & 3.6 & 3.3 & 5.3 & 5.5 \\
\hline 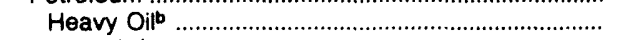 & 1.1 & 40.3 & 11.7 & .5 & .2 & .4 & 2.1 & .7 \\
\hline 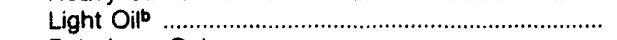 & .6 & 18.6 & 12.8 & 6.3 & 3.4 & 3.0 & 3.2 & 4.8 \\
\hline Petroleum Coke ........................................................ & - & & - & & & & & \\
\hline 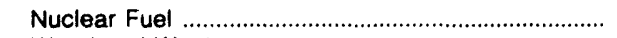 & - & - & - & 34.9 & 47.7 & 35.7 & 32.4 & 33.3 \\
\hline Wood and Waste & - & - & - & - & - & - & - & - \\
\hline Primary Energy — Five Sectore ……………................ & 712.2 & $1,451.0$ & $3,516.5$ & $4,037.6$ & $3,504.0$ & $3,589.7$ & $3,604.7$ & $3,815.5$ \\
\hline
\end{tabular}

-There are no direct fuel costs for hydroelectric, geothermal, centralized solar, or wind energy. Wood and other biomass fuels are not included, except those consumed at electric utilities.

b Heavy oil includes Grade Nos. 4, 5, and 6, and residual fuel oils. Light oil includes Grade No. 2 heating oil, kerosene, and jet fuel.

-No consumption, including cases where adjustments were made. See "Consumption Adjustments for Process Fuel and Intermediate Products."

- Value less than 0.05 million dollars.

Note: Totals may not equal sum of components due to independent rounding.

Sources: Data sources, estimation procedures, and assumptions are described in the "Documentation" section of this report. 


\begin{tabular}{|c|c|c|c|c|c|c|c|c|}
\hline Energy Source & 1970 & 1975 & 1980 & 1985 & 1986 & 1987 & 1988 & 1989 \\
\hline & \multicolumn{8}{|c|}{ Prices in Doliars per Million Btu } \\
\hline Coal & 0.26 & 0.75 & 1.37 & 1.48 & 1.39 & 1.33 & 1.26 & 1.22 \\
\hline Coking Coal & .38 & 1.60 & 1.81 & 1.93 & 1.75 & 1.63 & 1.73 & 1.75 \\
\hline 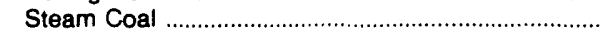 & .25 & .70 & 1.35 & 1.46 & 1.38 & 1.32 & 1.24 & 1.20 \\
\hline 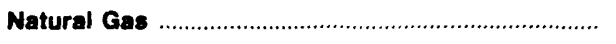 & .65 & 1.02 & 2.85 & 4.77 & 4.38 & 3.91 & 3.89 & 4.07 \\
\hline 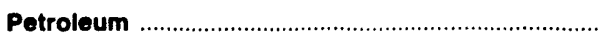 & 2.12 & 3.81 & 7.82 & 7.87 & 6.26 & 6.64 & 6.53 & 7.07 \\
\hline 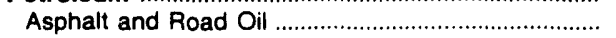 & .67 & 1.80 & 3.56 & 4.74 & 4.90 & 3.19 & 3.05 & 2.86 \\
\hline Aviation Gasoline & 2.17 & 3.45 & 9.02 & 9.99 & 8.41 & 7.55 & 7.41 & 8.28 \\
\hline Distillate Fuel & 1.21 & 2.58 & 6.41 & 6.81 & 5.39 & 5.91 & 5.81 & 6.49 \\
\hline Jet Fuel & .73 & 2.03 & 6.39 & 6.17 & 4.39 & 4.16 & 3.93 & 4.45 \\
\hline 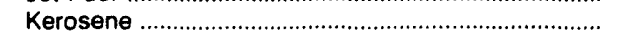 & 1.42 & 2.94 & 7.55 & 7.76 & 4.90 & 5.33 & 5.49 & 6.15 \\
\hline LPG & 1.90 & 3.60 & 5.85 & 6.90 & 7.22 & 8.09 & 7.37 & 6.56 \\
\hline 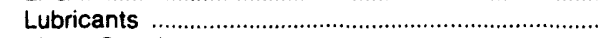 & 5.08 & 7.49 & 14.36 & 17.61 & 15.59 & 13.58 & 14.61 & 17.90 \\
\hline 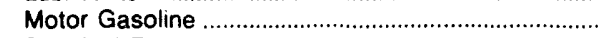 & 2.93 & 4.69 & 9.65 & 8.80 & 6.69 & 7.36 & 7.42 & 8.08 \\
\hline 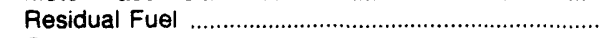 & .56 & 2.06 & 3.64 & 4.89 & 3.08 & 2.98 & 2.91 & 3.05 \\
\hline 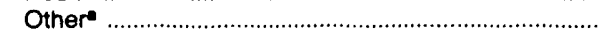 & 1.06 & 2.45 & 6.80 & 7.21 & 6.80 & 6.04 & 5.18 & 5.95 \\
\hline 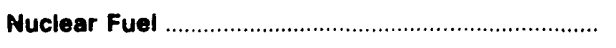 & - & - & - & - & - & - & - & - \\
\hline 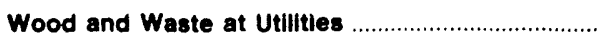 & - & - & - & - & - & - & - & - \\
\hline 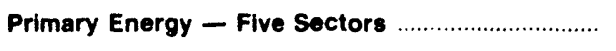 & .94 & 1.88 & 4.00 & 4.00 & 3.31 & 3.40 & 3.29 & 3.60 \\
\hline 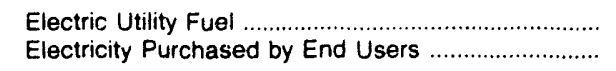 & $\begin{array}{r}.22 \\
3.37\end{array}$ & $\begin{array}{r}.64 \\
5.32\end{array}$ & $\begin{array}{r}1.32 \\
10.07\end{array}$ & $\begin{array}{r}1.43 \\
14.84\end{array}$ & $\begin{array}{r}1.34 \\
15.26\end{array}$ & $\begin{array}{r}1.26 \\
15.18\end{array}$ & $\begin{array}{r}1.20 \\
15.08\end{array}$ & $\begin{array}{r}1.14 \\
14.17\end{array}$ \\
\hline \multirow[t]{2}{*}{ Total Energy } & 1.66 & 3.27 & 6.73 & 8.02 & 7.25 & 7.36 & 7.30 & 7.47 \\
\hline & \multicolumn{8}{|c|}{ Expenditures in Millions of Dollars } \\
\hline (n) & 138.9 & 421.0 & 878.8 & $1,061.1$ & $1,045.4$ & 993.4 & $1,033.7$ & 933.9 \\
\hline 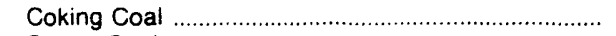 & 16.4 & 52.1 & 44.0 & 60.5 & 52.2 & 57.1 & 60.5 & 60.6 \\
\hline 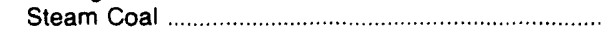 & 122.4 & 368.8 & 834.8 & $1,000.6$ & 993.2 & 936.2 & 973.2 & 873.3 \\
\hline Natural Gas & 136.7 & 185.7 & 511.8 & 722.4 & 630.7 & 587.4 & 637.0 & 689.6 \\
\hline Petroleum & 760.5 & $1,551.3$ & $3,867.9$ & $3,433.8$ & $2,716.2$ & $3,011.0$ & $3,176.9$ & $3,547.4$ \\
\hline 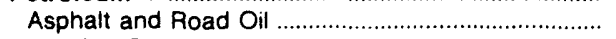 & 13.8 & 31.3 & 47.8 & 58.9 & 74.3 & 57.2 & 53.0 & 52.4 \\
\hline Aviation Gasoline & 3.6 & 2.2 & 5.1 & 3.3 & 3.6 & 2.4 & 2.3 & 2.2 \\
\hline Distillate Fuel & 58.0 & 164.1 & 855.7 & 863.2 & 640.8 & 705.8 & 835.7 & $1,063.4$ \\
\hline Jet Fuel & 12.6 & 24.6 & 104.4 & 119.3 & 87.8 & 113.5 & 110.8 & 127.7 \\
\hline Kerosene & 24.9 & 26.3 & 124.6 & 100.8 & 30.5 & 17.2 & 27.3 & 35.6 \\
\hline LPG & 67.5 & 143.7 & 216.4 & 179.9 & 170.2 & 224.4 & 214.1 & 182.2 \\
\hline 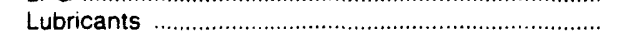 & 26.0 & 47.6 & 92.1 & 102.7 & 88.9 & 87.5 & 90.9 & 114.2 \\
\hline 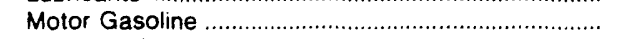 & 517.3 & $1,005.6$ & $2,019.1$ & $1,845.7$ & $1,494.7$ & $1,658.9$ & $1,722.4$ & $1,841.4$ \\
\hline 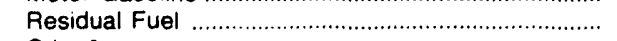 & 3.2 & 11.1 & 20.9 & 13.4 & 11.8 & 11.9 & 8.1 & 8.8 \\
\hline 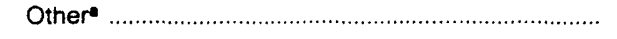 & 33.5 & 94.9 & 381.9 & 146.5 & 113.6 & 132.0 & 112.3 & 119.6 \\
\hline (2) & - & - & - & - & - & - & - & - \\
\hline 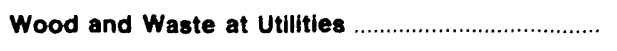 & - & - & - & - & - & - & - & - \\
\hline 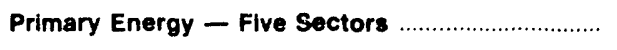 & $1,036.0$ & $2,158.0$ & $5,258.5$ & $5,217.2$ & $4,392.3$ & $4,591.7$ & $4,847.5$ & $5,170.9$ \\
\hline 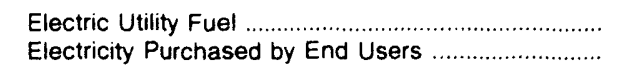 & $\begin{array}{l}-90.6 \\
354.9\end{array}$ & $\begin{array}{r}-309.8 \\
852.2\end{array}$ & $\begin{array}{r}-743.7 \\
1,698.6\end{array}$ & $\begin{array}{r}-883.4 \\
2,528.3\end{array}$ & $\begin{array}{r}-886.1 \\
2,552.6\end{array}$ & $\begin{array}{r}-832.6 \\
2.596 .4\end{array}$ & $\begin{array}{r}-884.5 \\
2.747 .0\end{array}$ & $\begin{array}{r}-768.0 \\
2,792.2\end{array}$ \\
\hline Total Energyb & $1,300.3$ & $2,700.5$ & $6,213.4$ & $6,862.1$ & $6,058.8$ & $6,355.5$ & $6,710.0$ & $7,195.1$ \\
\hline
\end{tabular}

Includes petroleum coke used at electric utilities.

-There are no direct fuel costs for hydroelectric, geothermal, centralized solar, or wind energy. Wood and other biomass fuels are not included, except those consumed at electric utilities.

- No consumption, including cases where adjustments were made. See "Consumption Adjustments for Process Fuel and Intermediate Products."

Note: Expenditure totals may not equal sum of components due to independent rounding.

Sources: Data sources, estimation procedures, and assumptions are described in the "Documentation" section of this report. 


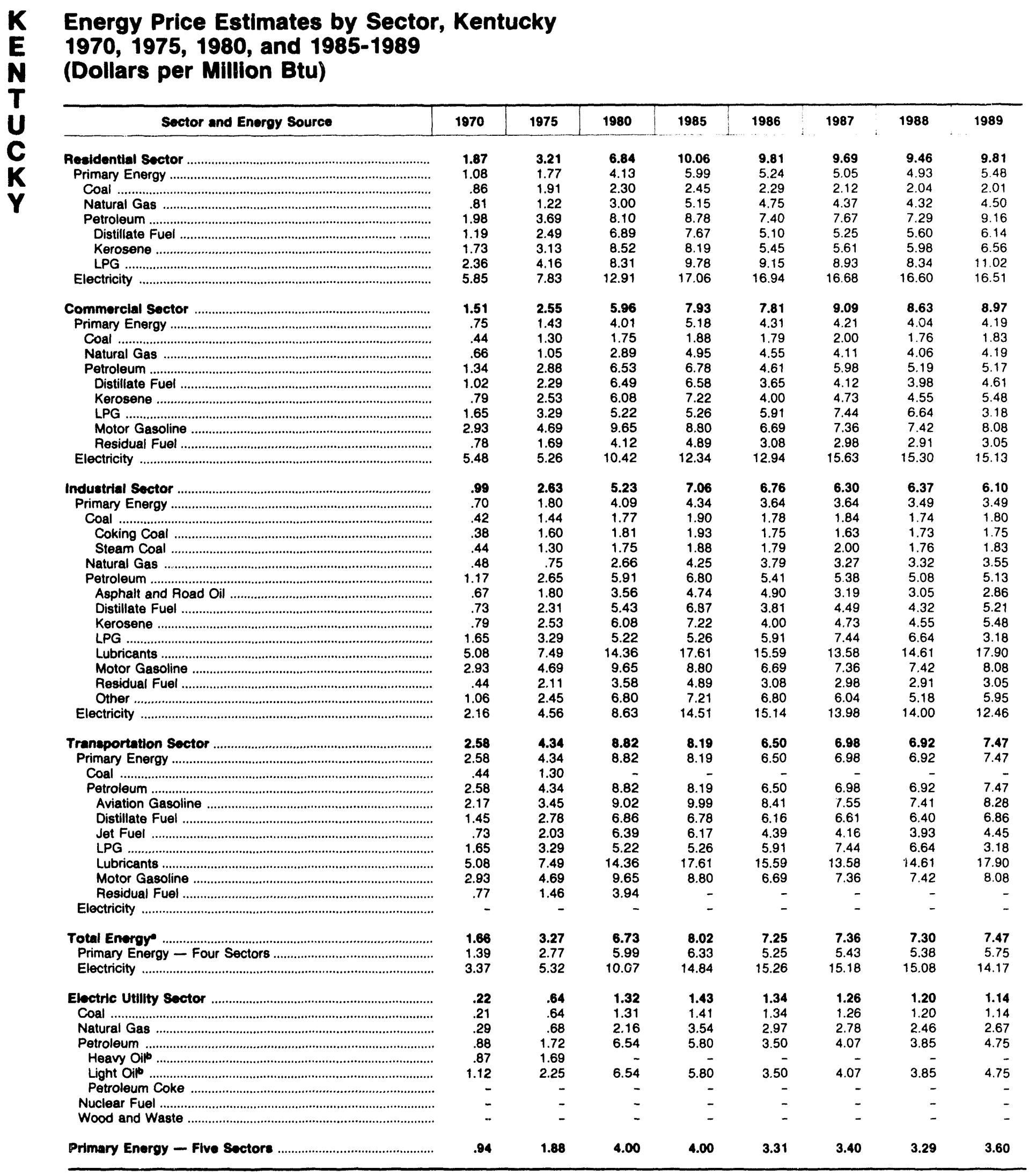

-There are no direct fuel costs for hydroelectric, geothermal, centralized solar, or wind energy. Wood and other biomass fuels are not included, except those consumed at electric utilities.

b Heavy oil includes Grade Nos. 4, 5, and 6, and residual fuel oils. Light oil includes Grade No. 2 heating oil, kerosene, and jet fuel.

-No consumption, including cases where adjustments were made. See "Consumption Adjustments for Process Fuel and intermediate Products." Sources: Data sources, estimation procedures, and assumptions are described in the "Documentation" section of this report. 


\begin{tabular}{|c|c|c|c|c|c|c|c|c|}
\hline Soctor and Energy Source & 1970 & 1975 & 1980 & 1985 & 1986 & 1987 & 1988 & 1989 \\
\hline 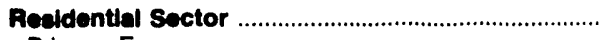 & 268.1 & 441.0 & 986.8 & $1,358.4$ & $1,311.6$ & $1,332.5$ & $1,397.6$ & $1, \mathbf{4 4 3 . 7}$ \\
\hline 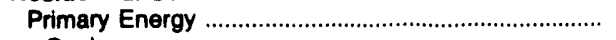 & 128.6 & 185.0 & 410.9 & 512.2 & 426.8 & 417.3 & 445.6 & 490.7 \\
\hline Cool & 3.8 & 4.6 & 5.6 & 5.2 & 5.7 & 5.3 & 6.3 & 3.9 \\
\hline 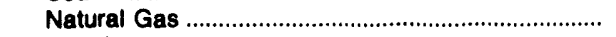 & 71.6 & 97.1 & 224.9 & 318.9 & 292.7 & 267.7 & 286.8 & 304.6 \\
\hline 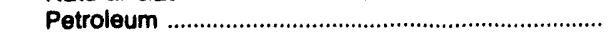 & 53.1 & 83.2 & 180.4 & 188.1 & 128.4 & 144.4 & 152.5 & 182.2 \\
\hline 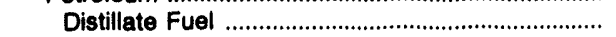 & 2.8 & 6.4 & 32.9 & 36.8 & 20.3 & 23.2 & 28.9 & 26.7 \\
\hline 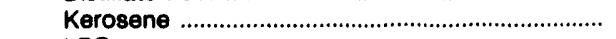 & 20.4 & 19.0 & 84.6 & 58.9 & 20.9 & 12.5 & 19.7 & 23.5 \\
\hline LPG & 30.0 & 57.8 & 63.0 & 92.5 & 87.2 & 108.7 & 103.9 & 132.0 \\
\hline 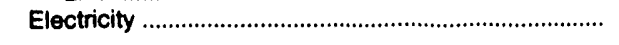 & 139.6 & 256.0 & 575.9 & 846.2 & 884.7 & 915.1 & 952.0 & 953.0 \\
\hline Commerclal Soctor ........................................................... & 111.2 & 193.0 & 563.7 & 668.4 & 650.6 & 744.0 & 782.5 & 798.2 \\
\hline 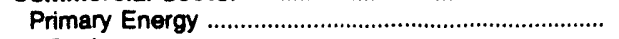 & 46.5 & 76.6 & 263.8 & 269.7 & 212.9 & 197.6 & 217.5 & 210.2 \\
\hline Coal & 3.5 & 5.6 & 7.7 & 7.4 & 8.4 & 9.2 & 10.1 & 6.6 \\
\hline 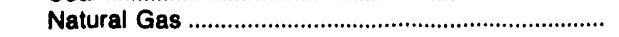 & 28.3 & 40.8 & 114.9 & 172.1 & 154.4 & 141.8 & 150.4 & 157.2 \\
\hline 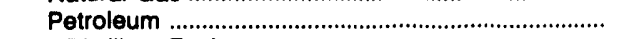 & 14.6 & 30.2 & 141.1 & 90.2 & 50.1 & 46.5 & 57.0 & 46.3 \\
\hline 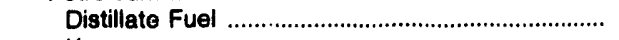 & 5.0 & 12.2 & 99.6 & 58.3 & 21.8 & 12.8 & 22.6 & 17.4 \\
\hline 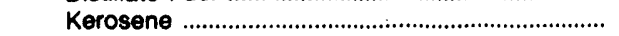 & 1.8 & 3.0 & 21.4 & 5.7 & 3.4 & 1.6 & 3.3 & 5.5 \\
\hline 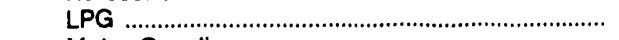 & 3.7 & 8.1 & 7.0 & 8.8 & 9.9 & 16.0 & 14.6 & 6.7 \\
\hline 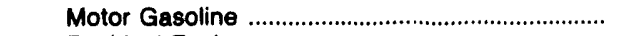 & 4.1 & 6.8 & 12.7 & 17.5 & 14.2 & 16.2 & 15.8 & 16.7 \\
\hline 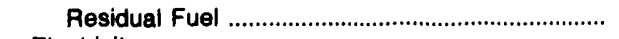 & .1 & .1 & .5 & & .7 & & .8 & \\
\hline 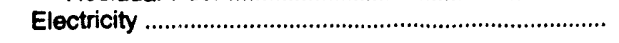 & 64.8 & 116.4 & 299.9 & 398.7 & 437.7 & 546.5 & 565.0 & 588.0 \\
\hline 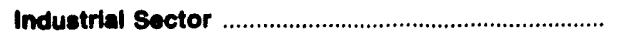 & 340.8 & 920.0 & $1,991.8$ & $2,336.0$ & $2,024.1$ & $1,985.9$ & $2,045.5$ & $2,109.5$ \\
\hline 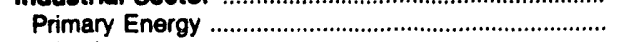 & 190.3 & 440.1 & $1,168.9$ & $1,052.6$ & 794.1 & 851.1 & 815.5 & 858.3 \\
\hline Coal & 44.0 & 102.3 & 134.6 & 178.3 & 151.3 & 152.5 & 138.4 & 162.6 \\
\hline 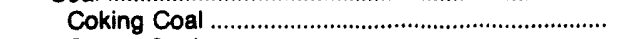 & 16.4 & 52.1 & 44.0 & 60.5 & 52.2 & 57.1 & 60.5 & 60.6 \\
\hline 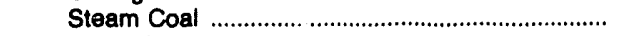 & 27.5 & 50.1 & 90.6 & 117.9 & 99.2 & 95.4 & 77.9 & 102.0 \\
\hline 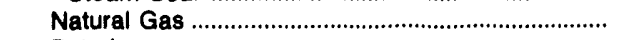 & 34.3 & 47.5 & 167.8 & 227.4 & 182.3 & 176.9 & 198.6 & 226.9 \\
\hline Petroleum & 112.0 & 290.3 & 866.6 & 646.9 & 460.5 & 521.7 & 478.4 & 468.8 \\
\hline 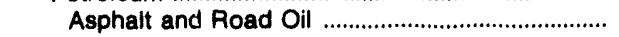 & 13.8 & 31.3 & 47.8 & 58.9 & 74.3 & 57.2 & 53.0 & 52.4 \\
\hline Distillate Fuel ................................................... & 8.9 & 44.7 & 203.6 & 224.3 & 110.1 & 142.4 & 131.0 & 145.2 \\
\hline 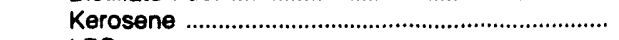 & 2.7 & 4.2 & 18.6 & 36.3 & 6.1 & 3.2 & 4.4 & 6.6 \\
\hline 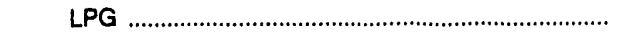 & 33.5 & 77.0 & 146.2 & 76.3 & 71.0 & 97.7 & 93.5 & 42.5 \\
\hline 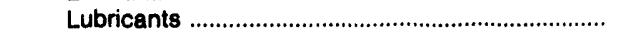 & 14.6 & 23.5 & 46.9 & 52.4 & 45.3 & 44.6 & 46.3 & 58.2 \\
\hline 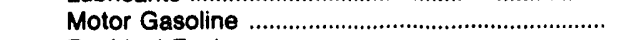 & 3.2 & 4.8 & 4.5 & 39.0 & 28.9 & 32.6 & 30.6 & 35.6 \\
\hline 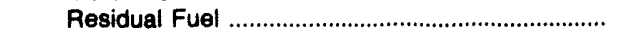 & 1.8 & 9.9 & 17.1 & 13.4 & 11.1 & 11.9 & 7.4 & 8.8 \\
\hline Other & 33.5 & 94.9 & 381.9 & 146.5 & 113.6 & 132.0 & 112.3 & 119.6 \\
\hline 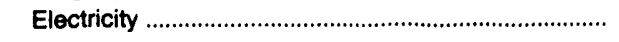 & 150.5 & 479.9 & 822.8 & $1,283.4$ & $1,230.1$ & $1,134.8$ & $1,230.0$ & $1,251.2$ \\
\hline 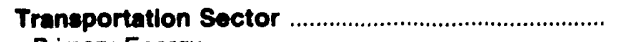 & 580.1 & $1,146.5$ & $2,671.1$ & $2,499.4$ & $2,072.5$ & $2,293.1$ & $2,484.5$ & $2,843.7$ \\
\hline $\begin{array}{l}\text { Primary Energy } \\
\text { Coal }\end{array}$ & 580.1 & $1,146.5$ & $2,671.1$ & $2,499.4$ & $2,072.5$ & $2,293.1$ & $2,484.5$ & $2,843.7$ \\
\hline 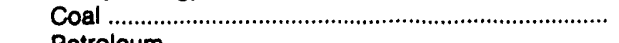 & .1 & & & - & - & - & & \\
\hline 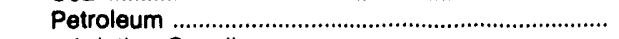 & $58 \mathrm{C} .0$ & $1,146.5$ & $2,671.1$ & $2,499.4$ & $2,072.5$ & $2,293.1$ & $2,484.5$ & $2,843.7$ \\
\hline 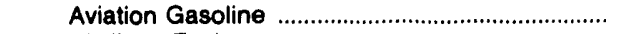 & 3.6 & 2.2 & 5.1 & 3.3 & 3.6 & 2.4 & 2.3 & 2.2 \\
\hline 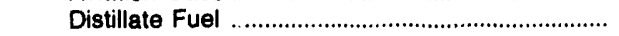 & 41.4 & 100.8 & 511.0 & 534.7 & 483.8 & 522.1 & 648.7 & 867.7 \\
\hline 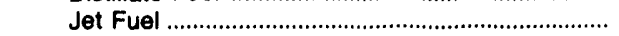 & 12.6 & 24.6 & 104.4 & 119.3 & 87.8 & 113.5 & 110.8 & 127.7 \\
\hline LPG & .3 & .8 & .2 & 2.4 & 2.1 & 2.1 & 2.1 & 1.0 \\
\hline 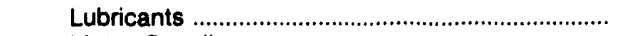 & 11.4 & 24.1 & 45.1 & 50.3 & 43.6 & 42.9 & 44.5 & 56.0 \\
\hline 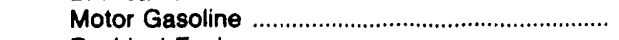 & 510.0 & 994.0 & $2,002.0$ & $1,789.3$ & 1.451 .6 & $1,610.1$ & $1,676.0$ & $1,789.1$ \\
\hline 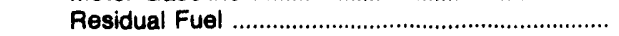 & .7 & • & 3.4 & - & - & - & - & - \\
\hline 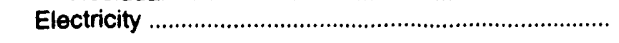 & - & - & - & - & - & - & - & - \\
\hline 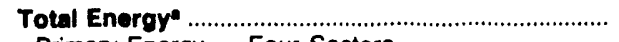 & $1,300.3$ & $2,700.5$ & $6,213.4$ & $6,862.1$ & $6,058.8$ & $6,355.5$ & $6,710.0$ & $7,195.1$ \\
\hline $\begin{array}{l}\text { Primary Energy - Four Sectors } \\
\text { Electricity }\end{array}$ & $\begin{array}{l}945.4 \\
354.9\end{array}$ & $1,848.2$ & $4,514.8$ & $4,333.9$ & $\begin{array}{l}3,506.2 \\
2,552.6\end{array}$ & $\begin{array}{l}3,759.1 \\
2,596.4\end{array}$ & $\begin{array}{l}3,963.0 \\
2,747.0\end{array}$ & $\begin{array}{l}4,402.8 \\
2,792.2\end{array}$ \\
\hline 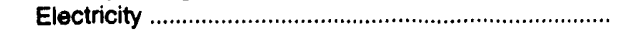 & 354.9 & 852.2 & $1,698.6$ & $2,528.3$ & $2,552.6$ & $2,596.4$ & $2,747.0$ & $2,792.2$ \\
\hline Electric Utility Soctor & 90.6 & 309.8 & 743.7 & 883.4 & 886.1 & 832.6 & 884.5 & $\mathbf{7 6 8 . 0}$ \\
\hline 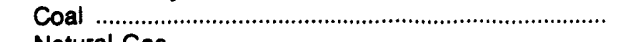 & 87.4 & 308.4 & 730.9 & 870.2 & 879.9 & 826.3 & 878.9 & 760.8 \\
\hline 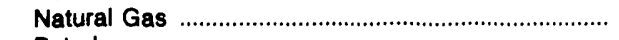 & 2.5 & .2 & 4.2 & 4.1 & 1.3 & 1.0 & 1.1 & .9 \\
\hline Petroleum & .7 & 1.2 & 8.6 & 9.1 & 4.8 & 5.3 & 4.5 & 6.4 \\
\hline 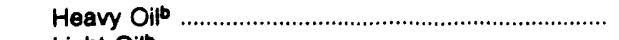 & .7 & 1.1 & $\ddot{0}$ & - & - & & - & - \\
\hline 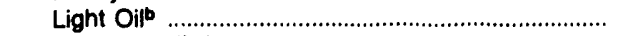 & • & .1 & 8.6 & 9.1 & 4.8 & 5.3 & 4.5 & 6.4 \\
\hline 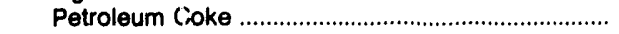 & - & - & - & - & - & - & - & - \\
\hline 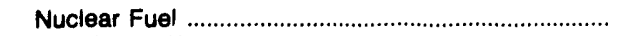 & - & - & - & - & - & - & - & - \\
\hline Wood and Waste & - & - & - & - & - & - & - & - \\
\hline Primary Energy - Flye Sectors ………….................... & $1,036.0$ & $2,158.0$ & $5,258.5$ & $5,217.2$ & $4,392.3$ & 4,591.7 & $4,847.5$ & $5,170.9$ \\
\hline
\end{tabular}

-There are no direct fuel costs for hydroelectric, geothermal, centralizerd solar, or wind energy. Wood and other biomass fuels are not included, except those consumed at electric utilities.

bHeavy oil includes Grade Nos. 4, 5, and 6, and residual fuel oils. Light oil includes Grade No. 2 heating oil, kerosene, and jet fuel.

- No consumption, including cases where adjustments were made. See "Consumption Adjustments for Process Fuel and Intermediate Products."

-Value iess than 0.05 million dollars.

Note: Totals may not equal sum of components due to independent rounding.

Sources: Data sources, estimation procedures, and assumptions are described in the "Documentation" section of this report. 


\section{Energy Price and Expenditure Estimates by Source, Louisiana \\ O 1970, 1975, 1980, and 1985-1989}

\begin{tabular}{|c|c|c|c|c|c|c|c|c|}
\hline Energy Source & 1970 & 1975 & 1980 & 1985 & 1986 & 1987 & 1988 & 1989 \\
\hline & \multicolumn{8}{|c|}{ Prices in Dollars per Million Btu } \\
\hline Coal & - & - & 1.26 & 2.14 & 1.80 & 1.66 & 1.57 & 1.62 \\
\hline 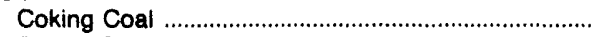 & - & - & - & - & - & - & - & - \\
\hline 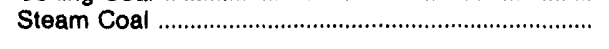 & - & - & 1.26 & 2.14 & 1.80 & 1.66 & 1.57 & 1.62 \\
\hline 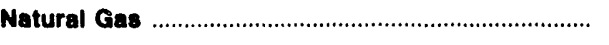 & 0.27 & 0.75 & 1.61 & 3.09 & 2.15 & 2.00 & 2.14 & 2.13 \\
\hline Petroleum & 1.48 & 2.80 & 5.98 & 6.05 & 4.73 & 5.04 & 4.84 & 5.26 \\
\hline 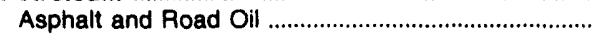 & .66 & 1.78 & 3.62 & 4.17 & 4.57 & 3.15 & 3.14 & 2.89 \\
\hline 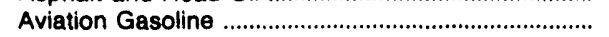 & 2.17 & 3.45 & 9.02 & 9.99 & 8.41 & 7.55 & 7.41 & 8.28 \\
\hline 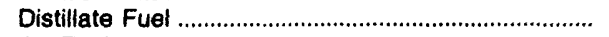 & .86 & 2.34 & 6.02 & 5.01 & 4.84 & 5.45 & 5.61 & 6.12 \\
\hline 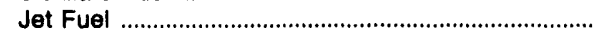 & .72 & 2.01 & 6.34 & 5.70 & 3.67 & 3.90 & 3.61 & 4.12 \\
\hline Kerosene …………………………………….. & .60 & 2.02 & 5.53 & 3.49 & 3.68 & 4.82 & 5.19 & 5.77 \\
\hline LPG & 1.12 & 2.52 & 5.30 & 5.13 & 5.69 & 5.85 & 5.78 & 5.51 \\
\hline 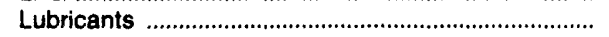 & 5.08 & 7.49 & 14.36 & 17.61 & 15.59 & 13.58 & 14.61 & 17.90 \\
\hline Motor Gasoline ........................................................ & 2.86 & 4.40 & 9.89 & 9.36 & 7.04 & 7.14 & 7.25 & 7.90 \\
\hline 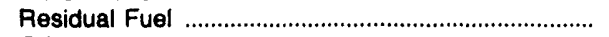 & .45 & 1.62 & 3.31 & 3.83 & 1.68 & 2.62 & 2.24 & 2.40 \\
\hline Other" & .71 & 2.41 & 6.28 & 6.57 & 3.81 & 4.62 & 3.86 & 4.71 \\
\hline 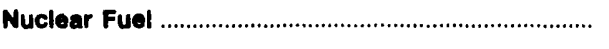 & - & - & - & .86 & 1.04 & .97 & .92 & .90 \\
\hline 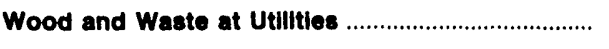 & - & - & - & - & - & - & - & - \\
\hline Primary Energy - Five Sectors ……......................... & .65 & 1.60 & 3.70 & 4.46 & 3.30 & 3.38 & 3.34 & 3.44 \\
\hline 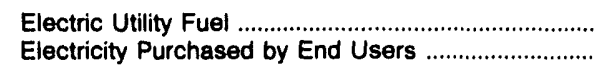 & $\begin{array}{r}.21 \\
4.69\end{array}$ & $\begin{array}{r}.73 \\
6.24\end{array}$ & $\begin{array}{r}2.19 \\
11.49\end{array}$ & $\begin{array}{r}2.46 \\
18.25\end{array}$ & $\begin{array}{r}1.67 \\
17.40\end{array}$ & $\begin{array}{r}1.46 \\
17.33\end{array}$ & $\begin{array}{r}1.44 \\
17.30\end{array}$ & $\begin{array}{r}1.51 \\
17.64\end{array}$ \\
\hline 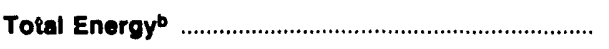 & .94 & 2.03 & 4.50 & 6.22 & 5.00 & 5.08 & 5.07 & 5.18 \\
\hline
\end{tabular}

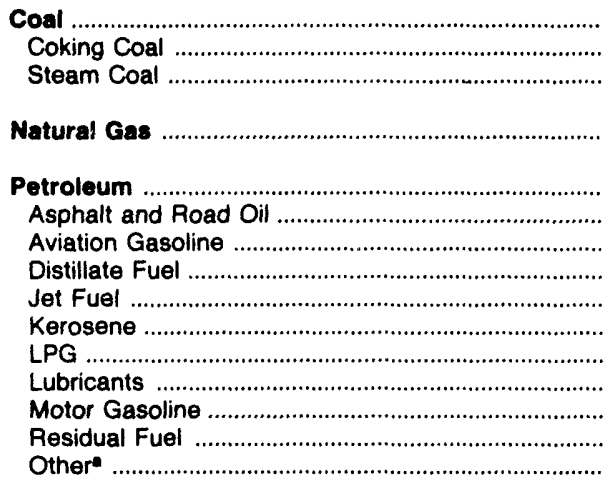

Expenditures In Millions of Doliars

Nuclear Fuel

Wood and Waste at Utilities

Primary Energy - Five Soctore

\begin{tabular}{|c|c|c|c|c|c|c|c|}
\hline - & - & 3.1 & 340.0 & 308.6 & 286.7 & 332.9 & 335.6 \\
\hline - & - & - & - & - & - & - & - \\
\hline - & - & 3.1 & 340.0 & 308.6 & 286.7 & 332.9 & 335.6 \\
\hline 376.4 & $1,036.2$ & $2,396.3$ & 3,152.5 & $2,249.7$ & $2,095.4$ & $2,203.4$ & $2,430.6$ \\
\hline 866.1 & 2,706.2 & $8,193.6$ & $7,122.0$ & $5,671.5$ & $6,253.4$ & $6,321.6$ & $6,374.8$ \\
\hline $\begin{array}{l}9.7 \\
4.9\end{array}$ & 33.2 & 46.8 & 50.8 & 54.3 & 47.5 & 49.9 & 44.4 \\
\hline 59.1 & 5.1 & 11.0 & 8.0 & 1.0 & 5.0 & $\begin{array}{r}4.0 \\
707\end{array}$ & 4.8 \\
\hline 234 & 268.9 & 752.1 & 500.0 & 984.5 & $1,162.3$ & $1,2 / 0.6$ & $1,319.7$ \\
\hline 8.5 & 07.9 & 306.8 & 410 & 369.3 & 415.1 & 435.5 & 518.2 \\
\hline & 28.1 & 179.2 & & $\begin{array}{r}63.3 \\
\end{array}$ & $\begin{array}{r}30.9 \\
1\end{array}$ & 138.0 & 700 \\
\hline 49.0 & $\begin{array}{l}4011.4 \\
829\end{array}$ & $\begin{array}{r}1,012.5 \\
174.1\end{array}$ & $\begin{array}{r}1,190.0 \\
194.3\end{array}$ & $\begin{array}{r}7,731.2 \\
168.2\end{array}$ & $\begin{array}{r}1,701.5 \\
165.6\end{array}$ & $\begin{array}{r}1,051.9 \\
171.9\end{array}$ & 215.9 \\
\hline 523.4 & $1,018.8$ & $2,449.2$ & $2,424.2$ & $1,847.2$ & $1,804.3$ & $1,862.1$ & $1,944.6$ \\
\hline 31.1 & 280.0 & $1,265.9$ & 975.2 & 352.2 & 707.8 & 625.3 & 628.4 \\
\hline 57.7 & 440.3 & $1,995.3$ & 846.3 & 694.1 & 813.4 & 711.8 & 852.2 \\
\hline - & - & - & 22.9 & 119.4 & 128.5 & 135.7 & 119.8 \\
\hline - & - & - & - & - & - & - & - \\
\hline $1,342.5$ & $3,742.4$ & $10,593.0$ & $10,637.4$ & $8,349.1$ & $8,763.9$ & $8,993.6$ & $9,260.7$ \\
\hline $\begin{array}{l}-72.9 \\
435.9\end{array}$ & $\begin{array}{r}-303.4 \\
710.5\end{array}$ & $\begin{array}{r}-1,079.1 \\
1,899.6\end{array}$ & $\begin{array}{r}-1,168.2 \\
3,664.5\end{array}$ & $\begin{array}{r}-942.4 \\
3,408.9\end{array}$ & $\begin{array}{r}-811.9 \\
3,379.5\end{array}$ & $\begin{array}{r}-886.9 \\
3,430.5\end{array}$ & $\begin{array}{r}-881.3 \\
3.604 .0\end{array}$ \\
\hline $1,705.6$ & $4,149.5$ & $11,413.5$ & $13,133.7$ & $10,815.6$ & $11,331.6$ & $11,537.1$ & $11,983.4$ \\
\hline
\end{tabular}

Electric Utility Fuel

Electricity Purchased by End Users

Total Energy

- Includes petroleum coke used at electric utilities.

bThere are no direct fuel costs for hydroelectric, geothermal, centralized solar, or wind energy. Wood and other biomass fuels are not included, except those consumed at electric utilities.

-No consumption, including cases where adjustments were made. See "Consumption Adjustments for Process Fuel and Intermediate Products."

Note: Expenditure totals may not equal sum of components due to independent rounding.

Sources: Data sources, estimation procedures, and assumptions are described in the "Documentation" section of this report. 


\begin{tabular}{|c|c|c|c|c|c|c|c|c|}
\hline Sector and Energy Source & 1970 & 1975 & 1980 & 1985 & 1986 & 1987 & 1988 & 1989 \\
\hline Residential Sector & 2.29 & 3.31 & 7.84 & 12.97 & 13.47 & 13.30 & 13.42 & 14.00 \\
\hline Primary Energy & .90 & 1.55 & 3.56 & 5.59 & 5.81 & 5.59 & 5.81 & 6.10 \\
\hline 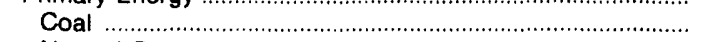 & - & - & 2.97 & - & - & - & - & - \\
\hline 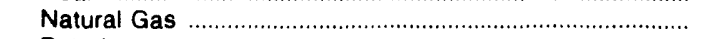 & .75 & 1.33 & 3.28 & 5.47 & 5.56 & 5.35 & 5.51 & 5.72 \\
\hline Petroleum & 2.19 & 4.36 & 8.52 & 7.10 & 8.61 & 8.80 & 9.40 & 9.76 \\
\hline 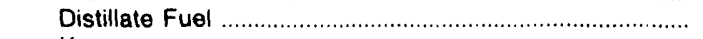 & .96 & 2.24 & 6.65 & 3.24 & 5.05 & 5.00 & 6.69 & 5.62 \\
\hline 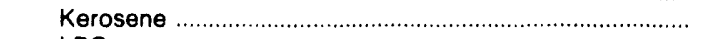 & 1.60 & 3.40 & - & 4.77 & 7.44 & 7.37 & 9.85 & 8.28 \\
\hline LPG & 2.20 & 4.39 & 8.54 & 7.68 & 8.98 & 9.13 & 9.28 & 10.44 \\
\hline Electricity & 6.58 & 7.96 & 13.81 & 20.27 & 20.75 & 21.05 & 20.89 & 21.50 \\
\hline Commercial Sector & 1.62 & 2.89 & 5.54 & 11.33 & 10.96 & 11.32 & 11.69 & 11.92 \\
\hline 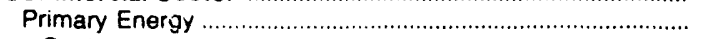 & .48 & 1.25 & 3.40 & 4.22 & 3.94 & 4.10 & 4.42 & 4.03 \\
\hline 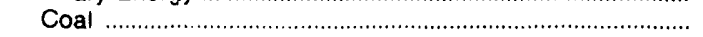 & - & - & 1.24 & - & - & - & 1.85 & - \\
\hline Natural Gas & .37 & .77 & 2.60 & 5.09 & 5.05 & 4.78 & 4.94 & 5.04 \\
\hline 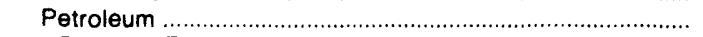 & 1.04 & 2.19 & 3.77 & 3.53 & 3.09 & 3.47 & 3.96 & 3.09 \\
\hline Distillate Fuel & .89 & 2.14 & 6.36 & 2.85 & 3.20 & 3.95 & 4.66 & 4.19 \\
\hline Kerosene & .59 & 2.01 & 5.53 & 3.35 & 3.40 & 4.42 & 4.97 & 5.17 \\
\hline LPG & 1.06 & 2.44 & 5.22 & 5.08 & 5.62 & 5.79 & 5.70 & 5.36 \\
\hline Motor Gasoline & 2.86 & 4.49 & 9.89 & 9.36 & 7.04 & 7.14 & 7.25 & 7.90 \\
\hline 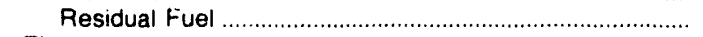 & .49 & 1.76 & 3.55 & 4.12 & 2.14 & 2.67 & 1.91 & 2.29 \\
\hline Electricity & 5.07 & 6.99 & 12.08 & 20.24 & 19.24 & 19.25 & 19.56 & $19 \cap 9$ \\
\hline Industrial Sector & .48 & 1.40 & 3.30 & 4.59 & 3.45 & 3.51 & 3.48 & 3.44 \\
\hline Frimary Energy & .42 & 1.32 & 3.07 & 3.95 & 2.93 & 3.02 & 2.98 & 2.91 \\
\hline Coal & - & - & 1.24 & 1.46 & 1.83 & 2.03 & 1.85 & 1.55 \\
\hline 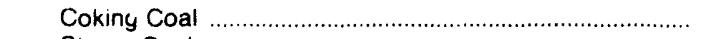 & - & - & - & - & - & - & - & - \\
\hline 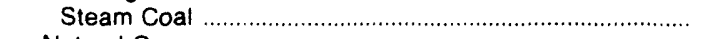 & - & - & 1.24 & 1.46 & 1.83 & 2.03 & 1.85 & 1.55 \\
\hline Natural Gas & .23 & .74 & 1.24 & 2.92 & 1.84 & 1.73 & 1.91 & 1.89 \\
\hline 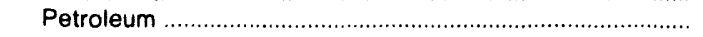 & .97 & 2.41 & 5.67 & 5.11 & 4.27 & 4.53 & 4.16 & 4.49 \\
\hline 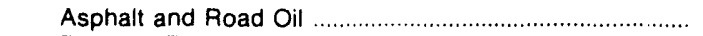 & .66 & 1.78 & 3.62 & 4.17 & 4.57 & 3.15 & 3.14 & 2.89 \\
\hline 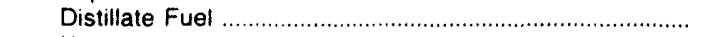 & .51 & 1.81 & 4.89 & 2.96 & 3.00 & 3.90 & 4.39 & 4.57 \\
\hline Kerosene & .59 & 2.01 & 5.53 & 3.35 & 3.40 & 4.42 & 4.97 & 5.17 \\
\hline LPG & 1.06 & 2.44 & 5.22 & 5.08 & 5.62 & 5.79 & 5.70 & 5.36 \\
\hline 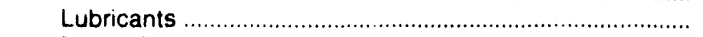 & 5.08 & 7.49 & 14.36 & 17.61 & 15.59 & 13.58 & 14.61 & 17.90 \\
\hline Motor Gasoline & 2.86 & 4.49 & 9.89 & 9.36 & 7.04 & 7.14 & 7.25 & 7.90 \\
\hline 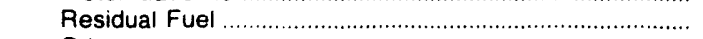 & .49 & 1.72 & 3.68 & 4.12 & 2.14 & 2.67 & 1.91 & 2.29 \\
\hline 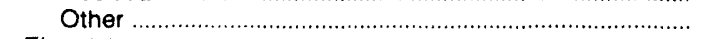 & .71 & 2.41 & 6.28 & 6.57 & 3.81 & 4.62 & 3.86 & 4.71 \\
\hline 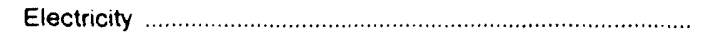 & 2.49 & 3.99 & 9.02 & 14.93 & 12.63 & 12.30 & 12.25 & 12.46 \\
\hline 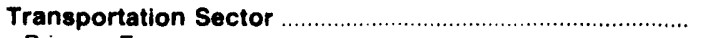 & 1.97 & 3.28 & $6.8^{7}$ & 7.21 & 5.27 & 5.59 & 5.52 & 5.98 \\
\hline 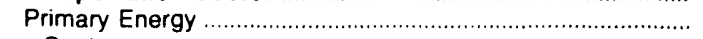 & 1.97 & 3.28 & $6.8:{ }^{\prime \prime}$ & 7.20 & 5.27 & 5.59 & 5.52 & 5.98 \\
\hline Coal & - & - & - & - & - & - & - & - \\
\hline 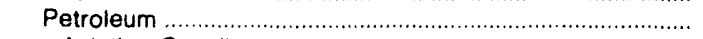 & 1.97 & 3.28 & 6.87 & 7.20 & 5.27 & 5.59 & 5.52 & 5.98 \\
\hline Aviation Gasoline & 2.17 & 3.45 & 9.02 & 9.99 & 8.41 & 7.55 & 7.41 & 8.28 \\
\hline 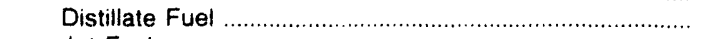 & 1.09 & 2.54 & 6.84 & 6.38 & 6.35 & 6.56 & 6.40 & 6.97 \\
\hline Jet Fuel . & .72 & 2.01 & 6.34 & 5.70 & 3.67 & 3.90 & 3.61 & 4.12 \\
\hline LPG & 1.06 & 2.44 & 5.22 & 5.08 & 5.62 & 5.79 & 5.70 & 5.36 \\
\hline 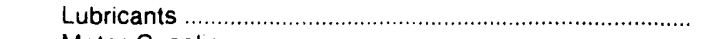 & 5.08 & 7.49 & 14.36 & 17.61 & 15.59 & 13.58 & 14.61 & 17.90 \\
\hline 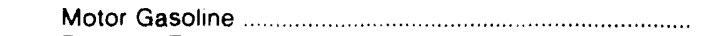 & 2.86 & 4.49 & 9.89 & 9.36 & 7.04 & 7.14 & 7.25 & 7.90 \\
\hline 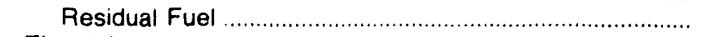 & .44 & 1.54 & 3.05 & 3.39 & 1.34 & 2.54 & 2.61 & 2.53 \\
\hline Electricity & 5.07 & 6.99 & 12.08 & 20.24 & 19.24 & 17.55 & 18.24 & 17.80 \\
\hline Total Energy* & .94 & 2.03 & 4.50 & 6.22 & 5.00 & 5.08 & 5.07 & 5.18 \\
\hline 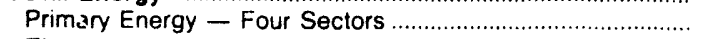 & .74 & 1.78 & 4.02 & 4.96 & 3.76 & 3.90 & 3.90 & 3.97 \\
\hline 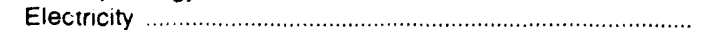 & 4.69 & 6.24 & 11.49 & 18.25 & 17.40 & 17.33 & 17.30 & 17.64 \\
\hline Electric Utility Sector & .21 & .73 & 2.19 & 2.46 & 1.67 & 1.46 & 1.44 & 1.51 \\
\hline Coal & - & - & - & 2.19 & 1.79 & 1.64 & 1.56 & 1.63 \\
\hline Natural Gas & .21 & .64 & 2.01 & 2.73 & 1.86 & 1.60 & 1.63 & 1.70 \\
\hline 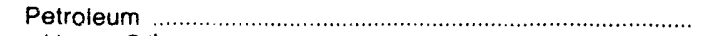 & .57 & 1.76 & 3.68 & 5.08 & 1.63 & 3.24 & 3.35 & 4.04 \\
\hline 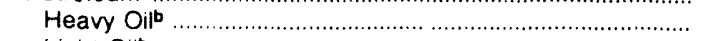 & .55 & 1.76 & 3.58 & 3.51 & 1.45 & 2.72 & 2.98 & 2.85 \\
\hline Light Oib & .60 & 1.92 & 4.37 & 5.84 & 3.20 & 3.63 & 4.42 & 5.01 \\
\hline 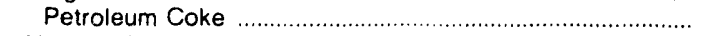 & - & - & - & - & - & - & - & - \\
\hline 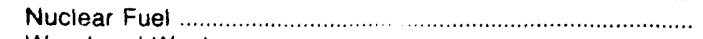 & - & - & - & .86 & 1.04 & .97 & .92 & .90 \\
\hline 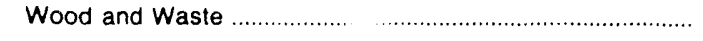 & - & - & - & - & - & - & - & - \\
\hline Primary Energy - Five Sectors & .65 & 1.60 & 3.70 & 4.46 & 3.30 & 3.38 & 3.34 & 3.44 \\
\hline
\end{tabular}

- There are no direct fuel costs for hydroelectric, geothermal, centralized solar, or wind energy. Wood and other biomass fuels are not included, except those consumed at electric utilities.

b Heavy oil includes Grade Nos. 4, 5, and 6, and residual fuel oils. Light oil includes Grade No. 2 heating oil, kerosene, and jet fuel.

-No consumption, including cases where adjustments were made. See "Consumption Adjustments for Process Fuel and Intermediate Products."

Sources: Data sources, estimation procedures, and assumptions are described in the "Documentation" section of this report. 


\section{Energy Expenditure Estimates by Sector, Louisiana \\ O 1970, 1975, 1980, and 1985-1989 \\ U (Million Dollars)}

\begin{tabular}{|c|c|c|c|c|c|c|c|c|}
\hline Sector and Energy Source & 1970 & 1975 & 1980 & 1985 & 1986 & 1987 & 1988 & 1989 \\
\hline $\begin{array}{l}\text { Reeldertial Soctor } \\
\text { Primary Energy }\end{array}$ & $\begin{array}{r}299.1 \\
89.4\end{array}$ & $\begin{array}{l}490.0 \\
166.2\end{array}$ & $\begin{array}{r}1,077.9 \\
285.0\end{array}$ & $\begin{array}{r}1,775.4 \\
380.4\end{array}$ & $\begin{array}{r}1,816.5 \\
381.8\end{array}$ & $\begin{array}{r}1,819.1 \\
383.4\end{array}$ & $\begin{array}{r}1,826.4 \\
391.4\end{array}$ & $\begin{array}{r}1,910.5 \\
405.5\end{array}$ \\
\hline Coal & 0.4 & & .1 & 000. & - & 000.4 & - & \\
\hline Natural Gas .. & 66.7 & 131.6 & 248.7 & 344.3 & 335.6 & 341.3 & 342.7 & 344.5 \\
\hline 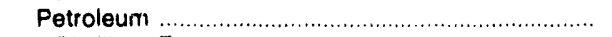 & 22.8 & 34.6 & 36.2 & 36.1 & 46.1 & 42.1 & 48.7 & 61.0 \\
\hline 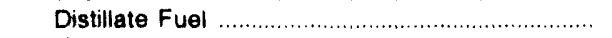 & & .1 & .2 & .2 & .3 & .1 & .1 & 3 \\
\hline 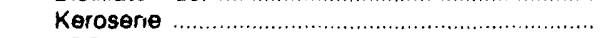 & 2 & .4 & & 4.5 & 8.9 & 6.4 & 11.7 & 15.4 \\
\hline & 22.5 & 34.0 & 36.0 & 31.5 & 37.0 & 35.6 & 36.8 & 45.3 \\
\hline Electricity . . & 209.6 & 323.8 & 792.9 & $1,395.0$ & $1,434.7$ & 1.435 .7 & $1,435.0$ & $1,505.0$ \\
\hline & 187.4 & $\begin{array}{r}318.5 \\
98.5\end{array}$ & $\begin{array}{l}981.0 \\
453.2\end{array}$ & $\begin{array}{r}1,441.5 \\
298.9\end{array}$ & $1,349.9$ & $1,311,2$ & $1,355.5$ & $1,362.7$ \\
\hline 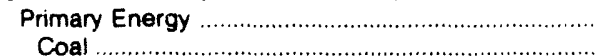 & 41.6 & 98.5 & $\begin{aligned} 453.2 \\
.1\end{aligned}$ & 298.9 & 263.0 & 248.4 & 266.6 & 232.9 \\
\hline 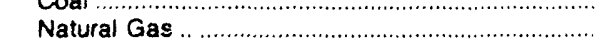 & $26 . \overline{6}$ & 40.5 & 107.7 & 159.7 & 146.9 & $138 . \overline{4}$ & $141.2^{\circ}$ & $140 \overline{0}$ \\
\hline Petroleum & 15.0 & 58.0 & 345.4 & 139.2 & 116.2 & $\begin{array}{l}38.4 \\
110.0\end{array}$ & 125.4 & $\begin{array}{r}140.0 \\
92.9\end{array}$ \\
\hline 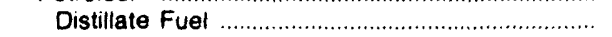 & 4.3 & 18.2 & 14.8 & 62.2 & 75.1 & 43.2 & 35.2 & 20.6 \\
\hline Kerosene & 1.5 & 5.3 & 17.2 & 11.6 & 5.4 & 8.3 & 56.6 & 10.5 \\
\hline LPG & 1.9 & 3.3 & 3.9 & 3.7 & 4.1 & 4.0 & 4.0 & 4.1 \\
\hline 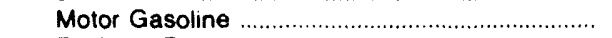 & 5.7 & 11.0 & 8.7 & 11.6 & 8.8 & 9.3 & 9.0 & 9.2 \\
\hline 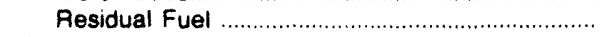 & 1.6 & 20.2 & 300.8 & 50.2 & 22.7 & 45.2 & 20.6 & 48.5 \\
\hline 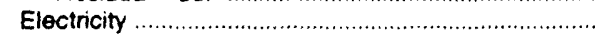 & 145.8 & 220.0 & 527.7 & $1,142.6$ & $1,086.9$ & $1,062.8$ & $1,088.8$ & $1,129.8$ \\
\hline Industrial sector .... & 590.8 & $1,873.5$ & $5,439.9$ & $5,942.2$ & $4,529.8$ & 4,823.1 & $4,809.6$ & $4,900.2$ \\
\hline Primary Energy ……………………………. & 510.3 & $1,706.9$ & $4,861.1$ & $4,815.5$ & $3,642.7$ & $3,942.2$ & $3,903.1$ & $3,931.2$ \\
\hline Coal & - & - & 2.9 & 15.8 & 11.5 & 17.6 & 19.5 & 22.0 \\
\hline 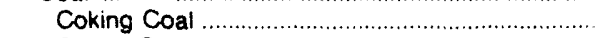 & - & - & - & & & - & - & \\
\hline 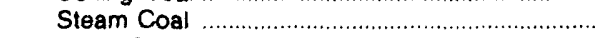 & - & - & 2.9 & 15.8 & 11.5 & 17.6 & 19.5 & 22.0 \\
\hline Natural Gas ....................... & 210.8 & 624.6 & $1,150.2$ & $1,833.1$ & $1,248.4$ & $1,203.7$ & $1,292.8$ & $1,510.8$ \\
\hline Petroleum & 299.5 & $1,082.3$ & $3,708.0$ & $2,966.5$ & $2,382.8$ & $2,720.9$ & $2,590.8$ & $2,398.4$ \\
\hline 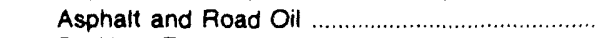 & 9.7 & 33.2 & 46.8 & 50.8 & 54.3 & 47.5 & 49.9 & 44.4 \\
\hline 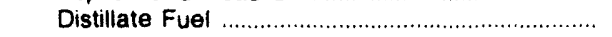 & 12.4 & 49.0 & 210.9 & 164.5 & 208.3 & 304.5 & 360.0 & 316.2 \\
\hline Kerosene & 6.8 & 22.0 & 161.9 & 18.5 & 49.0 & 16.2 & 69.6 & 30.1 \\
\hline LPG & 173.5 & 441.2 & 969.6 & $1,159.6$ & $1,088.5$ & $1,060.0$ & $1,009.3$ & 739.5 \\
\hline 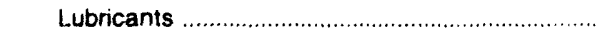 & 32.4 & 59.0 & 111.3 & 124.2 & 107.5 & 105.9 & 109.9 & 138.1 \\
\hline 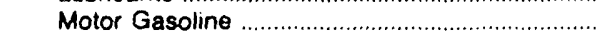 & 4.5 & 4.1 & 3.2 & 23.9 & 14.5 & 14.0 & 13.1 & 12.1 \\
\hline 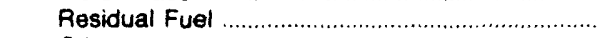 & 2.5 & 33.6 & 208.8 & 578.7 & 166.5 & 359.5 & 267.1 & 265.8 \\
\hline Other & 57.7 & 440.3 & $1,895.3$ & 846.3 & 694.1 & 813.4 & 711.8 & 852.2 \\
\hline 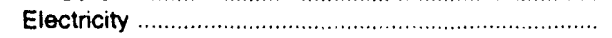 & $80 . \overline{5}$ & 166.6 & 578.8 & $1,126.7$ & 887.1 & 880.9 & 906.5 & 969.0 \\
\hline Transportation Sector & 628.3 & $1,467.5$ & $3,914.7$ & $3,974.5$ & $3,119.4$ & $3,378.2$ & $3,545.7$ & $3,810.0$ \\
\hline Primary Energy ……......................................... & 628.3 & $1,467.4$ & $3,914.6$ & $3,974.3$ & $3,119.2$ & $3,378.1$ & $3,545.5$ & $3,809.8$ \\
\hline Coal & & & & & & & & \\
\hline 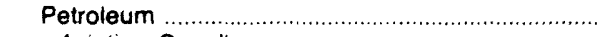 & 628.3 & $1,467.4$ & $3,914.6$ & $3,974.3$ & $3,119.2$ & $3,378.1$ & $3,545.5$ & $3,809.8$ \\
\hline Aviation Gasoline & 4.9 & 5.1 & 11.6 & 8.6 & 7.0 & 5.0 & 4.6 & 4.8 \\
\hline Distillate Fuel ……………………… & 42.1 & 200.6 & 496.3 & 749.4 & 699.4 & 813.1 & 871.6 & 974.0 \\
\hline Jet Fuel & 23.4 & 67.9 & 306.8 & 410.5 & 369.3 & 415.1 & 435.5 & 518.2 \\
\hline LPG & 1.4 & 2.8 & 3.0 & 1.9 & 1.7 & 1.9 & 1.7 & 1.5 \\
\hline 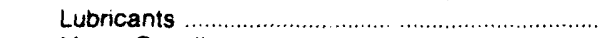 & 16.6 & 23.9 & 62.8 & 70.0 & 60.6 & 59.7 & 62.0 & 77.9 \\
\hline 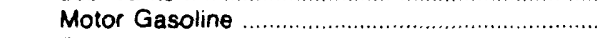 & 513.2 & $1,003.8$ & $2,437.3$ & $2,388.8$ & $1,823.9$ & $1,780.9$ & $1,839.9$ & $1,923.3$ \\
\hline 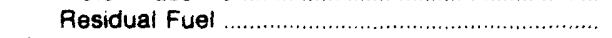 & 26.8 & 163.2 & 596.8 & 345.0 & 157.3 & 302.3 & 330.2 & 310.1 \\
\hline 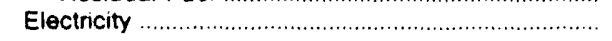 & & 1 & .1 & .2 & .2 & .1 & .2 & .2 \\
\hline Total Energye & $1,705.6$ & 4,149.5 & $11,413.5$ & $13,133.7$ & $10,815.6$ & $11,331.6$ & $11,537.1$ & $11,983.4$ \\
\hline Primany Energy - Four Sectors .................................... & $1,269.7$ & $3,439.0$ & $9,513.8$ & $9,469.2$ & $7,406.7$ & $7,952.0$ & $8,106.7$ & $8,379.4$ \\
\hline Electricity . . & 435.9 & 710.5 & $1,899.6$ & $3,664.5$ & $3,408.9$ & $3,379.5$ & $3,430.5$ & $3,604.0$ \\
\hline $\begin{array}{l}\text { Eloctric Utilty Sortor } \\
\text { Coal }\end{array}$ & 72.9 & 303.4 & $1,079.1$ & $1,168.2$ & 842.4 & 811.9 & 886.9 & 881.3 \\
\hline Coal & & & & 324.2 & 297.1 & 268.1 & 313.4 & 313.5 \\
\hline$\ldots$ & 72.3 & 239.5 & 889.7 & 815.3 & 518.7 & 412.0 & 426.6 & 435.3 \\
\hline 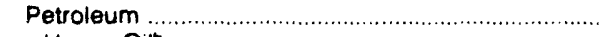 & .5 & 63.9 & 189.4 & 5.8 & 7.2 & 2.3 & 11.2 & 12.7 \\
\hline 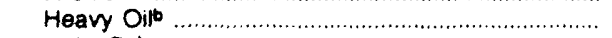 & 3 & 62.9 & 159.5 & 1.3 & 5.7 & .8 & 7.4 & 4.0 \\
\hline Light Oit . & .2 & 1.0 & 29.9 & 4.5 & 1.4 & 1.5 & 3.8 & 8.7 \\
\hline 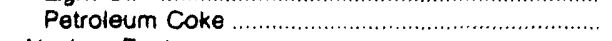 & - & - & - & - & & & - & \\
\hline 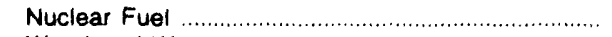 & - & - & - & 22.9 & 119.4 & 128.5 & 135.7 & 119.8 \\
\hline Wood and Waste & - & - & - & - & - & - & - & \\
\hline Primary Energy - Five Soctore & $1,342.5$ & $3,742.4$ & $10,593.0$ & $10,637.4$ & $8,349.1$ & $8,763.9$ & $8,993.6$ & $9,260.7$ \\
\hline
\end{tabular}

- There are no direct fuel costs for hydroelectric, geothermal, centralized solar, or wind energy. Wood and other biomass fuels are not included, except those consumed at electric utilities.

bHeavy oil includes Grade Nos. 4,5, and 6, and residual fuel oils. Light oil includes Grade No. 2 heating oll, kerosene, and jet fuel.

- No consumption, including cases where adjustments were made. See "Consumption Adjustments for Process Fuel and intermediate Products."

- Value less than 0.05 million dollars.

Note: Totals may not equal sum of components due to independent rounding.

Sources: Data sources, estimation procedures, and assumptions are described in the "Documentation" section of this report. 


\begin{tabular}{|c|c|c|c|c|c|c|c|c|}
\hline Energy Source & 1970 & 1975 & 1980 & 1985 & 1086 & 1987 & 1988 & 1989 \\
\hline & \multicolumn{8}{|c|}{ Prices in Dollars per Million Btu } \\
\hline Coal & 1.06 & 2.60 & 1.89 & 2.58 & 2.27 & 2.48 & 2.63 & 2.66 \\
\hline 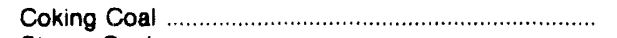 & - & - & - & - & - & - & - & - \\
\hline Steam Coal & 1.06 & 2.60 & 1.89 & 2.58 & 2.27 & 2.48 & 2.63 & 2.66 \\
\hline Natural Gas & 1.48 & 2.03 & 5.03 & 7.41 & 7.06 & 6.45 & 5.64 & 5.78 \\
\hline Potroleum & 1.46 & 3.04 & 7.00 & 7.48 & 5.51 & 5.97 & 5.79 & 6.52 \\
\hline 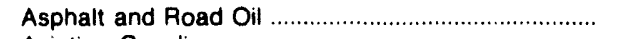 & .73 & 1.99 & 3.73 & 5.21 & 4.88 & 3.55 & 3.35 & 3.23 \\
\hline Aviation Gasoline & 2.17 & 3.45 & 9.02 & 9.99 & 8.41 & 7.55 & 7.41 & 8.28 \\
\hline 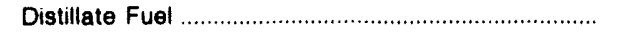 & 1.37 & 2.78 & 6.83 & 8.00 & 6.06 & 6.21 & 6.23 & 6.98 \\
\hline Jet Fuel . & .75 & 2.09 & 6.51 & 6.10 & 4.50 & 4.41 & 4.13 & 4.79 \\
\hline 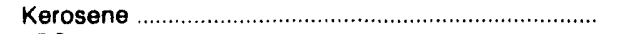 & 1.53 & 3.10 & 7.82 & 8.74 & 6.59 & 6.55 & 6.57 & 7.73 \\
\hline LPG & 2.37 & 4.20 & 7.86 & 10.95 & 10.49 & 10.64 & 10.59 & 10.31 \\
\hline 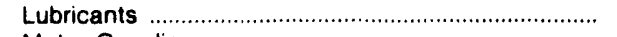 & 5.08 & 7.49 & 14.36 & 17.61 & 15.59 & 13.58 & 14.61 & 17.80 \\
\hline Motor Gasoline & 3.02 & 4.56 & 9.68 & 9.35 & 7.38 & 1.72 & 8.15 & 8.69 \\
\hline 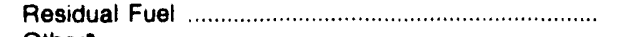 & .38 & 1.79 & 4.10 & 4.35 & 2.43 & 2.93 & 2.33 & 2.79 \\
\hline Other" & .89 & - & - & - & - & - & - & - \\
\hline Nuclear Fuel & - & .32 & .58 & .62 & .58 & .60 & .64 & .54 \\
\hline 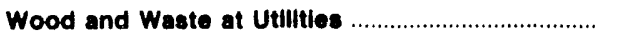 & - & - & - & - & - & - & - & - \\
\hline 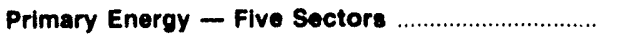 & 1.45 & 2.54 & 5.70 & 5.85 & 4.31 & 5.01 & 4.86 & 5.00 \\
\hline $\begin{array}{l}\text { Electric Utility Fuel } \\
\text { Electricity Purchased by End Users }\end{array}$ & $\begin{array}{r}.35 \\
5.92\end{array}$ & $\begin{array}{r}.71 \\
9.70\end{array}$ & $\begin{array}{r}1.82 \\
16.30\end{array}$ & $\begin{array}{r}1.60 \\
20.16\end{array}$ & $\begin{array}{r}1.11 \\
19.88\end{array}$ & $\begin{array}{r}1.50 \\
19.10\end{array}$ & $\begin{array}{r}1.27 \\
19.62\end{array}$ & $\begin{array}{r}1.21 \\
20.53\end{array}$ \\
\hline \multirow[t]{2}{*}{ Total Energy } & 1.95 & 3.77 & 8.44 & 9.70 & 7.86 & 8.26 & 8.03 & 9.13 \\
\hline & \multicolumn{8}{|c|}{ Expenditures in Millions of Dollars } \\
\hline Coal & 2.3 & 3.4 & 5.7 & 13.2 & 21.1 & 17.0 & 18.2 & 18.0 \\
\hline 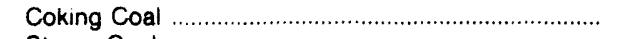 & 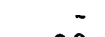 & - & - & - & - & - & - & - \\
\hline Steam Coal & 2.3 & 3.4 & 5.7 & 13.2 & 21.1 & 17.0 & 18.2 & 18.0 \\
\hline Natural Gas & 1.9 & 4.0 & 11.2 & 19.3 & 17.9 & 17.6 & 18.5 & 21.3 \\
\hline Petroleumn & 337.1 & 675.2 & $1,388.1$ & $1,440.7$ & $1,208.6$ & $1,291.2$ & $1,407.9$ & $1,475.5$ \\
\hline 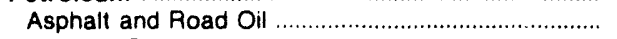 & 3.4 & 9.2 & 10.8 & 75.6 & 23.8 & 20.1 & 35.2 & 21.5 \\
\hline Aviation Gasoline & 1.0 & 1.2 & 3.7 & 2.1 & 2.4 & 2.0 & 2.5 & 2.9 \\
\hline 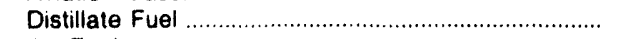 & 94.2 & 186.5 & 422.8 & 446.4 & 405.7 & 432.6 & 497.8 & 488.7 \\
\hline 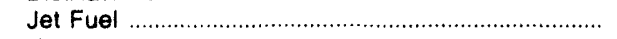 & 9.4 & 22.7 & 66.7 & 54.4 & 39.6 & 43.7 & 47.7 & 59.5 \\
\hline Kerosene & 15.5 & 18.2 & 22.4 & 23.4 & 12.7 & 15.1 & 17.2 & 20.3 \\
\hline LPG & 5.7 & 10.1 & 25.2 & 24.9 & 38.6 & 47.5 & 62.8 & 61.5 \\
\hline 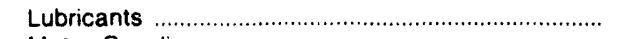 & 5.2 & 7.6 & 17.1 & 19.1 & 16.5 & 16.3 & 16.9 & 21.2 \\
\hline 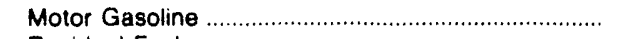 & 174.9 & 303.1 & 598.7 & 615.9 & 520.9 & 570.3 & 658.8 & 647.4 \\
\hline Residual Fuel . & 27.5 & 111.7 & 220.7 & 178.8 & 148.4 & 143.6 & 148.0 & 142.6 \\
\hline Other" & .4 & - & - & - & - & - & - & - \\
\hline 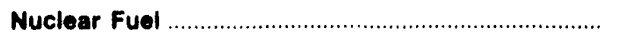 & - & 16.1 & 27.9 & 35.7 & 38.9 & 25.9 & 34.2 & 40.0 \\
\hline 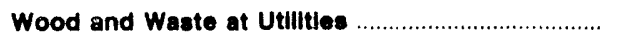 & - & - & - & - & - & - & - & - \\
\hline Primary Energy - Five Sectors .............................. & 341.3 & 698.6 & $1,432.8$ & $1,508.8$ & $1,286.6$ & $1,351.8$ & $1,558.6$ & $1,554.8$ \\
\hline $\begin{array}{l}\text { Electric Utility Fuel } \\
\text { Electricity Purchased by End Users }\end{array}$ & $\begin{array}{l}-10.6 \\
102.3\end{array}$ & $\begin{array}{l}-48.1 \\
216.1\end{array}$ & $\begin{array}{r}-129.8 \\
455.3\end{array}$ & $\begin{array}{r}-127.6 \\
675.7\end{array}$ & $\begin{array}{r}-105.0 \\
692.1\end{array}$ & $\begin{array}{r}-106.3 \\
698.4\end{array}$ & $\begin{array}{r}-107.3 \\
754.0\end{array}$ & $\begin{array}{r}-128.9 \\
801.0\end{array}$ \\
\hline Total Energyb & 433.1 & 866.7 & $1,758.3$ & $2,057.0$ & $1,873.6$ & $1,943.8$ & $2,205.5$ & $2,228.9$ \\
\hline
\end{tabular}

-Includes petroleum coke used at electric utilities.

- There are no direct fuel costs for hydroelectric, geothermal, centralized solar, or wind energy. Wood and other biomass fuels are not included, except those consumed at electric utilities.

-No consumption, including cases where adjustments were made. See "Consumption Adjustments for Process Fuel and Intermediate Products."

Note: Expenditure totals may not equal sum of components due to independent rounding.

Sources: Data sources, estimation procedures, and assumptions are described in the "Documentation" section of this report 


\begin{tabular}{|c|c|c|c|c|c|c|c|c|}
\hline Sector and Enorgy Source & 1970 & 1975 & 1980 & 1985 & 1986 & 1987 & 1988 & 1989 \\
\hline Residential Sector & 2.17 & 4.17 & 9.29 & 11.92 & 10.30 & 10.53 & 10.67 & 11.70 \\
\hline 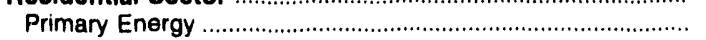 & 1.56 & 2.98 & 7.09 & 7.77 & 5.91 & 6.08 & 6.31 & 7.15 \\
\hline 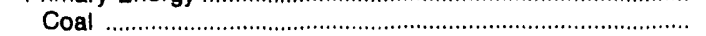 & 1.29 & 2.62 & 3.90 & 4.39 & 4.19 & 3.85 & 3.99 & 3.96 \\
\hline Natural Gas .......... & 1.96 & 2.59 & 6.20 & 8.76 & 8.26 & 7.73 & 7.07 & 7.14 \\
\hline 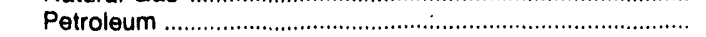 & 1.56 & 2.98 & 7.12 & 7.81 & 5.90 & 6.08 & 6.32 & 7.17 \\
\hline 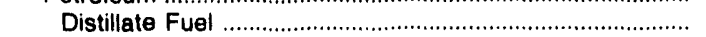 & 1.51 & 2.87 & 6.94 & 7.55 & 5.63 & 5.66 & 5.88 & 6.77 \\
\hline 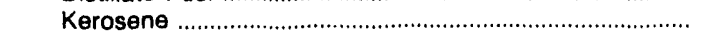 & 1.60 & 3.16 & 8.15 & 8.95 & 6.68 & 6.70 & 6.97 & 8.02 \\
\hline LPG & 3.04 & 4.78 & 10.04 & 11.45 & 9.79 & 10.37 & 10.37 & 10.37 \\
\hline 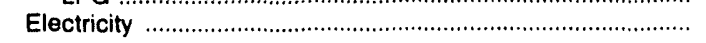 & 8.12 & 11.67 & 18.30 & 23.71 & 23.98 & 23.79 & 24.17 & 24.93 \\
\hline Commerclal Sector . & 2.40 & 5.09 & 9.16 & 12.79 & 10.62 & 10.93 & 9.20 & 11.05 \\
\hline 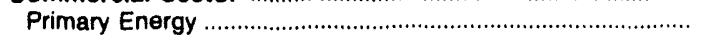 & 1.03 & 2.40 & 5.72 & 6.14 & 4.55 & 5.04 & 4.28 & 5.21 \\
\hline 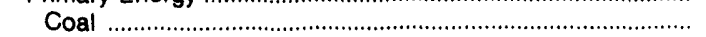 & .98 & 2.59 & 1.68 & 2.38 & 2.13 & 2.37 & 2.55 & 2.61 \\
\hline 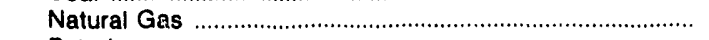 & 1.42 & 2.07 & 5.00 & 7.73 & 7.26 & 6.71 & 6.09 & 6.28 \\
\hline 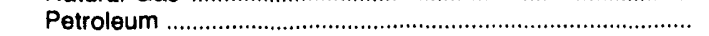 & 1.02 & 2.41 & 5.84 & 6.21 & 4.48 & 5.02 & 4.20 & 5.16 \\
\hline 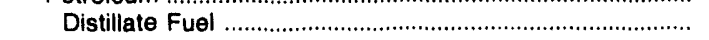 & 1.11 & 2.46 & 6.32 & 7.06 & 5.32 & 5.26 & 5.12 & 5.86 \\
\hline 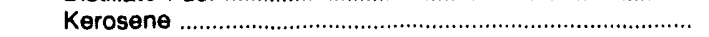 & .68 & 2.55 & 6.50 & 7.30 & 5.35 & 5.35 & 5.09 & 6.07 \\
\hline 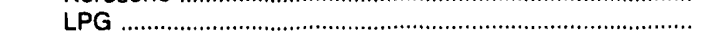 & 1.35 & 3.24 & 6.06 & 10.29 & 11.26 & 11.14 & 10.88 & 10.22 \\
\hline 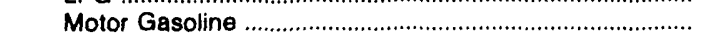 & 3.02 & 4.56 & 9.69 & 9.35 & 7.38 & 7.72 & 8.15 & 8.69 \\
\hline 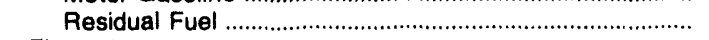 & .35 & 1.79 & 4.33 & 4.50 & 2.47 & 2.93 & 2.33 & 2.83 \\
\hline 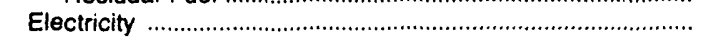 & 7.86 & 11.68 & 19.20 & 23.69 & 23.53 & 20.68 & 21.15 & 22.09 \\
\hline Industrial Sector & 1.03 & 2.64 & 6.32 & 7.72 & 5.83 & 6.61 & 6.13 & 7.55 \\
\hline 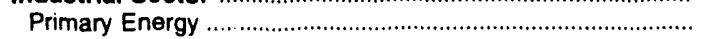 & .59 & 1.97 & 4.21 & 5.05 & 3.31 & 3.69 & 3.42 & 4.02 \\
\hline 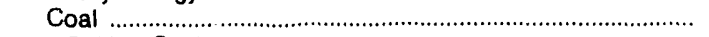 & .98 & 2.59 & 1.68 & 2.38 & 2.13 & 2.37 & 2.55 & 2.61 \\
\hline 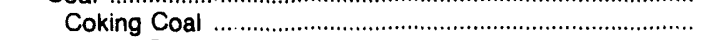 & - & - & - & - & - & - & - & \\
\hline 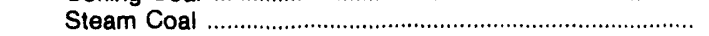 & .98 & 2.59 & 1.68 & 2.38 & 2.13 & 2.37 & 2.55 & 2.61 \\
\hline 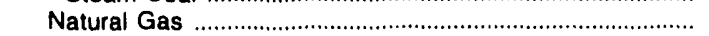 & .84 & 1.42 & 4.19 & 6.14 & 5.81 & 5.26 & 4.39 & 4.56 \\
\hline Petroleum & .58 & 1.97 & 4.38 & 5.33 & 3.51 & 3.88 & 3.52 & 4.29 \\
\hline 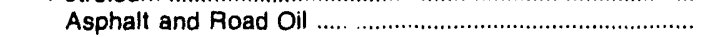 & .73 & 1.99 & 3.73 & 5.21 & 4.88 & 3.55 & 3.35 & 3.23 \\
\hline 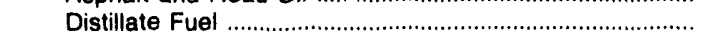 & .63 & 2.30 & 5.94 & 6.63 & 4.86 & 4.86 & 4.63 & 5.51 \\
\hline 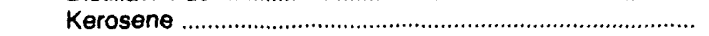 & .68 & 2.55 & 6.50 & 7.30 & 5.35 & 5.35 & 5.09 & 6.07 \\
\hline LPG & 1.35 & 3.24 & 6.06 & 10.29 & 11.26 & 11.14 & 10.88 & 10.22 \\
\hline 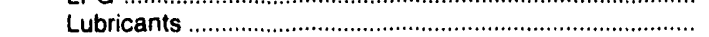 & 5.08 & 7.49 & 14.36 & 17.61 & 15.59 & 13.58 & 14.61 & 17.90 \\
\hline 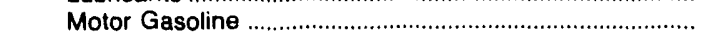 & 3.02 & 4.56 & 9.69 & 9.35 & 7.38 & 7.72 & 8.15 & 8.69 \\
\hline 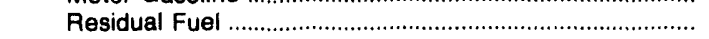 & .43 & 1.82 & 3.84 & 4.50 & 2.47 & 2.93 & 2.33 & 2.83 \\
\hline Other & .89 & & & - & - & - & & \\
\hline 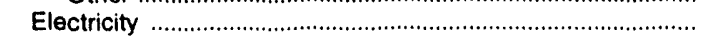 & 3.52 & 6.46 & 13.15 & 15.15 & 14.14 & 14.12 & 14.86 & 15.74 \\
\hline Transportation Sector & 2.28 & 3.95 & 8.99 & 9.06 & 7.15 & 7.42 & 7.58 & 8.15 \\
\hline 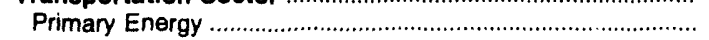 & 2.28 & 3.95 & 8.99 & 9.06 & 7.15 & 7.42 & 7.58 & 8.15 \\
\hline 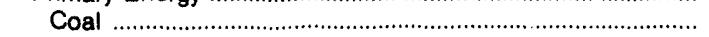 & .98 & 2.59 & - & - & - & - & & \\
\hline 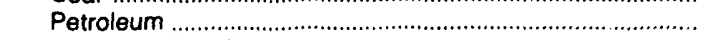 & 2.28 & 3.95 & 8.99 & 9.06 & 7.15 & 7.42 & 7.58 & 8.15 \\
\hline Aviation Gasoline ……1. & 2.17 & 3.45 & 9.02 & 9.99 & 8.41 & 7.55 & 7.41 & 8.28 \\
\hline 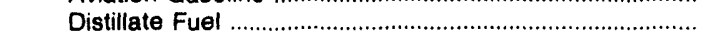 & 1.39 & 2.90 & 7.41 & 9.16 & 7.24 & 7.62 & 7.55 & 8.20 \\
\hline 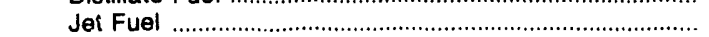 & .75 & 2.09 & 6.51 & 6.10 & 4.50 & 4.41 & 4.13 & 4.79 \\
\hline 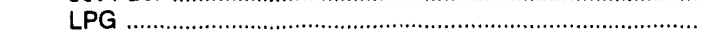 & 1.35 & 3.24 & 6.06 & 10.29 & 11.26 & 11.14 & 10.88 & 10.22 \\
\hline 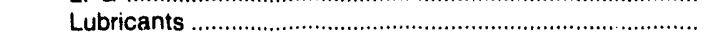 & 5.08 & 7.49 & 14.36 & 17.61 & 15.59 & 13.58 & 14.61 & 17.90 \\
\hline 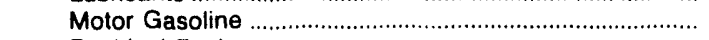 & 3.02 & 4.56 & 9.69 & 9.35 & 7.38 & 7.72 & 8.15 & 8.69 \\
\hline 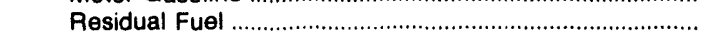 & .31 & 1.66 & 3.68 & 4.07 & 2.22 & 2.82 & 2.04 & $2 .-1$ \\
\hline 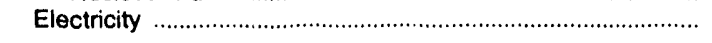 & - & - & - & - & - & - & - & - \\
\hline Total Energy" & 1.95 & 3.77 & 8.44 & 9.70 & 7.86 & 8.26 & 8.03 & 9.13 \\
\hline Primary Energy - Four Sectors .................................................. & 1.62 & 3.14 & 7.22 & 7.74 & 5.81 & 6.27 & 6.15 & 6.96 \\
\hline 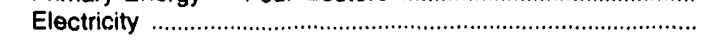 & 5.92 & 9.70 & 16.30 & 20.16 & 19.88 & 19.10 & 19.62 & 20.53 \\
\hline Eloctric Utillty Sector & .35 & .71 & 1.82 & 1.60 & 1.11 & 1.50 & 1.27 & 1.21 \\
\hline Coal & 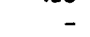 & - & - & - & - & - & - & - \\
\hline Natural Gas & - & - & - & - & - & - & - & \\
\hline Petroleum & .35 & 1.79 & 4.41 & 4.23 & 2.40 & 2.94 & 2.37 & 2.79 \\
\hline 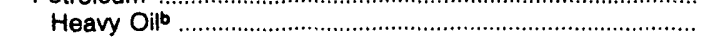 & .34 & 1.78 & 4.38 & 4.21 & 2.39 & 2.93 & 2.35 & 2.78 \\
\hline Light Oib & .41 & 2.48 & 6.33 & 5.89 & 3.49 & 4.19 & 4.43 & 4.14 \\
\hline 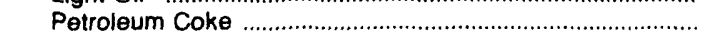 & - & - & - & - & - & - & - & \\
\hline 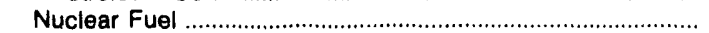 & - & .32 & .58 & .62 & .58 & .60 & .64 & .54 \\
\hline Word and Waste & - & - & - & - & - & - & - & - \\
\hline Primary Energy - Five Sectors & 1.45 & 2.54 & 5.70 & 5.85 & 4.31 & 5.01 & 4.86 & 8.00 \\
\hline
\end{tabular}

-There are no direct fuel costs for hydroelectric, geothermal, centralized solar, or wind energy. Wood and other biomass fuels are not included, except those consumed at electric utilities.

b Heavy oil includes Grade Nos. 4, 5, and 6, and residual fuel oils. Light oil includes Grade No. 2 heating oil, kerosene, and jet fuel.

- No consumption, including cases where adjustments were made. See "Consumption Adjustments for Process Fuel and Intermediate Products."

Sources: Data sources, estimation procedures, and assumptions are described in the "Documentation" section of this report. 


\begin{tabular}{|c|c|c|c|c|c|c|c|c|}
\hline Sector and Energy Source & 1970 & 1975 & 1980 & 1985 & 1986 & 1987 & 1988 & 1989 \\
\hline $\begin{array}{c}\text { Residential Sector } \\
\text { Primary Energy }\end{array}$ & $\begin{array}{r}137.9 \\
902\end{array}$ & 257.0 & 482.8 & 533.9 & 517.2 & $\begin{array}{l}532.1 \\
229.8\end{array}$ & 582.1 & $\begin{array}{l}525.7 \\
2847\end{array}$ \\
\hline $\begin{array}{l}\text { Primary Energy } \\
\text { Coal }\end{array}$ & $\begin{array}{r}90.2 \\
.7\end{array}$ & $\begin{array}{r}158.0 \\
.8\end{array}$ & $\begin{array}{r}295.6 \\
1.1\end{array}$ & $\begin{array}{r}257.3 \\
2.2\end{array}$ & $\begin{array}{r}224.5 \\
2.6\end{array}$ & $\begin{array}{r}229.8 \\
2.0\end{array}$ & $\begin{array}{r}260.2 \\
1.6\end{array}$ & $\begin{array}{r}284.7 \\
1.1\end{array}$ \\
\hline Natural Gas & 1.0 & 1.9 & 3.5 & 4.8 & 4.6 & 4.2 & 4.1 & 4.6 \\
\hline Petroleum & 88.4 & 155.3 & 291.0 & 250.3 & 217.3 & 223.5 & 254.5 & 279.0 \\
\hline Distillate Fuel & 69.1 & $12 ? .9$ & 257.7 & 214.6 & 186.4 & 179.9 & 204.6 & 223.9 \\
\hline 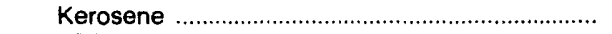 & 14.9 & 16.7 & 18.7 & 20.9 & 12.0 & 13.7 & 14.3 & 17.9 \\
\hline LPG & 4.4 & 10.7 & 14.6 & 14.8 & 18.8 & 29.8 & 35.6 & 37.3 \\
\hline 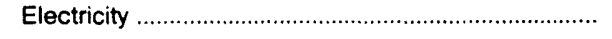 & 47.7 & 99.0 & 187.2 & 276.6 & 292.7 & 302.4 & 322.0 & 341.0 \\
\hline Commercial Sector & 39.9 & 93.9 & 210.3 & 269.3 & 282.1 & 261.5 & 295.2 & 308.0 \\
\hline 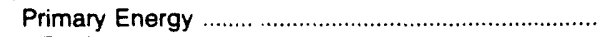 & 13.7 & 31.5 & 97.8 & 80.3 & 82.1 & 75.1 & 97.2 & 95.0 \\
\hline Coal & .4 & .7 & .5 & 1.6 & 2.0 & 1.7 & 1.4 & 1.0 \\
\hline Natural Gas & .6 & 1.1 & 4.4 & 9.1 & 9.1 & 8.7 & 9.1 & 10.5 \\
\hline Petroleum & 12.7 & 29.7 & 92.9 & 69.6 & 71.0 & 64.7 & 86.7 & 83.6 \\
\hline Distillate Fuel & 10.8 & 23.1 & 67.7 & 39.9 & 48.4 & 45.5 & 53.3 & 55.3 \\
\hline Kerosene & .3 & .6 & 2.6 & 1.9 & .4 & .7 & 2.1 & 1.6 \\
\hline LPG & .3 & 1.3 & 1.6 & 2.3 & 3.8 & 5.6 & 6.6 & 6.5 \\
\hline Motor Gasoline & .6 & 1.0 & 2.5 & 5.1 & 4.1 & 3.8 & 4.5 & 5.3 \\
\hline Residual Fuel & 6 & 3.7 & 18.6 & 20.4 & 14.3 & 9.1 & 20.2 & 14.9 \\
\hline 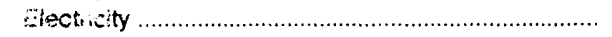 & 26.1 & 62.5 & 112.5 & 189.0 & 199.9 & 186.4 & 198.0 & 213.0 \\
\hline Industrial Sector & 55.3 & 151.1 & 317.1 & 405.4 & 353.6 & 350.6 & 408.0 & 393.2 \\
\hline Primary Energy & 26.9 & 96.5 & 161.4 & 195.2 & 154.1 & 140.9 & 174.0 & 146.2 \\
\hline Coal & 1.1 & 2.0 & 4.1 & 9.3 & 16.5 & 13.3 & 15.4 & 15.9 \\
\hline 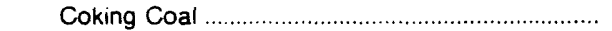 & - & - & - & - & - & - & - & - \\
\hline Steam Coal & 1.1 & 2.0 & 4.1 & 9.3 & 16.5 & 13.3 & 15.1 & 15.9 \\
\hline 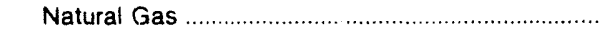 & .3 & 1.0 & 3.2 & 5.4 & 4.2 & 4.7 & 5.2 & 6.3 \\
\hline 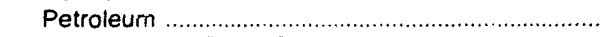 & 25.5 & 93.5 & 154.2 & 180.5 & 133.4 & 123.0 & 153.6 & 123.9 \\
\hline 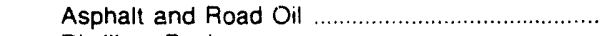 & 3.4 & 9.2 & 10.8 & 75.6 & 23.8 & 20.1 & 35.2 & 21.5 \\
\hline 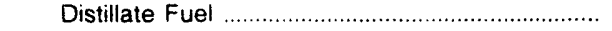 & 2.9 & 9.2 & 26.4 & 17.6 & 15.7 & 26.0 & 33.3 & 34.6 \\
\hline 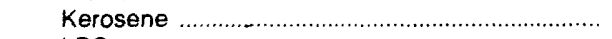 & .2 & .9 & 1.1 & .6 & .3 & .7 & .7 & .8 \\
\hline LPG & .9 & 3.0 & 8.9 & 7.3 & 15.2 & 11.5 & 19.7 & 16.7 \\
\hline Lubricants & 1.7 & 2.7 & 5.6 & 6.3 & 5.4 & 5.3 & 5.5 & 7.0 \\
\hline Motor Gasoline & 2.2 & 1.9 & 3.8 & 6.1 & 5.1 & 5.5 & 5.7 & 6.4 \\
\hline 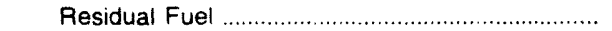 & 13.8 & 66.8 & 97.6 & 67.0 & 67.9 & 53.8 & 53.4 & 37.0 \\
\hline Other & .4 & - & - & - & - & - & - & - \\
\hline 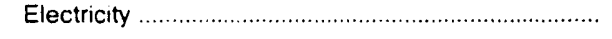 & 28.4 & 54.6 & 155.7 & 210.2 & 199.5 & 209.6 & 234.0 & 247.0 \\
\hline 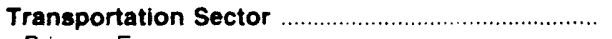 & 199.9 & 364.6 & $\mathbf{7 4 8 . 1}$ & 848.3 & 720.8 & 799.6 & 920.1 & 900.1 \\
\hline 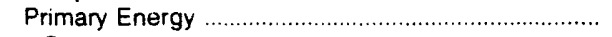 & 199.9 & 364.6 & 748.1 & 848.3 & 720.8 & 799.6 & 920.1 & 900.1 \\
\hline 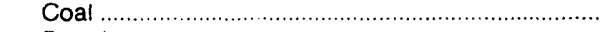 & • & 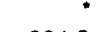 & - & - & - & - & - & - \\
\hline 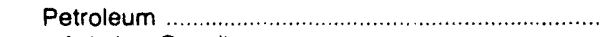 & 199.9 & 364.6 & 748.1 & 848.3 & 720.8 & 799.6 & 920.1 & 900.1 \\
\hline Aviation Gasoline & 1.0 & 1.2 & 3.7 & 2.1 & 2.4 & 2.0 & 2.5 & 2.9 \\
\hline 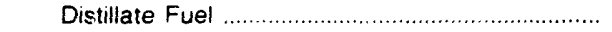 & 11.2 & 25.8 & 68.8 & 173.3 & 154.4 & 180.4 & 205.3 & 183.8 \\
\hline 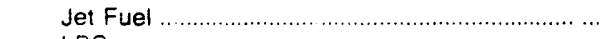 & 9.4 & 22.7 & 66.7 & 54.4 & 39.6 & 43.7 & 47.7 & 59.5 \\
\hline LPG & • & * & .2 & .4 & .7 & .5 & .9 & 1.0 \\
\hline Lubricants & 3.5 & 4.9 & 11.5 & 12.8 & 11.1 & 10.9 & 11.3 & 14.2 \\
\hline Motor Gasoline & 172.1 & 300.2 & 592.4 & 604.7 & 511.8 & 561.1 & 648.7 & 635.8 \\
\hline 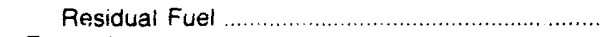 & 2.7 & 9.8 & 4.8 & .5 & .7 & 1.1 & 3.7 & 2.9 \\
\hline 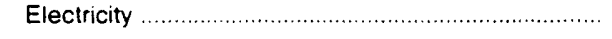 & - & - & - & - & - & - & - & - \\
\hline Total Energy" & 433.1 & 866.7 & $1,758.3$ & $2,057.0$ & $1,873.6$ & $1,943.8$ & $2,205.5$ & $2,226.9$ \\
\hline Primary Energy - Four Sectors .............................. & 330.7 & 650.6 & $1,303.0$ & $1,381.3$ & $1,181.6$ & $1,245.5$ & $1,451.5$ & $1,425.9$ \\
\hline 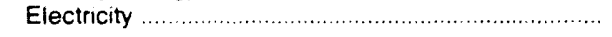 & 102.3 & 216.1 & 455.3 & 675.7 & 692.1 & 698.4 & 754.0 & 801.0 \\
\hline Electric Utility Sector & 10.6 & 48.1 & 129.8 & 127.6 & 105.0 & 106.3 & 107.3 & 128.9 \\
\hline 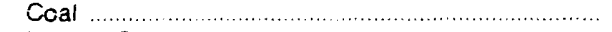 & - & - & - & - & - & - & - & - \\
\hline Natural Gas & - & - & - & - & - & - & - & - \\
\hline Petroleum & 10.6 & 32.0 & 101.9 & 91.9 & 66.1 & 80.4 & 73.0 & 88.9 \\
\hline Heavy O: $\mathbb{1}^{\mathbf{D}}$ & 10.3 & 31.4 & 99.7 & 90.9 & 65.5 & 79.6 & 71.7 & 87.8 \\
\hline 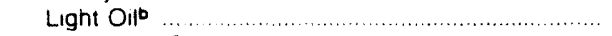 & .2 & .6 & 2.2 & 1.0 & .7 & .8 & 1.3 & 1.1 \\
\hline Petroieum Coke & - & - & - & - & - & - & - & - \\
\hline Nuclear Fuel & - & 16.1 & 27.9 & 35.7 & 38.9 & 25.9 & 34.2 & 40.0 \\
\hline Wood and Waste & - & - & .• & - & - & - & - & - \\
\hline Primary Energy - Five Sectors & 341.3 & 698.6 & $1,432.8$ & $1,508.8$ & 1,286.6 & $1,351.8$ & $1,558.8$ & $1,554.8$ \\
\hline
\end{tabular}

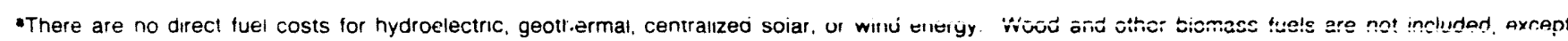
those consumed at electric utlities

DHeavy oil includes Grade Nns 4, 5. and 6. and residual fuel oils. Light oil includes Grade No. 2 heating oll, kerosene, and jet fuel

- No consumption, including ises where adjustments were made. See "Consumption Adjustments for Process Fuel and Intermediate Products."

- Value iess than 0.05 million duilars.

Noie. Totals may not equal sum of components due to independent rounding.

Sources Data sources. estimation procedures, and assumptions are described in the "Documentation" section of this report 


\begin{tabular}{|c|c|c|c|c|c|c|c|c|}
\hline Energy Source & 1970 & 1975 & 1980 & 1985 & 1986 & 987 & 1988 & 1989 \\
\hline & \multicolumn{8}{|c|}{ Prices In Dollars per Million Btu } \\
\hline 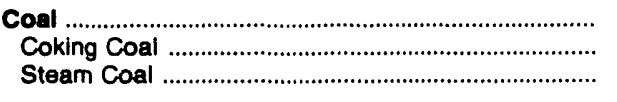 & $\begin{array}{r}0.45 \\
.58 \\
.34\end{array}$ & $\begin{array}{l}1.69 \\
2.14 \\
1.28\end{array}$ & $\begin{array}{l}1.77 \\
2.38 \\
1.50\end{array}$ & $\begin{array}{l}1.75 \\
1.88 \\
1.71\end{array}$ & $\begin{array}{l}1.67 \\
1.76 \\
1.64\end{array}$ & $\begin{array}{l}1.56 \\
1.59 \\
1.56\end{array}$ & $\begin{array}{l}1.57 \\
1.61 \\
1.56\end{array}$ & $\begin{array}{l}1.60 \\
1.67 \\
1.59\end{array}$ \\
\hline 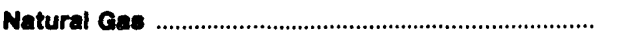 & 1.07 & 1.94 & 3.81 & 6.29 & 5.99 & $\mathbf{5 . 3 1}$ & 4.92 & 5.12 \\
\hline $\begin{array}{l}\text { Petroleum } \\
\text { Asphait and Road Oil } \\
\text { Aviation Gasoline } \\
\text { Distillate Fuel } \\
\text { Jet Fuel } \\
\text { Kerosene } \\
\text { LPG }\end{array}$ & $\begin{array}{r}1.61 \\
.60 \\
2.17 \\
1.20 \\
.73 \\
1.46 \\
1.87 \\
5.08 \\
2.85 \\
.43 \\
1.08\end{array}$ & $\begin{array}{l}3.29 \\
1.89 \\
3.45 \\
2.61 \\
2.04 \\
3.24 \\
3.71 \\
7.49 \\
4.86 \\
1.87 \\
2.00\end{array}$ & $\begin{array}{r}7.62 \\
3.73 \\
9.02 \\
6.87 \\
6.46 \\
7.88 \\
6.81 \\
14.36 \\
9.93 \\
4.04 \\
6.56\end{array}$ & $\begin{array}{r}8.10 \\
4.95 \\
9.99 \\
7.83 \\
5.80 \\
8.59 \\
11.27 \\
17.61 \\
9.50 \\
4.04 \\
5.56\end{array}$ & $\begin{array}{r}6.39 \\
4.18 \\
8.41 \\
6.13 \\
4.01 \\
7.08 \\
11.00 \\
15.59 \\
7.55 \\
2.29 \\
4.76\end{array}$ & $\begin{array}{r}6.49 \\
3.22 \\
7.55 \\
6.34 \\
4.01 \\
6.87 \\
10.83 \\
13.58 \\
7.80 \\
3.04 \\
4.44\end{array}$ & $\begin{array}{r}6.51 \\
3.16 \\
7.41 \\
6.32 \\
3.75 \\
6.64 \\
9.68 \\
14.61 \\
8.18 \\
2.28 \\
3.88\end{array}$ & $\begin{array}{r}6.94 \\
2.90 \\
8.28 \\
6.81 \\
4.38 \\
7.19 \\
13.90 \\
17.90 \\
8.84 \\
2.68 \\
4.72\end{array}$ \\
\hline 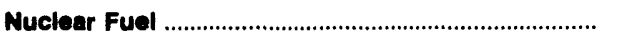 & - & .23 & .44 & .59 & .56 & .49 & .49 & .52 \\
\hline Wood and Waste at Utillties ......................................... & - & - & - & .78 & .32 & .95 & .87 & .82 \\
\hline Primary Energy - Flve Sectors ................................. & 1.16 & 2.62 & 4.88 & 5.35 & 4.31 & 4.35 & 4.25 & 4.96 \\
\hline 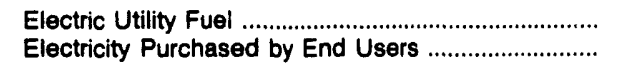 & $\begin{array}{r}.40 \\
5.76\end{array}$ & $\begin{array}{r}1.36 \\
11.19\end{array}$ & $\begin{array}{r}1.66 \\
15.47\end{array}$ & $\begin{array}{r}1.65 \\
18.60\end{array}$ & $\begin{array}{r}1.32 \\
18.66\end{array}$ & $\begin{array}{r}1.43 \\
17.61\end{array}$ & $\begin{array}{r}1.35 \\
17.12\end{array}$ & $\begin{array}{r}1.83 \\
17.53\end{array}$ \\
\hline \multirow[t]{2}{*}{ 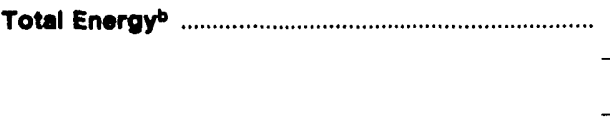 } & 1.78 & 4.02 & 7.67 & 9.09 & 8.21 & 7.95 & 7.97 & 8.60 \\
\hline & \multicolumn{8}{|c|}{ Expenditures in Mililions of Dollars } \\
\hline 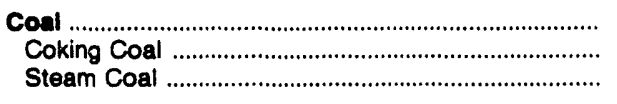 & $\begin{array}{r}139.6 \\
79.6 \\
60.0\end{array}$ & $\begin{array}{l}333.2 \\
200.6 \\
132.6\end{array}$ & $\begin{array}{l}416.8 \\
168.9 \\
247.9\end{array}$ & $\begin{array}{l}448.6 \\
107.4 \\
341.2\end{array}$ & $\begin{array}{r}458.2 \\
91.5 \\
366.7\end{array}$ & $\begin{array}{r}451.2 \\
82.3 \\
368.9\end{array}$ & $\begin{array}{r}473.4 \\
82.8 \\
390.6\end{array}$ & $\begin{array}{r}473.0 \\
77.4 \\
395.6\end{array}$ \\
\hline 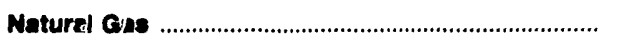 & 168.5 & 270.5 & 607.5 & 966.9 & 933.1 & 913.8 & 864.2 & 990.5 \\
\hline $\begin{array}{l}\text { Potroleum } \\
\text { Asphalt and Road Oil } \\
\text { Aviation Gasoline } \\
\text { Distillate Fuel } \\
\text { Jet Fuel } \\
\text { Kerosene } \\
\text { LPG } \\
\text { Lubricants } \\
\text { Motor Gasoline } \\
\text { Otheridual Fuel }\end{array}$ & $\begin{array}{r}851.3 \\
11.1 \\
3.4 \\
138.3 \\
18.1 \\
19.3 \\
13.0 \\
19.2 \\
556.7 \\
58.7 \\
13.4\end{array}$ & $\begin{array}{r}1,930.9 \\
40.8 \\
3.6 \\
317.1 \\
34.6 \\
21.9 \\
33.0 \\
34.6 \\
1,115.0 \\
314.0 \\
16.2\end{array}$ & $\begin{array}{r}3,996.4 \\
65.3 \\
7.9 \\
872.6 \\
126.3 \\
52.2 \\
51.5 \\
63.1 \\
2,296.3 \\
415.8 \\
45.4\end{array}$ & $\begin{array}{r}3,788.5 \\
148.6 \\
3.8 \\
807.7 \\
125.7 \\
27.5 \\
72.8 \\
70.4 \\
2,275.7 \\
191.9 \\
64.3\end{array}$ & $\begin{array}{r}3,027.8 \\
144.7 \\
4.3 \\
621.0 \\
86.7 \\
19.0 \\
57.4 \\
60.9 \\
1,860.2 \\
92.5 \\
81.1\end{array}$ & $\begin{array}{r}3,229.3 \\
102.9 \\
3.3 \\
668.0 \\
84.0 \\
27.0 \\
65.5 \\
60.0 \\
1,970.1 \\
151.8 \\
96.7\end{array}$ & $\begin{array}{r}3,352.1 \\
91.3 \\
3.5 \\
682.5 \\
93.7 \\
26.5 \\
64.2 \\
62.3 \\
2,114.9 \\
134.3 \\
81.9\end{array}$ & $\begin{array}{r}3,809.2 \\
86.7 \\
3.5 \\
816.8 \\
107.2 \\
20.0 \\
114.2 \\
78.2 \\
2,278.9 \\
226.4 \\
77.2\end{array}$ \\
\hline 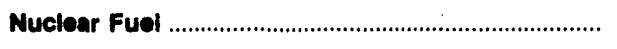 & - & 11.3 & 52.5 & 62.8 & 77.3 & 53.2 & 62.1 & 15.1 \\
\hline 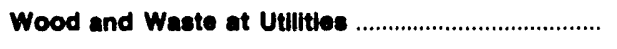 & - & - & - & .1 & .1 & .5 & .5 & .1 \\
\hline Primary Energy - Flve Sectore ..................................... & $1,159.4$ & $2,546.0$ & $5,073.2$ & $5,267.0$ & $4,496.6$ & $4,648.0$ & $4,752.3$ & $5,287.9$ \\
\hline 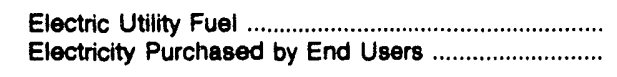 & $\begin{array}{l}-91.0 \\
442.4\end{array}$ & $\begin{array}{r}-352.5 \\
1,042.3\end{array}$ & $\begin{array}{r}-544.9 \\
1,825.5\end{array}$ & $\begin{array}{r}-536.2 \\
2,496.0\end{array}$ & $\begin{array}{r}-495.4 \\
2,671.2\end{array}$ & $\begin{array}{r}-517.2 \\
2,698.1\end{array}$ & $\begin{array}{r}-544.1 \\
2,779.1\end{array}$ & $\begin{array}{r}-646.8 \\
2,946.0\end{array}$ \\
\hline 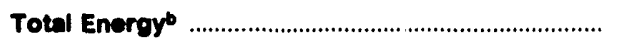 & $1,510.8$ & $3,235.7$ & $6,353.8$ & $7,226.8$ & $6,672.4$ & $8,828.8$ & $6,987.3$ & $7,587.2$ \\
\hline
\end{tabular}

Includes petroleum coke used at electric utilities.

-There are no direct fuel costs for hydroelectric, geothermal, centralized solar, or wind energy. Wood and other biomass fuels are not included, except those consumed at electric utilities.

-No consumption, including cases where adjustments were made. See "Consumption Adjustments for Process Fuel and Intermediate Products."

Note: Expenditure totais may not equal sum of components due to independent rounding.

Sources: Data sources, estimation procedures, and assumptions are described in the "Documentaiion" section of this report. 


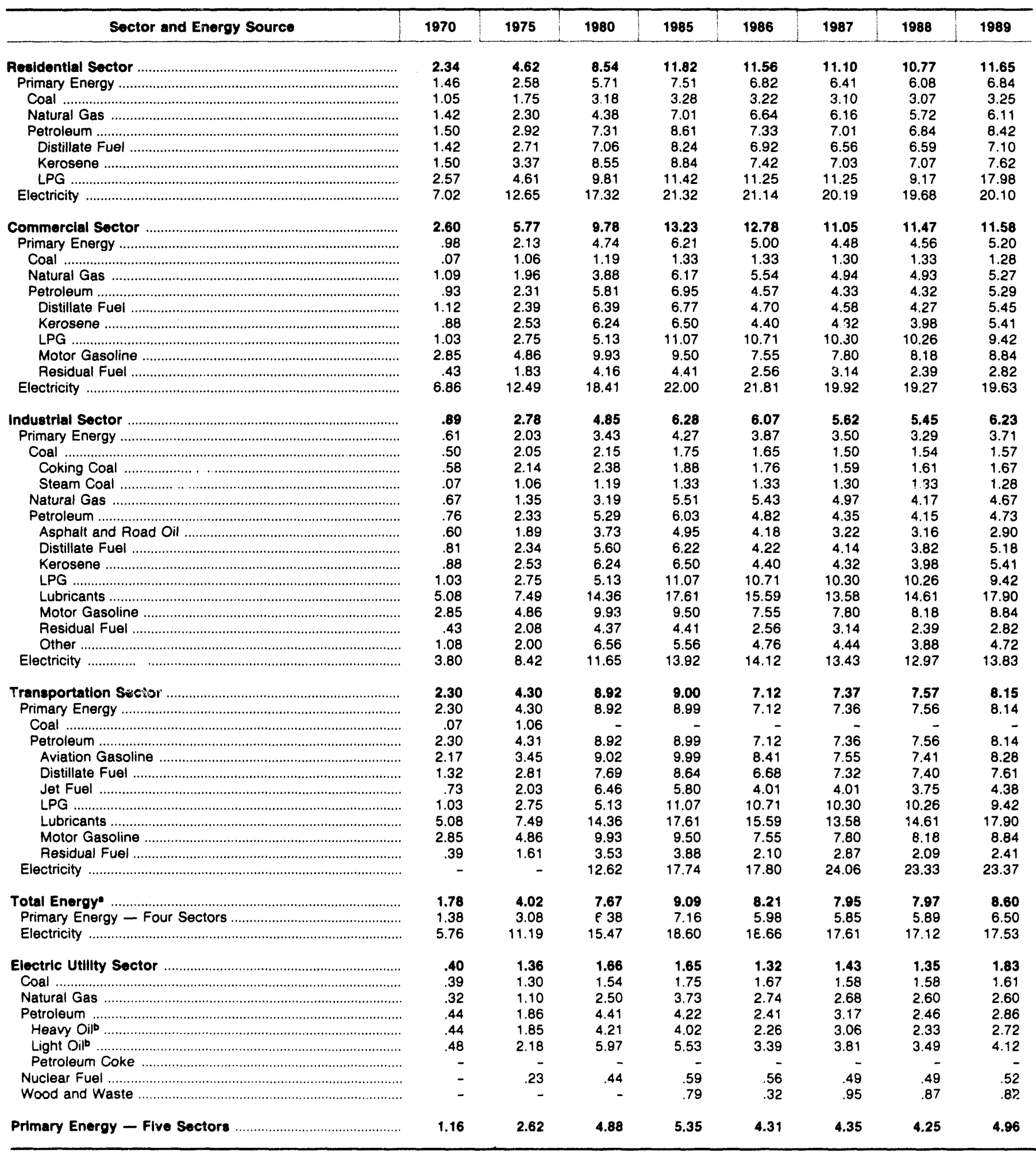

- There are no direct fuel costs for hydroelectric, geothermal, centralized solar, or wind energy. Wood and ather biomass fuels are not included, except those consumed at electric utilities.

bHeaw oil includes Grade Nos. 4, 5, and 6, and residual fuel oils. Light oil includes Grade No. 2 heating oil, kerosene, and jet fuel

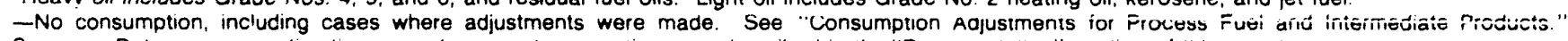

Sources: Data sources, estimation procedures, and assumptions are described in the "Documentation" section of this report 


\section{Energy Expenditure Estimates by Sector, Maryland \\ A $1970,1975,1980$, and $1985-1989$ \\ $\mathbf{R}$ (Million Dollars)}

\begin{tabular}{|c|c|c|c|c|c|c|c|c|}
\hline Sector and Energy Source & 1970 & 1975 & 1980 & 1985 & 1986 & 1987 & 1988 & 1989 \\
\hline Residentlal Sector & 387.3 & 752.6 & $1,450.2$ & $1,849.4$ & $1,883.7$ & $1,916.0$ & $1,969.3$ & $2,090.0$ \\
\hline 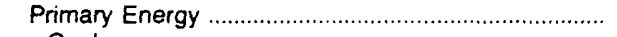 & 203.1 & 335.9 & 733.9 & 807.7 & 742.5 & 730.0 & 728.2 & 782.0 \\
\hline Coal & .9 & .6 & 1.2 & 3.6 & 3.7 & 4.9 & 3.5 & 1.6 \\
\hline 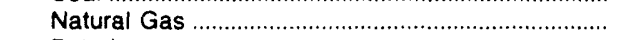 & 106.1 & 161.4 & 304.1 & 496.1 & 494.8 & 450.2 & 442.0 & 473.4 \\
\hline 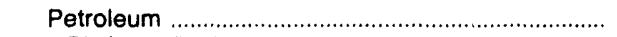 & 96.1 & 173.9 & 428.6 & 308.1 & 244.1 & 274.9 & 282.7 & 307.0 \\
\hline 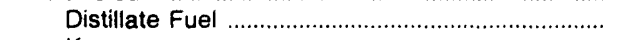 & 67.9 & 133.3 & 361.7 & 241.0 & 194.2 & 210.8 & 227.2 & 212.6 \\
\hline 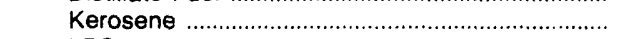 & 18.4 & 19.3 & 40.2 & 25.3 & 17.7 & 25.9 & 24.4 & 17.2 \\
\hline LPG & 9.8 & 21.3 & 26.7 & 41.8 & 32.2 & 38.1 & 31.2 & 77.3 \\
\hline 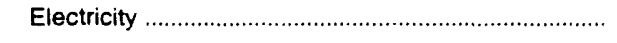 & 184.2 & 416.8 & 716.3 & $1,041.6$ & $1,141.2$ & $1,186.1$ & $1,241.0$ & $1,308.0$ \\
\hline Commerclal Sector & 204.9 & 480.9 & 849.7 & 977.8 & 967.2 & 963.9 & 961.9 & 951.8 \\
\hline 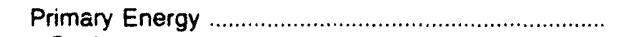 & 56.4 & 115.5 & 260.1 & 255.0 & 203.2 & 224.8 & 202.6 & 238.6 \\
\hline 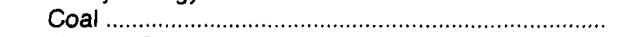 & .1 & .5 & .6 & 2.5 & 2.6 & 3.7 & 2.6 & 1.1 \\
\hline 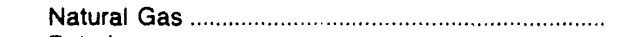 & 28.8 & 50.1 & 113.1 & 153.9 & 136.6 & 130.3 & 131.7 & 146.2 \\
\hline 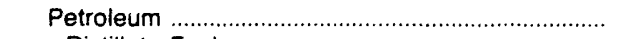 & 27.5 & 65.0 & 146.4 & 98.6 & 64.0 & 90.8 & 68.3 & 91.3 \\
\hline 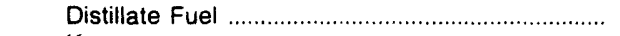 & 20.9 & 45.8 & 106.6 & 76.6 & 42.2 & 51.6 & 46.3 & 63.6 \\
\hline Kerosene & .3 & .5 & .7 & 1.5 & .6 & .3 & .7 & 1.3 \\
\hline LPG & .7 & 2.2 & 2.5 & 7.2 & 5.4 & 6.2 & 6.2 & 7.1 \\
\hline 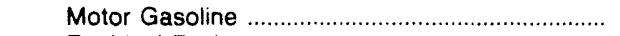 & 1.5 & 3.1 & 6.3 & 8.5 & 6.9 & 7.4 & 7.3 & 9.2 \\
\hline 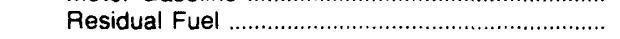 & 4.1 & 13.4 & 30.3 & 4.9 & 8.8 & 25.3 & 7.9 & 10.0 \\
\hline Electricity & 148.5 & 365.3 & 589.7 & 722.8 & 764.0 & 739.2 & 759.3 & 713.2 \\
\hline Industrial Sector & 294.9 & 731.8 & $1,247.0$ & $1,5>2.9$ & $1,526.3$ & $1,501.0$ & $1,451.1$ & $1,661.2$ \\
\hline 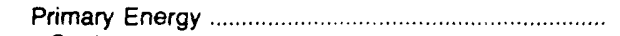 & 185.2 & 471.6 & 728.5 & 845.4 & 764.5 & 733.8 & 679.1 & 743.2 \\
\hline Coal & 81.4 & 209.4 & 150.1 & 131.0 & 115.4 & 113.7 & 105.5 & 99.1 \\
\hline 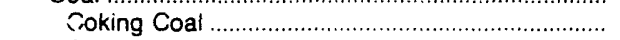 & 79.6 & 200.6 & 168.9 & 107.4 & 91.5 & 82.3 & 82.8 & 77.4 \\
\hline 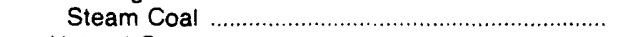 & 1.8 & 8.8 & 21.2 & 23.5 & 23.9 & 31.4 & 22.8 & 21.7 \\
\hline 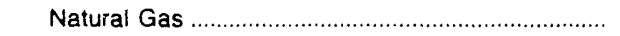 & 29.8 & 58.6 & 176.9 & 311.7 & 295.4 & 300.5 & 276.0 & 318.8 \\
\hline Petroleum & 74.0 & 203.6 & 361.5 & 402.8 & 353.7 & 319.7 & 297.6 & 325.4 \\
\hline 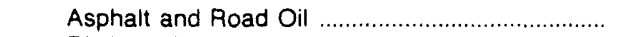 & 11.1 & 40.8 & 65.3 & 148.6 & 144.7 & 102.9 & 91.3 & 86.7 \\
\hline Distillate Fuel & 14.8 & 44.4 & 104.1 & 92.3 & 51.3 & 40.1 & 38.2 & 63.5 \\
\hline 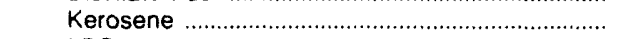 & .5 & 2.1 & 11.3 & .7 & .7 & .7 & 1.5 & 1.6 \\
\hline 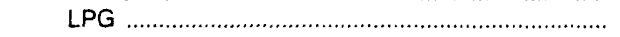 & 2.4 & 9.1 & 21.9 & 22.0 & 18.7 & 20.2 & 22.2 & 28.1 \\
\hline Lubricants . & 10.0 & 20.7 & 36.1 & 40.2 & 34.8 & 34.3 & 35.6 & 44.7 \\
\hline 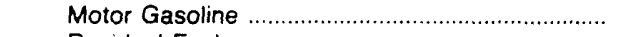 & 3.9 & 7.5 & 7.6 & 14.9 & 12.7 & 13.6 & 15.2 & 15.9 \\
\hline 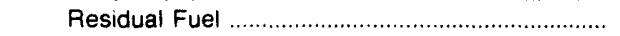 & 17.8 & 62.8 & 69.9 & 19.7 & 9.7 & 11.1 & 11.7 & 7.6 \\
\hline Other & 13.4 & 16.2 & 45.4 & 64.3 & 81.1 & 96.7 & 81.9 & 77.2 \\
\hline 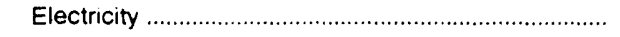 & 109.7 & 260.2 & 518.5 & 727.4 & 761.8 & 767.2 & 772.0 & 918.0 \\
\hline Transportation Sector & 623.7 & $1,270.4$ & $2,806.8$ & $2,826.8$ & $2,295.2$ & $2,447.9$ & $2,605.0$ & $2,884.1$ \\
\hline 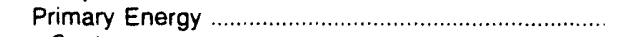 & 623.7 & $1,270.4$ & $2,805.9$ & $2,822.6$ & $2,291.0$ & $2,442.2$ & $2,598.2$ & $2,877.3$ \\
\hline 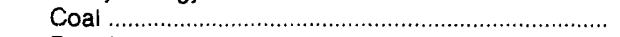 & & & & & & & & \\
\hline 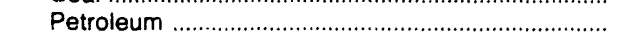 & 623.7 & $1,270.4$ & $2,805.9$ & $2,822.6$ & $2,291.0$ & $2,442.2$ & $2,598.2$ & $2,877.3$ \\
\hline 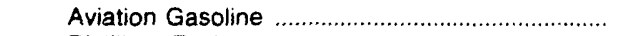 & 3.4 & 3.6 & 7.9 & 3.8 & 4.3 & 3.3 & 3.5 & 3.5 \\
\hline 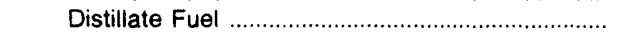 & 32.1 & 85.9 & 262.0 & 371.1 & 318.6 & 347.6 & 353.4 & 447.0 \\
\hline 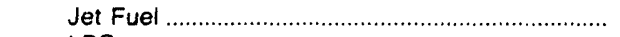 & 18.1 & 33.5 & 125.9 & 125.7 & 86.7 & 84.0 & 93.7 & 107.2 \\
\hline LPG & .1 & .5 & .5 & 1.8 & 1.1 & 1.0 & 1.7 & 1.7 \\
\hline 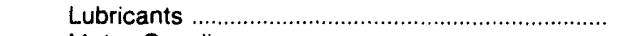 & 9.2 & 13.9 & 27.0 & 30.1 & 26.1 & 25.7 & 26.7 & 33.5 \\
\hline 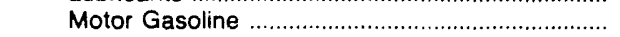 & 551.2 & $1,104.5$ & $2,282.4$ & $2,252.3$ & $1,840.6$ & $1,949.1$ & $2,092.4$ & 2.253 .8 \\
\hline 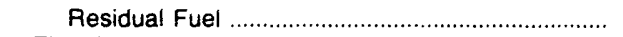 & 9.5 & 28.5 & 100.1 & 37.7 & 13.7 & 31.5 & 26.8 & 30.6 \\
\hline 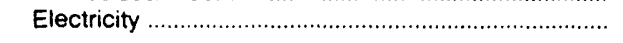 & - & - & .9 & 4.1 & 4.2 & 5.7 & 6.8 & 6.8 \\
\hline 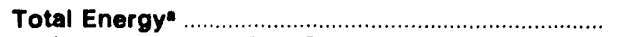 & $1,510.8$ & $3,235.7$ & $6,353.8$ & $7,226.8$ & $6,672.4$ & $6,828.8$ & $6,987.3$ & $7,587.2$ \\
\hline Primary Energy - Four Sectors ................................... & $1,058.4$ & $2,193.4$ & $4,528.3$ & $4,730.8$ & $4,001.2$ & $4,130.8$ & $4,208.2$ & $4,641.1$ \\
\hline 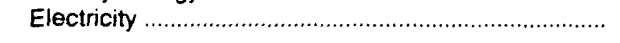 & 442.4 & $1,042.3$ & $1,825.5$ & $2,496.0$ & $2,671.2$ & $2,698.1$ & $2,779.1$ & $2,946.0$ \\
\hline Eloctric Utility Sector & 91.0 & 352.5 & $5+4.9$ & 536.2 & 495.4 & 517.2 & 544.1 & 646.8 \\
\hline Coal & 57.3 & 122.7 & 224.9 & 311.6 & 336.6 & 328.9 & 361.8 & 371.2 \\
\hline 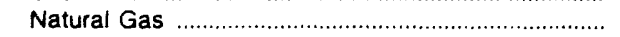 & 3.8 & .5 & 13.4 & 5.2 & 6.4 & 32.9 & 14.4 & 52.1 \\
\hline 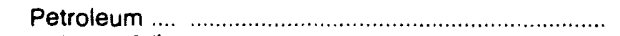 & 30.0 & 218.0 & 254.1 & 156.4 & 75.1 & 101.7 & 105.3 & 208.2 \\
\hline 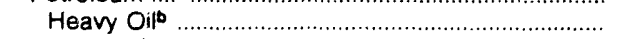 & 27.4 & 209.3 & 215.5 & 129.7 & 60.3 & 83.8 & 87.8 & 178.1 \\
\hline 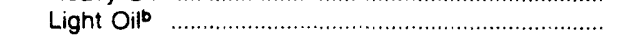 & 2.6 & 8.7 & 38.6 & 26.7 & 14.7 & 17.9 & 17.4 & 30.1 \\
\hline Petroleum Coke & - & & & - & & - & - & \\
\hline 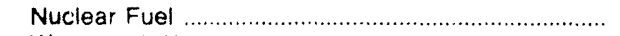 & - & 11.3 & 52.5 & 62.9 & 77.3 & 53.2 & 62.1 & 15.1 \\
\hline Wood and Waste & - & - & - & .1 & .1 & .5 & .5 & .1 \\
\hline Primary Energy - Five Sectors & $1,159.4$ & $2,546.0$ & $5,073.2$ & $5,267.0$ & $4,496.6$ & $4,648.0$ & $4,752.3$ & $5,287.9$ \\
\hline
\end{tabular}

-There are no direct fuel costs for hydroelectric, geothermal, centralized solar, or wind energy. Wood and other biomass fuels are not included, except those consumed at electric utilities.

- Heavy oil includes Grade Nos. 4, 5, and 6, and residual fuel oils. Light oil includes Grade No. 2 heating oil, kerosene, and iet fuel.

- Na sonsımptinn including cases where adiustments were made. See "Consumption Adjustments for Process Fuel and Intermediate Products."

- Value less than $\mathrm{j} .05$ million dollars.

Note: Totals may not squal sum of components due to independent rounding

Sources: Data sources, estimation frocedures, and assumptions are described in the "Documentation" section of this report 


\begin{tabular}{|c|c|c|c|c|c|c|c|c|}
\hline Energy Source & 1970 & 1975 & 1880 & 1985 & 1986 & 1987 & 1988 & 1989 \\
\hline & \multicolumn{8}{|c|}{ Prices In Dollars per Million Btu } \\
\hline Coal & 0.55 & 1.57 & 2.03 & 2.03 & 1.81 & 1.66 & 1.66 & 1.64 \\
\hline 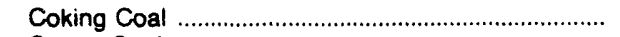 & - & - & - & - & - & - & - & - \\
\hline 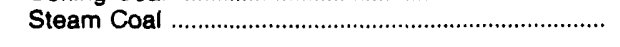 & .55 & 1.57 & 2.03 & 2.03 & 1.81 & 1.66 & 1.66 & 1.64 \\
\hline 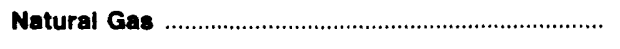 & 1.58 & 2.86 & 4.88 & 6.25 & 6.35 & 5.23 & 5.46 & 5.33 \\
\hline Petroleum & 1.24 & 2.85 & 6.60 & 7.37 & 5.26 & 5.67 & 5.62 & 6.26 \\
\hline 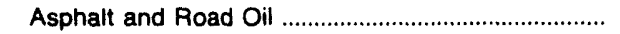 & .63 & 1.95 & 3.73 & 5.21 & 4.87 & 3.57 & 3.38 & 3.20 \\
\hline Aviation Gasoline & 2.17 & 3.45 & 9.02 & 9.99 & 8.41 & 7.55 & 7.41 & 8.28 \\
\hline 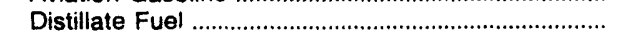 & 1.34 & 2.74 & 6.87 & 8.05 & 6.00 & 5.86 & 5.87 & 6.55 \\
\hline 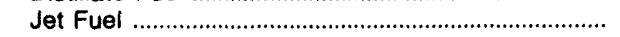 & .75 & 2.10 & 6.51 & 6.04 & 4.18 & 4.12 & 4.02 & 4.61 \\
\hline 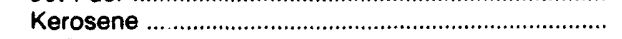 & 1.36 & 2.99 & 7.06 & 8.53 & 6.05 & 6.53 & 6.34 & 6.97 \\
\hline LPG & 2.31 & 3.74 & 6.62 & 11.75 & 10.09 & 11.05 & 11.01 & 12.04 \\
\hline 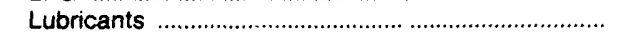 & 5.08 & 7.49 & 14.36 & 17.61 & 15.59 & 13.58 & 14.61 & 17.90 \\
\hline Motor Gasoline & 2.86 & 4.73 & 9.69 & 9.18 & 7.25 & 7.56 & 7.90 & 8.50 \\
\hline Residual Fuel & .39 & 1.95 & 3.84 & 4.00 & 2.29 & 2.75 & 2.27 & 2.64 \\
\hline 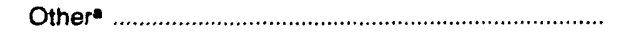 & 2.22 & 3.57 & 8.96 & 10.02 & 7.42 & 8.03 & 5.94 & 8.47 \\
\hline 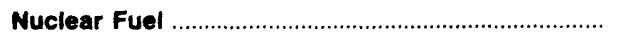 & .20 & .18 & .41 & .60 & .88 & .92 & .96 & .70 \\
\hline Wood and Waste at Utilities ..................................... & - & - & - & - & - & - & - & - \\
\hline 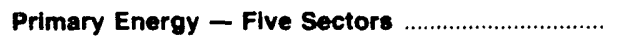 & 1.26 & 2.83 & 6.05 & 6.26 & 5.05 & 5.14 & 5.15 & 5.49 \\
\hline 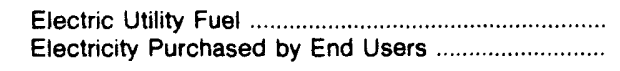 & $\begin{array}{r}.37 \\
7.29\end{array}$ & $\begin{array}{r}1.66 \\
13.93\end{array}$ & $\begin{array}{r}3.41 \\
21.13\end{array}$ & $\begin{array}{r}2.74 \\
24.34\end{array}$ & $\begin{array}{r}2.03 \\
22.70\end{array}$ & $\begin{array}{r}2.26 \\
23.46\end{array}$ & $\begin{array}{r}2.04 \\
22.86\end{array}$ & $\begin{array}{r}2.18 \\
24.39\end{array}$ \\
\hline \multirow[t]{2}{*}{ Total Energy } & 1.90 & 4.15 & 8.90 & 10.23 & 8.65 & 8.83 & 8.94 & 9.66 \\
\hline & \multicolumn{8}{|c|}{ Expenditures in Millions of Dollars } \\
\hline Coal & 11.7 & 38.5 & 46.4 & 223.8 & 180.9 & 195.2 & 194.2 & 198.4 \\
\hline 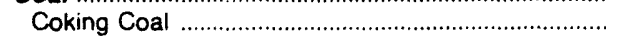 & - & - & - & - & - & - & - & - \\
\hline 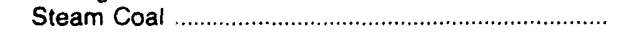 & 11.7 & 38.5 & 46.4 & 223.8 & 180.9 & 195.2 & 194.2 & 198.4 \\
\hline Natural Gas . & 234.1 & 441.3 & 901.9 & $1,395.1$ & $1,203.3$ & $1,215.6$ & $1,174.4$ & $1,367.2$ \\
\hline Petroleum & $1,542.1$ & $3,331.9$ & $6,056.8$ & $5,605.6$ & $4,474.9$ & $4,680.7$ & $4,755.9$ & $5,311.9$ \\
\hline 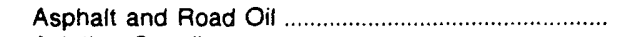 & 12.0 & 23.7 & 30.5 & 36.4 & 36.0 & 35.0 & 39.6 & 30.3 \\
\hline 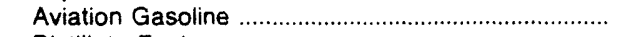 & 3.0 & 4.0 & 12.5 & 6.8 & 6.2 & 4.7 & 4.8 & 4.9 \\
\hline 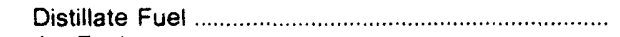 & 461.9 & 934.7 & $1,504.7$ & $1,550.3$ & $1,241.9$ & $1,290.7$ & $1,258.0$ & 1.540 .7 \\
\hline Jet Fuel & 33.3 & 95.0 & 315.8 & 238.4 & 163.4 & 182.9 & 212.1 & 260.8 \\
\hline 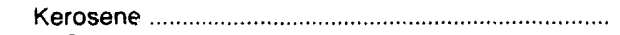 & 16.3 & 14.7 & 27.9 & 16.2 & 14.4 & 13.2 & 7.0 & 7.6 \\
\hline LPG & 15.9 & 32.2 & 51.7 & 72.3 & 83.5 & 99.8 & 96.7 & 117.5 \\
\hline Lubricants & 29.2 & 35.7 & 73.2 & 81.7 & 70.7 & 69.7 & 72.3 & 90.8 \\
\hline 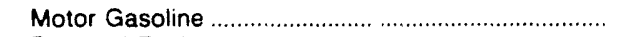 & 743.8 & $1,357.3$ & $2,619.1$ & $2,643.9$ & $2,147.8$ & $2,284.5$ & $2,465.8$ & $2,601.2$ \\
\hline Residual Fuel & 210.9 & 808.9 & $1,306.9$ & 826.0 & 624.0 & 604.8 & 518.5 & 561.4 \\
\hline 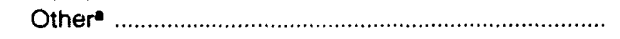 & 15.9 & 25.9 & 114.5 & 133.8 & 87.0 & 95.4 & 81.4 & 96.8 \\
\hline 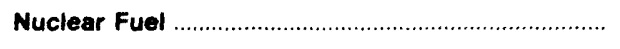 & 2.7 & 7.5 & 14.3 & 39.8 & 23.1 & 11.3 & 11.5 & 22.7 \\
\hline 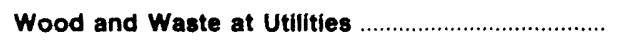 & - & - & - & - & - & - & - & - \\
\hline 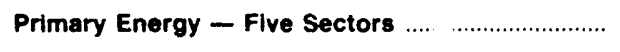 & $1,790.5$ & $3,819.2$ & $7,019.4$ & $7,264.4$ & $5,882.2$ & $6,102.8$ & $6,136.0$ & $6,900.2$ \\
\hline 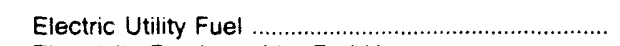 & -112.4 & -524.9 & $-1,191.4$ & $-1,011.3$ & -698.3 & -782.8 & -704.3 & -862.8 \\
\hline Electricity Purchased by End Users ............................ & 612.9 & $1,401.0$ & $2,398.4$ & $3,166.2$ & $3,101.9$ & $3,392.0$ & $3,489.0$ & $3,801.0$ \\
\hline Total Energyb & $2,291.0$ & $4,695.3$ & $8,226.4$ & $9,418.4$ & $8,285.7$ & $8,712.0$ & $8,920.7$ & $9,838.4$ \\
\hline
\end{tabular}

Includes petroleum coke used at electric utilities.

There are no direct fuel costs for hydroelectric, geothermal, centralized solar, or wind energy. Wood and other biomass fuels are not included, except those consumed at electric utilities.

-No consumption, including cases whsse adjustments were made. See "Consumption Adjustments for Process Fuel and Intermediate Products."

Note: Expenditure totals may not equai sum of components due to independent rounding.

Sources: Data sources, estimation procedures, and assumptions are described in the "Documentation" section of this report. 


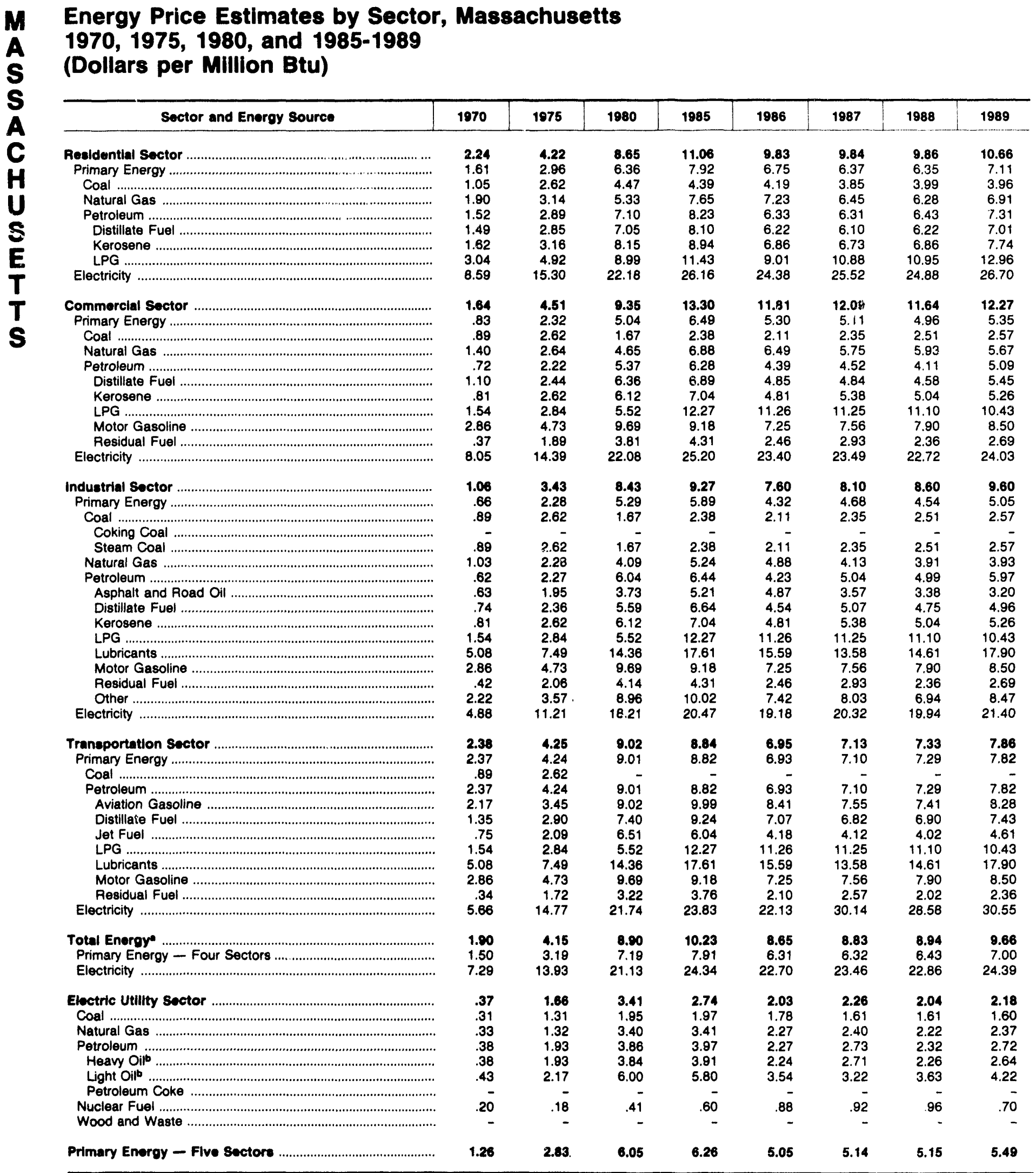

There are no direct fuel costs for hydroelectric, geothermal, centralized solar, or wind energy. Wood and other biomass fuels are not included, except those consumed at electric utilities.

bHeavy oil includes Grade Nos. 4, 5, and 6, and residual fuel oils. Light oil includes Grade No. 2 heating oil, kerosene, and jet fuel.

- No consumption, including cases where adjustments were made. See "Consumption Adjustmints for Process Fuel arid Intermediate Products."

Sources: Data sources, estimation procedures, and assumptions are described in the "Documentatisn" section of this report. 
Energy Expenditure Estimates by Sector, Massachusetts $1970,1975,1980$, and 1985-1989 (Million Dollars)

\begin{tabular}{|c|c|c|c|c|c|c|c|c|}
\hline Sector and Energy Source & 1970 & 1975 & 1980 & 1985 & 1986 & 1987 & 1988 & 1989 \\
\hline $\begin{array}{l}\text { Residential Sector } \\
\text { Primary Energy }\end{array}$ & $\begin{array}{l}793.8 \\
520.1\end{array}$ & $\begin{array}{r}1,501.2 \\
945.4\end{array}$ & $\begin{array}{l}2,362.9 \\
1,487.2\end{array}$ & $\begin{array}{l}2,829.2 \\
1,677.3\end{array}$ & $\begin{array}{l}2,612.3 \\
1,480.6\end{array}$ & $\begin{array}{l}2,684.3 \\
1,423.9\end{array}$ & $\begin{array}{l}2,755.0 \\
1,438.0\end{array}$ & $\begin{array}{l}3,163.8 \\
1,726.7\end{array}$ \\
\hline Coal & 2.6 & 3.3 & 5.2 & 7.2 & 4.1 & 3.1 & 3.2 & 2.7 \\
\hline 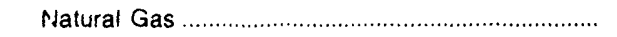 & 158.6 & 284.4 & 511.8 & 765.7 & 758.4 & 696.2 & 702.8 & 799.5 \\
\hline Petroleum ........... & 358.9 & 657.7 & 970.1 & 904.4 & 718.0 & 724.7 & 731.9 & 924.5 \\
\hline 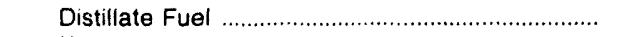 & 334.9 & 628.7 & 932.9 & 847.8 & 669.5 & 660.3 & 671.1 & 838.4 \\
\hline 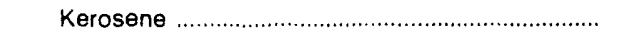 & 13.2 & 10.6 & 14.9 & 13.3 & 9.9 & 11.6 & 5.4 & 5.8 \\
\hline LPG & $10 . \bar{t}$ & 18.4 & 22.3 & 43.3 & 38.7 & 52.8 & 55.5 & 80.3 \\
\hline 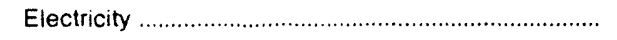 & $27 \% .7$ & 555.7 & 875.7 & $1,151.9$ & $1,131,8$ & $1,260.4$ & $1,317.0$ & $1,437.0$ \\
\hline $\begin{array}{c}\text { Commercial Sector } \\
\text { Primary Energy }\end{array}$ & $\begin{array}{l}390.4 \\
176.2\end{array}$ & $\begin{array}{l}964.2 \\
404.6\end{array}$ & $\begin{array}{r}1,645.4 \\
662.0\end{array}$ & $\begin{array}{r}1,942.5 \\
602.9\end{array}$ & $\begin{array}{r}1,858.0 \\
534.1\end{array}$ & $\begin{array}{r}1,921.5 \\
502.9\end{array}$ & $\begin{array}{r}1,981.3 \\
526.4\end{array}$ & $\begin{array}{r}2,184.1 \\
599.6\end{array}$ \\
\hline Coal …… & $1 \%$ & 2.6 & 2.0 & 3.8 & 1.9 & 1.5 & 2.1 & $\begin{array}{r}589.6 \\
1.9\end{array}$ \\
\hline 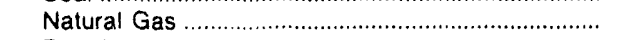 & 50.1 & 100.1 & 252.5 & 291.5 & 290.4 & 275.4 & 298.9 & 302.4 \\
\hline Petroleum & 124.4 & 301.8 & 407.5 & 307.6 & 241.7 & 226.0 & 225.4 & 295.4 \\
\hline 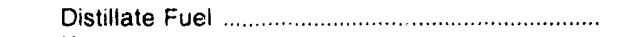 & 86.4 & 187.9 & 278.0 & 228.8 & 188.4 & 171.1 & 169.8 & 246.1 \\
\hline 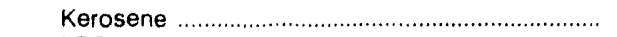 & .5 & .7 & 1.0 & 1.9 & 4.0 & .8 & .9 & .9 \\
\hline LPG & 1.0 & 1.9 & 2.4 & 8.2 & 8.5 & 9.6 & 9.9 & 11.4 \\
\hline 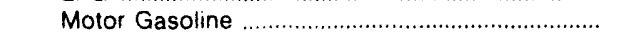 & 1.5 & 2.7 & 9.7 & 9.1 & 7.2 & 7.7 & 7.6 & 8.4 \\
\hline Residual Fuel & 35.0 & 108.6 & 116.3 & 59.5 & 33.5 & 36.8 & 37.2 & 28.6 \\
\hline Electricity & 214.2 & 559.6 & 983.4 & $1,338.5$ & $1,323.9$ & $1,418.6$ & $1,454.9$ & $1,584.5$ \\
\hline Industrial Sector & 282.4 & 666.6 & $1,003.2$ & $1,291.4$ & $1,137.3$ & $1,270.9$ & $1,141.4$ & $1,222.5$ \\
\hline 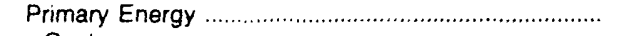 & 159.0 & 386.2 & 475.9 & 631.0 & 503.8 & 574.8 & 444.4 & 464.5 \\
\hline Coal & 3.2 & 6.9 & 4.0 & 10.4 & 5.9 & 9.8 & 8.8 & 7.3 \\
\hline 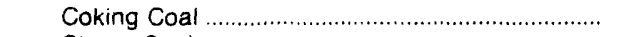 & - & - & & & & & & \\
\hline 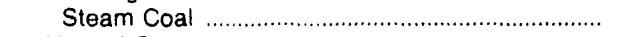 & 3.2 & 6.9 & 4.0 & 10.4 & 5.9 & 9.8 & 8.9 & 7.3 \\
\hline Natural Gas & 23.5 & 55.0 & 120.2 & 177.8 & 119.7 & 145.1 & 127.3 & 144.8 \\
\hline 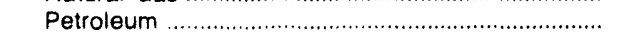 & 132.3 & 324.4 & 351.7 & 442.7 & 378.2 & 419.8 & 308.2 & 312.3 \\
\hline Asphalt and Road Oil & 12.0 & 23.7 & 30.5 & 36.4 & 36.0 & 35.0 & 39.6 & 30.3 \\
\hline 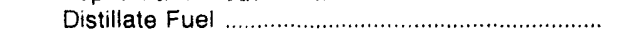 & 12.5 & 36.5 & 61.5 & 40.4 & 41.4 & 114.2 & 67.9 & 73.3 \\
\hline Kerosene & 2.5 & 3.4 & 12.0 & .8 & .5 & .8 & .6 & .8 \\
\hline LPG & 4.0 & 11.6 & 26.5 & 18.5 & 34.4 & 35.6 & 29.3 & 23.8 \\
\hline 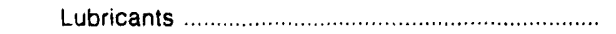 & 15.6 & 16.0 & 32.9 & 36.7 & 31.7 & 31.3 & 32.4 & 40.8 \\
\hline 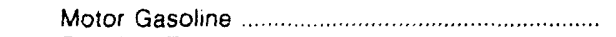 & 1.7 & 2.0 & 4.6 & 17.7 & 14.4 & 15.5 & 17.3 & 21.2 \\
\hline Residual Fuel & 68.1 & 205.3 & 69.3 & 158.4 & 132.8 & 92.1 & 39.7 & 25.3 \\
\hline Other & 15.8 & 25.8 & 114.5 & 133.8 & 87.0 & 95.4 & 81.4 & 96.8 \\
\hline 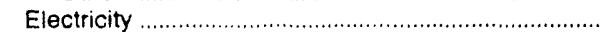 & 123.4 & 280.3 & 527.3 & 660.4 & 633.5 & 696.2 & 697.0 & 758.0 \\
\hline Transportation Sector & 824.4 & 1,563.4 & $3,214.8$ & $3,356.3$ & $2,678.1$ & $2,835.2$ & $3,043.1$ & $3,268.1$ \\
\hline Primary Energy & 822.8 & $1,558.0$ & $3,202.9$ & $3,342.0$ & $2,665.5$ & $2,818.4$ & $3,023.0$ & $3,246.6$ \\
\hline 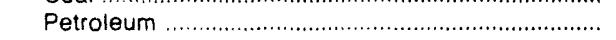 & 822.8 & $1,558.0$ & $3,202.9$ & $3,342.0$ & $2,665.5$ & $2,818.4$ & $3,023.0$ & $3,246.6$ \\
\hline 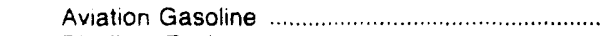 & 3.0 & 4.0 & 12.5 & 6.8 & 6.2 & 4.7 & 4.8 & 4.8 \\
\hline 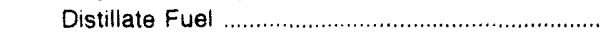 & 25.2 & 75.8 & 211.1 & 405.4 & 321.4 & 324.0 & 316.8 & 342.3 \\
\hline 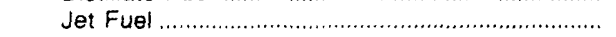 & 33.3 & 94.4 & 315.5 & 238.4 & 163.4 & 182.8 & 212.1 & 260.8 \\
\hline LPG & .2 & .3 & .5 & 2.4 & 2.0 & 1.8 & 2.0 & 2.0 \\
\hline Lubricants & 13.6 & 19.6 & 40.4 & 45.0 & 39.0 & 38.4 & 39.8 & 50.1 \\
\hline 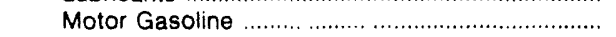 & 740.6 & $1,352.5$ & $2,604.7$ & $2,617.1$ & $2,126.1$ & $2,261.4$ & $2,440.9$ & $2,571.6$ \\
\hline 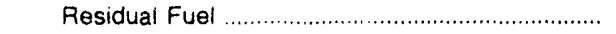 & 7.0 & 11.3 & 18.2 & 26.8 & 7.4 & 5.2 & 6.6 & 15.0 \\
\hline 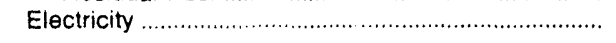 & 1.6 & 5.4 & 11.9 & 14.4 & 12.7 & 16.8 & 20.1 & 21.5 \\
\hline $\begin{array}{l}\text { Total Energy" } \\
\text { Primar Enera - Four Sectors }\end{array}$ & $2,291.0$ & $4,695.3$ & $8,226.4$ & $9,419.4$ & $\begin{array}{l}8,285.7 \\
5,83.8\end{array}$ & $8,712.0$ & $8,920.7$ & $\begin{array}{l}9,838.4 \\
60374\end{array}$ \\
\hline $\begin{array}{l}\text { Primary Energy - Four Sectors } \\
\text { Electricity }\end{array}$ & $\begin{array}{r}1.678 .0 \\
612.9\end{array}$ & $\begin{array}{l}3,294.2 \\
1,401.0\end{array}$ & $\begin{array}{l}5,828.0 \\
2,398.4\end{array}$ & $\begin{array}{l}6,253.2 \\
3,166.2\end{array}$ & $\begin{array}{l}5,183.8 \\
3,101.9\end{array}$ & $\begin{array}{l}5,320.0 \\
3,392.0\end{array}$ & $\begin{array}{l}5,431.7 \\
3,489.0\end{array}$ & $\begin{array}{l}6,037.4 \\
3,801.0\end{array}$ \\
\hline Electric Utillty Sector & 112.4 & 524.9 & $1,191.4$ & $1,011.3$ & 698.3 & 782.8 & 704.3 & 862.8 \\
\hline Coal …… & 4.2 & 25.6 & 35.2 & 202.4 & 169.0 & 180.8 & 180.0 & 186.5 \\
\hline 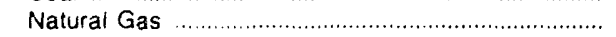 & 1.9 & 1.9 & 17.3 & 160.1 & 34.7 & 98.8 & 45.4 & 120.5 \\
\hline Petroleum ..... & 103.7 & 490.0 & $1,124.6$ & 609.0 & 471.5 & 491.8 & 467.3 & 533.1 \\
\hline 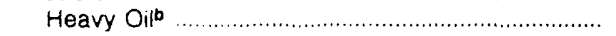 & 100.8 & 483.6 & $1,103.1$ & 581.3 & 450.3 & 470.7 & 434.9 & 492.5 \\
\hline Light Oilb ............... & 2.9 & 6.3 & 21.5 & 27.8 & 21.1 & 21.1 & 32.4 & 40.6 \\
\hline 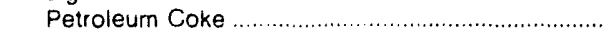 & & & & & & & & \\
\hline Nuclear Fuel & 2.7 & 7.5 & 14.3 & 39.8 & 23.1 & 11.3 & 11.3 & 22.7 \\
\hline Wood and Waste & & & & & & & & \\
\hline Primary Energy - Five Sectors & $1,790.5$ & $3,819.2$ & $7 ; 019.4$ & $7,264.4$ & $5,882.2$ & $6,102.8$ & $6,136.0$ & $6,900.2$ \\
\hline
\end{tabular}

-There are no direct fuel costs for hydroelectric, geothermal, centralized solar, or wind energy. Wood and other biomass fuels are not included, except those consumed at electric utilities

bHeavy oil includes Grade Nos. 4, 5, and 6, and residual fuel oils. Light oil includes Grade No. 2 heating oil, kerosene, and jet fuel.

- No consumption, including cases where adjustments were made. See "Consumption Adjustments for Process Fuel and Intermediate Products."

- Value less than 0.05 million dollars.

Note: Totals may not equal sum of components due to independent rounding.

Sources Data sources, estimation procedures, and assumptions are described in the "Documentation" section of this repont. 


\section{Energy Price and Expenditure Estimates by Source, Michigan I 1970, 1975, 1980, and 1985-1989}

Energy Source
Coal
Steam Coal

Nuclear Fuel

Wood and Waste at Utilities

Primary Energy - Five Sectors ...............................

Electric Utility Fuel ...................................................... Electricity Purchased by End Users ............................

\begin{tabular}{|c|c|c|c|c|c|c|c|}
\hline 1970 & 1975 & 1980 & 1985 & 1986 & 1987 & 1988 & 1989 \\
\hline \multicolumn{8}{|c|}{ Prices in Dollars per Million Btu } \\
\hline 0.44 & 1.23 & 1.71 & 1.92 & 1.84 & 1.76 & 1.76 & 1.72 \\
\hline .55 & 2.07 & 2.27 & 2.08 & 1.95 & 1.85 & 1.73 & 1.75 \\
\hline .42 & 1.04 & 1.62 & 1.90 & 1.83 & 1.75 & 1.76 & 1.72 \\
\hline .77 & 1.42 & 3.05 & 5.70 & 5.12 & 4.96 & 4.80 & 4.61 \\
\hline 2.00 & 3.72 & 8.48 & 8.67 & 6.55 & 6.80 & 6.67 & 7.43 \\
\hline .84 & 2.12 & 4.04 & 5.00 & 3.59 & 3.40 & 3.21 & 2.84 \\
\hline 2.17 & 3.45 & 9.02 & 9.99 & 8.41 & 7.55 & 7.41 & 8.28 \\
\hline 1.09 & 2.49 & 6.76 & 7.71 & 5.73 & 6.09 & 5.74 & 6.37 \\
\hline .74 & 2.08 & 6.38 & 6.09 & 4.20 & 4.09 & 3.85 & 4.39 \\
\hline .89 & 2.60 & 6.29 & 8.47 & 6.53 & 6.32 & 6.38 & 7.00 \\
\hline 1.89 & 3.76 & 6.62 & 8.86 & 8.21 & 7.91 & 7.73 & 10.13 \\
\hline 5.08 & 7.49 & 14.36 & 17.61 & 15.59 & 13.58 & 14.61 & 17.90 \\
\hline 2.71 & 4.72 & 10.09 & 9.10 & 6.79 & 7.13 & 7.15 & 7.77 \\
\hline .59 & 1.96 & 3.90 & 4.44 & 2.86 & 3.00 & 2.87 & 3.03 \\
\hline 1.20 & 2.87 & 7.84 & 8.30 & 5.75 & 6.50 & 5.53 & 6.07 \\
\hline .36 & .28 & .49 & .80 & .77 & .77 & .88 & .78 \\
\hline - & - & - & - & - & - & - & - \\
\hline 1.12 & 2.23 & 4.39 & 5.21 & 4.32 & 4.28 & 4.23 & 4.39 \\
\hline .38 & 1.02 & 1.58 & 1.72 & 1.66 & 1.60 & 1.60 & 1.52 \\
\hline 5.55 & 9.78 & 15.40 & 19.88 & 20.35 & 19.26 & 19.39 & 19.91 \\
\hline 1.70 & 3.33 & 6.61 & 8.48 & 7.49 & 7.61 & 7.44 & 7.78 \\
\hline
\end{tabular}

Total Energyb

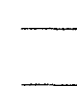

Coal

Coking Coal

Steam Coal

Natural Gas

Petroleum

Asphalt and Road Oil

Aviation Gasoline

Distillate Fuel

Jet Fuel...

Kerisene

LPG

Lubricants

Motor Gasoline

(1)

Residual Fuel

Other"

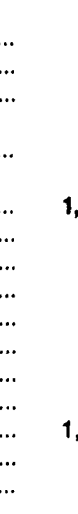

Muclear Fuel

Wood and Waste at Utilities

Primary Energy - Five Sectors

Electric Utility Fuel

Electricity Purchased by End Users

Total Enorgy

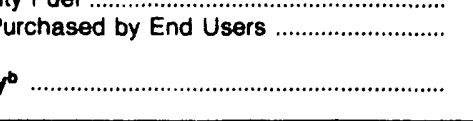

\begin{tabular}{|c|c|c|c|c|c|c|c|}
\hline $\begin{array}{r}363.6 \\
73.4 \\
290.3\end{array}$ & $\begin{array}{l}925.0 \\
290.3 \\
634.7\end{array}$ & $\begin{array}{r}1,299.2 \\
250.1 \\
1,049.1\end{array}$ & $\begin{array}{r}1,499.0 \\
149.7 \\
1,349.3\end{array}$ & $\begin{array}{r}1,496.4 \\
140.2 \\
1,356.1\end{array}$ & $\begin{array}{r}1,475.4 \\
53.0 \\
1,422.4\end{array}$ & $\begin{array}{r}1,459.6 \\
39.0 \\
1,420.6\end{array}$ & $\begin{array}{r}1,377.2 \\
46.4 \\
1,330.8\end{array}$ \\
\hline 620.2 & $1,235.6$ & $2,596.2$ & 3,954.1 & $3,376.6$ & 3,189.8 & $3,507.2$ & $3,517.3$ \\
\hline 1,922.9 & $4,015.0$ & $8,015.8$ & $7,205.5$ & $5,707.6$ & $6,034.3$ & $6,200.8$ & $6,854.6$ \\
\hline 21.5 & 54.6 & 94.0 & 92.2 & 80.6 & 79.0 & 61.2 & 72.8 \\
\hline 7.9 & 6.0 & 22.2 & 10.1 & 10.6 & 9.2 & 9.0 & 11.2 \\
\hline 240.6 & 610.6 & $1,087.9$ & $1,140.8$ & 883.6 & 897.7 & 923.9 & 922.8 \\
\hline 30.4 & 66.8 & 236.9 & 223.6 & 167.7 & 191.9 & 185.4 & 227.7 \\
\hline 15.7 & 19.9 & 44.0 & 37.0 & 15.7 & 13.3 & 15.4 & 22.2 \\
\hline 43.9 & 103.9 & 163.6 & 566.4 & 574.0 & 567.0 & 591.0 & 810.9 \\
\hline 97.4 & 124.9 & 285.2 & 318.1 & 275.4 & 271.2 & 281.5 & 353.6 \\
\hline $1,378.2$ & $2,686.4$ & $5,144.7$ & $4,465.3$ & $3,423.8$ & $3,706.1$ & $3,848.7$ & $4,127.1$ \\
\hline 33.7 & 217.0 & 315.1 & 70.6 & 61.0 & 53.1 & 77.6 & 85.3 \\
\hline 53.5 & 124.8 & 622.3 & 281.2 & 215.2 & 245.9 & 207.1 & 221.0 \\
\hline 1.5 & 22.2 & 85.1 & 117.0 & 102.1 & 119.2 & 169.0 & 178.2 \\
\hline- & - & - & - & - & - & - & - \\
\hline $2,908.2$ & $6,197,8$ & $11,996.2$ & $12,775.6$ & $10,682.7$ & $10,818.7$ & $11,336.6$ & $11,927.3$ \\
\hline $\begin{array}{r}-223.6 \\
1.041 .7\end{array}$ & $\begin{array}{r}-733.0 \\
2,139.6\end{array}$ & $\begin{array}{r}-1,246.3 \\
3,647.5\end{array}$ & $\begin{array}{r}-1,312.2 \\
4,993.3\end{array}$ & $\begin{array}{r}-1,294.4 \\
5,231.3\end{array}$ & $\begin{array}{r}-1,413.6 \\
5,127.9\end{array}$ & $\begin{array}{r}-1,448.5 \\
5,397.5\end{array}$ & $\begin{array}{r}-1,403.8 \\
5,572.4\end{array}$ \\
\hline 26.4 & 7,6045 & 4.397 .5 & $16,456.7$ & 14.619 .6 & 14533.1 & $15,285,6$ & 16.0958 \\
\hline
\end{tabular}

-Includes petroleum coke used at electric utilities.

bThere are no direct fuel costs for hydroelectric, geothermal, centralized solar, or wind energy. Wood and other biomass fuels are not included, except those consumed at electric utilities.

- No consumption, including cases where adjustments were made. See "Consumption Adjustments for Process Fuel and Intermediate Products."

Note: Expenditure totals may not equal sum of components due to independent rounding.

Sources: Data sources, estimation procedures, and assumptions are described in the "Documentation" section of this report. 


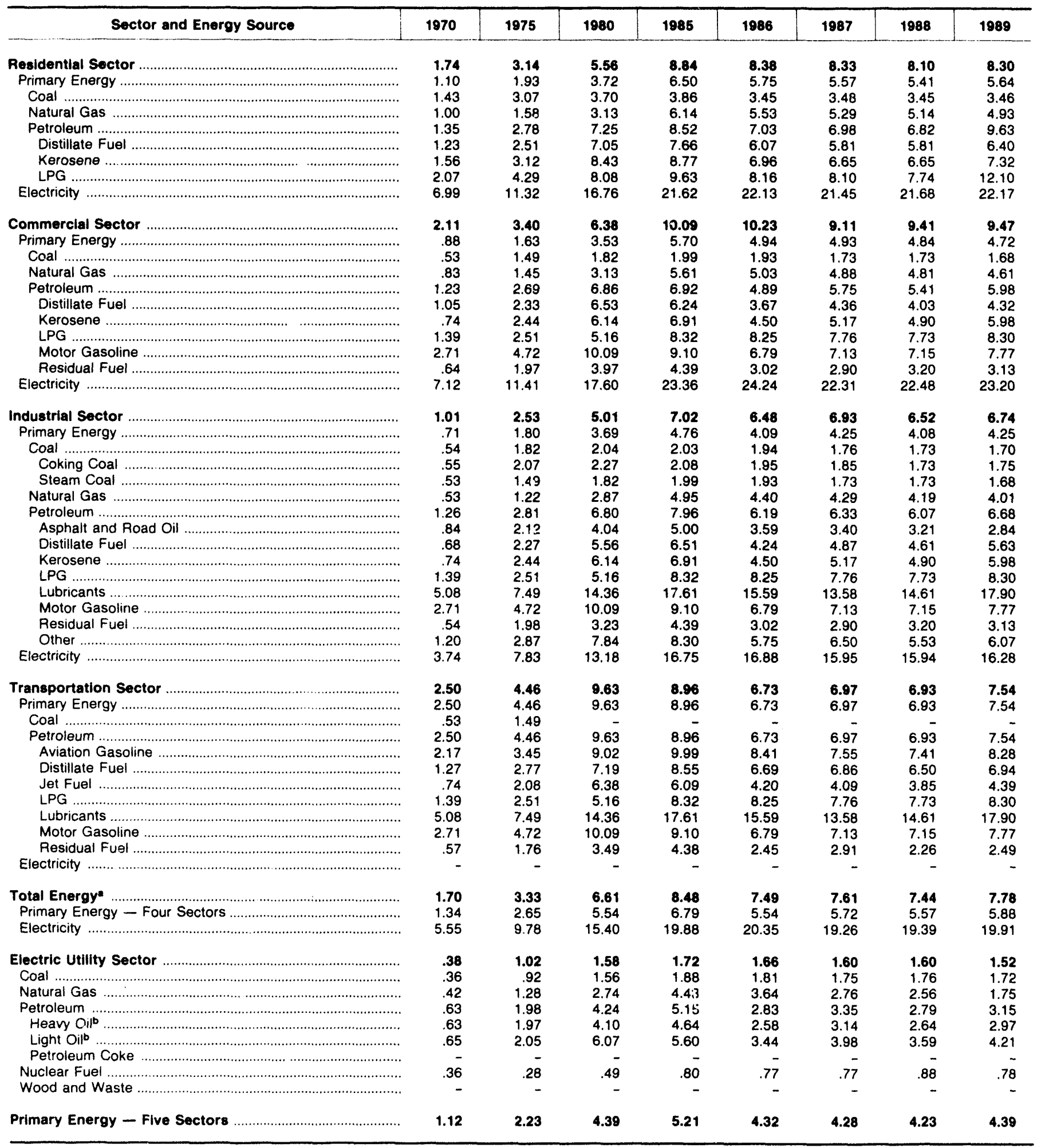

-There are no direct fuel costs for hydroelectric, geothermal, centralized solar, or wind energy. Wood and other biomass fuels are not included, except those consumed at electric utilities.

bHeavy oil includes Grade Nos. 4, 5, and 6, and residual fuel oils. Light oil incluies Grade No. 2 heating oil, kerosene, and jet fuel.

-No consumption, including cases where adjustments were made. See "Consumption Adjustments for Process Fuel and Intermediate Products."

Sources: Data sources, estimation procedures, and assumptions are described in the "Documentation" section of this report. 


\begin{tabular}{|c|c|c|c|c|c|c|c|c|}
\hline Sector and Energy Source & 1970 & 1975 & 1980 & 1985 & 1986 & 1987 & 1988 & 1989 \\
\hline 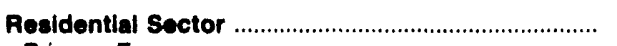 & 939.0 & $1,732.6$ & $3,000.8$ & $4,349.2$ & $4,100.5$ & $3,925.1$ & $4,241.5$ & $4,459.1$ \\
\hline 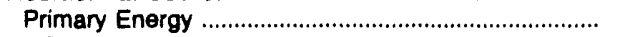 & 530.9 & 925.9 & 1.727 .5 & $2,704.0$ & $2,361.7$ & 2.166 .4 & 2.368 .5 & $2,544.0$ \\
\hline 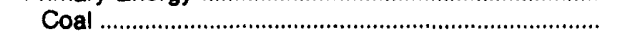 & 10.3 & 10.1 & 9.8 & 8.4 & 7.7 & 3.6 & 5.3 & 5.3 \\
\hline 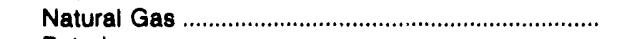 & 345.1 & 542.8 & $1,236.0$ & $2,143.5$ & $1,894.5$ & 1.716 .0 & $1,861.1$ & $1,877.1$ \\
\hline 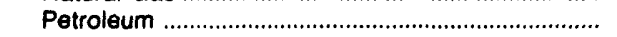 & 175.5 & 373.0 & 481.8 & 552.2 & 459.4 & 446.8 & 502.1 & 661.7 \\
\hline 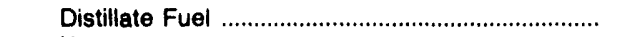 & 135.5 & 284.4 & 377.7 & 266.0 & 208.2 & 176.5 & 197.0 & 177.8 \\
\hline 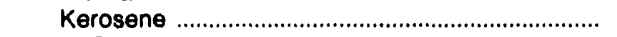 & 4.8 & 5.3 & 4.0 & 32.2 & 13.8 & 10.8 & 13.6 & 17.6 \\
\hline LPG & 35.2 & 83.3 & 100.1 & 254.0 & 237.5 & 259.5 & 291.6 & 466.3 \\
\hline 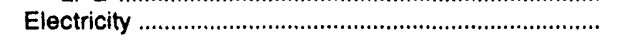 & 408.1 & 806.7 & $1,273.3$ & 1.645 .1 & $1,738.9$ & $1,758.7$ & $1,873.0$ & $1,915.0$ \\
\hline 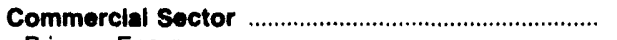 & 475.7 & 935.8 & $1,802.2$ & $2,548.9$ & $2, \mathbf{4 3 5 . 9}$ & $2,568.3$ & $2,586.9$ & $2,699.7$ \\
\hline 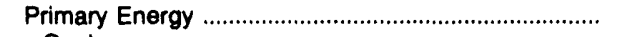 & 159.3 & 367.7 & 795.3 & $1,080.8$ & 853.0 & $1,057.1$ & 985.9 & 999.7 \\
\hline 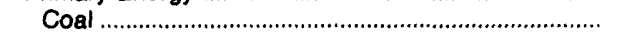 & 7.0 & 9.0 & 8.8 & 8.0 & 8.0 & 3.3 & 49 & 4.8 \\
\hline 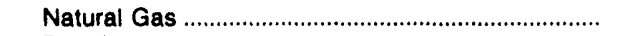 & 111.4 & 269.8 & 606.7 & 905.1 & 708.7 & 935.4 & 839.5 & 854.5 \\
\hline 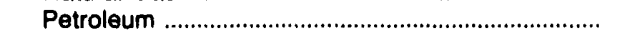 & 40.9 & 88.9 & 179.8 & 167.7 & 136.3 & 118.4 & 141.5 & 140.4 \\
\hline 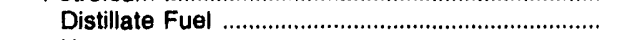 & 21.4 & 48.7 & 118.8 & 85.7 & 63.1 & 44.4 & 57.1 & 52.3 \\
\hline 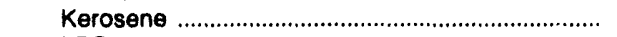 & 1.7 & 3.1 & .5 & .7 & .3 & .4 & .5 & 2.1 \\
\hline LPG & 4.2 & 8.6 & 11.3 & 38.7 & 42.4 & 43.9 & 51.4 & 56.5 \\
\hline 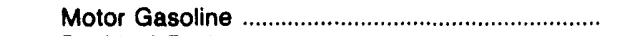 & 11.4 & 23.7 & 43.6 & 33.4 & 25.2 & 27.2 & 28.3 & 27.4 \\
\hline Residual Fuel .......................................................... & 2.2 & 4.8 & 5.6 & 9.1 & 5.3 & 2.6 & 4.2 & 2.3 \\
\hline 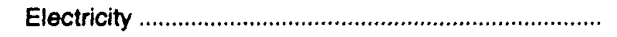 & 316.4 & 568.1 & $1,006.9$ & $1,468.2$ & $1,582.9$ & $1,511.3$ & $1,601.0$ & $1,700.0$ \\
\hline 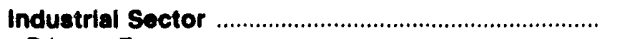 & 856.0 & $2,038.6$ & $3,741.3$ & $4,184.1$ & 3,923.0 & $3,521.8$ & $3,817.5$ & $3,912.1$ \\
\hline 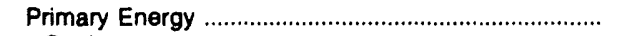 & 538.9 & $1,273.7$ & $2,373.9$ & $2,304.2$ & $2,013.5$ & $1,663.8$ & $1,894.0$ & $1,954.8$ \\
\hline 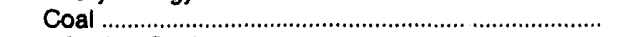 & 172.7 & 449.1 & 448.3 & 344.4 & 331.2 & 217.5 & 226.6 & 203.6 \\
\hline 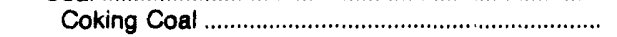 & 73.4 & 290.3 & 250.1 & 149.7 & 140.2 & 53.0 & 39.0 & 46.4 \\
\hline 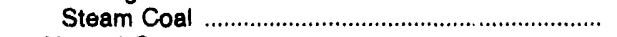 & 99.3 & 158.8 & 198.2 & 194.6 & 190.9 & 164.6 & 187.6 & 157.2 \\
\hline 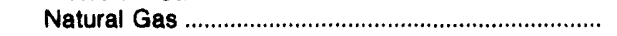 & 136.5 & 362.3 & 700.3 & 884.9 & 759.6 & 526.4 & 800.5 & 782.2 \\
\hline 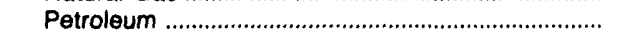 & 229.6 & 462.4 & $1,225.3$ & $1,074.9$ & 922.8 & 919.8 & 867.0 & 969.1 \\
\hline 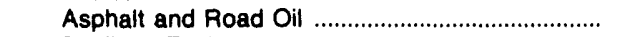 & 21.5 & 54.6 & 94.0 & 92.2 & 80.6 & 79.0 & 61.2 & 72.8 \\
\hline 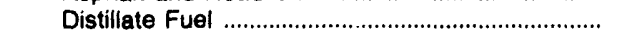 & 33.3 & 115.9 & 155.7 & 160.5 & 113.2 & 120.6 & 133.6 & 123.6 \\
\hline 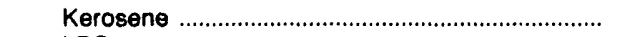 & 9.2 & 11.4 & 39.5 & 4.2 & 1.5 & 2.1 & 1.4 & 2.5 \\
\hline 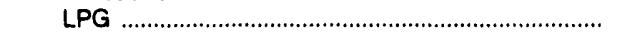 & 4.3 & 11.2 & 49.7 & 262.3 & 283.9 & 253.3 & 236.3 & 277.6 \\
\hline Lubricants .................................................... & 56.5 & 64.9 & 156.5 & 174.6 & 151.1 & 148.8 & 154.5 & 194.1 \\
\hline 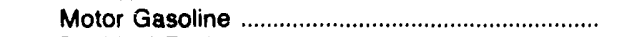 & 39.2 & 46.9 & 51.3 & 56.9 & 41.1 & 42.8 & 40.0 & 43.8 \\
\hline 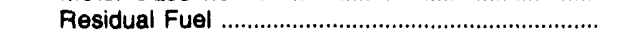 & 12.0 & 32.6 & 56.3 & 43.0 & 36.1 & 27.4 & 32.9 & 33.7 \\
\hline Other & 53.5 & 124.8 & 622.3 & 281.2 & 215.2 & 245.9 & 207.1 & 221.0 \\
\hline 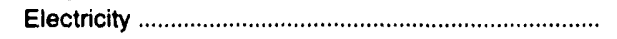 & 317.2 & 764.8 & $1,367.4$ & $1,880.0$ & $1,909.5$ & $1,858.0$ & 1.923 .4 & $1,957.3$ \\
\hline $\begin{array}{l}\text { Tranaportation Sector } \\
\text { Primary Energy }\end{array}$ & $\begin{array}{l}1,455.6 \\
1,4556\end{array}$ & $\begin{array}{l}2,897.5 \\
2,897.5\end{array}$ & $\begin{array}{l}5,853.1 \\
5853.1\end{array}$ & $\begin{array}{l}5,374.4 \\
5,374.4\end{array}$ & $\begin{array}{l}4,160.1 \\
4,160.1\end{array}$ & $\begin{array}{l}4,517.9 \\
45179\end{array}$ & $\begin{array}{l}4,639.7 \\
46397\end{array}$ & $5,024.9$ \\
\hline $\begin{array}{l}\text { Primary Energy . } \\
\text { Coal lon }\end{array}$ & $\begin{array}{r}1,455.6 \\
.3\end{array}$ & $\begin{array}{r}2,897.5 \\
.1\end{array}$ & $\begin{array}{r}5,853.1 \\
-\end{array}$ & $\begin{array}{r}5,374.4 \\
-\end{array}$ & $\begin{array}{r}4,160.1 \\
-\end{array}$ & $\begin{array}{l}4,517.9 \\
-\end{array}$ & $\begin{array}{r}4,639.7 \\
-\end{array}$ & $5,024.9$ \\
\hline 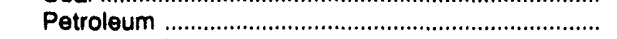 & $1,455.4$ & $2,897.5$ & $5,853.1$ & $5,374.4$ & $4,160.1$ & $4,517.9$ & $4,639.7$ & $5,024.9$ \\
\hline Aviation Gasoline ............................................... & 7.9 & 6.0 & 22.2 & 10.1 & 10.6 & 9.2 & 9.0 & 11.2 \\
\hline 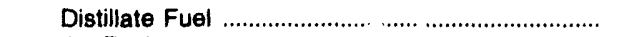 & 46.9 & 144.2 & 408.1 & 607.5 & 488.8 & 546.8 & 525.7 & 558.0 \\
\hline 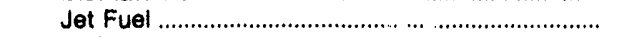 & 30.4 & 65.8 & 236.9 & 223.6 & 167.7 & 191.9 & 185.4 & 227.7 \\
\hline LPG & .3 & .9 & 2.4 & 11.3 & 10.2 & 10.4 & 11.7 & 10.6 \\
\hline 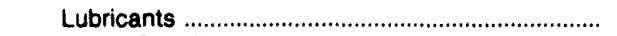 & 40.8 & 60.0 & 128.7 & 143.6 & 124.3 & 122.4 & 127.0 & 159.6 \\
\hline Motor Gasoline …………………………………. & $1,327.5$ & $2,615.8$ & $5,049.8$ & $4,375.0$ & $3,357.5$ & $3,636.2$ & $3,780.3$ & $4,055.9$ \\
\hline Residual Fuel .................................................................. & 1.5 & 4.7 & 5.1 & 3.3 & .9 & 1.1 & .4 & 1.9 \\
\hline 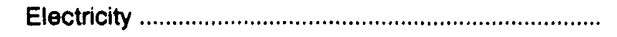 & - & - & - & - & - & - & - & \\
\hline 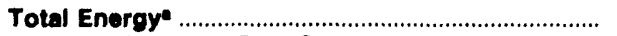 & $3,726.4$ & $7,604.5$ & $14,397.5$ & $16,456.7$ & $14,619.6$ & $14,533.1$ & $15,285.6$ & $16,095.8$ \\
\hline 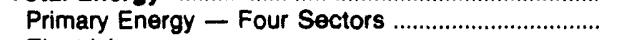 & $2,684.7$ & $5,464.9$ & $10,749.9$ & $11,463.4$ & $9,388.3$ & $9,405.2$ & $9,888.1$ & $10,523.5$ \\
\hline 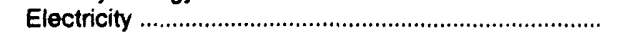 & $1,041.7$ & $2,139.6$ & $3,647.5$ & $4,993.3$ & $5,231.3$ & $5,127.9$ & $5,397.5$ & $5,572.4$ \\
\hline 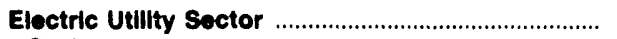 & 223.6 & 733.0 & $1,246.3$ & 1,31 & 1,29 & 1,41 & 18.5 & $1,403.8$ \\
\hline Coal & 173.4 & 456.8 & 832.3 & $1,138.3$ & $1,149.5$ & $1,250.9$ & $1,222.8$ & $1,163.5$ \\
\hline 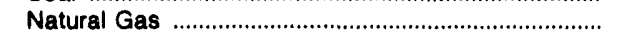 & 27.2 & 60.7 & 53.2 & 20.6 & 13.8 & 12.0 & 6.2 & 3.5 \\
\hline 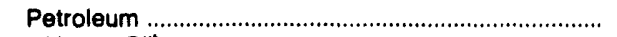 & 21.5 & 193.2 & 275.7 & 36.3 & 29.0 & 31.5 & 50.5 & 58.6 \\
\hline 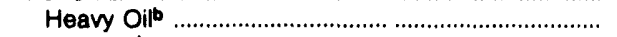 & 17.9 & 174.9 & 248.2 & 15.2 & 18.8 & 22.1 & 40.1 & 47.4 \\
\hline 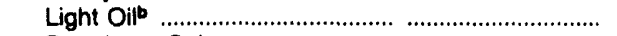 & 3.6 & 18.4 & 27.5 & 21.1 & 10.3 & 9.4 & 10.4 & 11.2 \\
\hline 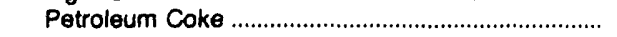 & & & & & - & & - & \\
\hline 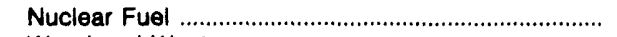 & 1.5 & 22.2 & 85.1 & 117.0 & 102.1 & 119.2 & 169.0 & 178.2 \\
\hline Wood and Waste & - & - & - & - & - & - & - & \\
\hline Primary Energy - Five Sectors . & $2,908.2$ & $6,197.8$ & $11,996.2$ & $12,775.6$ & $10,682.7$ & $10,818.7$ & $11,336.6$ & 11,9273 \\
\hline
\end{tabular}

-There are no direct fuel costs for hydroelectric, geothermal, centralized solar, or wind energy. Wood and other biomass fuels are not included, except those consumed at electric utilities.

bHeavy oil includes Grade Nos. 4, 5, and 6, and residual fuel oils. Light oil includes Grade No. 2 heating oil, kerosene, and jet fuel.

- No consumption, including cases where adjustments were made. See "Consumption Adjustments for Process Fuel and Internediate Products."

Note: Totals may not equal sum of components due to independent rounding.

Sources: Data sources, estimation procedures, and assumptions are described in the "Documentation" section of this report. 


\begin{tabular}{|c|c|c|c|c|c|c|c|c|}
\hline Energy Source & 1970 & 1976 & 1880 & 1985 & 1088 & 1987 & 1988 & 1989 \\
\hline & \multicolumn{8}{|c|}{ Prlces in Dollare per Million Btu } \\
\hline 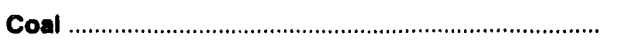 & 0.41 & 0.83 & 1.12 & 1.51 & 1.44 & 1.26 & 1.25 & 1.26 \\
\hline 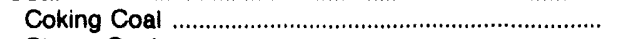 & .53 & 1.80 & - & - & - & - & - & - \\
\hline 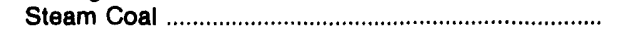 & .40 & .69 & 1.12 & 1.51 & 1.44 & 1.26 & 1.25 & 1.26 \\
\hline 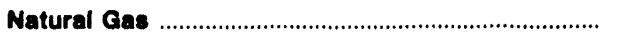 & .66 & 1.17 & 2.85 & 5.13 & 4.61 & 3.72 & 3.87 & 3.87 \\
\hline Petroleum & 2.02 & 3.58 & 7.90 & 8.45 & 6.52 & 8.71 & 6.79 & 7.59 \\
\hline Asphalt and Road Oil & .68 & 2.19 & 3.31 & 4.99 & 3.86 & 3.53 & 3.60 & 3.01 \\
\hline Aviation Gasoline & 2.17 & 3.45 & 9.02 & 9.99 & 8.41 & 7.55 & 7.41 & 8.28 \\
\hline 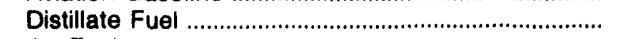 & 1.08 & 2.51 & 6.72 & 7.55 & 5.62 & 5.98 & 5.81 & 6.54 \\
\hline Jet Fuel & .75 & 2.09 & 6.47 & 5.93 & 4.04 & 4.15 & 3.94 & 4.47 \\
\hline Kerosene & 1.34 & 2.78 & 7.05 & 8.22 & 6.06 & 6.15 & 5.81 & 6.76 \\
\hline LPG & 1.80 & 3.67 & 5.82 & 8.08 & 7.91 & 6.78 & 7.34 & 8.73 \\
\hline 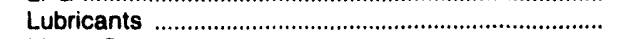 & 5.08 & 7.49 & 14.36 & 17.61 & 15.59 & 13.58 & 14.61 & 17.90 \\
\hline 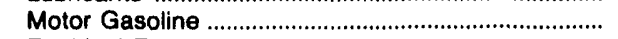 & 2.97 & 4.63 & 9.55 & 9.73 & 7.42 & 7.71 & 7.81 & 8.72 \\
\hline Residual Fuel & .59 & 1.80 & 3.52 & 4.06 & 2.88 & 2.43 & 2.36 & 2.38 \\
\hline Other & 2.10 & 2.73 & 5.71 & 7.48 & 10.53 & 9.08 & 6.89 & 6.94 \\
\hline 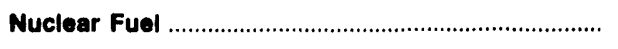 & - & .24 & .44 & .50 & .50 & .51 & .49 & .49 \\
\hline 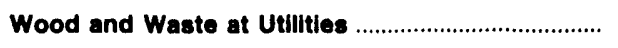 & .65 & .92 & 1.74 & - & - & .95 & .87 & .82 \\
\hline Primary Energy - Flve Sectors ................................. & 1.29 & 2.14 & 4.43 & 5.31 & 4.42 & 4.07 & 4.02 & 4.32 \\
\hline 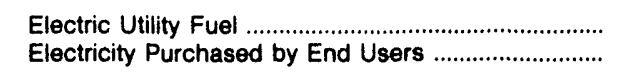 & $\begin{array}{r}.34 \\
6.10\end{array}$ & .52 & $\begin{array}{r}.91 \\
13.26\end{array}$ & $\begin{array}{r}1.09 \\
15.81\end{array}$ & $\begin{array}{r}1.03 \\
16.07\end{array}$ & $\begin{array}{r}.99 \\
15.72\end{array}$ & $\begin{array}{r}.99 \\
15.80\end{array}$ & $\begin{array}{r}1.01 \\
15.57\end{array}$ \\
\hline \multirow[t]{2}{*}{ Total Energyb } & 1.87 & 3.18 & 6.92 & 8.43 & 7.32 & 7.31 & 7.37 & 7.68 \\
\hline & \multicolumn{8}{|c|}{ Expenditures in Millions of Dollars } \\
\hline Coal tan. & 74.1 & 159.8 & 270.7 & 341.4 & 290.7 & 323.6 & 378.2 & 405.5 \\
\hline 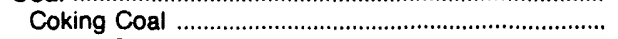 & 8.6 & 45.4 & & - & & - & - & - \\
\hline 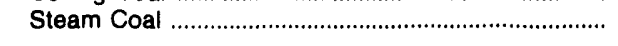 & 65.5 & 114.4 & 270.7 & 341.4 & 290.7 & 323.6 & 378.2 & 405.5 \\
\hline 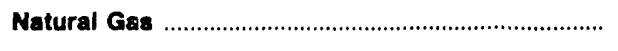 & 220.6 & 381.4 & 785.0 & $1,283.0$ & $1,084.8$ & 856.2 & $1,051.7$ & $1,108.7$ \\
\hline Petroleum & $1,005.3$ & $1,920.2$ & $3,810.6$ & $4,053.9$ & $3,184.6$ & $3,166.8$ & $3,303.9$ & $3,666.4$ \\
\hline 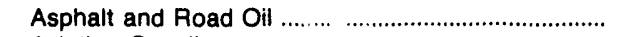 & 20.0 & 67.2 & 78.4 & 165.1 & 140.3 & 137.2 & 117.1 & 98.5 \\
\hline 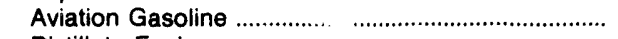 & 3.0 & 3.7 & 8.8 & 7.8 & 9.6 & 6.8 & 6.2 & 6.6 \\
\hline Distillate Fuel & 140.5 & 355.7 & 837.2 & 852.5 & 617.2 & 636.3 & 672.8 & 730.9 \\
\hline 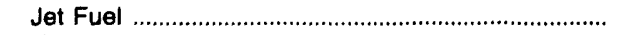 & 14.7 & 66.5 & 188.3 & 261.4 & 178.6 & 132.8 & 114.7 & 118.0 \\
\hline 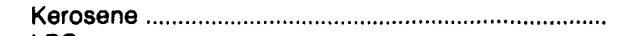 & 12.8 & 13.5 & 8.5 & 13.0 & 4.3 & 2.8 & 4.5 & 13.4 \\
\hline LPG , & 60.1 & 124.8 & 163.0 & 246.5 & 272.7 & 186.7 & 219.2 & 265.5 \\
\hline 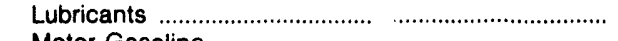 & 28.5 & $\begin{array}{r}45.6 \\
1170.9\end{array}$ & 97.5 & $\begin{array}{r}108.8 \\
205.8\end{array}$ & 94.2 & $\begin{array}{r}92.7 \\
18993\end{array}$ & $\begin{array}{r}96.3 \\
2005.6\end{array}$ & $\begin{array}{r}120.9 \\
2224.8\end{array}$ \\
\hline Motor Gasoline & $\begin{array}{r}688.9 \\
14.9\end{array}$ & 1.172 .9 & $2,319.4$ & $2,335.5$ & $1,784.2$ & $1,899.3$ & $2,005.6$ & $\begin{array}{r}2,224.8 \\
16.7\end{array}$ \\
\hline $\begin{array}{l}\text { Residual Fuei } \\
\text { Other" }\end{array}$ & $\begin{array}{l}14.9 \\
21.9\end{array}$ & $\begin{array}{l}38.4 \\
31.8\end{array}$ & $\begin{array}{l}56.3 \\
53.3\end{array}$ & $\begin{array}{l}19.2 \\
44.0\end{array}$ & $\begin{array}{l}28.7 \\
54.8\end{array}$ & $\begin{array}{l}14.4 \\
57.8\end{array}$ & $\begin{array}{l}15.9 \\
51.7\end{array}$ & $\begin{array}{l}16.7 \\
71.1\end{array}$ \\
\hline 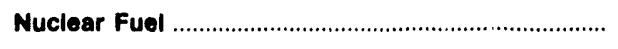 & - & 25.5 & 48.6 & 62.5 & 50.2 & 63.1 & 64.2 & $\mathbf{5 7 . 4}$ \\
\hline Wood and Waste at Utilities & .1 & - & * & - & - & .4 & 1.4 & 2.1 \\
\hline 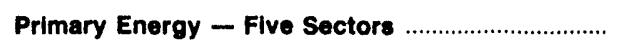 & $1,300.2$ & $2,487.0$ & $4,915.0$ & $5,740.8$ & $4,619.2$ & $4,410.2$ & $4,798.3$ & $5,240.2$ \\
\hline 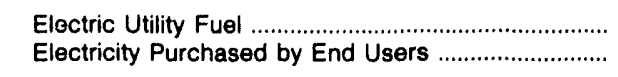 & $\begin{array}{r}-65.3 \\
427.5\end{array}$ & $\begin{array}{r}-144.2 \\
769.9\end{array}$ & $\begin{array}{l}-311.3 \\
1,481.2\end{array}$ & $\begin{array}{l}-355.5 \\
2,062.8\end{array}$ & $\begin{array}{r}-307.0 \\
2,097.2\end{array}$ & $\begin{array}{r}-362.5 \\
2,211.9\end{array}$ & $\begin{array}{r}-419.5 \\
2,433.7\end{array}$ & $\begin{array}{r}-432.7 \\
2,409.9\end{array}$ \\
\hline Total Energyb & $1,662.4$ & $3,112.6$ & $6,084.9$ & $7,448.2$ & $6,409.4$ & $6,259.6$ & $\mathbf{8} 813.5$ & $7,217.4$ \\
\hline
\end{tabular}

-Includes petroleum coke used at electric utilities.

bThere are no direct fuel costs for hydroelectric, geothermal, centralized solar, or wind energy. Wood and other biomass fuels are not included, except those consumed at electric utilities.

-No consumption, including cases where adjustments were made. See "Consumption Adjustments for Process Fuel and Intermediate Products."

- Value less than $J .25$ million dollars.

Note: Expenditure totals may not equal sum of components due to independent rounding.

Sources: Data sources, estimation procerlures, and assumptions are described in the "Documentation" section of this report. 
M Energy Price Estimates by Sector, Minnesota

I 1970, 1975, 1980, and 1985-1989

N (Dollars per Million Btu)

Sector and Energy Source
Residentlal Sector
Primary Energy
Coal
Natural Gas Getrolem
Pistillate Fuel
Kerosene
LPG
Electricity

Commerclal Sector

Primary Energy

Coal....

Natural Gas

Petroleum ...

Distillate Fuel

Kerosene

LPG

Motor Gasoline

Residual Fuel

Electricity

Industrial Sector

Primary Energy

Coal ....................

Steam Coal

Natural Gas

Petroleum

Asphalt and Road Oi

Distillate Fuel

K rosene ......

LPG

Lubricants............

Motor Gasoline

Residual Fuel

Other

Electricity

Transportation Secior

Primary Energy

Coal ...............

Aviation Gasoline

Distillate Fuel

Jet Fuel

LPG

Lubricants

Motor Gasoline

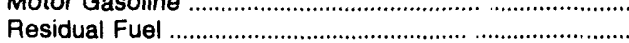

Electricity

Total Energy*

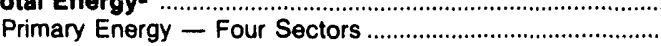

Electricity

Electric Utility Sector

Coal

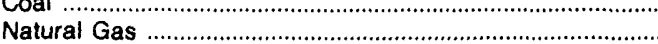

Petroleum

Heavy Oifb

Light Oilb

Petroleum Coke

Nuclear Fuel ...........

Wood and Waste

Primary Energy - Five Sectors

\begin{tabular}{l|}
1970 \\
\hline 2.17 \\
1.29 \\
1.55 \\
1.09 \\
1.54 \\
1.26 \\
1.52 \\
2.03 \\
7.30
\end{tabular}

1975

\subsection{8}

1.42

1.42
.76

.76
.47

.69

1.13

1.05

1.05
.89

1.20

2.97

.58

7.72

1.15

.83

2.36

2.97

.57

1.51
6.10

.34
.34

.34
.26

.73

.74
.85

.85
.28

.65

1.29
3.38
2.15

2.15
3.04

3.04
1.57

3.12

2.55

2.91

4.22
9.90

2.56

1.39

.87
1.16

2.61

2.34

2.54

2.61

4.63

1.97

10.38

1.77

1.33

1.80
.87

.87
83

2.69

2.19

2.39

2.54

2.61

7.49

4.63

1.74

2.80

6.73

4.13

4.13

.87

4.13

3.45

2.67

2.09

2.61

7.49

4.63

1.70

3.19

3.19
2.64

8.64

6.00

13.26

$.52 \quad .81$

1980

6.92

4.47

4.32

3.24

7.25

7.20

8.02

7.34
16.06

5.37

3.47

1.77

6.73

6.60

$4.8 \overline{7}$

9.55

2.95

5.14

3.68
1.77

$1.7 \overline{7}$

2.51

5.23

3.31
5.66

5.92

4.87

4.36

2.97

5.71

11.22

8.88

8.88

8.88

9.02

7.16
6.47

4.87

9.55

3.81

.62
.64

2.03

1.95

2.26

.54
.24
.92

.92

2.14

.81
1.04

1.99

4.86

4.46

5.80

44
1.74

1.74

4.43

1985

1986

1987

1988

1989

$\begin{array}{llllll}9.29 & 8.93 & 8.94 & 8.71 & 8.77\end{array}$

$6.29 \quad 5.65$

5.09

3.81

4.58

6.35

5.70

6.45

7.40
19.38

5.03

3.51

4.61

6.18

5.62

6.36
7.03

19.82

3.49

4.54

7.74

6.30

7.13

10.03

$\begin{array}{rr}7.79 & 7.53 \\ 19.01 & 19.41\end{array}$

7.42

$\begin{array}{ll}7.83 & 7.60\end{array}$

5.41

2.04

5.18

6.66

6.34

6.47

8.32

9.73

4.10

17.81

7.22

7.07

4.64
1.96

4.62

5.26

4.21

4.10

8.25
7.42

2.86

3.99

1.85

3.89

5.04

4.37
4.71

6.15

7.71

2.43

17.19

4.07

1.85

4.00

3.97

3.97
4.18

7.73

7.81

17.52

4.06

1.86

5.22

4.72

4.94

7.13

8.72

2.38

$\begin{array}{lllll}7.04 & 6.37 & 6.06 & 6.24 & 6.23\end{array}$

$\begin{array}{lllll}5.17 & 4.31 & 3.76 & 3.80 & 3.90\end{array}$

$2.04 \quad 1.96$

1.85

1.85

1.86

2.04

1.96

3.42

$1.8 \overline{5}$

2.55

1.85

2.79

2.84

$6.66 \quad 5.21$

3.86

3.92

$\begin{array}{ll}6.47 & 4.10 \\ 8.32 & 8.25\end{array}$

$17.61 \quad 15.59$

7.42

$\begin{array}{ll}9.10 & 2.86\end{array}$

$\begin{array}{ll}7.48 & 10.53\end{array}$

12.65

12.76

9.16

6.98

6.98

6.98 


Sector and Energy Source
Resldential Sector
Primary Energy
Coal
Natural Gas
Petroleum
Distillate Fuel
Kerosene
LPG

Commerclal Sector

Primary Energy .....

Coal

Natural Gas

Petroleum

Distillate Fusl

Kerosene

LPG

Motor Gasoline

Residual Fuel

Electricity

Industrial Sector

Primary Energy

Coal

Coking Coal

Steam Coal

Natural Gas

Petroleum

Asphalt and Roac

Distillate Fuel

Kerosene

LPG

Lubricants

Motor Gasoline

Residual Fuel

Other

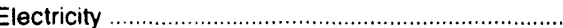

Transportation Sector

Primary Energy

Coál

Petroleum

Distillate Fuel

Jet Fuel

LPG

Lubricants

Motor Gasoline

Residual Fuel

Total Energy .

Primary Energy - Four Sectors .............................

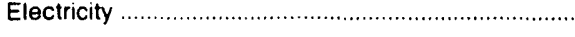

Electric Utility Sector

Coal

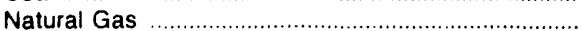

Petroleum.

Heavy Oilb
Light Oitb.

Nuclear Fuel

Wood and Waste

Primary Energy - Five Sectors
Aviation Gasoline

\begin{tabular}{|c|c|c|c|c|c|c|c|}
\hline 1970 & 1875 & 1980 & 1985 & 1986 & 1987 & 1988 & 1989 \\
\hline 455.3 & 730.9 & $1,315.8$ & $1,780.4$ & $1,684.1$ & $1,565.4$ & $1,791.0$ & $1,879.7$ \\
\hline $23 C .2$ & 395.8 & 672.0 & 920.1 & 816.0 & 650.7 & 777.0 & 885.7 \\
\hline 6.5 & 4.8 & 4.5 & 6.0 & 5.0 & 4.1 & 5.2 & 5.9 \\
\hline 111.5 & 179.5 & 333.8 & 618.7 & 545.1 & 411.9 & 508.9 & 534.3 \\
\hline 112.2 & 211.6 & 333.7 & 295.5 & 266.0 & 234.7 & 262.9 & 345.5 \\
\hline 52.9 & 107.6 & 249.5 & 173.6 & 141.0 & 129.1 & 143.2 & 164.9 \\
\hline 10.3 & 9.2 & 5.2 & 10.4 & 3.4 & 2.4 & 3.7 & 11.8 \\
\hline 48.9 & 94.8 & 79.0 & 111.5 & 121.6 & 103.2 & 116.0 & 168.8 \\
\hline 225.0 & 344.1 & 643.8 & 860.3 & 878.1 & 914.7 & $1,014.0$ & 994.0 \\
\hline 163.0 & 325.9 & 522.6 & $1,000.2$ & 881.3 & 784.0 & 904.2 & 918.1 \\
\hline 79.2 & 154.3 & 269.7 & 553.3 & 417.9 & 312.8 & 390.2 & 410.1 \\
\hline 3.7 & 2.5 & 3.4 & 5.6 & 4.7 & 3.7 & 5.1 & 5.9 \\
\hline 53.2 & 104.2 & 183.6 & 400.2 & 343.8 & 256.4 & 322.4 & 340.7 \\
\hline 22.3 & 47.6 & 82.7 & 147.5 & 69.4 & 52.7 & 62.7 & 63.5 \\
\hline 10.7 & 24.1 & 55.5 & 101.1 & 26.4 & 25.7 & 25.5 & 28.4 \\
\hline 1.3 & 1.7 & - & 1.3 & .1 & .1 & .1 & .1 \\
\hline 5.1 & 10.3 & 9.3 & 21.0 & 23.5 & 15.1 & 22.5 & 21.2 \\
\hline 3.7 & 8.6 & 17.1 & 17.1 & 12.7 & 9.7 & 9.9 & 8.7 \\
\hline 1.4 & 2.8 & .9 & 6.9 & 6.7 & 2.1 & 4.7 & 5.1 \\
\hline 83.7 & 171.6 & 252.8 & 446.9 & 463.3 & 471.2 & 514.0 & 508.0 \\
\hline 341.0 & 743.0 & $1,382.0$ & $1,678.4$ & $1,544.3$ & $1,519.6$ & $1,604.4$ & $1,640.2$ \\
\hline 222.2 & 488.9 & 797.4 & 922.7 & 788.5 & 693.5 & 698.7 & 732.3 \\
\hline 20.8 & 67.6 & 31.9 & 43.3 & 39.6 & 31.3 & 28.0 & 35.5 \\
\hline 8.6 & 45.4 & - & - & - & - & - & - \\
\hline 12.2 & 22.2 & 31.9 & 43.3 & 39.6 & 31.3 & 28.0 & 35.5 \\
\hline 40.6 & 83.5 & 251.7 & 259.4 & 190.8 & 176.0 & 210.1 & 224.2 \\
\hline 160.9 & 337.8 & 513.9 & 620.0 & 558.1 & 486.1 & 460.6 & 472.6 \\
\hline 20.0 & 67.2 & 78.4 & 165.1 & 140.3 & 137.2 & 117.1 & 98.5 \\
\hline 37.5 & 111.0 & 188.2 & 172.5 & 128.8 & 124.2 & 122.7 & 127.4 \\
\hline 1.2 & 2.5 & 3.3 & 1.3 & .7 & .2 & .7 & 1.5 \\
\hline 5.6 & 18.8 & 73.5 & 109.2 & 123.0 & 66.6 & 78.1 & 73.5 \\
\hline 9.1 & 11.4 & 28.2 & 31.4 & 27.2 & 26.8 & 27.8 & 35.0 \\
\hline 56.3 & 76.1 & 67.1 & 87.8 & 62.0 & 60.9 & 52.3 & 57.4 \\
\hline 9.4 & 19.0 & 22.0 & 8.7 & 21.2 & 12.2 & 11.0 & 11.4 \\
\hline 21.6 & 31.6 & 53.3 & 44.0 & 54.8 & 57.9 & 50.8 & 67.9 \\
\hline 118.8 & 254.2 & 584.6 & 755.7 & 755.8 & 826.1 & 905.7 & 907.9 \\
\hline 703.1 & $1,303.8$ & $2,864.6$ & $2,989.2$ & $2,289.7$ & $2,390.7$ & $2,514.0$ & $2,779.4$ \\
\hline 703.1 & $1,303.8$ & $2,864.6$ & $2,989.2$ & $2,289.7$ & $2,390.7$ & $2,514.0$ & $2,779.4$ \\
\hline • & " & - & - & - & - & - & - \\
\hline 703.1 & $1,303.8$ & $2,864.6$ & $2,989.2$ & $2,289.7$ & $2,390.7$ & $2,514.0$ & $2,779.4$ \\
\hline 3.0 & 3.7 & 8.8 & 7.8 & 9.6 & 6.8 & 6.2 & 6.6 \\
\hline 36.6 & 104.1 & 338.4 & 403.6 & 319.6 & 354.7 & 378.6 & 408.2 \\
\hline 14.7 & 66.5 & 188.3 & 261.4 & 178.6 & 132.8 & 1147 & 118.0 \\
\hline .4 & .9 & 1.2 & 4.8 & 4.5 & 1.7 & 2.5 & 2.0 \\
\hline 19.4 & 34.1 & 69.3 & 77.4 & 67.0 & 65.9 & 68.4 & 86.0 \\
\hline 628.9 & $1,088.1$ & $2,235.3$ & $2,230.6$ & $1,709.5$ & $1,828.6$ & 1.943 .4 & $2,158.7$ \\
\hline .1 & 6.2 & 23.2 & 3.6 & .8 & .1 & .1 & • \\
\hline - & - & - & - & - & - & - & - \\
\hline $\begin{array}{l}1,662.4 \\
1,234.8\end{array}$ & $\begin{array}{l}3,112.6 \\
2,342.8\end{array}$ & $\begin{array}{l}6,084.9 \\
4,603.7\end{array}$ & $\begin{array}{l}7,448.2 \\
5,385.4\end{array}$ & $\begin{array}{l}6,409.4 \\
4,312.2\end{array}$ & $\begin{array}{l}6,259.6 \\
4,047.7\end{array}$ & $\begin{array}{l}6,813.5 \\
4,379.8\end{array}$ & $\begin{array}{l}7,217.4 \\
4,807.5\end{array}$ \\
\hline 427.5 & 769.9 & $1,481.2$ & $2,062.8$ & $2,097.2$ & $2,211.9$ & $2,433.7$ & $2,409.9$ \\
\hline 65.3 & 144.2 & 311.3 & 355.5 & 307.0 & 362.5 & 419.5 & 432.7 \\
\hline 43.1 & 84.9 & 230.9 & 286.5 & 241.4 & 284.5 & 339.9 & 358.3 \\
\hline 15.3 & 14.2 & 16.0 & 4.7 & 5.1 & 11.8 & 10.4 & 9.5 \\
\hline 6.9 & 19.5 & 15.8 & 1.7 & 1.4 & 2.6 & 3.7 & 5.4 \\
\hline 3.9 & 10.4 & 10.1 & - & - & - & .1 & .1 \\
\hline 2.7 & 8.9 & 5.6 & 1.7 & 1.4 & 2.6 & 2.8 & 2.1 \\
\hline .2 & .2 & - & - & - & - & .8 & 3.2 \\
\hline - & 25.5 & 48.6 & 62.5 & 59.2 & 63.1 & 64.2 & 57.4 \\
\hline .1 & . & • & - & - & .4 & 1.4 & 2.1 \\
\hline $1,320.2$ & $2,487.0$ & $4,915.0$ & $5,740.8$ & $4,619.2$ & $4,410.2$ & $4,799.3$ & $5,240.2$ \\
\hline
\end{tabular}

- There are no direct fuel costs for hydroelectric, geothermal, centralized solar, or wind energy. Wood and jther biomass fuels are not included, except those consumed at electric utilities.

- Heavy oil includes Grade Nos. 4, 5, and 6, and residual fuel oils. Light oil includes Grade No. 2 heating oil, kerosene, and jet fuel.

- No consumption, including cases where adjustments were made. See "Consumption Adjustments for Process Fuel and intermediate Products"

- Value less than 0.05 million dollars.

Note: Totals may not equal sum of components due to independent rounding.

Sources: Data sources, estimation procedures, and assumptions are described in the "Documentation" section of this report 


\begin{tabular}{|c|c|c|c|c|c|c|c|c|}
\hline Energy Source & 1970 & 1975 & 1980 & 1985 & 1986 & 1987 & 1988 & 1989 \\
\hline & \multicolumn{8}{|c|}{ Prices in Dollars per Million Btu } \\
\hline Coul & 0.26 & 0.83 & 1.84 & 2.50 & 2.27 & 1.94 & 1.81 & 1.67 \\
\hline 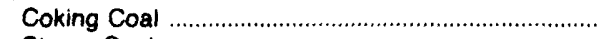 & - & - & - & - & - & - & - & - \\
\hline 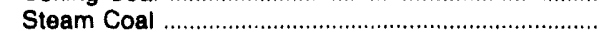 & .26 & .83 & 1.84 & 2.50 & 2.27 & 1.94 & 1.81 & 1.67 \\
\hline 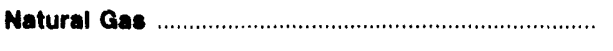 & .38 & .87 & 2.55 & 3.76 & 3.40 & 3.33 & 3.19 & 2.89 \\
\hline 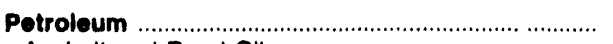 & 2.13 & 3.10 & 7.06 & 7.61 & 5.11 & 5.89 & 5.56 & 5.84 \\
\hline 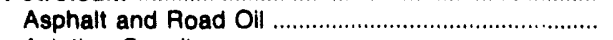 & .66 & 1.77 & 3.56 & 4.81 & 4.90 & 3.27 & 3.03 & 2.86 \\
\hline Aviation Gasoline & 2.17 & 3.45 & 9.02 & 9.99 & 8.41 & 7.55 & 7.41 & 8.28 \\
\hline 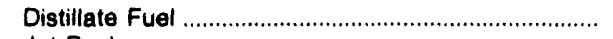 & 1.32 & 2.24 & 6.89 & 6.68 & 5.00 & 5.44 & 5.29 & 5.74 \\
\hline 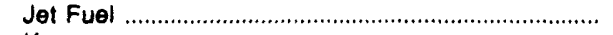 & .73 & 2.03 & 6.39 & 5.84 & 3.41 & 3.97 & 3.43 & 3.92 \\
\hline Kerosene & .83 & 2.03 & 6.99 & 8.43 & 5.52 & 5.57 & 4.49 & 4.48 \\
\hline LPG & 1.83 & 3.42 & 6.31 & 7.42 & 8.10 & 8.47 & 8.80 & 9.10 \\
\hline 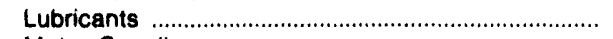 & 5.08 & 7.49 & 14.36 & 17.61 & 15.59 & 13.58 & 14.61 & 17.90 \\
\hline 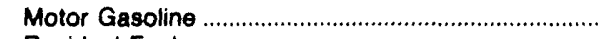 & 2.84 & 4.34 & $10.5 ?$ & 8.75 & 6.54 & 6.97 & 7.27 & 8.07 \\
\hline Residual Fuel & .45 & 1.67 & 2.84 & 4.10 & 2.09 & 2.01 & 2.28 & 2.66 \\
\hline 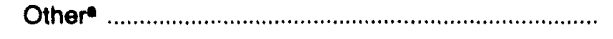 & .99 & 1.94 & 4.54 & 6.43 & 3.74 & 4.33 & 3.71 & 4.41 \\
\hline 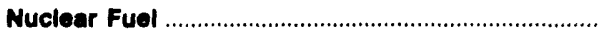 & - & - & - & 1.13 & 1.15 & .89 & 1.40 & 1.17 \\
\hline 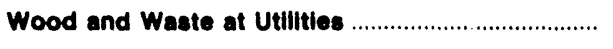 & - & - & - & - & - & - & - & - \\
\hline Primary Energy - Flve Sectors .................................. & 1.15 & 2.27 & 4.93 & 5.20 & 4.01 & 4.07 & 3.95 & 4.18 \\
\hline $\begin{array}{l}\text { Electric Utility Fuel } \\
\text { Electricity Purchased by End Users }\end{array}$ & $\begin{array}{r}.27 \\
4.44\end{array}$ & $\begin{array}{l}1.24 \\
7.58\end{array}$ & $\begin{array}{r}2.16 \\
13.69\end{array}$ & $\begin{array}{r}2.30 \\
17.05\end{array}$ & $\begin{array}{r}2.05 \\
18.01\end{array}$ & $\begin{array}{r}1.57 \\
17.87\end{array}$ & $\begin{array}{r}1.70 \\
18.21\end{array}$ & $\begin{array}{r}1.56 \\
18.25\end{array}$ \\
\hline \multirow[t]{2}{*}{ 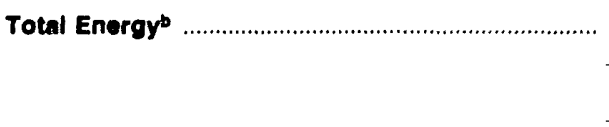 } & 1.71 & 3.20 & 7.33 & 8.20 & 6.80 & 7.33 & 7.01 & 7.13 \\
\hline & \multicolumn{8}{|c|}{ Expenditures in Millions of Dollars } \\
\hline Coal & 3.5 & 27.5 & 137.6 & 273.2 & 246.6 & 237.6 & 234.1 & 161.3 \\
\hline Coking Coal & - & - & - & - & - & - & - & - \\
\hline 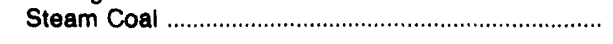 & 3.5 & 27.5 & 137.6 & 273.2 & 246.6 & 237.6 & 234.1 & 161.3 \\
\hline 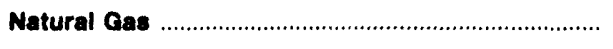 & 111.2 & 154.3 & $\mathbf{5 5 3 . 4}$ & 710.7 & 581.3 & 530.0 & 515.9 & $\mathbf{5 2 5 . 0}$ \\
\hline Petroleum & 522.4 & $1,105.2$ & $2,487.3$ & $2,385.7$ & $1,944.1$ & $2,082.5$ & $2,285.6$ & $2,563.1$ \\
\hline 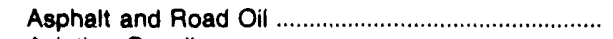 & 7.7 & 30.4 & 48.1 & 65.5 & 61.9 & 47.2 & 52.8 & 37.5 \\
\hline 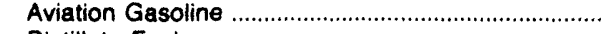 & 3.5 & 3.5 & 9.4 & 5.4 & 5.8 & 4.3 & 4.8 & 6.4 \\
\hline 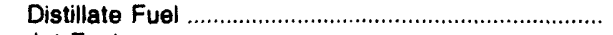 & 46.2 & 127.6 & 383.8 & 619.3 & 431.5 & 530.5 & 586.0 & 571.9 \\
\hline Jet Fuel . & 6.3 & 16.3 & 53.3 & 134.1 & 93.8 & 171.0 & 154.5 & 144.6 \\
\hline 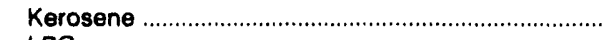 & 12.5 & 16.5 & 9.6 & 38.5 & 36.0 & 39.0 & 40.8 & 16.8 \\
\hline LPG & 59.4 & 102.3 & 125.9 & 133.3 & 106.9 & 117.6 & 136.6 & 196.9 \\
\hline 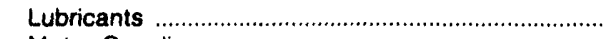 & 16.2 & 30.9 & 57.1 & 63.7 & 55.1 & 54.3 & 56.3 & 70.8 \\
\hline Motor Gasoline & 362.5 & 633.5 & $1,481.0$ & $1,267.2$ & 980.8 & $1,070.9$ & $1,126.9$ & $1,229.4$ \\
\hline 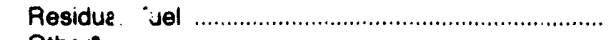 & 1.9 & 126.6 & 284.7 & 38.1 & 159.3 & 32.8 & 113.8 & 274.3 \\
\hline 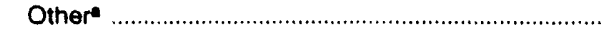 & 6.3 & 17.6 & 34.5 & 30.4 & 13.1 & 14.9 & 12.9 & 14.6 \\
\hline 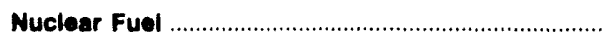 & - & - & - & 53.1 & 50.6 & 74.1 & 143.8 & 97.9 \\
\hline 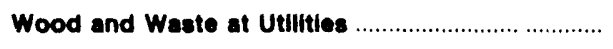 & - & - & - & - & - & - & - & - \\
\hline Primary Energy - Five Sectors ................................ & 637.1 & $1,287.0$ & $3,178.3$ & $3,432.7$ & $2,822.6$ & $2,924.1$ & $3,179.5$ & $3,347.4$ \\
\hline 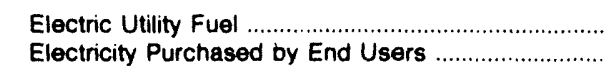 & $\begin{array}{l}-31.7 \\
225.9\end{array}$ & $\begin{array}{r}-154.7 \\
486.0\end{array}$ & $\begin{array}{r}-438.6 \\
1,075.9\end{array}$ & $\begin{array}{r}-476.1 \\
1,455.8\end{array}$ & $\begin{array}{r}-420.1 \\
1.582 .5\end{array}$ & $\begin{array}{r}-381.2 \\
1,606.2\end{array}$ & $\begin{array}{r}-453.7 \\
1,694.2\end{array}$ & $\begin{array}{r}-355.7 \\
1.786 .8\end{array}$ \\
\hline Total Energyb & 831.3 & $1,618.4$ & $3,815.6$ & $4,412.5$ & $3,985.0$ & $4,149.1$ & $4,420.0$ & $4,778.4$ \\
\hline
\end{tabular}

- Includes petroleum coke used at eler tric utilities.

bThere are no direct fuel costs for hyidrestectric, geothermal, centralized solar, or wind energy. Wood and other biomass fuels are not included, except those consumed at electric utilities.

- No consumption, including cases where adjustments were made. See "Consumption Adjustments for Process Fuel and Intermediate Products."

Note: Expenditure totals may not equal sum of components due to independent rounding.

Sources: Data sources, estimation procedures, and assumptions are described in the "Documentation" section of this report. 


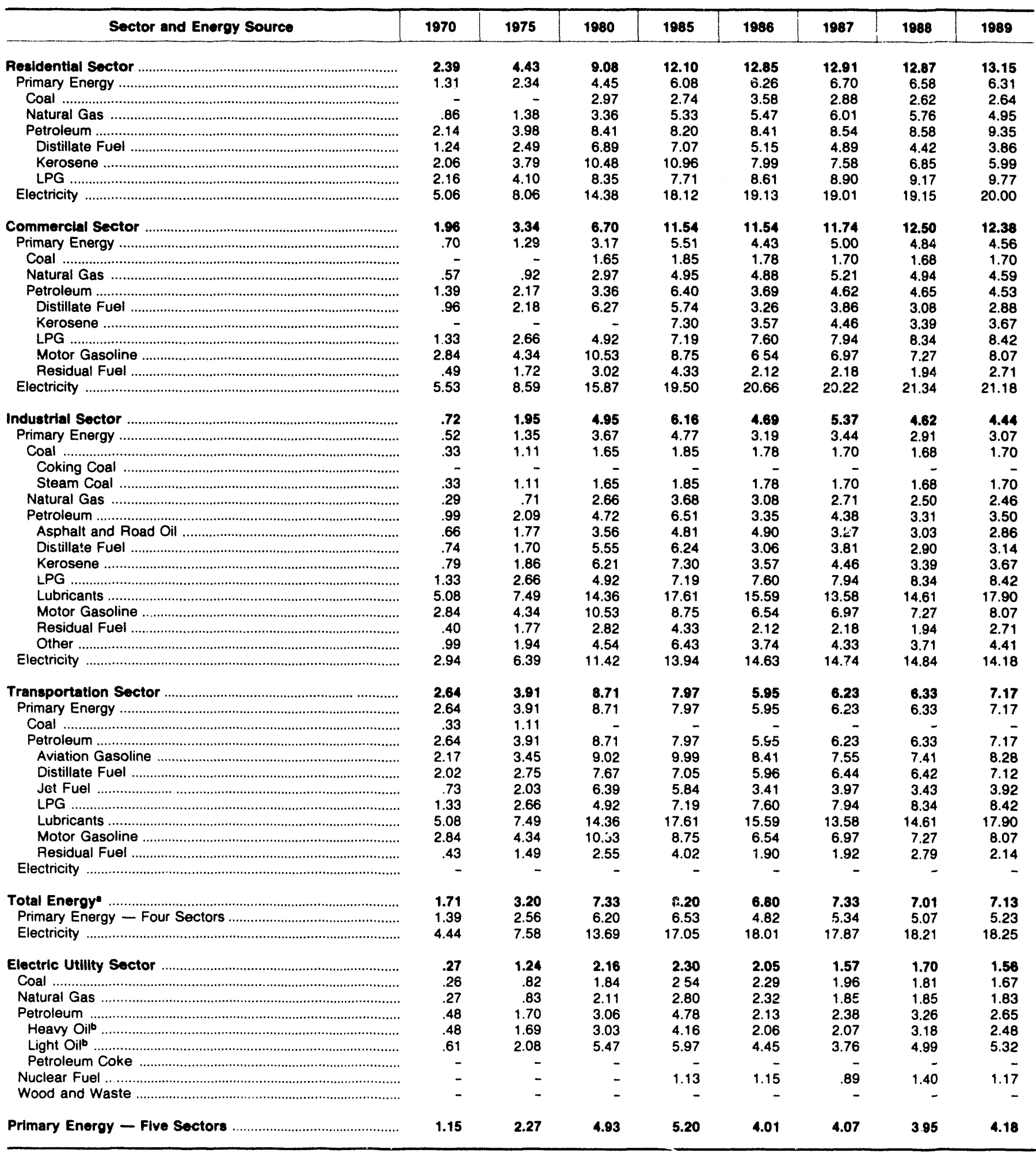

-There are no direct fuel costs for hydroelectric, geothermal, centralized solar, or wind energy. Wood and other biomass fuels are not included, except those consumed at electric utilities.

- Heavy oil includes Grade Nos. 4, 5, and 6, and residual fuel oils. Light oil includes Grade No. 2 heating oil, kerosene, and jet fuel.

-No consumption, including cases where adjustments were made. See "Consumption Adjustments for Proces. Fuel and Intermediate Products."

Sources: Data sources, estimation procedures, and assumptions ara described in the "Documentaticn" section of this report. 


\begin{tabular}{|c|c|c|c|c|c|c|c|c|}
\hline Sector and Energy Source & 1970 & 1975 & 1980 & 1985 & 1986 & 1987 & 1988 & 1989 \\
\hline Residential Sector & 194.5 & 334.1 & 661.9 & 863.2 & 930.5 & 972.3 & $1,002.1$ & $1,034.8$ \\
\hline 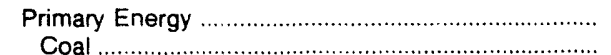 & 75.9 & 111.5 & 173.0 & 217.2 & 221.1 & 250.5 & 256.1 & 248.8 \\
\hline 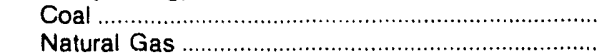 & - & - & & & .1 & .1 & .3 & \\
\hline 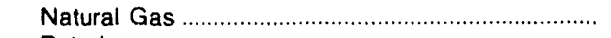 & 32.4 & 41.6 & 102.6 & 140.4 & 141.4 & 162.3 & 157.3 & 134.2 \\
\hline Petr:leum & 43.5 & 70.0 & 70.4 & 76.8 & 79.7 & 88.1 & 98.5 & 114.5 \\
\hline 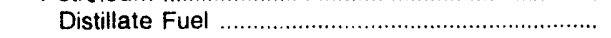 & .6 & 2.8 & .3 & .1 & .1 & .5 & .1 & .1 \\
\hline Kerosene …………………………………. & .9 & 2.7 & 2.6 & 15.6 & 23.0 & 18.9 & 19.7 & 7.8 \\
\hline LPG & 42.0 & 64.4 & 67.5 & 61.2 & 56.6 & 68.8 & 78.7 & 106.5 \\
\hline 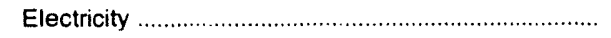 & 118.7 & 222.5 & 488.9 & 646.0 & 709.4 & 721.8 & 746.0 & 786.0 \\
\hline Commercial Sector & 77.5 & 161.8 & 420.3 & 560.0 & 569.3 & 576.8 & 601.6 & 637.5 \\
\hline 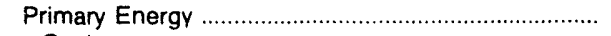 & 20.5 & 45.1 & 143.5 & 152.1 & 122.7 & 137.0 & 124.6 & 124.4 \\
\hline Coal & - & - & & & .1 & .1 & .3 & .1 \\
\hline 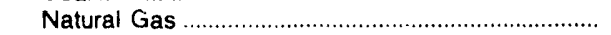 & 13.9 & 22.6 & 64.1 & 84.1 & 84.1 & 94.8 & 90.9 & 83.1 \\
\hline Petroleum & 6.7 & 22.5 & 79.4 & 68.0 & 38.5 & 42.0 & 33.4 & 41.3 \\
\hline 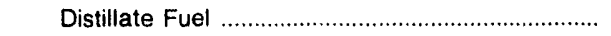 & .6 & 3.0 & .9 & 35.7 & 8.4 & 17.9 & 10.7 & 14.3 \\
\hline Kerosene & 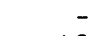 & - & - & 15.1 & 5.0 & 2.5 & 1.3 & 1.0 \\
\hline 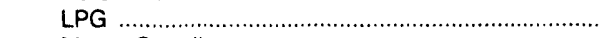 & 4.6 & 7.4 & 7.0 & 10.1 & 8.8 & 10.8 & 12.6 & 16.2 \\
\hline 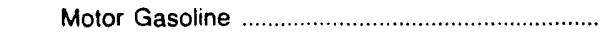 & 1.4 & 2.4 & 6.8 & 6.2 & 7.5 & 7.7 & 7.1 & 6.8 \\
\hline 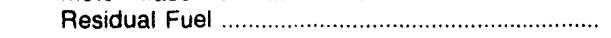 & .1 & 9.7 & 64.7 & 1.0 & 8.8 & 3.1 & 1.6 & 3.0 \\
\hline Electricity & 57.0 & 116.7 & 276.8 & 407.9 & 446.7 & 439.8 & 477.0 & 513.0 \\
\hline Industrial Sector & 150.1 & 371.8 & 815.4 & $1,175.2$ & $1,044.0$ & 946.1 & $1,023.2$ & $1,241.8$ \\
\hline 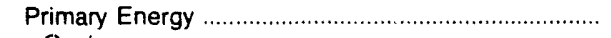 & 99.9 & 225.1 & 505.2 & 773.3 & 617.5 & 501.6 & 552.0 & 754.0 \\
\hline 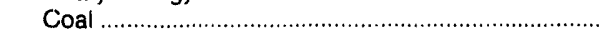 & .4 & .6 & 2.0 & 10.7 & 10.3 & 11.3 & 10.5 & 10.5 \\
\hline 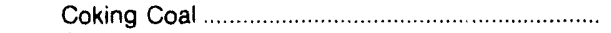 & - & - & - & - & - & - & - & - \\
\hline 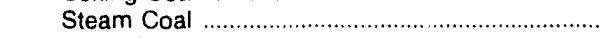 & .4 & .6 & 2.0 & 10.7 & 10.3 & 11.3 & 10.5 & 10.5 \\
\hline 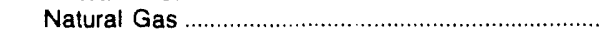 & 37.6 & 63.2 & 182.6 & 330.6 & 241.5 & 194.7 & 204.7 & 223.3 \\
\hline 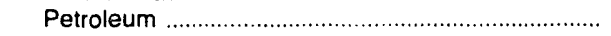 & 61.9 & 161.2 & 320.5 & 431.9 & 365.7 & 295.6 & 336.8 & 520.3 \\
\hline Asphalt and Road Oil ........................................... & 7.7 & 30.4 & 48.1 & 65.5 & 61.9 & 47.2 & 52.8 & 37.5 \\
\hline Distillate Fuel & 13.3 & 43.4 & 111.3 & 196.0 & 79.5 & 122.7 & 93.1 & 90.8 \\
\hline 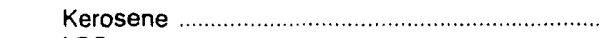 & 11.6 & 13.8 & 7.0 & 7.9 & 7.9 & 17.6 & 19.8 & 7.9 \\
\hline LPG & 10.5 & 25.9 & 48.6 & 56.5 & 36.8 & 33.5 & 41.2 & 70.6 \\
\hline 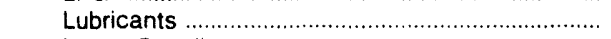 & 7.5 & 17.0 & 29.7 & 33.1 & 28.7 & 28.2 & 29.3 & 36.8 \\
\hline Motor Gasoline . & 4.6 & 5.0 & 4.1 & 34.5 & 21.6 & 23.0 & 24.2 & 23.8 \\
\hline 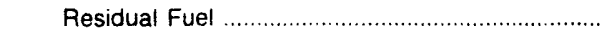 & .5 & 8.3 & 37.3 & 8.0 & 116.3 & 8.5 & 63.5 & 238.3 \\
\hline 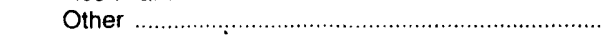 & 6.3 & 17.6 & 34.5 & 30.4 & 13.1 & 14.9 & 12.9 & 14.6 \\
\hline 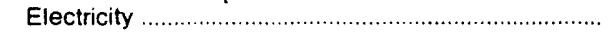 & 50.2 & 146.7 & 310.2 & 401.9 & 426.5 & 444.6 & 471.2 & 487.7 \\
\hline Transportation Sector & 409.1 & 750.7 & $1,918.0$ & $1,814.0$ & $1, \mathbf{4 4 1 . 2}$ & $1,653.9$ & $1,793.1$ & $1,864.4$ \\
\hline 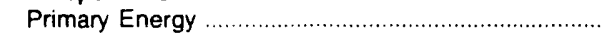 & 409.1 & 750.7 & $1,918.0$ & $1,814.0$ & $1,441.2$ & $1,653.9$ & $1,793.1$ & $1,864.4$ \\
\hline Coal & & & & - & - & - & - & - \\
\hline Petroleum & 409.1 & 750.7 & $1,918.0$ & $1,814.0$ & $1,441.2$ & $1,653.9$ & $1,793.1$ & $1,864.4$ \\
\hline Aviation Gasoline & 3.5 & 3.5 & 9.4 & 5.4 & 5.8 & 4.3 & 4.8 & 6.4 \\
\hline Distillate Fuel & 31.6 & 75.1 & 269.0 & 385.5 & 342.3 & 388.7 & 480.4 & 463.9 \\
\hline Jet Fuel & 6.3 & 16.3 & 53.3 & 134.1 & 93.8 & 171.0 & 154.5 & 144.6 \\
\hline LPG & 2.4 & 4.6 & 2.7 & 5.6 & 4.6 & 4.5 & 4.2 & 3.6 \\
\hline 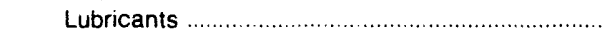 & 8.7 & 13.9 & 27.4 & 30.6 & 26.5 & 26.1 & 27.1 & 34.0 \\
\hline Motor Gasoline ……….................................. & 356.5 & 626.2 & 1.470 .2 & $1,226.6$ & 951.8 & $1,040.3$ & $1,095.6$ & $1,198.8$ \\
\hline Residual Fuel & • & 11.1 & 86.0 & 26.3 & 16.4 & 19.2 & 26.6 & 13.1 \\
\hline 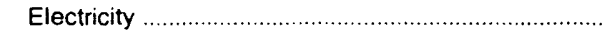 & - & - & - & - & - & - & - & - \\
\hline 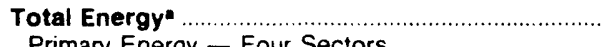 & 831.3 & $1,618.4$ & $3,815.6$ & $4,412.5$ & $3,985.0$ & $4,149.1$ & $4,420.0$ & $4,778.4$ \\
\hline $\begin{array}{l}\text { Primary Energy - Four Sectors } \\
\text { Electricity }\end{array}$ & $\begin{array}{l}605.4 \\
225.9\end{array}$ & $\begin{array}{r}1,132.4 \\
486.0\end{array}$ & $\begin{array}{l}2,739.7 \\
1,075.9\end{array}$ & $\begin{array}{l}2,956.7 \\
1,455.8\end{array}$ & $\begin{array}{l}2,402.5 \\
1,582.5\end{array}$ & $\begin{array}{l}2,542.9 \\
1,606.2\end{array}$ & $\begin{array}{l}2,725.8 \\
1,694.2\end{array}$ & $\begin{array}{l}2,991.6 \\
1,786.8\end{array}$ \\
\hline Electric Utility Sector & 31.7 & 154.7 & 438.6 & 476.1 & 420.1 & 381.2 & 453.7 & 355.7 \\
\hline Coal & 3.1 & 26.9 & 135.5 & 262.4 & 236.1 & 226.0 & 223.0 & 150.8 \\
\hline Natural Gas & 27.3 & 26.9 & 204.2 & 155.6 & 114.4 & 78.2 & 63.0 & 84.5 \\
\hline 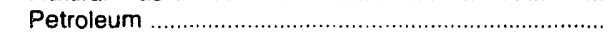 & 1.3 & 100.8 & 98.9 & 4.9 & 18.9 & 2.8 & 23.8 & 22.6 \\
\hline 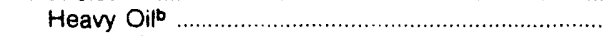 & 1.2 & 97.6 & 96.7 & 2.8 & 17.8 & 2.0 & 22.2 & 19.9 \\
\hline 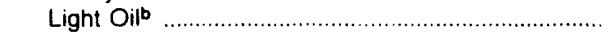 & • & 3.2 & 2.2 & 2.1 & 1.2 & .8 & 1.7 & 2.7 \\
\hline 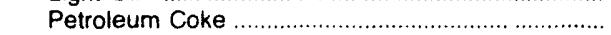 & - & - & - & - & - & - & & \\
\hline 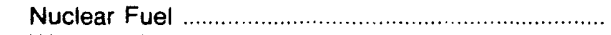 & - & - & - & 53.1 & 50.6 & 74.1 & 143.8 & 97.9 \\
\hline Wood and Waste & - & - & - & - & - & - & - & - \\
\hline Primary Energy - Five Sectors & 637.1 & $1,287.0$ & $3,178.5$ & $3,432.7$ & $2,822.6$ & $2,924.1$ & $3,179.5$ & $3,347.4$ \\
\hline
\end{tabular}

- There are no direct fuel costs for hydroelectric, geothermal, centralized solar, or wind energy. Wood and other biomass fuels are not included, except those consumed at electric utilities.

b Heavy oil includes Grade Nos. 4,5, and 6, and residual fuel oils. Light oil includes Grade No. 2 heating rill, kerosene, and jet fuel.

-No consumption, including cases where adjustments were made. See "Consumption Adjustments for Process Fuel and Intermediate Products."

- Value less than 0.05 million dollars.

Note: Totals may not equal sum of components due to independent rounding.

Sources: Data sources, estimation procedures, and assumptions are described in the "Documentation" section of this report 


\begin{tabular}{|c|c|c|c|c|c|c|c|c|}
\hline Energy Source & 1970 & 1975 & 1980 & 1985 & 1986 & 1987 & 1988 & 1989 \\
\hline & \multicolumn{8}{|c|}{ Prices in Dollars per Million Btu } \\
\hline 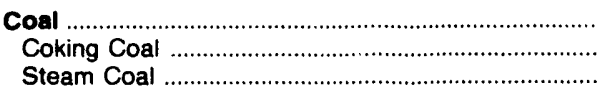 & $\begin{array}{r}0.29 \\
.38 \\
.28\end{array}$ & $\begin{array}{r}0.62 \\
1.60 \\
.60\end{array}$ & $\begin{array}{l}1.22 \\
1.81 \\
1.21\end{array}$ & $\begin{array}{l}1.51 \\
1.93 \\
1.51\end{array}$ & $\begin{array}{l}1.46 \\
1.75 \\
1.46\end{array}$ & $\begin{array}{l}1.40 \\
1.63 \\
1.40\end{array}$ & $\begin{array}{l}1.37 \\
1.70 \\
1.37\end{array}$ & $\begin{array}{r}1.35 \\
- \\
1.35\end{array}$ \\
\hline 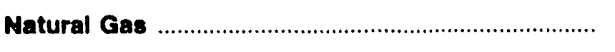 & .64 & 1.16 & 2.95 & 4.94 & 4.52 & 4.28 & 4.37 & 4.50 \\
\hline $\begin{array}{l}\text { Petroleum } \\
\text { Asphalt and Road Oil } \\
\text { Aviation Gasoline } \\
\text { Distillate Fuel } \\
\text { Jet Fuel } \\
\text { Kerosene } \\
\text { LPG } \\
\text { Lubricants } \\
\text { Motor Gasoline } \\
\text { Residual Fuel } \\
\text { Other }\end{array}$ & $\begin{array}{r}1.97 \\
.71 \\
2.17 \\
1.05 \\
.75 \\
.89 \\
1.79 \\
5.08 \\
2.73 \\
.56 \\
1.24\end{array}$ & $\begin{array}{l}3.66 \\
2.05 \\
3.45 \\
2.52 \\
2.09 \\
2.45 \\
3.06 \\
7.49 \\
4.55 \\
1.78 \\
2.57\end{array}$ & $\begin{array}{r}8.03 \\
3.59 \\
9.02 \\
6.61 \\
6.47 \\
6.43 \\
6.32 \\
14.36 \\
9.33 \\
3.33 \\
7.09\end{array}$ & $\begin{array}{r}7.85 \\
4.77 \\
9.99 \\
6.70 \\
5.90 \\
7.81 \\
8.08 \\
17.61 \\
8.56 \\
4.10 \\
7.43\end{array}$ & $\begin{array}{r}5.73 \\
3.81 \\
8.41 \\
5.26 \\
3.95 \\
5.81 \\
6.57 \\
15.59 \\
6.11 \\
2.25 \\
4.44\end{array}$ & $\begin{array}{r}6.19 \\
3.70 \\
7.55 \\
5.61 \\
4.13 \\
5.49 \\
6.70 \\
13.58 \\
6.81 \\
2.60 \\
4.81\end{array}$ & $\begin{array}{r}6.06 \\
3.53 \\
7.41 \\
5.37 \\
3.89 \\
5.16 \\
6.98 \\
14.61 \\
6.82 \\
2.13 \\
4.08\end{array}$ & $\begin{array}{r}6.74 \\
3.12 \\
8.28 \\
6.19 \\
4.36 \\
6.67 \\
7.42 \\
17.90 \\
7.45 \\
2.35 \\
4.73\end{array}$ \\
\hline 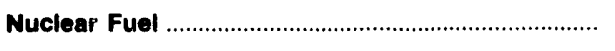 & - & - & - & .82 & .82 & .52 & .73 & .71 \\
\hline 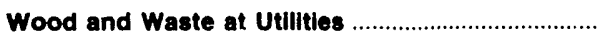 & - & - & - & - & - & - & - & - \\
\hline 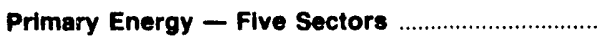 & 1.16 & 2.07 & 4.38 & 4.59 & 3.72 & 3.85 & 3.77 & 4.07 \\
\hline 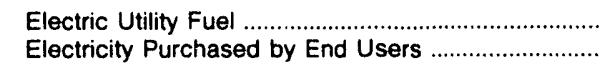 & 6.17 & $\begin{array}{r}.57 \\
8.64\end{array}$ & $\begin{array}{r}1.25 \\
13.91\end{array}$ & $\begin{array}{r}1.41 \\
17.16\end{array}$ & $\begin{array}{r}1.38 \\
18.45\end{array}$ & $\begin{array}{r}1.31 \\
18.90\end{array}$ & $\begin{array}{r}1.29 \\
18.94\end{array}$ & $\begin{array}{r}1.26 \\
18.92\end{array}$ \\
\hline \multirow{2}{*}{ 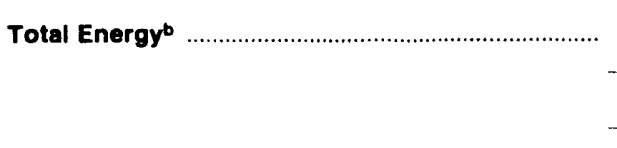 } & 1.83 & 3.32 & 7.19 & 8.28 & 7.31 & 7.66 & 7.60 & 8.01 \\
\hline & \multicolumn{8}{|c|}{ Expenditures in Millions of Dollars } \\
\hline $\begin{array}{l}\text { Coal } \\
\text { Coking Coal } \\
\text { Steam Coal }\end{array}$ & $\begin{array}{r}80.3 \\
3.1 \\
77.2\end{array}$ & $\begin{array}{r}266.8 \\
11.9 \\
254.9\end{array}$ & $\begin{array}{r}647.4 \\
9.6 \\
637.7\end{array}$ & $\begin{array}{r}800.8 \\
12.0 \\
788.8\end{array}$ & $\begin{array}{r}750.1 \\
10.8 \\
739.3\end{array}$ & $\begin{array}{r}741.4 \\
3.4 \\
738.0\end{array}$ & $\begin{array}{r}751.9 \\
751.9\end{array}$ & $\begin{array}{r}740.3 \\
- \\
740.3\end{array}$ \\
\hline 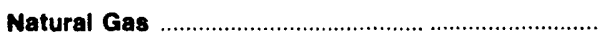 & 265.4 & 423.0 & 928.2 & $1,284.0$ & $1,089.0$ & 994.0 & $1,090.0$ & $1,160.6$ \\
\hline $\begin{array}{l}\text { Petroleum } \\
\text { Asphalt and Road Oil } \\
\text { Aviation Gasoline . } \\
\text { Distillate Fuel } \\
\text { Jet Fuel } \\
\text { Kerosene } \\
\text { LPG } \\
\text { Motor Gasoline } \\
\text { Residual Fuel } \\
\text { Other }\end{array}$ & $\begin{array}{r}1,120.8 \\
26.8 \\
2.0 \\
99.1 \\
34.1 \\
3.2 \\
79.5 \\
35.5 \\
803.2 \\
11.4 \\
26.1\end{array}$ & $\begin{array}{r}2,201.4 \\
73.3 \\
3.2 \\
261.8 \\
98.2 \\
3.9 \\
147.5 \\
58.3 \\
1,490.4 \\
21.7 \\
43.1\end{array}$ & $\begin{array}{r}4,681.5 \\
95.3 \\
7.4 \\
708.2 \\
229.5 \\
11.5 \\
211.7 \\
139.6 \\
2,889.0 \\
23.2 \\
366.2\end{array}$ & $\begin{array}{r}4,506.2 \\
135.9 \\
6.8 \\
765.2 \\
196.6 \\
10.1 \\
258.9 \\
155.8 \\
2,699.9 \\
22.4 \\
254.6\end{array}$ & $\begin{array}{r}3,400.3 \\
116.9 \\
6.9 \\
562.0 \\
149.9 \\
2.5 \\
218.6 \\
134.8 \\
2,034.0 \\
9.6 \\
165.1\end{array}$ & $\begin{array}{r}3,742.6 \\
106.8 \\
5.1 \\
629.7 \\
174.6 \\
2.0 \\
211.6 \\
132.8 \\
2,274.5 \\
11.6 \\
194.1\end{array}$ & $\begin{array}{r}3,857.4 \\
132.6 \\
6.1 \\
663.8 \\
160.8 \\
2.6 \\
248.8 \\
137.8 \\
2,326.8 \\
10.9 \\
167.3\end{array}$ & $\begin{array}{r}4,257.3 \\
94.1 \\
8.4 \\
798.1 \\
179.6 \\
4.7 \\
307.8 \\
173.1 \\
2,492.9 \\
10.4 \\
188.2\end{array}$ \\
\hline 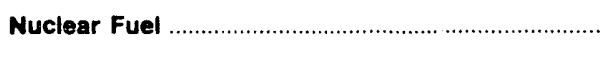 & - & - & - & 71.3 & 63.1 & 34.9 & 70.3 & 63.1 \\
\hline 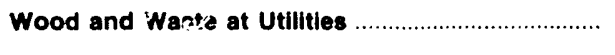 & - & - & - & - & - & - & - & - \\
\hline 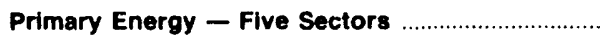 & $1,466.5$ & $2,891.1$ & $6,257.1$ & $6,662.4$ & $5,302.5$ & $5,512.9$ & $5,769.7$ & $6,221.4$ \\
\hline 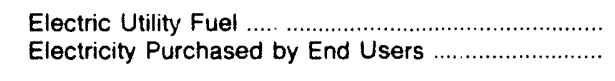 & $\begin{array}{l}-76.3 \\
542.4\end{array}$ & $\begin{array}{r}-234.0 \\
974.3\end{array}$ & $\begin{array}{r}-639.6 \\
2,022.4\end{array}$ & $\begin{array}{r}-811.7 \\
2.712 .0\end{array}$ & $\begin{array}{r}-761.7 \\
3.038 .6\end{array}$ & $\begin{array}{r}-733.5 \\
3,232.6\end{array}$ & $\begin{array}{r}-781.9 \\
3.377 .0\end{array}$ & $\begin{array}{r}-762.9 \\
3,396.0\end{array}$ \\
\hline Total Energy & $1,932.6$ & $3,631.4$ & $7,639.9$ & $8,562.7$ & $7,579.5$ & $8,012.0$ & $8,364.8$ & $8,854.5$ \\
\hline
\end{tabular}

Includes petroleum coke used at electric utilities.

bThere are no direct fuel costs for hydroelectric, geothermal, centralized solar, or wind energy. Wood and other biomass fuels are not included, except those consumed at electric utilities.

-No consumption, including cases where adjustments were made. See "Consumption Adjustments for Process Fuel and Intermediate Products."

- Value less than 0.05 million dollars.

Note: Expenditure totals may not equal sum of components due to independent rounding

Sources: Data sources, estimation procedures, and assumptions are described in the "Documentation" section of this report 


\begin{tabular}{|c|c|c|c|c|c|c|c|c|}
\hline Sector and Energy So: & 1970 & 1975 & 1980 & 1985 & 1986 & 1987 & 1988 & 1989 \\
\hline 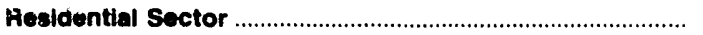 & 2.09 & 3.39 & 6.88 & 9.58 & 9.92 & 10.32 & 10.21 & 10.29 \\
\hline 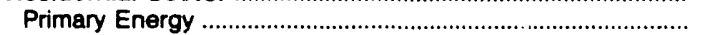 & 1.13 & 1.85 & 3.80 & 5.70 & 5.09 & 4.91 & 4.99 & 5.32 \\
\hline 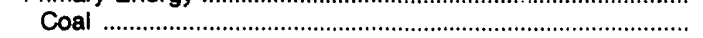 & .86 & 1.72 & 1.70 & 1.73 & 1.54 & 1.37 & 1.25 & 1.43 \\
\hline 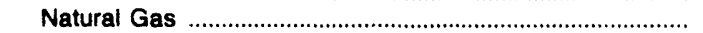 & .96 & 1.48 & 3.23 & 5.40 & 498 & 4.71 & 4.73 & 4.80 \\
\hline 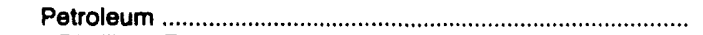 & 1.81 & 3.14 & 7.01 & 7.41 & 5.66 & 6.14 & 6.51 & 7.82 \\
\hline 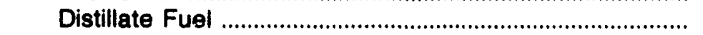 & 1.19 & 2.62 & 6.85 & 6.70 & 4.95 & 4.66 & 4.71 & 5.88 \\
\hline 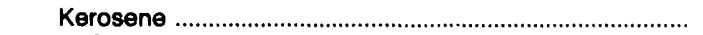 & 1.43 & 2.88 & 7.95 & 8.45 & 6.24 & 5.87 & 5.94 & 7.41 \\
\hline 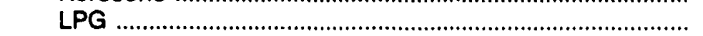 & 1.95 & 3.26 & 7.06 & 7.53 & 5.81 & 6.41 & 6.82 & 8.02 \\
\hline Electricity & 7.86 & 10.06 & 15.21 & 19.27 & 20.87 & 21.59 & 21.49 & 21.50 \\
\hline Commerclal Sector & 1.66 & 2.87 & 6.87 & 10.23 & 10.40 & 10.45 & 10.55 & 10.92 \\
\hline 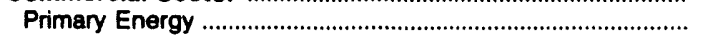 & .69 & 1.36 & 3.32 & 5.14 & 4.44 & 4.08 & 4.14 & 4.33 \\
\hline 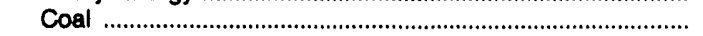 & .49 & 1.17 & 1.58 & 1.56 & 1.38 & 1.27 & 1.27 & 1.29 \\
\hline 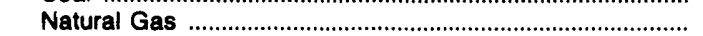 & .62 & 1.14 & 2.88 & 4.88 & 4.35 & 4.06 & 4.17 & 4.31 \\
\hline 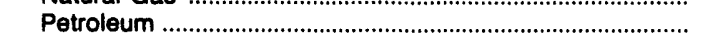 & .93 & 2.38 & 5.74 & 6.77 & 5.13 & 4.71 & 4.58 & 5.44 \\
\hline 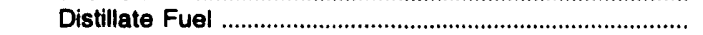 & 1.03 & 2.45 & 6.49 & 5.75 & 3.86 & 3.66 & 3.35 & 4.41 \\
\hline 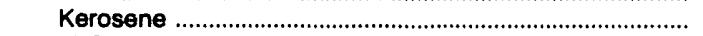 & .82 & 2.40 & 6.10 & 6.69 & 4.41 & 4.44 & 4.05 & 5.56 \\
\hline 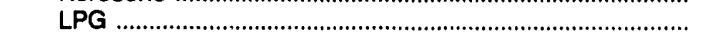 & 1.27 & 2.49 & 5.42 & 9.12 & 8.17 & 7.29 & 7.27 & 6.32 \\
\hline 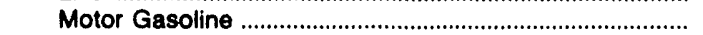 & 2.73 & 4.55 & 9.33 & 8.56 & 6.11 & 6.81 & 6.82 & 7.45 \\
\hline 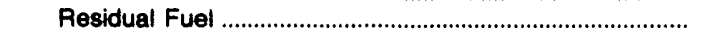 & .57 & 1.77 & 3.47 & 4.11 & 2.25 & 2.61 & 2.20 & 2.41 \\
\hline 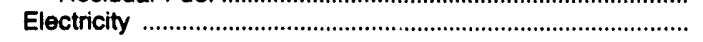 & 7.00 & 9.46 & 14.33 & 17.94 & 19.02 & 19.04 & 19.06 & 19.03 \\
\hline Industrial Soctor & 1.11 & 2.38 & 5.37 & 6.34 & 5.69 & 5.76 & 5.55 & 5.94 \\
\hline 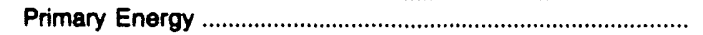 & .75 & 1.73 & 4.48 & 4.97 & 3.84 & 3.88 & 3.75 & 4.12 \\
\hline 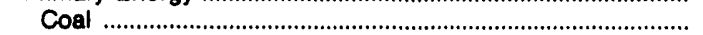 & .47 & 1.24 & 1.61 & 1.62 & 1.44 & 1.29 & 1.27 & 1.29 \\
\hline 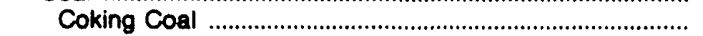 & .38 & 1.60 & 1.81 & 1.93 & 1.75 & 1.63 & 1.70 & - \\
\hline 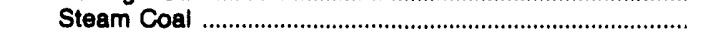 & .49 & 1.17 & 1.58 & 1.56 & 1.38 & 1.27 & 1.27 & 1.29 \\
\hline 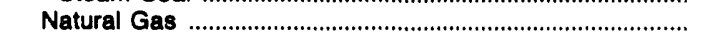 & .40 & .80 & 2.61 & 4.14 & 3.75 & 3.61 & 3.79 & 4.10 \\
\hline Petroleum & 1.16 & 2.66 & 6.32 & 6.80 & 4.82 & 4.87 & 4.45 & 4.99 \\
\hline 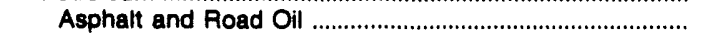 & .71 & 2.05 & 3.59 & 4.77 & 3.81 & 3.70 & 3.53 & 3.12 \\
\hline 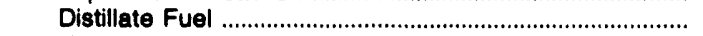 & .77 & 2.25 & 5.83 & 6.00 & 3.96 & 3.99 & 3.64 & 4.99 \\
\hline Kerosene ………1. & .82 & 2.40 & 6.10 & 6.69 & 4.41 & 4.44 & 4.05 & 5.56 \\
\hline LPG ...………… & 1.27 & 2.49 & 5.42 & 9.12 & 8.17 & 7.29 & 7.27 & 6.32 \\
\hline 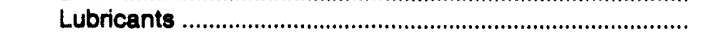 & 5.08 & 7.49 & 14.36 & 17.61 & 15.59 & 13.58 & 14.61 & 17.90 \\
\hline 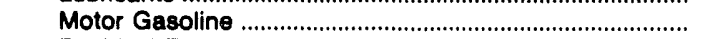 & 2.73 & 4.55 & 9.33 & 8.56 & 6.11 & 6.81 & 6.82 & 7.45 \\
\hline 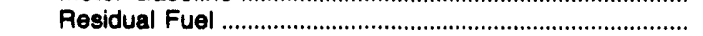 & .53 & 1.82 & 3.09 & 4.11 & 2.25 & 2.61 & 2.20 & 2.41 \\
\hline Other & 1.24 & 2.58 & 7.17 & 7.43 & 4.44 & 4.81 & 4.09 & 4.74 \\
\hline 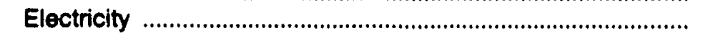 & 4.01 & 6.46 & $\$ 1.21$ & 13.14 & 14.02 & 14.36 & 14.45 & 14.50 \\
\hline 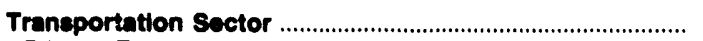 & 2.32 & 4.07 & 8.76 & 8.19 & 5.97 & 6.50 & 6.52 & 7.11 \\
\hline 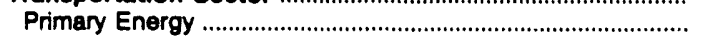 & 2.32 & 4.07 & 8.76 & 8.19 & 5.97 & 6.56 & 6.52 & 7.11 \\
\hline Coal & .49 & 1.17 & - & - & - & - & - & - \\
\hline 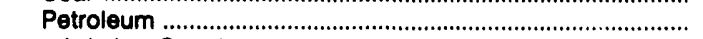 & 2.32 & 4.07 & 8.76 & 8.19 & 5.97 & 6.56 & 6.52 & 7.11 \\
\hline Aviation Gasoline ………………………………..... & 2.17 & 3.45 & 9.02 & 9.99 & 8.41 & 7.55 & 7.41 & 8.28 \\
\hline 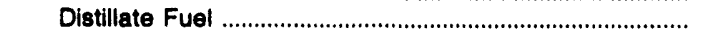 & 1.24 & 2.72 & 6.97 & 7.04 & 5.75 & 6.33 & 6.10 & 6.54 \\
\hline 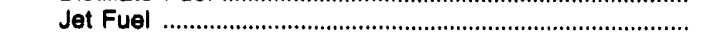 & .75 & 2.09 & 6.47 & 5.90 & 3.95 & 4.13 & 3.89 & 4.36 \\
\hline LPG & 1.27 & 2.49 & 5.42 & 9.12 & 8.17 & 7.29 & 7.27 & 6.32 \\
\hline 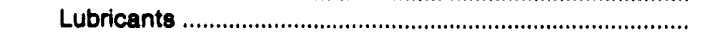 & 5.08 & 7.49 & 14.36 & 17.61 & 15.59 & 13.58 & 14.61 & 17.90 \\
\hline 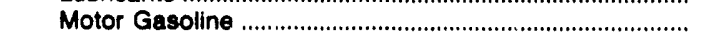 & 2.73 & 4.55 & 9.33 & 8.56 & 6.11 & 6.81 & 6.82 & 7.45 \\
\hline 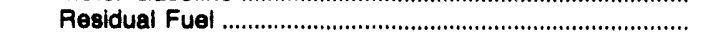 & .55 & 1.73 & 3.38 & 3.88 & 2.24 & - & 1.80 & 2.08 \\
\hline 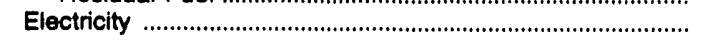 & - & - & - & - & - & - & - & - \\
\hline Total Energy & 1.83 & 3.32 & 7.19 & 8.28 & 7.31 & 7.66 & 7.80 & 8.01 \\
\hline 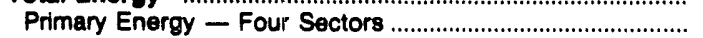 & 1.44 & 2.71 & 6.13 & 6.68 & 5.20 & 5.46 & 5.41 & 5.90 \\
\hline Eloctricity & 6.17 & 8.64 & 13.91 & 17.16 & 18.45 & 18.80 & 18.94 & 18.92 \\
\hline Eloctric Utllity Sector & & & 1.25 & 1.41 & 1.38 & 1.31 & 1.29 & 1.26 \\
\hline Coal ....1... & .25 & .54 & 1.19 & 1.50 & 1.47 & 1.41 & 1.38 & 1.35 \\
\hline 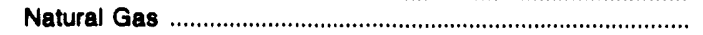 & .26 & .59 & 2.22 & 3.31 & 2.92 & 2.96 & 2.77 & 2.46 \\
\hline 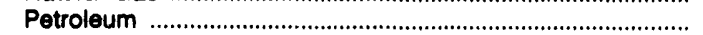 & .62 & 2.05 & 5.07 & 5.60 & 3.38 & 3.93 & 3.35 & 3.90 \\
\hline 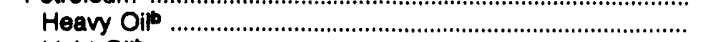 & .55 & 1.74 & 3.45 & 3.98 & 2.32 & 2.41 & 1.83 & 2.12 \\
\hline 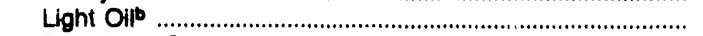 & .69 & 2.26 & 6.02 & 5.76 & 3.49 & 4.14 & 3.55 & 4.45 \\
\hline 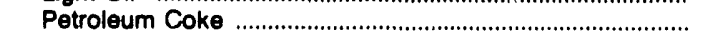 & - & .65 & .67 & 1.38 & 0.40 & - & .97 & .80 \\
\hline 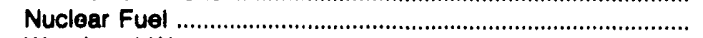 & - & - & - & .82 & .82 & .52 & .73 & .71 \\
\hline 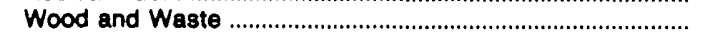 & - & - & - & - & - & - & - & - \\
\hline 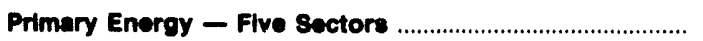 & 1.16 & 2.07 & 4.38 & 4.59 & 3.72 & 3.85 & 3.77 & 4.07 \\
\hline
\end{tabular}

-There are no direct fuel costs for hydroelectric, geothermal, centralized solar, or wind energy. Wood and other biomass fuels are not included, except those consumed at electric utilities.

-Heavy oil includes Grade Nos. 4, 5, and 6, and residual fuel oils. Light oil includes Grade No. 2 heating oil, kerosene, and jet fuel.

-No consumption, including cases where adjustments were made. See "Consumption Adjustments for Process Fuel and Intermediate Products."

Sources: Data sources, estimation procedures, and assumptions are described in the "Documentation" section of this report. 


\begin{tabular}{|c|c|c|c|c|c|c|c|c|}
\hline Sector and Energy Source & 1970 & 1975 & 1980 & 1985 & 1986 & 1987 & 1988 & 1989 \\
\hline 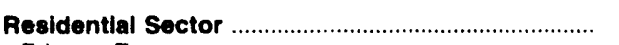 & 486.6 & 840.7 & $1,622.0$ & $2,116.3$ & $2,151.1$ & $2,206.3$ & $2,349.9$ & $2,407.8$ \\
\hline 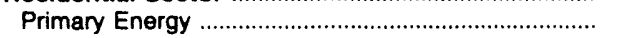 & 227.1 & 371.9 & 654.0 & 901.0 & 764.9 & 709.8 & 784.9 & 862.8 \\
\hline 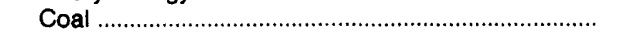 & .6 & 2.0 & 1.1 & 2.2 & 1.3 & 2.6 & 2.1 & 2.8 \\
\hline Natural Gas & 150.9 & 232.0 & 471.2 & 703.3 & 606.9 & 552.4 & 610.8 & 625.1 \\
\hline Petroleum & 75.5 & 137.9 & 181.7 & 195.5 & 156.8 & 154.8 & 172.0 & 235.0 \\
\hline 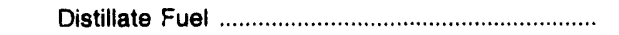 & 9.1 & 21.9 & 49.7 & 31.8 & 23.6 & 17.8 & 17.4 & 16.2 \\
\hline 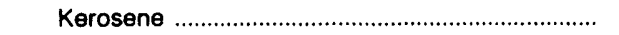 & .6 & .5 & 2.6 & 6.9 & 2.1 & 1.6 & 1.8 & 3.1 \\
\hline LPG & 65.9 & 115.5 & 129.4 & 156.7 & 131.1 & 135.5 & 152.8 & 215.6 \\
\hline Electricity & 259.5 & 468.8 & 967.9 & $1,215.3$ & $1,386.2$ & $1,496.5$ & $1,565.0$ & $1,545.0$ \\
\hline Commercial Sector & 227.1 & 400.9 & 943.7 & $1,333.9$ & $1,396.7$ & $1,444.8$ & $1,537.2$ & $1,559.4$ \\
\hline 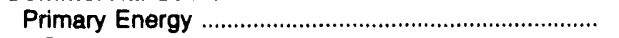 & 79.8 & 154.4 & 308.8 & 403.1 & 353.0 & 323.8 & 344.1 & 341.4 \\
\hline Coal & .6 & 2.5 & 1.8 & 3.6 & 2.1 & 4.4 & 4.1 & 4.7 \\
\hline 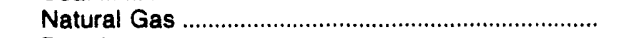 & 54.9 & 104.7 & 222.7 & 299.5 & 272.1 & 239.2 & 268.1 & 273.6 \\
\hline 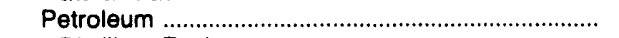 & 24.2 & 47.2 & 84.4 & 100.0 & 78.7 & 80.1 & 72.0 & 63.2 \\
\hline 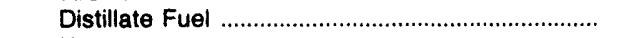 & 6.5 & 16.9 & 37.9 & 49.1 & 33.3 & 39.6 & 32.5 & 23.8 \\
\hline Kerosene & 2.0 & 2.4 & 5.9 & 1.9 & .2 & .1 & .3 & .4 \\
\hline LPG & 7.5 & 15.5 & 17.5 & 33.5 & 32.5 & 27.2 & 28.8 & 30.0 \\
\hline 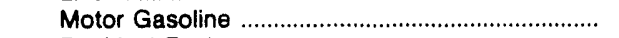 & 2.2 & 3.8 & 10.9 & 11.8 & 10.4 & 11.2 & 8.9 & 8.3 \\
\hline Residual Fuel & 6.0 & 8.5 & 12.1 & 3.8 & 2.2 & 2.0 & 1.5 & .7 \\
\hline 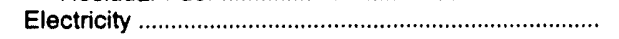 & 147.3 & 246.5 & 634.8 & 930.8 & $1,043.8$ & $1,121.0$ & $1,193.0$ & $1,218.0$ \\
\hline Industrial Sector & 340.3 & 690.4 & $1,525.7$ & $1,634.0$ & $1,359.7$ & $1,376.9$ & $1,412.4$ & $1,481.5$ \\
\hline 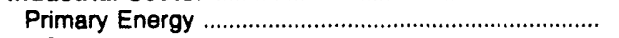 & 204.7 & 431.4 & $1,106.1$ & $1,068.1$ & 751.1 & 761.8 & 793.4 & 848.5 \\
\hline 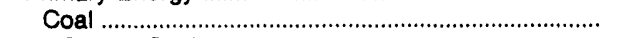 & 20.4 & 56.8 & 58.0 & 66.7 & 56.3 & 45.2 & 45.3 & 42.5 \\
\hline Coking Coal & 3.1 & 11.9 & 9.6 & 12.0 & 10.8 & 3.4 & $\cdot$ & - \\
\hline 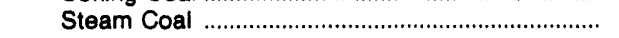 & 17.3 & 44.9 & 48.4 & 54.7 & 45.5 & 41.8 & 45.3 & 42.5 \\
\hline 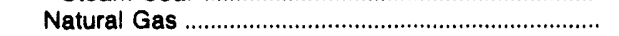 & 42.9 & 71.3 & 201.1 & 276.4 & 206.6 & 198.3 & 206.7 & 258.9 \\
\hline 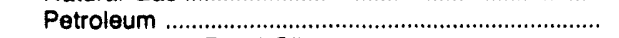 & 141.4 & 303.4 & 847.0 & 725.1 & 488.2 & 518.3 & 541.4 & 547.1 \\
\hline 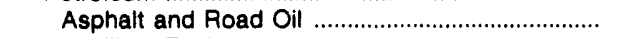 & 26.8 & 73.3 & 95.3 & 135.9 & 116.8 & 106.8 & 132.6 & 94.1 \\
\hline 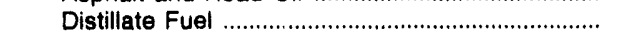 & 25.4 & 75.7 & 162.3 & 139.7 & 63.1 & 73.1 & 79.7 & 93.9 \\
\hline 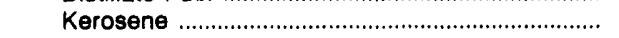 & .7 & 1.0 & 3.0 & 1.3 & .2 & .3 & .5 & 1.1 \\
\hline LPG & 5.6 & 15.8 & 63.4 & 62.8 & 49.4 & 45.1 & 63.9 & 58.9 \\
\hline 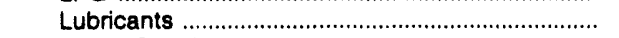 & 12.8 & 22.3 & 58.4 & 65.2 & 56.4 & 55.5 & 57.6 & 72.4 \\
\hline Motor Gasoline & 39.7 & 64.7 & 91.4 & 48.4 & 30.8 & 34.2 & 31.9 & 30.4 \\
\hline Residual Fuel & 4.4 & 7.5 & 7.5 & 17.3 & 6.4 & 9.2 & 7.9 & 8.1 \\
\hline Other & 26.1 & 43.0 & 365.8 & 254.6 & 165.1 & 194.1 & 167.3 & 188.2 \\
\hline Electricity & 135.6 & 259.0 & 419.6 & 565.9 & 608.6 & 615.0 & 619.0 & 633.0 \\
\hline $\begin{array}{l}\text { Transportation Sector } \\
\text { Primary Energy }\end{array}$ & $\begin{array}{l}878.6 \\
878.6\end{array}$ & $\begin{array}{l}1,699.5 \\
1,699.5\end{array}$ & $\begin{array}{l}3,548.5 \\
3,548.5\end{array}$ & $\begin{array}{l}3,478.4 \\
3,478.4\end{array}$ & $\begin{array}{l}2,671.9 \\
2,671.9\end{array}$ & $\begin{array}{l}2,984.0 \\
2,984.0\end{array}$ & $\begin{array}{l}3,065.3 \\
3,065.3\end{array}$ & $\begin{array}{l}3,405.8 \\
3,405.8\end{array}$ \\
\hline Coal & & & - & - & - & - & - & 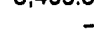 \\
\hline Petroleum & 878.5 & $1,689.5$ & $3,548.5$ & $3,478.4$ & $2,671.9$ & $2,984.0$ & $3,065.3$ & $3,405.8$ \\
\hline Aviation Gasoline & 2.0 & 3.2 & 7.4 & 6.8 & 6.9 & 5.1 & 6.1 & 8.4 \\
\hline 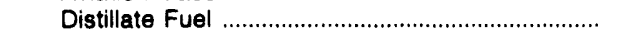 & 57.5 & 137.8 & 439.5 & 537.8 & 437.4 & 494.3 & 528.0 & 658.5 \\
\hline Jet Fuel & 34.1 & 98.2 & 229.5 & 196.6 & 149.9 & 174.6 & 160.8 & 179.6 \\
\hline LPG & .4 & .7 & 1.3 & 5.9 & 5.6 & 3.8 & 3.3 & 3.3 \\
\hline 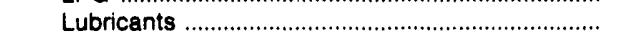 & 22.7 & 36.0 & 81.2 & 90.6 & 78.4 & 77.2 & 80.2 & 100.7 \\
\hline 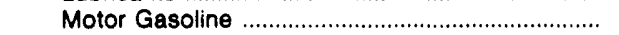 & 761.4 & $1,421.9$ & $2,786.6$ & $2,639.7$ & $1,992.9$ & $2,229.1$ & $2,286.0$ & $2,454.2$ \\
\hline 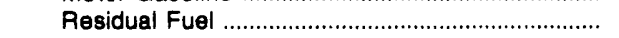 & .6 & 1.5 & 3.0 & 1.0 & .7 & - & 1.1 & 1.1 \\
\hline 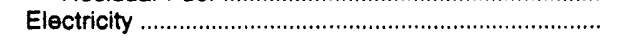 & - & - & - & - & - & - & - & - \\
\hline 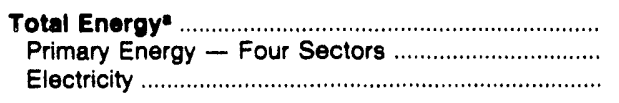 & $\begin{array}{r}1,932.6 \\
1,390.2 \\
542.4\end{array}$ & $\begin{array}{r}3,631.4 \\
2,657.2 \\
974.3\end{array}$ & $\begin{array}{l}7,639.9 \\
5,617.5 \\
2,022.4\end{array}$ & $\begin{array}{l}8,562.7 \\
5,850.7 \\
2,712.0\end{array}$ & $\begin{array}{l}7,579.5 \\
4,540.9 \\
3,038.6\end{array}$ & $\begin{array}{l}8,012.0 \\
4,779.4 \\
3,232.6\end{array}$ & $\begin{array}{l}8,364.8 \\
4,987.8 \\
3,377.0\end{array}$ & $\begin{array}{l}\mathbf{8 , 8 5 4 . 5} \\
5,458.5 \\
3,306.0\end{array}$ \\
\hline Electric Utillty Sector & 76.3 & 234.0 & 639.6 & 811.7 & 761.7 & 733.5 & 781.9 & $\begin{array}{l}762.9 \\
690.4\end{array}$ \\
\hline Natural Gas & $\begin{array}{l}58.6 \\
16.6\end{array}$ & $\begin{array}{r}205.4 \\
15.0\end{array}$ & $\begin{array}{r}586.4 \\
33.3\end{array}$ & $\begin{array}{r}728.4 \\
4.8\end{array}$ & $\begin{array}{r}690.4 \\
3.4\end{array}$ & $\begin{array}{r}689.2 \\
4.0\end{array}$ & $\begin{array}{r}700.4 \\
4.5\end{array}$ & $\begin{array}{r}690.4 \\
3.1\end{array}$ \\
\hline 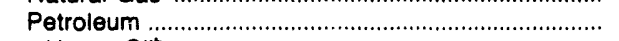 & 1.1 & 13.5 & 19.9 & 7.2 & 4.8 & 5.4 & 6.7 & 6.3 \\
\hline 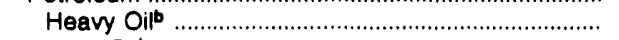 & .5 & 4.1 & 6 & .4 & .3 & .4 & .4 & .5 \\
\hline Light Oifb & 6 & 9.3 & 18.8 & 6.8 & 4.5 & 5.0 & $e .3$ & 5.7 \\
\hline 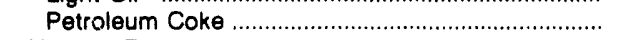 & - & .1 & .4 & 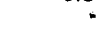 & - & - & 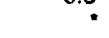 & .1 \\
\hline Nuclear Fuel & - & - & - & 71.3 & 63.1 & 34.9 & 70.3 & 63.1 \\
\hline Wood and Waste & - & - & - & - & - & - & - & - \\
\hline Primary Energy - Five Sectors ................................ & $1,466.5$ & $2,891.1$ & $6,257.1$ & $6,662.4$ & $5,302.5$ & $5,512.9$ & $5,769.7$ & $6,221.4$ \\
\hline
\end{tabular}

-There are no direct fuel costs for hydroelectric, geothermal, centralized solar, or wind energy. Wood and other biomass fuels are not included, except those consumed at electric utilities.

bHeavy oil includes Grade Nos. 4, 5, and 6, and residual fuel oils. Light oil includes Grade No. 2 heating oil, kerosene, and jet fuel.

-No consumption, including cases where adjustments were made. See "Consumption Adjustments for Process Fuel and Intermediate Products."

*Value less than 0.05 million dollars.

Note: Totals may not equal sum of components due to independent rounding.

Sources: Data sources, estimation procedures, and assumptions are describad in the "Documentation" section of this report. 


\begin{tabular}{|c|c|c|c|c|c|c|c|c|}
\hline Energy Source & 1970 & 1975 & 1980 & 1985 & 1986 & 1987 & 1988 & 1989 \\
\hline & \multicolumn{8}{|c|}{ Prices in Dollars per Mililon Btu } \\
\hline Coal & 0.22 & 0.33 & 0.53 & 0.75 & 0.74 & 0.68 & 0.57 & 0.61 \\
\hline 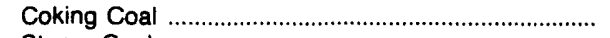 & - & - & - & - & - & - & - & - \\
\hline 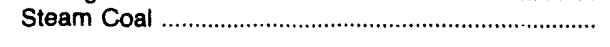 & .22 & .33 & .53 & .75 & .74 & .68 & .57 & 61 \\
\hline 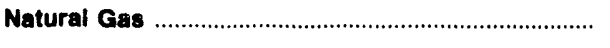 & .57 & 1.07 & 3.14 & 4.84 & 4.32 & 4.05 & 3.92 & 3.95 \\
\hline Petroleum & 1.89 & 3.41 & 7.13 & 7.28 & 5.97 & 6.38 & 6.36 & 6.72 \\
\hline 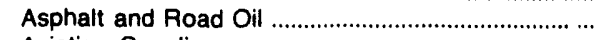 & .59 & 1.84 & 3.67 & 4.83 & 4.35 & 3.07 & 3.29 & 2.75 \\
\hline Aviation Gasoline & 2.17 & 3.45 & 9.02 & 9.99 & 8.41 & 7.55 & 7.41 & 8.28 \\
\hline Distillate Fuel & 1.14 & 2.58 & 6.59 & 6.39 & 5.68 & 6.40 & 6.32 & 6.51 \\
\hline Jet Fuel & .76 & 2.12 & 6.59 & 6.64 & 4.81 & 4.38 & 4.33 & 4.79 \\
\hline 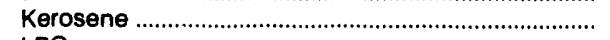 & .94 & 2.63 & - & 11.09 & 5.97 & 6.37 & 5.73 & 5.87 \\
\hline LPG & 2.10 & 3.62 & 6.49 & 7.61 & 6.62 & 6.15 & 6.15 & 7.22 \\
\hline 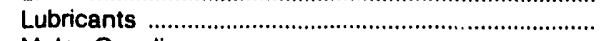 & 5.08 & 7.49 & 14.36 & 17.61 & 15.59 & 13.58 & 14.61 & 17.90 \\
\hline Motor Gasoline & 2.89 & 4.78 & 9.99 & 9.16 & 6.74 & 7.57 & 7.75 & 8.59 \\
\hline 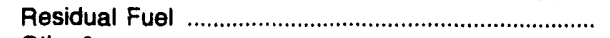 & .39 & 1.99 & 3.28 & 3.03 & 2.63 & 2.34 & 2.12 & 2.16 \\
\hline Other & .52 & 1.40 & 2.38 & 2.17 & 1.53 & 1.50 & 1.45 & 1.92 \\
\hline 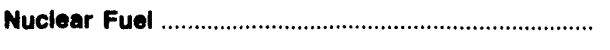 & - & - & - & - & - & - & - & - \\
\hline 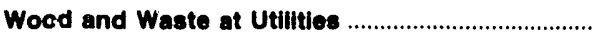 & .65 & .92 & 1.74 & .79 & .32 & .95 & .87 & .82 \\
\hline Primary Energy - Flve Sectors .................................. & 1.26 & 2.41 & 4.79 & 4.68 & 3.39 & 3.47 & 3.02 & 3.32 \\
\hline 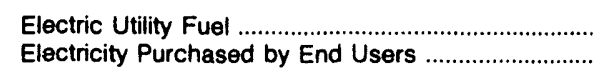 & $\begin{array}{r}.23 \\
2.88\end{array}$ & $\begin{array}{r}.34 \\
4.05\end{array}$ & $\begin{array}{r}.72 \\
5.80\end{array}$ & $\begin{array}{r}.72 \\
10.72\end{array}$ & $\begin{array}{r}.68 \\
11.18\end{array}$ & $\begin{array}{r}.66 \\
11.81\end{array}$ & $\begin{array}{r}.55 \\
12.09\end{array}$ & $\begin{array}{r}.59 \\
12.00\end{array}$ \\
\hline \multirow[t]{2}{*}{ 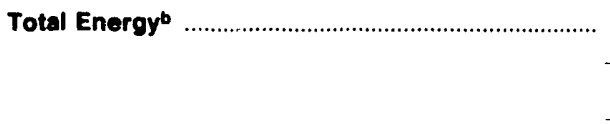 } & 1.56 & 2.78 & 6.02 & 7.42 & 6.72 & 7.04 & 7.03 & 7.13 \\
\hline & \multicolumn{8}{|c|}{ Expenditures In Millions of Dollars } \\
\hline Coal & 2.6 & 6.2 & 31.8 & 74.0 & 98.1 & 90.8 & 103.9 & 108.3 \\
\hline Coking Coal & - & - & - & - & - & - & - & - \\
\hline 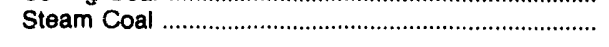 & 2.6 & 6.2 & 31.8 & 74.0 & 98.1 & 90.8 & 103.9 & 108.3 \\
\hline Natural Gas & 45.1 & 78.2 & 166.0 & 204.7 & 157.7 & 140.4 & 149.0 & 164.9 \\
\hline 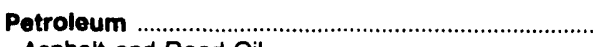 & 204.2 & 461.4 & $1,050.5$ & $1,075.4$ & 741.0 & 771.6 & 783.7 & 907.4 \\
\hline 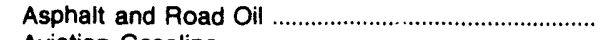 & 5.3 & 11.3 & 24.9 & 46.9 & 57.4 & 33.5 & 32.2 & 31.9 \\
\hline 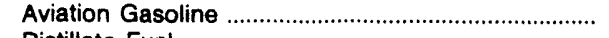 & .5 & 1.4 & 7.3 & 4.6 & 4.4 & 3.1 & 4.0 & 4.0 \\
\hline Distillate Fuel & 31.9 & 114.2 & 288.2 & 421.3 & 231.9 & 244.3 & 232.4 & 291.2 \\
\hline 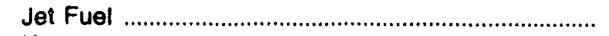 & 2.7 & 9.7 & 34.1 & 25.2 & 23.3 & 17.7 & 19.6 & 20.0 \\
\hline 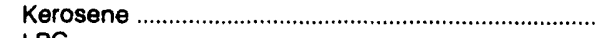 & 2.0 & 1.8 & - & .7 & .6 & .2 & .1 & .1 \\
\hline LPG & 9.9 & 17.1 & 41.9 & 43.8 & 34.1 & 34.9 & 31.0 & 43.2 \\
\hline Lubricants & 6.2 & 9.4 & 21.6 & 24.0 & 20.8 & 20.5 & 21.3 & 26.7 \\
\hline 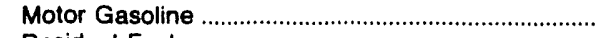 & 140.7 & 266.6 & 546.8 & 490.2 & 359.8 & 407.0 & 425.5 & 464.8 \\
\hline 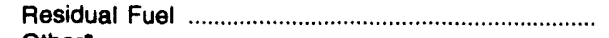 & .7 & 17.6 & 68.3 & 11.5 & 3.0 & 1.7 & 9.0 & 13.8 \\
\hline Other" & 4.4 & 12.3 & 17.4 & 7.1 & 5.5 & 8.6 & 8.7 & 11.6 \\
\hline 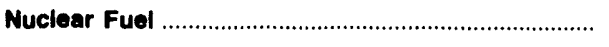 & - & - & - & - & - & - & - & - \\
\hline 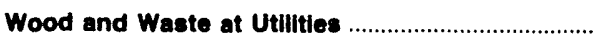 & .5 & .1 & .3 & .5 & .2 & .5 & .5 & .6 \\
\hline Primary Energy - Flve Sectors ................................. & 252.4 & 545.8 & $1,248.6$ & $1,354.7$ & 996.9 & $1,003.2$ & $1,037.1$ & $1,181.2$ \\
\hline $\begin{array}{l}\text { Electric Utility Fuel } \\
\text { Electricity Purchased by End Users }\end{array}$ & $\begin{array}{l}-3.4 \\
84.1\end{array}$ & $\begin{array}{r}-6.4 \\
119.8\end{array}$ & -44.3 & $\begin{array}{r}-69.2 \\
488.6\end{array}$ & $\begin{array}{r}-87.0 \\
515.8\end{array}$ & $\begin{array}{l}-85.9 \\
488.8\end{array}$ & $\begin{array}{l}-99.1 \\
521.6\end{array}$ & $\begin{array}{r}-102.8 \\
519.2\end{array}$ \\
\hline 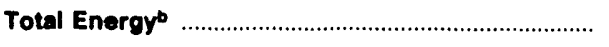 & 333.2 & 659.3 & $1,412.0$ & $1,774.1$ & $1,425.7$ & $1,406.2$ & $1,459.6$ & $1,597.6$ \\
\hline
\end{tabular}

- Includes petroleum coke used at electric utilities.

-There are no direct fuel costs for hydroelectric, geothermal, centralized solar, or wind energy. Wood and other biomass fuels are not included, except those consumed at electric utilities.

- No consumptiori, including cases where adjustments were made. See "Consumption Adjustments for Process Fuel and Intermediate Products." Note: Expenditure totals may not equal sum of components due to independent rounding.

Sources: Data sources, estimation procedures, and assumptions are described in the "Documentation" section of this report. 


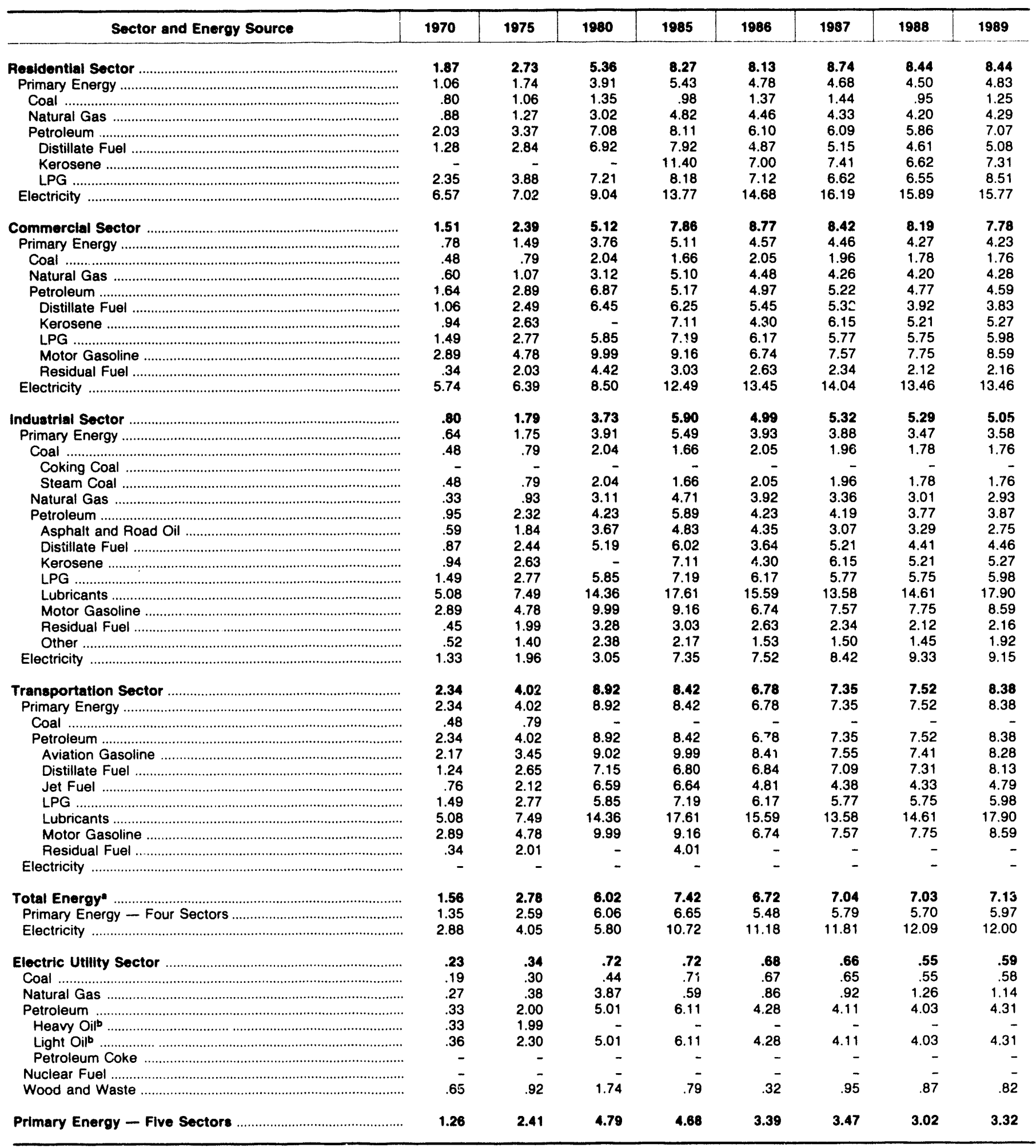

There are no direct fuel costs for hydroelectric, geothermal, centralized solar, or wind energy. Wood and other biomass fuels are not inclucied, except those consumed at electric utilities.

beavy oil includes Grade Nos. 4, 5, and 6, and residual fuel oils. Light oil includes Grade No. 2 heating oil, kerosene, and jet fuel.

- No consumption, including cases where adjustments were made. See "Consumption Adjustments for Process Fuel and Intermediate Products."

Sources: Data sources, estimation procedures, and assumptions are described in the "Documentation" section of this report. 


\section{Energy Expenditure Estimates by Sector, Montana \\ O 1970, 1975, 1980, and 1985-1989 \\ N (Million Dollars)}

\begin{tabular}{|c|c|c|c|c|c|c|c|c|}
\hline Sector and Energy Source & 1970 & 1975 & 1980 & 1965 & 1986 & 1987 & 1988 & 1989 \\
\hline 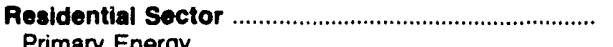 & 66.7 & 106.4 & $\begin{aligned} 187.8 \\
08.0\end{aligned}$ & 289.5 & 263.6 & 265.4 & 274.5 & 301.9 \\
\hline Primary Energy & $\begin{array}{r}32.3 \\
.1\end{array}$ & $\begin{array}{r}55.1 \\
.1\end{array}$ & $\begin{array}{r}98.0 \\
.1\end{array}$ & 129.7. & $\begin{array}{r}102.7 \\
.2\end{array}$ & $\begin{array}{r}92.0 \\
.1\end{array}$ & $\begin{array}{r}95.5 \\
.1\end{array}$ & $\begin{array}{r}115.9 \\
.5\end{array}$ \\
\hline 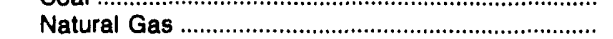 & 22.5 & 31.2 & 58.9 & 3.2 & 74.8 & 67.7 & 72.7 & 79.5 \\
\hline Petroleum & 9.7 & 23.8 & 38.9 & 36.4 & 27.7 & 24.2 & 22.8 & 35.9 \\
\hline 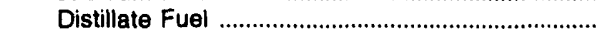 & 1.9 & 9.7 & 17.0 & 15.9 & 10.0 & 7.4 & 6.3 & 10.8 \\
\hline 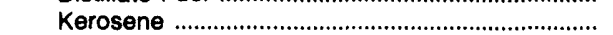 & - & - & - & .7 & .5 & & & \\
\hline 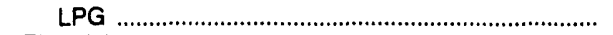 & 7.9 & 14.0 & 21.9 & 19.8 & 17.3 & 16.7 & 16.5 & 25.1 \\
\hline 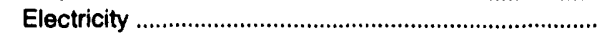 & 34.4 & 51.3 & 89.9 & 169.8 & 161.0 & 173.4 & 179.0 & 186.0 \\
\hline Commercial Soctor & 41.3 & 73.1 & 127.1 & 305.5 & 282.0 & 207.0 & 209.7 & 211.8 \\
\hline 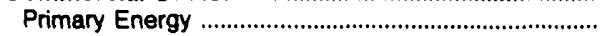 & 18.0 & 37.2 & 66.4 & 124.7 & 77.5 & 64.2 & 62.7 & 70.9 \\
\hline 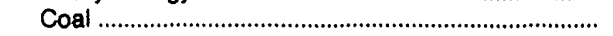 & .1 & .1 & .4 & .2 & .5 & .2 & .2 & 1.2 \\
\hline 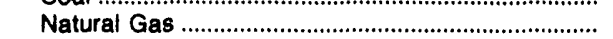 & 11.5 & 20.4 & 44.9 & 75.5 & 56.1 & 47.7 & 51.8 & 57.3 \\
\hline 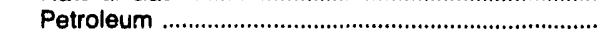 & 6.5 & 16.7 & 21.2 & 49.0 & 20.8 & 16.3 & 10.7 & 12.4 \\
\hline 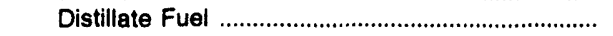 & 1.7 & 9.7 & 13.0 & 31.4 & 12.8 & 9.5 & 4.5 & 4.5 \\
\hline 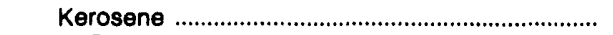 & .5 & .8 & - & & .1 & * & * & \\
\hline 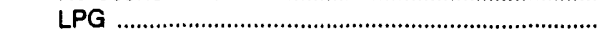 & .9 & 1.8 & 3.1 & 3.1 & 2.6 & 2.6 & 2.6 & 3.1 \\
\hline 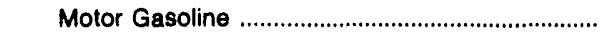 & 3.3 & 4.4 & 4.8 & 3.5 & 2.7 & 3.2 & 3.1 & 3.5 \\
\hline Residual Fuel ............................................... & & & .2 & 11.1 & 2.6 & 1.1 & .5 & 1.3 \\
\hline 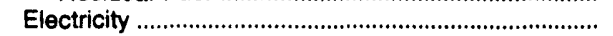 & 23.3 & 35.8 & 60.7 & 180.8 & 204.5 & 142.7 & 147.0 & 141.0 \\
\hline Industrial Sector & 67.2 & 156.8 & 329.7 & 495.4 & 336.8 & 343.3 & 356.6 & 402.6 \\
\hline Primary Energy ………………………………....... & 40.7 & 124.1 & 272.6 & 357.4 & 186.6 & 170.6 & 161.1 & 210.4 \\
\hline 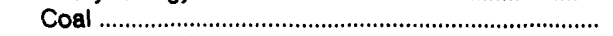 & .3 & .8 & 6.0 & 6.8 & 11.7 & 6.7 & 7.0 & 6.4 \\
\hline 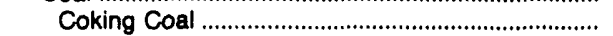 & - & - & - & - & - & - & - & - \\
\hline 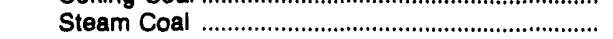 & .3 & .8 & 6.0 & 6.8 & 11.7 & 6.7 & 7.0 & 6.4 \\
\hline 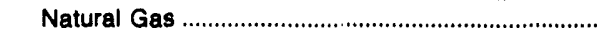 & 10.4 & 26.1 & 45.2 & 35.7 & 26.3 & 24.4 & 24.1 & 27.6 \\
\hline 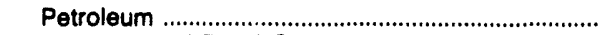 & 30.0 & 97.2 & 221.4 & 315.0 & 148.6 & 139.5 & 129.9 & 176.4 \\
\hline Asphalt and Road Oll ................................................ & 5.3 & 11.3 & 24.9 & 46.8 & 57.4 & 33.5 & 32.2 & 31.9 \\
\hline Distillate Fuel ............................................... & 6.5 & 35.5 & 58.2 & 203.3 & 45.1 & 54.7 & 41.6 & 72.3 \\
\hline 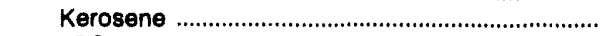 & 1.5 & 1.0 & - & & $\cdot$ & .2 & .1 & \\
\hline 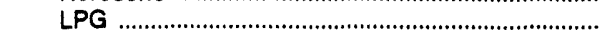 & .9 & .8 & 15.9 & 19.7 & 13.2 & 14.9 & 11.2 & 14.1 \\
\hline 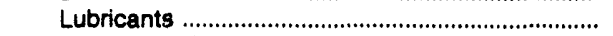 & 1.4 & 2.1 & 4.5 & 5.0 & 4.3 & 4.2 & 4.4 & 5.5 \\
\hline 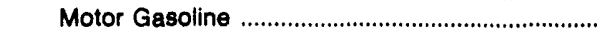 & 9.6 & 19.4 & 32.5 & 32.6 & 22.6 & 22.8 & 23.4 & 28.4 \\
\hline 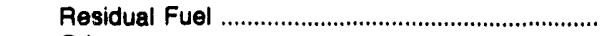 & .4 & 14.9 & 68.1 & .4 & .4 & .6 & 8.4 & 12.6 \\
\hline Other & 4.4 & 12.3 & 17.4 & 7.1 & 5.5 & 8.6 & 8.7 & 11.6 \\
\hline 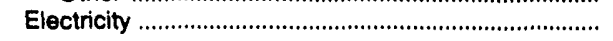 & 26.5 & 32.6 & 57.1 & 138.0 & 150.2 & 172.7 & 195.6 & 192.2 \\
\hline $\begin{array}{c}\text { Traneportation Sector } \\
\text { Primary Energy }\end{array}$ & $\begin{array}{l}157.0 \\
157.9\end{array}$ & $\begin{array}{l}323.1 \\
323.1\end{array}$ & $\begin{array}{l}767.3 \\
767.3\end{array}$ & $\begin{array}{l}673.7 \\
6737\end{array}$ & $\begin{array}{l}543.3 \\
543.3\end{array}$ & $\begin{array}{l}590.5 \\
590.5\end{array}$ & $\begin{array}{l}618.8 \\
618.8\end{array}$ & $\begin{array}{l}681.2 \\
6812\end{array}$ \\
\hline Coal lo & & 323.1 & & 0.1 & 040.0 & 000. & $\begin{array}{l}618.8 \\
-\end{array}$ & 681.2 \\
\hline 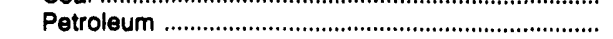 & 157.9 & 323.1 & 767.3 & 673.7 & 543.3 & 590.5 & 618.8 & 681.2 \\
\hline 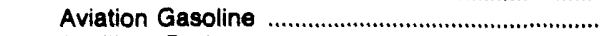 & .5 & 1.4 & 7.3 & 4.6 & 4.4 & 3.1 & 4.0 & 4.0 \\
\hline Distillate Fuel ............................................ & 21.9 & 59.2 & 198.3 & 169.4 & 163.5 & 171.7 & 178.4 & 202.0 \\
\hline 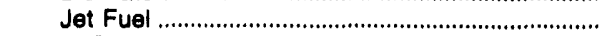 & 2.7 & 9.7 & 34.1 & 25.2 & 23.3 & 17.7 & 19.6 & 20.0 \\
\hline LPG & .2 & .5 & 1.0 & 1.2 & 1.0 & .7 & .8 & 1.0 \\
\hline 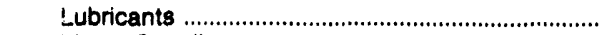 & 4.7 & 7.3 & 17.1 & 19.1 & 16.5 & 16.3 & 16.9 & 21.2 \\
\hline 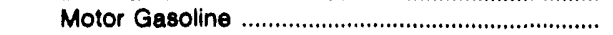 & 127.7 & 242.8 & 508.5 & 454.2 & 334.6 & 381.1 & 388.9 & 432.9 \\
\hline 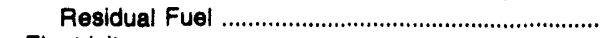 & .3 & 2.0 & - & • & - & - & - & - \\
\hline 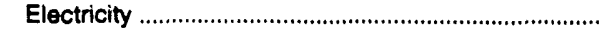 & - & - & - & - & - & - & - & - \\
\hline Total Energy & 333.2 & 659.3 & & & $1,425.7$ & & & $1,597.6$ \\
\hline 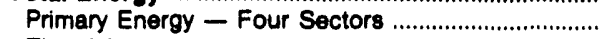 & 249.0 & 539.5 & $1,204.3$ & $1,285.4$ & 910.0 & 817.3 & 938.0 & $1,078.4$ \\
\hline 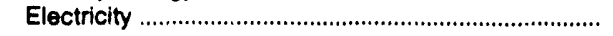 & 84.1 & 118.8 & 207.7 & 488.6 & 515.8 & 488.8 & 521.6 & 519.2 \\
\hline Electrlc Utility Sector & 3.4 & 6.4 & 44.3 & 60.2 & 87.0 & 85.9 & 99.1 & 102.8 \\
\hline Coal & 2.2 & 5.2 & 25.3 & 67.1 & 85.7 & 83.8 & 96.7 & 100.2 \\
\hline 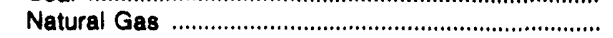 & .7 & .5 & 17.0 & .3 & .4 & .5 & .4 & .5 \\
\hline Petroleum & .1 & .7 & 1.7 & 1.4 & .6 & 1.1 & 1.5 & 1.5 \\
\hline 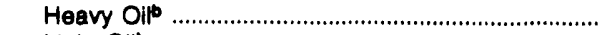 & .1 & .7 & - & - & - & - & - & \\
\hline 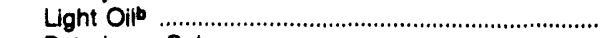 & - & $\cdot$ & 1.7 & 1.4 & .6 & 1.1 & 1.5 & 1.5 \\
\hline 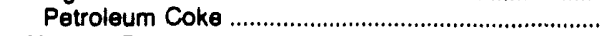 & - & - & - & - & - & - & - & \\
\hline 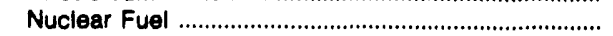 & - & - & - & - & - & - & - & - \\
\hline Wood and Waste & .5 & .1 & 3 & .5 & 2 & .5 & .5 & 6 \\
\hline Primary Energy - Five Snctore .................................... & 252.4 & 546.0 & $1,248.6$ & $1,354.7$ & 908.9 & $1,003.2$ & $1,037.1$ & $1,181.2$ \\
\hline
\end{tabular}

-There are no direct fuel costs for hydroelectric, geothermal, centralized soler, or wind energy. Wood and other biomass fuels are not included, except those consumed at electric utilities.

bHeavy oil includes Grade Nos. 4, 5, and 6, and residual fuel oils. Light oll includes Grecis No. 2 heating oil, kerosene, and jet fuel.

-No consumption, including cases where adjustments were made. Soe "Consumption Adjustments for Process Fuel and Intermediate Products."

- Value less than 0.05 million dollars.

Note: Totals may not equal sum of components due to independent rounding.

Sources: Data sources, estimation procedures, and assumptions are described in the "Documentation" section of this report. 


\begin{tabular}{|c|c|c|c|c|c|c|c|c|}
\hline Energy Source & 1970 & 1975 & 1980 & 1985 & 1986 & 1987 & 1988 & 1989 \\
\hline & \multicolumn{8}{|c|}{ Prices in Dollars per Million Btu } \\
\hline Coal & 0.32 & 0.86 & 1.27 & 1.17 & 1.08 & 0.98 & 0.87 & 0.85 \\
\hline Coking Coal . & - & - & - & - & - & - & - & - \\
\hline 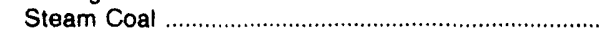 & .32 & .86 & 1.27 & 1.17 & 1.08 & .98 & .87 & .85 \\
\hline Natural Gas & .50 & .90 & 2.40 & 4.43 & 4.08 & 3.71 & 3.81 & 3.84 \\
\hline Petroleum n. & 2.12 & 3.74 & 8.26 & 8.29 & 6.46 & 6.66 & 6.25 & 7.27 \\
\hline Asphalt and Road Oil & .71 & 2.07 & 3.89 & 5.04 & 3.93 & 3.85 & 3.54 & 3.09 \\
\hline 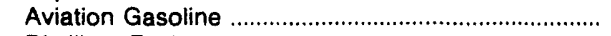 & 2.17 & 3.45 & 9.02 & 9.99 & 8.41 & 7.55 & 7.41 & 8.28 \\
\hline Distillate Fuel & .95 & 2.38 & 6.24 & 6.86 & 5.53 & 5.87 & 5.37 & 6.37 \\
\hline Jet Fuel & .75 & 2.09 & 6.47 & 6.19 & 4.43 & 4.38 & 4.14 & 4.54 \\
\hline 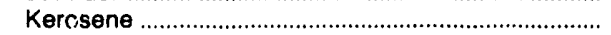 & 1.18 & 2.63 & 5.56 & 9.00 & 4.37 & 4.55 & 4.08 & 5.74 \\
\hline LPG & 1.56 & 3.07 & 5.70 & 7.00 & 7.20 & 6.88 & 6.93 & 7.37 \\
\hline 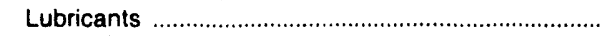 & 5.08 & 7.49 & 14.36 & 17.61 & 15.59 & 13.58 & 14.61 & 17.90 \\
\hline 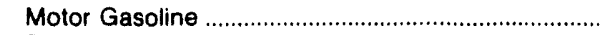 & 3.03 & 4.76 & 10.06 & 9.67 & 7.28 & 7.58 & 7.22 & 8.42 \\
\hline Residual Fuel & .48 & 1.74 & 3.21 & 4.28 & 2.72 & 2.53 & 2.17 & 2.04 \\
\hline Other & .51 & 1.40 & 2.44 & - & 3.95 & 4.85 & 4.00 & 4.86 \\
\hline 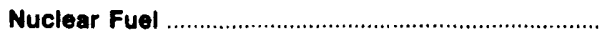 & - & .17 & .44 & .65 & .64 & .64 & .63 & .65 \\
\hline 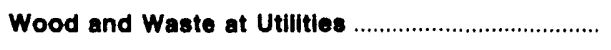 & - & - & - & - & - & - & - & - \\
\hline Primary Energy - Flve Sectors ........................... & 1.21 & 1.95 & 4.18 & 4.87 & 3.79 & 3.71 & 3.63 & 3.94 \\
\hline $\begin{array}{l}\text { Electric Utility Fuel } \\
\text { Electricity Purchased by End Users }\end{array}$ & $\begin{array}{r}.30 \\
5.12\end{array}$ & $\begin{array}{r}.50 \\
6.89\end{array}$ & $\begin{array}{r}1.00 \\
11.76\end{array}$ & $\begin{array}{r}1.01 \\
15.70\end{array}$ & $\begin{array}{r}.89 \\
15.62\end{array}$ & $\begin{array}{r}.82 \\
15.65\end{array}$ & $\begin{array}{r}.79 \\
15.52\end{array}$ & $\begin{array}{r}.78 \\
16.22\end{array}$ \\
\hline \multirow[t]{2}{*}{ Total Enorgy } & 1.72 & 2.89 & 6.51 & 8.06 & 7.12 & 7.13 & 6.87 & 7.61 \\
\hline & \multicolumn{8}{|c|}{ Expenditures in Millions of Dollare } \\
\hline Cool & $\$ 0.6$ & 28.4 & 119.4 & 135.5 & 118.5 & 114.1 & 121.7 & 112.7 \\
\hline 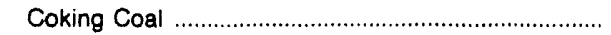 & - & - & & & & & - & - \\
\hline 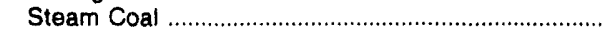 & 91.6 & 28.4 & 119.4 & 135.5 & 118.5 & 114.1 & 121.7 & 112.7 \\
\hline 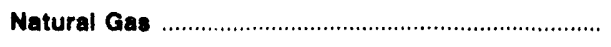 & 104.1 & 184.3 & 354.1 & 523.7 & 408.4 & 383.6 & 439.0 & 438.6 \\
\hline Potroleum & 404.8 & 773.8 & $1,561.0$ & $1,614.4$ & $1,278.7$ & 1,363.2 & $1,393.0$ & $1,532.0$ \\
\hline 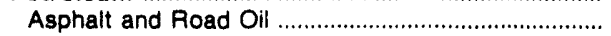 & 5.3 & 10.3 & 18.6 & 15.8 & 24.9 & 31.7 & 29.7 & 23.2 \\
\hline 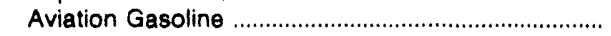 & 2.2 & 2.5 & 8.7 & 4.8 & 5.0 & 3.4 & 3.6 & 3.9 \\
\hline 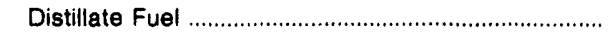 & 41.4 & 117.9 & 332.7 & 484.8 & 388.1 & 420.7 & 437.4 & 461.0 \\
\hline Jet Fuel & 7.3 & 19.3 & 56.2 & 45.9 & 32.8 & 32.8 & 34.2 & 37.1 \\
\hline 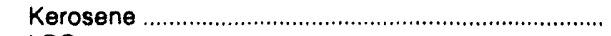 & 3.9 & 8.2 & 2.0 & 5.7 & 4.2 & 2.4 & 1.6 & .8 \\
\hline 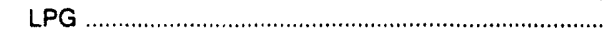 & 33.2 & 65.4 & 94.2 & 106.1 & 101.8 & 119.7 & 136.0 & 139.2 \\
\hline 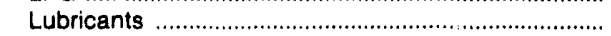 & 14.8 & 22.3 & 33.9 & 37.8 & 32.7 & 32.2 & 33.4 & 42.0 \\
\hline 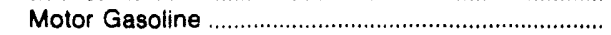 & 294.4 & 516.3 & $1,008.8$ & 901.2 & 679.4 & 710.3 & 708.3 & 814.2 \\
\hline Residual Fuel . & 2.3 & 11.2 & 4.3 & 2.0 & 5.0 & 4.4 & 6.0 & 5.9 \\
\hline Other" & .1 & .3 & .5 & - & 4.7 & 5.5 & 4.8 & 5.8 \\
\hline 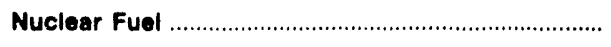 & - & 11.0 & 27.7 & 28.3 & 52.8 & 59.0 & 46.6 & 56.3 \\
\hline Wood and Waste at Utilties & - & - & - & - & - & - & - & - \\
\hline Primary Energy - Five Sectors & 518.6 & 997.4 & $2,062.2$ & $2,302.9$ & $1,858.4$ & $1,019.8$ & $2,000.2$ & $2,138.6$ \\
\hline $\begin{array}{l}\text { Electric Utility Fuel } \\
\text { Electricity Purchased by End Users }\end{array}$ & $\begin{array}{l}-22.3 \\
170.3\end{array}$ & $\begin{array}{r}-68.1 \\
271.2\end{array}$ & $\begin{array}{r}-164.7 \\
550.6\end{array}$ & $\begin{array}{r}-158.7 \\
841.2\end{array}$ & $\begin{array}{r}-167.8 \\
846.3\end{array}$ & $\begin{array}{r}-169.4 \\
864.2\end{array}$ & $\begin{array}{r}-166.5 \\
914.0\end{array}$ & $\begin{array}{r}-168.8 \\
972.0\end{array}$ \\
\hline (2) & 668.6 & $1,200.5$ & $2,448.1$ & $2,985.4$ & $2,537.0$ & $2,614.5$ & $2,747.7$ & $2,941.7$ \\
\hline
\end{tabular}

- Includes petroleum coke used at electric utilities.

- There are no direct fuel costs for hydroelectric, geothermal, centralized solar, or wind energy. Wood and other biomass fuels are not included, except those consumed at electric utilities.

- No consumption, including cases where adjustments were made. See "Consumption Adjustments for Process Fuel and Intermediate Products."

Note: Expenditure totals may not equal sum of components due to independent rounding.

Sources: Data sources, estimation procedures, and assumptions are described in the "Documentation" section of this report. 


\section{N Energy Price Estimates by Sector, Nebraska \\ E $1970,1975,1980$, and 1985-1989 \\ B (Dollars per Million Btu)}

\begin{tabular}{|c|c|c|c|c|c|c|c|c|}
\hline Sector and Energy Source & 1970 & 1975 & 1980 & 1985 & 1986 & 1987 & 1988 & 1989 \\
\hline $\begin{array}{l}\text { Resldential Sector } \\
\text { Primary Energy } \\
\text { Coal . } \\
\text { Natural Gas } \\
\text { Petroleum } \\
\text { Distillate Fuel } \\
\text { Kerosene } \\
\text { LPG } \\
\text { Electricity }\end{array}$ & $\begin{array}{r}1.84 \\
1.04 \\
1.08 \\
.84 \\
1.69 \\
1.19 \\
1.39 \\
1.78 \\
6.21\end{array}$ & $\begin{array}{l}2.95 \\
1.74 \\
2.16 \\
1.29 \\
3.39 \\
2.62 \\
2.74 \\
3.57 \\
8.13\end{array}$ & $\begin{array}{r}5.83 \\
3.32 \\
3.60 \\
2.78 \\
6.84 \\
6.85 \\
7.55 \\
6.82 \\
13.22\end{array}$ & $\begin{array}{r}8.78 \\
5.45 \\
2.76 \\
5.10 \\
7.42 \\
7.92 \\
9.74 \\
7.12 \\
17.30\end{array}$ & $\begin{array}{r}8.55 \\
4.70 \\
2.40 \\
4.62 \\
5.24 \\
4.88 \\
6.00 \\
5.35 \\
17.27\end{array}$ & $\begin{array}{r}8.73 \\
4.55 \\
2.43 \\
4.43 \\
5.22 \\
4.50 \\
5.54 \\
5.35 \\
17.52\end{array}$ & $\begin{array}{r}8.63 \\
4.65 \\
2.49 \\
4.53 \\
5.31 \\
4.27 \\
5.25 \\
5.48 \\
17.42\end{array}$ & $\begin{array}{r}8.96 \\
4.97 \\
2.42 \\
4.54 \\
7.37 \\
5.37 \\
6.61 \\
7.82 \\
18.05\end{array}$ \\
\hline $\begin{array}{l}\text { Commorcial Soctor } \\
\text { Primary Energy } \\
\text { Coal . } \\
\text { Natural Gas } \\
\text { Petroleum } \\
\text { Distillate Fuel } \\
\text { Kerosene } \\
\text { LPG } \\
\text { Motor Gasoline } \\
\text { Residual Fuel } \\
\text { Electricity }\end{array}$ & $\begin{array}{r}1.36 \\
.59 \\
.16 \\
.52 \\
1.09 \\
1.03 \\
.79 \\
1.09 \\
3.03 \\
.50 \\
4.87\end{array}$ & $\begin{array}{r}2.36 \\
1.17 \\
.81 \\
1.00 \\
2.60 \\
2.45 \\
2.39 \\
2.46 \\
4.76 \\
1.75 \\
6.96\end{array}$ & $\begin{array}{r}5.01 \\
2.62 \\
1.69 \\
2.33 \\
6.81 \\
6.49 \\
5.17 \\
5.19 \\
10.06 \\
3.22 \\
12.86\end{array}$ & $\begin{array}{l}8.33 \\
4.71 \\
2.46 \\
4.29 \\
7.20 \\
6.79 \\
8.12 \\
6.92 \\
9.67 \\
- \\
16.78\end{array}$ & $\begin{array}{r}8.22 \\
4.09 \\
1.70 \\
3.95 \\
5.47 \\
3.49 \\
4.17 \\
8.25 \\
7.28 \\
- \\
16.55\end{array}$ & $\begin{array}{r}8.31 \\
3.93 \\
1.63 \\
3.76 \\
5.49 \\
3.54 \\
4.41 \\
7.76 \\
7.58 \\
2.60 \\
16.43\end{array}$ & $\begin{array}{r}8.03 \\
3.92 \\
1.56 \\
3.81 \\
5.32 \\
3.04 \\
3.77 \\
7.73 \\
7.22 \\
2.13 \\
16.22\end{array}$ & $\begin{array}{r}8.55 \\
3.97 \\
1.47 \\
3.82 \\
5.68 \\
4.03 \\
5.22 \\
7.13 \\
8.42 \\
2.02 \\
16.93\end{array}$ \\
\hline $\begin{array}{l}\text { Industrial Sector } \\
\text { Primary Energy } \\
\text { Coal } \\
\text { Coking Coal } \\
\text { Steam Coal } \\
\text { Natural Gas } \\
\text { Petroleum } \\
\text { Asphalt and Road Oil } \\
\text { Distillate Fuel } \\
\text { Kerosene } \\
\text { LPG }\end{array}$ & $\begin{array}{r}.88 \\
.69 \\
.16 \\
. \\
.16 \\
.32 \\
1.26 \\
.71 \\
.73 \\
.79 \\
1.09 \\
5.08 \\
3.03 \\
.40 \\
.51 \\
3.42\end{array}$ & $\begin{array}{r}1.77 \\
1.47 \\
.81 \\
.81 \\
.69 \\
2.92 \\
2.07 \\
2.25 \\
2.39 \\
2.46 \\
7.49 \\
4.76 \\
1.74 \\
1.40 \\
4.96\end{array}$ & $\begin{array}{r}4.48 \\
3.85 \\
1.69 \\
1 . \\
1.69 \\
2.21 \\
5.84 \\
3.89 \\
4.94 \\
5.17 \\
5.19 \\
14.36 \\
10.06 \\
3.13 \\
2.44 \\
8.71\end{array}$ & $\begin{array}{r}6.41 \\
5.61 \\
2.46 \\
- \\
2.46 \\
3.67 \\
7.37 \\
5.04 \\
7.09 \\
8.12 \\
6.92 \\
17.61 \\
9.67 \\
4.28 \\
11.47\end{array}$ & $\begin{array}{r}5.27 \\
4.22 \\
1.70 \\
-\overline{0} \\
1.70 \\
3.28 \\
4.93 \\
3.93 \\
3.64 \\
4.17 \\
8.25 \\
15.59 \\
7.28 \\
2.81 \\
3.95 \\
11.42\end{array}$ & $\begin{array}{r}5.06 \\
4.10 \\
1.63 \\
- \\
1.63 \\
2.81 \\
5.14 \\
3.85 \\
3.85 \\
4.41 \\
7.76 \\
13.58 \\
7.58 \\
2.60 \\
4.85 \\
11.34\end{array}$ & $\begin{array}{r}4.87 \\
3.88 \\
1.56 \\
- \\
1.56 \\
2.90 \\
4.67 \\
3.54 \\
3.30 \\
3.77 \\
7.73 \\
14.61 \\
7.22 \\
2.13 \\
4.00 \\
11.28\end{array}$ & $\begin{array}{r}5.43 \\
4.23 \\
1.47 \\
1.47 \\
1.47 \\
2.96 \\
5.28 \\
3.09 \\
4.56 \\
5.22 \\
7.13 \\
17.90 \\
8.42 \\
2.02 \\
4.86 \\
12.34\end{array}$ \\
\hline 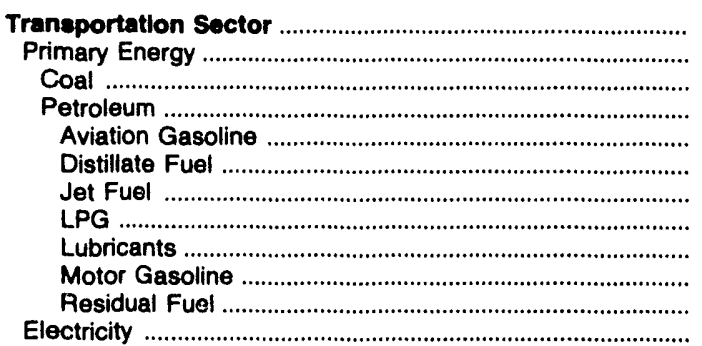 & $\begin{array}{r}2.51 \\
2.51 \\
.16 \\
2.51 \\
2.17 \\
1.14 \\
.75 \\
1.09 \\
5.08 \\
3.03 \\
.50\end{array}$ & $\begin{array}{r}4.15 \\
4.15 \\
.81 \\
4.15 \\
3.45 \\
2.50 \\
2.09 \\
2.46 \\
7.49 \\
4.76 \\
1.74\end{array}$ & $\begin{array}{r}9.20 \\
9.20 \\
- \\
9.20 \\
9.02 \\
7.06 \\
6.47 \\
5.19 \\
14.36 \\
10.06 \\
- \\
-\end{array}$ & $\begin{array}{r}8.70 \\
8.70 \\
- \\
8.70 \\
9.99 \\
6.68 \\
6.19 \\
6.92 \\
17.61 \\
9.67 \\
-\end{array}$ & $\begin{array}{r}7.10 \\
7.10 \\
- \\
7.10 \\
8.41 \\
6.79 \\
4.43 \\
8.25 \\
15.59 \\
7.28 \\
2.32 \\
\end{array}$ & $\begin{array}{r}7.32 \\
7.32 \\
- \\
7.32 \\
7.55 \\
7.02 \\
4.38 \\
7.76 \\
13.58 \\
7.58 \\
2.29 \\
-\end{array}$ & $\begin{array}{r}6.90 \\
6.90 \\
- \\
6.90 \\
7.41 \\
6.47 \\
4.14 \\
7.73 \\
14.61 \\
7.22 \\
-\end{array}$ & $\begin{array}{r}8.01 \\
8.01 \\
- \\
8.01 \\
8.28 \\
7.39 \\
4.54 \\
7.13 \\
17.90 \\
8.42 \\
- \\
-\end{array}$ \\
\hline $\begin{array}{l}\text { Total Energye . . Four Sectors } \\
\text { Primary Energy - Fon } \\
\text { Electricity . }\end{array}$ & $\begin{array}{l}1.72 \\
1.40 \\
5.12\end{array}$ & $\begin{array}{l}2.89 \\
2.48 \\
6.89\end{array}$ & $\begin{array}{r}6.51 \\
5.77 \\
11.76\end{array}$ & $\begin{array}{r}8.06 \\
6.77 \\
15.70\end{array}$ & $\begin{array}{r}7.12 \\
5.60 \\
15.62\end{array}$ & $\begin{array}{r}7.13 \\
5.62 \\
15.65\end{array}$ & $\begin{array}{r}6.87 \\
5.38 \\
15.52\end{array}$ & $\begin{array}{r}7.61 \\
6.03 \\
16.22\end{array}$ \\
\hline $\begin{array}{l}\text { Electric Utllity Soctor } \\
\text { Coal } \\
\text { Natural Gas } \\
\text { Petroleum } \\
\text { Heavy Oib } \\
\text { Light Oilb }\end{array}$ & $\begin{array}{l}.30 \\
.35 \\
.27 \\
.54 \\
.49 \\
.63\end{array}$ & $\begin{array}{r}.50 \\
.87 \\
.63 \\
1.77 \\
1.73 \\
1.85\end{array}$ & $\begin{array}{l}1.00 \\
1.24 \\
1.82 \\
4.14 \\
3.21 \\
6.19\end{array}$ & $\begin{array}{r}1.01 \\
1.11 \\
3.58 \\
5.89 \\
5.89\end{array}$ & $\begin{array}{r}.89 \\
1.04 \\
3.21 \\
3.05 \\
2.32 \\
3.87\end{array}$ & $\begin{array}{r}.82 \\
.95 \\
2.56 \\
2.87 \\
2.29 \\
3.98\end{array}$ & $\begin{array}{r}.79 \\
.84 \\
2.69 \\
2.83 \\
2.34 \\
3.47\end{array}$ & $\begin{array}{r}.78 \\
.83 \\
2.36 \\
3.09 \\
2.15 \\
4.35\end{array}$ \\
\hline $\begin{array}{l}\text { Petroleum Coke } \\
\text { Nuclear Fuel } \\
\text { Wood and Waste }\end{array}$ & $\overline{-}$ &.$\overline{-17}$ & 44 & .65 & .64 & .64 & 63 & .65 \\
\hline Primary Energy - Five Sectore & 1.21 & 1.95 & 4.18 & 4.87 & 3.79 & 3.71 & 3.63 & 3.94 \\
\hline
\end{tabular}

-There are no direct fuel costs for hydroelectric, geothermal, centralized solar, or wind energy. Wood and other biomass fuels are not included, except those consumed at electric utilities.

bHeavy oil includes Grade Nos. 4, 5, and 6, and residual fuel oils. Light oil includes Grade No. 2 heating oil, kerosene, and jet fuel.

- No consumption, including cases where adjustments were made. See "Consumption Adjustments for Process Fuel and Intermediate Products."

Sources: Data sources, estimation procedures, and assumptions are described in the "Documentation" section of this report. 


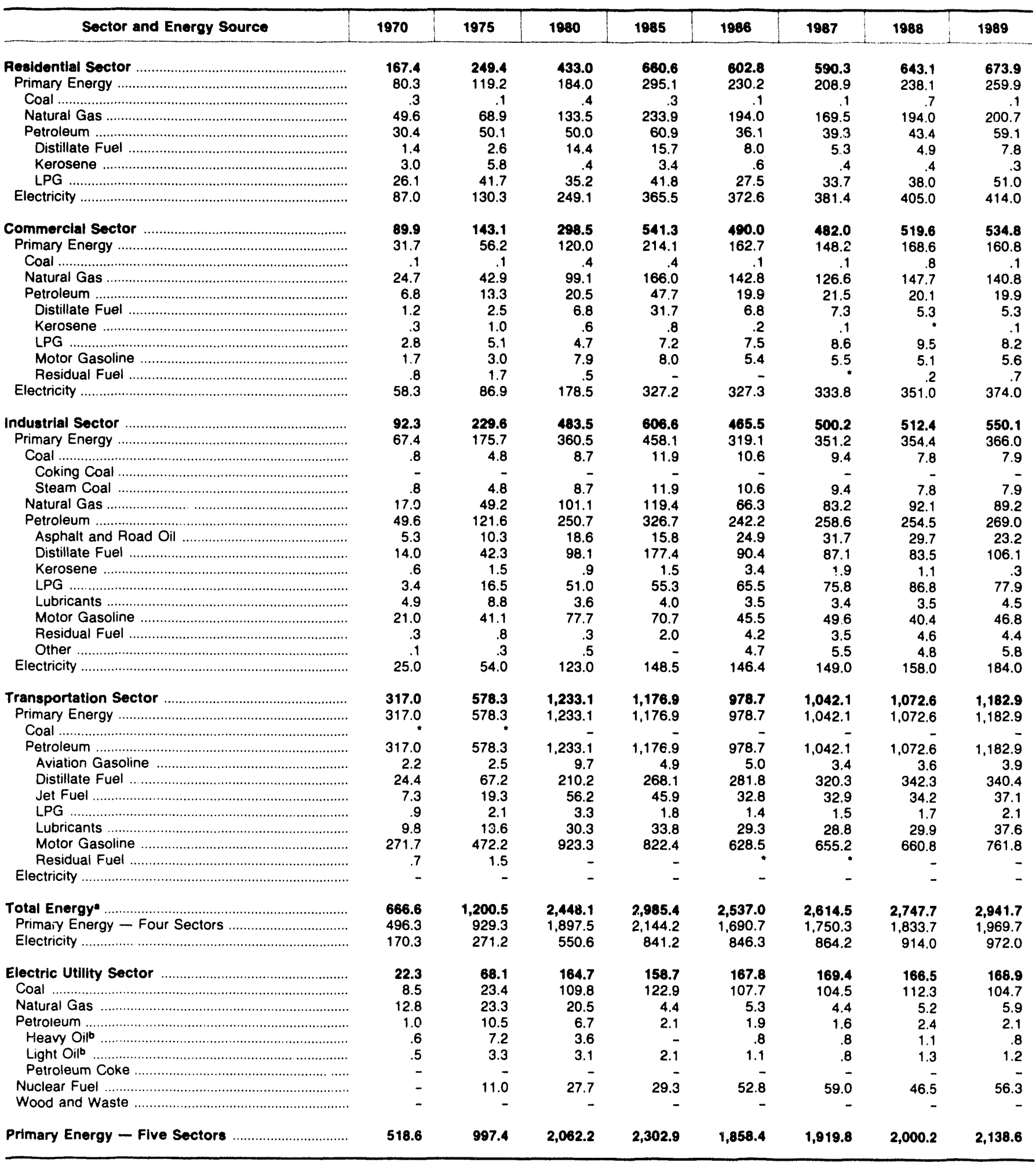

-There are no direct fuel costs for hydroelectric, geothermal, centralized solar, or wind energy. Wood and other biomass fuels are not included, except those consumed at electric utilities.

beavy o:l includes Grade Nos. 4, 5, and 6, and residual fuel oils. Light oil includes Grade No. 2 heating oil, kerosene, and jet fuel.

-No consumption, including cases where adjustments were made. See "Consumption Adjustments for Process Fuel and Intermediate Products."

- Value less than 0.05 million dollars.

Note: Totals may not equal sum of components due to independent rounding

Sources: Data sources, estimation procedures, and assumptions are described in the "Documentation" section of this report. 
N Energy Price and Expenditure Estimates by Source, Nevada

E 1970, 1975, 1980, and 1985-1989

\begin{tabular}{|c|c|c|c|c|c|c|c|c|}
\hline Energy Source & 1970 & 1975 & 1980 & 1985 & 1986 & 1987 & 1988 & 1989 \\
\hline & \multicolumn{8}{|c|}{ Prices in Dollars per Million Btu } \\
\hline Coal & 0.37 & 0.35 & 1.06 & 1.62 & 1.40 & 1.40 & 1.37 & 1.49 \\
\hline 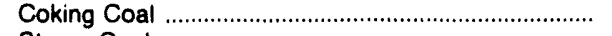 & - & $\bar{r}$ & - & - & - & - & - & - \\
\hline Steam Coal & .37 & .35 & 1.06 & 1.62 & 1.40 & 1.40 & 1.37 & 1.49 \\
\hline 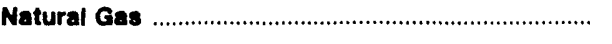 & .61 & 1.31 & 3.10 & 5.44 & 4.60 & 4.25 & 4.49 & 3.84 \\
\hline Petroloum & 1.95 & 3.40 & 7.67 & 7.79 & 5.84 & 5.84 & 6.05 & 6.98 \\
\hline 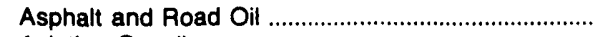 & .59 & 1.83 & 3.69 & 4.78 & 4.34 & 3.27 & 3.28 & 2.73 \\
\hline Aviation Gasoline & 2.17 & 3.45 & 9.02 & 9.99 & 8.41 & 7.55 & 7.41 & 8.28 \\
\hline 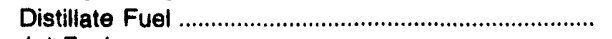 & 1.29 & 2.75 & 6.97 & 7.32 & 5.44 & 5.70 & 5.83 & 6.92 \\
\hline 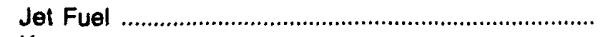 & .76 & 2.12 & 6.59 & 6.22 & 4.25 & 4.27 & 4.08 & 4.79 \\
\hline 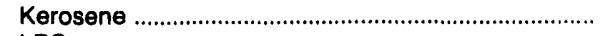 & .77 & 2.42 & - & 14.41 & 12.71 & 8.60 & 9.20 & 12.04 \\
\hline LPG & 2.91 & 4.03 & 7.07 & 11.51 & 10.95 & 10.29 & 10.13 & 12.60 \\
\hline 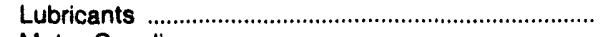 & 5.08 & 7.49 & 14.36 & 17.61 & 15.59 & 13.58 & 14.61 & 17.90 \\
\hline 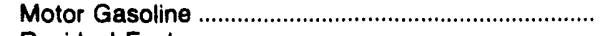 & 3.07 & 4.74 & 9.96 & 8.77 & 6.75 & 6.79 & 7.32 & 8.19 \\
\hline 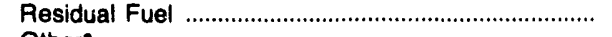 & .58 & 1.98 & 3.58 & 4.13 & 2.02 & 2.38 & 2.39 & 2.72 \\
\hline Other" & .43 & - & 4.04 & 3.39 & - & - & - & - \\
\hline 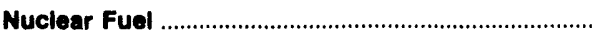 & - & - & - & - & - & - & - & - \\
\hline 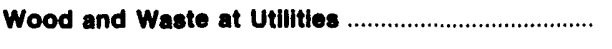 & - & - & - & - & - & - & - & - \\
\hline 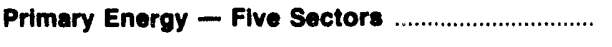 & 1.32 & 1.82 & 4.69 & 4.90 & 3.59 & 3.69 & 3.70 & 4.20 \\
\hline 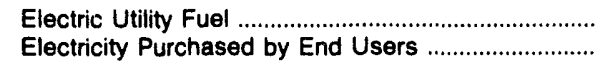 & $\begin{array}{r}.36 \\
3.89\end{array}$ & $\begin{array}{r}.59 \\
6.86\end{array}$ & $\begin{array}{r}1.68 \\
13.18\end{array}$ & $\begin{array}{r}1.79 \\
16.75\end{array}$ & $\begin{array}{r}1.47 \\
17.16\end{array}$ & $\begin{array}{r}1.47 \\
15.45\end{array}$ & $\begin{array}{r}1.47 \\
15.93\end{array}$ & $\begin{array}{r}1.62 \\
15.46\end{array}$ \\
\hline \multirow[t]{2}{*}{ 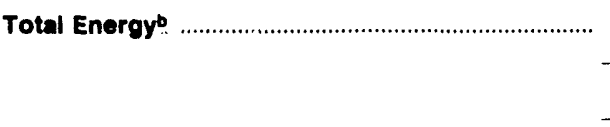 } & 1.96 & 3.52 & 8.18 & 9.04 & 7.82 & 7.38 & 7.75 & 8.22 \\
\hline & \multicolumn{8}{|c|}{ Expenditures in Millions of Dollars } \\
\hline 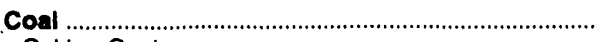 & 6.4 & 35.8 & 99.1 & 204.2 & 225.5 & 217.1 & 250.5 & 253.1 \\
\hline 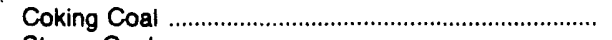 & - & - & - & - & - & - & - & - \\
\hline Steam Coal & 6.4 & 35.8 & 99.1 & 204.2 & 225.5 & 217.1 & 250.5 & 253.1 \\
\hline 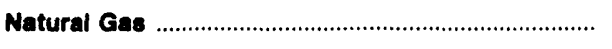 & 34.5 & 85.5 & 191.5 & 222.8 & 162.3 & 173.6 & 213.0 & 245.9 \\
\hline 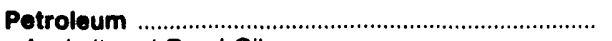 & 176.6 & 393.5 & $1,125.9$ & $1,056.6$ & 825.9 & 904.7 & $1,004.0$ & $1,216.8$ \\
\hline 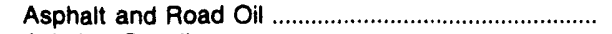 & 2.4 & 10.2 & 15.0 & 26.8 & 16.3 & 18.7 & 20.3 & 25.3 \\
\hline 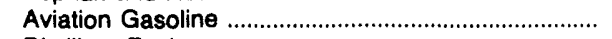 & 2.0 & 3.4 & 9.4 & 5.3 & 5.2 & 3.8 & 4.5 & 4.9 \\
\hline 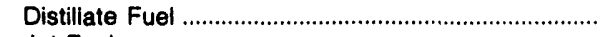 & 21.2 & 41.1 & 160.9 & 230.4 & 172.6 & 214.8 & 229.6 & 299.9 \\
\hline 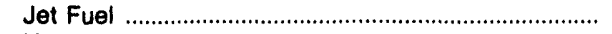 & 19.2 & 69.2 & 266.2 & 197.0 & 140.5 & 152.5 & 145.3 & 162.6 \\
\hline 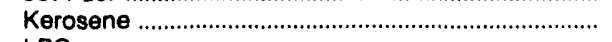 & .1 & 4 & - & 4.6 & 1.2 & .6 & 2.0 & 2.9 \\
\hline LPG & 9.2 & 7.4 & 22.9 & 44.3 & 40.5 & 33.3 & 37.8 & 71.9 \\
\hline 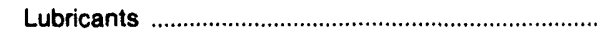 & 3.2 & 5.4 & 9.4 & 10.5 & 9.1 & 9.0 & 9.3 & 11.7 \\
\hline Motor Gasoline & 118.7 & 239.7 & 587.0 & 535.6 & 432.9 & 465.1 & 541.6 & 626.3 \\
\hline 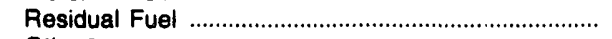 & .5 & 16.7 & 55.0 & 2.1 & 7.6 & 6.8 & 13.7 & 11.3 \\
\hline 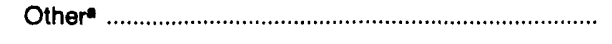 & • & - & .1 & .1 & - & - & - & - \\
\hline 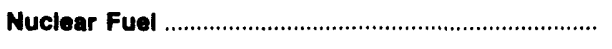 & - & - & - & - & - & - & - & - \\
\hline 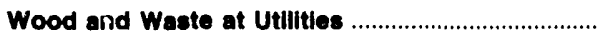 & - & - & - & - & - & - & - & - \\
\hline 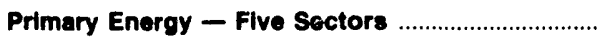 & 217.5 & 514.7 & $1,416.5$ & $1,483.5$ & $1,213.7$ & $1,295.4$ & $1,467.5$ & $1,715.8$ \\
\hline $\begin{array}{l}\text { Electric Utility Fuel } \\
\text { Electricity Purchased by End Users }\end{array}$ & $\begin{array}{r}-15.1 \\
75.7\end{array}$ & $\begin{array}{l}-79.8 \\
179.0\end{array}$ & $\begin{array}{r}-226.1 \\
468.2\end{array}$ & $\begin{array}{r}-238.1 \\
634.3\end{array}$ & $\begin{array}{r}-249.1 \\
655.8\end{array}$ & $\begin{array}{r}-239.1 \\
638.7\end{array}$ & $\begin{array}{r}-290.8 \\
717.3\end{array}$ & $\begin{array}{r}-315.8 \\
773.1\end{array}$ \\
\hline Total Energyb & 278.1 & 613.9 & $1,658.6$ & $1,879.7$ & $1,620.3$ & $1,695.0$ & $1,894.0$ & $2,173.2$ \\
\hline
\end{tabular}

- Includes petroleum coke used at electric utilities.

bThere are no direct fuel costs for hydroelectric, geothermal, centralized solar, or wind energy. Wood and other biomass fuels are not included, except those consumed at electric utilities.

- No consumption, including cases where adjustments were made. See "Consumption Adjustments for Process Fuel and Intermediate Products."

"Value less than 0.05 million dollars.

Note: Expenditure totals may not equal sum of components due to independent rounding.

Sources: Data sources, estimation procedures, and assumptions are described in the "Documentation" section of this report. 


\begin{tabular}{|c|c|c|c|c|c|c|c|c|}
\hline Sector and Energy Source & 1970 & 1975 & 1980 & 1985 & 1986 & 1987 & 1988 & 1989 \\
\hline $\begin{array}{l}\text { Roaldentlal Sector } \\
\text { Prim ary Energy } \\
\text { Coal } \\
\text { Natural Gas } \\
\text { Petroleum } \\
\text { Distillate Fuel } \\
\text { Kerosene }\end{array}$ & $\begin{array}{r}2.70 \\
1.76 \\
1.31 \\
1.39 \\
2.51 \\
1.27 \\
.- \\
3.52 \\
4.0\end{array}$ & $\begin{array}{l}4.30 \\
2.18 \\
1.55 \\
1.83 \\
3.72 \\
2.82 \\
4.90 \\
7.54\end{array}$ & $\begin{array}{r}8.74 \\
4.58 \\
5.13 \\
3.87 \\
8.31 \\
6.92 \\
9.28 \\
14.21\end{array}$ & $\begin{array}{r}12.59 \\
7.69 \\
4.54 \\
6.63 \\
10.80 \\
7.55 \\
15.04 \\
12.40 \\
18.83\end{array}$ & $\begin{array}{r}12.39 \\
6.72 \\
4.49 \\
5.62 \\
10.26 \\
7.26 \\
14.46 \\
11.84 \\
19.28\end{array}$ & $\begin{array}{r}11.09 \\
6.05 \\
4.11 \\
5.29 \\
8.68 \\
5.79 \\
11.54 \\
11.19 \\
17.05\end{array}$ & $\begin{array}{r}11.70 \\
6.57 \\
3.83 \\
5.89 \\
9.03 \\
6.02 \\
11.99 \\
10.98 \\
17.58\end{array}$ & $\begin{array}{r}11.32 \\
6.96 \\
4.14 \\
5.38 \\
12.74 \\
8.26 \\
16.46 \\
14.68 \\
16.78\end{array}$ \\
\hline 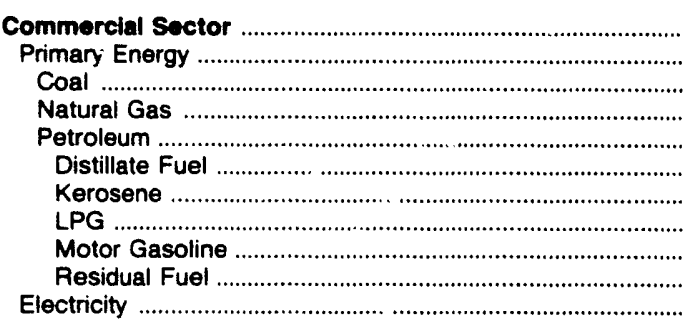 & $\begin{array}{r}2.15 \\
.77 \\
.52 \\
.70 \\
1.34 \\
1.12 \\
.77 \\
1.16 \\
3.07 \\
.62 \\
4.74\end{array}$ & $\begin{array}{r}3.88 \\
1.59 \\
.82 \\
1.45 \\
2.99 \\
2.62 \\
2.42 \\
2.45 \\
4.74 \\
2.00 \\
8.01\end{array}$ & $\begin{array}{r}7.73 \\
4.29 \\
1.36 \\
3.68 \\
6.78 \\
6.60 \\
4- \\
4.99 \\
9.96 \\
3.53 \\
15.39\end{array}$ & $\begin{array}{r}11.29 \\
6.19 \\
1.58 \\
5.77 \\
8.17 \\
7.76 \\
9.06 \\
9.62 \\
8.77 \\
4.80 \\
18.24\end{array}$ & $\begin{array}{r}10.75 \\
4.92 \\
1.66 \\
4.63 \\
5.86 \\
5.32 \\
4.51 \\
9.06 \\
6.75 \\
2.30 \\
18.66\end{array}$ & $\begin{array}{r}9.37 \\
4.49 \\
1.53 \\
4.27 \\
5.08 \\
4.60 \\
4.65 \\
8.52 \\
6.79 \\
2.64 \\
16.59\end{array}$ & $\begin{array}{r}10.02 \\
4.85 \\
1.49 \\
4.64 \\
5.76 \\
5.09 \\
4.96 \\
8.58 \\
7.32 \\
2.32 \\
16.94\end{array}$ & $\begin{array}{r}10.09 \\
4.84 \\
1.62 \\
4.30 \\
7.47 \\
6.73 \\
6.59 \\
10.08 \\
8.19 \\
2.52 \\
16.85\end{array}$ \\
\hline $\begin{array}{l}\text { Industrial Sector } \\
\text { Primary Energy } \\
\text { Coal } \\
\text { Coking Coal } \\
\text { Steam Coal } \\
\text { Natural Gas } \\
\text { Petroleum } \\
\text { Asphalt and Road Oil } \\
\text { Distillate Fuel } \\
\text { Kerosene }\end{array}$ & $\begin{array}{r}1.02 \\
.75 \\
.52 \\
.52 \\
.52 \\
.52 \\
1.04 \\
.59 \\
.96 \\
.77 \\
1.16 \\
5.08 \\
3.07 \\
.49 \\
.43 \\
2.14\end{array}$ & $\begin{array}{r}2.18 \\
1.61 \\
.82 \\
-8 \overline{-} \\
.82 \\
1.06 \\
2.25 \\
1.83 \\
2.25 \\
2.42 \\
2.45 \\
7.49 \\
4.74 \\
1.83 \\
4.23\end{array}$ & $\begin{array}{r}7.20 \\
3.67 \\
1.36 \\
1 . \\
1.36 \\
2.83 \\
5.10 \\
3.69 \\
5.56 \\
4- \\
4.99 \\
14.36 \\
9.96 \\
3.75 \\
4.04 \\
11.63\end{array}$ & $\begin{array}{r}8.10 \\
5.73 \\
1.58 \\
1.58 \\
1.58 \\
4.05 \\
7.01 \\
4.78 \\
7.97 \\
9.06 \\
9.62 \\
17.61 \\
8.77 \\
4.80 \\
3.39 \\
12.91\end{array}$ & $\begin{array}{r}7.59 \\
4.02 \\
1.66 \\
- \\
1.68 \\
3.76 \\
4.48 \\
4.34 \\
3.97 \\
4.51 \\
9.06 \\
15.59 \\
6.75 \\
2.30 \\
13.36\end{array}$ & $\begin{array}{r}6.78 \\
3.80 \\
1.53 \\
- \\
1.53 \\
3.72 \\
4.18 \\
3.27 \\
4.09 \\
4.65 \\
8.52 \\
13.58 \\
6.79 \\
2.64 \\
12.46\end{array}$ & $\begin{array}{r}6.86 \\
4.00 \\
1.49 \\
- \\
1.49 \\
3.84 \\
4.37 \\
3.28 \\
4.37 \\
4.56 \\
8.58 \\
14.61 \\
7.32 \\
2.32 \\
13.01\end{array}$ & $\begin{array}{r}7.18 \\
4.66 \\
- \\
- \\
- \\
4.83 \\
5.21 \\
2.73 \\
5.80 \\
6.59 \\
10.08 \\
17.90 \\
8.19 \\
2.52 \\
12.99\end{array}$ \\
\hline 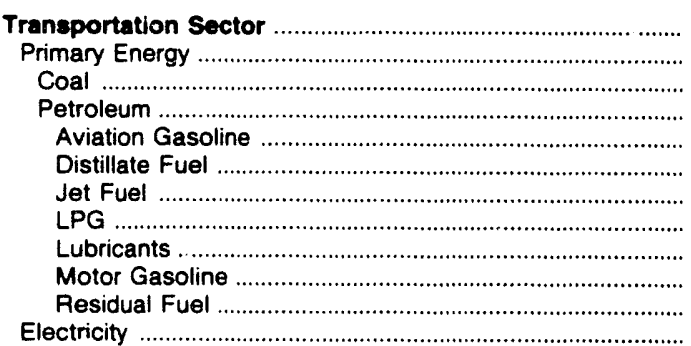 & $\begin{array}{r}2.08 \\
2.08 \\
.52 \\
2.08 \\
2.17 \\
1.50 \\
.76 \\
1.16 \\
5.08 \\
3.07 \\
.60 \\
-\end{array}$ & $\begin{array}{r}3.66 \\
3.66 \\
.82 \\
3.66 \\
3.45 \\
3.01 \\
2.12 \\
2.45 \\
7.49 \\
4.74 \\
2.36\end{array}$ & $\begin{array}{r}8.44 \\
8.44 \\
- \\
8.44 \\
9.02 \\
7.36 \\
6.59 \\
4.99 \\
14.36 \\
9.96 \\
-\end{array}$ & $\begin{array}{r}7.79 \\
7.79 \\
- \\
7.79 \\
9.99 \\
6.97 \\
6.22 \\
9.62 \\
17.61 \\
8.77 \\
-\end{array}$ & $\begin{array}{r}5.96 \\
5.96 \\
- \\
5.96 \\
8.41 \\
6.01 \\
4.25 \\
9.06 \\
15.59 \\
6.75 \\
1.86\end{array}$ & $\begin{array}{r}6.07 \\
6.07 \\
- \\
6.07 \\
7.55 \\
6.59 \\
4.27 \\
8.52 \\
13.58 \\
6.79 \\
-\end{array}$ & $\begin{array}{r}6.40 \\
6.40 \\
- \\
6.40 \\
7.41 \\
6.87 \\
4.08 \\
8.58 \\
14.61 \\
7.32 \\
-\end{array}$ & $\begin{array}{r}7.29 \\
7.29 \\
- \\
7.29 \\
8.28 \\
7.76 \\
4.79 \\
10.08 \\
17.90 \\
8.19 \\
-\end{array}$ \\
\hline $\begin{array}{l}\text { Total Energy" } \\
\text { Primary Energy - Four Sectors } \\
\text { Electricity }\end{array}$ & $\begin{array}{l}1.96 \\
1.65 \\
3.89\end{array}$ & $\begin{array}{l}3.52 \\
2.93 \\
6.86\end{array}$ & $\begin{array}{r}8.18 \\
7.12 \\
13.18\end{array}$ & $\begin{array}{r}9.04 \\
7.33 \\
16.75\end{array}$ & $\begin{array}{r}7.82 \\
5.71 \\
17.16\end{array}$ & $\begin{array}{r}7.38 \\
5.61 \\
15.45\end{array}$ & $\begin{array}{r}7.75 \\
5.90 \\
15.93\end{array}$ & $\begin{array}{r}8.22 \\
6.54 \\
15.46\end{array}$ \\
\hline $\begin{array}{l}\text { Eloctric Utility Sector } \\
\text { Coal . } \\
\text { Natural Gas } \\
\text { Petroleum } \\
\text { Heavy Oib } \\
\text { Light Ojb } \\
\text { Petroleum Coke } \\
\text { Nuclear Fuel } \\
\text { Wood and Waste }\end{array}$ & $\begin{array}{l}.36 \\
.31 \\
.38 \\
.62 \\
.61 \\
.70 \\
- \\
- \\
-\end{array}$ & $\begin{array}{r}.59 \\
.34 \\
1.09 \\
2.00 \\
1.98 \\
2.47 \\
- \\
-\end{array}$ & $\begin{array}{r}1.68 \\
1.05 \\
2.59 \\
3.60 \\
3.58 \\
5.58 \\
- \\
-\end{array}$ & $\begin{array}{r}1.79 \\
1.62 \\
4.07 \\
4.91 \\
3.71 \\
6.12 \\
- \\
= \\
-\end{array}$ & $\begin{array}{r}1.47 \\
1.39 \\
3.04 \\
2.06 \\
1.97 \\
3.94 \\
- \\
-\end{array}$ & $\begin{array}{r}1.47 \\
1.40 \\
2.58 \\
2.51 \\
2.36 \\
4.41 \\
- \\
-\end{array}$ & $\begin{array}{r}1.47 \\
1.36 \\
2.72 \\
2.51 \\
2.40 \\
4.05 \\
- \\
- \\
-\end{array}$ & $\begin{array}{r}1.62 \\
1.52 \\
2.11 \\
2.97 \\
2.74 \\
5.19 \\
- \\
-\end{array}$ \\
\hline 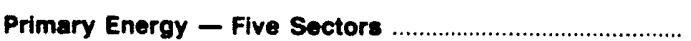 & 1.32 & 1.82 & 4.69 & 4.90 & 3.59 & 3.69 & 3.70 & 4.20 \\
\hline
\end{tabular}

-There are no direct fuel costs for hydroelectric, geothermal, centralized solar, or wind energy. Wood and other biomass fuels are not included, except those consumed at electric utilities.

bHeavy oil includes Grade Nos. 4, 5, and 6, and residual fuel oils. Light oil includes Grade No. 2 heating oil, kerosene, and jet fuel.

-No consumption, including cases where adjustments were made. See "Consumption Adjustments for Process Fuel and Intermediate Products."

Sources: Data sources, estimation procedures, and assumptions are described in the "Documentation" section of this report. 
N Energy Expenditure Estimates by Sector, Nevada

E $1970,1975,1980$, and 1985-1989

V (Million Dollars)

\begin{tabular}{|c|c|c|c|c|c|c|c|c|}
\hline Sector and Energy Source & 1970 & 1975 & 1980 & 1985 & 1986 & 1987 & 1988 & 1989 \\
\hline $\begin{array}{l}\text { Residential Sector } \\
\text { Primary Energy } \ldots \ldots\end{array}$ & $\begin{array}{l}52.6 \\
22.4\end{array}$ & $\begin{array}{r}104.0 \\
31.9\end{array}$ & $\begin{array}{r}255.1 \\
75.9\end{array}$ & $\begin{array}{l}403.0 \\
137.9\end{array}$ & $\begin{array}{l}383.9 \\
114.3\end{array}$ & $\begin{array}{l}374.4 \\
110.5\end{array}$ & $\begin{array}{l}426.0 \\
128.0\end{array}$ & $\begin{array}{l}449.3 \\
153.3\end{array}$ \\
\hline Coal ..1. & .7 & .1 & .2 & .1 & .1 & .1 & .1 & .1 \\
\hline 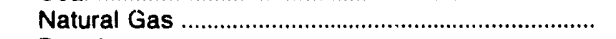 & 10.9 & 21.6 & 53.6 & 88.7 & 72.9 & 75.1 & 89.7 & 93.0 \\
\hline 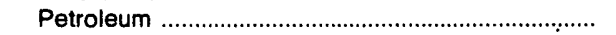 & 10.7 & 10.1 & 22.1 & 49.2 & 41.3 & 35.4 & 38.3 & 60.2 \\
\hline 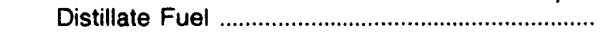 & 2.4 & 4.4 & 7.5 & 12.5 & 10.4 & 11.0 & 10.2 & 12.1 \\
\hline 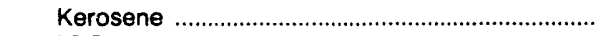 & - & - & - & 4.3 & 1.1 & .4 & 1.6 & 2.2 \\
\hline LPG & 8.3 & 5.8 & 14.6 & 32.5 & 29.8 & 24.0 & 26.5 & 45.9 \\
\hline 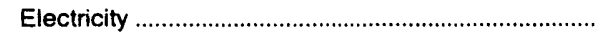 & 30.3 & 72.1 & 179.2 & 265.1 & 269.5 & 263.9 & 298.0 & 296.0 \\
\hline Commerclal Sector & 43.7 & 106.6 & 151.1 & 310.4 & 298.6 & 296.1 & 322.4 & 338.4 \\
\hline 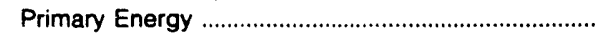 & 10.3 & 28.1 & 57.9 & 98.3 & 78.7 & 84.5 & 89.4 & 91.4 \\
\hline Coal & .5 & .1 & .1 & & $\therefore$ & & & \\
\hline Natural Gas & 7.3 & 23.2 & 39.6 & 74.9 & 56.2 & 59.0 & 68.7 & 67.1 \\
\hline 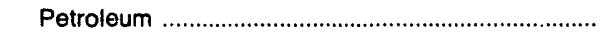 & 2.5 & 4.8 & 18.3 & 23.4 & 22.4 & 25.5 & 20.6 & 24.2 \\
\hline Distillate Fuel & 1.0 & 2.0 & 13.6 & 14.6 & 15.3 & 19.2 & 13.5 & 14.8 \\
\hline 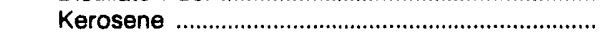 & & .2 & - & .2 & * & • & .3 & .3 \\
\hline LPG & .5 & .5 & 1.4 & 4.4 & 4.0 & 3.2 & 3.7 & 5.6 \\
\hline 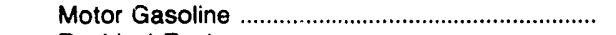 & 8 & 1.7 & 3.2 & 3.8 & 2.9 & 3.0 & 3.1 & 3.5 \\
\hline 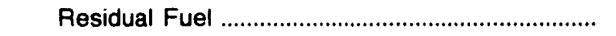 & .1 & .4 & .2 & .2 & .2 & .1 & * & . \\
\hline 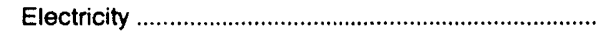 & 33.4 & 78.6 & 93.2 & 212.0 & 219.9 & 211.6 & 233.0 & 247.0 \\
\hline 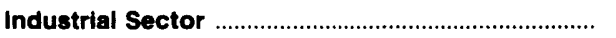 & 29.7 & 66.4 & 273.4 & 299.0 & 247.3 & 258.3 & 309.6 & 420.7 \\
\hline 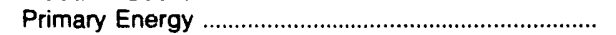 & 17.7 & 38.1 & 77.6 & 141.8 & 81.0 & 95.1 & 123.2 & 190.5 \\
\hline 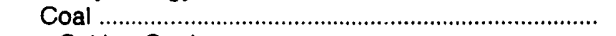 & .9 & 1.5 & 4.6 & 4.1 & 4.1 & 4.0 & 4.2 & - \\
\hline Coking Coal & - & - & - & - & - & - & - & - \\
\hline 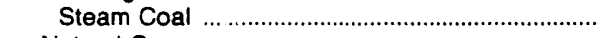 & .9 & 1.5 & 4.6 & 4.1 & 4.1 & 4.0 & 4.2 & - \\
\hline 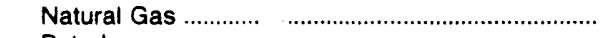 & 5.8 & 11.4 & 21.9 & 24.2 & 12.1 & 20.7 & 24.8 & 35.3 \\
\hline 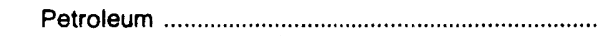 & 11.0 & 25.2 & 51.1 & 113.5 & 64.8 & 70.4 & 94.2 & 155.2 \\
\hline 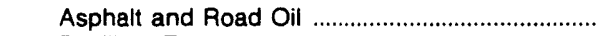 & 2.4 & 10.2 & 15.0 & 26.8 & 16.3 & 18.7 & 20.3 & 25.3 \\
\hline 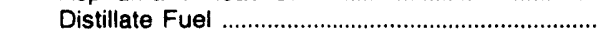 & 4.7 & 9.3 & 21.1 & 71.1 & 34.4 & 38.1 & 58.7 & 99.7 \\
\hline Kerosene & • & .2 & - & .1 & * & .1 & .1 & .4 \\
\hline LPG & .4 & 1.0 & 6.9 & 6.4 & 5.9 & 5.5 & 7.0 & 19.8 \\
\hline 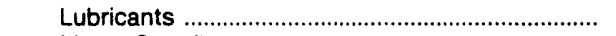 & .7 & 1.2 & 2.2 & 2.4 & 2.1 & 2.1 & 2.2 & 2.7 \\
\hline 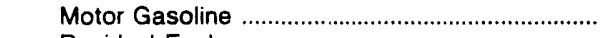 & 2.7 & 2.9 & 5.8 & 6.0 & 4.9 & 5.5 & 5.6 & 6.4 \\
\hline 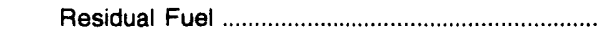 & .1 & .5 & * & .7 & 1.2 & .5 & .5 & .9 \\
\hline Other & & - & .1 & .1 & - & - & - & - \\
\hline 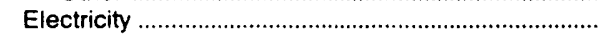 & 12.0 & 28.3 & 195.8 & 157.2 & 166.3 & 163.2 & 186.4 & 230.1 \\
\hline $\begin{array}{c}\text { Transportation Sector } \\
\text { Primary Energy }\end{array}$ & $\begin{array}{l}152.1 \\
152.1\end{array}$ & $\begin{array}{l}336.9 \\
336.9\end{array}$ & $\begin{array}{l}978.9 \\
978.9\end{array}$ & $\begin{array}{l}\mathbf{8 6 7 . 4} \\
\mathbf{8 6 7 . 4}\end{array}$ & $\begin{array}{l}690.6 \\
690.6\end{array}$ & $\begin{array}{l}766.2 \\
766.2\end{array}$ & $\begin{array}{l}836.1 \\
836.1\end{array}$ & $\begin{array}{l}964.8 \\
964.8\end{array}$ \\
\hline Coal & & & & 001.4 & & & - & \\
\hline Petroleum ……1-1) & 152.1 & 336.9 & 978.9 & 867.4 & 690.6 & 766.2 & 836.1 & 964.8 \\
\hline Aviation Gasoline & 2.0 & 3.4 & 9.4 & 5.3 & 5.2 & 3.8 & 4.5 & 4.9 \\
\hline 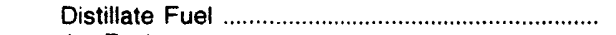 & 13.0 & 24.7 & 118.0 & 130.3 & 111.9 & 145.7 & 145.5 & 171.2 \\
\hline 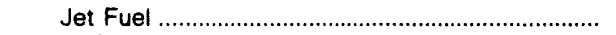 & 19.2 & 69.2 & 266.2 & 197.0 & 140.5 & 152.5 & 145.3 & 162.6 \\
\hline LPG & & .1 & .1 & 1.0 & .8 & .6 & .6 & .6 \\
\hline 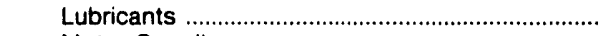 & 2.6 & 4.2 & 7.3 & 8.1 & 7.0 & 6.9 & 7.2 & 9.0 \\
\hline 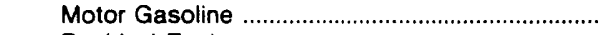 & 115.3 & 235.2 & 578.0 & 525.7 & 425.0 & 456.6 & 532.9 & 616.5 \\
\hline 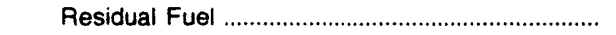 & • & .1 & - & - & .1 & - & - & - \\
\hline 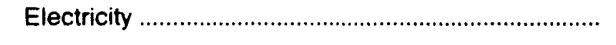 & - & - & - & - & $\cdot$ & - & - & - \\
\hline Total Energy & 278.1 & 613.9 & $1,658.6$ & $1,879.7$ & $1,620.3$ & $1,695.0$ & $1,894.0$ & $2,173.2$ \\
\hline Primary Energy - Four Sectors .................................... & 202.5 & 434.9 & $1,190.4$ & $1,245.4$ & 964.5 & $1,056.3$ & $1,176.7$ & $1,400.0$ \\
\hline 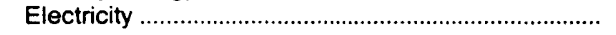 & 75.7 & 179.0 & 468.2 & 634.3 & 655.8 & 638.7 & 717.3 & 773.1 \\
\hline Electric Utility Sector & 15.1 & 79.8 & 226.1 & 238.1 & 249.1 & 259.1 & 290.8 & 315.8 \\
\hline Coal & 4.3 & 34.1 & 94.2 & 199.9 & 221.2 & 213.0 & 246.2 & 253.0 \\
\hline Natural Gas & 10.5 & 29.3 & 76.4 & 35.0 & 21.1 & 18.9 & 29.8 & 50.4 \\
\hline 「 atroleum & .4 & 16.5 & 55.5 & 3.1 & 6.8 & 7.2 & 14.8 & 12.4 \\
\hline 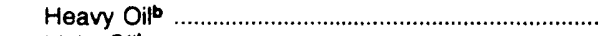 & 3 & 15.7 & 54.8 & 1.2 & 6.2 & 6.3 & 13.2 & 10.4 \\
\hline 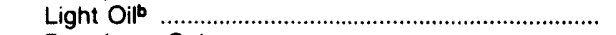 & .1 & .8 & .7 & 1.9 & .6 & .9 & 1.6 & 2.0 \\
\hline Petroleum Coke & - & - & - & - & - & - & - & - \\
\hline 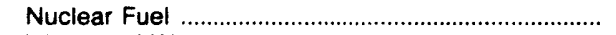 & - & - & - & - & - & - & - & - \\
\hline 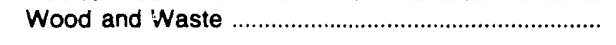 & - & - & - & - & - & - & - & - \\
\hline Primary Energy - Five Sectors .................................. & 217.5 & 514.7 & $1,416.5$ & $1,483.5$ & 1,213.7 & $1,295.4$ & $1,467.5$ & $1,715.8$ \\
\hline
\end{tabular}

-There are no direct fuel costs for hydrcelectric, geothermal, centralized solar, or wind energy. Wood and other biomass fuels are not included, except those consumed at electric utilities.

b Heavy oil includes Grade Nos. 4, 5, and 6, and residual fuel oils. Light oil includes Grade No. 2 heating oil, kerosene, and jet fuel.

-No consumption, including cases where adjustments were made. See "Consumption Adjustments for Process Fuel and intermediate Products."

- Value less than 0.05 million dollars.

Note: Totals may not equal sum of components due to independent rounding

Sources: Data sources, estimation procedures, and assumptions are described in the "Documentation" section of this report. 


\begin{tabular}{|c|c|c|c|c|c|c|c|c|}
\hline Energy Source & 1970 & 1975 & 1980 & 1985 & 1986 & 1987 & 1988 & 1989 \\
\hline & \multicolumn{8}{|c|}{ Prices in Dollars per Million Btu } \\
\hline Coal & 0.37 & 1.22 & 1.60 & 2.03 & 2.01 & 1.93 & 1.79 & 1.75 \\
\hline 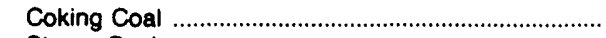 & - & - & - & - & - & - & - & - \\
\hline Steam Coal & .37 & 1.22 & 1.60 & 2.03 & 2.01 & 1.93 & 1.79 & 1.75 \\
\hline 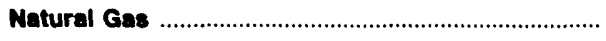 & 1.65 & 2.24 & 4.27 & 6.44 & 6.53 & 5.75 & 5.50 & 6.06 \\
\hline Petroleum & 1.60 & 3.24 & 7.31 & 7.84 & 5.58 & 5.99 & 5.89 & 6.57 \\
\hline 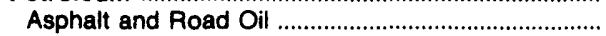 & .70 & 2.05 & 4.00 & 5.20 & 4.86 & 3.54 & 3.34 & 3.17 \\
\hline Aviation Gasoline & 2.17 & 3.45 & 9.02 & 9.99 & 8.41 & 7.55 & 7.41 & 8.28 \\
\hline 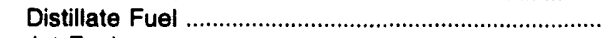 & 1.38 & 2.80 & 6.97 & 7.51 & 5.52 & 5.54 & 5.66 & 6.33 \\
\hline Jet Fuel & .75 & 2.10 & 6.51 & 6.53 & 4.93 & 4.18 & 4.22 & 4.83 \\
\hline 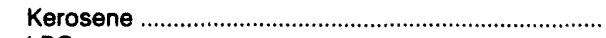 & 1.50 & 3.09 & 8.05 & 8.70 & 6.34 & 6.32 & 6.47 & 7.37 \\
\hline LPG & 2.06 & 3.53 & 6.89 & 11.40 & 10.85 & 10.67 & 10.55 & 10.32 \\
\hline Lubricants & 5.08 & 7.49 & 14.36 & 17.61 & 15.59 & 13.58 & 14.61 & 17.90 \\
\hline Motor Gasoline & 2.92 & 4.54 & 10.11 & 9.26 & 7.32 & 7.60 & 7.82 & 8.60 \\
\hline Residual Fuel & .42 & 1.85 & 3.82 & 3.76 & 1.99 & 2.65 & 1.93 & 2.29 \\
\hline Other & 1.89 & 4.36 & 8.89 & 11.55 & 11.59 & 11.45 & 9.75 & 14.58 \\
\hline 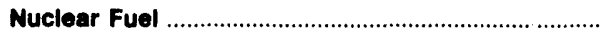 & - & - & - & - & - & - & - & - \\
\hline Wood and Waste at Uttilies & - & - & - & - & - & - & - & - \\
\hline 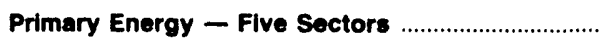 & 1.41 & 2.89 & 6.17 & 6.41 & 5.15 & 5.34 & 5.21 & 5.81 \\
\hline 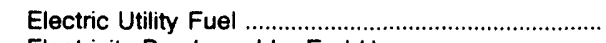 & .36 & 1.43 & 2.68 & 2.46 & 1.95 & 2.20 & 1.83 & 2.01 \\
\hline 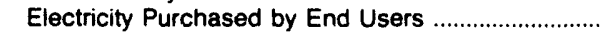 & 6.74 & 12.68 & 19.55 & 23.28 & 22.14 & 23.25 & 24.24 & 24.49 \\
\hline \multirow[t]{2}{*}{ Total Energy' } & 2.20 & 4.39 & 9.60 & 10.81 & 9.16 & 9.21 & 9.76 & 10.39 \\
\hline & \multicolumn{8}{|c|}{ Expenditures in Millions of Dollars } \\
\hline Coal & 10.1 & 31.9 & 46.9 & 80.5 & 50.4 & 60.9 & 58.7 & 55.3 \\
\hline Coking Coal & - & - & - & - & - & - & - & - \\
\hline Steam Coal & 10.1 & 31.9 & 46.9 & 80.5 & 50.4 & 60.9 & 58.7 & 55.3 \\
\hline 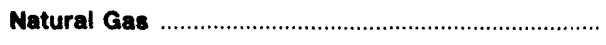 & 11.2 & 17.2 & 41.0 & 69.7 & 68.8 & 70.7 & 73.2 & 86.0 \\
\hline 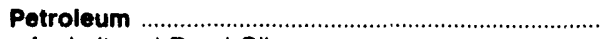 & 225.4 & 443.1 & 975.9 & 959.2 & 821.3 & 940.1 & 942.1 & $1,087.7$ \\
\hline 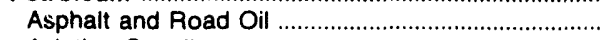 & 2.5 & 5.9 & 6.7 & 29.5 & 17.8 & 18.3 & 9.5 & 15.6 \\
\hline Aviation Gasoline & .4 & .6 & 1.8 & 1.2 & 1.6 & 1.1 & 1.4 & 1.4 \\
\hline 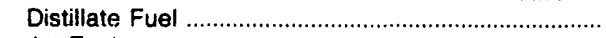 & 61.9 & 116.9 & 236.1 & 229.4 & 185.7 & 243.5 & 224.4 & 278.6 \\
\hline 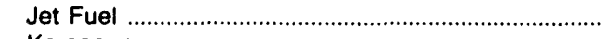 & 4.2 & 10.3 & 27.3 & 18.4 & 16.5 & 14.6 & 16.6 & 20.0 \\
\hline 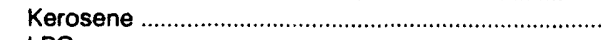 & 6.6 & 8.1 & 15.5 & 20.2 & 6.9 & 9.6 & 8.3 & 11.0 \\
\hline LPG & 6.5 & 18.7 & 31.3 & 63.3 & 67.0 & 76.7 & 83.3 & 99.2 \\
\hline 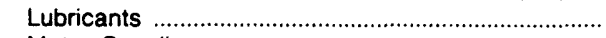 & 2.2 & 3.2 & 7.3 & 8.1 & 7.0 & 6.9 & 7.2 & 9.0 \\
\hline Motor Gasoline & 124.4 & 223.4 & 498.1 & 502.8 & 427.7 & 471.6 & 506.7 & 554.9 \\
\hline Residual Fuel & 14.7 & 53.2 & 135.5 & 73.4 & 77.8 & 84.3 & 72.6 & 80.4 \\
\hline 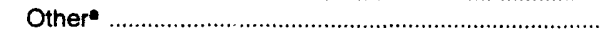 & 1.9 & 2.9 & 16.3 & 13.0 & 13.2 & 13.5 & 12.0 & 17.7 \\
\hline 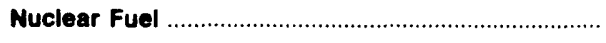 & - & - & - & - & - & - & - & - \\
\hline Wood and Waste at Utilities & - & - & - & - & - & - & - & - \\
\hline 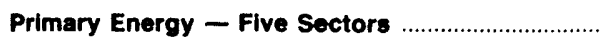 & 246.7 & 492.2 & $1,063.9$ & $1,109.4$ & 940.6 & $1,071.7$ & $1,074.0$ & $1,229.0$ \\
\hline $\begin{array}{l}\text { Electric Utility Fuel } \\
\text { Electricity Purchased by End Users }\end{array}$ & $\begin{array}{r}-15.6 \\
83.5\end{array}$ & $\begin{array}{r}-58.2 \\
207.7\end{array}$ & $\begin{array}{r}-150.9 \\
394.5\end{array}$ & $\begin{array}{r}-131.5 \\
588.4\end{array}$ & $\begin{array}{r}-104.3 \\
594.8\end{array}$ & $\begin{array}{r}-118.2 \\
664.3\end{array}$ & $\begin{array}{r}-115.5 \\
731.9\end{array}$ & $\begin{array}{r}-127.3 \\
759.1\end{array}$ \\
\hline Total Energyb & 314.6 & 641.7 & $1,307.4$ & $1,566.3$ & $1,431.0$ & $1,617.9$ & $1,690.5$ & $1,860.8$ \\
\hline
\end{tabular}

Includes petroleum coke used at electric utilities.

bThere are no direct fuel costs for hydroelectric, geothermal, centralized solar, or wind energy. Wood and other biornass fuels are not included, except those consumed at electric utilities.

-No consumption, including cases where adjustments were made. See "Consumption Adjustments for Process Fuel and Intermediate Products."

Note: Expenditure totals may not equal sum of components due to independent rounding.

Sources: Data sources, estimation procedures, and assumptions are described in the "Documentation" section of this report 
N Energy Price Estimates by Sector, New Hampshire

E $1970,1975,1980$, and 1985-1989

W (Dollars per Million Btu)

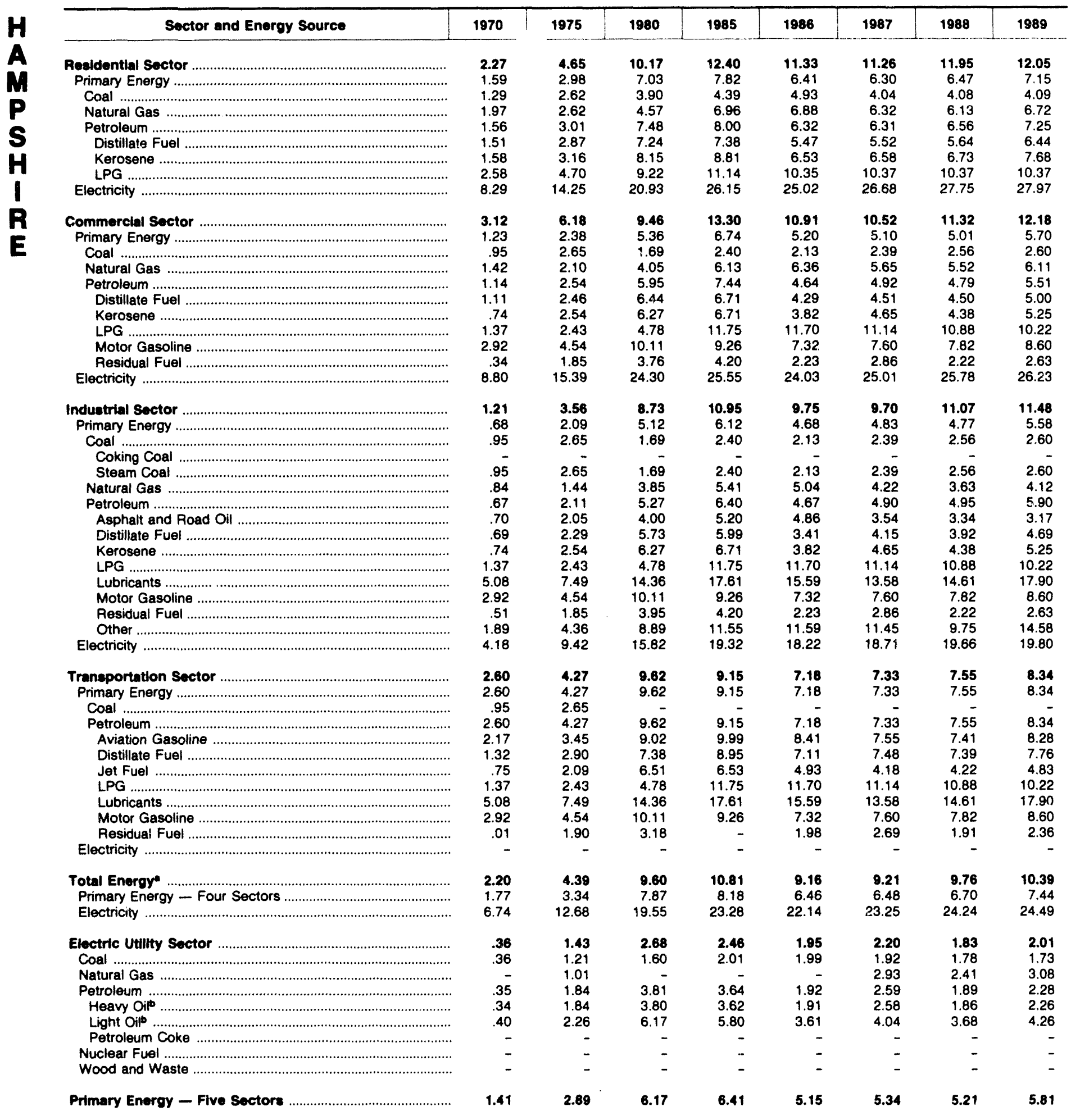

-There are no direct fuel costs for hydroelectric, geothermal, centralized solar, or wind energy. Wood and other biomass fuels are not included, except those consumed at electric utilities.

beavy oil includes Grade Nos. 4, 5, and 6, ai d residual fuel oils. Light oil includes Grade No. 2 heating oil, kerosene, and jet fuel

-No consumption, including cases where alliustments were made. See "Consumption Adjustments for Process Fuel and Intermediate Prnducts."

Sources: Data sources, estirination procedures and assumptions are described in the "Documentation" section of this report. 


Sector and Energy Source
Residential Sector
Primary Energy
Coal
Natural Gas
Petroleum
Distillate Fuel
Kerosene
LPG

Commercial Sector

Primary Energy

Coal.

Natural Gas

Petroleum

Distillate Fuel

Kerosene

LPG

Motor Gasoline

Residual Fuel

Electricity

Industrial Sector

Primary Energy

Coal

Coking Coal

Steam Coal

Natural Gas

Petroleum

Asphait and Road Oil

Distillate Fuel

Kerosene

LPG

Lubricants

Motor Gasoline

Residual Fuel

Otner

Electricity .

Transportation Sector

Primary Energy

Coal .....

Petroleum

Aviation Gasoline

Distillate Fliel

Jet Fuel.....

LPG

Lubricants

Motor Gasoline

Residual Fuel

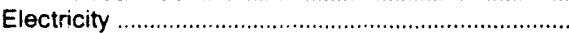

Total Energy

Primary Energy - Four Sectors

Electric Utility Sector

Coal

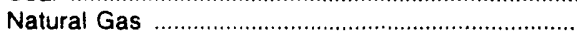

Petroleum ......

Heavy Oib

Light Oilb

Petroleum Coke

Nuclear Fuel

Wood and Waste

Primary Energy - Flve Sectors

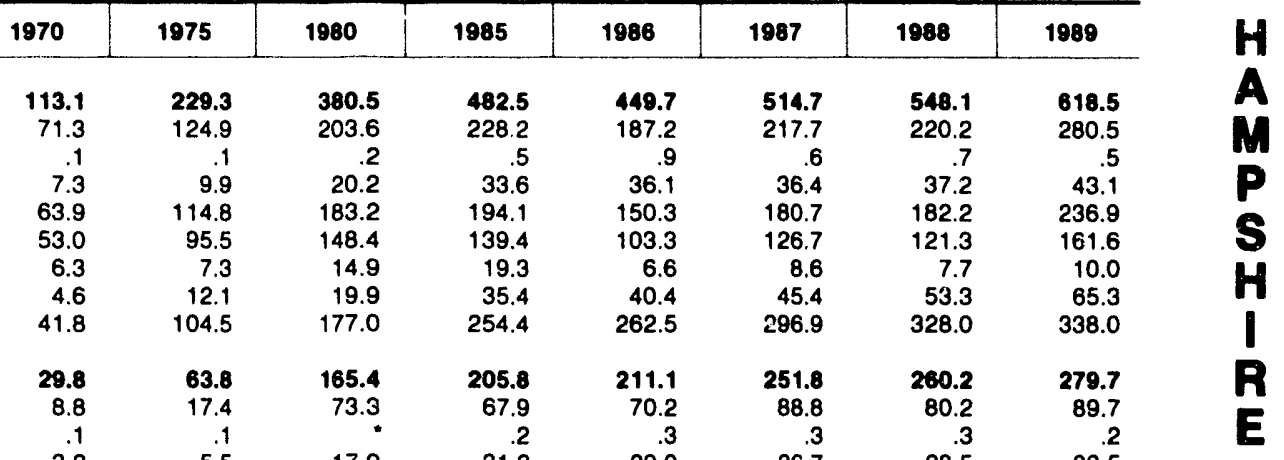

5.5
11.7

17.0

56.3

$\begin{array}{lll}4.1 & 8.5 & 39.2\end{array}$

.3

1.8
6.2

6.2
8.8

31.2
36.5

21.5

29.0
40.9

22.4

26.7
61.8

28.5

51.4

30.2

33.5

56.0
34.6

.8

6.6

.2
8.1

5.6
4.6

6.1
1.6
137.9

8.6

11.4

9.9
5.8

5.1
3.6

3.6
163.0

5.0

205.2

$\begin{array}{ll}39.8 & 10 \\ 19 . i & 5\end{array}$

79.7

304.0

140.9

302.0

298.2

273.4
82.0

97.6
.2

74.2

.2
.
.7

2.4

.

3.9

18.2

2.5

2.0

1.4

.5

.6
9.1
1.9

20.7

1.6
48.3

2.4
5.0

100.6

$\begin{array}{rr}75.5 & 29.5\end{array}$

18.6

13.4

20.6

556.4

573.9

496.9

$556.4 \quad 573.9$

496.9

$131.9 \quad 241.5$

556.4

$573 . \overline{9}$

$496 . \overline{9}$

$\begin{array}{rr}1.8 & 1.2 \\ 29.5 & 54.1\end{array}$

27.0

4.2

54.1

1.6
52.6

16.5

5.2

$\begin{array}{rr}1.7 & 2.2\end{array}$

490.5

18.4
5.8
493.6

.5
5.1
419.5
1.1

7.5

89.9

18.3

12.9
.4

22.1

1.9

2.6
18.2

13.5

204.4

$\begin{array}{ll}.1 & 1.0 \\ - & \end{array}$

.1
7.3

66.8

9.5

11.3

19.7

2.0

2.8
9.3

224.0

5.8
3.5

190.0

322.7

1.0

1.0
9.4

8.4

15.6

14.7

22.0

2.5

4.1
4.4

4.4
17.7

231.0

549.4
549.4
-
549.4
1.1
59.3
14.6
.6
5.0
463.9
5.0
-

584.0

639.8

$584.0 \quad 639.8$

$584 . \overline{0} \quad 639 . \overline{8}$

$1.4 \quad 1.4$

16.6

$\begin{array}{rr}16.6 & 20.0 \\ .5 & \end{array}$

5.2
498.1
1.9

6.5
545.0

$314.6 \quad 64$

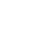

$1,566.3$

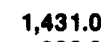

$1,617.9$

953.6

$1,690.5$

$1,860.8$

$\begin{array}{rrrrr}977.9 & 836.3 & 953.6 & 958.6 & 1,101.7 \\ 588.4 & 594.3 & 664.3 & 731.9 & 759.1\end{array}$

$\begin{array}{llllr}588.4 & 594.3 & 664.3 & 731.9 & 759.1\end{array}$

958.

$1,101.7$

$\begin{array}{llllllll}15.6 & 58.2 & 150.9 & 131.5 & 104.3 & 118.2 & 115.5 & 127.3\end{array}$

$\begin{array}{rrrr}15.6 & 58.2 & 150.9 & 131.5 \\ 9.7 & 31.3 & 46.3 & 77.4\end{array}$

$49.1 \quad 59.9$

$57.7 \quad 53.5$

(9.7

$$
26.7
$$

$104 . \overline{6}$

104.0

77.4

$55 . \overline{2}$

54.5

58.3

.1
57.7
56.3
1.3

53.5
.1
73.7

53.

.7

.7

72.2

1.5

- There are no direct fuel costs for hydroelectric, geothermal cent those consumed at electric utilities.

- Heavy oil includes Grade Nos. 4, 5, and 6, and residual fuel oils. Light oil includes Grade No. 2 heating oil, kerosene, and jet fuel.

No consumption Grade Nos. 4, 5, and 6, and residual tuel oils. Light oil includes Grade No. 2 heating oil, kerosene, and jet fuel.

- Value less than 0.05 million dollars

Note: Totals may not equal sum of components due to independent rounding.

Sources: Data sources, estimation procedures, and assumptions are described in the "Documentation" section of this report. 


\section{N Energy Price and Expenditure Estimates by Source, New Jersey E 1970, 1975, 1980, and 1985-1989}

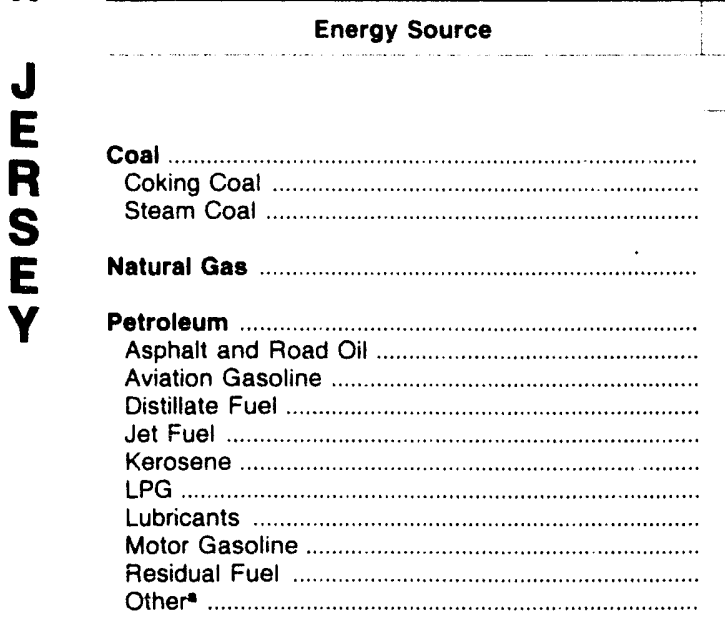

1970

1975

1980

1985

1986

1987

1988

1989

Prices in Dollare per Million Btu

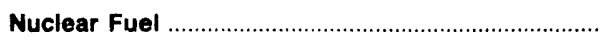

Wood and Waste at Utilities

Primary Energy - Five Sectors .............................

\section{Electric Utility Fuel .......}

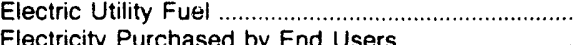

Total Energyb

.

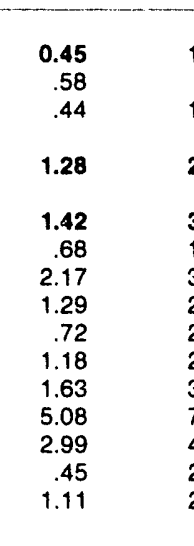

1.58

1.80

1.70

1.71

$\begin{array}{rrr}1.5 \overline{8} & 1.8 \overline{1} & 1.92 \\ & 1.8 \overline{2}\end{array}$

$$
1.80
$$

1.76

$1.71 \quad 1.63$

2.29

4.15

6.18

5.86

5.04

1.71

1.63

3.27

$\begin{array}{ll}3.27 & 7.17\end{array}$

1.89
3.45

3.45

2.03

2.03

3.59

7.49

4.79

2.08

2.70

3.65
9.02

6.75

6.26

6.35

5.72

14.36

9.94

4.53
7.07

7.51

4.89

9.99

7.92

5.76

8.70

11.82

17.61

8.95

4.33

\subsection{8}

\subsection{6}

8.41

6.23

3.84

6.55
11.53

11.53

15.59

6.89

2.34

5.85

$\mathbf{5 . 8 5}$
3.39

7.55

6.05

4.01

5.86

11.04

13.58

7.25

3.02
5.38

4.97

5.11

.18

4.64

5.79

5.79
3.07
7.41

7.41

6.44

6.00

3.79

5.53

10.62

14.61

7.58
2.42

2.42
4.50

$.71 \quad .73$

.81

.68

.66

\section{1}

2.99

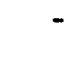

620

5

$\begin{array}{lll}.42 & 1.71 & 2.67\end{array}$

6.20
1.90

5.05

4.78

5.19

0.24

13.61

28.18

1.47

1.49

1.30

1.45

1.95

4.26

8.34

9.67

8.29

8.07

8.12

8.68

Expenditures in Millions of Dollars

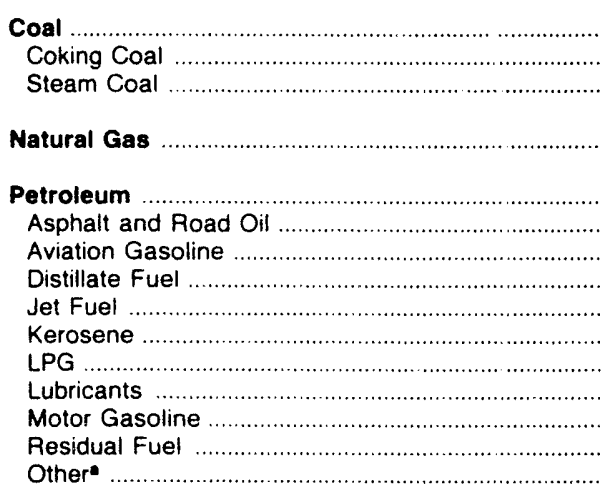

Nuclear Fuel

Wood and Waste at Utilities

Primary Energy - Five Sectors

Electric Utility Fuel

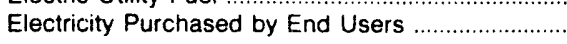

Total Energy ${ }^{b}$

Expenditures in Millions of Dollars

\begin{tabular}{|c|c|c|c|c|c|c|c|}
\hline 55.6 & 95.9 & 124.5 & 198.1 & 140.2 & 158.9 & 138.5 & 152.9 \\
\hline 5.3 & - & - & - & - & - & - & - \\
\hline 50.4 & 95.9 & 124.5 & 198.1 & 140.2 & 158.9 & 138.5 & 152.9 \\
\hline 413.8 & 556.5 & $1,434.3$ & $2,371.8$ & $2,097.3$ & $2,150.0$ & $2,087.1$ & $2,360.1$ \\
\hline $2,001.9$ & $4,088.3$ & $8,944.3$ & $8,651.6$ & $6,777.4$ & $7,042.4$ & $6,979.8$ & $7,739.2$ \\
\hline 26.3 & 62.9 & 105.7 & 153.6 & 153.5 & 119.3 & 88.4 & 72.4 \\
\hline 1.7 & 1.6 & 3.8 & 9.3 & 6.8 & 7.7 & 5.7 & 5.3 \\
\hline 468.7 & 947.8 & $2,072.7$ & $1,861.2$ & $1,629.6$ & $1,542.6$ & $1,612.5$ & $1,700.8$ \\
\hline 26.9 & 71.4 & 308.7 & $1,430.6$ & 852.8 & 984.5 & 876.3 & $1,084.9$ \\
\hline 12.2 & 19.6 & 61.0 & 31.4 & 23.0 & 25.1 & 20.0 & 26.8 \\
\hline 40.9 & 95.0 & 134.3 & 236.0 & 234.5 & 259.1 & 237.3 & 175.2 \\
\hline 60.2 & 79.1 & 206.5 & 230.4 & 199.5 & 196.4 & 203.9 & 256.1 \\
\hline $1,040.8$ & $1,951.3$ & $3,797.7$ & $3,546.4$ & $2,921.4$ & $3,090.8$ & $3,230.9$ & $3,625.8$ \\
\hline 215.4 & 575.0 & $1,419.1$ & 554.4 & 327.8 & 316.2 & 273.4 & 293.4 \\
\hline 108.7 & 284.7 & 834.8 & 598.3 & 428.5 & 500.7 & 431.6 & 498.4 \\
\hline 7.6 & 6.1 & 27.9 & 135.8 & 117.2 & 197.0 & 175.7 & 162.2 \\
\hline
\end{tabular}

\begin{tabular}{rrrrrrrr}
$2,479.0$ & $4,746.7$ & $10,531.1$ & $11,357.3$ & $9,132.1$ & $9,548.3$ & $9,381.1$ & $10,414.5$ \\
-182.1 & -451.6 & -881.5 & -730.2 & -479.1 & -655.2 & -574.2 & -654.6 \\
799.5 & $1,966.1$ & $3,538.5$ & $5,148.3$ & $5,228.8$ & $5,017.5$ & $5,265.5$ & $5,583.2$ \\
$3,096.4$ & $6,261.3$ & $13,188.1$ & $15,775.5$ & $13,881.8$ & $13,910.6$ & $14,072.5$ & $15,343.0$ \\
\hline
\end{tabular}

Includes petroleum coke used at electric utilities.

bThere are no direct fuel costs tor hydroelectric, geothermal, centralized solar, or wind energy. Wood and other biomass fuels are not included, except those consumed at electric utilities.

-No consumption, including cases where adjustments were made. See "Consumption Adjustments for Process Fuel and Intermediate Products."

Note: Expenditure totals may not equal sum of components due to independent rounding.

Sources: Data sources, estimation procedures, and assumptions are described in the "Documentation" section of this report. 


\begin{tabular}{|c|c|c|c|c|c|c|c|c|}
\hline Sector and Energy Source & 1970 & 1975 & 1980 & 1985 & 1986 & 1987 & 1988 & 1989 \\
\hline Residential Sector & 2.28 & 4.50 & 8.97 & 12.10 & 11.62 & 10.80 & 10.66 & 10.98 \\
\hline 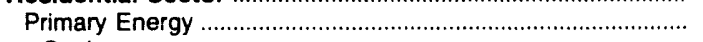 & 1.62 & 2.75 & 6.02 & 7.68 & 7.13 & 6.52 & 6.35 & 6.61 \\
\hline 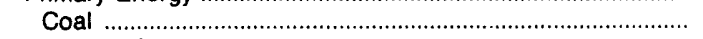 & 1.13 & 2.09 & 3.17 & 3.07 & 2.82 & 2.80 & 2.82 & 2.93 \\
\hline 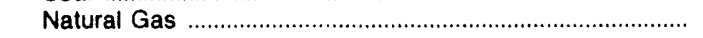 & 1.84 & 2.61 & 4.90 & 7.33 & 7.17 & 6.44 & 6.17 & 6.35 \\
\hline 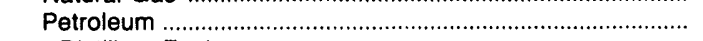 & 1.46 & 2.86 & 7.14 & 8.22 & 7.12 & 6.66 & 6.68 & 7.14 \\
\hline 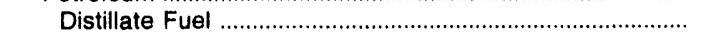 & 1.43 & 2.81 & 7.06 & 8.09 & 6.89 & 6.44 & 6.48 & 7.02 \\
\hline 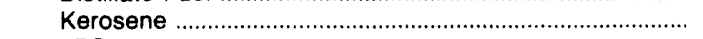 & 1.72 & 3.51 & 9.27 & 10.06 & 8.57 & 8.01 & 8.05 & 8.72 \\
\hline 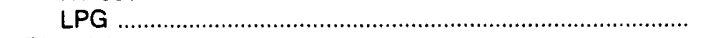 & 3.00 & 4.94 & 9.83 & 10.95 & 12.42 & 11.74 & 10.21 & 8.96 \\
\hline 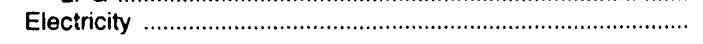 & 7.83 & 15.77 & 24.08 & 32.24 & 31.19 & 29.01 & 28.70 & 29.53 \\
\hline Commercial Sector & 2.01 & 5.19 & 9.22 & 14.18 & 12.97 & 11.99 & 11.73 & 12.29 \\
\hline 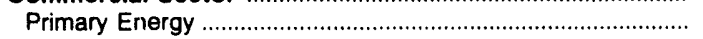 & .99 & 2.34 & 5.14 & 6.45 & 5.47 & 5.06 & 4.84 & 5.26 \\
\hline 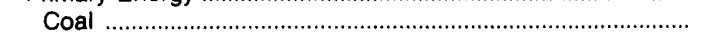 & .23 & 1.27 & 1.49 & 1.74 & 1.62 & 1.60 & 1.56 & 1.58 \\
\hline 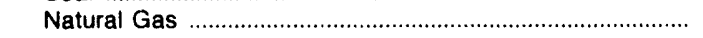 & 1.38 & 2.26 & 4.45 & 6.49 & 6.17 & 5.41 & 5.11 & 5.17 \\
\hline 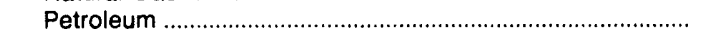 & .83 & 2.39 & 5.51 & 6.49 & 4.57 & 4.53 & 4.41 & 5.45 \\
\hline 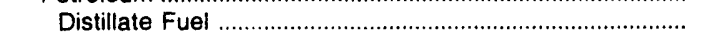 & 1.14 & 2.48 & 6.47 & 6.94 & 4.73 & 4.55 & 4.59 & 5.41 \\
\hline 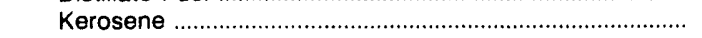 & .79 & 2.50 & 5.81 & 6.23 & 4.30 & 4.50 & 4.22 & 4.86 \\
\hline LPG & 1.43 & 3.38 & 5.15 & 12.00 & 11.32 & 10.90 & 10.74 & 9.53 \\
\hline 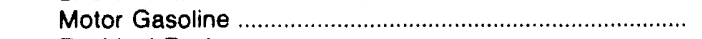 & 2.99 & 4.79 & 9.94 & 8.95 & 6.89 & 7.25 & 7.58 & 8.48 \\
\hline 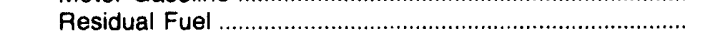 & .45 & 2.04 & 4.66 & 4.56 & 2.67 & $3 . i 2$ & 2.54 & 2.97 \\
\hline 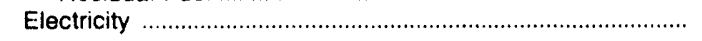 & 7.62 & 14.97 & 22.49 & 29.02 & 28.40 & 25.49 & 25.00 & 25.97 \\
\hline Industrial Sector & $\mathbf{1 . 1 8}$ & $\mathbf{3 . 4 3}$ & 6.99 & 9.47 & 7.81 & 7.33 & 7.11 & 7.67 \\
\hline 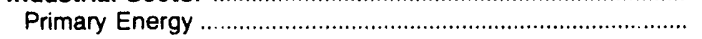 & .86 & 2.47 & 5.59 & 6.81 & 5.04 & 5.08 & 4.67 & 5.04 \\
\hline Coal & .40 & 1.27 & 1.49 & 1.74 & 1.62 & 1.60 & 1.56 & - \\
\hline Coking Coal & .58 & & - & - & - & - & - & - \\
\hline 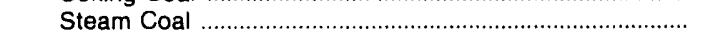 & .23 & 1.27 & 1.49 & 1.74 & 1.62 & 1.60 & 1.56 & - \\
\hline 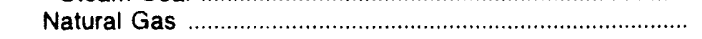 & .68 & 1.65 & 3.63 & 5.39 & 4.27 & 3.98 & 3.78 & 3.88 \\
\hline 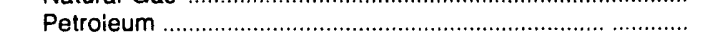 & .93 & 2.61 & 5.97 & 7.74 & 5.43 & 5.65 & 5.14 & 5.80 \\
\hline 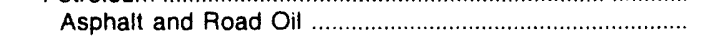 & .68 & 1.89 & 3.65 & 4.89 & 4.16 & 3.39 & 3.07 & 2.71 \\
\hline 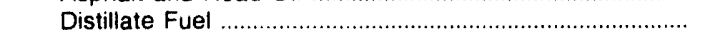 & .76 & 2.36 & 5.48 & 6.16 & 4.25 & 4.45 & 4.17 & 4.81 \\
\hline Kerosene & .79 & 2.50 & 5.81 & 6.23 & 4.30 & 4.50 & 4.22 & 4.86 \\
\hline LPG & 1.43 & 3.38 & 5.15 & 12.00 & 11.32 & 10.90 & 10.74 & 9.53 \\
\hline Lubricants & 5.08 & 7.49 & 14.36 & 17.61 & 15.59 & 13.58 & 14.61 & 17.90 \\
\hline 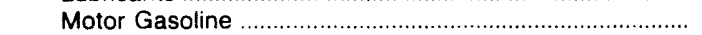 & 2.99 & 4.79 & 9.94 & 8.95 & 6.89 & 7.25 & 7.58 & 8.48 \\
\hline 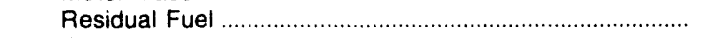 & .48 & 2.15 & 4.69 & 4.56 & 2.67 & 3.12 & 2.54 & 2.97 \\
\hline Other & 1.11 & 2.70 & 7.07 & 7.92 & 4.64 & 5.38 & 4.50 & 5.27 \\
\hline 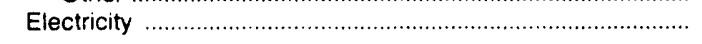 & 3.89 & 10.03 & 16.96 & 22.54 & 22.09 & 19.68 & 19.83 & 21.00 \\
\hline 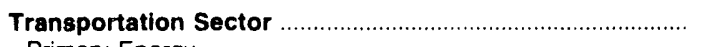 & 2.39 & 4.32 & 8.60 & 7.56 & 5.73 & 6.03 & 6.19 & 6.82 \\
\hline Primary Energy & 2.39 & 4.32 & 8.60 & 7.55 & 5.72 & 6.01 & 6.17 & 6.80 \\
\hline Coal & .23 & 1.27 & & - & - & - & - & - \\
\hline 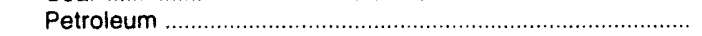 & 2.39 & 4.32 & 8.60 & 7.55 & 5.72 & 6.01 & 6.17 & 6.80 \\
\hline 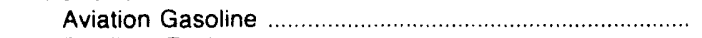 & 2.17 & 3.45 & 9.02 & 9.99 & 8.41 & 7.55 & 7.41 & 8.28 \\
\hline 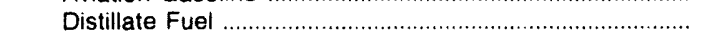 & 1.57 & 3.21 & 7.34 & 8.51 & 6.96 & 6.83 & 6.80 & 7.22 \\
\hline Jet Fuel & .72 & 2.01 & 6.27 & 5.76 & 3.84 & 4.01 & 3.79 & 4.34 \\
\hline LPG & 1.43 & 3.38 & 5.15 & 12.00 & 11.32 & 10.90 & 10.74 & 9.53 \\
\hline Lubricants & 5.08 & 7.49 & 14.36 & 17.61 & 15.59 & 13.58 & 14.61 & 17.90 \\
\hline 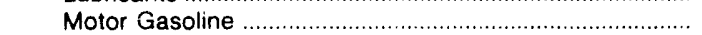 & 2.99 & 4.79 & 9.94 & 8.95 & 6.89 & 7.25 & 7.58 & 8.48 \\
\hline 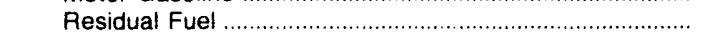 & .41 & 1.81 & 3.94 & 4.17 & 2.16 & 2.87 & 2.17 & 2.57 \\
\hline Electricity . . & 4.62 & 11.14 & 14.91 & 21.28 & 18.46 & 48.18 & 46.87 & 47.21 \\
\hline Total Energya & 1.95 & 4.26 & 8.34 & 9.67 & 8.29 & 8.07 & 8.12 & 8.68 \\
\hline 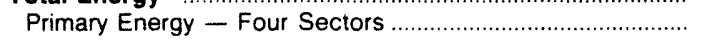 & 1.57 & 3.24 & 6.82 & 7.34 & 5.83 & 5.83 & 5.79 & 6.29 \\
\hline Electricity . & 6.24 & 13.61 & 21.26 & 28.18 & 27.56 & 25.16 & 24.97 & 25.95 \\
\hline Electric Utillity Sector & .42 & 1.71 & 2.67 & 1.90 & 1.47 & 1.49 & 1.30 & 1.45 \\
\hline Coal & .45 & 1.59 & 1.80 & 1.92 & 1.81 & 1.77 & 1.72 & 1.76 \\
\hline 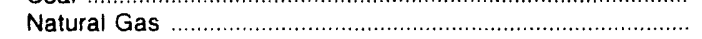 & .39 & .95 & 3.01 & 3.97 & 2.51 & 2.55 & 2.25 & 2.46 \\
\hline 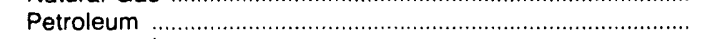 & .45 & 2.12 & 4.98 & 4.62 & 2.44 & 3.29 & 2.68 & 3.24 \\
\hline 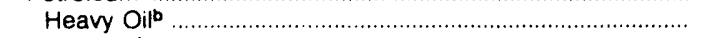 & .45 & 2.12 & 4.79 & 4.41 & 2.38 & 3.12 & 2.51 & 3.03 \\
\hline Light Oilb & .45 & 2.14 & 5.93 & 6.24 & 3.20 & 4.10 & 3.53 & 4.08 \\
\hline 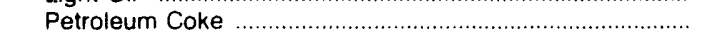 & - & & - & - & - & - & - & - \\
\hline 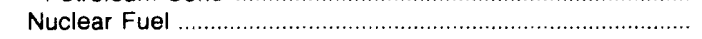 & .20 & .18 & .34 & .71 & .73 & .81 & 68 & .66 \\
\hline Wood and Waste & - & - & - & - & - & - & - & - \\
\hline Primary Energy - Five Sectors. & 1.31 & 2.99 & 6.04 & 6.20 & 5.05 & 4.86 & 4.78 & 5.19 \\
\hline
\end{tabular}

-There are no direct fuel costs for hydroelectric, geothermal, centralized solar, or wind energy. Wood and other biomass fuels are not included, except those consumed at electric utilities.

bHeavy oil includes Grade Nos. 4, 5, and 6, and residual fuel oils. Light oil includes Grade No. 2 heating oil, kerosene, and jet fuel

- No consumption, including cases where adjustments were made. See "Consumption Adjustments for Process Fuel and intermediate Products."

Sources: Data sources. estimation procedures, and assumptions are described in the "Documentation" section of this report. 


\section{N Energy Expenditure Estimates by Sector, New Jersey \\ E $1970,1975,1980$, and $1985-1989$ \\ W (Million Dollars)}

\begin{tabular}{|c|c|c|c|c|c|c|c|c|}
\hline Soctor and Enorgy Source & 1970 & 1975 & 1980 & 1985 & 1986 & 1987 & 1988 & 1989 \\
\hline $\begin{array}{l}\text { Resldential Sector } \\
\text { Primary Energy }\end{array}$ & $\begin{array}{l}882.8 \\
558.7\end{array}$ & $\begin{array}{r}1,657.8 \\
877.8\end{array}$ & $\begin{array}{l}3,062.8 \\
1,721.3\end{array}$ & $\begin{array}{l}3,937.6 \\
2,048.0\end{array}$ & $\begin{array}{l}3,848.3 \\
1,923.3\end{array}$ & $\begin{array}{l}3,739.9 \\
1,828.6\end{array}$ & $\begin{array}{l}3,893.4 \\
1,870.4\end{array}$ & $4,068.9$ \\
\hline Primary Energy & 2.4 & 2.2 & 2.4 & $\begin{array}{r}2,048.0 \\
4.4\end{array}$ & $\begin{array}{r}1,923.3 \\
2.5\end{array}$ & $\begin{array}{r}1,828.6 \\
1.3\end{array}$ & $\begin{array}{r}1,870.4 \\
1.1\end{array}$ & $\begin{array}{r}1,983.9 \\
.7\end{array}$ \\
\hline 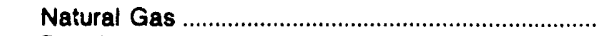 & 264.7 & 348.4 & 691.2 & $1,130.9$ & $1,163.4$ & 1.113 .0 & $1,147.1$ & $1,273.0$ \\
\hline Petroleum & 291.6 & 527.3 & 1.027 .7 & 912.7 & 757.4 & 714.3 & 722.2 & 710.3 \\
\hline 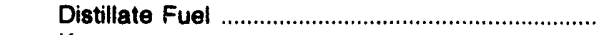 & 274.6 & 501.0 & 985.9 & 852.0 & 693.4 & 654.5 & 659.9 & 650.9 \\
\hline 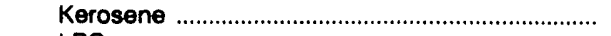 & 7.5 & 8.6 & 13.8 & 23.4 & 15.9 & 13.3 & 9.9 & 13.8 \\
\hline LPG & 9.5 & 17.7 & 28.0 & 37.3 & 48.1 & 46.4 & 52.3 & 45.6 \\
\hline 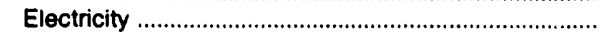 & 324.1 & 780.0 & $1,341.5$ & $1,889.6$ & $1,925.0$ & $1,911.3$ & $2,023.0$ & $2,085.0$ \\
\hline 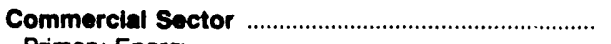 & 478.8 & $1,085.2$ & $2,259.8$ & 2,955.7 & $2,999.8$ & $2,057.1$ & $2,990.3$ & $3,316.8$ \\
\hline 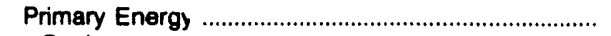 & 197.9 & 378.0 & 964.5 & 885.1 & 850.6 & 797.5 & 812.9 & 936.4 \\
\hline Coal & .3 & .9 & .8 & 1.9 & 1.0 & .5 & .4 & .3 \\
\hline 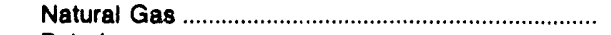 & 79.3 & 124.2 & 278.0 & 553.5 & 543.1 & 523.3 & 530.9 & 622.1 \\
\hline Petroleum & 118.2 & 252.9 & 685.8 & 329.7 & 306.5 & 273.7 & 281.6 & 314.0 \\
\hline 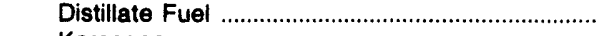 & 74.0 & 149.4 & 345.2 & 227.9 & 245.0 & 206.3 & 211.4 & 257.4 \\
\hline 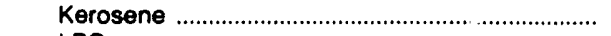 & 1.3 & 2.4 & 1.3 & 1.2 & 1.3 & 1.6 & 1.3 & 3.5 \\
\hline LPG & .8 & 2.1 & 2.6 & 7.2 & 7.7 & 7.6 & 9.7 & 8.6 \\
\hline 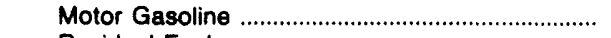 & 9.6 & 15.9 & 15.5 & 31.0 & 23.6 & 25.3 & 25.8 & 29.8 \\
\hline 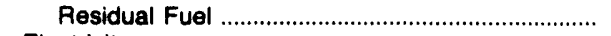 & 32.5 & 83.0 & 321.1 & 62.3 & 28.8 & 32.9 & 33.5 & 14.7 \\
\hline 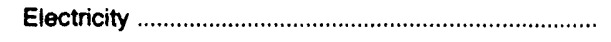 & 280.9 & 707.2 & $1,295.3$ & $2,070.5$ & $2,149.3$ & $2,059.6$ & $2,177.4$ & $2,380.4$ \\
\hline Industrial Sector & 561.0 & $1,277.1$ & $3,001.3$ & $2,932.5$ & $2,497.2$ & $2,492.4$ & $2,342.3$ & $2,446.4$ \\
\hline 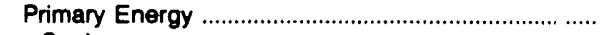 & 366.9 & 799.8 & $2,101.2$ & $1,750.7$ & $1,348.5$ & $1,461.1$ & $1,290.8$ & $1,342.2$ \\
\hline 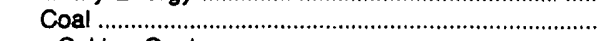 & 7.5 & 2.0 & 1.2 & 15.0 & 10.6 & 13.0 & 10.2 & - \\
\hline 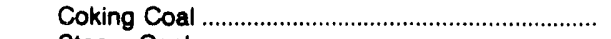 & 5.3 & - & - & - & - & - & - & - \\
\hline 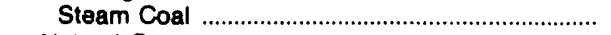 & 2.2 & 2.0 & 1.2 & 15.0 & 10.6 & 13.0 & 10.2 & - \\
\hline 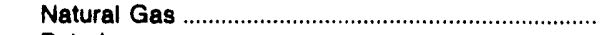 & 51.4 & 75.5 & 217.5 & 433.0 & 294.9 & 315.7 & 290.6 & 324.5 \\
\hline Petroleum & 308.0 & 722.4 & $1,882.5$ & $1,302.8$ & $1,043.0$ & $1,132.4$ & 990.0 & $1,017.7$ \\
\hline 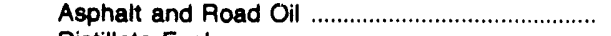 & 26.3 & 62.9 & 105.7 & 153.6 & 153.5 & 119.3 & 88.4 & 72.4 \\
\hline 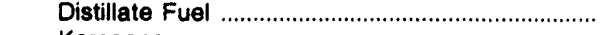 & 38.6 & 109.5 & 230.9 & 89.3 & 83.3 & 76.5 & 77.5 & 96.8 \\
\hline 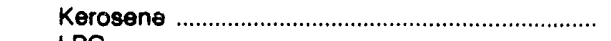 & 3.4 & 8.7 & 45.9 & 6.7 & 5.8 & 10.2 & 8.8 & 9.5 \\
\hline LPG & 30.0 & 73.9 & 102.9 & 187.8 & 175.4 & 202.3 & 172.5 & 118.6 \\
\hline 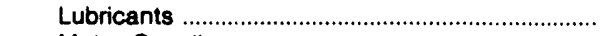 & 42.5 & 51.6 & 144.5 & 161.2 & 139.5 & 137.4 & 142.6 & 179.1 \\
\hline 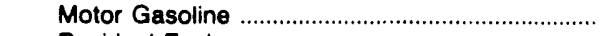 & 6.3 & 5.9 & 7.7 & 21.7 & 16.9 & 19.6 & 20.9 & 22.3 \\
\hline 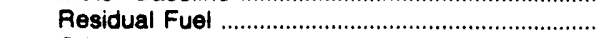 & 52.1 & 125.3 & 410.2 & 84.1 & 40.1 & 66.5 & 47.7 & 20.6 \\
\hline Other & 108.7 & 284.7 & 834.8 & 598.3 & 428.5 & 500.7 & 431.6 & 498.4 \\
\hline Electricity & 194.0 & 477.3 & 900.1 & $1,181.8$ & $1,148.7$ & $1,031.3$ & $1,051.5$ & $1,104.2$ \\
\hline 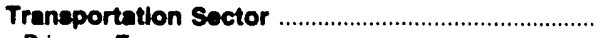 & $1,173.9$ & $2,241.2$ & $4,864.2$ & $5,949.7$ & $4,536.5$ & $4,821.3$ & $4,846.5$ & $5,510.9$ \\
\hline 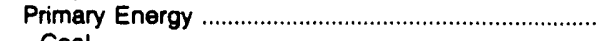 & $1,173.4$ & $2,239.5$ & $4,862.6$ & $5,943.3$ & $4,530.7$ & $4,805.9$ & $4,832.9$ & $5,497.3$ \\
\hline 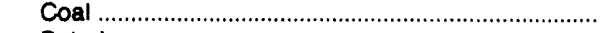 & & & & & & & & \\
\hline 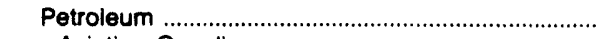 & 1.173 .4 & $2,239.5$ & $4,862.6$ & $5,943.3$ & $4,530.7$ & $4,805.9$ & $4,832.9$ & $5,497.3$ \\
\hline Aviation Gasoline & 1.7 & 1.6 & 3.8 & 9.3 & 6.8 & 7.7 & 5.7 & 5.3 \\
\hline 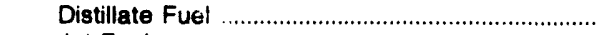 & 78.3 & 166.5 & 438.1 & 667.6 & 595.0 & 580.8 & 629.6 & 645.2 \\
\hline Jet Fuel & 26.9 & 64.9 & 284.6 & $1,430.6$ & 852.8 & 984.5 & 876.3 & $1,084.9$ \\
\hline LPG & .6 & 1.2 & .8 & 3.7 & 3.3 & 2.7 & 2.7 & 2.4 \\
\hline 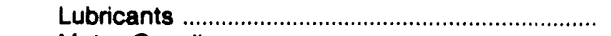 & 17.7 & 27.5 & 62.1 & 69.3 & 60.0 & 59.0 & 61.3 & 77.0 \\
\hline 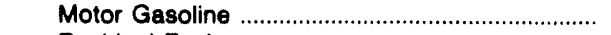 & $1,024.9$ & $1,929.5$ & $3,774.5$ & $3,493.6$ & $2,880.9$ & $3,045.9$ & $3,184.2$ & $3,573.7$ \\
\hline 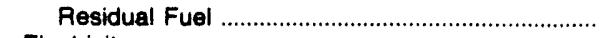 & 23.3 & 48.3 & 298.7 & 269.3 & 132.0 & 125.2 & 73.1 & 108.7 \\
\hline 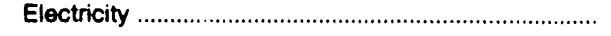 & .5 & 1.7 & 1.6 & 6.4 & 5.8 & 15.4 & 13.6 & 13.7 \\
\hline Total Enorgy" & $3,096.4$ & $6,261.3$ & $13,188.1$ & $15,775.5$ & $13,881.8$ & $13,910.6$ & $14,072.5$ & $15,343.0$ \\
\hline Primary Energy - Four Sectors ..................................... & $2,296.9$ & $4,295.2$ & $9,649.5$ & $10,627.2$ & $8,653.0$ & $8,893.1$ & $8,807.0$ & $9,759.8$ \\
\hline 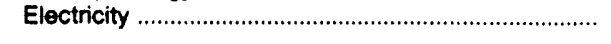 & 799.5 & $1,966.1$ & $3,538.5$ & $5,148.3$ & $5,228.8$ & $5,017.5$ & $5,265.5$ & $5,583.2$ \\
\hline 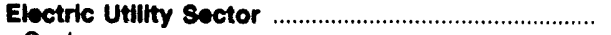 & 182.1 & 451.6 & 881.5 & 730.2 & 479.1 & 655.2 & 574.2 & 654.6 \\
\hline Coal & 45.4 & 90.8 & 120.2 & 176.8 & 126.1 & 144.2 & 126.9 & 151.9 \\
\hline Natural Gas & 18.4 & 8.4 & 247.6 & 254.5 & 95.9 & 197.9 & 118.4 & 140.5 \\
\hline Petroleum & 110.6 & 346.2 & 485.8 & 163.1 & 139.9 & 116.1 & 153.2 & 199.9 \\
\hline 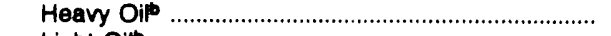 & 107.5 & 318.4 & 389.1 & 138.7 & 126.9 & 91.6 & 119.1 & 149.5 \\
\hline 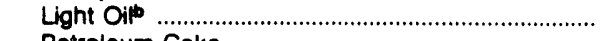 & 3.2 & 27.9 & 96.7 & 24.4 & 13.0 & 24.5 & 34.1 & 50.5 \\
\hline Petroleum Coke & - & - & - & - & & & & \\
\hline 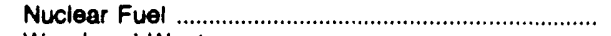 & 7.6 & 6.1 & 27.9 & 135.8 & 117.2 & 197.0 & 175.7 & 162.2 \\
\hline Wood and Waste & & - & & - & - & - & - & \\
\hline Primary Energy - Five Sectore ………………............ & $2,479.0$ & $4,746.8$ & $10,531.1$ & $11,357.3$ & $9,132.1$ & 9,548.3 & $9,381.1$ & $10,414.5$ \\
\hline
\end{tabular}

-There are no direct fuel costs for hydroelectric, geothermal, centralized solar, or wind energy. Wood and other biomass fuels are not included, except those consumed at electric utilities.

Heavy oil includes Grade Nos. 4, 5, and 6, and residual fuel oils. Light oil includes Grade No, 2 heating oil, kerosene, and jet fuel.

-No consumption, including cases where adjustments were made. See "Consumption Adjustments for Process Fuel and Intermediate Produr.ts." - Value less than 0.05 million dollars.

Note: Totals may not equal sum of components due to independent rounding.

Sources: Data sources, estimation procedures, and assumptions are described in the "Documentation" section of this report. 


\begin{tabular}{|c|c|c|c|c|c|c|c|c|}
\hline Energy Source & 1970 & 1875 & 1880 & 1885 & 1886 & 1987 & 1988 & 1989 \\
\hline & \multicolumn{8}{|c|}{ Pricea In Dollars per Million Btu } \\
\hline 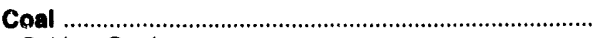 & 0.14 & 0.23 & 0.56 & 1.09 & 1.14 & 1.21 & 1.18 & 1.23 \\
\hline Coking Coal & - & - & - & - & - & - & - & - \\
\hline 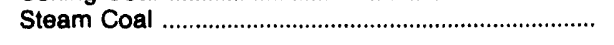 & .14 & .23 & .56 & 1.09 & 1.14 & 1.21 & 1.18 & 1.23 \\
\hline 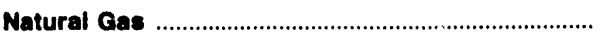 & .39 & .75 & 2.66 & 4.60 & 4.24 & 3.68 & 3.44 & 3.61 \\
\hline Potroleum & 1.92 & 3.43 & 7.80 & 7.61 & 5.83 & 6.24 & 6.15 & 6.73 \\
\hline 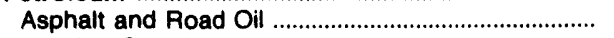 & .58 & 1.84 & 3.67 & 4.79 & 4.35 & 3.19 & 3.39 & 2.87 \\
\hline Aviation Gasoline & 2.17 & 3.45 & 9.02 & 9.99 & 8.41 & 7.55 & 7.41 & 8.28 \\
\hline 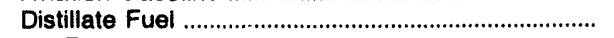 & 1.07 & 2.42 & 6.80 & 6.53 & 4.87 & 5.50 & 5.31 & 6.34 \\
\hline Jet Fuel & .76 & 2.12 & 6.59 & 6.24 & 4.39 & 4.28 & 4.17 & 4.79 \\
\hline Kerosene & 1.03 & 2.26 & 6.91 & 7.15 & 4.25 & 4.85 & 4.17 & 5.00 \\
\hline LPG & 1.35 & 3.17 & 5.84 & 8.22 & 6.89 & 7.21 & 6.35 & 6.32 \\
\hline 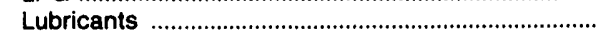 & 5.08 & 7.49 & 14.36 & 17.61 & 15.59 & 13.58 & 14.61 & 17.90 \\
\hline Motor Gasoline & 2.94 & 4.72 & 9.58 & 9.14 & 7.02 & 7.46 & 7.44 & 8.24 \\
\hline 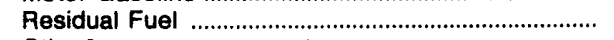 & .34 & 1.66 & 3.80 & 3.99 & 2.08 & 2.82 & 1.82 & 1.97 \\
\hline 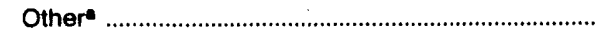 & .50 & 1.37 & 2.65 & 2.59 & 2.84 & 3.26 & 2.85 & 3.61 \\
\hline 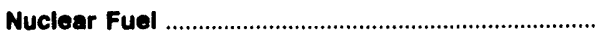 & - & - & - & - & - & - & - & - \\
\hline 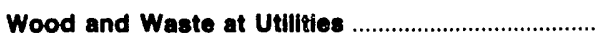 & - & - & - & - & - & - & - & - \\
\hline Primary Energy - Five Sectors ................................... & .85 & 1.63 & 3.71 & 4.05 & 3.42 & 3.48 & 3.38 & 3.67 \\
\hline 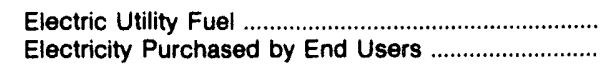 & $\begin{array}{r}.20 \\
5.62\end{array}$ & $\begin{array}{r}.45 \\
7.99\end{array}$ & $\begin{array}{r}1.02 \\
15.52\end{array}$ & $\begin{array}{r}1.33 \\
21.20\end{array}$ & $\begin{array}{r}1.31 \\
22.14\end{array}$ & $\begin{array}{r}1.29 \\
21.83\end{array}$ & $\begin{array}{r}1.25 \\
21.76\end{array}$ & $\begin{array}{r}1.32 \\
21.55\end{array}$ \\
\hline \multirow{2}{*}{ 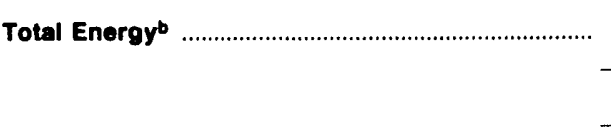 } & 1.46 & 2.88 & 7.08 & 8.98 & 7.78 & 7.80 & 7.67 & 8.13 \\
\hline & \multicolumn{8}{|c|}{ Expenditures in Millions of Dollars } \\
\hline 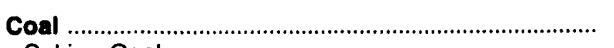 & 14.3 & 30.0 & 114.2 & 293.7 & 274.3 & 315.2 & 312.9 & 345.0 \\
\hline 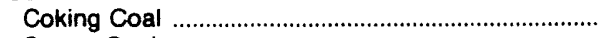 & - & - & - & - & - & - & - & - \\
\hline 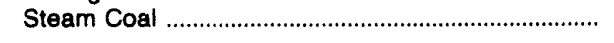 & 14.3 & 30.0 & 114.2 & 293.7 & 274.3 & 315.2 & 312.9 & 345.0 \\
\hline 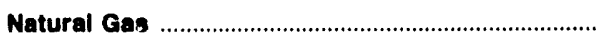 & 80.7 & 134.8 & 394.1 & 350.8 & 303.8 & 280.8 & 320.8 & 355.3 \\
\hline Petroleum & 292.3 & 653.3 & $1,507.8$ & $1,606.9$ & $1,187.4$ & $1,307.7$ & $1,284.3$ & $1,511.3$ \\
\hline 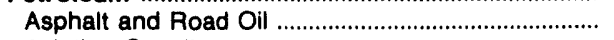 & 4.6 & 19.9 & 27.7 & 47.7 & 46.7 & 43.7 & 47.6 & 31.7 \\
\hline 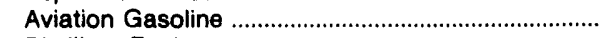 & 1.2 & 1.4 & 7.6 & 4.8 & 4.4 & 3.3 & 2.1 & 4.0 \\
\hline 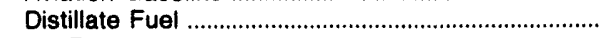 & 33.6 & 94.7 & 315.6 & 323.9 & 275.5 & 341.2 & 316.2 & 331.4 \\
\hline 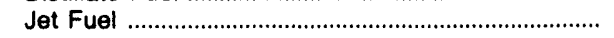 & 12.9 & 30.9 & 96.0 & 97.7 & 66.8 & 70.0 & 64.1 & 74.7 \\
\hline 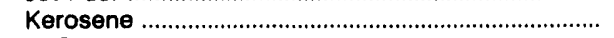 & 5.8 & 8.4 & 52.5 & 72.4 & 22.1 & 26.0 & 21.8 & 20.4 \\
\hline LPG & 22.0 & 41.3 & 98.6 & 101.1 & 45.6 & 42.2 & 38.3 & 168.8 \\
\hline Lubricants & 8.3 & 14.4 & 28.9 & 32.2 & 27.9 & 27.5 & 28.5 & 35.8 \\
\hline 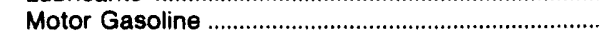 & 202.9 & 409.2 & 850.8 & 859.3 & 674.8 & 740.5 & 755.8 & 817.1 \\
\hline 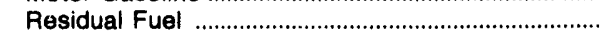 & .4 & 31.0 & 23.5 & 65.7 & 21.1 & 10.4 & 7.4 & 24.3 \\
\hline 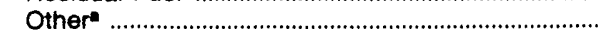 & .5 & 2.0 & 6.6 & 2.0 & 2.6 & 2.9 & 2.6 & 3.1 \\
\hline 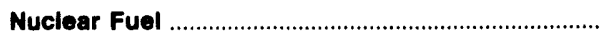 & - & - & - & - & - & - & - & - \\
\hline 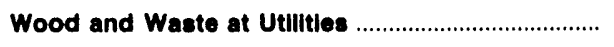 & - & - & - & - & - & - & - & - \\
\hline Primary Energy - Five Sectors ................................. & 387.3 & 818.1 & $2,016.1$ & $2,251.4$ & $1,765.6$ & $1,903.6$ & $1,918.0$ & $2,211.7$ \\
\hline 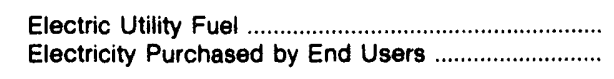 & $\begin{array}{l}-32.0 \\
106.6\end{array}$ & $\begin{array}{l}-95.4 \\
179.5\end{array}$ & $\begin{array}{r}-268.0 \\
460.2\end{array}$ & $\begin{array}{r}-392.6 \\
836.0\end{array}$ & $\begin{array}{r}-342.2 \\
874.2\end{array}$ & $\begin{array}{r}-359.9 \\
893.3\end{array}$ & $\begin{array}{r}-359.3 \\
923 . C\end{array}$ & $\begin{array}{r}-407.0 \\
958.2\end{array}$ \\
\hline Total Energy & 461.9 & 902.3 & $2,208.2$ & $2,694.8$ & $2,297.6$ & $2,437.1$ & $2,481.7$ & $2,762.9$ \\
\hline
\end{tabular}

Includes petroleum coke used at electric utilities

bThere are no direct fuel costs for hydroelectric, geothermal, centralized solar, or wind energy. Wood and other biomass fuels are not included, except those consumed at electric utilities.

-No consumption, including cases where adjustments were made. See "Consumption Adjustments for Process Fuel and Intermediate Products."

Note: Expenditure totals may not equal sum of components due to independent rounding.

Sources: Data sources, estimation procedures, and assumptions are described in the "Documentation" section of this report 


\begin{tabular}{|c|c|c|c|c|c|c|c|c|}
\hline Sector and Energy Source & 1970 & 1975 & 1980 & 1985 & 1986 & 1987 & 1988 & 1989 \\
\hline Residential Sector & 1.78 & 3.06 & 6.66 & 10.93 & 10.83 & 10.16 & 10.40 & 11.06 \\
\hline 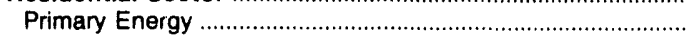 & 1.00 & 1.64 & 3.78 & 6.53 & 5.56 & 5.03 & 5.08 & 5.81 \\
\hline Coal & .90 & - & 2.54 & 2.83 & 2.78 & 2.40 & 2.12 & 2.43 \\
\hline 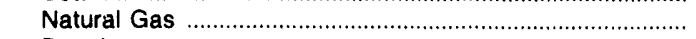 & .86 & 1.24 & 3.17 & 5.59 & 5.28 & 4.61 & 4.87 & 5.45 \\
\hline Petroleum & 1.60 & 4.12 & 7.29 & 8.60 & 6.86 & 7.15 & 6.34 & 7.39 \\
\hline 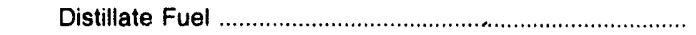 & .98 & 2.82 & 6.79 & 6.92 & 5.09 & 4.81 & 4.74 & 5.34 \\
\hline 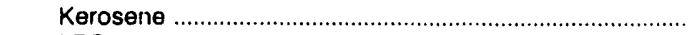 & 1.49 & 3.05 & 7.95 & 8.62 & 6.34 & 5.99 & 5.91 & 6.65 \\
\hline LPG & 1.61 & 4.16 & 7.19 & 8.62 & 7.16 & 7.78 & 6.51 & 7.48 \\
\hline 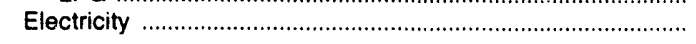 & 8.15 & 10.47 & 18.89 & 25.48 & 26.43 & 26.39 & 26.42 & 26.41 \\
\hline Commercial Sector & 1.37 & 2.74 & 6.79 & 12.06 & 11.41 & 10.99 & 9.34 & 10.45 \\
\hline 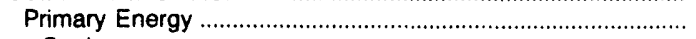 & .50 & .94 & 3.50 & 5.72 & 4.06 & 3.85 & 3.26 & 3.74 \\
\hline Coal & .56 & - & .88 & 1.41 & 1.38 & 1.40 & 1.33 & 1.18 \\
\hline 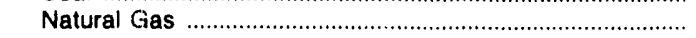 & .44 & .74 & 2.79 & 5.34 & 4.04 & 3.67 & 3.08 & 3.54 \\
\hline 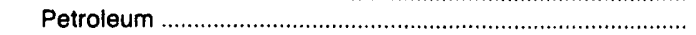 & 1.34 & 3.03 & 6.85 & 6.63 & 4.23 & 4.44 & 4.04 & 4.92 \\
\hline 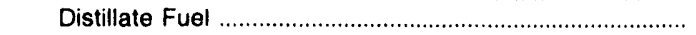 & .92 & 2.62 & 6.57 & 5.62 & 3.23 & 3.78 & 3.34 & 3.89 \\
\hline 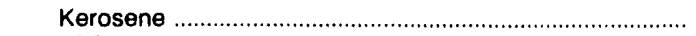 & 1.01 & 2.22 & 6.80 & 6.75 & 3.33 & 4.19 & 3.67 & 4.73 \\
\hline LPG & 1.12 & 2.60 & 5.36 & 7.27 & 6.51 & 6.06 & 6.11 & 6.00 \\
\hline 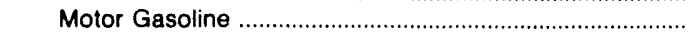 & 2.94 & 472 & 9.58 & 9.14 & 7.02 & 7.46 & 7.44 & 8.24 \\
\hline 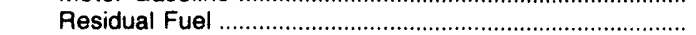 & - & - & - & 4.00 & - & 1.70 & $8.44-$ & 0.24 \\
\hline 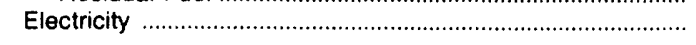 & 5.78 & 7.90 & 15.95 & 22.57 & 23.41 & 22.49 & 23.10 & 22.68 \\
\hline Industrial Sector & .65 & 1.52 & 4.95 & 7.07 & 5.86 & 6.10 & 5.81 & 5.82 \\
\hline 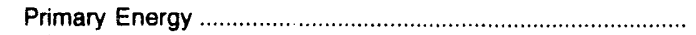 & .49 & 1.27 & 4.06 & 5.28 & 3.46 & 3.78 & 3.52 & 4.11 \\
\hline Coal & .56 & - & .88 & 1.41 & 1.38 & 1.40 & 1.33 & - \\
\hline 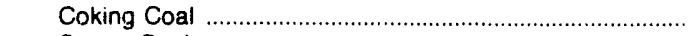 & - & - & - & - & - & - & - & - \\
\hline Steam Coal & .56 & - & .88 & 1.41 & 1.38 & 1.40 & 1.33 & - \\
\hline Natural Gas & .25 & .58 & 2.46 & 3.67 & 3.04 & 3.30 & 3.16 & 2.97 \\
\hline Petroleum & 1.01 & 2.13 & 5.34 & 5.56 & 3.56 & 3.89 & 3.64 & 4.40 \\
\hline Asphalt and Road Oil & .58 & 1.84 & 3.67 & 4.79 & 4.35 & 3.19 & 3.39 & 2.87 \\
\hline 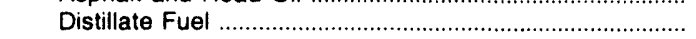 & .95 & 2.05 & 6.42 & 6.11 & 3.02 & 3.80 & 3.32 & 4.28 \\
\hline 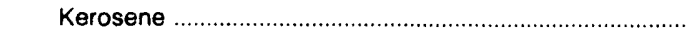 & 1.01 & 2.22 & 6.80 & 6.75 & 3.33 & 4.19 & 3.67 & 4.73 \\
\hline LPG & 1.12 & 2.60 & 5.36 & 7.27 & 6.51 & 6.06 & 6.11 & 6.00 \\
\hline 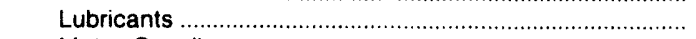 & 5.08 & 7.49 & 14.36 & 17.61 & 15.59 & 13.58 & 14.61 & 17.90 \\
\hline Motor Gasoline & 2.94 & 4.72 & 9.58 & 9.14 & 7.02 & 7.46 & 7.44 & 8.24 \\
\hline 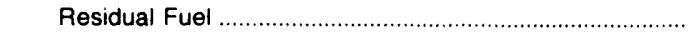 & .41 & 1.60 & 3.82 & 4.00 & 2.09 & 2.86 & 1.81 & 1.96 \\
\hline Other & .50 & 1.37 & 2.65 & 2.59 & 2.84 & 3.26 & 2.85 & 3.61 \\
\hline 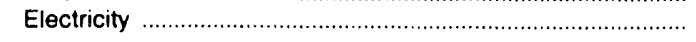 & 3.44 & 5.54 & 12.11 & 16.01 & 16.64 & 16.56 & 15.56 & 15.53 \\
\hline Transportation Sector & 2.28 & 4.03 & 8.69 & 8.47 & 6.65 & 7.03 & 6.99 & 7.73 \\
\hline 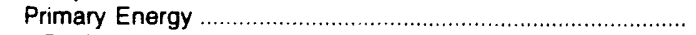 & 2.28 & 4.03 & 8.69 & 8.47 & 9.65 & 7.03 & 6.99 & 7.73 \\
\hline Coal & .56 & - & - & - & - & - & - & - \\
\hline 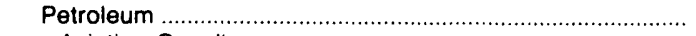 & 2.28 & 4.03 & 8.69 & 8.47 & 6.65 & 7.03 & 6.99 & 7.73 \\
\hline 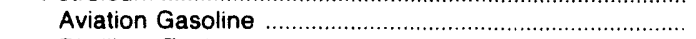 & 2.17 & 3.45 & 9.02 & 9.99 & 8.41 & 7.55 & 7.41 & 8.28 \\
\hline Distillate Fuel ........ & 1.15 & 2.62 & 6.97 & 6.98 & 6.29 & 6.89 & 6.67 & 7.30 \\
\hline Jet Fuel & .76 & 2.12 & 6.59 & 6.24 & 4.39 & 4.28 & 4.17 & 4.79 \\
\hline LPG & 1.12 & 2.60 & 5.36 & 7.27 & 6.51 & 6.06 & 6.11 & 6.00 \\
\hline 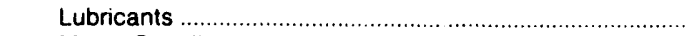 & 5.08 & 7.49 & 14.36 & 17.61 & 15.59 & 13.58 & 14.61 & 17.90 \\
\hline 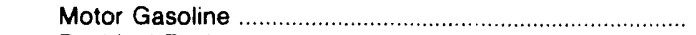 & 2.94 & 4.72 & 9.58 & 9.14 & 7.02 & 7.46 & 7.44 & 8.24 \\
\hline Residual Fuel ........ & .38 & - & - & - & - & 1.70 & - & - \\
\hline 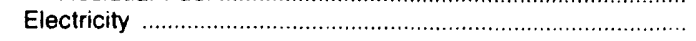 & - & - & - & - & - & - & - & - \\
\hline Total Energy" .................................. & 1.46 & 2.88 & 7.08 & $8.9 p$ & 7.78 & 7.90 & 7.67 & 8.13 \\
\hline 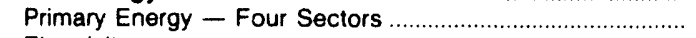 & 1.20 & 2.49 & 6.20 & 7.14 & 5.56 & 5.77 & 5.54 & 6.11 \\
\hline Electricity & 5.62 & 7.99 & 15.52 & 21.20 & 22.14 & 21.83 & 21.76 & 21.55 \\
\hline Eloctric Utility Sector & .20 & .45 & 1.02 & 1.33 & 1.31 & 1.29 & 1.25 & 1.32 \\
\hline 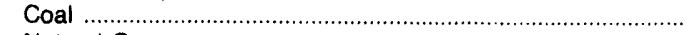 & .14 & .23 & .56 & 1.09 & 1.13 & 1.21 & 1.18 & 1.24 \\
\hline Natural Gas & .30 & .69 & 2.47 & 3.48 & 3.31 & 2.36 & 2.14 & 2.13 \\
\hline 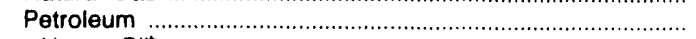 & .23 & 1.70 & 5.21 & 4.98 & 2.69 & 3.58 & 3.17 & 3.99 \\
\hline 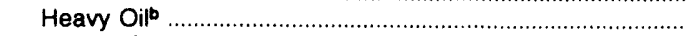 & .23 & 1.70 & 3.70 & 3.71 & 1.65 & 2.14 & 1.97 & 2.74 \\
\hline 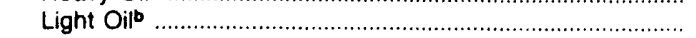 & .27 & 1.89 & 6.53 & 6.20 & 3.79 & 4.47 & 4.22 & 4.98 \\
\hline Petroleum Coke & - & - & - & - & - & - & - & - \\
\hline 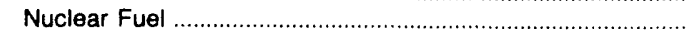 & - & - & - & - & - & - & - & - \\
\hline 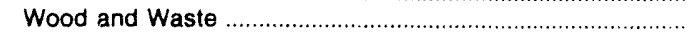 & - & - & - & - & - & - & - & - \\
\hline Primary Energy - Five Sectors & .85 & 1.63 & 3.71 & 4.05 & 3.42 & 3.48 & 3.38 & 3.67 \\
\hline
\end{tabular}

-There are no direct fuel costs for hydroelectric, geothermal, centralized solar, or wind energy. Wood and other biomass fuels are not included, except those consumed at electric utilities.

b Heavy oil includes Grade Nos. 4, 5, and 6, and residual fuel oils. Light oil includes Grade No. 2 heating oil, kerosene, and jet fuel.

-No consumption, including cases where adjustments were made. See "Consumption Adjustments for Procens Fuel and Intermediate Products."

Sources: Data sources, estimation procedures, and assumptions are described in the "Documentation" section of this report. 


\begin{tabular}{|c|c|c|c|c|c|c|c|c|}
\hline Sector and Energy Source & 1970 & 1975 & 1980 & 1985 & 1986 & 1987 & 1988 & 1989 \\
\hline $\begin{array}{l}\text { Pesidential Sector } \\
\text { Primary Energy }\end{array}$ & $\begin{array}{l}82.0 \\
41.0\end{array}$ & $\begin{array}{r}127.1 \\
57.2\end{array}$ & $\begin{array}{l}292.1 \\
134.1\end{array}$ & $\begin{array}{l}497.2 \\
227.9\end{array}$ & $\begin{array}{l}459.8 \\
176.3\end{array}$ & $\begin{array}{l}477.5 \\
179.8\end{array}$ & $\begin{array}{l}483.2 \\
177.2\end{array}$ & $\begin{array}{l}512.6 \\
200.7\end{array}$ \\
\hline 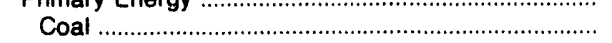 & . & - & .8 & .2 & .1 & .1 & 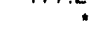 & $\begin{array}{r}200.1 \\
.2\end{array}$ \\
\hline 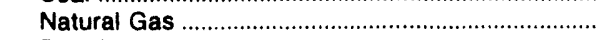 & 28.6 & 37.0 & 95.0 & 133.4 & 137.3 & 137.1 & 145.6 & 152.4 \\
\hline 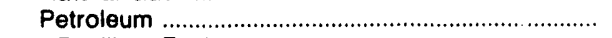 & 12.4 & 20.2 & 38.3 & 94.3 & 38.9 & 42.6 & 31.5 & 48.1 \\
\hline 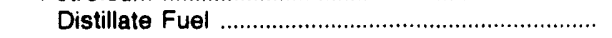 & $\cdot$ & .1 & .4 & 8 & 1.0 & .4 & .3 & .3 \\
\hline 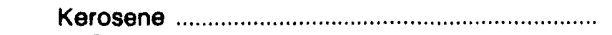 & .2 & .5 & 6.0 & 18.7 & 10.1 & 11.7 & 7.0 & 3.9 \\
\hline LPG & 12.2 & 19.6 & 31.9 & 74.8 & 27.8 & 30.5 & 24.2 & 43.9 \\
\hline 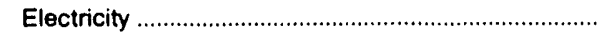 & 41.0 & 69.9 & 158.1 & 269.4 & 283.5 & 297.7 & 306.0 & 312.0 \\
\hline 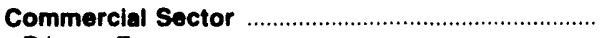 & 62.7 & 99.4 & 296.3 & 510.1 & 497.9 & 506.4 & 554.1 & 573.8 \\
\hline 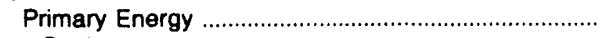 & 19.0 & 25.4 & 112.4 & 150.9 & 110.0 & 109.6 & 134.1 & 132.8 \\
\hline 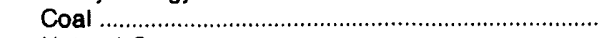 & * & - & .5 & .1 & .1 & .1 & .1 & .1 \\
\hline 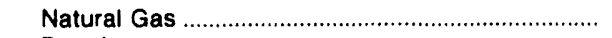 & 15.7 & 18.2 & 71.7 & 97.2 & 90.2 & 79.2 & 102.7 & 105.9 \\
\hline 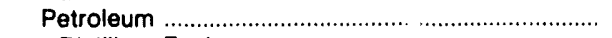 & 3.2 & 7.2 & 40.1 & 53.5 & 19.7 & 30.3 & 31.3 & 26.7 \\
\hline 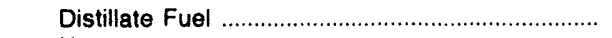 & 6 & 2.7 & 5.1 & 14.8 & 7.6 & 15.6 & 10.9 & 11.5 \\
\hline Kerosene & $\cdot$ & .1 & 25.4 & 21.8 & 3.3 & 5.8 & 11.8 & 3.9 \\
\hline LPG & 1.5 & 2.2 & 4.2 & 11.1 & 4.5 & 4.2 & 4.0 & 6.2 \\
\hline 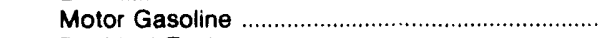 & 1.1 & 2.3 & 5.5 & 5.4 & 4.3 & 4.8 & 4.6 & 5.2 \\
\hline 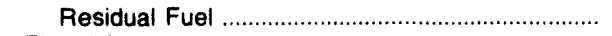 & - & - & - & .3 & - & - & - & - \\
\hline 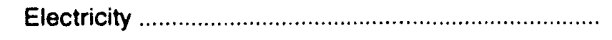 & 43.7 & 74.0 & 184.0 & 359.2 & 387.8 & 396.8 & 420.0 & 441.0 \\
\hline 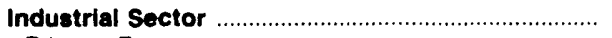 & 76.8 & 165.0 & 436.1 & 549.4 & 391.7 & 402.9 & 385.7 & 512.9 \\
\hline 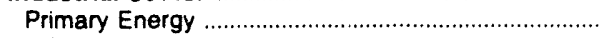 & 54.9 & 129.4 & 318.0 & 341.9 & 188.9 & 204.1 & 188.7 & 307.7 \\
\hline Coal & .1 & - & .2 & 2.5 & 2.8 & 1.5 & 1.5 & - \\
\hline 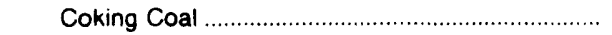 & - & - & - & - & - & - & - & - \\
\hline 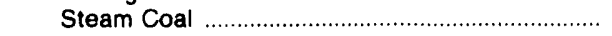 & .1 & - & .2 & 2.5 & 2.8 & 1.5 & 1.5 & - \\
\hline Natural Gas & 18.7 & 32.8 & 84.5 & 21.1 & 6.8 & 19.8 & 26.3 & 36.8 \\
\hline 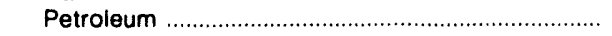 & 36.0 & 96.5 & 233.4 & 318.3 & 179.3 & 182.8 & 160.9 & 270.9 \\
\hline 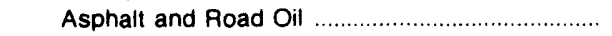 & 4.6 & 19.9 & 27.7 & 47.7 & 46.7 & 43.7 & 47.6 & 31.7 \\
\hline Distillate Fuel . & 11.7 & 27.5 & 82.1 & 130.5 & 66.7 & 89.0 & 69.1 & 55.9 \\
\hline Kerosene & 5.5 & 7.8 & 21.1 & 31.9 & 8.7 & 8.6 & 3.1 & 12.6 \\
\hline LPG & 7.3 & 17.5 & 61.9 & 12.9 & 11.4 & 5.9 & 8.5 & 116.1 \\
\hline 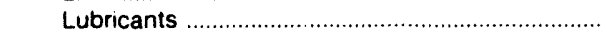 & 3.2 & 5.4 & 10.3 & 11.5 & 10.0 & 9.8 & 10.2 & 12.8 \\
\hline Motor Gasoline & 3.0 & 3.6 & 4.2 & 17.3 & 12.6 & 12.9 & 13.0 & 15.0 \\
\hline 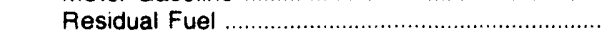 & .3 & 12.8 & 19.4 & 64.4 & 20.6 & 10.0 & 6.9 & 23.7 \\
\hline Other & .5 & 2.0 & 6.6 & 2.0 & 2.6 & 2.9 & 2.6 & 3.1 \\
\hline 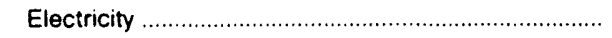 & 21.9 & 35.6 & 118.1 & 207.5 & 202.9 & 198.8 & 196.9 & 205.2 \\
\hline 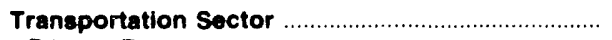 & 240.4 & 510.8 & $1,183.6$ & $1,138.2$ & 948.1 & $1,050.3$ & $1,058.8$ & $1,163.5$ \\
\hline Primary Energy & 240.4 & 510.8 & $1,183.6$ & $1,138.2$ & 948.1 & $1,050.3$ & $1,058.8$ & 1.163 .5 \\
\hline 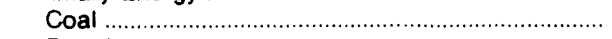 & $\cdot$ & - & - & - & - & - & - & - \\
\hline 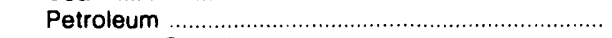 & 240.4 & 510.8 & $1,183.6$ & $1,138.2$ & 948.1 & $1,050.3$ & $1,058.8$ & $1,163.5$ \\
\hline 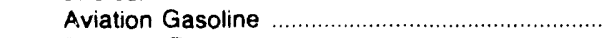 & 1.2 & 1.4 & 7.6 & 4.8 & 4.4 & 3.3 & 2.1 & 4.0 \\
\hline 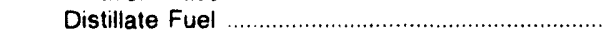 & 21.2 & 64.0 & 219.7 & 176.1 & 199.2 & 234.9 & 234.5 & 262.3 \\
\hline Jet Fuel . . & 12.9 & 30.9 & 96.0 & 97.7 & 66.8 & 70.0 & 64.1 & 74.7 \\
\hline LPG & 1.0 & 2.0 & .6 & 2.3 & 1.9 & 1.5 & 1.7 & 2.5 \\
\hline 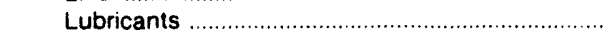 & 5.1 & 9.0 & 18.6 & 20.7 & 17.9 & 17.7 & 18.3 & $\quad 23.0$ \\
\hline Motor Gasoline & 198.9 & 403.4 & 841.2 & 836.6 & 657.8 & 722.8 & 738.1 & 796.9 \\
\hline 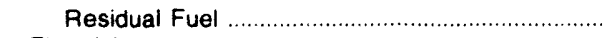 & $\cdot$ & - & - & - & - & - & - & - \\
\hline 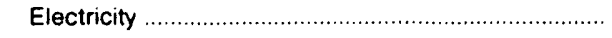 & - & - & - & - & - & - & - & - \\
\hline 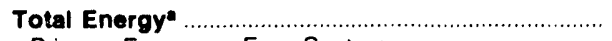 & 461.9 & 902.3 & $2,208.2$ & $2,684.8$ & $2,297.6$ & $2,437.1$ & $2,481.7$ & $2,762.9$ \\
\hline 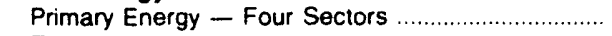 & 355.2 & 722.7 & $1,748.0$ & $1,858.8$ & 1.423 .3 & $1,543.7$ & $1,558.7$ & $1,804.7$ \\
\hline 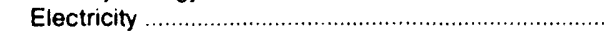 & 106.6 & 1795 & 460.2 & 836.0 & 874.2 & 893.3 & 923.0 & 958.2 \\
\hline Electric Utility Sector & 32.0 & 95.4 & 268.0 & 392.6 & 342.2 & 359.9 & 359.3 & 407.0 \\
\hline Coal & 14.2 & 30.0 & 112.8 & 290.9 & 271.3 & 313.5 & 311.3 & 344.7 \\
\hline 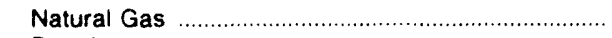 & 17.7 & 46.8 & 142.9 & 99.1 & 69.5 & 44.6 & 46.2 & 60.3 \\
\hline 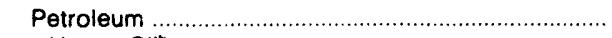 & .1 & 18.6 & 12.3 & 2.6 & 1.4 & 1.8 & 1.8 & 2.0 \\
\hline 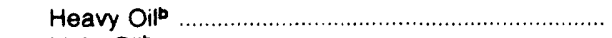 & .1 & 18.2 & 4.1 & .9 & 4 & .4 & .5 & 6 \\
\hline 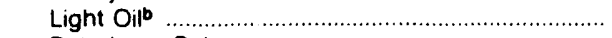 & • & .4 & 8.2 & 1.6 & 9 & 1.4 & 1.3 & 1.4 \\
\hline 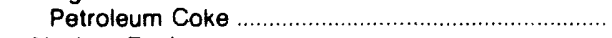 & - & - & - & - & - & - & - & - \\
\hline 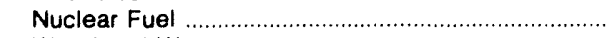 & - & - & - & - & - & - & - & - \\
\hline Wood and Waste & - & - & - & - & - & - & - & - \\
\hline 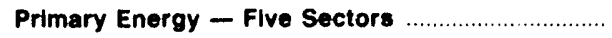 & 387.3 & 818.1 & $2,016.1$ & $2,251.4$ & $1,765.6$ & $1,903.6$ & $1,918.0$ & $2,211.7$ \\
\hline
\end{tabular}

-There are no direct fuel costs for hydroelectric, geothermal, centralized solar, or wind energy. Wood and other biomass fueis are not included, except those consumed at electric utilities.

Heavy oil includes Grade Nos. 4, 5, and 6, and residual fuel oils. Light oll includes Grade No. 2 heating oil, kerosene, and jet fuel.

- No consumption, including cases where adjustments were made. See "Consumption Adjustments for Process Fuel and Intermediate Products."

"Value less than 0.05 million dollars.

Note: Totals may not equal sum of components due to independent rounding

Sources: Data sources, estimation procedures, and assumptions are described in the "Documentation" section of this report. 


\section{N Energy Price and Expenditure Estimates by Source, New York $1970,1975,1980$, and 1985-1989}

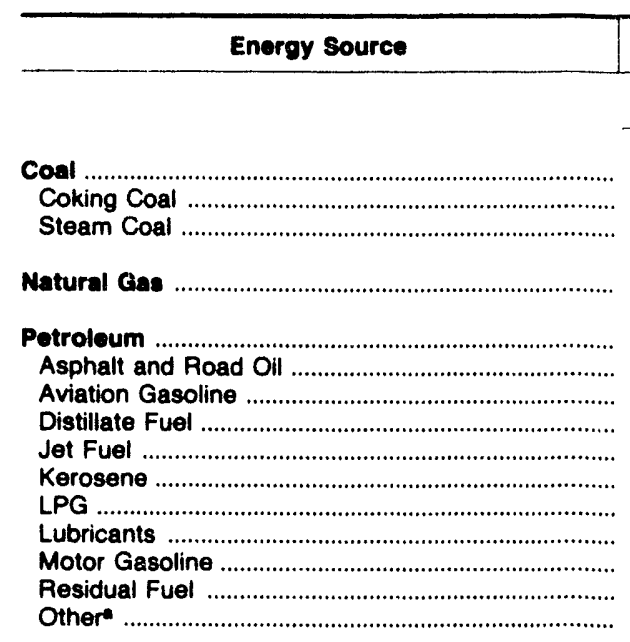

Nuclear Fuel

Wood and Waste at Utilitice

Primary Energy - Flve Sectors

Electric Utility Fuel.

Electricity Purchased by End Users

Total Energyb

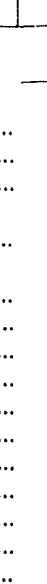

1970

1975

1980

980

1985

1986

1987

1988

1989

Prices in Dollars per Million Btu

\begin{tabular}{|c|c|c|c|c|c|c|c|}
\hline 0.51 & 1.53 & 1.78 & 1.81 & 1.72 & 1.61 & 1.62 & 1.63 \\
\hline .58 & 2.14 & 2.38 & 1.88 & 1.76 & 1.59 & 1.61 & 1.67 \\
\hline .49 & 1.27 & 1.56 & 1.80 & 1.71 & 1.62 & 1.62 & 1.62 \\
\hline 1.07 & 2.16 & 4.10 & 5.94 & 5.65 & 5.06 & 5.09 & 5.38 \\
\hline 1.36 & 2.96 & 6.93 & 7.45 & 5.45 & 5.76 & 5.47 & 6.15 \\
\hline .70 & 1.84 & 3.67 & 4.81 & 4.34 & 3.73 & 3.18 & 2.81 \\
\hline 2.17 & 3.45 & 9.02 & 9.99 & 8.41 & 7.55 & 7.41 & 8.28 \\
\hline 1.24 & 2.66 & 6.78 & 7.92 & 6.25 & 6.09 & 6.11 & 6.64 \\
\hline .72 & 2.02 & 6.27 & 6.51 & 4.42 & 4.55 & 4.15 & 4.70 \\
\hline 1.39 & 3.06 & 7.78 & 8.22 & 6.72 & 6.55 & 6.68 & 6.75 \\
\hline 2.24 & 3.99 & 7.43 & 11.39 & 10.48 & 10.12 & 10.00 & 11.03 \\
\hline 5.08 & 7.49 & 14.36 & 17.61 & 15.59 & 13.58 & 14.61 & 17.90 \\
\hline 2.92 & 4.80 & 10.26 & 8.79 & 6.60 & 6.99 & 7.16 & 7.82 \\
\hline .43 & 1.93 & 4.10 & 4.35 & 2.57 & 3.09 & 2.52 & 2.97 \\
\hline 1.11 & 2.66 & 7.07 & 6.55 & 4.32 & 5.68 & 4.81 & 5.54 \\
\hline .20 & .31 & .56 & .67 & .61 & .63 & .57 & .65 \\
\hline - & - & 1.74 & - & - & .95 & - & - \\
\hline 1.17 & 2.60 & 5.45 & 5.87 & 4.79 & 4.77 & 4.58 & 5.02 \\
\hline $\begin{array}{r}.42 \\
6.72\end{array}$ & $\begin{array}{r}1.53 \\
14.04\end{array}$ & $\begin{array}{r}2.68 \\
19.64\end{array}$ & $\begin{array}{r}2.54 \\
26.97\end{array}$ & $\begin{array}{r}1.79 \\
26.07\end{array}$ & $\begin{array}{r}2.01 \\
25.56\end{array}$ & $\begin{array}{r}1.80 \\
25.09\end{array}$ & $\begin{array}{r}2.06 \\
26.08\end{array}$ \\
\hline 1.81 & 4.11 & 8.08 & 10.49 & 9.24 & 9.14 & 9.11 & 9.94 \\
\hline
\end{tabular}

Expenditures in Millions of Dollars

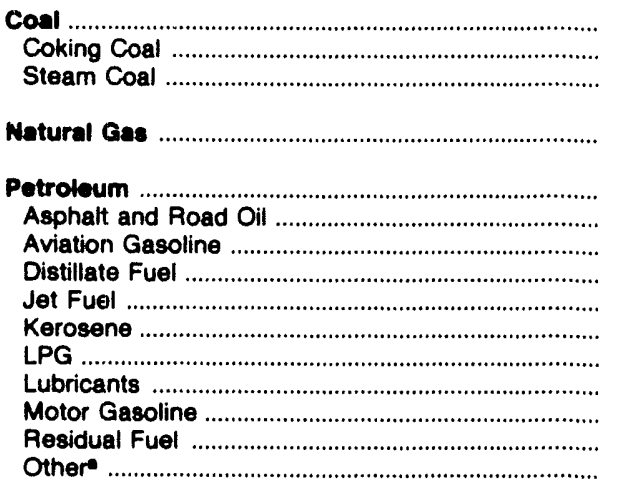

Othere

Nuctear Fuel

Wood and Waste at Utilitios

Primary Energy - Five Soctore

Eloctric Utility Fuel.

Electricity Purchased by End Users

Total Energyb

....

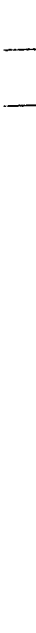

\begin{tabular}{|c|c|c|c|c|c|c|c|}
\hline 308.4 & 477.1 & 558.6 & 546.0 & 435.4 & 473.5 & 539.1 & 589.7 \\
\hline 96.4 & 197.8 & 197.6 & 58.5 & 58.0 & 61.6 & 72.6 & 74.1 \\
\hline 212.0 & 279.3 & 361.0 & 487.6 & 377.4 & 411.9 & 466.6 & 515.6 \\
\hline 771.3 & $1,255.2$ & $3,087.1$ & $4,637.2$ & $4,213.2$ & $3,999.6$ & $4,090.8$ & $4,627.0$ \\
\hline $3,608.3$ & $7,617.9$ & $14,904.3$ & $11,911.0$ & $9,307.1$ & $10,029.8$ & $9,889.2$ & $10,784.3$ \\
\hline 26.0 & 69.9 & 121.3 & 230.1 & 185.3 & 162.0 & 168.7 & 92.7 \\
\hline 2.7 & 4.8 & 14.6 & 11.1 & 10.9 & 4.8 & 3.9 & 3.7 \\
\hline 803.3 & $1,626.9$ & $2,862.3$ & $2,861.6$ & $2,568.6$ & $2,590.2$ & $2,684.5$ & $2,963.2$ \\
\hline 155.5 & 441.7 & $1,275.3$ & 139.0 & 91.8 & 72.8 & 113.6 & 158.9 \\
\hline 55.3 & 90.3 & 101.8 & 112.3 & 59.1 & 88.5 & 92.1 & 89.7 \\
\hline 37.2 & 70.3 & 139.0 & 203.1 & 189.1 & 194.9 & 195.6 & 235.6 \\
\hline 67.8 & 88.5 & 182.2 & 203.2 & 175.9 & 173.2 & 179.8 & 225.9 \\
\hline $2,005.9$ & $3,368.0$ & $6,865.7$ & $6,299.5$ & $4,740.2$ & $5,234.5$ & $5,018.8$ & $5,480.3$ \\
\hline 409.7 & $1,740.1$ & $2,964.1$ & $1,631.0$ & $1,125.3$ & $1,335.8$ & $1,284.3$ & $1,366.3$ \\
\hline 44.8 & 117.4 & 378.0 & 219.9 & 160.9 & 173.0 & 147.9 & 168.1 \\
\hline 9.2 & 44.9 & 118.3 & 175.2 & 146.3 & 154.6 & 148.3 & 159.1 \\
\hline - & - & .2 & - & - & - & - & - \\
\hline $4,687.1$ & 9,395.1 & $18,668.6$ & $17,269.4$ & $14,102.0$ & $14,657.6$ & $14,667.4$ & $16,160.1$ \\
\hline $\begin{array}{r}-335.3 \\
2,006.4\end{array}$ & $\begin{array}{r}-1,326.8 \\
4,580.0\end{array}$ & $\begin{array}{r}-2,427.3 \\
7,044.4\end{array}$ & $\begin{array}{l}-2,320.5 \\
10,369.9\end{array}$ & $\begin{array}{l}-1,558.8 \\
10,220.2\end{array}$ & $\begin{array}{r}-1,917.7 \\
10,328.0\end{array}$ & $\begin{array}{r}-1,892.7 \\
10,706.6\end{array}$ & $\begin{array}{l}-2,303.0 \\
11,352.8\end{array}$ \\
\hline $6,368.1$ & $12,648.2$ & $23,285.7$ & $25,318.8$ & $22,763.5$ & $23,067.9$ & $23,481.3$ & $25,209.8$ \\
\hline
\end{tabular}

Includes petroleum coke used at electric utilities.

bThere are no direct fuel costs for hydroelectric, geothermal, centralized solar, or wind energy. Wood and other biomass fuels are not included, except those consumed at electric utilities.

- No consumption, including cases where adjustments were made. See "Consumption Adjustments for Process Fuel and Intermediate Products."

"Value less than 0.05 million dollars.

Note: Expenditure totals may not equal sum of components due to independent rounding

Sources: Data sources, estimation procedures, and assumptions are described in the "Documentation" section of this report. 


\begin{tabular}{|c|c|c|c|c|c|c|c|c|}
\hline Sector and Energy Source & 1970 & 1975 & 1980 & 1985 & 1986 & 1987 & 1988 & 1989 \\
\hline Residential Sector ................ & $2: 19$ & 4.40 & 8.42 & 12.03 & 11.14 & 10.76 & 10.65 & 11.55 \\
\hline 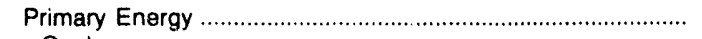 & 1.43 & 2.70 & 5.80 & 7.88 & 7.14 & 6.63 & 6.45 & 7.18 \\
\hline Coal & 1.43 & 2.78 & 3.26 & 3.61 & 3.39 & 3.27 & 3.29 & 3.36 \\
\hline 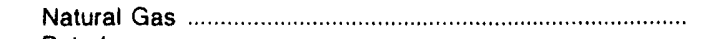 & 1.37 & 2.50 & 4.85 & 7.54 & 7.26 & 6.68 & 6.32 & 7.01 \\
\hline 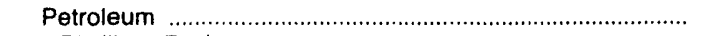 & 1.48 & 2.89 & 7.22 & 8.55 & 7.03 & 6.61 & 6.69 & 7.51 \\
\hline Distillate Fuel & 1.43 & 2.81 & 7.08 & 8.35 & 6.83 & 6.39 & 6.47 & 7.18 \\
\hline 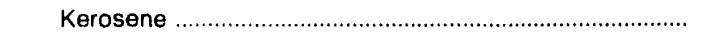 & 1.56 & 3.28 & 8.49 & 9.30 & 7.61 & 7.12 & 7.21 & 8.00 \\
\hline LPG & 2.70 & 4.48 & 9.12 & 11.12 & 9.96 & 9.64 & 9.53 & 11.54 \\
\hline Electricity & 8.83 & 16.44 & 23.08 & 31.84 & 30.86 & 30.81 & 30.67 & 32.03 \\
\hline Commercial Sector & 1.99 & 5.42 & 9.27 & 14.30 & 12.87 & 12.48 & 12.21 & 13.29 \\
\hline Primary Energy & .80 & 2.11 & 4.70 & 5.95 & 4.95 & 4.69 & 4.67 & 5.22 \\
\hline 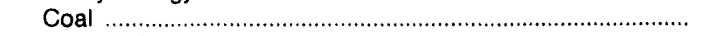 & .48 & 1.36 & 1.67 & 1.91 & 1.74 & 1.76 & 1.70 & 1.75 \\
\hline 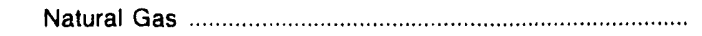 & 1.17 & 1.97 & 4.17 & 5.95 & 5.61 & 5.02 & 5.24 & 5.46 \\
\hline 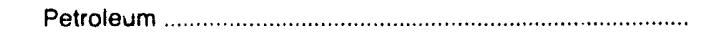 & .67 & 2.19 & 5.09 & 6.09 & 4.45 & 4.45 & 4.12 & 4.99 \\
\hline 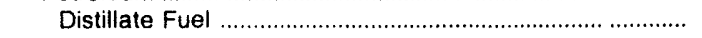 & 1.14 & 2.48 & 6.48 & 7.03 & 5.34 & 5.24 & 5.12 & 5.53 \\
\hline 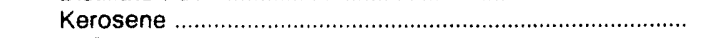 & .73 & 2.51 & 5.68 & 6.56 & 4.41 & 4.64 & 4.67 & 5.03 \\
\hline LPG & 1.45 & 3.09 & 5.79 & 11.95 & 11.62 & 11.29 & 11.21 & 9.73 \\
\hline Motor Gasoline & 2.92 & 4.80 & 10.26 & 8.79 & 6.60 & 6.99 & 7.16 & 7.82 \\
\hline 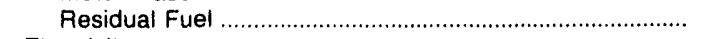 & .42 & 1.90 & 4.18 & 4.64 & 2.92 & 3.22 & 2.67 & 3.12 \\
\hline Electricity & 7.80 & 16.57 & 23.21 & 30.86 & 29.62 & 27.96 & 27.13 & 27.99 \\
\hline Industrlal Sector & .96 & 2.85 & 5.40 & 7.06 & 6.45 & 6.41 & 6.29 & 6.97 \\
\hline 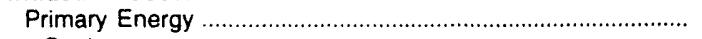 & 69 & 2.06 & 3.98 & 4.74 & 3.98 & 3.85 & 3.73 & 4.01 \\
\hline Coal & .53 & 1.82 & 2.08 & 1.90 & 1.75 & 1.68 & 1.66 & 1.71 \\
\hline Coking Coal & .58 & 2.14 & 2.38 & 1.88 & 1.76 & 1.59 & 1.61 & 1.67 \\
\hline 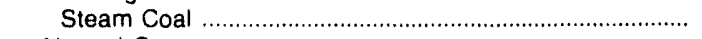 & .48 & 1.36 & 1.67 & 1.91 & 1.74 & 1.76 & 1.70 & 1.75 \\
\hline 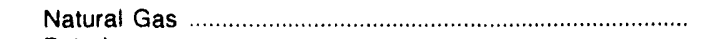 & .68 & 1.47 & 3.43 & 5.13 & 4.78 & 4.16 & 4.56 & 4.69 \\
\hline 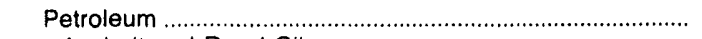 & .80 & 2.35 & 5.33 & 6.25 & 4.78 & 5.02 & 4.53 & 5.33 \\
\hline 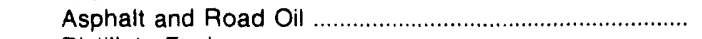 & .70 & 1.84 & 3.67 & 4.81 & 4.34 & 3.73 & 3.18 & 2.81 \\
\hline 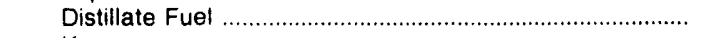 & .70 & 2.36 & 5.36 & 6.13 & 4.12 & 4.33 & 4.36 & 4.70 \\
\hline 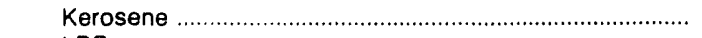 & .73 & 2.51 & 5.68 & 6.56 & 4.41 & 4.64 & 4.67 & 5.03 \\
\hline LPG & 1.45 & 3.09 & 5.79 & 11.95 & 11.62 & 11.29 & 11.21 & 9.73 \\
\hline Lubricants & 5.08 & 7.49 & 14.36 & 17.61 & 15.59 & 13.58 & 14.61 & 17.90 \\
\hline Motor Gasoline & 2.92 & 4.80 & 10.26 & 8.79 & 6.60 & 6.99 & 7.16 & 7.82 \\
\hline 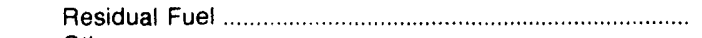 & .49 & 2.01 & 3.78 & 4.64 & 2.92 & 3.22 & 2.67 & 3.12 \\
\hline Other & 1.11 & 2.66 & 7.07 & 6.55 & 4.32 & 5.68 & 4.81 & 5.54 \\
\hline 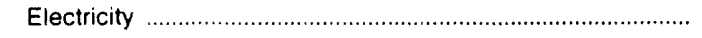 & 3.51 & 7.97 & 12.11 & 15.34 & 14.43 & 14.74 & 14.47 & 15.52 \\
\hline Transportation Sector & 2.14 & 4.02 & 8.86 & 8.84 & 6.70 & 7.06 & 7.13 & 7.83 \\
\hline Primary Energy & 2.12 & 3.95 & 8.82 & 8.74 & 6.57 & 6.92 & 6.99 & 7.69 \\
\hline 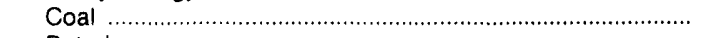 & .48 & 1.36 & - & - & - & - & - & - \\
\hline 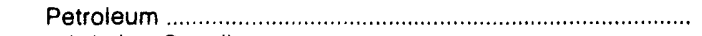 & 2.12 & 3.95 & 8.82 & 8.74 & 6.57 & 6.92 & 6.99 & 7.69 \\
\hline 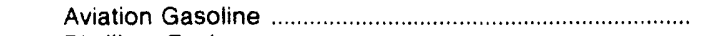 & 2.17 & 3.45 & 9.02 & 9.99 & 8.41 & 7.55 & 7.41 & 8.28 \\
\hline 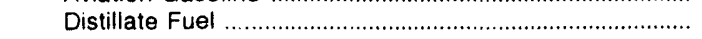 & 1.44 & 2.84 & 7.45 & 8.48 & 6.59 & 6.74 & 6.78 & 7.35 \\
\hline 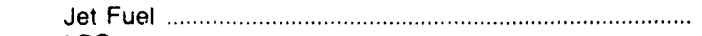 & .72 & 2.01 & 6.27 & 6.51 & 4.42 & 4.55 & 4.15 & 4.70 \\
\hline LPG & 1.45 & 3.09 & 5.79 & 11.95 & 11.62 & 11.29 & 11.21 & 9.73 \\
\hline Lubricants & 5.08 & 7.49 & 14.36 & 17.61 & 15.59 & 13.58 & 14.61 & 17.90 \\
\hline Motor Gasoline & 2.92 & 4.80 & 10.26 & 8.79 & 6.60 & 6.99 & 7.16 & 7.82 \\
\hline 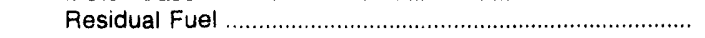 & .37 & 1.67 & 3.53 & 4.08 & 2.27 & 2.86 & 2.20 & 2.66 \\
\hline 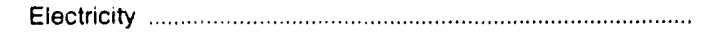 & 4.82 & 13.66 & 15.02 & 19.65 & 19.51 & 22.59 & 21.39 & 22.65 \\
\hline Total Energy a & 1.81 & 4.11 & 8.08 & 10.49 & 9.24 & 9.14 & 9.11 & 9.94 \\
\hline 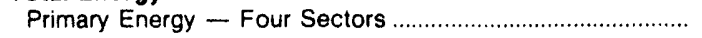 & 1.36 & 2.93 & 6.44 & 7.37 & 6.05 & 6.01 & 5.94 & 6.60 \\
\hline Electricity & 6.72 & 14.04 & 19.64 & 26.97 & 26.07 & 25.56 & 25.09 & 26.08 \\
\hline Electric Utllity Sector & .42 & 1.53 & 2.68 & 2.54 & 1.79 & 2.01 & 1.80 & 2.06 \\
\hline 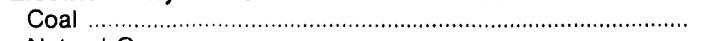 & .47 & 1.18 & 1.47 & 1.72 & 1.65 & 1.54 & 1.58 & 1.57 \\
\hline 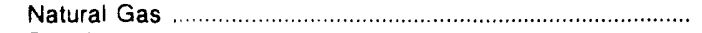 & .38 & .88 & 2.67 & 3.48 & 2.24 & 2.44 & 2.24 & 2.35 \\
\hline 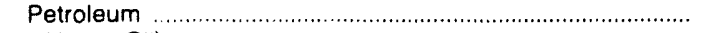 & .42 & 1.95 & 4.26 & 4.29 & 2.50 & 3.09 & 2.53 & 3.03 \\
\hline (1) & .42 & 1.94 & 4.25 & 4.26 & 2.47 & 3.06 & 2.49 & 2.95 \\
\hline 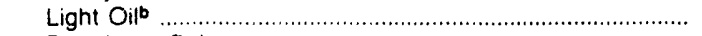 & .44 & 2.16 & 5.63 & 6.11 & 3.81 & 4.25 & 3.97 & 4.48 \\
\hline 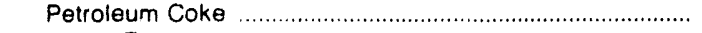 & - & - & - & - & - & - & - & - \\
\hline 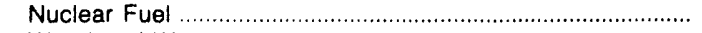 & .20 & .31 & .56 & 67 & .61 & .63 & .57 & .65 \\
\hline 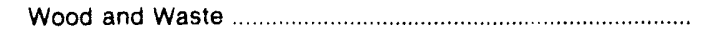 & - & - & 1.74 & - & - & .95 & - & - \\
\hline Primary Energy - Five Sectors & 1.17 & 2.60 & 5.45 & 5.87 & 4.79 & 4.77 & 4.58 & 5.02 \\
\hline
\end{tabular}

-There are no direct fuel costs for hydroelectric, geothermal, centralized solar, or wind energy. Wood and other biomass fuels are not included, except those consumed at electric utilities.

b Heavy oil includes Grade Nos. 4, 5, and 6, and residual fuel oils. Light oil includes Grade No. 2 heating oil, kerosene, and jet fuel.

- No consumption, including cases where adjustments were made. See "Consumption Adjustments for Process Fuel and Intermediate Products."

Sources: Daia sources, estimation procedures, and assumptions are described in the "Documentation" section of this report. 


\section{N Energy Expenditure Estimates by Sector, New York \\ E $1970,1975,1980$, and 1985-1989 \\ W (Million Dollars)}

\section{$\mathbf{Y}$
$\mathbf{O}$
$\mathbf{R}$
$\mathrm{K}$}

Sector and Energy Source
Residential Sector
Primary Energy
Coal
Natural Gas
Petroleum
Kerosene Futile Fuel
Electricity

Commercial Sector

Primary Energy

Coal.

Natural Gas

Petroleum

Distillate Fuel

Kerosene

LPG

Motor Gasoline

Residual Fuel

Electricity

Industrial Sector

Primary Energy

Coa

Coking Coal

Steam Coal

Natural Gas

Petroleum

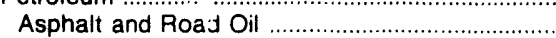

Distillate Fuel .

Kerosene

LPG .

Lubricants

Residual Fuel

Other

Transportation Sector.

Primary Energy

Coal.

Petroleum

Aviation Gasoline

Distillate Fuel

Jet Fuel

LPG

Lubricants .

Motor Gasoline

Residual Fuel

Electricity

Total Energy"

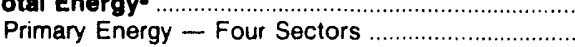

Electricity

Eloctric Utillty Sector

Coal

Natural Gas

Petroleum

Heavy Oitb

Light Oilb

Petroleum Coke

.

Nuclear Fuel .......

Wood and Waste

Primary Energy - Five Sectors

.

1970

$1,844.5$

$1,076.5$

12.8
484.5

484.5

579.2

501.4
49.4

49.4
28.3

768.0

$1,325.0$

439.9

3.2

166.0

270.8

135.5

2.6

2.7

16.1

113.8

885.0

899.6

577.5

164.5

96.4

68.1

80.0

333.0

26.0

68.8

3.3

5.6
30.9

30.9
50.3

103.2

44.8

322.1

$2,299.1$
2,267

$2,267.8$

$2,267.6$

2.7
89.5

89.5
155.5

155.5
.6

36.9
939.4

$1,939.4$
43.0

31.3

6,368.1

$4,361.7$

$2,006.4$

\section{5.}

127.6
40.9

40.9

157.7

149.6
8.1

149.6
8.1
-

$9 . \overline{2}$

$4,697.1$

$1975 \mid 1980$

$3,490.4$

$1,879.9$

14.2

830.2

$1,035.4$

914.6

69.6

51.2

$1,610.5$

$3,056.9 \quad 5,194.8$

$919.0 \quad 1,982.8$

6.2

256.7

656.0

273.8

6.0

29.3

340.7

$2,137.9$

$1,960.2$

$1,225.7$

283.3

197.8

85.5

156.0

786.3
69.9

69.9
216.9

14.8

11.4

45.3

34.1

276.6

117.4

734.6

$4,140.7$

$4,043.8$

$4,043.7$

4.8
173.7

173.7
423.1

i. 4

43.1

$3,304.6$

93.0

7.0

690.4

$1,285.4$

546.7

5.4
9.4

9.4

55.7
668.1

668.1

3,376.6

$2,058.5$

304.2

197.6

106.6

398.4

$1,355.9$

121.3

289.8

13.4

43.8

89.5
82.7

337.3

378.0

1,318.1

$8,915.6$

$8,810.0$

$8,810 . \overline{0}$

14.6

447.5

$1,274.5$

92.7

6.727 .2

$\begin{array}{r}651.7 \\ \hline\end{array}$

105. 6

$12,648.2$

$8,068.3$

23,285.7

$16,241.3$

$7,044.4$

1,326.8

173.3

$2,427.3$

233.8

343.4

$1,096.4$

$1,029.8$

66.6

$1,731.5$

$1,706.9$

24.5

118.3

44.9
-

$9,395.1$

1985

1985

1986

1988

1989

\section{$7,771.2$}

$7,612.3$

$4,212.6$

17.7

$2,478.1$

$4,056.0$

7,579.3

$3,869.5$

14.6

$2,511.7$

$1,527.2$

$1,355.5$

$1,506.7$

76.9

133.1

$3,558.6$

$7,132.6$

$1,971.9$

10.4
$1,010.8$

$\begin{array}{r}950.7 \\ \hline\end{array}$

484.4

14.5

25.2

88.3

338.3

$5,160.7$

$3,158.8$

1,658.4

180.4

58.5

121.9

526.2

951.8

230.1

172.1

20.9

39.9

99.8

56.5

1126

219.9

$1,500.4$

7,256.3

$7,106.0$

$7,106.0$

11.1

669.3

139.0
4.9

103.4
$6,154.6$

23.6
150.3

48.3
123.4

$2,300.8$

$1,347.9$

74.3

132.0

$3,709.8$

$6,942.0$

$1,814.2$

$6,707.0$

$1,676.0$

11.1

966.2
836.9

512.3

2.9

25.4

64.3

232.1 


\begin{tabular}{|c|c|c|c|c|c|c|c|c|}
\hline Ergrgy Source & 1970 & 1975 & 1980 & 1985 & 1986 & 1987 & 1988 & 1989 \\
\hline & \multicolumn{8}{|c|}{ Prices in Dollars per Million Btu } \\
\hline Coal & 0.43 & 1.12 & 1.58 & 1.97 & 1.85 & 1.79 & 1.77 & 1.77 \\
\hline Coking Coal & - & - & - & - & - & - & - & - \\
\hline 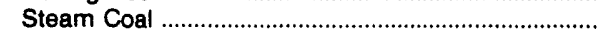 & .43 & 1.12 & 1.58 & 1.97 & 1.85 & 1.79 & 1.77 & 1.77 \\
\hline 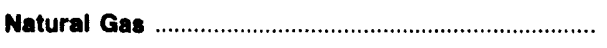 & .69 & 1.57 & 3.55 & 5.29 & 4.77 & 4.66 & 4.46 & 4.56 \\
\hline 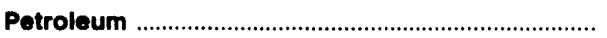 & 1.94 & 3.68 & 8.15 & 8.26 & 6.18 & 6.53 & 6.38 & 7.27 \\
\hline 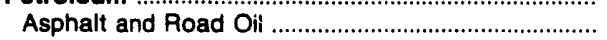 & .67 & 1.80 & 3.57 & 4.94 & 4.22 & 3.23 & 3.18 & 2.89 \\
\hline 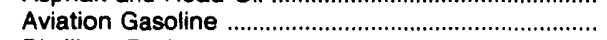 & 2.17 & 3.45 & 9.02 & 9.99 & 8.41 & 7.55 & 7.41 & 8.28 \\
\hline 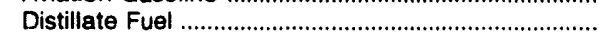 & 1.13 & 2.74 & 6.80 & 7.52 & 5.75 & 5.71 & 5.60 & 6.37 \\
\hline Jet Fuel & .73 & 2.03 & 6.46 & 5.77 & 3.85 & 3.96 & 3.71 & 4.30 \\
\hline Kerosene & 1.32 & 2.86 & 7.67 & 8.44 & 6.53 & 5.55 & 5.68 & 6.72 \\
\hline LPG & 1.89 & 3.24 & 6.11 & 9.73 & 9.03 & 8.71 & 8.76 & 8.82 \\
\hline 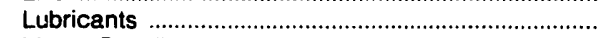 & 5.08 & 7.49 & 14.36 & 17.61 & 15.59 & 13.58 & 14.61 & 17.90 \\
\hline 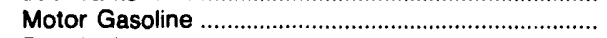 & $? .82$ & 4.55 & 9.91 & 9.03 & 6.69 & 7.33 & 7.26 & 8.10 \\
\hline 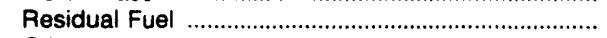 & .46 & 1.90 & 3.72 & 4.44 & 2.71 & 2.94 & 2.33 & 2.65 \\
\hline Other" & 1.12 & 2.57 & 7.38 & 7.10 & 4.71 & 5.84 & 4.91 & 5.68 \\
\hline 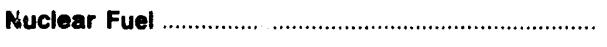 & - & .29 & .36 & .54 & .53 & .56 & .59 & .55 \\
\hline Wood and Waste at Utilities & - & - & - & - & - & - & - & - \\
\hline 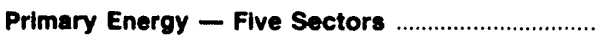 & 1.20 & 2.47 & 4.63 & 4.75 & 3.79 & 3.87 & 3.82 & 4.07 \\
\hline Electric Utility Fuel & .41 & 1.05 & 1.48 & 1.56 & 1.46 & 1.29 & 1.30 & 1.31 \\
\hline Electricity Purchased by End Users .............................. & 4.17 & 7.92 & 11.72 & 17.46 & 18.16 & 18.05 & 18.03 & 18.33 \\
\hline \multirow[t]{2}{*}{ 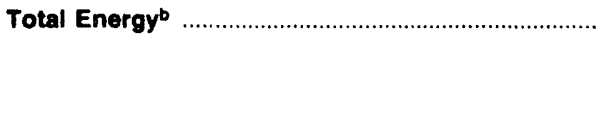 } & 2.02 & 4.11 & 8.02 & 9.62 & 8.47 & 8.69 & 8.57 & 9.31 \\
\hline & \multicolumn{8}{|c|}{ Expenditures in Millions of Dollars } \\
\hline Coal nan. & 210.3 & 533.2 & 985.6 & $1,084.5$ & $1,077.4$ & 894.0 & 912.8 & 982.3 \\
\hline Coking Coal & - & - & - & - & - & - & - & - \\
\hline 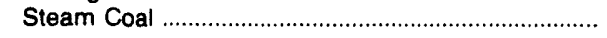 & 210.3 & 533.2 & 985.6 & $1,084.5$ & $1,077.4$ & 894.0 & 912.8 & 982.3 \\
\hline 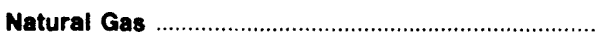 & 102.8 & 178.1 & 529.3 & 705.5 & 644.6 & 677.9 & $66 \AA .9$ & 718.8 \\
\hline Petroleum . & $1,202.1$ & $2,354.7$ & $5,421.0$ & $5,484.6$ & $4,419.2$ & $4,786.2$ & $4,910.7$ & $5,234.2$ \\
\hline 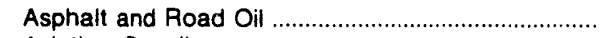 & 16.1 & 36.4 & 73.3 & 113.2 & 127.0 & 86.3 & 94.8 & 72.3 \\
\hline 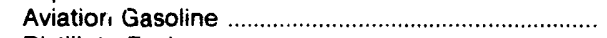 & 1.7 & 3.8 & 9.8 & 8.8 & 9.6 & 8.3 & 8.8 & 9.6 \\
\hline 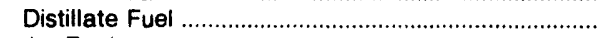 & 149.3 & 339.0 & 955.5 & $1,087.3$ & 924.9 & 943.6 & $1,028.8$ & $1,003.9$ \\
\hline 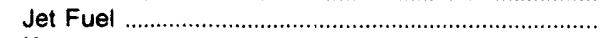 & 18.7 & 42.3 & 185.3 & 213.6 & 152.9 & 171.2 & 172.1 & 184.0 \\
\hline 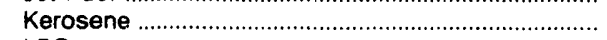 & 86.6 & 94.7 & 141.7 & 103.6 & 73.9 & $66 . C$ & 71.4 & 62.7 \\
\hline LPG & 39.1 & 77.6 & 179.1 & 269.9 & 245.9 & 270.5 & 254.1 & 311.9 \\
\hline 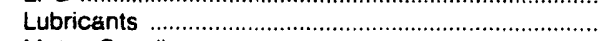 & 26.2 & 42.9 & 105.0 & 17.2 & 101.4 & 99.9 & 103.7 & 130.2 \\
\hline 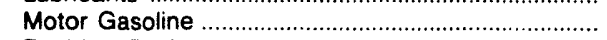 & 835.7 & $1,599.1$ & $3,448.9$ & $3,362.0$ & $2,601.7$ & $2,947.9$ & $3,015.8$ & $3,306.4$ \\
\hline Residual Fuel & 19.7 & 92.9 & 211.1 & 121.8 & 68.9 & 66.9 & 53.8 & 31.2 \\
\hline 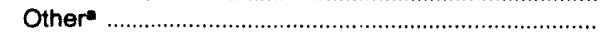 & 9.0 & 26.1 & 111.2 & 87.3 & 112.8 & 125.6 & 107.4 & 121.9 \\
\hline 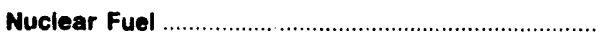 & - & 4.4 & 22.9 & 111.8 & 115.7 & 171.2 & 183.3 & 173.4 \\
\hline 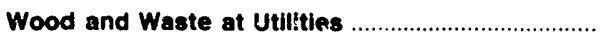 & - & - & - & - & - & - & - & - \\
\hline Primary Energy - Flve Sectors ................................ & $1,515.2$ & $3,070.4$ & $6,958.8$ & $7,386.4$ & $6,256.9$ & $6,529.3$ & $6,669.7$ & 7,108.6 \\
\hline 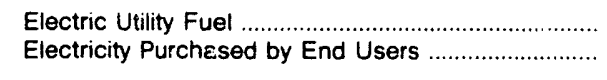 & $\begin{array}{r}-190.7 \\
576.2\end{array}$ & $\begin{array}{r}-473.6 \\
1.393 .1\end{array}$ & $\begin{array}{r}-967.2 \\
2,553.8\end{array}$ & $\begin{array}{r}-1,097.2 \\
4,305.3\end{array}$ & $\begin{array}{r}-1,079.1 \\
4,797.8\end{array}$ & $\begin{array}{r}-960.0 \\
5,051.7\end{array}$ & $\begin{array}{r}-989.4 \\
5,228.3\end{array}$ & $\begin{array}{r}-1,058.0 \\
5,479.4\end{array}$ \\
\hline . & $i, 900.6$ & $3,990.0$ & $8,545.4$ & $10,594.5$ & $9,975.6$ & $10,621.0$ & $10,908.6$ & $11,530.1$ \\
\hline
\end{tabular}

- Includes petroleum coke used at electric utilities

bThere are no direct fuel costs for hydroelectric, geothermal, centralized solar, or wind energy. Wood and other biomass fuels are not included, except those consumed at electric utilities.

-No consumption, including cases where adjustments were made. See "Consumption Adjustments for Process Fuel and Intermediate Products."

Note: Expenditure totals may not equal sum of components due to independent rounding.

Sources: Data sources, estimation procedures, and assumptions are described in the "Documentation" section of this report. 


\begin{tabular}{|c|c|c|c|c|c|c|c|c|}
\hline Sector and Energy Source & 1970 & 1975 & 1980 & 1985 & 1986 & 1987 & 1988 & 1989 \\
\hline Residential Sector & 2.41 & 5.15 & 9.62 & 14.47 & 14.61 & 14.40 & 14.45 & 15.33 \\
\hline 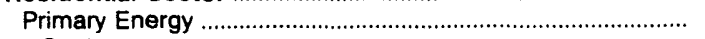 & 1.40 & 2.69 & 6.15 & 7.73 & 6.81 & 6.32 & 6.20 & 7.00 \\
\hline Coal & 1.14 & 2.06 & 2.70 & 2.75 & 2.60 & 2.69 & 2.53 & 2.87 \\
\hline Natural Gas & 1.27 & 1.99 & 4.06 & 6.38 & 6.41 & 6.46 & 6.07 & 6.35 \\
\hline Petroleum & 1.44 & 2.97 & 7.30 & 8.69 & 7.19 & 6.33 & 6.40 & 7.58 \\
\hline 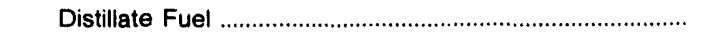 & 1.31 & 2.71 & 6.95 & 8.02 & 6.35 & 5.30 & 5.54 & 6.36 \\
\hline 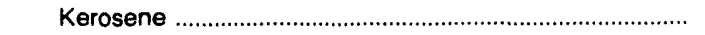 & 1.40 & 2.96 & 7.96 & 8.73 & 6.91 & 5.77 & 6.03 & 6.92 \\
\hline 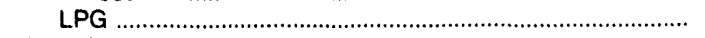 & 2.28 & 4.32 & 7.67 & 10.27 & 9.44 & 9.20 & 8.81 & 9.63 \\
\hline Electricity & 5.45 & 9.31 & 13.91 & 20.48 & 21.17 & 21.59 & 21.89 & 22.45 \\
\hline Commercial Sector & 2.51 & 5.16 & 8.50 & 12.85 & 13.12 & 12.54 & 12.20 & 12.92 \\
\hline 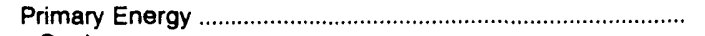 & .98 & 2.01 & 4.73 & 6.14 & 5.03 & 4.80 & 4.48 & 5.04 \\
\hline Coal & .53 & 1.53 & 1.71 & 1.90 & 1.83 & 1.75 & 1.74 & 1.71 \\
\hline 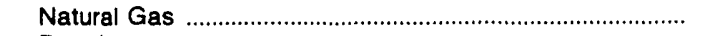 & .94 & 1.71 & 3.67 & 5.65 & 5.39 & 5.05 & 4.80 & 4.99 \\
\hline 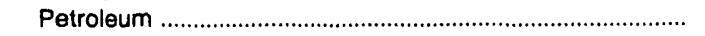 & 1.23 & 2.66 & 6.58 & 7.28 & 4.96 & 4.82 & 4.41 & 5.51 \\
\hline 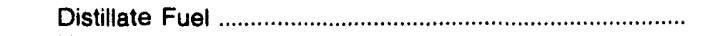 & 1.02 & 2.34 & 6.33 & 6.90 & 4.14 & 3.81 & 3.44 & 4.45 \\
\hline 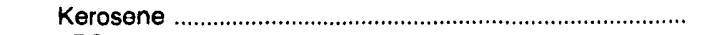 & .77 & 2.37 & 6.12 & 6.99 & 4.48 & 4.01 & 3.72 & 5.07 \\
\hline 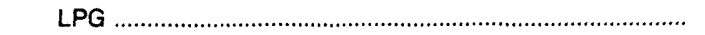 & 1.41 & 2.67 & 5.24 & 9.33 & 8.70 & 8.32 & 8.71 & 7.90 \\
\hline 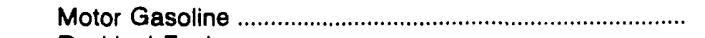 & 2.82 & 4.55 & 9.91 & 9.03 & 6.69 & 7.33 & 7.26 & 8.10 \\
\hline 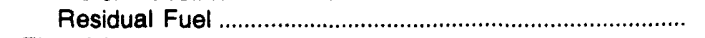 & .67 & 1.79 & 3.80 & 4.46 & 2.74 & 2.95 & 2.36 & 2.75 \\
\hline 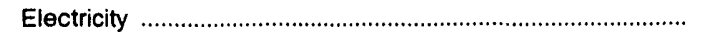 & 4.60 & 8.46 & 12.28 & 18.18 & 18.79 & 18.45 & 18.35 & 18.44 \\
\hline Industrlal Sector & 1.06 & 2.99 & 5.34 & 7.35 & 6.59 & 6.55 & 6.42 & 6.83 \\
\hline 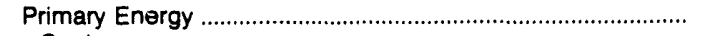 & .68 & 1.91 & 4.02 & 4.88 & 3.87 & 3.76 & 3.53 & 3.80 \\
\hline 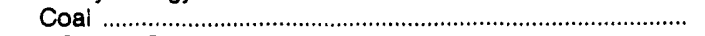 & .53 & 1.53 & 1.71 & 1.90 & 1.83 & 1.75 & 1.74 & 1.71 \\
\hline 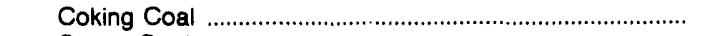 & - & - & - & - & - & - & - & - \\
\hline 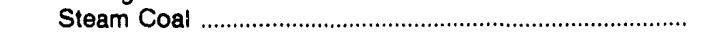 & .53 & 1.53 & 1.71 & 1.90 & 1.83 & 1.75 & 1.74 & 1.71 \\
\hline 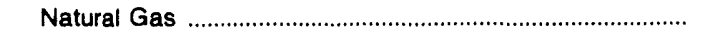 & .50 & 1.34 & 3.32 & 4.75 & 3.87 & 3.65 & 3.47 & 3.53 \\
\hline Petroleum & .86 & 2.31 & 5.02 & 6.62 & 4.90 & 4.96 & 4.56 & 5.42 \\
\hline 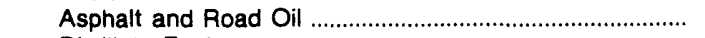 & .67 & 1.80 & 3.57 & 4.94 & 4.22 & 3.23 & 3.18 & 2.89 \\
\hline 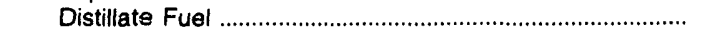 & .71 & 2.19 & 5.49 & 6.92 & 4.43 & 3.97 & 3.68 & 5.03 \\
\hline 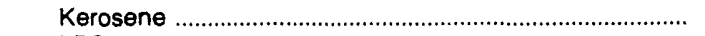 & .77 & 2.37 & 6.12 & 6.99 & 4.48 & 4.01 & 3.72 & 5.07 \\
\hline 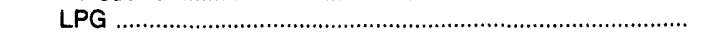 & 1.41 & 2.67 & 5.24 & 9.33 & 8.70 & 8.32 & 8.71 & 7.90 \\
\hline 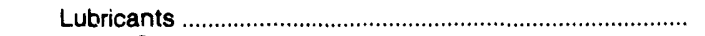 & 5.08 & 7.49 & 14.36 & 17.61 & 15.59 & 13.58 & 14.61 & 17.90 \\
\hline 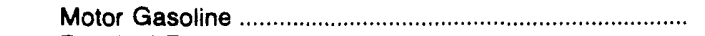 & 2.82 & 4.55 & 9.91 & 9.03 & 6.69 & 7.33 & 7.26 & 8.10 \\
\hline 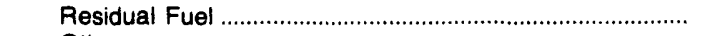 & .45 & 1.92 & 3.72 & 4.46 & 2.74 & 2.95 & 2.36 & 2.75 \\
\hline 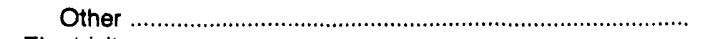 & 1.12 & 2.57 & 7.38 & 7.10 & 4.71 & 5.84 & 4.91 & 5.68 \\
\hline 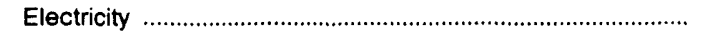 & 2.76 & 6.36 & 9.28 & 13.83 & 14.39 & 13.80 & 13.60 & 13.83 \\
\hline 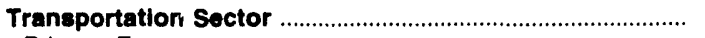 & 2.52 & 4.27 & 9.35 & 8.62 & 6.46 & 6.97 & 6.84 & 7.65 \\
\hline 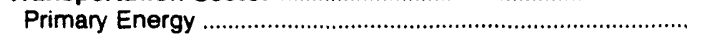 & 2.52 & 4.27 & 9.35 & 8.62 & 6.46 & 6.97 & 6.84 & 7.65 \\
\hline Coal & .53 & 1.53 & - & - & - & - & - & \\
\hline 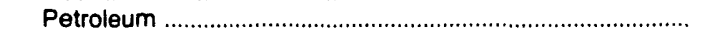 & 2.52 & 4.27 & 9.35 & 8.62 & 6.46 & 6.97 & 6.84 & 7.65 \\
\hline 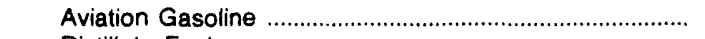 & 2.17 & 3.45 & 9.02 & 9.99 & 8.41 & 7.55 & 7.41 & 8.28 \\
\hline 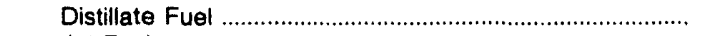 & 1.30 & 3.12 & 7.34 & 7.66 & 6.26 & 6.70 & 6.41 & 7.05 \\
\hline 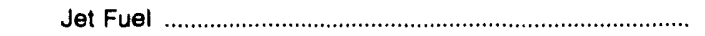 & .73 & 2.03 & 6.46 & 5.77 & 3.85 & 3.96 & 3.71 & 4.30 \\
\hline LPG & 1.41 & 2.67 & 5.24 & 9.33 & 8.70 & 8.32 & 8.71 & 7.90 \\
\hline Lubricants & 5.08 & 7.49 & 14.36 & 17.61 & 15.59 & 13.58 & 14.61 & 17.90 \\
\hline 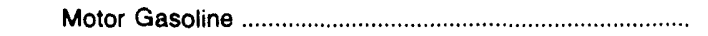 & 2.82 & 4.55 & 9.91 & 9.03 & 6.69 & 7.33 & 7.26 & 8.10 \\
\hline Residual Fuel & .27 & 1.56 & 3.43 & 3.78 & 1.82 & 2.86 & 2.10 & 2.39 \\
\hline Electricity & - & - & - & - & - & - & - & - \\
\hline 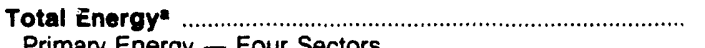 & 2.02 & $\begin{array}{l}4.11 \\
3.27\end{array}$ & 8.02 & $\begin{array}{l}9.62 \\
7.36\end{array}$ & $\begin{array}{l}8.47 \\
5.66\end{array}$ & $\begin{array}{l}8.69 \\
5.91\end{array}$ & $\begin{array}{l}8.57 \\
5.78\end{array}$ & $\begin{array}{l}9.31 \\
6.44\end{array}$ \\
\hline $\begin{array}{l}\text { Primary Energy - Four Sectors } \\
\text { Electricity }\end{array}$ & $\begin{array}{l}1.65 \\
4.17\end{array}$ & $\begin{array}{l}3.27 \\
7.92\end{array}$ & $\begin{array}{r}7.07 \\
11.72\end{array}$ & $\begin{array}{r}7.36 \\
17.46\end{array}$ & $\begin{array}{r}5.66 \\
18.16\end{array}$ & $\begin{array}{r}5.91 \\
18.05\end{array}$ & 18.03 & 18.33 \\
\hline Electric Utility Sector & .41 & 1.05 & 1.48 & 1.56 & 1.46 & 1.29 & 1.30 & 1.31 \\
\hline 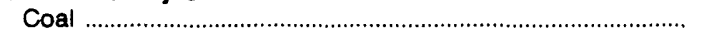 & .41 & 1.07 & 1.57 & 1.98 & 1.85 & 1.79 & 1.78 & 1.77 \\
\hline Natural Gas & .37 & 1.41 & 3.15 & 4.78 & 2.74 & 3.09 & 3.37 & 3.46 \\
\hline 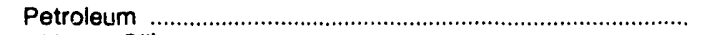 & .79 & 1.89 & 5.82 & 5.68 & 3.48 & 3.91 & 3.49 & 3.99 \\
\hline 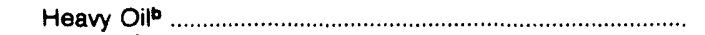 & .69 & 1.78 & 3.82 & - & - & - & - & \\
\hline Light Oilb & .83 & 2.22 & 5.82 & 5.68 & 3.48 & 3.91 & 3.49 & 3.99 \\
\hline 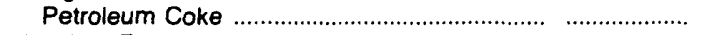 & - & - & - & - & - & - & - & - \\
\hline 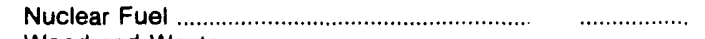 & - & .29 & .36 & .54 & .53 & 56 & .59 & .55 \\
\hline 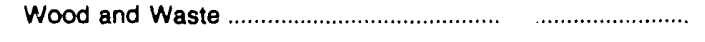 & - & - & - & - & - & - & - & - \\
\hline Primary Energy — Five Sectors & 1.20 & 2.47 & 4.63 & 4.75 & 3.79 & 3.87 & 3.82 & 4.07 \\
\hline
\end{tabular}

- There are no direct fuel costs for hydroelectric, geothermal, centralized solar, or wind energy. Wood and other biomass fuels are not included, except those consumed at elect-ic utilities.

DHeavy oil includes Grade Nos. 4, 5, and 6, and residual fuel oils. Light oil includes Grade No. 2 heating oil, kerosene, and jet fuel.

- No consumption, including cases where adjustments were made. See "Consumption Adjustments for Process Fuel and Intermediate Products."

Sources: Data sources, estimation procedures, and assumptions are described in the "Documentation" section of this report. 


\begin{tabular}{|c|c|c|c|c|c|c|c|c|}
\hline Sector and Energy Source & 1970 & 1975 & 1980 & 1985 & 1986 & 1987 & 1988 & 1989 \\
\hline 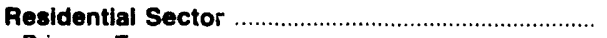 & 483.8 & 897.9 & $1,789.6$ & $2,509.9$ & $2,708.2$ & $2,927.1$ & $3,019.8$ & $3,180.3$ \\
\hline 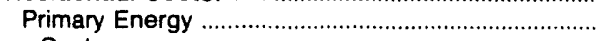 & 211.3 & 294.6 & 633.0 & 633.4 & 576.8 & 605.9 & 613.8 & 669.3 \\
\hline 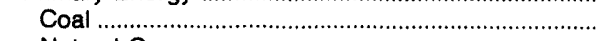 & 4.1 & 6.3 & 3.9 & 4.7 & 3.5 & 3.8 & 4.5 & 3.8 \\
\hline 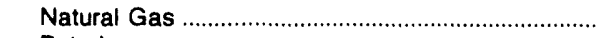 & 35.6 & 55.6 & 139.6 & 189.1 & 209.8 & 239.1 & 239.9 & 253.2 \\
\hline 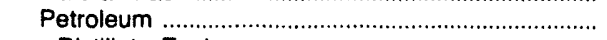 & 171.6 & 232.8 & 489.4 & 439.6 & 363.4 & 362.9 & 369.4 & 412.3 \\
\hline 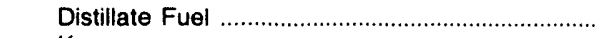 & 65.9 & 114.6 & 285.2 & 228.0 & 184.1 & 175.5 & 185.0 & 173.1 \\
\hline Kerosene & 79.8 & 82.2 & 124.0 & 89.6 & 66.0 & 60.1 & 64.4 & 57.7 \\
\hline LPG & 25.9 & 36.0 & 80.2 & 121.9 & 113.3 & 127.3 & 120.0 & 181.4 \\
\hline 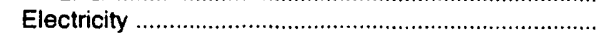 & 272.5 & 603.3 & $1,156.6$ & $1,876.5$ & $2,131.4$ & $2,321.2$ & $2,406.0$ & $2,511.0$ \\
\hline 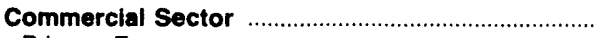 & 196.5 & 420.8 & 827.5 & $1,507.7$ & $1,587.8$ & $1,668.9$ & $\mathbf{1 , 7 2 7 . 6}$ & $1,819.6$ \\
\hline 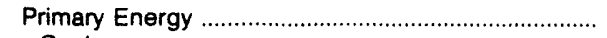 & 44.3 & 83.8 & 230.1 & 318.8 & 250.7 & 276.8 & 280.7 & 292.7 \\
\hline Coal & 3.6 & 8.6 & 4.6 & 6.0 & 4.6 & 4.6 & 5.8 & 4.2 \\
\hline 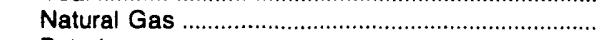 & 20.7 & 37.7 & 97.1 & 146.2 & 141.7 & 156.4 & 160.4 & 170.4 \\
\hline 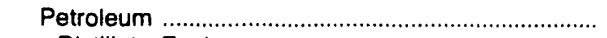 & 20.0 & 37.4 & 128.3 & 166.7 & 104.4 & 115.9 & 114.5 & 118.1 \\
\hline 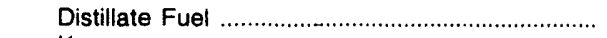 & 10.1 & 19.4 & 61.7 & 106.4 & 58.4 & 65.1 & 61.9 & 61.0 \\
\hline 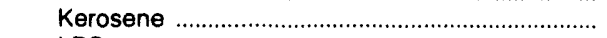 & 1.0 & 1.6 & 4.1 & 4.4 & 2.2 & 1.8 & 2.5 & 2.5 \\
\hline LPG & 2.8 & 3.9 & 9.7 & 19.5 & 18.4 & 20.3 & 20.9 & 26.3 \\
\hline 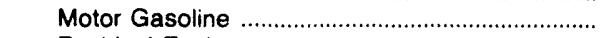 & 5.3 & 9.9 & 41.1 & 30.0 & 22.8 & 27.8 & 26.1 & 26.6 \\
\hline 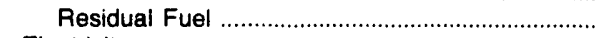 & .8 & 2.6 & 11.7 & 6.3 & 2.6 & .8 & 3.1 & 1.7 \\
\hline 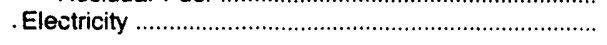 & 152.2 & 337.0 & 597.4 & $1,188.9$ & $1,337.2$ & $1,392.0$ & $1,447.0$ & $1,526.9$ \\
\hline Industrial Sector & 319.6 & 879.1 & $1,836.4$ & $2,385.7$ & $2,350.8$ & $2,286.8$ & $2,262.3$ & $2,359.1$ \\
\hline 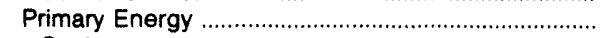 & 168.2 & 426.3 & $1,036.6$ & $1,145.8$ & $1,021.6$ & 948.4 & 887.0 & 917.5 \\
\hline 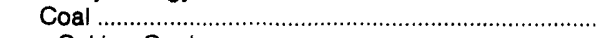 & 28.7 & 53.2 & 57.3 & 106.1 & 116.2 & 110.3 & 109.4 & 108.5 \\
\hline Coking Coal & - & - & - & - & - & - & - & - \\
\hline 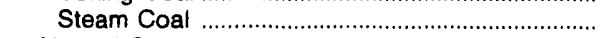 & 28.7 & 53.2 & 57.3 & 106.1 & 116.2 & 110.3 & 109.4 & 108.5 \\
\hline 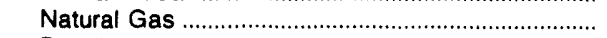 & 38.4 & 84.6 & 287.1 & 367.2 & 289.8 & 278.7 & 258.9 & 289.3 \\
\hline 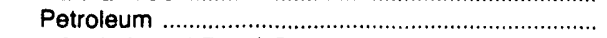 & 101.0 & 288.5 & 692.2 & 672.5 & 615.6 & 559.3 & 518.7 & 519.8 \\
\hline 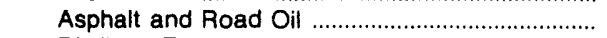 & 16.1 & 36.4 & 73.3 & 113.2 & 127.0 & 86.3 & 94.8 & 72.3 \\
\hline Distillate Fuel & 18.6 & 54.6 & 132.0 & 130.5 & 118.4 & 87.6 & 79.5 & 103.8 \\
\hline 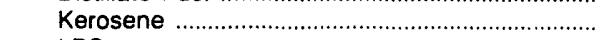 & 5.8 & 10.9 & 13.7 & 9.6 & 5.7 & 4.1 & 4.5 & 2.6 \\
\hline LPG & 10.1 & 36.6 & 88.2 & 123.7 & 110.0 & 119.6 & 109.5 & 100.5 \\
\hline 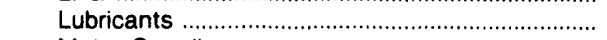 & 10.1 & 20.2 & 49.7 & 55.5 & 48.0 & 47.3 & 49.1 & 61.7 \\
\hline Motor Gasoline & 14.9 & 18.7 & 26.8 & 39.5 & 28.6 & 31.6 & 28.2 & 35.6 \\
\hline 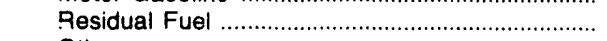 & 16.5 & 85.1 & 197.3 & 113.3 & 65.0 & 57.1 & 45.6 & 21.4 \\
\hline 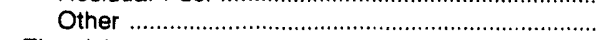 & 9.0 & 26.1 & 111.2 & 87.3 & 112.8 & 125.6 & 107.4 & 121.9 \\
\hline 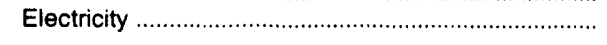 & 151.4 & 452.8 & 799.8 & $1,239.9$ & $1,329.2$ & $1,338.5$ & $1,375.3$ & $1,441.5$ \\
\hline Transportation Sector & 900.7 & $1,792.1$ & $4,092.0$ & $4,191.2$ & $3,328.7$ & $3,738.2$ & $3,898.8$ & 4,171.2 \\
\hline 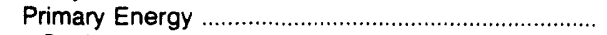 & 900.7 & $1,792.1$ & $4,092.0$ & $4,191.2$ & $3,328.7$ & $3,738.2$ & $3,898.8$ & $4,171.2$ \\
\hline Coal & $\dot{-1}$ & * & - & - & - & - & - & - \\
\hline 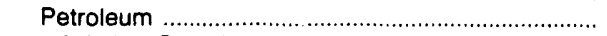 & 900.7 & $1,792.1$ & $4,092.0$ & $4,191.2$ & $3,328.7$ & $3,738.2$ & $3,898.8$ & $4,171.2$ \\
\hline 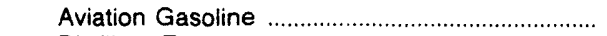 & 1.7 & 3.8 & 9.8 & 8.8 & 9.6 & 8.3 & 8.8 & 9.6 \\
\hline 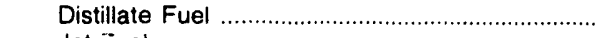 & 47.8 & 149.2 & 457.5 & 607.6 & 556.9 & 605.5 & 693.0 & 653.0 \\
\hline 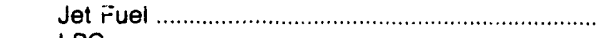 & 18.7 & 42.3 & 185.3 & 213.6 & 152.9 & 171.2 & 172.1 & 184.0 \\
\hline LPG & .3 & 1.1 & 1.0 & 4.8 & 4.2 & 3.2 & 3.7 & 3.7 \\
\hline Lubricants & 16.1 & 22.6 & 55.3 & 61.7 & 53.4 & 52.6 & 54.6 & 68.6 \\
\hline 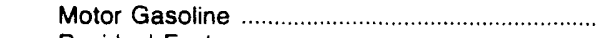 & 815.5 & $1,570.5$ & $3,381.0$ & $3,292.5$ & $2,550.3$ & $2,888.5$ & $2,961.5$ & $3,244.2$ \\
\hline 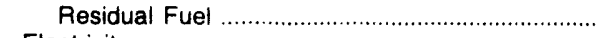 & .6 & 2.6 & 2.1 & 2.2 & 1.3 & 8.9 & 5.1 & 8.1 \\
\hline 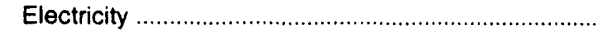 & - & - & - & - & - & - & - & - \\
\hline 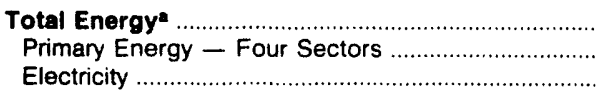 & $\begin{array}{r}1,900.6 \\
1,324.5 \\
576.2\end{array}$ & $\begin{array}{l}3,990.0 \\
2,596.8 \\
1,393.1\end{array}$ & $\begin{array}{l}\mathbf{8 , 5 4 5 . 4} \\
5,991.6 \\
2,553.8\end{array}$ & $\begin{array}{r}10,594.5 \\
6,289.2 \\
4,305.3\end{array}$ & $\begin{array}{l}9,975.6 \\
5,177.8 \\
4,797.8\end{array}$ & $\begin{array}{r}10,621.0 \\
5,569.3 \\
5,051.7\end{array}$ & $\begin{array}{r}10,908.6 \\
5,680.3 \\
5,228.3\end{array}$ & $\begin{array}{r}11,530.1 \\
6,050.7 \\
5,479.4\end{array}$ \\
\hline Electric Utility Sector .... & 190.7 & 473.6 & 967.2 & $1,097.2$ & $1,079.1$ & 960.0 & 989.4 & $1,058.0$ \\
\hline Coal & 173.8 & 465.1 & 919.7 & 967.8 & 953.1 & 775.2 & 793.1 & 865.7 \\
\hline 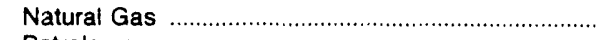 & 8.0 & .1 & 5.5 & 2.9 & 3.2 & 3.7 & 3.7 & 6.0 \\
\hline 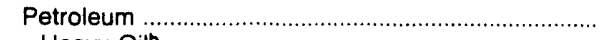 & 8.9 & 3.9 & 19.0 & 14.7 & 7.1 & 9.9 & 9.3 & 12.9 \\
\hline 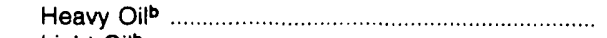 & 1.9 & 2.6 & $*$ & - & - & - & - & - \\
\hline (2) & 6.9 & 1.2 & 19.0 & 14.7 & 7.1 & 9.9 & 9.3 & 12.9 \\
\hline 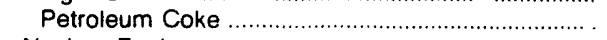 & - & - & - & - & - & - & - & - \\
\hline 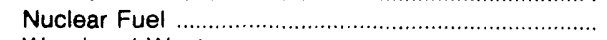 & - & 4.4 & 22.9 & 111.8 & 115.7 & 171.2 & 183.3 & 173.4 \\
\hline Wood and Waste & - & - & - & - & - & - & - & - \\
\hline 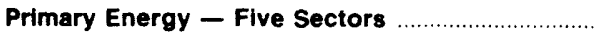 & $1,515.2$ & $3,070.4$ & $6,958.8$ & $7,386.4$ & $6,256.9$ & $6,529.3$ & $6,669.7$ & $7,108.6$ \\
\hline
\end{tabular}

-There are no direct fuel costs for hydroelectric, geothermal, centralized solar, or wind energy. Wood and other biomass fuels are not included, except those consumed at electric utilities.

b Heavy oil includes Grade Nos. 4, 5, and 6, and residual fuel oils. Light oil includes Grade No. 2 heating oil, kerosene, and jet fuel.

-No consumption, including cases where adjustments were made. See "Consumption Adjustments for Process Fuel and Intermediate Products."

"Value less than 0.05 million dollars.

Note: Totals may not equal sum of components due to independent rounding.

Sources: Data sources, estimation procedures, and assumptions are described in the "Documentation" section of this report. 


\section{N Energy Price and Expenditure Estimates by Source, North Dakota \\ O 1970, 1975, 1980, and 1985-1989}

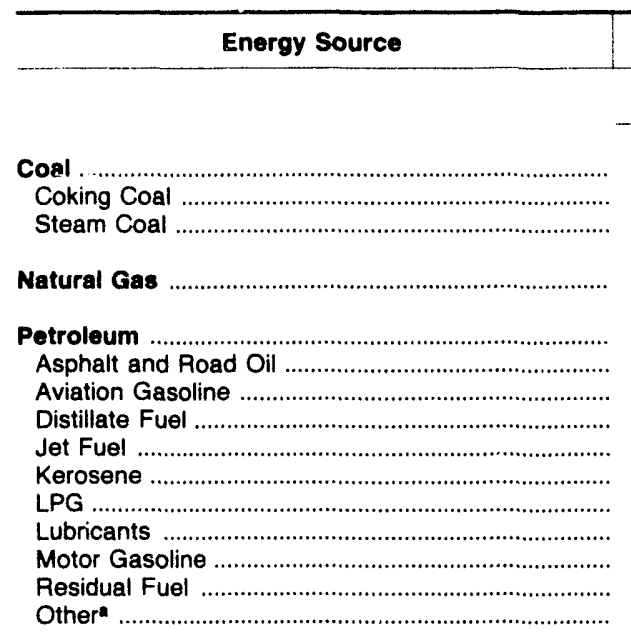

\begin{tabular}{r}
1970 \\
\hline 0.34 \\
\hline 1.83 \\
2. \\
1.07 \\
1.47 \\
5.08 \\
2.89 \\
\end{tabular}

Nuclear Fuel

Wood and Waste at Utilities

Primary Energy - Five Sectors

Electric Utility Fuel ...

Electricity Purchased by End Users

Total Energyb

\begin{tabular}{l|l}
\hline 970 & 1975 \\
\hline
\end{tabular}

1980

1985

1986

1987

1988

1989

Prices in Dollars per Million Btu

\begin{tabular}{|c|c|c|c|c|c|c|c|c|}
\hline \multirow[t]{2}{*}{ Total Energyb } & 1.97 & 3.47 & 7.28 & 7.07 & 5.90 & 5.86 & 5.90 & 6.05 \\
\hline & \multicolumn{8}{|c|}{ Expenditures in Millions of Dollars } \\
\hline Coal & 19.5 & 28.8 & 110.2 & 436.0 & 444.2 & 430.8 & 424.0 & 436.6 \\
\hline 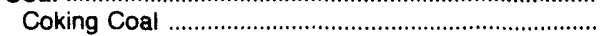 & - & - & & & & & & \\
\hline 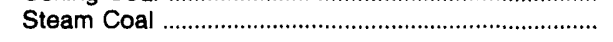 & 19.5 & 28.8 & 110.2 & 436.0 & 444.2 & 430.8 & 424.0 & 436.6 \\
\hline 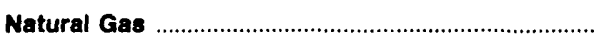 & 14.9 & 31.1 & 77.6 & 118.4 & 103.3 & 89.4 & 103.4 & 105.4 \\
\hline Petroleum & 201.2 & 393.6 & 938.4 & 897.3 & 676.5 & 693.3 & 694.7 & 771.9 \\
\hline Asphalt and Road Oil ............................................. & 7.3 & 14.6 & 19.2 & 35.9 & 23.7 & 22.0 & 22.4 & 19.2 \\
\hline Aviation Gasoline ........................................... & 1.0 & 1.5 & 2.9 & .2 & 1.6 & 1.1 & 1.2 & 1.3 \\
\hline 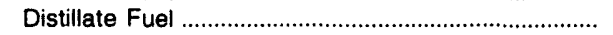 & 30.9 & 68.8 & 312.6 & 303.8 & 210.9 & 227.5 & 212.4 & 249.9 \\
\hline 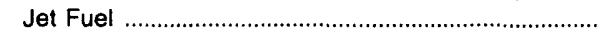 & 8.3 & 20.9 & 59.7 & 58.3 & 43.5 & 31.5 & 31.0 & 34.5 \\
\hline Kerosene & 2.0 & 1.1 & .5 & 1.0 & .4 & .2 & .3 & .4 \\
\hline 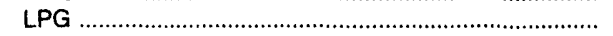 & 12.1 & 19.3 & 29.0 & 23.2 & 64.9 & 43.5 & 42.5 & 49.3 \\
\hline 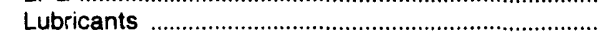 & 5.1 & 7.2 & 15.5 & 17.2 & 14.9 & 14.7 & 15.3 & 19.2 \\
\hline 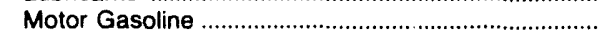 & 130.2 & 247.6 & 480.1 & 446.7 & 311.7 & 348.8 & 363.7 & 391.7 \\
\hline 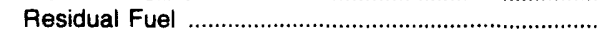 & 3.2 & 10.0 & 13.6 & 8.5 & 4.1 & 2.8 & 4.4 & 4.8 \\
\hline Other" & .8 & 2.6 & 5.3 & 2.3 & .8 & 1.3 & 1.4 & 1.7 \\
\hline Nuclear Fuel & - & - & - & - & - & - & - & - \\
\hline 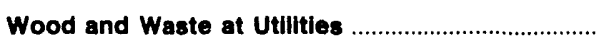 & - & - & - & - & - & - & - & - \\
\hline Primary Energy - Five Sectors .................................. & 235.6 & 453.5 & $1,126.3$ & $1,451.7$ & $1,224.0$ & $1,213.5$ & $1,222.1$ & $1,314.0$ \\
\hline $\begin{array}{l}\text { Electric Utility Fuel } \\
\text { Electricity Purchased by End Users }\end{array}$ & $\begin{array}{r}-12.3 \\
67.3\end{array}$ & $\begin{array}{r}-15.8 \\
108.0\end{array}$ & $\begin{array}{r}-87.9 \\
210.2\end{array}$ & $\begin{array}{r}-203.4 \\
407.5\end{array}$ & $\begin{array}{r}-188.0 \\
401.2\end{array}$ & $\begin{array}{r}-183.8 \\
383.2\end{array}$ & $\begin{array}{r}-201.8 \\
407.3\end{array}$ & $\begin{array}{r}-189.4 \\
400.6\end{array}$ \\
\hline$\ldots$ & 290.6 & 545.7 & $1,248.6$ & $1,655.8$ & $1,437.2$ & $1,412.9$ & $1,427.6$ & $1,325.1$ \\
\hline
\end{tabular}

- Includes petroleum coke used at electric utilities.

bThere are no direct fuel costs for hydroelectric, geothermal, centralized solar, or wind energy. Wood and other biomass fuels are not included, except those consumed at electric utilities.

-No consumption, including cases where adjustments were made. See "Consumption Adjustments for Process Fuel and Intermediate Products."

Note: Expenditure totals may not equal sum of components due to independent rounding.

Sources: Data sources, estimation procedures, and assumptions are described in the "Documentation" section of this report. 


\begin{tabular}{|c|c|c|c|c|c|c|c|c|}
\hline Sector and Energy Source & 1970 & 1975 & 1980 & 1985 & 1986 & 1987 & 1988 & 1989 \\
\hline Residential Sector & 2.54 & 3.95 & 7.52 & 10.16 & 9.41 & 9.83 & 9.35 & $\mathbf{9 . 4 7}$ \\
\hline 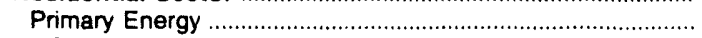 & 1.37 & 2.25 & 5.12 & 6.02 & 5.05 & 5.14 & 4.78 & 5.23 \\
\hline Coal & 1.56 & 3.09 & 1.96 & 1.74 & 1.53 & 1.45 & 1.15 & 1.13 \\
\hline 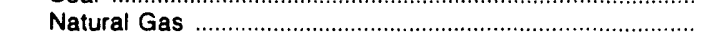 & .99 & 1.51 & 3.66 & 5.26 & 5.08 & 5.22 & 4.88 & 4.46 \\
\hline 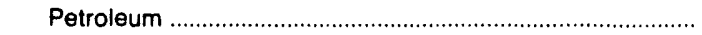 & 1.62 & 3.02 & 7.04 & 7.62 & 5.32 & 5.26 & 4.91 & 6.36 \\
\hline 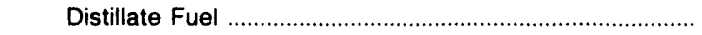 & 1.28 & 2.55 & 6.92 & 7.48 & 4.84 & 5.34 & 4.66 & 5.55 \\
\hline Kerosene & 1.65 & 2.69 & 7.39 & 8.09 & 5.24 & 5.77 & 5.04 & 6.00 \\
\hline LPG & 2.07 & 3.51 & 7.48 & 8.46 & 6.13 & 5.13 & 5.24 & 7.32 \\
\hline 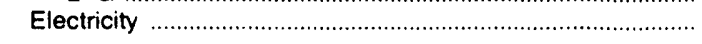 & 7.80 & 9.18 & 13.14 & 18.02 & 18.20 & 18.44 & 18.26 & 18.01 \\
\hline Commerclal Sector & 1.74 & 2.23 & 5.39 & 8.76 & 8.63 & 9.10 & 8.49 & 7.99 \\
\hline 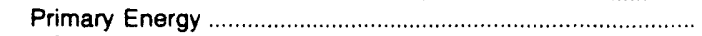 & .89 & 1.43 & 4.06 & 5.03 & 4.41 & 4.44 & 4.16 & 3.95 \\
\hline Coal & .74 & 1.26 & 2.63 & 3.21 & 3.11 & 2.80 & 2.66 & 2.74 \\
\hline Natural Gas & .67 & 1.11 & 3.26 & 4.81 & 4.59 & 4.53 & 4.25 & 3.99 \\
\hline Petroleum & 1.45 & 2.23 & 5.63 & 6.44 & 4.46 & 4.73 & 4.50 & 4.46 \\
\hline Distillate Fuel ......... & 1.06 & 2.34 & 6.45 & 6.42 & 3.47 & 4.19 & 3.31 & 4.17 \\
\hline 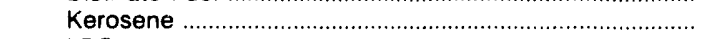 & - & - & - & 6.98 & 3.76 & 4.76 & 3.74 & 4.91 \\
\hline 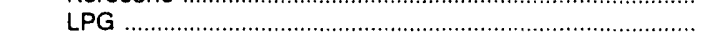 & 1.30 & 2.63 & 5.23 & 8.32 & 8.25 & 5.37 & 4.95 & 3.60 \\
\hline 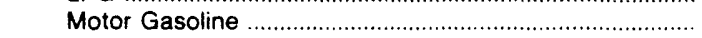 & 2.83 & 4.69 & 9.97 & 9.64 & 6.92 & 7.53 & 8.05 & 8.88 \\
\hline 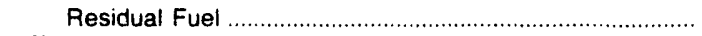 & .84 & 1.69 & 3.78 & 3.49 & 1.92 & 1.61 & 2.49 & 2.58 \\
\hline 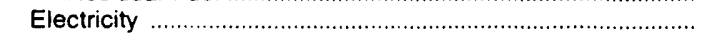 & 6.62 & 7.84 & 12.16 & 17.54 & 17.53 & 17.25 & 17.41 & 16.95 \\
\hline Industrial Sector & 1.52 & 3.12 & 5.87 & $4 . \overline{9} \mathrm{c}$ & 4.19 & 3.90 & 3.75 & 3.85 \\
\hline 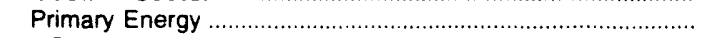 & 1.30 & 2.73 & 5.37 & 4.32 & 3.59 & 3.35 & 3.12 & 3.28 \\
\hline Coal & .74 & 1.26 & 2.63 & 3.21 & 3.11 & 2.80 & 2.66 & 2.74 \\
\hline 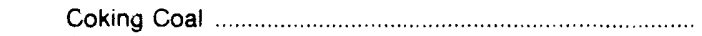 & - & - & - & - & - & - & - & - \\
\hline 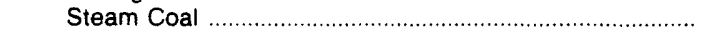 & .74 & 1.26 & 2.63 & 3.21 & 3.11 & 2.80 & 2.66 & 2.74 \\
\hline Natural Gas & .38 & 1.00 & 2.58 & 4.19 & 3.92 & 3.09 & 3.23 & 3.38 \\
\hline Petroleum & 1.45 & 3.16 & 6.07 & 6.68 & 4.68 & 4.84 & 4.33 & 4.72 \\
\hline Asphalt and Road Oil & .79 & 2.08 & 3.85 & 5.17 & 4.07 & 3.74 & 3.53 & 3.13 \\
\hline Distillate Fuel ...... & .79 & 2.72 & 5.50 & 6.70 & 3.61 & 4.57 & 3.60 & 4.71 \\
\hline 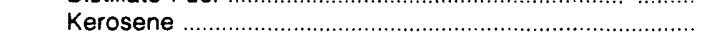 & .85 & 2.90 & 5.76 & 6.98 & 3.76 & 4.76 & 3.74 & 4.91 \\
\hline 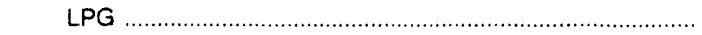 & 1.30 & 2.63 & 5.23 & 8.32 & 8.25 & 5.37 & 4.95 & 3.60 \\
\hline Lubricants & 5.08 & 7.49 & 14.36 & 17.61 & 15.59 & 13.58 & 14.61 & 17.90 \\
\hline Notor Gasoline & 2.83 & 4.69 & 9.97 & 9.64 & 6.92 & 7.53 & 8.05 & 8.88 \\
\hline 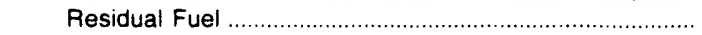 & .94 & 1.94 & 3.19 & 3.49 & 1.92 & 1.61 & 2.49 & 2.58 \\
\hline Other & .51 & 1.40 & 2.48 & 2.31 & 1.53 & 1.50 & 1.45 & 1.92 \\
\hline 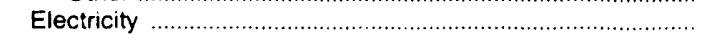 & 5.95 & 8.00 & 9.94 & 15.27 & 15.60 & 15.05 & 14.44 & 14.56 \\
\hline Transportation Sector .... & 2.19 & 3.95 & 8.74 & 8.65 & 6.64 & 7.17 & 7.49 & 8.24 \\
\hline 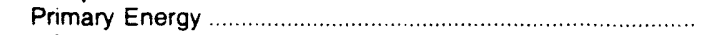 & 2.19 & 3.95 & 8.74 & 8.65 & 6.64 & 7.17 & 7.49 & 8.24 \\
\hline Coal & .74 & 1.26 & - & - & - & - & - & - \\
\hline 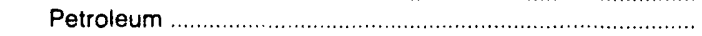 & 2.19 & 3.95 & 8.74 & 8.65 & 6.64 & 7.17 & 7.49 & 8.24 \\
\hline Aviation Gasoline & 2.17 & 3.45 & 9.02 & 9.99 & 8.41 & 7.55 & 7.41 & 8.28 \\
\hline 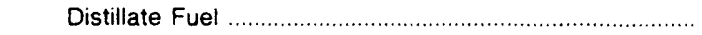 & 1.33 & 2.67 & 7.23 & 7.12 & 6.46 & 6.97 & 7.14 & 7.73 \\
\hline Jet Fuel & .75 & 2.09 & 6.47 & 6.44 & 4.90 & 4.64 & 4.37 & 4.77 \\
\hline LPG & 1.30 & 2.63 & 5.23 & 8.32 & 8.25 & 5.37 & 4.95 & 3.60 \\
\hline Lubricants & 5.08 & 7.49 & 14.36 & 17.61 & 15.59 & 13.58 & 14.61 & 17.90 \\
\hline 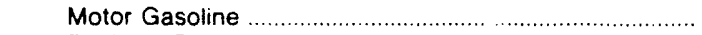 & 2.83 & 4.69 & 9.97 & 9.64 & 6.92 & 7.53 & 8.05 & 8.88 \\
\hline Residual Fuel & .83 & - & - & - & 2.34 & - & - & - \\
\hline 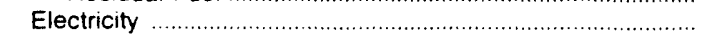 & - & - & - & - & - & - & - & - \\
\hline Total Energy & 1.97 & 3.47 & 7.28 & 7.07 & 5.90 & 5.86 & 5.90 & 6.05 \\
\hline 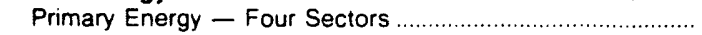 & 1.62 & 3.03 & 6.75 & 5.93 & 4.70 & 4.71 & 4.68 & 4.93 \\
\hline Electricity & 7.04 & 8.57 & 11.96 & 17.11 & 17.30 & 17.15 & 16.93 & 16.74 \\
\hline Electric Utility Sector & .25 & .27 & .57 & .89 & .83 & .80 & .71 & .70 \\
\hline 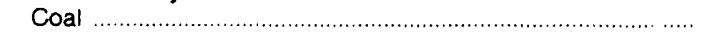 & .25 & .26 & .56 & .88 & .82 & .79 & .70 & .69 \\
\hline Natural Gas & .35 & .66 & 2.47 & 4.74 & 5.22 & 4.43 & 4.29 & 4.39 \\
\hline 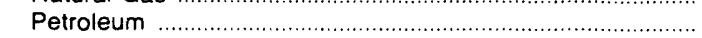 & .96 & 1.94 & 6.07 & 5.52 & 3.71 & 4.19 & 3.81 & 4.41 \\
\hline 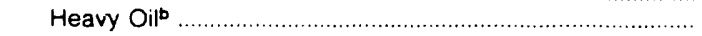 & .90 & 1.93 & - & - & - & - & - & - \\
\hline Light OilD & 1.23 & 2.12 & 6.07 & 5.52 & 3.71 & 4.19 & 3.81 & 4.41 \\
\hline Petroleum Coke & - & - & - & - & - & - & - & - \\
\hline Nuclear Fuel .... . . . . . & - & - & - & - & - & - & - & - \\
\hline Wood and Waste & - & - & - & - & - & - & - & - \\
\hline Primary Energy - Five Sectors . & 1.26 & 2.23 & 3.66 & 3.31 & 2.73 & 2.70 & 2.43 & 2.63 \\
\hline
\end{tabular}

-There are no direct fuel costs for hydroelectric, geothermal, centralized solar, or wind energy. Wood and other biomass fuels are not included, except those consumed at electric utilities.

Heavy oil includes Grade Nos. 4, 5, and 6, and residual fuel olls. Light oil includes Grade No. 2 heating oil, kerosene, and jet fuel.

-No consumption, including cases where adjustments were made. See "Consumption Adjustments for Process Fuel and Intermediate Products."

Sources: Data sources, estimation procedures, and assumptions are described in the "Documentation" section of this report. 


\section{N Energy Expenditure Estimates by Sector, North Dakota \\ O 1970, 1975, 1980, and 1985-1989 \\ R (Million Dollars)}

\begin{tabular}{|c|c|c|c|c|c|c|c|c|}
\hline Sector and Energy Source & 1970 & 1975 & 1980 & 1985 & 1986 & 1987 & 1988 & 1989 \\
\hline Residential Sector & 66.8 & 104.4 & 210.1 & 302.8 & 286.3 & 265.0 & 287.1 & 297.8 \\
\hline 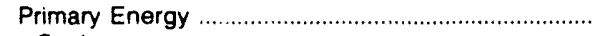 & 29.6 & 44.9 & 100.0 & 117.7 & 102.8 & 89.7 & 97.1 & 109.8 \\
\hline Coal & 1.2 & 2.3 & 1.3 & 1.6 & 1.2 & .7 & .7 & .9 \\
\hline Natural Gas & 8.4 & 15.4 & 37.1 & 57.9 & 49.5 & 44.1 & 47.1 & 46.0 \\
\hline Petroleum . & 20.0 & 27.3 & 61.6 & 58.2 & 52.1 & 44.9 & 49.2 & 62.9 \\
\hline Distillate Fuel & 8.2 & 11.5 & 47.3 & 48.8 & 29.8 & 27.8 & 26.2 & 29.5 \\
\hline 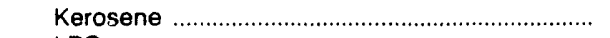 & 1.8 & .3 & .2 & .9 & .2 & .2 & .2 & .4 \\
\hline 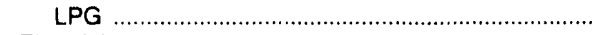 & 10.0 & 15.4 & 14.0 & 8.5 & 22.1 & 16.9 & 22.8 & 33.0 \\
\hline 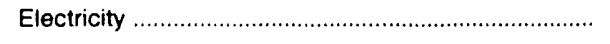 & 37.2 & 59.5 & 110.1 & 185.1 & 183.5 & 175.4 & 190.0 & 188.0 \\
\hline Commercial Sector & 27.9 & 48.0 & 127.8 & 203.1 & 183.3 & 168.3 & 175.8 & 174.4 \\
\hline 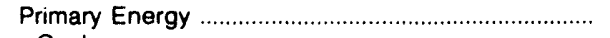 & 12.2 & 27.5 & 80.3 & 81.9 & 63.5 & 52.3 & 57.8 & 59.3 \\
\hline Coal & 1.0 & 1.7 & 3.2 & 5.4 & 4.7 & 2.5 & 3.2 & 4.1 \\
\hline 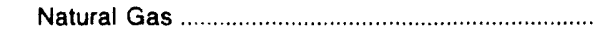 & 5.8 & 13.7 & 37.8 & 51.7 & 43.5 & 37.6 & 44.0 & 44.5 \\
\hline Petroleum & 5.4 & 12.0 & 39.2 & 24.8 & 15.3 & 12.3 & 10.7 & 10.8 \\
\hline 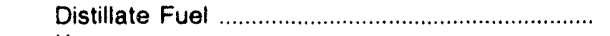 & 1.5 & 2.4 & 24.1 & 18.1 & 6.3 & 5.9 & 3.0 & 4.5 \\
\hline 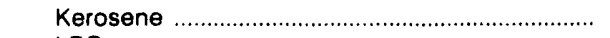 & - & - & - & $\cdot$ & - & • & & \\
\hline 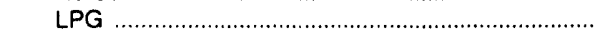 & 1.1 & 2.0 & 1.7 & 1.5 & 5.2 & 3.1 & 3.8 & 2.9 \\
\hline 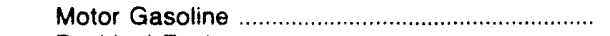 & 2.2 & 2.3 & 3.8 & 3.5 & 2.6 & 2.9 & 3.1 & 2.8 \\
\hline 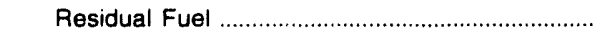 & .5 & 5.2 & 9.5 & 1.7 & 1.1 & .4 & .8 & .6 \\
\hline 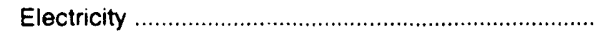 & 15.7 & 21.5 & 47.5 & 121.2 & 119.9 & 116.0 & 118.0 & 115.0 \\
\hline Industrial Sector & 77.3 & 143.2 & 279.1 & 561.4 & 524.4 & 504.6 & 465.7 & 512.1 \\
\hline 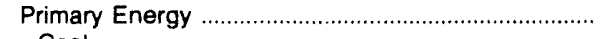 & 63.0 & 116.2 & 226.5 & 460.2 & 426.5 & 412.8 & 366.3 & 414.6 \\
\hline Coal & 5.4 & 9.4 & 20.2 & 228.0 & 251.5 & 245.1 & 219.3 & 244.0 \\
\hline 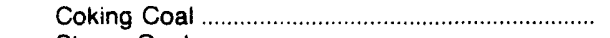 & - & - & & - & - & - & - & - \\
\hline 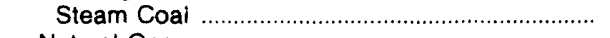 & 5.4 & 9.4 & 20.2 & 228.0 & 251.5 & 245.1 & 219.3 & 244.0 \\
\hline Natural Gas & .7 & 1.9 & 2.6 & 8.7 & 10.3 & 7.7 & 12.3 & 15.0 \\
\hline Petroleum & 56.9 & 105.0 & 203.7 & 223.5 & 164.7 & 159.9 & 134.7 & 155.5 \\
\hline 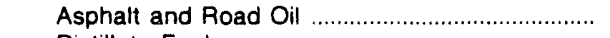 & 7.3 & 14.6 & 19.2 & 35.9 & 23.7 & 22.0 & 22.4 & 19.2 \\
\hline 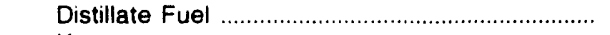 & 10.0 & 25.6 & 78.8 & 1084 & 64.6 & 68.4 & 51.5 & 76.3 \\
\hline 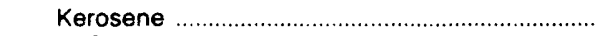 & .3 & .8 & .3 & .1 & .2 & • & .1 & \\
\hline LPG & 1.0 & 1.8 & 13.0 & 12.8 & 36.8 & 23.2 & 15.5 & 13.1 \\
\hline 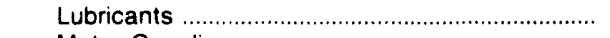 & .9 & 1.0 & 2.3 & 2.5 & 2.2 & 2.2 & 2.2 & 2.8 \\
\hline 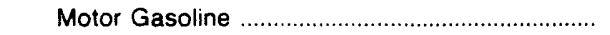 & 34.4 & 54.1 & 80.7 & 54.7 & 33.6 & 40.6 & 37.9 & 38.2 \\
\hline 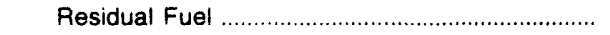 & 2.3 & 4.6 & 4.1 & 6.8 & 2.9 & 2.4 & 3.6 & 4.2 \\
\hline Other & .8 & 2.6 & 5.3 & 2.3 & .8 & 1.3 & 1.4 & 1.7 \\
\hline 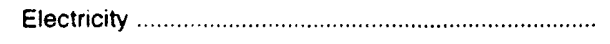 & 14.3 & 27.0 & 52.6 & 101.1 & 97.9 & 91.8 & 99.3 & 97.5 \\
\hline Transportation Sector & 118.6 & 249.1 & 631.5 & 588.5 & 443.1 & 474.9 & 499.0 & 540.8 \\
\hline Primary Energy . & 118.6 & 249.1 & 631.5 & 588.5 & 443.1 & 474.9 & 499.0 & 540.8 \\
\hline Coal & & & - & - & - & - & - & - \\
\hline Petroleum & 118.6 & 249.1 & 631.5 & 588.5 & 443.1 & 474.9 & 499.0 & 540.8 \\
\hline Aviation Gasoline & 1.0 & 1.5 & 2.9 & .2 & 1.6 & 1.1 & 1.2 & 1.3 \\
\hline 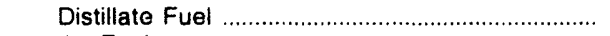 & 11.1 & 29.2 & 159.9 & 126.3 & 108.9 & 124.1 & 130.8 & 137.7 \\
\hline 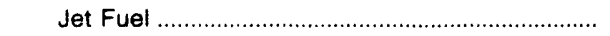 & 8.3 & 20.9 & 59.7 & 58.3 & 43.5 & 31.5 & 31.0 & 34.5 \\
\hline LPG & & & .2 & .4 & .8 & .3 & .4 & .3 \\
\hline 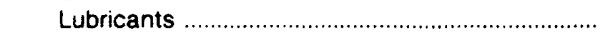 & 4.2 & 6.2 & 13.2 & 14.7 & 12.7 & 12.5 & 13.0 & 16.4 \\
\hline Motor Gasoline & 93.6 & 191.2 & 395.6 & 388.6 & 275.5 & 305.4 & 322.7 & 350.6 \\
\hline 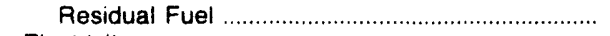 & .2 & - & - & - & " & - & - & - \\
\hline Electricity & - & - & - & - & - & - & - & - \\
\hline & 290.6 & 545.7 & $1,248.6$ & $\begin{array}{l}1,655.8 \\
1,658\end{array}$ & $1,437.2$ & $1,412.9$ & $1,427.6$ & $1,525.1$ \\
\hline 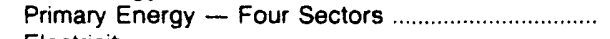 & $\begin{array}{r}223.3 \\
67.3\end{array}$ & $\begin{array}{l}437.7 \\
108.0\end{array}$ & $1,038.4$ & $1,2.48 .3$ & $1,036.0$ & $1,029.7$ & $1,020.3$ & $1,124.5$ \\
\hline Electricity & 67.3 & 108.0 & 210.2 & 407.5 & 401.2 & 383.2 & 407.3 & 400.6 \\
\hline Electric Utility Sector & 12.3 & 15.8 & 87.9 & 203.4 & 188.0 & 183.8 & 201.8 & 189.4 \\
\hline Coal & 12.0 & 15.4 & 85.5 & 201.1 & 186.8 & 182.5 & 200.8 & 187.6 \\
\hline Natural Gas & .1 & .1 & • & • & * & • & & \\
\hline Petroleum & .2 & .2 & 2.4 & 2.4 & 1.2 & 1.2 & 1.0 & 1.9 \\
\hline Heavy Oib & .1 & .2 & - & - & - & - & - & - \\
\hline Light Oib & - & - & 2.4 & 2.4 & 1.2 & 1.2 & 1.0 & 1.9 \\
\hline Petroleum Coke & - & - & - & - & - & - & - & - \\
\hline 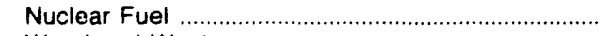 & - & - & - & - & - & - & - & - \\
\hline Wood and Waste & - & - & - & - & - & - & - & - \\
\hline Primary Energy - Five Sectors & 235.6 & 453.5 & $1,126.3$ & $1,451.7$ & $1,224.0$ & $1,213.5$ & $1,222.1$ & $1,314.0$ \\
\hline
\end{tabular}

-There are no direct fuel costs for hydroelectric, geothermal, centralized solar, or wind energy. Wood and other biomass fuels are not included, except those consumed at electric utilities.

b Heavy oil includes Grade Nos. 4, 5, and 6, and residual fuel oils. Light oil includes Grade No. 2 heating oil, kerosene, and jet fuel.

-No consumption, including cases where adjustments were made. See "Consumption Adjustments for Process Fuel and Intermediate Products."

- Value less than 0.05 million dollars.

Note: Tutals may not equal sum of components due to independent rounding.

Sources: Data sources, estimation procedures, and assumptions are described in the "Documentation" section of this report. 


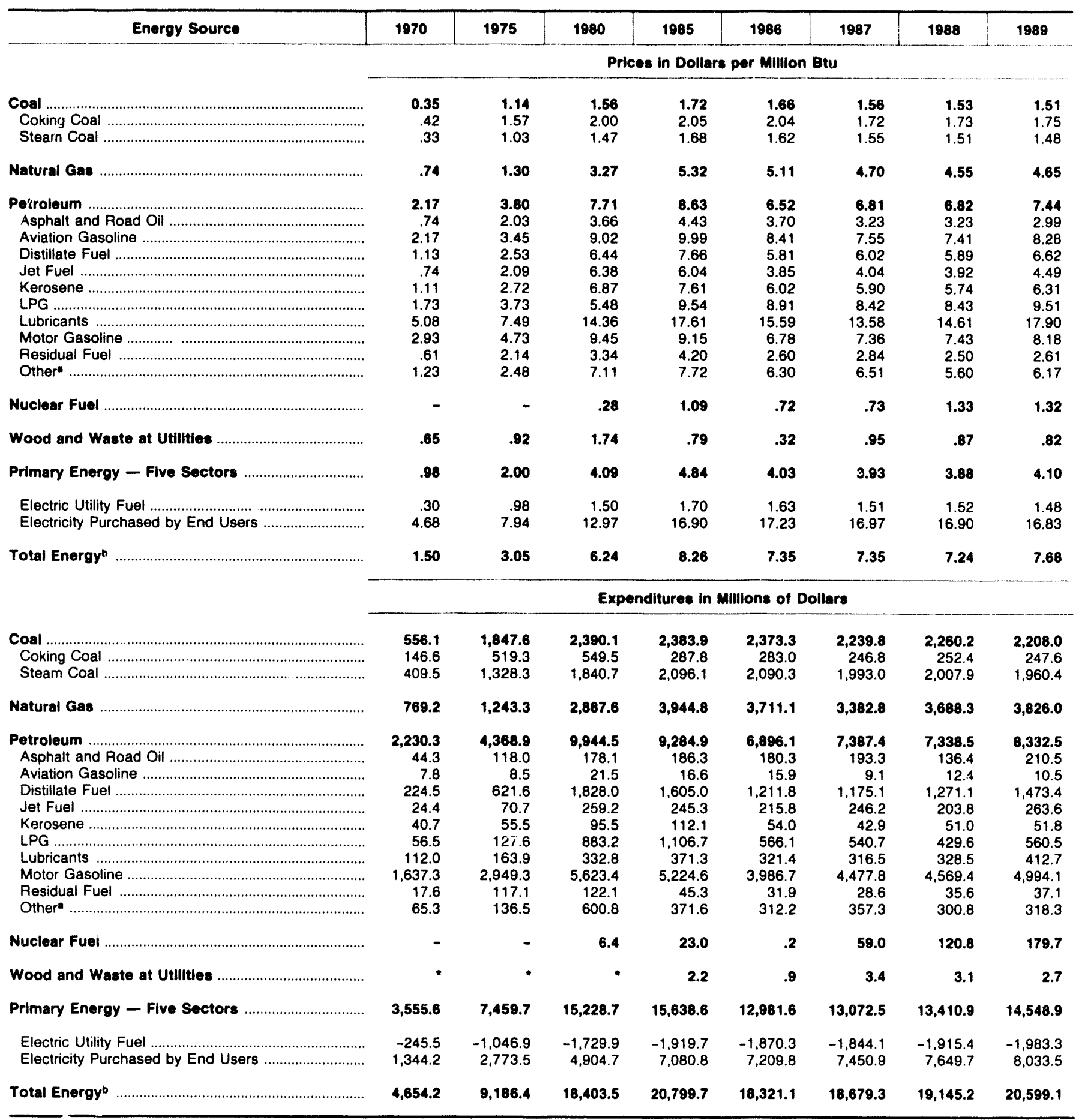

Includes petroleum coke used at electric utilities.

bThere are no direct fuel costs for hydroelectric, geothermal, centralized solar, or wind energy. Wood and other biomass fuels are not included, except those consumed at electric utilities.

-No consumption, including cases where adjustments were made. See "Consumptir,n Adjustments for Process Fuel and Intermediate Products."

"Value less than 0.05 million dollars.

Note: Expenditure totals may not equal sum of components due to independent rounding.

Sources: Data sources, estimation procedures, and assumptions are described in the "Iocumentation" section of this report. 


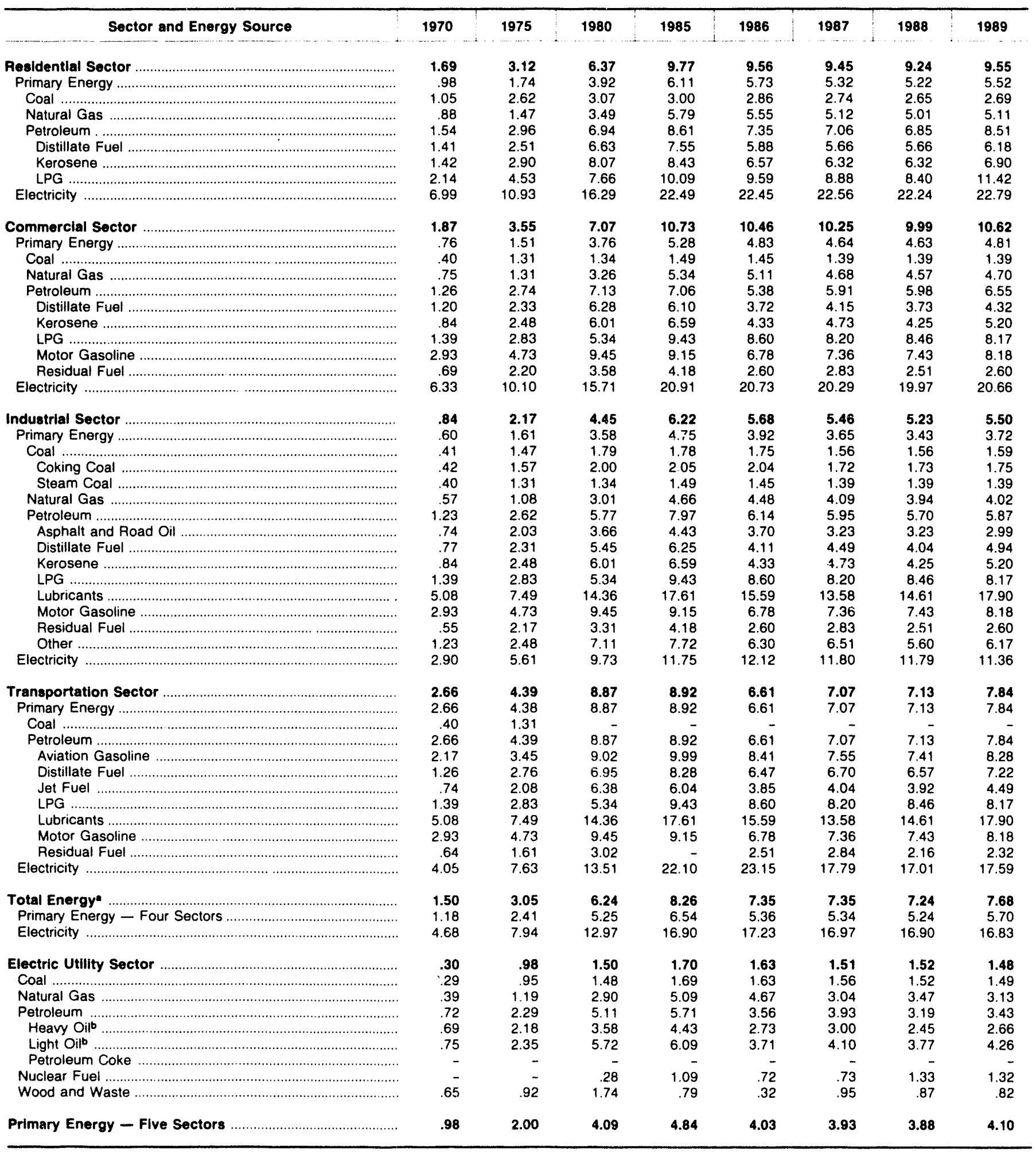

-There are no direct fuel costs for hydroelectric, geothermal, centralized solar, or wind energy. Wood and other biomass fuels are not included, except those consumed at electric utilities.

bHeavy oil includes Grade Nos. 4, 5, and 6, and residual tuel oils. Light oil includes Grade No. 2 heating oil, kerosene, and jet fuel.

-No consumption, including cases where adjustments were made. See "Consumption Adjustments for Process Fuel and Intermediate Products."

Sources: Data sources, estimation procedures, and assumptions are described in the "Documentation" section of this report. 


\begin{tabular}{|c|c|c|c|c|c|c|c|c|}
\hline Sector and Energy Source & 1970 & 1975 & 1980 & 1985 & 1986 & 1987 & 1988 & 1989 \\
\hline $\begin{array}{c}\text { Residential Sector } \\
\text { Primary Energy }\end{array}$ & $1,090.9$ & $1,980.1$ & $3,675.4$ & $5,070.6$ & $5,014.5$ & $4,948.5$ & $5,169.1$ & $5,419.8$ \\
\hline 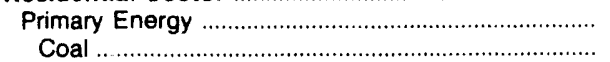 & 559.8 & 940.4 & $1,815.5$ & 2.466 .2 & $2,316.2$ & $2,122.4$ & $2,231,1$ & $2,402.8$ \\
\hline Coal & 13.8 & 23.3 & 14.0 & 21.7 & 25.3 & 18.8 & 16.4 & 12.4 \\
\hline 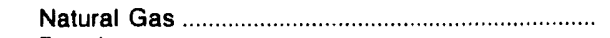 & 414.0 & 643.4 & $1,396.3$ & 1.978 .7 & $1,898.7$ & $1,746.7$ & $1,826.7$ & $1,910.7$ \\
\hline Petroleum & 132.0 & 273.6 & 405.3 & 465.8 & 392.2 & 356.9 & 388.0 & 479.7 \\
\hline Distillate Fuel & 76.5 & 157.8 & 286.8 & 196.7 & 157.0 & 137.1 & 153.4 & 164.6 \\
\hline 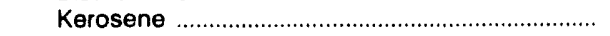 & 24.1 & 33.8 & 46.5 & 68.4 & 44.4 & 33.8 & 40.5 & 37.0 \\
\hline LPG & 31.5 & 82.0 & 72.0 & 200.7 & 190.7 & 186.0 & 194.1 & 278.1 \\
\hline 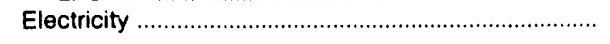 & 531.1 & $1,039.7$ & $1,859.9$ & $2,604.3$ & $2,698.4$ & $2,826.2$ & $2,938.0$ & $3,017.0$ \\
\hline Commerclal Sector .... & 545.7 & 1,023.2 & $2,031.3$ & $3,062.9$ & $3,073.4$ & $3,100.7$ & $3,249.1$ & $3,407.8$ \\
\hline 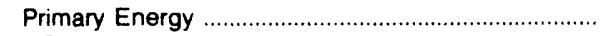 & 176.6 & 332.5 & 781.1 & 980.8 & 917.9 & 901.1 & 978.4 & 977.2 \\
\hline Coal & 9.5 & 21.5 & 11.2 & 19.7 & 23.7 & 17.6 & 15.8 & 11.7 \\
\hline 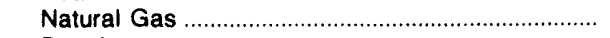 & 140.0 & 227.6 & 551.1 & 799.0 & 743.7 & 718.7 & 754.3 & 791.4 \\
\hline 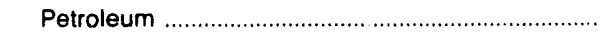 & 27.1 & 83.5 & 218.8 & 162.1 & 150.4 & 164.7 & 208.3 & 174.0 \\
\hline 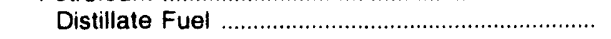 & 13.0 & 29.0 & 94.8 & 72.4 & 46.1 & 51.1 & 48.5 & 42.4 \\
\hline 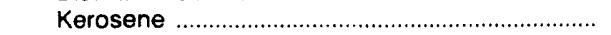 & .7 & 1.5 & 4.4 & 25.0 & 4.7 & 4.5 & 5.7 & 7.7 \\
\hline LPG & 3.6 & 9.0 & 8.9 & 33.1 & 30.2 & 30.3 & 34.5 & 35.1 \\
\hline 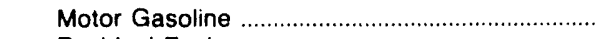 & 6.2 & 23.7 & 102.2 & 29.0 & 66.3 & 77.9 & 118.2 & 88.5 \\
\hline 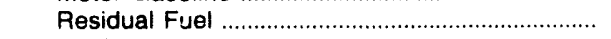 & 3.6 & 20.1 & 8.5 & 2.6 & 3.1 & .9 & 1.3 & .4 \\
\hline 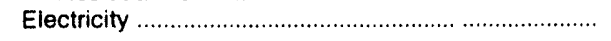 & 369.2 & 690.7 & $1,250.2$ & $2,082.1$ & $2,155.5$ & $2,199.6$ & $2,270.7$ & $2,430.6$ \\
\hline Industrial Sector & $1,259.7$ & $2,883.2$ & $5,821.9$ & $6,027.0$ & $5,120.0$ & $5,046.3$ & $5,025.7$ & $5,362.4$ \\
\hline 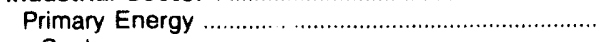 & 816.3 & $1,841.3$ & $4,029.3$ & $3,635.8$ & $2,767.3$ & $2,623.4$ & $2,587.1$ & $2,779.0$ \\
\hline 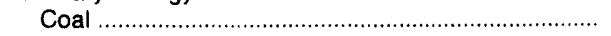 & 301.9 & 815.2 & 723.6 & 473.5 & 470.0 & 438.4 & 457.6 & 407.2 \\
\hline 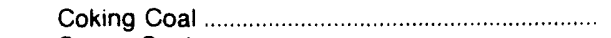 & 146.6 & 519.3 & 549.5 & 287.8 & 283.0 & 246.8 & 252.4 & 247.6 \\
\hline 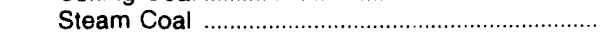 & 155.3 & 296.0 & 174.1 & 185.7 & 187.0 & 191.6 & 205.2 & 159.6 \\
\hline 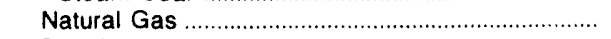 & 206.6 & 366.0 & 926.5 & $1,163.5$ & $1,065.9$ & 914.7 & $1,103.9$ & 1.120 .8 \\
\hline 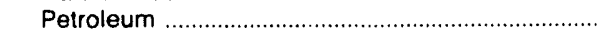 & 307.9 & 660.1 & $2,379.2$ & $1,998.8$ & $1,231.4$ & $1,270.2$ & $1,025.7$ & $1,251.0$ \\
\hline Asphalt and Road Oil ............................................ & 44.3 & 118.0 & 178.1 & 186.3 & 180.3 & 193.3 & 136.4 & 210.5 \\
\hline 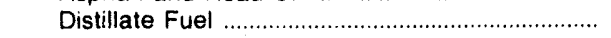 & 50.5 & 149.7 & 396.8 & 243.0 & 139.2 & 138.3 & 121.7 & 150.9 \\
\hline 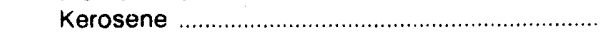 & 15.9 & 20.2 & 44.5 & 18.6 & 4.9 & 4.7 & 4.8 & 7.1 \\
\hline 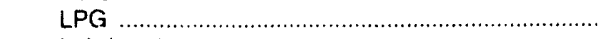 & 20.7 & 34.7 & 797.9 & 856.2 & 329.7 & 313.1 & 188.0 & 233.6 \\
\hline 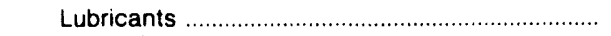 & 73.7 & 90.2 & 208.6 & 232.8 & 201.5 & 198.4 & 205.9 & 258.7 \\
\hline 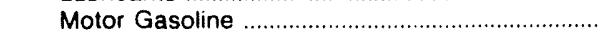 & 29.7 & 37.7 & 57.3 & 51.6 & 36.3 & 39.8 & 40.1 & 43.7 \\
\hline 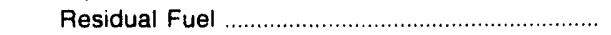 & 7.9 & 73.0 & 95.1 & 38.8 & 27.3 & 25.4 & 28.1 & 28.1 \\
\hline Other & 65.3 & 136.5 & 600.8 & 371.6 & 312.2 & 357.3 & 300.8 & 318.3 \\
\hline 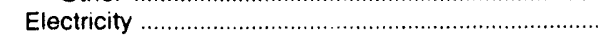 & 443.4 & $1,042.0$ & $1,792.6$ & $2,391.2$ & $2,352.8$ & $2,422.9$ & $2,438.6$ & $2,583.4$ \\
\hline $\begin{array}{c}\text { Transportation Sector } \\
\text { Primary Energy }\end{array}$ & $\begin{array}{l}1,757.9 \\
1.757 .3\end{array}$ & $\begin{array}{l}3,299.8 \\
3,298.7\end{array}$ & $\begin{array}{l}6,874.9 \\
6,872.9\end{array}$ & $\begin{array}{l}6,639.3 \\
6,636.1\end{array}$ & $\begin{array}{l}\mathbf{5 , 1 1 3 . 2} \\
5,110.0\end{array}$ & $\begin{array}{l}5,583.8 \\
5,581.5\end{array}$ & $\begin{array}{l}5,701.2 \\
5,698.9\end{array}$ & $\begin{array}{l}6,409.1 \\
6,406.7\end{array}$ \\
\hline 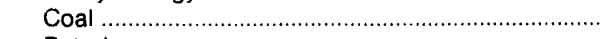 & .4 & .1 & & - & - & - & -5 & $0 \overline{7}$ \\
\hline Petroleum & $1,756.8$ & $3,298.6$ & $6,872.9$ & $6,636.1$ & $5,110.0$ & $5,581,5$ & $5,698.9$ & $6,406.7$ \\
\hline Aviation Gasoline ……………………………........ & 7.8 & 8.5 & 21.5 & 16.6 & 15.9 & 9.1 & 12.4 & 10.5 \\
\hline 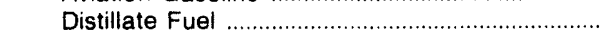 & 81.2 & 251.5 & 994.9 & $1,074.9$ & 858.8 & 836.2 & 935.9 & $1,102.9$ \\
\hline Jet Fuel & 24.4 & 69.2 & 259.2 & 245.3 & 215.8 & 246.2 & 203.8 & 263.6 \\
\hline 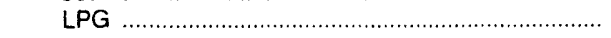 & .7 & 1.9 & 4.4 & 16.7 & 15.5 & 11.3 & 13.0 & 13.7 \\
\hline Lubricants & 38.3 & 73.7 & 124.2 & 138.5 & 119.9 & 118.1 & 122.5 & 154.0 \\
\hline Motor Gasoline & $1,601.5$ & $2,887.8$ & $5,463.9$ & $5,144.0$ & $3,884.0$ & $4,360.1$ & $4,411.1$ & $4,861.9$ \\
\hline 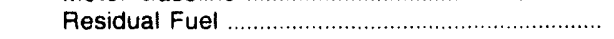 & 3.1 & 6.0 & 4.8 & - & 1 & .6 & .2 & .2 \\
\hline 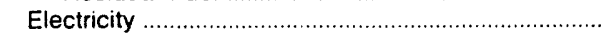 & .6 & 1.2 & 2.0 & 3.2 & 3.2 & 2.3 & 2.3 & 2.4 \\
\hline Total Energya & $4,654.2$ & $9,186.4$ & $18,403.5$ & $20,799.7$ & $18,321.1$ & $18,679.3$ & $19,145.2$ & $20,599.1$ \\
\hline Primary Energy - Four Sectors .................................. & $3,310.0$ & 6.412 .8 & $13,498.9$ & $13,718.9$ & $11,111.3$ & $11,228.4$ & $11,495.5$ & $12,565.6$ \\
\hline 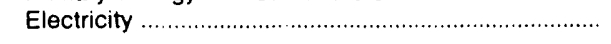 & $1,344.2$ & $2,773.5$ & $4,904.7$ & $7,080.8$ & $7,209.8$ & $7,450.9$ & $7,649.7$ & $8,033.5$ \\
\hline Electric Utlity Sector & 245.5 & $1,046.9$ & $1,729.9$ & $1,919.7$ & $1,870.3$ & $1,844.1$ & $1,915.4$ & $1,983.3$ \\
\hline 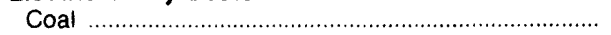 & 230.5 & 987.4 & 1.641 .4 & $1,869.0$ & $1,854.3$ & $1,764.9$ & $1,770.4$ & $1,776.7$ \\
\hline Natural Gas & 8.6 & 6.3 & 13.7 & 3.6 & 2.9 & 2.7 & 3.4 & 3.1 \\
\hline 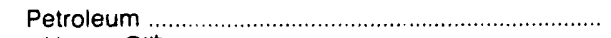 & 6.4 & 53.2 & 68.3 & 22.0 & 12.1 & 14.1 & 17.7 & 21.1 \\
\hline 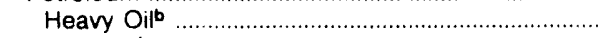 & 3.0 & 18.0 & 13.6 & 3.9 & 1.4 & 1.7 & 6.0 & 8.5 \\
\hline 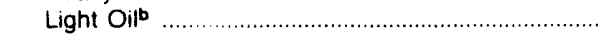 & 3.4 & 35.2 & 54.7 & 18.0 & 10.7 & 12.4 & 11.7 & 12.6 \\
\hline Petroleum Coke & - & - & - & & - & - & - & \\
\hline Nuclear Fuel & - & - & 6.4 & 23.0 & .2 & 59.0 & 120.8 & 179.7 \\
\hline Wood and Waste & • & - & & 2.2 & .9 & 3.4 & 3.1 & 2.7 \\
\hline Primáry Energy - Five Sectors. & $3,555.6$ & $7,459.7$ & $15,228.7$ & $15,638.6$ & $12,981.6$ & $13,072.5$ & $13,410.9$ & $14,548.9$ \\
\hline
\end{tabular}

- There are no direct fuel costs for hydroelectric, geothermal, centralized solar, or wind energy. Wood and other biomass fuels are not included, except those consumed at electric utilities.

bHeavy oil includes Grade Nos. 4, 5, and 6, and residual fuel oils. Light oil includes Grade No. 2 heating oil, kerosene, and jet fuel.

-No consumption, includirig cases where adjustments were made. See "Consumption Adjustments for Process Fuel and Intermediate Products."

*Value less than 0.05 million dollars.

Note: Totals may not equal sum of components due to independent rounding.

Sources: Data sources, estimation procedures, and assumptions are described in the "Documentation" section of this report. 


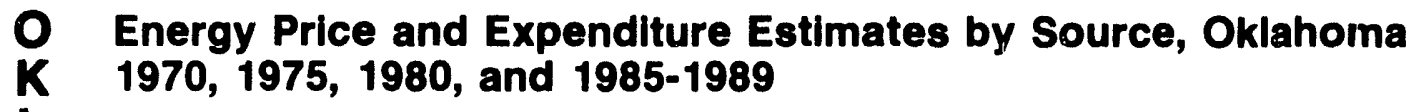

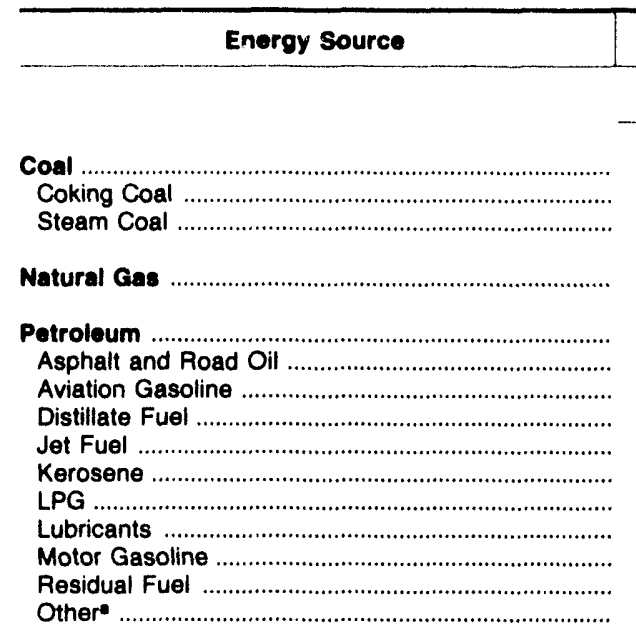

1970

Nuclear Fuel

Wood and Waste at Utilities

Primary Energy - Five Sectors

Electric Utility Fuel

Electricity Purchased by End Users

Total Enorgyb

1975

1880

1985

1986

1987

1988

1989

Prices in Dollare per Million Btu

$\begin{array}{rrrrrrrr}0.33 & 0.96 & 1.25 & 1.69 & 1.65 & 1.58 & 1.50 & 1.29 \\ -33 & .96 & 1.25 & 1.69 & 1.65 & 1.58 & 1.50 & 1.29 \\ .35 & .75 & 1.96 & 3.41 & 3.11 & 2.76 & 2.80 & 2.87 \\ 1.97 & 3.52 & 7.97 & 7.43 & 6.04 & 6.33 & 6.15 & 6.77 \\ .68 & 1.86 & 3.60 & 4.20 & 4.58 & 3.44 & 3.24 & 3.15 \\ 2.17 & 3.45 & 9.02 & 9.99 & 8.41 & 7.55 & 7.41 & 8.28 \\ .90 & 2.36 & 6.77 & 5.54 & 5.36 & 5.63 & 5.52 & 6.21 \\ .72 & 2.01 & 6.34 & 5.87 & 3.94 & 3.90 & 3.75 & 4.27 \\ .66 & 2.41 & 6.52 & 4.19 & 4.97 & 4.01 & 4.89 & 6.17 \\ 1.42 & 2.91 & 6.03 & 7.26 & 7.84 & 7.33 & 5.59 & 5.79 \\ 5.08 & 7.49 & 14.36 & 17.61 & 15.59 & 13.58 & 14.61 & 17.90 \\ 2.82 & 4.52 & 9.79 & 8.76 & 6.30 & 7.08 & 7.12 & 7.79 \\ .50 & 1.58 & 3.23 & 3.41 & 2.10 & 2.18 & 1.81 & 2.03 \\ .58 & 1.45 & 2.90 & 3.36 & 3.04 & 2.89 & 2.44 & 2.99\end{array}$

\begin{tabular}{|c|c|c|c|c|c|c|c|c|}
\hline \multirow[t]{2}{*}{ Total Energy } & 1.83 & 3.06 & 6.43 & 7.58 & 6.97 & 6.60 & 6.48 & 6.73 \\
\hline & \multicolumn{8}{|c|}{ Expenditures in Millions of Dollars } \\
\hline 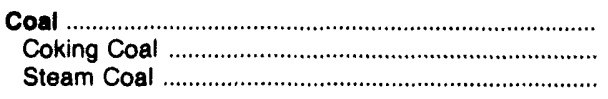 & $\begin{array}{r}0.1 \\
.1\end{array}$ & $\begin{array}{r}0.5 \\
\overline{5}\end{array}$ & 132.5 & $\begin{array}{r}400.2 \\
400 . \overline{2}\end{array}$ & $\begin{array}{r}360.2 \\
360 . \overline{2}\end{array}$ & $\begin{array}{r}379.9 \\
379.9\end{array}$ & $\begin{array}{r}404.1 \\
404 . \overline{1}\end{array}$ & $\begin{array}{r}346.5 \\
346 . \overline{5}\end{array}$ \\
\hline 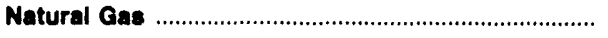 & 152.7 & 392.2 & $1,200.5$ & $1,633.3$ & $1,416.3$ & $1,305.6$ & $1,307.5$ & $1,352.6$ \\
\hline $\begin{array}{l}\text { Potroleum } \\
\text { Asphalt and Road Oil } \\
\text { Aviation Gasoline . } \\
\text { Distillate Fuel } \\
\text { Jet Fuel } \\
\text { Kerosene } \\
\text { LPG } \\
\text { Lubricants } \\
\text { Motor Gasoline } \\
\text { Residual Fuel } \\
\text { Other }\end{array}$ & $\begin{array}{r}636.9 \\
20.7 \\
4.9 \\
28.7 \\
17.2 \\
4.1 \\
50.3 \\
19.2 \\
481.9 \\
2.2 \\
7.7\end{array}$ & $\begin{array}{r}1,329.1 \\
69.9 \\
5.4 \\
128.1 \\
43.2 \\
4.5 \\
99.1 \\
36.8 \\
913.4 \\
5.7 \\
23.1\end{array}$ & $\begin{array}{r}3,202.7 \\
115.1 \\
14.9 \\
478.2 \\
170.5 \\
12.6 \\
196.2 \\
118.1 \\
2,038.2 \\
13.1 \\
45.7\end{array}$ & $\begin{array}{r}3,274.6 \\
111.7 \\
11.0 \\
592.0 \\
190.6 \\
4.1 \\
270.5 \\
131.8 \\
1,940.7 \\
3.4 \\
18.9\end{array}$ & $\begin{array}{r}2,360.9 \\
99.8 \\
10.6 \\
434.6 \\
129.8 \\
2.2 \\
210.8 \\
114.1 \\
1,342.7 \\
4.2 \\
12.2\end{array}$ & $\begin{array}{r}2,427.0 \\
62.4 \\
6.8 \\
457.6 \\
161.8 \\
1.3 \\
168.3 \\
112.3 \\
1,436.3 \\
3.5 \\
15.7\end{array}$ & $\begin{array}{r}2,429.3 \\
76.7 \\
6.5 \\
479.1 \\
150.7 \\
2.2 \\
122.9 \\
116.6 \\
1,454.1 \\
5.7 \\
14.9\end{array}$ & $\begin{array}{r}2,721.9 \\
57.5 \\
6.9 \\
533.6 \\
220.5 \\
4.5 \\
139.9 \\
146.5 \\
1,590.1 \\
5.0 \\
17.4\end{array}$ \\
\hline 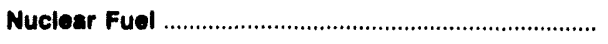 & - & - & - & - & - & - & - & - \\
\hline Wood and Waste at Utilities & - & - & - & - & - & - & - & - \\
\hline Primary Energy - Five Sectore .................................. & 789.6 & $1,721.8$ & $4,644.7$ & $5,308.2$ & $4,137.4$ & $4,112.5$ & $4,140.9$ & $4,420.8$ \\
\hline 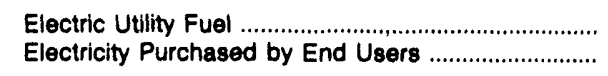 & $\begin{array}{l}-46.8 \\
311.7\end{array}$ & $\begin{array}{r}-190.0 \\
509.6\end{array}$ & $\begin{array}{r}-727.3 \\
1,211.3\end{array}$ & $\begin{array}{r}-988.5 \\
2,141.2\end{array}$ & $\begin{array}{r}-883.8 \\
2,158.5\end{array}$ & $\begin{array}{r}-882.1 \\
2,0+4.1\end{array}$ & $\begin{array}{r}-900.4 \\
2,047.7\end{array}$ & $\begin{array}{r}-891.0 \\
2,040.0\end{array}$ \\
\hline 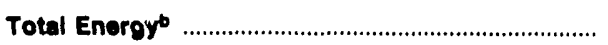 & $1,054.5$ & $2,041.4$ & $5,028.7$ & $8,400.9$ & $5,412.1$ & $5,244.5$ & $5,288.2$ & $5,569.9$ \\
\hline
\end{tabular}

Includes petroleum coke used at electric utilities.

bThere are no direct fuel costs for hydroelectric, geothermal, centralized solar, or wind energy. Wood and other blomass fuels are not included, except those consumed at electric utilities.

- No consumption, including cases where adjustments were made. See "Consumption Adjustments for Process Fuel and Intermediate Products."

Note: Expenditure totals may not equal sum of components due to independent rounding.

Sources: Data sources, estimation procedures, and assumptions are described in the "Documentation" section of this report. 
Energy Price Estimates by Sector, Oklahoma

1970 , 1975, 1980, and 1985-1989

(Dollars per Million Btu)

\begin{tabular}{|c|c|c|c|c|c|c|c|c|}
\hline Sector and Energy Source & 1970 & 1975 & 1980 & 1985 & 1986 & 1987 & 1988 & 1989 \\
\hline 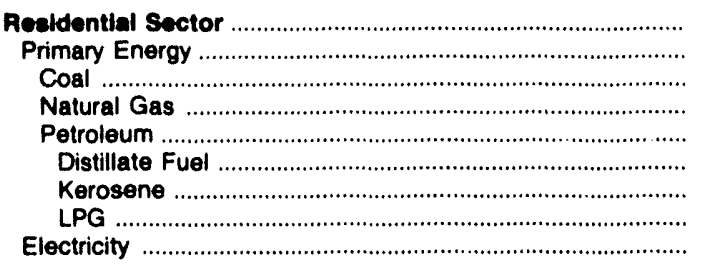 & $\begin{array}{r}2.24 \\
.99 \\
.90 \\
.81 \\
1.60 \\
.89 \\
1.41 \\
1.60 \\
7.42\end{array}$ & $\begin{array}{l}3.19 \\
1.62 \\
1.58 \\
1.22 \\
3.12 \\
2.22 \\
2.88 \\
3.13 \\
8.22\end{array}$ & $\begin{array}{r}6.40 \\
2.84 \\
2.54 \\
2.46 \\
7.29 \\
6.60 \\
7.95 \\
7.29 \\
13.50\end{array}$ & $\begin{array}{r}10.01 \\
4.92 \\
2.83 \\
4.49 \\
7.56 \\
3.73 \\
4.57 \\
7.78 \\
19.37\end{array}$ & $\begin{array}{r}11.06 \\
5.12 \\
2.78 \\
4.90 \\
6.92 \\
5.41 \\
6.63 \\
6.95 \\
20.69\end{array}$ & $\begin{array}{r}10.64 \\
4.67 \\
2.40 \\
4.49 \\
6.28 \\
3.64 \\
4.45 \\
6.33 \\
19.76\end{array}$ & $\begin{array}{r}10.25 \\
4.58 \\
2.12 \\
4.36 \\
6.74 \\
4.81 \\
5.89 \\
6.80 \\
19.74\end{array}$ & $\begin{array}{r}10.34 \\
4.64 \\
2.43 \\
4.40 \\
6.71 \\
6.14 \\
7.52 \\
6.70 \\
20.02\end{array}$ \\
\hline $\begin{array}{l}\text { Commercial Sector } \\
\text { Primary Energy } \\
\text { Coal . } \\
\text { Natural Gas } \\
\text { Petroleum } \\
\text { Distillate Fuel } \\
\text { Kerosene } \\
\text { LPG } \\
\text { Motor Gasoline } \\
\text { Residual Fuel } \\
\text { Electricity }\end{array}$ & $\begin{array}{r}1.68 \\
.61 \\
- \\
.51 \\
1.18 \\
.82 \\
.62 \\
1.12 \\
2.82 \\
.47 \\
5.50\end{array}$ & $\begin{array}{r}2.96 \\
1.24 \\
.94 \\
.94 \\
2.58 \\
2.12 \\
2.37 \\
2.56 \\
4.52 \\
1.46 \\
6.73\end{array}$ & $\begin{array}{r}6.12 \\
2.74 \\
1.39 \\
2.30 \\
7.20 \\
6.31 \\
6.42 \\
5.72 \\
9.79 \\
3.42 \\
11.91\end{array}$ & $\begin{array}{r}10.52 \\
4.50 \\
1.79 \\
4.32 \\
5.41 \\
3.20 \\
4.05 \\
7.01 \\
8.76 \\
- \\
18.02\end{array}$ & $\begin{array}{r}11.27 \\
4.51 \\
1.84 \\
4.31 \\
6.07 \\
3.83 \\
4.75 \\
8.25 \\
6.30 \\
- \\
18.48\end{array}$ & $\begin{array}{r}10.72 \\
4.24 \\
1.96 \\
4.05 \\
5.34 \\
2.86 \\
3.77 \\
7.76 \\
7.08 \\
2.18 \\
17.13\end{array}$ & $\begin{array}{r}9.35 \\
4.01 \\
1.88 \\
3.92 \\
4.68 \\
3.42 \\
4.49 \\
4.95 \\
7.12 \\
1.80 \\
16.67\end{array}$ & $\begin{array}{r}10.01 \\
4.10 \\
1.42 \\
3.86 \\
5.35 \\
4.53 \\
5.91 \\
5.33 \\
7.79 \\
2.01 \\
16.87\end{array}$ \\
\hline $\begin{array}{l}\text { Industrial Sector } \\
\text { Primary Energy } \\
\text { Coal ... } \\
\text { Coking Coal } \\
\text { Steam Coal I } \\
\text { Natural Gas } \\
\text { Petroleum } \\
\text { Asphalt and Road Oil } \\
\text { Distillate Fuel } \\
\text { Kerosene } \\
\text { LPG } \\
\text { Lubricants } \\
\text { Motor Gasoline } \\
\text { Residual Fuel } \\
\text { Other . } \\
\text { Electricity . }\end{array}$ & $\begin{array}{r}.76 \\
.53 \\
- \\
- \\
- \\
.25 \\
.82 \\
.68 \\
.54 \\
.62 \\
1.12 \\
5.08 \\
2.82 \\
.53 \\
.58 \\
3.13\end{array}$ & $\begin{array}{r}1.70 \\
1.39 \\
.94 \\
- \\
.94 \\
.72 \\
2.08 \\
1.86 \\
2.09 \\
2.37 \\
2.56 \\
7.49 \\
4.52 \\
1.65 \\
1.45 \\
4.29\end{array}$ & $\begin{array}{r}3.86 \\
3.23 \\
1.39 \\
- \\
1.39 \\
2.11 \\
4.92 \\
3.60 \\
5.68 \\
6.42 \\
5.72 \\
14.36 \\
9.79 \\
3.22 \\
2.90 \\
9.31\end{array}$ & $\begin{array}{r}4.92 \\
3.83 \\
1.79 \\
- \\
1.79 \\
3.23 \\
5.05 \\
4.20 \\
3.34 \\
4.05 \\
7.01 \\
17.61 \\
8.76 \\
3.39 \\
3.36 \\
13.33\end{array}$ & $\begin{array}{r}4.70 \\
3.49 \\
1.84 \\
- \\
1.84 \\
2.57 \\
5.66 \\
4.58 \\
3.92 \\
4.75 \\
8.25 \\
15.59 \\
6.30 \\
2.11 \\
3.04 \\
13.05\end{array}$ & $\begin{array}{r}3.75 \\
2.74 \\
1.96 \\
- \\
1.96 \\
1.96 \\
5.05 \\
3.44 \\
3.11 \\
3.77 \\
7.76 \\
13.58 \\
7.08 \\
2.18 \\
2.89 \\
11.19\end{array}$ & $\begin{array}{r}3.53 \\
2.47 \\
1.88 \\
- \\
1.88 \\
1.70 \\
4.30 \\
3.24 \\
3.71 \\
4.49 \\
4.95 \\
14.61 \\
7.12 \\
1.80 \\
2.44 \\
10.75\end{array}$ & $\begin{array}{r}3.67 \\
2.65 \\
- \\
- \\
- \\
1.93 \\
5.15 \\
3.15 \\
4.88 \\
5.91 \\
5.33 \\
17.90 \\
7.79 \\
2.01 \\
2.99 \\
10.73\end{array}$ \\
\hline 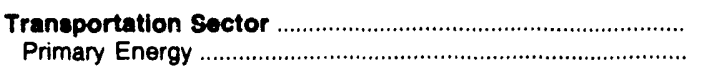 & $\begin{array}{l}2.44 \\
2.44\end{array}$ & $\begin{array}{l}4.11 \\
4.11\end{array}$ & $\begin{array}{l}9.12 \\
9.12\end{array}$ & $\begin{array}{l}8.27 \\
8.27\end{array}$ & $\begin{array}{l}6.11 \\
6.11\end{array}$ & $\begin{array}{l}6.62 \\
6.62\end{array}$ & $\begin{array}{l}6.59 \\
6.59\end{array}$ & $\begin{array}{l}7.13 \\
7.13\end{array}$ \\
\hline $\begin{array}{l}\text { Peal } \\
\text { Aviation Gasoline } \\
\text { Distillate Fuel } \\
\text { Jet Fuel } \\
\text { LPG } \\
\text { Lubricants } \\
\text { Motor Gasoline }\end{array}$ & $\begin{array}{r}- \\
2.44 \\
2.17 \\
1.11 \\
.72 \\
1.12 \\
5.08 \\
2.82\end{array}$ & $\begin{array}{r}.94 \\
4.11 \\
3.45 \\
2.61 \\
2.01 \\
2.56 \\
7.49 \\
4.52\end{array}$ & $\begin{array}{r}9.12 \\
9.02 \\
7.30 \\
6.34 \\
5.72 \\
14.36 \\
9.79\end{array}$ & $\begin{array}{r}- \\
8.27 \\
9.99 \\
7.15 \\
5.87 \\
7.01 \\
17.61 \\
8.76\end{array}$ & $\begin{array}{r}6.11 \\
8.41 \\
5.92 \\
3.94 \\
8.25 \\
15.59 \\
6.30\end{array}$ & $\begin{array}{r}6.62 \\
7.55 \\
6.45 \\
3.90 \\
7.76 \\
13.58 \\
7.08\end{array}$ & $\begin{array}{r}- \\
6.59 \\
7.41 \\
6.17 \\
3.75 \\
4.95 \\
14.61 \\
7.12\end{array}$ & $\begin{array}{r}- \\
7.13 \\
8.28 \\
6.64 \\
4.27 \\
5.33 \\
17.90 \\
7.79\end{array}$ \\
\hline Residual Fuel & .46 & 1.79 & - & - & - & - & - & - \\
\hline Electricity & - & - & - & - & - & - & $\sim$ & - \\
\hline 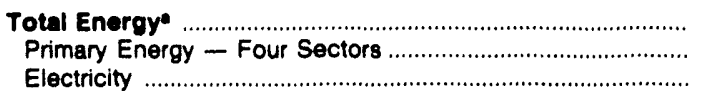 & $\begin{array}{l}1.83 \\
1.42 \\
5.76\end{array}$ & $\begin{array}{l}3.08 \\
2.59 \\
6.64\end{array}$ & $\begin{array}{r}6.43 \\
5.62 \\
11.80\end{array}$ & $\begin{array}{r}7.58 \\
5.83 \\
17.23\end{array}$ & $\begin{array}{r}6.97 \\
4.96 \\
17.83\end{array}$ & $\begin{array}{r}6.60 \\
4.80 \\
16.48\end{array}$ & $\begin{array}{r}6.48 \\
4.68 \\
16.20\end{array}$ & $\begin{array}{r}6.73 \\
5.03 \\
16.28\end{array}$ \\
\hline $\begin{array}{l}\text { Electric Utility sector } \\
\text { Coal } \\
\text { Natural Gas } \\
\text { Petroleum } \\
\text { Heavy Oifb } \\
\text { Light Oib }\end{array}$ & $\begin{array}{l}.19 \\
.39 \\
.19 \\
.50 \\
.46 \\
.56\end{array}$ & $\begin{array}{r}.61 \\
.43 \\
.61 \\
1.75 \\
1.45 \\
1.92\end{array}$ & $\begin{array}{l}1.63 \\
1.23 \\
1.74 \\
5.29 \\
3.44 \\
5.30\end{array}$ & $\begin{array}{l}2.30 \\
1.68 \\
2.95 \\
5.34 \\
3.73 \\
5.54\end{array}$ & $\begin{array}{l}2.17 \\
1.64 \\
2.68 \\
2.81 \\
1.50 \\
2.90\end{array}$ & $\begin{array}{l}2.08 \\
1.56 \\
2.67 \\
3.89 \\
2.76 \\
3.91\end{array}$ & $\begin{array}{l}2.04 \\
1.48 \\
2.81 \\
3.35 \\
2.50 \\
3.42\end{array}$ & $\begin{array}{l}2.02 \\
1.36 \\
2.93 \\
3.94 \\
2.91 \\
4.15\end{array}$ \\
\hline $\begin{array}{l}\text { Petroleum Coke } \\
\text { Nuclear Fuel } \\
\text { Wood and Waste }\end{array}$ & - & - & - & - & - & - & - & - \\
\hline Primary Energy - Flve Sectore & 1.03 & 1.90 & 4.04 & 4.59 & 3.89 & 3.75 & 3.66 & 3.87 \\
\hline
\end{tabular}

-There are no direct fuel costs for hydroelectric, geothermal, centralized solar, or wind energy. Wood and other biomass fuels are not included, except those consumed at electric utilities.

b Heavy oil includes Grade Nos. 4, 5, and 6, and residual fuel oils. Light oil includes Grade No. 2 heating oil, kerosene, and jet fuel.

- No consumption, including cases where adjustments were made. See "Consumption Adjustments for Process Fuel and Intermediate Products."

Sources: Data sources, estimation procedures, and assumptions are described in the "Documentation" section of this report. 


\section{- Energy Expenditure Estimates by Sector, Oklahoma \\ K 1970, 1975, 1980, and 1985-1989 \\ L (Million Dollars)}

Sector and Energy Source
Residentlal Sector
Primary Energy
Coal
Natural Gas
Petroleum
Distillate Fuel
Kerosene
LPG
Electricity

\section{Commercial Sector}

Primary Energy.

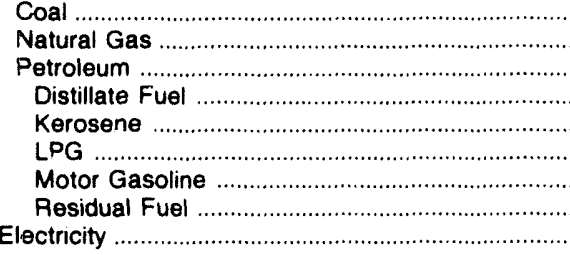

Electricity

\section{Industrial Sector}

Primary Energy

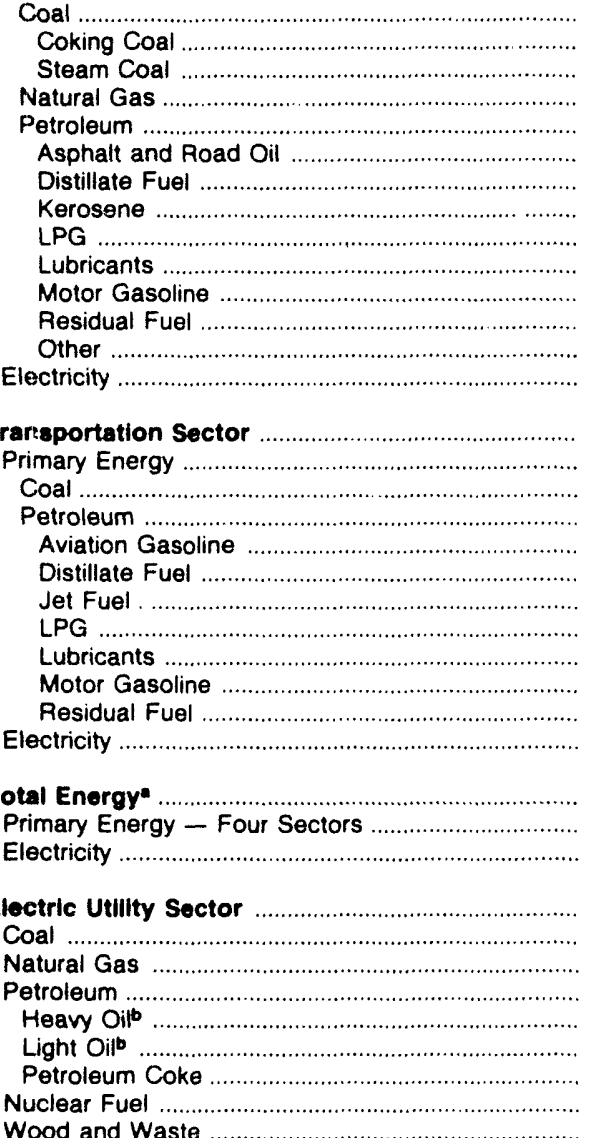

Wood and Waste

Primary Energy - Five Sectors

\begin{tabular}{|c|c|c|c|c|c|c|c|}
\hline 1970 & 1975 & 1980 & 1985 & 1986 & 1987 & 1988 & 1989 \\
\hline 285.3 & 422.0 & 804.6 & $1,396.2$ & $1,375.8$ & $1,291.9$ & $1,354.1$ & $1,340.4$ \\
\hline 100.7 & 163.3 & 237.8 & 444.6 & 394.4 & 342.3 & 379.2 & 378.4 \\
\hline " & • & .6 & .1 & .1 & $\bullet$ & .1 & • \\
\hline 65.1 & 97.3 & 188.5 & 348.3 & 333.7 & 297.1 & 325.3 & 323.1 \\
\hline 35.5 & 66.0 & 48.6 & 96.3 & 60.6 & 45.2 & 53.7 & 55.3 \\
\hline 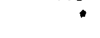 & .2 & .6 & 1.8 & .9 & .2 & .8 & • \\
\hline .4 & .4 & .9 & 1.2 & .3 & .5 & .7 & .9 \\
\hline 35.1 & 65.4 & 47.1 & 93.3 & 59.3 & 44.5 & 52.2 & 54.4 \\
\hline 184.6 & 258.7 & 566.8 & 951.6 & 981.5 & 949.6 & 975.0 & 962.0 \\
\hline 115.4 & 219.6 & 509.6 & 944.0 & 926.0 & 843.3 & 918.0 & 876.9 \\
\hline 32.5 & 63.1 & 143.8 & 224.1 & 191.3 & 165.7 & 228.0 & 192.8 \\
\hline - & • & .6 & .1 & .1 & .1 & .2 & • \\
\hline 22.9 & 39.1 & 108.4 & 179.8 & 160.9 & 135.5 & 194.8 & 151.7 \\
\hline 9.6 & 24.0 & 34.8 & 44.2 & 30.3 & 30.0 & 32.9 & 41.2 \\
\hline .5 & 5.0 & 11.6 & 13.1 & 6.3 & 6.8 & 12.4 & 16.8 \\
\hline .8 & 1.4 & .5 & .7 & .1 & .1 & 1.0 & 3.2 \\
\hline 4.3 & 9.4 & 6.5 & 14.8 & 12.4 & 9.6 & 6.7 & 7.6 \\
\hline 3.4 & 6.3 & 15.5 & 15.6 & 11.4 & 13.3 & 12.8 & 12.8 \\
\hline .6 & 1.8 & .6 & - & - & .2 & .1 & .7 \\
\hline 82.9 & 156.5 & 365.8 & 719.9 & 734.7 & 677.6 & 690.0 & 684.0 \\
\hline 122.6 & 352.2 & $1,110.5$ & $1,519.3$ & $1,253.1$ & $1,084.8$ & 981.5 & $1,062.6$ \\
\hline 78.3 & 257.7 & 831.9 & $1,049.7$ & 810.9 & 697.9 & 598.8 & 668.6 \\
\hline- & .4 & 7.8 & 32.6 & 30.2 & 25.3 & 22.5 & - \\
\hline- & - & - & - & - & - & - & - \\
\hline - & .4 & 7.8 & $32 \digamma$ & 30.2 & 25.3 & 22.5 & - \\
\hline 18.1 & 66.6 & 310.6 & 486.9 & 369.8 & 346.9 & 269.4 & 334.7 \\
\hline 60.2 & 190.6 & 513.5 & 530.1 & 410.9 & 325.8 & 307.0 & 333.8 \\
\hline 20.7 & 69.9 & 115.1 & 111.7 & 99.8 & 62.4 & 76.7 & 57.5 \\
\hline 6.3 & 49.3 & 122.5 & 134.9 & 79.2 & 53.0 & 68.2 & 78.9 \\
\hline 2.9 & 2.7 & 11.1 & 2.2 & 1.7 & .7 & .5 & .4 \\
\hline 8.7 & 19.7 & 137.7 & 158.1 & 135.2 & 112.4 & 61.7 & 75.8 \\
\hline 5.1 & 12.4 & 50.4 & 56.3 & 48.7 & 48.0 & 49.8 & 62.6 \\
\hline 7.6 & 10.4 & 18.4 & 44.9 & 30.0 & 30.5 & 29.7 & 37.1 \\
\hline 1.2 & 3.2 & 12.5 & 3.2 & 4.1 & 3.2 & 5.6 & 4.1 \\
\hline 7.7 & 23.1 & 45.7 & 18.9 & 12.2 & 15.7 & 14.9 & 17.4 \\
\hline 44.2 & 94.5 & 278.6 & 469.7 & 442.2 & 386.9 & 382.7 & 394.0 \\
\hline 531.2 & $1,047.7$ & $2,604.0$ & $2,601.3$ & $1,857.1$ & $2,024.4$ & $2,034.5$ & $2,290.1$ \\
\hline 531.2 & $1,047.7$ & $2,604.0$ & $2,601.3$ & $1,857.1$ & $2,024.4$ & $2,034.5$ & $2,290.1$ \\
\hline- & • & - & - & - & - & - & - \\
\hline 531.2 & $1,047.7$ & $2,604.0$ & $2,601.3$ & $1,857.1$ & $2,024.4$ & $2,034.5$ & $2,290.1$ \\
\hline 4.9 & 5.4 & 14.9 & 11.0 & 10.6 & 6.8 & 6.5 & 6.9 \\
\hline 21.7 & 73.0 & 341.7 & 439.7 & 346.3 & 396.1 & 396.7 & 436.5 \\
\hline 17.2 & 43.2 & 170.5 & 190.6 & 129.8 & 161.8 & 150.7 & 220.5 \\
\hline 2.2 & 4.5 & 4.9 & 4.4 & 3.8 & 2.8 & 2.2 & 2.0 \\
\hline 14.1 & 24.4 & 67.7 & 75.5 & 65.4 & 64.4 & 66.8 & 84.0 \\
\hline 470.9 & 896.7 & $2,004.2$ & $1,880.2$ & $1,301.2$ & $1,392.6$ & 1.411 .7 & $1,540.2$ \\
\hline .2 & .5 & - & - & - & - & - & - \\
\hline- & - & - & - & - & - & - & - \\
\hline $\begin{array}{r}1,054.5 \\
742.8\end{array}$ & $\begin{array}{l}2,041.4 \\
1,531.8\end{array}$ & $\begin{array}{l}5,028.7 \\
3,817.5\end{array}$ & $\begin{array}{l}6,460.9 \\
4,319.7\end{array}$ & $\begin{array}{l}5,412.1 \\
3,253.6\end{array}$ & $\begin{array}{l}5,244.5 \\
3,230.4\end{array}$ & $\begin{array}{l}5,288.2 \\
3,240.5\end{array}$ & $\begin{array}{l}5,569.9 \\
3,529.9\end{array}$ \\
\hline 311.7 & 509.6 & $1,211.3$ & $2,141.2$ & $2,158.5$ & $2,014.1$ & $2,047.7$ & $2,040.0$ \\
\hline $46: 8$ & 190.0 & $\begin{array}{l}727.3 \\
123.5\end{array}$ & $\begin{array}{l}988.5 \\
367.4\end{array}$ & $\begin{array}{l}883.8 \\
329.8\end{array}$ & $\begin{array}{l}882.1 \\
354.5\end{array}$ & $\begin{array}{l}900.4 \\
381.3\end{array}$ & $\begin{array}{l}891.0 \\
346.5\end{array}$ \\
\hline 46.5 & 189.1 & 602.0 & 618.3 & 551.9 & 526.0 & 518.0 & 543.1 \\
\hline 4 & .9 & 1.8 & 2.7 & 2.0 & 1.6 & 1.2 & 1.5 \\
\hline .2 & .3 & • & .2 & .1 & • & .1 & .2 \\
\hline .2 & 6 & 1.8 & 2.5 & 1.9 & 1.5 & 1.1 & 1.3 \\
\hline- & - & - & - & - & - & - & - \\
\hline - & - & - & - & - & - & - & - \\
\hline - & - & - & - & - & - & - & - \\
\hline 789.6 & $1,721.8$ & $4,544.7$ & $5,308.2$ & $4,137.4$ & $4,112.5$ & $4,140.9$ & $4,420.9$ \\
\hline
\end{tabular}

- There are no direct fuel costs for hydroelectric, geothermal, centralized solar, or wind energy. Wood and other biomass fuels are not included, except those consumed at electric utilities.

b Heavy oil includes Grade Nos. 4, 5, and 6, and residual fuel oils. Light oil includes Grade No. 2 heating oil, kerosene, and jet fuel.

-No consumption, including cases where adjustments were made. See "Consumption Adjustments for Process Fuel and intermediate Products."

"Value less than 0.05 million dollars.

Note: Totais may not equal sum of components due to independent rounding.

Sources: Data sources, estimation procedures, and assumptions are described in the "Documentation" section of this report 


\begin{tabular}{|c|c|c|c|c|c|c|c|c|}
\hline Enorgy Source & 1970 & 1975 & 1980 & 1985 & 1986 & 1987 & 1988 & 1989 \\
\hline & \multicolumn{8}{|c|}{ Prices in Dollars per Million Btu } \\
\hline Coal & 0.41 & 1.04 & 1.72 & 2.15 & 2.47 & 2.43 & 2.43 & 1.25 \\
\hline 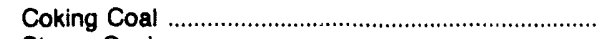 & - & - & - & - & - & - & - & - \\
\hline 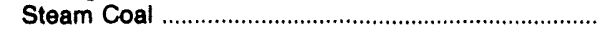 & .41 & 1.04 & 1.72 & 2.15 & 2.47 & 2.43 & 2.43 & 1.25 \\
\hline 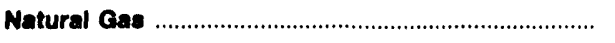 & .81 & 1.44 & 4.69 & 5.60 & 4.94 & 4.55 & 4.80 & 4.17 \\
\hline 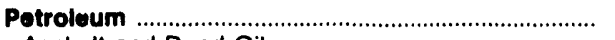 & 1.88 & 3.50 & 7.88 & 8.05 & 5.86 & 6.08 & 6.21 & 7.02 \\
\hline 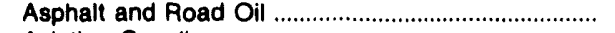 & .58 & 1.78 & 3.60 & 4.42 & 4.47 & 4.27 & 3.95 & 3.19 \\
\hline Aviation Gasoline & 2.17 & 3.45 & 9.02 & 9.99 & 8.41 & 7.55 & 7.41 & 8.28 \\
\hline 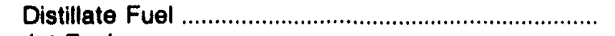 & 1.21 & 2.62 & 6.62 & 7.98 & 5.32 & 5.75 & 5.88 & 6.77 \\
\hline 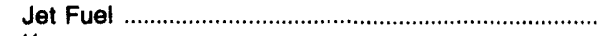 & .73 & 2.04 & 6.21 & 6.16 & 4.24 & 4.33 & 4.04 & 4.76 \\
\hline 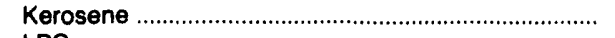 & 1.48 & 2.84 & 7.61 & 8.51 & 6.20 & 6.28 & 5.76 & 7.46 \\
\hline LPG & 2.21 & 4.17 & 7.09 & 9.16 & 9.65 & 8.75 & 8.55 & 10.19 \\
\hline 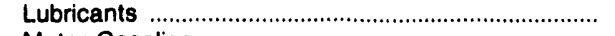 & 5.08 & 7.49 & 14.36 & 17.61 & 15.59 & 13.58 & 14.61 & 17.90 \\
\hline 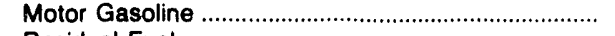 & 2.83 & 4.45 & 9.75 & 8.87 & 6.92 & 7.12 & 7.48 & 8.50 \\
\hline 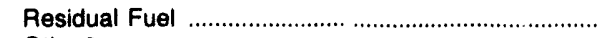 & .51 & 2.06 & 3.92 & 4.91 & 2.60 & 2.61 & 2.31 & 2.54 \\
\hline 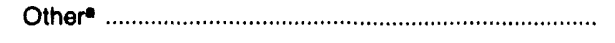 & .81 & 2.44 & 6.53 & 9.99 & 2.80 & 2.43 & 2.19 & 2.55 \\
\hline 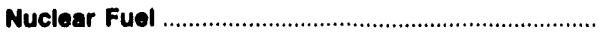 & - & .20 & .36 & .54 & .52 & .45 & .46 & .46 \\
\hline 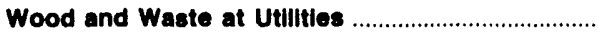 & .65 & .92 & 1.74 & - & - & - & .87 & .82 \\
\hline Primary Energy - Five Sectors .............................. & 1.63 & 2.98 & 6.30 & 6.34 & 4.85 & 5.23 & 5.17 & 5.65 \\
\hline 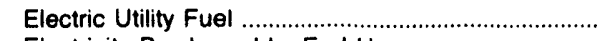 & .48 & 2.04 & .59 & .67 & .52 & .45 & .47 & .99 \\
\hline 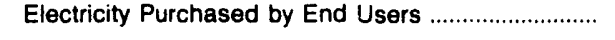 & 2.90 & 4.13 & 7.59 & 13.08 & 12.95 & 12.88 & 12.61 & 12.49 \\
\hline \multirow[t]{2}{*}{ Total Energy } & 1.87 & 3.23 & 7.36 & 8.83 & 7.35 & 7.46 & 7.50 & 7.92 \\
\hline & \multicolumn{8}{|c|}{ Expenditures in Mililions of Dollars } \\
\hline 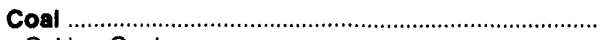 & 1.2 & 2.8 & 20.8 & 21.6 & 7.2 & 9.0 & 7.6 & 8.5 \\
\hline 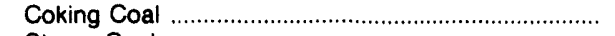 & - & - & - & - & - & - & - & - \\
\hline 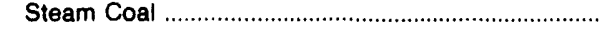 & 1.2 & 2.8 & 20.8 & 21.6 & 7.2 & 9.0 & 7.6 & 8.5 \\
\hline 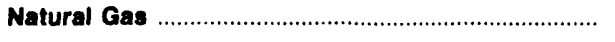 & 68.7 & 139.9 & 320.9 & 432.9 & 340.2 & 337.2 & 388.0 & 429.7 \\
\hline 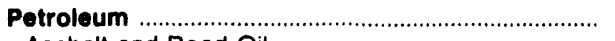 & 541.4 & $1,050.5$ & $2,609.2$ & $2,511.9$ & $1,922.8$ & $2,033.0$ & $2,174.7$ & $2,465.3$ \\
\hline 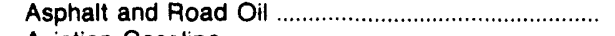 & 8.3 & 38.0 & 59.2 & 83.3 & 65.9 & 60.6 & 63.5 & 59.4 \\
\hline 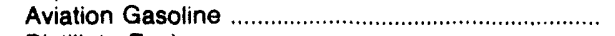 & 3.3 & 3.0 & 11.8 & 7.1 & 8.2 & 4.8 & 3.7 & 4.2 \\
\hline 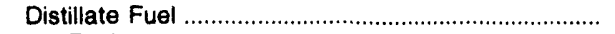 & 89.2 & 199.4 & 643.9 & 715.0 & 461.1 & 543.0 & 563.9 & 640.6 \\
\hline 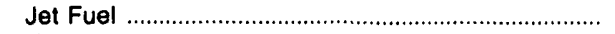 & 8.6 & 24.0 & 86.5 & 74.3 & 62.5 & 71.4 & 72.7 & 90.9 \\
\hline Kerosene & 1.8 & 3.6 & 4.8 & 3.5 & .4 & .2 & .9 & 3.4 \\
\hline LPG & 10.2 & 10.0 & 31.7 & 47.0 & 60.1 & 49.0 & 49.4 & 60.3 \\
\hline 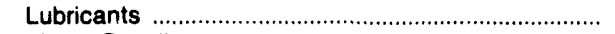 & 23.7 & 30.8 & 65.4 & 73.0 & 63.2 & 62.2 & 64.6 & 81.1 \\
\hline Motor Gasoline & 371.2 & 675.3 & $1,562.9$ & $1,353.9$ & $1,088.0$ & $1,144.3$ & $1,263.3$ & $1,423.0$ \\
\hline Residual Fuel & 18.5 & 45.4 & 100.0 & 125.5 & 93.4 & 70.9 & 69.0 & 75.8 \\
\hline 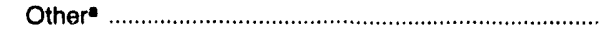 & 6.6 & 20.9 & 42.7 & 29.3 & 20.0 & 26.6 & 23.8 & 26.5 \\
\hline 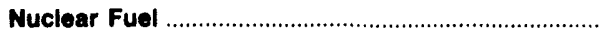 & - & " & 21.4 & 40.6 & 39.5 & 21.1 & 31.4 & 26.1 \\
\hline 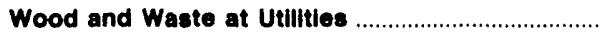 & .3 & * & 2.9 & - & - & - & .9 & .2 \\
\hline Primary Energy - Five Sectors ............................ & 611.6 & $1,193.2$ & $2,975.1$ & $3,006.9$ & $2,309.7$ & $2,400.2$ & $2,602.4$ & $2,929.8$ \\
\hline $\begin{array}{l}\text { Electric Utility Fuel } \\
\text { Electricity Purchased by End Users }\end{array}$ & $\begin{array}{r}-.8 \\
248.3\end{array}$ & $\begin{array}{r}-.4 \\
458.4\end{array}$ & $\begin{array}{l}-41.1 \\
950.4\end{array}$ & $\begin{array}{r}-54.5 \\
1.573 .1\end{array}$ & $\begin{array}{r}-39.6 \\
1549.2\end{array}$ & $\begin{array}{r}-21.1 \\
1657.4\end{array}$ & $\begin{array}{r}-32.3 \\
16920\end{array}$ & $\begin{array}{r}-75.6 \\
17740\end{array}$ \\
\hline & & & & & & & & \\
\hline 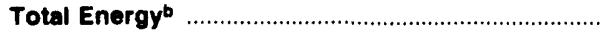 & 859.1 & $1,651.1$ & $3,884.5$ & $4,525.5$ & $3,819.3$ & $4,036.5$ & $4,262.2$ & $4,628.2$ \\
\hline
\end{tabular}

Includes petroleum coke used at electric utilities.

bThere are no direct fuel costs for hydroelectric, geothermal, centralized solar, or wind energy. Wood and other biomass fuels are not included, except those consumed at electric utilities.

-No consumption, including cases where adjustments were made. See "Consumption Adjustments for Process Fuel and Intermediate Products."

Value less than 0.05 million dollars.

Note: Expenditure totals may not equal sum of components due to independent rounding.

Sources: Data sources, estimation procedures, and assumptions are described in the "Documentation" section of this report 
O Energy Price Estimates by Sector, Oregon

R $1970,1975,1980$, and 1985-1989

E (Dollars per Million Btu)

G

\begin{tabular}{|c|c|c|c|c|c|c|c|c|}
\hline Sector and Energy Source & 1970 & 1975 & 1980 & 1985 & 1986 & 1987 & 1988 & 1989 \\
\hline $\begin{array}{l}\text { Residentlal Sector } \\
\text { Primary Energy } \\
\text { Coal ... } \\
\text { Natural Gas } \\
\text { Fetroleum } \\
\text { Distillate Fuel } \\
\text { Kerosene } \\
\text { LPG } \\
\text { Electricity }\end{array}$ & $\begin{array}{r}2.47 \\
1.53 \\
.95 \\
1.45 \\
1.63 \\
1.41 \\
2.79 \\
2.66 \\
3.65\end{array}$ & $\begin{array}{l}3.78 \\
2.42 \\
1.14 \\
2.11 \\
3.03 \\
2.80 \\
3.82 \\
5.27 \\
5.27\end{array}$ & $\begin{array}{l}8.04 \\
6.20 \\
4.26 \\
5.36 \\
7.35 \\
7.02 \\
9.80 \\
9.00 \\
9.37\end{array}$ & $\begin{array}{r}10.77 \\
6.95 \\
3.67 \\
6.73 \\
7.27 \\
7.00 \\
9.85 \\
8.73 \\
13.72\end{array}$ & $\begin{array}{r}10.67 \\
6.24 \\
3.79 \\
6.47 \\
5.92 \\
5.08 \\
7.14 \\
10.89 \\
13.84\end{array}$ & $\begin{array}{r}10.93 \\
6.23 \\
3.76 \\
6.44 \\
5.89 \\
5.23 \\
7.36 \\
9.83 \\
14.07\end{array}$ & $\begin{array}{r}10.87 \\
6.24 \\
3.37 \\
6.64 \\
5.54 \\
5.11 \\
7.19 \\
9.16 \\
14.02\end{array}$ & $\begin{array}{r}10.81 \\
6.30 \\
3.66 \\
5.98 \\
6.90 \\
5.78 \\
8.14 \\
15.93 \\
13.97\end{array}$ \\
\hline $\begin{array}{l}\text { Commercial Sector } \\
\text { Primary Energy } \\
\text { Coal ... } \\
\text { Natural Gas } \\
\text { Petroleum } \\
\text { Distillate Fuel } \\
\text { Kerosene } \\
\text { LPG } \\
\text { Motor Gasoline } \\
\text { Residual Fuel } \\
\text { Electricity }\end{array}$ & $\begin{array}{r}2.29 \\
1.16 \\
.35 \\
1.22 \\
1.14 \\
1.22 \\
.93 \\
1.12 \\
2.83 \\
.79 \\
3.90\end{array}$ & $\begin{array}{l}3.67 \\
2.21 \\
1.04 \\
1.79 \\
2.68 \\
2.60 \\
2.58 \\
2.76 \\
4.45 \\
2.45 \\
5.20\end{array}$ & $\begin{array}{l}7.29 \\
5.56 \\
2.24 \\
4.88 \\
6.39 \\
6.71 \\
6.54 \\
5.40 \\
9.75 \\
4.90 \\
8.86\end{array}$ & $\begin{array}{r}10.98 \\
6.29 \\
2.48 \\
6.06 \\
6.74 \\
6.42 \\
6.50 \\
9.45 \\
8.87 \\
4.12 \\
14.96\end{array}$ & $\begin{array}{r}10.52 \\
5.14 \\
2.47 \\
5.47 \\
4.63 \\
4.59 \\
3.96 \\
9.06 \\
6.92 \\
2.10 \\
14.82\end{array}$ & $\begin{array}{r}10.53 \\
4.84 \\
2.42 \\
5.17 \\
4.36 \\
3.96 \\
4.66 \\
8.28 \\
7.12 \\
2.65 \\
15.00\end{array}$ & $\begin{array}{r}10.15 \\
4.96 \\
2.42 \\
5.24 \\
4.50 \\
4.16 \\
4.47 \\
8.38 \\
7.48 \\
2.55 \\
14.19\end{array}$ & $\begin{array}{r}10.03 \\
4.69 \\
2.37 \\
4.64 \\
4.83 \\
4.77 \\
5.18 \\
8.55 \\
8.50 \\
2.39 \\
14.17\end{array}$ \\
\hline $\begin{array}{l}\text { Industrial Sector } \\
\text { Primary Energy } \\
\text { Coal ... } \\
\text { Coking Coal } \\
\text { Steam Coal } \\
\text { Natural Gas } \\
\text { Petroleum } \\
\text { Asphalt and Road Oil } \\
\text { Distillate Fuel } \\
\text { Kerosene } \\
\text { LPG } \\
\text { Lubricants } \\
\text { Motor Gasoline } \\
\text { Residual Fuel } \\
\text { Other } \\
\text { Electricity }\end{array}$ & $\begin{array}{r}.78 \\
.67 \\
.35 \\
- \\
.35 \\
.46 \\
.83 \\
.58 \\
.80 \\
.93 \\
1.12 \\
5.08 \\
2.83 \\
.33 \\
.81 \\
1.26\end{array}$ & $\begin{array}{r}1.77 \\
1.65 \\
1.04 \\
- \\
1.04 \\
.92 \\
2.25 \\
1.78 \\
2.29 \\
2.58 \\
2.76 \\
7.49 \\
4.45 \\
1.85 \\
2.44 \\
2.13\end{array}$ & $\begin{array}{r}4.67 \\
4.68 \\
2.24 \\
- \\
2.24 \\
4.21 \\
5.06 \\
3.60 \\
5.62 \\
6.54 \\
5.40 \\
14.36 \\
9.75 \\
3.39 \\
6.53 \\
4.65\end{array}$ & $\begin{array}{r}7.17 \\
5.84 \\
2.48 \\
- \\
2.48 \\
4.65 \\
7.01 \\
4.42 \\
8.72 \\
6.50 \\
9.45 \\
17.61 \\
8.87 \\
4.12 \\
9.99 \\
10.32\end{array}$ & $\begin{array}{r}5.70 \\
3.85 \\
2.47 \\
-\overline{2} \\
2.47 \\
3.73 \\
4.00 \\
4.47 \\
2.84 \\
3.96 \\
9.06 \\
15.59 \\
6.92 \\
2.10 \\
2.80 \\
10.08\end{array}$ & $\begin{array}{r}5.80 \\
3.84 \\
2.42 \\
- \\
2.42 \\
3.30 \\
4.32 \\
4.27 \\
4.18 \\
4.66 \\
8.28 \\
13.58 \\
7.12 \\
2.65 \\
2.43 \\
9.91\end{array}$ & $\begin{array}{r}5.81 \\
3.92 \\
2.42 \\
- \\
2.42 \\
3.64 \\
4.21 \\
3.95 \\
4.00 \\
4.47 \\
8.38 \\
14.61 \\
7.48 \\
2.55 \\
2.19 \\
9.82\end{array}$ & $\begin{array}{r}5.82 \\
3.91 \\
- \\
- \\
- \\
3.40 \\
4.42 \\
3.19 \\
4.64 \\
5.18 \\
8.55 \\
17.90 \\
8.50 \\
2.38 \\
2.55 \\
9.68\end{array}$ \\
\hline $\begin{array}{l}\text { Transportation Sactor } \\
\text { Primary Energy } \\
\text { Coal foldent } \\
\text { Petroleum } \\
\text { Aviation Gasoline } \\
\text { Distillate Fuel } \\
\text { Jet Fuel } \\
\text { LPG } \\
\text { Lubricants } \\
\text { Motor Gasoline } \\
\text { Residual Fuel } \\
\text { Electricity }\end{array}$ & $\begin{array}{r}2.41 \\
2.41 \\
.35 \\
2.41 \\
2.17 \\
1.34 \\
.73 \\
1.12 \\
5.08 \\
2.83 \\
.71 \\
-\end{array}$ & $\begin{array}{r}3.98 \\
3.98 \\
1.04 \\
3.98 \\
3.45 \\
2.69 \\
2.04 \\
2.76 \\
7.49 \\
4.45 \\
2.21 \\
-\end{array}$ & $\begin{array}{r}8.81 \\
8.81 \\
8.81 \\
9.02 \\
6.96 \\
6.21 \\
5.40 \\
14.36 \\
9.75 \\
4.14 \\
-\end{array}$ & $\begin{array}{r}8.36 \\
8.36 \\
- \\
8.36 \\
9.99 \\
8.27 \\
6.16 \\
9.45 \\
17.61 \\
8.87 \\
5.02 \\
-\end{array}$ & $\begin{array}{r}6.32 \\
6.32 \\
- \\
6.32 \\
8.41 \\
6.16 \\
4.24 \\
9.06 \\
15.59 \\
6.92 \\
2.85 \\
-\end{array}$ & $\begin{array}{r}6.53 \\
6.53 \\
- \\
6.53 \\
7.55 \\
6.63 \\
4.33 \\
8.28 \\
13.58 \\
7.12 \\
2.61 \\
17.04\end{array}$ & $\begin{array}{r}6.71 \\
6.71 \\
- \\
6.71 \\
7.41 \\
6.81 \\
4.04 \\
8.38 \\
14.61 \\
7.48 \\
2.26 \\
13.54\end{array}$ & $\begin{array}{r}7.64 \\
7.64 \\
7.64 \\
8.28 \\
7.77 \\
4.76 \\
8.55 \\
17.90 \\
8.50 \\
2.57 \\
14.24\end{array}$ \\
\hline 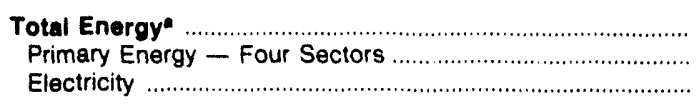 & $\begin{array}{l}1.87 \\
1.63 \\
2.90\end{array}$ & $\begin{array}{l}3.23 \\
2.98 \\
4.13\end{array}$ & $\begin{array}{l}7.36 \\
7.29 \\
7.59\end{array}$ & $\begin{array}{r}8.83 \\
7.53 \\
13.08\end{array}$ & $\begin{array}{r}7.35 \\
5.68 \\
12.95\end{array}$ & $\begin{array}{r}7.46 \\
5.77 \\
12.88\end{array}$ & $\begin{array}{r}7.50 \\
5.92 \\
12.61\end{array}$ & $\begin{array}{r}7.92 \\
6.46 \\
12.48\end{array}$ \\
\hline $\begin{array}{l}\text { Electrlc UtIlity Sector } \\
\text { Coal ... } \\
\text { Natural Gas . } \\
\text { Petroleum . } \\
\text { Heavy Oib . } \\
\text { Light Oilb } \\
\text { Petroleum Coke } \\
\text { Nuclear Fuel } \\
\text { Wood and Waste }\end{array}$ & $\begin{array}{r}.48 \\
- \\
.37 \\
.80 \\
.80 \\
.83 \\
- \\
- \\
.65\end{array}$ & $\begin{array}{r}2.04 \\
- \\
1.27 \\
2.31 \\
- \\
2.31 \\
- \\
.20 \\
.92\end{array}$ & $\begin{array}{r}.59 \\
1.41 \\
4.29 \\
6.53 \\
- \\
6.53 \\
- \\
.36 \\
1.74\end{array}$ & $\begin{array}{r}.67 \\
2.00 \\
- \\
5.67 \\
- \\
5.67 \\
- \\
.54 \\
-\end{array}$ & $\begin{array}{r}.52 \\
- \\
2.81 \\
3.24 \\
- \\
3.24 \\
.5 \overline{2} \\
-\end{array}$ & $\begin{array}{r}.45 \\
- \\
4.10 \\
4.10 \\
- \\
.45 \\
-\end{array}$ & $\begin{array}{r}.47 \\
- \\
- \\
4.03 \\
- \\
4.03 \\
- \\
.46 \\
.87\end{array}$ & $\begin{array}{r}.90 \\
1.56 \\
2.92 \\
4.53 \\
4.53 \\
.46 \\
.82\end{array}$ \\
\hline 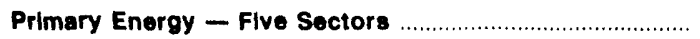 & 1.63 & 2.88 & 6.30 & 6.34 & 4.85 & 5.23 & 5.17 & 5.65 \\
\hline
\end{tabular}

-There are no direct fuel costs for hydroelectric, geothermal, centralized solar, or wind energy. Wood and other biomass fuels are not included, except those consumed at electric utilities.

beavy oil includes Grade Nos. 4, 5, and 6, and residual fuel oils. Light oil includes Grade No. 2 heating oil, kerosene, and jet fuel.

-No consumption, including cases where adjustments were made. See "Consumption Adjustments for Process Fuel and Intermediate Products."

Sources: Data sources, estimation procedures, and assumptions are described in the "Documentation" section of this report 


\begin{tabular}{|c|c|c|c|c|c|c|c|c|}
\hline Sector and Energv Source & 1970 & 1975 & 1980 & 1985 & 1986 & 1987 & 1988 & 1989 \\
\hline $\begin{array}{l}\text { Residential Sector } \\
\text { Primary Energy }\end{array}$ & $\begin{array}{r}188.1 \\
65.4\end{array}$ & $\begin{array}{l}327.8 \\
110.3\end{array}$ & $\begin{array}{l}640.2 \\
207.3\end{array}$ & $\begin{array}{l}946.3 \\
266.2\end{array}$ & $\begin{array}{l}856.4 \\
208.6\end{array}$ & $\begin{array}{l}852.8 \\
194.4\end{array}$ & $\begin{array}{l}894.2 \\
208.1\end{array}$ & $\begin{array}{l}945.9 \\
227.0\end{array}$ \\
\hline Coal & .2 & .1 & .6 & .1 & 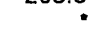 & .1 & .1 & .2 \\
\hline 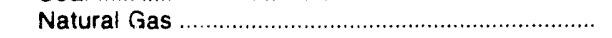 & 29.8 & 63.1 & 103.1 & 148.8 & 126.1 & 124.1 & 141.4 & 139.3 \\
\hline Petroleum & 35.3 & 47.1 & 103.5 & 117.4 & 82.5 & 70.2 & 66.6 & 87.5 \\
\hline 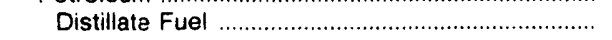 & 25.6 & 39.0 & 82.5 & 96.8 & 60.5 & 53.2 & 54.9 & 63.6 \\
\hline 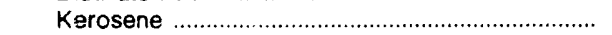 & 1.0 & 1.0 & 2.1 & 2.4 & .3 & .1 & .5 & 2.9 \\
\hline LPG & 8.7 & 7.1 & 19.0 & 18.1 & 21.8 & 16.9 & 11.2 & 21.0 \\
\hline Electricity & 122.8 & 217.4 & 432.9 & 680.0 & 647.8 & 658.3 & 686.0 & 719.0 \\
\hline 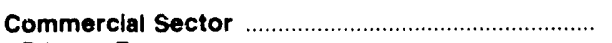 & 126.0 & 225.8 & 509.3 & 715.3 & 668.4 & 691.4 & 697.8 & 705.8 \\
\hline Primary Energy & 37.3 & 69.7 & 193.4 & 187.7 & 145.2 & 139.4 & 149.2 & 144.2 \\
\hline 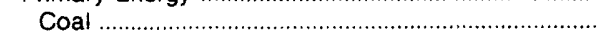 & .2 & .2 & .6 & .1 & 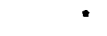 & .1 & .2 & .2 \\
\hline 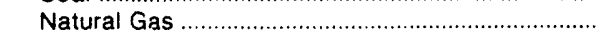 & 14.5 & 29.6 & 77.5 & 118.9 & 94.2 & 88.8 & 98.7 & 97.2 \\
\hline Petroleum & 22.6 & 39.8 & 115.3 & 68.6 & 50.9 & 51.5 & 50.4 & 46.8 \\
\hline Distillate Fuel & 11.5 & 18.8 & 70.0 & 51.8 & 35.8 & 37.4 & 36.8 & 29.9 \\
\hline Kerosene & 2 & .5 & 1.4 & 1.0 & .1 & 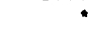 & .3 & .3 \\
\hline LPG & .6 & .7 & 2.0 & 3.5 & 3.2 & 2.5 & 1.8 & 2.0 \\
\hline Motor Gasoline & 3.7 & 5.1 & 14.9 & 10.8 & 8.5 & 9.1 & 9.3 & 9.8 \\
\hline 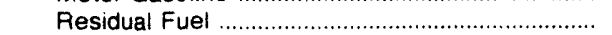 & 6.6 & 14.8 & 27.0 & 1.6 & 3.3 & 1.5 & 2.1 & 4.7 \\
\hline Electricity & 88.7 & 156.1 & 316.0 & 527.6 & 523.2 & 552.0 & 548.6 & 561.6 \\
\hline Industrial Sector & 119.0 & 278.9 & 674.9 & 854.0 & 720.4 & 812.1 & 843.6 & 895.7 \\
\hline Primary Energy & 82.1 & 194.1 & 473.3 & 488.6 & 342.2 & 365.5 & 386.6 & 402.7 \\
\hline Coßl . & .8 & 2.5 & 8.5 & 7.5 & 7.1 & 8.8 & 7.2 & - \\
\hline 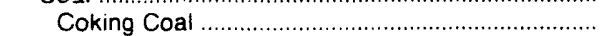 & - & - & - & - & - & - & - & - \\
\hline Steam Coal & .8 & 2.5 & 8.5 & 7.5 & 7.1 & 8.8 & 7.2 & - \\
\hline Natural Gas & 23.9 & 47.2 & 138.8 & 165.2 & 119.9 & 124.3 & 147.9 & 154.0 \\
\hline F'etroleum & 57.4 & 144.4 & 326.0 & 315.9 & 215.2 & 232.4 & 231.4 & 248.7 \\
\hline Asphalt and Road Oil & 8.3 & 38.0 & 59.2 & 83.3 & 65.9 & 60.6 & 63.5 & 59.4 \\
\hline Distillate Fuel & 14.8 & 35.1 & 128.4 & 128.5 & 41.0 & 74.1 & 67.9 & 78.4 \\
\hline Kerosene & .6 & 2.1 & 1.4 & .1 & 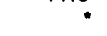 & 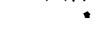 & .1 & .2 \\
\hline LPG & .8 & 2.1 & 9.5 & 19.5 & 29.6 & 24.0 & 30.7 & 32.4 \\
\hline Lubricants & 8.7 & 8.6 & 19.3 & 21.5 & 18.6 & 18.3 & 19.0 & 23.9 \\
\hline Motor Gasoline & 10.7 & 13.1 & 21.4 & 22.4 & 18.2 & 18.0 & 16.4 & 21.3 \\
\hline Residual Fuel & 7.0 & 24.5 & 44.2 & 11.3 & 21.9 & 10.8 & 10.0 & 6.5 \\
\hline Other & 6.6 & 20.9 & 42.7 & 29.3 & 20.0 & 26.6 & 23.8 & 26.5 \\
\hline 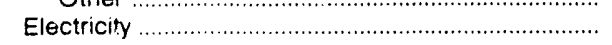 & 36.8 & 84.8 & 201.6 & 365.4 & 378.2 & 446.7 & 457.0 & 493.0 \\
\hline 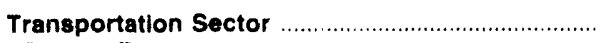 & 426.0 & 818.7 & $2,060.1$ & $2,009.9$ & $1,574.1$ & $1,680.2$ & $1,826.6$ & $2,080.8$ \\
\hline Primary Energy & 426.0 & 818.7 & $2,060.1$ & $2,009.9$ & $1,574.1$ & $1,679.8$ & $1,826.3$ & $2,080.4$ \\
\hline 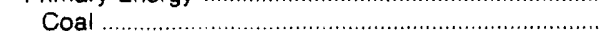 & 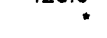 & . & - & -10000 & - & - & - & - \\
\hline Petroleum & 426.0 & 818.7 & $2,060.1$ & $2,009.9$ & $1,574.1$ & $1,679.8$ & $1,826.3$ & $2,080.4$ \\
\hline Aviation Gasoline & 3.3 & 3.0 & 11.8 & 7.1 & 8.2 & 4.8 & 3.7 & 4.2 \\
\hline Distillate Fuel & 37.4 & 106.2 & 358.9 & 437.8 & 323.8 & 378.3 & 404.2 & 466.7 \\
\hline Jet Fuel & 8.6 & 24.0 & 86.5 & 74.3 & 62.5 & 71.4 & 72.7 & 90.9 \\
\hline LPG & .1 & .1 & 1.3 & 5.9 & 5.5 & 5.6 & 5.7 & 4.9 \\
\hline Lubricants & 15.0 & 22.3 & 46.1 & 51.5 & 44.6 & 43.9 & 45.5 & 57.2 \\
\hline Motor Gasoline & 356.7 & 657.1 & $1,526.7$ & $1,320.7$ & $1,061.3$ & $1,117.2$ & $1,237.5$ & $1,391.9$ \\
\hline Residua' Fuel & 4.8 & 6.1 & 28.8 & 112.6 & 68.2 & 58.6 & 57.0 & 64.6 \\
\hline 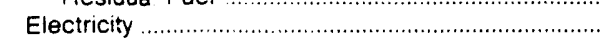 & - & - & - & - & - & .4 & .4 & .4 \\
\hline 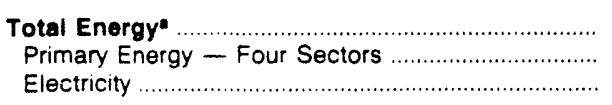 & $\begin{array}{l}859.1 \\
610.8 \\
248.3\end{array}$ & $\begin{array}{r}1,651.1 \\
1,192.8 \\
458.4\end{array}$ & $\begin{array}{r}3,884.5 \\
2,934.1 \\
950.4\end{array}$ & $\begin{array}{l}\mathbf{4}, \mathbf{5 2 5 . 5} \\
2,952.4 \\
1,573.1\end{array}$ & $\begin{array}{l}3,819.3 \\
2,270.1 \\
1 . \therefore: 9.2\end{array}$ & $\begin{array}{l}4,036.5 \\
2,379.2 \\
1,657.4\end{array}$ & $\begin{array}{l}4,262.2 \\
2,570.2 \\
1,692.0\end{array}$ & $\begin{array}{l}4,628.2 \\
2,854.2 \\
1,774.0\end{array}$ \\
\hline Electric Utility Sector & .8 & .4 & 41.1 & 54.5 & 39.6 & 21.1 & 32.3 & 75.6 \\
\hline Coal & - & - & 11.2 & 13.9 & - & - & - & 8.1 \\
\hline Natural Gas & .4 & $\cdot$ & 1.4 & - & $\cdot$ & - & - & 39.2 \\
\hline 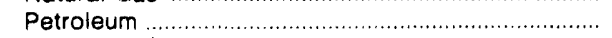 & .1 & .4 & 4.2 & .1 & .1 & * & • & 2.0 \\
\hline 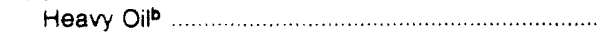 & .1 & - & - & - & - & - & - & - \\
\hline 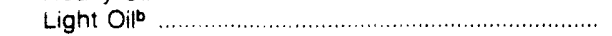 & * & .4 & 4.2 & .1 & .1 & • & - & 2.0 \\
\hline Petroleum Coke & - & - & - & - & - & - & - & - \\
\hline 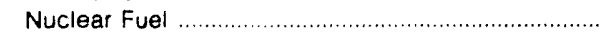 & - & * & 21.4 & 40.6 & 39.5 & 21.1 & 31.4 & 26.1 \\
\hline 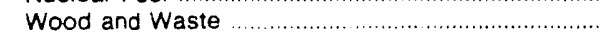 & .3 & • & 2.9 & - & - & - & .9 & .2 \\
\hline Primary Energy - Five Sectors …....................... & 611.6 & $1,193.2$ & $2,975.1$ & $3,006.9$ & $2,309.7$ & $2,400.2$ & $2,602.4$ & $2,929.8$ \\
\hline
\end{tabular}

- There are no direct fuel costs for hyc'roelectric, geothermal, centralized solar, or wird energy. Wood and other biomass fuels are ne included, except those consumed at electric utitities.

bHeavy oil includes Grade Nos. 4, 5, and 6, anci residual fuel oils. Light oil includes Grade No. 2 heating oil, kerosene, and jet fuel.

- No consumption, including cases where adjustments were made. See "Consumption Adjustments for Process Fuel and Intermediate Products." - Value less than 0.05 million dollar.

Note: Totals may not equal sum o components due to independent roundir,g.

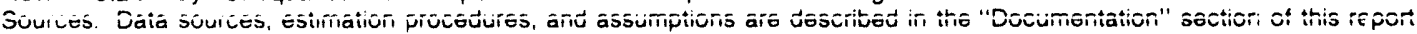




\section{P Energy Price and Expenditure Estimates by Source, Pennsylvania \\ E $1970,1975,1980$, and 1985- 1989}

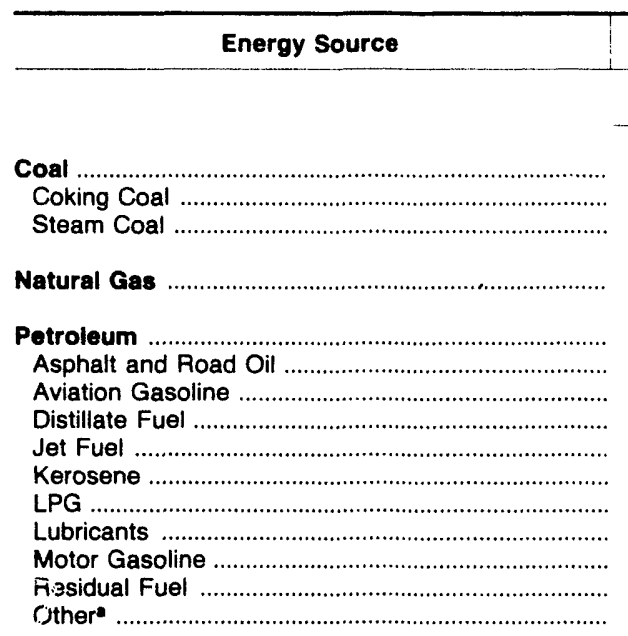

Nuclear Fuel $1 !) 70$ 1975

1980

1985

1986

1987

1988

1989

Prices in Dollars per Million Btu

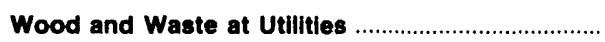

Primary Energy - Five Sectors ..............................

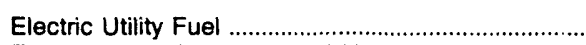

Electricity Purchased by End Users ...

$\begin{array}{rrr}0.39 & 1.21 & 1.59 \\ .44 & 1.52 & 2.20\end{array}$

1.59

$\begin{array}{rrr}0.39 & 1.21 & 1.59 \\ .44 & 1.52 & 2.20\end{array}$

2.20
1.35

1.64
1.88

\subsection{5}

1.76

1.52

1.49

1.58

1.59
1.47

$\begin{array}{ll}1.50 & 1.50 \\ 1.61 & 1.67\end{array}$

.87

1.53

3.37

5.74

5.38

4.84

4.70

1.47

1.71
.78

\subsection{5}

1.97
3.45

2.17
1.17

1.17
.72

.72
1.39

1.39
1.82

5.08

2.92

.47

1.20

3.45
2.65

2.01

2.88

3.39

7.49

4.72

2.02
2.28

7.67

3.72

3.72
9.02

6.70

6.27

7.75

6.16
14.36

14.36
9.71

4.30

6.56

\subsection{9}

4.95

9.99

7.73
5.84

5.84

8.28

9.85
17.61

17.61
9.01

4.36

4.36
7.25

\subsection{4}

4.01

8.41
5.87

3.91

6.34

8.87

15.59
6.54

6.54

2.44
5.79

6.16

3.25

7.55

5.88

6.04

6.04
8.49

8.49
13.58

7.03

2.96

$.25 \quad .42$

$\begin{array}{llllllllll}.21 & .25 & .42 & .92 & .92 & .78 & .78 & .84 & .88\end{array}$

$\begin{array}{llllllllll}.21 & .25 & .42 & .92 & .92 & .78 & .78 & .84 & .88\end{array}$

$\begin{array}{llllllllll}.21 & .25 & .42 & .92 & .92 & .78 & .78 & .84 & .88\end{array}$

$6.08 \quad 6.79$

$3.04 \quad 2.81$

$7.41 \quad 8.28$

$\begin{array}{ll}5.84 & 6.32 \\ 3.85 & 4.44\end{array}$

$\begin{array}{ll}3.15 & 6.85\end{array}$

$\begin{array}{ll}6.15 & 6.85 \\ 8.86 & 9.33\end{array}$

$14.61 \quad 17.90$

$7.01 \quad 7.79$

$2.34 \quad 2.81$

$4.94 \quad 5.82$

Total Energyb

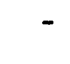

\section{.97}

.34

5.23

1.47

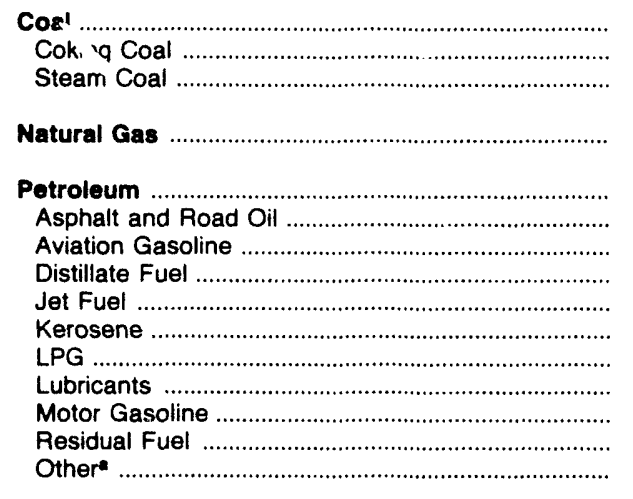

Nuclear Fuel .

Woos and Waste at Utilities

Primary Energy - Five Sectors

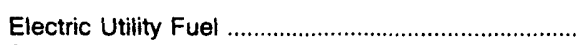
Electricity Purchased by End Users

Total Energyb

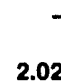

\section{6}

317.5

339.1

653.4

\section{$2,449.7$}

$2,449.7$
34.0

34.0
7.3
429.1

429.1
36.9

33.6

32.7

118.5

$1,559.6$

157.4

40.7

1.1

$3,760.8$

$-296.5$

$1,330.2$

$4,794.5$
.93

10.37

3.25

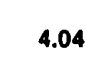

1.55

1.55
15.17

4.44

1.61

21.24

8.49

3.56

1.35

21.86

7.72

Expenditures in Millio $B$ of Dollars

\section{1,991.7 2,594.6}

913.7

$1,078.1$

$1,005.0$

$1,589.6$

$964.8 \quad 2,489.5$

$4,716.0 \quad 10,487.6$

74.2

7.4
$1,039.9$

039.9
97.3

55.5

76.5

152.1

$2,695.2$

441.3

76.8

44.3

127.1
15.3
$2,665.1$

2,665.1

360.1
121.5

121.5
163.8

354.4

$5,507.0$

798.1

375.1

55.4

$2,307.2$

$2,307.2$
492.9

492.9
$1,814.4$

$2,042.7$
330.9

330.9

$2,051.5$
357.1

$1,694.3$

$3,444.8$

$3,147.9$

$2,944.4$

$6,965.9$

$9,187.0$

161.3

10.5

$2,418.9$

334.6

75.7

243.1

395.4

$4,825.9$

443.4

278.3

158.5

10.7

$\therefore 850.0$

219.3

69.5

268.8

342.3

$3,576.6$

288.4

181.8

262.2

336.6

7,487.2

141.9

5.6
$1,976.3$

239.6

57.2

249.3

337.0

$3,927.4$

345.6

207.1

293.8

3.48

3.76

1.35

20.92

1.38
21.68

$\begin{array}{ll}7.35 & 7.88\end{array}$ 


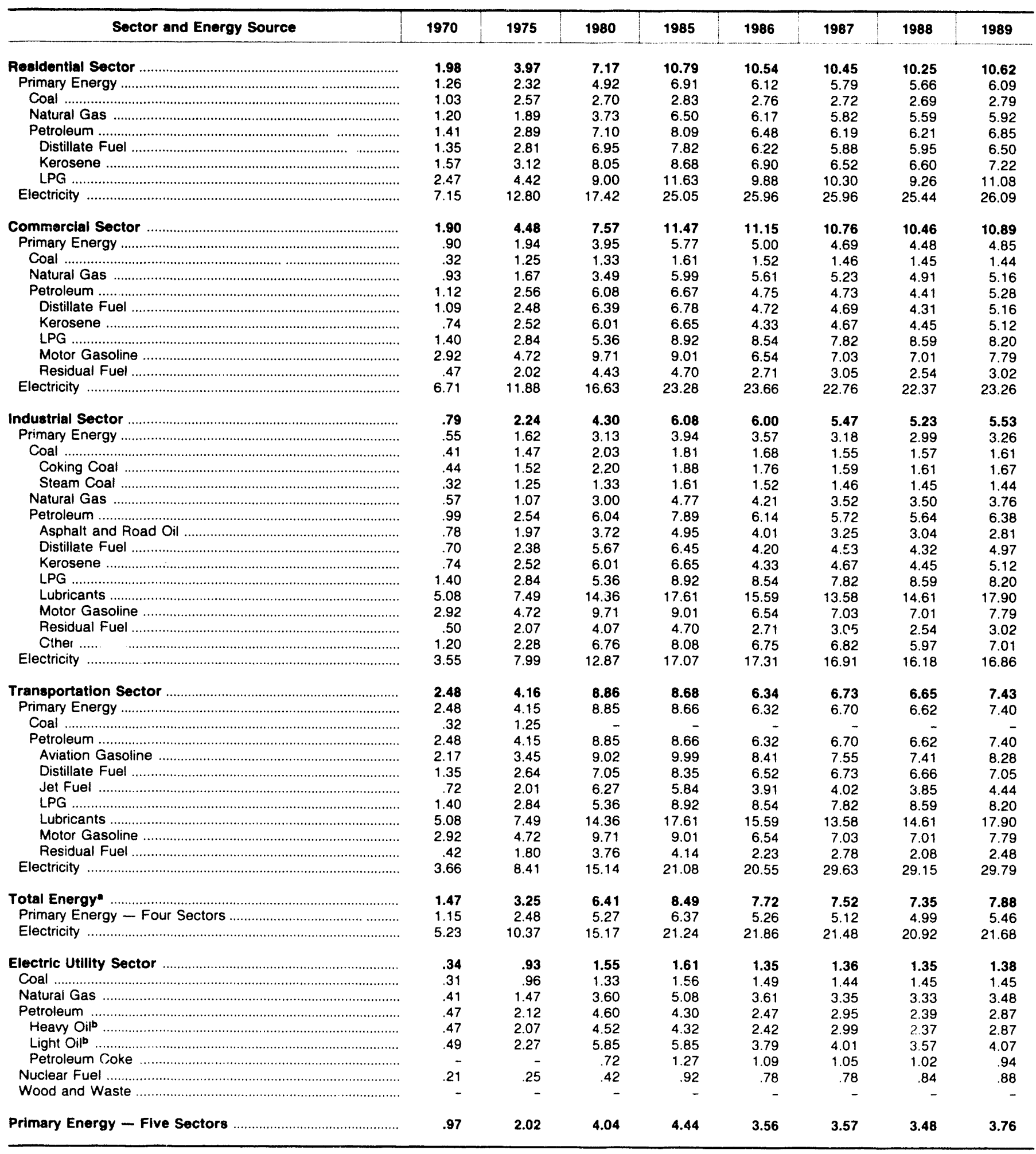

-There are no direct fuel costs for hydroelectric, geothermal, centralized solar, or wind energy. Wood and other biomass fuels are not included, except those consumed at electric utilities.

b Heavy oil includes Grade Nos. 4, 5, and 6, and residual fuel oils. Light oil includes Grade No. 2 heating oil, kerosene, and jet fuel

-No consumption, including cases where adjustments were made. See "Consumption Adjustments for Process Fuel and Intermediate Products."

Sources: Data sources, estimation procedures, and assumptions are described in the "Documentation" section of this report. 


\section{P Energy Expenditure Estimates by Sector, Pennsylvania \\ E 1970, 1975, 1980, and 1985-1989 \\ N (Million Dollars)}

Sector and Energy Source
Residential Sector
Primary Energy
Coal I
Natural Gas
Petroleum
Distillate Fuel
Kerosene
LPG
Electricity

Transportation Sector

Primary Energy

Coal

Petroleum

Aviation Gasoline

Distillate Fuel

Jet Fuel

LPG

Lubricants

Motor Gasoline

Residual Fuel

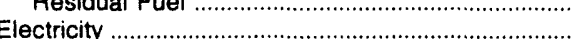

1970

1975

1980

1985

1986

1987

1988

1989

$\begin{array}{rl}1,269.9 & 2,383.3 \\ 708.4 & 1,174.8\end{array}$

\section{$4,325.2$}

708.4

$1,174.8$

$2,437.1$

\section{$5,630.3$}

$5,526.8$

$5,515.9$

$5,701.5$

$6,104.9$

367.4

527.3

$5,098.2$
$1,098.5$

292.7

587.6

$1,287.5$

$1,127.1$

35.8

107.8

29.9

17.6
561.5

34.7

52.6

$1,208.5$

$1,888.1$

$2,836.9$

$2,494.4$

$2,348.9$

$1,644.9$

$1,149.2$

48.5

$1,629.4$
816.6

986.4

683.8

53.5

$2,418.5$

$2,710.0$

$1,514.6$
780.9

$1,551.9$

818.4

652.8

63.6
99.2

$2,793.4$

59.0

73.8
$3,032.4$

45.5

82.5
$3,166.9$

50.5

82.9

1,969.1

507.0
198.6

$1,111.1$

12.3

95.9

90.4

34.4

1.2
1.8

37.6

15.4

308.4

2,958.8

23.3

734.4

169.1

23.0

164.3

422.8

288.6

2.5

3.9

32.5

46.0
754.2

$1,003.5$

$2,992.1$

24.3

714.6

899.7

2,958.1

$3,283.0$

$1,662.4$

995.2

50.6
109.3

$3,034.0 \quad 3,340.7$

264.6

665.6

2071

164.9

6.6

5.5

16.0

6.1

13.4

21.2

42.4
$1,234.7$

29.1
$1,955.3$

$1,311.7$

$3,119.2$

$5,470.9$

$5,306.3$

$2,027.0$

$1,085.7$

$3,506.1$

$1,155.8$

381.8
317.5

64.3

913.7

1.005 .0

150.8

957.9

$1,392.4$
127.1

674.7

285.3

34.0

38.9

2.5
12.6

12.6
77.6

18.1

60.9

40.7

458.4

144.8

358.4

17.1

36.2

102.4

27.2

196.0

76.8
.092 .1

$2,958.1$
856.6

$3,034.0$
864.2

$3,340.7$

29.8
622.2

204.5

26.3

999.7

$648.4 \quad 708.9$

$189.6 \quad 263.6$

154.4

5.0

11.3

15.8

17.9

15.8
2.092 .4

16.2
$2,101.5$

140.2

219.3

4.9
13.6

4.0

17.4

18.5

13.4
2.169 .7

7.6
$2,340.9$

$\mathbf{4 , 7 4 4 . 6} \quad \mathbf{4 , 8 3 7 . 3}$

$4,773.2$

$5,086.0$

$2,875.9$

$2,875.4$
492.9

492.9

154.6

$1,077.2$

$1,151.2$

161.3

212.3

$\begin{array}{ll}7.1 & 5.9\end{array}$

102.8

240.1

29.9

153.1

373.8
1.964 .8

124.3
267.9

60.4

$4,744.6$

$4,773.2$

$2,496.1$

$483.5 \quad 509.3$

330.9

152.5

850.2

991.0

158.5

357.1

152.2

800.7

$1,039.0$

141.9

202.6

5.5

179.1

231.9

152.0
228.3

48.4

46.9

272.3

58.8

176.0

$2,419.9$

200.3

$2,488.3$

583.8

437.4

590.2

$146.4 \quad 148.0$

$803.4 \quad 909.0$

$878.8 \quad 996.9$

$\begin{array}{ll}110.4 & 125.1\end{array}$

182.3

4.1

94.7

237.0

3.6
107.4

107.4
297.8

$51.1 \quad 54.9$

$40.2 \quad 28.5$

28.5
197.4

$3,116.1$

$6,961.5$

$6,275.9$

$4,740.0$

$5,241.1$

2.589 .8

$1,704.0$

$3,110.5$

$6,952.3$

$6,251.8$

$4,715.8$

$5,208.3$

$\mathbf{5 , 4 4 4 . 4}$

$5,833.8$

$6,952 . \overline{3} \quad 6,251 . \overline{8}$

$4,715 . \overline{8}$

5,2083

10.5

10.7

254.4

885.1

977.0

811.7

929.9

239.6

3.7

127.5

49.7

2.9

114.3

$2,635.5 \quad 5,461.1 \quad 4,744.3$

51.7
24.1

110.4

$3,517.6$

108.7

41.4
24.2

$3,861.1$

59.6
32.8

5,410 .

$5,798.7$

$5,410 . \overline{1} \quad 5,798 . \overline{7}$

7.1

7.4

965.3

255.1

974.6

112.8 


\begin{tabular}{|c|c|c|c|c|c|c|c|c|}
\hline Energy Source & 1970 & 1975 & 1980 & 1985 & 1986 & $198 ?$ & 1988 & 1989 \\
\hline & \multicolumn{8}{|c|}{ Prices in Dollars per Million Btu } \\
\hline Coal & 0.94 & 2.64 & 2.37 & 3.03 & 2.30 & 3.09 & 2.54 & 2.70 \\
\hline 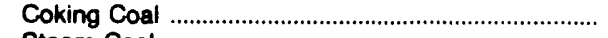 & - & - & - & - & - & - & - & - \\
\hline 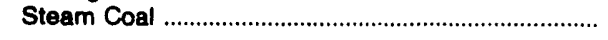 & .94 & 2.64 & 2.37 & 3.03 & 2.30 & 3.09 & 2.54 & 2.70 \\
\hline 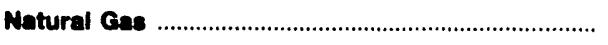 & 1.38 & 2.74 & 5.09 & 6.66 & 6.77 & 5.49 & 5.94 & 6.21 \\
\hline Petroleum & 1.41 & 3.16 & 7.58 & 7.72 & 6.15 & 6.45 & 6.43 & 7.32 \\
\hline 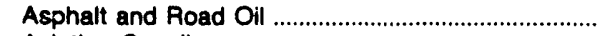 & .66 & 1.86 & 3.58 & 5.18 & 4.86 & 3.54 & 3.34 & 3.17 \\
\hline Aviation Gasoline & 2.17 & 3.45 & 9.02 & 9.99 & 8.41 & 7.55 & 7.41 & 8.28 \\
\hline 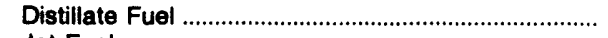 & 1.35 & 2.76 & 7.06 & 8.08 & 6.37 & 6.24 & 6.37 & 7.06 \\
\hline 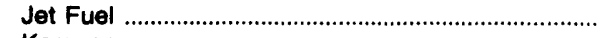 & .75 & 2.09 & 6.51 & 6.10 & 4.25 & 4.18 & 4.04 & 4.75 \\
\hline 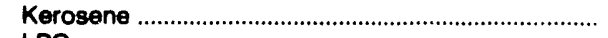 & 1.50 & 2.98 & 7.44 & 8.89 & 6.88 & 6.80 & 6.70 & 7.65 \\
\hline LPG & 1.77 & 3.50 & 6.57 & 11.69 & 10.60 & 11.19 & 11.00 & 9.25 \\
\hline 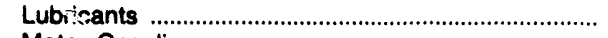 & 5.08 & 7.49 & 14.36 & 17.61 & 15.59 & 13.58 & 14.61 & 17.90 \\
\hline 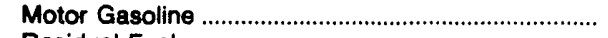 & 2.90 & 4.50 & 9.72 & 9.13 & 7.36 & 7.96 & 8.40 & 9.11 \\
\hline 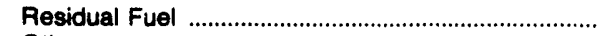 & .43 & 1.92 & 4.03 & 4.59 & 2.61 & 3.02 & 2.43 & 2.82 \\
\hline Other & 1.65 & 3.71 & 7.67 & 8.68 & 10.73 & 10.73 & 10.84 & 10.00 \\
\hline 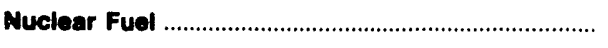 & - & - & - & - & - & - & - & - \\
\hline 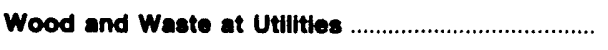 & - & - & - & - & - & - & - & - \\
\hline Primary Energy - Five Sectore ................................... & 1.40 & 3.10 & 7.04 & 7.48 & 6.25 & 6.21 & 6.22 & 7.02 \\
\hline 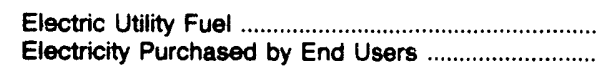 & $\begin{array}{r}.43 \\
6.85\end{array}$ & $\begin{array}{r}1.84 \\
13.78\end{array}$ & $\begin{array}{r}3.91 \\
20.67\end{array}$ & $\begin{array}{r}3.82 \\
24.73\end{array}$ & $\begin{array}{r}2.39 \\
23.40\end{array}$ & $\begin{array}{r}2.50 \\
23.93\end{array}$ & $\begin{array}{r}2.28 \\
23.28\end{array}$ & $\begin{array}{r}2.65 \\
24.24\end{array}$ \\
\hline \multirow[t]{2}{*}{ Total Energy } & 1.91 & 4.16 & 9.05 & 9.77 & 8.70 & 8.69 & 8.63 & 9.59 \\
\hline & \multicolumn{8}{|c|}{ Expenditures in Millions of Dollars } \\
\hline Coal (n) & 0.2 & 0.4 & 0.4 & 0.6 & 1.6 & 0.4 & 11.1 & 1.8 \\
\hline Coking Coal & - & - & - & - & - & - & - & - \\
\hline 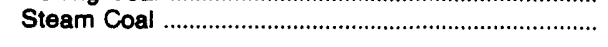 & .2 & .4 & .4 & .6 & 1.6 & .4 & 11.1 & 1.8 \\
\hline 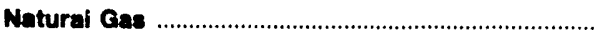 & 35.2 & 64.3 & 142.7 & 204.6 & 182.9 & 202.3 & 187.0 & 215.8 \\
\hline Petroleum & 233.7 & 432.6 & 790.5 & 840.0 & 689.4 & 737.4 & 765.4 & 779.1 \\
\hline 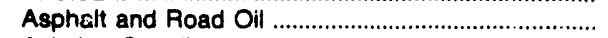 & 4.1 & 16.5 & 24.7 & 102.3 & 47.7 & 41.7 & 38.6 & 33.7 \\
\hline Aviation Gasoline & 1.6 & 5.0 & 12.2 & 1.5 & 1.5 & 1.6 & 1.7 & 1.9 \\
\hline 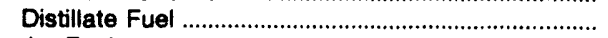 & 67.9 & 128.5 & 207.0 & 209.6 & 196.7 & 220.1 & 220.3 & 242.8 \\
\hline Jet Fuel & .6 & 3.2 & 12.8 & 17.1 & 9.3 & 12.5 & 14.5 & 19.4 \\
\hline 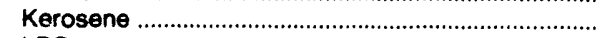 & 3.7 & 2.2 & 3.5 & 3.1 & 3.3 & 2.4 & 2.0 & 1.3 \\
\hline LPG & 2.5 & 6.5 & 7.1 & 20.9 & 22.5 & 25.5 & 22.8 & 17.6 \\
\hline 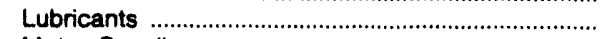 & 3.9 & 4.4 & 11.5 & 12.8 & 11.1 & 10.9 & 11.3 & 14.2 \\
\hline 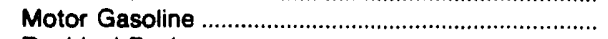 & 122.0 & 211.9 & 429.7 & 415.5 & 345.4 & 381.1 & 409.6 & 424.3 \\
\hline Residual Fuel & 25.7 & 52.9 & 63.9 & 51.0 & 48.0 & 37.2 & 40.9 & 20.8 \\
\hline Other & 1.8 & 1.9 & 18.0 & 6.2 & 4.0 & 4.4 & 3.5 & 3.1 \\
\hline 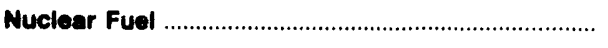 & - & - & - & - & - & - & - & - \\
\hline 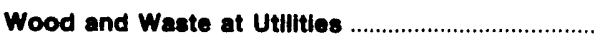 & - & - & - & - & - & - & - & - \\
\hline Primary Energy - Flve Sectors ................................. & 269.1 & 497.4 & 933.6 & $1,045.2$ & 874.0 & 940.1 & 963.6 & 996.7 \\
\hline Electric Utility Fuel & -9.3 & -18.1 & -47.5 & -27.4 & -22.3 & -26.8 & -22.4 & -17.7 \\
\hline 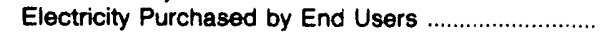 & 90.7 & 209.3 & 361.9 & 458.2 & 451.6 & 485.1 & 494.0 & 526.0 \\
\hline Total Energy & 350.5 & 688.6 & $1,248.0$ & $1,476.0$ & $1,303.3$ & $1,398.4$ & $1,435.1$ & $1,505.0$ \\
\hline
\end{tabular}

- Includes petroleum coke used at electric utilities.

-There are no direct fuel costs for hydroelectric, geothermal, centralized solar, or wind energy. Wood and other biomass fuels are not included, except those consumed at electric utilities.

-No consumption, including cases where adjustments were made. See "Consumption Adjustments for Process Fuel and Intermediate Products."

Note: Expenditure totals may not equal sum of components due to independent rounding.

Sources: Data sources, estimation procedures, and assumptions are described in the "Documentation" section of this report 


\section{R Energy Price Estimates by Sector, Rhode Island \\ H $1970,1975,1980$, and 1985-1989 (Dollars per Million Btu)}

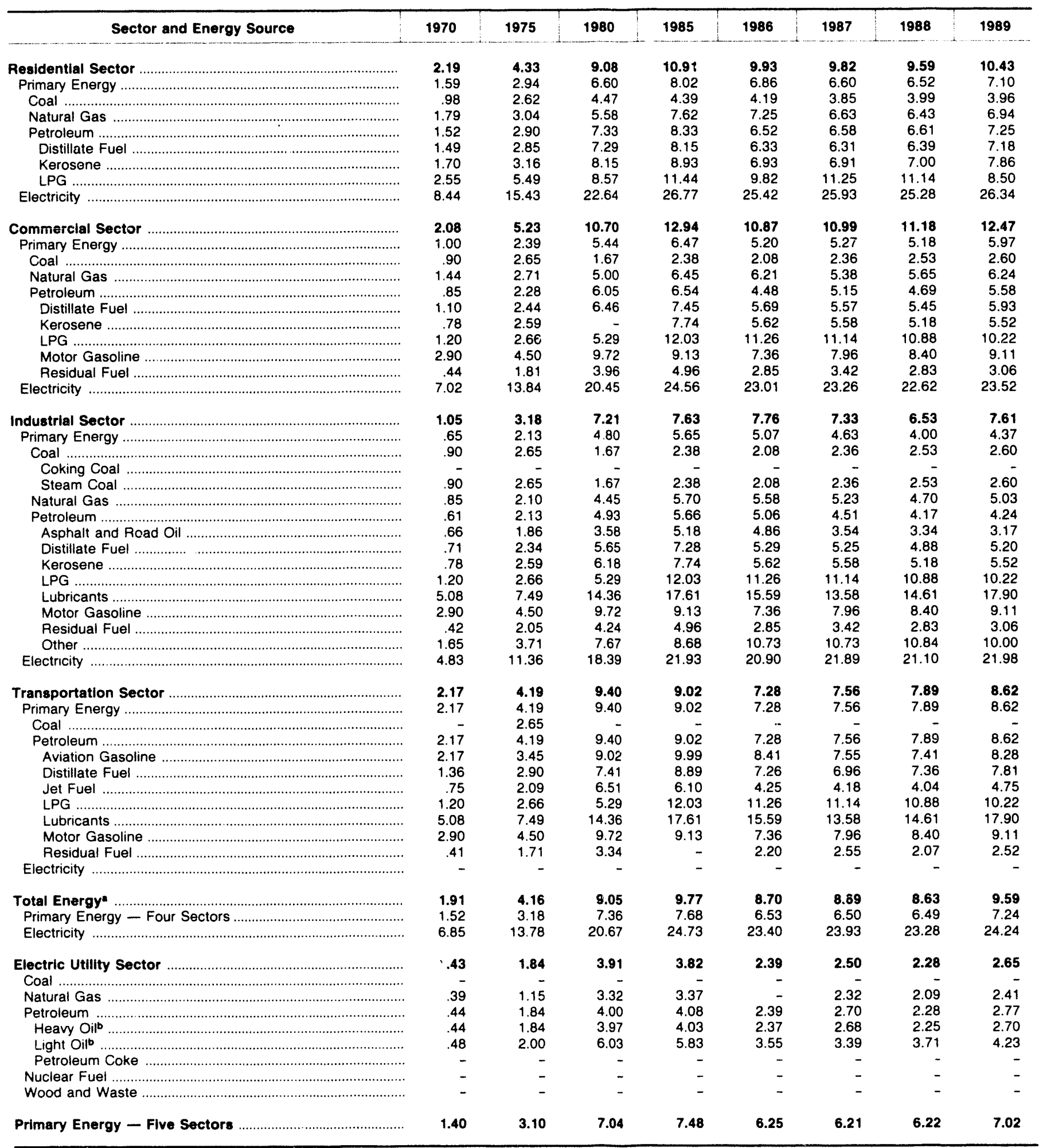

-There are no direct fuel costs for hydroelectric, geothermal, centralized solar, or wind energy. Wood and other biomass fuels are not included, except those consumed at electric utilities.

beavy oil includes Grade Nos. 4, 5, and 6, and residual fuel oils. Light oil includes Grade No. 2 heating oil, kerosene, and jet fuel.

-No consumption, including cases where adjustments were made. See "Consumption Adjustments for Process Fuel and Intermediate Products."

Sources: Data sources, estimation procedures, and assumptions are described in the "Documentation" section of this report. 


\begin{tabular}{|c|c|c|c|c|c|c|c|c|}
\hline Sector and Energy Source & 1970 & 1975 & 1980 & 1985 & 1986 & 1987 & 1988 & 1989 \\
\hline $\begin{array}{c}\text { Residential Sector } \\
\text { Primary Energy }\end{array}$ & 117.5 & 223.2 & 367.9 & 475.5 & 422.7 & 439.5 & 463.8 & 486.7 \\
\hline Primary Energy & $\begin{array}{r}77.5 \\
.1\end{array}$ & $\begin{array}{r}134.5 \\
.1\end{array}$ & $\begin{array}{r}225.8 \\
.2\end{array}$ & $\begin{array}{r}295.5 \\
.3\end{array}$ & $\begin{array}{r}243.7 \\
.3\end{array}$ & $\begin{array}{r}246.1 \\
.2\end{array}$ & 263.8 & 273.7 \\
\hline Natural Gas . & 21.9 & 40.2 & 79.5 & 118.0 & 120.2 & 114.0 & 116.7 & $\begin{array}{r}.2 \\
130.4\end{array}$ \\
\hline 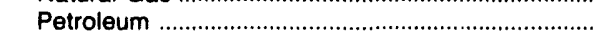 & 55.5 & 94.1 & 146.1 & 177.2 & 123.2 & 131.8 & 146.9 & 143.2 \\
\hline Distillate Fuel & 50.7 & 89.6 & 140.0 & 162.4 & 110.5 & 117.4 & 134.1 & 132.9 \\
\hline 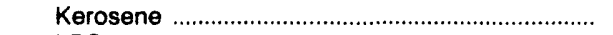 & 3.2 & 1.6 & 2.5 & 3.0 & 3.2 & 2.3 & 1.8 & 1.2 \\
\hline 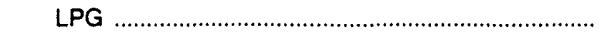 & 1.5 & 3.0 & 3.6 & 11.8 & 9.5 & 12.2 & 11.1 & 9.0 \\
\hline 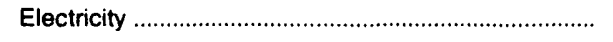 & 40.0 & 88.7 & 142.1 & 180.0 & 179.0 & 193.4 & 200.0 & 213.0 \\
\hline Commercial Sector & 51.1 & 113.0 & 197.1 & 266.6 & 264.1 & 282.5 & 281.7 & 301.9 \\
\hline 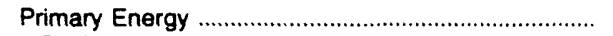 & 20.3 & 39.1 & 65.1 & 85.6 & 86.1 & 92.4 & 85.8 & 90.8 \\
\hline Coal & .1 & .1 & • & .1 & .1 & .1 & .1 & .1 \\
\hline Natural Gas & 7.5 & 11.6 & 34.5 & 50.6 & 42.9 & 52.0 & 48.4 & 56.2 \\
\hline 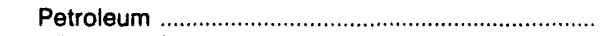 & 12.8 & 27.3 & 30.6 & 34.9 & 43.0 & 40.3 & 37.2 & 34.6 \\
\hline 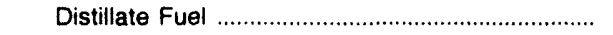 & 9.4 & 19.3 & 23.2 & 19.2 & 26.7 & 28.9 & 25.6 & 26.9 \\
\hline 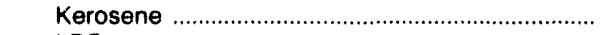 & • & & - & .1 & .1 & .1 & * & .1 \\
\hline LPG & .1 & .3 & .4 & 2.2 & 1.9 & 2.1 & 1.9 & 1.9 \\
\hline 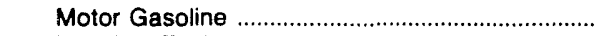 & .6 & 1.0 & 2.5 & 1.5 & 1.3 & 1.5 & 1.6 & 1.8 \\
\hline 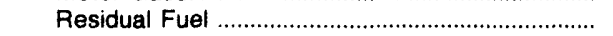 & 2.7 & 6.9 & 4.5 & 12.0 & 12.9 & 77 & 8.1 & 3.8 \\
\hline 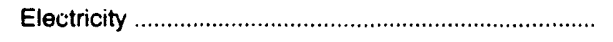 & 30.8 & 74.4 & 132.0 & 151.0 & 178.1 & 190.2 & 196.0 & 211.0 \\
\hline Industrial Sector & 44.6 & 113.1 & 194.3 & 278.2 & 206.4 & 217.8 & 204.6 & 192.2 \\
\hline 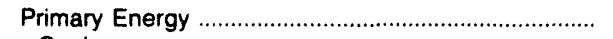 & 24.7 & 67.0 & 106.6 & 180.9 & 111.8 & 116.2 & 106.6 & 90.2 \\
\hline Coal & • & .1 & .2 & .2 & 1.2 & .1 & 10.9 & 1.6 \\
\hline 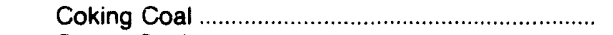 & - & - & - & - & - & - & - & - \\
\hline 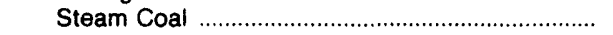 & • & .1 & .2 & .2 & 1.2 & .1 & 10.9 & 1.6 \\
\hline Natural Gas & 5.0 & 12.4 & 23.1 & 27.2 & 19.8 & 23.6 & 21.5 & 23.9 \\
\hline 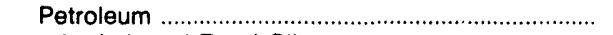 & 19.7 & 54.4 & 83.3 & 153.5 & 90.7 & 92.5 & 74.2 & 64.8 \\
\hline Asphalt and Road Oil & 4.1 & 16.5 & 24.7 & 102.3 & 47.7 & 41.7 & 38.6 & 33.7 \\
\hline 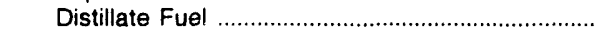 & 2.8 & 6.0 & 13.6 & 10.5 & 8.8 & 16.6 & 7.7 & 9.4 \\
\hline Kerosene & .4 & .6 & 1.1 & * & • & .1 & .2 & \\
\hline LPG & .7 & 2.9 & 2.9 & 6.2 & 10.5 & 10.8 & 9.1 & 6.0 \\
\hline 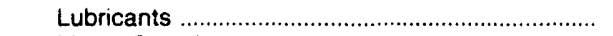 & 1.5 & 1.8 & 5.4 & 6.0 & 5.2 & 5.1 & 5.3 & 6.7 \\
\hline 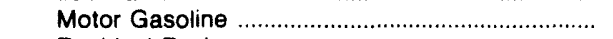 & • & .1 & .1 & 1.3 & 1.2 & 1.2 & 1.5 & 1.7 \\
\hline 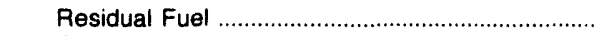 & 8.3 & 24.7 & 17.4 & 21.1 & 13.2 & 12.6 & 8.3 & 4.2 \\
\hline Other & 1.8 & 1.9 & 18.0 & 6.2 & 4.0 & 4.4 & 3.5 & 3.1 \\
\hline 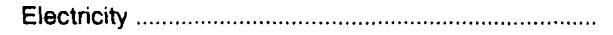 & 19.9 & 46.2 & 87.8 & 97.3 & 94.6 & 101.6 & 98.0 & 102.0 \\
\hline Transportation Sector & 137.4 & 238.7 & 488.7 & 455.7 & 410.1 & 458.6 & 485.0 & 524.2 \\
\hline 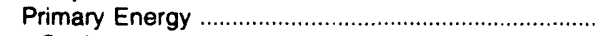 & 137.4 & 238.7 & 488.7 & 455.7 & $\Delta 10.1$ & 458.6 & 485.0 & 524.2 \\
\hline Coal & - & & - & - & - & - & - & - \\
\hline 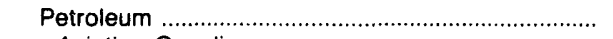 & 137.4 & 238.7 & 488.7 & 455.7 & $410: 1$ & 458.6 & 485.0 & 524.2 \\
\hline Aviation Gasoline & 1.6 & 5.0 & 12.2 & 1.5 & 1.5 & 1.6 & 1.7 & 1.9 \\
\hline 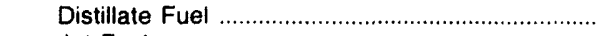 & 4.8 & 13.3 & 29.2 & 16.9 & 50.0 & 56.7 & 52.0 & 72.7 \\
\hline Jet Fuel & .6 & 3.2 & 12.8 & 17.1 & 9.3 & 12.5 & 14.5 & 19.4 \\
\hline 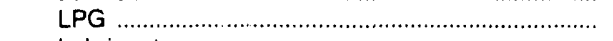 & .1 & .3 & .2 & .7 & .6 & .3 & .6 & .6 \\
\hline 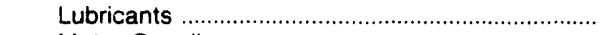 & 2.4 & 2.6 & 6.1 & 6.8 & 5.9 & 5.8 & 6.0 & 7.6 \\
\hline Motor Gasoline & 121.4 & 210.8 & 427.1 & 412.7 & 342.8 & 378.4 & 406.6 & 420.8 \\
\hline 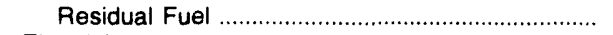 & 6.5 & 3.5 & 1.2 & - & .1 & 3.4 & 3.5 & 1.2 \\
\hline 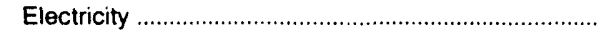 & - & - & - & - & - & - & - & - \\
\hline Total Energy• & 350.5 & 688.6 & $1,248.0$ & $1,476.0$ & $1,303.3$ & $1,398.4$ & $1,435.1$ & $1,505.0$ \\
\hline Primary Energy - Four Sectors .................................... & 259.8 & 479.3 & 886.1 & $1,017.8$ & 851.7 & 913.2 & 941.1 & 979.0 \\
\hline 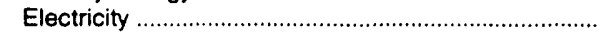 & 90.7 & 209.3 & 361.9 & 458.2 & 451.6 & 485.1 & 494.0 & 526.0 \\
\hline Electric Utllity Sector . & 9.3 & 18.1 & 47.5 & 27.4 & 22.3 & 26.8 & 22.4 & 17.7 \\
\hline Coal & - & - & & & - & & - & \\
\hline Natural Gas & .9 & * & 5.7 & 8.8 & - & 12.8 & .4 & 5.3 \\
\hline - & 8.4 & 18.1 & 41.8 & 18.6 & 22.3 & 14.1 & 22.0 & 12.4 \\
\hline 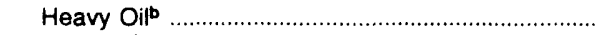 & 8.2 & 17.8 & 40.8 & 17.9 & 21.7 & 13.6 & 21.1 & 11.5 \\
\hline 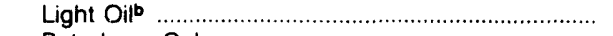 & .2 & .3 & 1.0 & .7 & .6 & .5 & .9 & .9 \\
\hline Petroleum Coke & - & - & - & - & - & - & - & - \\
\hline 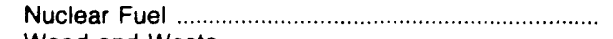 & - & - & - & - & - & - & - & - \\
\hline Wood and Waste & - & - & - & - & - & - & - & - \\
\hline 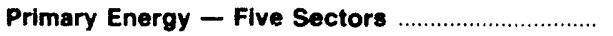 & 269.1 & 497.4 & 933.6 & $1,045.2$ & 874.0 & 940.1 & 963.6 & 996.7 \\
\hline
\end{tabular}

- There are no direct fuel costs for hydroelectric, geothermal, centralized solar, or wind energy. Wood and other biomass fuels are not included, except those consumed at electric utilities.

beavy oil includes Grade Nos. 4, 5, and 6, and residual fuel oils. Light oil includes Grade No. 2 heating oil, kerosene, and jet fuel.

-No consumption, including cases where adjustments were made. See "Consumption Adjustments for Process Fuel and Intermediate Products."

- Value less than 0.05 million dollars.

Note: Totals may not equal sum of components due to independent rounding.

Sources: Data sources, estimation procedures, and assumptions are described in the "Documentation" section of this report. 


\begin{tabular}{|c|c|c|c|c|c|c|c|c|}
\hline Energy Source & 1970 & 1975 & 1980 & 1985 & 1986 & 1987 & 1988 & 1989 \\
\hline & \multicolumn{8}{|c|}{ Prices in Dollare per Million Btu } \\
\hline 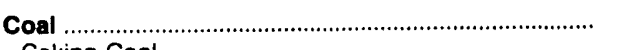 & 0.47 & 1.25 & 1.60 & 1.88 & 1.82 & 1.74 & 1.75 & 1.71 \\
\hline Coking Coal & $.4 \overline{7}$ & $1 . \overline{5}$ & $1.60^{-}$ & $1 . \overline{8}$ & $1.8 \overline{2}$ & $1 . \overline{4}$ & $1.7 \overline{5}$ & $1.7 \overline{1}$ \\
\hline 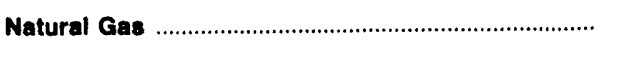 & .57 & 1.16 & 3.07 & 5.06 & 4.40 & 4.60 & 4.25 & 4.22 \\
\hline Petroleum & 1.88 & 3.43 & 8.08 & 8.04 & 6.08 & 6.35 & 6.21 & 7.05 \\
\hline 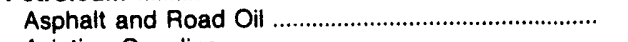 & .67 & 1.78 & 3.58 & 4.99 & 4.18 & 3.20 & 3.17 & 2.90 \\
\hline 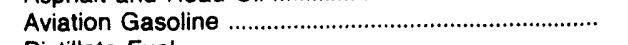 & 2.17 & 3.45 & 9.02 & 9.99 & 8.41 & 7.55 & 7.41 & 8.28 \\
\hline 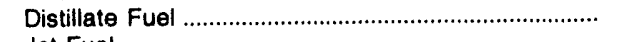 & 1.03 & 2.68 & 6.84 & 7.06 & 5.76 & 5.82 & 5.69 & 6.15 \\
\hline 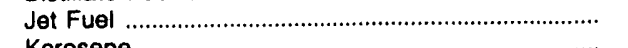 & .73 & 2.03 & 6.46 & 6.11 & 4.23 & 4.35 & 4.19 & 4.74 \\
\hline LPG & $\begin{array}{l}2.04 \\
5.08\end{array}$ & $\begin{array}{l}3.56 \\
7.49\end{array}$ & $\begin{array}{r}5.77 \\
14.36\end{array}$ & $\begin{array}{r}9.87 \\
17.61\end{array}$ & $\begin{array}{r}9.80 \\
15.59\end{array}$ & $\begin{array}{r}8.30 \\
13.58\end{array}$ & $\begin{array}{l}10.17 \\
14.61\end{array}$ & $\begin{array}{r}9.27 \\
17.90\end{array}$ \\
\hline Lubricants & $\begin{array}{l}3.08 \\
2.75\end{array}$ & 4.35 & 10.18 & 8.84 & $\begin{array}{r}15.59 \\
6.40\end{array}$ & $\begin{array}{r}7.08 \\
7.08\end{array}$ & $\begin{array}{r}14.01 \\
7.08\end{array}$ & 7.84 \\
\hline 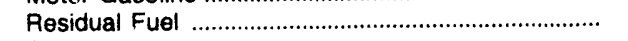 & .42 & 1.40 & 3.43 & 4.32 & 2.49 & 2.94 & 2.30 & 2.65 \\
\hline Other & 1.23 & 3.62 & 6.90 & 6.38 & 4.94 & 4.94 & 4.24 & 5.76 \\
\hline 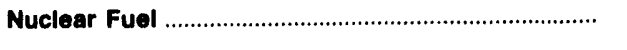 & .19 & .19 & .44 & .62 & .61 & .64 & .59 & .58 \\
\hline 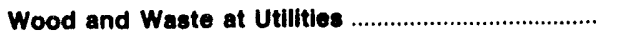 & - & - & - & - & - & - & - & - \\
\hline Primary Energy - Five Sectors ………........................ & 1.20 & 1.87 & 4.09 & 3.78 & 3.00 & 3.01 & 2.92 & 3.21 \\
\hline $\begin{array}{l}\text { Electric Utility Fuel } \\
\text { Electricity Purchased by End Users }\end{array}$ & $\begin{array}{r}.42 \\
3.98\end{array}$ & $\begin{array}{r}.56 \\
7.72\end{array}$ & $\begin{array}{r}1.14 \\
11.11\end{array}$ & $\begin{array}{r}1.11 \\
15.99\end{array}$ & $\begin{array}{r}1.03 \\
16.25\end{array}$ & $\begin{array}{r}1.03 \\
16.41\end{array}$ & $\begin{array}{r}1.00 \\
16.40\end{array}$ & $\begin{array}{r}.99 \\
16.53\end{array}$ \\
\hline \multirow[t]{2}{*}{ Total Energy $\mathbf{b}^{\mathrm{b}}$} & 1.82 & 3.79 & 7.26 & 8.89 & 7.92 & 8.16 & 8.02 & 6.52 \\
\hline & \multicolumn{8}{|c|}{ Expenditures in Millions of Dollare } \\
\hline Coal & 65.3 & 174.7 & 392.2 & 493.6 & 480.6 & 513.0 & 526.9 & 514.1 \\
\hline 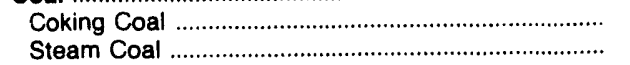 & $65 . \overline{3}$ & & $392 . \overline{2}$ & $493 . \overline{6}$ & $480 . \overline{6}$ & $513 . \overline{0}$ & $526 . \overline{9}$ & $514 . \overline{1}$ \\
\hline 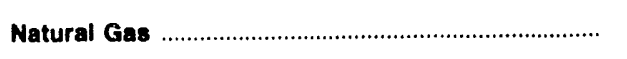 & 91.4 & 143.3 & 441.2 & 495.3 & 435.6 & 488.0 & 479.3 & 495.2 \\
\hline Potroleum & 568.3 & $1,172.2$ & $2,946.2$ & $2,762.9$ & $2,162.3$ & $2,314.8$ & $2,314.0$ & 2,722.1 \\
\hline 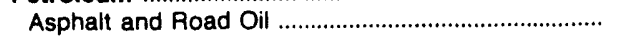 & 9.9 & 28.9 & 36.4 & 45.3 & 57.4 & 51.6 & 69.3 & 44.5 \\
\hline 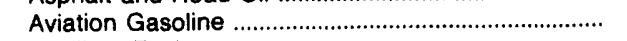 & 2.5 & 2.5 & 6.8 & 6.9 & 6.6 & 4.6 & 4.7 & 5.0 \\
\hline 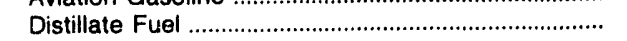 & 56.7 & 130.7 & 424.9 & 482.8 & 392.7 & 401.8 & 417.9 & 447.8 \\
\hline 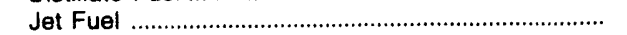 & 12.4 & 29.5 & 107.1 & 105.3 & 72.7 & 75.4 & 73.4 & 80.3 \\
\hline 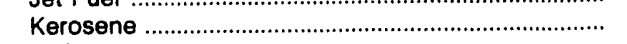 & 19.3 & 17.5 & 60.7 & 30.1 & 24.4 & 25.5 & 25.3 & 25.4 \\
\hline LPG & 22.6 & 42.3 & 67.4 & 113.5 & 104.4 & 104.9 & 135.1 & 131.8 \\
\hline 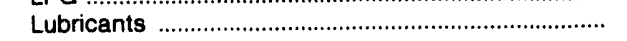 & 11.9 & 21.0 & 47.3 & 52.8 & 45.7 & 45.0 & 46.7 & 58.7 \\
\hline 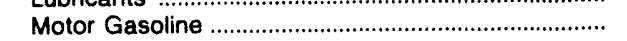 & 415.8 & 809.3 & $1,899.0$ & $1,751.7$ & $1,321.6$ & $1,448.6$ & $1,393.6$ & $1,754.5$ \\
\hline 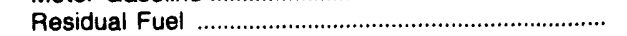 & 14.2 & 67.5 & 155.3 & 59.3 & 24.7 & 30.7 & 35.0 & 24.7 \\
\hline 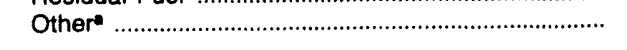 & 3.0 & 23.0 & 141.3 & 115.2 & 112.1 & 126.8 & 113.1 & 149.3 \\
\hline Nuclear Fuel & • & 40.6 & $\mathbf{8 3 . 4}$ & 214.6 & 233.9 & 289.0 & 257.5 & 251.7 \\
\hline 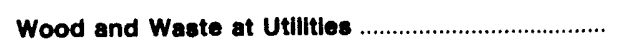 & - & - & - & - & - & - & - & - \\
\hline 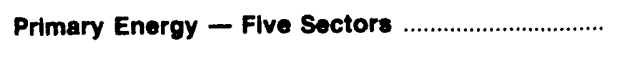 & 725.1 & $1,530.8$ & $3,863.1$ & $3,966.2$ & $3,312.4$ & $3,584.9$ & $3,577.7$ & 3,983.1 \\
\hline 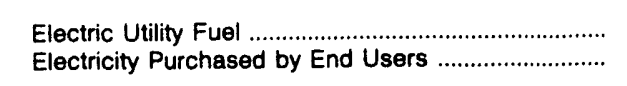 & $\begin{array}{l}-65.0 \\
294.7\end{array}$ & $\begin{array}{r}-205.4 \\
782.8\end{array}$ & $\begin{array}{r}-467.6 \\
1,412.5\end{array}$ & $\begin{array}{r}-601.3 \\
2,523.7\end{array}$ & $\begin{array}{r}-598.7 \\
2,741.1\end{array}$ & $\begin{array}{r}-670.6 \\
2,909.0\end{array}$ & $\begin{array}{r}-677.1 \\
2,955.0\end{array}$ & $\begin{array}{r}-672.7 \\
3,038.0\end{array}$ \\
\hline Total Energy' & 954.7 & $2,108.1$ & $4,808.0$ & $5,888.6$ & $5,454.8$ & $5,823.4$ & $5,855.6$ & $6,348.4$ \\
\hline
\end{tabular}

Includes petroleum coke used at electric utilities.

bThere are no direct fuel costs for hydroelectric, geothermal, centralized solar, or wind energy. Wood anc uther biomass fuels are not included, except those consumed at electric utilities.

- No consumption, including cases where adjustments were made. See "Consumption Adjustments for Process Fuel and Intermediate Products." - Value less than 0.05 million dollars.

Note: Expenditure totals may not equal sum of components due to independent rounding.

Sources: Data sources, estimation procedures, and assumptions are described in the "Documentation" section of this report. 


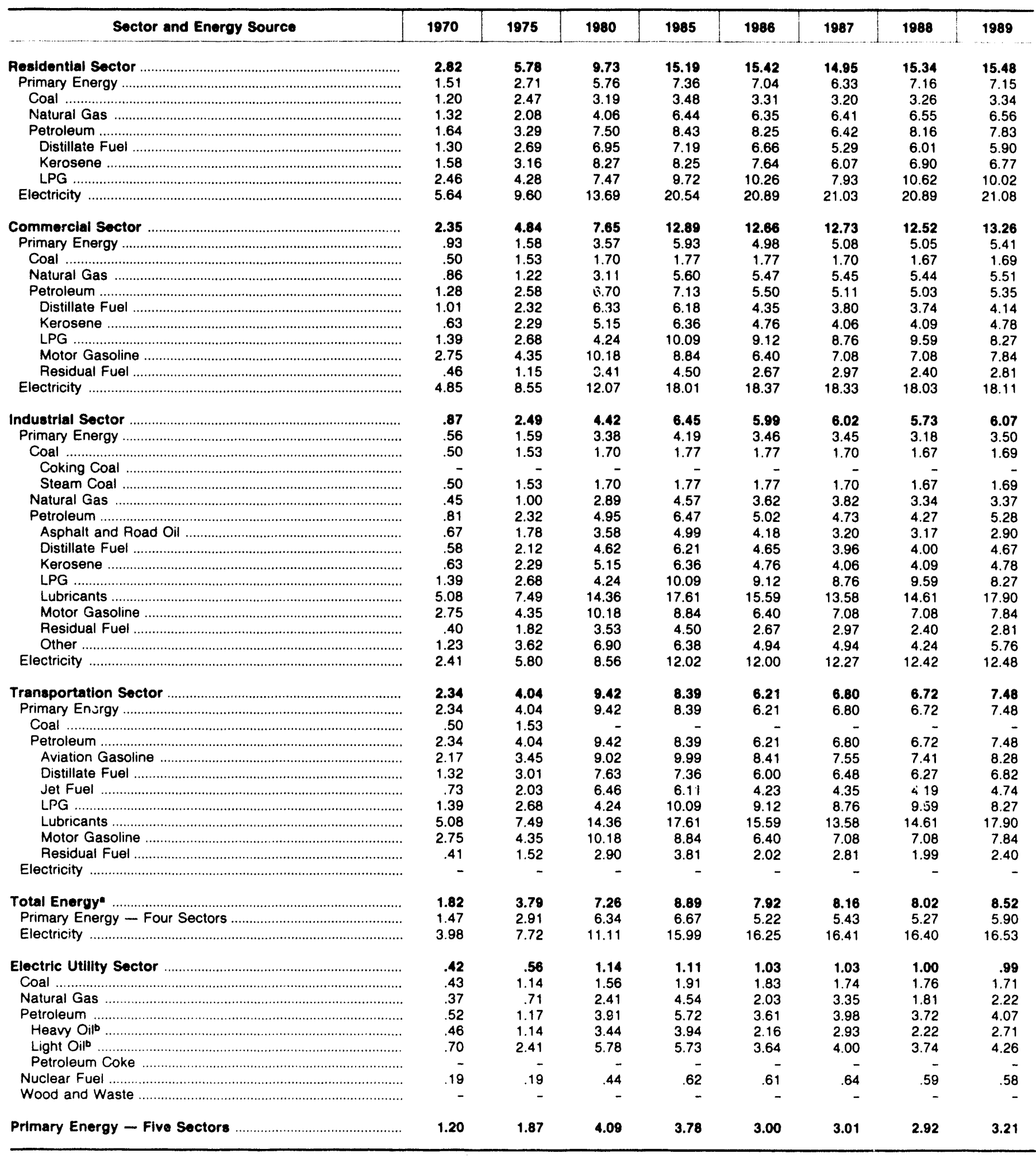

-There are no direct fuel costs for hydroelectric, geothermal, centralized solar, or wind energy. Wood and other biomass fuels are not included, except those consumed at electric utilities.

beavy oil includes Grade Nos. 4, 5, and 6, and residual fuel oils. Light oil includes Grade No. 2 heating oil, kerosene, and jet fuel.

-No consumption, including cases where adjustments were made. See "Consumption Adjustments for Process Fuel and Intermediate Products."

Sources: Data sources, estimation procedures, and assumptions are described in the "Documentation" section of this report. 


\section{S Energy Expenditure Estimates by Sector, South Carolina \\ O 1970, 1975, 1980, and 1985-1989 \\ U (Million Dollars)}

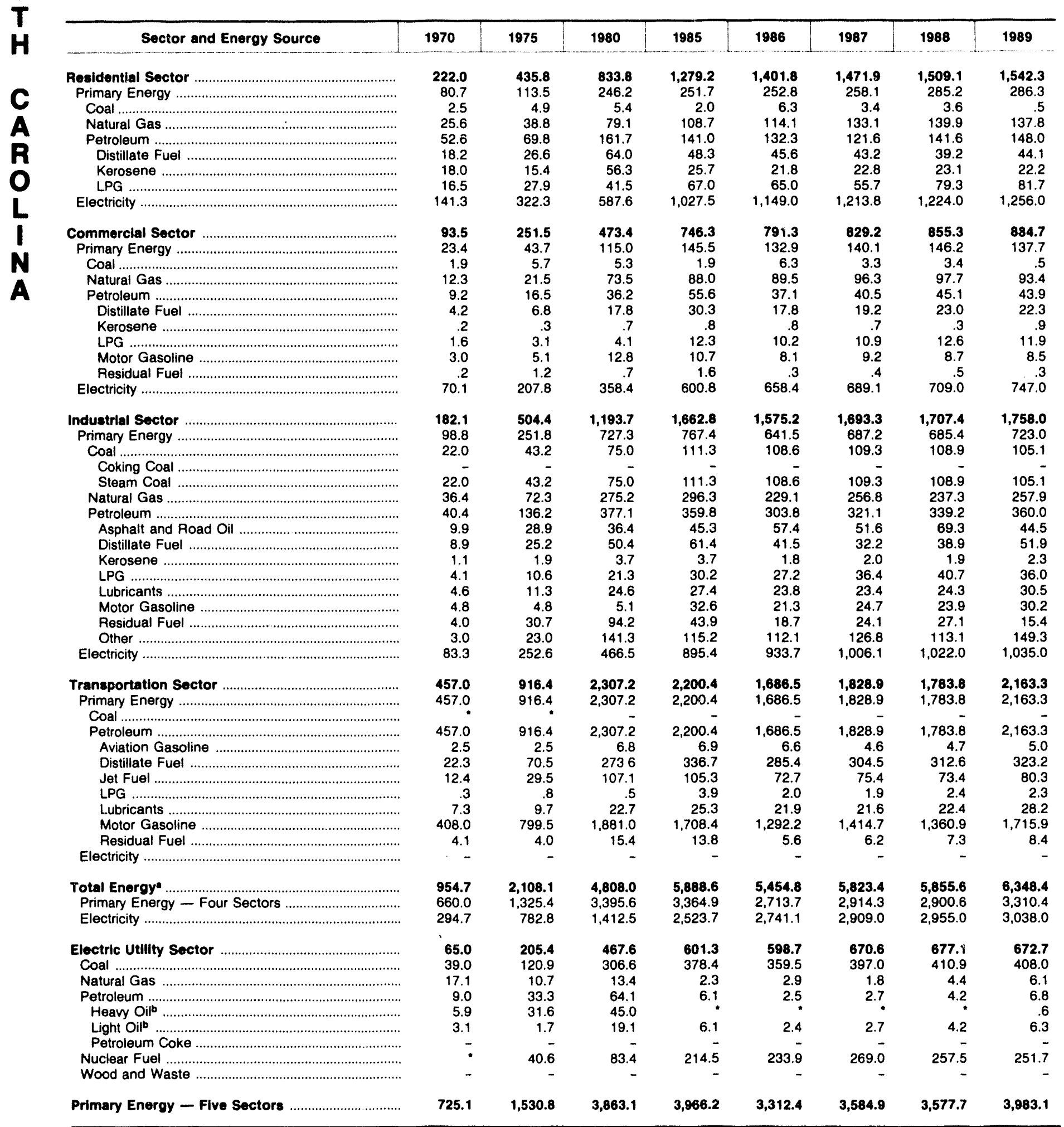

-There are no direct fuel costs for hydroelectric, geothermal, centralized solar, or wind energy. Wood and other biomass fuels are not included, except those consumed at electric utilities.

bHeavy oil includes Grade Nos. 4, 5, and 6, and residual iuel oils. Light oil includes Grade No. 2 heating oil, kerosene, and jet fuel.

-No consumption, including cases where adjustments were made. See "Consumption Adjustments for Process Fuel and Intermediate Products."

"Value less than 0.05 million dollars.

Note: Totals may not equal sum of components due to independent rounding.

Sources: Data sources, estimation procedures, and assumptions are described in the "Documentation" section of this report. 


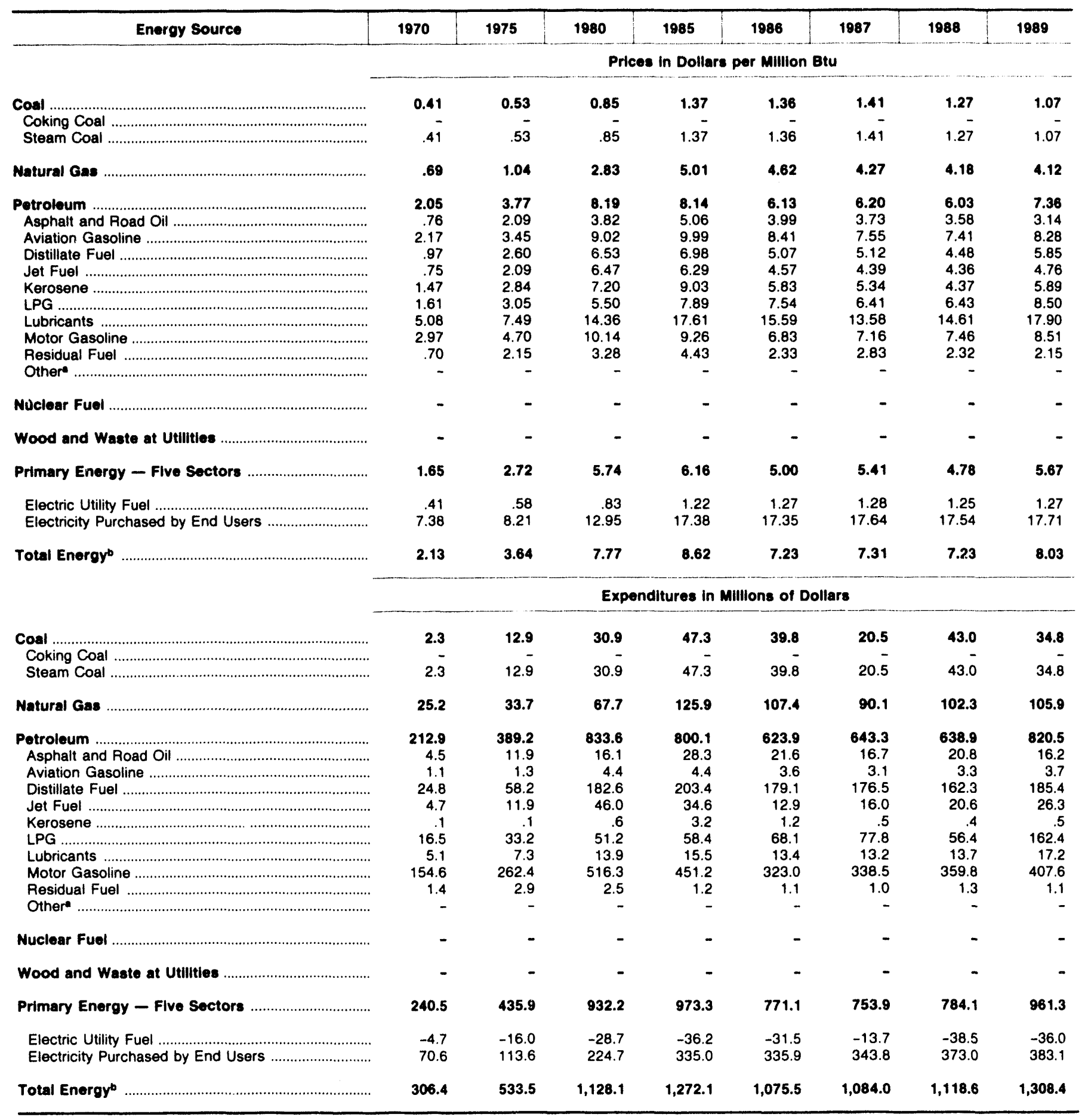

-includes petroleum coke used at electric utilities.

-There are no direct fuel costs for hydroelectric, geothermal, centralized solar, or wind energy. Wood and other biomass fuels are not included, except those consumed at electric utilities.

-No consumption, including cases where adjustments were made. See "Consumption Adjustments for Process Fuel and Intermediate Products."

Note: Expenditure totals may not equal sum of components due to independent rounding.

Sources: Data sources, estimation procedures, and assumptions are described in the "Documentation" section of this report. 


\section{S Energy Price Estimates by Sector, South Dakota \\ O $1970,1975,1980$, and $1985-1989$ \\ U (Dollars per Million Btu)}

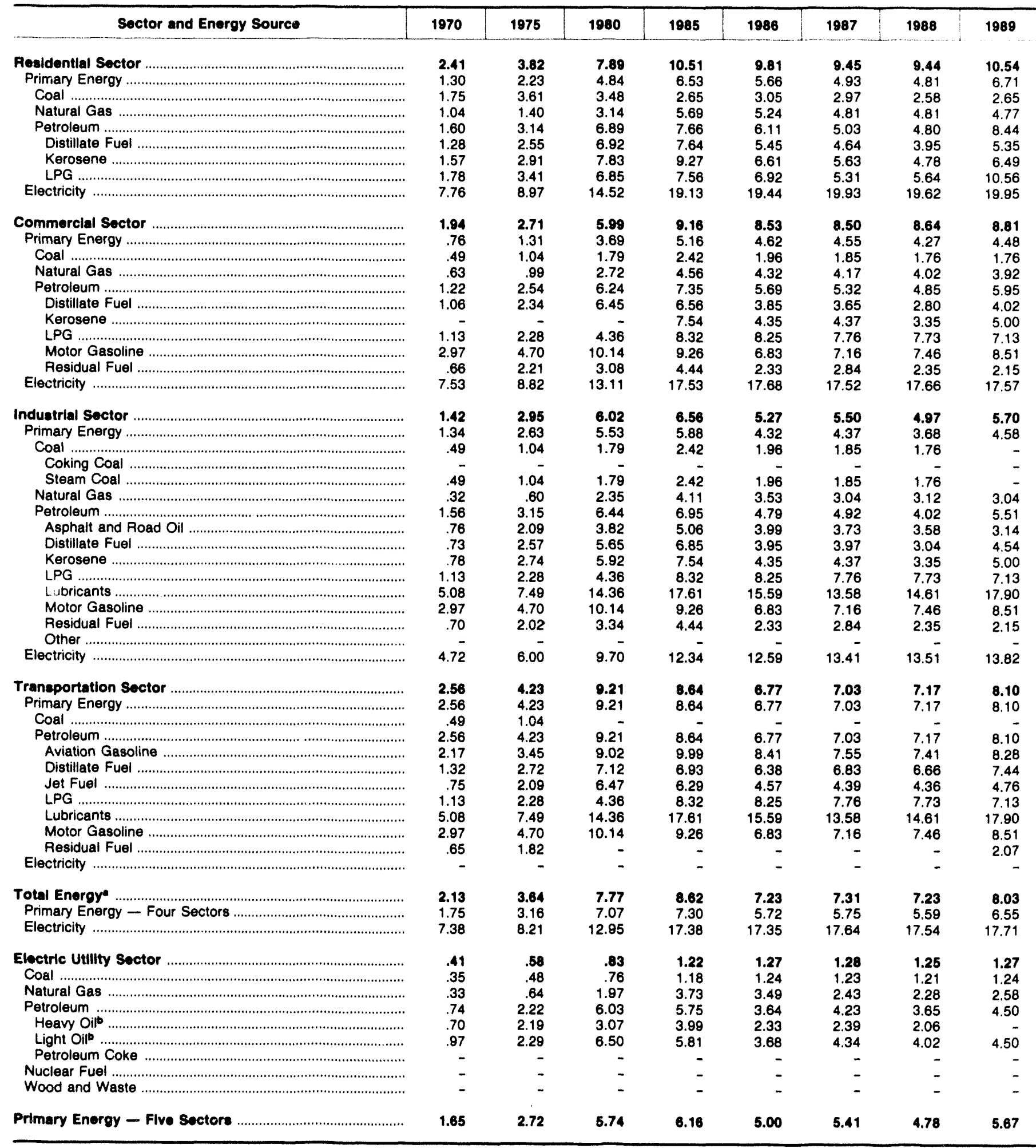

-There are no direct fuel costs for hydroelectric, geothermal, centralized solar, or wind energy. Wood and other biomass fuels are not included, except those consumed at electric utilities.

- Heavy oil includes Grade Nos. 4, 5, and 6, and residual fuel oils. Light oil includes Grade No. 2 heating oil, kerosene, and jet fuel.

- No consumption, including cases where adjustments were made. See "Consumption Adjustments for Process Fuel and Intermediate Products."

Sources: Data sources, estimation procedures, and assumptions are described in the "Documentation" section of this report. 


\begin{tabular}{|c|c|c|c|c|c|c|c|c|}
\hline Sector and Energy Source & 1970 & 1975 & 1980 & 1985 & 1936 & 1987 & 1988 & 1989 \\
\hline 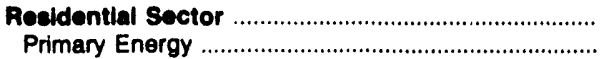 & $\begin{array}{l}76.0 \\
34.0\end{array}$ & $\begin{array}{r}114.4 \\
51.1\end{array}$ & $\begin{array}{r}223.8 \\
93.9\end{array}$ & $\begin{array}{l}313.8 \\
133.2\end{array}$ & $\begin{array}{l}306.3 \\
123.6\end{array}$ & $\begin{array}{l}286.7 \\
104.5\end{array}$ & $\begin{array}{l}300.0 \\
104.9\end{array}$ & $\begin{array}{l}363.3 \\
164.3\end{array}$ \\
\hline 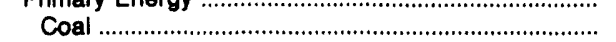 & .4 & .5 & .4 & .3 & .5 & .1 & .1 & \\
\hline 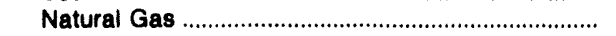 & 14.3 & 16.7 & 33.1 & 65.3 & 55.6 & 45.2 & 52.5 & 55.0 \\
\hline 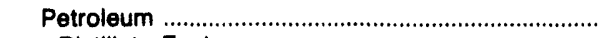 & 19.4 & 33.9 & 60.5 & 67.6 & 67.5 & 59.2 & 52.4 & 109.2 \\
\hline 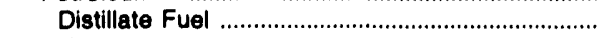 & 5.7 & 8.5 & 30.7 & 33.1 & 33.0 & 23.1 & 21.1 & 28.1 \\
\hline Kerosene & .1 & 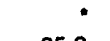 & .4 & 2.8 & .9 & .4 & .3 & .3 \\
\hline LPG & 13.5 & 25.3 & 29.3 & 31.7 & 33.6 & 35.6 & 30.9 & 80.8 \\
\hline 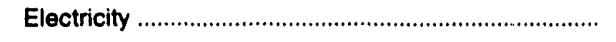 & 42.0 & 63.3 & 129.9 & 180.7 & 182.7 & 182.3 & 195.0 & 199.0 \\
\hline Commercial Sector & 36.7 & 49.4 & 95.3 & 180.2 & 155.8 & 155.0 & 158.9 & 163.8 \\
\hline 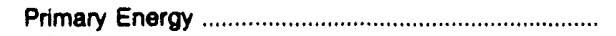 & 11.6 & 19.5 & 44.4 & 68.7 & 59.1 & 57.7 & 52.9 & 55.7 \\
\hline Coal & .2 & .3 & .4 & .5 & .6 & .1 & .1 & .1 \\
\hline 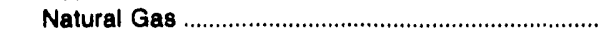 & 7.2 & 11.4 & 23.1 & 46.0 & 39.8 & 34.6 & 34.4 & 35.2 \\
\hline Petroleum & 4.2 & 7.8 & 20.9 & 22.2 & 18.7 & 23.0 & 18.4 & 20.4 \\
\hline 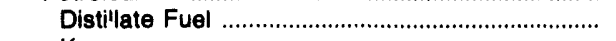 & 1.9 & 3.1 & 13.7 & 10.6 & 6.1 & 8.8 & 5.6 & 5.2 \\
\hline 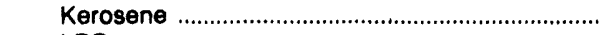 & - & - & - & & • & & $\cdot$ & \\
\hline LPG & 1.5 & 3.0 & 3.3 & 6.2 & 7.1 & 9.2 & 7.5 & 9.6 \\
\hline 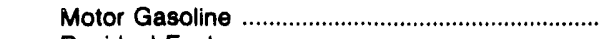 & .8 & 1.4 & 3.5 & 4.8 & 5.4 & 4.9 & 4.9 & 5.3 \\
\hline 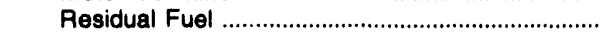 & .1 & .3 & .4 & .6 & .1 & .1 & .4 & .4 \\
\hline 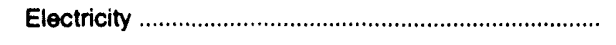 & 24.1 & 29.9 & 51.0 & 111.5 & 96.7 & 97.4 & 106.0 & 108.1 \\
\hline Industrial Sector & 57.5 & 107.6 & 227.6 & 218.6 & 206.0 & 210.2 & 201.0 & 258.4 \\
\hline 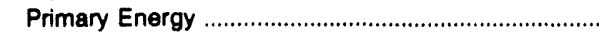 & 53.0 & 87.3 & 183.9 & 175.7 & 149.5 & 146.1 & 129.0 & 182.4 \\
\hline Coal & • & 1.2 & 4.4 & 11.7 & 8.2 & 7.4 & 6.1 & - \\
\hline 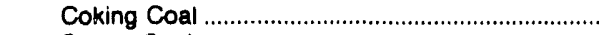 & - & - & - & - & - & - & - & - \\
\hline 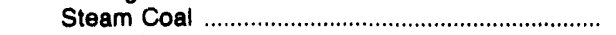 & • & 1.2 & 4.4 & 11.7 & 8.2 & 7.4 & 6.1 & - \\
\hline Natural Gas & 2.2 & 3.5 & 11.0 & 14.6 & 11.9 & 10.1 & 14.8 & 15.3 \\
\hline 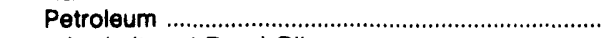 & 50.7 & 82.6 & 168.5 & 148.5 & 129.4 & 128.6 & 108.1 & 167.1 \\
\hline 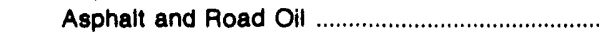 & 4.5 & 11.9 & 16.1 & 28.3 & 21.6 & 16.7 & 20.8 & 16.2 \\
\hline 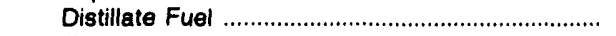 & 9.9 & 24.5 & 54.0 & 66.6 & 58.6 & 55.4 & 47.3 & 54.1 \\
\hline 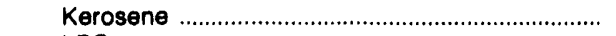 & & & .2 & .3 & .3 & .1 & .1 & .2 \\
\hline LPG & 1.3 & 4.5 & 17.5 & 19.6 & 26.5 & 31.6 & 17.4 & 71.4 \\
\hline Lubricants & .4 & .9 & .3 & .3 & .3 & .3 & .3 & .4 \\
\hline 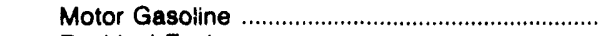 & 34.5 & 40.1 & 78.5 & 33.8 & 21.3 & 23.7 & 21.3 & 24.2 \\
\hline 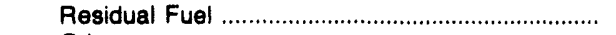 & .2 & .7 & 2.0 & .5 & .9 & .9 & .8 & .7 \\
\hline Other & - & - & - & - & - & - & - & - \\
\hline 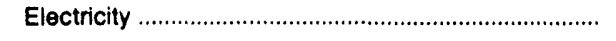 & 4.5 & 20.4 & 43.8 & 42.9 & 56.5 & 64.1 & 72.0 & 76.0 \\
\hline 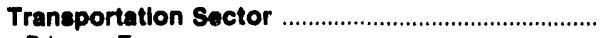 & 137.2 & 262.1 & 581.3 & 559.5 & 407.4 & 432.0 & 458.7 & 522.9 \\
\hline 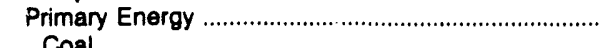 & 137.2 & 262.1 & 581.3 & 559.5 & 407.4 & 432.0 & 458.7 & 522.9 \\
\hline Coal Petroloum & & & & & - & & $\overline{-}$ & $5 \bar{c}$ \\
\hline 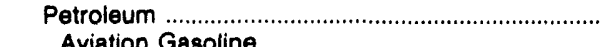 & 137.2 & 262.1 & 581.3 & 559.5 & 407.4 & 432.0 & 458.7 & 522.9 \\
\hline 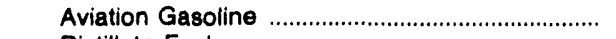 & 1.1 & 1.3 & 4.4 & 4.4 & 3.6 & 3.1 & 3.3 & 3.7 \\
\hline 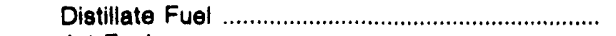 & 7.1 & 21.1 & 82.0 & 91.8 & 80.6 & 88.7 & 87.2 & 97.2 \\
\hline 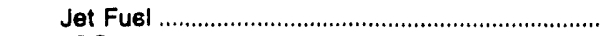 & 4.7 & 11.9 & 46.0 & 34.6 & 12.9 & 16.0 & 20.6 & 26.3 \\
\hline 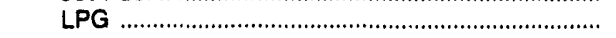 & .2 & .5 & 1.1 & .9 & .9 & 1.4 & .7 & .6 \\
\hline 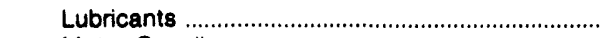 & 4.7 & 6.3 & 13.6 & 15.2 & 13.1 & 12.8 & 13.4 & 16.8 \\
\hline 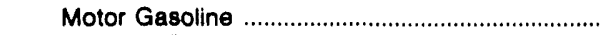 & 119.3 & 220.8 & $4 *: 3$ & 412.7 & 296.3 & 310.0 & 333.6 & 378.2 \\
\hline 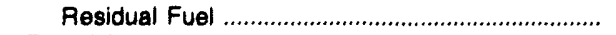 & * & • & - & - & - & - & - & • \\
\hline 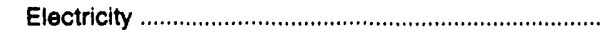 & - & - & - & - & - & - & - & - \\
\hline 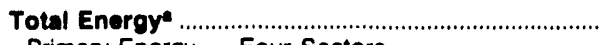 & 306.4 & 533.5 & $1,128.1$ & $1,272.1$ & $1,075.5$ & $1,084.0$ & $1,118.6$ & $1,308.4$ \\
\hline Primary Energy - Four Sectors ..................................... & 235.8 & 419.9 & 903.5 & 937.1 & 739.6 & 740.2 & 745.6 & 925.3 \\
\hline 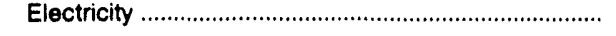 & 70.6 & $\$ 13.6$ & 224.7 & 335.0 & 335.8 & 343.8 & 373.0 & 383.1 \\
\hline 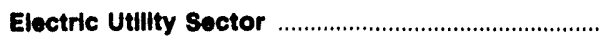 & 4.7 & 16.0 & 28.7 & 36.2 & 31.5 & 13.7 & 38.5 & 36.0 \\
\hline Coal & 1.8 & 11.0 & 25.8 & 34.8 & 30.5 & 12.8 & 36.7 & 34.7 \\
\hline Natural Gas & 1.5 & 2.1 & .5 & .1 & .1 & .2 & .5 & .3 \\
\hline Petroleum & 1.5 & 2.9 & 2.4 & 1.3 & 8 & 6 & 1.3 & .9 \\
\hline 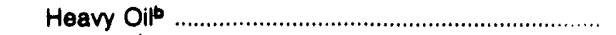 & i.2 & 2.0 & .2 & • & - & * & .1 & - \\
\hline 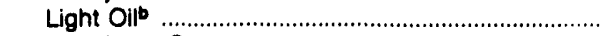 & .3 & .9 & 2.2 & 1.3 & .8 & .5 & 1.1 & .9 \\
\hline 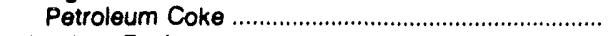 & - & - & - & - & - & - & - & - \\
\hline 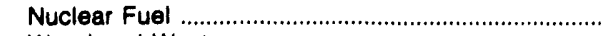 & - & - & - & - & - & - & - & - \\
\hline Wood and Waste & - & - & - & - & - & - & - & - \\
\hline Primary Energy - Five Sectors ………………..... & 240.5 & 435.9 & $\mathbf{8 3 2 . 2}$ & 973.3 & 771.1 & 753.9 & 784.1 & 961.3 \\
\hline
\end{tabular}

-There are no direct fuel costs for hydroelectric, geothermal, centralized solar, or wind energy. Wood and other biomass fuels are not included, except those consumed at electric utilities.

bHeavy oil includes Grade Nos, 4, 5, and 6, and residual fuel oils. Light oil includes Grade No. 2 heating oil, kerosene, and jet fuel.

-No consumption, including cases where adjustments were made. See "Consumption Adjustments for Process Fuel and Intermediate Products."

- Value less than 0.05 million dollars.

Note: Totals may not equal sum of components due to independent rounding.

Sources: Data sources, estimation procedures, and assumptions are described in the "Documentation" section of this report. 


\begin{tabular}{|c|c|c|c|c|c|c|c|c|}
\hline Energy source & 1870 & 1975 & 1980 & 1985 & 1986 & 1987 & 1888 & 1989 \\
\hline & \multicolumn{8}{|c|}{ Pricec In Dollars per Million Btu } \\
\hline 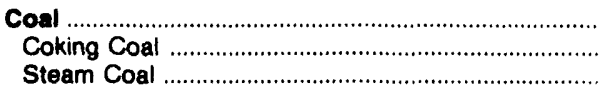 & $\begin{array}{r}0.26 \\
.38 \\
.25\end{array}$ & $\begin{array}{r}0.91 \\
1.60 \\
.91\end{array}$ & $\begin{array}{l}1.54 \\
1.81 \\
1.54\end{array}$ & $\begin{array}{l}1.55 \\
1.93 \\
1.55\end{array}$ & $\begin{array}{l}1.44 \\
1.75 \\
1.44\end{array}$ & $\begin{array}{l}1.38 \\
1.63 \\
1.38\end{array}$ & $\begin{array}{l}1.35 \\
1.70 \\
1.35\end{array}$ & $\begin{array}{l}1.36 \\
1.74 \\
1.35\end{array}$ \\
\hline 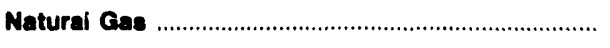 & .54 & .83 & 2.69 & 4.46 & 4.20 & 3.69 & 3.75 & 3.87 \\
\hline $\begin{array}{l}\text { Petroloum } \\
\text { Asphait and Road Oil } \\
\text { Aviation Gasoline . } \\
\text { Distillate Fuel } \\
\text { Jet Fuel } \\
\text { Kerosene } \\
\text { LPG } \\
\text { Lubricants } \\
\text { Motor Gasoline } \\
\text { Residual Fuel } \\
\text { Othere }\end{array}$ & $\begin{array}{r}2.06 \\
.67 \\
2.17 \\
1.11 \\
.73 \\
1.19 \\
1.92 \\
5.08 \\
2.84 \\
.36 \\
.80\end{array}$ & $\begin{array}{l}3.76 \\
1.79 \\
3.45 \\
2.68 \\
2.03 \\
2.93 \\
3.44 \\
7.49 \\
4.58 \\
1.78 \\
2.28\end{array}$ & $\begin{array}{r}8.37 \\
3.56 \\
9.02 \\
6.80 \\
6.39 \\
7.21 \\
6.42 \\
14.36 \\
9.89 \\
3.36 \\
6.31\end{array}$ & $\begin{array}{r}7.89 \\
4.76 \\
9.99 \\
6.44 \\
5.83 \\
6.68 \\
8.98 \\
17.51 \\
8.85 \\
4.80 \\
6.96\end{array}$ & $\begin{array}{r}6.40 \\
4.89 \\
8.41 \\
5.85 \\
3.81 \\
5.15 \\
8.68 \\
15.59 \\
7.01 \\
2.83 \\
4.17\end{array}$ & $\begin{array}{r}6.69 \\
3.24 \\
7.55 \\
6.23 \\
4.01 \\
5.25 \\
8.73 \\
13.58 \\
7.44 \\
2.88 \\
4.83\end{array}$ & $\begin{array}{r}6.60 \\
3.05 \\
7.41 \\
6.00 \\
5.82 \\
5.31 \\
8.93 \\
14.61 \\
7.43 \\
2.64 \\
3.99\end{array}$ & $\begin{array}{r}7.27 \\
2.86 \\
8.28 \\
6.63 \\
4.34 \\
6.02 \\
9.48 \\
17.90 \\
8.25 \\
2.68 \\
4.84\end{array}$ \\
\hline 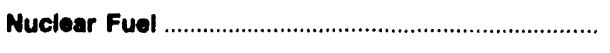 & - & - & .38 & .78 & - & - & .83 & .89 \\
\hline 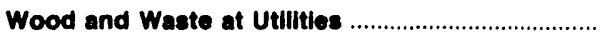 & - & - & - & - & - & - & - & - \\
\hline Primary Energy - Five Sectors ................................. & 1.02 & 2.13 & 4.43 & 4.28 & 3.80 & 3.96 & 3.77 & 3.91 \\
\hline 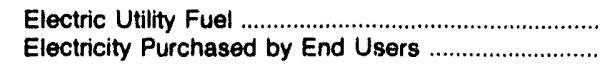 & $\begin{array}{r}.23 \\
2.85\end{array}$ & $\begin{array}{r}.89 \\
5.83\end{array}$ & $\begin{array}{r}1.57 \\
10.67\end{array}$ & $\begin{array}{r}1.41 \\
14.65\end{array}$ & $\begin{array}{r}1.43 \\
15.59\end{array}$ & $\begin{array}{r}1.38 \\
15.98\end{array}$ & $\begin{array}{r}1.30 \\
16.19\end{array}$ & $\begin{array}{r}1.23 \\
15.91\end{array}$ \\
\hline \multirow[t]{2}{*}{ 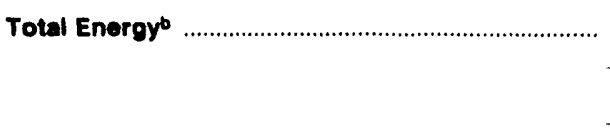 } & 1.71 & 3.54 & 7.30 & 8.15 & 7.51 & 7.67 & 7.73 & 8.03 \\
\hline & \multicolumn{8}{|c|}{ Expendltures in Millions of Dollars } \\
\hline 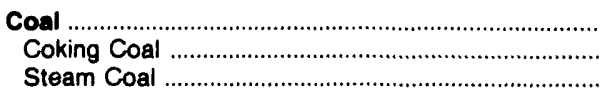 & $\begin{array}{r}103.1 \\
2.5 \\
100.7\end{array}$ & $\begin{array}{r}431.0 \\
8.9 \\
422.1\end{array}$ & $\begin{array}{r}888.2 \\
5.0 \\
883.2\end{array}$ & $\begin{array}{r}929.3 \\
8.0 \\
921.3\end{array}$ & $\begin{array}{r}876.0 \\
8.1 \\
867.9\end{array}$ & $\begin{array}{r}825.7 \\
6.8 \\
819.0\end{array}$ & $\begin{array}{r}822.4 \\
3.6 \\
818.8\end{array}$ & $\begin{array}{r}765.2 \\
3.5 \\
761.7\end{array}$ \\
\hline 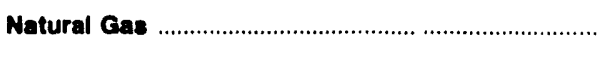 & 123.6 & 186.1 & 570.9 & 813.4 & 742.6 & 689.5 & 747.2 & 797.4 \\
\hline $\begin{array}{l}\text { Potroleum } \\
\text { Asphalt and Road Oil } \\
\text { Aviation Gasoline } \\
\text { Distillate Fuel } \\
\text { Jet Fuel } \\
\text { Kerosene } \\
\text { LPG } \\
\text { Lubricants } \\
\text { Motor Gasoline } \\
\text { Residual Fuel } \\
\text { Othere }\end{array}$ & $\begin{array}{r}831.3 \\
16.2 \\
1.3 \\
70.8 \\
13.6 \\
27.9 \\
23.0 \\
25.4 \\
625.1 \\
1.1 \\
1.1 \\
26.9\end{array}$ & $\begin{array}{r}1,873.0 \\
44.8 \\
1.2 \\
272.8 \\
45.1 \\
38.0 \\
48.8 \\
60.3 \\
1,292.7 \\
4.3 \\
64.9\end{array}$ & $\begin{array}{r}4,370.7 \\
79.8 \\
13.2 \\
759.3 \\
149.8 \\
62.7 \\
65.3 \\
108.1 \\
2,853.4 \\
28.2 \\
250.9\end{array}$ & $\begin{array}{r}4,307.9 \\
139.3 \\
7.8 \\
835.9 \\
160.1 \\
63.8 \\
114.8 \\
120.6 \\
2,698.2 \\
12.9 \\
154.5\end{array}$ & $\begin{array}{r}3,655.4 \\
134.9 \\
8.5 \\
771.9 \\
127.8 \\
14.1 \\
124.6 \\
104.4 \\
2,221.8 \\
9.3 \\
138.1\end{array}$ & $\begin{array}{r}3,911.5 \\
98.3 \\
7.1 \\
818.9 \\
129.0 \\
17.6 \\
110.4 \\
102.8 \\
2,460.3 \\
4.7 \\
162.4\end{array}$ & $\begin{array}{r}3,747.3 \\
81.8 \\
6.8 \\
823.4 \\
91.3 \\
27.0 \\
144.7 \\
106.7 \\
2,316.9 \\
6.1 \\
142.6\end{array}$ & $\begin{array}{r}4,227.4 \\
108.1 \\
7.6 \\
901.5 \\
106.8 \\
29.8 \\
163.7 \\
134.0 \\
2,600.6 \\
7.9 \\
167.4\end{array}$ \\
\hline 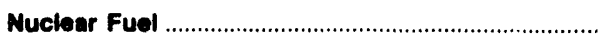 & - & - & 2.1 & 81.1 & - & - & 34.9 & 149.2 \\
\hline Wood and Waste at Utilities & - & - & - & - & - & - & - & - \\
\hline 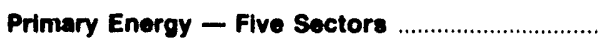 & $1,058.0$ & $2,490.0$ & $5,831.9$ & $6,131.6$ & $5,274.0$ & $5,426.7$ & $5,351.8$ & $5,939.3$ \\
\hline 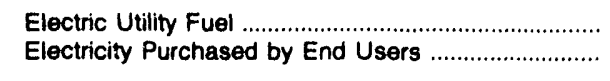 & $\begin{array}{r}-60.9 \\
504.6\end{array}$ & $\begin{array}{r}-376.4 \\
1,357.0\end{array}$ & $\begin{array}{r}-804.8 \\
2,656.5\end{array}$ & $\begin{array}{r}-846.9 \\
3,409.7\end{array}$ & $\begin{array}{r}-716.0 \\
3,559.5\end{array}$ & $\begin{array}{r}-682.9 \\
3,754.3\end{array}$ & $\begin{array}{r}-718.6 \\
3,975.4\end{array}$ & $\begin{array}{r}-773.6 \\
4,030.7\end{array}$ \\
\hline Total Energy & $1,481.7$ & $3,470.6$ & $7,683.7$ & $8,694.4$ & $8,117.5$ & $8,498.1$ & $8,608.6$ & $9,186.5$ \\
\hline
\end{tabular}

- Includes petroleum coke used at electric utilities.

-There are no direct fuel costs for hydroelectric, geothermal, centralized solar, or wind energy. Wood and other biomass fuels are not included, except those consumed at electric utilities.

-No consumption, including cases where adjustments were made. See "Consumption Adjustments for Process Fuel and Intermediate Products."

Note: Expenditure totals may not equal sum of components due to independent rounding.

Sources: Data sources, estimation procedures, and assumptions are described in the "Documentation" section of this report. 


\begin{tabular}{|c|c|c|c|c|c|c|c|c|}
\hline Sector and Energy Source & 1970 & 1975 & 1980 & 1985 & 1986 & 1987 & 1988 & 1989 \\
\hline Reoldential Sector & 2.15 & 4.46 & 7.79 & 10.87 & 11.57 & 11.93 & 12.06 & 12.06 \\
\hline Primary Energy & 1.15 & 1.93 & 3.73 & 5.70 & 5.45 & 5.14 & 5.23 & 5.58 \\
\hline Coal & .74 & 1.75 & 1.97 & 1.85 & 1.67 & 1.79 & 1.52 & 1.61 \\
\hline 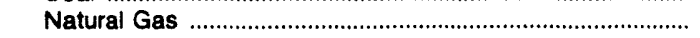 & .89 & 1.25 & 2.85 & 4.96 & 4.81 & 4.53 & 4.51 & 4.68 \\
\hline Petroleum & 1.82 & 3.56 & 7.95 & 8.07 & 8.09 & 7.90 & 8.07 & 8.93 \\
\hline 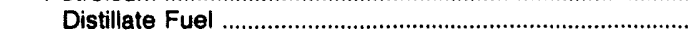 & 1.24 & 2.49 & 6.89 & 6.59 & 5.30 & 5.25 & 5.53 & 5.90 \\
\hline Kerosene & 1.62 & 3.38 & 9.09 & 7.09 & 5.71 & 5.65 & 5.95 & 6.35 \\
\hline LPG & 2.16 & 3.84 & 7.65 & 9.23 & 8.96 & 9.09 & 9.06 & 10.25 \\
\hline Electricity & 3.34 & 6.62 & 10.43 & 14.28 & 15.26 & 16.03 & 16.74 & 16.65 \\
\hline Commerclal Sector & 1.87 & 3.48 & 7.97 & 8.92 & 9.38 & 9.31 & 9.29 & 9.91 \\
\hline 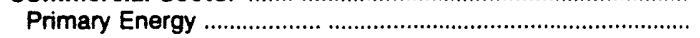 & .75 & 1.38 & 3.57 & 5.06 & 4.51 & 4.30 & 4.33 & 4.48 \\
\hline Coal & .35 & 1.17 & 1.39 & 1.60 & 1.54 & 1.43 & 1.40 & 1.40 \\
\hline Natural Gas & .70 & 1.09 & 2.95 & 4.75 & 4.53 & 4.20 & 4.25 & 4.35 \\
\hline Petroleum & $\therefore 46$ & 2.88 & 7.05 & 6.05 & 4.73 & 5.16 & 5.33 & 6.19 \\
\hline 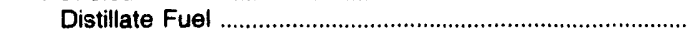 & 1.06 & 2.29 & 6.49 & 5.66 & 3.80 & 4.12 & 3.93 & 4.43 \\
\hline Kerosene & .78 & 2.32 & 6.16 & 5.87 & 3.93 & 4.46 & 4.23 & 4.97 \\
\hline LPG & 1.27 & 2.39 & 4.96 & 8.66 & 8.30 & 8.25 & 8.71 & 8.23 \\
\hline Motor Gasoline & 2.84 & 4.58 & 9.89 & 8.85 & 7.01 & 7.44 & 7.43 & 8.25 \\
\hline Residual Fuel & .42 & 1.77 & 3.44 & 4.80 & 2.83 & 2.88 & 2.62 & 2.69 \\
\hline Electricity & 4.97 & 8.27 & 13.29 & 17.05 & 17.96 & 17.67 & 18.03 & 18.03 \\
\hline Industrial Sector & .93 & 2.56 & 5.11 & 6.82 & 6.52 & 6.35 & 6.36 & 6.24 \\
\hline Primary Energy & .54 & 1.39 & 3.37 & 3.91 & 3.35 & 3.06 & 2.97 & 3.14 \\
\hline 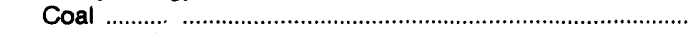 & .35 & 1.22 & 1.41 & 1.61 & 1.55 & 1.44 & 1.40 & 1.41 \\
\hline 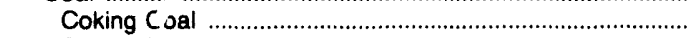 & .38 & 1.60 & 1.81 & 1.93 & 1.75 & 1.63 & 1.70 & 1.74 \\
\hline Steam Coal & .35 & 1.17 & 1.39 & 1.60 & 1.54 & 1.43 & 1.40 & 1.40 \\
\hline Natural Gas & .38 & .73 & 2.54 & 4.11 & 3.77 & 3.05 & 3.16 & 3.27 \\
\hline Petroleum & .86 & 2.29 & 5.54 & 6.42 & 4.92 & 4.76 & 4.42 & 4.75 \\
\hline 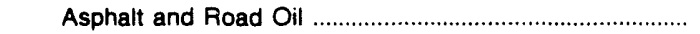 & .67 & 1.79 & 3.56 & 4.76 & 4.89 & 3.24 & 3.05 & 2.86 \\
\hline 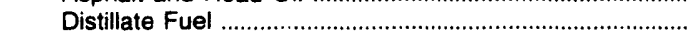 & .72 & 2.11 & 5.50 & 5.91 & 3.96 & 4.49 & 4.26 & 5.01 \\
\hline Kerosene & .78 & 2.32 & 6.16 & 5.87 & 3.93 & 4.46 & 4.23 & 4.97 \\
\hline LPG & 1.27 & 2.39 & 4.96 & 8.66 & 8.30 & 8.25 & 8.71 & 8.23 \\
\hline Lubricants & 5.08 & 7.49 & 14.36 & 17.61 & 15.59 & 13.58 & 14.61 & 17.90 \\
\hline 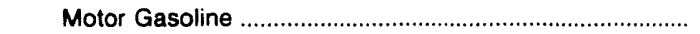 & 2.84 & 4.58 & 9.89 & 8.85 & 7.01 & 7.44 & 7.43 & 8.25 \\
\hline 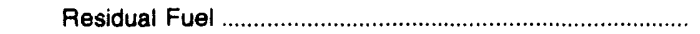 & .36 & 1.89 & 3.36 & 4.80 & 2.83 & 2.88 & 2.62 & 2.69 \\
\hline Other & .80 & 2.28 & 6.31 & 6.96 & 4.17 & 4.83 & 3.99 & 4.84 \\
\hline 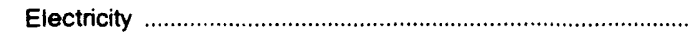 & 2.05 & 4.87 & 9.71 & 14.22 & 15.12 & 15.39 & 15.15 & 14.51 \\
\hline Transportation Sector & 2.49 & 4.19 & 9.20 & 8.29 & 6.73 & 7.10 & 7.07 & 7.80 \\
\hline Primary Energy & 2.49 & 4.19 & 9.20 & 8.29 & 6.73 & 7.10 & 7.07 & 7.80 \\
\hline Coal & .35 & 1.17 & - & - & - & - & - & - \\
\hline 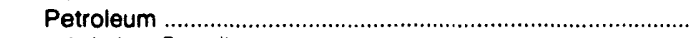 & 2.49 & 4.19 & 9.20 & 8.29 & 6.73 & 7.10 & 7.07 & 7.80 \\
\hline Aviation Gasoline & 2.17 & 3.45 & 9.02 & 9.99 & 8.41 & 7.55 & 7.41 & 8.28 \\
\hline 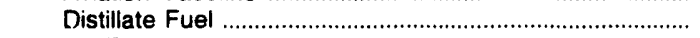 & 1.28 & 3.02 & 7.25 & 6.73 & 6.47 & 6.75 & 6.49 & 6.96 \\
\hline 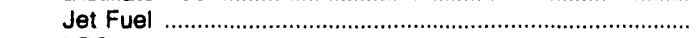 & .73 & 2.03 & 6.39 & 5.83 & 3.81 & 4.01 & 3.82 & 4.34 \\
\hline LPG & 1.27 & 2.39 & 4.96 & 8.66 & 8.30 & 8.25 & 8.71 & 8.23 \\
\hline Lubricants & 5.08 & 7.49 & 14.36 & 17.61 & 15.59 & 13.58 & 14.61 & 17.90 \\
\hline Motor Gasoline & 2.84 & 4.58 & 9.89 & 8.85 & 7.01 & 7.44 & 7.43 & 8.25 \\
\hline 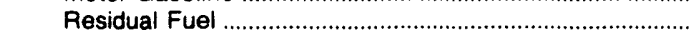 & .42 & 1.67 & 3.45 & - & - & 1.92 & 3.01 & 2.37 \\
\hline 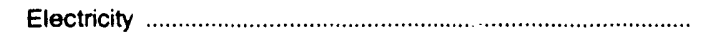 & 4.97 & 8.27 & 13.29 & 17.05 & 17.96 & 19.96 & 20.34 & 19.95 \\
\hline 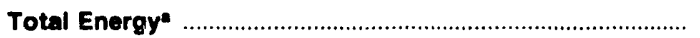 & 1.71 & 3.54 & 7.30 & 8.15 & 7.51 & 7.67 & 7.73 & 8.03 \\
\hline 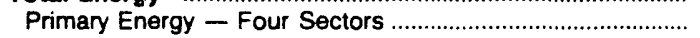 & 1.42 & 2.82 & 6.25 & 6.34 & 5.35 & 5.43 & 5.33 & 5.80 \\
\hline Electricity & 2.85 & 5.83 & 10.67 & 14.65 & 15.59 & 15.98 & 16.19 & 15.91 \\
\hline Electric Utillty Sector & .23 & .89 & 1.57 & 1.41 & 1.43 & 1.38 & 1.30 & 1.23 \\
\hline Coal & .23 & .87 & 1.56 & 1.54 & 1.42 & 1.37 & 1.34 & 1.34 \\
\hline 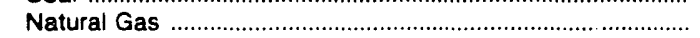 & .25 & - & 2.33 & - & - & - & 2.39 & 2.74 \\
\hline Petroleum & - & 2.19 & 6.39 & 5.85 & 3.37 & 3.93 & 3.51 & 3.95 \\
\hline 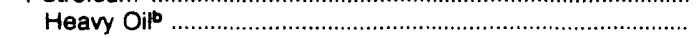 & - & - & - & - & - & - & - & - \\
\hline Light Oib & - & 2.9 & 6.30 & 5.85 & 3.37 & 3.93 & 3.51 & 3.95 \\
\hline 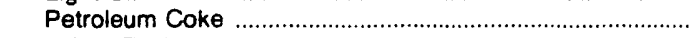 & - & - & - & - & - & - & - & - \\
\hline 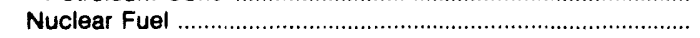 & - & - & .38 & .78 & - & - & 83 & .89 \\
\hline Wood and Waste & - & - & - & - & - & - & - & - \\
\hline 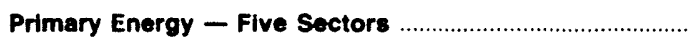 & 1.02 & 2.13 & 4.43 & 4.28 & 3.90 & 3.96 & 3.77 & 3.91 \\
\hline
\end{tabular}

-There are no direct fuel costs for hydroelectric, geothermal, centralized solar, or wind energy. vivod and other biomass fuels are not included, except those consumed at electric utilities.

bHeavy oil includes Grade Nos. 4, 5, and 6, and residual fuel oils. Light oil includes Grade No. 2 heating oil, kerosene, and jet fuel.

-No consumption, including cases where adjustments were made. See "Consumption Adjustments for Process Fuel and Intermediate Products."

Sources: Data sources, estimation procedures, and assumptions are described in the "Documentation" section of this report. 


\section{T Energy Expenditure Estimates by Sector, Tennessee

\begin{tabular}{|c|c|c|c|c|c|c|c|c|}
\hline Sector and Energy Source & 1970 & 1975 & 1980 & 1985 & 1986 & 1987 & 1988 & 1989 \\
\hline & 288.7 & $\begin{array}{l}650.3 \\
129.7\end{array}$ & $\begin{array}{r}1,149.1 \\
216.5\end{array}$ & $\begin{array}{r}1,570.9 \\
326.3\end{array}$ & $\begin{array}{r}1,638.2 \\
290.6\end{array}$ & $\begin{array}{r}1,792.0 \\
290.5\end{array}$ & $\begin{array}{r}1,939.4 \\
3425\end{array}$ & $\begin{array}{r}1,993.1 \\
382.1\end{array}$ \\
\hline Primary Energy . Coal & $\begin{array}{r}84.5 \\
3.3\end{array}$ & $\begin{array}{r}129.7 \\
4.7\end{array}$ & $\begin{array}{r}216.5 \\
3.9\end{array}$ & $\begin{array}{r}326.3 \\
2.7\end{array}$ & $\begin{array}{r}290.6 \\
1.1\end{array}$ & $\begin{array}{r}290.5 \\
1.5\end{array}$ & $\begin{array}{r}342.5 \\
2.5\end{array}$ & $\begin{array}{r}382.1 \\
3.1\end{array}$ \\
\hline 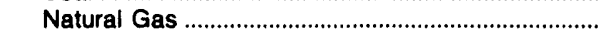 & 42.5 & 56.8 & 129.8 & 202.0 & 199.9 & 203.5 & 221.7 & 237.3 \\
\hline Petroleum & 38.7 & 68.1 & 82.8 & 121.6 & 89.6 & 85.5 & 118.3 & 141.7 \\
\hline 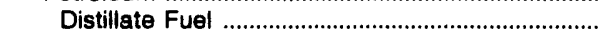 & 1.2 & 3.4 & 12.4 & 9.9 & 5.1 & 7.0 & 7.3 & 8.4 \\
\hline 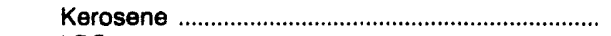 & 18.6 & 25.3 & 28.3 & 45.1 & 10.8 & 12.7 & 19.0 & 24.0 \\
\hline LPG & 18.9 & 39.5 & 42.2 & 66.5 & 73.7 & 65.9 & 92.1 & 109.3 \\
\hline 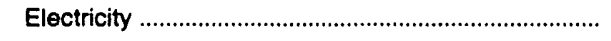 & 204.2 & 520.6 & 932.6 & $1,244.6$ & $1,347.6$ & $1,501.5$ & $1,597.0$ & $1,611.0$ \\
\hline Commercial Sector & 153.4 & 289.4 & 853.7 & 931.1 & 859.7 & 865.4 & 917.6 & $1,032.3$ \\
\hline 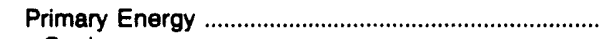 & 45.6 & 79.4 & 209.2 & 357.8 & 263.8 & 250.5 & 272.7 & 279.3 \\
\hline Coal & 2.9 & 5.8 & 5.1 & 4.3 & 1.8 & 2.2 & 4.2 & 4.8 \\
\hline 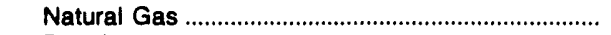 & 30.4 & 47.9 & 132.1 & 213.2 & 199.0 & 191.1 & 200.8 & 213.6 \\
\hline 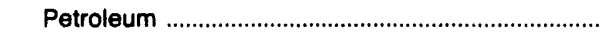 & 12.2 & 25.7 & 72.0 & 140.3 & 62.8 & 57.1 & 67.6 & 60.8 \\
\hline 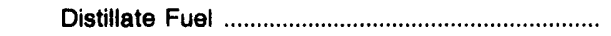 & 2.6 & 7.9 & 38.4 & 101.7 & 31.2 & 27.9 & 25.2 & 17.1 \\
\hline 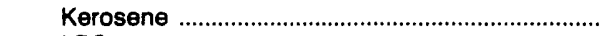 & 1.8 & 3.4 & 3.6 & 8.5 & 2.0 & 2.8 & 5.2 & 4.7 \\
\hline 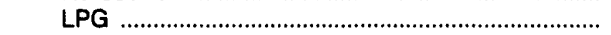 & 2.0 & 4.3 & 4.8 & 11.0 & 12.1 & 10.6 & 15.6 & 15.5 \\
\hline 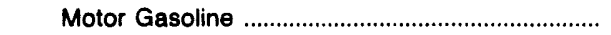 & 5.9 & 10.1 & 24.2 & 15.6 & 14.8 & 14.6 & 20.2 & 22.3 \\
\hline 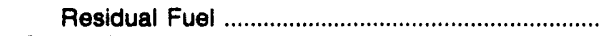 & & & 1.0 & 3.5 & 2.8 & 1.3 & 1.3 & 1.1 \\
\hline 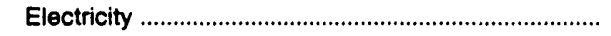 & 107.8 & 210.0 & 644.5 & 573.3 & 595.9 & 614.9 & 644.9 & 753.0 \\
\hline Industrlal Sector ... & 339.7 & 977.9 & $2,073.2$ & $2,702.7$ & $2,587.0$ & $2,534.6$ & $2,615.2$ & $2,626.5$ \\
\hline 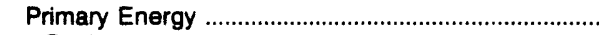 & 147.1 & 351.6 & 993.8 & $1,110.9$ & 971.0 & 896.7 & 881.7 & 959.8 \\
\hline Coal ........ & 20.3 & 60.8 & 94.4 & 164.6 & 161.6 & 144.2 & 140.0 & 141.2 \\
\hline 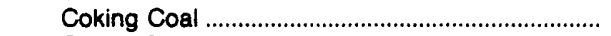 & 2.5 & 8.9 & 5.0 & 8.0 & 8.1 & 6.8 & 3.6 & 3.5 \\
\hline 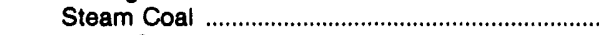 & 17.8 & 52.0 & 89.4 & 156.6 & 153.5 & 137.5 & 136.4 & 137.6 \\
\hline Natural Gas & 46.3 & 81.4 & 306.4 & 398.1 & 343.7 & 294.8 & 324.1 & 346.4 \\
\hline 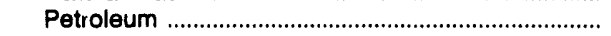 & 80.5 & 209.4 & 593.1 & 548.2 & 465.7 & 457.7 & 417.5 & 472.2 \\
\hline 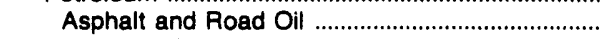 & 16.2 & 44.8 & 79.8 & 139.3 & 134.9 & 98.3 & 81.8 & 108.1 \\
\hline Distillate Fuel .......................................... & 13.3 & 57.6 & 136.3 & 119.5 & 84.5 & 90.8 & 83.9 & 68.7 \\
\hline Kerosene …………………………………... & 7.5 & 9.4 & 30.8 & 10.3 & 1.3 & 2.1 & 2.8 & 1.1 \\
\hline LPG & 1.7 & 3.9 & 17.1 & 30.5 & 31.5 & 30.1 & 31.4 & 33.1 \\
\hline 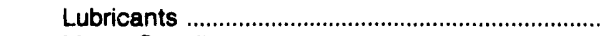 & 10.3 & 23.7 & 49.2 & 54.9 & 47.5 & 46.8 & 48.6 & 61.0 \\
\hline 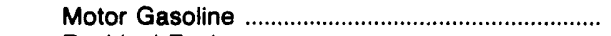 & 3.5 & 2.8 & 1.3 & 29.9 & 21.3 & 23.8 & 21.9 & 26.2 \\
\hline 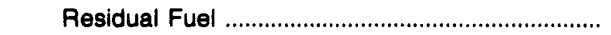 & 1.1 & 2.3 & 27.0 & 9.4 & 6.5 & 3.4 & 4.5 & 6.5 \\
\hline Other & 26.9 & 64.9 & 250.9 & 154.5 & 138.1 & 162.4 & 142.6 & 167.4 \\
\hline 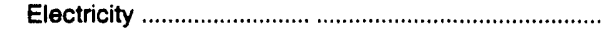 & 192.6 & 626.3 & $1,079.4$ & $1,591.8$ & $1,616.0$ & $1,637.9$ & $1,733.5$ & $1,666.7$ \\
\hline 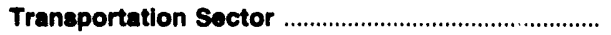 & 698.9 & $1,553.0$ & $3,607.6$ & $3,489.7$ & $3,032.7$ & $3,306.2$ & $3,136.4$ & $3,544,5$ \\
\hline 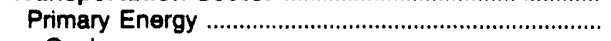 & 699.9 & $1,553.0$ & $3,607.6$ & $3,489.7$ & $3,032.7$ & $3,306.1$ & $3,136.4$ & $3,544.5$ \\
\hline Coal & & & & & & & & \\
\hline 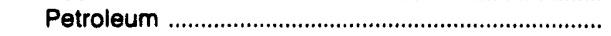 & 699.8 & $1,553.0$ & $3,60 \% .6$ & $3,489.7$ & $3,032.7$ & $3,306.1$ & $3,136.4$ & $3,544.5$ \\
\hline 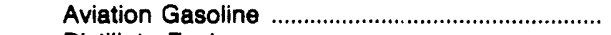 & 1.3 & 1.2 & 13.2 & 7.8 & 8.5 & 7.1 & 6.8 & 7.6 \\
\hline 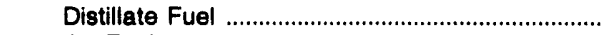 & 53.6 & 187.1 & 557.1 & 596.7 & 646.5 & 688.2 & 699.5 & 799.1 \\
\hline 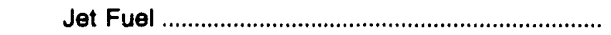 & 13.6 & 45.1 & 149.8 & 160.1 & 127.8 & 129.0 & 91.3 & 106.8 \\
\hline 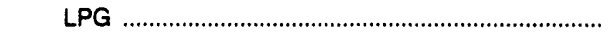 & .5 & 1.1 & 1.1 & 6.7 & 7.3 & 3.8 & 5.6 & 5.7 \\
\hline 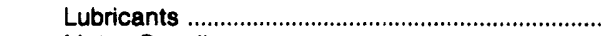 & 15.1 & 36.6 & 58.9 & 65.7 & 56.9 & 56.0 & 58.1 & 73.0 \\
\hline 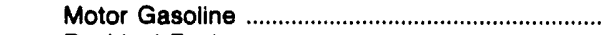 & 615.7 & $1,279.8$ & $2,827.3$ & $2,652.7$ & $2,185.7$ & $2,422.0$ & $2,274.8$ & $2,552.1$ \\
\hline 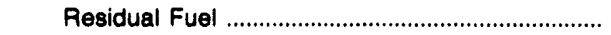 & * & 2.0 & .1 & - & - & . & .3 & .2 \\
\hline 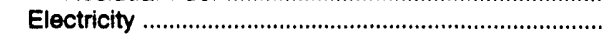 & * & & & - & - & - & & \\
\hline 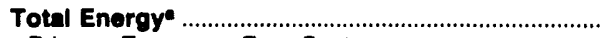 & $1,481.7$ & $3,470.6$ & $7,683.7$ & $8,694.4$ & $8,117.5$ & $8,498.1$ & $8,608.6$ & $9,196.5$ \\
\hline 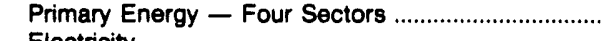 & 977.1 & $2,113.6$ & $5,027.2$ & $5,284.7$ & $4,558.1$ & $4,743.9$ & $4,633.2$ & $5,165.7$ \\
\hline 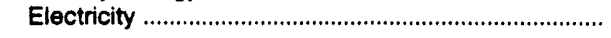 & 504.6 & $1,357.0$ & $2,656.5$ & $3,409.7$ & $3,559.5$ & $3,754.3$ & $3,975.4$ & $4,030.7$ \\
\hline 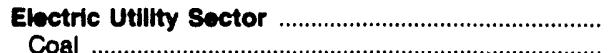 & 80.9 & 376.4 & 804.8 & $\begin{array}{l}846.8 \\
7577\end{array}$ & 716.0 & 682.9 & $\begin{array}{l}718.6 \\
6757\end{array}$ & 773.6 \\
\hline Coal & $\begin{array}{r}76.5 \\
4.4\end{array}$ & 359.6 & $\begin{array}{r}784.9 \\
2.6\end{array}$ & 757.7 & 711.4 & 677.8 & $\begin{array}{r}675.7 \\
.6\end{array}$ & $\begin{array}{r}616.1 \\
.1\end{array}$ \\
\hline Petroleum & - & 16.7 & 15.1 & 8.1 & 4.5 & 5.1 & 7.5 & 8.1 \\
\hline 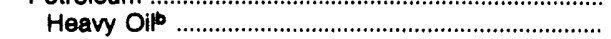 & - & - & - & - & - & - & - & - \\
\hline 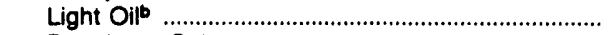 & - & 16.7 & 15.1 & 8.1 & 4.5 & 5.1 & 7.5 & 8.2 \\
\hline 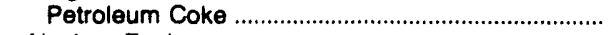 & - & - & - & - & - & - & - & \\
\hline Nuclear Fuel & - & - & 2.1 & 81.1 & - & - & 34.9 & 149.2 \\
\hline Wood and Waste & - & - & - & - & - & - & - & - \\
\hline 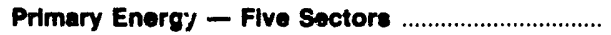 & $1,058.0$ & $2,490.0$ & $5,831.8$ & $6,131.6$ & $5,274.0$ & $5,426.7$ & $5,351.8$ & $5,939.3$ \\
\hline
\end{tabular}

-There are no direct fuel costs for hydroelectric, geothermal, centralized solar, or wind energy. Wood and other biomass fuels are not included, except those consumed at electric utilities.

bHeavy oil includes Grade Nos. 4, 5, and 6, and residual fuel oils. Light oil includes Grade No. 2 heating oil, kerosene, and jet fuel

-No consumption, including cases where adjustments were made. See "Consumption Adjustments for Process Fuel and Intermediate Products." - Value less than 0.05 million dollars.

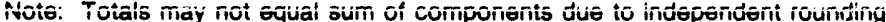

Sources: Data sources, estimation procedures, and assumptions are described in the "Documentation" section of this report. 
Energy Price and Expenditure Estimates by Source, Texas $1970,1975,1980$, and $1985-1989$

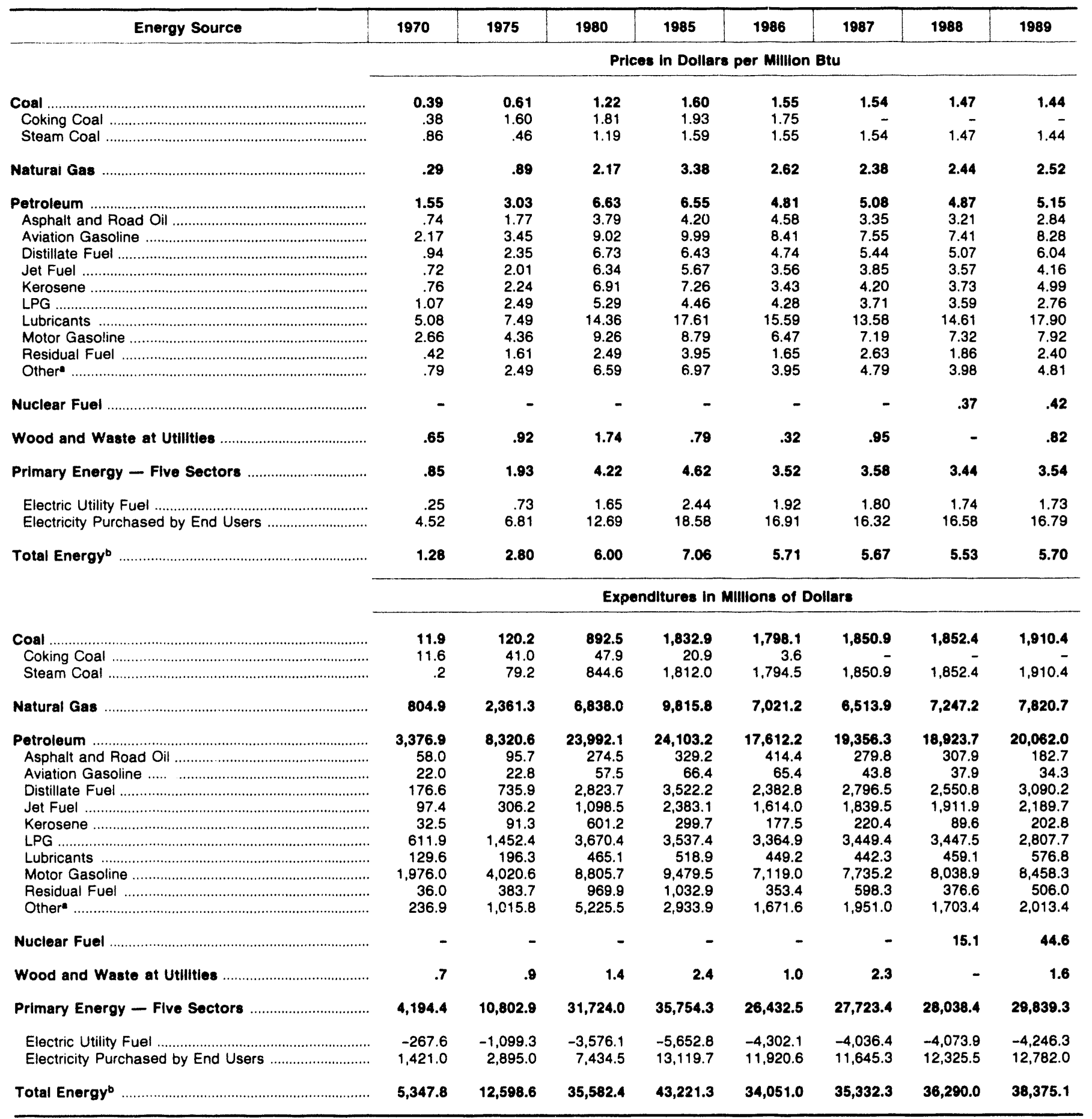

- Includes petroleum coke used at electric utilities.

bThere are no direct fuel costs for hydroelectric, geothermal, centralized solar, or wind energy. Wood and other biomass fuels are not included, except those consumed at electric utilities.

- No consumption, including cases where adjustments were made. See "Consumption Adjustments for Process Fuel and Intermediate Products."

Note: Expenditure totals may not equal sum of components due to independent rounding.

Sources: Data sources, estimation procedures, and assumptions are described in the "Documentation" section of this report. 


\begin{tabular}{|c|c|c|c|c|c|c|c|c|}
\hline Sector and Energy Source & 1970 & 1975 & 1980 & 1985 & 1986 & 1987 & 1988 & 1989 \\
\hline Residential Sector & 2.48 & 4.08 & 8.55 & 13.78 & 13.03 & 12.53 & 12.99 & 13.14 \\
\hline 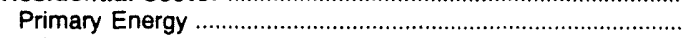 & 1.06 & 1.79 & 3.69 & 5.96 & 5.42 & 5.29 & 5.44 & 5.89 \\
\hline 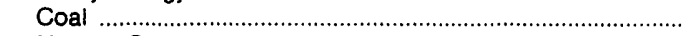 & .90 & - & 2.54 & 2.83 & 2.78 & 2.40 & 2.12 & 2.43 \\
\hline Natural Gas …………………………………………... & .90 & 1.48 & 3.31 & 5.55 & 5.09 & 5.00 & 5.16 & 5.34 \\
\hline Petroleum & 1.70 & 3.45 & 7.43 & 8.50 & 7.75 & 7.24 & 7.37 & 9.72 \\
\hline Distillate Fuel & .98 & 2.24 & 6.51 & 6.99 & 4.57 & 4.69 & 4.41 & 5.42 \\
\hline 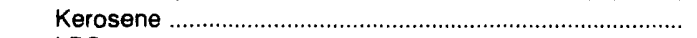 & 1.29 & 3.01 & 8.35 & 8.40 & 5.50 & 5.63 & 5.30 & 6.51 \\
\hline 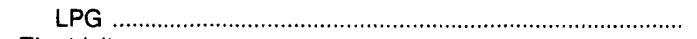 & 1.71 & 3.50 & 7.39 & 8.53 & 8.08 & 7.60 & 7.88 & 10.02 \\
\hline 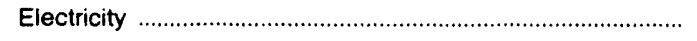 & 6.31 & 8.74 & 14.92 & 21.99 & 20.18 & 19.74 & 20.16 & 20.45 \\
\hline Commercial Sector & 1.91 & 3.92 & 7.77 & 11.98 & 10.80 & 9.92 & 10.20 & 10.29 \\
\hline 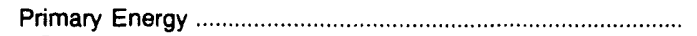 & .56 & 1.41 & 3.89 & 5.30 & 3.86 & 3.96 & 3.82 & 4.04 \\
\hline 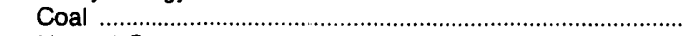 & .86 & - & .89 & 1.60 & 1.50 & 1.27 & 1.31 & 1.27 \\
\hline 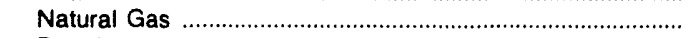 & .44 & 1.02 & 2.90 & 4.70 & 4.00 & 4.09 & 4.03 & 3.95 \\
\hline Petroleum & 1.01 & 2.37 & 6.24 & 6.34 & 3.55 & 3.78 & 3.38 & 4.22 \\
\hline Distillate Fuel & .90 & 2.14 & 6.23 & 6.15 & 3.13 & 3.70 & 3.07 & 4.04 \\
\hline 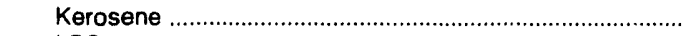 & .76 & 2.23 & 6.89 & 7.07 & 3.28 & 4.04 & 3.21 & 4.87 \\
\hline 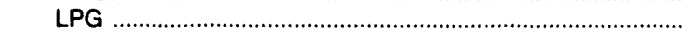 & 1.00 & 2.42 & 5.22 & 4.30 & 4.15 & 3.59 & 3.47 & 2.53 \\
\hline 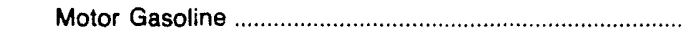 & 2.66 & 4.36 & 9.26 & 8.79 & 6.47 & 7.19 & 7.32 & 7.92 \\
\hline 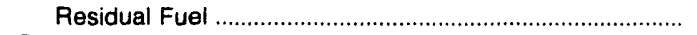 & .48 & 1.87 & 2.53 & 3.87 & 2.00 & 2.67 & 1.90 & 2.33 \\
\hline 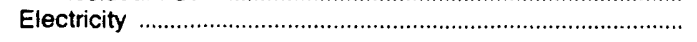 & 5.19 & 7.59 & 14.12 & 20.06 & 18.28 & 17.45 & 17.76 & 17.92 \\
\hline Industrial Sector & .63 & 1.95 & 4.74 & 5.09 & 4.03 & 3.76 & 3.57 & 3.50 \\
\hline 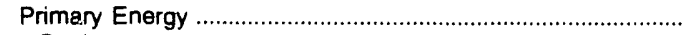 & .53 & 1.73 & 4.34 & 4.31 & 3.28 & 3.10 & 2.91 & 2.85 \\
\hline Coal & .38 & 1.20 & 1.28 & 1.64 & 1.51 & 1.27 & 1.31 & 1.27 \\
\hline 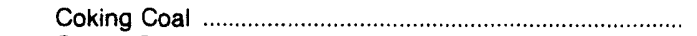 & 38 & 1.60 & 1.81 & 1.93 & 1.75 & - & - & - \\
\hline 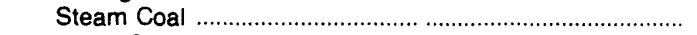 & .86 & 1.01 & .89 & 1.60 & 1.50 & 1.27 & 1.31 & 1.27 \\
\hline 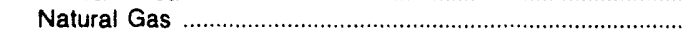 & .20 & .92 & 2.24 & 3.07 & 2.36 & 1.98 & 2.11 & 2.17 \\
\hline Petroleum & .96 & 2.41 & 5.92 & 5.44 & 4.05 & 4.01 & 3.66 & 3.54 \\
\hline 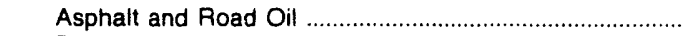 & .74 & 1.77 & 3.79 & 4.20 & 4.58 & 3.35 & 3.21 & 2.84 \\
\hline Distillate Fuel & .66 & 2.02 & 6.09 & 6.39 & 2.97 & 3.65 & 2.90 & 4.40 \\
\hline 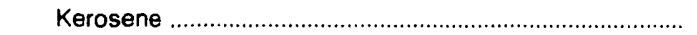 & .76 & 2.23 & 6.89 & 7.07 & 3.28 & 4.04 & 3.21 & 4.87 \\
\hline LPG & 1.00 & 2.42 & 5.22 & 4.30 & 4.15 & 3.59 & 3.47 & 2.53 \\
\hline 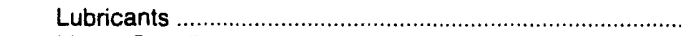 & 5.08 & 7.49 & 14.36 & 17.61 & 15.59 & 13.58 & 14.61 & 17.90 \\
\hline 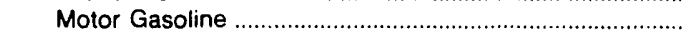 & 2.66 & 4.36 & 9.26 & 8.79 & 6.47 & 7.19 & 7.32 & 7.92 \\
\hline 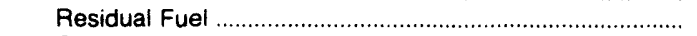 & .37 & 1.51 & 3.69 & 3.87 & 2.00 & 2.67 & 1.90 & 2.33 \\
\hline Other & .79 & 2.49 & 6.59 & 6.97 & 3.95 & 4.79 & 3.98 & 4.81 \\
\hline Electricity & 2.51 & 4.70 & 9.99 & 14.15 & 12.51 & 11.82 & 11.87 & 11.96 \\
\hline Transportation Sector & 2.07 & 3.54 & 7.42 & 7.46 & $\mathbf{5 . 4 3}$ & 6.06 & 5.92 & 6.49 \\
\hline 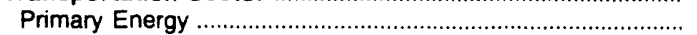 & 2.07 & 3.54 & 7.42 & 7.46 & 5.43 & 6.06 & 5.92 & 6.49 \\
\hline Coal & .86 & 1.01 & - & - & - & - & - & - \\
\hline 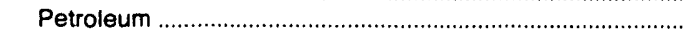 & 2.07 & 3.54 & 7.42 & 7.46 & 5.43 & 6.06 & 5.92 & 6.49 \\
\hline 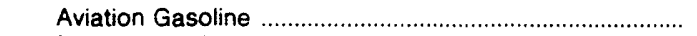 & 2.17 & 3.45 & 9.02 & 9.99 & 8.41 & 7.55 & 7.41 & 8.28 \\
\hline 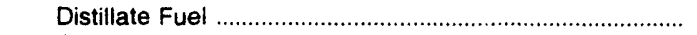 & 1.05 & 2.49 & 7.09 & 6.50 & 5.84 & 6.60 & 6.44 & 6.97 \\
\hline 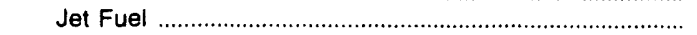 & .72 & 2.01 & 6.34 & 5.67 & 3.56 & 3.85 & 3.57 & 4.16 \\
\hline LPG & 1.00 & 2.42 & 5.22 & 4.30 & 4.15 & 3.59 & 3.47 & 2.53 \\
\hline Lubricants & 5.08 & 7.49 & 14.36 & 17.61 & 15.59 & 13.58 & 14.61 & 17.90 \\
\hline 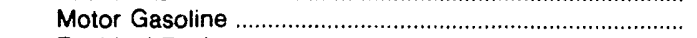 & 2.66 & 4.36 & 9.26 & 8.79 & 6.47 & 7.19 & 7.32 & 7.92 \\
\hline 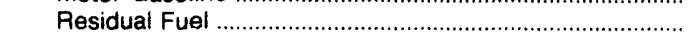 & .42 & 1.63 & 2.15 & 4.02 & 1.42 & 2.58 & 1.83 & 2.41 \\
\hline Electricity & - & - & - & - & - & - & - & - \\
\hline Total Energy" & 1.28 & 2.80 & 6.00 & 7.06 & 5.71 & 5.67 & 5.53 & 5.70 \\
\hline 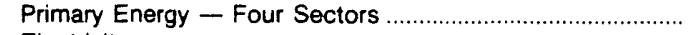 & 1.02 & 2.38 & 5.26 & 5.56 & 4.21 & 4.29 & 4.12 & 4.28 \\
\hline Electricity & 4.52 & 6.81 & 12.69 & 18.58 & 16.91 & 16.32 & 16.58 & 16.79 \\
\hline Electrlc Utllity Sector & .25 & .73 & 1.65 & 2.44 & 1.92 & 1.80 & 1.74 & 1.73 \\
\hline Coal & - & .23 & 1.21 & 1.59 & 1.55 & 1.55 & 1.47 & 1.45 \\
\hline 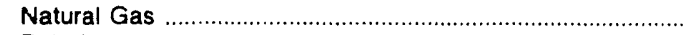 & .24 & .76 & 1.84 & 3.15 & 2.27 & 2.06 & 2.09 & 2.15 \\
\hline Petroleum & .51 & 1.89 & 3.35 & 4.90 & 2.35 & 3.26 & 2.69 & 3.51 \\
\hline 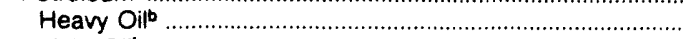 & .47 & 1.89 & 2.59 & 4.36 & 1.55 & 2.76 & 2.08 & 2.74 \\
\hline Light Oilb & .63 & 2.03 & 3.83 & 5.57 & 3.03 & 3.87 & 3.43 & 4.20 \\
\hline Petroleum Coke & - & - & - & - & - & - & - & - \\
\hline 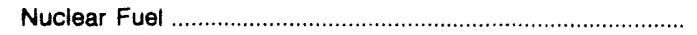 & - & - & - & - & - & - & .37 & .42 \\
\hline Wood and Waste & .65 & 92 & 1.74 & .79 & 32 & .95 & - & .82 \\
\hline Primary Energy - Five Sectors & .85 & 1.93 & 4.22 & 4.62 & 3.52 & 3.58 & 3.44 & 3.54 \\
\hline
\end{tabular}

-There are no direct fuel costs for hydroelectric, geothermal, centralized solar, or wind energy. Wood and other biomass fuels are not included, except those consumed at electric utilities.

bHeavy oil includes Grade Nos. 4, 5, and 6, and residual fuel oils. Light oil includes Grade No. 2 heating oil, kerosene, and jet fuel.

-No consumption, including cases where adjustments were made. See "Consumption Adjustments for Process Fuel and Intermediate Products."

Sources: Data sources, estimation procedures, and assumptions are described in the "Documentation" section of this report. 


\begin{tabular}{|c|c|c|c|c|c|c|c|c|}
\hline Sector and Energy Source & 1970 & 1975 & 1980 & 1985 & 1986 & 1987 & 1988 & 1989 \\
\hline Residential Sector & $1,015.6$ & $1,726.1$ & $3,852.3$ & $6,915.1$ & $6,244.9$ & $6,345.9$ & $6,676.7$ & $7,166.7$ \\
\hline 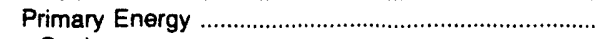 & 314.3 & 506.5 & 942.0 & 1.533 .2 & $1,260.2$ & $1,337.0$ & $1,362.7$ & $1,610.7$ \\
\hline Coal & & & & & .3 & .6 & .8 & .3 \\
\hline 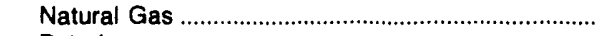 & 213.8 & 353.8 & 765.9 & $1,226.8$ & $1,032.8$ & $1,097.7$ & $1,127.5$ & $1,277.1$ \\
\hline 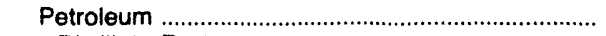 & 100.5 & 152.7 & 176.1 & 306.3 & 227.0 & 238.7 & 234.4 & 333.4 \\
\hline 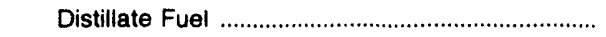 & .8 & 3.5 & .3 & 1.6 & .6 & 2.2 & .8 & .4 \\
\hline Kerosene ………………………………… & .2 & .7 & 9.4 & 50.2 & 19.3 & 29.9 & 31.9 & 18.5 \\
\hline LPG & 99.5 & 148.5 & 166.4 & 254.5 & 207.2 & 206.5 & 201.7 & 314.4 \\
\hline 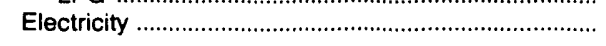 & 701.2 & $1,219.6$ & $2,910.3$ & $5,381.8$ & $4,984.7$ & $5,009.0$ & $5,314.0$ & $5,556.0$ \\
\hline Commerclal Sector & 511.7 & $1,115.4$ & $3,079.0$ & $5,428.3$ & $4,695.7$ & $4,787.1$ & $4,980.7$ & $5,255.2$ \\
\hline 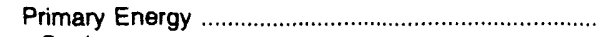 & 106.4 & 238.3 & 956.5 & $1,312.3$ & 868.7 & $1,067.3$ & $1,010.7$ & $1,132.1$ \\
\hline Coal & & - & & .2 & .3 & .6 & .9 & .2 \\
\hline 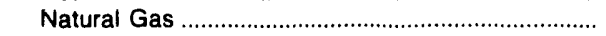 & 66.3 & 122.6 & 504.3 & 741.3 & 613.0 & 666.7 & 734.8 & 757.0 \\
\hline Petroleum & 40.1 & 115.7 & 452.2 & 570.8 & 255.4 & 400.0 & 275.0 & 374.9 \\
\hline 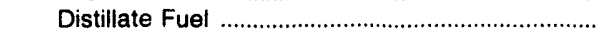 & 4.4 & 20.8 & 103.1 & 343.3 & 98.7 & 176.2 & 99.9 & 91.6 \\
\hline 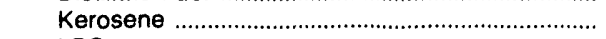 & 15.6 & 53.1 & 126.9 & 94.1 & 44.3 & 29.7 & 13.6 & 114.3 \\
\hline LPG & 10.3 & 18.1 & 20.7 & 22.6 & 18.8 & 17.2 & 15.7 & 14.0 \\
\hline 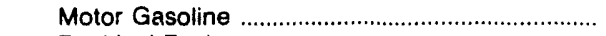 & 9.7 & 15.7 & 160.5 & 90.2 & 70.9 & 86.5 & 94.0 & 97.0 \\
\hline 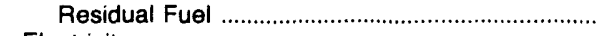 & .2 & 7.9 & 40.9 & 20.7 & 22.6 & 90.4 & 51.8 & 58.1 \\
\hline Electricity ......-1-1- & 405.2 & 877.2 & $2,122.5$ & $4,116.0$ & $3,827.0$ & $3,719.8$ & $3,970.0$ & $4,123.0$ \\
\hline Industrial Sector ... & $1,515.0$ & $4,523.8$ & $16,082.6$ & $16,414.1$ & $12,388.1$ & $12,336.8$ & $12,536.4$ & $12,729.7$ \\
\hline 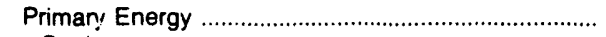 & $1,200.5$ & $3,725.6$ & $13,681.0$ & $12,792.2$ & $9,279.2$ & $9,420.3$ & $9,494.8$ & $9,626.7$ \\
\hline Coal & 11.8 & 93.3 & 80.9 & 138.5 & 108.7 & 78.4 & 68.3 & 79.1 \\
\hline 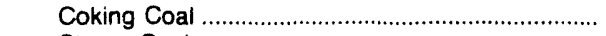 & 11.6 & 41.0 & 47.9 & 20.9 & 3.6 & - & - & - \\
\hline 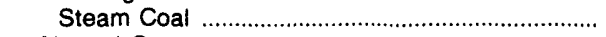 & .2 & 52.3 & 32.9 & 117.6 & 105.1 & 78.4 & 68.3 & 79.1 \\
\hline 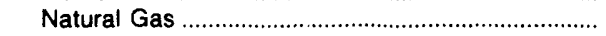 & 258.3 & 834.9 & $2,840.6$ & $3,940.8$ & $2,775.0$ & $2,507.9$ & $3,131.1$ & $3,507.5$ \\
\hline Petroleum & 930.4 & $2,797.4$ & $10,759.5$ & $8,712.9$ & 6.395 .5 & $6,833.9$ & $6,295.4$ & $6,040.1$ \\
\hline Asphalt and Road Oil .................................................... & 58.0 & 95.7 & 274.5 & 329.2 & 414.4 & 279.8 & 307.9 & 182.7 \\
\hline 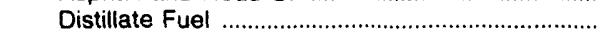 & 33.9 & 168.1 & 701.9 & $1,016.2$ & 474.3 & 558.1 & 466.0 & 644.2 \\
\hline Kerosene & 16.7 & 37.5 & 464.9 & 155.4 & 113.9 & 160.8 & 44.1 & 70.0 \\
\hline 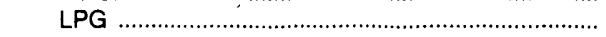 & 481.0 & $1,241.2$ & 3.470 .9 & $3,251.4$ & $3,128.9$ & $3,219.9$ & $3,224.0$ & $2,474.9$ \\
\hline 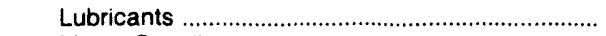 & 79.6 & 117.3 & 298.8 & 333.4 & 288.6 & 284.2 & 295.0 & 370.6 \\
\hline 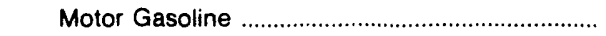 & 19.7 & 22.8 & 22.9 & 217.1 & 154.3 & 159.1 & 158.8 & 187.7 \\
\hline 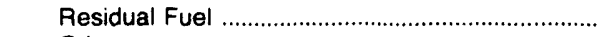 & 4.5 & 99.0 & 300.1 & 476.4 & 149.4 & 221.0 & 96.1 & 96.5 \\
\hline Other & 236.9 & $1,015.8$ & $5,225.5$ & $2,933.9$ & $1,671.6$ & $1,951.0$ & $1,703.4$ & $2,013.4$ \\
\hline 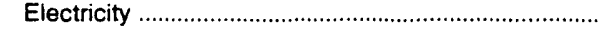 & 314.5 & 798.2 & $2,401.7$ & $3,621.9$ & $3,108.9$ & $2,916.5$ & $3,041.6$ & $3,103.0$ \\
\hline Transportation Sector & $2,305.6$ & $5,233.4$ & $12,568.5$ & $14,463.9$ & $10,722.4$ & $11,862.4$ & $12,096.3$ & $13,223.5$ \\
\hline 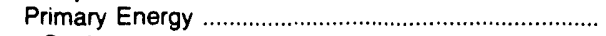 & $2,305.6$ & $5,233.4$ & $12,568.5$ & $14,463.9$ & $10,722.4$ & $11,862.4$ & $12,096.3$ & $13,223.5$ \\
\hline Coal & & & & & & & & \\
\hline Petroleum & $2,305.5$ & $5,233.3$ & $12,568.5$ & $14,463.9$ & $10,722.4$ & $11,862.4$ & $12,096.3$ & $13,223.5$ \\
\hline 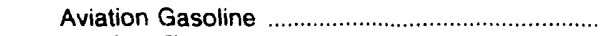 & 22.0 & 22.8 & 57.5 & 66.4 & 65.4 & 43.8 & 37.9 & 34.3 \\
\hline 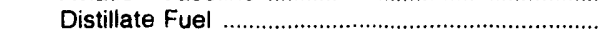 & 137.5 & 542.6 & $1,993.2$ & $2,136.1$ & $1,800.9$ & $2,048.6$ & $1,971.0$ & $2,297.4$ \\
\hline Jet Fuel & 97.4 & 306.2 & $1,098.5$ & $2,383.1$ & $1,614.0$ & $1,839.5$ & 1.911 .9 & $2,189.7$ \\
\hline 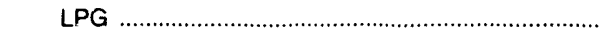 & 21.1 & 44.6 & 12.4 & 8.8 & 10.0 & 5.8 & 6.0 & 4.4 \\
\hline 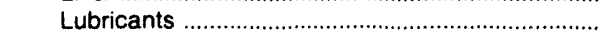 & 50.0 & 78.9 & 166.3 & 185.5 & 160.6 & 158.2 & 164.2 & 206.3 \\
\hline 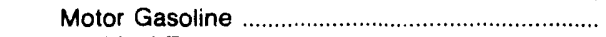 & $1,946.6$ & $3,982.0$ & $8,622.3$ & $9,172.2$ & $6,893.7$ & $7,489.6$ & $7,786.0$ & $8,173.6$ \\
\hline 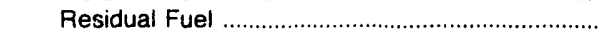 & 30.9 & 256.2 & 618.1 & 511.6 & 177.7 & 277.0 & 219.2 & 318.0 \\
\hline 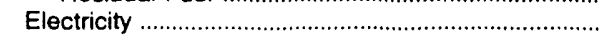 & - & - & - & - & & & & \\
\hline Total Energy" ………………………......... & $5,347.8$ & $12,598.6$ & $35,582.4$ & $43,221.3$ & $34,051.0$ & $35,332.3$ & $36,290.0$ & $38,375.1$ \\
\hline 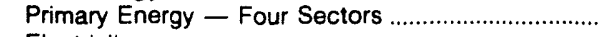 & $3,926.8$ & $9,703.7$ & $28,147.9$ & $30,101.5$ & $22,130.4$ & $23,687.0$ & $23,964.5$ & $25,593.1$ \\
\hline 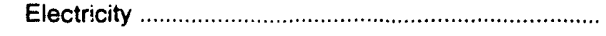 & $1,421.0$ & $2,895.0$ & $7,434.5$ & $13,119.7$ & $11,920.6$ & $11,645.3$ & $12,325.5$ & $12,782.0$ \\
\hline Electric Utility Sector & 267.6 & $1,099.3$ & $3,576.1$ & $5,652.8$ & $4,302.1$ & $4,036.4$ & $4,073.9$ & $4,246.3$ \\
\hline & & 26.9 & 811.7 & $1,694.0$ & $1,688.7$ & $1,771.4$ & $1,782.4$ & $1,830.8$ \\
\hline 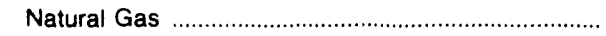 & 266.5 & $1,050.0$ & $2,727.1$ & $3,907.0$ & $2,600.4$ & $2,241.5$ & $2,253.8$ & $2,279.2$ \\
\hline Petroleum & .5 & 21.5 & 35.9 & 49.3 & 12.0 & 21.3 & 22.6 & 90.0 \\
\hline 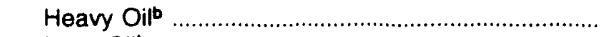 & .3 & 20.6 & 10.7 & 24.2 & 3.6 & 9.9 & 9.6 & 33.4 \\
\hline Light Oilb & .2 & .9 & 25.1 & 25.1 & 8.3 & 11.4 & 13.1 & 56.6 \\
\hline Petroleum Coke ………………………………......... & - & - & - & - & - & - & & \\
\hline 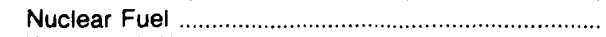 & - & - & - & - & - & - & 15.1 & 44.6 \\
\hline Wood and Waste & .7 & .9 & 1.4 & 2.4 & 1.0 & 2.3 & - & 1.6 \\
\hline Primary Energy — Five Sectors ……………............. & $4,194.4$ & $10,802.9$ & $31,724.0$ & $35,754.3$ & $26,432.5$ & $27,723.4$ & $28,038.4$ & $29,839.3$ \\
\hline
\end{tabular}

-There are no direct fuel costs for hydroelectric, geothermal, centralized solar, or wind energy. Wood and other biomass fuels are not included, except those consumed at electric utilities.

bHeavy oil includes Grade Nos. 4, 5, and 6, and residual fuel oils. Light oil includes Grade No. 2 heating oil, kerosene, and jet fuel.

- No consumption, including cases where adjustments were made. See "Consumption Adjustments for Process Fuel and Intermediate Products." - Value less than 0.05 million dollars.

Note: Totals may not equal sum of components due to independent rounding.

Sources: Data sources, estimation procedures, and assumptions are described in the "Documentation" section of this report. 


\section{U Energy Price and Expenditure Estimates by Source, Utah $1970,1975,1980$, and 1985-1989}

\begin{tabular}{|c|c|c|c|c|c|c|c|c|}
\hline Energy Source & 1970 & 1975 & 1980 & 1985 & 1986 & 1987 & 1988 & 1989 \\
\hline & \multicolumn{8}{|c|}{ Prices in Dollars per Million Btu } \\
\hline Coal ................... & 0.38 & 0.92 & 1.35 & 1.48 & 1.46 & 1.25 & 1.34 & 1.31 \\
\hline 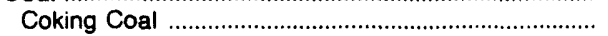 & .43 & 1.38 & 1.97 & 1.93 & 1.75 & - & 2.02 & 1.92 \\
\hline 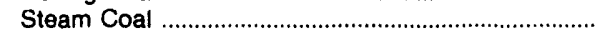 & .28 & .55 & 1.16 & 1.39 & 1.42 & 1.25 & 1.26 & 1.25 \\
\hline 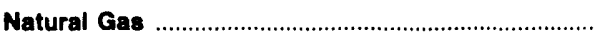 & .57 & 1.07 & 2.33 & 4.01 & 4.41 & 4.16 & 3.94 & 3.99 \\
\hline 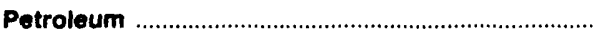 & 1.67 & 3.28 & 7.48 & 7.72 & 5.87 & 6.09 & 6.35 & 6.75 \\
\hline 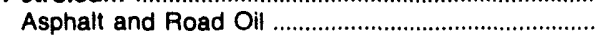 & .57 & 1.79 & 3.65 & 4.85 & 4.38 & 3.23 & 3.32 & 2.73 \\
\hline Aviation Gasoline ………………………………. & 2.17 & 3.45 & 9.02 & 9.99 & 8.41 & 7.55 & 7.41 & 8.28 \\
\hline 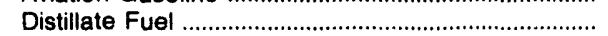 & 1.09 & 2.61 & 6.54 & 6.69 & 5.43 & 5.78 & 5.85 & 6.67 \\
\hline 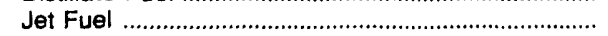 & .76 & 2.12 & 6.59 & 6.25 & 4.05 & 4.19 & 3.95 & 4.60 \\
\hline 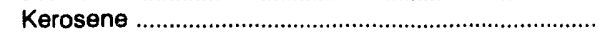 & .76 & 2.42 & 5.82 & 10.38 & 4.70 & 5.75 & 5.57 & 8.89 \\
\hline 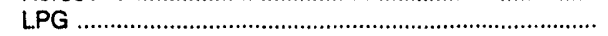 & 2.12 & 4.37 & 5.68 & 8.69 & 6.84 & 6.70 & 6.81 & 8.48 \\
\hline 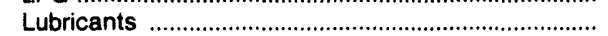 & 5.08 & 7.49 & 14.36 & 17.61 & 15.59 & 13.58 & 14.61 & 17.90 \\
\hline 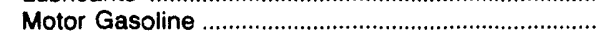 & 2.75 & 4.52 & 9.80 & 9.09 & 6.78 & 7.44 & 7.71 & 8.24 \\
\hline 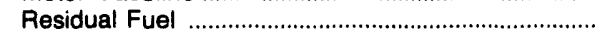 & .39 & 1.71 & 3.70 & 3.94 & 2.40 & 2.16 & 1.98 & 1.66 \\
\hline Other & .51 & 1.41 & 2.85 & 3.68 & 1.53 & 1.50 & 1.45 & 1.92 \\
\hline Nuclear Fuel & - & - & - & - & - & - & - & - \\
\hline 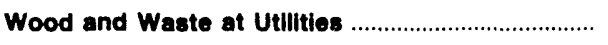 & - & - & - & - & - & - & - & - \\
\hline Primary Energy - Five Sectors ............................ & 1.02 & 2.04 & 4.05 & 4.28 & 3.77 & 3.37 & 3.26 & 3.33 \\
\hline Electric Utility Fuel & 25 & .51 & 1.20 & 1.39 & 1.42 & 1.27 & 1.27 & 1.25 \\
\hline 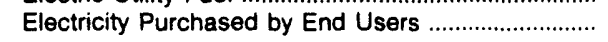 & 5.31 & 7.06 & 13.11 & 19.05 & 19.56 & 19.15 & 18.41 & 17.10 \\
\hline \multirow[t]{2}{*}{ Total Energy $b$} & 1.31 & 2.59 & 5.77 & 7.16 & 6.86 & 7.18 & 6.69 & 6.69 \\
\hline & \multicolumn{8}{|c|}{ Expenditures in Millions of Dollars } \\
\hline Coal & 30.1 & 107.0 & 227.1 & 295.0 & 275.0 & 342.0 & 451.3 & 452.0 \\
\hline 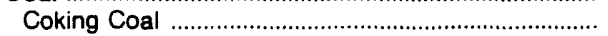 & 22.7 & 71.7 & 77.9 & 64.8 & 36.9 & & 63.8 & 60.6 \\
\hline 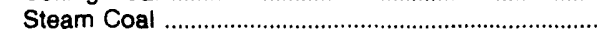 & 7.4 & 35.3 & 149.2 & 230.2 & 238.1 & 342.0 & 387.5 & 391.4 \\
\hline Natural Gas & 61.5 & 113.6 & 255.6 & 439.9 & 365.0 & 348.4 & 376.3 & 406.0 \\
\hline Petroleum ……….................... & 252.9 & 612.1 & $1,399.3$ & $1,318.2$ & $1,076.1$ & $1,153.6$ & $1,197.3$ & $1,251.1$ \\
\hline 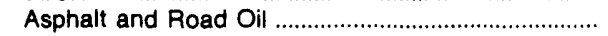 & 6.0 & 14.5 & 35.8 & 50.8 & 37.7 & 30.6 & 23.5 & 30.3 \\
\hline Aviation Gasoline …………………………… & 1.9 & 2.8 & 6.3 & 4.7 & 4.7 & 3.8 & 4.2 & 4.4 \\
\hline 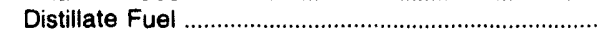 & 32.4 & 137.5 & 319.7 & 231.5 & 231.3 & 227.9 & 249.6 & 239.9 \\
\hline 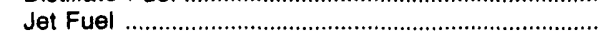 & 7.6 & 22.4 & 96.4 & 133.0 & 98.4 & 116.8 & 110.4 & 131.7 \\
\hline 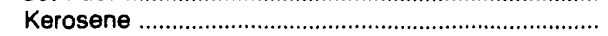 & 1.1 & 2.0 & 3.4 & 2.1 & .5 & .8 & .7 & 3 \\
\hline LPG & 6.7 & 15.4 & 23.8 & 47.3 & 36.0 & 36.6 & 32.6 & 43.4 \\
\hline 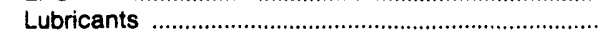 & 7.9 & 10.5 & 26.1 & 29.1 & 25.2 & 24.8 & 25.7 & 32.3 \\
\hline 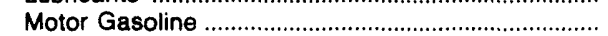 & 177.5 & 357.3 & 799.6 & 775.3 & 624.7 & 687.1 & 735.7 & 749.2 \\
\hline 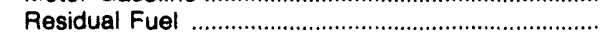 & 10.3 & 43.5 & 74.8 & 34.7 & 14.6 & 21.7 & 11.6 & 14.6 \\
\hline Othere & 1.5 & 6.2 & 13.4 & 9.7 & 3.0 & 3.5 & 3.3 & 4.9 \\
\hline 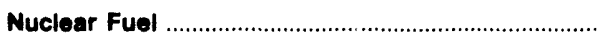 & - & - & - & - & - & - & - & - \\
\hline 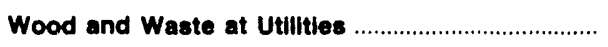 & - & - & - & - & - & - & - & - \\
\hline Primary Energy - Five Sectors & 344.5 & 832.7 & $1,882.0$ & $2,053.0$ & $1,716.1$ & $1,844.0$ & $2,024.8$ & $2,109.1$ \\
\hline $\begin{array}{l}\text { Electric Utility Fuel } \\
\text { Electricity Purchased by End Users }\end{array}$ & $\begin{array}{r}-6.4 \\
92.0\end{array}$ & $\begin{array}{r}-26.2 \\
186.9\end{array}$ & $\begin{array}{r}-141.2 \\
469.3\end{array}$ & $\begin{array}{r}-208.0 \\
830.7\end{array}$ & $\begin{array}{r}-222.5 \\
852.9\end{array}$ & $\begin{array}{r}-331.1 \\
861.6\end{array}$ & $\begin{array}{r}-367.6 \\
895.9\end{array}$ & $\begin{array}{r}-367.7 \\
853.9\end{array}$ \\
\hline Total Energy & 430.2 & 993.4 & $2,210.1$ & $2,675.8$ & $2,346.5$ & $2,374.5$ & $2,553.0$ & $2,595.3$ \\
\hline
\end{tabular}

Includes petroleum coke used at electric utilities.

bThere are no direct fuel costs for hydroelectric. geothermal, centralized solar, or wind energy. Wood and other biomass fuels are not included, except those consumed at electric utilities.

-No consumption, including cases where adjustments were made. See "Consumption Adjustments for Process Fuel and Intermediate Products." Note: Expenditure totals may not equal sum of components due to independent rounding.

Sources: Data sources, estimation procedures, and assumptions are described in the "Documentation" section of this report 


\begin{tabular}{|c|c|c|c|c|c|c|c|c|}
\hline Sector and Energy Source & 1970 & 1975 & 1980 & 1985 & 1986 & 1987 & 1988 & 1989 \\
\hline $\begin{array}{l}\text { Reatdential Sector } \\
\text { Primary Energy } \\
\text { Coal } \\
\text { Natural Gas } \\
\text { Petroleum } \\
\text { Distillate Fuel } \\
\text { Kerosene } \\
\text { LPG }\end{array}$ & $\begin{array}{r}1.62 \\
.99 \\
.76 \\
.91 \\
2.06 \\
1.28 \\
2.62 \\
2.31 \\
6.69\end{array}$ & $\begin{array}{l}2.37 \\
1.49 \\
1.33 \\
1.28 \\
4.31 \\
2.84 \\
5.16 \\
5.77 \\
8.84\end{array}$ & $\begin{array}{r}4.64 \\
2.69 \\
3.02 \\
2.51 \\
8.08 \\
6.89 \\
8.68 \\
16.92\end{array}$ & $\begin{array}{r}7.70 \\
4.69 \\
3.46 \\
4.52 \\
9.10 \\
7.25 \\
16.24 \\
9.25 \\
22.80\end{array}$ & $\begin{array}{r}8.37 \\
4.93 \\
3.42 \\
4.89 \\
6.50 \\
4.04 \\
9.06 \\
6.93 \\
23.27\end{array}$ & $\begin{array}{r}8.73 \\
4.68 \\
2.62 \\
4.60 \\
6.63 \\
3.73 \\
8.35 \\
7.34 \\
23.28\end{array}$ & $\begin{array}{r}8.75 \\
4.77 \\
2.63 \\
4.73 \\
6.59 \\
3.86 \\
8.63 \\
7.49 \\
22.88\end{array}$ & $\begin{array}{r}8.42 \\
4.92 \\
3.01 \\
4.73 \\
9.58 \\
5.43 \\
12.17 \\
12.23 \\
21.68\end{array}$ \\
\hline $\begin{array}{l}\text { Commercial Sector } \\
\text { Primary Energy } \\
\text { Coal } \\
\text { Natural Gas } \\
\text { Petroleum } \\
\text { Distillate Fuel } \\
\text { Kerosene } \\
\text { LPG } \\
\text { Motor Gasoline } \\
\text { Residual Fuel } \\
\text { Electricity }\end{array}$ & $\begin{array}{r}1.78 \\
.70 \\
.29 \\
.63 \\
.84 \\
1.06 \\
.71 \\
1.20 \\
2.75 \\
.27 \\
5.32\end{array}$ & $\begin{array}{r}3.30 \\
1.94 \\
.74 \\
1.60 \\
2.22 \\
2.49 \\
2.35 \\
2.30 \\
4.52 \\
1.55 \\
7.15\end{array}$ & $\begin{array}{r}7.70 \\
4.30 \\
1.07 \\
5.12 \\
5.14 \\
6.42 \\
5.82 \\
4.36 \\
9.80 \\
3.69 \\
13.22\end{array}$ & $\begin{array}{r}11.59 \\
4.37 \\
1.28 \\
4.57 \\
6.23 \\
6.42 \\
7.84 \\
8.22 \\
9.09 \\
3.94 \\
20.09\end{array}$ & $\begin{array}{r}12.61 \\
4.00 \\
1.29 \\
4.99 \\
4.47 \\
4.52 \\
3.74 \\
6.76 \\
6.78 \\
2.40 \\
20.49\end{array}$ & $\begin{array}{r}10.01 \\
3.94 \\
1.08 \\
4.61 \\
3.38 \\
3.85 \\
4.66 \\
6.22 \\
7.44 \\
2.16 \\
20.06\end{array}$ & $\begin{array}{r}9.63 \\
3.45 \\
1.12 \\
3.77 \\
3.53 \\
3.28 \\
4.56 \\
6.21 \\
7.71 \\
1.98 \\
19.91\end{array}$ & $\begin{array}{r}9.83 \\
3.56 \\
1.19 \\
3.83 \\
4.39 \\
4.09 \\
5.89 \\
6.68 \\
8.24 \\
1.66 \\
18.87\end{array}$ \\
\hline 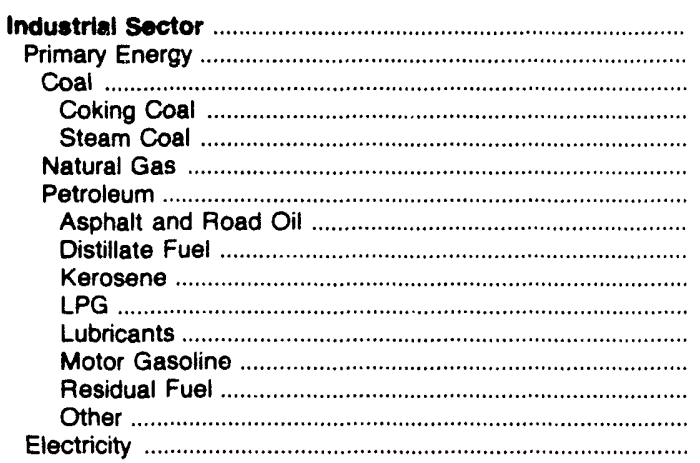 & $\begin{array}{r}.56 \\
.46 \\
.40 \\
.43 \\
.29 \\
.32 \\
.76 \\
.57 \\
.66 \\
.71 \\
1.20 \\
5.08 \\
2.75 \\
.60 \\
.51\end{array}$ & $\begin{array}{r}1.60 \\
1.38 \\
1.26 \\
1.38 \\
.74 \\
.73 \\
2.04 \\
1.79 \\
2.18 \\
2.35 \\
2.30 \\
7.49 \\
4.52 \\
1.78 \\
1.41 \\
5.39\end{array}$ & $\begin{array}{r}3.46 \\
2.74 \\
1.77 \\
1.97 \\
1.07 \\
2.08 \\
4.44 \\
3.65 \\
5.49 \\
5.82 \\
4.36 \\
14.36 \\
9.80 \\
3.71 \\
2.85 \\
10.22\end{array}$ & $\begin{array}{r}4.47 \\
3.21 \\
1.77 \\
1.93 \\
1.28 \\
3.01 \\
5.49 \\
4.85 \\
6.34 \\
7.84 \\
8.22 \\
17.61 \\
9.09 \\
3.94 \\
3.68 \\
14.36\end{array}$ & $\begin{array}{r}4.61 \\
2.88 \\
1.62 \\
1.75 \\
1.29 \\
3.16 \\
3.86 \\
4.38 \\
3.02 \\
3.74 \\
6.76 \\
15.59 \\
6.78 \\
2.40 \\
1.53 \\
14.90\end{array}$ & $\begin{array}{r}5.10 \\
2.97 \\
1.08 \\
1.08 \\
2.96 \\
3.67 \\
3.23 \\
3.77 \\
4.66 \\
6.22 \\
13.58 \\
7.44 \\
2.16 \\
1.50 \\
14.35\end{array}$ & $\begin{array}{r}4.14 \\
2.62 \\
1.75 \\
2.02 \\
1.12 \\
2.87 \\
3.71 \\
3.32 \\
3.69 \\
4.56 \\
6.21 \\
14.61 \\
7.71 \\
1.98 \\
1.45 \\
13.27\end{array}$ & $\begin{array}{r}3.91 \\
2.69 \\
1.68 \\
1.92 \\
1.19 \\
3.04 \\
3.71 \\
2.73 \\
4.77 \\
5.89 \\
6.68 \\
17.90 \\
8.24 \\
1.66 \\
1.92 \\
11.77\end{array}$ \\
\hline $\begin{array}{l}\text { Traneportation Sector } \\
\text { Primary Energy } \\
\text { Coal } \\
\text { Petroleum } \\
\text { Aviation Gasoline } \\
\text { Distillate Fuel } \\
\text { Jet Fuel } \\
\text { LPG } \\
\text { Lubricants } \\
\text { Motor Gasoline } \\
\text { Residual Fuel }\end{array}$ & $\begin{array}{r}2.28 \\
2.28 \\
.29 \\
2.28 \\
2.17 \\
1.32 \\
.76 \\
1.20 \\
5.08 \\
2.75 \\
.26\end{array}$ & $\begin{array}{r}3.97 \\
3.97 \\
.74 \\
3.97 \\
3.45 \\
2.97 \\
2.12 \\
2.30 \\
7.49 \\
4.52 \\
1.84\end{array}$ & $\begin{array}{r}8.82 \\
8.82 \\
- \\
8.82 \\
9.02 \\
7.02 \\
6.59 \\
4.36 \\
14.36 \\
9.80 \\
-\end{array}$ & $\begin{array}{r}8.28 \\
8.28 \\
- \\
8.28 \\
9.99 \\
6.82 \\
6.25 \\
8.22 \\
17.61 \\
9.09 \\
-\end{array}$ & $\begin{array}{r}6.39 \\
6.39 \\
- \\
6.39 \\
8.41 \\
6.80 \\
4.05 \\
6.76 \\
15.59 \\
6.78 \\
-\end{array}$ & $\begin{array}{r}6.78 \\
6.78 \\
- \\
6.78 \\
7.55 \\
6.97 \\
4.19 \\
6.22 \\
13.58 \\
7.44 \\
-\end{array}$ & $\begin{array}{r}6.98 \\
6.98 \\
- \\
6.98 \\
7.41 \\
7.27 \\
3.95 \\
6.21 \\
14.61 \\
7.71 \\
-\end{array}$ & $\begin{array}{r}7.52 \\
7.52 \\
- \\
7.52 \\
8.28 \\
7.82 \\
4.60 \\
6.68 \\
17.90 \\
8.24 \\
2.73\end{array}$ \\
\hline 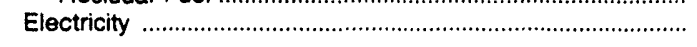 & - & - & - & - & - & - & - & - \\
\hline $\begin{array}{l}\text { Total Energy" } \\
\text { Primary Energy - Four Sectors } \\
\text { Electricity }\end{array}$ & $\begin{array}{l}1.31 \\
1.08 \\
5.31\end{array}$ & $\begin{array}{l}2.59 \\
2.26 \\
7.06\end{array}$ & $\begin{array}{r}5.77 \\
5.01 \\
13.11\end{array}$ & $\begin{array}{r}7.16 \\
5.59 \\
19.05\end{array}$ & $\begin{array}{r}6.86 \\
5.00 \\
19.56\end{array}$ & $\begin{array}{r}7.18 \\
5.29 \\
19.15\end{array}$ & $\begin{array}{r}6.69 \\
4.98 \\
18.41\end{array}$ & $\begin{array}{r}6.69 \\
5.15 \\
17.10\end{array}$ \\
\hline $\begin{array}{l}\text { Electric Utllity Soctor } \\
\text { Coal } \\
\text { Natural Gas } \\
\text { Petroleum } \\
\text { Heavy Oib } \\
\text { Light Oib }\end{array}$ & $\begin{array}{l}.25 \\
.23 \\
.31 \\
.26 \\
.26 \\
.32\end{array}$ & $\begin{array}{r}.51 \\
.48 \\
.61 \\
1.59 \\
1.54 \\
2.31\end{array}$ & $\begin{array}{l}1.20 \\
1.14 \\
2.00 \\
5.00 \\
3.69 \\
6.23\end{array}$ & $\begin{array}{l}\mathbf{1 . 3 9} \\
1.37 \\
4.12 \\
5.02 \\
3.71 \\
5.67\end{array}$ & $\begin{array}{l}\mathbf{1 . 4 2} \\
1.41 \\
5.10 \\
3.55 \\
2.09 \\
3.62\end{array}$ & $\begin{array}{r}1.27 \\
1.25 \\
5.07 \\
4.12 \\
- \\
4.12\end{array}$ & $\begin{array}{r}1.27 \\
1.27 \\
2.83 \\
3.98 \\
- \\
3.98\end{array}$ & $\begin{array}{r}1.25 \\
1.24 \\
3.14 \\
4.45 \\
- \\
4.45\end{array}$ \\
\hline $\begin{array}{l}\text { Petroleum Coke } \\
\text { Nuclear Fuel } \\
\text { Wood and Waste }\end{array}$ & - & - & - & - & - & - & - & - \\
\hline Primary Energy — Five Sectors & 1.02 & 2.04 & 4.05 & 4.28 & 3.77 & 3.37 & 3.26 & 3.33 \\
\hline
\end{tabular}

- There are no direct fuel costs for hydroelectric, geothermal, centralized solar, or wind energy. Wood and other biomass fuels are not included, except those consumed at electric utilities.

bHeavy oil includes Grade Nos. 4, 5, and 6, and residual fuel oils. Light oil includes Grade No. 2 heating oil, kerosene, and jet fuel.

- No consumption, including cases where adjustments were made. See "Consumption Adjustments for Process Fuel and Intermediate Products."

Sources: Data sources, estimation procedures, and assumptions are described in the "Documentation" section of this report. 
U Energy Expenditure Estimates by Sector, Utah

T 1970, 1975, 1980, and 1985-1989

A (Million Dollars)

H

\begin{tabular}{|c|c|c|c|c|c|c|c|c|}
\hline Sector and Energy Source & 1970 & 1975 & 1980 & 1985 & 1986 & 1987 & 1988 & 1989 \\
\hline 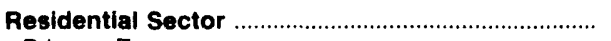 & 84.4 & 167.5 & 359.4 & 629.9 & 607.5 & 544.8 & 564.1 & 571.9 \\
\hline Primary Energy & 45.9 & 92.3 & 179.4 & 319.8 & 290.8 & 228.7 & 240.1 & 263.9 \\
\hline Coal & .7 & 1.5 & 5.8 & 7.2 & 5.3 & 2.7 & 4.2 & 5.6 \\
\hline 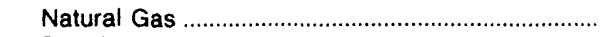 & 37.9 & 72.8 & 158.0 & 285.3 & 267.4 & 206.4 & 215.9 & 232.2 \\
\hline 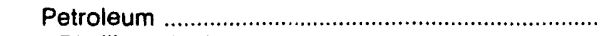 & 7.2 & 18.1 & 15.6 & 27.3 & 18.1 & 19.6 & 20.1 & 26.2 \\
\hline 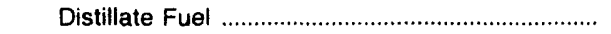 & 1.1 & 5.9 & 4.5 & 3.1 & 1.8 & 2.2 & 3.0 & 5.8 \\
\hline 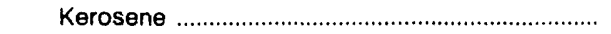 & .1 & .1 & - & 1.0 & .2 & .3 & .3 & .2. \\
\hline LPG & 6.0 & 12.1 & 11.1 & 23.2 & 16.2 & 17.0 & 16.9 & 20.3 \\
\hline 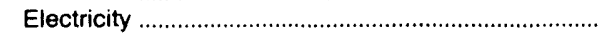 & 38.5 & 75.2 & 179.9 & 310.1 & 316.7 & 316.1 & 324.0 & 308.0 \\
\hline Commercial Sector & 49.0 & 106.9 & 216.4 & 395.8 & 385.7 & 441.0 & 440.5 & 423.7 \\
\hline Primary Energy & 14.8 & 46.4 & 74.7 & 80.8 & 58.4 & 108.2 & 98.5 & 90.7 \\
\hline 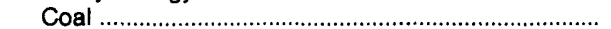 & .5 & 1.5 & 3.8 & 4.9 & 3.7 & 2.0 & 3.3 & 4.1 \\
\hline Natural Gas & 6.0 & 9.2 & 1.8 & 41.7 & 21.9 & 73.8 & 73.1 & 68.7 \\
\hline 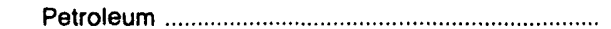 & 8.2 & 35.8 & 69.0 & 34.2 & 32.8 & 32.4 & 22.1 & 17.9 \\
\hline 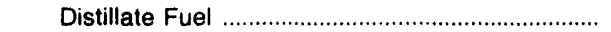 & 3.2 & 18.8 & 38.4 & 20.2 & 24.0 & 16.5 & 13.3 & 10.9 \\
\hline 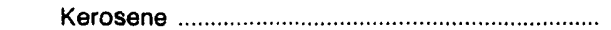 & .2 & .4 & 1.1 & .9 & .1 & .4 & .1 & .1 \\
\hline LPG & .6 & .9 & 1.0 & 3.6 & 2.8 & 2.5 & 2.5 & 2.0 \\
\hline Motor Gasoline & 2.9 & 5.0 & 4.1 & 4.2 & 3.2 & 3.6 & 3.6 & 3.9 \\
\hline 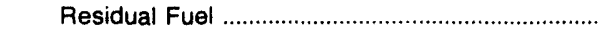 & 1.4 & 10.7 & 24.4 & 5.2 & 2.7 & 9.3 & 2.6 & 1.0 \\
\hline Electricity & 34.3 & 60.5 & 141.7 & 315.0 & 327.3 & 332.9 & 342.0 & 333.0 \\
\hline Industrial Sector & 89.2 & 268.0 & 524.1 & 565.3 & 449.7 & 404.4 & 502.7 & $\mathbf{5 2 6 . 5}$ \\
\hline Primary Energy & 70.0 & 216.8 & 376.4 & 359.6 & 240.8 & 191.9 & 272.8 & 313.5 \\
\hline Coal & 26.4 & 31.2 & 89.9 & 78.3 & 47.6 & 12.1 & 79.1 & 79.1 \\
\hline 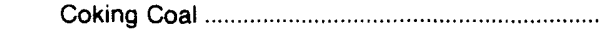 & 22.7 & 71.7 & 77.9 & 64.8 & 36.9 & - & 63.8 & 60.6 \\
\hline Steam Coal & 3.6 & 9.5 & 12.0 & 13.5 & 10.7 & 12.1 & 15.3 & 18.4 \\
\hline Natural Gas & 16.5 & 29.9 & 86.0 & 111.8 & 74.4 & 66.7 & 86.8 & 103.0 \\
\hline Petroleum & 27.1 & 105.7 & 200.6 & 169.5 & 118.9 & 113.0 & 106.9 & 131.5 \\
\hline 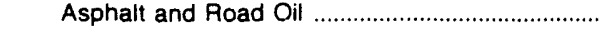 & 6.0 & 14.5 & 35.8 & 50.8 & 37.7 & 30.6 & 23.5 & 30.3 \\
\hline Distillate Fuel & 6.0 & 40.9 & 70.9 & 40.8 & 34.2 & 33.7 & 41.2 & 42.8 \\
\hline 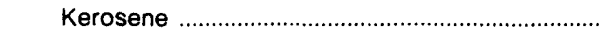 & .8 & 1.5 & 2.2 & .2 & .2 & .1 & .3 & * \\
\hline LPG & .1 & 2.3 & 11.5 & 18.4 & 15.5 & 15.9 & 12.0 & 20.0 \\
\hline 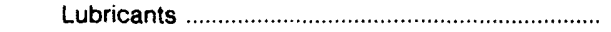 & 2.9 & 3.3 & 9.2 & 10.3 & 8.9 & 8.8 & 9.1 & 11.4 \\
\hline 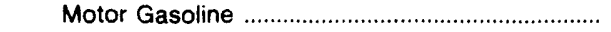 & 3.8 & 6.3 & 8.5 & 10.5 & 7.5 & 7.9 & 8.5 & 8.4 \\
\hline 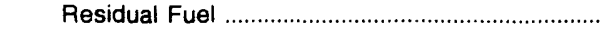 & 6.0 & 30.5 & 49.1 & 28.9 & 11.8 & 12.4 & 8.9 & 13.6 \\
\hline Other & 1.5 & 6.2 & 13.4 & 9.7 & 3.0 & 3.5 & 3.3 & 4.9 \\
\hline 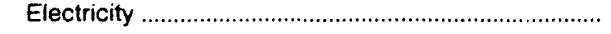 & 19.2 & 51.2 & 147.7 & 205.7 & 208.9 & 212.6 & 229.9 & 212.9 \\
\hline 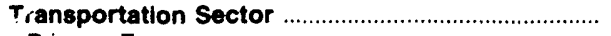 & 207.5 & 451.0 & $1,110.3$ & $1,084.8$ & 903.5 & 984.2 & $1,045.8$ & $1,073.3$ \\
\hline 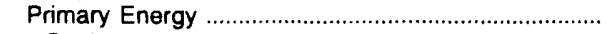 & 207.5 & 451.0 & $1,110.3$ & $1,084.8$ & 903.5 & 984.2 & $1,045.8$ & $1,073.3$ \\
\hline 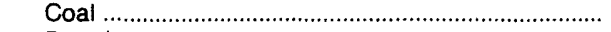 & & * & - & - & - & - & - & - \\
\hline 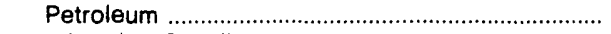 & 207.5 & 451.0 & $1,110.3$ & $1,084.8$ & 903.5 & 984.2 & $1,045.8$ & $1,073.3$ \\
\hline 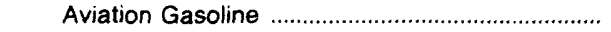 & 1.9 & 2.8 & 6.3 & 4.7 & 4.7 & 3.8 & 4.2 & 4.4 \\
\hline Distillate Fuel & 22.1 & 71.7 & 203.5 & 165.5 & 168.6 & 170.9 & 189.7 & 178.1 \\
\hline Jet Fuel & 7.6 & 22.4 & 96.4 & 133.0 & 98.4 & 116.8 & 110.4 & 131.7 \\
\hline LPG & • & .1 & .2 & 2.0 & 1.5 & 1.1 & 1.2 & 1.1 \\
\hline 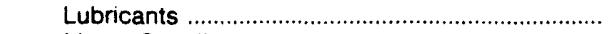 & 5.0 & 7.2 & 16.9 & 18.8 & 16.3 & 16.0 & 16.6 & 20.9 \\
\hline Motor Gasoline & 170.9 & 346.0 & 787.0 & 760.7 & 614.0 & 675.5 & 723.6 & 736.9 \\
\hline 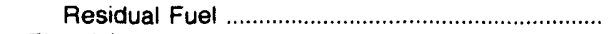 & • & .8 & - & - & - & - & - & $\bullet$ \\
\hline 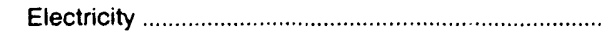 & - & - & - & - & - & - & - & - \\
\hline Total Energy" & 430.2 & 993.4 & $2,210.1$ & $2,675.8$ & $2,346.5$ & $2,374.5$ & $2,553.0$ & $2,595.3$ \\
\hline Primary Energy - Four Sectors & 338.2 & 806.5 & $1,740.8$ & $1,845.0$ & $1,493.6$ & $1,512.9$ & $1,657.1$ & $1,741.4$ \\
\hline Electricity & 92.0 & 186.9 & 469.3 & 830.7 & 852.9 & 861.6 & 895.9 & 853.9 \\
\hline Electric Utllity Sector & 6.4 & 26.2 & 141.2 & 208.0 & 222.5 & 331.1 & 367.6 & 367.7 \\
\hline Coal & 2.5 & 22.8 & 127.6 & 204.6 & 218.4 & 325.1 & 364.7 & 363.3 \\
\hline Natural Gas & 1.0 & 1.8 & 9.8 & 1.0 & 1.3 & 1.4 & .6 & 2.2 \\
\hline Petroleum & 2.9 & 1.6 & 3.8 & 2.4 & 2.8 & 4.5 & 2.4 & 2.2 \\
\hline 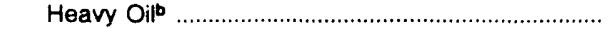 & 2.8 & 1.5 & 1.4 & .6 & .1 & - & - & - \\
\hline 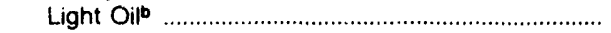 & - & .1 & 2.4 & 1.8 & 2.7 & 4.5 & 2.4 & 2.2 \\
\hline Petroleum Coke & - & - & - & - & - & - & - & - \\
\hline 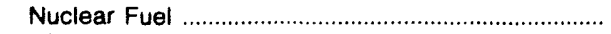 & - & - & - & - & - & - & - & - \\
\hline Wood and Waste & - & - & - & - & - & - & - & - \\
\hline 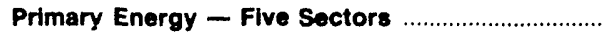 & 344.5 & 832.7 & $1,882.0$ & $2,053.0$ & $1,716.1$ & $1,844.0$ & $2,024.8$ & $2,109.1$ \\
\hline
\end{tabular}

-There are no direct fuel costs for hydroelectric, geothermal, centralized solar, or wind energy. Wood and other biomass fuels are not included, except those consumed at electric utilities.

byeavy oil includes Grade Nos. 4, 5, and 6, and residual fuel oils. Light oil includes Grade No. 2 heating oil, kerosene, and jet fuel.

-No consumption, including cases where adjustments were made. See "Consumption Adjustments for Process Fuel and Intermediate Products."

- Value less than 0.05 million dollars.

Note: Totals may not equal sum of components due to independent rounding.

Sources: Data sources, estimation procedures, and assumptions are described in the "Documentation" section of this report. 


\begin{tabular}{|c|c|c|c|c|c|c|c|c|}
\hline Energy Source & 1970 & 1975 & 1980 & 1985 & 1986 & 1987 & 1988 & 1989 \\
\hline & \multicolumn{8}{|c|}{ Prices in Dollars per Million Btu } \\
\hline Coal & 0.72 & 2.35 & 2.46 & 2.84 & 2.62 & 3.37 & 3.40 & 3.12 \\
\hline Coking Coal & - & - & - & - & - & - & - & - \\
\hline 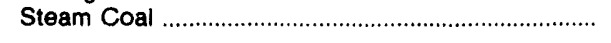 & .72 & 2.35 & 2.46 & 2.84 & 2.62 & 3.37 & 3.40 & 3.12 \\
\hline 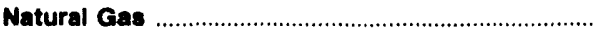 & 1.41 & 1.87 & 5.62 & 5.59 & 5.00 & 4.92 & 4.51 & 4.56 \\
\hline 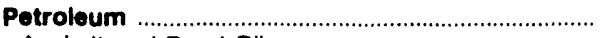 & 1.97 & 3.63 & 8.39 & 8.87 & 6.97 & 6.94 & 7.08 & 8.03 \\
\hline 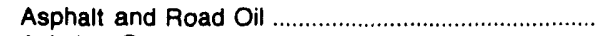 & .68 & 1.91 & 3.66 & 5.20 & 4.86 & 3.55 & 3.39 & 3.17 \\
\hline 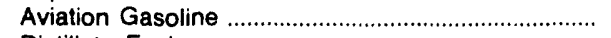 & 2.17 & 3.45 & 9.02 & 9.99 & 8.41 & 7.55 & 7.41 & 8.28 \\
\hline 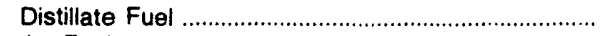 & 1.37 & 2.77 & 7.01 & 8.13 & 6.36 & 6.22 & 6.23 & 6.74 \\
\hline Jet Fuel & .75 & 2.22 & 6.55 & 6.10 & 4.25 & 4.18 & 4.04 & 5.33 \\
\hline Kerosene & 1.53 & 3.03 & 7.82 & 8.79 & 6.63 & 6.57 & 6.26 & 7.11 \\
\hline LPG & 2.15 & 4.12 & 7.56 & 11.71 & 10.37 & 10.36 & 9.37 & 12.20 \\
\hline Lubricants & 5.08 & 7.49 & 14.36 & 17.61 & 15.59 & 13.58 & 14.61 & 17.90 \\
\hline 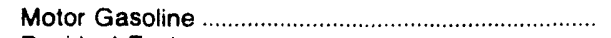 & 3.09 & 4.69 & 10.12 & 9.53 & 7.47 & 7.58 & 7.87 & 8.84 \\
\hline 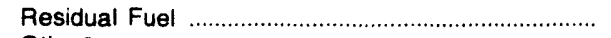 & .66 & 1.92 & 4.05 & 4.54 & 2.92 & 3.15 & 2.54 & 2.82 \\
\hline 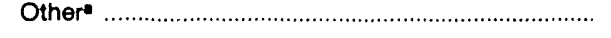 & 4.14 & - & - & - & 10.73 & 10.73 & 10.84 & 10.00 \\
\hline 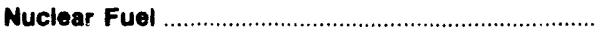 & - & .31 & .58 & .64 & .70 & .69 & .71 & .62 \\
\hline 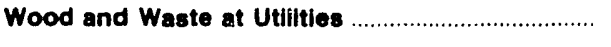 & - & - & 1.74 & .79 & .32 & .95 & .87 & .82 \\
\hline 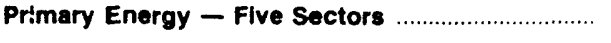 & 1.91 & 2.40 & 5.64 & 5.86 & 5.28 & 4.70 & 4.65 & 5.35 \\
\hline 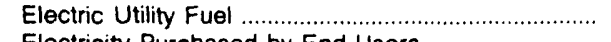 & .72 & .36 & .69 & .72 & .73 & .73 & .74 & .66 \\
\hline Electricity Purchased by End Users .............................. & 6.05 & 10.33 & 14.33 & 20.81 & 22.51 & 24.16 & 23.69 & 23.46 \\
\hline \multirow[t]{2}{*}{ Total Energy } & 2.39 & 4.40 & 9.28 & 10.57 & 9.24 & 9.51 & 9.60 & 10.34 \\
\hline & \multicolumn{8}{|c|}{ Expenditures in Millions of Dollars } \\
\hline Coal & 1.5 & 1.7 & 1.3 & 5.6 & 1.7 & 1.1 & 1.0 & 0.7 \\
\hline 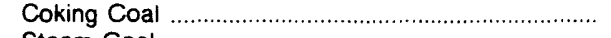 & - & - & - & - & - & - & - & - \\
\hline 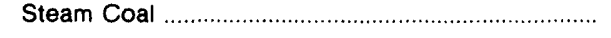 & 1.5 & 1.7 & 1.3 & 5.6 & 1.7 & 1.1 & 1.0 & .7 \\
\hline Natural Gas & 3.8 & 7.5 & 22.2 & 27.7 & 24.7 & 25.1 & 24.8 & 27.6 \\
\hline 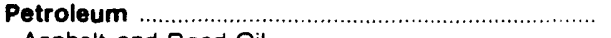 & 144.8 & 248.3 & 512.9 & 564.6 & 451.0 & 491.8 & 520.9 & 590.3 \\
\hline 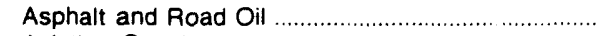 & 1.2 & 4 & 1.0 & 11.4 & 13.5 & 11.6 & 8.9 & 9.5 \\
\hline Aviation Gasoline & .2 & .2 & 1.1 & 1.1 & 1.2 & 8 & .6 & .7 \\
\hline 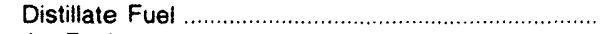 & 45.7 & 75.0 & 167.3 & 198.5 & 147.1 & 158.4 & 169.5 & 181.7 \\
\hline 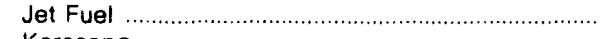 & .5 & 2.2 & 5.6 & 6.7 & 3.1 & 4.2 & 3.2 & 6.6 \\
\hline 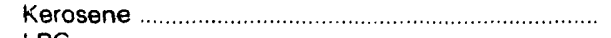 & 4.4 & 5.4 & 12.5 & 13.0 & 7.2 & 6.7 & 7.5 & 7.1 \\
\hline LPG & 4.4 & 12.8 & 18.5 & 34.0 & 33.3 & 40.0 & 41.0 & 71.3 \\
\hline 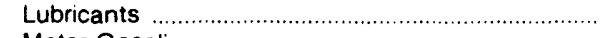 & 2.0 & 2.5 & 5.9 & 6.5 & 5.7 & 5.6 & 5.8 & 7.3 \\
\hline Motor Gasoline & 82.5 & 140.2 & 288.9 & 290.9 & 234.0 & 259.4 & 281.3 & 304.2 \\
\hline Residual Fuel & 3.7 & 9.6 & 12.0 & 2.4 & 5.5 & 4.7 & 2.8 & 1.6 \\
\hline 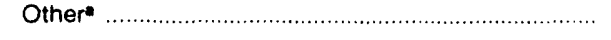 & .3 & - & - & - & .4 & .5 & .4 & .3 \\
\hline Nuclear Fuel & - & 12.0 & 18.7 & 20.8 & 15.5 & 26.3 & 31.3 & 24.1 \\
\hline 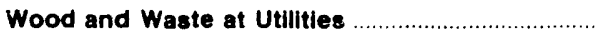 & - & - & .9 & 2.3 & .3 & 1.5 & .9 & 1.6 \\
\hline Primary Energy - Five Sectors ................................ & 150.2 & 269.5 & 556.0 & 621.0 & 493.3 & 545.9 & 578.9 & 644.3 \\
\hline $\begin{array}{l}\text { Electric Utility Fuel } \\
\text { Electricity Purchased by End Users }\end{array}$ & $\begin{array}{l}-2.2 \\
53.9\end{array}$ & $\begin{array}{l}-14.5 \\
105.6\end{array}$ & $\begin{array}{l}-23.4 \\
193.1\end{array}$ & $\begin{array}{l}-26.1 \\
285.1\end{array}$ & $\begin{array}{l}-17.3 \\
286.2\end{array}$ & $\begin{array}{l}-29.2 \\
337.8\end{array}$ & $\begin{array}{l}-33.9 \\
357.0\end{array}$ & $\begin{array}{l}-27.0 \\
366.0\end{array}$ \\
\hline & & & & & & & & \\
\hline Total Energy ${ }^{b} \ldots \ldots$ & 201.9 & 360.5 & 725.7 & 880.0 & 762.2 & 854.4 & 902.1 & 983.4 \\
\hline
\end{tabular}

Includes petroleum coke used at electric utilities.

bThere are no direct fuel costs for hydroelectric, geothermal, centralized solar, or wind energy. Wood and other biomass fuels are not included, except those consumed at electric utilities.

-No consumption, including cases where adjustments were made. See "Consumption Adjustments for Process Fuel and Intermediate Products." Note: Expenditure totals may not equal sum of components due io independent rounding.

Sources: Data sources, estimation procedures, and assumptions are described in the "Documentation" section of this report. 


\begin{tabular}{|c|c|c|c|c|c|c|c|c|}
\hline Sector and Energy Source & 1970 & 1975 & 1980 & 1985 & 1986 & 1987 & 1988 & 1989 \\
\hline $\begin{array}{l}\text { Resldential Sector } \\
\text { Primary Energy } \\
\text { Coal .. } \\
\text { Natural Gas ... } \\
\text { Petroleum } \\
\text { Distillate Fuel } \\
\text { Kerosene } \\
\text { LPG }\end{array}$ & $\begin{array}{l}2.24 \\
1.58 \\
1.37 \\
1.97 \\
1.57 \\
1.51 \\
1.63 \\
2.55 \\
6.68\end{array}$ & \begin{tabular}{r|}
4.52 \\
3.04 \\
2.62 \\
2.62 \\
3.07 \\
2.87 \\
3.16 \\
4.72 \\
11.47
\end{tabular} & $\begin{array}{r}9.65 \\
7.43 \\
4.42 \\
6.30 \\
7.56 \\
7.32 \\
8.15 \\
9.28 \\
15.76\end{array}$ & $\begin{array}{r}11.22 \\
8.37 \\
4.91 \\
6.33 \\
8.65 \\
8.08 \\
8.98 \\
11.79 \\
21.20\end{array}$ & $\begin{array}{r}10.27 \\
7.02 \\
4.71 \\
6.36 \\
7.12 \\
6.49 \\
7.22 \\
10.02 \\
23.15\end{array}$ & $\begin{array}{r}11.64 \\
6.89 \\
4.37 \\
6.31 \\
6.99 \\
6.23 \\
6.92 \\
10.04 \\
27.72\end{array}$ & $\begin{array}{r}11.38 \\
6.70 \\
4.51 \\
5.71 \\
6.84 \\
6.19 \\
6.88 \\
8.95 \\
26.66\end{array}$ & $\begin{array}{r}12.06 \\
8.05 \\
4.48 \\
5.70 \\
8.33 \\
6.79 \\
7.54 \\
12.77 \\
26.24\end{array}$ \\
\hline $\begin{array}{l}\text { Commercial Sector } \\
\text { Primary Energy } \\
\text { Coal } \\
\text { Natural Gas } \\
\text { Petroleum } \\
\text { Distillate Fuel } \\
\text { Kerosene } \\
\text { LPG } \\
\text { Motor Gasoline } \\
\text { Residual Fuel } \\
\text { Electricity }\end{array}$ & $\begin{array}{r}2.18 \\
1.06 \\
.87 \\
1.43 \\
1.04 \\
1.11 \\
.92 \\
1.37 \\
3.09 \\
.79 \\
6.78\end{array}$ & $\begin{array}{r}4.51 \\
2.33 \\
2.60 \\
2.10 \\
2.35 \\
2.46 \\
2.65 \\
2.92 \\
4.69 \\
1.91 \\
11.34\end{array}$ & $\begin{array}{r}9.00 \\
5.90 \\
1.65 \\
6.22 \\
5.93 \\
6.48 \\
6.39 \\
5.59 \\
10.12 \\
4.09 \\
15.56\end{array}$ & $\begin{array}{r}12.81 \\
6.80 \\
2.38 \\
5.76 \\
7.97 \\
7.56 \\
7.24 \\
11.46 \\
9.53 \\
4.54 \\
24.02\end{array}$ & $\begin{array}{r}12.73 \\
5.69 \\
2.12 \\
5.19 \\
5.97 \\
5.77 \\
4.71 \\
11.26 \\
7.47 \\
2.92 \\
25.65\end{array}$ & $\begin{array}{r}13.35 \\
5.51 \\
2.36 \\
4.94 \\
5.78 \\
5.29 \\
5.21 \\
11.14 \\
7.58 \\
3.15 \\
24.53\end{array}$ & $\begin{array}{r}13.38 \\
5.60 \\
2.53 \\
4.72 \\
5.98 \\
5.42 \\
4.95 \\
10.88 \\
7.87 \\
2.54 \\
24.44\end{array}$ & $\begin{array}{r}13.41 \\
6.02 \\
2.60 \\
4.81 \\
6.49 \\
5.91 \\
5.69 \\
10.22 \\
8.84 \\
2.84 \\
24.23\end{array}$ \\
\hline 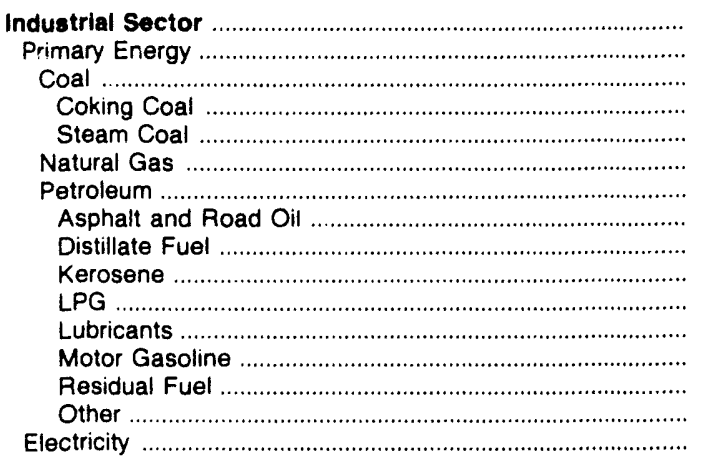 & $\begin{array}{r}1.68 \\
.90 \\
.87 \\
- \\
.87 \\
.85 \\
.90 \\
.68 \\
.84 \\
.92 \\
1.37 \\
5.08 \\
3.09 \\
.53 \\
4.14 \\
4.52\end{array}$ & $\begin{array}{r}3.68 \\
2.26 \\
2.60 \\
- \\
2.60 \\
1.44 \\
2.45 \\
1.91 \\
2.38 \\
2.65 \\
2.92 \\
7.49 \\
4.69 \\
1.93 \\
-\end{array}$ & $\begin{array}{r}7.51 \\
5.31 \\
1.65 \\
1.65 \\
4.94 \\
5.45 \\
3.66 \\
5.84 \\
6.39 \\
5.59 \\
14.36 \\
10.12 \\
4.01 \\
11.37\end{array}$ & $\begin{array}{r}10.90 \\
6.18 \\
2.38 \\
-\overline{2} \\
2.38 \\
4.91 \\
6.64 \\
5.20 \\
6.70 \\
7.24 \\
11.46 \\
17.61 \\
9.53 \\
4.54 \\
- \\
18.40\end{array}$ & $\begin{array}{r}10.08 \\
4.80 \\
2.12 \\
2.12 \\
3.57 \\
5.07 \\
4.86 \\
4.36 \\
4.71 \\
11.26 \\
15.59 \\
7.47 \\
2.92 \\
10.73 \\
20.05\end{array}$ & $\begin{array}{r}9.14 \\
4.68 \\
2.36 \\
- \\
2.36 \\
3.52 \\
4.91 \\
3.55 \\
4.82 \\
5.21 \\
11.14 \\
13.58 \\
7.58 \\
3.15 \\
10.73 \\
19.79\end{array}$ & $\begin{array}{r}9.10 \\
4.34 \\
2.53 \\
- \\
2.53 \\
3.00 \\
4.64 \\
3.39 \\
4.58 \\
4.95 \\
10.88 \\
14.61 \\
7.87 \\
2.54 \\
10.84 \\
19.39\end{array}$ & $\begin{array}{r}9.48 \\
4.71 \\
2.60 \\
- \\
2.60 \\
3.05 \\
5.15 \\
3.17 \\
5.26 \\
5.69 \\
10.22 \\
17.90 \\
8.84 \\
2.84 \\
10.00 \\
19.21\end{array}$ \\
\hline 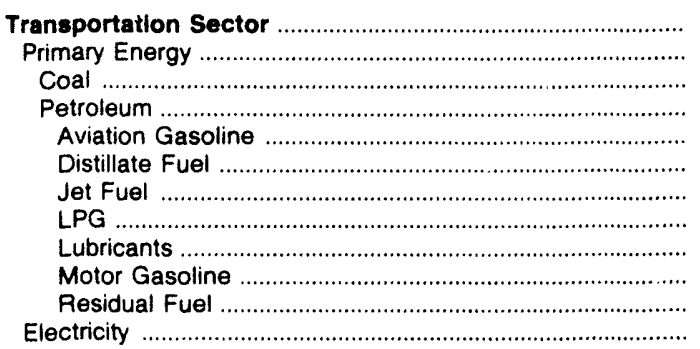 & $\begin{array}{r}2.94 \\
2.94 \\
.87 \\
2.94 \\
2.17 \\
1.43 \\
.75 \\
1.37 \\
5.08 \\
3.09 \\
.76 \\
-\end{array}$ & $\begin{array}{l}4.49 \\
4.49 \\
2.60 \\
4.49 \\
3.45 \\
2.90 \\
2.09 \\
2.92 \\
7.49 \\
4.69 \\
1.84 \\
-\end{array}$ & $\begin{array}{r}9.72 \\
9.72 \\
9.72 \\
9.02 \\
7.41 \\
6.51 \\
5.59 \\
14.36 \\
10.12 \\
- \\
-\end{array}$ & $\begin{array}{r}9.46 \\
9.46 \\
- \\
9.46 \\
9.99 \\
9.30 \\
6.10 \\
11.46 \\
17.61 \\
9.53 \\
- \\
-\end{array}$ & $\begin{array}{r}7.48 \\
7.48 \\
- \\
7.48 \\
8.41 \\
7.51 \\
4.25 \\
11.26 \\
15.59 \\
7.47 \\
-\end{array}$ & $\begin{array}{r}7.50 \\
7.50 \\
- \\
7.50 \\
7.55 \\
7.35 \\
4.18 \\
11.14 \\
13.58 \\
7.58 \\
2.64\end{array}$ & $\begin{array}{r}7.78 \\
7.78 \\
7.78 \\
7.41 \\
7.51 \\
4.04 \\
10.88 \\
14.61 \\
7.87 \\
- \\
-\end{array}$ & $\begin{array}{r}8.64 \\
8.64 \\
- \\
8.64 \\
8.28 \\
7.88 \\
5.33 \\
10.22 \\
17.90 \\
8.84 \\
2.60 \\
-\end{array}$ \\
\hline $\begin{array}{l}\text { Total Energy" } \\
\text { Primary Energy - Four Sectors } \\
\text { Electricity }\end{array}$ & $\begin{array}{l}2.39 \\
1.96 \\
6.05\end{array}$ & $\begin{array}{r}4.40 \\
3.55 \\
10.33\end{array}$ & $\begin{array}{r}9.28 \\
8.23 \\
14.33\end{array}$ & $\begin{array}{r}10.57 \\
8.55 \\
20.81\end{array}$ & $\begin{array}{r}9.24 \\
6.83 \\
22.51\end{array}$ & $\begin{array}{r}9.51 \\
6.81 \\
24.16\end{array}$ & $\begin{array}{r}9.60 \\
6.91 \\
23.69\end{array}$ & $\begin{array}{r}10.34 \\
7.76 \\
23.46\end{array}$ \\
\hline $\begin{array}{l}\text { Electric Utillty Sector } \\
\text { Coal } \\
\text { Natural Gas . } \\
\text { Petroleum } \\
\text { Heavy Oib } \\
\text { Light Oilb } \\
\text { Petroleum Coke } \\
\text { Nuclear Fuel } \\
\text { Wood and Waste }\end{array}$ & $\begin{array}{r}.72 \\
.49 \\
- \\
.91 \\
.83 \\
.92 \\
- \\
- \\
-\end{array}$ & $\begin{array}{r}.36 \\
2.05 \\
1.17 \\
2.41 \\
1.95 \\
2.42 \\
. \\
. \\
-\end{array}$ & $\begin{array}{r}.69 \\
1.73 \\
4.50 \\
6.28 \\
- \\
6.28 \\
- \\
.58 \\
1.74\end{array}$ & $\begin{array}{r}.72 \\
2.03 \\
4.84 \\
5.83 \\
- \\
5.83 \\
- \\
.64 \\
.79\end{array}$ & $\begin{array}{r}.73 \\
1.88 \\
2.26 \\
3.55 \\
- \\
3.55 \\
- \\
.70 \\
.32\end{array}$ & $\begin{array}{r}.73 \\
- \\
- \\
3.39 \\
- \\
3.39 \\
.69 \\
.95\end{array}$ & $\begin{array}{r}.74 \\
- \\
- \\
3.71 \\
- \\
3.71 \\
- \\
.71 \\
.87\end{array}$ & $\begin{array}{r}.66 \\
- \\
2.39 \\
4.23 \\
- \\
4.23 \\
.62 \\
.82\end{array}$ \\
\hline 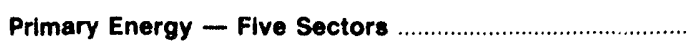 & 1.91 & 2.40 & 5.64 & 5.86 & 5.28 & 4.70 & 4.65 & 5.35 \\
\hline
\end{tabular}

-There are no direct fuel costs for hydroelectric, gecthermal, centralized solar, or wind energy. Wood and other biomass fuels are not included, except those consumed at electric utilities.

bHeavy oil includes Grade Nos. 4, 5, and 6, and residual fuel oils. Light oil includes Grade No. 2 heating oil, kerosene, and jet fuel.

-No consumption, incliding cases where adjustments were made. See "Consumption Adjustments for Process Fuel and Intermediate Products."

Sources: Data sources, estimation procedures, and assumptions are described in the "Documentation" section of this report. 


\begin{tabular}{|c|c|c|c|c|c|c|c|c|}
\hline Sector and Energy Source & 1970 & 1975 & 1980 & 1985 & 1986 & 1987 & 1988 & 1989 \\
\hline $\begin{array}{c}\text { Resldentlal Sector } \\
\text { Primary Energy }\end{array}$ & $\begin{array}{l}71.8 \\
44.1\end{array}$ & $\begin{array}{r}125.1 \\
69.3\end{array}$ & $\begin{array}{l}219.8 \\
124.1\end{array}$ & $\begin{array}{l}265.3 \\
154.1\end{array}$ & $\begin{array}{l}201.0 \\
109.7\end{array}$ & $\begin{array}{l}245.4 \\
112.2\end{array}$ & $\begin{array}{l}260.4 \\
117.5\end{array}$ & $\begin{array}{l}310.4 \\
161.4\end{array}$ \\
\hline Coal & .6 & .5 & .7 & 2.3 & .7 & .7 & .6 & .3 \\
\hline 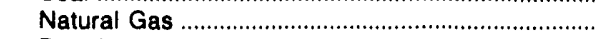 & 2.1 & 3.0 & 8.1 & 9.1 & 10.0 & 10.4 & 10.6 & 11.9 \\
\hline 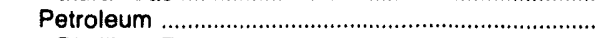 & 41.4 & 65.8 & 115.3 & 142.7 & 98.9 & 101.1 & 106.3 & 149.2 \\
\hline 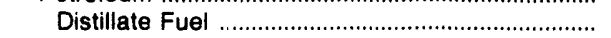 & 34.0 & 51.9 & 92.5 & 104.5 & 69.9 & 68.1 & 70.0 & 85.5 \\
\hline 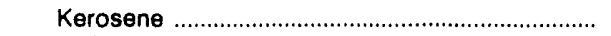 & 4.0 & 4.2 & 10.6 & 11.9 & 6.0 & 5.6 & 5.5 & 5.8 \\
\hline 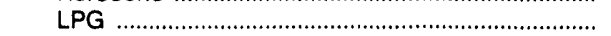 & 3.4 & 9.7 & 12.1 & 26.3 & 23.0 & 27.4 & 30.8 & 57.9 \\
\hline 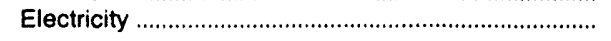 & 27.7 & 55.8 & 95.8 & 111.2 & 91.3 & 133.3 & 143.0 & 149.0 \\
\hline Commerclal Sector & 23.2 & 45.0 & 88.3 & 120.1 & 122.6 & 157.3 & 165.8 & 173.3 \\
\hline 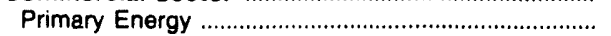 & 9.1 & 17.6 & 39.3 & 41.5 & 35.5 & 38.1 & 40.8 & 46.3 \\
\hline Coal & .2 & .4 & .2 & 1.6 & .2 & .2 & .2 & .1 \\
\hline 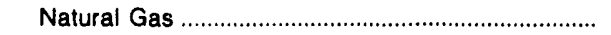 & .8 & 1.6 & 5.1 & 9.0 & 8.6 & 8.9 & 9.1 & 9.9 \\
\hline 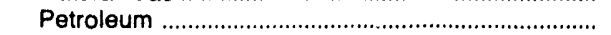 & 8.1 & 15.6 & 34.1 & 31.0 & 26.6 & 28.9 & 31.5 & 36.3 \\
\hline Distillate Fuel & 5.1 & 9.1 & 23.4 & 23.3 & 18.1 & 20.1 & 21.8 & 24.9 \\
\hline 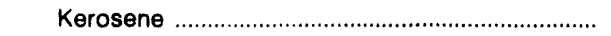 & .1 & .2 & 1.6 & .7 & .8 & .6 & .8 & .9 \\
\hline LPG & 3 & 1.1 & 1.3 & 4.5 & 4.6 & 5.4 & 6.6 & 8.2 \\
\hline 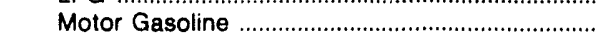 & .4 & .7 & 1.7 & 2.0 & 1.6 & 1.6 & 1.6 & 1.7 \\
\hline 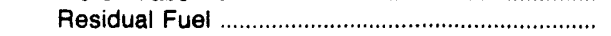 & 2.1 & 4.5 & 6.1 & .5 & 1.6 & 1.3 & .7 & .7 \\
\hline 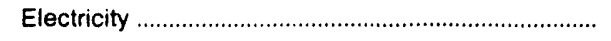 & 14.1 & 27.4 & 49.0 & 78.6 & 87.1 & 119.2 & 125.0 & 127.1 \\
\hline Industrlal Sector & 20.9 & 40.5 & 88.1 & 146.2 & 156.5 & 133.5 & 131.9 & 135.0 \\
\hline 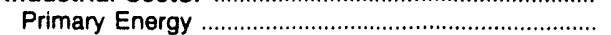 & 8.8 & 18.2 & 39.7 & 50.8 & 48.7 & 48.2 & 43.0 & 45.0 \\
\hline 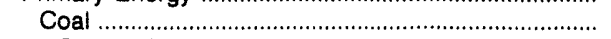 & .1 & .1 & .1 & .3 & .2 & .1 & .2 & .3 \\
\hline 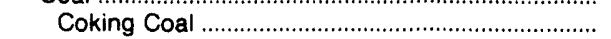 & - & - & - & - & - & - & - & - \\
\hline 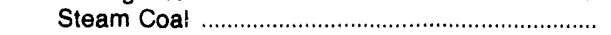 & .1 & .1 & .1 & .3 & .2 & .1 & .2 & .3 \\
\hline 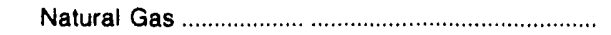 & .9 & 2.2 & 7.9 & 9.1 & 6.1 & 5.8 & 5.2 & 5.7 \\
\hline 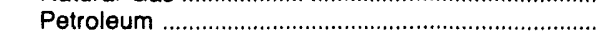 & 7.8 & 15.8 & 31.7 & 41.4 & 42.5 & 42.2 & 37.6 & 39.0 \\
\hline Asphalt and Road Oil . & 1.2 & .4 & 1.0 & 11.4 & 13.5 & 11.6 & 8.9 & 9.5 \\
\hline Distillate Fuel & 2.3 & 5.1 & 17.1 & 17.5 & 12.8 & 13.3 & 15.4 & 15.4 \\
\hline Kerosene & .2 & 1.0 & 3 & .5 & .4 & .6 & 1.1 & .4 \\
\hline LPG & .6 & 1.9 & 5.0 & 2.8 & 5.4 & 6.8 & 3.3 & 4.9 \\
\hline Lubricants ................................................. & .5 & .5 & 1.3 & 1.5 & 1.3 & 1.3 & 1.3 & 1.6 \\
\hline 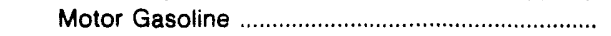 & 1.1 & 1.9 & 1.0 & 5.8 & 4.7 & 4.8 & 5.1 & 5.9 \\
\hline 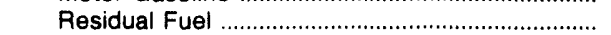 & 1.5 & 5.1 & 5.9 & 2.0 & 3.9 & 3.4 & 2.1 & .8 \\
\hline Other & .3 & - & - & - & .4 & .5 & .4 & .3 \\
\hline 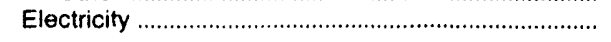 & 12.1 & 22.3 & 48.4 & 95.3 & 107.8 & 85.3 & 89.0 & 90.0 \\
\hline Transportation Sector & 86.0 & 149.9 & 329.5 & 348.4 & 282.1 & 318.2 & 343.8 & 364.7 \\
\hline 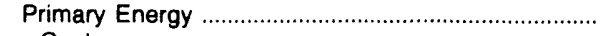 & 86.0 & 149.9 & 329.5 & 348.4 & 282.1 & 318.2 & 343.8 & 364.7 \\
\hline Coal & & & - & - & - & - & - & - \\
\hline Petroleum ………1-1) & 86.0 & 149.9 & 329.5 & 348.4 & 282.1 & 318.2 & 343.8 & 364.7 \\
\hline Aviation Gasoline & .2 & .2 & 1.1 & 1.1 & 1.2 & .8 & .6 & .7 \\
\hline 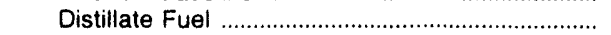 & 2.9 & 8.5 & 32.7 & 52.0 & 45.4 & 55.5 & 60.6 & 54.7 \\
\hline Jet Fuel & .5 & 1.5 & 4.9 & 6.7 & 3.1 & 4.2 & 3.2 & 6.6 \\
\hline 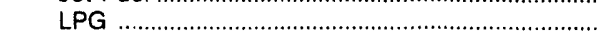 & 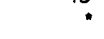 & & & .4 & .3 & .4 & .3 & .3 \\
\hline 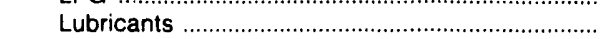 & 1.5 & 2.1 & 4.5 & 5.1 & 4.4 & 4.3 & 4.5 & 5.6 \\
\hline Motor Gasoline .............................................. & 81.0 & 137.6 & 286.2 & 283.1 & 227.7 & 253.0 & 274.6 & 296.6 \\
\hline 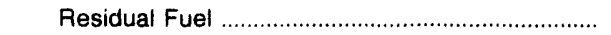 & * & $\cdot$ & - & - & - & .1 & - & .1 \\
\hline 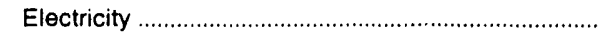 & - & - & - & - & - & - & - & - \\
\hline Total Energy" & 201.9 & 360.5 & 725.7 & 880.0 & 762.2 & 854.4 & 902.1 & 983.4 \\
\hline Primary Energy - Four Sectors ……………….......... & 147.9 & 255.0 & 532.6 & 594.9 & 476.0 & 516.6 & 545.1 & 617.4 \\
\hline 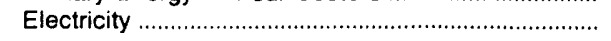 & 53.9 & 105.6 & 193.1 & 285.1 & 286.2 & 337.8 & 357.0 & 366.0 \\
\hline Electric Utility Sector & 2.2 & 14.5 & 23.4 & 26.1 & 17.3 & 29.2 & 33.9 & 27.0 \\
\hline Coal & .7 & .7 & .4 & 1.4 & .6 & - & - & - \\
\hline Natural Gas …1... & - & .7 & 1.1 & .5 & $\cdot$ & - & - & .1 \\
\hline Petroleum & 1.6 & 1.2 & 2.3 & 1.1 & .9 & 1.4 & 1.6 & 1.2 \\
\hline Heavy Oib & .1 & • & & - & - & - & - & - \\
\hline Light Oilb & 1.4 & 1.2 & 2.3 & 1.1 & .9 & 1.4 & 1.6 & 1.2 \\
\hline 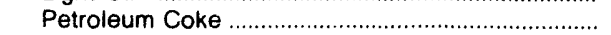 & - & - & - & - & - & - & - & - \\
\hline 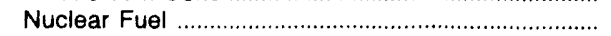 & - & 12.0 & 18.7 & 20.8 & 15.5 & 26.3 & 31.3 & 24.1 \\
\hline Wood and Waste & - & - & .9 & 2.3 & .3 & 1.5 & .9 & 1.6 \\
\hline Primary Energy - Five Sectors ……………......... & 150.2 & 269.5 & 556.0 & 621.0 & 493.3 & 545.9 & 578.9 & 644.3 \\
\hline
\end{tabular}

-There are no direct fuel costs for hydrcelectric, geothermal, centralized solar, or wind er.argy. Wood and other biomass fuels are not included, except those consumed at electric utilities.

b Heavy oil includes Grade Nos. 4, 5, and 6, and residual fuel oils. Light oil includes Grade No. 2 heating oil, kerosene, and jet fuel.

-No consumption, including cases where adjustments were made. See "Consumption Adjustments for Process Fuel and Intermediate Products."

"Value less than 0.05 million dollars.

Note: Totals may not equal sum of components due to independent rounding.

Sources: Data sources, estimation procedures, and assumptions are described in the "Documentation" section of this report. 


\section{Energy Price and Expenditure Estimates by Source, Virginia I $1970,1975,1980$, and 1985-1989}

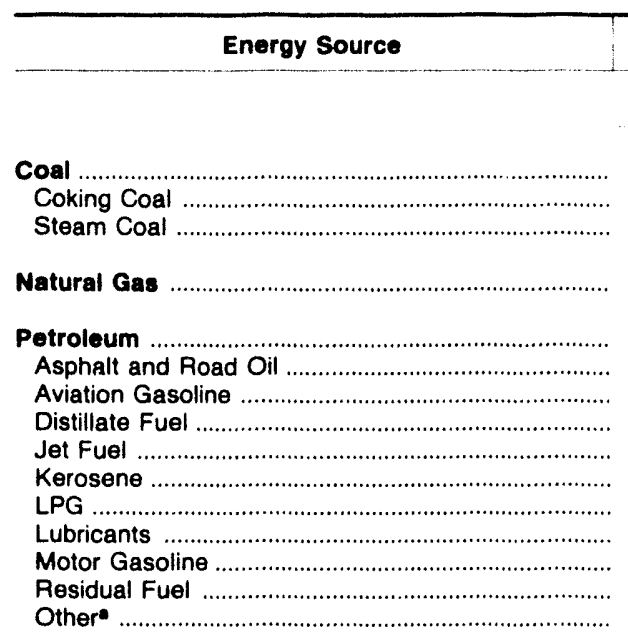

\begin{tabular}{|c|c|c|c|c|c|c|c|}
\hline 1970 & 1975 & 1980 & 1985 & 1986 & 1987 & 1988 & 1989 \\
\hline \multicolumn{8}{|c|}{ Prices In Dollars per Milition Btu } \\
\hline 0.44 & 1.30 & 1.72 & 1.80 & 1.70 & 1.60 & 1.57 & 1.57 \\
\hline .40 & - & 1.86 & 1.93 & 1.75 & 1.72 & 1.73 & 1.75 \\
\hline .41 & 1.30 & 1.71 & 1.78 & 1.69 & 1.59 & 1.56 & 1.56 \\
\hline .96 & 1.71 & 3.62 & 5.68 & 5.06 & 4.53 & 4.40 & 4.95 \\
\hline 1.49 & 3.16 & 7.47 & 8.16 & 5.96 & 6.41 & 6.43 & 6.97 \\
\hline .68 & 1.82 & 3.60 & 4.93 & 4.21 & 3.29 & 3.25 & 2.96 \\
\hline 2.17 & 3.45 & 9.02 & 9.99 & 8.41 & 7.55 & 7.41 & 8.28 \\
\hline 1.14 & 2.60 & 6.84 & 7.77 & 5.91 & 6.09 & 6.00 & 6.54 \\
\hline .73 & 2.03 & 6.46 & 5.79 & 3.83 & 4.05 & 3.77 & 4.34 \\
\hline 1.36 & 2.93 & 7.59 & 8.12 & 6.50 & 6.00 & 6.03 & 6.58 \\
\hline 1.95 & 3.63 & 6.33 & 9.90 & 9.26 & 9.20 & 9.06 & 8.44 \\
\hline 5.08 & 7.49 & 14.36 & 17.61 & 15.59 & 13.58 & 14.61 & 17.90 \\
\hline 2.85 & 4.77 & 9.97 & 9.33 & 6.97 & 7.60 & 7.85 & 8.55 \\
\hline .31 & 1.80 & 3.75 & 4.25 & 2.13 & 3.01 & 2.23 & 2.68 \\
\hline .73 & 2.66 & 7.25 & 8.26 & 5.75 & 6.47 & 5.53 & 6.05 \\
\hline - & .28 & .74 & .55 & .53 & .56 & .53 & .39 \\
\hline- & - & - & - & - & - & - & - \\
\hline 1.17 & 2.52 & 5.31 & 5.12 & 4.04 & 4.27 & 4.21 & 4.68 \\
\hline .35 & 1.24 & 2.00 & 1.18 & 1.14 & 1.23 & 1.11 & 1.28 \\
\hline 4.91 & 9.63 & 15.77 & 17.06 & 16.97 & 17.03 & 16.64 & 17.26 \\
\hline 1.80 & 3.97 & 8.02 & 8.82 & 7.60 & 7.74 & 7.71 & 8.39 \\
\hline
\end{tabular}

Nuclear Fuel

Wood and Waste at Utilities

Primary Energy - Five Sectors

Electric Utility Fuel

Electricity Purchased by End Users

Total Energy

1975

1980

1985

1986

1987

1988

1989

Prices In Dollars per Militon Btu

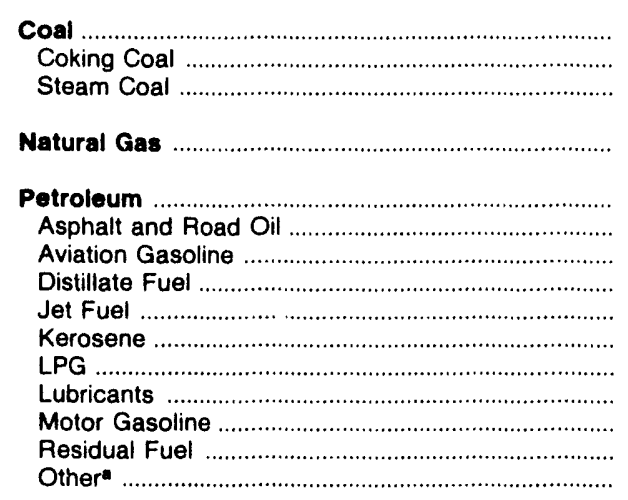

Nuclear Fuel.

Wood and Waste at Utilitles

Primary Energy - Five Sectors

Electric Utility Fuel

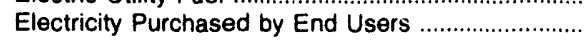

Total Energyb

Expenditures in Millions of Dollars

\begin{tabular}{|c|c|c|c|c|c|c|c|}
\hline 113.6 & 220.7 & 398.1 & 531.3 & 512.0 & 538.9 & 537.5 & 569.1 \\
\hline .3 & - & 33.0 & 45.7 & 42.0 & 43.4 & 42.8 & 44.0 \\
\hline 113.3 & 220.7 & 365.1 & 485.5 & 470.0 & 495.5 & 494.7 & 525.1 \\
\hline 126.6 & 205.0 & 548.0 & 783.7 & 706.0 & 707.2 & 701.7 & 851.4 \\
\hline $1,105.2$ & $2,590.3$ & $5,723.9$ & $5,384.6$ & $4,232.8$ & $4,763.9$ & $4,953.0$ & $5,293.4$ \\
\hline 10.2 & 28.1 & 62.6 & 132.0 & 124.2 & 96.2 & 77.6 & 82.4 \\
\hline 3.9 & 4.4 & 9.9 & 6.6 & 6.6 & 2.8 & 2.8 & 3.1 \\
\hline 163.6 & 344.3 & 980.1 & $1,139.9$ & 976.9 & $1,039.6$ & $1,138.9$ & $1,106.3$ \\
\hline 44.9 & 131.9 & 444.2 & 357.1 & 283.6 & 327.8 & 331.6 & 383.1 \\
\hline 38.9 & 37.7 & 73.9 & 84.1 & 52.5 & 48.8 & 48.1 & 49.0 \\
\hline 17.8 & 41.2 & 70.2 & 136.4 & 113.2 & 132.1 & 142.0 & 142.3 \\
\hline 22.2 & 33.3 & 82.9 & 92.5 & 80.1 & 78.9 & 81.8 & 102.8 \\
\hline 727.8 & $1,484.6$ & $3,092.9$ & $3,086.1$ & $2,387.4$ & $2,782.4$ & $2,936.2$ & $3,183.1$ \\
\hline 65.0 & 462.4 & 575.1 & 191.9 & 122.6 & 157.1 & 111.2 & 152.6 \\
\hline 11.0 & 22.4 & 332.0 & 157.9 & 85.8 & 98.4 & 82.8 & 88.6 \\
\hline - & 27.7 & 92.8 & 131.5 & 120.4 & 108.9 & 119.0 & 60.1 \\
\hline - & - & - & - & - & - & - & - \\
\hline $1,345.4$ & $3,043.6$ & $6,762.6$ & $6,831.0$ & $5,571.4$ & $6,118.9$ & $6,311.2$ & $6,774.1$ \\
\hline $\begin{array}{r}-101.4 \\
494.4\end{array}$ & $\begin{array}{r}-455.1 \\
1,280.5\end{array}$ & $\begin{array}{r}-726.4 \\
2,581.5\end{array}$ & $\begin{array}{r}-514.6 \\
3,343.0\end{array}$ & $\begin{array}{r}-510.6 \\
3,643.1\end{array}$ & $\begin{array}{r}-538.8 \\
3,868.1\end{array}$ & $\begin{array}{r}-522.4 \\
3,964.8\end{array}$ & $\begin{array}{r}-568.3 \\
4,307.7\end{array}$ \\
\hline $1,738.5$ & $3,869.0$ & $8,617.8$ & $9,659.4$ & $8,703.8$ & $9,448.2$ & $9,753.7$ & $10,513.5$ \\
\hline
\end{tabular}

-Includes petroleum coke used at electric utilities.

bThere are no direct fuel costs for hydroelectric, geothermal, centralized solar, or wind energy. Wood and other biomass fuels are not included, except those consumed at electric utilities.

-No consumption, including cases where adjustments were made. See "Consumption Adjustments for Process Fuel and Intermediate Products."

Note: Expenditure totals may not equal sum of components due to independent rounding.

Sources: Data sources, estimation procedures, and assumptions are described in the "Documentation" section of this report. 


\begin{tabular}{|c|c|c|c|c|c|c|c|c|}
\hline Sector and Energy Source & 1970 & 1975 & 1980 & 1985 & 1986 & 1987 & 1988 & 1989 \\
\hline 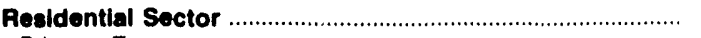 & 2.45 & 5.19 & 10.23 & 12.71 & 12.30 & 12.10 & 11.96 & 12.97 \\
\hline 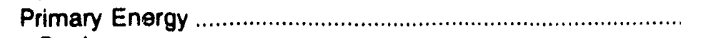 & 1.45 & 2.60 & 5.75 & 7.43 & 6.49 & 5.98 & 5.97 & 6.69 \\
\hline 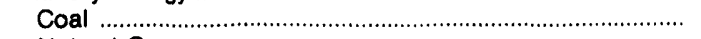 & 1.34 & 2.73 & 3.85 & 3.92 & 3.59 & 3.53 & 3.49 & 3.59 \\
\hline 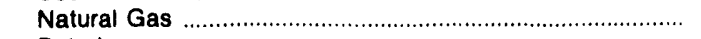 & 1.45 & 2.20 & 4.20 & 6.76 & 6.24 & 5.63 & 5.58 & 6.33 \\
\hline 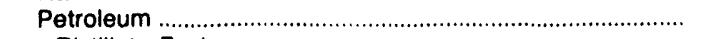 & 1.45 & 2.88 & 7.32 & 8.35 & 6.87 & 6.50 & 6.52 & 7.26 \\
\hline 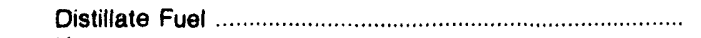 & 1.37 & 2.69 & 7.10 & 7.89 & 6.43 & 5.93 & 6.01 & 6.49 \\
\hline 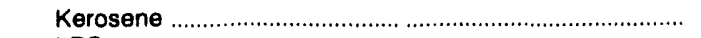 & 1.44 & 2.99 & 7.96 & 8.30 & 6.76 & 6.24 & 6.32 & 6.83 \\
\hline LPG & 2.27 & 4.43 & 8.07 & 10.48 & 9.86 & 10.08 & 9.81 & 11.03 \\
\hline 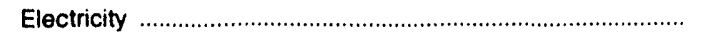 & 6.11 & 11.05 & 17.80 & 19.49 & 19.48 & 19.62 & 19.29 & 20.33 \\
\hline Commercial Sector & 2.53 & $\mathbf{5 . 5 0}$ & 10.06 & 12.16 & 11.62 & 11.33 & 11.12 & 11.81 \\
\hline 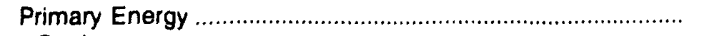 & .94 & 1.93 & 4.30 & 5.82 & 4.70 & 4.31 & 4.21 & 4.75 \\
\hline Coal & .42 & 1.47 & 1.64 & 1.69 & 1.61 & 1.55 & 1.53 & 1.56 \\
\hline 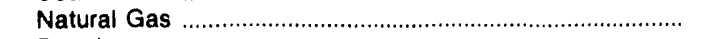 & .94 & 1.69 & 3.71 & 5.76 & 5.07 & 4.46 & 4.28 & 4.74 \\
\hline Petroleum & 1.18 & 2.60 & 6.32 & 6.81 & 4.64 & 4.65 & 4.67 & 5.31 \\
\hline 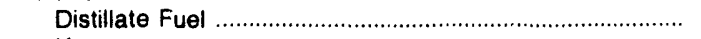 & 1.08 & 2.37 & 6.46 & 6.54 & 4.62 & 4.31 & 4.12 & 4.72 \\
\hline Kerosene & .65 & 2.36 & 5.94 & 6.58 & 4.55 & 4.38 & 4.20 & 5.22 \\
\hline LPG & 1.48 & 2.79 & 4.60 & 9.35 & 8.72 & 8.45 & 8.49 & 6.02 \\
\hline Motor Gasoline & 2.85 & 4.77 & 9.97 & 9.33 & 6.97 & 7.60 & 7.85 & 8.55 \\
\hline 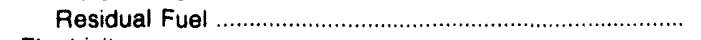 & .32 & 1.85 & 3.91 & 4.29 & 2.40 & 3.05 & 2.32 & 2.72 \\
\hline 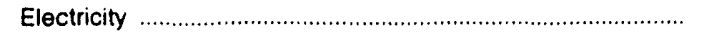 & 4.84 & 9.49 & 15.79 & 17.35 & 17.21 & 17.12 & 16.55 & 16.89 \\
\hline 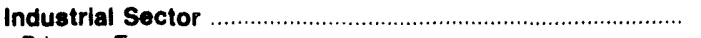 & .81 & 2.50 & 4.83 & 5.40 & 4.72 & 4.56 & 4.48 & 4.81 \\
\hline 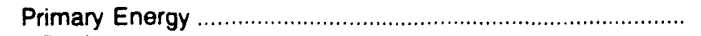 & .57 & 1.74 & 3.77 & 4.08 & 3.25 & 3.10 & 2.95 & 3.18 \\
\hline Coal & .42 & 1.47 & 1.69 & 1.74 & 1.64 & 1.59 & 1.57 & 1.60 \\
\hline 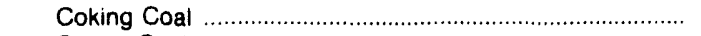 & .40 & - & 1.86 & 1.93 & 1.75 & 1.72 & 1.73 & 1.75 \\
\hline Steam Coal & .42 & 1.47 & 1.64 & 1.69 & 1.61 & 1.55 & 1.53 & 1.56 \\
\hline Natural Gas & .49 & 1.08 & 2.99 & 4.60 & 3.81 & 3.50 & 3.22 & 3.76 \\
\hline 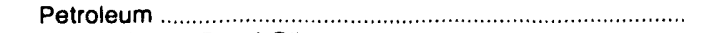 & .78 & 2.18 & 5.58 & 6.59 & 4.95 & 4.86 & 4.69 & 4.98 \\
\hline 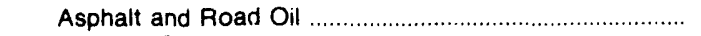 & .68 & 1.82 & 3.60 & 4.93 & 4.21 & 3.29 & 3.25 & 2.96 \\
\hline 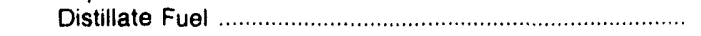 & .60 & 2.19 & 5.33 & 6.10 & 4.22 & 4.06 & 3.89 & 4.84 \\
\hline Kerosene & .65 & 2.36 & 5.94 & 6.58 & 4.55 & 4.38 & 4.20 & 5.22 \\
\hline LPG & 1.48 & 2.79 & 4.60 & 9.35 & 8.72 & 8.45 & 8.49 & 6.02 \\
\hline 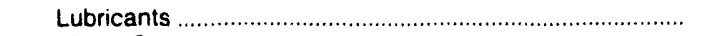 & 5.08 & 7.49 & 14.36 & 17.61 & 15.59 & 13.58 & 14.61 & 17.90 \\
\hline Moto: Gasoline & 2.85 & 4.77 & 9.97 & 9.33 & 6.97 & 7.60 & 7.85 & 8.55 \\
\hline 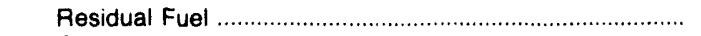 & .34 & 1.81 & 3.58 & 4.29 & 2.40 & 3.05 & 2.32 & 2.72 \\
\hline Other & .93 & 2.66 & 7.25 & 8.26 & 5.75 & 6.47 & 5.53 & 6.05 \\
\hline 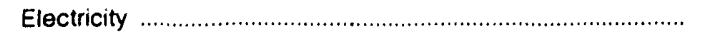 & 3.08 & 7.37 & 12.19 & 12.47 & 12.08 & 12.12 & 11.95 & 12.33 \\
\hline Transportation Sector & 1.95 & 3.91 & 8.73 & 8.55 & 6.33 & 6.90 & 6.94 & 7.59 \\
\hline 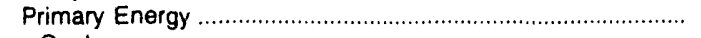 & 1.95 & 3.91 & 8.73 & 8.54 & 6.33 & 6.90 & 6.93 & 7.59 \\
\hline 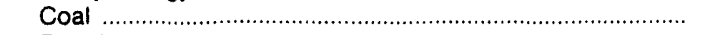 & .42 & 1.47 & - & - & - & - & - & - \\
\hline 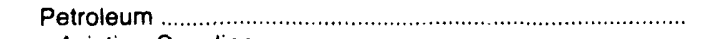 & 1.95 & 3.91 & 8.73 & 8.54 & 6.33 & 6.90 & 6.93 & 7.59 \\
\hline 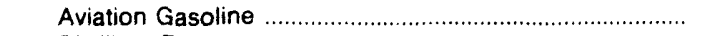 & 2.17 & 3.45 & 9.02 & 9.99 & 8.41 & 7.55 & 7.41 & 8.28 \\
\hline 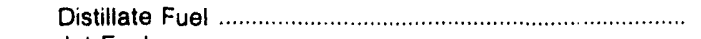 & 1.25 & 2.72 & 7.27 & 8.34 & 6.34 & 6.93 & 6.74 & 7.26 \\
\hline 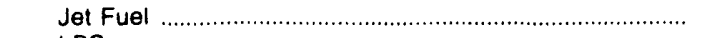 & .73 & 2.03 & 6.46 & 5.79 & 3.83 & 4.05 & 3.77 & 4.34 \\
\hline LPG & 1.48 & 2.79 & 4.60 & 9.35 & 8.72 & 8.45 & 8.49 & 6.02 \\
\hline 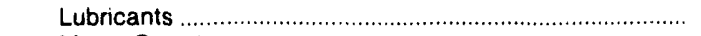 & 5.08 & 7.49 & 14.36 & 17.61 & 15.59 & 13.58 & 14.61 & 17.90 \\
\hline Motor Gasoline & 2.85 & 4.77 & 9.97 & 9.33 & 6.97 & 7.60 & 7.85 & 8.55 \\
\hline 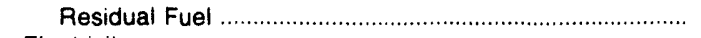 & .30 & 1.61 & 3.32 & 4.18 & 1.92 & 2.83 & 1.99 & 2.35 \\
\hline Electricity & - & - & 14.65 & 17.33 & 18.20 & 16.57 & 16.10 & 15.09 \\
\hline Total Energy^ & 1.80 & 3.97 & 8.02 & 8.82 & 7.60 & $\mathbf{7 . 7 4}$ & 7.71 & 8.39 \\
\hline 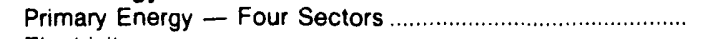 & 1.44 & 3.08 & 6.62 & 7.03 & 5.44 & 5.62 & 5.64 & 6.18 \\
\hline 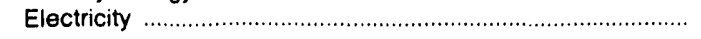 & 4.91 & 9.63 & 15.77 & 17.06 & 16.97 & 17.03 & 16.64 & 17.26 \\
\hline Electric Utility Sector & .35 & 1.24 & 2.00 & 1.18 & 1.14 & 1.23 & 1.11 & 1.28 \\
\hline Coal & 38 & 1.14 & 1.71 & 1.80 & 1.71 & 1.58 & 1.55 & 1.55 \\
\hline 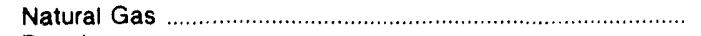 & .29 & .99 & 2.89 & 3.44 & 1.93 & 2.42 & 2.05 & 2.48 \\
\hline 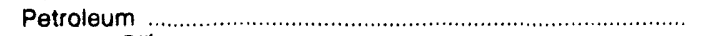 & .32 & 1.85 & 4.03 & 4.60 & 2.16 & 3.11 & 2.38 & 2.88 \\
\hline 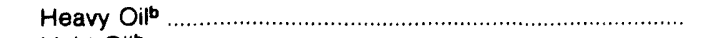 & .31 & 1.84 & 3.94 & 4.37 & 2.09 & 3.06 & 2.30 & 2.78 \\
\hline 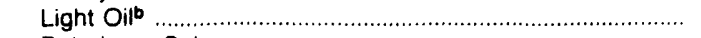 & .35 & 2.18 & 5.86 & 5.57 & 3.39 & 3.73 & 3.38 & 4.01 \\
\hline 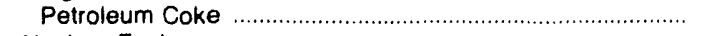 & 35 & - & - & - & - & - & - & - \\
\hline 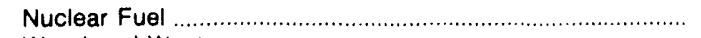 & - & .28 & .74 & .55 & .53 & .56 & .53 & .39 \\
\hline Wood and Waste & - & - & - & - & - & - & - & - \\
\hline 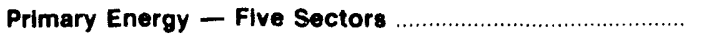 & 1.17 & 2.52 & 5.31 & 5.12 & 4.04 & 4.27 & 4.21 & 4.68 \\
\hline
\end{tabular}

- There are no direct fuel costs for hydroelectric, geothermal, centralized solar, or wind energy. Wood and other biomass fuels are not included, except those consumed at electric utilities.

bHeavy oil includes Grade Nos. 4, 5, and 6, and residual fuel oils. Light oil includes Grade No. 2 heating oil, kerosene, and jet fuel.

- No consumption, including cases where adjustments were made. See "Consumption Adjustments for Process Fuel and intermediate Products."

Sources: Data sources, estimation procedures, and assumptions are described in the "Documentation" section of this report. 


\section{Energy Expenditure Estimates by Sector, Virginia \\ I 1970, 1975, 1980, and 1985-1989 \\ R (Million Dollars)}

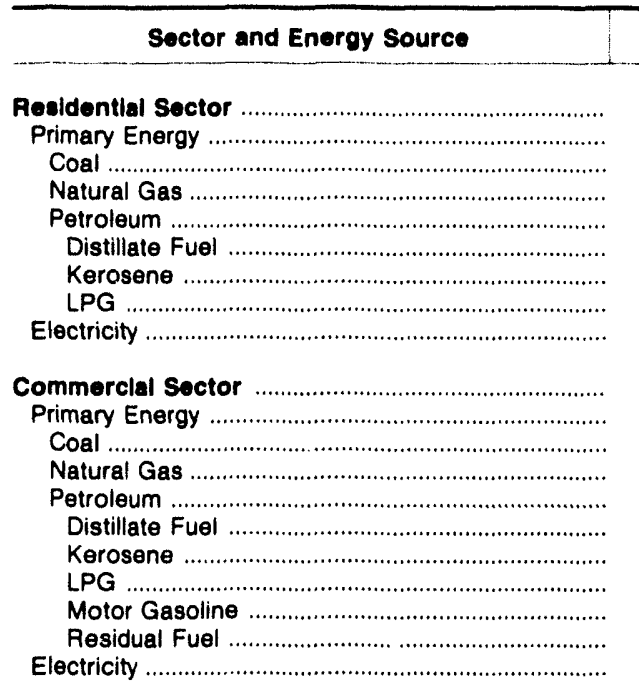

Industrial Sector

Primary Energy

Coal .....

Coking Coal

Steam Coal

Natural Gas

Petroleum

Asphalt and Road OII

Distillate Fuel

Kerosene

LPG

Lubricants

Motor Gasoline

Residual Fuel

Other

Electricity

Transportation Sector

Primary Energy

Coal

Petroleum

Aviation Gasoline

Distillate Fuel

Jet Fuel

LPG

Lubricants .....

Motor Gasolin

Residual Fuel

Electricity

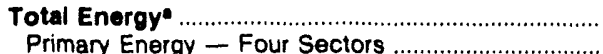

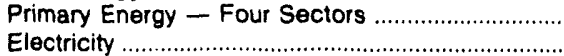

Electric Utllity Sector

Coal

Natural Gas

Petroleum ....

Heavy Oilb

Light Oilb

Petroleum Coke

Nuclear Fuel

Wood and Waste

Primary Energy - Five Sectors

1970

1970

446.8
206.2

5.3
73.8

73.8

127.1

77.7
37.1

37.1
12.3

240.5

228.6

50.2

3.1

28.9
18.2

18.2
13.1

13.1
.3
1.4

1.4
3.1

3.1
.2

178.4

207.4

131.9

42.1

.3

41.8
22.5

22.5
67.2

10.2

15.3

1.5
3.8

8.9

9.8

8.6
8.6

9.2
75.5

1975

1985

1987

1988

1989

$$
319.8
$$

$\begin{array}{rr}918.4 & 1,851.9\end{array}$

7.3
109.5

$2,235.8$

$2,368.0$

$2,475$.

5559.0

$2,810.3$

203.0

142.4

34.9
25.7

598.6

653.6
6.4

735.2

233.9

413.3

342.4

383.5

305.3
63.4

63.4
44.7

$1,198.3$

236.3
77.0

70.2

$1,500.6$

690.4
7.7
334.3

675.7

10.1

324.8

348.4

340.9

229.4

48.1

57.0

$1,677.6$

67.2

$1,799.3$

$1,161.9$
247.7

103.5

$1,622.4$

$1,684.8$

304.0

$1,763.0$

302.6

7.4

5.0

203.3

55.5

40.8

26.8

144.9

97.8
61.5

139.0

93.7

1.5

4.5

19.4

10.9
914.2

3.6
11.0

22.4

2.9

453.5

914.2

577.1

348.8
97.0

$1,474.8$

$1,007.3$

148.4

33.0

115.4

161.8

697.0

62.6

111.0

9.0

20.3

36.8

14.6

110.9

332.0

8.3
$1,272.8$

$1,564.4$

$1,564.4$
997.9

183.7

45.7

138.0

232.5

581.8

132.0

105.7

3.5

52.5

33.6

55.5

157.8
566.4

228.3

6.4
186.8

186.8
110.8

110.8
76.1

1.9

8.9

14.5

9.3
380.7

$1,380.7$

$1,364.8$
784.3

175.8

42.0

133.8

183.2

425.3

124.2

80.7

2.5
46.0

35.5

25.2

25.5

85.8
580.5

$4,236.8$

$4,129.1$

$4,127.6$

$4,233.6$

$3,286.3$

3,282.

$4,127.6$

$4,233.6$

$3,282.1$

$1,816.5$

4.9
4.9

9.9
475.3

6.6

693.2
357.1

2.7

131.9

444.2

2.7
51.5

46.1

$3,058.9$

?.030.1

$1,465.4$

64.4

92.3

92.3

6.6
571.4

283.6

1.4

44.6

$2,377.6$
27.0

27.0

182.8

182.8
111.6

65.2

2.8

10.0

13.3

$1,460.4$

$1,409.6$

805.4

184.4

43.4

141.0
195.3

425.7

96.2

82.3

1.8

52.7

35.0

29.5

29.9

98.4

604.1

$3,800.5$

$3,796.3$

$3,796 . \overline{3}$

$\begin{array}{r}2.8 \\ \hline\end{array}$

655.5

2.2

43.9

$2,732.6$

31.5

$\mathbf{9 , 6 5 9 . 4}$

8,617.8

$6,036.3$

$6,316.4$

$5,060.8$

$\mathbf{9 , 4 4 8 . 2}$

$5,580.1$

$3,868.1$

$2,588.6$
$1,280.5$

$3,343.0$

510.6

101.4

455.1

726.4

514.8

238.2
7.3

330.9

558.8

$388.1-5.5$

$361.0 \quad 35.7$

$27.1 \quad 11.0$

$92 . \overline{8}$

$131 . \overline{5}$

3.7
66.3 
Energy Price and Expenditure Estimates by Source, Washington 1970, 1975, 1980, and 1985-1989

\begin{tabular}{|c|c|c|c|c|c|c|c|c|}
\hline Emorgy Source & 1970 & 1975 & 1980 & 1985 & 1986 & 1987 & 1988 & 1989 \\
\hline & \multicolumn{8}{|c|}{ Prices in Dollars per Million Btu } \\
\hline Conl & 0.54 & 0.61 & 1.15 & 1.74 & 1.78 & 1.73 & 1.62 & 1.62 \\
\hline Coking Coal & - & - & - & - & - & - & - & - \\
\hline 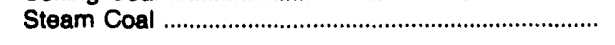 & .54 & .61 & 1.15 & 1.74 & 1.78 & 1.73 & 1.62 & 1.62 \\
\hline 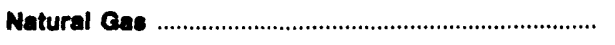 & .71 & 1.60 & 4.48 & 5.23 & 4.53 & 3.81 & 3.96 & 4.02 \\
\hline 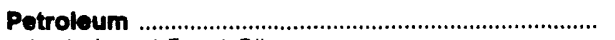 & 1.71 & 3.24 & 7.10 & 7.78 & 5.67 & 5.85 & 5.72 & 6.23 \\
\hline 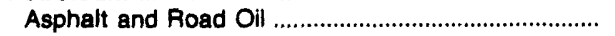 & .66 & 1.77 & 3.70 & 4.17 & 3.94 & 3.21 & 3.41 & 3.29 \\
\hline 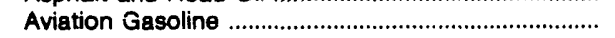 & 2.17 & 3.45 & 9.02 & 9.99 & 8.41 & 7.55 & 7.41 & 8.28 \\
\hline 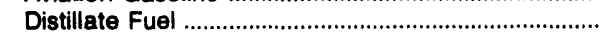 & 1.18 & 2.55 & 6.68 & 7.80 & 5.95 & 6.22 & 6.31 & 7.06 \\
\hline Jet Fuel & .73 & 2.04 & 6.21 & 6.03 & 4.01 & 4.02 & 3.79 & 4.50 \\
\hline 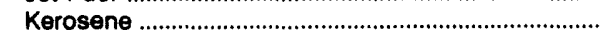 & 1.62 & 3.07 & 8.53 & 6.82 & 4.25 & 4.49 & 4.62 & 5.72 \\
\hline LPG & 2.50 & 4.46 & 6.78 & 9.09 & 9.23 & 8.98 & 9.01 & 9.30 \\
\hline 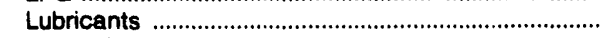 & 5.08 & 7.49 & 14.36 & 17.61 & 15.59 & 13.58 & 14.61 & 17.90 \\
\hline 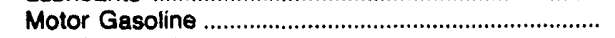 & 2.92 & 4.62 & 9.92 & 9.31 & 7.29 & 7.36 & 7.40 & 8.03 \\
\hline 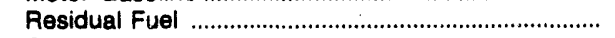 & .32 & 1.93 & 3.24 & 4.82 & 2.64 & 2.60 & 2.23 & 2.20 \\
\hline Other" & .58 & 1.45 & 2.83 & 3.24 & 2.83 & 2.06 & 1.87 & 2.24 \\
\hline 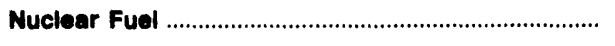 & .18 & .24 & .43 & .71 & .70 & .70 & .49 & .48 \\
\hline 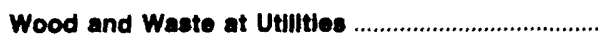 & .65 & - & - & .79 & .32 & .85 & .87 & .82 \\
\hline Primary Energy - Five Sectore ................................ & 1.42 & 2.51 & 5.83 & 5.96 & 4.72 & 4.76 & 4.65 & 5.03 \\
\hline 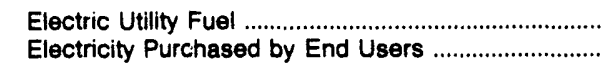 & $\begin{array}{r}.18 \\
2.02\end{array}$ & $\begin{array}{r}.46 \\
2.77\end{array}$ & $\begin{array}{r}.91 \\
4.16\end{array}$ & $\begin{array}{l}1.17 \\
9.18\end{array}$ & $\begin{array}{l}1.06 \\
9.41\end{array}$ & $\begin{array}{l}1.28 \\
9.80\end{array}$ & $\begin{array}{r}1.13 \\
10.13\end{array}$ & $\begin{array}{r}1.31 \\
10.34\end{array}$ \\
\hline \multirow[t]{2}{*}{ Total Energy' } & 1.59 & 2.81 & 5.98 & 7.75 & 6.44 & 6.55 & 6.63 & 6.98 \\
\hline & \multicolumn{8}{|c|}{ Expenditures in Millions of Dollars } \\
\hline Coal & 3.2 & 46.9 & 104.2 & $163 . \mathrm{A}$ & 112.7 & 165.3 & 160.9 & 157.5 \\
\hline 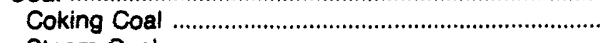 & - & - & $\overline{0}$ & - & $\overline{7}$ & $\overline{-}$ & $\operatorname{sen}^{-}$ & $\bar{c}$ \\
\hline 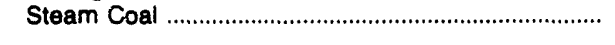 & 3.2 & 46.9 & 104.2 & 163.4 & 112.7 & 165.3 & 160.9 & 157.5 \\
\hline 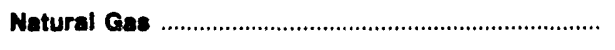 & 97.2 & 242.3 & 530.5 & 686.4 & 510.2 & 476.0 & 551.3 & 627.7 \\
\hline Potroloum & 811.5 & $1,628.6$ & $3,833.8$ & $4,132.9$ & $3,481.3$ & $3,595.2$ & $3,601.9$ & $4,173.9$ \\
\hline 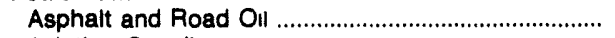 & 10.2 & 34.1 & 50.3 & 56.4 & 62.9 & 48.3 & 43.5 & 57.1 \\
\hline 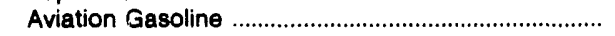 & 3.8 & 4.8 & 16.2 & 10.2 & 9.7 & 10.5 & 8.0 & 7.9 \\
\hline 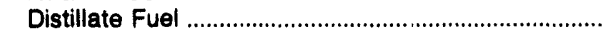 & 123.0 & 248.4 & 715.7 & 923.8 & 801.8 & 766.2 & 772.8 & 864.4 \\
\hline 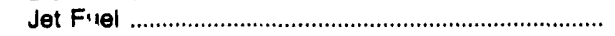 & 43.3 & 160.7 & 419.5 & 522.2 & 385.1 & 421.2 & 440.3 & 522.5 \\
\hline 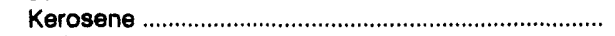 & 2.2 & 6.0 & 5.8 & 49.4 & 5.9 & 7.1 & 34.3 & 37.9 \\
\hline LPG & 15.3 & 11.5 & 33.4 & 72.9 & 76.0 & 92.9 & 74.7 & 98.3 \\
\hline 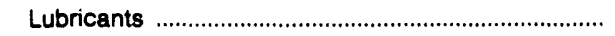 & 20.5 & 28.2 & 61.2 & 68.3 & 59.1 & 58.2 & 60.4 & 75.9 \\
\hline Motor Gasoline & 553.3 & 994.2 & $2,222.4$ & $2,151.5$ & $1,798.4$ & $1,977.9$ & $1,974.4$ & $2,268.1$ \\
\hline 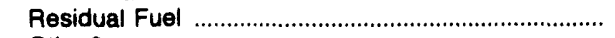 & 17.9 & 82.8 & 327.7 & 241.0 & 260.8 & 172.4 & 154.5 & 187.0 \\
\hline Other & 22.0 & 57.9 & 81.5 & 37.2 & 21.7 & 40.5 & 39.0 & 54.8 \\
\hline 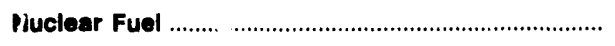 & 5.2 & 8.7 & 9.6 & 61.4 & 63.7 & 41.8 & 31.6 & 31.7 \\
\hline Wood and Waste at Utilities & * & - & - & 2.3 & .6 & 3.4 & 3.4 & 3.2 \\
\hline Primary Energy - Five Sectors ……........................... & 917.2 & $1,926.4$ & $4,578.1$ & $5,046.3$ & $4,168.6$ & $4,281.7$ & $4,349.0$ & $4,993.9$ \\
\hline 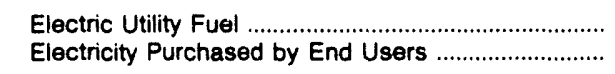 & $\begin{array}{r}-5.2 \\
316.8\end{array}$ & $\begin{array}{l}-46.5 \\
523.9\end{array}$ & $\begin{array}{l}-85.7 \\
95 \vdots 4\end{array}$ & $\begin{array}{r}-203.6 \\
2,331.7\end{array}$ & $\begin{array}{r}-155.9 \\
2,312.1\end{array}$ & $\begin{array}{r}-194.0 \\
2.427 .3\end{array}$ & $\begin{array}{r}-182.5 \\
2,800.5\end{array}$ & $\begin{array}{r}-221.6 \\
2,983.8\end{array}$ \\
\hline Total Energy' & $1,228.8$ & $2,403.8$ & $5,435 . i$ & $7,174.4$ & $6,324.8$ & $6,515.1$ & $6,966.9$ & $7,756.1$ \\
\hline
\end{tabular}

-Includes petroleum coke used at electric utilities.

There are no direct fuel costs for hydroelectric, geothermal, centralized solar, or wind energy. Wood and other biomass fuels are not included, except those consumed at electric utilities.

-No consumption, including cases where adjustments were made. See "Consumption Adjustments for Process Fuel and Intermediate Products."

* Value less than 0.05 million dollars.

Note: Expenditure totais may not equal sum of components due to independent rounding

Sources: Data source's, estimation procedures, and assumptions are described in the "Documentation" section of this report. 


\section{W Energy Price Estimates by Sector, Washington \\ A 1970, 1975, 1980, and 1985-1989 \\ S (Dollars per Million Btu)}

\begin{tabular}{|c|c|c|c|c|c|c|c|c|}
\hline Sector and Energy Source & 1970 & 1975 & 1980 & 1985 & 1986 & 1987 & 1988 & 1989 \\
\hline Residential Sector & 2.12 & 3.23 & 5.73 & 9.53 & 9.45 & 9.83 & 9.90 & 10.18 \\
\hline 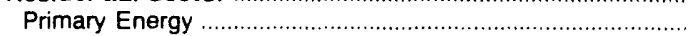 & 1.47 & 2.54 & 5.99 & 6.83 & 5.96 & 5.80 & 5.73 & 6.03 \\
\hline 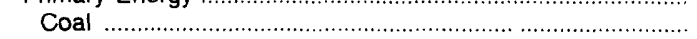 & .95 & 1.14 & 4.26 & 3.67 & 3.79 & 3.76 & 3.37 & 3.66 \\
\hline 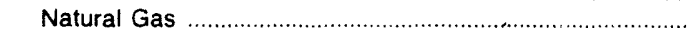 & 1.33 & 2.18 & 5.05 & 6.35 & 5.76 & 5.25 & 5.36 & 5.32 \\
\hline Petroleum & 1.57 & 2.97 & 7.40 & 7.89 & 6.34 & 6.67 & 6.50 & 7.61 \\
\hline Distillate Fuel & 1.40 & 2.80 & 7.27 & 7.76 & 5.95 & 6.10 & 6.03 & 6.71 \\
\hline 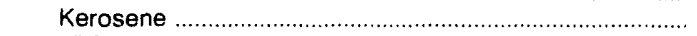 & 2.47 & 3.61 & 9.80 & 9.92 & 7.61 & 7.80 & 7.70 & 8.58 \\
\hline LPG & 3.05 & 5.73 & 8.12 & 8.46 & 9.77 & 10.26 & 10.31 & 13.86 \\
\hline 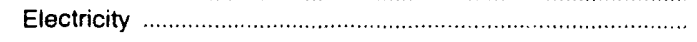 & 3.12 & 3.94 & 5.56 & 11.14 & 11.45 & 12.20 & 12.45 & 12.69 \\
\hline Commercial Sector & 1.90 & 2.94 & 5.35 & 8.03 & 8.24 & 8.14 & 7.92 & 8.42 \\
\hline 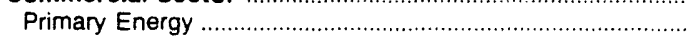 & 1.12 & 2.06 & 5.03 & 5.69 & 4.83 & 4.51 & 4.50 & 4.80 \\
\hline Coal & .52 & $\begin{array}{r}.00 \\
.90\end{array}$ & 2.28 & 2.30 & 2.22 & 2.36 & 2.34 & 2.38 \\
\hline 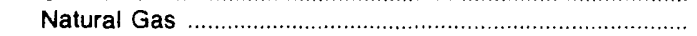 & 1.05 & 1.75 & 4.59 & 5.24 & 4.91 & 4.36 & 4.47 & 4.54 \\
\hline 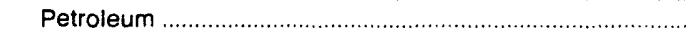 & 1.21 & 2.87 & 6.76 & 6.61 & 4.87 & 4.89 & 4.68 & 5.54 \\
\hline Distillate Fuel . & 1.21 & 2.60 & 6.90 & 6.53 & 4.63 & 4.55 & 4.53 & 5.31 \\
\hline 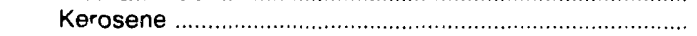 & .84 & 2.31 & 7.04 & 6.59 & 4.01 & 4.33 & 4.42 & 5.48 \\
\hline LFS & 1.19 & 2.67 & 5.60 & 9.34 & 9.06 & 8.52 & 8.58 & 8.09 \\
\hline 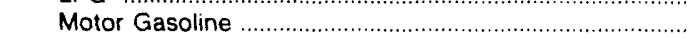 & 2.92 & 4.62 & 9.92 & 9.31 & 7.29 & 7.36 & 7.40 & 8.03 \\
\hline 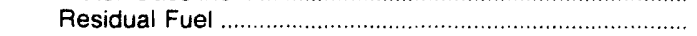 & .33 & 2.45 & 3.61 & 4.05 & 2.07 & 2.58 & 2.01 & 2.33 \\
\hline Electricity & 3.21 & 4.10 & 5.67 & 10.57 & 10.95 & 10.92 & 11.01 & 11.47 \\
\hline Industrial Sector & .67 & 1.51 & 3.39 & 5.52 & 4.68 & 4.66 & 5.24 & 5.35 \\
\hline 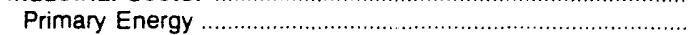 & .55 & 1.57 & 4.06 & 5.00 & 3.61 & 3.35 & 3.27 & 3.53 \\
\hline Coal & .52 & .90 & 2.28 & 2.30 & 2.22 & 2.36 & 2.34 & 2.38 \\
\hline 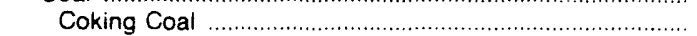 & - & - & - & - & - & - & - & 2.00 \\
\hline 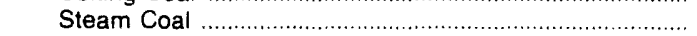 & .52 & .90 & 2.28 & 2.30 & 2.22 & 2.36 & 2.34 & 2.38 \\
\hline Natural Gas & .38 & 1.29 & 4.09 & 4.58 & 3.49 & 2.78 & 2.89 & 2.84 \\
\hline Petroleum & .60 & 1.84 & 4.16 & 5.58 & 3.80 & 3.86 & 3.67 & 4.13 \\
\hline Asphalt and Road Oil ................... & .66 & 1.77 & 3.70 & 4.17 & 3.94 & 3.21 & 3.41 & 3.29 \\
\hline 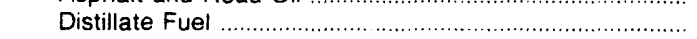 & .73 & 2.05 & 6.06 & 6.20 & 3.78 & 4.08 & 4.16 & 5.16 \\
\hline Kerosene & .84 & 2.31 & 7.04 & 6.59 & 4.01 & 4.33 & 4.42 & 5.48 \\
\hline 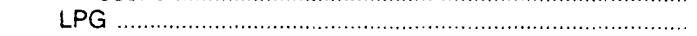 & 1.19 & 2.67 & 5.60 & 9.34 & 9.06 & 8.52 & 8.58 & 8.09 \\
\hline 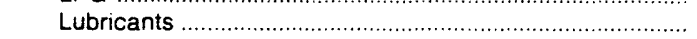 & 5.08 & 7.49 & 14.36 & 17.61 & 15.59 & 13.58 & 14.61 & 17.90 \\
\hline 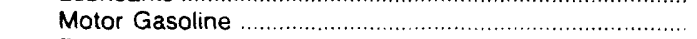 & 2.92 & 4.62 & 9.92 & 9.31 & 7.29 & 7.36 & 7.40 & 8.03 \\
\hline 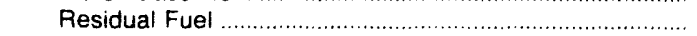 & .33 & 1.78 & 3.36 & 4.05 & 2.07 & 2.58 & 2.01 & 2.33 \\
\hline Other & .58 & 1.45 & 2.83 & 3.24 & 2.83 & 2.06 & 1.87 & 2.24 \\
\hline 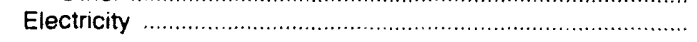 & .97 & 1.37 & 2.26 & 6.23 & 6.29 & 6.71 & 7.68 & 7.77 \\
\hline Transportation Sector ........... & 2.23 & 3.73 & 7.86 & 8.19 & 5.97 & 6.16 & 6.02 & 6.55 \\
\hline 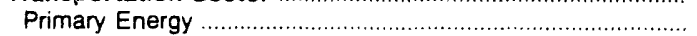 & 2.23 & 3.73 & 7.86 & 8.19 & 5.97 & 6.16 & 6.02 & $\begin{array}{l}0.00 \\
6.55\end{array}$ \\
\hline Coal & .52 & .90 & - & - & - & - & - & \\
\hline Petroleum & 2.23 & 3.73 & 7.86 & 8.19 & 5.97 & 6.16 & 6.02 & 6.55 \\
\hline 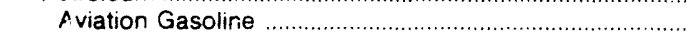 & 2.17 & 3.45 & 9.02 & 9.99 & 8.41 & 7.55 & 7.41 & 8.28 \\
\hline 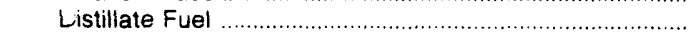 & 1.32 & 2.65 & 6.72 & 8.77 & 6.71 & 7.22 & 7.24 & 7.92 \\
\hline Jet Fuel & .73 & 2.04 & 6.21 & 6.03 & 4.01 & 4.02 & 3.79 & 4.50 \\
\hline LPG & 1.19 & 2.67 & 5.60 & 9.34 & 9.06 & 8.52 & 8.58 & 8.09 \\
\hline 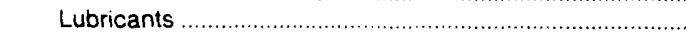 & 5.08 & 7.49 & 14.36 & 17.61 & 15.59 & 13.58 & 14.61 & 17.90 \\
\hline 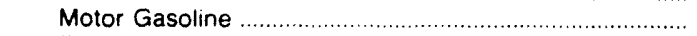 & 2.92 & 4.62 & 9.92 & 9.31 & 7.29 & 7.36 & 7.40 & 8.03 \\
\hline Residual Fuel ....…… & .30 & 2.14 & 3.15 & 5.01 & 2.86 & 2.60 & 2.27 & 2.18 \\
\hline 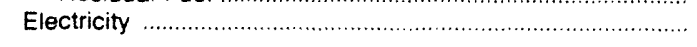 & 2.16 & 3.20 & 4.26 & 8.28 & 8.39 & 7.63 & 8.49 & 9.19 \\
\hline Total Energy" ................................ & 1.59 & 2.81 & 5.98 & 7.75 & 6.44 & 6.55 & 6.63 & 6.98 \\
\hline 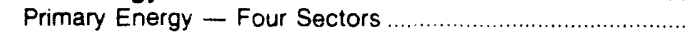 & 1.48 & 2.83 & 6.58 & 7.21 & 5.45 & 5.47 & 5.38 & 5.80 \\
\hline Electricity ……… & 2.02 & 2.77 & 4.16 & 9.18 & 9.41 & 9.80 & 10.13 & 10.34 \\
\hline Electric Utility Sector ............ & .18 & .46 & .91 & 1.17 & 1.06 & 1.28 & 1.13 & 1.31 \\
\hline 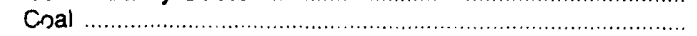 & - & .57 & .96 & 1.65 & 1.69 & 1.67 & 1.55 & 1.56 \\
\hline ivatural Gas & - & - & 3.43 & 4.54 & 2.81 & 2.50 & 3.05 & 5.02 \\
\hline 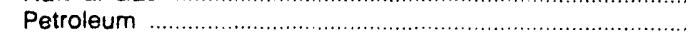 & .33 & 2.49 & 3.93 & 5.72 & 2.79 & 4.03 & 3.98 & 2.95 \\
\hline 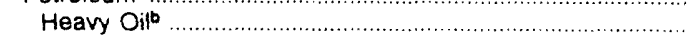 & .32 & 2.50 & 3.58 & - & 3.16 & 2.82 & 2.66 & 2.54 \\
\hline 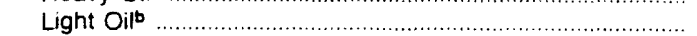 & .37 & 2.41 & 6.40 & 5.72 & 2.76 & 4.10 & 4.03 & 4.52 \\
\hline 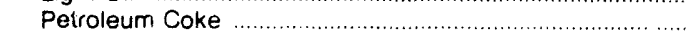 & - & - & - & - & - & - & 4.00 & 4.52 \\
\hline 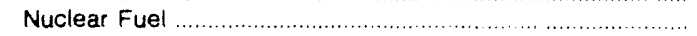 & .18 & .24 & .43 & .71 & .70 & .70 & .49 & .48 \\
\hline Wood and Waste & .65 & - & - & .79 & 32 & .95 & $\begin{array}{l}40 \\
.87\end{array}$ & $\begin{array}{l}.40 \\
.82\end{array}$ \\
\hline Primary Energy - Five Sectors . & 1.42 & 2.51 & 5.83 & 5.96 & 4.72 & 4.76 & 4.65 & 5.03 \\
\hline
\end{tabular}

2There are no direct fuel costs for hydroelectric geothermal, centralized solar, or wind energy. Wood and other biomass fuels are not included, except those consumed at electric utilities.

b Heavy oil includes Grade Nos. 4, 5, and 6, and residual fuel oils. Light oil includes Grade No. 2 heating oil, kerosene, and jet fuel.

-No consumption, including cases where adjustments were made. See "Consumption Adjustments for Process Fuel and Intermediate Products."

Sources: Data sources, estimation procedures. and assumbtions are described in the "Dnrumantatinn" sention of this report. 


\begin{tabular}{|c|c|c|c|c|c|c|c|c|}
\hline Sector and Energy Source & 1970 & 1975 & 1980 & 1985 & 1986 & 1987 & 1988 & 1989 \\
\hline Residential Sector & 280.8 & 427.3 & 794.5 & $1,450.0$ & $1,343.4$ & $1,372.2$ & $1,482.5$ & $1,596.6$ \\
\hline 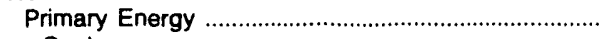 & 117.3 & 169.4 & 330.6 & 388.2 & 308.1 & 299.4 & 326.5 & 355.6 \\
\hline 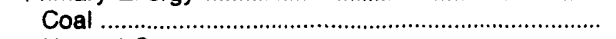 & 3 & .2 & 5.5 & 6.5 & 2.6 & 1.5 & 3.0 & 2.5 \\
\hline 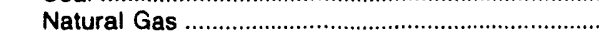 & 44.8 & 78.1 & 158.0 & 217.8 & 179.1 & 161.5 & 192.4 & 210.6 \\
\hline 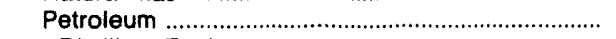 & 72.2 & 91.1 & 167.2 & 163.9 & 126.4 & 136.3 & 131.1 & 142.5 \\
\hline 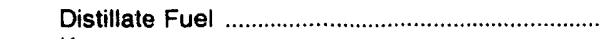 & 57.4 & 78.3 & 144.9 & 140.0 & 106.4 & 107.7 & 106.2 & 107.3 \\
\hline 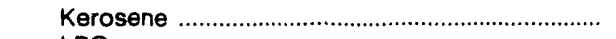 & 1.6 & 4.2 & 3.6 & 5.1 & .7 & .6 & 3.6 & 4.3 \\
\hline 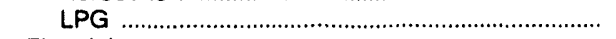 & 13.2 & 8.6 & 18.7 & 18.8 & 19.2 & 28.0 & 21.3 & 30.9 \\
\hline 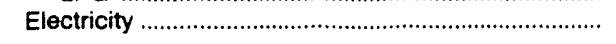 & 163.5 & 258.0 & 463.8 & $1,061.8$ & $1,035.3$ & $1,072.8$ & $1,156.0$ & $1,241.0$ \\
\hline Commercial Sector & 116.7 & 242.3 & 502.6 & $1,082.4$ & 949.5 & 966.3 & $1,064.8$ & $1,092.3$ \\
\hline 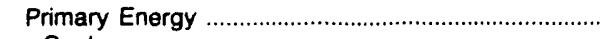 & 43.0 & 97.0 & 234.8 & 398.7 & 246.6 & 232.2 & 287.2 & 284.6 \\
\hline 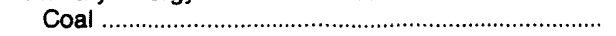 & .3 & .2 & 5.4 & 7.6 & 2.8 & 1.8 & 3.9 & 3.1 \\
\hline 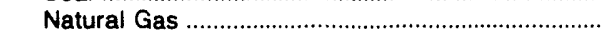 & 20.4 & 58.2 & 148.7 & 193.3 & 161.7 & 145.6 & 168.3 & 180.2 \\
\hline 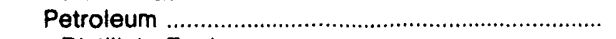 & 22.3 & 38.6 & 80.7 & 197.8 & 82.0 & 84.7 & 114.9 & 101.3 \\
\hline 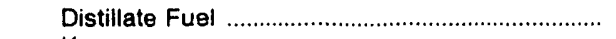 & 15.7 & 23.0 & 43.1 & 162.4 & 65.2 & 61.7 & 69.8 & 52.8 \\
\hline 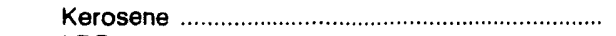 & .1 & .3 & .7 & 8.1 & .4 & 6.4 & 30.1 & 33.2 \\
\hline 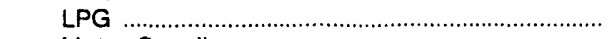 & .9 & .7 & 2.3 & 3.7 & 3.1 & 4.1 & 3.1 & 3.2 \\
\hline 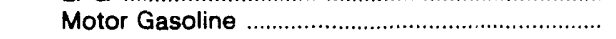 & 4.7 & 9.1 & 24.9 & 17.4 & 11.8 & 12.1 & 10.8 & 11.0 \\
\hline 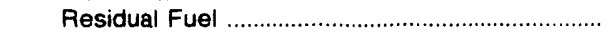 & 1.0 & 5.5 & 9.7 & 6.2 & 1.4 & .4 & 1.1 & 1.2 \\
\hline 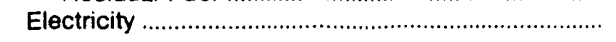 & 73.6 & 145.3 & 267.8 & 683.8 & 702.9 & 734.1 & 777.7 & 807.6 \\
\hline Industrial Sector & 197.4 & 443.7 & 898.4 & $1,228.0$ & $1,068.5$ & $1,103.6$ & $1,323.0$ & $1,498.9$ \\
\hline & 117.7 & 323.2 & 676.7 & 642.2 & 494.9 & 483.6 & 456.6 & 564.1 \\
\hline 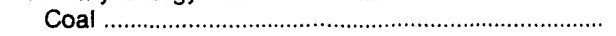 & 2.7 & 9.8 & 16.2 & 10.3 & 16.4 & 13.9 & 12.3 & 11.8 \\
\hline 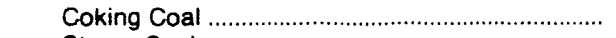 & - & - & & - & - & - & - & - \\
\hline 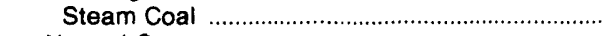 & 2.7 & 9.8 & 16.2 & 10.3 & 16.4 & 13.9 & 12.3 & 11.8 \\
\hline 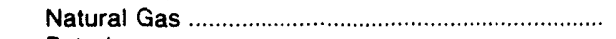 & 32.0 & 106.0 & 220.5 & 274.9 & 169.0 & 168.6 & 185.1 & 193.8 \\
\hline Petroleum & 83.0 & 207.3 & 440.0 & 357.0 & 309.5 & 301.1 & 259.2 & 358.5 \\
\hline Asphalt and Road Oil ............................................... & 10.2 & 34.1 & 50.3 & 56.4 & 62.9 & 48.3 & 43.5 & 57.1 \\
\hline 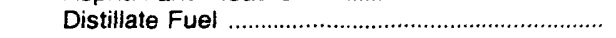 & 19.6 & 44.8 & 150.7 & 99.4 & 75.3 & 86.6 & 68.6 & 110.1 \\
\hline 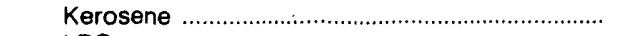 & .5 & 1.5 & 1.5 & 36.2 & 4.8 & .1 & .6 & .3 \\
\hline LPG & 1.1 & 1.8 & 10.5 & 40.4 & 43.6 & 52.8 & 42.4 & 58.1 \\
\hline 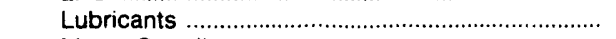 & 8.2 & 8.7 & 17.6 & 19.6 & 17.0 & 16.7 & 17.3 & 21.8 \\
\hline 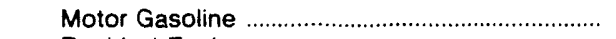 & 8.4 & 10.6 & 14.5 & 33.8 & 28.4 & 28.4 & 26.3 & 29.4 \\
\hline 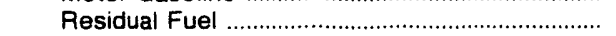 & 13.1 & 47.9 & 113.3 & 34.0 & 55.9 & 27.8 & 21.5 & 27.0 \\
\hline Other & 22.0 & 57.9 & 81.5 & 37.2 & 21.7 & 40.5 & 39.0 & 54.8 \\
\hline 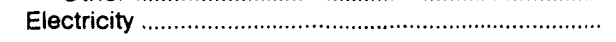 & 79.7 & 120.6 & 221.7 & 585.8 & 573.5 & 620.0 & 866.5 & 934.8 \\
\hline Transportation Sector & 634.0 & $1,290.3$ & $3,240.3$ & $3,414.0$ & $2,963.4$ & $3,072.9$ & $3,096.6$ & $3,568.3$ \\
\hline 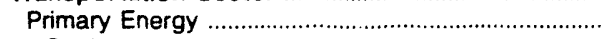 & 633.9 & $1,290.3$ & $3,240.3$ & $3,413.6$ & $2,963.1$ & $3,072.6$ & $3,096.3$ & $3,568.0$ \\
\hline Coal & & & - & - & - & - & & \\
\hline Petroleum & 633.9 & $1,290.3$ & $3,240.3$ & $3,413.6$ & $2,963.1$ & $3,072.6$ & $3,096.3$ & $3,568.0$ \\
\hline Aviation Gasoline & 3.8 & 4.8 & 16.2 & 10.2 & 9.7 & 10.5 & 8.0 & 7.9 \\
\hline Distillate Fuel & 30.3 & 102.2 & 375.8 & 521.4 & 554.4 & 509.7 & 527.9 & 593.1 \\
\hline 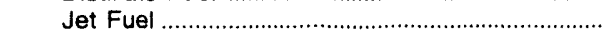 & 43.3 & 160.7 & 419.5 & 522.2 & 385.1 & 421.2 & 440.3 & 522.5 \\
\hline 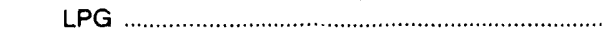 & .2 & .4 & 1.9 & 10.0 & 10.1 & 8.0 & 8.0 & 6.2 \\
\hline Lubricants & 12.3 & 19.4 & 43.6 & 48.7 & 42.2 & 41.5 & 43.1 & 54.1 \\
\hline 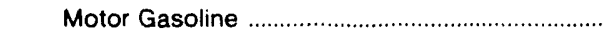 & 540.2 & 974.5 & $2,183.1$ & $2,100.2$ & $1,758.2$ & $1,937.4$ & $1,937.2$ & $2,227.8$ \\
\hline 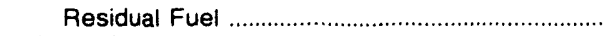 & 3.8 & 28.3 & 200.3 & 200.8 & 203.5 & 144.2 & 131.9 & 156.3 \\
\hline 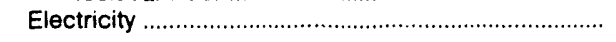 & & & & .4 & .3 & .3 & .3 & .4 \\
\hline 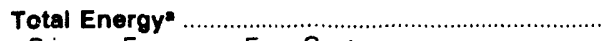 & $1,228.8$ & $2,403.8$ & $5,435.7$ & $7,174.4$ & $6,324.8$ & $6,515.1$ & $6,966.9$ & $7,756.1$ \\
\hline Primary Energy - Four Sectors ................................... & 911.9 & $1,879.9$ & $4,482.4$ & $4,842.7$ & $4,012.7$ & $4,087.8$ & $4,166.5$ & $4,772.3$ \\
\hline Electricity & 316.8 & 523.9 & 953.4 & $2,331.7$ & $2,312.1$ & $2,427.3$ & $2,800.5$ & $2,983.8$ \\
\hline Electric Utility Sector & 5.2 & 46.5 & 95.7 & 203.6 & 155.9 & 194.0 & 182.5 & 221.6 \\
\hline Coal & - & 36.7 & 77.1 & 139.0 & 90.9 & 148.1 & 141.6 & 140.0 \\
\hline 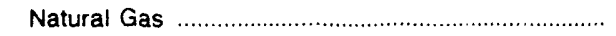 & - & - & 3.3 & .4 & .4 & .2 & 5.5 & 43.2 \\
\hline Petroleum & - & 1.2 & 5.7 & .6 & 3 & .4 & .4 & 3.5 \\
\hline 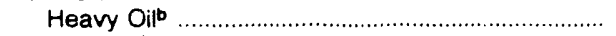 & - & 1.1 & 4.5 & - & - & • & • & 2.4 \\
\hline Light Oilb & - & .1 & 1.1 & .6 & .3 & .4 & .4 & 1.1 \\
\hline 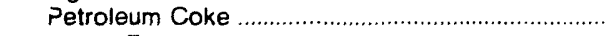 & - & - & - & - & - & - & - & - \\
\hline 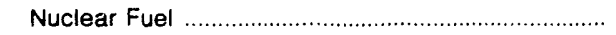 & 5.2 & 8.7 & 9.6 & 61.4 & 63.7 & 41.8 & 31.6 & 31.7 \\
\hline Wood and Waste & & - & & 2.3 & .6 & 3.4 & 3.4 & 3.2 \\
\hline Primary Energy - Five Sectors & 917.2 & $1,926.4$ & $4,578.1$ & $5,046.3$ & $4,168.6$ & $4,281.7$ & $4,349.0$ & $4,993.9$ \\
\hline
\end{tabular}

-There are no direct fuel costs for hydroelectric, geothermal, centralized solar, or wind energy. Wood and other biomass fuels are not included, except those consumed at electric utilities.

bHeavy oil includes Grade Nos. 4, 5, and 6, and residual fuel oils. Light oil includes Grade No. 2 heating oil, kerosene, and jet fuel.

-No consumption, including cases where adjustments were made. See "Consumption Adjustments for Process Fuel and Intermediaie Products."

- Value less than 005 millinn dollars

Note: Totals may not equal sum of components due to independent rounding.

Sources: Data sources, estimation procedures, and assumptions are described in the "Documentation" section of this report. 


\begin{tabular}{|c|c|c|c|c|c|c|c|c|}
\hline Energy Source & 1970 & 1975 & 1880 & 1985 & 1986 & 1987 & 1988 & 1989 \\
\hline & \multicolumn{8}{|c|}{ Prices in Dollars per Million Btu } \\
\hline 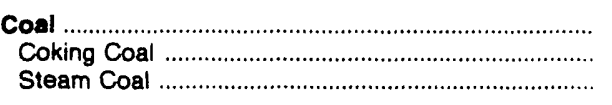 & $\begin{array}{r}0.31 \\
.40 \\
.28\end{array}$ & $\begin{array}{r}1.02 \\
1.51 \\
.94\end{array}$ & $\begin{array}{l}1.46 \\
1.86 \\
1.41\end{array}$ & $\begin{array}{l}1.61 \\
1.93 \\
1.59\end{array}$ & $\begin{array}{l}1.50 \\
1.75 \\
1.49\end{array}$ & $\begin{array}{l}1.43 \\
1.72 \\
1.41\end{array}$ & $\begin{array}{l}1.44 \\
1.73 \\
1.42\end{array}$ & $\begin{array}{l}1.43 \\
1.75 \\
1.41\end{array}$ \\
\hline Natural Gas & .62 & 1.16 & 3.18 & 5.28 & 4.99 & 4.48 & 4.08 & 4.07 \\
\hline $\begin{array}{l}\text { Petroleum } \\
\text { Asphalt and Road Oil } \\
\text { Aviation Gasoline } \\
\text { Distillate Fuel } \\
\text { Jet Fuel } \\
\text { Kerosene }\end{array}$ & $\begin{array}{r}1.77 \\
.68 \\
2.17 \\
1.40 \\
.73 \\
1.49 \\
1.67 \\
5.08 \\
2.86 \\
.58 \\
.87\end{array}$ & $\begin{array}{l}3.62 \\
1.81 \\
3.45 \\
3.36 \\
2.05 \\
2.83 \\
3.25 \\
7.49 \\
4.61 \\
1.89 \\
2.87\end{array}$ & $\begin{array}{r}8.06 \\
3.58 \\
9.02 \\
7.24 \\
6.46 \\
8.19 \\
6.16 \\
14.36 \\
9.96 \\
3.33 \\
7.19\end{array}$ & $\begin{array}{r}8.41 \\
4.91 \\
9.99 \\
8.01 \\
6.87 \\
7.93 \\
9.08 \\
17.61 \\
9.19 \\
4.01 \\
7.37\end{array}$ & $\begin{array}{r}5.87 \\
4.19 \\
8.41 \\
5.87 \\
5.14 \\
6.34 \\
7.97 \\
15.59 \\
7.00 \\
2.57 \\
3.95\end{array}$ & $\begin{array}{r}6.31 \\
3.23 \\
7.55 \\
5.97 \\
4.72 \\
6.09 \\
8.18 \\
13.58 \\
7.34 \\
2.81 \\
4.81\end{array}$ & $\begin{array}{r}6.12 \\
3.35 \\
7.41 \\
5.88 \\
4.45 \\
6.08 \\
10.20 \\
14.61 \\
7.52 \\
2.38 \\
3.98\end{array}$ & $\begin{array}{r}6.97 \\
3.13 \\
8.28 \\
6.62 \\
4.89 \\
6.90 \\
7.77 \\
17.90 \\
8.52 \\
2.65 \\
4.82\end{array}$ \\
\hline 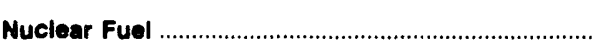 & - & - & - & - & - & - & - & - \\
\hline 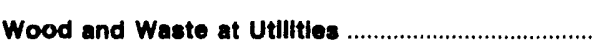 & .65 & - & - & - & - & - & - & - \\
\hline Primary Energy - Five Sectors …............................ & .68 & 1.58 & 3.16 & 3.20 & 2.61 & 2.64 & 2.58 & 2.74 \\
\hline 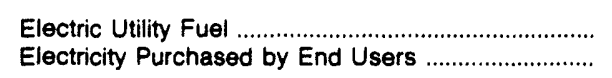 & $\begin{array}{r}.26 \\
3.96\end{array}$ & $\begin{array}{r}.88 \\
8.30\end{array}$ & $\begin{array}{r}1.43 \\
10.58\end{array}$ & $\begin{array}{r}1.62 \\
14.19\end{array}$ & $\begin{array}{r}1.50 \\
14.81\end{array}$ & $\begin{array}{r}1.43 \\
14.77\end{array}$ & $\begin{array}{r}1.45 \\
14.10\end{array}$ & $\begin{array}{r}1.43 \\
14.04\end{array}$ \\
\hline \multirow[t]{2}{*}{ Total Energy ${ }^{b}$} & 1.13 & 2.79 & 5.78 & 7.35 & 6.00 & 6.10 & 5.78 & 6.13 \\
\hline & \multicolumn{8}{|c|}{ Expenditures In Millions of Dollars } \\
\hline $\begin{array}{l}\text { Coal } \\
\text { Coking Coal } \ldots \ldots \ldots \ldots \ldots\end{array}$ & $\begin{array}{r}187.2 \\
55.3 \\
131.9\end{array}$ & $\begin{array}{l}833.9 \\
178.3 \\
655.6\end{array}$ & $\begin{array}{r}1,253.8 \\
190.2 \\
1,063.6\end{array}$ & $\begin{array}{r}1,399.0 \\
72.4 \\
1,326.6\end{array}$ & $\begin{array}{r}1,315.2 \\
89.0 \\
1,226.2\end{array}$ & $\begin{array}{r}1,241.8 \\
81.0 \\
1,160.8\end{array}$ & $\begin{array}{r}1,310.5 \\
94.3 \\
1,225.2\end{array}$ & $\begin{array}{r}1,326.2 \\
91.5 \\
1,234.7\end{array}$ \\
\hline 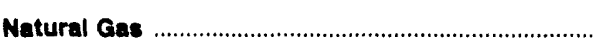 & 108.3 & 171.0 & 415.1 & 510.6 & 472.4 & 444.9 & 434.9 & 474.7 \\
\hline $\begin{array}{l}\text { Petroleum } \\
\text { Asphalt and Road Oil } \\
\text { Aviation Gasoline } \\
\text { Distillate Fuel } \\
\text { Jet Fuel } \\
\text { Kerosene } \\
\text { LPG } \\
\text { Motor Gasoline } \\
\text { Residual Fuel } \\
\text { Other }\end{array}$ & $\begin{array}{r}383.5 \\
3.9 \\
.9 \\
31.9 \\
1.2 \\
2.7 \\
7.8 \\
21.1 \\
237.6 \\
7.5 \\
69.0\end{array}$ & $\begin{array}{r}927.6 \\
11.3 \\
1.0 \\
114.2 \\
2.8 \\
5.2 \\
18.1 \\
31.2 \\
467.7 \\
26.2 \\
250.0\end{array}$ & $\begin{array}{r}2,380.6 \\
17.0 \\
3.0 \\
441.1 \\
12.9 \\
23.0 \\
77.7 \\
58.4 \\
1,014.2 \\
24.8 \\
708.4\end{array}$ & $\begin{array}{r}1,915.1 \\
14.0 \\
1.9 \\
451.7 \\
9.0 \\
14.2 \\
27.2 \\
65.2 \\
894.0 \\
14.8 \\
423.1\end{array}$ & $\begin{array}{r}1,352.0 \\
15.7 \\
2.1 \\
261.1 \\
6.3 \\
10.7 \\
26.1 \\
56.4 \\
686.3 \\
8.5 \\
278.8\end{array}$ & $\begin{array}{r}1,501.6 \\
11.5 \\
1.3 \\
312.3 \\
5.6 \\
10.3 \\
27.3 \\
55.5 \\
743.9 \\
5.3 \\
328.6\end{array}$ & $\begin{array}{r}1,517.7 \\
19.5 \\
1.4 \\
310.6 \\
6.2 \\
9.3 \\
38.1 \\
57.6 \\
781.3 \\
5.8 \\
287.8\end{array}$ & $\begin{array}{r}1,757.6 \\
16.0 \\
1.6 \\
388.4 \\
10.6 \\
9.7 \\
36.5 \\
72.4 \\
871.5 \\
5.0 \\
345.0\end{array}$ \\
\hline Nuclear Fuel . . & - & - & - & - & - & - & - & - \\
\hline 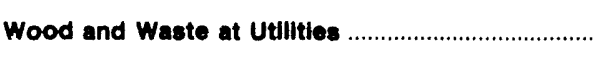 & * & - & - & - & - & - & - & - \\
\hline Primary Energy - Five Sectore ……......................... & 679.1 & $1,932.5$ & $4,049.5$ & $3,824.6$ & $3,139.7$ & $3,188.2$ & $3,272.1$ & $3,558.5$ \\
\hline 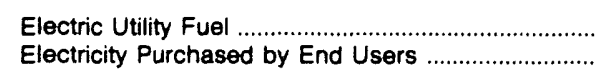 & $\begin{array}{l}-89.9 \\
204.3\end{array}$ & $\begin{array}{r}-531.0 \\
477.3\end{array}$ & $\begin{array}{r}-997.7 \\
748.8\end{array}$ & $\begin{array}{r}-1,261.8 \\
1,000.4\end{array}$ & $\begin{array}{r}-1,156.2 \\
1,029.6\end{array}$ & $\begin{array}{r}-1,093.2 \\
1,053.0\end{array}$ & $\begin{array}{r}-1,148.1 \\
1,068.2\end{array}$ & $\begin{array}{r}-1,155.4 \\
1,084.8\end{array}$ \\
\hline Total Energyb & 793.5 & $1,878.8$ & $3,800.5$ & $3,563.2$ & $3,013.1$ & $3,148.1$ & $3,192.2$ & $3,488.0$ \\
\hline
\end{tabular}

- Includes petroleum coke used at electric utilities.

bThere are no direct fuel costs for hydroelectric, geothermal, centralized solar, or wind energy. Wood and other biomass fuels are not included, except those consumed at electric utilities.

- No consumption, including cases where adjustments were made. See "Consumption Adjustments for Process Fuel and Intermediate Products."

- Value less than 0.05 million dollars.

Note: Expenditure totals may not equal sum of components due to independent rounding.

Sources: Data sources, estimation procedures, and assumptions are described in the "Documentation" section of this report. 


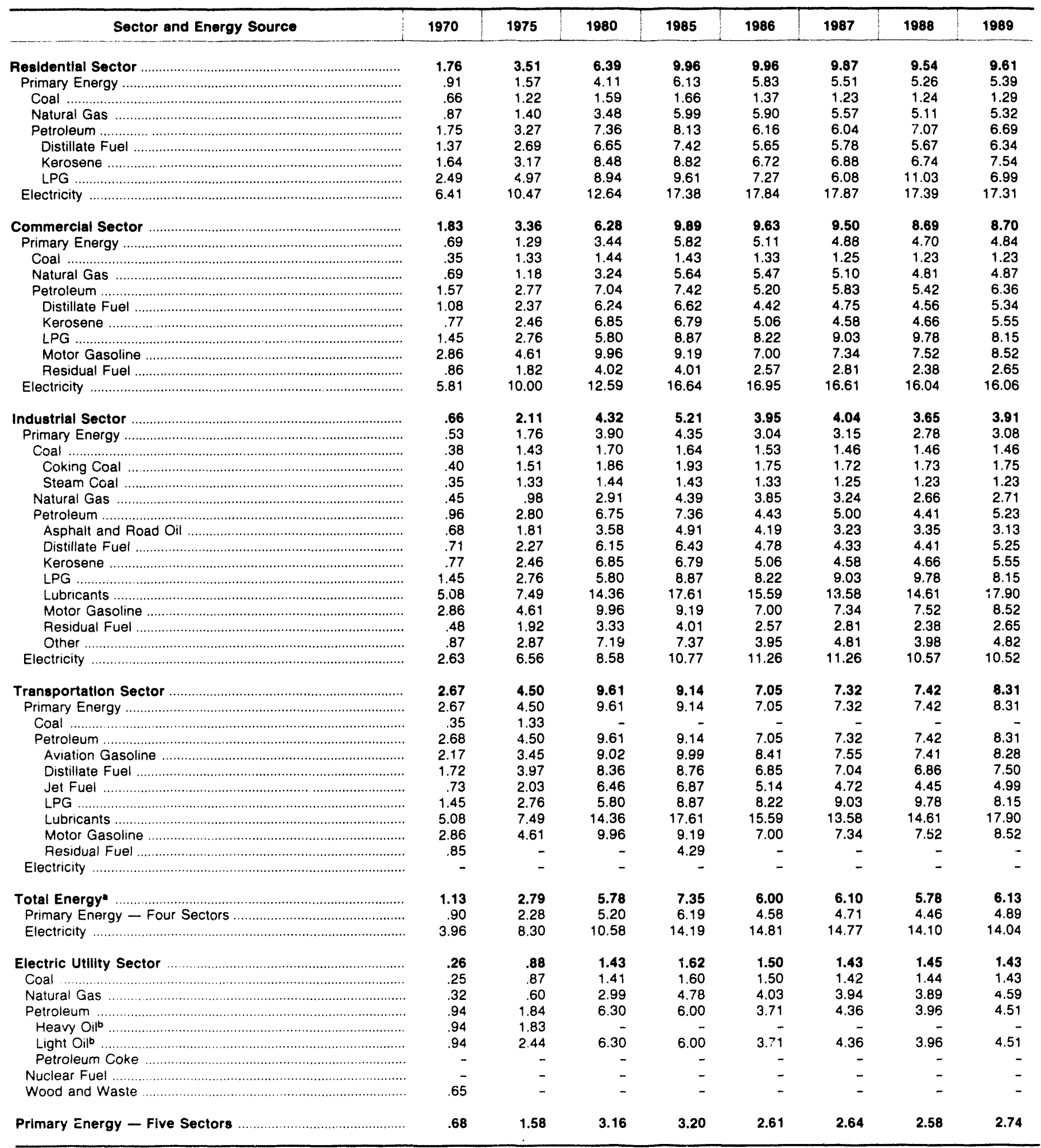

- There are no direct fuel costs for hydroelectric, geothermal, centralized solar, or wind energy. Wood and other biomass fuels are not included, except those consumed at electric utilities.

bHeavy oil includes Grade Nos. 4, 5, and 6, and residual fuel oils. Light oil includes Grade No. 2 heating oil, kerosene, and jet fuel.

-No consumption, including cases where adjustments were made. See "Consumption Adjustments for Process Fuel and Intermediate Products."

Sources: Data sources, estimation procedures, and assumptions are described in the "Documentation" section of this report. 


\section{W Energy Expenditure Estimates by Sector, West Virginia \\ E $1970,1975,1980$, and 1985-1989 \\ S (Million Dollars)}

\begin{tabular}{|c|c|c|c|c|c|c|c|c|}
\hline Sector and Energy Source & 1970 & 1975 & 1980 & 1985 & 1986 & 1987 & 1988 & 1989 \\
\hline Residential Sector & 135.3 & 273.1 & 538.5 & 670.8 & 689.7 & 691.5 & 696.5 & 707.3 \\
\hline 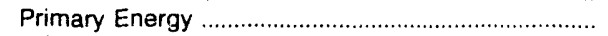 & 59.7 & 95.2 & 253.6 & 272.8 & 264.6 & 249.5 & 248.5 & 256.3 \\
\hline 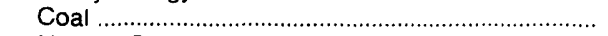 & 1.1 & 2.4 & 2.1 & 1.2 & 1.4 & 1.5 & 1.2 & 1.7 \\
\hline 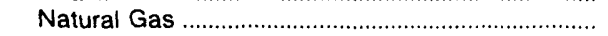 & 51.7 & 74.5 & 173.6 & 234.7 & 229.8 & 213.1 & 207.3 & 212.7 \\
\hline 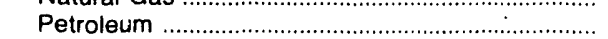 & 7.0 & 18.3 & 77.9 & 36.8 & 33.4 & 34.9 & 40.0 & 41.8 \\
\hline 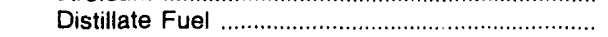 & 2.0 & 9.1 & 45.3 & 20.0 & 18.4 & 21.4 & 18.9 & 23.8 \\
\hline 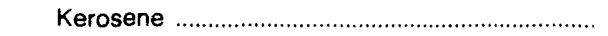 & 2.5 & 3.1 & 19.6 & 8.9 & 8.8 & 7.7 & 7.0 & 7.2 \\
\hline LPG & 2.5 & 6.1 & 13.0 & 8.0 & 6.3 & 5.9 & 14.0 & 10.8 \\
\hline 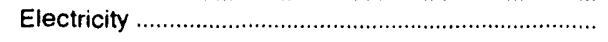 & 75.6 & 177.9 & 284.9 & 398.1 & 425.1 & 442.0 & 448.0 & 451.0 \\
\hline Commercial Sector & 62.5 & 137.8 & 252.4 & 400.6 & 397.1 & 391.9 & 414.1 & 433.3 \\
\hline 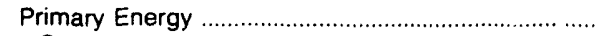 & 18.1 & 40.3 & 95.2 & 147.2 & 130.0 & 122.3 & 145.1 & 158.4 \\
\hline 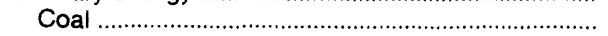 & 1.0 & 4.9 & 3.5 & 1.8 & 2.4 & 2.9 & 2.3 & 3.0 \\
\hline 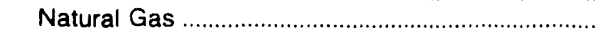 & 15.3 & 30.2 & 73.4 & 103.7 & 94.2 & 92.0 & 116.1 & 122.1 \\
\hline 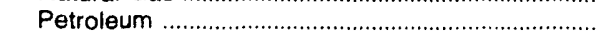 & 1.8 & 5.2 & 18.3 & 41.7 & 33.4 & 27.4 & 26.7 & 33.3 \\
\hline 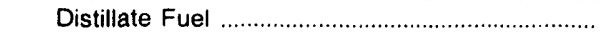 & .6 & 2.9 & 9.5 & 23.3 & 19.3 & 12.5 & 9.5 & 15.4 \\
\hline 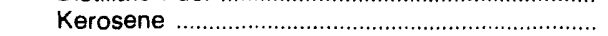 & .1 & .1 & 1.4 & 2.2 & .8 & .9 & 1.0 & 1.2 \\
\hline 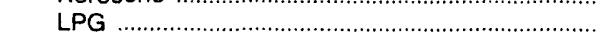 & 3 & .6 & 1.5 & 1.3 & 1.2 & 1.5 & 2.2 & 2.2 \\
\hline 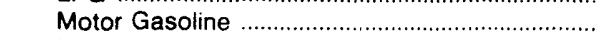 & .8 & 1.4 & 5.7 & 14.8 & 12.0 & 12.4 & 12.2 & 13.8 \\
\hline 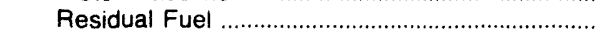 & • & .1 & .1 & .1 & .1 & & 1.9 & .6 \\
\hline 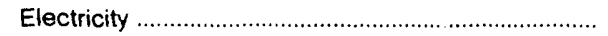 & 44.4 & 97.5 & 157.1 & 253.4 & 267.0 & 269.6 & 269.0 & 275.0 \\
\hline Industrial Sector & 327.8 & 905.7 & $1,731.5$ & $1,262.0$ & $1,066.8$ & $1,109.2$ & $1,084.3$ & $1,201.2$ \\
\hline 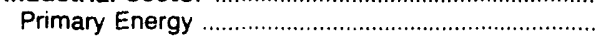 & 243.5 & 703.8 & $1,424.9$ & 913.1 & 729.3 & 767.8 & 733.1 & 842.3 \\
\hline 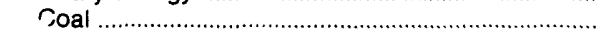 & 97.9 & 304.0 & 275.7 & 147.6 & 164.7 & 154.9 & 176.4 & 177.2 \\
\hline 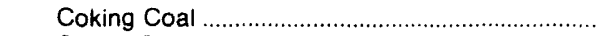 & 55.3 & 178.3 & 190.2 & 72.4 & 89.0 & 81.0 & 94.3 & 91.5 \\
\hline 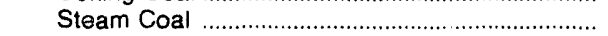 & 42.6 & 125.7 & 85.6 & 75.2 & 75.7 & 73.9 & 82.1 & 85.7 \\
\hline 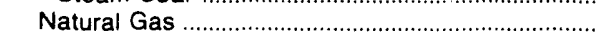 & 41.2 & 66.1 & 167.9 & 171.6 & 147.3 & 138.8 & 111.2 & 139.3 \\
\hline 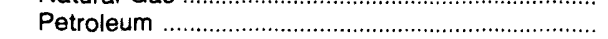 & 104.5 & 333.7 & 981.2 & 593.8 & 417.4 & 474.0 & 445.5 & 525.7 \\
\hline 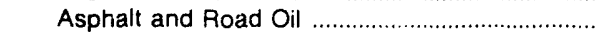 & 3.9 & 11.3 & 17.0 & 14.0 & 15.7 & 11.5 & 19.5 & 16.9 \\
\hline 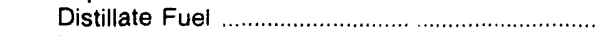 & 4.5 & 19.1 & 125.3 & 69.7 & 51.4 & 63.6 & 66.2 & 78.8 \\
\hline 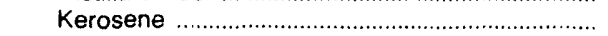 & .2 & 2.0 & 2.0 & 3.1 & 1.1 & 1.8 & 1.3 & 1.4 \\
\hline 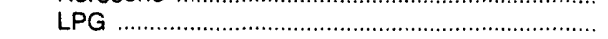 & 5.0 & 11.2 & 62.9 & 17.4 & 18.2 & 19.5 & 21.3 & 22.9 \\
\hline Lubricants .......... & 15.4 & 20.3 & 36.6 & 40.9 & 35.4 & 34.8 & 36.1 & 45.4 \\
\hline 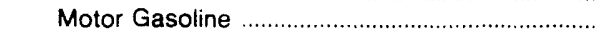 & 1.7 & 1.9 & 4.3 & 11.1 & 8.4 & 9.1 & 9.3 & 11.1 \\
\hline 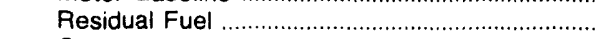 & 4.8 & 17.9 & 24.7 & 14.7 & 8.4 & 5.2 & 3.9 & 4.3 \\
\hline 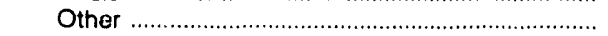 & 69.0 & 250.0 & 708.4 & 423.1 & 278.8 & 328.6 & 287.8 & 345.0 \\
\hline 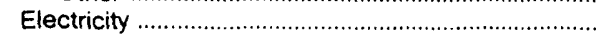 & 84.3 & 201.9 & 306.7 & 348.9 & 337.5 & 341.4 & 351.2 & 358.9 \\
\hline Transportation Sector & 267.9 & 562.1 & $1,278.1$ & $1,229.7$ & 859.5 & 955.5 & 997.3 & $1,146.2$ \\
\hline 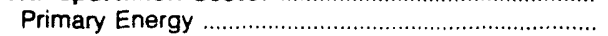 & 267.9 & 562.1 & $1,278.1$ & $1,229.7$ & 859.5 & 955.5 & 997.3 & $1,146.2$ \\
\hline 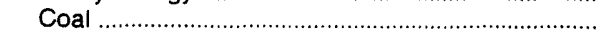 & .1 & & - & - & - & - & & \\
\hline 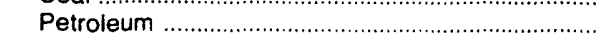 & 267.7 & 562.1 & $1,278.1$ & $1,229.7$ & 859.5 & 955.5 & 997.3 & $1,146.2$ \\
\hline 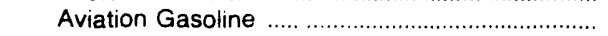 & .9 & 1.0 & 3.0 & 1.9 & 2.1 & 1.3 & 1.4 & 1.6 \\
\hline 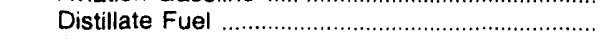 & 24.8 & 83.0 & 236.1 & 325.9 & 163.8 & 205.2 & 207.7 & 259.9 \\
\hline Jet Fuel & 1.2 & 2.7 & 12.8 & 9.0 & 6.3 & 5.6 & 6.2 & 10.6 \\
\hline 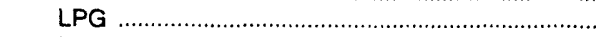 & .1 & .1 & .3 & .5 & .5 & .4 & .6 & .5 \\
\hline 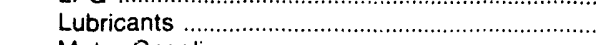 & 5.7 & 10.9 & 21.8 & 24.3 & 21.0 & 20.7 & 21.5 & 27.0 \\
\hline 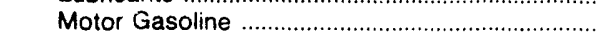 & 235.1 & 464.3 & $1,004.2$ & 868.1 & 665.9 & 722.3 & 759.8 & 846.6 \\
\hline 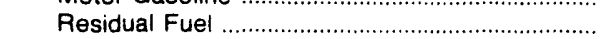 & $?$ & - & - & ? & - & - & - & - \\
\hline 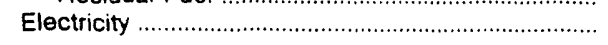 & - & - & - & - & - & - & - & - \\
\hline Total Energy" ...………… & 793.5 & $1,878.8$ & $3,800.5$ & $3,563.2$ & $3,013.1$ & $3,148.1$ & $3,192.2$ & $3,488.0$ \\
\hline Primary Energy - Four Sectors .................................... & 589.2 & $1,401.5$ & $3,051.8$ & $2,562.8$ & $1,983.5$ & $2,095.1$ & $2,124.0$ & $2,403.1$ \\
\hline Electricity . . & 204.3 & 477.3 & 748.8 & $1,000.4$ & 1.029 .6 & 1.053 .0 & $1,068.2$ & $1,084.9$ \\
\hline 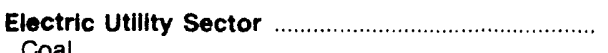 & 89.9 & . 531.0 & 997.7 & $1,261.8$ & $1,156.2$ & $1,093.2$ & $1,148.1$ & $1,155.4$ \\
\hline Coal & 87.1 & 522.5 & 972.5 & $1,248.3$ & $1,146.7$ & $1,082.5$ & $1,139.6$ & $1,144.3$ \\
\hline 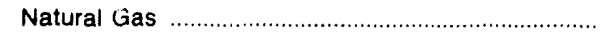 & .2 & .1 & .2 & .6 & 1.2 & .9 & 3 & 6 \\
\hline 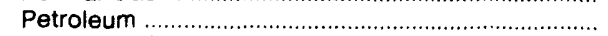 & 2.6 & 8.3 & 25.1 & 12.9 & 8.2 & 9.7 & 8.2 & 10.6 \\
\hline (2) & 2.5 & 8.2 & - & - & - & - & - & \\
\hline 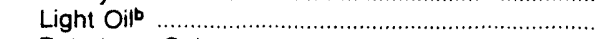 & & .2 & 25.1 & 12.9 & 8.2 & 9.7 & 8.2 & 10.6 \\
\hline 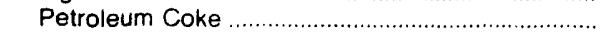 & - & - & - & - & - & - & - & - \\
\hline 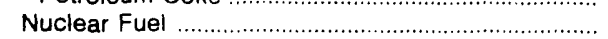 & - & - & - & - & - & - & - & - \\
\hline Wood and Waste & - & - & - & - & - & - & - & - \\
\hline Primary Energy - Five Sectors. & 679.1 & $1,932.5$ & $4,049.5$ & $3,824.6$ & $3,139.7$ & $3,188.2$ & $3,272.1$ & $3,558.5$ \\
\hline
\end{tabular}

- There are no direct fuel costs for hydroelectric, geothermal, centralized solar, or wind energy. Wood and other biomass fuels are not included, except those consumed at electric utilities.

bHeavy oil includes Grade Nos. 4, 5, and 6, and residual fuel oils. Light oil includes Grade No. 2 heating oil, kerosene, and jet fuel.

-No consumption, includirig cases where adjustments were made. See "Consumption Adjustments for Process Fuel and Intermediate Products." - Value less than 0.05 million dollars.

Note: Totals may not equal sum of components due to independent roundina.

Sources: Data sources, estimation procedures, and assumptions are described in the "Documentation" section of this report. 
Energy Price and Expenditure Estimates by Source, Wisconsin $1970,1975,1980$, and 1985-1989

\begin{tabular}{|c|c|c|c|c|c|c|c|c|}
\hline Energy Source & 1970 & 1975 & 1980 & 1985 & 1986 & 1987 & 1988 & 1989 \\
\hline & \multicolumn{8}{|c|}{ Prices in Dollars per Million Btu } \\
\hline Cosl & 0.51 & 1.05 & 1.44 & 1.76 & 1.68 & 1.53 & 1.53 & 1.50 \\
\hline 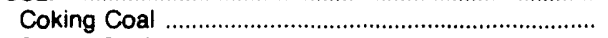 & .53 & 1.80 & 2.27 & 2.08 & - & - & - & - \\
\hline 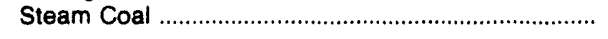 & .51 & 1.03 & 1.43 & 1.76 & 1.68 & 1.53 & 1.53 & 1.50 \\
\hline 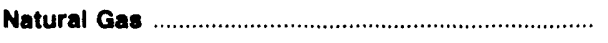 & .79 & 1.30 & 3.43 & 5.37 & 5.14 & 5.03 & 4.88 & 4.61 \\
\hline Potroleum & 1.89 & 3.65 & 8.14 & 8.70 & 6.69 & 6.97 & 6.85 & 7.72 \\
\hline 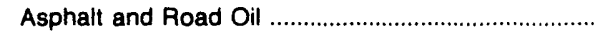 & .76 & 2.05 & 3.85 & 4.87 & 4.09 & 3.37 & 3.34 & 2.94 \\
\hline Aviation Gasoline & 2.17 & 3.45 & 9.02 & 9.99 & 8.41 & 7.55 & 7.41 & 8.28 \\
\hline 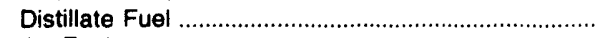 & 1.07 & 2.47 & 6.59 & 7.66 & 5.78 & 6.15 & 5.96 & 6.66 \\
\hline 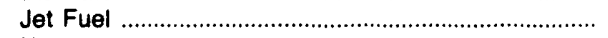 & .74 & 2.08 & 6.38 & 6.19 & 4.45 & 4.28 & 4.11 & 4.63 \\
\hline 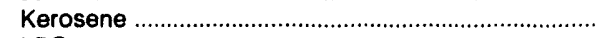 & 1.17 & 2.71 & 7.05 & 8.15 & 5.95 & 6.26 & 5.84 & 6.41 \\
\hline LPG & 1.88 & 3.60 & 6.51 & 8.56 & 8.13 & 7.36 & 7.07 & 10.31 \\
\hline 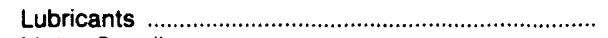 & 5.08 & 7.49 & 14.36 & 17.61 & 15.59 & 13.58 & 14.61 & 17.90 \\
\hline 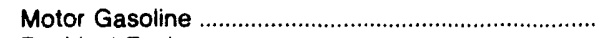 & 2.65 & 4.54 & 9.43 & 9.33 & 7.00 & 7.49 & 7.55 & 8.29 \\
\hline 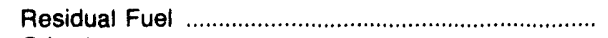 & .57 & 1.79 & 3.48 & 4.57 & 2.97 & 2.55 & 2.45 & 2.40 \\
\hline Othere & 1.89 & 3.36 & 9.30 & 9.71 & 8.93 & 9.20 & 8.02 & 10.19 \\
\hline 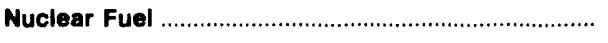 & .15 & .32 & .47 & .58 & .51 & .48 & .43 & .45 \\
\hline 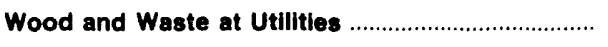 & .65 & - & 1.74 & .79 & .32 & .95 & .87 & .82 \\
\hline 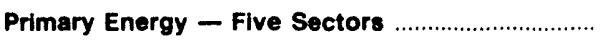 & 1.16 & 2.12 & 4.43 & 5.07 & 4.20 & 4.18 & 4.18 & 4.47 \\
\hline 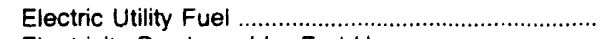 & .39 & .71 & 1.25 & 1.42 & 1.33 & 1.23 & 1.21 & 1.21 \\
\hline 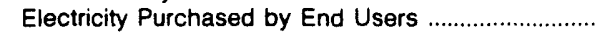 & 6.02 & 8.88 & 13.34 & 16.87 & 16.97 & 16.41 & 16.14 & 15.99 \\
\hline \multirow[t]{2}{*}{ Total Energy } & 1.75 & 3.29 & 6.86 & 8.62 & 7.73 & 7.70 & 7.52 & 7.80 \\
\hline & \multicolumn{8}{|c|}{ Expenditures in Millions of Dollars } \\
\hline 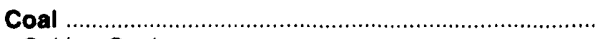 & 196.2 & 285.6 & 472.2 & 636.0 & 623.8 & 590.9 & 601.6 & 589.5 \\
\hline Coking Coal & 5.0 & 12.0 & 12.3 & .1 & - & - & - & - \\
\hline 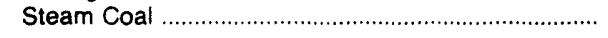 & 191.2 & 273.6 & 459.9 & 635.9 & 623.8 & 590.5 & 601.6 & 589.5 \\
\hline 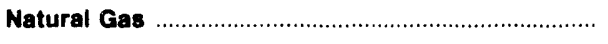 & 267.1 & 474.2 & $1,184.8$ & $1,634.5$ & $1,408.5$ & $1,374.5$ & $1,515.0$ & $1,491.3$ \\
\hline 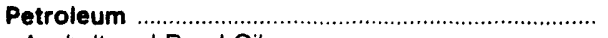 & 955.4 & $1,899.8$ & $3,853.2$ & $3,917.5$ & $3,028.1$ & $3,123.8$ & $3,337.4$ & $3,800.9$ \\
\hline 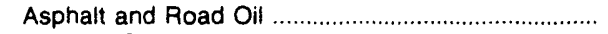 & 23.6 & 41.1 & 77.1 & 54.6 & 55.8 & 53.5 & 75.8 & 74.3 \\
\hline Aviation Gasoline & 3.6 & 3.0 & 5.6 & 5.1 & 4.6 & 3.2 & 3.5 & 5.4 \\
\hline 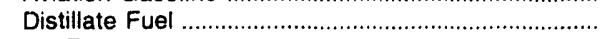 & 161.6 & 382.3 & 863.2 & $1,007.8$ & 738.9 & 756.9 & 838.7 & 942.2 \\
\hline Jet Fuel & 6.7 & 26.0 & 86.1 & 57.8 & 39.1 & 34.8 & 30.9 & 34.9 \\
\hline 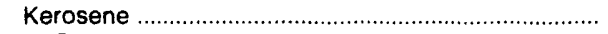 & 19.9 & 15.0 & 8.9 & 16.4 & 3.2 & 3.6 & 2.1 & 2.5 \\
\hline LPG & 54.3 & 112.5 & 143.2 & 263.7 & 242.9 & 204.6 & 228.6 & 366.7 \\
\hline 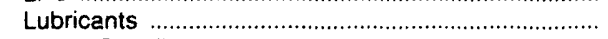 & 30.6 & 41.9 & 88.8 & 99.0 & 85.7 & 84.4 & 87.6 & 110.1 \\
\hline 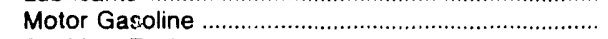 & 633.6 & $1,230.6$ & $2,457.8$ & $2,280.9$ & $1,743.8$ & $1,864.8$ & $1,967.6$ & $2,139.3$ \\
\hline 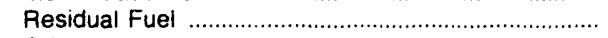 & 8.8 & 19.3 & 27.6 & 10.3 & 18.1 & 14.6 & 14.1 & 16.4 \\
\hline 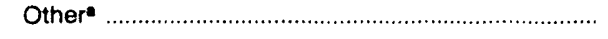 & 12.6 & 28.0 & 94.7 & 121.8 & 96.0 & 103.3 & 88.5 & 109.1 \\
\hline 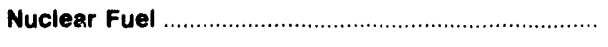 & .3 & 36.6 & 50.3 & 69.2 & 62.2 & 59.1 & 52.7 & 52.1 \\
\hline 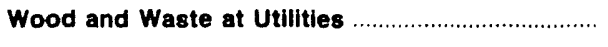 & .1 & - & 1.1 & .7 & .4 & 1.5 & 1.5 & 1.5 \\
\hline 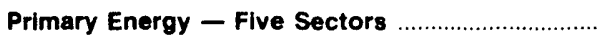 & $1,419.1$ & $2,696.2$ & $5,561.7$ & $6,257.9$ & $5,122.9$ & $5,149.8$ & $5,508.1$ & $5,935.3$ \\
\hline 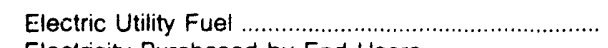 & -109.2 & -245.2 & .494 .9 & -612.9 & -595.6 & -571.4 & -574.9 & -565.8 \\
\hline Electricity Purchased by End Users ............................. & 501.0 & 932.2 & $1,669.5$ & $2,601.0$ & $2,674.4$ & $2,491.2$ & $2,609.1$ & $2,613.8$ \\
\hline Total Energy & $1,810.9$ & $3,383.2$ & $6,736.3$ & $8,246.0$ & $7,201.7$ & $7,069.6$ & $7,542.4$ & $7,983.3$ \\
\hline
\end{tabular}

- Includes petroleum coke used at electric utilities.

bThere are no direct fuel costs for hydroelectric, geothermal, centralized solar, or wind energy. Wood and other biomass fuels are not included, except those consumed at electric utilities.

-No consumption, including cases where adjustments were made. See "Consumption Adjustments for Process Fuel and Intermediate Products."

Note: Expenditure totals may not equal sum of components due to independent rounding.

Sources: Data sources, estimation procedures, and assumptions are described in the "Documentation" section of this report. 


\begin{tabular}{|c|c|c|c|c|c|c|c|c|}
\hline Sector and Energy Source & 1970 & 1975 & 1980 & 1985 & 1986 & 1987 & 1988 & 1989 \\
\hline Residentlal Sector & 2.06 & 3.48 & 6.85 & 9.99 & 9.73 & 9.50 & 9.14 & 9.57 \\
\hline Primary Energy & 1.33 & 2.24 & 4.78 & 6.88 & 6.25 & 6.07 & 5.95 & 6.55 \\
\hline 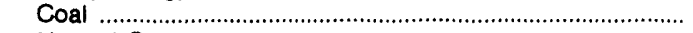 & 1.63 & 3.10 & 3.92 & 4.26 & 3.86 & 3.72 & 3.83 & 3.84 \\
\hline 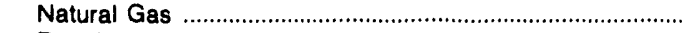 & 1.22 & 1.71 & 3.81 & 6.41 & 6.14 & 5.94 & 5.84 & 5.61 \\
\hline 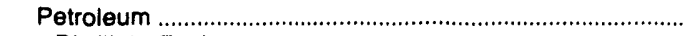 & 1.42 & 2.94 & 6.82 & 7.88 & 6.47 & 6.37 & 6.18 & 8.72 \\
\hline 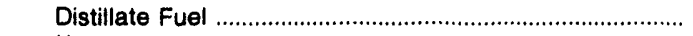 & 1.21 & 2.57 & 6.60 & 7.44 & 5.72 & 5.69 & 5.59 & 6.14 \\
\hline Kerosene & 1.47 & 2.97 & 8.11 & 8.45 & 6.50 & 6.45 & 6.35 & 6.97 \\
\hline LPG & 2.07 & 4.15 & 7.69 & 8.72 & 8.05 & 7.69 & 7.29 & 12.12 \\
\hline 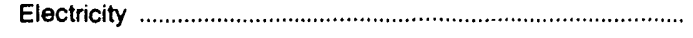 & 6.75 & 10.04 & 15.04 & 19.73 & 20.07 & 19.69 & 19.52 & 19.56 \\
\hline 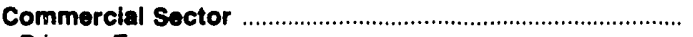 & 2.05 & 3.55 & 6.92 & 9.49 & 10.02 & 9.45 & 9.06 & 8.82 \\
\hline 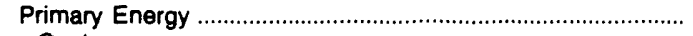 & .84 & 1.50 & 3.80 & 5.54 & 4.98 & 4.79 & 4.67 & 4.62 \\
\hline 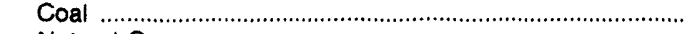 & .66 & 1.51 & 1.47 & 2.11 & 2.02 & 1.90 & 1.95 & 1.87 \\
\hline 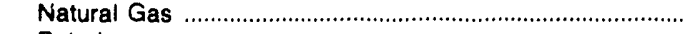 & .82 & 1.29 & 3.43 & 5.14 & 4.96 & 4.74 & 4.64 & 4.42 \\
\hline Petroleum & 1.08 & 2.43 & 6.21 & 6.80 & 5.11 & 5.22 & 4.96 & 5.36 \\
\hline 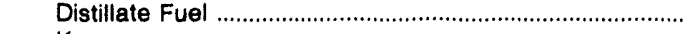 & 1.04 & 2.39 & 6.30 & 6.43 & 4.29 & 4.63 & 4.40 & 5.15 \\
\hline 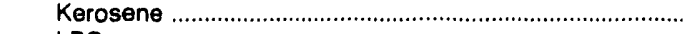 & .83 & 2.41 & 5.72 & 6.63 & 4.34 & 4.90 & 4.15 & 5.45 \\
\hline LPG & 1.35 & 2.63 & 5.33 & 8.32 & 8.25 & 6.78 & 6.69 & 6.59 \\
\hline 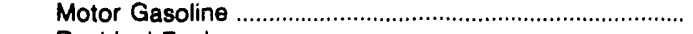 & 2.65 & 4.54 & 9.43 & 9.33 & 7.00 & 7.49 & 7.55 & 8.29 \\
\hline 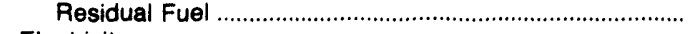 & .59 & 1.66 & 4.31 & 4.50 & 2.90 & 2.55 & 2.46 & 2.40 \\
\hline Electricity & 7.28 & 10.13 & 15.25 & 18.90 & 18.78 & 17.93 & 17.63 & 17.33 \\
\hline Industrial Sector & 1.01 & 2.18 & 4.61 & 6.44 & 6.06 & 5.94 & 5.66 & 5.63 \\
\hline 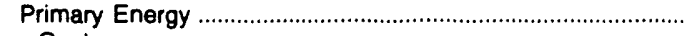 & .76 & 1.66 & 3.67 & 4.84 & 4.26 & 4.27 & 4.01 & 4.00 \\
\hline Coal & .65 & 1.55 & 1.55 & 2.11 & 2.02 & 1.90 & 1.95 & 1.87 \\
\hline Coking Coal & .53 & 1.80 & 2.27 & 2.08 & - & - & - & - \\
\hline 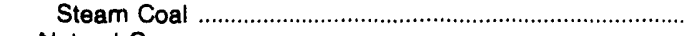 & .66 & 1.51 & 1.47 & 2.11 & 2.02 & 1.90 & 1.95 & 1.87 \\
\hline 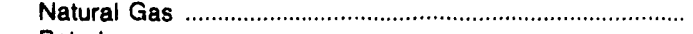 & .54 & 1.03 & 3.12 & 4.44 & 4.18 & 4.32 & 4.05 & 3.71 \\
\hline 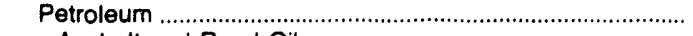 & 1.16 & 2.71 & 6.09 & 7.85 & 6.11 & 5.83 & 5.24 & 5.81 \\
\hline 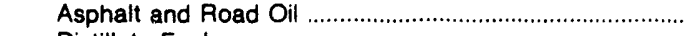 & .76 & 2.05 & 3.85 & 4.87 & 4.09 & 3.37 & 3.34 & 2.94 \\
\hline 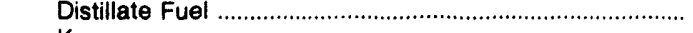 & .76 & 2.23 & 5.18 & 6.30 & 4.13 & 4.66 & 3.94 & 5.19 \\
\hline 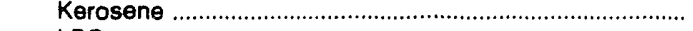 & .83 & 2.41 & 5.72 & 6.63 & 4.34 & 4.90 & 4.15 & 5.45 \\
\hline LPG & 1.35 & 2.63 & 5.33 & 8.32 & 8.25 & 6.78 & 6.69 & 6.59 \\
\hline 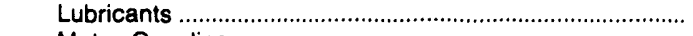 & 5.08 & 7.49 & 14.36 & 17.61 & 15.59 & 13.58 & 14.61 & 17.90 \\
\hline 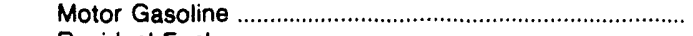 & 2.65 & 4.54 & 9.43 & 9.33 & 7.00 & 7.49 & 7.55 & 8.29 \\
\hline 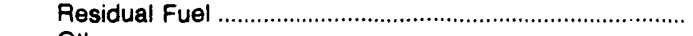 & .57 & 2.06 & 3.31 & 4.50 & 2.90 & 2.55 & 2.46 & 2.40 \\
\hline Other & 2.31 & 3.43 & 9.34 & 9.81 & 8.93 & 9.20 & 8.02 & 10.19 \\
\hline Electricity & 4.23 & 6.63 & 10.10 & 12.64 & 12.71 & 12.31 & 12.01 & 11.91 \\
\hline 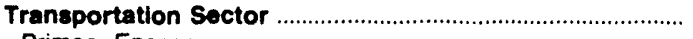 & 2.49 & 4.24 & 8.99 & 9.18 & 6.95 & 7.41 & 7.42 & 8.12 \\
\hline 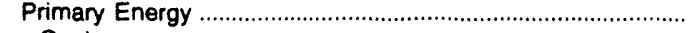 & 2.49 & 4.24 & 8.99 & 9.18 & 6.95 & 7.41 & 7.42 & 8.12 \\
\hline 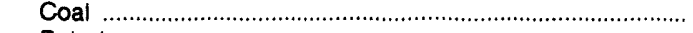 & .66 & 1.51 & - & - & - & - & - & - \\
\hline Petroleum & 2.49 & 4.24 & 8.99 & 9.18 & 6.95 & 7.41 & 7.42 & 8.12 \\
\hline 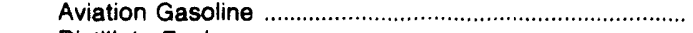 & 2.17 & 3.45 & 9.02 & 9.99 & 8.41 & 7.55 & 7.41 & 8.26 \\
\hline 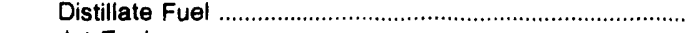 & 1.33 & 2.62 & 7.28 & 8.69 & 6.69 & 7.15 & 7.00 & 7.51 \\
\hline 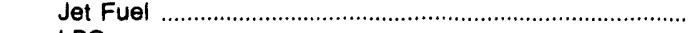 & .74 & 2.08 & 6.38 & 6.19 & 4.45 & 4.28 & 4.11 & 4.63 \\
\hline LPG & 1.35 & 2.63 & 5.33 & 8.32 & 8.25 & 6.78 & 6.69 & 6.59 \\
\hline 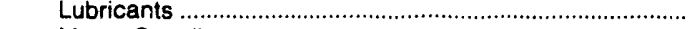 & 5.08 & 7.49 & 14.36 & 17.61 & 15.59 & 13.58 & 14.61 & 17.90 \\
\hline Motor Gasoline & 2.65 & 4.54 & 9.43 & 9.33 & 7.00 & 7.49 & 7.55 & 8.29 \\
\hline Residual Fuel & .55 & 1.44 & 3.80 & 4.71 & 3.60 & - & 2.37 & 2.66 \\
\hline Electricity & - & - & - & - & - & - & - & - \\
\hline 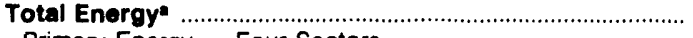 & 1.75 & 3.29 & 6.86 & 8.62 & 7.73 & 7.70 & 7.52 & 7.80 \\
\hline 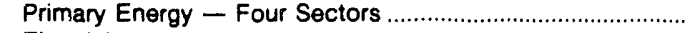 & 1.38 & 2.65 & 5.91 & 7.04 & 5.85 & 5.97 & 5.86 & 6.25 \\
\hline Electricity & 6.02 & 8.88 & 13.34 & 16.87 & 16.97 & 16.41 & 16.14 & 15.99 \\
\hline 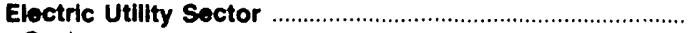 & 39 & .71 & 1.25 & 1.42 & 1.33 & 1.23 & 1.21 & 1.21 \\
\hline 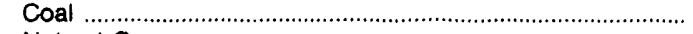 & .39 & .86 & 1.42 & 1.71 & 1.62 & 1.47 & 1.46 & 1.45 \\
\hline 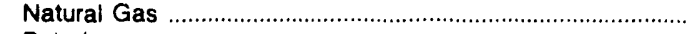 & .42 & .82 & 2.94 & 4.11 & 3.74 & 3.92 & 3.25 & 3.10 \\
\hline 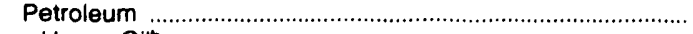 & .54 & 1.93 & 5.35 & 5.12 & 3.60 & 3.97 & 3.65 & 4.10 \\
\hline (1) & .56 & 1.65 & 4.28 & - & - & - & - & - \\
\hline 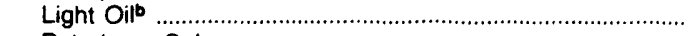 & .67 & 2.30 & 5.58 & 5.48 & 3.60 & 3.97 & 3.65 & 4.10 \\
\hline 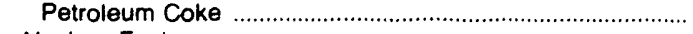 & .36 & .72 & 1.17 & 1.38 & - & - & - & - \\
\hline 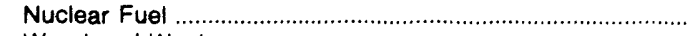 & .15 & .32 & .47 & .58 & .51 & .48 & .43 & .45 \\
\hline Wood and Waste & .65 & - & 1.74 & .79 & .32 & .95 & .87 & .82 \\
\hline Primary Energy - Five Sectors & 1.16 & 2.12 & 4.43 & 5.07 & 4.20 & 4.18 & 4.18 & 4.47 \\
\hline
\end{tabular}

- There are no direct fuel costs for hydroelectric, geothermal, centralized solar, or wind energy. Wood and other biomass fuels are not included, except those consumed at electric utilities.

beavy oil includes Grade Nos. 4, 5, and 6, and residual fuel oils. Light oil includes Grade No. 2 heating oil, kerosene, and jet fuel.

-No consumption, including cases where adjustments were made. See "Consumption Adjustments for Process Fuel and Intermediate Products."

Sources: Data sources, estimation procedures, and assumptions aie described in the "Documentation" section of this report 
Energy Expenditure Estimates by Sector, Wisconsin

1970, 1975, 1980, and 1985-1989

(Million Dollars)

\begin{tabular}{|c|c|c|c|c|c|c|c|c|}
\hline Sector and Energy Source & 1970 & 1975 & 1980 & 1985 & 1986 & 1987 & 1988 & 1989 \\
\hline $\begin{array}{l}\text { Pesidential Sector } \\
\text { Primary Energy } \\
\text { Coal .... } \\
\text { Natural Gas . } \\
\text { Petroleum } \\
\text { Distillate Fuel } \\
\text { Kerosene } \\
\text { LPG } \\
\text { Electricity }\end{array}$ & $\begin{array}{r}512.5 \\
286.3 \\
15.5 \\
131.2 \\
139.5 \\
82.3 \\
13.4 \\
43.9 \\
226.2\end{array}$ & $\begin{array}{r}882.0 \\
478.4 \\
11.9 \\
209.5 \\
257.0 \\
164.8 \\
8.9 \\
83.2 \\
403.6\end{array}$ & $\begin{array}{r}1,575.9 \\
878.3 \\
1.7 \\
473.2 \\
403.4 \\
313.4 \\
5.7 \\
84.3 \\
697.6\end{array}$ & $\begin{array}{r}2,301.2 \\
1,203.5 \\
1.0 \\
751.6 \\
450.9 \\
278.4 \\
14.2 \\
158.2 \\
1,097.7\end{array}$ & $\begin{array}{r}2,181.1 \\
1,047.6 \\
1.1 \\
687.3 \\
359.2 \\
214.2 \\
2.6 \\
142.3 \\
1.133 .5\end{array}$ & $\begin{array}{r}1,987.0 \\
950.2 \\
2.9 \\
617.9 \\
329.4 \\
191.5 \\
3.3 \\
134.7 \\
1,036.7\end{array}$ & $\begin{array}{r}2,169.8 \\
1,078.8 \\
2.6 \\
714.7 \\
361.6 \\
212.4 \\
1.7 \\
147.5 \\
1,091.0\end{array}$ & $\begin{array}{r}2,286.8 \\
1,201.8 \\
.6 \\
716.3 \\
484.8 \\
193.0 \\
1.7 \\
290.2 \\
1,085.0\end{array}$ \\
\hline 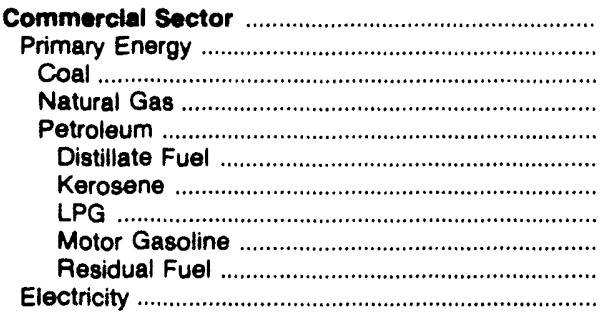 & $\begin{array}{r}229.6 \\
76.1 \\
11.7 \\
45.5 \\
18.8 \\
11.5 \\
.6 \\
5.0 \\
.8 \\
.9 \\
153.5\end{array}$ & $\begin{array}{r}425.5 \\
137.1 \\
10.7 \\
88.6 \\
37.8 \\
24.9 \\
.6 \\
9.3 \\
1.2 \\
1.8 \\
288.4\end{array}$ & $\begin{array}{r}868.0 \\
346.5 \\
1.2 \\
266.9 \\
78.5 \\
61.8 \\
1.8 \\
10.3 \\
3.8 \\
.8 \\
521.5\end{array}$ & $\begin{array}{r}1,322.7 \\
543.0 \\
.9 \\
378.3 \\
163.9 \\
118.8 \\
1.0 \\
26.6 \\
13.9 \\
3.6 \\
779.6\end{array}$ & $\begin{array}{r}1,152.8 \\
363.0 \\
1.1 \\
277.1 \\
84.8 \\
43.1 \\
.1 \\
25.7 \\
10.3 \\
5.5 \\
789.8\end{array}$ & $\begin{array}{r}1,106.2 \\
361.4 \\
2.8 \\
276.0 \\
82.6 \\
48.4 \\
.1 \\
21.0 \\
11.2 \\
1.9 \\
744.8\end{array}$ & $\begin{array}{r}1,179.4 \\
401.4 \\
2.4 \\
313.3 \\
85.7 \\
46.2 \\
.1 \\
23.9 \\
11.4 \\
4.1 \\
778.0\end{array}$ & $\begin{array}{r}1,194.1 \\
418.1 \\
.5 \\
311.2 \\
106.4 \\
60.5 \\
.2 \\
27.9 \\
12.1 \\
5.7 \\
776.0\end{array}$ \\
\hline $\begin{array}{l}\text { Industrial Sector } \\
\text { Primary Energy } \\
\text { Coal ... } \\
\text { Coking Coal . } \\
\text { Steam Coal } \\
\text { Natural Gas } \\
\text { Petroleum } \\
\text { Asphalt and Road Oil } \\
\text { Distillate Fuel } \\
\text { Kerosene } \\
\text { LPG } \\
\text { Lubricants } \\
\text { Motor Gasoline } \\
\text { Residual Fuel } \\
\text { Other . } \\
\text { Electricity . }\end{array}$ & $\begin{array}{r}410.3 \\
289.0 \\
78.0 \\
5.0 \\
73.0 \\
77.3 \\
133.6 \\
23.6 \\
35.1 \\
5.9 \\
5.0 \\
13.6 \\
34.4 \\
3.9 \\
12.1 \\
121.3\end{array}$ & $\begin{array}{r}747.8 \\
507.6 \\
84.6 \\
12.0 \\
72.6 \\
159.5 \\
263.5 \\
41.1 \\
92.9 \\
5.5 \\
19.1 \\
19.3 \\
48.4 \\
9.3 \\
27.9 \\
240.2\end{array}$ & $\begin{array}{r}1,411.1 \\
960.7 \\
84.6 \\
12.3 \\
72.3 \\
404.2 \\
471.9 \\
77.1 \\
108.3 \\
1.3 \\
47.0 \\
43.3 \\
80.9 \\
19.4 \\
94.7 \\
450.4\end{array}$ & $\begin{array}{r}1,796.4 \\
1,072.7 \\
104.7 \\
.1 \\
104.6 \\
499.2 \\
468.8 \\
54.6 \\
112.5 \\
1.2 \\
71.7 \\
48.3 \\
55.7 \\
3.2 \\
121.7 \\
723.7\end{array}$ & $\begin{array}{r}1,685.1 \\
934.0 \\
100.6 \\
-\overline{-} \\
100.6 \\
437.5 \\
395.9 \\
55.8 \\
82.5 \\
.5 \\
69.8 \\
41.8 \\
39.2 \\
10.3 \\
96.0 \\
751.0\end{array}$ & $\begin{array}{r}1,648.4 \\
938.7 \\
86.9 \\
- \\
86.9 \\
472.1 \\
379.7 \\
53.5 \\
83.8 \\
.2 \\
45.7 \\
41.1 \\
39.3 \\
12.7 \\
103.3 \\
709.7\end{array}$ & $\begin{array}{r}1,692.5 \\
952.3 \\
89.7 \\
- \\
89.7 \\
478.2 \\
384.5 \\
75.8 \\
79.6 \\
.2 \\
53.2 \\
42.7 \\
34.5 \\
9.9 \\
88.5 \\
740.1\end{array}$ & $\begin{array}{r}1,729.4 \\
976.5 \\
86.7 \\
- \\
86.7 \\
457.3 \\
432.6 \\
74.3 \\
101.5 \\
.6 \\
45.1 \\
53.6 \\
37.8 \\
10.6 \\
109.1 \\
752.9\end{array}$ \\
\hline $\begin{array}{l}\text { Traneportation Sector } \\
\text { Primary Energy } \\
\text { Coal . } \\
\text { Petroleum foum } \\
\text { Aviation Gasoline } \\
\text { Distillate Fuel } \\
\text { Jet Fuel } \\
\text { LPG } \\
\text { Lubricants } \\
\text { Motor Gasoline } \\
\text { Residual Fuel } \\
\text { Electricity . }\end{array}$ & $\begin{array}{r}658.5 \\
658.5 \\
.1 \\
658.4 \\
3.6 \\
32.3 \\
6.7 \\
.4 \\
17.0 \\
598.4 \\
\end{array}$ & $\begin{array}{r}1,328.0 \\
1,328.0 \\
1,328.0 \\
3.0 \\
92.4 \\
25.5 \\
.9 \\
22.6 \\
1,181.0 \\
2.6 \\
\end{array}$ & $\begin{array}{r}2,881.3 \\
2,881.3 \\
2,881.3 \\
5.6 \\
363.6 \\
86.1 \\
1.6 \\
45.5 \\
2,373.2 \\
5.6 \\
-\end{array}$ & $\begin{array}{r}2,825.7 \\
2,825.7 \\
- \\
2,825.7 \\
5.1 \\
490.1 \\
57.8 \\
7.2 \\
50.8 \\
2,211.3 \\
3.5 \\
-\end{array}$ & $\begin{array}{r}2,182.7 \\
2,182.7 \\
- \\
2,182.7 \\
4.6 \\
393.6 \\
39.1 \\
5.0 \\
44.0 \\
1,694.2 \\
2.2 \\
-\end{array}$ & $\begin{array}{r}2,328.1 \\
2,328.1 \\
- \\
2,328.1 \\
3.2 \\
429.2 \\
34.8 \\
3.3 \\
43.3 \\
1,814.3 \\
- \\
-\end{array}$ & $\begin{array}{r}2,500.6 \\
2,500.6 \\
- \\
2,500.6 \\
3.5 \\
495.5 \\
30.9 \\
4.0 \\
44.9 \\
1,921.7 \\
.1 \\
-\end{array}$ & $\begin{array}{r}2,773.1 \\
2,773.1 \\
- \\
2,773.1 \\
5.4 \\
583.3 \\
34.9 \\
3.6 \\
56.5 \\
2,089.4 \\
.1 \\
-\end{array}$ \\
\hline 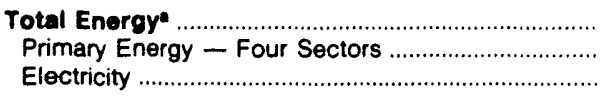 & $\begin{array}{r}1,810.9 \\
1,309.9 \\
501.0\end{array}$ & $\begin{array}{r}3,383.2 \\
2,451.0 \\
932.2\end{array}$ & $\begin{array}{l}6,736.3 \\
5,066.8 \\
1,669.5\end{array}$ & $\begin{array}{l}\mathbf{8 , 2 4 6 . 0} \\
5,644.9 \\
2,601.0\end{array}$ & $\begin{array}{l}7,201.7 \\
4,527.3 \\
2,674.4\end{array}$ & $\begin{array}{l}7,069.6 \\
4,578.4 \\
2,491.2\end{array}$ & $\begin{array}{l}7,542.4 \\
4,933.2 \\
2,609.1\end{array}$ & $\begin{array}{l}7,983.3 \\
5,369.5 \\
2,613.8\end{array}$ \\
\hline 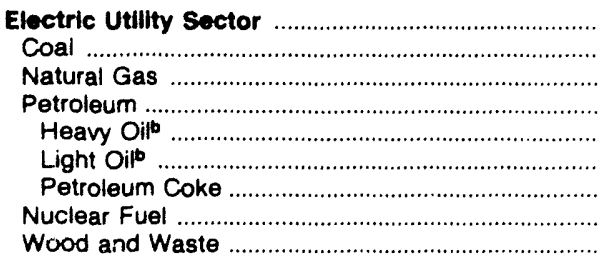 & $\begin{array}{r}109.2 \\
90.8 \\
13.1 \\
5.0 \\
4.0 \\
.5 \\
.5 \\
.3 \\
.1\end{array}$ & $\begin{array}{r}245.2 \\
178.3 \\
16.7 \\
13.6 \\
5.7 \\
7.7 \\
.2 \\
36.6 \\
-\end{array}$ & $\begin{array}{r}494.9 \\
384.7 \\
40.6 \\
18.1 \\
1.8 \\
16.2 \\
.1 \\
50.3 \\
1.1\end{array}$ & $\begin{array}{r}612.9 \\
529.4 \\
5.4 \\
8.2 \\
- \\
8.0 \\
.2 \\
69.2 \\
.7\end{array}$ & $\begin{array}{r}585.6 \\
520.9 \\
6.7 \\
5.4 \\
- \\
5.4 \\
- \\
62 . \overline{4} \\
4\end{array}$ & $\begin{array}{r}571.4 \\
498.4 \\
8.5 \\
4.0 \\
-\overline{0} \\
4.0 \\
- \\
59.1 \\
1.5\end{array}$ & $\begin{array}{r}574.9 \\
506.9 \\
8.9 \\
4.9 \\
- \\
4.9 \\
- \\
52.7 \\
1.5\end{array}$ & $\begin{array}{r}565.8 \\
501.7 \\
6.4 \\
4.0 \\
- \\
4.0 \\
-\overline{5} \\
52.1 \\
1.5\end{array}$ \\
\hline 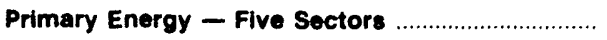 & $1,419.1$ & $2,696.2$ & $5,561.7$ & $6,257.9$ & $5,122.9$ & $5,149.8$ & $5,508.1$ & $5,935.3$ \\
\hline
\end{tabular}

-There are no direct fuel costs for hydroelectric, geothermal, centralized solar, or wind energy. Wood and other biomass fuels are not included, except those consumed at electric utilities.

bHeavy oil includes Grade Nos. 4, 5, and 6, and residual fuel oils. Light oil includes Grade No. 2 heating oil, kerosene, and jet fuel.

-No consumption, including cases where adjustments were made. See "Consumption Adjustments for Process Fuel and Intermediate Products."

- Value less than 0.05 million dollars.

Note: Totals may not equal sum of components due to independent rounding.

Sources: Data sources. estimation procedures. and assumptions are described in the "Documentation" sertionn of this rennrt 


\begin{tabular}{|c|c|c|c|c|c|c|c|c|}
\hline Energy Source & 1970 & 1975 & 1980 & 1985 & 1986 & 1987 & 1988 & 1989 \\
\hline & \multicolumn{8}{|c|}{ Prices In Dollars per Million Btu } \\
\hline Coal & 0.16 & 0.31 & 0.70 & 1.00 & 0.88 & 0.91 & 0.88 & 0.89 \\
\hline Coking Coal & - & - & - & - & - & - & - & - \\
\hline 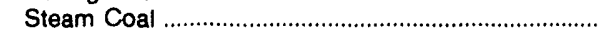 & .16 & .31 & .70 & 1.00 & .98 & .91 & .88 & .89 \\
\hline 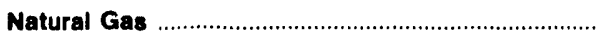 & .38 & .71 & 2.45 & 4.28 & 4.01 & 3.93 & 3.75 & 3.82 \\
\hline 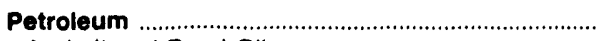 & 1.73 & 3.28 & 7.16 & 7.49 & 5.95 & 5.73 & 5.80 & 6.85 \\
\hline Asphalt and Road Oil & .58 & 1.80 & 3.66 & 4.82 & 4.36 & 3.21 & 3.40 & 2.88 \\
\hline Aviation Gasoline & 2.17 & 3.45 & 9.02 & 9.99 & 8.41 & 7.55 & 7.41 & 8.28 \\
\hline 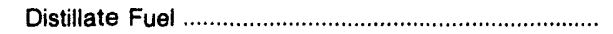 & 1.11 & 2.51 & 6.44 & 7.32 & 5.81 & 5.53 & 5.80 & 6.66 \\
\hline Jet Fisel & .76 & 2.12 & 6.59 & 6.53 & 4.56 & 4.61 & 4.62 & 5.06 \\
\hline Kerosene & .96 & 2.47 & 5.76 & 9.88 & 5.00 & 4.15 & 4.53 & 5.40 \\
\hline LPG & 1.64 & 3.46 & 5.77 & 8.13 & 7.21 & 6.34 & 6.21 & 7.46 \\
\hline Lubricants . . & 5.08 & 7.49 & 14.36 & 17.61 & 15.59 & 13.58 & 14.61 & 17.90 \\
\hline Motor Gasoline & 2.93 & 4.77 & 10.28 & 8.87 & 6.72 & 6.88 & 6.84 & 7.79 \\
\hline Residual Fuel & .55 & 1.71 & 3.56 & 3.14 & 2.06 & 1.83 & 1.65 & 1.63 \\
\hline Other" & .51 & 1.39 & 2.53 & 2.37 & 1.53 & 1.50 & 1.45 & 1.92 \\
\hline 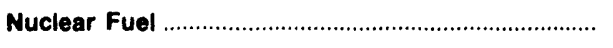 & - & - & - & - & - & - & - & - \\
\hline 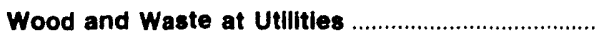 & - & - & - & - & - & - & - & - \\
\hline 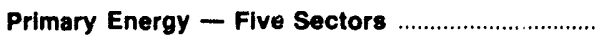 & .84 & 1.51 & 3.00 & 2.55 & 2.29 & 2.05 & 2.02 & 2.29 \\
\hline 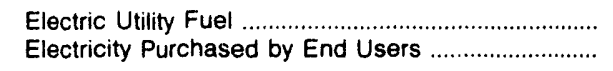 & $\begin{array}{r}.14 \\
4.53\end{array}$ & $\begin{array}{r}.26 \\
4.63\end{array}$ & $\begin{array}{r}.59 \\
7.45\end{array}$ & $\begin{array}{r}.93 \\
12.54\end{array}$ & $\begin{array}{r}.93 \\
13.13\end{array}$ & $\begin{array}{r}.88 \\
12.91\end{array}$ & $\begin{array}{r}.85 \\
12.81\end{array}$ & $\begin{array}{r}.85 \\
12.66\end{array}$ \\
\hline \multirow[t]{2}{*}{ Total Energyb } & 1.31 & 2.53 & 5.79 & 6.80 & 6.05 & 5.92 & 5.96 & 6.45 \\
\hline & \multicolumn{8}{|c|}{ Expenditures in Millions of Dollars } \\
\hline Coal & 10.2 & 39.8 & 187.2 & 406.6 & 328.3 & 389.3 & 390.6 & 374.6 \\
\hline 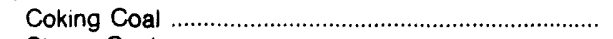 & - & - & - & - & - & - & - & - \\
\hline 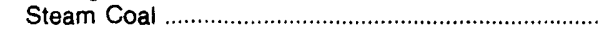 & 10.2 & 39.8 & 187.2 & 406.6 & 328.3 & 389.3 & 390.6 & 374.6 \\
\hline Natural Gas & 28.4 & 36.4 & 91.6 & 176.5 & 148.5 & 125.2 & 131.2 & 135.2 \\
\hline Petroleum & 152.0 & 359.5 & $1,097.6$ & 844.1 & 619.9 & 667.7 & 690.1 & 805.3 \\
\hline Asphalt and Road Oil & 4.2 & 7.2 & 28.2 & 53.6 & 46.5 & 31.3 & 23.6 & 17.6 \\
\hline 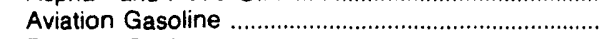 & 2.8 & 3.8 & 4.9 & 2.6 & 2.1 & 1.9 & 2.0 & 1.6 \\
\hline 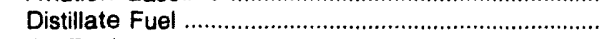 & 32.7 & 111.2 & 496.4 & 326.9 & 233.4 & 282.4 & 318.0 & 379.4 \\
\hline Jet Fuel & .5 & 1.5 & 6.0 & 5.6 & 3.7 & 5.2 & 5.0 & 4.5 \\
\hline Kerosene . & 1.8 & 2.4 & 2.0 & 1.3 & .2 & .2 & .2 & .1 \\
\hline LPG & 10.8 & 21.6 & 42.7 & 55.5 & 52.3 & $5 ? .0$ & 42.5 & 63.1 \\
\hline 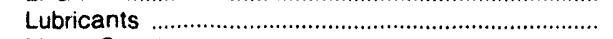 & 3.5 & 7.0 & 18.1 & 20.2 & 17.5 & 17.2 & 17.9 & 22.5 \\
\hline 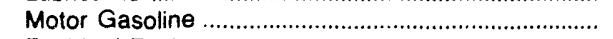 & 90.8 & 184.4 & 458.9 & 357.2 & 252.3 & 262.6 & 267.0 & 309.1 \\
\hline 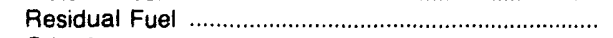 & 2.7 & 13.6 & 24.0 & 15.4 & 8.6 & 5.9 & 9.8 & 1.7 \\
\hline 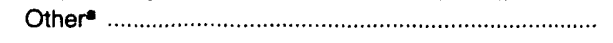 & 2.1 & 6.9 & 16.4 & 5.8 & 3.3 & 4.0 & 4.1 & 5.6 \\
\hline Nuclear Fuel & - & - & - & - & - & - & - & - \\
\hline 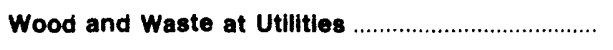 & - & - & - & - & - & - & - & - \\
\hline 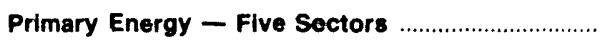 & 190.6 & 435.7 & $1,376.3$ & $1,427.2$ & $1,096.7$ & $1,182.2$ & $1,211.8$ & $1,315.1$ \\
\hline Electric Utility Fuel & -8.9 & -30.3 & -140.7 & -346.3 & -283.5 & -345.3 & -350.0 & -328.6 \\
\hline Electricity Purchased by End Users ............................ & 46.9 & 70.0 & 176.1 & 427.3 & 433.6 & 449.3 & 465.3 & 466.9 \\
\hline Total Energy & 228.5 & 475.4 & $1,411.7$ & $1,508.2$ & $1,246.8$ & $1,286.2$ & $1,327.2$ & $1,453.4$ \\
\hline
\end{tabular}

- Includes petroleumi coke used at electric utilities.

bThere are no direct fuel costs for hydroelectric, geothermal, centralized solar, or wind energy. Wood and other biomass fuels are not included, except those consumed at electric utilities.

-No consumption, including cases where adjustments were made. See "Consumption Adjustments for Process Fuel and Intermediate Products."

Note: Expenditure totals may not equal sum of components due to independent rounding.

Sources: Data sources, estimation procedures, and assumptions are described in the "Documentation" section of this report. 


\begin{tabular}{|c|c|c|c|c|c|c|c|c|}
\hline Sector and Energy Source & 1970 & 1975 & 1980 & 1985 & 1986 & 1987 & 1988 & 1989 \\
\hline 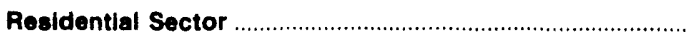 & 1.45 & 2.78 & 5.58 & 8.12 & 8.13 & 8.13 & 7.84 & 8.57 \\
\hline 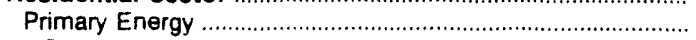 & .89 & 1.83 & 3.42 & 5.21 & 4.94 & 4.89 & 4.45 & 5.11 \\
\hline 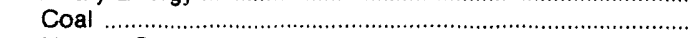 & .66 & .99 & .87 & 2.29 & 1.37 & 1.44 & .95 & 1.25 \\
\hline Natural Gas & .67 & 1.09 & 2.66 & 4.92 & 4.69 & 4.45 & 4.25 & 4.46 \\
\hline Petroleum & 1.93 & 4.13 & 7.23 & 7.35 & 6.70 & 6.33 & 6.09 & 10.04 \\
\hline Distillate Fuel & 1.28 & 2.84 & 6.94 & 10.07 & 5.72 & 3.73 & 4.29 & 5.43 \\
\hline 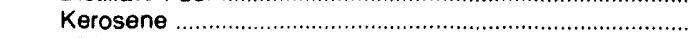 & 1.70 & 3.17 & - & 12.66 & 7.20 & 4.69 & 5.40 & 6.83 \\
\hline LPG & 1.96 & 4.20 & 7.25 & 7.51 & 6.75 & 6.44 & 6.20 & 10.48 \\
\hline 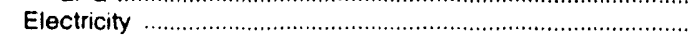 & 7.52 & 7.58 & 11.66 & 16.60 & 17.66 & 17.90 & 17.11 & 17.71 \\
\hline 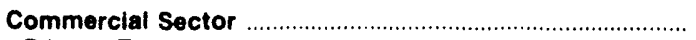 & 1.10 & 1.86 & 5.94 & 8.47 & 8.09 & 8.02 & 7.24 & 8.22 \\
\hline 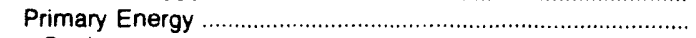 & .55 & 1.08 & 3.95 & 5.07 & 4.26 & 3.94 & 3.32 & 3.93 \\
\hline 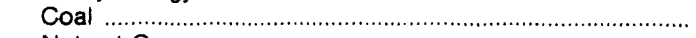 & .42 & .90 & 1.72 & 1.89 & 1.47 & 1.35 & 1.34 & 1.36 \\
\hline Natural Gas & .43 & .72 & 2.50 & 4.83 & 4.43 & 4.23 & 3.90 & 4.10 \\
\hline 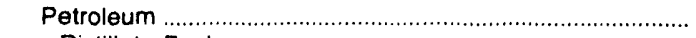 & 1.32 & 2.77 & 6.70 & 6.22 & 4.54 & 4.06 & 2.95 & 5.03 \\
\hline 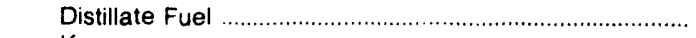 & 1.06 & 2.49 & 6.47 & 7.95 & 6.40 & 3.85 & 3.65 & 4.09 \\
\hline 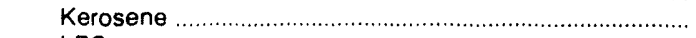 & .86 & 2.42 & 5.76 & 8.15 & 4.56 & 4.02 & 4.38 & 5.08 \\
\hline LPG & 1.22 & 2.48 & 5.07 & 8.38 & 7.52 & 6.22 & 6.21 & 6.47 \\
\hline Motor Gasoline & 2.93 & 4.77 & 10.28 & 8.87 & 6.72 & 6.88 & 6.84 & 7.79 \\
\hline Residual Fuel . & .55 & 2.03 & 3.59 & 3.14 & 2.06 & 1.83 & 1.65 & 1.62 \\
\hline 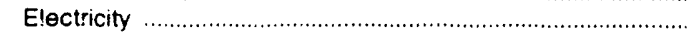 & 5.28 & 5.48 & 11.21 & 15.38 & 16.02 & 15.72 & 15.84 & 15.45 \\
\hline Industrlal Sector & .75 & 1.66 & 3.79 & 5.29 & 4.64 & 4.43 & 4.62 & 4.71 \\
\hline 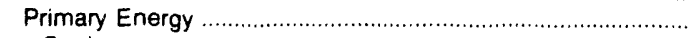 & .54 & 1.46 & 3.62 & 4.21 & 3.17 & 2.74 & 2.78 & 3.02 \\
\hline 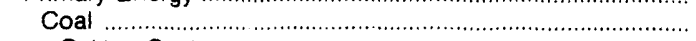 & 42 & .90 & 1.72 & 1.89 & 1.47 & 1.35 & 1.34 & 1.36 \\
\hline Coking Coal & - & - & - & - & - & - & - & - \\
\hline 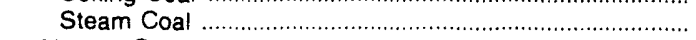 & .42 & .90 & 1.72 & 1.89 & 1.47 & 1.35 & 1.34 & 1.36 \\
\hline 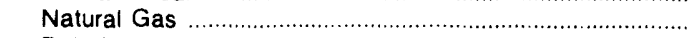 & .24 & .55 & 2.32 & 3.38 & 3.17 & 3.20 & 3.19 & 3.06 \\
\hline 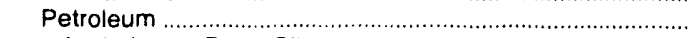 & .95 & 2.27 & 4.91 & 6.46 & 4.68 & 3.86 & 4.03 & 4.74 \\
\hline 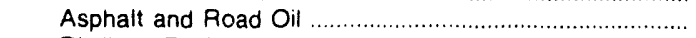 & .58 & 1.80 & 3.66 & 4.82 & 4.36 & 3.21 & 3.40 & 2.88 \\
\hline 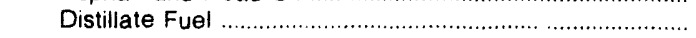 & .80 & 2.30 & 5.44 & 7.65 & 4.28 & 3.77 & 4.11 & 4.77 \\
\hline 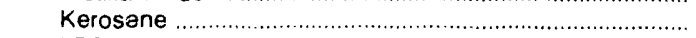 & .86 & 2.42 & 5.76 & 8.15 & 4.56 & 4.02 & 4.38 & 5.08 \\
\hline LPG & 1.22 & 2.48 & 5.07 & 8.38 & 7.52 & 6.22 & 6.21 & 6.47 \\
\hline 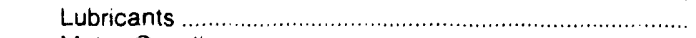 & 5.08 & 7.49 & 14.36 & 17.61 & 15.59 & 13.58 & 14.61 & 17.90 \\
\hline Motor Gasoline & 2.93 & 4.77 & 10.28 & 8.87 & 6.72 & 6.88 & 6.84 & 7.79 \\
\hline Residual Fuel & .55 & 1.65 & 3.55 & 3.14 & 2.06 & 1.83 & 1.65 & 1.62 \\
\hline Other & .51 & 1.39 & 2.53 & 2.37 & 1.53 & 1.50 & 1.45 & 1.92 \\
\hline Electricity & 3.23 & 3.44 & 5.12 & 10.15 & 10.68 & 10.68 & 10.67 & 10.49 \\
\hline Transportation Sector & 2.19 & 3.95 & 8.94 & 8.25 & 6.84 & 6.74 & 6.72 & 7.68 \\
\hline Primary Energy & 2.19 & 3.95 & 8.94 & 8.25 & 6.84 & 6.74 & 6.72 & 7.68 \\
\hline Coal & .42 & .90 & - & - & - & - & - & - \\
\hline 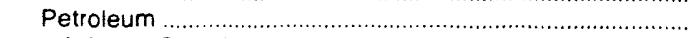 & 2.19 & 3.95 & 8.94 & 8.25 & 6.84 & 6.74 & 6.72 & 7.68 \\
\hline 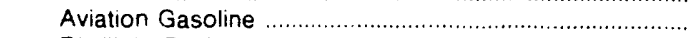 & 2.17 & 3.45 & 9.02 & 9.99 & 8.41 & 7.55 & 7.41 & 8.28 \\
\hline 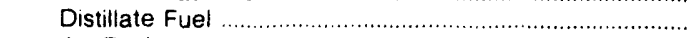 & 1.31 & 2.70 & 7.39 & 7.05 & 6.77 & 6.46 & 6.48 & 7.41 \\
\hline Jet Fuel & .76 & 2.12 & 6.59 & 6.53 & 4.56 & 4.61 & 4.62 & 5.06 \\
\hline LPG & 1.22 & 2.48 & 5.07 & 8.38 & 7.52 & 6.22 & 6.21 & 6.47 \\
\hline 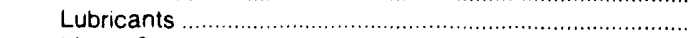 & 5.08 & 7.49 & 14.36 & 17.61 & 15.59 & 13.58 & 14.61 & 17.90 \\
\hline 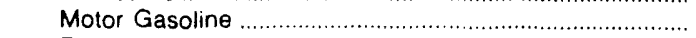 & 2.93 & 4.77 & 10.28 & 8.87 & 6.72 & 6.88 & 6.84 & 7.79 \\
\hline 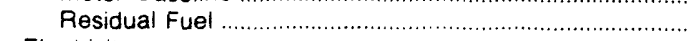 & .54 & - & - & 4.01 & - & - & - & 2.73 \\
\hline Electricity & - & - & - & - & - & - & - & - \\
\hline 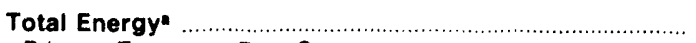 & 1.31 & 2.53 & 5.79 & 6.80 & 6.05 & 5.92 & 5.96 & 6.45 \\
\hline 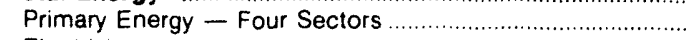 & 1.11 & 2.35 & 5.61 & 5.76 & 4.70 & 4.59 & 4.03 & 5.24 \\
\hline 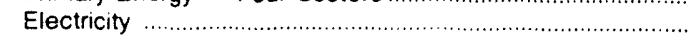 & 4.53 & 4.63 & 7.45 & 12.54 & 13.13 & 12.91 & 12.81 & 12.66 \\
\hline Electric Utility Sector & .14 & .26 & .59 & .93 & .93 & .88 & .85 & .85 \\
\hline 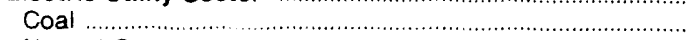 & .14 & .25 & .57 & .92 & .92 & .87 & .84 & .85 \\
\hline 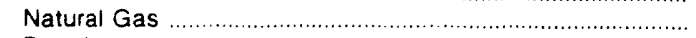 & .22 & .94 & 4.61 & 4.33 & 3.80 & 3.26 & 3.68 & 3.48 \\
\hline (1) & .67 & 2.01 & 6.98 & 6.00 & 3.87 & 4.10 & 3.80 & 4.15 \\
\hline (n) & .58 & 1.99 & - & - & - & - & - & - \\
\hline (n) & .76 & 2.44 & 6.98 & 6.00 & 3.87 & 4.10 & 3.80 & 4.15 \\
\hline Petroleum Coke & - & - & - & - & - & - & - & - \\
\hline Nuclear Fuel & - & - & - & - & - & - & - & - \\
\hline Wood and Waste & - & - & - & - & - & - & - & - \\
\hline 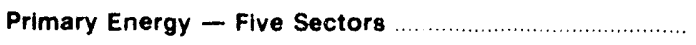 & .84 & 1.51 & 3.00 & 2.55 & 2.29 & 2.05 & 2.02 & 2.29 \\
\hline
\end{tabular}

-There are no direct fuel costs for hydroelectric, geothermal, centralized solar, or wind energy. Wood and other biomass fuels are not included, except those consumed at electric utilities.

bHeavy oll includes Grade Nos. 4, 5, and 6, and residual fuel oils. Light oil includes Grade No. 2 heating oil, kerosene, and jet fuel.

-No consumption, including cases where adjustments were made. See "Consumption Adjustments for Process Fuel and Intermediate Products."

Sources: Data sources, estimation procedures, and assumptions are described in the "Documentation" section of this report. 


\section{W Energy Expenditure Estimates by Sector, Wyoming \\ Y $1970,1975,1980$, and 1985-1989 \\ O (Million Dollars)}

\begin{tabular}{|c|c|c|c|c|c|c|c|c|}
\hline Sector and Energy Source & 1970 & 1975 & 1980 & 1985 & 1986 & 1987 & 1988 & 1989 \\
\hline Residentlal Sector & 35.7 & 51.3 & 102.2 & 196.8 & 185.4 & 181.8 & 176.2 & 183.6 \\
\hline 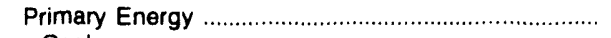 & 20.2 & 28.3 & 46.1 & 94.0 & 84.3 & 82.0 & 73.2 & 79.6 \\
\hline Coal & .1 & .3 & .6 & 1.5 & .8 & .9 & .8 & 1.0 \\
\hline 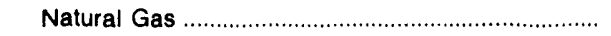 & 12.3 & 12.3 & 27.5 & 74.2 & 62.8 & 49.9 & 52.2 & 55.5 \\
\hline Petroleum & 7.9 & 15.6 & 18.1 & 18.3 & 20.7 & 31.2 & 20.2 & 23.0 \\
\hline 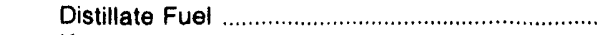 & .1 & .4 & .9 & 3.0 & .9 & .7 & .8 & 1.1 \\
\hline 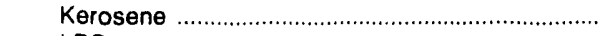 & .4 & .2 & - & .7 & $\cdot$ & " & • & • \\
\hline LPG & 7.4 & 15.0 & 17.1 & 14.7 & 19.7 & 30.4 & 19.4 & 22.0 \\
\hline Electricity ……… & 15.5 & 23.0 & 56.1 & 102.8 & 101.1 & 99.8 & 103.0 & 104.0 \\
\hline 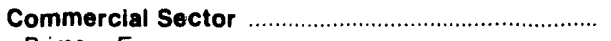 & 21.2 & 27.9 & $\mathbf{8 4 . 0}$ & 203.5 & 190.7 & 172.2 & 175.1 & 167.2 \\
\hline 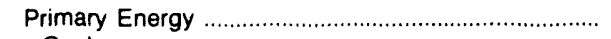 & 9.4 & 13.4 & 40.4 & 81.7 & 67.8 & 55.4 & 55.1 & 50.1 \\
\hline Coal & .1 & .5 & 2.1 & 2.3 & 1.7 & 1.6 & 2.2 & 2.1 \\
\hline 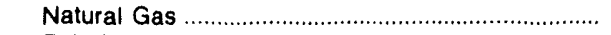 & 6.1 & 6.9 & 13.2 & 46.4 & 37.4 & 37.7 & 35.8 & 37.0 \\
\hline Petroleum & 3.2 & 5.9 & 25.1 & 33.0 & 28.7 & 16.1 & 17.2 & 11.0 \\
\hline Distillate Fuel & .2 & .9 & 16.1 & 20.4 & 14.6 & 6.1 & 5.7 & 5.9 \\
\hline 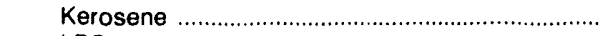 & .7 & .6 & .8 & .3 & • & • & .1 & \\
\hline LPG & .8 & 1.6 & 2.1 & 2.9 & 3.9 & 5.2 & 3.4 & 2.4 \\
\hline Motor Gasoline & 1.3 & 1.8 & 5.5 & 3.1 & 4.3 & 2.6 & 2.4 & 2.6 \\
\hline 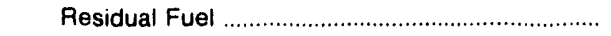 & .2 & 1.1 & .6 & 6.3 & 6.0 & 2.1 & 5.5 & \\
\hline 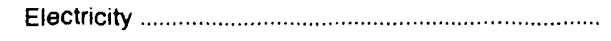 & 11.8 & 14.5 & 43.5 & 121.8 & 122.9 & 116.8 & 120.0 & 117.0 \\
\hline Industrial Sector & 59.2 & 154.8 & 490.1 & 578.4 & 466.8 & 453.4 & 451.3 & 486.8 \\
\hline Primary Energy & 39.7 & 122.4 & 413.6 & 375.7 & 257.2 & 220.7 & 209.0 & 240.9 \\
\hline 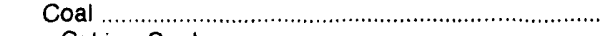 & 1.7 & 10.6 & 49.6 & 62.2 & 45.6 & 44.6 & 41.0 & 46.1 \\
\hline 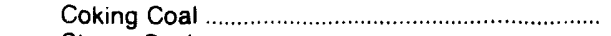 & - & - & - & - & - & - & - & - \\
\hline Steam Coal & 1.7 & 10.6 & 49.6 & 62.2 & 45.6 & 44.6 & 41.0 & 46.1 \\
\hline Natural Gas & 9.5 & 16.7 & 50.0 & 55.3 & 47.8 & 37.3 & 42.5 & 42.4 \\
\hline Petroleum & 28.5 & 95.0 & 314.0 & 258.2 & 163.8 & 138.9 & 125.5 & 152.5 \\
\hline Asphalt and Road Oil & 4.2 & 7.2 & 28.2 & 53.6 & 46.5 & 31.3 & 23.6 & 17.6 \\
\hline 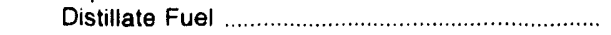 & 8.9 & 47.3 & 198.0 & 122.6 & 61.2 & 58.3 & 53.2 & 63.6 \\
\hline 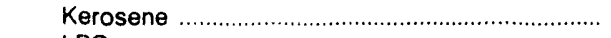 & .8 & 1.6 & 1.3 & .3 & .1 & .1 & .1 & \\
\hline LPG & 2.1 & 4.0 & 22.0 & 36.7 & 27.5 & 20.4 & 18.7 & 38.1 \\
\hline 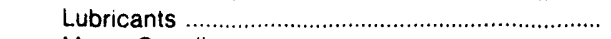 & .9 & 2.1 & 5.0 & 5.6 & 4.8 & 4.8 & 4.9 & 6.2 \\
\hline 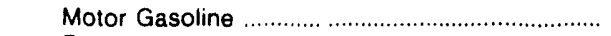 & 8.5 & 14.8 & 19.7 & 24.7 & 17.7 & 16.3 & 16.6 & 19.7 \\
\hline 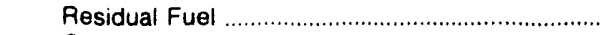 & .9 & 11.1 & 23.4 & 9.1 & 2.6 & 3.8 & 4.3 & 1.7 \\
\hline Other & 2.1 & 6.9 & 16.4 & 5.8 & 3.3 & 4.0 & 4.1 & 5.6 \\
\hline 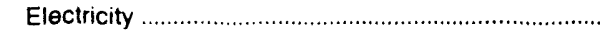 & 19.6 & 32.5 & 76.5 & 202.7 & 209.6 & 232.7 & 242.3 & 245.9 \\
\hline Transportation Sector & 112.4 & 211.4 & 735.4 & 529.5 & 403.9 & 478.8 & 524.5 & 615.9 \\
\hline Primary Energy & 112.4 & 241.4 & 735.4 & 529.5 & 403.9 & 478.8 & 524.5 & 615.9 \\
\hline Coal & & & - & & & - & - & - \\
\hline Petroleum & 112.3 & 241.4 & 735.4 & 529.5 & 403.9 & 478.8 & 524.5 & 615.9 \\
\hline 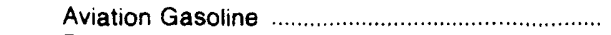 & 2.8 & 3.8 & 4.9 & 2.6 & 2.1 & 1.9 & 2.0 & 1.6 \\
\hline Distillate Fuel & 23.4 & 62.4 & 276.4 & 176.1 & 154.0 & 214.5 & 255.6 & 305.9 \\
\hline Jet Fuel & .5 & 1.5 & 6.0 & 5.6 & 3.7 & 5.2 & 5.0 & 4.5 \\
\hline LPG & .4 & 1.1 & 1.4 & 1.2 & 1.1 & 1.0 & 1.0 & .7 \\
\hline 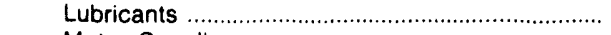 & 2.6 & 4.9 & 13.1 & 14.6 & 12.7 & 12.5 & 12.9 & 16.3 \\
\hline 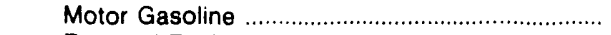 & 81.0 & 167.8 & 433.7 & 329.4 & 230.3 & 243.7 & 248.0 & 286.8 \\
\hline 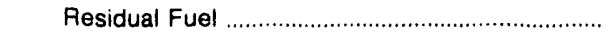 & 1.6 & - & - & * & - & - & - & . \\
\hline 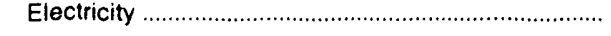 & - & - & - & - & - & - & - & - \\
\hline Total Energy" & 228.5 & 475.4 & $1,411.7$ & $1,508.2$ & $1,246.8$ & $1,286.2$ & $1,327.2$ & $1,453.4$ \\
\hline Primary Energy - Four Sectors .................................. & 181.7 & 405.4 & $1,235.6$ & $1,080.9$ & 813.2 & 836.9 & 861.9 & 986.5 \\
\hline 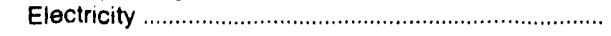 & 46.9 & 70.0 & 176.1 & 427.3 & 433.6 & 449.3 & 465.3 & 466.8 \\
\hline Electric Utillty Sector & 8.9 & 30.3 & 140.7 & 346.3 & 283.5 & 345.3 & 350.0 & 328.6 \\
\hline Coal & 8.3 & 28.4 & 134.9 & 340.7 & 280.2 & 342.2 & 346.6 & 325.4 \\
\hline 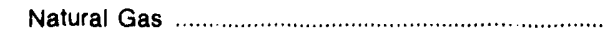 & .5 & .4 & .9 & 6 & .5 & .3 & .7 & .3 \\
\hline Petroleum & .1 & 1.5 & 5.0 & 5.0 & 2.8 & 2.8 & 2.7 & 2.9 \\
\hline Heavy Oib & • & 1.4 & - & - & - & - & - & - \\
\hline 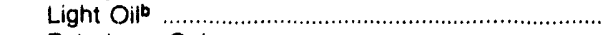 & 1 & .1 & 5.0 & 5.0 & 2.8 & 2.8 & 2.7 & 2.8 \\
\hline Petroleum Coke & - & - & - & - & - & - & - & - \\
\hline Nuclear Fuel & - & - & - & - & - & - & - & - \\
\hline Wood and Waste & - & - & - & - & - & - & - & - \\
\hline Primary Energy - Five Sectors & 190.6 & 435.7 & $1,376.3$ & $1,427.2$ & $1,096.7$ & $1,182.2$ & $1,211.8$ & $1,315.1$ \\
\hline
\end{tabular}

- There are no direct fuel costs for hydroelectric, geothermal, centralized solar, or wind energy. Wood and other biomass fuels are not included, except those consumed at electric utilities.

bHeavy oil includes Grade Nos. 4, 5, and 6, and residual fuel oils. Light oil includes Grade No. 2 heating oil, kerosene, and jet fuel.

- No consumption, including cases where adjustments were made. See "Consumption Adjustments for Process Fuel and Intermediate Products."

- Value less than 0.05 million dollars.

Note: Totals may not equal sum of components due to independent rounding.

Sources: Data sources, estimation procedures, and assumptions are described in the "Documentation" section of this report. 


\section{Documentation}

This section documents data sources and procedures used to develop the estimates presented in the data tables. Information is provided for each of the four major energy sources: coal, natural gas, petroleum, and electricity. A section is included to describe adjustments for consumption of process fuel and intermediate products, and the last section summarizes the changes incorporated into this report since its previous release.

Energy consumption estimates used in the State Energy Price and Expenditure Data System (SEPEDS) are from the Energy Information Administration (EIA) State Energy Data Report. Consumption Estimates, 1960-1989, DOE/EIA-0214(89), published in May 1991. The data system from which the consumption report is developed is called the State Energy Data System (SEDS). Full documentation of SEDS data sources and the methods used to estimate energy consumption are described in the consumption report. The methods used to estimate corresponding prices and expenditures are discussed in the following paragraphs and in the remaining documentation of this report. Many of the price estimates used in SEPEDS were developed by the Battelle Pacific Northwest Laboratories under contract to EIA.

Price data in the State Energy Price and Expenditure Report are expressed in dollars per million Btu. If the source data are in physical units, they are divided by the appropriate conversion factors to generate the Btu prices. Estimated prices are used only when specific State-level prices are not available for a given energy source and sector. In some cases, prices for energy consumed in one sector in a State are assigned to another sector in the same State. Specific examples are: commercial and transportation steam coal use industrial steam coal prices; commercial kerosene uses industrial kerosene prices; commercia! and transportation liquefied petroleum gases (LPG), including ethane, use industrial LPG prices; transportation lubricants uses industrial lubricants prices; and commercial and industrial motor gasoline use transportation motor gasoline prices.

In addition, there are a few cases where State-level prices could not be identified for any economic sector for a given energy source for some or all years. In these instances, a national-level price is used for all States for a given year. The procedures for estimating these national-level prices are presented in the body of the documentation under each energy source as appropriate. The cases where a national-level price is assigned to all States in all years are: transportation use of aviation gasoline; industrial and transportation use of lubricants; wood and waste used at electric utilities; and the components of other petroleum products.

Finally, within a given energy source and sector where price data are usually available, there are some cases of missing prices. Two general approaches are used to assign or estimate prices in cases where consumption occurs but no price is directly available from the data sources. The first approach is to assign an adjacent State price or the simple average of adjacent States' prices. When this approach is not feasible, the consumption-weighted price from the Census division or Census region in which the State is located is assigned. Nuclear fuel price estimation procedures are unique and are described in the nuclear fuel documentation.

Three State groupings used in the report--U.S. Census regions and divisions, Federal regions, and Petroleum Administration for Defense districts--are shown in Figures 9,10 , and 11 , respectively, on the following pages. Throughout the documentation, the term "States" : $n$ cludes the District of Columbia. States are often designated by the two-letter postal code abbreviations shown on the maps. 
Figure 9. U.S. Census Regions and Divisions

$\mathbf{O}$
$\mathbf{C}$
$\mathbf{U}$
$\mathbf{M}$
$\mathbf{E}$
$\mathbf{N}$
$\mathbf{T}$
$\mathbf{A}$
$\mathbf{T}$
$\mathbf{U}$
$\mathbf{O}$
$\mathbf{N}$

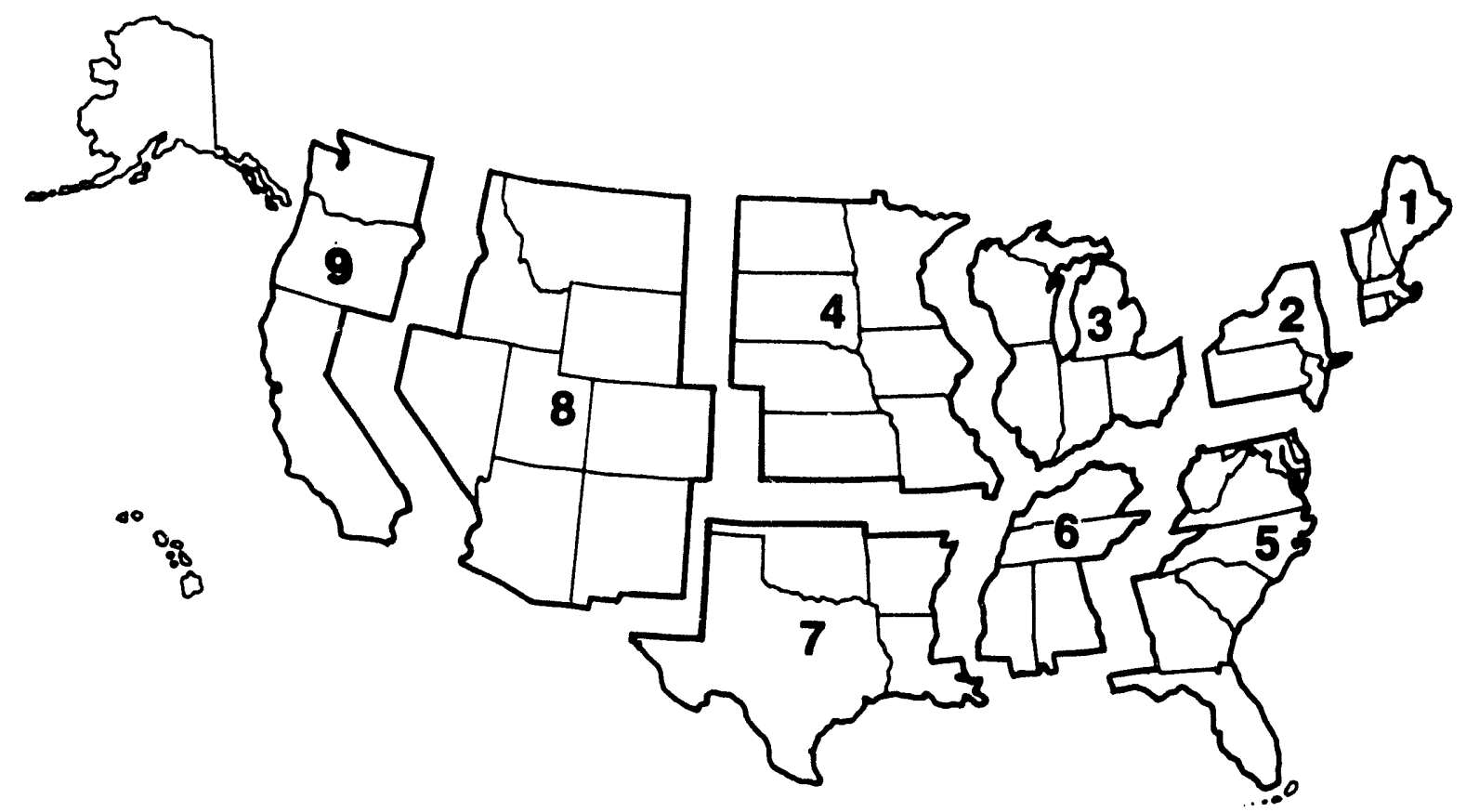

Region 1

Region 2

Midwest

Region 3

South

Region 4

Northeast

Division 3

Division 5

Division 1

(East North Central)

(South Atlantic)

Division 8

(New England)

Connecticut (CT)

Illinois (IL)

Maine (ME)

Massachusetts (MA)

Indiana (IN)

Michigan (MI)

New Hampshire (NH)

Ohio $(\mathrm{OH})$

Rhode Island (RI)

Vermont (VT)

Division 2

(Middle Atlantic)

New Jersey (NJ)

New York (NY)

Pennsylvania (PA)

Wisconsin (WI)

Division 4

(West North Central)

lowa (IA)

Kansas (KS)

Minnesota (MN)

Missouri (MO)

Nebraska (NE)

North Dakota (ND)

South Dakota (SD)
Delaware (DE)

District of Columbia (DC)

Florida (FL)

Georgia (GA)

Maryland (MD)

North Carolina (NC)

South Carolina (SC)

Virginia (VA)

West Virginia (WV)

\section{Division 6}

(East South Central)

Alabama (AL)

Kentucky (KY)

Mississippi (MS)

Tennessee (TN)

\section{Division 7}

(West South Central)

Arkansas (AR)

Louisiana (LA)

Oklahoma (OK)

Texas (TX)

\section{(Mountain)}

Arizona ( $A Z$ )

Colorado (CO)

Idaho (ID)

Montana (MT)

Nevada (NV)

New Mexico (NM)

Utah (UT)

Wyoming (WY)

Division 9

(Pacific)

Alaska (AK)

California (CA)

Hawaii (HI)

Oregon (OR)

Washington (WA) 


\section{Region 1}

New England

Connecticut (CT)

Maine (ME)

Massachusetts (MA)

New Hampshire (NH)

Rhode Island (RI)

Vermont (VT)

Region 2

New York/

New Jersey

New Jersey (NJ)

New York (NY)

\section{Region 3}

\section{Mid Atlantic}

Delaware (DE)

District of Columbia (DC)

Maryland (MD)

Pennsylvania (PA)

Virginia (VA)

West Virginia (WV)
Region 4

South Atlantic

Alabama (AL)

Florida (FL)

Georgia (GA)

Kentucky (KY)

Mississippi (MS)

North Carolina (NC)

South Carolina (SC)

Tennessee (TN)

Region 5

Midwest

Illinois (IL)

Indiana (IN)

Michigan (MI)

Minnesota (MN)

Ohio (OH)

Wisconsin (WI)
Region 6

Southwest

Arkansas (AR)

Louisiana (LA)

New Mexico (NM)

Oklahoma (OK)

Texas (TX)

\section{Region 7}

Central

Iowa (IA)

Kansas (KS)

Missouri (MO)

Nebraska (NE)
Region 8

North Central

Colorado (CO)

Montana (MT)

North Dakota (ND)

South Dakota (SD)

Utah (UT)

Wyoming (WY)

Region 9

West

Arizona ( $A Z$ )

California (CA)

Hawaii (HI)

N. ada (NV)

Region 10

Northwest

Alaska (AK)

Idaho (ID)

Oregon (OR)

W'ashington (WA) 


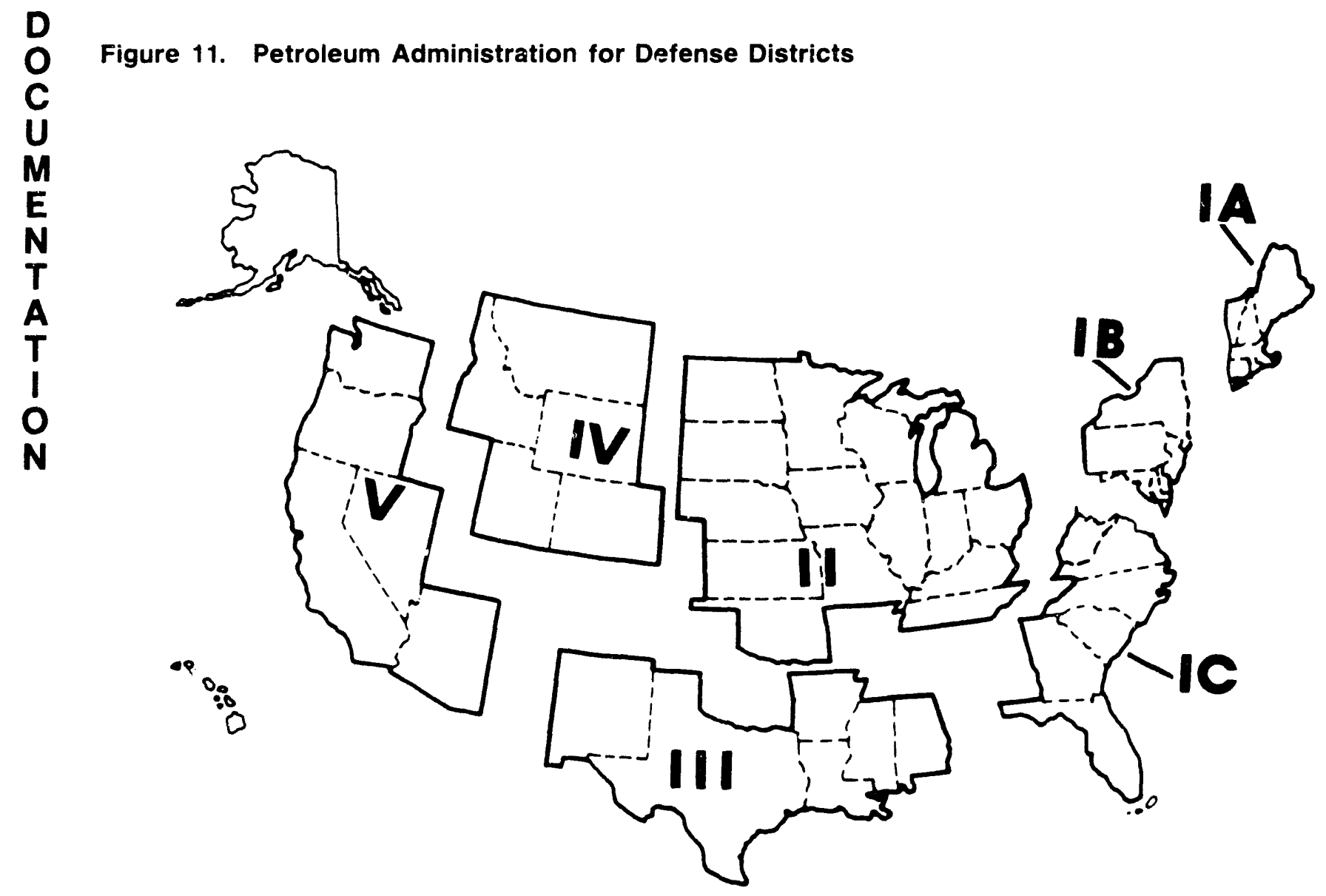

District IA

Connecticut (CT)

Maine (ME)

Massachusetts (MA)

New Hampshire (NH)

Rhode Island (RI)

Vermont (VT)

\section{District IB}

Delaware (DE)

District of Columbia (DC)

Maryland (MD)

New Jersey (NJ)

New York (NY)

Pennsylvania (PA)

District IC

Florida (FL)

Georgia (GA)

North Carulina (NC)

South Carolina (SC)

Virginia (VA)

West Virginia (WV)

\section{District II}

Illinois (IL)

Indiana (IN)

Iowa (IA)

Kansas (KS)

Kentucky (KY)

Michigan (MI)

Minnesota (MN)

Missouri (MO)

Nebraska (NE)

North Dakota (ND)

Ohio $(\mathrm{OH})$

Oklahoma (OK)

South Dakota (SD)

Tennessee (TN)

Wisconsin (WI)
District III

Alabama (AL)

Arkansas (AR)

Louisiana (LA)

Mississippi (MS)

New Mexico (NM)

Texas (TX)

District IV

Colorado (CO)

Idaho (ID)

Montana (MT)

Utah (UT)

Wyoming (WY)
District V

Alaska (AK)

Arizona (AZ)

California (CA)

Hawaii (HI)

Nevada (NV)

Orezon (OR)

Washington (WA) 


\section{Coal}

Coal prices are developed for the following categories: coking coal; steam coal (all noncoking coal); and coal coke, imports and exports.

Coking coal, used in the industrial sector only, is a high-quality bituminous coal that is used to make coal coke. Steam coal includes anthracite, bituminous coal not used for coking, subbituminous coal, and lignite. Steam coal may be used in all sectors. In the industrial sector, coal consumption is the sum of coking coal and steam coal. The industrial coal price is the quantityweighted average price of these two components.

Imports and exports of coal coke are confined to the national level and are accounted for in the industrial sector. Coal coke imports and exports are reported separately, and their prices and expenditures are not merged with other coal prices and expenditures.

\section{Coking Coal}

Coking coal is generally more expensive than steam coal; therefore, it is identified separately in developing the price estimates. Coking coal prices are those paid at coke plants for coal received, and probably include any taxes (although this is not clear from the sources' data collection forms).

\section{Physical Unit Prices: All Years}

Source publications contain physical unit prices for States or for groups of States. Individual State prices are used directly for their respective States. Group prices are assigned to each State within the group as shown in Table 15.

Wherever individual State prices or State group prices are unavailable, prices are assigned from an adjacent or nearby State as shown in Table 16.
Table 15. Coking Coal Group Price Assignments

\begin{tabular}{lll}
\hline State & Years & Assigned Group Price \\
\hline CA & $1970-1982$ & CA, CO, UT \\
CO & $1970-1982$ & CA, CO, UT \\
KY & $1970-1987$ & KY, MO, TN, TX \\
MD & 1970,1971 & MD, NJ, NY \\
& $1972-1982$ & MD, NY \\
MI & 1979 & MI, MN, WI \\
& $1980-1987$ & MI, WI \\
MN & $1970-1978$ & MN, WI \\
& 1979 & MI, MN, WI \\
MO & $1970-1987$ & KY, MO, TN, TX \\
NJ & 1970 & MD, NJ, NY \\
NY & 1970,1971 & MD, NJ, NY \\
& $1972-1982$ & MD, NY \\
TN & $1970-1987$ & KY, MO, TN, TX \\
TX & $1970-1986$ & KY, MO, TN, TX \\
UT & $1970-1982$ & CA, CO, UT \\
VA & $1978-1982$ & VA, WV \\
WI & $1970-1978$ & MN, WI \\
& 1979 & MI, MN, WI \\
WV & $1980-1986$ & MI, WI \\
\hline
\end{tabular}

Table 16. Coking Coal Adjacent State Price Assignments

\begin{tabular}{lll}
\hline State & Years & Assigned Prices \\
\hline IL & $1986,1988,1989$ & IN \\
KY & 1988.1989 & OH \\
MD & $1983-1989$ & PA \\
MI & 1988,1989 & OH \\
MO & 1988 & AL \\
NY & $1983-1989$ & PA \\
TN & 1988,1989 & AL \\
UT & $1983-1986$ & TX \\
& 1988,1989 & IN \\
VA & $1983-1986$ & KY \\
& $1987-1989$ & OH \\
WV & $1983-1986$ & KY \\
& $1987-1989$ & UH \\
\hline
\end{tabular}


Btu prices for States are calculated from the physical unit prices and the constant conversion factor for coking coal. U.S. Btu prices are calculated as the average of the State Btu prices, weighted by consumption data from the State Energy Data System (SEDS).

\section{Data Sources}

Prices

1981 forward: Energy Information Administration, Quarterly Coal Report, October-December issue, Table A3.

1977-1980: Energy Information Administration, Coke and Coal Chemicals, Table 19 (1977), Table 15 (1978), and Table 7 (1979, 1980).

1970-1976: Bureau of Mines, U.S. Department of the Interior, Minerals Yearbook, "Coke and Coal Chemicals" chapter, Table 22.

\section{Consumption}

1970 forward: Energy Information Administration, State Energy Data System, coking coal consumption.

\section{Conversion Factor: All Years}

26.80 million Btu per short ton

\section{Steam Coal}

Steam coal is used in all sectors. Price data are generally available in the electric utility, residential, and industrial sectors. However, no price data are directly available in the transportation and commercial sectors. The industrial sector steam coal prices are assigned as price estimates in these two sectors. Data sources and computational procedures for estimating coal prices are discussed by sector. Estimates of the amount of steam coal consumed by sector are from SEDS and are adjusted for process fuel consumption in the industrial sector. See the "Consumption Adjustments for Process Fuel and Intermediate Products" section in this documentation.

\section{Btu Prices: 1973 Forward}

State Btu prices, including all taxes, are from Cost and Quality of Fuels (C\&Q) for 1973 forward and are converted from cents to dollars per million Btu. Where individual State prices are unavailable, quantityweighted Census division prices are assigned as follows: CT/1975-1979; OK/1973, 1974; OR/1983, 1989; and VT/1980, 1983-1986. In addition, the RI/1974 price is assigned from MA, the $\mathrm{OK} / 1975$ price is assigned from the simple average of $\mathrm{CO}, \mathrm{KS}, \mathrm{MO}, \mathrm{NM}$, $\mathrm{TX}$, and the DC/1976 price is assigned from the simple average of MD, VA.

\section{Btu Prices: 1970-1972}

Btu prices for States are from the Statistical Yearbook and are converted from cents to dollars. Delaware, $\mathrm{DC}$, and $\mathrm{MD}$ are each assigned the combined price for the three States. The steam coal electric utility sector AK price for 1971 is estimated as discussed below.

\section{Alaska Prices: All Years}

Since $C \& Q$ data do not cover $\mathrm{AK}$, Btu prices for $\mathrm{AK}$ are from other sources or estimated for 1971 and for 1973 forward. For the years 1970, 1972, 1974, 1976, 1977, and 1979-1988, prices are directly from the Statistical Yearbook. Prices for 1971, 1973, 1975, and 1978 are estimated from the Statistical Yearbook prices for the United States and the average ratio of AK-to-U.S. prices for the years when AK prices are available. The 1971 and 1973 estimated prices are based on the average ratio for 1970 and 1972, while the 1975 and 1978 estimated prices are based on the average ratio for 1974 , 1976, 1977, and 1979.

\section{U.S. Prices: All Years}

U.S. Btu prices are calculated as the average of the State Btu prices, weighted by consumption data from SEDS.

\section{Data Sources}

Prices

1973 forward: Energy Information Administration, Cost and Quality of Fuels for Electric Utility Plants, Table 3 (1973-1979), Table 51 (1980-1982), Table 50 (1983, 1984), Tatic 40 (1985 furwalci).

1970 forward: Edison Electric Institute. Statistical Yearbook of the Electric Utility Industry, table titled, 
"Analysis of Fuel for Electric Generation: Total Electric Utility Industry."

\section{Consumption}

1970 forward: Energy Information Administration, State Energy Data System, electric utility sector coal consumption.

\section{Conversion Factors: All Years}

Btu prices are directly from the data sources; no explicit conversion factors are used.

\section{Residential Sector}

Residential sector steam coal prices are the average delivered prices for coal purchased by residential customers and include taxes.

\section{Prices: 1979 Forward}

Residential steam coal Btu prices for 1979 and subsequent years are computed using an equation estimated by regressing the residential steam coal prices generated for 1974-1978 from Gas Househeating Survey (GHS) on average spot coal Btu prices from $C \& Q$. The $C \& Q$ prices in cents per million Btu must be changed to dollars per million Btu before they are used to estimate the prices for 1979 forward.

Several States are missing $C \& Q$ coal spot prices, both in the 1974-1978 data and in the 1979 forward data used to calculate prices. For these missing data, $C \& Q$ prices are assigned from other States for use in the regression as shown in Table 17.

Wherever individual State prices are unavailable, prices are assigned from adjacent or nearby States, as follows:

\begin{tabular}{lll} 
State & Years & Assigned Prices \\
\hline AR & $\begin{array}{l}1980,1982,1984,1985, \\
1987-1989\end{array}$ & AL \\
& $\begin{array}{l}1987-1989 \\
\text { AZ }\end{array}$ & UT \\
CA & $1979-1985$ & NV \\
& $1987-1989$ & WA \\
FL & $1980-1985,1987-1989$ & GA \\
LA & $1980,1932,1984$ & AL \\
MS & $1979,1980,1983,1984$, & AL \\
& $1987-1989$ & \\
MT & $1986-1989$ & WY \\
NM & $1979-1989$ & CU \\
OK & $1979-1989$ & CO \\
OR & $1979.1980,1982-1989$ & WA \\
TX & $1980-1982,1985-1989$ & CO
\end{tabular}

Table 17. Residential Sector Spot Coal Price Assignments for Prices Missing in $C \& Q$

\begin{tabular}{|c|c|c|}
\hline State & Years & Assigned Prices \\
\hline $\mathrm{CO}$ & 1979,1981 & KS \\
\hline \multirow[t]{3}{*}{ CT } & 1975 & NY \\
\hline & $1976-1979$ & NH \\
\hline & $1980-1987$ & MA \\
\hline $\mathrm{DC}$ & $1976-1989$ & MD \\
\hline \multirow[t]{4}{*}{ ID } & $1974,1979-1982$ & NV \\
\hline & $1975-1977$ & $\mathrm{SD}$ \\
\hline & 1978 & ND \\
\hline & $1983-1989$ & $\mathrm{CO}$ \\
\hline \multirow[t]{2}{*}{$\mathbf{M A}$} & 1975 & VT \\
\hline & $1976-1979$ & NH \\
\hline \multirow[t]{3}{*}{$\mathrm{ME}$} & $1974,1975,1981,14: 3$ & VT \\
\hline & $1976-1980,1982$ & $\mathrm{NH}$ \\
\hline & $1984-1989$ & MA \\
\hline \multirow[t]{3}{*}{$\mathrm{MT}$} & $1974,1975,1978$ & ND \\
\hline & 1976,1977 & $\mathrm{SD}$ \\
\hline & 1979.1982 & NV \\
\hline \multirow{2}{*}{ ND } & 1976,1977 & $\mathrm{SD}$ \\
\hline & $1979-1988$ & $\mathrm{MN}$ \\
\hline \multirow{2}{*}{ NH } & $1974,1975,1981,1983$ & VT \\
\hline & 1984,1985 & MA \\
\hline NV & $\begin{array}{l}1975-1978,1983-1985, \\
1987-1989\end{array}$ & $\mathrm{CO}$ \\
\hline \multirow[t]{4}{*}{ RI } & 1974 & CT \\
\hline & 1975 & VT \\
\hline & $1976-1979$ & NH \\
\hline & $1980-1989$ & MA \\
\hline \multirow[t]{2}{*}{ SD } & 1978,1984 & ND \\
\hline & $\begin{array}{l}1979-1983,1986,1987, \\
1989\end{array}$ & $\mathrm{MN}$ \\
\hline \multirow[t]{2}{*}{ UT } & $1975-1978,1980,1983$ & $\mathrm{CO}$ \\
\hline & 1979 & NV \\
\hline \multirow[t]{2}{*}{ VT } & 1976,1980 & $\mathrm{NH}$ \\
\hline & 1984-1989 & MA \\
\hline \multirow[t]{2}{*}{ WA } & $1974-1978,1983-1985$ & $\mathrm{CO}$ \\
\hline & $1979-1982$ & NV \\
\hline WY & $\begin{array}{l}\text { 1974-1976, 1978, } 1982, \\
1983,1985\end{array}$ & $\mathrm{CO}$ \\
\hline
\end{tabular}

In addition, the following States are assigned the simple average of the prices of adjacent States:

\begin{tabular}{lll} 
State & Years & Averaged Prices \\
\hline AR & 1981 & MO, OK, TN, TX \\
& 1983 & MO, MS, OK, TN \\
AZ & $1982,1984,1985$ & CA, NM, NV, UT \\
MS & 1985 & AL, AR, TN \\
OR & 1981 & CA, ID, NV, WA
\end{tabular}

AK's Btu prices for 1981-1986 are estimated from the WA State prices during that period. The average ratio 
C of AK-to-WA prices during 1970-1979 is applied to the WA price each year to estimate the AK price for the year.

\section{Prices: 1971-1978}

For 1971-1978. Btu steam coal prices are calculated using data from $G H S$. The price for a State is equal to the simple average of the city/utility price observations for that State. For 1971 and 1972, GHS reports physical unit prices rather than Btu prices, (as published for 1973-1978) and therefore, the State-level conversion factors for this sector from SEDS are used to convert to Btu prices for those years.

The combined DC, DE and MD price is assigned to each of the three States. A simple average of price observations in CT, MA, ME, NH, RI, and VT is assigned to each of these States. To impute other missing prices in the 1971-1978 period, simple averages of adjacent State prices are used as listed here:

\begin{tabular}{lll} 
State & Years & $\begin{array}{l}\text { Assigned or } \\
\text { Averaged Prices }\end{array}$ \\
\hline AL & 1971 & TN \\
GA & 1971 & NC, TN \\
& 1972 & AL, NC, TN \\
ID & 1977 & MT, UT, WY \\
KS & 1971,1972 & CO, MO \\
MN & 1971 & IA, ND, WI \\
& 1972 & IA, WI \\
MT & 1971 & ID, ND, WY \\
& 1972,1973 & ID, WY \\
ND & 1972 & IA, WI \\
& 1973 & MN, SD \\
& 1974 & MN, MT, SD \\
NE & 1971,1972 & CO, IA, MO, WY \\
& 1975 & CO, IA, KS, MO, SD, \\
& $1971,1972,1974$, & DE, NY, PA \\
NJ & 1977,1978 & \\
& $1971,1972,1975$ & ID, UT \\
NV & 1973 & ID, OR, UT \\
SC & 1971,1972 & NC \\
SD & 1971 & IA, ND, WY \\
& 1972 & IA, WY \\
UT & 1974,1978 & CO, ID, NV, WY \\
WA & $1971,1972,1974$ & ID \\
& 1977 & MT, UT, WY \\
WV & 1971,1972 & KY, MD, OH, PA, \\
& & VA
\end{tabular}

Wherever individual State prices are still unavailable, prices are assigned from adjacent or nearby States as follows:

\begin{tabular}{lll} 
State & Years & Assigned Prices \\
\hline AR & 1977,1978 & AL \\
CA & $1971.1972,1974,1978$ & NV \\
DE & 1971,1972 & MD \\
MS & 1978 & AL \\
NM & 1971 & CO \\
OK & $1971-1978$ & CO \\
OR & $1971-1978$ & WA \\
TX & $1971-1974,1977$ & CO
\end{tabular}

AK physical unit prices for 1971-1977 are estimated using the ratio of AK-to-U.S. utility sector prices.

\section{Prices: 1970}

Since State-level coal price data for 1970 are not available from either $G H S$ or $C \& Q$, the 1970 residential sector coal prices are calculated using the 1971-1978 data from the Statistical Yearbook for the 39 States with some reported coal use from 1971-1983 and regression analysis.

For estimating the 1970 prices, States missing Statistical Yearbook data are assigned prices as follows: ID/1970-1978 from MT; MA/1976-1978 from CT; $\mathrm{ME} / 1970-1978$ from NH; RI/1973, 1975-1978 from CT; and WA/1970-1972 from OR. DC, DE, and MD are all assigned the combined Statistical Yearbook price for those States. Wherever individual State prices are unavailable, prices are assigned from an adjacent or nearby State as follows: CA from NV; NM from $\mathrm{CO}$; $\mathrm{OK}$ from $\mathrm{CO}$; OR from WA; and TX from $\mathrm{CO}$.

AK physical unit prices are estimated using the ratio of AK-to-U.S. utility sector prices.

\section{U.S. Prices: All Years}

U.S. Btu prices are calculated as the average of the State Btu prices, weighted by consumption data from SEDS.

\section{Data Sources}

Prices

1974 forward: Energy Information Administration, Cost and Quality of Fuels for Electric Plants, average spot coal prices, Table 2 (1974-1979); Table 44 (1980-1982); Table 49 (1983, 1984); and Table 39 (1985 forvard).

1971-1978: American Gas Association, Gas Househeating Survey, table titled, "Competitive Fuel Prices." 
1970-1978: Edison Electric Institute. Statistical Yearbook of the Electric Utility Industry. Table 43S.

\section{Consumption}

1970 forward: Energy Information Administration, State Energy Data System, residential sector coal consumption.

\section{Conversion Factors: 1971, 1972}

Energy Information Administration, Stcte Energy Data Report. Consumption Estimates, 1960-1989, Table D8.

\section{Commercial Sector}

Commercial sector prices are assigned from industrial steam coal prices. Although there was no industrial steam coal consumption in 1989 in NJ, NM, NV, OK, $\mathrm{OR}$, or $\mathrm{SD}$, industrial sector prices are calculated from physical unit prices available in the source documents to be assigned to the commercial sector consumption in those States. Physical unit industrial steam coal prices are not available for DC/1980-1989 so the MD/1980-1989 prices are assigned. Physical unit industrial prices are not available for RI/1982, 1983, and MA prices are assigned for those years. The Btu price for RI/1982 is calculated using a conversion factor that is the simple average of the 1981 and 1983 conversion factors. U.S. Btu prices are calculated as the average of the State Btu prices, weighted by consumption data from SEDS.

\section{Industrial Sector}

For 1980 forward, prices are based on the average price of coal receipts at manufacturing plants. For 1970-1979, prices are based on the average cost of coal sold to manufacturing firms.

\section{Physical Unit Prices: 1980 Forward}

For 1984 forward, State prices are from the Quarterly Coal Report ( $Q C R)$. The annual price is estimated as the average of the quarterly prices weighted by the quarterly receipts. For 1980-19x.3. prices are directly from EIA-3 data. Published prices in average cost per ton are used directly when available, while missing prices are estimated using simple averages or price assignments from adjacent States. Although it is not clear from the data sources, the prices probably inclide taxes.
Missing State data are estimated using simple averages of the published data for adjacent States. In a few cases, only a single adjacent State price is available for the estimation. The adjacent States used for estimations are shown in Table 18 on page 184.

\section{Physical Unit Prices: 1971, 1974-1979}

For 1971 and 1974-1979, available cost and quantity of hitumino is coal, lignite, and anthracite from Annual Survey of Manufactures (ASM) or Census of Manufactures $(C M)$ are used to calculate prices as average cost per unit of sales for covered States. (States with undisclosed data are not considered covered). It is not clear from the data sources whether the prices include taxes.

For States with industrial steam coal use and for which $A S M$ or $C M$ data are not available in 1971 and 1974-1979, adjacent State simple averages of available $A S M / C M$ data are used to impute prices. The assigned prices from adjacent States are are shown in Table 19 on page 184 .

\section{Physical Unit Prices: 1970, 1972, 1973}

Steam coal industrial sector prices for 1970,1972 , and 1973 (years for which no $A S M / C M$ prices are available) are estimated using regression techniques. Values for the independent variable are steam coal electric utility sector physical unit prices and values for the dependent variable are the steam coal industrial physical unit prices (from $A S M$ or estimated as described above) for 1971, and 1974-1977. A few States are assigned electric utility prices for the dependent variable in the regression as shown below:

\begin{tabular}{lll} 
State & Years & Assigned Prices \\
\hline AR & 1972 & MO, TN \\
& $1973,1976,1977$ & MO \\
CA & $1970-1977$ & NV \\
CT & $1975-1977$ & NY \\
DC & 1976,1977 & MD \\
ID & $1970-1977$ & MT \\
MA & 1976,1977 & NH \\
ME & $1970-1977$ & NH \\
OK & $1973-1975$ & KS \\
OR & $1973-1977$ & WA \\
TX & 1970 & NM \\
WA & $1970-1972$ & OR
\end{tabular}


Table 18. Industrial Sector Steam Coal Price Estimates for 1980 Forward

\begin{tabular}{|c|c|c|}
\hline State & Years & $\begin{array}{l}\text { Prices l'sed } \\
\text { in the Estimation }\end{array}$ \\
\hline \multirow[t]{2}{*}{$\mathrm{AZ}$} & 1980 & $\mathrm{CA}, \mathrm{UT}$ \\
\hline & $1981,1984,1985$ & CA, CO, UT \\
\hline $\mathrm{CO}$ & 1980 & KS, UT \\
\hline $\mathrm{CT}$ & $1981-1989$ & New England \\
\hline DC & $1980-1981$ & $\mathrm{MD}$ \\
\hline DE & $1980-1989$ & $\mathrm{MD}$ \\
\hline FL & 1980 & $A L, G A$ \\
\hline HI & $1982-1989$ & $\mathrm{CA}$ \\
\hline LA & $1980-1985,1989$ & AR, TX \\
\hline \multirow[t]{2}{*}{ MA } & $1980-1983$ & NY \\
\hline & $1984-1989$ & New England \\
\hline \multirow[t]{2}{*}{$\mathrm{ME}$} & $1980-1983$ & NY \\
\hline & $1984-1989$ & New England \\
\hline MS & $1980-1989$ & AL. AR. TN \\
\hline \multirow[t]{2}{*}{ MT } & 1983 & ID, WY \\
\hline & $1984-1989$ & ID \\
\hline \multirow[t]{2}{*}{ ND } & 1980-1982 & MN, MT \\
\hline & $1983-1988$ & MN \\
\hline \multirow[t]{2}{*}{ NE } & 1980 & IA, KS. MO \\
\hline & $1982,1983,1986-1989$ & $\begin{array}{l}\text { CO, IA, KS, } \\
\text { MO, WY }\end{array}$ \\
\hline \multirow[t]{2}{*}{ NH } & $1980-1983$ & NY \\
\hline & $1984-1989$ & New England \\
\hline $\mathrm{NJ}$ & $1980-1989$ & NY, PA \\
\hline \multirow[t]{4}{*}{ NM } & 1980 & TX, UT \\
\hline & $1981,1984-1988$ & CO, OK, TX \\
\hline & 1982,1983 & $\begin{array}{l}\text { AZ. CO, OK, } \\
\text { TX }\end{array}$ \\
\hline & 1989 & AZ. CO. TX \\
\hline \multirow[t]{2}{*}{ NV } & $1980,1981,1984-1989$ & CA, ID, UT \\
\hline & 1983 & $\begin{array}{l}\text { AZ, CA. ID, } \\
\text { UT }\end{array}$ \\
\hline OK & 1980,1989 & $\begin{array}{l}\text { AR, KS, MO, } \\
\text { TX }\end{array}$ \\
\hline \multirow[t]{2}{*}{ OR } & $1980,1981,1983-1989$ & CA. ID, WA \\
\hline & 1982 & $\begin{array}{l}\text { CA. ID, NV, } \\
\text { WA }\end{array}$ \\
\hline $\mathrm{RI}$ & $1980,1981,1984-1989$ & New England \\
\hline \multirow[t]{5}{*}{ SD } & 1980 & IA, MN, MT \\
\hline & 1981 & $\begin{array}{l}\text { IA, MN, MT, } \\
\text { NE }\end{array}$ \\
\hline & 1982 & $\begin{array}{l}\text { IA, MN, MT, } \\
\text { WY }\end{array}$ \\
\hline & $1983,1986-1989$ & IA, MN, WY \\
\hline & 1984. 1985 & IA, MN, NE \\
\hline \multirow[t]{2}{*}{ VT } & $1980-1983$ & NY \\
\hline & $1984-1989$ & New England \\
\hline WV & 1980 & $\begin{array}{l}\text { KY, MD, OH, } \\
\text { PA, VA }\end{array}$ \\
\hline \multirow[t]{3}{*}{ WY } & 1980 & ID, MT, UT \\
\hline & 1981 & $\begin{array}{l}\text { CO, ID, MT, } \\
\text { NE, UT }\end{array}$ \\
\hline & 1984. 1985 & $\begin{array}{l}\text { CO. ID. NE. } \\
\text { UT }\end{array}$ \\
\hline
\end{tabular}

Table 19. Industrial Sector Steam Coal Price Estimates for 1971 and 1974-1979

\begin{tabular}{lll}
\hline State & Years & Prices Used \\
\hline iR the Estimation \\
\hline & $1971,1974,1975$ & MO, TN \\
AZ & 1979 & MO, TN, TX \\
& 1971 & CA, NV, UT \\
CO & $1974-1978$ & CA, UT \\
& $1974-1978$ & KS, NE, UT \\
CT & $1974-1978$ & UT \\
& 1979 & MA, NY \\
DC & $1971,1974-1979$ & NY \\
DE & $1971,1974-1979$ & MD, VA \\
FL & 1979 & MD, NJ, PA \\
ID & 1974 & AL, GA \\
& $1975-1978$ & OR, UT \\
& 1979 & UT \\
KS & 1979 & UT, WA \\
LA & 1978 & MO \\
& 1979 & AR \\
MA & 1979 & TX \\
ME & $1975-1978$ & NY \\
& 1979 & MAA \\
MS & $1971,1974-1975,1979$ & NY \\
& $1976-1978$ & AL, TN \\
MT & $1974-1978$ & AL, AR, TN \\
& 1979 & MN, NE. UT \\
ND & $1974-1979$ & MN, UT \\
NE & 1979 & MN \\
NH & $1971,1974-1979$ & IA, MO \\
NM & 1971 & MA \\
& $1974,1976-1978$ & CO, OK, TX, \\
& 1979 & UT \\
NV & 1974 & UT UT \\
& $1975-1979$ & CA, OR, UT \\
OK & 1974,1975 & CA, UT \\
& $1976-1978$ & KS, MO \\
OR & 1979 & AR, KS, MO \\
& $1975-1978$ & MO, TX \\
RI & 1979 & CA \\
SD & $1971,1974-1979$ & CA, WA \\
& 1971,1974 & MA \\
TX & $1975-1978$ & IA \\
& 1979 & IA, MN, NE \\
VT & 1974,1975 & IA, MN \\
WA & $1971,1974-1979$ & KS \\
& $1975-1978$ & AR, KS \\
& $1974-1978$ & MA \\
& & CA, OR \\
CA & NE, UT \\
& & UT \\
\hline
\end{tabular}


Wherever individual State prices remain unavailable after the estimation using the above regression techniques, prices are assigned from adjacent or nearby States as follows:

\begin{tabular}{lll} 
State & Years & Assigned Prices \\
\hline NH & $1970,1972,1973$ & MA \\
RI & $1970,1972,1973$ & MA \\
SD & $1970,1972,1973$ & IA \\
VT & $1970,1972,1973$ & MA
\end{tabular}

Steam coal industrial sector prices are estimated for Alaska based on the assumption that the ratio of the State price to the U.S. price for the industrial sector is the same as the ratio found in the electric utility sector for each respective year from 1970-1979.

\section{Btu Prices: All Years}

Btu prices for States are calculated from the physical unit prices and the conversion factors, which vary by State and by year. U.S. Btu prices are calculated as the average of the State Btu prices, weighted by consumption data from SEDS, adjusted for process fuel and coking coal consumption.

\section{Data Sources}

\section{Prices}

1984 forward: Energy Information Administration, Quarterly Coal Report, Tables 16 and 17 (1984 1st quarter through 1985 2nd quarter and 1986, 1987, and 1988 1st quarters); Tables 18 and 19 (1985 3rd and 4th quarters, 1986, 1987, and 1988 2nd quarters through 4th quarters, and 1989 1st quarter through 4th quarter).

1980-1983: Energy Information Administration, "Quarterly Coal Consumption Report: Manufacturing Plants," (Form EIA-3). Only published data are used from Table 25 (1980), Table 11 (1981, 1982), and Table 2 (1983).

1971, 1974-1979: Bureau of the Census, U.S. Department of Commerce, Annual Survey of Manufactures and Census of Marufactures, Table 4 (1971) and Table 3 (1974-1979).

1970, 1972, 1973: Steam coal electric utility physical unit prices.

\section{Consumption}

1970 forward: Energy Information Administration, State Energy Data System. industrial sector coal consumption.

\section{Conversion Factors: All Years}

Energy Information Administration, State Energy Data Report, Consumption Estimates 1960-1989, Tables D10 and D11.

The $\mathrm{NH} / 1976$ conversion factor is assigned from $\mathrm{NH} / 1975$, and the VT/1986 conversion factor is assigned from VT/1985.

\section{Transportation Sector}

Transportation sector steam coal prices are assigned from industrial sector steam coal prices. U.S. Btu prices are calculated as the average of the State Btu prices, weighted by consumption data from the State Energy Data System (SEDS).

\section{Coal Coke, Imports and Exports}

Imports and exports of coal coke are components of total U.S. energy consumption and are accounted for in the industrial sector. Prices and values of imports and exports are developed only for the United States; no attempt is made to estimate State-level prices or expenditures. Prices are f.a.s. (free alongside ship) values and do not include taxes. The quantities of U.S. coal coke imports and exports are from SEDS.

\section{Physical Unit Prices: All Years}

For 1980 forward, the Coke Plant Report, the Quarterly Coal Report, and Bureau of the Census computer tapes provide physical unit coal coke import and export prices in dollars per short ton. For 1970-1979, Coke and Coal Chemicals, International Coal, and the Minerals Yearbook provide coal coke import and export physical unit quantities and values in short tons and dollars, respectively. Values are equivalent to expenditures.

\section{Btu Prices: All Years}

For 1980 forward, Btu prices are computed by dividing the physical unit prices by the conversion factor. For 1970-1979, physical unit prices are computed by dividing the import and export values by their respective quantities, and Btu prices are computed by dividing the physical unit prices by the conversion factor. 


\section{Data Sources}

A Prices

L 1989: Bureau of the Census, U.S. Department of Commerce, computer tapes for "Monthly Report IM 145" and "Monthly Report EM 522."

O 1981-1988: Energy Information Administration, Quarterly Coal Report, October-December issue, Tables A11 and A13 (1981-1985) and Tables A10 and A12 (1986-1988).

1980: Energy Information Administration, Coke Plant Report, Tables 7 and 8.

1978-1979: Energy Information Administration, Coke and Coal Chemicals 1979, Tables 5 and 6.

1977: National Coal Association, International Coal 1980, tables titled, "U.S. Imports of Solid Fuels and
Customs Value" and "U.S. Exports of Coke and Value."

1976: Energy Information Administration, Coke and Coal Chemicals, Tables 19 and 20.

1970-1975: Bureau of Mines, U.S. Department of the Interior, Minerals Yearbook, "Coke and Coal Chemicals" chapter, Tables 19 and 20.

\section{Consumption}

1970 forward: Energy Information Administration, State Energy Data System, U.S. imports and exports of coal coke.

Conversion Factor: All Years

24.8 million Btu per short ton 
Natural gas prices are developed for the residential, commercial, industrial, and electric utility sectors. The transportation sector is not covered here because natural gas used for transportation is consumed solely in pipeline operations and is discussed in the section called "Consumption Adjustments for Process Fuel and Intermediate Products." Reported natural gas prices are retail prices for sales of natural gas to ultimate users. In general, taxes are included in the prices. However, taxes collected by a utility from an end user and turned over to a government authority are not included in the revenues reported in the Natural Gas Annual or Natural Gas Production and Consumption and are not included in the prices. Taxes paid by the utility (rather than the end user) are considered operating costs and are passed on to the end user as part of the rate. Therefore, Federal, State, business, and property taxes are typically included in the prices, while sales and other pointof-purchase taxes are not.

Estimates of the amount of natural gas consumed by the residential, commercial, industrial, and electric utility sectors are from the State Energy Data System (SEDS). Estimates for the industrial sector are adjusted to remove estimated refinery consumption and lease

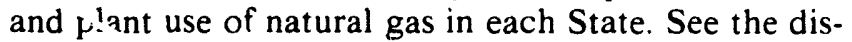
cussion under ' Consumption Adjustments for Process Fuel and Intermediate Products" in this report.

\section{Residential, Commercial, and Industrial Sectors}

\section{Prices: 1987 Forward}

All natu $\because$ l gas physical unit prices by State for the residential, commercial, and industrial sectors are from the Natural Gas Annual (! $V A$ ).

\section{Prices: 1970-1986}

All natural gas physical unit prices for the residential, commercial, and industrial sectors are calculated from value and quantity of sales data from the Natural Gas Annual or its predecessor report Noturn! Gos Production and Consumption. State prices are calculated directly from the data sources as average revenue per unit of sales by natural gas utilities. Prices for each of the three sectors are calculated by dividing the value of natural gas, reported in thousands of dollars, by the quantity of natural gas sold, as reported in million cubic feet.

For 1970-1979, both the value and quantity of sales data from the $N G A$ are reported as composites for MD and $\mathrm{DC}$ and for ME, NH, and VT. In each case, the combined prices are assigned to each of the States in the composite.

\section{Btu Prices: All Years}

State Btu prices for all years are calculated using the physical unit price series and the State-level nonelectric utility conversion factors. U.S. Btu prices are calculated as the average of the State Btu prices, weighted by consumption data from SEDS, adjusted for process fuel consumption in the industrial sector.

\section{Data Sources}

\section{Prices}

1980 forward: Energy Information Administration, Natural Gas Annıal Volume 1, Table 11 (1980), Table 14 (1981-1985), Table 15 (1986), Table 19 (1987), and Table 22 (1988, 1989).

1970-1979: Bureau of Mines, U.S. Department of the Interior, Natural Gas Production and Consumption, Table $6(1970,1979)$ and Table 7 (1971-1978).

\section{Consumption}

1970 forward: Energy Information Administration, State Energy Data System, residential, commercial, and industrial natural gas consumption.

\section{Conversion Factors: Al/ Years}

Energy Information Administration, Siute Energy Duia Report, Consumption Estimates, 1960-1989. Tables D4 and D5. 


\section{N Electric Utility Sector}

\section{Prices: 1973, 1974, 1983 Forward}

$\mathbf{R}$ Prices for States are reported by Cost and Quality of A Fuels for Electric Utility Plants $(C \& Q)$ for gas consumed L at steam-electric plants only. Btu prices are from $C \& Q$, converted from cents to dollars per million Btu.

Where individual State prices are unavailable from $C \& Q$, they are developed from the $N G A$. Prior to 1987 , physical unit prices are calculated by dividing the value of natural gas, reported in thousands of dollars, by the quantity of natural gas sold, reported in million cubic feet. For 1987 forward, physical unit prices are directly from the $N G A$. Table 20 lists the States and years for which $N G A$ data are used. Btu prices are calculated from the physical unit prices using Statelevel electric utility conversion factors.

Table 20. Natural Gas Electric Utility Sector Prices from NGA, 1973-1988

\begin{tabular}{ll}
\hline State & Years \\
\hline AK & $1973-1989$ \\
CT & $1974-1976$ \\
ID & $1983-1986$ \\
MD & $1973,1974,1983-1985$ \\
NC & $1983-1989$ \\
ND & $1973,1974,1976-1986$ \\
NH & $1973,1974,1977,1987-1989$ \\
PA & 1973 \\
RI & 1976,1980 \\
SC & 1977 \\
SD & $1983-1989$ \\
TN & $1976,1980,1981,1983,1988,1989$ \\
UT & 1988,1989 \\
VT & $1983-1985$ \\
WA & $1978,1983-1985,1988,1989$ \\
WY & 1973,1975
\end{tabular}

Note: $N G A$ includes predecessor publications.

Prices are not available from either $C \& Q$ or $N G A$ for CT/1973: ID/1974, 1987; NH/1983; OR/1983, 1984, 1986, 1989; and VT/1989. In these cases, quantityweighted Census division prices from $C \& Q$ are assigned. In addition, prices for VT/1986 and WA/1986, 1987 use quantity-weighted Census division prices from $C \& Q$ for a more consistent price level than available from the $N G A$.
Prices: $1980-1982$

State Btu and physical unit prices for 1980-1982 are from $C \& Q$ for all reporting plants. Physical unit prices are directly from the data source, while Btu prices are converted from cents to dollars per million Btu.

\section{Prices: 1975-1979}

State prices are reported separately by $C \& Q$ for gas consumed at steam-electric plants and gas consumed at combustion turbine and internal combustion units. Weighted-average Btu prices are calculated using the two $C \& Q$ prices and the respective gas deliveries for steam-electric and combustion use. The NH/1977 price is not available from $C \& Q$. A combined price is computed from value and quantity of sales data from the $N G A$ for ME, NH, and VT and assigned to NH/1977.

\section{Prices: 1970-1972}

State prices for 1970-1972 are from Natural Gas Production and Consumption and are calculated similarly to prices for the residential, commercial, and industrial sectors. Prices, as average revenue per unit of sales, are computed from value and quantity of sales data from the source reports. A combined price is reported for NH and VT for 1971 and 1972, and each of these States is assigned the combined price. State Btu prices are calculated from the physical unit prices using the State-level electric utility conversion factors.

\section{U.S. Prices: Al/ Years}

U.S. Btu prices are calculated as the average of the State Btu prices, weighted by consumption data from SEDS.

\section{Data Sources}

\section{Prices}

1973 forward: Energy Information Administration, Cost and Quality of Fuels for Electric Utility Plants, tables numbered as listed here:

\begin{tabular}{llc} 
Years & Price Data & Volume Data \\
\hline 1973,1974 & Table 10 & Table 9 \\
$1975-1979$ & Table 10, 16 & Table 9, 15 \\
$1980-1982$ & Table 48 & - \\
1983,1984 & Table 53 & - \\
$1985-1987$ & Table 43 & - \\
1988,1989 & Table 44 & -
\end{tabular}

1980 forward: Energy Information Administration, Natural Gas Annual Volume 1, Table 11 (1980), Table 
14 (1981-1985), Table 15 (1986), Table 19 (1987), and Table 22 (1988, 1989).

1976-1979: Energy Information Administration, Energy Data Reports, Natural Gas Production and Consumption, Table 7 (1976-1978) and Table 6 (1979).

1970-1975: Bureau of Mines, U.S. Department of the Interior, Natural Gas Production and Consumption, Table 6 (1970) and Table 7 (1971-1975).

\section{Consumption}

1970 forward: Energy Information Administration, State Energy Data System, electric utility natural gas consumption.

\section{Conversion Factors}

Btu prices that are calculated directly from Cost and Quality of Fuels for Electric Uility Plants $(C \& Q)$ require no conversion factors. When Natural Gas Annual $(N G A)$ data are used to develop prices that are missing from $C \& Q$ (Table 9), conversion factors are used from the following source.

1970 forward: Energy Information Administration, State Energy Data Report, Consumption Estimates, 1960-1989, Tables D2 and D3. 


\section{Petroleum}

\section{Asphalt and Road Oil}

Asphalt and road oil prices are developed for the industrial sector only. Estimates of the amount of asphalt and road oil consumed by the industrial sector are from SEDS. The asphalt and road oil prices reflect construction use of asphalt and road oil, defined as part of the industrial sector. Taxes are included.

\section{Physical Unit Prices: All Years}

Asphalt prices in physical units are developed from monthly reports in the Engineering News Record, a construction industry weekly magazine published by McGraw-Hill, Inc. The source data consist of monthly reports from correspondents in 20 U.S. cities with price quotes for tank cars, drums, or both, for the three major types of asphalt products: asphalt cement (AC-20), asphalt emulsion, and asphalt cutback.

When only one price is available, that price is used. For i , 86 forward, the tank car price is used. For 1970-1985, when both tank car and drum prices are available, a simple average of the two prices is used.

Asphalt prices are developed by calculating a simple average annual price from the monthly prices for each city for the three products. City prices are assigned to States. CA, OH, and PA have prices from two cities; in these cases, simple averages of the two city prices are used. No States have prices from more than two cities. States with no prices are assigned a Census division simple average price state average asphalt prices are calculated as the quantity-weighted average prices of the three products for each State. If there is no Census division price, the simple average of the prices for the other Census divisions within that Census region is used. Quantity data for 1970-1980 are from the Bureau of Mines and EIA reports on sales of asphalt. Quantity data for 1981 forward are from the Report on Sales of Asphalt in the U.S. published by the Asphalt Institute. Non-paving asphalts are assumed to have the price of asphalt cement.

For 1970-1982, asphalt and road oil are estimated as separate data series. Asphalt prices are estimated as discussed above. Road oll prices are assumed to equal asphalt emulsion prices because specific prices are not available from any source.
Btu Prices: All Years

Asphalt prices in dollars per ton are converted to dollars per gallon by dividing by 235 gallons per ton for asphalt cement, 241 gallons per ton for emulsion, or 248.6 gallons per ton for cutback. These prices are then multiplied by 42 gallons per barrel and divided by 6.636 million Btu per barrel to get dollars per million Btu. Road oil unit prices of dollars per ton are converted to dollars per million Btu using the constant conversion factors of 5.5 barrels per ton and $6.636 \mathrm{mil}$ lion Btu per barrel.

U.S. Btu prices are calculated as the average of the State Btu prices, weighted by consumption data from SEDS

\section{Data Sources}

\section{Prices}

1970 forward: McGraw-Hill Inc., Engineering News Record.

Quantities for Calculating Weighted Average Prices 1981 forward: Asphalt Institute, Report on Sales of Asphalt in the U.S.

1978-1980: Energy Information Administration, Energy Data Reports, Sales of Asphalt, Table 2.

1977: Energy Information Administration, Energy Data Reports, Asphalt Sales, Annual, Table 2.

1971-1976: Bureau of Mines, U.S. Department of the Interior, Mineral Industry Survey, Asphalt Sales, Annual, Table 2.

1970: Bureau of Mines, U.S. Department of the Interior, Mineral Industry Survey, Asphalt Shipments, Annual, Table 2.

\section{Consumption}

1970 forward: Energy Information Administration, State Energy Data System, industrial sector, asphalt and road oil consumption. 
235 gallons per ton of asphalt cement; 241 gallons per ton of emulsion; 248.6 gallons per ton of cutback; 42 gallons per barrel; 5.5 barrels per ton of road oil; 6.636 million Btu per barrel.
Aviation gasoline prices are developed for the transportation sector. Estimates of the amount of aviation gasoline consumed by the transportation secior are from SEDS. Aviation gasoline prices are national averages, excluding taxes, developed from several sources, depending on the years. In all cases, physical unit prices are developed and then converted to Btu prices.

\section{Physical Unit Prices: 1976 Forward}

Aviation gasoline prices for 1978 forward are assumed to be the national average refiners sales prices to end users published in the EIA's Annual Energy Review. The 1976 and 1977 prices are assumed to be the national average retail prices published in the EIA's Monthly Energy Review.

\section{Physical Unit Prices: 1970-1975}

For 1970-1975, aviation gasoline nrices are not available and are estimated by calculating the percent difference between the 1976 national aviation gasoline price and the national average motor gasoline price. That percent difference is applied to the motor gasoline prices for 1970-1975 to derive an aviation gasoline price estimate for each year.

\section{Btu Prices: All Years}

Aviation gasoline. Btu prices are calculated by converting the physical unit prices from cents per gallon to dollars per barrel (42 gallons per barrel) and, then, to dollars per million Btu (5.048 million Btu per barrel).

\section{Data Sources}

\section{Prices}

1978 forward: Energy Information Administration, $A n$ nual Energy Review 1990, Trable 71, row titled "Sales Prices to End Users: Aviation Gasoline."

1976, 1977: Energy Information Administration, Monthly Energy Review, April 1984, page 106, column titled "Aviation Gasoline, Retail."

1970-1975: Energy Information Administration, Annual Energy Review 1989. Table 70, column titled "Motor Gasoline, Leaded Regular, Nominal."

\section{Consumption}

1970 forward: Energy Informatiun Administration, State Energy Data System, transportation sector, aviation gasoline consumption. 
Distillate fuel prices are developed for all sectors. Distillate fuel in the transportation sector is assumed to be diesel fuel. Estimates of the amount of distillate fuel consumed in each sector are from SEDS. Estimated consumption for the industrial sector is adjusted to remove the estimated refinery consumption of distillate fuel in each State. See the discussion under "Consumption Adjustments for Process Fuel and Intermediate Products" in this report.

\section{Residential Sector}

Residential distillate prices are developed from a variety of data sources, depending on the years involved. In all cases, physical unit prices for States are developed first, then Btu prices are calculated using the physical unit prices and the conversion factor. The prices contained in this series are the retail prices paid by consumers for residential heating oil. Beginning with 1983, taxes are added to the prices. It is not clear from the data sources whether taxes are included in the prices for previous years.

\section{Physical Unit Prices: 1983 Forward}

For 1983 forward, physical unit distillate prices in cents per gallon (excluding taxes) are directly available for 24 States from the Petroleum Marketing Annual (PMA). State-level prices for the remaining 27 States are estimated using price data from the Gas Househeating Survey (GHS), SEDS consumption data, and PMA Petroleum Administration for Defense (PAD) district prices. The estimation procedures are described below.

1. State prices in cents per gallon are generally available from the $P M A$ for the following 24 States: AK, CT, DC, DE, ID, IL, IN, MA, MD, ME, MI, MN, NH, NJ, NY, OH, OR, PA, RI, VA, VT, WA, WI, and WV. In 1986, IL is assigned the PAD District II price. Prices for these States are converted from cents to dollars per gallon, and State general sales taxes from the Bureau of the Census, State Government Tax Collections (SGTC) are added.

2. For the "emaining 27 States that do not have prices in the $P M A$, prices are estimated using $G H S$ fuel oil prices, SEDS consumption data, and PMA PAD District prices for Districts II, III, IV, and V and Subdistrict IC (all the States in PAD Subdistricts IA and IB have pubiished prices). The following steps are used to estimate the prices: 
a. Distillate prices from the $P M A$ for PAD Districts II, III, IV, and V and Subdistrict IC are converted from cents per gallon to dollars per gallon.

b. The $G H S$ lists fuel oil prices by company for the principal city served in dollars per million Btu, including State sales taxes. A simple average of the city-level prices is used to derive a State-level price for each of the 27 States without $P M A$ prices. These GHS State averages are converted from dollars per million Btu to dollars per gallon using the GHS conversion factor of 7.194 gallons per million Btu. State general sales taxes from SGTC are subtracted to give State averages comparable to the $P M A$ prices.

c. The GHS State prices derived in Step b. are combined into PAD district averages using SEDS consumption to weight each State's values. This procedure gives GHS consumption-weighted average prices for PAD Districts II, III, IV, and V and Subdistrict IC that are comparable to the volume-weighted PAD district prices published in the PMA. The GHS PAD district averages are calculated using only the available States; if a State does not appear in the survey, it is not included in the PAD calculation.

d. Adjustment factors, ratios of the PMA PAD district price divided by the $G H S$ derived PAD district price, are calculated for PAD Districts II, III, IV, and V, and Subdistrict IC.

e. Prices for the 27 States not published in the $P M A$ are calculated by multiplying the $G H S$ State prices derived in Step b. by the appropriate PAD district adjustment factor from Step d. and adding State general sales taxes from the $S G T C$.

f. Stares that do not have prices in either the $P M A$ or the GHS are assigned a PMA PAD district price, and State general sales taxes from the SGTC are added. The States with assigned prices are as follows:

\begin{tabular}{lll} 
State & Years & Prices Assigned \\
\hline KS & 1986,1989 & PAD District II \\
OK & 1985,1989 & PAD District II \\
SD & 1986 & PAD District II \\
LA & 1985 & PAD District III \\
MS & $1983,1985,1986$ & PAD District III \\
NM & $1984-1989$ & PAD District III \\
AR & 1988 & PAD District III \\
UT & 1985 & PAD District IV \\
CA & 1984 & PAD District V \\
HI & $1983-1989$ & PAD District V
\end{tabular}

\section{Physical Unit Prices: 1978-1982}

Procedures for the 1978-1982 period are similar to those for 1983 forward except for changes in data sources. Annual physical unit prices are either directly from the Monthly Energy Review (MER) or are calculated from monthly regional price data, also from the $M E R$. These data were collected on EIA Form 9A (formerly EIA Form 9 and FEA Form P112-M-1) Price data from Platt's Oil Price Handbook and Oilmanac (Platt's) and SEDS consumption data for 1978-1982 are used to compute State prices when only regional data are available. These calculations are described step-by-step below.

1. Annual State physical unit prices are available from the $M E R$ for the same 24 States covered by the $P M A$ in 1983 forward. These 24 States compose all of Federal Regions 1, 2, 3, 5, and 10 (see Figure 10 on page 177). Prices for these States exclude taxes and are converted to dollars per gallon.

2. Of the remaining 27 States, the 22 in Federal Regions $4,7,8$, and 9 have annual prices estimated from the monthly Federal regional prices published in the $M E R$. No regional prices are available for Federal Region 6 for the 1978-1982 period, and some monthly prices are missing in regions 7,8 , and 9 in 1980, 1981, and 1982 .

a. Missing monthly prices for Federal regions are estimated with assigned prices as follows: the Region 9 November 1980 price is assigned for December 1980; an average of the Region 7 July and October 1982 prices is assigned to August and September 1982; an average of Region 8 June and September 1982 prices is assigned to July and August 1982; and an average of Region 3 August and October 1982 prices is assigned for the missing September 1982 price. Imputation of missing Region 6 prices for 1978-1982 and missing Region 9 prices for 1981 and 1982 is discussed later.

b. The simple average of monthly State-level normal heating degree-day data is averaged for all the States within each of the 10 Federal regions and used to estimate average Federal region heating degree-days. $\mathrm{AK}, \mathrm{DC}$, and $\mathrm{HI}$ are assigned the monthly heating degree-days from $M N, M D$, and $F L$, respectively.

c. Weighted average annual physical unit distillate prices for the residential sector are calculated for Federal Regions 4, 7, 8, and 9 (except for Region 9 in 1981 and 1982) using the regional normal heating degree-days and the monthly regional prices from the $M E R$.

d. In 1981, only March and May prices are available for Federal Region 9. To estimate the average annual price for this region, the relationship between the U.S. annual heating oil 
price (from the $M E R$ ) and the U.S. March and May prices is expressed as a ratio and used with the Region 9 March and May prices to estimate the 1981 annual Region 9 price.

e. City-level prices from Platt's are assigned to States as shown in Table 21 on nage 196. The assigned State-level Platt's prices for States are consumption-weighted into Federal regions using residential sector consumption data from SEDS.

f. Adjustment factors, ratios of the regional $M E R$ distillate prices to the regional Platt's-based distillate prices, are calculated for Federal Regions 4, 7, 8, and 9 (except for 1982).

g. Since there are no monthly regional distillate prices from the $M E R$ for Federal Region 6 for 1978-1982 and Federal Region 9 for 1982, the adjustment factors for these regions are based on the adjustment factors for previous time periods. The Region 6 adjustment factor for each of the years in the 1978-1982 period is equal to 1.1313 , which is the average of the adjustment factor for the West South Central Census Division for 1976 and 1977. The Region 9 adjustment factor for 1982 is equal to 1.1995 , which is the average adjustment factor for Region 9 from 1978 to 1981 .

h. The residential sector distillate State prices for the 27 States in Federal Regions 4, 6, 7, 8, and 9 are calculated by multiplying the regional adjustment factors for each year and the State-level assigned Platt's prices.

\section{Physical Unit Prices: 1975-1977}

For the years 1975-1977, no State-level data are available, and regional data from EIA Form 9A are available only at the Census division level, except for Federal region prices for November and December of 1977. Using a methodology similar to that described above for the allocation of regional data to States, adjustment factors are calculated at the regional level and applied to Platt's price data assigned to States.

1. Monthly regional price data for 1975 and 1976 are reported in the $M E R$ only for Census divisions. In 1977, however, monthly price data are reported for Census divisions for January through October and ic . Federal regions for November and December. The Federal region prices for November and December are assigned to their respective States and reaggregated into Census divisions in order to create a consistent set of monthly Census division prices for 1977 . Annual residential sector distillate consumprion data from SEDS are used to do the reaggregation.

2. The Census division monthly price data from the $M E R$ for 1975,1976 . and the first 10 months of
1977 are used with the estimated Census division price data for November and December 1977 to estimate State-level prices.

a. Missing monthly prices in the East South Central Division for June and November 1975, and the Mountain Division for March and July 1975 are estimated using an average of the prices for the month preceding and the month following the missing month. Missing November and December West South Central Division prices in 1977 are estimated with the assignment of the October price to both months. No monthly price data are available for the West South Central Division in 1975; Step "f." below discusses how the calculations are handled for this division.

b. The monthly State-level normal heating degree-day data are averaged for the States within each Census division to estimate regional monthly heating degree-days. AK, DC, and $\mathrm{HI}$ are assigned the monthly heating degree-days from $M N, M D$, and FL, respectively.

c. Weighted average annual distillate prices for Census divisions are calculated using the monthly Census division price data from the $M E R$ and the normal heating degree-days estimated for Census divisions.

d. City-level No. 2 fuel oil refinery and terminal prices from Platt's for 1975-1977 are assigned to States as shown in Table 21 on page 196. The assigned Platt's prices for States are consumption-weighted into Census divisions using residential sector consumption data from SEDS.

e. Adjustment factors, ratios of the $M E R$ distillate Census division prices to the Platt's distillate Census division prices, are calculated.

f. Since there are no $1975 M E R$ price data for the West South Central Division from which to calculate an adjustment factor, the 1975 adjustment factor for this region is assumed to be equal to the simple average of the West South Central adjustment factors for 1976 and 1977 (i.e., 1.1313).

g. The residential sector distillate State prices for all States are calculated by multiplying the regional adjustment factors for each year by the State-level assigned Platt's prices.

\section{Physical Unit Prices: 1970-1974}

There are no regional or State-level distillate price data directly available for the $1970-1974$ period. To estimate State prices, regional average prices are first derived from the relationship between U.S. prices and Federal region prices for 1975 to 1980 . State prices are then 
Table 21. Platt's Prices for No. 2 Fuel Assigned to States, 1970-1982

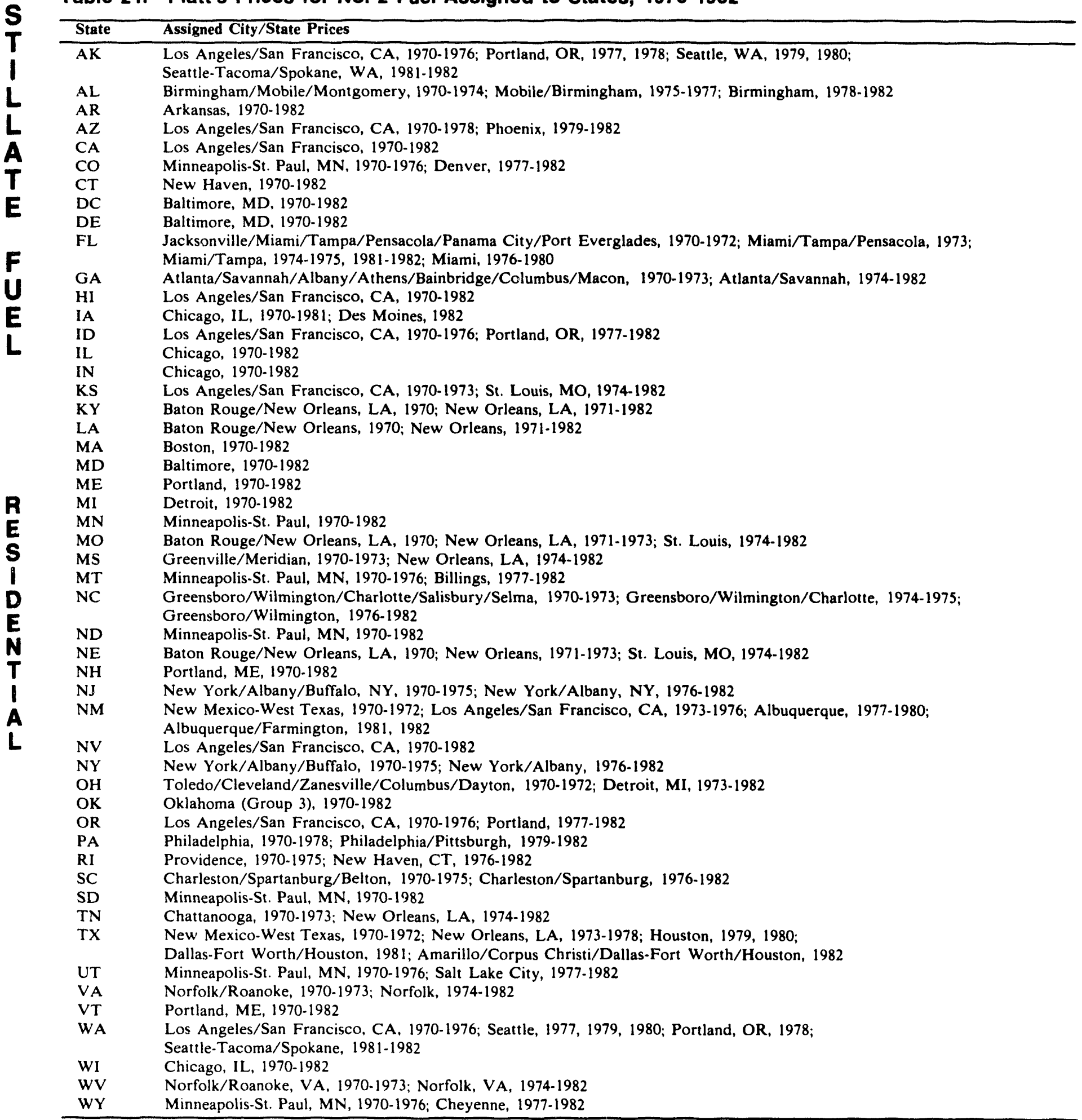

estimated from the regional prices using a methodology similar to that described for 1978-1982.

1. The first step in the estimation of residential distillate prices for the 1970-1974 time period, is to develop an equation that uses U.S. prices to estimate prices for Federal regions. Regression techniques are used for this purpose. U.S. prices for 1975-1980 from the Annual Energy Review $(A E R)$ are used as the independent variable for developing the equation; annual Federal region prices are used as the dependent variable. Federal region prices for 1978-1980 are calculated above, but $M E R$ prices for 1975-1977 are for Census 
divisions. To convert these annual Census division prices into Federal region prices, the estimated State prices for 1975-1977 are aggregated into Federal regions using SEDS consumption data.

2. Regression techniques are applied to the pooled Federal region price data (dependent variable) and the U.S. prices from the $A E R$ (independent variable) for 1975-1980. U.S. prices for 1970-1974 are input to estimate annual Federal region prices for 1970-1974.

3. City-level prices from Platt's for 1970-1974 are assigned to States as shown in Table 21 on page 196. The assigned State-level Platt's prices are consumption-weighted into Federal regions using residential sector distillate consumption data from SEDS.

4. Adjustment factors, ratios of the regional $M E R$ distillate Federal region prices to the Platt's-based distillate Federal region prices, are calculated.

5. The residential sector distillate prices for all States are calculated by multiplying the regional adjustment factors for each year by the Statelevel assigned Platt's prices.

\section{Btu Prices: All Years}

Btu prices for States are calculated by converting the physical unit prices from dollars per gallon to dollars per barrel (42 gallons per barrel) and then to dollars per million Btu (5.825 million Btu per barrel). U.S. Btu prices are calculated as the average of the State Btu prices, weighted by consumption data from SEDS.

\section{Data Sources}

\section{Prices}

1986 forward: Energy Information Administration, $\mathrm{Pe}$ troleum Marketing Annual, Table 37 (1986-1988) and Table 39 (1989), column titled, "To Residential Consumers."

1983 forward: American Gas Association, Gas Househeating Survey, Appendix 2, "Competitive Fuel Prices," column titled, "Fuel Oil."

1983 forward: Bureau of the Census, U.S. Department of Commerce, State Government Tax Collections, table titled, "State Government Excises on General Sales, Motor Fuel, and Cigarettes, Beginning and End of Fiscal Year."

1983-1985: Energy Information Administration, Petroleum Marketing Annual 1985, Volume 1, Table 26.
1970-1982: McGraw-Hill, Inc., Platt's Oil Price Handbook and Oilmanac, refinery and terminal prices for No. 2 fuel oil, average of highs and lows.

1975-1982: National Oceanic and Atmospheric Administration, U.S. Department of Commerce, State, Regional, and National Monthly and Seasonal Heating Degree Days Weighted by Population (1980 Census), Historical Climatology Series 5-1, table titled, "1951-80 State Pop. Wgt'd Heating Degree Days."

1975-1982: Energy Information Administration, Monthly Energy Review, table titled, "Residential Heating Oil Prices by Region," February 1978, page 67 (1975, 1976); April 1980, page 83 (1977, 1978); July 1982, page 87 (1979-1982).

1970-1982: Energy Information Administration, $A n$ nual Energy Review 1988. Table 67, "Motor Gasoline and Residential Heating Oil Prices, 1949-1988."

\section{Consumption}

1970 forward: Energy Information Administration, State Energy Data System, residential sector distillate consumption.

\section{Conversion Factor: All years}

5.825 million Btu per barrel

\section{Commercial Sector}

Commercial sector distillate prices are estimated by two methods, one for prices 1983 forward and the other for 1970-1982 prices. For 1983 forward, retail prices paid by commercial/institutional establishments (excluding taxes) are from Petroleum Marketing Annual (PMA). State general sales taxes from the Bureau of the Census are added. For 1970-1982, commercial distillate prices are based on markups calculated from $E n$ ergy Prices: 1960-73 and refinery and terminal (wholesale) prices from Platt's. It is not clear from the data sources whether these prices include taxes. For both time periods, physical unit prices are calculated from the data sources, and Btu prices are computed using the physical unit prices and the conversion factor.

\section{Physical Unit Prices: 1983 Forward}

For 1983 forward, physical unit distillate prices in cents per gallon (excluding taxes) are directly available for 24 States from the PMA. State-level prices for the remaining 27 States are estimated using price data from the Gas Househeating Survey (GHS), SEDS consumption data, and $P M A$ Petroleum Administration for 
Defense (PAD) district prices. The estimation procedures are described below.

1. State prices in cents per gallon are generally available from the $P M A$ for the following 24 States: AK, CT, DC, DE, ID, IL, IN, MA, MD, ME, MI, MN, NH, NJ, NY, OH, OR, PA, RI, VA, VT, WA, WI, and WV. Prices for these States are converted from cents to dollars per gallon, and State general sales taxes from the Bureau of the Census, State Government Tax Collections (SGTC) are added.

2. For the remaining 27 States that do not have prices in the $P M A$, prices are estimated using GHS fuel oil prices, SEDS consumption data, and PMA PAD district prices for Districts II, III, IV, and V and Subdistrict IC (all the States in PAD Subdistricts IA and IB have published prices). The following steps are used to estimate the prices:

a. Distillate prices from the PMA for PAD Districts II, III, IV, and V and Subdistrict IC are converted from cents per gallon to dollars per gallon.

b. The GHS lists fuel oil prices by company for the principal city served in dollars per million Btu, including State sales taxes. A simple average of the city-level prices is used to derive a State-level price for each of the 27 States without PMA prices. These GHS State averages are converted from dollars per million Btu to dollars per gallon using the GHS conversion factor of 7.194 gallons per million Btu. State general sales taxes from SGTC are subtracted to give State averages comparable to the $P M A$ prices.

c. The GHS State prices derived in Step b. are combined into PAD district averages using SEDS consumption to weight each State's values. This procedure gives GHS consumption-weighted average prices for PAD Districts II, III, IV, and V and Subdistrict IC that are comparable to the volume-weighted PAD district prices published in the PMA. The GHS PAD district averages are calculated using only the available States; if a State does not appear in the survey, it is not included in the PAD calculation.

d. Adjustment factors, ratios of the PMA PAD district price divided by the GHS derived PAD district price, are calculated for PAD Districts II, III, IV, and V, and Subdistrict IC.

e. Prices for the 27 States not published in the $P M A$ are calculated by multiplying the $G H S$ State prices derived in Step b. by the appropriate PAD district adjustment factor from Step d. and adding State general sales taxes from the SGTC. f. States that do not have prices in either the $P M A$ or the $G H S$ are assigned a PMA PAD district price, and State general sales taxes from the SGTC are added. The States with assigned prices are as follows:

\begin{tabular}{lll} 
State & Years & Prices Assigned \\
\hline KS & 1986,1989 & PAD District II \\
OK & 1985,1989 & PAD District II \\
SD & 1986 & PAD District II \\
LA & 1985 & PAD District III \\
MS & $1983,1985,1986$ & PAD District III \\
NM & $1984-1989$ & PAD District III \\
AR & 1988 & PAD District III \\
UT & 1985 & PAD District IV \\
CA & 1984 & PAD District V \\
HI & $1983-1989$ & PAD District V
\end{tabular}

\section{Physical Unit Prices: 1970-1982}

Commercial sector distillate physical unit prices for 1970-1982 are calculated using Platt's prices assigned to States and commercial sector markups estimated from Energy Prices: 1960-73.

1. The first step is to compute the markups. Energy Prices contains single price estimates for small commercial users and two price estimates for large commercial users for 10 cities: Boston, MA; Albany, NY; New York, NY; Charlotte, NC; Washington, DC; Chicago, IL; Detroit MI; Minneapolis/St. Paul, MN; St. Louis, MO; and Seattle, WA. First, a simple average of the two large commercial prices is calculated for each city except for Albany and New York. In this case, all four large commercial prices are averaged together, since cities are assigned to their respective States.

2. For the nine States covered by the Energy Prices data (noted in Step 1), the markup of the reported prices from Energy Prices over the assigned Platt's prices (Table 21 on page 196) and the markup of the residential prices calculated above for 1970-1972 over the Platt's prices are calculated.

3. At this point, residential and commercial sector retail markups have been computed for nine States for each of the years 1970-1972. The next step is to calculate the average retail markup for the 3-year period for each sector. A simple average of the markup ratios is calculated.

4. The average commercial and residential sector retail markups for the nine available States are assigned as shown in Table 22 on page 199.

5. To translate the average commercial and residential markups for 1970-1972 into the estimated commercial sector retail markups to be used for 1970-1982, the relationship between these two markups is used with the residential markups 
calculated for all States for each year. The calculation of the residential markups is the same procedure used in Step 2 above.

6. The commercial sector adjustment factors for each State for each of the years 1970-1982 are multiplied by the corresponding Platt's prices for 1970-1982 to calculate the final commercial sector physical unit prices.

\section{Btu Prices: All Years}

Btu prices for States are calculated by converting the physical unit prices from cents to dollars per gallon, then to dollars per barrel (42 gallons per barrel), and finally, to dollars per million Btu (5.825 million Btu per barrel). U.S. prices are calculated as the average of the State Btu prices, weighted by consumption data from SEDS.

\section{Data Sources}

\section{Prices}

1986 forward: Energy Information Administration, Petroleum Marketing Annual, Table 37 (1986-1988) and Table 39 (1989), column titled, "To Commerical/ Institutional Consumers."

1983 forward: American Gas Association, Gas Househeating Survey, Appendix 2, "Competitive Fuel Prices," column titled, "Fuel Oil."

1983 forward: Bureau of the Census, U.S. Department of Commerce, State Government Tax Collections, table titled, "State Government Excises on General Sales, Motor Fuel, and Cigarettes: Beginning and End of Fiscal Year."

1983-1985: Energy Information Administration, Petroleum Marketing Annual 1985, Volume 1, Table 26.

1970-1982: McGraw-Hill, Inc., Platt's Oil Price Handbook and Oilmanac, refinery and terminal prices for No. 2 fuel oil, average of highs and lows.

1970-1982: Foster Associates, Inc., 1974, Energy Prices 1960-73, Tables 4-c and 5-b.

\section{Consumption}

1970 forward: Energy Information Administration, State Energy Data System, commercial sector distillate consumption.

\section{Conversion Factor: All Years}

Table 22. Average Retail Markup Price Assignments, 1970-1972

\begin{tabular}{|c|c|}
\hline State & City Price Assignments \\
\hline AK & Seattle, WA \\
\hline$A L$ & Charlotte, NC \\
\hline AR & St. Louis, MO \\
\hline $\mathrm{AZ}$ & Seattle, WA \\
\hline $\mathrm{CA}$ & Seattle, WA \\
\hline $\mathrm{CO}$ & Minneapolis/St. Paul, MN \\
\hline CT & Boston, MA \\
\hline DC & Washington, DC \\
\hline $\mathrm{DE}$ & Washington, DC \\
\hline FL & Charlotte, NC \\
\hline GA & Charlotte, NC \\
\hline HI & Seattle, WA \\
\hline IA & St. Louis, MO \\
\hline ID & Seattle, WA \\
\hline IL & Chicago, IL \\
\hline IN & Chicago, IL \\
\hline KS & St. Louis, MO \\
\hline KY & Chicago, IL \\
\hline LA & St. Louis, MO \\
\hline MA & Boston, MA \\
\hline MD & Washington, DC \\
\hline ME & Boston, MA \\
\hline MI & Detroit, MI \\
\hline MN & Minneapolis/St. Paul, MN \\
\hline MO & St. Louis, MO \\
\hline MS & Charlotte, NC \\
\hline MT & Minneapolis/St. Paul, MN \\
\hline NC & Charlotte, NC \\
\hline
\end{tabular}

NE St. Louis, MO

NH Boston, MA

NJ Albany and New York, NY

NM Seattle, WA

NV Seattle, WA

NY Albany and New York, NY

$\mathrm{OH} \quad$ Detroit, $\mathrm{MI}$

OK St. Louis, MO

OR Seattle, WA

PA Albany and New York, NY

RI Boston, MA

SC Charlotte, NC

SD Minneapolis/St. Paul, MN

TN Chicago, IL

TX St. Louis, MO

UT Minneapolis/St. Paul, MN

VA Washington, DC

VT Boston, MA

WA Seattle, WA

WI Chicago, IL

WV Washington, DC

WY Minneapolis/St. Paul, MN

5.825 million Btu per barrel 


\section{Electric Utility Sector}

The electric utility price for distillate fuel is the average delivered cost of No. 2 fuel oil receipts at electric utilities (See Light Oil). For 1973 forward, these prices are from Cost and Quality of Fuels for Electric Utility Plants; for 1970-1972, prices from the Statistical Yearbook of the Electric Utility Industry are used with regression analysis. Btu prices are developed directly from the data sources and include all applicable taxes.

\section{Prices: 1973 Forward}

Btu prices for the years 1973 forward are based directly on the Btu prices reported in Cost and Quality of Fuels (C\&Q). For 1973, 1974, and 1980 forward, Btu prices are directly from the data source. For 1975-1979, weighted average Btu prices are calculated from prices and consumption data reported separately for steamelectric plants and for combustion turbine and internal combustion units. Because 1973 was the first year $C \& Q$ was published, there are many States with missing data for that year. The estimation of the missing 1973 State prices follows the same procedure as prices for 1970-1972 and is discussed under that section.

Wherever individual State prices are unavailable, quantity-weighted Census division prices from $C \& Q$ are assigned as follows:

\begin{tabular}{lll}
$\mathbf{U}$ & State & Years \\
\cline { 2 - 3 } $\mathbf{T}$ & CA & $1983-1985,1987,1988$ \\
$\mathbf{L}$ & CT & 1973 \\
$\mathbf{I}$ & DC & 1973 \\
$\mathbf{Y}$ & DE & 1973 \\
& ID & $1973,1974,1976,1980-1989$ \\
& MD & 1973 \\
& ME & 1973,1974 \\
MT & $1973-1975,1977,1983$ \\
& NH & 1973,1974 \\
NJ & 1973,1974 \\
OR & 1987,1988 \\
RI & $1976-1989$ \\
SD & 1973,1974 \\
TN & 1973 \\
VT & $1973,1974,1978,1983-1989$ \\
WA & $1973-1977$ \\
WV & 1973 \\
WY & 1973
\end{tabular}

For 1973, 1974, and 1983 forward, Btu prices in cents per million Btu are from $C \& Q$ and converted to dollars per million Btu. For 1975-1979, Btu prices in cents per million Btu are from $C \& Q$ with their corresponding delivered quantities, weighted into overall prices, and converted to dollars per million Btu. For 1980-1982, Btu prices are reported in $C \& Q$ for all reporting plants and are converted to dollars per million Btu. U.S. Btu prices for all years are calculated as the average of the State Btu prices, weighted by consumption data from SEDS.

\section{Prices: 1970-1972}

Btu prices for 1970-1972 (and AK and HI for 1973) are estimated using data from Statistical Yearbook of the Electric Utility Industry. U.S. prices are then computed using the State-level prices and the electric utility distillate consumption data from SEDS.

1. Regression techniques are used to arrive at the equation for estimating electric utility sector distillate prices for the 1970-1972 (or 1973) period. AL is treated as the reference State. The regression equation uses Statistical Yearbook State-level prices for 1974-1980 as the independent variable and the State-level prices calculated above for 1974-1980 as the dependent variable. Substituting Btu prices for 1970-1972 (or 1973) from the Statistical Yearbook into the regression equation yields the estimated electric utility sector Statelevel distillate prices.

2. Wherever individual State prices are unavailable, quantity-weighted Census division prices are assigned as follows: ID/1970-1972; TN/1970; and WA/1970, 1971.

3. U.S. Btu prices are calculated as the average of the State Btu prices, weighted by consumption data from SEDS.

\section{Alaska and Hawaii Prices}

Since $C \& Q$ does not cover $\mathrm{AK} / 1973$ forward or HI/1973-1982, prices for these States are estimated. The ratio of the reported $C \& Q$ U.S. price and the Statistical Yearbook U.S. price is multiplied by the Statistical Yearbook State price for each year to estimate the price. AK prices for 1971, 1975, and 1978, for which Statistical Yearbook data are missing, are estimated from the average ratio of AK prices to U.S. prices in adjacent years (e.g., 1975 is calculated as the average of the 1974 and 1976 prices). Prices for 1970-1973 are calculated as described in the "Prices: 1970-1972" section. Prices for HI/1983 forward are from $C \& Q$.

\section{Data Sources}

\section{Prices}

1973 forward: Energy Information Administration, Cost and Quality of Fuels for Electric Utility Plants, Table 6 (1973, 1974); Tables 5, 6, 12, 13 (1975-1979); Table 45 (1980-1982); Table 51 (1983, 1984); and Table 41 (1985 forward). 
1970 forward: Edison Electric Institute, Statistical Yearbook of the Electric Utility Industry, table titled, "Analysis of Fuel for Electric Generation-Total Electric Utility Industry," (1970-1988); and table titled, "Fossil Fuels Used for Electric Generation Total Electric Utility Industry," (1989).

\section{Consumption}

1970 forward: Energy Information Administration, State Energy Data System, electric utility sector distillate consumption.

\section{Conversion Factors}

Btu prices are calculated directly from data sources; no explicit conversion factors are needed for any years for the electric utility sector.

\section{Industrial Sector}

The industrial sector distillate prices are developed using several different data sources and estimation methods, depending on the years involved. For 1983 forward, sales prices of No. 2 fuel oil to industrial consumers (excluding taxes) are from the Petroleum Marketing Annual (PMA). State general sales taxes from the Bureau of the Census are added. For 1970-1982, prices are the average cost of distillate to manufacturing firms; it is not clear from the data sources whether these prices include taxes.

\section{Physical Unit Prices: 1983 Forward}

For 1983 forward, physical unit distillate prices in cents per gallon (excluding taxes) are directly available for 24 States from the PMA. State-level prices for the remaining 27 States are estimated using price data from the Gas Househeating Survey (GHS), SEDS consumption data, and PMA Petroleum Administration for Defense (PAD) district prices. The estimation procedures are described below.

1. State prices in cents per gallon are generally available from the $P M A$ for the following 24 States: AK, CT, DC, DE, ID, IL, IN, MA, MD, ME, MI, MN, NH, NJ, NY, OH, OR, PA, RI, VA, VT, WA, WI, and WV. Prices for these States are converted from cents to dollars per gallon, and State general sales taxes from the $\mathrm{Bu}$ reau of the Census, State Government Tax Collections (SGTC) are added. In the instances when these States have no prices in the PMA (DC/1989, ID/1984, 1986, OR/1984-1987, and AK/1988), prices are obtained as described below.
2. For the remaining 27 States that do not have prices in the $P M A$, prices are estimated using GHS fuel oil prices, SEDS consumption data, and PMA PAD district prices for Districts II, III, IV, and $V$ and Subdistrict IC (all the States in PAD Subdistricts IA and IB have published prices). The following steps are used to estimate the prices:

a. Distillate prices from the $P M A$ for PAD Districts II, III, IV, and V and Subdistrict IC are converted from cents per gallon to dollars per gallon.

b. The $G H S$ lists fuel oil prices by company for the principal city served in dollars per million Btu, including State sales taxes. A simple average of the city-level prices is used to derive a State-level price for each of the 27 States without PMA prices. These GHS State averages are converted from dollars per million Btu to dollars per gallon using the GHS conversion factor of 7.194 gallons per million Btu. State general sales taxes from SGTC are subtracted to give State averages comparable to the $P M A$ prices.

c. The GHS State prices derived in Step b. are combined into PAD district averages using SEDS consumption to weight each State's values. This procedure gives GHS consumption-weighted average prices for PAD Districts II, III, IV, and V and Subdistrict IC that are comparable to the volume-weighted PAD district prices published in the PMA. The GHS PAD district averages are calculated using only the available States; if a State does not appear in the survey, it is not included in the PAD calculation.

d. Adjustment factors, ratios of the PMA PAD district price divided by the GHS derived PAD district price, are calculated for PAD Districts II, III, IV, and V, and Subdistrict IC.

e. Prices for the 27 States not published in the $P M A$ are calculated by multiplying the $G H S$ State prices derived in Step b. by the appropriate PAD district adjustment factor from Step d. and adding State general sales taxes from the SGTC.

f. States that do not have prices in either the $P M A$ or the $G H S$ are assigned a PMA PAD district price, and State general sales taxes from the SGTC are added. The States with assigned prices are as follows: 


\begin{tabular}{lll} 
State & Years & Prices Assigned \\
\hline KS & 1986,1989 & PAD District II \\
OK & 1985,1989 & PAD District II \\
SD & 1986 & PAD District II \\
LA & 1985 & PAD District III \\
MS & $1983,1985,1986$ & PAD District III \\
NM & $1984-1989$ & PAD District III \\
AR & 1988 & PAD District III \\
UT & 1985 & PAD District IV \\
CA & 1984 & PAD District V \\
HI & $1983-1989$ & PAD District V \\
AK & 1988 & PAD District V
\end{tabular}

\section{Physical Unit Prices: 1982}

In 1984, the Bureau of the Census announced that State-level fuel cost and quantity information would no longer be published in either the Annual Survey of Manufactures (ASM) or Census of Manufactures (CM). In addition, the $P M A$, the source for 1983 forward industrial sector distillate price data, did not contain 1982 prices. Because of this lack of price data, the 1982 industrial sector distillate prices are estimated, based on the relationship of industrial sector prices to electric utility sector prices for 1978-1981. The 1983 prices are not used in the estimation because they exclude taxes, while the 1978-1981 prices may include taxes.

1. In order to calculate the average ratios of industrial-to-electric utility distillate prices, electric utility price assignments are made for: AK/1978-1982 from WA; ID/1979-1982 from MT; RI/1978-1982 from CT; and VT/1978 from ME.

2. The average 1978-1981 ratios of industrialto-electric utility sector distillate prices are calculated for each State.

3. Prices for 1982 are estimated by multiplying the average ratios by the electric utility data for 1982 .

\section{Physical Unit Prices: 1971, 1974-1981}

For the years 1971 and 1974-1981, industrial sector distillate prices are calculated directly from cost and quantity data from the Annual Survey of Manufactures (ASM) or Census of Manufactures (CM) for all States where data are available.

There are no missing prices for 1971. Six States are missing some $A S M$ cost and quantity data for the 1974-1981 period. Cost and quantity data for these States are estimated as the simple average of the cost and quantity data for their adjacent States. The States, the years for which data are estimated, and the adjacent States used to make the estimation are as follows:

\begin{tabular}{lll} 
State & Years & State Prices Used \\
\hline HI & $1979-1981$ & CA \\
ND & $1979-1981$ & MN, MT, SD \\
NM & $1974-1979$ & AZ, CO, TX \\
NV & $1974-1981$ & AZ, CA, ID, OR, UT \\
OK & $1974-1978$ & AR, CO, KS, MO, TX \\
WY & $1974-1981$ & CO, ID, MT, NE, SD, UT
\end{tabular}

\section{Physical Unit Prices: 1970, 1972, 1973}

Since $A S M$ and $C M$ data are not available for these years, the prices must be estimated. Physical unit prices are based on the ratio of $1971 C M$ prices to the 1971 assigned Platt's prices (Table 21 on page 196). The resulting ratios for each State are used with the Platt's assigned prices for 1970, 1972, and 1973 to impute prices.

1. The first step is to calculate State-level ratios between prices calculated from the $1971 C M$ cost and quantity data and the 1971 assigned Platt's prices. There are no missing States in either of these two sets of prices.

2. State-level physical unit prices for 1970,1972 , and 1973 are estimated by multiplying the 1971 ratio by the assigned State-level Platt's prices for each respective year.

\section{Btu Prices: All Years}

Btu prices for States are calculated by converting the physical unit prices from cents to dollars per gallon, then to dollars per barrel (42 gallons per barrel) and, finally to dollars per million Btu (5.825 million Btu per barrel). U.S. Btu prices are calculated as the average of the State Btu prices, weighted by consumption data from SEDS, adjusted for process fuel consumption.

\section{Data Sources}

\section{Prices}

1986 forward: Energy Information Administration, $P e$ troleum Marketing Annual, Table 37 (1986-1988) and Table 39 (1989), column titled, "To Industrial Corısumers."

1983 forward: American Gas Association, Gas Househeating Survey. Appendix 2, "Competitive Fuel Prices," column titled, "Fuel Oil."

1983 forward: Bureau of the Census, U.S. Department of Commerce, State Government Tax Collections, table titled, "State Government Excises on General Sales, Motor Fuel, and Cigarettes: Beginning and End of Fiscal Year." 
1983-1985: Energy Information Administration, Petroleum Marketing Annual 1985, Volume 1, Table 26.

1970-1982: McGraw-Hill, Inc., Platt's Oil Price Handbook and Oilmanac, refinery and terminal prices for No. 2 fuel oil, average of highs and lows.

1971, 1977, and 1981: Bureau of the Census, U.S. Department of Commerce, Census of Manufactures, Table 4 (1971) and Table 3 (1977, 1981).

1974-1976 and 1978-1980: Bureau of the Census, U.S. Department of Commerce, Annual Survey of Manufactures, Table 3.

\section{Consumption}

1970 forward: Energy Information Administration, State Energy Data System, industrial sector distillate consumption.

\section{Conversion Factor: All Years}

5.825 million Btu per barrel

\section{Transportation Sector}

Consumption of distillate fuel in the transportation sector includes distillate fuel used for vessel bunkering and for military and railroad use, plus on-highway diesel fuel use. Since on-highway diesel fuel use accounts for the largest portion of this sector--increasing from 55 percent in 1970 to 76 percent in 1989--prices and expenditures are calculated using diesel prices. State physical unit prices for 1986 forward are from the $P e$ troleum Marketing Annual (PMA). Physical unit prices for earlier years are calculated using $P M A$ prices and consumption data from Highway Statistics to weight monthly or quarterly prices from Agricultural Prices into annual prices. For all years, physical unit prices are calculated from the data sources, while Btu prices are calculated from the physical unit prices and the conversion factor.

\section{Physical Unit Prices: 1986 Forward}

For 24 States, diesel fuel physical unit prices for 1986 forward are based on the annual State-level price data available from the $P M A$ and monthly tax rate information from Highway Statistics. For AK, CT, DE, DC, ID, IL, IN, ME, MD, MA, MI, MN, NH, NJ, NY, OH, OR, PA, RI, VT, VA, WA, WI, and WV, the $P M A$ provides physical unit prices, excluding taxes. State and Federal diesel fuel taxes are added to $P M A$ prices to derive final physical unit prices, which are converted to dollars per gallon. In cases where the tax rate is not constant through the year, the months' rates are averaged, weighted by the number of months each rate was in effect. State and local sales and other general taxes are not included.

For the remaining 27 States for which no State-level physical unit prices are published, the PMA PAD district prices for diesel fuel and motor gasoline and State motor gasoline prices are used. The State diesel fuel price is estimated as the ratio of the PAD district diesel fuel price to the PAD district motor gasoline price times the State motor gasoline price. State and Federal taxes are added as described above. The use of the ratio assumes that the relationship between the motor gasoline State and PAD district prices is similar to that of the diesel fuel State and PAD district prices.

\section{Physical Unit Prices: 1983-1985}

Diesel fuel physical unit prices for 1983-1985 are based on the annual State-level price data available from the $P M A$ and monthly State and Federal tax rate information from Highway Statistics for 24 States. The prices for the remaining 27 States are calculated using Agricultural Prices as outlined in the 1977-1982 methodology.

For the 24 States of AK, CT, DE, DC, ID, IL, IN, MA, MD, ME, MI, MN, NH, NJ, NY, OH, OR, PA, RI, VA, VT, WA, WI, and WV, the PMA provides physical unit prices, excluding taxes. In 1983-1985, the DC price is missing, and the MD price is assigned. In 1983, RI has no price and the PAD District IA average is assigned. A simple average of monthly State and Federal taxes is calculated as a combined average tax and added to the $P M A$ price for a final physical unit price. State and local sales and other general taxes are not included.

\section{Physical Unit Prices: 1977-1982}

Monthly prices from Agricultural Prices and monthly special fuels consumption data from Highway Statistics are collected for the States. MD prices are assigned to DC. Prices include State and local per gallon taxes. Federal taxes and State and local sales and other general taxes are not included.

The volume-weighted annual diesel physical unit prices for States and the United States are calculated using the monthly Agricultural Prices price data, weighted by the monthly Highway Statistics consumption data. AK/1977-1982 prices are assigned the quantityweighted Census division prices. 
Physical Unit Prices: 1970-1976

Quarterly prices from Agriculturai Prices and monthly special fuels consumption data from Highway Statistics are collected for the States. Prices include State and local per gallon taxes. Federal taxes and State and local sales taxes und other general taxes are not included.

1. Prices for 1970-1972 are reported in cents per gallon and must be converted to dollars per gallon. Prices for 1973-1976 are already reported in dollars per gallon.

2. For 1971-1973, State-level prices are not available for CT, MA, ME, NH, RI, and VT. Each is assigned the New England price for the 3 years.

3. The third quarter DE price is assigned to the missing fourth quarter DE price in 1972.

4. The combined MD/DE piices reported in 1973 are assigned to each of the States.

5. For 1970-1976, MD (or MD/DE) prices are assigned to DC.

The monthly special fuels consumption for 1970-1976 are converted into quarterly consumption by summing the months for each quarter.

The consumption-weighted annual diesel physical unit prices for the States are calculated using the quarterly weights and quarterly prices. For 1970-1972, the quarterly prices from Agriculture Prices are converted from cents per gallon to dollars per gallon. For 1973 forward, the prices are already in dollars per gallon in the source. AK/1970-1976 prices are assigned the consumptionweighted Census division prices.

\section{Btu Prices: Al/ Years}

Btu prices for States are calculated by converting the physical unit prices from cents per gallon to dollars per barrel (42 gallons per barrel) and, then, to dollars per million Btu (5.825 million Btu per barrel). U.S. Btu prices are calculated as the average of the State Btu prizes, weighted by consumption from SEDS.

\section{Data Sources}

\section{Pricas}

1986 forward: Energy Information Administration, $P e$ troleum Marketing Annual. Table 36 (1986-1988) and Table 38 (1989), column titled, "Sales to End Users, Through Company-Operated Retail Outlets," for diesel fuel prices; and Table 29 (1986-1988) and Table 30 (1989), column titled "All Refiners, Sales to End Users, Through Company Outlets," for motor gasoline prices. colurnn titled, "Sales to End Users, Sales Through Company-Operated Retail Outlets."

1970-1985: Crop Reporting Board, U.S. Department of Agriculture, Agriculture Prices, tables generally titled, "Motor Supplies: Average Price Paid by Farmers for Motor Fuel" for 1970-1979, and "Diesel Fuel: Average Price Paid by States" for 1980-1985.

1970 forward: Federal Highway Administration, U.S. Department of Transportation, Highway Statistics, Table MF-121T for State tax rates, Table FE-101 for Federal tax rates, and Table MF- 25 for special fuels consumption data. Table MF-25 is not included in the 1976 volume but is publicly available directly from the Federal Highway Administration.

\section{Consumption}

1970 forward: Energy Information Administration, State Energy Data System, transportation sector distillate consumption.

\section{Conversion Factor: All Years}

5.825 million Btu per barrel 
For all years, the price of heavy oil consumed at electric utilities is the average cost of No. 6 fuel oil (residual fuel oil) as reported in Cost and Quality of Fuels for Electric Utility Plants. (See Residual Fuel Electric Utility Sector).
Jet fuel prices are estimated for all years for the transportation sector and for 1972-1982 for the electric utility sector. After 1982, electric utility use of jet fuel is reported as light oil. See the "Light Oil (Utilities)" section elsewhere in this Documentation.

\section{Transportation Sector}

Consumption estimates from SEDS are developed for two types of jet fuel: kerosene type (used primarily by commercial aircraft and accounting for about 80 percent of all jet fuel) and naphtha type (used primarily by military aircraft and accounting for the remaining 20 percent). Prices are developed for kerosene-type jet fuel and are used as the price for both iypes of jet fuel. Taxes are not included in the prices 1983 forward. It is not clear from the data sources whether taxes are included in the 1970-1982 prices.

\section{Physical Unit Prices: 1985 Forward}

Transportation sector jet fuel prices for 1985 forward are based on data from the EIA's Petroleum Marketing Annual (PMA). Annual prices to end users are available for all States in most cases. Prices are converted to dollars per gallon. States without prices are assigned adjacent State or PAD subdistrict prices as follows:

\begin{tabular}{lll} 
Year & States & Price Assignments \\
\hline 1985 & DC & MD \\
& ME, RI, VT & PADD IA \\
1986 & DC & MD \\
& RI, VT & PADD IA \\
1987 & DC & MD \\
& NH, RI, VT & PADD IA \\
1988 & DC & MD \\
& RI, VT & PADD IA
\end{tabular}

\section{Physical Unit Prices: 1983, 1984}

Prices for 1983 and 1984 are based on data from EIA's Petroleum Marketing Monthly (PMM). Monthly jet fuel prices in cents per gallon (excluding taxes) are available from the $P M M$ for the PAD districts and subdistricts for January 1983 through August 1984. The monthly prices for each of the PAD districts or subdistricts (IA, IB, IC, II, III, IV, and V) are assigned to each State within a district whenever the State monthly price is unavailable. State-level data are available from September 1984 through December 1984. Annual prices are the simple averages of the monthly prices 


\begin{tabular}{|c|c|c|}
\hline State & Months & $\begin{array}{l}\text { Price } \\
\text { Assignments }\end{array}$ \\
\hline CT & September & PADD IA \\
\hline DC & September-December & MD \\
\hline ID & September & PADD IV \\
\hline KS & November, December & PADD II \\
\hline ME & September-December & PADD IA \\
\hline MS & October & PADD III \\
\hline ND & December & PADD II \\
\hline NE & October & PADD II \\
\hline NH & $\begin{array}{l}\text { September, } \\
\text { November, December }\end{array}$ & PADD IA \\
\hline NM & October-December & PADD III \\
\hline OR & November & PADD V \\
\hline RI & September-December & PADD IA \\
\hline VT & September-December & PADD IA \\
\hline WV & September & PADD IC \\
\hline
\end{tabular}

\section{Physical Unit Prices: 1976-1982}

State-level jet fuel prices for 1976-1982 are calculated from the Producer Prices and Price Indexes (PPI) monthly indices for Census divisions and the jet fuel base prices by State for July 1975. The monthly price for each Census division is equal to the PPI monthly index times the jet fuel base price for July 1975 for that Census division. Census division monthly prices are assigned to each State within the Census division, and annual jet fuel prices are computed as simple averages of the monthly State prices. It is not clear whether the jet fuel base prices for July 1975 include taxes.

\section{Physical Unit Prices: 1970-1975}

Jet fuel physical unit State-level prices for the 1970-1975 period are based on U.S. annual wholesale prices from the $P P I$ and the relationship of these prices to wholesale kerosene prices reported in Platt's. The U.S. prices are converted to Census division prices which are then assigned directly to States. It is not clear whether these prices include taxes.

Preliminary U.S. jet fuel prices from the PPI for 1973-1980 are calculated using the annual jet fuel price indices, the jet fuel U.S. base price for July $1975(0.276$ dollars per gallon), and the U.S. index for July 1975 (235.8) The index for 1973 is assumed to be equal to a simple average of the 11 available monthly indices.

The calculated preliminary U.S. jet fuel prices from the $P P I$ are used as the dependent variable in a regression equation ror 1973-1980 where the wholesale kerosene prices from Platt's are the independent variable.
The regression equation is used to estimate U.S. annual jet fuel prices for 1970-1972.

Jet fuel prices for Census divisions are estimated using the preliminary U.S. prices derived above for 1970-1975 (calculated directly from the PPI data for 1973-1975 and estimated for 1970-1972). These prices are used as inputs to a regression equation which establishes a linear relationship 'uetween preliminary U.S. prices and Census division prices for the years 1970-1975. Census division prices are assigned to each State within the Census division.

\section{Btu Prices: All Years}

Btu prices for States are calculated from the physical unit prices and the Btu conversion factor. U.S. Btu prices are calculated as the average of the State Btu prices, weighted by consumption data from SEDS.

\section{Data Sources}

Prices

1985 forward: Energy Information Administration, $P e$ troleum Marketing Annual, Table 21, column titled, "Kerosene-Type Jet Fuel" (1985); and Table 33 (1986-1988) and Table 35 (1989), column titled, "Kerosene-Type Jet Fuel, Sales to End Users."

1983, 1984: Energy Information Administration, Petroleum Marketing Monthly, Table 14 (January 1984), Table 19 (February-September 1984), and Table 25 (October 1984-January 1985).

1973-1982: Bureau of Labor Statistics, U.S. Department of Labor, Producer Prices and Price Indexes, Supplement, table titled, "Producer price indexes for refined petroleum products by region."

1970-1975: McGraw Hill, Inc., Platt's Oil Price Handbook and Oilmanac, 57th Edition, page 480.

\section{Consumption}

1970 forward: Energy Information Administration, State Energy Data System, transportation sector jet fuel consumption.

\section{Conversion Factor: All Years}

5.670 million Btu per barrel 


\section{Electric Utility Sector}

Jet fuel electric utility consumption estimates are from SEDS for 1972-1982 only. For 1970 and 1971, no parallel series is available, and for the years after 1982, the series is part of light oil. All applicable taxes are included in the prices.

\section{Btu Prices: 1975-1982}

For the States that consumed kerosene-type jet fuel at electric utilities during these years, the Btu prices are directly from Cost and Quality of Fuels for Electric Utility Plants $(C \& Q)$.

\section{Btu Prices: 1972-1974}

Because $C \& Q$ prices are not available for 1972-1974, prices are estimated from $C \& Q$ prices for 1975 and 1976 and Agricultural Prices data for 1972-1976.

1. Simple annual averages of Agricultural Prices quarterly values are calculated for 1972-1976. New England Census Division prices are assigned to CT, MA, ME, NH, RI, and VT.

2. The average annual prices based on Agricultural Prices values for 1975 and 1976 are used as the independent variables in a regression where the dependent variables are State-level prices based on $C \& Q$ prices for 1975 and 1976.

3. State-level price estimates for 1972-1974 are derived from the results of the regression analysis and the Agricultural Prices values for 1972-1974.

\section{U.S. Btu Prices: A/l Years}

U.S. Btu prices are calculated as the average of the State Btu prices, weighted by consumption data from SEDS.

\section{Data Sources}

\section{Prices}

1975-1982: Energy Information Administration, Cost and Quality of Fuels for Electric Utility Plants, Tables 6 and 13 (1975), Table 13 (1976-1979), and Table 47 (1980-1982).

1972-1976: Crop Reporting Board, U.S. Department of Agriculture, Agriculture Prices, table titled, "Household Supplies: Average Prices Paid by Farmers for Lawn Mowers and Petroleum Products."

\section{Consumption}

1972-1982: Energy Information Administration, State
Energy Data System, electric utility sector kerosenetype jet fuel consumption.

\section{Conversion Factors: All Years}

Becau:o Btu prices are available directly from the data sources, in conversion factors are used. 


\section{Kerosene}

Kerosene prices are developed for the residential and industrial sectors, and the industrial sector prices are assigned to the commercial sector. Estimates of the amount of kerosene consumed by the residential, commercial, and industrial sectors are from State Energy Data System (SEDS).

\section{Residential Sector}

\section{Physical Unit Prices: 1985 Forward}

In July 1985, the Bureau of Labor Statistics ceased publication of the Producer Prices and Price Indexes (PPI) data series that is used to estimate State Energy Price and Expenditure Data System residential kerosene prices for earlier years. An alternative approach is therefore applied that maintains continuity with the previously published prices. This approach is used to estimate physical unit prices for all States with residential kerosene consumption.

The approach used to estimate residential kerosene prices for $198^{5}$ forward is based on the fact that average U.S. kerosene and distillate fuel prices from 1970 through 1984 are highly correlated. Residential kerosene prices for 1985 forward are estimated by applying the percentage changes in residential distillate fuel prices for each year to the residential kerosene prices for the previous year in each State. Taxes are included in the prices.

\section{Physical Unit Prices: 1977-1984}

Because Agricultural Prices kerosene price data are not available after 1976, monthly Census division Producer Prices and Price Indexes (PPI) prices and price indices are used as the basis for the residential kerosene series from 1974 to 1984 . To maintain consistency in the series, the $P P I$ prices are multiplied by an adjustment factor that accounts for the relationship between $P P I$ and Agricultural Prices data for quarters in which the two series overlap. In the description of computational procedures below, the adjustment factor is derived first, the PPI prices for 1977-1984 are estimated, and the final kerosene physical unit and Btti prices for States are calculated. The final residential sector kerosene prices approximate the average prices paid by farmers. It is not clear from the data sources whether these prices include taxes.

The first step is to compute the adjustment factor relating PPI and Agricultural Prices daia.
1. Monthly PPI prices for the 18 months covered from July 1975 through December 1976 are calculated from the July 1975 base prices and monthly indices for Census divisions.

2. The calculated Census division monthly prices are assigned to each State within the respective Census division.

3. Volume-weighted quarterly $P P I$-based prices for States are calculated using the monthly volume weights developed from Retail Sales and Inventories sales data for "other distillate fuel oil."

4. The adjustment factor relating PPI and Agricultural Prices data is calculated as the simple average of the ratios of the quarterly kerosene price by State from Agricultural Prices to the calculated quarterly $P P I$-based kerosene prices by' State.

The next step is the calculation of monthly State-level prices from PPI kerosene Census division data for 1977-1984.

1. Monthly Census division PPI prices are calculated using the July 1975 base prices and the monthly price indices for 1977-1984. The missing monthly indices for February, June, July, and October 1980 for the East South Central Division are assumed to be equal to the index for the preceding month.

2. Census division monthly prices are assigned to each State within the respective Census division.

The next step is the calculation of annual physical unit State prices.

1. Annual $P P I$-based physical unit prices for States are computed from the monthly PPI prices and the monthly consumption weights.

2. Final residential kerosene prices for States are estimated as the product of the annual PPI-based State price and the adjustment factor calculated above.

3. The AK/1984 price is estimated by applying the percentage change in the distillate fuel residential price to the kerosene residential price for the previous year.

\section{Physical Unit Prices: 1970-1976}

Physical unit prices for States are calculated from quarterly price data in Agricultural Prices and consumption weights derived from Retail Sales and Inventories of Fuel Oil. It is not clear from the data sources whether the prices include taxes.

The quarterly physical unit price data from Agricultural Prices for 1970-1976 are published in several different forms. The first step in the calculation of prices for these years is to organize the published Agricultural 
1. For 1971-1973, no quarterly prices are available for CT, MA, ME, NH, RI, and VT. Each of these States is assigned the quarterly prices reported for the New England Census Division.

2. For 1973, combined MD/DE quarterly prices are reported instead of separate State prices. For this year, the combined prices are assigned to both States.

3. Missing quarterly NV/1972 prices are estimated by calculating the average ratio between the quarterly NV prices to the quarterly U.S. prices reported in Agricultural Prices for 1971 and 1973 (eight quarters). The derived ratio is applied to the Agricultural Prices quarterly prices for the United States for 1972.

4. No prices are reported for AK, DC/1970-1976. Quarterly weighted Census division prices are assigned to $A K$, and $M D$ prices are assigned to DC for all 7 years.

In order to weight the quarterly prices from Agricultural Prices into annual State prices, monthly quantity weights are calculated from Retail Sales and Inventories of Fuel Oil. This assumes that the "other distillate oil" consumption data by PAD districts is kerosene.

1. Monthly weights are computed using simple averaging of all available "other distillate oil" sales data for each month for each PAD district. Since data are available from November 1978 to March 1981 , some months have averages based on three data points while others are based on one or two data points. For example, the average weight for March is the simple average of the 1979, 1980, and 1981 March volumes published in Retail Sales and Inventories of Fuel Oil.

2. Each month's share of average annual sales is calculated by PAD district from the average monthly sales figures. These shares, which become the monthly weights, are then assigned to each State within its respective district.

Final State annual kerosene physical unit prices are calculated as the weighted average of the Agricultural Prices quarterly prices. The monthly weights (shares) are converted to quarterly weights by summing the shares for months within a particular quarter. These same weights are used with the State-level price data for each year from 1970 to 1976.

\section{Alaska Btu Prices: 1970-1979, 1983}

Kerusene residential prices for AK are estimated based on the assumption that the ratio of AK-to-U.S. kerosene residential prices is the same as the ratio of AK-to-U.S. distillate fuel residential prices.
Btu prices for States are calculated from the physical unit prices and the conversion factor. U.S. Btu prices are calculated as the average of the State Btu prices, weighted by consumption data from SEDS.

\section{Data Sources}

\section{Prices}

1984 forward: Residential sector distillate fuel price estimates (in physical units) from the State Energy Price and Expenditure Data System.

1975-1984: Bureau of Labor Statistics, U.S. Department of Labor, Producer Prices and Price Indexes, Supplement, table titled, "Producer Price Indexes for refined petroleum products by region."

1978-1981: Energy Information Administration, Retail Sales and Inventories of Fuel Oil, Table 2.

1970-1976: Crop Reporting Board, U.S. Department of Agriculture, Agricultural Prices, table titled, "Household Supplies: Average Price Paid by Farmers for Lawn Mowers and Petroleum Products."

\section{Consumption}

1970 forward: Energy Information Administration, State Energy Data System, residential sector kerosene consumption.

\section{Conversion Factor: All Years}

5.670 million Btu per barrel

\section{Commercial Sector}

State prices from the industrial sector are assigned to the commercial sector. Although there is no industrial consumption of kerosene in DC in 1989, an industrial price is calculated to be assigned to the commercial sector consumption.

\section{Industrial Sector}

The industrial sector kerosene prices are based on wholesale price and price index data and on the industrial sector distillate prices. The procedures vary slightly for 1985 forward, 1975-1984, and 1970-1974. In all years, physical unit prices are calculated first, 
then Btu prices are computed using the physical unit prices and the conversion factor. Prices approximate an average kerosene price for the manufacturing sector.

\section{Physical Unit Prices: 1985 Forward}

The discontinuation of the published PPI data from the Bureau of Labor Statistics affected the estimation of industrial sector kerosene prices. To estimate prices for 1985 forward, the percentage change in industrial sector distillate fuel prices for each year is applied to the industrial sector kerosene prices for the previous year on a State-by-State basis. Taxes are included in the prices.

\section{Physical Unit Prices: 1975-1984}

Physical unit industrial kerosene prices for 1975.1984 are estimated from $P P I$ base prices and indices for kerosene and No. 2 distillate oil, and from the industrial sector distillate prices in physical units. The ratio of $P P I$ kerosene prices to $P P I$ distillate prices is used as an adjustment factor to estimate kerosene prices. It is not clear from the data sources whether taxes are included in the prices.

Annual wholesale prices are calculated from PPI annual indices for kerosene and No. 2 distillate fuel oil and their respective July 1975 base prices for Census divisions. Annual average distillate price indices for 1976 are estimated as the simple average of monthly indices. Census division prices for both kerosene and fuel oil No. 2 are assigned to each State within the respective Census divisions. The industrial sector physical unit kerosene prices for States are computed using the distillate industrial physical unit prices and the ratio of PPI kerosene prices to PPI fuel oil No. 2 prices.

\section{Physical Unit Prices: 1970-1974}

Physical unit State-level prices for 1970-1974 are estimated from the distillate industrial prices and the average ratio of kerosene to distillate prices from PPI for 1975-1978. The average annual wholesale price ratio between kerosene and fuel oil No. 2 (distillate) is calculated from PPI-based data for the years 1975-1978. State-level kerosene industrial physical unit prices are calculated as the product of the ratios and the industrial sector distillate prices for 1970-1974. It is not clear from the data sources whether taxes are included in these prices.

\section{Btu Prices: All Years}

Btu prices for States are computed by converting the physical unit prices in dollars per gallon to dollars per barrel (42 gallons per barrel) and, then, to dollars per million Btu (5.670 million Btu per barrel). U.S. Btu prices are calculated as the average of the State Btu prices, weighted by consumption data from SEDS.

\section{Data Sources}

\section{Prices}

1970 forward: Industrial sector distillate fuel price estimates (in physical units) from the State Energy Price and Expenditure Data System.

1975-1985: Bureau of Labor Statistics, U.S. Department of Labor, Producer Prices and Price Indexes, Supplement, table titled "Producer price indexes for refined petroleum products by region."

\section{Consumption}

1970 forward: Energy Information Administration, State Energy Data System, industrial sector kerosene consumption.

\section{Conversion Factor: All Years}

5.670 million Btu per barrel 


\section{Light Oil (Utilities)}

Beginning with 1980, the price of light oil consumed at electric utilities is the average delivered cost of No. 2 fuel oil as reported in Cost and Quality of Fuels for Electric Utility Plants. For earlier years, the price is the consumption-weighted average of the kerosene-type jet fuel price and No. 2 fuel oil. (See also Distillate Fuel Electric Utility Sector and Jet Fuel Electric Utility Sector).

\section{Liquefied Petroleum Gases}

Liquefied petroleum gases (LPG) prices are developed for the residential and the industrial sectors. State prices for the commercial and transportation sectors are assigned from the industrial sector. Estimates of the amount of LPG consumed by sector are from the State Energy Data System (SEDS), and are adjusted for process fuel and intermediate product consumption in the industrial sector. See the discussion under "Consumption Adjustments for Process Fuel and Intermediate Products" in this report.

\section{Residential Sector}

Residential sector prices are the average delivered prices of LPG to residential consumers in areas where natural gas is available as a competing fuel. Taxes are included in the prices. For 1973 forward, Btu prices are reported in the data source. For 1970-1972, physical unit prices are calculated first and Btu prices are calculated from the physical unit prices and the conversion factors. Prices for AK and $\mathrm{HI}$ in all years are estimated by a different methodology described in a separate section that follows.

\section{Btu Prices: 1973 Forward}

Propane prices by city are reported in the Gas Househeating Survey (GHS) directly in dollars per million Btu. The simple average of available city prices is used as the State annual average. Outlier prices are omitted from consideration for Arkansas Louisiana Gas for AR/1989, City of Long Beach for CA/1989, Public Service Electric \& Gas for NJ/1989, Central Hudson Gas and Electric for NY/1979-1981, Orange \& Rockland Utilities for NY/1989, Pike County Light \& Power for PA/1989, and Northwestern Public Service for $\mathrm{SD} / 1989$.

To estimate missing prices (other than $\mathrm{AK}$ and $\mathrm{HI}$, which are described in a separate section that follows), simple averages of adjacent States' prices are used. The adjacent State assignments are shown in Table 23 on page 212. Estimated data for one State are not used to estimate prices for another State.

\section{Physical Unit Prices: 1971, 1972}

Physical unit residential LPG prices are based on the city-level propane prices reported in $G H S$ in cents per gallon. Prices for missing States are estimated. The GHS prices are the average delivered prices for propane purchased by residential consumers as of December 31 . 


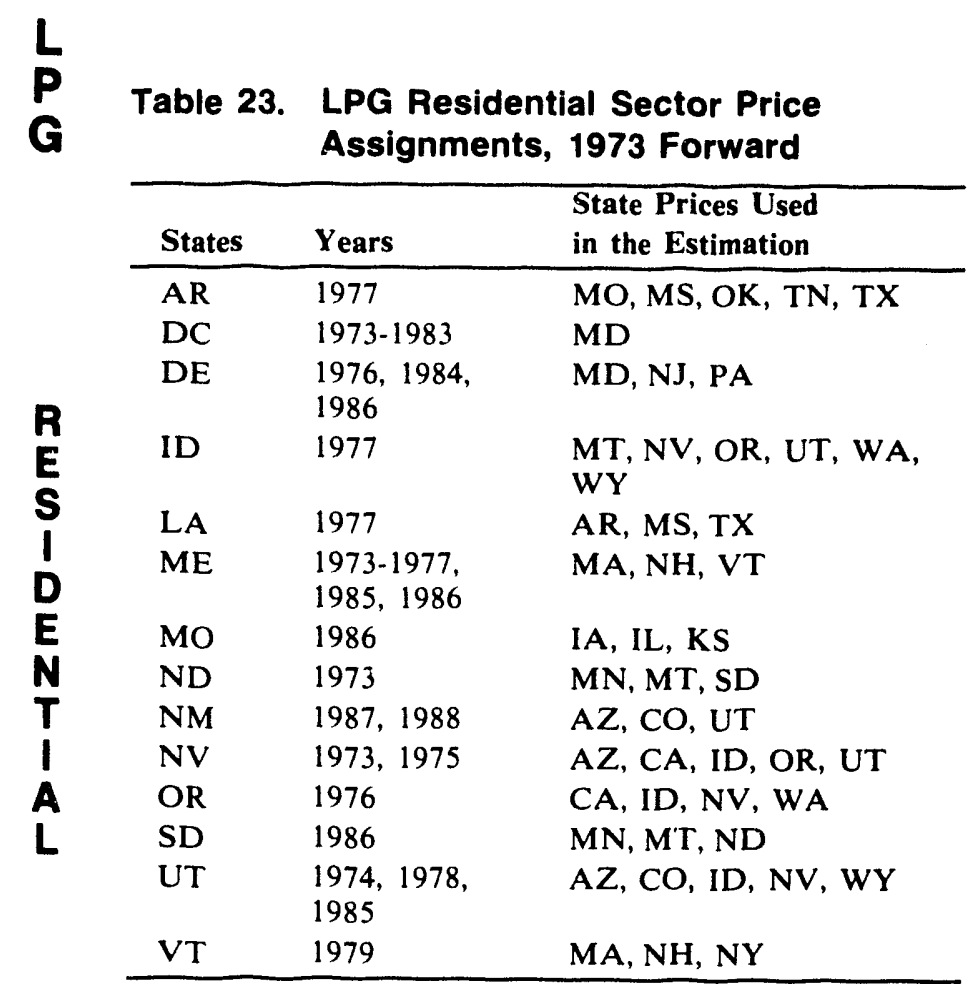

1. City-level propane prices from $G H S$ are assigned to their respective States. The AL/1971 price for the Phoenix City Utilities System is omitted as an outlier.

2. Physical unit prices for a State are calculated directly from the available city/utility price observations reported in GHS. Final physical unit prices are equal to the simple average of the price observations for each State.

3. MD prices are assigned for missing DC prices. AK and $\mathrm{HI}$ prices are discussed in a separate section that follows.

\section{Physical Unit Prices: 1970}

Since GHS did not publish LPG prices prior to 1971, the residential sector LPG prices for 1970 are estimated. To maintain continuity with the GHS prices for 1971 forward, prices for 1970 are estimated using simple regression analysis. The relationship between GHS data for 1971 and 1972 and corresponding Agricultural Prices data is the basis for the estimation.

1. Before regression analysis can be applied, Agricultural Prices data for 1970-1972 are prepared for 49 States (no AK or HI prices are available).

a. State-level prices for small purchases, representing residential end users, for 1970-1972 are published by Agricultural Prices in cents per pound. When price per pound data are not available, price per gallon data, representing larger volume purchases, are used. These prices per gallon are multiplied by 0.543 , the average ratio of price per pound to price per gallon for the United States for 1970-1972, to create uniform input data in price per pound.

b. For 1971 and 1972, the price reported for the New England Region is assigned to CT, MA, ME, NH, RI, and VT.

c. Data in cents per pound are converted to dollars per gallon by multiplying by the propane conversion factor of 4.2 pounds per gallon, from the Petroleum Products Handbook and dividing by 100 .

d. Missing prices use adjacent States' average prices as shown below:

\begin{tabular}{lll} 
State & Years & State Prices Used \\
\hline NV & 1970, & AZ, CA, ID, UT \\
& 1971 & \\
OR & $1971-1972$ & CA, ID, \\
UT & 1972 & AZ, CO, ID, NV, WY \\
WA & $1970-1972$ & CA, ID
\end{tabular}

e. MD prices are assigned for missing DC prices. $\mathrm{AK}$ and $\mathrm{HI}$ prices are discussed below.

2. The physical unit GHS prices and Agricultural Prices data for 1971-1972 (excluding AK and HI) are used with simple regression analysis to estimate final phy'sical unit LPG residential prices.

\section{Alaska and Hawaii Prices: All Years}

Prices cannot be estimated for AK and $\mathrm{HI}$ using adjacent State price assignments. Missing prices for these two States are estimated by computing ratios of the AK or HI prices to the U.S. prices from the GHS for years when AK or HI data are available and applying these ratios to the U.S. prices in years where prices need to be estimated.

1. GHS prices for AK are available in 1972 and 1980. The 1972 AK-to-US ratio is used to estimate prices for 1970, 1971, and 1973-1979. The 1980 AK-to-US price ratio is used to estimate prices for 1981-1989.

2. $G H S$ prices for $H I$ are available in 1971, 1977-1979, and 1989. The 1971 HI-to-US GHS is used to estimate prices for 1970 and 1972-1974. The average ratio of the HI-to-US prices for 1977-1979 is used to estimate prices for 1975, 1976 and 1980-1984. The $1989 \mathrm{HI}$-to-US ratio is used to extimate prices for 1985-1988. 
Btu Prices: All Years

For 1973 forward, Btu prices for States are directly from the data sources. For 1970-1972, Btu prices for States are calculated by converting the physical unit prices by using the factors cited in Table 25 on page 215 . U.S. Btu prices are calculated as the average of the State Btu prices, weighted by consumption data from SEDS.

\section{Data Sources}

Prices

1971 forward: American Gas Association, Gas Househeating Survey (GHS), Appendix 2, "Competitive Fuel Prices."

1970-1972: Crop Reporting Board, U.S. Department of Agriculture, Agricultural Prices, table titled, "Average Price Paid by Farmers for Lawn Mowers and Petroleum Products, Specified Dates, by State," L.P. Gas column.

\section{Consumption}

1970 forward: Energy Information Administration, State Energy Data System, residential sector LPG consumption.

\section{Conversion Factors}

1970-1972: Energy Information Administration, State Energy Data Report, Consumption Estimates, 1960-1989. Table D1, as shown in Table 25 on page 215 .

1970-1972: 4.2 pounds per gallon from Guthrie, Virgil, ed., 1960. Petroleum Products Handbook. John Wiley and Sons, Inc., New York, New York: pages 3-5.

Conversion factors are not necessary for other years because Btu prices are available directly from the data sources.

\section{Commercial Sector}

State prices from the industrial sector are assigned to the commercial sector.

\section{Industrial Sector}

The industrial sector LPG prices are estimated from average $L P G$ prices to manufacturing firms, average LPG prices to farmers, and refiner and gas plant operator sales to end users. Prices for 1985 forward are from the Petroleum Marketing Annual (PMA). Prices for 1978-1981 are from the Anmual Survey of Manufactures (ASM) or the Census of Manufactures (CM), and prices for 1970-1977 and 1982-1984 are derived from $A g r i c u l t u r a l$ Prices and scaled to the $A S M /$ $C M$ prices using the ratio of $A S M / C M$ to Agricultural Prices LPG prices for the years 1978-1981, when both price series were available. Taxes are included in the 1970-1984 estimates but are not included in the prices for 1985 forward.

\section{Physical Unit Prices: 1985 Forward}

Industrial sector LPG physical unit State prices for 1985 forward are estimated using physical unit annual prices in the Petroleum Marketing Annual (PMA). Where prices are not available, the PAD district price is assigned as follows:

\begin{tabular}{lllll} 
State & Years & & State & Years \\
\cline { 1 - 2 } \cline { 5 - 5 } AK & $1986-1988$ & & ND & 1986 \\
AL & $1985-1988$ & & NE & $1986-1989$ \\
AZ & $1986-1989$ & & NH & $1987-1989$ \\
DC & $1985-1989$ & & NV & $1985-1988$ \\
DE & $1986-1989$ & & OK & 1986,1987 \\
GA & 1985 & & OR & 1986 \\
HI & $1985-1989$ & & RI & $1986-1989$ \\
IA & 1986 & & SD & $1985-1989$ \\
ID & 1986 & & UT & $1986-1988$ \\
KS & $1986-1989$ & & VT & $1986-1989$ \\
MA & 1986 & & WA & $1986-1989$ \\
MD & 1988 & WI & 1985,1986 \\
ME & $1986-1989$ & WV & 1989 \\
MI & $1985-1988$ & WY & 1987,1988 \\
MN & 1985,1986, & &
\end{tabular}

When a PAD district price is not available, a consumption-weighted price is calculated using available prices for States within the district and the SEDS industrial sector consumption for the States. PAD district price calculations are made for PAD District $V$ for 1985 using AK, CA, OR, and WA consumptionweighted prices, for PAD Subdistrict IA for 1986 using CT and NH consumption-weighted prices, and for PAD Subdistrict IA for 1987-1988 using CT and MA consumption-weighted prices.

\section{Physical Unit Prices: 1982-1984, 1970-1977}

Industrial sector LPG physical unit prices for 1982-1984 and 1970-1977 are estimated based on the relationship between State-level LPG prices from $A g$ ricultural Prices and the prices calculated from Annual Survey of Manufactures (ASM) or Census of Manufac- 
1. Before the adjustment factor relating Agricultural Prices and $A S M / C M$ data is computed, monthly Agricultural Prices data are converted into annual prices and missing data are estimated.

a. Annual LPG prices are calculated as simple averages of the monthly prices from Agricultural Prices for the years 1977-1984. The only States missing data are $W V / 1977-1981$ and AK, DC, HI/1977-1984. WV is assigned the simple average of the KY, MD, OH, $\mathrm{PA}$, and VA prices. $\mathrm{AK}, \mathrm{DC}$, and $\mathrm{HI}$ prices are discussed below.

b. The average ratio of $A S M / C M$-based final prices for 1978-1981 and the 1978-1981 Agricultural Prices annual prices is calculated for 48 States (excluding AK, DC, and $\mathrm{HI}$ ) as the simple average of the ratio over the 4 years. This average ratio is used as an adjustment factor.

2. Final industrial sector LPG prices for $1982-1984$ and 1970-1977 are estimated using the State-level adjustment factors and annual average LPG prices from Agricultural Prices for these years.

a. Annual average LPG prices are calculated for 1982-1984 and 1970-1977 as the simple average. of the monthly prices.

b. Agricultural Prices published annual average prices in dollars per gallon for all States in 1975 and 1976. For DE/1970-1974, MD/1970-1974, VA/1970-1974, and WV/1970-1972, only prices for small volume purchases in cents per pound were published. These are converted to cents per gallon by multiplying by 1.96 , the average ratio of cents per gallon to cents per pound for the United States for 1970-1974.

c. For 1970-1972, Agricultural Prices are converted from cents per gallon to dollars per gallon.

d. For 1971-1973, the New England price per gallon reported by Agricultural Prices is assigned to CT, MA, ME, NH, RI, and VT.

e. MD prices are assigned to DC/1970-1972, 1974-1977, and 1982-1984. The combined $\mathrm{MD} / \mathrm{DE} / 1973$ price is assigned to $\mathrm{MD}, \mathrm{DE}$, DC.

f. Excluding AK and HI, States missing Agricultural Prices LPG prices are assigned the simple average price of adjacent States. The States with missing data and the adjacent State assignments are shown in Table 24.

g. Industrial sector LPG physical unit prices for 1970-1977 and 1982-1984 for all States (except $\mathrm{AK}, \mathrm{DC}$, and $\mathrm{HI}$ ) are calculated using the estimated anıual Agricultural Prices data for the respective year and the State-level average ratios as adjustment factors.

3. AK/1982-1984 and HI/1982-1984, 1970-1977 prices are estimated by using the relationship between $A S M / C M$ based prices for these States and the U.S. price reported by Agricultural Prices (1979-1981 for AK and 1978-1981 for HI). The average ratio for the available years for the two States is calculated and used with the Agricultural Prices U.S. prices for the years to be estimated.

Table 24. LPG Industrial Sector Price Assignments, 1970-1976

\begin{tabular}{lll}
\hline State & Years & $\begin{array}{l}\text { State Prices Used } \\
\text { in the Estimation }\end{array}$ \\
\hline CT & 1974 & NY \\
MA & 1974 & NY \\
ME & 1974 & NY \\
NH & 1974 & NY \\
NV & $1970-1971$ & AZ, CA, ID, UT \\
& $1973-1974$ & AZ, CA, ID \\
OR & $1970-1974$ & CA, ID \\
RI & 1974 & NY \\
& $1975-1976$ & CT, MA, NY \\
UT & 1972 & AZ, CO, ID, NV, WY \\
& $1973-1974$ & AZ, CO, ID, WY \\
VT & 1974 & NY \\
WA & $1970-1974$ & CA, ID \\
\hline
\end{tabular}

\section{Physical Unit Prices: 1978-1981}

For 1978-1981, the industrial sector LPG prices are either calculated directly from cost and quantity data from the $A S M$ or the $C M$ or are estimated using the relationship of $A S M / C M$ data to LPG price data from Agricultural Prices.

1. For 1978-1981, industrial sector physical unit prices for LPG are calculated as the average cost per unit from cost and quantity data published in $A S M / C M$. Since sales are reported in pounds, the prices are converted to dollars per gallon. The conversion factor of 4.5 pounds per gallon is from $A S M / C M$.

2. The AK price for 1978 is the consumptionweighted average Census division price. In addition, four States have prices estimated as the simple average of the prices of adjacent States, and $\mathrm{DC}$ is assigned the MD price as follows: 


\begin{tabular}{lll} 
State & Years & State Prices Used \\
\hline AR & 1978 & LA, MC, MS, OK, TX \\
DC & $1978-1981$ & MD \\
LA & 1980 & AR, MS, TX \\
NM & $1979-1981$ & AZ, CO, OK, TX \\
WY & $1978-1981$ & CO, ID, MT, ND, NE, \\
& & SD, UT
\end{tabular}

\section{Btu Prices: All Years}

Btu prices for States and the United States are calculated from the physical unit prices and the conversion factors shown in Table 25. U.S. Btu prices are calculated as the average of the State Btu prices, weighted by consumption data from SEDS, adjusted for process fuel and intermediate product consumption.

\section{Data Sources}

\section{Prices}

1985 forward: Energy Information Administration, $P e$ troleum Marketing Annual, Table 21 (1985), Table 33 (1986-1988), and Table 35 (1989).

1970-1984: Crop Reporting Board, U.S. Department of Agriculture, Agricultural Prices, tables titled, "Average Price Paid by Farmers for Lawn Mowers and Petroleum Products, Specified Dates, by State," L.P. Gas column (1970-1976); "Household Supplies: Average Price by Farmers" (1977-1979); "L.P. Gas: Average Price Paid by States" (1980); and "L.P. Gas: Average Price Paid by Months by States" (1981-1984).

1981: Bureau of the Census, U.S. Department of Commerce, 1982 Census of Manufactures, Fuels and Electric Energy Consumed, Part 2, States and Standard Metropolitan Statistical Areas by Major Industry Groups, Table 3 , State-level quantity and cost of liquefied petroleum gases.

1978-1980: Bureau of the Census, U.S. Department of Commerce, Annual Survey of Manufactures, Fuels and Electric Energy Consumed, States by Industry Group and Standard Metropolitan Statistical Areas by Major Industry Group. Table 3, State-level quantity and cost of liquefied petroleum gases.

\section{Consumption}

1970 forward: Energy Information Administration, State Energy Data System, industrial sector LPG consumption.

\section{Conversion Factors}

1970 forward: Energy Information Administration, State Energy Data Report, Consumption Estimates, 1960-1989, Table D1, as shown in Table 25.
1978-1981: 4.5 pounds per gallon from Annual Survey of Manufactures. Appendix C.

Table 25. I PG Btu Conversion Factors, 1970 Forward (Million Btu per Barrel)

\begin{tabular}{lllll}
\hline Year & $\begin{array}{l}\text { Conversion } \\
\text { Factor }\end{array}$ & & Year & $\begin{array}{l}\text { Conversion } \\
\text { Factor }\end{array}$ \\
\cline { 1 - 2 } \cline { 5 - 5 } 1970 & 3.779 & & 1980 & 3.674 \\
1971 & 3.772 & & 1981 & 3.643 \\
1972 & 3.760 & & 1982 & 3.615 \\
1973 & 3.746 & & 1983 & 3.614 \\
1974 & 3.730 & & 1984 & 3.599 \\
1975 & 3.715 & & 1985 & 3.603 \\
1976 & 3.711 & & 1986 & 3.640 \\
1977 & 3.677 & & 1987 & 3.659 \\
1978 & 3.669 & & 1988 & 3.652 \\
1979 & 3.680 & 1989 & 3.683 \\
\hline
\end{tabular}

\section{Transportation Sector}

State prices from the industrial sector are assigned to the transportation sector. 


\section{Lubricants}

Lubricant prices are developed for the industrial sector and assigned to the transportation sector. State-level prices are not available for either sector; national-level prices are developed and assigned to all States. Taxes are not included in the prices. Estimates of lubricant consumption by the industrial and transportation sectors are from the State Energy Data System (SEDS).

\section{Physical Unit Prices: 1989}

Data from the Bureau of the Census that are used to develop lubricant price estimates for 1970-1988 were not available in time to be used for the 1989 price. An alternative estimate was therefore developed using the ratio of the lubricant price to the crude oil price in 1988. The 1989 lubricant price is estimated to be the price of lubricants in 1988 times 1.22 (the ratio of the crude oil price in 1989 to the crude oil price in 1988).

\section{Physical Unit Prices: 1970-1988}

Prices of lubricants are estimated from three product categories:

1. Lubricating oils made in refineries (SIC 29117.21) and not made in refineries (SIC 29920.21).

2. Lubricating greases made in refineries (SIC 29117.31) and not made in refineries (SIC 29920.31).

3. Lubricating oils and greases, not specifically known (n.s.k.), made in refineries (SIC 29117.00) and not made in refineries (SIC 29920.00 for establishments with 10 employees or more and SIC 29920.02 for establishments with fewer than 10 employees).

For years where Census of Manufactures (CM) data are available (1967, 1972, 1977, and 1982), total shipments are calculated by adding the shipments for the three product categories. Shipments for the third product category are estimated for 29920.00 and 29920.02 by dividing their value of shipments sum by the weighted average cost of the product categories 29920.21 and 29920.31

Total shipments in each year for which $C M$ data are available is divided by the estimated SEDS total lubricants consumption (in physical units) for that year to establish a shipments-to-consumption ratio. Ratios for the years not covered by the $C M$ (i.e., 1968-1971, 1972-1976, and 1978-1981) are estimated by linear interpolation, and the 1982 value is used for 1983 forward. Total shipments for the years not covered by the $C M$ are estimated by multiplying SEDS consumption by the appropriate shipment-to-consumption ratio.
Estimated shipment prices are calculated by dividing the value of shipments shown in the $C M$ or the Annual Survey of Manufactures by the estimated shipments for each product category. The shipment prices are used to represent wholesale prices.

End-user prices in dollars per barrel are estimated by multiplying the shipment prices by trade ratio factors representing the wholesale-to-retail markup. The trade ratio factors are obtained from Bureau of Economic Analysis computer tapes showing 1972 and 1977 data, the only years for which data are available. For 1972 , the sum of the "purchasers value" is divided by the total "producers value" for the sum of the three product categories. A similar calculation is made for 1977 , but the terms "purchase value" and "basic value" are used in the source data. The 1972 ratio is used for 1970-1972, the 1977 ratio is used for 1977 forward, and linearly interpolated values are used for 1973-1976.

\section{Btu Prices: All Years}

Btu prices are obtained by dividing the prices in dollars per barrel by the conversion factor, 6.065 million Btu per barrel.

\section{Data Sources}

\section{Prices}

1989: Lubricant price estimate for 1988 from the State Energy Price and Expenditure Data System.

1989: Energy Information Administration, Arinual Energy Review 1990, Table 70, "Crude Oil Refiner Acquisition Cost," column titled, "Composite, Nominal."

1970, 1971, 1973-1976, 1978-1981, 1983-1988: Bureau of the Census, U.S. Department of Commerce, Annual Survey of Manufactures; Lubricating Oils and Greases (Industries 29117 and 29920).

1972, 1977, and 1982: Bureau of the Census, U.S. Department of Commerce, Census of Manufactures, Petroleum Refining; Lubricating Oils and Greases (Industries 29117 and 29920).

1972 and 1977: Bureau of Economic Analysis, U.S. Department of Commerce, Input-Output Table Work Tapes for SIC Codes 29117 and 29920).

\section{Consumption}

1970 forward: Energy Information Administration, State Energy Data System, lubricants consumption.

\section{Conversion Factor: All Years}

6.065 million Btu per barrel 


\section{Motor Gasoline}

Motor gasoline prices are developed for the transportation sector, and the transportation sector prices are assigned to the commercial and industrial sectors. Motor gasoline consumed in privately-owned vehicles is accounted for in the transportation sector. Estimates of motor gasoline consumed by the transportation, commercial, and industrial sectors are from SEDS. Prices in this series are retail prices (usually service station prices) including taxes.

\section{Physical Unit Prices: 1983 Forward}

Motor gasoline physical unit prices for 1983 forward are based on annual State-level prices from the Petroleum Marketing Annual (PMA) except for certain State and year prices, noted below, that are derived from the Consumer Prices: Energy (CPI). State and Federal motor gasoline taxes are added to the prices from the $P M A$; all taxes are included in the CPI data. In cases where the tax rate is not constant through the year, the months' rates are averaged, weighted by the number of months each rate was in effect. Due to the lack of uniformity in application, State and local general Sales taxes are not included.

The average sales price (excluding taxes) of finished motor gasoline to end users through company outlets is used, under the assumption that this price most closely approximates retail motor gasoline prices. Finished motor gasoline includes leaded and unleaded motor gasoline and gasohol.

State-level prices that are not available from the $P M A$ are assigned as listed:

\begin{tabular}{lll} 
State & Years & Source \\
\hline AK & $1983-1986$ & CPI \\
CT & 1989 & $P M A$, PAD Subdistrict IA \\
DC & $1983-1989$ & $C P I$ \\
HI & $1983-1986$ & $C P I$ \\
HI & $1987-1989$ & See text below. \\
MD & $1985-1989$ & $C P I$ (Baltimore) \\
ME & $1985-1988$ & PMA, PAD Subdistrict IA \\
SD & 1987 & $P M A$, PAD District II \\
VT & 1989 & $P M A$, PAD Subdistrict IA \\
WY & 1985 & $P M A$, PAD District IV
\end{tabular}

Monthly State tax information and Federal tax information are from Highway Statistics. The monthly State taxes are averaged to create an average annual tax for each State which is combined with the Federal tax to adjust the $P M A$ price.

Monthly CPI city prices weighted by monthly consumption from Highway Statistics for AK/1983-1986, $\mathrm{HI} / 1983-1986$, DC/1983 forward, and MD/1985 for- ward are used to calculate annual prices for those States. $C P I$ prices already include taxes.

$$
\text { . }
$$

Collection of motor gasoline price data for HI by the Bureau of Labor Statistics stopped in December 1986. The HI/1987-1989 prices are derived as follows: the average ratio of the $\mathrm{HI} C P I$ price (with $\mathrm{HI}$ State and Federal taxes subtracted) to the PAD District $V$ price (with HI State and Federal taxes added) for the years 1983-1986 is multiplied by the PAD District V price for the current year and then taxes are added to estimate the $\mathrm{HI} / 1987-1989$ prices.

\section{Phy'sical Unit Prices: 1982}

Monthly physical unit motor gasoline prices for 1982 are from the Platt's Oil Price Handbook and Oilmanac (Platt's) table, "AAA 'Fuel Gauge' Report," the CPI, or both. Table 26 on page 218 summarizes price data availability by source. The Platt's prices are reported for both leaded and unleaded motor gasoline and for both full service and self service for all States except AK and HI. All available Platt's prices for 1982 are used in the calculation of motor gasoline prices. The continuity of these prices with prices published by Platt's in previous years suggests that taxes are included, but this is not explicitly indicated.

The available CPI monthly physical unit motor gasoline prices for 1982 are for all types of motor gasoline and cover 25 States as shown in Table 27 on page 219. The $C P I$ prices are assigned to any State that has a county included in the Standard Metropolitan Statistical Area (SMSA) definitions used by the Bureau of Labor Statistics. These "all types" prices cover leaded regular, unleaded regular, and leaded premium, including taxes. All the available CPI prices for 1982 are also used in the calculation of motor gasoline prices. Complete monthly data exist for the 25 States covered by the CPI. The CPI Detailed Report of April 1986 explicitly states that Federal, State, and local taxes are included.

To combine the product-specific Platt's prices with the "all types" prices published in the CPI, the Platt's prices are weighted into "all types" prices using annual U.S. share data for leaded and unleaded motor gasoline (no breakdowns for regular and premium are possible with current data limitations). The share data come from the Monthly Energy Review (MER).

Motor gasoline price data reported by Platt's for 1982 cover the following months: February, April, June, August, November, and December. The missing 6 months are assigned prices as follows: January is assigned the February price, and the other missing months are assigned the average price of the preceding and succeeding months. A missing February price for $\mathrm{MO}$ is assumed to be equal to the April price, and a missing price for $O R$ is assumed to be equal to the average of the April and August prices. 
Table 26. Summary of Motor Gasoline Price Data by Year, 1970-1982

Years

$1979-1981$

$1979-1981$

1978

1976, 1977

1974,1975

$1970-1973$

\begin{tabular}{ll} 
Source & Grades Covered \\
\hline Platt's & $\begin{array}{l}\text { leaded } \\
\text { unleaded } \\
\text { leaded regular } \\
\text { leaded premium } \\
\text { unleaded regular }\end{array}$ \\
Platt's & $\begin{array}{l}\text { leaded regular } \\
\text { leaded premium } \\
\text { unleaded regular } \\
\text { unleaded premium } \\
\text { leaded regular } \\
\text { leaded premium } \\
\text { unleaded regular }\end{array}$ \\
CPI &
\end{tabular}

Platt's CPI

Platt's

CPI

Platt's

Platt's

\begin{tabular}{l} 
Composite \\
Price \\
\hline no \\
no \\
yes \\
yes \\
yes \\
no \\
no \\
no \\
no \\
yes \\
yes \\
yes
\end{tabular}

leaded regular

leaded regular leaded premium unleaded regular

leaded regular

leaded regular

leaded premium

unleaded regular

leaded regular

leaded regular

leaded premium

leaded regular
Missing States, All Sources

none

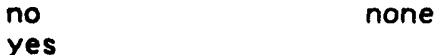

yes

yes

yes

no AK

no

no

no

no

no

no

no

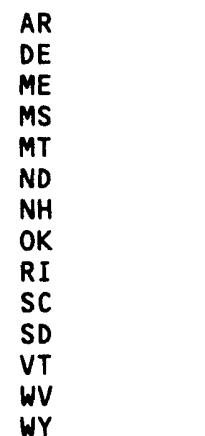

none

AK

$A K, H I$
For States with data from Platt's only, prices by product type (leaded and unleaded) are first calculated as the simple average of full-service and self-service prices for that product for each month and State. The resulting prices are then weighted into monthly composite prices using U.S. leacisd and unleaded shares of motor gasoline product supplied from the $M E R$. The following 26 States have data only frcm Platt's: AL, AR, AZ, CT, DE, IA, ID, LA, ME, MS, MT, NC, ND, NE, NH, NM, NV, OK, RI, SC, SD, TN, UT, VT, WV, and WY.

Platt's reports two prices for each motor gasoline product for each year: one full-service price and one selfservice price. These two prices are combined using a simple average into a single product price for each State for each month.

The unleaded U.S. share of total motor gasoline consumption is reported in the $M E R$ as 52.1 percent in 1982. Assuming that the remaining motor gasoline consumption is leaded, the leaded portion of total consumption is 47.9 percent. These shares are used for ail
States and months to calculate the composite prices from the leaded and unleaded prices.

For AK and HI, the only States with data only from the $C P I$, the all types monthly prices reported are used directly as monthly composite prices.

For States with price data from both Platt's and the $C P I$, the Platt's data are first combined into product type prices and weighted with the $M E R$ shares. The resulting combined prices for all motor gasoline types are averaged together with the combined CPI city prices assigned to the respective month and State. The following 23 States have monthly composite prices computed in this way: CA, CO, DC, FL, GA, IL, IN, KS, KY, MA, MD, MI, MN, MO, NJ, NY, OH, OR, PA, TX, VA, WA, and WI.

1. Leaded and unleaded gasoline prices are calculated as simple averages of full-service and selfservice prices from Platt's, and are then weighted into a composite price using $M E R$ shares of leaded and unleaded motor gasoline consumptiôn. 
2. Monthly "all types" motor gasoline prices covering leaded regular, leaded premium, and unleaded regular are directly from the $C P I$. If there is more than one $C P I$ price observation for a month and State, the CPI prices are simple averages.

3. Using a simple average, the composite Platt's prices are combined with the "all types" CPI prices for that State. The resulting prices are the monthly composite prices for 1982 .

Table 27. Motor Gasoline Price Assignments from Consumer Prices: Energy, 1978-1982

\begin{tabular}{|c|c|}
\hline State & City Price Assignments \\
\hline AK & Anchorage \\
\hline CA & $\begin{array}{l}\text { Los Angeles-Long Beach-Anaheim, } \\
\text { San Diego, San Francisco, Oakland }\end{array}$ \\
\hline $\mathrm{CO}$ & Denver-Boulder \\
\hline DC & Washington \\
\hline FL & Miami \\
\hline GA & Atlanta \\
\hline HI & Honolulu \\
\hline IL & $\begin{array}{l}\text { Chicago-Northwestern Indiana, } \\
\text { St. Louis }\end{array}$ \\
\hline IN & $\begin{array}{l}\text { Chicago-Northwestern Indiana, } \\
\text { Cincinnati }\end{array}$ \\
\hline KS & Kansas City \\
\hline KY & Cincinnati \\
\hline MA & Boston \\
\hline MD & Baltimore, Washington \\
\hline MI & Detroit \\
\hline $\mathrm{MN}$ & Minneapolis-St. Paul \\
\hline MO & St. Louis, Kansas City \\
\hline NJ & $\begin{array}{l}\text { New York-Northeastern NJ, } \\
\text { Philadelphia }\end{array}$ \\
\hline NY & $\begin{array}{l}\text { New York-Northeastern NJ, } \\
\text { Buffalo }\end{array}$ \\
\hline $\mathrm{OH}$ & Cincinnati, Cleveland \\
\hline OR & Portland \\
\hline PA & $\begin{array}{l}\text { Philadelphia, } \\
\text { Northeastern PA, Pittsburgh }\end{array}$ \\
\hline TX & Dallas-Ft. Worth, Houston \\
\hline VA & Washington \\
\hline WA & Seattle-Everett, Portland \\
\hline WI & Milwaukee, Minneapolis-St. Paul \\
\hline
\end{tabular}

Note: All types of motor gasoline are included.

Annual physical unit prices for all States are 6 . culated from the monthly motor gasoline prices calculated above and weighted by the monthly motor gasoline consumption volumes for States from Highway Statistics.
Physical Unit Prices: 1979-1981

For 1979-1981, Platt's monthly motor gasoline prices are from a table titled "Platt's/Lundberg Summary." Prices are available for cities by product type, by grade, and by type of service (full service, self service). Four products and grades of motor gasoline are covered: leaded regular, unleaded regular, leaded premium, and unleaded premium. These data cover 37 States. Although it is not clear whether taxes are included in the prices, the continuity of the series with 1978 prices suggests that taxes are included. The CPI reports "all types" prices, including taxes, for the cities listed in Table 27. Platt's city price assignments to States for 1979-1981 are shown in Table 28.

Table 28. Motor Gasoline Price Assignments from Platt's, 1979-1981

\begin{tabular}{ll}
\hline State & City Price Assignments \\
\hline AL & Birningham \\
AZ & Phoenix, Tucson \\
CA & Bakersfield, Fresno, Los Angeles, \\
& Sacramento, San Diego, \\
& San Francisco, Stockton \\
CO & Denver \\
CT & New Havf,n \\
DC & Washingion \\
FL & Miami, Timpa-St. Petersburg \\
GA & Atlanta \\
IA & Des Moines \\
ID & Boise \\
IL & Chicago \\
IN & Indianapolis \\
KY & Louisville \\
LA & New Orleans \\
MA & Boston \\
MD & Baltimore \\
MI & Detroit \\
MN & Minneapolis \\
MO & Kansas City, St. Louis \\
NC & Charlotte \\
NE & Omaha \\
NJ & Newark \\
NM & Albuquerque \\
NV & Las Vegas, Reno \\
NY & Long Island, Rochester \\
OH & Cincinnati \\
OR & Portland \\
PA & Philadelphia, Pittsburgh \\
TN & Memphis \\
TX & El Paso, Houston \\
UT & Salt Lake City \\
VA & Norfolk \\
WA & Seattle, Spokane \\
WI & Milwaukee \\
\hline &
\end{tabular}


The computation of monthly composite prices for 1979-1981 varies, depending on the available data sources for each State. Monthly composite prices are estimated for the 14 States which do not have reported price data from either data source. If both Platt's and the $C P I$ report prices for a city, the $C P I$ price is used.

1. For States with city price observations only from Platt's, prices for leaded and unleaded motor gasoline are combined using simple averaging, regardless of the type of service, and converted to dollars per gallon. The leaded and unleaded prices are then weighted together into a monthly composite price. The following 12 States have prices only from Platt's for 1979-1981: AL, AZ, CT, IA, ID, LA, NC, NE, NM, NV, TN, and UT.

a. The Platt's prices for 1981 end in September of that year; monthly prices by grade and service type for October, November, and December are assumed to be equal to the corresponding September prices.

b. Leaded and unleaded prices are calculated for each State by simple averaging of all prices available for each product (leaded, unleaded), regardless of service type or grade of motor gasoline (regular, premium). All city prices for each State are averaged together.

c. Leaded and unleaded shares of total motor gasoline consumption for the United States are calculated from the $M E R$ for each year 1979-1981. The monthly product type prices are weighted into composite prices using the national leaded and unleaded shares as weights.

2. For States with city price observations only from the CPI, the monthly "all types" prices are used directly for States with only one price observation per month. For States with multiple observations, monthly prices are combined by simple averaging. States with $C P I$ data only are: $\mathrm{AK}$, CO, DC, GA, HI, IL, KS, MA, MD, MI, MN, $\mathrm{MO}, \mathrm{NJ}, \mathrm{OH}, \mathrm{OR}, \mathrm{PA}$, and WI.

3. For the eight States with price observations from both Platt's and the CPI (CA, FL, IN, KY, NY, TX, VA, and WA), monthly composite prices for 1979-1981 are calculated using three steps:

a. The Platt's prices are combined into single "all types" prices as described above, usin $E$ leaded and unleaded grades of motor gasoline shares as weights.

b. The $C P I$ prices are combined by State.

c. Using simple averaging, the composite Platt's price for each State is combined with the "all types" CPI price for that State. The resulting prices are the monthly composite prices for 1979-1981.
4. Fourteen States are not covered by price data from either Platt's or the CPI in 1979-1981. These States are AR, DE, ME, MS, MT, ND, NH, OK, RI, SC, SD, VT, WV, and WY. Monthly composite prices for these States are estimated using the monthly State-level composite prices for 1982 and Census region monthly prices from the CPI for 1979-1982:

a. The ratio between the 1982 State prices and the 1982 CPI Census region prices corresponding to each State is calculated for use as an adjustment factor in 1979, 1980, and 1981.

b. The monthly price for each of the 14 missing States is assumed to be the product of the 1982 Census region adjustment factor for that State times the monthly motor gasoline price for that Census region from the CPI.

Annual physical unit prices for all States are calculated from the monthly motor gasoline prices calculated above and weighted by the monthly motor gasoline consumption volumes for States from Highway Statistics.

\section{Physical Unit Prices: 1978}

The Platt's monthly leaded regular motor gasoline prices cover all States except AK and HI. The Platt's city assignments to States are shown in Table 29 on page 221 . In 1978, the $C P I$ motor gasoline coverage was expanded from 21 States to 25 States (28 SMSAs) and an "all types" price is published that covers leaded regular, leaded premium, and unleaded regular. The CPI SMSA assignments to States for 1978-1982 are shown in Table 27 on page 219 . Both the $C P I$ and the Platt's prices include taxes.

Since both sources report a single price for each city or SMSA, product weights are not needed to compute monthly composite prices. Instead, city price observations are assigned to States as shown in Table 27 on page 219 and Table 29 on page 221 , and are combined using simple averaging by State and month. If both Platt's and the $C P I$ cover a city/SMSA, the CPI price is used. Platt's prices are converted to dollars per gallon; the CPI prices are already expressed in dollars. All States are covered by the data sources, so no imputation is required for 1978 . The following 26 States have prices only from Platt's: AL, AR, AZ, CT, DE, IA, ID, LA, ME, MS, MT, NC, ND, NE, NH, NM, NV, OK, RI, SC, SD, TN, UT, VT, WV, and WY. The following 19 States are covered only by the CPI: AK, CA, CO, DC, FL, GA, HI, IL, MA, MD, MI, MN, MO, NJ, NY, OH, OR, PA, and WI. Six States have price data from both sources: IN, KS, KY, TX, VA, and WA.

Annual physical unit prices for all States are calculated from the monthly motor gasoline prices calculated 
Table 29. Motor Gasoline Price Assignments from Platt's, 1970-1978

\begin{tabular}{ll}
\hline State & City Price Assignments \\
\hline AL & Birmingham \\
AR & Little Rock \\
AZ & Phoenix \\
CA & Los Angeles, San Francisco \\
CO & Denver \\
CT & Hartford \\
DC & Washington \\
DE & Wilmington \\
FL & Miami \\
GA & Atlanta \\
IA & Des Moines \\
ID & Boise \\
IL & Chicago \\
IN & Indianapolis \\
KS & Wichita \\
KY & Louisville \\
LA & New Orleans \\
MA & Boston \\
MD & Baltimore \\
ME & Portland (ME) \\
MI & Detroit \\
MN & Twin Cities \\
MO & St. Louis \\
MS & Jackson \\
MT & Great Falls \\
NC & Charlotte \\
ND & Fargo \\
NE & Omaha \\
NH & Manchester \\
NJ & Newark \\
NM & Albuquerque \\
NV & Reno \\
NY & Buffalo, New York \\
OH & Cincinnati, Cleveland \\
OK & Tulsa \\
OR & Portland (OR) \\
PA & Philadelphia \\
RI & Providence \\
SC & Charleston (SC) \\
SD & Huron \\
TN & Memphis \\
TX & Dallas, El Paso, Houston \\
UT & Salt Lake City \\
VA & Norfolk \\
VT & Burlington \\
WA & Seattle, Spokane \\
WI & Milwaukee \\
WV & Charleston (WV) \\
WY & Cheyennie \\
\hline & \\
&
\end{tabular}

above and weighted by the monthly motor gasoline consumption volumes for States from Highway Statistics.
The calculation of monthly composite State prices for 1976 and 1977 depends upon the source of data; different procedures are used for States with only Platt's data, States with only CPI data, and States with both Platt's and CPI data. If both data sources cover a city, the $C P I$ price is used. City price assignments to States are given in Table 29 for Platt's and in Table 30 for the CPI. Prices from both sources include taxes. AK is the only State for which prices need to be estimated.

\section{Table 30. Motor Gasoline Price Assignments from Consunver Prices: Energy, 1974-1977}

\begin{tabular}{ll}
\hline State & City Price Assignments \\
\hline CA & Los Angeles-Long Beach, San Diego, \\
& San Francisco-Oakland \\
DC & Washington \\
GA & Atlanta \\
HI & Honolulu \\
IL & Chicago, St. Louis \\
IN & Cincinnati, Chicago \\
KS & Kansas City \\
KY & Cincinnati \\
MA & Boston \\
MD & Baltimore, Washington \\
MI & Detroit \\
MN & Minneapolis-St. Paul \\
MO & St. Louis, Kansas City \\
NJ & New York-Northeastern NJ, \\
& Philadelphia \\
NY & Buffalo, New York-Northeastern NJ \\
OH & Cincinnati, Cleveland \\
PA & Philadelphia, Pittsburgh \\
TX & Dallas, Houston \\
VA & Washington \\
WA & Seattle \\
WI & Milwaukee, Minneapolis-St. Paul \\
\hline
\end{tabular}

Note: Prices are available separately for leaded regular, leaded premium, and unleaded regular (1976, 1977); "all types" prices are not available.

For States with data from Platt's only, the monthly prices reported in Platt's are either used directly or combined using simple averaging if there is more than one price observation for a State in a given month. The reported prices in cents per gallon are converted to dollars per gallon. Prices for the following 29 States are calculated using this procedure and cover only leaded regular motor gasoline: $\mathrm{AL}, \mathrm{AR}, \mathrm{AZ}, \mathrm{CO}, \mathrm{CT}$, DE, FL, IA, ID, LA, ME, MS, MT, NC, ND, NE, NH, NM, NV, OK, OR, RI, SC, SD, TN, UT, VT, $W V$, and WY.

If State-level motor gasoline prices for 1976 and 1977 are available only from the CPI, monthly composite 
prices are calculated as weighted averages of leaded and unleaded prices. Prices for 15 States are calculated using data only from the CPI: CA, DC, GA, HI, IL, MA, MD, MI, MN, MO, NJ, NY, OH, PA, and WI.

1. The weights used in this process are nationallevel shares of leaded and unleaded motor gasoline product supplied. For 1977, the leaded and unleaded share of 0.725 and 0.275 , respectively, are from the MER. For 1976, MER data for 1977-1984 are used to estimate the unleaded share using simple regression. The unleaded percentages for 1977-1984 are converted to shares and used to estimate leaded and unleaded shares of motor gasoline. The resulting 1976 leaded share is 0.744 and the unleaded share is 0.256 .

2. The next step is to calculate monthly composite leaded and unleaded prices for each State. If more than one $C F I$ price observation is available for a particular grade of motor gasoline (leaded or unleaded) for a State in a given month, the $C P I$ observations are combined by grade using simple averaging. Regular and premium prices are averaged for an estimate of State-level leaded prices.

3. Final monthly composite prices for 1976 and 1977 are calculated using the leaded and unleaded composite prices calculated above and the $M E R$-based leaded and unleaded sharus as volume weights.

For States with price data from both Platt's and the $C P I$, all price observations are averaged together by product type. If both sources report prices for a city, the $C P I$ price is used. Once composite leaded and unleaded prices have been calculated separately for each State, the leaded and unleaded consumption shares are used to weight the product-type prices into the final monthly composite motor gasoline prices. Six States are calculated with data from both Platt's and the CPI: IN, KS, KY, TX, VA, and WA.

1. Monthly leaded composite prices are calculated by combining Platt's prices with the CPI prices for leaded regular and premium motor gasoline by month, since the Platt's prices cover only regular leaded fuel (Table 26 on page 218). If both data sources cover a city, the $C P I$ prices are used.

2. Since the $C P I$ is the only source of unleaded gasoline price data for 1976-1977, monthly unleaded composite prices are calculated from CPI data only.

3. Final monthly composite prices for the six States with price data from both Platt's and the CPI are calculated using annual U.S. leaded and unleaded shares and leaded and unleaded monthly composite prices.

Prices for 1976 and 1977 for AK, the only State not covered by price data from either data source, are es- timated based on the average relationship between the State and the national average price for years in which data are available. The national average price used for these estimations is a simple average of the prices of the 49 States for which data are available in all years (i.e., excluding $\mathrm{AK}$ and $\mathrm{HI}$ for all years). Annual prices for AK are estimated based on the average AK-to-U.S. price relationship for 1978 and 1979.

Annual physical unit prices (excluding AK) are calculated from the monthly motor gasoline prices calculated above and weighted by the monthly motor gasoline consumption volumes for States from Highway Statistics.

\section{Physical Unit Prices: 1974, 1975}

The Platt's price data for 1974-1975 cover only leaded regular motor gasoline. Beginning in 1974, motor gasoline price data are also available from the CPI for selected SMSAs. An SMSA price is assigned to each State with courities included in the definition of that SMSA; for the years 1974-197?, prices for 23 SMSAs cover 21 States. The State assignments of SMSA prices for 1974-1977 are given in Table 30 on page 221. For 1974 and 1975, CPI prices are reported separately for leaded regular and leaded premium motor gasoline. According to the CPI Detailed Report, April 1986, these prices include taxes; the Platt's prices also include taxes. AK is the only State not covered by either of these two data sources; prices for AK are estimated for 1974 and 1975.

The Platt's regular leaded prices and the $C P I$ regular and premium leaded motor gasoline prices, including taxes, are assigned to their respective States as shown in Table 29 on page 221 and Table 30 on page 221. If both sources cover a city, the $C P I$ price is used. The following 29 States are covered only by Platt's: AL, AR, AZ, CO, CT, DE, FL, IA, ID, LA, ME, MS, MT, NC, ND, NE, NH, NM, NV, OK, OR, RI, SC, SD, TN, UT, VT, WV, and WY. The following 15 States are covered only by CPI: CA, DC, GA, HI, IL, MA, MD, MI, MN, MO, NJ, NY, OH, PA, and WI. The following six States have both Platt's and CPI data for the cities within these States: IN, KS, KY, TX, VA, and WA.

All price observations in a State, regardless of grade or data source, are added together and divided by the number of observations. As part of this calculation, Platt's prices are converted from cents per gallon to dollars per gallon.

Neither Platt's nor the CPI reports price data for AK. The methodology of the estimation of annual AK prices is the same as used in 1976 and 1977.

Annual physical unit prices for the remaining 50 States (excluding $\mathrm{AK}$ ) are calculated from the monthly motor gasoline prices calculated above and weighted by the 
monthly motor gasoline consumption volumes for States from Highway Statistics.

\section{Physical Unit Prices: 1970-1973}

Monthly motor gasoline physical unit prices for 1970-1973 are available only from Platt's, where city prices covering 49 States are reported. These prices, as shown in Table 26 on page 218 , are for leaded regular motor gasoline only and include taxes.

Monthly average city prices from Platt's are assigned to the State in which the city is located. Platt's city price assignments to States are given in Table 29 on page 221 .

Monthly composite prices for 1970-1973 are equal to the reported monthly Platt's prices or, if more than one city is available for a given State in a certain month, a simple average of the assigned city prices. The reported prices are converted from cents to dollars per gallon.

Platt's does not report data for either AK or $\mathrm{HI}$ for 1970-1973. The methodology of the estimation of AK and HI prices is the same as used for 1976 and 1977.

Annual physical unit prices (excluding $\mathrm{AK}$ and $\mathrm{HI}$ ) are calculated from the monthly motor gasoline prices weighted by the monthly motor gasoline consumption volumes for States from Highway Statistics.

\section{Btu Prices: All Years}

Btu prices for States are computed by converting the physical unit prices in dollars per gallon to dollars per barrel (42 gallons per barrel) and, then, to dollars per million Btu (5.253 million Btu per barrel). U.S. Btu prices are calculated as the average of the State Btu prices, weighted by consumption data from SEDS.

\section{Data Sources}

\section{Prices}

1986 forward: Energy Information Administration, Petroleum Marketing Annual, Table 29 (1986-1988) and Table 30 (1989), column titled, “All Refiners, Sales to End Users, Through Company Outlets."

1983-1985: Energy Information Administration, Petroleum Marketing Annual 1985, Volume 1, Table 16, column titled, "All Refiners and Gas Plant Operators, Sales to End-Users, Through Company Outlets."

1974 forward: Bureau of Labor Statistics, U.S. Department of Labor, Consumer Prices: Energy, computer printouts of monthly gasoline prices.
1970-1982: McGraw-Hill, Inc., Platt's Oil Price Handbook and Oilmanac; table titled "AAA 'Fuel-gauge' Report" (1982); table titled, "Platt's/Lundberg Summary," (1979-1981); and table titled, "Service Station Prices: Gasoline (Including Taxes)," (1970-1978).

1974-1982: Bureau of Labor Statistics, CPI Detailed Report, April 1986, Technical Notes, page 110.

1982: Energy Information Administration, Form EIA-25 "Prime Supplier Monthly Report" computer tape, unpublished data.

1976-1984: Energy Information Administration, Monthly Energy Review, January 1985, table titled, "Petroleum: Finished Motor Gasoline Supply and Disposition."

\section{Taxes}

1970 forward: Federal Highway Administration, U.S. Department of Transportation, Highway Statistics, Tables MF-26 (1973 forward); MF-121T for State taxes (1983 forward); FE-101 for Federal taxes (1983 forward); and MF-23 and MF-25 (1970-1972).

\section{Consumption}

1970 forward: Energy Information Administration, State Energy Data System, transportation sector, motor gasoline consumption.

\section{Conversion Factor: All Years}

5.253 million Btu per barrel 


\section{Petroleum Coke (Utilities)}

Petroleum coke is consumed by the electric utility and industrial sectors. The portion of petroleum coke consumed by the industrial sector ( 98 percent in 1989) is included in the category "other petroleum products," which is discussed in a subsequent section. The remaining petroleum coke is used for electricity generation in 12 States over the period 1970 forward. A maximum of seven States reported consumption in any single year. Estimates of the annual consumption of petroleum coke by electric utilities are from SEDS. Estimates of the deliveries of petroleum coke, used in the price calculation described below, are from the Cost and Quality of Fuels for Electric Utility Plants $(C \& Q)$. The $C \& Q$ prices are the delivered costs of the fuels and include all taxes, transportation, and other charges paid by the utilities.

\section{Btu Prices: 1972 Forward}

The principal data source for petroleum coke used by electric utilities is an EIA public use computer tape covering the period 1972 forward. From 1972-1982, steam plants with a maximum capacity of 25 megawatts were included. For 1983 and subsequent years, the reporting threshold was raised to 50 megawatts capacity. In addition to the computer tape, the data are also published, but not for the full period. From 1978-1982, Cost and Quality of Fuels for Electric Utility Plants was published monthly and annually; data for calculating petroleum coke prices are in only the monthly reports. For 1983 and subsequent years, $C \& Q$ was published annually and includes petroleum coke prices for individual States and for the Nation.

The computer tape, Cost and Quality of Fuels for Electric Utility Plants (Form 423) Historic Files, shows quantity in short tons, estimated Btu per pound, and price in dollars per million Btu. The data are presented by plant, by State, and by month. The Btu price is calculated as the annual sum of the unit prices, weighted by the total Btu in each reported delivery, divided by the annual sum of the Btu delivered to all electric utility plants within the State. The States and years listed below have prices assigned as follows:

\begin{tabular}{lll} 
State & Years & Assigned Prices \\
\hline DE & 1981 forward & PA \\
KS & 1975 & MO \\
MO & 1983,1985 & MN \\
NY & 1974 & PA \\
WI & 1985 & MN
\end{tabular}

The high DE prices prior to 1981 are actual reported prices.
Btu Prices: 1970, 1971

For the years 1970 and 1971, prices are estimated using the Gross National Product Implicit Price Deflator. The deflator for 1970 or 1971 is divided by the 1972 deflator and the quotient is multiplied by the 1972 price for each State to develop the price estimates for 1970 and 1971. The deflators are 42.0 in 1970, 44.4 in 1971, and 46.5 in 1972.

Although SEDS has a consumption estimate for $\mathrm{NJ}$ in 1971, there are no NJ price data for any year in the Form 423 Historic Files. Form 423 data for PA in 1972 are used to estimate a PA price for 1971, which is assigned to NJ. The Form 423 PA prices for 1972 and 1971 do no appear in SEPEDS since SEDS has no petroleum coke consumption in PA for those years.

\section{U.S. Btu Prices: All Years}

U.S. Btu prices are calculated as the average of the State Btu prices, weighted by consumption data from SEDS.

\section{Data Sources}

\section{Prices}

1972 forward: Energy Information Administration, Public Use Tape, "Cost and Quality of Fuels for Electric Utility Plants (Form 423) Historic Files," as published in the following reports:

- 1983 forward: Energy Information Administration, Cost and Quality of Fuels for Electric Utility Plants, Table 20 (1983, 1984), Table $12(1985$ forward).

- 1978-1982: Energy Information Administration, Cost and Quality of Fuels for Electric Utility Plants, table titled, "Wood Chips, Refuse, and Petroleum Coke Used as Fuel by Steam-Electric Units."

1970-1972: Energy Information Administration, Annual Energy Review 1989, Appendix C. GNP Dollars and Deflators.

\section{Consumption}

1970 forward: Energy Information Administration, State Energy Data System, electric utility petroleum coke consumption.

\section{Conversion Factors: All Years}

No conversion factors are required; Blu prices are cal. culated directly from data sources. 
Residual fuel prices are developed for the electric utility, industrial, commercial, and transportation sectors. Estimates of the amount of residual fuel consumed by sector are from SEDS and are adjusted for process fuel consumption in the industrial sector. See the "Consumption Adjustments for Process Fuel and Intermediate Products" section in this documentation.

\section{Electric Utility Sector}

The electric utility price for residual fuel (heavy oil) is the average delivered cost of No. 6 fuel oil receipts at electric utilities. Prices include taxes. For 1973 forward, Btu prices are developed directly from the data sources. For 1970-1972, prices are estimated using simple regression analysis. All taxes, transportation, and other charges paid by utilities are included in the prices.

\section{Btu Prices: 1973 Forward}

Electric utility sector residual fuel prices for 1973 forward are from Cost and Quality of Fuels for Electric Utility Plants (C\&Q). For 1973-1979, Btu prices are calculated as the weighted average of contract and spct prices for No. 6 fuel oil. Table 31 lists the States and years for which consumption is indicated by SEDS but no price is shown in $C \& Q$. For these States, the Census division price, as shown in $C \& Q$, is assigned as the State price.

For 1983 forward, $C \& Q$ reports prices for steam electric plants of 50 megawatt capacity or greater. For 1980-1982, $C \& Q$ prices cover all reporting plants of 25 megawatt capacity or greater.

AK/1973-1989 and HI/1973-1982 prices are developed using the ratio of the $C \& Q$ U.S. price and the Statistical Yearbook U.S. price multiplied by the Statistical Yearbook State price. AK/1973, 1975, 1978 prices are based on the average ratio of U.S. and AK prices for 1974, 1976 and 1977, and 1979 and 1980. The U.S. prices for 1973, 1975, and 1978, are multiplied by the average ratio for the years that have prices.

\section{Btu Prices: 1970-1972}

State-level Btu prices for 1970-1972 are estimated using regression techniques and price data from the Statistical Yearbook. The regression equations use Statistical Yearbook State-level prices for 1973-1980 as the independent variable and the State-level prices calculated above (including the estimations for $\mathrm{AK}$ and $\mathrm{HI}$ ) as the dependent variable. Pacific regional price averages are
Table 31. Residual Fuel Electric Utility Price Assignments from Census Division Prices, 1970 Forward

\begin{tabular}{ll}
\hline State & Years of Assigned Prices \\
\hline AL & $1975-1979$ \\
AR & 1987 \\
AZ & 1984,1985 \\
CO & $1982,1987,1989$ \\
DC & $1982-1989$ \\
IA & $1970-1985$ \\
IN & $1970-1979$ \\
KS & $1980,1981,1985-1987,1989$ \\
KY & $1970-1979$ \\
MN & $1984,1985,1987-1989$ \\
MT & $1970-1979$ \\
NC & $1976,1977,1979,1980,1982,1984$ \\
ND & $1970-1979$ \\
NE & $1981-1983$ \\
NM & $1979-1982,1989$ \\
NV & 1983,1985 \\
OK & $1977,1978,1980,1982-1987,1989$ \\
OR & $1970,1973,1974$ \\
SC & $1983,1985-1989$ \\
SD & $1981-1988$ \\
TN & 1979 \\
UT & $1982,1983,1986$ \\
VT & $1970-1979$ \\
WA & $1970,1971,1975-1978,1981-1983,1986-1988$ \\
WV & $1970-1977,1979$ \\
WY & $1970-1979$ \\
\hline
\end{tabular}

assigned for the missing WA/1970, 1971 prices. The average of 1970 and 1972 AK Statistical Yearbook prices is substituted for the missing 1971 AK price, and a similar calculation is made for the 1973, 1975, and 1978 prices.

\section{U.S. Btu Prices: All Years}

U.S. Btu prices are calculated as the average of the State Btu prices, weighted by consumption data from SEDS.

\section{Data Sources}

\section{Prices}

1973 forward: Energy Information Administration, Cost and Quality of Fuels for Electric Utility Plants, Table 6(1973-1979), Table 45 (1980-1982), Table 51 (1983, 1984), and Table 41 (1985 forward).

1970 forward: Edison Electric Institute, Statistical Yearbook of the Electric Utility Industry, Table 43 (1970-1979), Table 26 (1980-1983), Table 28 (1984-1986), and Table 29 (1987 forward). 
1970 forward: Energy Information Administration, State Energy Data System, electric utility sector heavy oil consumption.

\section{Conversion Factors: All Years}

Because Btu prices are available directly from the data sources, no conversion factors are used.

\section{Industrial Sector}

The industrial sector residual fuel prices for 1984 forward are developed from refiner/reseller prices of residual fuel as published in the Petroleum Marketing Annual. Residual fuel prices for 1970-1983 are calculated or estimated using average costs of residual fuel to manufacturing firms published in two Bureau of the Census reports and Platt's Oil Price Handbook and Oilmanac. Price data in these sources are available for the years 1971 and 1974-1981; prices for 1970, 1972, 1973, 1982, and 1983 are estimated. Prices for all years include taxes.

\section{Physical Unit Prices: 1984 Forward}

A

Residual fuel industrial sector physical unit prices are calculated using refiner/reseller prices to end users from the Petroleum Marketing Annual (PMA). The States that do not have $P M A$ prices are assigned their PAD district or subdistrict price as shown in Table 32, with the exception of AK. AK industrial residual fuel price for 1984 forward is based on the WA industrial residual fuel price and the ratio of the AK-to-WA industrial distillate fuel prices for each year. Tax data from Bureau of the Census publications are added to develop final sector prices.

Table 32. Residual Fuel Industrial Sector Price Assignments, 1984 Forward

\begin{tabular}{lll}
\hline State & Years & PAD Prices Assigned \\
\hline AR & 1985 & District III \\
AZ & $1984-1989$ & District V \\
CO & 1986,1988 & District IV \\
ID & $1985,1986,1989$ & District IV \\
MS & 1988 & District III \\
ND & 1988,1989 & District II \\
NM & $1984-1986$ & District III \\
NV & 1986,1988 & District V \\
CR & 1989 & District V \\
UT & 1989 & District IV \\
WV & 1984 & Subdistrict IC \\
WY & 1989 & District IV \\
\hline
\end{tabular}

Physical Unit Prices: 1982, 1983

After 1981, the Annual Survey of Manufacturers and the Census of Manufacturers (ASM/CM) ceased publication of fuel-specific State-level residual fuel data from which prices can be calculated. Prices for 1982 and 1983 are estimated from the average relationship between the $A S M / C M$-based prices generated for 1978-1981 and the assigned Platt's No. 6 fuel oil prices for 1978-1981 (Table 33 on page 227). These average ratios are calculated at the State-level for all States except AK, which shows no industrial sector residual fuel use reported in SEDS for 1982 and 1983. Physical unit residual fuel industrial prices for 1982 and 1983 are calculated using the assigned Platt's prices for 1982 and 1983 (Table 33 on page 227) and the State-level average ratios.

\section{Physical Unit Prices: 1971, 1974-1981}

For the years 1971 and 1974-1981, industrial sector residual prices are calculated directly from cost and quantity data reported by the $A S M / C M$. For all States with available cost and quantity data, prices are equal to the average cost of residual fuel to manufacturers. Taxes are included in the published cost data. Missing data for these years are assigned from the average prices of adjacent States as shown below:

\begin{tabular}{lll} 
State & Years & State Prices Used \\
\hline AK & 1980,1981 & HI, WA \\
DC & $1979-1981$ & MD, VA \\
MT & $1974-1979$ & ID, ND, SD \\
ND & 1980 & MN, MT, SD \\
NM & 1971, & AZ, CO, TX \\
& $1974-1981$ & \\
NV & $1974-1978$ & AZ, CA, ID, OR, UT \\
OK & $1974-1978$, & AR, CO, KS, MO, TX \\
& 1980 & \\
SD & 1981 & IA, MN, MT, ND, NE \\
WY & 1971, & CO, NE, UT
\end{tabular}

\section{Physical Unit Prices: 1970, 1972, 1973}

Since $A S M / C M$ data are not available for 1970,1972 , or 1973, prices for these years must be estimated. Physical unit prices are based on the ratio of the $1971 C M$ prices to the 1971 assigned No. 6 fuel oil prices from Platt's Oil Price Handbook and Oilmanac (Table 33 on page 227). The estimated $1971 C M$ prices for NM and WY are used in the calculations. The resulting ratios for each State are used with the Platt's assigned prices for 1970,1972 , and 1973 to estimate prices. 
Table 33. No. 6 Fuel Oil Price Assignments from Platt's, 1970-1983

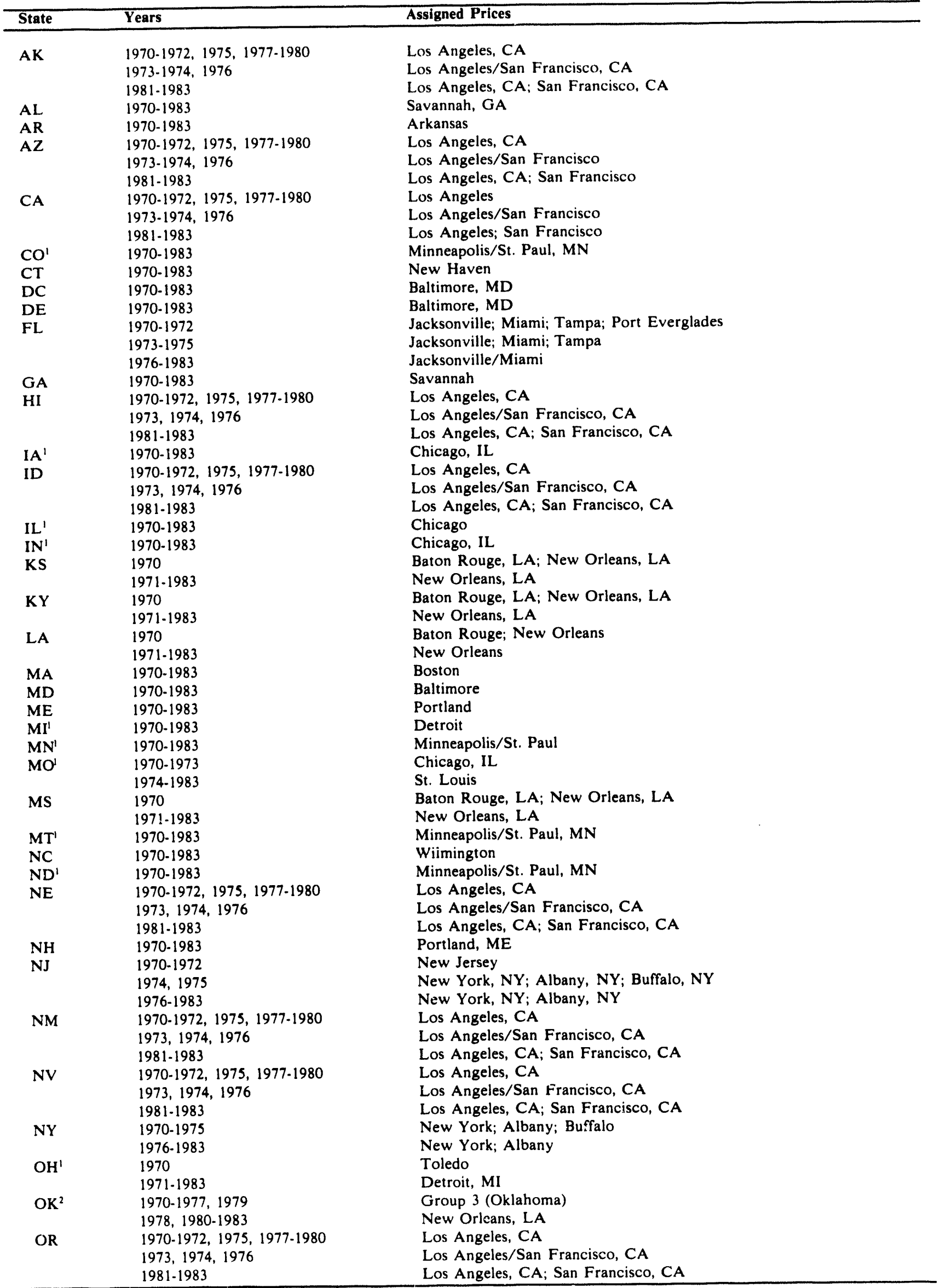

Jacksonville; Miami; Tampa

sonville/Miami

Los Angeles/San Francisco, CA

(San Francisco, CA 


\begin{tabular}{lll}
\hline State & Years & Assigned Prices \\
\hline Continued & $1970-1983$ & Philadelphia \\
PA & $1970-1975$ & Providence \\
RI & $1976-1983$ & New Haven, CT \\
& $1970-1983$ & Charleston \\
SC & $1970-1983$ & Minneapolis/St. Paul, MN \\
SD' & 1970 & Baton Rouge, LA; New Orleans, LA \\
TN & $1971-1983$ & New Orleans, LA \\
& $1970-1972$ & New Mexico/West Texas \\
TX & $1973-1983$ & New Orleans, LA \\
& $1970-1983$ & Minneapolis/St. Paul, MN \\
UT' & $1970-1983$ & Norfolk \\
VA & $1970-1983$ & Portland, ME \\
VT & $1970-1972,1975,1978,1979$ & Los Angeles, CA \\
WA & $1973,1974,1976$ & Los Angeles/San Francisco, CA \\
& $1980-1983$ & Seattle/Tacoma \\
WI' & $1970-1983$ & Chicago, IL \\
WV & $1970-1983$ & Norfolk, VA \\
WY' & $1970-1983$ & Minneapolis/St. Paul, MN \\
\hline
\end{tabular}

'Raw data from Platt's must be converted from cents per gallon to dollars per barrel.

${ }^{2}$ As shown in Platt's.

\section{Btu Prices: Al/ Years}

Btu prices for States are calculated from the physical unit prices and the conversion factor. U.S. Btu prices are calculated as the average of the State Btu prices, weighted by consumption data from SEDS, adjusted for process fuel consumption.

\section{Data Sources}

\section{Prices}

1984 forward: Energy Information Administration, Petroleum Marketing Annual 1989, Table A4.

1984 forward: Industrial sector distillate fuel price estimates (in physical units) from the SEPER data system (AK and WA only).

1970-1983: McGraw-Hill, Inc., Platt's Oil Price Handbook and Oilmanac, refinery and terminal prices for No. 6 fuel oil, average of highs and lows.

1971, 1977, 1981: Bureau of the Census, U.S. Department of Commerce, Census of Manufactures, Fuels and Electric Energy Consumed, Part 2, Table 3. (Dates shown on the report covers are, respectively, 1972, 1977, and 1982.)

1974-1976 and 1978-1980: Bureau of the Census, U.S. Department of Commerce, Annual Survey of Manufactures, Fuels and Electric Energy Consumed, States by Industry Group. Table 3.

\section{Taxes}

1987 forward: Bureau of the Census, U.S. Department of Commerce, State Government Tax Collections, Table 8 , column titled, "Percentage rate, September 1."
1984-1986: Bureau of the Census, U.S. Department of Commerce, Statistical Abstract of the United States, table titled, "State Government Tax Collections and Excise Taxes," column titled, "Excise Taxes, General sales and gross receipts."

\section{Consumption}

1970 forward: Energy Information Administration, State Energy Data System, industrial sector residual consumption.

\section{Conversion Factor: All Years}

6.287 million Btu per barrel

\section{Commercial Sector}

For 1984 forward, State-level commercial sector residual fuel prices are developed from refiner/reseller prices of residual fuel to end users published in the Petroleum Marketing Annual. For 1970-1983, commercial sector residual fuel prices are estimated for all States from national-level residual fuel prices and the State-level electric utility sector residual fuel prices. State and Federal taxes are included in the final prices for all years.

\section{Physical Unit Prices: 1984 Forward}

Commerical sector residual fuel physical unit prices are calculated using refiner/reseller prices to end users from the Petroleum Marketing Annual (PMA). The 
States that do not have $P M A$ prices are assigned their PAD district or subdistrict price as shown in Table 34, with the exception of $\mathbf{A K}$. AK commercial residual fuel price for 1984 forward is based on the WA commerical residual fuel price and the ratio of the AKto-WA commercial distillate fuel prices for each year. Tax data from Bureau of the Census publications are added to develop final sector prices.

\section{Physical Unit Prices: 1976-1983}

The commercial sector residual fuel physical unit prices for 1976-1983 are estimated from the electric utility sector residual prices for corresponding years and the tax-adjusted U.S. average retail residual fuel prices.

1. The first step in the estimation of the commercial residual fuel physical unit State prices is to convert the State-level excise tax rates reported in the Bureau of the Census publications into the volume-weighted average U.S. sales tax rate using commercial residual consumption data from SEDS.

2. A preliminary U.S. residual fuel oil price, including taxes, is computed using the average U.S. tax rate estimated above and the annual average U.S. residual fuel price to end users (average retail price excluding taxes) from the Monthly Energy Review (MER).

3. Commercial sector physical unit residual fuel prices for States are computed using the electric utility sector residual fuel prices. To do this calculation, the ratio of the State-level and U.S. prices in the commercial sector is assumed to be the same as the ratio of State and U.S. prices in the electric utility sector. Some States are missing electric utility prices for 1976-1983; these are estimated using adjacent States' average prices as listed in Table 35.

Table 34. Residual Fuel Commercial Sector Price Assignments, 1984 Forward

\begin{tabular}{lll}
\hline State & Years & PAD Prices Assigned \\
\hline AZ & $1984,1985,1988$ & District V \\
CO & 1986 & District IV \\
ID & $1985,1986,1989$ & District IV \\
MS & 1988 & District III \\
ND & 1988,1989 & District II \\
NM & 1984,1985 & District III \\
NV & 1986,1988 & District V \\
OR & 1989 & District V \\
UT & 1989 & District IV \\
WV & 1984 & Subdistrict IC \\
WY & 1989 & District IV \\
\hline
\end{tabular}

Table 35. Resldual Fuel Commercial Sector Price Assignments, 1970-1983

\begin{tabular}{lll}
\hline State & Years & $\begin{array}{l}\text { State Prices Used } \\
\text { in the Estimation }\end{array}$ \\
\hline AL & $1970-1974,1980$, & FL, GA, MS \\
& 1982,1983 & \\
ID & $1980,1981,1983$ & CA, CO \\
& 1982 & CA \\
IN & $1980-1983$ & IL, MI, OH \\
MT & $1980,1981,1983$ & CO, MN \\
& 1982 & MN \\
NC & 1981,1983 & GA, VA \\
ND & 1980,1983 & MN, SD \\
& 1981,1982 & MN \\
OR & $1975-1983$ & CA \\
SC & 1984 & GA \\
TN & $1970-1978$, & AR, GA, MO, MS, VA \\
& $1980-1983$ & \\
VT & $1980-1983$ & ME, NH, NY \\
Wi & 1982,1983 & IL, MI, MN \\
WV & $1980-1983$ & MD, OH, PA, VA \\
WY & 1980 & CO, NE, SD, UT \\
& 1981,1983 & CO \\
& 1982 & MN \\
\hline
\end{tabular}

\section{Physical Unit Prices: 1970-1975}

Since no national or State-level retail residual prices are available from published data sources, commercial sector residual prices for 1970-1975 are estimated. The estimation method is based on the assumption that the average ratio of State-to-U.S. prices is the same in the commercial and electric utility sectors. The average ratio for 1976-1979 of the MER U.S. tax-adjusted prices to the electric utility sector U.S. prices is calculated and used as an adjustment factor with State-level electric utility sector prices for 1970-1975.

1. The average ratio of the $M E R$ tax-adjusted U.S. prices and the electric utility sector U.S. prices is calculated for 1976-1979.

2. State-level commercial sector residual fuel prices are calculated using the electric utility sector physical unit price series for 1970-1975 and the average ratio computed above. Price assignments for States missing electric utility sector data are shown in Table 35.

\section{Btu Prices: All Years}

Btu prices for States are calculated from the physical unit prices and the conversion factor. U.S. Btu prices are calculated as the average of the State Btu prices, weighted by consumption data from SEDS. 


\section{Data Sources}

Prices

1984 forward: Energy Information Administration, Petroleum Marketing Annual 1989, Table A4.

1984 forward: Commercial sector distillate fuel price estimates (in physical units) from the SEPER data system (AK and WA only).

1978-1983: Energy Information Administration, Monthly Energy Review, December 1988, table titled, "Refiner Sales Prices of Residual Fuel Oil," column Average Sales to End Users.

1976, 1977: Energy Information Administration, Monthly Energy Review, December 1983, table titled, "Average No. 6 Residual Fuel Oil Prices," column Average, Retail.

1970-1983: Electric utility sector residual fuel price estimates (in physical units) from the $S E P E R$ data system.

\section{Taxes}

1987 forward: Bureau of the Census, U.S. Department of Commerce, State Government Tax Collections, Table 8 , column titled, "Percentage rate, September 1."

1976-1986: Bureau of the Census, U.S. Department of Commerce, Statistical Abstract of the United States, table titled, "State Government Tax Collections and Excise Taxes," column titled, "Excise Taxes, General sales and gross receipts."

\section{Consumption}

1970 forward: Energy Information Administration, State Energy Data System, commercial sector residual fuel consumption.

\section{Conversion Factor: All Years}

6.287 million Btu per barrel

\section{Transportation Sector}

Residual fuel is consumed in the transportation sector for vessel bunkering, military use, and railroads. In 1970 , vessels consumed 74 percent of the transportation use of residual fuel, and the military and railroads accounted for 24 percent and 2 percent, respectively. By 1989 , vessel use had grown to 98 percent, military use had dropped to 2 percent, and the railroads' share was insignificant. Prices are developed for vessel bunkering, and electric utility prices are assigned to the military and railroad uses. Tax adjustments are made as described below. The transportation sector average price for each State and year is the consumptionweighted average of the prices of the three uses.

\section{Physical Unit Prices: All Years}

Vessel Bunkering. Physical unit prices are calculated from actual or estimated U.S. bunker $C$ prices and corresponding residual fuel electric utility sector State and U.S. prices. The ratio of U.S. bunker C price to U.S. residual fuel electric utility sector price is multiplied by the State residual fuel electric utility sector price to obtain the State bunker C price. Taxes are calculated as described for the commercial sector (1976-1983) and added, so that final State vessel bunkering price estimates include taxes. Other procedures are described separately by groups of years.

1. For 1982 forward, national average prices for residual fuel with sulfur content greater than 1 percent are from the Annual Energy Review and used as proxies for bunker $\mathrm{C}$ prices.

2. For 1975-1981, national average bunker C prices are available from the Monthly Petroleum Product Price Report (MPPPR). Annual average U.S. prices for 1975 and 1976 are calculated as the simple average of the monthly prices for each respective year because annual average prices are not shown in the MPPPR.

3. For 1970-1974, no U.S. bunker C prices are available. To estimate State-level prices for these years, the average ratio of published bunker $C$ prices and electric utility sector prices for 1975-1979 is calculated and multiplied by the State-level electric utility prices for 1970-1974.

Missing State prices are assigned adjacent States' average prices as shown in Table 36 on page 231 .

Military and Railroad Use. For all years, electric utility sector residual fuel prices are assigned to military and railroad uses. The electric utility prices include taxes. Since the military does not pay State taxes, their prices are adjusted to remove taxes. Estimation of the tax adjustment is described under the commercial sector.

In some cases States have no reported consumption of residual fuel oil in the electric utility sector and, therefore, no final price in that sector. Since the electric utilities sector prices are used as a base in the calculation of prices in the other sectors, intermediate prices are calculated for OR in 1971 and for all States that need prices in 1987 forward.

Average Prices. Transportation sector prices are the average of bunker fuel, military, and railroad prices, weighted by each category's share of total transportation deliveries or sales as developed in SEDS. 
Table 36. Residual Fuel Transportation Sector Price Assignments, 1970-1986

\begin{tabular}{lll}
\hline State & Years & $\begin{array}{l}\text { State Prices Used } \\
\text { in the Estimation }\end{array}$ \\
\hline AL & $1970-1974$, & FL, GA, MS \\
CT & $1981-1986$ & NH, VT \\
GA & 1978 & KY, MS \\
IL & 1978 & IA, IN, WI \\
IN & $1980-1986$ & IL, MI, OH \\
KS & 1975 & MO, NE \\
MD & 1978 & DE, PA \\
ME & 1975 & VT \\
MN & 1986 & IL, MI \\
NC & 1975 & GA \\
& 1978 & KY \\
& 1981,1983, & GA, VA \\
OH & 1985,1986 & IN, MI \\
OR & 1975 & CA \\
SC & $1975-1986$ & GA \\
& 1978,1984 & AL, FL \\
TN & 1981,1982 & AR, GA, MO, MS, VA \\
VA & 1975 & GA \\
WA & 1984,1985 & CA \\
WI & $1982-1984$ & IL, MI, MN \\
& 1986 & IL, MI \\
\hline
\end{tabular}

\section{Btu Prices: All Years}

Btu prices for States are calculated from the physical unit prices and the residual fuel conversion factor. U.S. Btu prices are calculated as the average of the State Btu prices, weighted by consumption data from SEDS.

\section{Data Sources}

\section{Prices}

1982 forward: Energy Information Administration, $A$ nual Energy Review 1990, Table 71, row titled, "Sales Prices to End Users, Residual Fuel Oil, Greater Than 1 Percent Sulfur Content."

1976-1981: Energy Information Administration, Monthly Petroleum Product Price Report, Table 3.

1975: National Energy Information Center, Federal Energy Administration, Monthly Petroleum Product Price Report, Table 3.

1970-1986: Electric utility sector residual fue! price estimates (in physical units) from SEPEDS.

\section{Taxes}

1987 forward: Bureau of the Census, U.S. Department of Commerce, State Government Tax Collections, Table 8, column titled, "Percentage rate, September 1."

1976-1986: Bureau of the Census, U.S. Department of Commerce, Statistical Abstract of the United States, table titled, "State Government Tax Collections and Excise Taxes," column titled, "Excise Taxes, General sales and gross receipts."

\section{Consumption}

1970 forward: Energy Information Administration, State Energy Data System, transportation sector residual fuel consumption and transportation sector deliveries including the subcategories for vessel bunkering, military, and railroad uses.

\section{Conversion Factor: All Years}

6.287 million Btu per barrel 


\section{Other Petroleum}

Sixteen separate products are included in the category called other petroleum:

1. Aviation gasoline blendirg components

2. Crude oil

3. Miscellaneous products $(*)$

4. Motor gasoline blending components

5. Natural gasoline, including isopentane (1970-1983)

6. Pentanes plus (1984 forward)

7. Petrochemical feedstocks, naphtha (*)

8. Petrochemical feedstocks, other oils $\left({ }^{*}\right)$

9. Petrochemical feedstocks, still gas (1970-1985) $\left(^{*}\right)$

10. Petroleum coke $\left(^{*}\right)$

11. Plant condensate (1970-1983)

12. Special naphthas $(*)$

13. Still gas

14. Unfinished oils

15. Unfractionated stream (1970-1983)

16. Waxes $\left(^{*}\right)$

\section{Physical Unit Prices: All Years}

Of the 16 products, national-level prices are de 'eloped for the 7 noted with asterisks $\left({ }^{*}\right)$ and described below are assigned to the industrial sector. Only national-level prices are developed because State-level price information is not available, and taxes are not included in any of the estimates. Expenditures for the other nine products are complectly removed as process fuel or intermediate products. See the "Consumption Adjustments for Process Fuel and Intermediate Products" section of this documentation.

Starting in 1984, three products--natural gasoline, plant condensate, and unfractionated stream--are dropped, and pentanes plus is added in the Energy Information Administration (EIA) reporting system. Natural gasoline (including isopentane) and plant condensate are reported together as the new product, pentanes plus. Unfractionated stream is dropped because its components are reported separately as liquefied petroleum gases.

\section{Miscellaneous Products}

The products in this subcategory vary $\mathrm{f}$ om the inexpensive (absorption oils similar to kerose ne) to the very expensive (hydraulic fluids). The prir z estimates are based on the evidence presented in th: Minerals Yearbooks of the 1970's indicating that the greater part of the miscellaneous product line consists of finished pet- rockemicals, especially the aromatic hydrocarbons: benzene, toluene, and the xylenes.

Price estimates for 1972, 1977, 1982, and 1987 are from Census of Manufactures (CM) data on quantity and value of aromatics (product codes 2911054 and 2911056) and "other finished petroleum products" (product code 2911098) shipped by industry 2911. The ratio of miscellaneous-products-to-crude-oil price for these 4 years varies widely. Specifically, the ratio equals 1.90 for $1972,2.15$ for $1977,1.56$ for 1982 , and 1.99 for 1987 . Thus, to estimate miscellaneous products prices, the following ratios are used:

1970 - 1974: 1.90 times the crude-oil price 1975 - 1979: 2.15 times the crude-oil price 1980 - 1984: 1.56 times the crude-oil price. 1985 forward: 1.99 times the crude-oil price.

\section{Price Data Sources}

1970 forward: Energy Information Administration, $A n$ nual Energy Review 1990, Table 70, "Crude Oil Refiner Acquisition Cost," column titled, "Composite, Nominal."

1972, 1977, 1982, 1987: Bureau of the Census, U.S. Department of Commerce, Census of Manufactures, data for industry 2911 on "Quantity and Value of Shipments by All Producers" as shown in Table 6a from MC77. I-29A, Product Codes 2911054, 2911056, and 2911098 (1972 and 1977); and Table 6a from MC87-I-29A, Product Codes 2911D55 and 2911D57 (1982 and 1987).

\section{Petrochemical Feedstocks, Naphtha}

Napthas for petrochemical feedstock use are those oils with boiling points less than $401^{\circ} \mathrm{F}$. Consumer prices for 1978-1980 are derived from the special Annual Survey of Manufactures (ASM) series on "Hydrocarbon, Coal, and Coke Materials Consumed," using data for industries 2869 (industrial crganic chemicals) and 2821 (plastics materials, synthetic resins, and nonvulcanizable elastomers). A price estimate for 1982 is obtained from the $C M$, based on data for industry 2869 only. Since the ratio of petrochemicalnaphtha-to-crude-oil price is reasonably constant in $1978,1979,1980$, and 1982, the simple average of the four ratios, 1.23 , is used to estimate prices for petrochemical feedstocks, naphthas, for all other years.

\section{Price Data Sources}

1970-1977, 1981, 1983 forward: Energy Information Administration, Annual Energy Review 1990. Table 70, "Crude Oil Refiner Acquisition Cost," column i tled, "Composite, Nominal."

1982: Bureau of the Census, U.S. Department of Commerce, 1982 Census of Manufactures, $M 82-1-28 F-3(P)$, nage 6 , industry 2869 . 
1980: Bureau of the Census, U.S. Department of Commerce, 1980 Annual Survey of Manufactures, M80(AS) -4.3, page 9 , industry 2821 .

1978, 1979: Bureau of the Census, U.S. Department of Commerce, 1979 Annual Survey of Manufactures, M79(AS) - 4.3, page 8, industries 2821 and 2869

\section{Petrochemical Feedstocks, Other Oils}

Petrochemical feedstocks referred to as "other oils" or "gas oils" are those oils with boiling points equal to or greater than $401^{\circ} \mathrm{F}$. Consumer prices for 3 years are obtained from the data on gas oils presented in the special $A S M$ series on hydrocarbons consumed, using data on industry 2865 (cyclic crudes and intermediates). The other-oils-to-crude-oil price ratio is quite stable, and the average ratio for the 3-year period, 1.607 , is used to estimate prices for petrochemical feedstocks, other oils, for all other years.

\section{Price Data Sources}

1970-1977, 1981 forward: Energy Information Administration, Annual Energy Review 1990, Table 70, "Crude Oil Refiner Acquisition Cost," column titled, "Composite, Nominal."

1979, 1980: Bureau of the Census, U.S. Department of Commerce, 1980 Annual Survey of Manufactures, M80(AS)-4.3, pag - 9, industry 2865.

1978: Bureau of the Census, U.S. Department of Commerce, 1979 Annual Survey of Manufactures, M79(AS)-4.3, page 8, industry 2865.

\section{Petrochemical Feedstocks, Still Gas (1970-1985)}

The source data for still gas is a mixture of consumer prices and producer prices for industries 2869 and 2911 (petroleum refining). The still-gas-to-crude-oil price ratio is somewhat variable because still gas is a highly variable gaseous mixture. Value and quantity are available for 1972, 1977-1980, and 1982. In imputing prices for years when data from the $C M$ or $A S M$ are not available, the average still-gas-to-crude-oil price ratio, 0.759 , is used. After 1985, EIA data series no longer report feedstock and refinery use of still gas separately and all SEDS industrial consumption is removed from $S E P E R$. See the "Consunntion Adjustments for Process Fuel and Intermediate Products" section of this documentation.

\section{Price Data Sources}

1970, 1971, 1981, 1983-1985: Energy Information Administration, Annual Energy Review 1990, Table 70 , "Crude Oil Refiner Acquisition Cost," column titled, "Composite, Nominal."
1982: Bureau of the Census, U.S. Department of Commerce, 1987 Census of Manufactures, MC87-I-29A, Table 6a, industry 2911.

1979, 1980: Bureau of the Census, U.S. Department of Commerce, 1980 Annual Survey of Manufactures, M80(AS)-4.3, page 9, industry 2869.

1978: Bureau of the Census, U.S. Department of Commerce, 1979 Annual Survey of Manufactures, M79(AS)-4.3, page 28, industry 2869.

1972, 1977: Bureau of the Census, U.S. Department of Commerce, 1977 Census of Manufactures, MC77-1-29A, page 29A-20, industry 2911.

\section{Petroleum Coke}

Petroleum coke is consumed by the industrial and electric utility sectors. The portion of petroleum coke consumed by the electric utility sector (about 2 percent in 1989) is described under the heading "Petroleum Coke (Electric Utility)." The remaining petroleum coke is marketed to industrial consumers in two forms, calcined and uncalcined. On a unit basis, the calcined coke is about three times as expensive as the uncalcined. Unit prices are calculated by divicing the value exported by the quantity exporter, weighted by type of petroleum coke. The physical anit conversion factor for petroleum coke is 5 barrels per short ton.

\section{Price Data Sources}

1978 forward: Bureau of the Census, U.S. Department of Commerce, FT-446, U.S. Exports Schedule B, Commodity by Country, Petroleum Coke, Except Calcined, 5213150, and Petroleum Coke, Calcined, 5175120.

1970-1977: Bureau of the Census, U.S. Department of Commerce, December issues of FT-410, U.S. Exports Schedule B. Commodity by Country, Petroleum Coke, Except Calcined, 3329420, and Petroleum Coke, Calcined, 3329410.

\section{Special Naphthas}

Prices for special naphthas are the simple average of the "varnish makers and painters naphtha" average price and the "solvent naphtha" average price calculated from the city prices published in the Chemical Marketing Reporter. For 1984 forward, the prices are averaged from the first issue of each month; for 1974, 1979 , and 1980, when petroleum prices were increasing rapidly, prices are averaged from 10 randomly-selected issues; and for all other years, prices are averaged from at least five randomly-selected issues.

\section{Price Data Source}

1970 forward: Schnell Publishing Co., Inc., Chemical Marketing Reporter, selected monthly issues. 


\section{Waxes}

The product-supplied information covers fully refined crystalline wax, other refined crystalline wax, and microcrystalline wax. A composite price index for these three waxes is listed in the Bureau of Labor Statistics publication Producer Prices and Producer Price Indexes since April 1974. Producer prices for 1967 for the three waxes are available from data in the 1967 Census of Manufactures. A weighted-average price for 1967 of $\$ 15.75$ per barrel is obtained by summing the values of shipments of the three waxes aiid dividing the sum by the total quantity shipped. Price estimates for 1974-1985 are derived by multiplying the published price indices by the estimated 1967 base price. For 1985, a simple average of monthly price indices for January through June is applied because the index for petroleum wax was discontinued after June 1985. Similarly, the index for 1974 is estimated by averaging the October, November, and December values available for that year. Price estimates for 1970-1973, and 1986 forward are obtained by dividing the value of exports by the quantity exported. The physical unit conversion factors for wax are 280 pounds per barrel and 2.2046 pounds per kilogram.

\section{Price Data Sources}

1987 forward: Bureau of the Census, U.S. Department of Commerce, December issue of Report No. EM-546 (1987), EM-522 (1988), and EM-545 (1989), titled U.S. Exports, Schedule B, Commodity by Country for Paraffin Wax and Other Petroleum Waxes Unblended incl Microcrystalline Wax (Commodity no. 4925200).

1986: Bureau of the Census, U.S. Department of Commerce, December issue of EM-546, U.S. Exports, Schedule $B$, Commodity by Country for Paraffin Wax, Crystalline, Fully Refined (Commodity no. 4925210), Paraffin Wax, Crystalline, Except Fully Refined (Commodity no. 4925220), and Petroleum Waxes, NSPF incl Microcrystalline Wax (Commodity no. 4925240)

1974-1985: Bureau of Labor Statistics, U.S. Department of Labor, Producer Prices and Producer Price Indexes, Annual Supplement, Commodity Code 0577.

1970. 1973: Bureau of the Census, U.S. Department of Commerce, December issues of FT-410, U.S. Exports, Schedule B. Commodity by Country for Paraffin Wax, Crystalline, Fully Refined (Commodity no. 3326220), Paraffin Wax, Crystalline, Except Fully Refined (Commodity no. 3326230), and Microcrystalline Wax (Commodity no. 3326210 ).
(1967: Bureau of the Census, U.S. Department of Commerce, Census of Manufactures, 1967, page 29A-15, quantity and value of shipments of waxes.)

\section{Btu Prices: All Years}

Btu prices for the seven petroleum products are calculated by converting physical unit prices from dollars per barrel to dollars per million Btu using the conversion factors show'n in Table 37. U.S. prices are developed for each product and assigned to the industrial sector in States and years where there is consumption. The State-level "other petroleum" price is the average of the seven petroleum products, weighted by SEDS consumption data. The variable State prices reflect the different mix of products consumed. U.S. Btu prices are calculated as the average of the State Btu prices, weighted by SEDS consumption data. All State and U.S. SEDS consumption data are adjusted to remove process fuel and intermediate product consumption.

Table 38 shows national-level estimated prices and expenditures by the other petroleum product components for the years 1970, 1975, 1980, and 1985 forward.

\section{Conversion Factors: All Years}

Factors used to convert price data from physical unit prices to Btu prices are shown in Table 37.

Table 37. Conversion Factors for Other Petroleum Products (Million Btu per Barrel)

\begin{tabular}{lc}
\hline Petroleum Product & Heat Rate \\
\hline Miscellaneous Products & 5.796 \\
Petrochemical Feedstocks & \\
Naphtha & 5.248 \\
Other Oils & 5.825 \\
Still Gas & 6.000 \\
Petroleum Coke & 6.024 \\
Special Naphthas & 5.248 \\
Waxes & 5.537 \\
\hline
\end{tabular}


Table 38. Other Petroleum Price and Expenditure Estimates for the Industrial Sector, United States, 1970, 1975, 1980, and 1985-1989

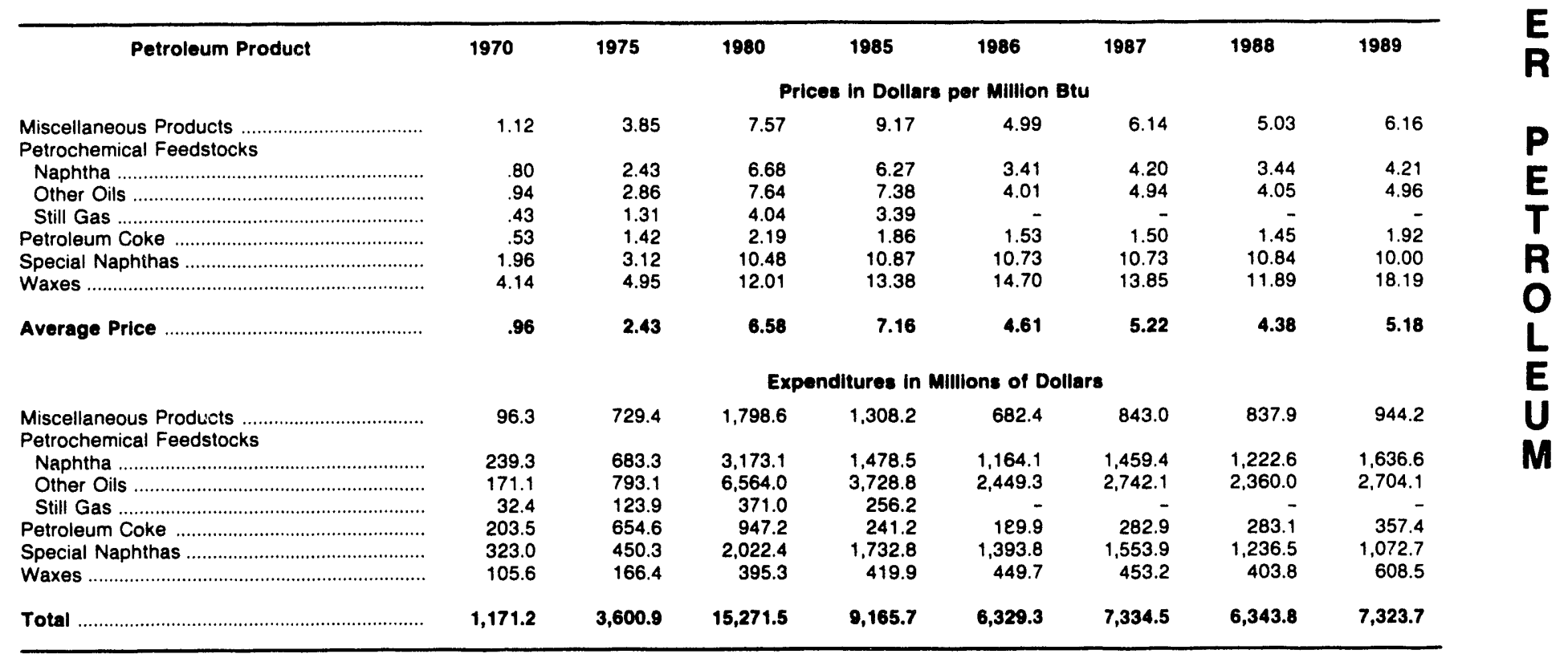

-Consumption data for this series are not available after 1985. See text.

Note: Expenditure totals may not equal sum of components due to independent rounding.

Source: State Energy Price and Expenditure Data System 1989. 


\section{Electricity Consumed by End-Use Sectors}

Electricity prices are retail prices for sales to ultimate users. Prices are developed for the residential, commercial, industrial, and transportation sectors. In general, taxes are included in the prices. However, taxes collected by a utility from an end user and turned over to a government authority are not included in the revenues reported in the Electric Power Annual or the Statistical Yearbook and are not included in the prices. Taxes paid by the utility (rather than the end user) are considered operating costs and are passed on to the end user as part of the rate. Therefore, Federal, State, business, and property taxes are typically included in the prices, while sales and other point of purchase taxes are not.

Consumption is based on sales by electric utilities to ultimate users. Electricity consumption data by State for the residential, commercial, industrial, and transportation sectors are obtained from SEDS. Consumption of electricity in the industrial sector is adjusted for estimated refinery use in each State. See the discussion under "Consumption Adjustments for Process Fuel and Intermediate Products" in this report.

\section{Conversion Factor: All Years}

3,412 Btu per kilowatthour

\section{Physical Unit Prices: 1987 Forward}

For 1987 forward, physical unit prices for States are calculated for all four sectors as the average revenue per unit of sales for all electric utilities reported in a State. The calculation of physical unit prices uses the revenues and sales data from the Electric Power Annual. Data for the residential and industrial sectors are drawn from their respective columns. Data for the transportation sector are from the column titled "Other" that includes sales to railroads and railways. SEDS consumption is used to determine the portion of the data in the column titled "Other" that is not sold to the transportation sector. These data are added to the column titled "Commercial" to arrive at the revenues and sales data for the commercial sector.

For 1987 forward, prices are based on preliminary revenues and sales data in the given year, and are replaced with revised data in the following year. There are no missing prices in any of the four sectors.

\section{Physical Unit Prices: 1970-1986}

For 1970-1986, preliminary physical unit prices for States are calculated for all four sectors as the average revenue per unit of sales for all electric utilities reported in a State. The calculation of physical prices uses the revenues and sales data from the Statistical Yearbook for each year in the series. Data for the residential sector and industrial sector are drawn from their respective columns. The commercial sector is the sum of the columns titled "Commercial," "Street and Highway Lighting," "Other Public Authorities," and "Interdepartmental." The transportation sector is the column titled "Railroads and Railways."

For 1980-1986, prices are based on preliminary revenues and sales data in the given year, and are replaced with revised data in the following year. The only exception to this rule is the revenues data for $A R / 1981$; preliminary data are used in this case because of an apparent error in the revised data.

For 1970-1981, MD prices are assigned to DC. There are no other missing prices for the residential, commercial, and industrial sectors.

In the transportation sector, numerous price assignments are made due to the lack of sector-specific price data. Generally, electricity usage in the transportation sector is small; the sector's electricity use ranged from 0.1 percent to 0.2 percent of total U.S. electricity consumption in 1970 through 1986. From 1970-1986, only 15 States used measurable amounts of electricity in the transportation sector (CA, DC, FL, GA, IL, LA, MA, MD, NJ, NY, OH, PA, TN, VA, and WA). A few individual State prices are unavailable and are assigned the commercial sector prices: LA/1970-1986, and TN/1970-1986. (Prices are available for LA/1970, 1972,1973 , but those prices are replaced by commercial sector prices to maintain a consistent series for the State.) In addition, MA/1985, 1986 transportation prices are estimated by multiplying the MA 
commercial prices by the average of the ratios of the commercial-to-transportation sector prices for 1980-1984. Similarly, the VA/1977 transportation price is estimated by multiplying the VA/1977 commercial price by the average of the ratios of the commercialto-transportation sectors prices for 1978-1982.

In order to reconcile national-level electricity prices based on the Statistical Yearbook with the EIA nationallevel electricity prices published in the Annual Energy Review (AER), yearly adjustment factors are calculated for the residential, commercial, and industrial sectors, as follows: a preliminary U.S. price for each sector is calculated as the average of the State prices, weighted by SEDS consumption. These preliminary U.S. prices are divided by the national-level electricity prices published in the $A E R$. and the quotient is used as an adjustment factor. The preliminary State prices are multiplied by the adjustment factor to produce the final physical unit State prices in those sectors. Since no transportation sector prices are published in the $A E R$, no adjustments are made to that sector and the final physical unit prices are derived solely from the Statistical Yearbook sales and revenue data. The annual adjustment factors for the residential, commercial, and industrial sectors are shown in Table 39.

Table 39. Annual Electrici:y Price Adjustment Factors

\begin{tabular}{cccc}
\hline Year & Residential & Commercial & Industrial \\
\hline 1970 & 1.05121 & 1.05712 & 1.06832 \\
1971 & 1.05632 & 1.05926 & 1.05504 \\
1972 & 1.05271 & 1.05514 & 1.05765 \\
1973 & 1.06626 & 1.06188 & 1.05991 \\
1974 & 1.09572 & 1.08098 & 1.08732 \\
1975 & 1.09257 & 1.08145 & 1.07665 \\
1976 & 1.07753 & 1.07755 & 1.06891 \\
1977 & 1.06746 & 1.07675 & 1.06820 \\
1978 & 1.06654 & 1.08273 & 1.06861 \\
1979 & 1.06986 & 1.08349 & 1.06441 \\
1980 & 1.04457 & 1.06109 & 1.06781 \\
1981 & 1.05821 & 1.06943 & 1.06523 \\
1982 & 1.06654 & 1.06351 & 1.05597 \\
1983 & 1.05421 & 1.05301 & 1.05537 \\
1984 & 0.99693 & 1.01924 & 0.99015 \\
1985 & 1.00010 & 1.02008 & 0.98355 \\
1986 & 0.99854 & 1.01518 & 0.98618 \\
\hline
\end{tabular}

Source: ElA calculations based on data from the Annual Energy Review and the Statistical Yearbook of the Electric Utility Industry.

\section{Btu Prices: All Years}

Btu prices for States are calculated by dividing the physical unit prices by the conversion factor. U.S. Btu prices are calculated as the average of the State Btu prices, weighted by consumption data from SEDS adjusted for process fuel consumption in the industrial sector.

\section{Data Sources}

\section{Prices}

1987 forward: Energy Information Administration, Eltctric Power Annual 1988, Tables 19 and 21 (1987 data); Electric Power Annual 1989, Tables 27 and 29 (1988, 1989).

1970-1986: Edison Electric Institute, Statistical Yearbook of the Electric Utility Industry, tables titled, "Revenues: Total Electric Utility Industry" and "Energy Sales: Total Electric Utility Industry."

1970-1986: Energy Information Administration, Annual Energy Review 1989, Table 95, "Retail Prices of Electricity Sold by Electric Utilities, 1960-1989."

\section{Consumption}

1970 forward: Energy Information Administration, State Energy Data System, electricity consumption by end-use sector. 
Nuclear Fuel for Generation of Electricity

Nuclear fuel prices are developed for the electric utility sector. State-level data on the amount of electricity generated from nuclear power plants are from SEDS. Utilities operating nuclear plants report fuel costs per kilowatthour to the U.S. Department of Energy annually. These data are published on a plant-by-plant basis and include all taxes, transportation, and handling costs paid by the utilities. The State where the nuclear power plant is located is assigned the reported nuclear fuel cost. For States with two or more nuclear power plants, the average fuel cost, weighted by generation, is attributed to the State.

\section{Physical Unit Prices: All Years}

State-level nuclear fuel prices are estimated in two steps: (1) the total cost of fuels consumed at all nuclear power plants in a State is divided by their total generation of electricity, and (2) the cost per output created in step 1 is divided by the U.S.-level thermal conversion factor to create the price in dollars per million Btu. Occasionally, the fuel costs at nuclear power plants include trivial amounts of non-nuclear fuels, which are necessary to continue essential plant operations during refueling or maintenance of the reactor.

For States where generation and fuel cost data are not available for years in which $S E D R$ shows nuclear electric power generation, a price is estimated by another method. The estimation method using regional prices that is applied to other energy sources in SEPER is not suitable for nuclear fuels. For this reason, the ratio of the change in the national nuclear fuel price is used to interpolate or to project nuclear fuel prices for missing years within a State. The ratio of national prices used in the interpolation or projection of missing prices is the ratio before missing prices are assigned. The States and years for which nuclear prices are estimated are shown in Table 40.

$\mathrm{CO} / 1985$ generation is negative indicating that the nuclear power plant used more energy than it supplied. In this case the fuel price and expenditures are set to zero. In 1986 forward, there is generation but plant data are not sufficient to calculate prices. The 1986 forward prices for $\mathrm{CO}$ are estimated by calculating the percentage change in the U.S. average prices each year and applying that percentage to the previous year's CO price.

For $\mathrm{MO} / 1985$, a large credit resulting from litigation is assigned to fuel costs, creating an artificially low price. The MO/1986 uranium price, which is in the range of other nuclear fuel plants, is used to estimate the 1985 price by applying the ratio of the 1986-to-1985 national prices.
Table 40. Nuclear Fuel Price Assignments

\begin{tabular}{ll}
\hline State & Years \\
\hline Alabama & $1973,1974,1976$ \\
Arizona & 1985 \\
Arkansas & 1980 \\
Colorado & $1977,1978,1982-1984,1986-1989$ \\
Georgia & $1974,1975,1978$ \\
Maine & 1972 \\
Mississippi & 1984 \\
Missouri & 1984,1985 \\
North Carolina & 1982 \\
Ohio & 1986 \\
Oregon & 1975 \\
South Carolina & 1970,1985 \\
Tennessee & 1980 \\
Washington & $1970-1987$ \\
Wisconsin & 1970 \\
\hline
\end{tabular}

The 1985 Historical Plant Costs and Annual Production Expenses for Selected Electric Plants has a footnote for the Duke Power Catawba plant in SC stating that the reported production expenses represent only 12.5 percent of the actual production expenses. The production expenses used in the calculation for the Catawba plant are adjusted accordingly.

TN/1986, 1987 generation levels are negative indicating that the nuclear power plant used more energy than it supplied. As with $\mathrm{CO} / 1985$, the fuel price and expenditure are set to zero.

Prices for GA/1978, NC/1982. and $\mathrm{OH} / 1986$ are assigned because the prices calculated using reported data are significantly different from prices for other years for these States.

There are no prices available for WA/1970-1987; national prices are assigned for those years.

\section{Btu Prices: All Years}

Unit prices for nuclear fuel in dollars per kilowatthour of energy output are converted to dollars per million Btu of equivalent energy input using the annual thermal conversion factors shown in Table 41 on page 240 .

\section{Data Sources}

Prices

1988 forward: Energy Information Administration, Electric Plant Cost and Power Production Expenses, Table 6.

1982-1987: Energy Information Administration, Historical Plant Costs and Annual Production Expenses for 
Selected Electric Plants, Table 18 (1982-1984), and Table 20 (1985-1987).

1979-1981: Energy Information Administration, Ther mal Electric Plant Construction Cost and Annual Production Expenses, pages 267-279 (1979), Table 11 (1980 and 1981).

1975-1978: Energy Information Administration, Steam Electric Plant Construction Cost and Annual Production Expenses, "Section II-Nuclear Plants."

1970-1974: Federal Power Commission, Steam Electric Plant Construction Costs and Annual Production Expenses, data sheets for Nuclear Plants (1970-1973), and "Section II-Nuclear Plants" (1974).

\section{Consumption}

1970 forward: Energy Information Administration, State Energy Data System, electricity generated by nuclear power.

\section{Conversion Factors}

1970 forward: Energy Information Administration, State Energy Data Report, Consumption Estimates, 1960-1989, Table D1, as shown in Table 41.

Table 41. Nuclear Electric Power Btu Conversion Factors, 1970 Forward (Thousand Btu per Kilowatthour)

\begin{tabular}{lllll}
\hline Year & $\begin{array}{l}\text { Conversion } \\
\text { Factor }\end{array}$ & & Year & $\begin{array}{l}\text { Conversion } \\
\text { Factor }\end{array}$ \\
\hline 1970 & 10.977 & & 1980 & 10.908 \\
1971 & 10.837 & & 1981 & 11.030 \\
1972 & 10.792 & & 1982 & 11.073 \\
1973 & 10.903 & & 1983 & 10.905 \\
1974 & 11.161 & & 1984 & 10.843 \\
1975 & 11.013 & & 1985 & 10.813 \\
1976 & 11.047 & & 1986 & 10.799 \\
1977 & 10.769 & & 1987 & 10.776 \\
1978 & 10.941 & & 1988 & 10.743 \\
1979 & 10.879 & & 1989 & 10.724 \\
\hline
\end{tabular}

\section{Wood and Waste for Generation of Electricity}

Wood and waste prices are developed for the electric utility sector; data for wood and waste consumed by other sectors are not available. State-level data on the amount of electricity generated at electric utilities from wood and waste are from SEDS and are collected on Form EIA-759 (formerly, FPC-4). All electric utilities are required to report on EIA-759 but no price data are collected. National-level wood and waste prices are developed for the electric utility sector and are used for each State consuming wood or waste at electric utilities. Taxes are included in the prices for all years.

\section{Prices: All Years}

1983 Forward. The national price in dollars per million Btu is obtained by telephone surveys of selected electric utilities that report use of wood and waste for generating electricity. The price used is the weightedaverage price of those electric utilities able to provide price and consumption data.

During the telephone surveys, some anomalies became apparent that are unique to wood and waste as fuel for utilities. In some cases, there was no charge for the fuel, and in one case involving refuse-derived fuel, the electric utility charged a "tipping fee" for accepting truckloads of waste. Instead of paying for the fuel, the electric utility was paid to take the fuel. Handling refuse-derived fuel is more labor intensive than handling conventional fossil fuels, and the labor expenses are included in the plant's operating costs, not the fuel costs. In addition, the price is very erratic because the demand is relatively small and the pricing mechanism, even for a single facility, may change from year to year. A price or quantity change by a single major user affects the national price more significantly than in any another fuel.

1978-1982. National prices are derived from data collected on FPC Form 423 and published monthly by EIA in Cost and Quality of Fuels for Electric Utility Plants $(C \& Q)$ in a table titled, "Wood Chips, Refuse, and Petroleum Coke Used as Fuel by Steam-Electric Plants." For these years, fossil-fueled plants with a combined capacity of 25 megawatts or greater were required to report on FPC Form 423. Annual prices of wood and waste sold to electric utilities are developed as quantity-weighted monthly prices for those plants where wood and waste were used as fuel. Beginning in 1983, the reporting threshhold was raised to 50 megawatts, and no plants reported use of wood or waste in that or subsequent years.

A detailed review of data in $C \& Q$ showed that some entries were in error by factors of 10,100 , or 1,000 . 
Accordingly, the following corrections were made. For 1982, the February, March, and April quantities for the Florida Power Corporation were divided by 1,000 to make them $0.08,0.04$, and 0.06 thousand short tons, respectively. The March, April, and May costs for Northern State Power were multiplied by 100 to make them $\$ 0.70$ per million Btu. For the 5 months from November 1979 through March 1980, the reported quantities of wood delivered to Burlington Electric $\mathrm{Co}$. were divided by 10 , to place them in the range 7.98 to 9.39 thousand short tons. For the 8 months from June 1978 through January 1979, seed corn delivered to the Logansport Indiana Electric Department were included in wood and waste. For February 1978, the reported quantity of wood delivered to the United Power Associates was divided by 1,000 to make it 0.09 thousand short tons.

1970-1977. The annual prices for wood chips are derived by deflating the 1978 price using the Gross National Product (GNP) implicit price deflator based on 1982 dollars. The deflators are:
$1970 . .42 .0$
1973. . . 49.5
1976. . . 63.1
$1971 \ldots .44 .4$
1974. . . .54.0
1977. . . 67.3
1972. . . .46.5
$1975 \ldots .59 .3$
1978. . . .72.2

Data Sources

Prices

1983 forward: Energy Information Administration, telephone survey of selected electric utilities that use wood and waste for generating electricity.

1978-1982: Energy Information Administration, Cost and Quality of Fuels for Electric Utility Plants, table titled, "Wood Chips, Refuse, and Petroleum Coke Used as Fuel by Steam-Electric Plants."

\section{Consumption}

1970 forward: Energy Information Administration, State Energy Data System, electricity generated from consumption of wood and waste. 


\section{Consumption Adjustments for Process Fuel and Intermediate Products}

\begin{abstract}
Almost all aspects of energy production, processing, and distribution consume energy as an inherent part of those activities. Familiar examples include drilling for oil and gas, refining crude oil into finished petroleum products, transporting natural gas and petroleum by pipeline, and generating and delivering electricity to end users. Energy that is consumed to make energy more useable to the end user is called "process fuel." Energy products that are subsequently incorporated into another energy product for end-use consumption are called "intermediate products." Motor gasoline blending components are a familiar example of intermediate products which are consumed as part of the finished motor gasoline sold at service stations and other outlets.
\end{abstract}

The end user does not consume either process fuel or intermediate products directly, but he does pay for them, because the cost to the processor or distributor is passed on to the end user. In estimating energy expenditures, as is done in this report, process fuel and intermediate product consumption must be subtracted from the end-use sector in which they are included, either the industrial or transportation sector. If their use is not removed, the expenditures would be double counted, first by the "processor" (producer, processor, or transporter) and again by the end user.

Process fuel consumption adjustments are incorporated into the data tables in SEPER for:

1. Fuel (petroleum, natural gas, coal) and electricity consumed at refineries

2. Crude oil lease, plant, and pipeline fuel

3. Natural gas lease and plant fuel

4. Natural gas pipeline fuel

5. Electrical system energy losses (i.e., energy consumed in the generation, transmission, and distribution of electricity)

Intermediate product consumption adjustments are incorporated into SEPER for:

1. Aviation gasoline blending components

2. Motor gasoline blending components

3. Natural gasoline (1970-1983)

4. Pentanes plus (1984 forward)

5. Plant condensate (1970-1983)
6. Unfinished oils

7. Unfractionated stream (1970-1983)

Starting in 1984, natural gasoline (including isopentane) and plant condensate are reported together as the new product, pentanes plus, and the components of unfractionated stream are reported separately under liquefied petroleum gases.

Table 43 shows estimates of the quantities of energy consumed by State as process fuel and intermediate products in 1988 and 1989. Estimates for 1970 through 1989 are available on the SEPEDS personal computer diskettes.

\section{Procedures}

Refinery Fuel. Refinery fuel consumption of distillate fuel, residual fuel, liquefied petroleum gases, other petroleum (crude oil, petroleum coke, and still gas), natural gas, coal, and electricity is individually estimated for each source and subtracted from its SEDS industrial sector total. Because crude oil consumption is not an individual fuel in SEDS for 1970-1982, the small amounts of crude oil that were used at refineries during those years were allocated to residual and distillate fuels consumed at refineries. The allocation from crude oil refinery use to residual and distillate fuels refinery use was made according to each fuel's share of the total crude oil used directly (including losses) as residual and distillate fuels (Petroleum Supply Annual Volume 1, Table 2). Over this period, residual fuel ranged from a low share of 75 percent in 1971 to a high share of 92 percent in 1976. Refinery consumption of still gas, excluding still gas consumed as petrochemical feedstocks, is subtracted from the SEDS industrial sector total for 1970-1985. Beginning in 1986, EIA data series no longer report refinery fuel and feedstock use separately and all SEDS industrial still gas consumption is removed from SEPER.

Refinery fuel consumption data are available in the data sources by Petroleum Administration for Defense District (PADD) (1981 forward) and by State or group of States (1970-1980). Where State-level consumption data are not available, the State-level estimates are derived by allocating the district's or group's total consumption to the individual States within the district or 
group to the States that had operating refineries in a given year. Individual fuels are allocated to the refining States according to each State's share of the refining States' total industrial sector fuel consumption during the year. In some instances, estimated refinery fuel consumption exceeds the SEDS estimate for total industrial consumption of a fuel within a State. When this occurs, the excess refinery fuel consumption is reallocated as shown in Table 42.

Table 42. Reallocations of Excess Refinery Fuel Consumption

\begin{tabular}{|c|c|c|c|c|}
\hline Year & Fuel & $\begin{array}{r}\text { Thousand } \\
\text { Barrels }\end{array}$ & $\begin{array}{c}\text { Excess } \\
\text { in: }\end{array}$ & $\begin{array}{c}\text { Re- } \\
\text { allocated to: }\end{array}$ \\
\hline 1971 & $\begin{array}{l}\text { Residual } \\
\text { Fuel }\end{array}$ & 294 & Kansas & Oklahoma \\
\hline 1973 & $\begin{array}{l}\text { Residual } \\
\text { Fuel }\end{array}$ & 45 & $\begin{array}{l}\text { Group 4: } \\
\text { Kentucky, } \\
\text { Tennessee }\end{array}$ & Illinois \\
\hline 1979 & LPG & 173 & Montana & Wyoming \\
\hline 1983 & $\begin{array}{l}\text { Petroleum } \\
\text { Coke }\end{array}$ & 3.091 & PADD 1 & PADD II \\
\hline 1984 & Crude Oil & 80 & PADD II & PADD III \\
\hline 1984 & $\begin{array}{l}\text { Petroleum } \\
\text { Coke }\end{array}$ & 992 & PADD I & PADD II \\
\hline 1985 & $\begin{array}{l}\text { Petroleum } \\
\text { Coke }\end{array}$ & 80 & PADD II & PADD III \\
\hline 1985 & Crude Oil & 719 & PADD I & PADD II \\
\hline 1986 & $\begin{array}{l}\text { Petroleum } \\
\text { Coke }\end{array}$ & 1,297 & PADD I & PADD II \\
\hline 1988 & Crude Oil & 8 & PADD III & PADD V \\
\hline 1989 & $\begin{array}{l}\text { Crude Oil } \\
\text { Crude Oil }\end{array}$ & $\begin{array}{r}9 \\
15\end{array}$ & $\begin{array}{l}\text { PADD I } \\
\text { PADD III }\end{array}$ & $\begin{array}{l}\text { PADD V } \\
\text { PADD V }\end{array}$ \\
\hline
\end{tabular}

Source: ElA calculations based on data from the State Energy Data Report and the Petroleum Supply Annual.

Intermediate Products. Aviation gasoline blending components, motor gasoline blending components, natural gasoline (1970-1983), pentanes plus (1984 forward), plant condensate (1970-1983), unfinished oils, and unfractionated stream (1970-1983) are used at refineries and blending plants to make end-use petroleum products, particularly motor gasoline. Accordingly, consumption of these products is completely removed from SEPER.

Crude Oil Lease, Plant, and Pipeline Fuel. Industrial crude oil remaining after refinery fuel consumption is removed is assumed to be used as lease, plant, and pipeline fuel. Since these are process fuel uses, the remaining crude oil is removed from SEDS industrial sector consumption.
Natural Gas Lease and Plant Fuel. Natural gas consumed as lease and plant fuel is process fuel and is subtracted from SEDS industrial sector natural gas totals by State and year.

Natural Gas Pipeline Fuel. Transportation sector natural gas consumption shown in SEDS is used to power pipelines. As such, it is process fuel and is completely removed from the SEPER expenditure calculations.

Electrical System Energy Losses. The amount of energy lost during generation, transmission, and distribution of electricity (including plant use and unaccounted for electrical energy) is process fuel and is subtracted from sectoral energy consumption estimates used in SEPER. The energy losses are "paid for" when residential, commercial, industrial, and transportation sector consumers buy the electricity produced at electric utilitięs.

\section{Data Sources}

Capacity of Petroleum Refineries

1982 forward: Energy Information Administration, $P e$ troleum Supply Annual Volume 1, table titled, "Number and Capacity of Operable Petroleum Refineries," column heading, "Crude Capacity, Barrels per Calendar Day, Operating" (1982-1985), "Atmospheric Crude Oil Distillation Capacity, Barrels per Calendar Day, Operating" (1986 forward).

1979-1981: Energy Information Administration, Energy Data Reports, Petroleum Refineries in the United States and U.S. Territories, table titled, "Number and Capacity of Petroleum Refineries," column heading, "Crude Capacity, Barrels per Calendar Day, Operating."

1978: Energy Information Administration, Energy Data Reports, Petroleum Refineries in the United States and Puerto Rico, table titled, "Number and Capacity of Petroleum Refineries," column heading, "Crude Capacity, Barrels per Calendar Day, Operating."

1970-1977: Bureau of Mines, U.S. Department of the Interior, Mineral Industry Surveys, Petroleum Refineries in the United States and Puerto Rico, table titled, "Number and Capacity of Petroleum Refineries," column heading, "Crude Capacity, Barrels per Calendar Day, Operating."

\section{Fuel Consumed at Refineries}

1981 forward: Energy Information Administration, $P e$ troleum Supply Annual Volume 1, table titled, "Fuels Consumed at Refineries by PAD District."

1976-1980: Energy Information Administration, Energy Data Reports, Crude Petroleum. Petroleum Products, and Natural Gas Liquids, table titled, "Fuels Con- 
sumed for All Purposes at Refineries in the United States, by States."

1970-1975: Bureau of Mines, U.S. Department of the Interior, Mineral Industry Surveys, Crude Petroleum, Petroleum Products, and Natural Gas Liquids, tables titled, "Fuels Consumed for All Purposes at Refineries in the United States, by States."

1970 forward: Energy Information Administration, State Energy Data System, industrial sector consumption estimates for aviation gasoline blending components, crude oil, motor gasoline blending components, natural gasoline (1970-1983), pentanes plus (1984 forward), petroleum coke, plant condensate (1970-1983), still gas (excluding still gas consumed as petrochemical feedstocks, 1970-1985), unfinished oil, and unfractionated stream (1970-1983).
Natural Gas Lease, Plant, and Pipeline Fuel Use

1980 forward: Energy Information Administration, Natural Gas Annual, Table 10 (1980) and Table 13 (1981, 1982); Natural Gas Annual Volume 1, Table 13 (1983-1987) and Table 16 (1988); Natural Gas Annual, Table 16 (1989).

1977-1979: Energy Information Administration, Energy Data Reports, "Natural Gas Production and Consumption," Table $6(1977,1978)$ and Table 5 (1979).

1970-1976: Bureau of Mines, U.S. Department of the Interior, Mineral Industry Surveys, "Natural Gas Production and Consumption," Table 5 (1970) and Table $6(1971-1976)$. 


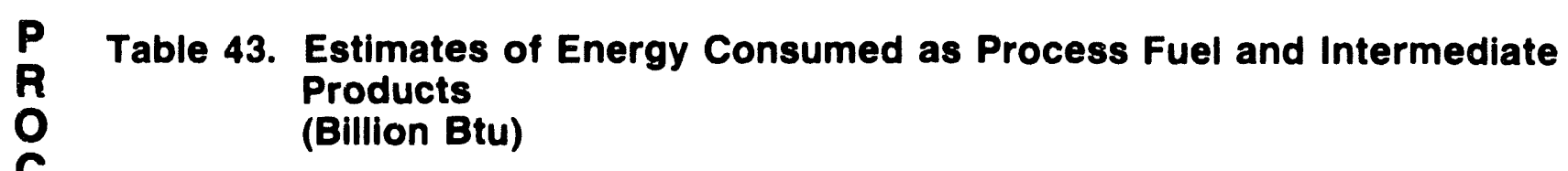

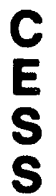

(Billion Btu)

Rofinorios

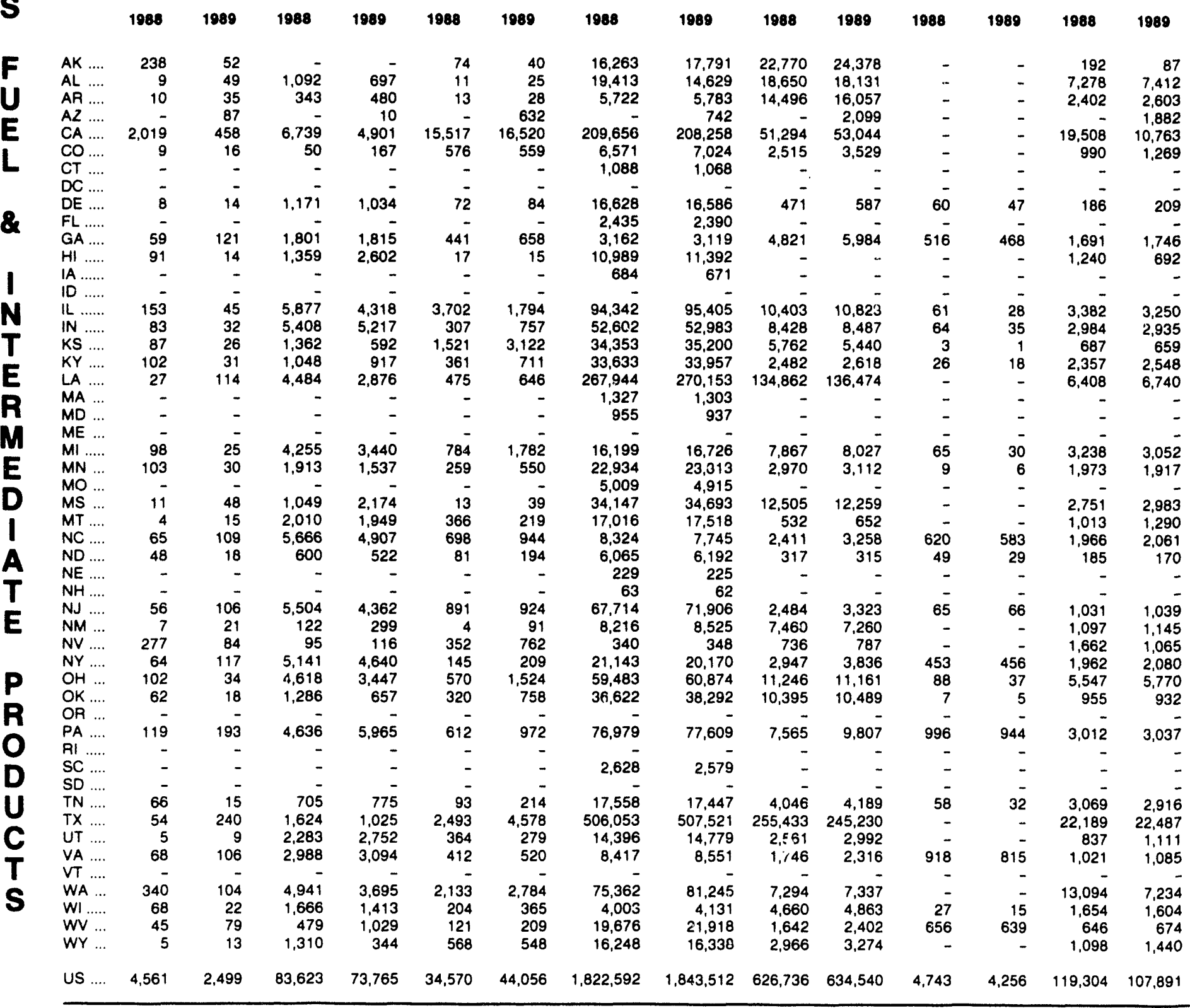

See footnotes at end of table. 
Table 43. Estimates of Energy Consumed as Process Fuel and Intermediate

Products (Continued)

(Billion Btu)

\begin{tabular}{|c|c|c|c|c|c|c|c|c|c|c|c|c|}
\hline & \multicolumn{2}{|c|}{$\begin{array}{c}\text { Total } \\
\text { Refineries }\end{array}$} & \multicolumn{2}{|c|}{$\begin{array}{l}\text { Crude Oll Lease, } \\
\text { Plant, and Pipeline Fuel }\end{array}$} & \multicolumn{2}{|c|}{$\begin{array}{l}\text { Natural Gas } \\
\text { Lease and Plant Fuel }\end{array}$} & \multicolumn{2}{|c|}{$\begin{array}{l}\text { Natural Gas } \\
\text { Pipeline Fuel }\end{array}$} & \multicolumn{2}{|c|}{$\begin{array}{c}\text { Electrical System } \\
\text { Energy Losses }\end{array}$} & \multicolumn{2}{|c|}{ Total } \\
\hline & 1988 & 1989 & 1988 & 1989 & 1988 & 1989 & 1988 & 1989 & 1988 & 1989 & 1988 & 1989 \\
\hline AK .... & 39,536 & 42,348 & 14,564 & 10,016 & 154,248 & 191,895 & 1,968 & 1,873 & 35,288 & 36,937 & 245,603 & 283,069 \\
\hline AL $\ldots$. & 46,451 & 40,943 & - & - & 6,847 & 6,337 & 12,465 & 13,940 & 436,410 & 445,330 & 502,174 & 506,550 \\
\hline AR .... & 22,985 & 24,985 & - & - & 12,197 & 11,688 & 7,564 & 9,740 & 193,963 & 199,638 & 236,708 & 246,052 \\
\hline AZ .... & - & 5,453 & - & - & 5 & 36 & 18,967 & 19,217 & 299,863 & 311,857 & 318,835 & 336,563 \\
\hline CA $\ldots .$. & 304,733 & 293,944 & 70,604 & 48,541 & 23,434 & 20,288 & 19,747 & 19,911 & $1,545,974$ & $1,562,353$ & $1,964,491$ & $1,945,036$ \\
\hline co .... & 10,711 & 12,564 & - & - & 11,701 & 12,370 & 7,929 & 12,090 & 226,035 & 230,666 & 256,376 & 267,690 \\
\hline CT .... & 1,088 & 1,068 & - & - & - & - & 555 & 587 & 207,452 & 209,431 & 209,095 & 211,086 \\
\hline DC .... & - & - & - & - & - & - & 239 & 251 & 72,273 & 73,876 & 72,511 & 74,128 \\
\hline DE .... & 18,596 & 18,560 & - & - & - & - & 4 & 4 & 58,124 & 61,727 & 76,724 & 80,291 \\
\hline FL ....... & 2,435 & 2,390 & - & - & 7,856 & 9,708 & 3,635 & 4,512 & $1,003,547$ & $1,059,795$ & $1,017,472$ & $1,076,405$ \\
\hline GA .... & 12,492 & 13,910 & - & - & - & - & 7,098 & 7,244 & 572,862 & 589.768 & 592.451 & 610,921 \\
\hline $\mathrm{HI} \ldots . .$. & 13,694 & 14,715 & - & - & - & - & - & - & 55,570 & 58,623 & 69,265 & 73,339 \\
\hline IA ....... & 684 & 671 & - & - & - & - & 10,663 & 10,561 & 222,203 & 219,747 & 233,550 & 230,980 \\
\hline ID ..... & - & - & - & - & - & - & 4,173 & 5,095 & 132,265 & 136,392 & 136,437 & 141,487 \\
\hline IL ....... & 117,921 & 115,663 & - & - & 63 & 53 & 13,028 & 13,869 & 847,900 & 836,524 & 978,912 & 966,110 \\
\hline IN ..... & 69,877 & 70,445 & - & - & 12 & 10 & 9,399 & 8,593 & 552,282 & 557,862 & 631,570 & 636,910 \\
\hline KS .... & 43,774 & 45,041 & - & - & 45,267 & 43,335 & 41,846 & 43,005 & 199,018 & 197,626 & 329,904 & 329,007 \\
\hline KY ... & 40,010 & 40,801 & - & - & 3,051 & 2,437 & 21,574 & 21,414 & 416,690 & 447,624 & 481,325 & 512,276 \\
\hline LA .... & 414,200 & 417,003 & - & - & 283,726 & 267,088 & 58,913 & 51,959 & 462,402 & 473,297 & $1,219,242$ & $1,209,347$ \\
\hline MA ... & 1,327 & 1,303 & - & - & 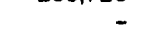 & - & 2,038 & 2,334 & 344,636 & 349,631 & 348,001 & 353,268 \\
\hline MD ... & 955 & 937 & - & - & 1 & 1 & 2,655 & 2,260 & 366,496 & 376,996 & 370,106 & $.380,194$ \\
\hline ME ... & - & - & - & - & - & - & 6 & 6 & 86.791 & 87,510 & 86,798 & 87,516 \\
\hline MI ..... & 32,506 & 33,082 & - & - & 8,577 & 8,418 & 19,105 & 17,697 & 635,820 & 634,691 & 696,008 & 693,888 \\
\hline MN ... & 30,162 & 30,966 & - & - & - & - & 11,335 & 11,960 & 352,345 & 351,534 & 393,841 & 394,460 \\
\hline MO ... & 5,009 & 4,915 & - & - & - & - & 4,931 & 5,205 & 402,580 & 402,588 & 412,520 & 412,708 \\
\hline MS ... & 50,476 & 52,194 & - & - & 7,209 & 5,178 & 35,040 & 35,069 & 216,362 & 226,345 & 309,087 & 318,786 \\
\hline MT .... & 20,940 & 21,644 & - & - & 2,041 & 1,799 & 2,298 & 2,460 & 99,719 & 99,961 & 124,998 & 125,863 \\
\hline NC .... & 19.750 & 19,606 & - & - & - & - & 5,356 & 5,859 & 659,115 & 675,100 & 684,220 & 700,566 \\
\hline ND .... & 7,344 & 7,440 & - & - & 4,235 & 3,554 & 1,819 & 1,893 & 54,760 & 54,046 & 68,158 & 66,934 \\
\hline NE .... & 229 & 225 & - & - & 46 & 34 & 4,620 & 4,848 & 132,983 & 134,445 & 137,879 & 139,552 \\
\hline $\mathrm{NH} \ldots$ & 63 & 62 & - & - & - & - & 23 & 19 & 68,178 & 69,537 & 68,263 & 69,618 \\
\hline NJ .... & 77,745 & 81,726 & - & - & - & - & 2,936 & 4,098 & 478,591 & 484,907 & 559,271 & 570,731 \\
\hline NM ... & 16,906 & 17,341 & - & - & 44,784 & 14,354 & 39,832 & 55,028 & 98,284 & 102,324 & 199,806 & 219,047 \\
\hline NV .... & 3,463 & 3,162 & - & - & - & - & 194 & 740 & 105,442 & 114,556 & 109,099 & 118,458 \\
\hline NY ... & 31,856 & 31,510 & - & - & 810 & 622 & 4,893 & 5,447 & 968,120 & 981,191 & $1,005,679$ & $1,018,770$ \\
\hline $\mathrm{OH} \ldots$ & 81,654 & 82,848 & - & - & 5,064 & 4,039 & 10,404 & 10,832 & $1,034,948$ & $1,083,905$ & $1,132,070$ & $1,181,624$ \\
\hline OK .... & 49,648 & 51,152 & - & - & 104,718 & 92,174 & 28,782 & 37,270 & 287,609 & 283,232 & 470,756 & 463,829 \\
\hline OR ... & & - & - & .. & 118 & 61 & 8,237 & 8,820 & 302,913 & 318,527 & 311,268 & 327,408 \\
\hline PA .... & 93,920 & 98,528 & - & - & 5,162 & 4,944 & 38,322 & 28,351 & 869,865 & 871,720 & $1,007,269$ & $1,003,542$ \\
\hline RI ..... & & - & - & - & - & - & 122 & 119 & 47,924 & $48,6 \% 1$ & 48,046 & 48,790 \\
\hline SC .... & 2,628 & 2,579 & - & - & - & - & 2,560 & 2,648 & 406,907 & 412,190 & 412,095 & 417,417 \\
\hline SD .... & - & - & - & - & 125 & 114 & 133 & 132 & 48,041 & 48,509 & 48,299 & 48,755 \\
\hline TN .... & 25,595 & 25,588 & - & - & 62 & 46 & 17,619 & 18,380 & 561,501 & 574,868 & 604,776 & 618,882 \\
\hline TX .... & 787,845 & 781,081 & - & - & 331,711 & 303,531 & 111,839 & 111,432 & $1,728,657$ & $1,757,679$ & $2,960,051$ & $2,953,722$ \\
\hline UT .... & 20,446 & 21,922 & - & - & 18,257 & 17,622 & 1,472 & 1,127 & 111,778 & 114,530 & 151,953 & 155,202 \\
\hline VA .... & $15,57 \mathrm{C}$ & 16,487 & - & - & 558 & 442 & 8,575 & 6,090 & 540,259 & 562,228 & 564,962 & 585,247 \\
\hline VT .... & - & - & - & - & - & - & - & 5 & 34,026 & 35,000 & 34,026 & 35,005 \\
\hline WA ... & 103,165 & 102,399 & - & - & - & - & 4,055 & 4,498 & 653,661 & 663,354 & 760,881 & 770,251 \\
\hline WI ..... & 12,282 & 12,413 & - & - & - & - & 4,349 & 4,200 & 368,821 & 370,240 & 385,452 & 386,853 \\
\hline$W V \ldots$ & 23,265 & 26,951 & - & - & 8,986 & 9,026 & 14,219 & 12,118 & 172,506 & 174,860 & 218,977 & 222,955 \\
\hline WY ... & 22,194 & 21,957 & - & - & 42,857 & 42,582 & 5,960 & 5,663 & 84,483 & 85.975 & 155,496 & 156,177 \\
\hline US .... & $2,696,129$ & $2,710,519$ & 85,167 & 58,557 & $1,133,729$ & $1,103,784$ & 633,494 & 650,476 & $19,864,238$ & $20,259,924$ & $24,412,758$ & $24,783,260$ \\
\hline
\end{tabular}

- In this table, other petroleum consists of: crude oil, petroleum coke, and still gas consumed as process fuel; aviation gasoline blending components, motor gasoline blending components, pentanes plus, and unfinished oils used as intermediate products.

Electricity is converted at the rate of 3,412 Btu per kilowatthour.

'Equals the sum of energy sources on the first page of this table.

-No consumption.

Sources: Energy Information Administration. See "Consumption Adjustments for Process Fuel and Intermediate Products" in text. 


\section{Summary of Changes Since the State Energy Price and Expenditure Report 1988}

In this section, revisions in the State Energy Price and Expenditure Data System (SEPEDS) that appear in the tables of this State Energy Price and Expenditure Report (SEPER) are summarized by energy source. Revisions to prices and expenditures are noted, as well as revisions to consumption data that are used in the calculation of weighted-average prices. This section covers data revisions in all years contained in SEPEDS, 1970-1989, although selected years are published in $S E P E R$. Users of the SEPEDS personal computer (PC) diskettes may notice data changes that do not appear in the report due to the level of rounding in the published tables.

Price and expenditure revisions occur for several reasons: data sources for prices change; price estimation methodologies are revised or price assignment and estimation procedures are updated; data entries are corrected; or consumption estimates are revised. The first three kinds of changes affect State-level and U.S. prices directly. The fourth, a revised estimated consumption, affects only State prices that are estimated by consumption-weighted averaging of adjacent States and, similarly, affects U.S. average prices. Consumption estimates used in SEPER are from the State Energy Data System (SEDS), published as the State Energy Data Report, Consumption Estimates, 1960-1989 (SEDR), which contains full documentation of the consumption estimation procedures. Consumption revisions are described in SEDR's Appendix E "Summary of Changes" section. Since energy expenditure estimates depend on both the price and consumption estimates (including the consumption adjustments for process fuel and intermediate products), revision of either or both may cause revisions to the expenditures series.

\section{Coal}

Commercial Sector. Coal consumption in the District of Columbia commercial sector in 1988, which was missed in the previous edition, is included with a price of $\$ 1.31$ per million Btu giving an expenditure of 0.7 million dollars. Thic change increased the DC total cual àviage pricc in 1989 from $\$ 1.07$ to $\$ 1.92$ per mil lion Btu and affected the U.S. average commercial and total coal prices insignificantly.

Industrial Sector. Btu prices for the industrial sector in Hawaii from 1982 through 1988 are revised due to the implementation of the Hawaii conversion factor instead of the California factor previously used. This change lowered Hawaii's industrial sector and total coal prices and expenditures by 5 percent to 10 percent for 1982 through 1988 . The U.S. average values for industrial and total coal prices and expenditures were affected insignificantly by these changes.

\section{Natural Gas}

Industrial Sector. Consumption of natural gas as lease and plant fuel in California and West Virginia in 1988 were revised in SEDS, as documented in SEDR Appendix $E$. These consumption changes cause expenditure adjustments of less than 1 percent that are not visible in the published tables but may be noticed on SEPEDS PC diskettes.

\section{Petroleum}

\section{Asphalt and Road Oil}

Industrial Sector. Asphalt and road oil consumption changed for all States in 1979, 1984, 1985, 1987, and 1988 as documented in the SEDR Appendix E. These changes slightly affect the U.S. average price per million Btu in inose 5 years. Although users of the SEPEDS PC diskettes may notice the adjustments, only the revisions to U.S. prices in 1987 and 1988 can be seen in the published report. The combined changes to State consumption and U.S. average prices cause expenditures revisions in all States and the U.S. totals in those 5 years. 
Transportation Sector. Estimates of aviation gasoline consumption by the military were revised in SEDS for all States in 1970-75 and for Maryland and the District of Columbia in 1976-88. The most significant change in SEPEDS is that the District of Columbia price is eliminated for all years, because aviation gasoline consumption is now assumed to be zero in SEDS, and the consumption that was previously reported for the District of Columbia is added to Maryland. Consequently, although Maryland aviation gasoline prices are not changed, Maryland expenditures are increased by 80 percent to 100 percent for all years. The consumption revisions to all States in 1970-75 caused all States' expenditures to change, most by less than 20 percent. The exceptions are Maine and Vermont with expenditure increases of 20 percent to 30 percent and Tennessee with expenditure decreases of 30 percent to 40 percent during those years.

\section{Jet Fuel}

Transportation Sector Expenditures. Although jet fuel prices did not change for any States or years, SEDS consumption revisions caused numerous expenditures and consumption-weighted U.S. average prices to be revised. Estimates of jet fuel consumption by the military were revised in SEDS for all States in 1970-75 and 1988 and for Maryland and the District of Columbia in 1976-87. The military use of jet fuel in the District of Columbia is now assumed to be zero in SEDS, and the consumption that was previously reported for the District of Columbia is added to Maryland. Some commercial use of jet fuel is still reported for the District of Columbia causing jet fuel prices and expenditures to drop to zero in $1975-77$ and remain with small expenditures in other years. Maryland expenditures are increased by about 25 percent for $1970-79$, by 5 percent or less in 1980-87, and by 16 percent in 1988. The consumption revisions to all States in 1970-75 caused all States' expenditures to change, most by less than 20 percent. The exceptions are Maine, where expenditures doubled, and North Dakota, where expenditures tripled, during those years.

Total Jet Fuel Prices. The consumption weighted average prices for total jet fuel used by both the transportation and electric utility sectors are revised for the United States in 1970-75 and 1988 and for 16 States in 1972-75. The State-level revisions occur in States that have electric utility sector consumption of jet fuel that affect the consumption-weighted price. These small price adjustments do not show in the published tables but may be noticed on the SEPEDS PC diskettes.
Residential Sector. Liquefied petroleum gases (LPG) prices for Alaska and Hawaii in the residential sector are recalculated for all years to implement a revised methodology. Alaska's residential LPG prices and expenditures decreased about 4 percent in 1970, 1971, and 1973-79 but increased about 3 percent in 1981-88. Hawaii's residential LPG prices and expenditures increased in 1970 and $1972-74$ by about 25 percent; decreased by 9 percent in 1975, 1976, and 1980-84; and increased by 3 percent in 1985-88.

The District of Columbia prices in $1979-83$ are revised to correct the Maryland price assignments in those years. The revisions cause prices and expenditures for residential use of LPG in the District of Columbia to decrease 20 percent to 30 percent in 1979 and 1981-83 and 2 percent in 1980 .

The average prices and expenditures for total LPG consumption in Alaska, Hawaii, and the District of Columbia are similarly affected but by smaller percentages. The revisions described above affect the U.S. average residential and total prices and expenditures for $L P G$, but by insignificant amounts.

\section{Lubricants}

Industrial and Transportation Sectors. The national average lubricants price that is used for all States is revised for 1987 from $\$ 12.70$ to $\$ 13.58$ per million Btu reflecting revised U.S. Department of Commerce, Bureau of the Census, value of shipments for lubricating oils and greases. The 1988 average price is revised from $\$ 10.41$ to $\$ 14.61$ per million Btu by using Bureau of Census value of shipments data not available previously. These revisions caused a 7-percent increase in prices and expenditures for both sectors and for total lubricants in all States in 1987 and a 40-percent increase to all prices and expenditures in 1988.

\section{Other Petroleum Products}

Industrial Sector. The U.S. Department of Commerce, Bureau of the Census, Census of Manufactures Industry Series are used to develop prices for SEPEDS miscellaneous petroleum products (1970-89) and still gas used as petrochemical feedstocks (1970-85). The Census data are also used to estimate several of the other petroleum products State-level consumption in SEDS. The recently published 1987 Census of Manufactures contains revisions to the 1982 Census. The incorporation of the new 1987 and revised 1982 data cause numerous revisions to other petroleum product prices and expenditures.

Still gas consumption as a petrochemical feedstock was reported by EIA separately from still gas consumed at 
refineries for 1970-85. The prices for still gas used as a petrochemical feedstock are developed using data from the 1982 Census of Manufactures Industry Series. Incorporating the revised 1982 Census data causes price decreases in 1970-85 of 2 percent or less (with the exception of a 13-percent decrease in 1982) in the U.S. average prices that are assigned to all States, with proportional decreases in each State's expenditures.

Miscellaneous petroleum product U.S. average prices, which are assigned to all States, are revised from 1980 forward reflecting the revised 1982 Census and new 1987 Census data. A review of the miscellaneous product prices for all years and the transfer to PC software caused slight revisions in the $1970-79$ prices due to differences in rounding during the recalculation. Prices for all States increased by less than 1 percent in 1970-79, by about 1 percent in $1980-84$, and by 28 percent in 1985-88.

Consumption of petrochemical feedstocks, special naphthas, waxes, pentanes plus, and miscellaneous petroleum products were revised in SEDS for 1986-88 due to the incorporation of data from the 1987 Census of Manufactures Industry Series. Although the individual product prices are not changed (with the exception of miscellaneous petroleum products prices discussed above), revisions to the consumption data cause the weighted average prices for the combined other petroleum products to be changed as the product mix is adjusted for most States in 1986-88. The most significant changes to prices occur in Vermont and Nebraska where there now is estimated consumption of other petroleum products, and therefore a price, when previous reports had estimated no consumption. Prices increase in Rhode Island and New Mexico by 80 percent to 100 percent and in Kansas and Arkansas by 40 percent to 70 percent in 1986-88. Prices decrease by 40 percent to 60 percent in Mississippi and Utah in 1986-88.

Industrial sector expenditures for other petroleum products combined in all States and all years are affected by the SEPEDS price revisions and by the changes in SEDS consumption estimates. Revisions prior to 1986 are 1 percent or less for all States, except in 1982 and 1985. In 1982, Alaska, Arizona, and Nevada expenditures for other petroleum products are 13 percent lower and New Mexico is 7 percent lower reflecting the revisions to still gas prices. In 1985, expenditures are lower by as much as 5 percent in a number of States. The revisons to SEDS consumption in 1986-88 cause larger revisions to other petroleum product expenditures. Expenditures are lower by 35 percent to 55 percent in Colorado, Mississippi, Oregon, and Utah but are almost five times higher in New Mexico during 1986-88. Expenditures are increased by 70 percent to 80 percent in North Carolina and by 40 percent to 60 percent in Kansas, Maryland, and Minnesota in 1986-88.
Residual Fuel

The transfer of SEPEDS residual fuel price estimates from EIA's mainframe computer to the PC uncovered several data errors that apparently had been resident in the database since its initial development in the early 1980's. Data from the new tables in EIA's Petroleum Marketing Annual caused commercial and industrial sector price revisions in 1984-88.

Commercial Sector. Prices for Alaska in 1970-72, 1974-78, 1980, and 1983 are revised to correct data entry errors, causing changes of 1 percent or less except for the 10 percent increase in 1980. Corrections of data entry errors also cause Connecticut prices to increase by 11 percent in 1970-72 and by 2 percent in 1978 . Correction of ir.consistent treatment of methodology causes prices to change in 1975 by 2 percent or less in the District of Columbia, Illinois, Kansas, Maine, North Carolina, New Hampshire, Nevada, Ohio, South Carolina, South Dakota, Virginia, Wisconsin, and Wyoming, and by 5 percent in North Dakota. The same type of correction to 1978 prices causes 2-percent changes to occur in Connecticut, the District of Columbia, Georgia, Maryland, North Carolina, Nevada, Oklahoma, South Carolina, South Dakota, Tennessee, Virginia, and Wisconsin, and a 7-percent change in West Virginia. Corrections to adjacent State price assignments in 1983 cause Montana and North Dakota prices to increase by $\$ .08$ and $\$ .03$ per million Btu, respectively. Prices for all States are revised for 1984 forward due to additional data from new tables in the source publication. Price revisions ranged from $\$ .02$ per million Btu for Delaware in 1986 to $\$ 2.08$ per million Btu for Oregon in 1984, with most revisions less than $\$ 1.00$ per million Btu. All the price revisions cause proportonal revisions to expenditures. The $1970-83$ revisions cause changes to the U.S. average prices and expenditures that are too small to be seen in the published tables. Changes to the U.S. averages are more noticeable in the 1984-88 revisions.

Industrial Sector. Prices for Wyoming in 1974-77 are revised to correct adjacent State calculations. The Wyoming price increases range from $\$ .31$ per million Btu in 1974 to $\$ .01$ per million Btu in 1976 . Prices for all States are revised for 1984 forward due to additional data from new tables in the source publication. Price revisions range from less than $\$ .01$ per million Btu to $\$ 1.51$ per million Btu. All the price revisions cause proportional revisions to expenditures. The 1974-83 revisions cause insignificant adjustments in the U.S. average prices and expenditures. Changes to the U.S. averages are more noticeable in the $1984-88$ revisions.

Transportation Sector. Correction of data entry errors causes prices for residual fuel used for transportation to increase in Connecticut by 11 percent in 1970-72, to decrease in North Carolina by 56 percent in 1970, to decrease in New Hampshire by 97 percent in 1979, and to decrease in Oregon by i 8 percent in 1972 . Correction of calculation errors causes 1975 prices to be revised 
Changes described above are reflected in total refinery fuel (including intermediate products) and process fuel consumption. The revisions are generally less than 0.5 percent with the exception of refinery fuel consumption in 1986-88. Refinery fuel consumption changes are less than 5 percent for most States and are nearly the same percentage in each year, 1986-88, due to the reallocation of pentanes plus. The largest changes occur in Maryland and Nebraska, which previously had no estimated refinery fuel consumption, and in Rhode Island where consumption estimates are revised to zero. In all 3 years, refinery fuel consumption decreases in Connecticut by 36 percent, in lowa by 55 percent, and in New Hampshire by 22 percent, and increases in North Carolina by 13 percent. 


\title{
Appendix A
}

\author{
Additional \\ Measures of \\ Energy \\ Consumption, \\ Expenditures, and \\ Prices
}




\section{Additional Measures of Energy Consumption, Expenditures, and Prices}

The annual editions of the Energy Information Administration (EIA) State Energy Price and Expenditure Report (SEPER) provide detailed est:mates of energy prices and expenditures, with expenditures given in nominal dollars. Energy expenditures are derived as the product of energy prices and energy consumption with the latter adjusted to exclude energy used or lost by energy industries. The fully adjusted measure of energy consumption is designated as net energy consumption and represents the energy sold to end users of energy in the domestic economy. The energy price and net energy consumption data underlying the current dollar energy expenditure estimates can also be used to derive three additional energy related measures: energy expenditures in constant 1982 dollars, an implicit energy price deflator, and a fixed-weight energy price index. These measures complement, but are not intended to replace, existing EIA data series.

In this section, the methodology used to derive the three additional energy-related measures is presented, and the additional measures are used to examine changes in energy consumption, expenditures, and prices throughout the 1970-89 period. For purposes of analysis, information is presented for three shorter periods, as well as for the 20-year period, 1970-89. The first period, 1970-73, was chosen to allow analysis of energy use and its relation to Gross National Product (GNP) over a base period, before the sharp price increases that began in the last months of 1973 . The second period, 1973-81, includes the years of rapid price increases, which led to efforts to conserve energy and increase energy efficiency. The third period, 1981-89, was selected to see whether the decline in real energy prices after 1981 resulted in a moderation of efforts to increase energy efficiency, relative to the earlier periods.

\section{Methodology}

\section{Net Energy Consumption}

The net energy consumption data ${ }^{4}$ used in SEPER differ from the total energy consumption data currently published by EIA. The total (gross) consumption data include all energy sources before deduction of intermediate energy used or lost by energy industries in producing, processing, converting, generating, and distributing energy to end users. The net consumption data exclude certain categories of energy use, as outlined below.

The major category of energy used or lost by energy industries is the losses incurred in the generation, transmission, and distribution of utility electricity. Additional important losses include a power plant's own use of electricity, unaccounted-for electric system energy losses, fuels and purchased electricity consumed by petroleum refineries, natural gas lease and plant fuel, natural gas used as pipeline fuel, and crude oil consumed as lease, plant, and pipeline fuel.

In addition to the exclusion of energy used or lost, two other adjustments are made to the total energy consumption data: industrial hydroelectricity (used as a power source by the company generating the power, and not selling it) is excluded, and several intermediate petroleum products, which are used in the production of other petroleum products or reflect petroleum supply-consumption balance entries, are also excluded. The fully adjusted measure thus derived is designated "net energy consumption" (Table A1). Table A6 in this appendix shows the reconciliation of total (gross) and net energy consumption data.

\footnotetext{
${ }^{4}$ The detailed estimates of net energy, classified by energy form and major end-use sector, at both State and national levels, are included in

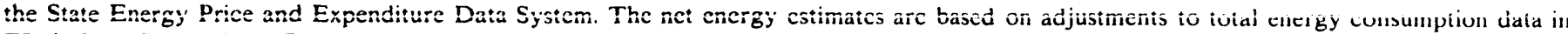
EIA's State Energy Data Report, Consumption Estimates, 1960-1989 DOE/EIA-0214(89) (Washington, DC, May 1991).
} 
A Constant-Dollar Energy Expenditures

The energy expenditures series presented in the Appendix is the constant-dollar counterpart to the nominal-dollar expenditure series published in the present report. ${ }^{5}$ The constant-dollar series is the product of fixed 1982 energy prices, at purchaser's value in dollars per million Btu, and net energy consumption in Btu for each year. Since it is derived by holding 1982 prices constant, it is comparable to the constantdollar GNP measures. In addition, the constant-dollar energy expenditure series has been used along with the constant-dollar GNP series to derive an expenditure per GNP index, which is a measure of the energy intensity of the economy (Table A2).

\section{Energy Price Measures}

The development of the constant-dollar energy expenditure series makes it possible to derive nominal and real implicit energy price deflators. The methodology used to derive the nominal implicit energy price deflator is comparable to the methodology used to derive the GNP implicit price deflator: the current-dollar expenditure series is divided by the constant-dollar energy expenditure series. The real implicit energy price deflator is then obtained by dividing the nominal price deflator by the GNP implicit price deflator.

Implicit price deflators, however, present a problem as indicators of changes in price because they also re. flect changes in product mix (except for direct comparisons between any given year and the base year, with the product mix of the given year held constant). Therefore, although they are convenient, their usefulness is limited. In order to compare price data for any year with price data for any other year, it is necessary to develop fixed-weight energy price indices. In concept, a fixed-weight price index excludes the effect of changes in product mix.

The methodology used to develop the fixed-weight energy' price indices presented in this section is comparable to that used to develop the GNP fixed-weight price index. The nominal fixed-weight energy price index is a measure of the average price of net energy consumption for each given year relative to the average price of net energy consumption in 1982, the weight base year. The composition of net energy consumption is held constant at 1982 weights for each year in the series. The 1982 weights consist of detailed energy source and end-use sector categories (cells) for each State and the relative distribution of net energy consumption among the various States.

Table A2. Energy Expenditures and

\begin{tabular}{c|cccc}
\hline Year & $\begin{array}{c}\text { Total } \\
\text { (trillion Btu) }\end{array}$ & $\begin{array}{c}\text { Net } \\
\text { (trillion Btu) }\end{array}$ & $\begin{array}{c}\text { Net/Total } \\
\text { (percent) }\end{array}$ \\
\hline $1970 \ldots$ & 66,334 & 50,138 & 75.6 \\
$1971 \ldots$ & 67,789 & 50,968 & 75.2 \\
$1972 \ldots$ & 71,275 & 53,273 & 74.7 \\
$1973 \ldots$ & 74,351 & 55,344 & 74.4 \\
$1974 \ldots$ & 72,528 & 53,409 & 73.6 \\
$1975 \ldots$ & 70,569 & 51,553 & 73.1 \\
$1976 \ldots$ & 74,392 & 54,276 & 73.0 \\
$1977 \ldots$ & 76,317 & 55,287 & 72.4 \\
$1978 \ldots$ & 78,158 & 56,419 & 72.2 \\
$1979 \ldots$ & 78,920 & 56,935 & 72.1 \\
$1980 \ldots$ & & & \\
$1981 \ldots$ & 75,985 & 54,140 & 71.3 \\
$1982 \ldots$ & 74,022 & 52,703 & 71.2 \\
$1983 \ldots$ & 70,806 & 49,827 & 70.4 \\
$1984 \ldots$ & 70,486 & 49,093 & 69.6 \\
& 74,042 & 51,847 & 70.0 \\
$1985 \ldots$ & 74,019 & & \\
$1986 \ldots$ & 74,232 & 51,694 & 69.8 \\
$1987 \ldots$ & 76,792 & 51,824 & 69.8 \\
$1988 \ldots$ & 80,247 & 53,363 & 69.5 \\
$1989 \ldots$ & 81,342 & 55,802 & 69.5 \\
& & 56,526 & 69.5 \\
\hline
\end{tabular}

Note: Calculations are based on unrounded data.

Sources: Total Consumption -- EIA, State Energy Data Report Consumption Estimates, 1960-1989. DOE/EIA-0214(89) (Washington, DC, May 1991), p. 23, "Total" (trillion Btu).

Tiet Consumption -. EIA, State Energy Price and Expenditure Data System 1989

Net/Total Consumption -- Net Btu divided by Total Btu.

\section{Expenditure/GNP Index, 1970-1989}

\begin{tabular}{cccc}
\hline Year & $\begin{array}{c}\text { Constant-Dollar } \\
\text { Expenditures } \\
\text { (million 1982 dollars) }\end{array}$ & $\begin{array}{c}\text { Constant-Dollar } \\
\text { Expenditures/GNP } \\
\text { Index (1982=100) }\end{array}$ \\
\hline $1970 \ldots$ & 378,389 & 116.7 \\
$1971 \ldots$ & 391,010 & 117.3 \\
$1972 \ldots$ & 413,788 & 118.2 \\
$1973 \ldots$ & 433,185 & 117.6 \\
$1974 \ldots$ & 420,225 & 114.7 \\
$1975 \ldots$ & 415,139 & 114.8 \\
$1976 \ldots$ & 439,201 & 115.8 \\
$1977 \ldots$ & 452,514 & 114.0 \\
$1978 \ldots$ & 464,944 & 111.2 \\
$1979 \ldots$ & 465,027 & 108.6 \\
$1980 \ldots$ & 446,545 & 104.4 \\
$1981 \ldots$ & 440,446 & 101.0 \\
$1982 \ldots$ & 424,808 & 100.0 \\
$1983 \ldots$ & 426,333 & 96.9 \\
$1984 \ldots$ & 446,609 & 95.1 \\
$1985 \ldots$ & 449,972 & 92.7 \\
$1986 \ldots$ & 457,036 & 91.6 \\
$1987 \ldots$ & 471,473 & 91.4 \\
$1988 \ldots$ & 490,993 & 91.1 \\
$1989 \ldots$ & 497,491 & 90.0 \\
& & \\
\hline
\end{tabular}

Note: Calculations are based on unrounded data

Sources: Expenditures -. EIA, State Energy Price and Expenditure Data System 1989.

Expenditures/GNP Index .. Expenditures/GNP, converted to index, $1982=100.0$. (GNP data appear in column 4 of Table A5.)

\footnotetext{
${ }^{5}$ Although the Appendix does not provide estimates at the State level, the national estimates of constant dollar energy expenditures are based on detailed energy consumption and price data for energy products, classified by end-use sector, for each State.
} 
The nominal fixed-weight energy price index is derived by (a) multiplying 1982 net energy consumption in Btu for each of the cells by the related end-use price for each year, (b) aggregating the hypothetical expenditures derived by this procedure to the individual State and national levels for each year, and (c) dividing the hypothetical expenditures for each year by the actual expenditures in 1982. The real fixed-weight energy price index is then obtained by dividing the nominal fixed-weight energy price index by the GNP fixedweight price index (Table A3).

Table A3. Fixed-Weight Energy Price Indices, 1970-1989 $(1982=100)$

\begin{tabular}{|c|c|c|}
\hline \multirow[b]{2}{*}{ Year } & \multicolumn{2}{|c|}{ Fixed-Weight Price Index } \\
\hline & Nominal & Real \\
\hline $\begin{array}{l}1970 \ldots \\
1971 \ldots \\
1972 \ldots \\
1973 \ldots \\
1974 \ldots\end{array}$ & $\begin{array}{l}22.9 \\
23.9 \\
24.6 \\
26.5 \\
36.8\end{array}$ & $\begin{array}{l}48.6 \\
49.1 \\
48.8 \\
50.0 \\
64.4\end{array}$ \\
\hline $\begin{array}{l}1975 \ldots \\
1976 \ldots \\
1977 \ldots \\
1978 \ldots \\
1979 \ldots\end{array}$ & $\begin{array}{l}41.6 \\
44.4 \\
49.0 \\
51.9 \\
64.1\end{array}$ & $\begin{array}{l}67.3 \\
68.2 \\
71.6 \\
71.3 \\
81.3\end{array}$ \\
\hline $\begin{array}{l}1980 \ldots \\
1981 \ldots \\
1982 \ldots \\
1983 \ldots \\
1984 \ldots\end{array}$ & $\begin{array}{r}83.8 \\
96.9 \\
100.0 \\
97.6 \\
97.2\end{array}$ & $\begin{array}{r}97.4 \\
103.0 \\
100.0 \\
93.8 \\
89.7\end{array}$ \\
\hline $\begin{array}{l}1985 \ldots \\
1986 \ldots \\
1987 \ldots \\
1988 \ldots \\
1989 \ldots\end{array}$ & $\begin{array}{l}97.0 \\
83.4 \\
83.5 \\
82.7 \\
87.3\end{array}$ & $\begin{array}{l}86.6 \\
72.6 \\
70.2 \\
66.7 \\
67.4\end{array}$ \\
\hline
\end{tabular}

Notes: Indices are based on 1982 quantity weights. Calculations are based on unrounded data.

Sources: Nominal Fixed-Weight Price Index -- Based on net energy consumption and end-use price data from EIA, State Energy Price and Expenditure Data System 1989

Real Fixed-Weight Price Index .. Nominal fixed-weight price index/GNP fixed-weight price index. (GNP data appear in column 6 of Table A5.)

In order to avoid some of the problems involved in interpreting the movement in the energy implicit deflator, because the measure reflects both price and product mix changes, only the fixed-weight energy price data are analyzed in this section. However, the implicit energy price deflators are presented in Table A5.

\section{Energy Consumption, Expenditures, and Prices}

\section{The Decline in the Net/Total Ratio}

Over time, the amount of energy used or lost by energy industries has accounted for an increasing proportion of total energy. In 1970, energy used or lost by energy industries amounted to about 24.4 percent of total energy consumed. By 1989, it had risen to 30.5 percent. Of course, as the proportion of energy used or lost rose, the ratio of net energy consumption--energy actually delivered to end users--to total energy consumption registered a concomitant decline (Table A1). The rate of decline in the ratio averaged about 0.4 percent per year for the $1970-89$ period.

\section{The Shift to Higher-Priced Products}

When the effect on constant-dollar energy expenditures of the decline in the proportion of energy actually delivered to end users is taken into account by basing energy expenditure and price series on net energy consumption, it becomes possible to examine changes in the product mix. The difference between the rate of increase in constant-dollar energy expenditures and that of net energy consumption is an implicit measure of the rate of change in the mix of products supplied to end users (Table A4).

When the rate of change in constant-dollar energy expenditures exceeds the rate of change in net energy consumption, the shift in product mix is towards higher-priced products. And, in fact, throughout the 1970-89 period, constant-dollar energy expenditures rose more rapidly--or fell more slowly--than net energy consumption. For the period as a whole, the product mix effect averaged 0.8 percent per year. But the product mix effect was not uniform over the period; it declined from 1.3 percent per year in $1970-73$ to 0.8 percent per year in 1973-81 and 0.7 percent per year in 1981-89.

An examination of changes in the shares of electricity and motor gasoline over the 20-year period illustrates the product mix effect. On a dollar-per-net-Btu basis, electricity and motor gasoline are relatively higherpriced sources of energy. (In 1982, the base year used in calculating constant-dollar energy expenditures, the prices per million net Btu of electricity and motor gasoline averaged $\$ 18.16$ and $\$ 10.39$, respectively. By comparison, the average price for all other energy sources was $\$ 5.52$ per million net Btu.) In 1970, electricity accounted for 9.3 percent of net energy consumption and motor gasoline accounted for 22.1 percent. By 1989, the shares of these higher-priced energy products had grown to 15.8 percent, and 24.9 percent, respectiveiy. 
Table A4. Rates of Change in Energy Expendltures and Consumption, 1970-1989 (Percent)

\begin{tabular}{l|c|c|c}
\hline Period & $\begin{array}{c}\text { Constant- } \\
\text { Dollar } \\
\text { Expenditures }\end{array}$ & $\begin{array}{c}\text { Net Energy } \\
\text { Consump- } \\
\text { tion }\end{array}$ & $\begin{array}{c}\text { Product } \\
\text { Mix } \\
\text { Effect }\end{array}$ \\
\hline $1970-73 \ldots$ & 4.6 & 3.3 & 1.3 \\
$1973-81 \ldots$ & 0.2 & -0.6 & 0.8 \\
$1981-89 \ldots$ & 1.5 & 0.9 & 0.7 \\
$1970-89 \ldots$ & 1.5 & 0.6 & 0.8 \\
\hline
\end{tabular}

'Constant-dollar expenditure rate minus net energy consumption rate. Product mix effect based on unrounded data.

Note: Rates of change are average annual rates.

Source: Based on data in Tables A1 and A2.

\section{The Decline in Energy Intensity}

A second analytical use of the constant-dollar energy expenditure series is an examination of changes in the energy intensity of the economy. The change in the ratio of constant-dollar energy expenditures to constant-dollar GNP is one measure of energy intensity.

By definition, if the energy intensity of the economy were constant, the rate of change in constant-dollar energy expenditures would be identical to the rate of change in GNP. During the $1970-89$ period, however, the rates of change differed. Furthermore, the change in the energy expenditure/GNP ratio was not uniform over the period. In 1970-73, the energy expenditure/ GNP ratio actually increased 0.3 percent per year (Figure $\mathrm{A} 1$ ).

In 1973-81, the decline in energy intensity, as measured by the energy expenditure/GNP ratio, averaged 1.9 percent. The decline is largely attributable to dramatic increases in real energy prices. Such increases, by promoting energy conservation and energy efficiency, would tend to reduce energy consumption, and hence constant-dollar expenditures, relative to growth in the economy.
Figure A1. Rates of Change in Constant-Dollar Energy Expenditure/GNP Index, 1970-1989

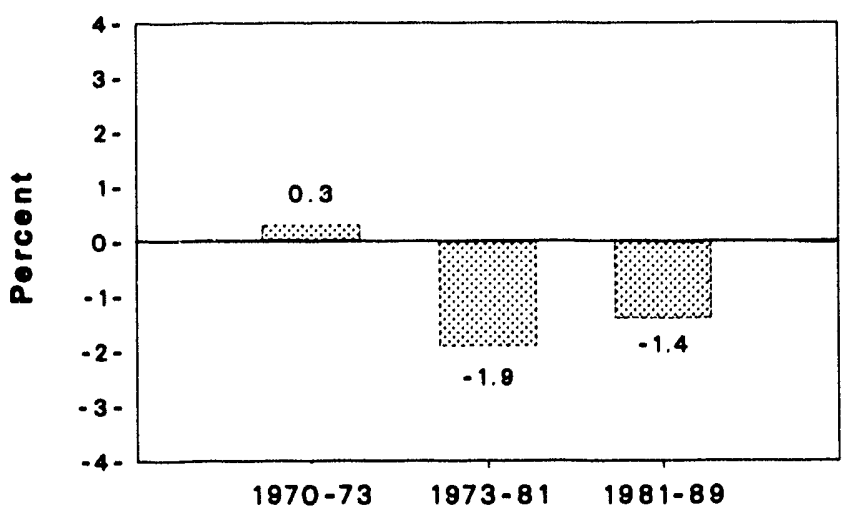

Note: Rates of change ere average annual retes of change.

Source: Based on data in Table A2.

In 1981-89, the energy intensity continued to decline, averaging 1.4 percent per year, despite a substantial decline in the real price of energy. The price decline might have been expected to lead to an increase in energy expenditures relative to the growth in the economy. This apparent anomaly--the continuation of the decline in energy intensity although at a slower rate, despite the price decline--can be explained by the continuing replacement of machinery, vehicles, appliances, structures, and other energy-consuming units with more efficient units. As the proportion of more efficient units rose, average energy efficiency increased. For example, U.S. passenger car efficiency increased faster in 1981-89 than in 1973-81. From 1973 to 1981, the average number of miles traveled per gallon of fuel consumed rose from 13.30 to 15.94 , an average annual increase of 2.3 percent. From 1981 to 1989 , the number rose from 15.94 to 20.54 , an average annual increase of 3.2 percent. $^{6}$

\section{Changes in Energy Prices}

For the 1970-89 period as a whole, the fixed-weight energy price index, in nominal terms, registered a sizeable increase of 7.3 percent per year. That average, however, masked significant variations between periods. In 1970-73, prices increased at the average annual rate of 5.0 percent, in nominal terms (Figure A2). In 1973-81, the rate jumped to 17.6 percent as world events--notably the Arab oil embargo of 1973-74 and the Iranian crisis in 1979--boosted the price of oil. In 
1981-89, energy prices declined an average of 1.3 percent per year, in nominal terms.

However, removing the effects of general inflation results in much lower rates of increase for the first two periods and substantially higher rates of decline for the period after 1981. The 5.0-percent-per-year increase in nominal terms in 1970-73 is an increase of only 0.9 percent per year in real terms. Similarly, the increase in 1973-81 is reduced from 17.6 percent per year, in nominal terms, to somewhat more than half that rate, 9.5 percent, in real terms.

\section{Figure A2. Rates of Change in Fixed-Weight Energy Price Indices, 1970-1989}
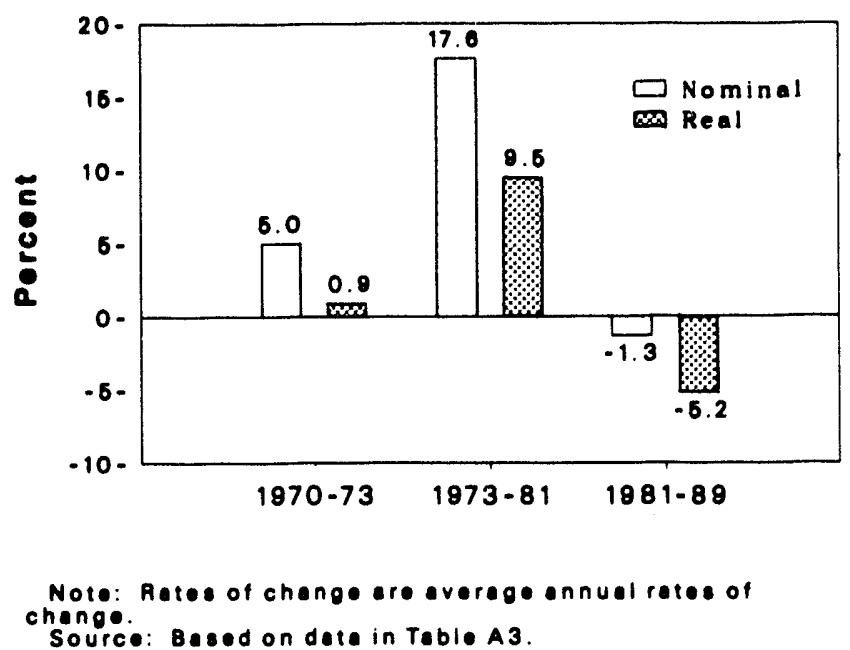

In the period after 1981 , the rate of decline in real terms is much larger than the nominal rate of decline, due to continued increases in the GNP fixed-weight price index (although the rates of increase in the GNP measure were reduced substantially). The decline in nominal energy prices of 1.3 percent per year in 1981-89 is a 5.2-percent-per-year reduction in real terms. Considering only the nominal rates of change in energy prices conceals the magnitude of the post-1981 decline. By 1989, the average price of energy, in real terms, was about 35 percent above the level of real energy prices in 1973, prior to the period of dramatic increase in energy prices. This represents a sharp reduction from the level reached in 1981 when real energy prices were more than double the 1973 level.

\section{Summary of Major Findings}

This appendix presents three additional measures of energy, as well as examples of the kinds of analysis that the series makes possible. The major analytical findings of this appendix are as follows:

- The proportion of total energy consumption actually delivered to end users declined throughout 1970-89, from 75.6 percent to 69.5 percent.

- During the 20-year period, there was a shift towards higher-priced energy products in the mix of products delivered to end users.

- The energy intensity of the economy declined in response to the sharp increase in real energy prices in 1973-81 and the decline continued in 1981-89, despite a sharp decrease in real prices after 1981. The apparent anomaly is explained in large part by the effect of replacement of energy-consuming stock with more energy efficient units.

- Real energy prices increased dramatically between 1973 and 1981 , at a rate of 9.5 percent per year, with the level of real energy prices more than doubling over the period. A major part of the increase was offset, however, by the decline in real energy prices in the following period, at a rate of 5.2 percent per year, so that the level of real energy prices in 1989 was reduced to 35 percent above the 1973 level. 
Table A5. Energy and GNP Measures, 1970-1989

\begin{tabular}{|c|c|c|c|c|c|c|}
\hline \multirow[b]{2}{*}{ Year } & \multicolumn{3}{|c|}{ Energy Measures } & \multicolumn{3}{|c|}{ GNP Measures } \\
\hline & $\begin{array}{l}\text { Current-Dollar } \\
\text { Expenditures } \\
\text { (million dollars) }\end{array}$ & $\begin{array}{l}\text { Nominal Implicit } \\
\text { Price Deflator } \\
(1982=100) \\
\end{array}$ & $\begin{array}{c}\text { Real Implicit } \\
\text { Price Deflator } \\
(1982=100) \\
\end{array}$ & $\begin{array}{c}\text { GNP } \\
\text { (billion } \\
\text { constant } \\
1982 \text { dollars) } \\
\end{array}$ & $\begin{array}{c}\text { GNP } \\
\text { Implicit Price } \\
\text { Deflator } \\
(1982=100) \\
\end{array}$ & $\begin{array}{l}\text { GNP } \\
\text { Fixed-Weight } \\
\text { Price Index } \\
(1982=100)\end{array}$ \\
\hline 1970. & 82,579 & 21.8 & 52.0 & $2,416.2$ & 42.0 & 47.2 \\
\hline $1971 \ldots$ & 89,781 & 23.0 & 51.7 & $2,484.8$ & 44.4 & 48.8 \\
\hline $1972 \ldots$ & 97,802 & 23.6 & 50.8 & $2,608.5$ & 46.5 & 50.3 \\
\hline 1973. & 111,616 & 25.8 & 52.1 & 2.744 .1 & 49.5 & 53.1 \\
\hline 1974. & 153,077 & 36.4 & 67.5 & $2,729.3$ & 54.0 & 57.2 \\
\hline 1975. & 171,784 & 41.4 & 69.8 & $2,695.0$ & 59.3 & 61.8 \\
\hline 1976. & 193,661 & 44.1 & 69.9 & $2,826.7$ & 63.1 & 65.1 \\
\hline 1977. & 220,221 & 48.7 & 72.3 & $2,958.6$ & 67.3 & 68.4 \\
\hline $1978 \ldots$ & 238,877 & 51.4 & 71.2 & $3,115.2$ & 72.2 & 72.7 \\
\hline $1979 \ldots$ & 296,991 & 63.9 & 81.3 & $3,192.4$ & 78.6 & 78.8 \\
\hline 1980 & 373,901 & 83.7 & 97.7 & $3,187.1$ & 85.7 & 86.1 \\
\hline 1981 & 426,447 & 96.8 & 103.0 & $3,248.8$ & 94.0 & 94.1 \\
\hline 1982 & 424,808 & 100.0 & 100.0 & $3,166.0$ & 100.0 & 100.0 \\
\hline 1983 & 415,749 & 97.5 & 93.9 & $3,279.1$ & 103.9 & 104.1 \\
\hline 1984 & 433,584 & 97.1 & 90.1 & $3,501.4$ & 107.7 & 108.3 \\
\hline $1985 \ldots$ & 435,901 & 96.9 & 87.4 & $3,618.7$ & 110.9 & 111.9 \\
\hline 1986. & 381,490 & 83.5 & 73.3 & $3,717.9$ & 113.8 & 114.9 \\
\hline 1987 & 393,838 & 83.5 & 71.2 & $3,845.3$ & 117.4 & 118.9 \\
\hline 1988. & 407,749 & 83.0 & 68.5 & $4,016.9$ & 121.3 & 123.9 \\
\hline 1989. & 436,643 & 87.8 & 69.5 & $4,117.7$ & 126.3 & 129.5 \\
\hline
\end{tabular}

Sources: Current-Dollar Energy Expenditures--EIA, State Energy Price and Expenditure Data System 1989.

Nominal Energy Implicit Price Deflator--Current-dollar expenditures/constant-dollar expenditures (column 1, Table A2).

Real Energy Implicit Price Deflator--Nominal energy implicit price deflator/GNP implicit price deflator. The calculation is based on the unrounded nominal energy implicit price deflator sipries.

GNP.- Economic Report of the President (Washington. DC, February 1991), p. 288, Table B-2, "Gross national product in 1982 dollars, 1929-1990," col. I.

GNP Implicit Price Deflator--Same source as above, p. 290, Table B-3, "Implicit price deflators for gross national product, 1929-1990," col. 1.

GNP Fixed-Weight Price Index--Same source as above, p. 292, Table B-4, "Fixed-weighted price indexes for gross national product, 1982 weights, 1959-1990," col. 1. 
Table A6. Total (Gross) - Net Energy Balance, 1970-1989 (Trillion Btu)

\begin{tabular}{|c|c|c|c|c|c|c|c|c|c|}
\hline Year & $\begin{array}{c}\text { Total } \\
\text { (Gross) }\end{array}$ & Net & $\begin{array}{l}\text { Electric } \\
\text { Utility } \\
\text { Losses }\end{array}$ & $\begin{array}{c}\text { Refinery } \\
\text { Use }\end{array}$ & $\begin{array}{c}\text { Crude } \\
\text { Oil } \\
\end{array}$ & $\begin{array}{c}\text { Natural } \\
\text { Gas } \\
\text { Lease } \\
\text { and Plant } \\
\end{array}$ & $\begin{array}{c}\text { Natural } \\
\text { Gas } \\
\text { Pipeline }\end{array}$ & $\begin{array}{l}\text { Indus- } \\
\text { trial } \\
\text { Hydro- } \\
\text { electric }\end{array}$ & $\begin{array}{l}\text { Total } \\
\text { Loss }\end{array}$ \\
\hline 1970 & 66,334 & 50,138 & 11,517 & 2,464 & - & 1,442 & 740 & 34 & 16,197 \\
\hline $1971 \ldots$ & 67.789 & 50,968 & 12,127 & 2,442 & - & 1,456 & 761 & 34 & 16,821 \\
\hline $1972 \ldots$ & 71.275 & 53,273 & 13,110 & 2,576 & $\cdot$ & 1,497 & 786 & 34 & 18,003 \\
\hline 1973 & 74,351 & 55,344 & 13,999 & 2,690 & - & 1,539 & 745 & 35 & 19,007 \\
\hline $1974 \ldots$ & 72,528 & 53,409 & 14,198 & 2,683 & $\cdot$ & 1,520 & 684 & 33 & 19,119 \\
\hline 1975 & 70,569 & 51,553 & 14,384 & 2,571 & - & 1,434 & 595 & 32 & 19,017 \\
\hline $1976 \ldots$ & 74,392 & 54,276 & 15,255 & 2,590 & - & 1,679 & 559 & 33 & 20,116 \\
\hline 1977 & 76,317 & 55,287 & 16,060 & 2,687 & - & 1,706 & 544 & 33 & 21,030 \\
\hline $1978 \ldots$ & 78,158 & 56,419 & 16,850 & 2,623 & - & 1,694 & 541 & 32 & 21,740 \\
\hline $1979 \ldots$ & 78,920 & 56,935 & 17,063 & 2.742 & $\cdot$ & 1,534 & 613 & 34 & 21,985 \\
\hline $1980 \ldots$ & 75,985 & 54,140 & 17,387 & 2,718 & $\cdot$ & 1,058 & 650 & 33 & 21,845 \\
\hline 1981 & 74,022 & 52,703 & 17,464 & 2,204 & - & 959 & 660 & 33 & 21,320 \\
\hline $1982 \ldots$ & 70,806 & 49,827 & 17,100 & 2,089 & - & 1,144 & 614 & 33 & 20,979 \\
\hline 1983 & 70,486 & 49,093 & 17,583 & 2,122 & 140 & 1,010 & 505 & 33 & 21,394 \\
\hline $1984 \ldots$ & 74,042 & 51,847 & 18,115 & 2,255 & 134 & 1,113 & 545 & 33 & 22,195 \\
\hline 1985 & 74,019 & 51,694 & 18,596 & 2,046 & 127 & 1,001 & 521 & 33 & 22,324 \\
\hline 1986 & 74.232 & 51,824 & 18,532 & 2,286 & 103 & 954 & 501 & 32 & 22,409 \\
\hline 1987 & 76,792 & 53,363 & 19,107 & 2,485 & 72 & 1.194 & 538 & 32 & 23,429 \\
\hline 1988 & 80,247 & 55,802 & 19,864 & 2,696 & 85 & 1,134 & 633 & 32 & 24,445 \\
\hline $1989 \ldots$ & 81,342 & 56,526 & 20,260 & 2,711 & 59 & 1,104 & 650 & 33 & 24,816 \\
\hline
\end{tabular}

Note: Totals may not equal sum of components due to independent rounding.

Sources: Total (Gross)--EIA, State Energy Data Report, Consumption Estimates, 1960-1989, DOE/EIA-0214(89) (Washington, DC, May 1991), p. 23, col. titled, "Total" (Trillion Btu).

Net--EIA, State Energy Price and Expenditure Data System, 1989.

Electric Utility Losses--EIA, State Energy Data Report. Consumption Estimates, 1960-1989, pp. 18-21, sum of four end-use sectors', "Electrical System Energy Losses" (Trillion Btu).

Refinery Use--EIA, State Energy Price and Expenditure Data System, 1989

Crude Oil Lease, Plant, and Pipeline Use--EIA, State Energy Price and Expenditure Data System, 1989.

Natural Gas Lease and Plant--EIA, State Energy Price and Expenditure Data System, 1989.

Natural Gas Pipeline--EIA, State Energy Data Report. Consumption Estimates, 1960-1989, p. 27, col. titled, "Natural Gas" (Trillion Btu)

Industrial Hydroelectric--EIA, State Energy Data Report, Consumption Estimates, 1960-1989, p. 26, col. titled, "Industrial Hydropower" (Trillion Btu)

Total Loss--Sum of Electric Utility Losses, Refinery Use, Crude Oil as Lease, Plant, and Pipeline Fuel, Natural Gas Lease and Plant, Natural Gas Pipeline, and Industrial Hydroelectric. 


\section{Glossary}

Asphalt: A dark-brown-to-black cement-like material containing bitumens as the predominant constituents obtained by petroleum processing. The definition includes crude asphalt as well as the following finished products: cements, fluxes, the asphalt content of emulsions (exclusive of water), and petroleum distillates blended with asphalt to make cutback asphalts.

ASTM: The American Society for Testing and Materials.

Aviation Gasoline Blending Components: Naphthas that are used for blending or compounding into finished aviation gasoline (e.g., straight-run gasoline, alkylate, and reformate). Excluded are oxygenates (alcohols and ethers), butane, and pentanes plus.

Aviation Gasoline: All special grades of gasoline for use in aviation reciprocating engines, as given in ASTM Specification D910 and Military Specification MILG-5572. Aviation gasoline includes blending components.

Barrel (petroleum): A unit of volume equal to 42 U.S. gallons.

British Thermal Unit (Btu): The quantity of heat needed to raise the temperature of 1 pound of water by $1^{\circ} \mathrm{F}$ at or near $39.2^{\circ} \mathrm{F}$. See Heat Content of a Quantity of Fuel, Gross and Heat Content of a Quantity of Fuel, Net.

Butane: A normally gaseous straight-chain or branched-chain hydrocarbon $\left(\mathrm{C}_{4} \mathrm{H}_{10}\right)$. It is extracted from natural gas or refinery gas streams. It includes isobutane and normal butane and is designated in ASTM Specification D1835 and Gas Processors Association Specifications for commercial butane.

- Isobutane: A normally gaseous branched-chain hydrocarbon. It is a colorless paraffinic gas that boils at a temperature of $10.9^{\circ} \mathrm{F}$. It is extracted from natural gas or refinery gas streams.

- Normal Butane: A normally gaseous straightchain hydrocarbon. It is a colorless paraffinic gas that boils at a temperature of $31.1^{\circ} \mathrm{F}$. It is extracted from natural gas or refinery gas streams.

Butylene: An olefinic hydrocarbon $\left(\mathrm{C}_{4} H_{8}\right)$ recovered from refinery processes.
Coal: Includes all ranks of coal--anthracite, bituminous coal, subbituminous coal, and lignite--conforming to ASTM Specification D388.

- Coking coal: Coal that meets the requirements for making coal coke. It must be low in ash and sulfur and form a coke that is capable of supporting the charge of iron ore and limestone in a blast furnace. Coking coal is usually a blend of two or more bituminous coais.

- Steam coal: In this report, steam coal represents all noncoking coal.

Coal Coke: A hard, porous product made from baking bituminous coal in ovens at temperatures as high as $2,000^{\circ} \mathrm{F}$. It is used both as a fuel and as a reducing agent in smelting iron ore in a blast furnace.

Coke Plants: Plants where coal is carbonized in slot or beehive ovens for the manufacture of coke.

\section{Coking Coal: See Coal.}

Commercial Sector: The commercial sector, as defined economically, consists of business establishments that are not engaged in transportation or in manufacturing or other types of industrial activity (agriculture, mining, or construction). Commercial establishments include hotels, motels, restaurants, wholesale businesses, retail stores, laundries, and other service enterprises; religious and nonprofit organizations; health, social, and educational institutions; and Federal, State, and local governments. Street lights, pumps, bridges, and public services are also included if the establishment operating them is considered commercial. SIC codes used to classify an establishment as commercial are 50 through 87, 89, and 91 through 97.

Conversion Factor: A number that translates units of one system into corresponding values of another system. Conversion factors can be used to translate physical units of measure for various fuels into Btu equivalents.

Crude Oil Used Directly: Crude oil consumed as fuel by crude oil pipelines and on crude oil leases.

Cubic Foot (natural gas): A unit of volume equal to 1 cubic foot at a pressure base of 14.73 pounds standard per square inch absolute and a temperature base of $60^{\circ} \mathrm{F}$. 
Degree-Day Normals: Simple arithmetic averages of monthly or annual degree-days over a long period of time (usually the 30-year period 1951-1980). The averages may be simple degree-day normals or populationweighted degree-day normals.

Degree-Days, Cooling (CDD): The number of degrees per day that the daily average temperature is above $65^{\circ} \mathrm{F}$. The daily average temperature is the mean of the maximum and minimum temperatures for a 24 -hour period.

Degree-Days, Heating (HDD): The number of degrees per day that the daily average temperature is below $65^{\circ} \mathrm{F}$. The daily average temperature is the mean of the maximum and minimum temperatures for a 24 -hour period.

Degree-Days, Population-Weighted: Heating or cooling degree-days weighted by the population of the area in which the degree-days are recorded. To compute State population-weighted degree-days, each State is divided into from one to nine climatically homogeneous divisions, which are assigned weights based on the ratio of the population of the division to the total population of the State. Degree-day readings for each division are multiplied by the corresponding population weight for each division and those products are then summed to arrive at the State population-weighted degree-day figure. To compute national population-weighted degreedays, the Nation is divided into nine Census regions comprised of from three to eight States, which are assigned weights based on the ratio of the population of the region to the total population of the Nation. Degree-day readings for each region are multiplied by the corresponding population weight for each region and those products are then summed to arrive at the national population-weighted degree-day figure.

Diesel Fuel: Fuel used for internal combustion in diesel engines; usually that fraction of crude oil that distills after kerosene. (See Distillate Fuel Oil.)

Distillate Fuel Oil: A general classification for one of the petroleum fractions produced in conventional distillation operations. Included are products known as No. 1, No. 2, and No. 4 fuel oils and No. 1, No. 2, and No. 4 diesel fuels. It is used primarily for space heating, on- and off-highway diesel engine fuel (including railroad engine fuel and fuel for agricultural machinery), and electric power generation.

Electrical System Energy Losses: The amount of energy lost during generation, transmission, and distribution of electricity, including plant and unaccounted-for uses.

Electricity Generation: The process of producing electric energy or transforming other forms of energy into electric energy. Also the amount of electric energy produced or expressed in watthours (Wh).
Electricity Sales: The amount of kilowatthours sold in a given period of time; usually grouped by classes of service, such as residential, commercial, industrial, and other. "Other" sales include sales for public street and highway lighting and other sales to public authorities, sales to railroads and railways, and interdepartmental sales.

Electric Power Plant: A station containing prime movers, electric generators, and auxiliary equipment for converting mechanical, chemical, and/or fission energy into electric energy.

Electric Utility Sector: Privately and publicly owned establishments that generate electricity primarily for use by the public.

End-Use Sectors: The residential, commercial, industrial, and transportation sectors of the econoniy.

Energy: The capacity for doing work as measured by the capability of doing work (potential energy) or the conversion of this capability to motion (kinetic energy). Energy has several forms, some of which are easily convertible and can be changed to another form useful for work. Most of the world's convertible energy comes from fossil fuels that are burned to produce heat that is then used as a transfer medium to mechanical or other means in order to accomplish tasks. Electrical energy is usually measured in kilowatthours, while heat energy is usually measured in British thermal units.

Ethane: A normally gaseous straight-chain hydrocarbon $\left(C_{2} H_{6}\right)$. It is a colorless, paraffinic gas that boils at a temperature of $-127.48^{\circ} \mathrm{F}$. It is extracted from natural gas and refinery gas streams.

Ethylene: An olefinic hydrocarbon $\left(\mathrm{C}_{2} \mathrm{H}_{4}\right)$ recovered from refinery processes or petrochemical processes.

Expenditures: In this report, "expenditures" refers to the money directly spent by consumers to purchase energy. Expendit_. ss equal the amount of energy used by the consumer times the price per unit paid by the consumer. In calculating the amount of energy used, process fuel and intermediate products are not included.

Exports: Shipments of goods from the 50 States and the District of Columbia to foreign couritries and to Puerto Rico, the Virgin Islands, and other U.S. possessions and territories.

Federal Energy Regulatory Commission (FERC): The Federal agency with jurisdiction over interstate electricity sales, wholesale electric rates, hydroelectric licensing, natural gas pricing, oil pipeline rates, and gas pipeline certification. FERC is an independent regulatory agency within the Department of Energy and is the successor to the Federal Power Commission. 
Federal Power Commission (FPC): The predecessor agency of the Federal Energy Regulatory Commission. The Fideral Power Commission was created by an Act of Congress under the Federal Water Power Act on June 10, 1920. It was charged originally with regulating the eleciric power and natural gas industries. It was abolished on September 30, 1977, when the Department of Energy was created. Its functions were divided between the Department of Energy and the Federal Energy Regulatory Commission, an independent regulatory agency.

Fossil Fuel: Any naturally occurring organic fuei, such as petroleum, coal, and natural gas.

Gasonol: A blend of finished motor gasoline (leaded or unleaded) and alcohol (generally ethanol but sometimes methanol) limited to 10 percent by volume of alcohol. Gasohol is included in finished leaded and unleaded motor gasoline.

Geothermal Energy (as used at electric utilities): Hot water or steam extracted from geothermal reservoirs in the Earth's crust and supplied to steam turbines at electric utilities that drive generators to produce electricity.

Gross National Product (GNP): The total value of goods and services produced by the Nation's economy, before deduction of depreciation charges and other allowances for capital consumption. It includes the total purchases of goods and services by private consumers and government, gross private domestic capital investment, and net foreign trade.

Gross National Product (GNP) Implicit Price Deflator: The implicit rrice deflator, published by the U.S. Department of Commerce. Bureau of Economic Analysis. is used to convert nominal figures to real figures.

Heat Content of a Quantity of Fuel, Gross: The total amount of heat released when a fuel is burned. Coal, crude oil, and natural gas all include chemical compounds of carbon and hydrogen. When those fuels are burned, the carbon and hydrogen combine with oxygen in the air to produce carbon dioxide and water. Some of the energy released in burning goes into transforming the water into steam and is usually lost. The amount of heat spent in transforming the water into steam is counted as part of gross heat content but is not counted as part of net heat content. Also referred to as the higher heating value. Btu conversion factors typically used in EIA represent gross heat content.

Heat Content of a Quantity of Fuel, Net: The amount of useable heat energy released when a fuel is burned under conditions similar to those in which it is normally used. Also reterred to as the lower heatıng value. Btu conversion factors typically used in EIA represent gross heat content.
Heavy Oil: The fuel oils remaining after the lighter oils have been distilled off during the refining process. Except for start-up and flame stabilization, virtually all petroleum used in steam-electric power plants is heavy oil.

Hydroelectric Power: The production of electricity from the kinetic energy of falling water.

Imports: Receipts of goods into the 50 States and the District of Columbia from foreign countries and from Puerto Rico, the Virgin Islands, and other U.S. possessions and territories.

Industrial Sector: The industrial sector comprises manufacturing industries, which make up the largest part of the sector, along with mining, construction, agriculture, fisheries, and forestry. Establishments in the sector range from steel mills, to small farms, to companies assembling electronic components. The SIC codes used to classify establishments as industrial are 1 through 39.

Jet Fuel: The term includes kerosene-type jet fuel and naphtha-type jet fuel. Kerosene-type jet fuel is a kerosene-quality product used primarily for commercial turbojet and turboprop aircraft engines. Naphthatype jet fuel is a fuel in the heavy naphthas range used primarily for military turbojet and turboprop aircraft engines.

Kerosene: A petroleum distillate that has a maximum distillation temperature of $401^{\circ} \mathrm{F}$ at the 10 -percent recovery point, a final boiling point of $572^{\circ} \mathrm{F}$, and a minimum flash point of $100^{\circ} \mathrm{F}$. Included are the two grades designated in ASTM D3699 (No. 1-K and No. $2-\mathrm{K}$ ) and all grades of kerosene called range or stove oil. Kerosene is used in space heaters, cook stoves, and water heaters; it is suitable for use as an illuminant when burned in wick lamps.

Lease and Plint Fuel: Natural gas used in well, field, and lease ope.ations (such as gas used in drilling operations, heat ers. dehydrators, and field compressors), and as fuel in natural gas processing plants.

Light Oil: Lighter fuel oils distilled off during the refining process. Virtually all petroleum used in internal combustion and gas-turbine engines is ligitt oil.

Liquefied Petroleum Gases (LPG): Ethane, ethylene, propane, propylene, normal butane, butylene, and isobutane produced at refineries or natural gas processing plants. including plants that fractionate new natural gas plant liquids.

Lubricants: Substances used to reduce friction between bearing surfaces or as process materials either incorporated into other materials used as processing aids in the manufacturing of other prociucis or as carriers of other materials. Petroleum lubricants may be produced either from distillates or residues. Other substances 
may be added to impart or improve certain required properties. Excluded are byproducts of lubricating oil refining, such as aromatic extracts derived from solvent extraction or tars derived from deasphalting. Included are all grades of lubricating oils from spindle oil to cylinder oil and those used in greases. Lubricants categories are paraffinic and naphthenic.

Miscellaneous Petroleum Products: All finished petroleum products not classified elsewhere--for example, petrolatum, lube refining byproducts (aromatic extracts and tars), absorption oils, ram-jet fuel, petroleum rocket fuels, synthetic natural gas feedstocks, and specialty oils.

Motor Gasoline: A complex mixture of relatively volatile nydrocarbons, with or without small quantities of additives, obtained by blending appropriate refinery streams to form a fuel suitable for use in spark-ignition engines. Motor gasoline includes both leaded and unleaded grades of finished motor gasoline, blending components, and gasohol.

Motor Gasoline Blending Components: Naphthas that will be used for blending or compounding into finished motor gasoline (e.g., straight-run gasoline, alkylate, and reformate). Excluded are oxygenates (alcohols and ethers), butane, and pentanes plus.

Natural Gas: A mixture of hydrocarbons (principally methane) and small quantities of various nonhydrocarbons existing in the gaseous phase or in solution with crude oil in underground reservoirs.

Natural Gasoline: A mixture of hydrocarbons, mostly pentanes and heavier, extracted from natural gas that meets specifications for natural gasoline set by the Gas Processors Association. Includes isopentane.

Nominal Price: The price paid for a product or service at the time of the transaction.

Nuclear Electric Power: Electricity generated by an electric power plant whose turbines are driven by steam generated in a reactor by heat from the fissioning of nuclear fuel.

Nuclear Electric Power Plant: A single-unit or multiunit facility in which heat produced in one or more reactors by the fissioning of nuclear fuel is used to drive one or more steam turbines.

Nuclear Fuel: Fissionable materials that have been enriched to a composition that. when placed in a nuclear reactor, will support a self-sustaining fission chain reaction, producing heat in a controlled manner for process use.

Pentanes Plus: A mixture of hydrocarbons, mostly pentanes and heavier, extracted from natura! gas. Includes isopentane. natural gasoline, and plant condensate.
Petrochemical Feedstocks: Chemical feedstocks derived from petroleum principally for the manufacture of chemicals, synthetic rubber, and a variety of plastics. The categories reported are naphthas less than $401^{\circ} \mathrm{F}$ endpoint and other oils equal to or greater than $401^{\circ} \mathrm{F}$ endpoint.

Petroleum: A generic term applied to oil and oil products in all forms, such as crude oil, lease condensate, unfinished oils, petroleum products, natural gas plant liquids, and nonhydrocarbon compounds blended into finished petroleum products.

Petroleum Coke: A residue that is the final product of the condensation process in cracking. The product is either marketable petroleum coke or catalyst petroleum coke.

Petroleum Coke, Catalyst: The carbonaceous residue that is deposited on and deactivates the catalyst used in many catalytic operations (e.g., catalytic cracking). Carbon is deposited on the catalyst, thus deactivating the catalyst. The catalyst is reactivated by burning of $\mathrm{i}^{\circ}$ the carbon, which is used as a fuel in the refining process. That carbon or coke is not recoverable in a concentrated form.

Petroleum Coke, Marketable: Those grades of coke produced in delayed or fluid cokers that may be recovered as relatively pure carbon. Marketable petroleum coke may be sold as is or further purified by calcining.

Petroleum Products: Products obtained from the processing of crude oil (including lease condensate), natural gas, and other hydrocarbon compounds. Petroleum products include unfinished oils, liquefied petroleum gases, pentanes plus, aviation gasoline, motor gasoline, naphtha-type jet fuel, kerosene-type jet fuel, kerosene, distillate fuel oil, residual fuel oil, petrochemical feedstocks, special naphthas, lubricants, waxes, petroleum coke, asphalt, road oil, still gas, and miscellaneous products.

Photovoltaic and Solar Thermal Energy (as used at electric utilities): Energy radiated by the sun as electromagnetic waves (electromagnetic radiation) that is converted at electric utilities into electricity by means of solar (photovoltaic) cells or concentrating (focusing) collectors.

Plant Condensate: One of the natural gas liquids, mostly pentanes and heavier hydrocarbons, recovered and separated as liquids at gas inlet separators or scrubbers in processing plants.

Primary Energy: All energy consumed by end users, excluding electricity but including the energy consumed at electric utilities to generate electricity. (In estimating encrgy expenditures, there are no fuelassociated expenditures for hydroelectric power, geothermal energy, solar energy, or wind energy, and 
the quantifiable expenditures for process fuel and intermediate products are excluded.)

Process Fuel: All energy consumed in the acquisition, processing, and transportation of energy. Quantifiable process fuel includes three categories: natural gas lease and plant operations, natural gas pipeline operations, and oil refinery operations.

Propane: A normally gaseous straight-chain hydrocarbon $\left(C_{3} H_{8}\right)$. It is a colorless paraffinic gas that boils at a temperature of $-43.67^{\circ} \mathrm{F}$. It is extracted from natural gas or refinery gas streams. It includes all products designated in ASTM Specification D1835 and Gas Processors Association Specifications for commercial propane and HD-5 propane.

Propylene: An olefinic hydrocarbon $\left(\mathrm{C}_{3} \mathrm{H}_{6}\right)$ recovered from refinery or petrochemical processes.

Real Price: A price that has been adjusted to remove the effect of changes in the purchasing power of the dollar. A real price usually reflects buying power relative to a base year.

Refinery (petroleum): An installation that manufactures finished petroleum products from crude oil, unfinished oils, natural gas liquids, other hydrocarbons, and alcohol.

Residential Sector: The residential sector is considered to consist of all private residences, whether occupied or vacant, owned or rented, including single-family homes, multifamily housing units, and mobile homes. Secondary homes, such as stimmer homes, are also included. Institutional housing, such as school dormitories, hospitals, and military barracks generally are not included in the residential sector; they are included in the commercial sector. The SIC code used to classify an establishment as residential is 88 (Household).

Residual Fuel Oil: The heavier oils that remain after the distillate fuel oils and lighter hydrocarbons are distilled away in refinery operations and that conform to ASTM Specifications D396 and 975. Included are No. 5 , a residual fuel oil of medium viscosity; Navy Special, for use in steam-powered vessels in government service and in shore power plants; and No. 6, which includes Bunker $\mathrm{C}$ fuel oil and is used for commercial and industrial heating, electricity generation, and to power ships. Imports of residual fuel oil include imported crude oil burned as fuel.

Road Oil: Any heavy petroleum oil, including residual asphaltic oil, used as a dust palliative and surface treatment on roads and highways. It is generally produced in six grades, from 0 , the most liquid, to 5 , the most viscous.

Short Ton (coal): A unit of weight equal to 2,000 pounds.
SIC: See Standard Industrial Classification.

Special Naphthas: All finished products within the naphtha boiling range that are used as paint thinners, cleaners, or solvents. Those products are refined to a specified flash point. Special naphthas include all commercial hexane and cleaning solvents conforming to ASTM Specifications D1836 and D484, respectively. Naphthas to be blended or marketed as motor gasoline or aviation gasoline, or that are to be used as petrochemical and synthetic natural gas (SNG) feedstocks, are excluded.

Standard Industrial Classification (SIC): A set of codes developed by the Office of Management and Budget which categorizes industries into groups with similar economic activities.

Steam Coal: See Coal.

Still Gas (refinery gas): Any form or mixture of gas produced in refineries by distillation, cracking, reforming, and other processes. The principal constituents, are methane, ethane, ethylene, normal butane, butylene, propane, and propylene. It is used primarily as refinery fuel and petrochemical feedstock.

Total Energy: All energy consumed by end users including electricity but excluding the energy consumed at electric utilities to generate electricity. (In estimating energy expenditures, there are no fuel-associated expenditures for hydroelectric power, geothermal energy, solar power, or wind energy, and the quantifiable expenditures for process fuel and intermediate products are excluded.)

Transportation Sector: Private and public "ehicles that move people and commodities. Included are automobiles, trucks, buses, motorcycles, railroads and railways (including streetcars), aircraft, ships, barges, and natural gas pipelines. The SIC codes used to classify establishments as belonging to the transportation sector are 40 through 49

Unfinished Oils: All oils requiring further refinery processing, except those requiring only mechanical blending. Included are naphthas and lighter oils, kerosene and light gas oils, heavy gas oils, and residuum.

Unfractionated Streams: Mixtures of unsegregated natural gas liquid components, excluding those in plant condensate. This product is extracted from natural gas.

United States: Unless otherwise noted, "United States" in this publication means the 50 States and the District of Columbia. U.S. exports include shipments to U.S. territories, and imports include receipts from U.S. territories.

Waxes: Solid or semisolid materials derived from petroleum distillates or residues. Waxes are light-colored, more or less translucent crystalline masses, slightly 


\title{
Order Form
}

\section{State Energy Data Report, Consumption Estimates, 1960-1989}

\author{
Published: May 1991 \\ Energy Information Administration \\ DOE/EIA-0214(89) \\ Price per copy: $\$ 23.00$
}

The State Energy Data Report, Consumption Estimates, 1960-1989 presents annual energy consumption estimates for the 50 States, the District of Columbia, and the United States. The estimates are provided by type of energy (refined petroleum products, natural gas, coal, and electricity) and by major consuming sectors (residential, commercial, industrial, transportation, and electric utilities) in physical units and in British thermal units. The 475-page report includes technical documentation describing the data sources and estimation procedures used.

Company or Personal Name:

Additional Address/Attention Line:

Street Address:

City, State, ZIP Code:

Daytime Phone Number (area code first):

***** Please include payment with this order form. Allow 2 weeks for delivery.

Number of copies $x \$ 23.00=\$$ (total due). (Foreign orders add 25\%.)

$\square$ Check payable to Superintendent of Documents

$\square$ Money order payable to Superintendent of Documents

$\square$ Charge to Deposit Account No. Order No.

$\square$ Charge to: $\square$ VISA $\square$ MasterCard $\square$ Choice Number

Signature Expiration Date (Month/Year)

Address inquiries and mail order form to: National Energy Information Center; Energy Information Administration; Room IF-048; Washington, DC 20585. (Telephone, 202-586-8800; TDD, 202-586-1181; Hours, 8 a.m.-5 p.m., eastern time, M-F.) 


\section{Order Form}

\section{State Energy Price and Expenditure Report 1989}

Published: September 1991

Energy Information Administration

DOE/EIA-0376(89)

Price per copy: $\$ 14.00$

The State Energy Price and Expenditure Report 1989 presents energy price and expenditure estimates for the 50 States, the District of Columbia, and the United States for 1970, 1975, 1980, and 1985 through 1989. The estimates are provided by energy source (petroleum, natural gas, coal, and electricity) and by major consuming sector (residential, commercial, industrial, transportation, and electric utilities). The 270-page report includes technical documentation describing the data sources and estimation procedures used.

Company or Personal Name:

Additional Address/Attention Line:

Street Address:

City, State, ZIP Code:

Daytime Phone Number (area code first):

***** Please include payment with this order form. Allow 2 weeks for delivery.

Number of copies $x \$ 14.00=\$$ (total due). (Foreign orders add 25\%.)

$\square$ Check payable to Superintendent of Documents

$\square$ Money order payable to Superintendent of Documents

$\square$ Charge to Deposit Account No. _ _ _ _ _ _ Order No.

$\square$ Charge to: $\square$ VISA $\square$ MasterCard $\square$ Choice Number

Signature Expiration Date (Month/Year) 1

Address inquiries and mail order form to: National Energy Information Center; Energy Information Administration; Room 1F-048; Washington, DC 20585. (Telephone, 202-586-8800; TDD, 202-586-1181; Hours, 8 a.m.-5 p.m., eastern time, $M-F$.) 


\section{Other EIA Multifuel Historical Energy Data Reports}

The State Energy Data Report (DOE/EIA-0214) presents estimates of annual energy consumption at the State and national levels by major sector (i.e., residential, commercial, industrial, transportation, and electric utilities) and by principal energy type for 1960 forward. The report includes documentation of the consumption estimates for each source of energy, the sources of all data, and a summary of changes made to historical data in the report since its previous release.

The Monthly Energy Review (DOE/EIA-0035) presents current monthly data on production, consumption, stocks, imports, exports, and prices of the principal energy commodities in the United States. Also included are data on international production of crude oil, consumption of petroleum products, petroleum stocks, and production of electricity from nuclear-powered facilities.

The Historical Monthly Energy Review (DOE/EIA-0035(73-88)) presents monthly data from January 1973 through December 1988 for most of the series that are published for current months only in the Monthly Energy Review.

The Annual Energy Review (DOE/EIA-0384) presents long-term historical annual energy data. Most series begin in 1949. U.S. energy consumption, production, trade, and prices are included. Major sections of the report are energy overview, consumption indicators, financial indicators, energy resources, petroleum, natural gas, coal, electricity, nuclear energy, renewable energy, and international energy.

The International Energy Annual (DOE/EIA-0219) presents annual data for production, consumption, imports, and exports of primary energy commodities in more than 190 countries, dependencies, and areas of special sovereignty. Also included are prices of crude oil and petroleum products in selected countries. The data presented are derived largely from national publications, international organizations, and other authoritative sources. The data are converted to units of measurement and thermal values familiar to the American public.

The International Petroleum Statistics Report (DOE/EIA-0520) presents current monthly international petroleum data on production, consumption, imports, and stocks. Included are oil consumption and stocks for specific countries in the Organization for Economic Cooperation and Development (OECD). Also provided are the oil supply/consumption balances for the world in quarterly intervals and oil imports by OECD countries.

For further information, contact the:

National Energy Information Center, EI-231

Energy Information Administration

1000 Independence Avenue, S.W.

Washington, DC 20585

202-586-8800 (TDD 202-586-1181)

Hours: 8 a.m. -5 p.m., eastern time, M-F 

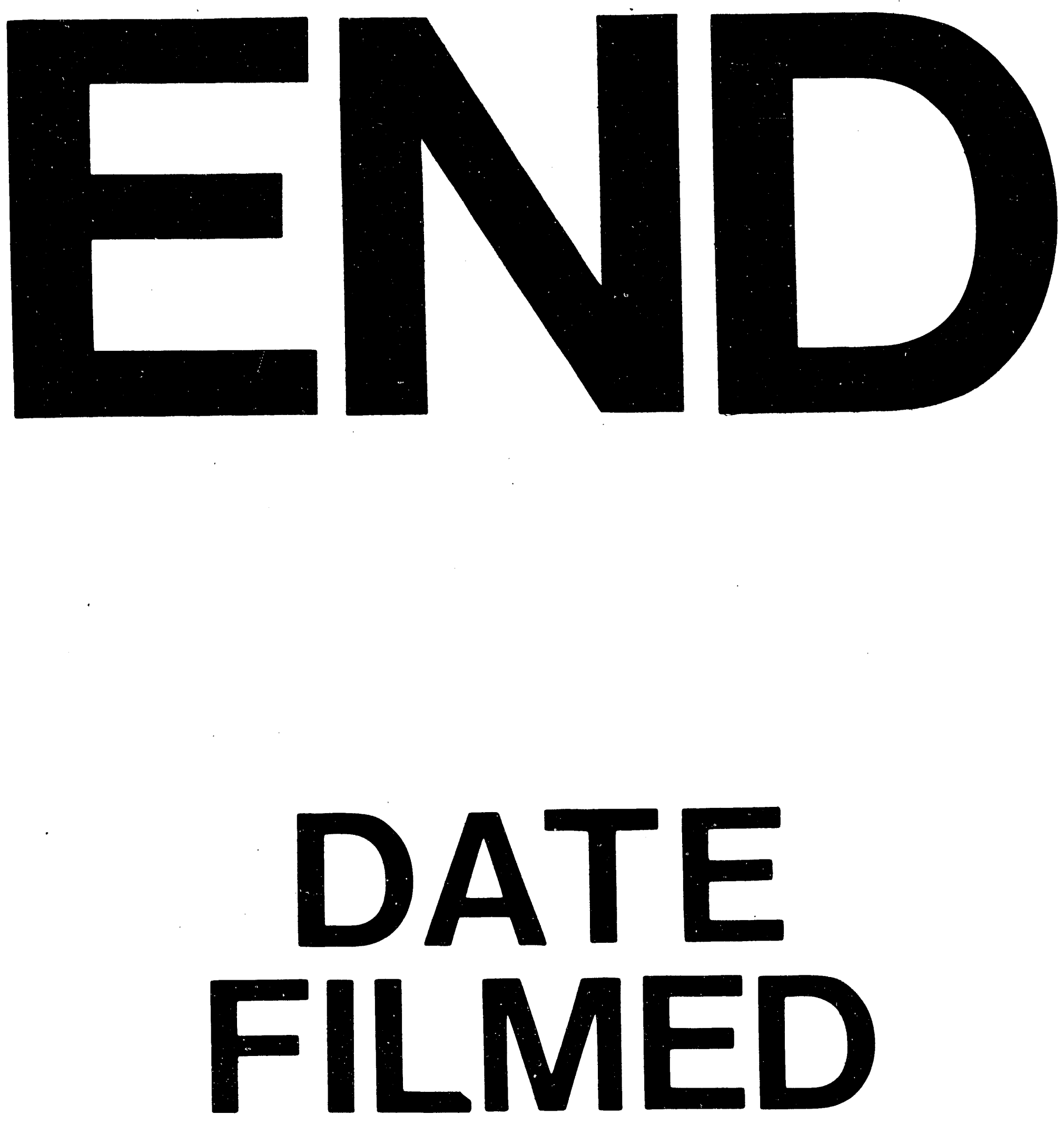

i

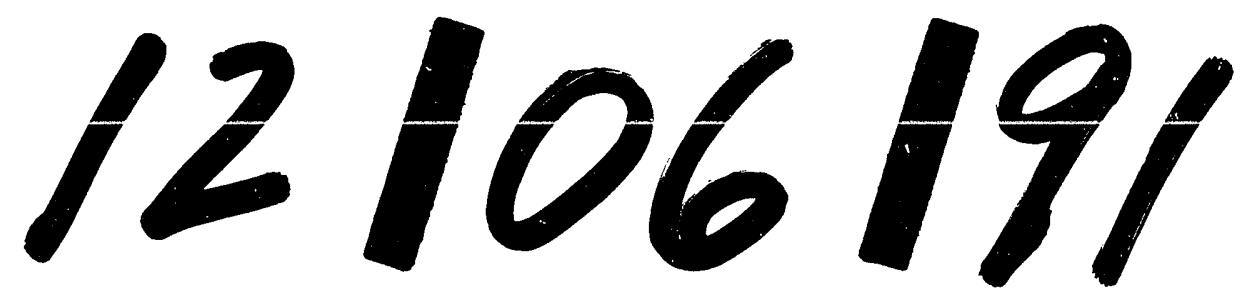


